
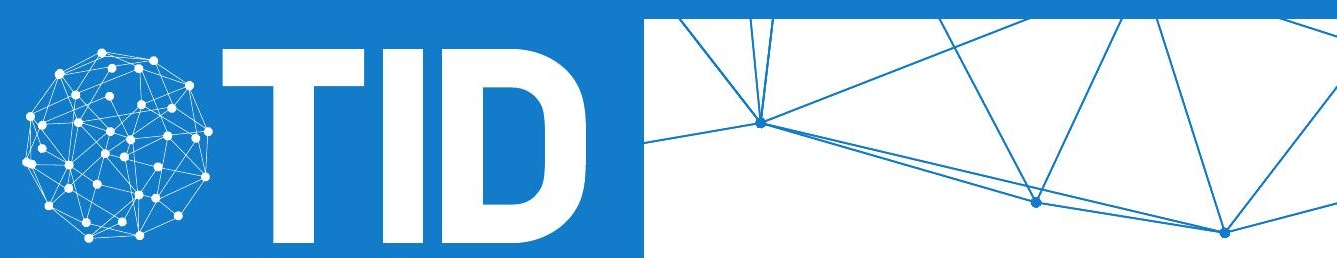

Tobacco Induced Diseases

\title{
Abstract Book
}

\section{TH WORLD CONFERENCE ON TOBACCO OR HEALTH}

7-9 MARCI 2018, CAPE TOWN, SOUTH AFRICA 


\section{Aims and Scope}

Tobacco Induced Diseases encompasses all aspects of research related to the prevention and control of tobacco use at a global level. Preventing diseases attributable to tobacco is only one aspect of the journal, whose overall scope is to provide a forum for the publication of research articles that can contribute to reducing the burden of tobacco induced diseases globally. To address this epidemic we believe that there must be an avenue for the publication of research/ policy activities on tobacco control initiatives that may be very important at a regional and national level. This approach provides a very important "hands on" service to the tobacco control community at a global scale - as common problems have common solutions. Hence, we see ourselves as "connectors" within this global community.

The journal hence encourages the submission of articles from all medical, biological and psychosocial disciplines, ranging from medical and dental clinicians, through health professionals to basic biomedical and clinical scientists.

Full Journal Title:

Tobacco Induced Diseases

\section{Abbreviated Title:}

Tob. Induc. Dis.

ISSN (electronic): $1617-9625$

Publishing model:

Open Access

\section{Society:}

The International Society for the Prevention of Tobacco Induced Diseases

Publisher:

EU European Publishing

Editors:

See Editorial Board Page

Publisher Address:

Science and Technological Park of Crete, Greece

\section{Peer Review:}

Double Blind

Impact factor:

Licenses:

CC-BY

Publication Frequency:

Continuous

\section{Publication Medium:}

Electronic Only

Publication website:

http://www.tobaccoinduceddiseases.org/ 


\section{Editorial Board}

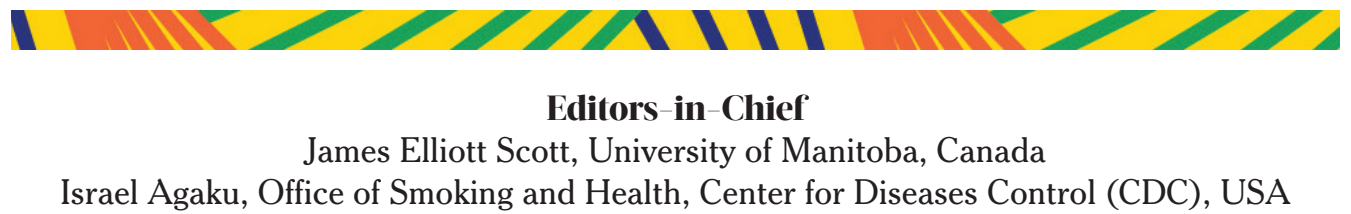

\section{Associate Editors}

Taru H Kinnunen, Harvard University, USA

Xing Li Wang, Baylor College of Medicine, USA

Athanasios Zavras, Boston University, USA

Parimal Chowdhury, University of Arkansas for Medical Sciences, USA

David Scott, University of Louisville, USA

\section{Editorial Board}

Olalekan Ayo-Yusuf, Sefako Makgatho Health Sciences University, South Africa

Sophia S Chan, The University of Hong Kong, Hong Kong

Gregory Connolly, Northeastern University, USA

Elif Dagli, Turkish Thoracic Society, Turkey

Silvio De Flora, University of Genoa, Italy

Kim DaeHyun, Keimyung University Dongsan Medical Center, South Korea

Filippos Filippidis, Imperial College London, UK

Geoffrey Fong, University of Waterloo, Canada

Erika S Froelicher, University of California San Francisco, USA

Stanton Glantz, University of California, USA

Giuseppe Gorini, Cancer Research \& Prevention Institute, Italy

Prakash C Gupta, Healis - Sekhsaria Institute of Public Health, India

Takashi Hanioka, Fukuoka Dental College, Japan

Wojciech Hanke, Nofer Institute of Occupational Medicine, Poland

Asgeir R Helgason, Karolinska Institute, Sweden

Christos Lionis, University of Crete, Greece

Maria Jose Lopez, Public Health Agency of Barcelona, Spain

Karl E Lund, Norwegian Institute for Alcohol and Drug Research, Norway

Toshitaka Nakahara, Kyoto University School of Medicine, Japan

Rima Nakkash, American University of Beirut, Lebanon

Evridiki Patelarou, Kings College London, UK

Kinga Polanska, Nofer Institute of Occupational Medicine, Poland

Lars Ramstrom, Institute for Tobacco Studies, Sweden

Hana Ross, University of Cape Town, South Africa

Kazunari Satomura, Kyoto University, Japan

Steve Sussman, University of Southern California, USA

Tai Hing Tai Lam, University of Hong Kong, Hong Kong

Witold Zatonski, The Maria Sklodowsska-Curie Memorial Cancer Center, Poland

Strategic Development Editor

Constantine Vardavas, Strategic Development Editor

\section{Publications Management}

Chryssi Igoumenaki, Publications Manager

Katerina Papathanasaki, Editorial Manager 


\section{Abstract Book \\ 17TI WORLD CONFERENCE ON TOBACCO OR HEALTH \\ 7-9 MARCII 2018, CAPE TOWN, SOUTH AFRICA}

www.wctoh.org

Disclaimer: This supplement has been created within the context of a memorandum of understanding between "EUEP European Publishing" and "The Union". Responsibility for the scientific content of the supplement is allocated to the Scientific Committee of the WCTOH. All authors are responsible for the content of their abstracts and retain copyright of their abstract under an Open Access, Creative Commons License (CC-BY-4.0). Each abstract is citable and identifiable through its individual Digital Object Identifier (DOI). 


\section{Table of Contents}

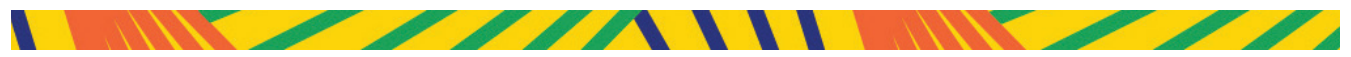

\section{Wednesday, 7 March 2018}

\subsection{Priorities and challenges for advancing the WHO FCTC}

PS-500-1 A quantitative analysis of the 2015 WHO MPOWER report

PS-501-1 Attitude towards plain packaging of tobacco products among

the adult population in Bangladesh: a mixed method approach.

PS-502-1 Policy progress of health warnings on tobacco products as per Article 11 of WHO FCTC: a global analysis........................ 1 PS-503-1 Global Policy progress in Article 16 of WHO FCTC on cigarettes (CIG) and smokeless tobacco (SLT) ............................. 1 PS-504-1 Awareness and implementation of cigarette and other tobacco products act in selected communities in Northern India .......2 PS-505-1 Are strong tobacco control policies associated with bans on the retail sales of electronic nicotine delivery systems?............ 2 PS-506-1 Global policy progress in Article 20 of World Health Organization's

Framework Convention on Tobacco Control (WHO FCTC) on Smokeless Tobacco (SLT) 3

\subsection{Integrating tobacco control into Iealth systems, global firameworks and strategies to reach WHO FCTC and SDG's targets.}

SUS The United Nations and its agencies, and their relationship with tobacco industry - an analysis of positions, policy and practice .......... 3

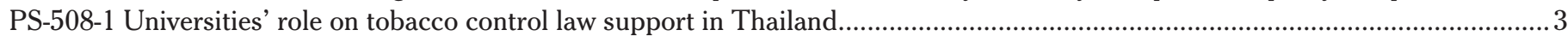

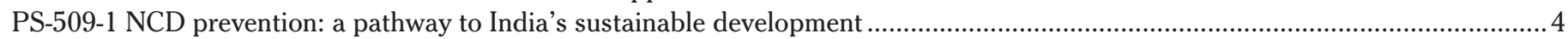
PS-510-1 Assessment of 2016 Taiwan tobacco control performance based on WHO MPOWER guidelines ....................................... 4 PS-511-1 Empowering librarians on effective tobacco demand reduction through public libraries in Sri Lanka ................................. 4 PS-512-1 Upgrade needed: South African tobacco control policy and the WHO-framework convention on tobacco control .................5 PS-513-1 Prevalence, perceptions and predictors of smokeless tobacco use in a rural community in South Eastern Nigeria .................5 PS-514-1 Tobacco control and sustainable development goals in Brazil..... PS-515-1 Effectiveness of tobacco cessation intervention among patients visiting primary care settings in India: a quasi-experimental study

\subsection{Using data: Opportunities and gaps in measuring WHO FCTC impact on global health and development}

PS-516-1 Usage of big data for global tobacco control: exploring the trends in quantitative research from 2006 to 2015 .................... 6 PS-517-1 Willingness to quit tobacco smoking and its correlates among Indian smokers - findings from GATS India, 2009-10........... 7 PS-518-1 The Data on Alcohol and Tobacco in Africa (DATA) project..... PS-519-1 Impact of tobacco control policies on smoking prevalence and quit ratios in 27 European Union countries from $2006-2014$

PS-520-1 Trends in tobacco use among Indian adults 1998 - 99 to 2015 - 16: results from nationally

representative data of the national family health surveys.....

\subsection{Setting the research agenda for translating science to policy and new tools for policy impact assessments}

SUS Cost-effectiveness and budget impact analysis of smoking cessation interventions in chronic obstructive pulmonary disease patients in Thailand.

PS-521-1 Restaurant smoking sections in South Africa and the perceived impact of the proposed smoke-free laws: evidence from a nationally representative survey .....

PS-522-1 Comparative jurisdictional analysis of municipal smoke-free policies in Canada .....

PS-523-1 Gaps in tobacco control research in accordance with WHO FCTC guidelines: a systematic literature review in Thailand ............ PS-524-1 Developing synthetic birth cohort-specific smoking histories using Global Adult Tobacco Surveys - an example from India ......... 10 PS-525-1 Chemical profiling of smokeless tobacco and their disease association.

\subsection{The End Game - Pathways to turning vision into reality.}

FO-335-1 Modelling how to achieve 5\% adult smoking prevalence by 2025: a regional approach ................................................. 10

FO-336-1 Achieving an end to government tobacco tax revenues: another challenge for the tobacco endgame? ............................... 11

FO-337-1 The tobacco reduction targets act: a legislated phase out for combustible cigarettes........................................................ 11

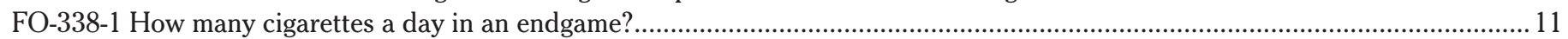

FO-339-1 Should we pay all smokers to quit smoking? A mixed methods survey exploring 
New Zealand smokers' perceptions of financial incentives

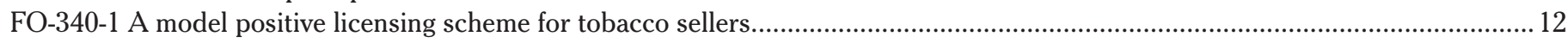

FO-341-1 How tobacco control advocates and organizational leaders in California view endgame policies ..................................... 12

PS-526-1 California policymakers' perspectives on tobacco endgame approaches ...................................................................... 13

PS-527-1 Ban on Gutka in India: symbolic Victory or actual end-game for smokeless tobacco.

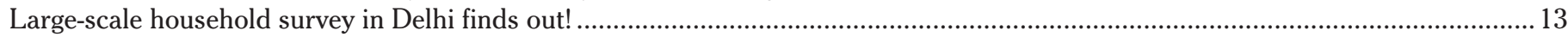

PS-528-1 Redesigning government regulations towards a tobacco endgame strategy: a comparative law approach ........................... 14

PS-529-1 Seeking patterns of countries banning sales of electronic nicotine delivery systems

at different stages of the tobacco epidemic: an exploratory analysis ...

PS-530-1 Baseline and Endline study helped administration to declare 8 districts compliant

to various sections of Indian Tobacco Control Act in a scientific manner .................................................................................. 14

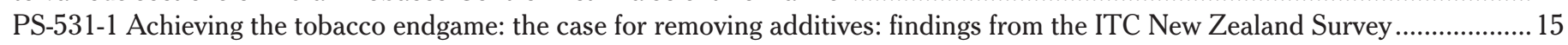

PS-532-1 Decreasing temptation: attitudes to tobacco sales by alcohol-licensed premises and policy implications ............................. 15

PS-533-1 The effect of tobacco control policies on smoking prevalence using

the SimSmoke Ireland model: influence of interventions 2010-2016 on reaching End Game 2025

PS-534-1 Regulating Shisha/ waterpipe smoking \& E-cigarettes by regulating nicotine as poison: a success story from India ............ 16

\subsection{Promoting transnational cooperation and Partnerships: models of Intergovernmental and nongovernmental collaboration across multiple sectors for high level commitments}

PS-535-2 Policy and advocay in tobacco control in Romania of the Romanian Society of Pneumology .......................................... 16 PS-536-2 Smoking habits and anti-smoking acts awareness among general public in Gurgaon, Haryana, India............................... 17 PS-537-2 Reconciling profits and pandemics? An analysis of attitudes to commercial sector engagement in health policy and research ..... 17 PS-538-2 Initiatives of Sirajganj district administration to tobacco control in Bangladesh .............................................................. 17 PS-539-2 Mobile app to identify gaps in implementation and provide evidences for effective policy change..................................... 18

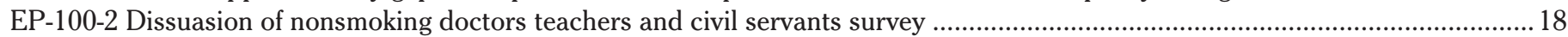
EP-101-2 Is ban on smokeless tobacco products effective in controlling the menace: a case study from Punjab, India....................... 18

\subsection{International tobacco trade and health diplomacy: understanding the nexus between the two and domestic international disputes}

SUS A "win-win situation overall": lessons from tobacco industry influence of the negotiation of the North American Free Trade Agreement .....

PS-540-2 Intellectual property arguments in tobacco industry legal challenges: lessons from recently concluded cases...

\subsection{Building leadership capacity and broader alliances for tobacco control policy advocacy}

PS-541-2 Second hand smoke "a threat to child's health and development"

PS-542-2 Sensitization of Ministries, Departments and Agencies (MDAs) on tobacco

control measures of the public health ACT 851 and WHO FCTC Article 5.3 in Ghana

PS-543-2 Story from Indonesia: the media advocacy strategy on restraining "The Tobacco Bill Draft" ...........................................21

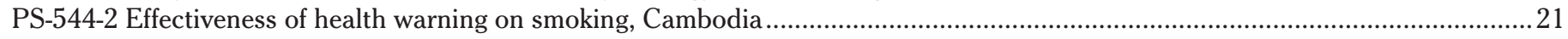

PS-545-2 Asia Pacific Mayors Alliance for Tobacco Control (AP-CAT): a breakthrough to gain political will ................................21

PS-546-2 To encounter the challenges of tobacco industry: concerted community effort to advocate for policy change......................22

PS-547-2 JHSPH scholar's program: strengthening the next generation of global tobacco control leaders ........................................22

PS-549-2 Balparishad - a health assembly of young leaders advocating for tobacco control around schools and in the communities...........22 PS-550-2 Capacity building for female community health workers - an effective tool for tobacco control and empowerment...............23 PS-551-2 Strengthening smokeless tobacco control in Myanmar: challenges and the way forward...................................................23

PS-552-2 Advocacy with police department for effective implementation of tobacco control polices ................................................24

PS-553-2 Advocacy by children to remove surrogate products advertisements on the public transport buses in Mumbai ..................24

PS-554-2 Evaluation of a training workshop on tobacco cessation: capacity building initiative in India .............................................24

PS-555-2 Efforts made for Tobacco Control (NTCP) implementation in all health institution,

Govt. offices and Districts Basti Tobacco Free State of Uttar Pradesh ..........................................................................................25

PS-556-2 Compliance COTPA 2003 and other supportive law for tobacco free district Kanpur Nagar in State of Uttar Pradesh .......25 PS-557-2 Brazilian Virtual Health Library of Prevention and Cancer Control: facebook fanpage and tobacco control.......................25 PS-558-2 Citizen Oversight Groups (Veedurías), a suitable instrument to advance

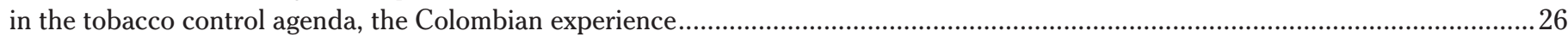

PS-559-2 True progress is tobacco-free campaign: engaging civil society to implement FCTC Article 5.3 in the Philippines..............26

\subsection{Tobacco industry interference: New tactics and counter measures}

FO-314-2 Building up echo chambers and framing messages: comparison of tobacco industry's think tank activities .......................27 FO-315-2 Influencing decision makers - model of interference of the tobacco industry in Brazil.....................................................27

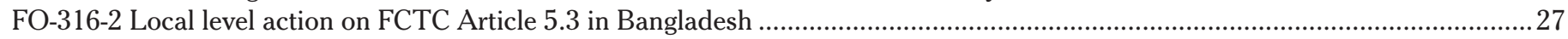

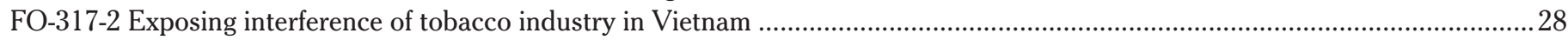

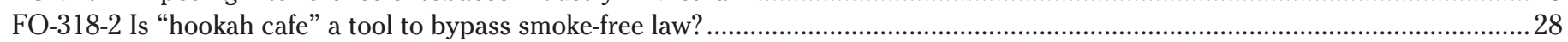


FO-319-2 Monitoring and exposing tobacco industry tactics in implementation of 85 percent pictorial health warnings . .28

FO-320-2 Analysis of media reports to understand tobacco industry tactics in undermining tobacco tax policies in Bangladesh.

PS-560-2 Effective implementation of Article 5.3 of the WHO framework convention

on tobacco control to curb tobacco industry interference...

PS-561-2 Emotional attachment to personal tobacco packages might affect quitting behaviour.

Results from a cross-sectional survey among tobacco users ......

PS-562-2 One unhealthy commodities industry? Understanding links across tobacco,

alcohol and ultra-processed food manufacturers and their implications for tobacco control and the SDGS .......................................30

PS-566-2 The filter fraud: debunking the myth of "Safer" as a key new strategy of tobacco control ..................................................30

PS-567-2 Use of electronic games as tobacco cross boarder advertisement ...................................................................................31

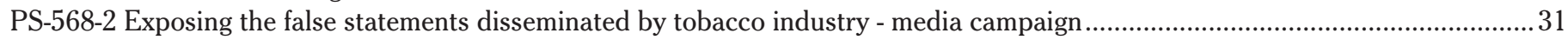

PS-569-2 Widespread violations of point-of-sale advertisements of tobacco products law around schools in India ........................... 31

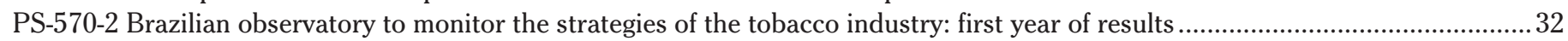

PS-572-2 Ongoing relationships between the tobacco industry and universities: an insidious obstacle to tobacco control................... 32

PS-573-2 Strategies and impacts of Japanese tobacco industry in shutting down

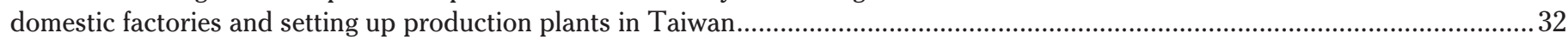

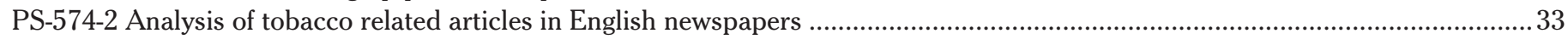

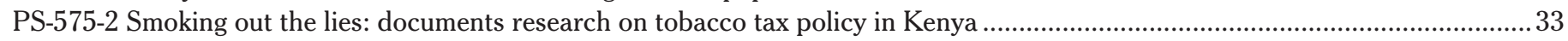

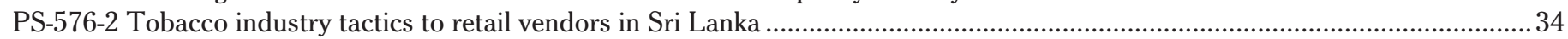

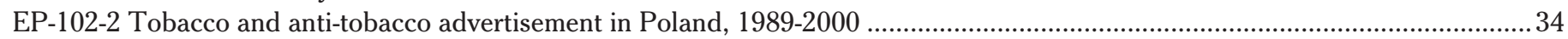

EP-103-2 Tobacco industry securing connections with political elites: a case study from Sri Lanka ..............................................34

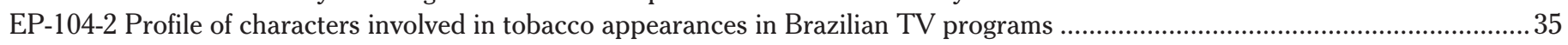

EP-105-2 Hostage to fortune: an empirical study of the tobacco industry's business strategies since the advent of E-cigarettes .......... 35

EP-106-2 Stopping Tobacco Industry Interference (TII): bottom-up approach to achieve national policy on FCTC Article 5.3 in India ....... 36

EP-107-2 Ending corporate giving to halt tobacco industry interference in the ASEAN region ......................................................3 36

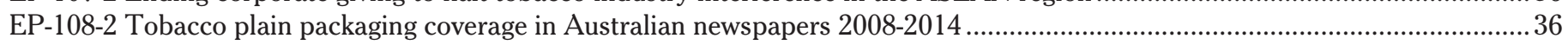

EP-109-2 Tobacco control law written by JTI: a case study of flagrant industry interference in Kyrgyzstan ......................................3 37

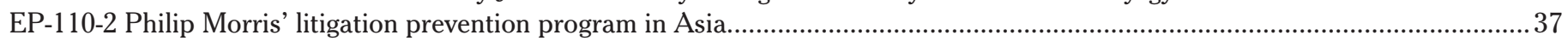

EP-111-2 Challenges in the implementation of the National Tobacco Control Law in Argentina ....................................................37

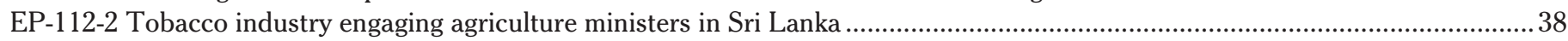

EP-113-2 Why do violations of FCTC Article 5.3 occur?: The case of tobacco industry incentives in Turkey ..................................38

\subsection{Implementing sustainable funding mechanisms for tobacco control}

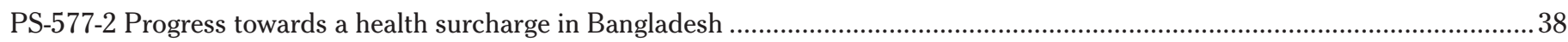

PS-578-2 Pooling local public health budgets to achieve exceptional value for money: the case of the North East of England ............39

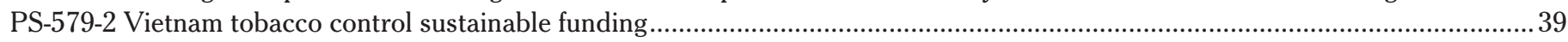

PS-580-2 Member states of the FCTC can generate self-sustaining funding by applying

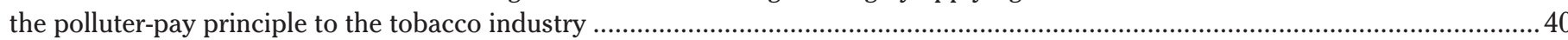

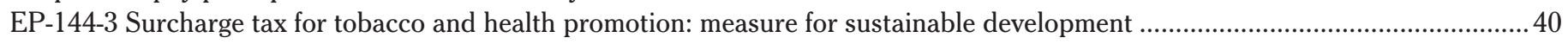

\subsection{Pricing, Taxation and countering illicit trade: Innovations and reform strategies}

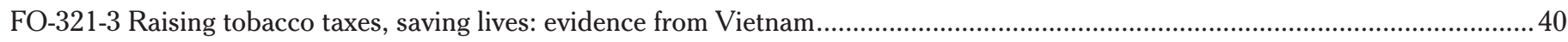

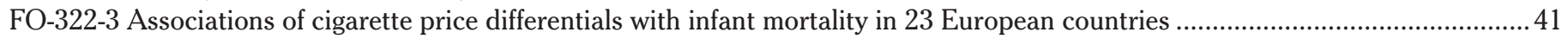

FO-323-3 Tobacco Tax Index 2017: assessing implementation of WHO FCTC Article 6 in ASEAN countries .......................................4 41

FO-324-3 The price sensitivity of choice and consumption of cigarette by brand categories

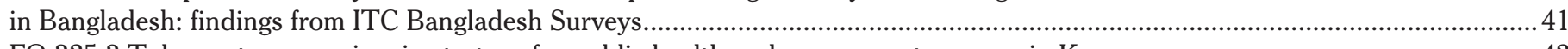

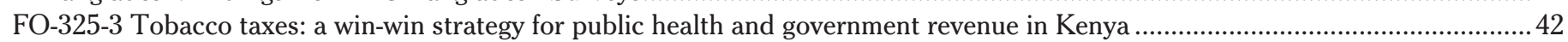

FO-342-3 Exposing industry-driven retail incentive programs that undermine tobacco tax policies .................................................42

FO-343-3 Understanding tobacco industry pricing strategy: evidence from China's 2015 Tax Reform ..............................................42

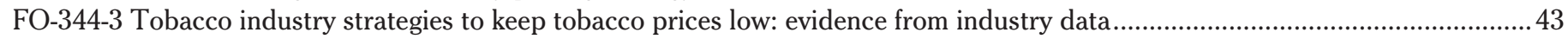

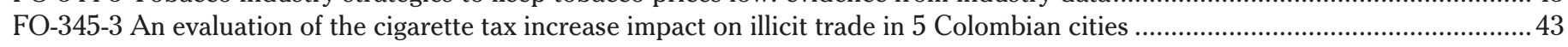

FO-346-3 Consumption of illicit cigarettes in India: findings from a retail survey .........................................................................4

FO-347-3 Converging combustible and electronic cigarette prices:

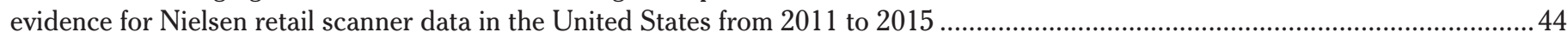

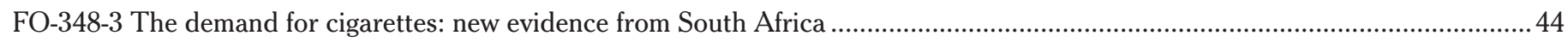

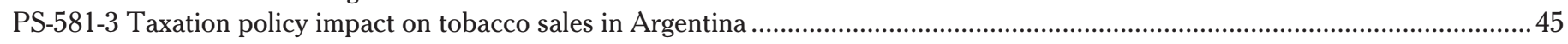

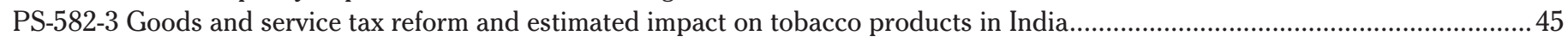

PS-583-3 Implementing a sub-national strategic framework to reduce the illicit tobacco market to support national strategies ........... 45

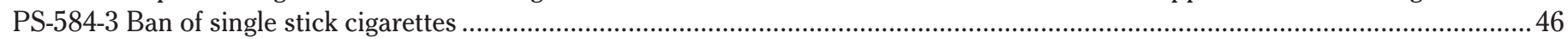

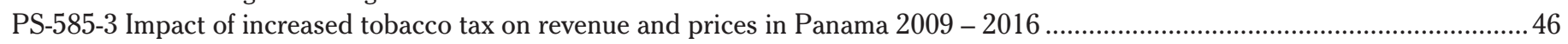

PS-586-3 Monitoring tobacco taxation in the Philippines at the TAP (Tobacco Action Patrol) of a finger........................................46

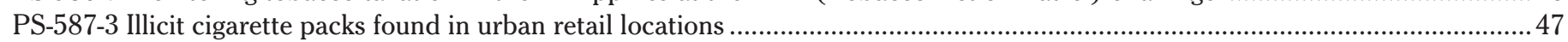

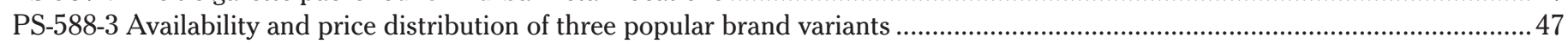

PS-589-3 The impact of a 2018 tax increase on illicit cigarette trade in Mongolia - baseline results ...................................................47 
PS-590-3 Assessing market competition in the Philippine cigarette industry.....

EP-130-3 Gender analysis on drivers of cigarette consumption in Kenya: two-part model approach..............................................48

EP-131-3 Implementation of the WHO FCTC article 6 - price and tax measures to reduce the demand for tobacco in Ghana ........... 49

EP-132-3 Measuring illicit cigarette consumption with tobacco surveillance data:

evidence from the Global Adult Tobacco Survey for Mexico .

EP-133-3 Public health and fiscal impacts of radical decrease of ad valorem excise share in the mixed tobacco excise system .......... 49

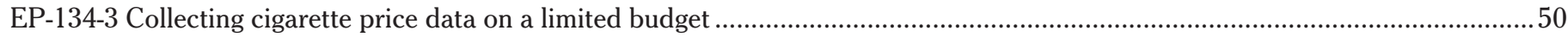

EP-135-3 \#OneTobaccoTax: social media campaign for unified tobacco taxes under Goods and Services Tax in India.....................50

EP-136-3 The counter productivity of a stagnant tobacco tax on national tobacco control efforts in Nigeria .....................................50

EP-137-3 Characterization of cigarrete smuggling distribution in Colombia ..................................................................................51

EP-138-3 Increase in tobacco tax does not lead to increase in illicit cigarette consumption

in Hong Kong: findings from a top-down approach against tobacco industry-funded results .......................................................5

EP-140-3 Implementing effective pricing and taxation measures for prevention and control of smokeless tobacco............................51

EP-141-3 Analyzing current and future levels of cigarette affordability under the Philippine Sin Tax Law (RA 10351) ...................52

EP-142-3 Social cost benefit analysis of tobacco control policies in the Netherlands ...................................................................52

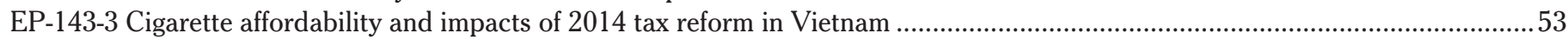

\subsection{Advances in protection from tobacco pollution, second- and third-hand smoke}

RF-1204-3 Exposure to secondhand smoke infiltration at home amongst Hong Kong residents: a population-based study ................53

RF-1205-3 Implementation of smoke free policy in restaurant setting - experience of a NGO in Vietnam ........................................53

RF-1206-3 Smoke free policy in bars and restaurants in Kampala: a cross sectional study .........................................................54

RF-1208-3 Advancing smoke-free public spaces: the challenge of water-pipe in the Canadian context ...........................................54

PS-591-3 Parental perceptions of exposure to tobacco smoke: a new tool to shed light on parental smoking behavior ........................54

PS-593-3 Exposure to second-hand smoke at work in the European Union, 2006 - 2014 ........................................................55

PS-594-3 Did local smoke free policy in Indonesia prevent youth from smoking?......................................................................55

PS-595-3 A cross-country comparison of secondhand smoke exposure in public places

among adults in five African countries - The Global Adult Tobacco Survey, 2012 - 2015 ...........................................................5 56

PS-596-3 Trends in SHS exposure and smokers' support for smoke-free laws in China:

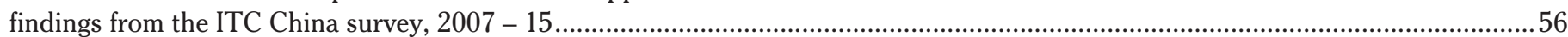

PS-597-3 Knowledge, attitude and practice regarding smoking among Police in Bangladesh .........................................................57

PS-598-3 Beliefs and rules about vaping in home and smoke-free public places: findings from the ITC 4-country project .................57

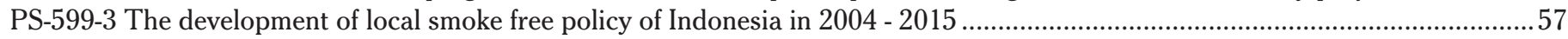

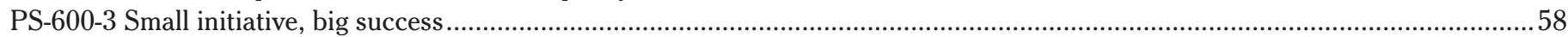

PS-601-3 What kind of factors influence the effective enforcement of smoke-free law in China? ......................................................58

PS-602-3 Tobacco control policies and perinatal and child health: a systematic review and meta-analysis ......................................58

PS-603-3 Results from unannounced visits to check compliance with smoke-free regulations in three types of indoor places in Beijing.......59

PS-604-3 A pre-post evaluation of and public support for smoke-free policies

at the 2016 Rio Olympics: findings from the ITC Brazil survey, $2012-17 \ldots$

PS-605-3 Impact of the smoke-free legislation on the incidence and mortality of AMI and stroke

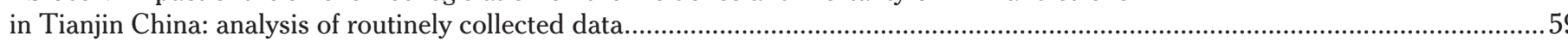

PS-606-3 Strengthening tobacco-free worksite policies in India

PS-607-3 The long road to smokefree bars in the Netherlands: findings from the ITC Netherlands Survey 2008-2016 .....................60

PS-608-3 Promoting tobacco control law enforcement in China through applications

of "Government Information Disclosure" and media advocacy ....

PS-609-3 Do European smokers opt for partial or total bans on smoking in homes and cars?

Findings from the ITC 6 European Country Survey (EUREST-PLUS Project).......

PS-610-3 Togo smokefree compliance monitoring in public places after 3.5 years of implementation of the law (2017) ...................61

PS-611-3 Smoking ban in public places in Japan - adverse legacy of the 2020 Olympic Paralympic Games?.....................................62

EP-115-3 Youth exposure to second hand smoke: a study among secondary school students

in public schools in an urban district in Lagos state Nigeria

EP-116-3 Secondhand smoke exposure at home in China .........

EP-117-3 Exposure to secondhand smoke and intention to quit

among South African smokers during 2007-2016: implications for policy...

EP-118-3 Building on the success of ten years of comprehensive smokefree legislation in England.................................................63

EP-119-3 Exposure to second-hand smoke and support for smoke-free policies in an urban district in Lagos, Nigeria ..................... 64

EP-120-3 Children Learning About Second-hand Smoke (CLASS II): a large pilot cluster randomised controlled trial.....................64

EP-121-3 Second-hand smoke exposure among undergraduates and support for smoke free campuses in Lagos, Nigeria ..................64

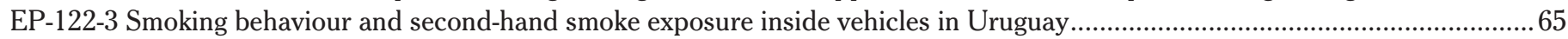

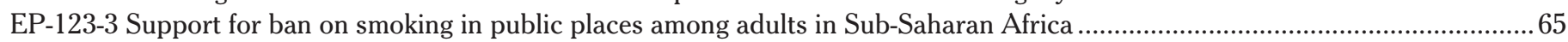

EP-124-3 Awareness and perception of the state tobacco control law prohibiting smoking

in public places among selected stakeholders in Ekiti State, Nigeria......

EP-125-3 Intervention for mothers during pregnancy to reduce exposure

to second-hand smoke (IMPRESS): a pilot randomized controlled trial in Bangladesh......

EP-126-3 Self-reported smoking patterns and compliance of cigarette smokers

after passage of the statewide smoking law in Lagos, Nigeria. 
EP-127-3 City sustained free of hookah bars by applying different acts and continued monitoring by law enforcers..

EP-128-3 Exposure to second hand smoke and community knowledge on its health effects

in rural and urban settings in Unguja Island - Zanzibar.....

EP-129-3 Awareness and support for the statewide smoking law among cigarette smokers in Lagos State, Nigeria

\subsection{Mind the gaps: loopholes in the Ban on Sponsorships, Advertising and Promotions}

FO-328-3 How tobacco companies in the United Kingdom responded to standardised packaging of cigarettes and rolling tobacco .68

FO-334-3 Tobacco packaging design for reducing tobacco use: cochrane systematic review.

RF-1200-3 Point of sale display ban in Scotland: retailers' views of the effects

on customer transactions, sales, youth and feelings about selling tobacco........

RF-1201-3 Effectiveness of TAPS bans and public support for point-of-sale (POS) bans

in Brazil: findings from the ITC Brazil Survey, 2009-17.

RF-1202-3 Exposures and responses to point-of-sale tobacco displays and support

for banning displays in Hong Kong: a population-based cross-sectional survey ......

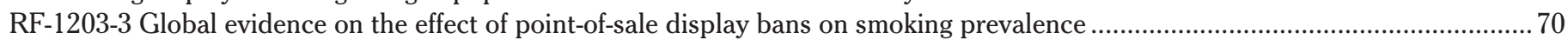

RF-1207-3 Point of sale tobacco advertising compliance with legislation in 3 large cities in Argentina ........................................... 70

RF-1210-3 Monitoring of compliance with the national tobacco advertising law in 11 cities in China ............................................70

RF-1211-3 Student advocacy efforts to remove tobacco advertising from their school environment, Jakarta \& Bandung, Indonesia ............ 71

PS-613-3 Awareness of tobacco advertising, promotion and sponsorship

in four states: findings from TCP India survey - Wave 1 and Wave 2.

PS-614-3 Denormalising tobacco at a Danish music festival.

PS-616-3 Removing tar information from cigarette packages

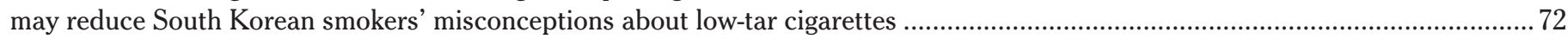

PS-617-3 Collaboration between stakeholders: TAPS free model rail station ..........................................................................72

PS-618-3 Effects of 85\% pictorial health warnings on manufactured cigarette package: a cross-sectional survey at retail shops......... 73

PS-619-3 "Pro-tobacco propaganda": a case study of tobacco industry-sponsored elementary schools in China............................... 73

PS-620-3 Monitoring of tobacco advertising and promotion in Lagos, Nigeria:

observational assessment to inform proposed regulations to the National Tobacco Control Act.....................................................73

PS-621-3 Monitoring compliance with the ban on tobacco advertising and promotion at the point of sale in Bangladesh (2017) ...... 74

PS-622-3 Menace of surrogate advertising of Gutka-tobacco brand advertisement

and resultant rampant selling bypassing Gutka ban in State of U.P

PS-623-3 E-cigarette marketing online: characteristics of websites serving the Indian market .......................................................75

PS-624-3 Study on violation of tobacco control law (Tobacco Advertisement, Promotion and Sponsorship) in TV drama ................. 75

PS-625-3 Big Tobacco, Tiny Targets: discrediting tobacco industry tactics targeting kids

through ads by school communities in 5 cities in Indonesia

\subsection{Innovations in media educational campaigns and health warning labels on cigarettes and other tobacco products}

FO-329-3 Over-time changes in reactions to pictorial health warning labels and association

with quitting behavior among adult smokers in Thailand: findings from ITC Thailand survey (2005 - 2012) .................................. 76

FO-330-3 Identifying credible attribution statements for sources on pictorial health warning labels in China................................... 76

FO-331-3 Indonesian adolescents' reactions to different text and image types of pictorial health warnings for cigarette packages ..... 76

FO-332-3 Health warnings effects on the decision to quit. GATS analysis from Argentina........................................................... 77

FO-333-3 Using message adaptation and pre-testing to achieve resource efficiencies

for best practice lung health communication programs in low- and middle-income country settings ..............................................77

PS-626-3 The dispute between health and market: a communicational analysis of the cigarette package in Argentina ......................77

PS-628-3 An innovative approach to improving implementation of pack warnings ....................................................................78

PS-629-3 Impact of pictorial health warnings on Vietnamese male smokers' knowledge of smoking harms .....................................78

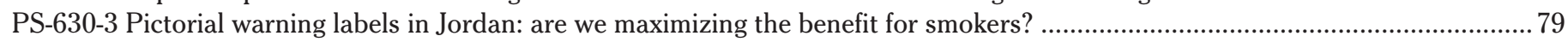

PS-631-3 Evaluating the impact of health warnings in Brazil

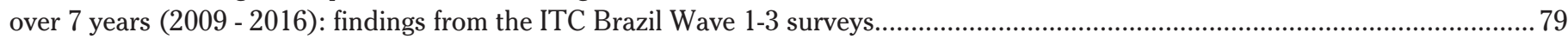

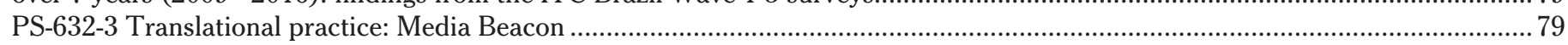

PS-633-3 Perceived effectiveness of larger graphic health warnings and plain packaging

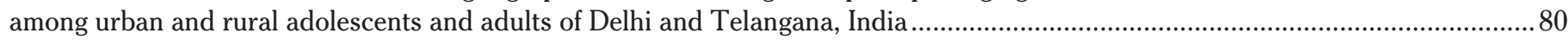

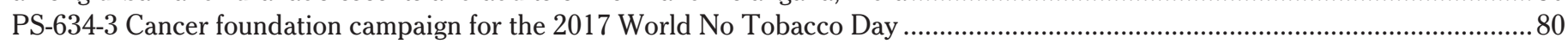

PS-635-3 Impact of textual framing and depictions of harm on indicators

of cigarette pictorial health warning label effectiveness in China.....

PS-636-3 The role of digital and social media within mass media anti-smoking campaigns ...........................................................8

PS-637-3 Implementation of graphic health warnings in Bangladesh - current status and challenges ..............................................8 81

PS-638-3 Urgent need for implementing media education campaigns and health warnings in Parties with high burden of SLT use ..82

PS-639-3 Connecting the region through infographics and social media in tobacco control campaigns ..........................................82

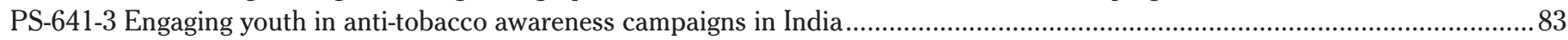

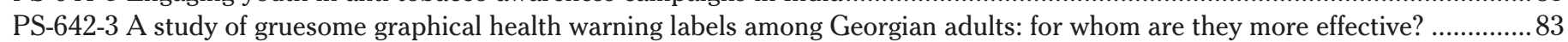




\subsection{Opportunities for regulating tobacco products' content and emissions to reduce harm and addiction potential}

PS-643-3 MADES study: evaluation plan of the new tobacco product directive 2014/40/UE in Italy ...............................................8 84

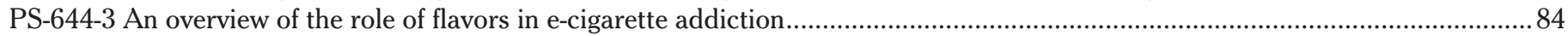

PS-645-3 Concentrations of hazardous chemicals in mainstream aerosol generated by heat-not-burn tobacco..................................8 84

PS-646-3 Prevalence of lower harm perceptions of cigarette product characteristics:

findings from 10 low-middle-income countries from the ITC project.

PS-647-3 E-liquid product labels: the good, the bad, and the ugly......

\subsection{Prevalence of tobacco and nicotine-related products' use: Traditional and novel products}

PS-666-6 Reasons for e-cigarette use and perceptions of harm in Brazil:

findings from the ITC Brazil Wave 2 (2012-13) and 3 (2016-17) surveys

FO-307-4 Use of electronic cigarettes across 13 ITC countries with different regulatory environments ..........................................8. 86

FO-308-4 Awareness and use of electronic cigarettes among youth: evidence from the Global Youth Tobacco Survey 2012 - 2015...........87

FO-309-4 Awareness, use, sales and interest of heat-not-burn tobacco products in Italy .............................................................8. 87

FO-310-4 Does use of electronic nicotine delivery systems predict current cigarette use among young adults? .................................88

FO-311-4 Effect of seeing e-cigarettes in small shops on probability of e-cigarette experimentation

by 1 year follow up in adolescents in Scotland, UK....

FO-312-4 Use of flavored cigarettes in the first few puffs: a step toward smoking initiation

and nicotine addiction? Data from a national survey among Brazilian adolescents

FO-313-4 Tobacco cigarette use versus electronic cigarette use: determinants of smoking and vaping behavior ...............................89

PS-648-4 The use of flavour-changing capsule cigarettes among smokers in the United Kingdom ..................................................89

PS-650-4 Socioeconomic differences in smoking declining? Result from population-based surveys in 1978 - 2016 in Finland ............ 90

PS-651-4 Dual use of combustible and electronic cigarettes: patterns and associations between products .......................................90

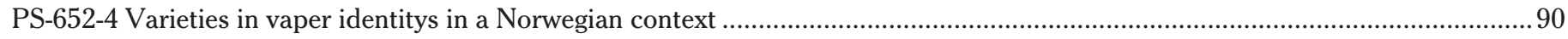

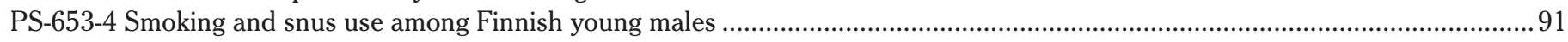

PS-654-4 Factors associated with ever-use of e-cigarette among college students in the Republic of Korea ......................................91

PS-655-4 Electronic cigarette effectiveness to quit smoking in the representative Italian population PASSI survey, $2014-2016 \ldots . . . . .91$

PS-656-4 Perceptions, work environment, and job stress related with tobacco use among fishermen in remote Island, Rebun Japan ..........92

PS-657-4 E-cigarettes or vaping: examining perceptions of use and associated harm among current users in Australia and Bangladesh ..... 92

PS-658-4 E-cigarettes or vaping: is there any difference in perceptions of use and associated harm

among the current users between a developed and a developing country? ...............................................................................9.

PS-659-4 Prevalence and characteristics of e-cigarette use among adults in Malaysia...................................................................93

PS-660-4 Prevalence of flavored cigarettes and e-cigarettes in Uruguay: findings from the Wave 5 of the ITC Uruguay survey.......... 93

PS-661-4 Contrasting trends of smoking cessation status: insights from the stages of change theory

using data from the global adult tobacco survey ....

PS-662-4 Health care professional and cigarette cessation volunteers knowledge, attitude and practice on e-cigarettes ....................94

PS-663-4 Smoking prevalence and smoking behavior change among the soldiers in the Korean military .........................................94

PS-664-4 Association of tobacco control policies with cigarette smoking

among school age youth 13 to 15 in the Philippines, $2000-2015$.....

PS-665-4 Prevalence and willingness to pay for home-made cigarettes (HC) and industry cigarettes (IC) of Thai men .................... 95

PS-667-4 Characteristics and factors associated with tobacco use: findings of Kenya Global Adult Tobacco Survey, 2014................96

PS-668-4 Amendment of tobacco hazards prevention act in Taiwan: brought about a reduction

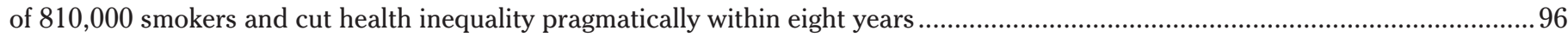

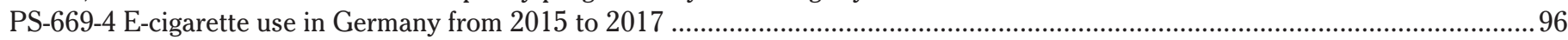

PS-670-4 "Chewing of tobacco laced areca-nuts" - is nothing but "Chewing of tiny-cancer-causing-bombs

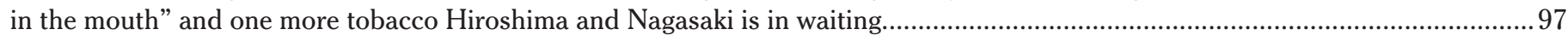

PS-671-4 Passive exposure to e-cigarette emissions: irritation symptoms, severity and duration ....................................................97

PS-672-4 Cigarette brand preferences of adolescent and adult smokers in the United States .........................................................97

PS-673-4 "It Didn't Fill the Void": reasons for e-cigarette and tobacco dual use ...........................................................................98

PS-674-4 Comparison of smoking prevalence and initiation between physician and general people of Bangladesh..........................98

PS-675-4 Novel tobacco products in Japan - their risks and impacts to tobacco control policy and the society .................................98

PS-676-4 Marketing tobacco at the point of sale: a survey of 675 tobacco shops in Mumbai, India..................................................99

PS-677-4 Prevalence and factors that influence smokeless tobacco use among adults

in pastoralist communities of Borena zone, Ethiopia: mixed method study ....

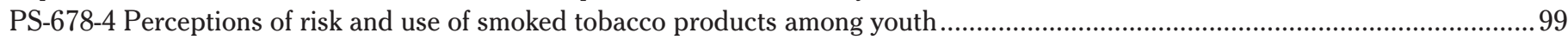

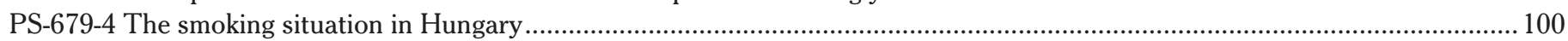

PS-680-4 Prevalence and determinants of tobacco use among youth in Myanmar:

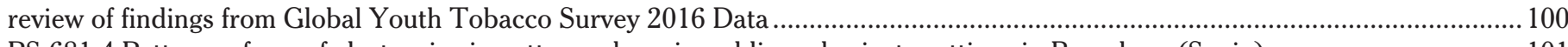

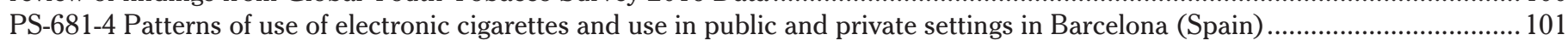

EP-160-4 Electronic cigarettes use and perception amongst medical students, a cross sectional survey from Sindh, Pakistan .......... 101

EP-161-4 Prevalence of tobacco consumption among Auto Rickshaw Drivers in Chennai City, Tamil Nadu, India ......................... 101

EP-162-4 Use of e-cigarettes among smokers and recent quitters: results from the ITC New Zealand Survey ................................ 102

EP-163-4 Prevalence of e-cigarette in China: preliminary findings from two surveys...................................................................... 102

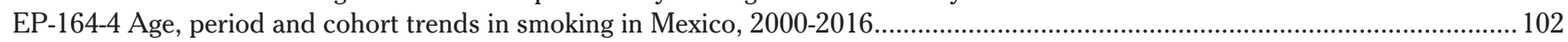


EP-165-4 Increase of electronic cigarette use and awareness in Brazil: findings from a country

that has strict regulatory requirements for electronic cigarette sales, import, and advertising ......

EP-166-4 Use of roll-your-own tobacco among smokers in New Zealand: results from the ITC New Zealand Survey ...................... 103

EP-167-4 E-cigarettes use among urban male tobacco smokers age 15 years or older in China ..................................................... 104

EP-168-4 Smoking prevalence among inpatients with drug resistant tuberculosis in KwaZulu-Natal, South Africa.......................... 104

EP-169-4 Prevalence and patterns of use of smokeless tobacco in Mumbai schools................................................................. 104

EP-170-4 E-cigarette perceptions, use frequency and susceptibility among young adults in Hong Kong......................................... 105

EP-171-4 Transition from experimentation with tobacco and nicotine products to regular smoking in Europe ................................. 105

EP-172-4 Dual use of tobacco and cannabis: significant change in use

between Prince Edward Island (PEI) and Canadian students (2008/2009 to 2014/2015) ......................................................... 105

EP-173-4 Portrayal of orthodox and unorthodox uses of electronic cigarettes in YouTube videos ............................................... 106

\subsection{Burden and Disparities in tobacco-related diseases: Non-communicable diseases (NCDs) and communicable diseases (TB and IIIV/AIDS)}

SUS Correlates of tobacco use and disease burden - an analysis of the World Health Organisation South East Asian Countries..... 106 SUS Effect of elevated fasting serum glucose after smoking cessation on cardiovascular disease and mortality: an eight-year longitudinal study

SUS Association between alpha1-antitrypsin (a1-AT) levels, lipid profile

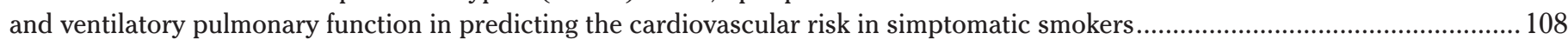

SUS Tobacco use among urban residents with diagnosed major Non-communicable diseases in Bangladesh................................. 108

PS-682-4 Tobacco related morbidity among patients hospitalized at Kenyatta National Referral Hospitals Kenya.......................... 108

PS-683-4 Reported health effects of passive smoking by bar workers in Ibadan, Nigeria ............................................................ 109

PS-684-4 Physical and psychological health problems associated with tobacco consumption among professional drivers in India .. 109

PS-685-4 Risk of cancers associated with tobacco use in India: a systematic review and meta-analysis ........................................... 109

PS-686-4 Projections of burden of tobacco related cancers: a new approach

for measuring incidence cases for India and its states - Till 2025

PS-687-4 Prevalence and correlates of dry nasal snuff use among HIV-infected adult women in South Africa ................................ 110

PS-689-4 Physical activity level and its relationship with tobacco use among youth: a cross-sectional survey in North India ........... 110

PS-690-4 Effect of smokeless tobacco on vascular changes following flap surgery - a doppler study ........................................... 111

\subsection{New tools for monitoring policy impact and tobacco use surveillance}

PS-691-4 How to monitor WHO-MPOWER policies implementation at sub national level

in low resource settings? Experience from the state of Himachal Pradesh in India..

PS-692-4 Impact of India's National Tobacco Control Programme

on bidi and cigarette consumption: a difference-in-differences analysis .....

PS-693-4 Classification and regression tree for characterising smoking patterns

among adults: evidence from global adult tobacco survey, Bangladesh

PS-694-4 Computerized tablet based versus traditional paper-based survey methods:

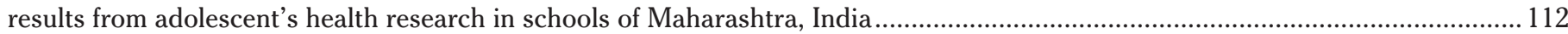

PS-695-4 Mapping of tobacco use among people visiting mass gathering (Kumbh Mela) in India.................................................. 113

PS-696-4 A sudden surge in outpatient volume of the smoking cessation clinic

after the implementation of Beijing tobacco control regulation .....

PS-697-4 Implementing section - 6b of Cigarettes and Other Tobacco Products Act 2004,

prohibiting the sale around educational institutions using technology based software - safe zone.....

PS-698-4 Building capacity for tobacco-free schools in rural Maharashtra: the Salaam Mumbai model.

\subsection{Tobacco dependence treatment and smoking cessation: Innovations in Practice and Opportunities for scaling}

PS-699-4 Tobacco cesssation - as we want it! An interview study with young people .

PS-700-4 Third times the C.H.A.R.M.S: a socioecological thirdhand smoke cessation pilot study.......

PS-701-4 Empowering smoke-free environment through self-verbalization and cognitive

re-appraisal psychotherapies among university participants in a smoke-stoppage programme.....

PS-702-4 Experiences of young smokers in quitting smoking in twin cities of Pakistan: a phenomenological study...

PS-705-4 ABC in ED results of the "ASSET" study: outcomes of "Brief Interventions"

to stop smoking given in the emergency department of a central city hospital (2016).....

PS-706-4 Development of a guided imagery tobacco cessation intervention to be delivered by quitline and website......................... 117

PS-707-4 Integration of a personalized approach for tobacco use and dependence in a dentistry setting-preliminary results ........... 117

PS-708-4 "Lung age" - a motivational smoking cessation tool in smokers with chronic obstructive pulmonary disease..................... 118

PS-709-4 Self-perceived ability to cope with stress without smoking predicts

successful smoking cessation 12 months later in a quitline setting: a randomized trial.

PS-710-4 Scaling up the availability of tobacco dependence treatment training:

leveraging the experience of King Hussein Cancer Center to create new training hubs......

PS-711-4 Effect of community based tobacco cessation intervention in a rural community of Bangladesh...

PS-712-4 Long-term effectiveness, safety, perception, satisfaction and cost 
of electronic cigarette among single and dual users in Malaysia.....

PS-713-4 Evaluation of a national tobacco dependence treatment program in Uruguay 2017

PS-715-4 Readiness to quit addiction! A study among patients attending tertiary care hospital in Western India .

PS-716-4 Pregnant smokers' views on babyClear@: a package of measures

including universal carbon monoxide monitoring and opt-out referral to support their quit

PS-717-4 Analysis on current status and the evaluation criteria of smoking cessation clinics in China.........................................121

PS-719-4 Depression symptoms and quitting among a nationally representative sample of smokers from Africa .......................... 121

PS-720-4 Effect of healthcare practitioners' advice on the decision to quit. GATS analysis from Argentina .................................... 122

PS-721-4 Impact of behavioural intervention to the predictors of quit success

among smokers attending quit smoking services in Malaysia: 1- month, 3-months and 6-months follow-up...

PS-722-4 Effects of promoting smoking cessation program in drug dependence disorder patients

at the national institute on drug abuse treatment in Thailand....

PS-723-4 Efficacy of short message service (SMS) in smoking cessation program among

police personnel in Chennai city - an interventional study......

PS-724-4 The association between antitobacco advertising and calls to a tobacco Russian Quitline..

PS-725-4 Effect of nurse-based brief intervention for tobacco cessation among admitted

cardiac patients in a tertiary care hospital of Dhaka City, Bangladesh.

PS-726-4 Integrating worksite smoking cessation services into the construction trade: opportunities and challenges....................... 124

PS-727-4 Tobacco treatment education for health care professionals in behavioral health settings in Kansas, USA ....................... 124

PS-728-4 Reducing financial barrier of smoking cessation treatment can effectively

enhance prolonged abstinence: an experience from Taiwan.....

PS-729-4 Reduction of tobacco consumption in a rural area with a community based tobacco control program in Bangladesh ....... 125

PS-730-4 Moving towards a tobacco free workplace at a cement manufacturing plant in Chandrapur, Maharashtra ........................ 125

PS-731-4 Tobacco in my speciality - a pocket guide for specialists

PS-732-4 Impact of an online training program for brief intervention on smoking cessation

for health care workers in Bolivia, Guatemala and Paraguay......

PS-733-4 Smoking cessation therapy in Ethiopia: responsiveness and the predictors ................................................................ 127

PS-734-4 Predictors of intention to quit smokeless tobacco among adults in pastoral communities of Borena Zone, South Ethiopia ......... 127

PS-735-4 Does free or lower cost smoking cessation medication stimulate quitting?

Findings from the International Tobacco Control (ITC) Netherlands and United Kingdom Surveys

PS-736-4 Efficacy and safety of V. cinerea (L.) less. for smoking cessation:

a systematic review and meta-analysis of randomized controlled trials....

PS-737-4 Process and outcome evaluation of a novel online-only Tobacco Treatment Specialist (TTS) training program ............... 128

PS-740-4 Potential for sustainability of strategies for implementing tobacco use

treatment guidelines in the Vietnam public healthcare system: qualitative post intervention assessment ...................................... 128

PS-741-4 Smoking cessation intervention programme in four secondary boys' schools in Malaysia ............................................... 129

PS-742-4 The effectiveness of the smoking cessation telephone quitline service in Clalit Health Services, Israel............................. 129

PS-744-4 Perceptions of permanent and contractual employees about

a tobacco free workplace policy among four workplaces in Maharashtra, India

EP-175-4 Salivary thiocyanate as a biomarker for tobacco exposure - implications in diagnosis and tobacco cessation .................... 130

EP-176-4 Impact of weight change after quitting cigarettes on all-cause and cause-specific mortality

in middle-aged male smokers: national health screening cohort study .

EP-177-4 Factors associated with quit attempts and smoking cessation in Brazil:

findings from the International Tobacco Control Brazil Survey......

EP-178-4 Systematically scaling up cessation services provider referrals

through electronic medical records to improve patient acceptance rates ...

EP-179-4 2017 National Survey of Tobacco Cessation Clinics in China: evidence

from the combination of nationwidegovernment and clinic on-line survey........

EP-180-4 A hypothesis for an alternative treatment for tobacco addiction: phenelzine, a monoamine oxidase inhibitor .....

EP-181-4 Factors associated with intention to quit among tobacco users in India:

findings from TCP India survey - Wave 1 and Wave 2 ......

EP-182-4 Personal tobacco use and attitudes towards cessation among undergraduate health professional students in South Africa ........ 133

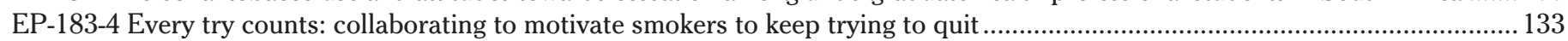

EP-184-4 Understanding the correlates of successful abstinence in a randomized

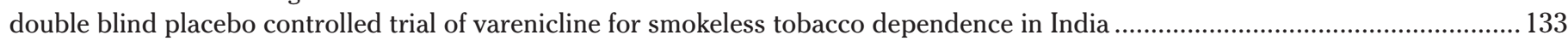

EP-185-4 Comparing 2 different intensities of active referral to smoking cessation services: a cluster randomized controlled trial... 134

EP-186-4 Health-care provider intervention and utilization of cessation assistance in low- and middle-income countries ................. 134

EP-187-4 Comparison of two approaches in achieving smoking abstinence among patients

in an outpatient clinic: a phase 2 randomized controlled trial ....

EP-188-4 The characteristics, conseling service and effectiveness of China Quitline

\subsection{Tobacco and Women: The gendered nature of tobacco}

FO-300-5 Smoking cessation in Argentina: a gender-based perspective from GATS results

FO-301-5 Attitudes, policy and behaviour change: the effect of attitudes towards smoke-free laws on quit attempts among smokers ......... 136

FO-302-5 A qualitative study exploring women's journeys to becoming smokers in the social context of urban India..... 
FO-306-5 The Vietnam Women's Union Testimonial Campaign - "Women create smoke-free homes"...... 136 PS-745-5 Designing effective cigarette health warning labels for women in China - findings from a 4 city study ............................ 137 PS-746-5 Most vulnerable victim of tobacco use and the key target of tobacco industry's

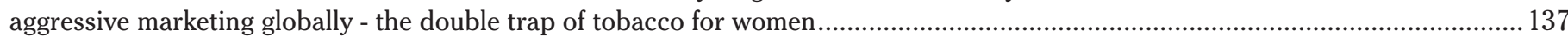

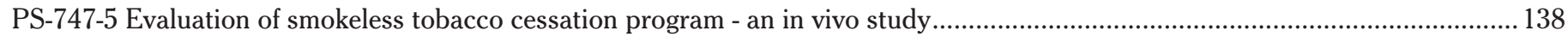
PS-748-5 Supporting young women to have smokefree pregnancies - BabyBe Smokefree .......................................................... 138 PS-749-5 Gender differences in tobacco use disorder phenotypes among smokers in the largest metropolitan area of South America ...... 138 PS-750-5 Second hand smoke exposure among pregnant women in Argentina and Uruguay ..........................................................139 PS-751-5 Gender differences in unassisted cessation attempts by South Australian smokers: 1998-2014 ...................................... 139 EP-145-5 Age of initiation of cigarette smoking and quit attempts among young women in India - evidence from Global Adult Tobacco Survey 2009 and 2017

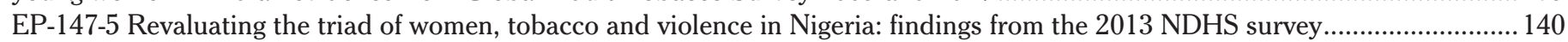
EP-149-5 Exposure to tobacco during pregnancy among mothers with low birth weight newborns in a rural area, Arua district, Uganda - 2016: a case control study.....

\subsection{Tobacco in vulnerable populations (e.g. indigenous populations)}

FO-303-5 Modeling smoking and depression comorbidity in the U.S .....

PS-752-5 Usages du tabac chez les travailleuses de sexe à Yaoundé. Perceptions, facteurs associés et risques sanitaires ................ 141

PS-753-5 Tobacco use, awareness and cessation among Malayali tribes, Yelagiri Hills, Tamil Nadu, India ................................... 142 PS-755-5 An assessment of oral health status, tobacco use and cancer awareness

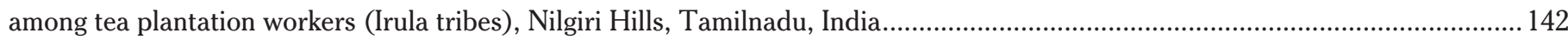
PS-756-5 Tailor-made smoke-free programme to clear the myths of smoking among Hong Kong elder people............................... 142 PS-757-5 The prevalence of secondhand smoke exposure among women and children in the deep South of Thailand.................... 143

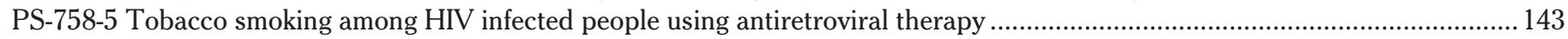
PS-759-5 Awareness about anti-smoking related laws and legislation in healthcare facilities of Delhi, India.................................. 143 PS-760-5 Tobacco chewing habits and risk of precancerous oral lesions among Paniya tribes of Wayanad, India - a cross sectional study.....

PS-761-5 Assessment of smoking/tobacco smoke exposure and provision of smoking cessation

advice/assistance by healthcare professionals at a women's and children's hospital - practices and barriers ................................... 144

PS-762-5 Tobacco packaging in India: assessing compliance with Health Warning Label (HWL) laws

and marketing appeals for cigarettes, bidis and smokeless products.

PS-763-5 Use of puppet shows for promoting smoke-free home and training children

about the harmful of smoke and care for themselves, in Tehran's kindergartens.........

PS-764-5 Perception of vulnerable populations of label warnings in Global Adult Tobacco Survey (GATS) - Panama ................... 145

PS-765-5 Antenatal tobacco use and iron deficiency anemia: formative research

to integrate tobacco cessation into antenatal care for low-income women in Mumbai, India......................................................... 146

PS-766-5 Generativity, self-rated health and smoking behavior of older people in Sri Lanka..................................................... 146

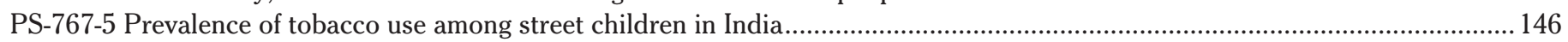

PS-768-5 Oral malignancies and tobacco related habits among Aranadar tribals in Kerala, India: a population based study ........... 146

\subsection{Tobacco, inequality and poverty}

FO-326-3 Poverty and smoking: assessing the financial impact of tobacco purchases on households' budgets in Argentina............. 147

FO-327-3 Lived experience of tobacco tax increase among socioeconomically disadvantaged smokers ......................................... 147

RF-1209-3 Smoke-free legislation and socioeconomic inequalities

in smoking-related morbidity and mortality among adults: a systematic review.....

PS-772-5 Completely given up sale of cigarette at a village in Sri Lanka.

\subsection{Tobacco farming: Opportunities and experiences in facilitating alternative livelihoods and preventing tobacco adverse environmental impact}

PS-773-5 Financing alternatives to tobacco growing: Philippine experience in implementing Art. 17 and 18

PS-774-5 Policy recommendations on tacing and reducing program mismatch

and perverse incentives present in earmarking sin tax to tobacco growing areas......

PS-775-5 Evaluating results of the program for tobacco leaf diversification in Brazil ................................................................. 149

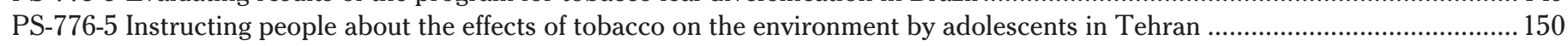

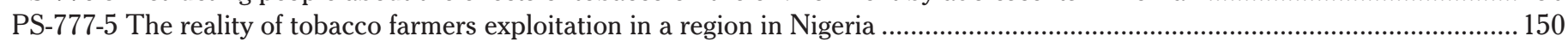

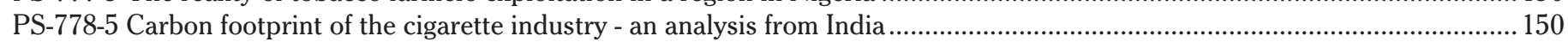

PS-779-5 Development of Indonesia's tobacco farming and business in the era of FCTC .......................................................... 151

PS-780-5 Elimination of tobacco growing is possible: a case study from Karnataka India .......................................................... 151

\subsection{Youth tobacco experience}

PS-782-5 Smoking facts and evidence among medical students from "Carol Davila"

University of Medicine and Pharmacy, Bucharest, Romania... 
PS-783-5 Photoaging smartphone app to reduce smoking prevalence in secondary schools: the smokerface randomized trial......... 152 PS-784-5 The ASSIST smoking prevention programme in adolescent social networks beyond the school setting: an exploratory study.... 152 PS-785-5 Does level of physical activity influence tobacco use behaviour among youth? A cross-sectional survey from India ........... 152

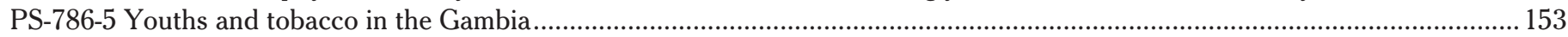

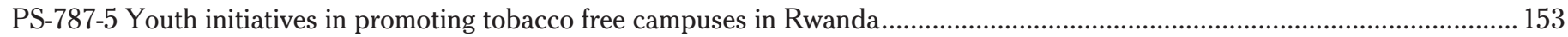

PS-788-5 Social differences in implementation of a school based smoking preventive intervention ............................................... 153 PS-789-5 Are school tobacco policies effective at reducing smoking among young people? A study on vocational schools in Denmark .... 154 PS-790-5 Knowledge, attitude and practice regarding use of tobacco

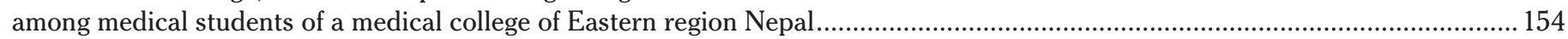
PS-791-5 Effect of school based smoking prevention program in Korea .....................................................................................154 PS-792-5 Is tobacco use as a gateway behavioral risk factor? Clustering of health

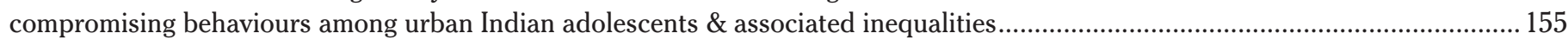
PS-793-5 Sale of tobacco products among minors in a satellite township near Kuala Lumpur, Malaysia. An observational study.... 155 PS-794-5 Assessing the predicting validity of the tobacco marketing receptivity among youth ....................................................... 156 PS-795-5 Use of e-cigarettes rising among junior and senior high school students in Taiwan....................................................... 156 PS-797-5 Tobacco and areca nut cessation programme for adolescent school students in Mumbai, India .................................... 157 PS-798-5 Youth involvement on tobacco control law implementation: success story .............................................................. 157 PS-799-5 Trend of easiness to purchase cigarettes among Korean adolescents:

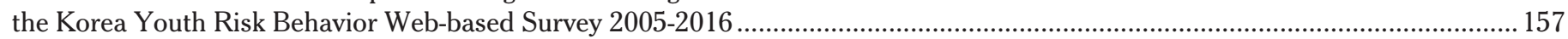
PS-800-5 The hidden burden of tobacco use among school adolescents in Ethiopia: call for action.............................................. 158 PS-801-5 Exposure to advertising of tobacco products at points of sale and consumption of tobacco in adolescents from 3 large cities in Argentina. Cross-sectional study. PS-802-5 Assessing knowledge and practice of tobacco retailers around primary and secondary schools, on selling tobacco products to and by minors in Kigali Rwanda.

EP-150-5 Did the Irish 2004 smokefree legislation contribute to the sharp decline in youth smoking?...

EP-151-5 Current and frequent cigarette smoking trends in South African learners.................................................................... 159

EP-152-5 Age-period-cohort effect of adolescent smoking in Korea: from 2006-2016 ................................................................ 160

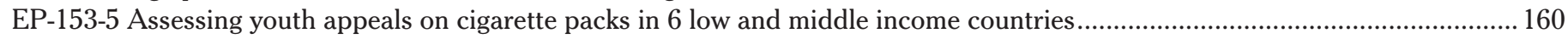

EP-154-5 The variation of tobacco use behavior among Beijing college students and middle school students from 2008 to 2014 .... 161

EP-155-5 A Stop Smoking in Schools Trial (ASSIST) a decade on: insights from a mixed method process evaluation..................... 161

EP-156-5 Decentralised public education campaigns for tobacco control by Malaysian

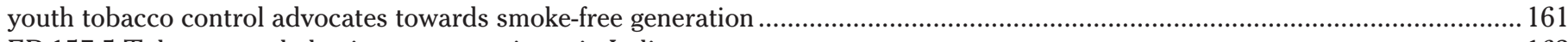

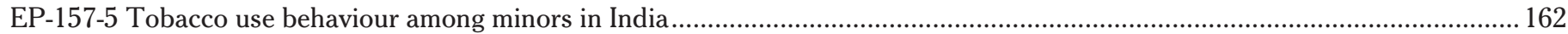

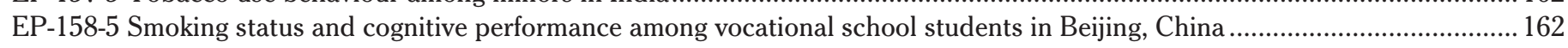

EP-159-5 Factors promoting initiation of cigarette smoking among adolescents in rural and urban areas in Oyo state, Nigeria ........ 162

\section{Thursday, 8 March 2018}

\subsection{Priorities and challenges for advancing the WHO FCTC}

RF-1212-1 Adoption of the Tobacco Control Regulations - Legislative Instrument (LI) 2247

to reduce the burden of NCDs and to advance WHO FCTC implementation in Ghana ...............................................................163

RF-1213-1 Perception of the adult population on packaging and labelling of cigarettes. Panama. Year: 2015 ................................. 163

RF-1217-1 Why does tobacco consumption increase in a MPOWER-compliant country? ............................................................. 164

RF-1218-1 Compliance with the Framework Convention on Tobacco Control: a 9-country study ................................................. 164

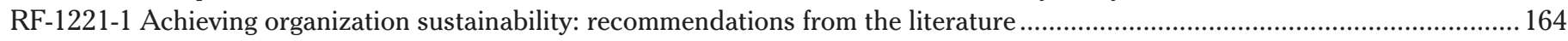

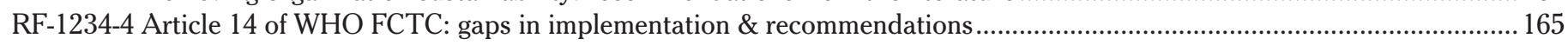

LB-1300-1 Compliance with tobacco control laws before and after the enactment of a national Tobacco Control Act in Ghana ....... 165

LB-1302-1 Role of WHO FCTC global knowledge hub on smokeless tobacco in smokeless tobacco control .................................. 166

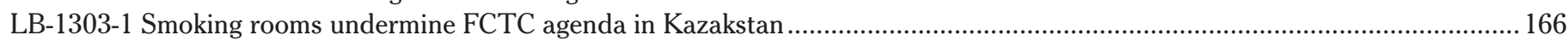

LB-1304-1 How MPOWER-ed are we towards effective implementation of WHO FCTC? ......................................................... 166

\subsection{Integrating tobacco control into I Health systems, global firameworks and strategies to reach WHO FCTC and SDG's targets.}

RF-1215-1 Promoting effective tobacco control through advancement of public health systems in Nigeria.................................... 167

RF-1216-1 Ten years of India's National Tobacco Control Programme: achievements, challenges and the way forward ................. 167

LB-1305-1 The impact of anti-smoking policies of the 2010-2014 Hungarian government - a comprehensive evaluation .................. 167

\subsection{Setting the research agenda for translating science to poliey and new tools for policy impact assessments}

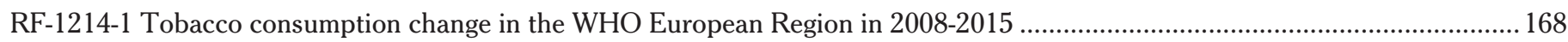

RF-1219-1 Tobacco marketing trends at the point-of-sale (POS) near schools in 9 middle and high-income countries..................... 168

RF-1220-1 Spatial distribution of tobacco outlets and related regulation within a restricted market framework .............................. 169

LB-1301-1 Network analysis to detect gaps in research on smokeless tobacco: implications for future policy ................................. 169 


\subsection{Promoting transnational cooperation and Partnerships: models of Intergovernmental and nongovernmental collaboration across multiple sectors for high level commitments}

FO-357-2 Multilateral development banks and bilateral donors and their responsibility

towards excluding tobacco investments and participation.....

\subsection{Building leadership capacity and broader alliances for tobacco control policy advocacy}

FO-355-5 Generating local evidence on tobacco industry tactics

to advance tobacco control objectives: a holistic approach embracing new technology .....

FO-360-2 Devolving tobacco control legislation to local governments

for enhanced capacity in tobacco control advocacy and implementation

LB-1312-2 To assess control measures for tobacco consumption in Zambia between 2014 and 2017. What are the gaps? .............. 171

LB-1315-2 Understanding tobacco control policy at the national level:

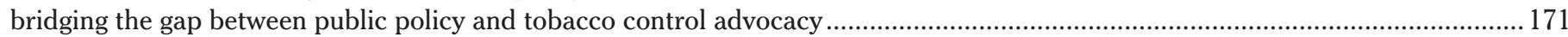

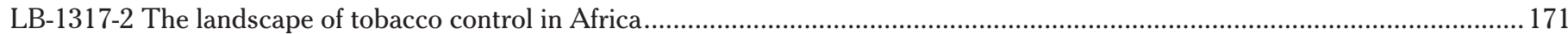

LB-1319-2 The struggle towards FCTC-compliant legislation in Burundi: an inspiring advocacy experience .................................. 172

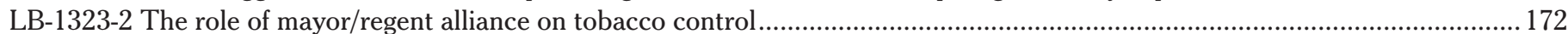

\subsection{Tobacco industry interference: New tactics and counter measures}

FO-356-2 The Philip Morris Genome Project: a guide to tracking alliances of the world's largest cigarette company

FO-358-2 The capsule trap - how tobacco companies communicate about flavor capsules in cigarette filters on the pack.....

FO-359-2 Promoting compliance and enforcement of the tobacco advertising ban

in China through legal actions and media advocacy by public interest lawyers

FO-361-2 Towards understanding the features of the waterpipe tobacco industry:

findings from two consecutive visits to the International Hookah Fair

LB-1313-2 Implementation of 85\% health warning labels in India: advocacy success factors..................................................... 174

LB-1314-2 'Alternative fact' narrative influencing tobacco control: a case study of tobacco industry interference in print media...... 174

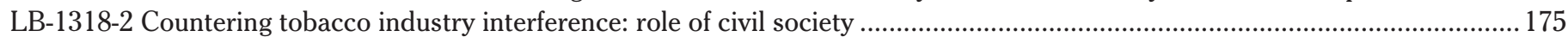

LB-1320-2 Possible consequences of relatively decreasing cigarette price in China from 1999 to 2015 ........................................ 175

LB-1322-2 Understanding tobacco industry tactics: a content analysis of newspaper coverage of tobacco issues in Pakistan .......... 175

\subsection{Pricing, Taxation and countering illicit trade: Innovations and reform strategies}

LB-1308-3 An assessment of metrics for progress in tobacco taxation in the WHO Global Tobacco Control Reports ..................... 176

LB-1337-3 Spatial and economic proximity of cigarette sales to school children in Mongolia.

\subsection{Advances in protection from tobacco pollution, second- and third-hand smoke}

LB-1306-3 A community led tobacco free city

LB-1307-3 Formative research to support the transition of multi-unit rental housing to smoke-free ........................................... 177

LB-1310-3 Empowering decision makers in Latin America: potential impact of three interventions for tobacco control.................. 177

LB-1311-3 The relationships between secondhand smoke exposure and blood cadmium,

lead and mercury concentration in the Korean population: findings from the 2008-2012 KNHANES.

LB-1338-3 Leaders for little lungs - children as advocates for clean air in the Western Cape province in South Africa.....

\subsection{Mind the gaps :loopholes in the Ban on Sponsorships, Advertising and Promotions}

FO-373-3 (Mis)perceptions related to Electronic Nicotine Delivery Systems (ENDS) and hookah: making a case for policy strengthening through a multi-stakeholder qualitative study from New Delhi, India ............................... 178

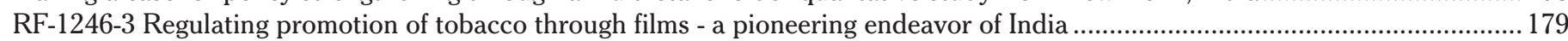

RF-1247-3 Exposure of smoking scenes in popular movies among Koreans from 2006-2015 ..................................................... 179

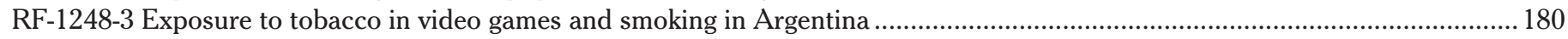

RF-1249-3 Pro-smoking apps for smartphones in the Republic of Korea: prevalence and potential risks ..................................... 180

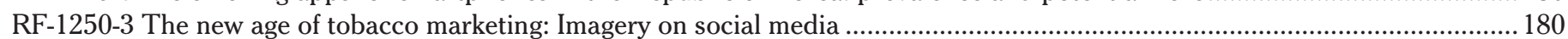

RF-1255-3 Use of social media to circumvent sales and advertising ban of electronic cigarettes .................................................... 181

RF-1256-3 Use of tobacco in Brazilian TV programs: status and potential influence on the low income population ........................ 181

RF-1257-3 Indian television channels become vehicle for tobacco advertisement,

promotion and sponsorship (TAPS) violations in India - results of a sub-national survey in a northern Indian city

\subsection{Innovations in media educational campaigns and health warning labels on cigarettes and other tobacco products}

FO-372-3 Do pictorial health warnings on waterpipe tobacco packs matter?

Recall effectiveness among waterpipe smokers \& non-smokers. 
RF-1251-3 Evaluation of the national tobacco control mass media campaign in China

RF-1252-3 The effectiveness and cost-effectiveness of tobacco control TV

mass media campaigns broadcast in Scotland (2003 - 2012) .

RF-1253-3 "Little Lungs" - using digital platforms, innovative creative,

and audience passion points to prevent smoking among U.S. youth .

RF-1254-3 Can Facebook help reduce smoking? A qualitative study to investigate

how to use social media for tobacco control among Australian Indigenous people

LB-1339-3 Mobilizing school children in "No Smoking, Be Charming!" media campaign in Taiwan..

\subsection{Opportunities for regulating tobacco products' content and emissions to reduce harm and addiction potential}

FO-369-3 Knowledge, attitudes and practices of Shisha smoking among youths in Kampala, Uganda. 184

FO-370-3 Effects of a real world menthol ban: changes in smoking behaviour and cigarette branding ........ 185

FO-371-3 The use of and beliefs about menthol cigarettes among Brazilian smokers:

findings from Wave 3 (2016-17) of the ITC Brazil Survey...

FO-375-3 Prevalence, perceptions and predictors of menthol cigarettes

among African smokers: findings from the ITC Kenya and Zambia Surveys

185

\subsection{Opportunities for regulating tobacco products' content and emissions to reduce harm and addiction potential}

LB-1309-3 Flavor capsule cigarette use, user profiles and perceptions in South Korea........ 186

\subsection{Prevalence of tobacco and nicotine related products' use: Traditional and novel products}

FO-376-4 A real-world assessment of indoor air quality (ultrafine particles) following e-cigarette use in two e-cigarette shops ........ 186 FO-377-4 Serum cotinine by socioeconomic status and exposure to second hand smoke in a national sample of South Africans..... 186 FO-382-4 Secondhand smoke exposure, perceived risks and knowledge of the national tobacco law

among non-smoking adults in outdoor motor parks in an urban area, Lagos, Nigeria .....

LB-1328-4 Pattern of smokeless tobacco use and cigarette smoking among adult South Africans during 2007-2016 .

LB-1331-4 Prevalence of Midwakh tobacco smoking in trend-setting Lebanon:

an indicator of potential spread across the Arab world?.

LB-1332-4 Antenatal tobacco smoke exposure: impact on infant birth outcomes

and lower respiratory tract infection in a South African birth cohort.

LB-1333-4 Use of electronic nicotine delivery systems (ENDS) among Chinese adults:

evidence from citywide representative surveys from 12 cities in China (2014 to 2016)...

LB-1334-4 Exposure to second-hand aerosol produced by electronic cigarettes: a systematic review ......

\subsection{Burden and Disparities in tobacco-related diseases: Non-communicable diseases (NCDs) and communicable diseases (TB and IIIV/AIDS)}

FO-378-4 Healthcare costs attributable to secondhand smoke exposure at home for U.S. adults

FO-379-4 Association of household tobacco exposure with recent respiratory symptoms

and medical services utilisation in Hong Kong young children under 2 years old

FO-380-4 Morbidity attributable to secondhand smoke exposure in children under 5 years old in Spain, 2015 ........................... 190

LB-1326-4 Patterns of lung cancer mortality in Russia over a 16-year period, 2000-2015...................................................... 190

LB-1327-4 Tobacco use among tuberculosis patients across 30 districts in India in 2013 and 2017 ........................................... 191

\subsection{New tools for monitoring policy impact and tobacco use surveillance}

FO-362-4 Smokeless tobacco use among adults in ten countries of SEA region

FO-363-4 Tobacco surveillance through electronic data collection on the Android

operating system - evidence from the Global Adult Tobacco Survey ....

FO-364-4 Using discrete choice experiments to characterize demand for waterpipe

tobacco smoking among university students in the Eastern Mediterranean region..

FO-365-4 Changes over time in illicit cigarette use in Brazil, overall and by educational level: proposed novel approach and results ......... 192

FO-366-4 Flavor capsule cigarettes in six countries: availability by brand, variant and flavor......................................................193

FO-368-4 Helping and harming: evaluating school tobacco programs and policies after 4 years in the COMPASS study................. 193

LB-1324-4 The Tobacco Control Policy (TCP) Tool: a web-based interface

to simulate the effects of tobacco policies in the United States

LB-1325-4 Does change in perception of tobacco risk affect smokers' behaviour? - An empirical analysis.....

\subsection{Tobacco dependence treatment and smoking cessation: Innovations in Practice and Opportunities for scaling}

RF-1235-4 Who is more likely to have a quit intention in Brazil's major cities? Findings from the ITC Brazil Wave 3 Survey .......... 194

RF-1236-4 Self-efficacy predicts the outcome of smoking cessation attempts ..... 
RF-1238-4 Feasibility and acceptability of e-cigarettes as an aid to quitting smoking among lung cancer patients: a pilot study........ 195 RF-1239-4 Creating the demand for tobacco cessation - an innovative approach to attract tobacco users contemplating to quit...... 196 RF-1240-4 What keeps healthcare professionals from advising their patients who smoke to quit? A large-scale cross-sectional study ....... 196 RF-1241-4 Encouraging physical activity for smokers: benefits beyond expectation ............................................................... 196

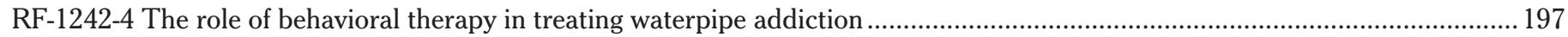
RF-1243-4 Roles of community pharmacists in providing smoking cessation services: a national survey in Thailand ...................... 197 RF-1244-4 The impact of nicotine metabolite ratio on pharmacotherapy effectiveness in real-world settings: a longitudinal analysis......... 198 RF-1245-4 Advice from healthcare providers and cessation aid utilization among current smokers from 28 countries: findings from the Global Adult Tobacco Survey (GATS), 2008 - 2015 ...................................................... 198 LB-1329-4 Systematic delivery of a smoking cessation intervention after discharge from a secondary care setting.......................... 198 LB-1330-4 Introducing SmokeBeat ${ }^{\mathrm{TM}}$ - an innovative cigarette smoking behavior monitoring solution.............................................199

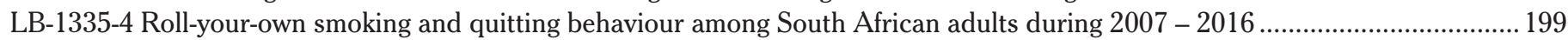

\subsection{Tobacco and Women: The gendered nature of tobacco}

LB-1350-5 Women's health, equality and empowerment in tobacco farming - findings from two counties in Yunnan, China............ 199 LB-1351-5 Labor input of women tobacco farmers in Tabora, Tanzania .............................................................................200 LB-1352-5 Smokeless tobacco, associated risk factors and women health: the study of garment workers in urban Mumbai, India...200 LB-1355-5 Women's awareness about toxic effect of tobacco consumption on health in India: Are they intending to quit too?........ 201

\subsection{Tobacco in vulnerable populations (e.g. indigenous populations)}

RF-1237-4 Smoking cessation practices among ethnic minorities. A case for ethnically-tailored smoking cessation services in Lagos State, Nigeria LB-1346-5 Attitudes towards implementing tobacco-free-schools in vocational schools

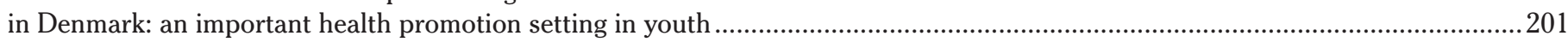

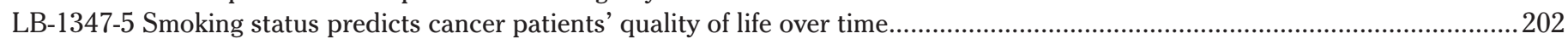

LB-1349-5 Withdrawal symptoms upon a short motivational 26-hour smoking abstinence program in psychiatric patients .............202

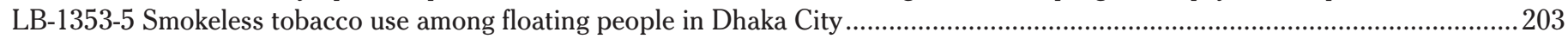

LB-1354-5 Investigating the practices of tobacco smoking in HIV-infected patients

attending HIV health centers in the Western Cape Province, South Africa: patterns and associated risk factors

\subsection{Tobacco, inequality and poverty}

LB-1348-5 Individual cost of smoking in a study population .203

LB-1358-5 Perceptions of plain tobacco packaging among adolescents and adults: DePICT, a French national survey ... 204

\subsection{Tobacco farming: Opportunities and experiences in facilitating alternative livelihoods and preventing tobacco adverse environmental impact}

LB-1321-5 An investigative journalism perspective on tobacco control in Tanzania .................................................................204

LB-1356-5 "I know the harm of growing this deadly crop but I am forced to continue" - Tobacco farmers' perspective from India.............204

LB-1357-5 Where do tobacco farmers live: association between Kenyan tobacco farmers' livelihood selection decision and their accessibility to nearby economic centers...

\subsection{Youth tobacco experience}

FO-349-5 Perceptions of cigarette and snus packages among youths and young adults in Norway ......

FO-350-5 The impact of a tobacco point-of-sale display ban on youth in the United Kingdom:

findings from a repeat cross-sectional survey pre-, mid- and post-implementation ...............................................................206

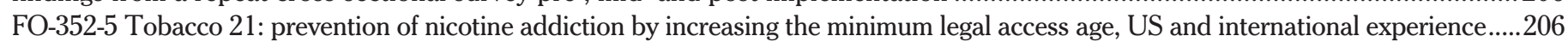

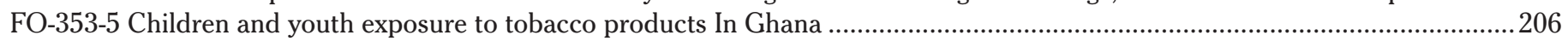
FO-354-5 Tobacco-free generation campus initiative: a program to increase the number of tobacco-free colleges in the United States...... 207 RF-1222-5 Differences in rates of tobacco product use among U.S. Hispanic youth ..................................................................207 RF-1223-5 Multilevel analysis of the individual and contextual factors of tobacco use among Mexican adolescents using the National Addiction Survey (NAS), 2011.

RF-1224-5 Youth attitudes and beliefs towards cigarette and waterpipe use in nine Middle Eastern Countries ...............................208

RF-1225-5 Testing the efficacy of a school-based tobacco and supari cessation intervention in Mumbai, India...............................208

RF-1226-5 Tobacco use among high schoolchildren: social and behavioral determinants in the Indian context ...............................209

RF-1227-5 Identifying misleading propaganda of Electronic Nicotine Delivery Systems (ENDS)

and hookah in New Delhi, India: building youth self-efficacy skills to resist new industry tactics ..................................................209

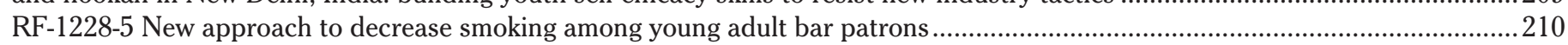

RF-1229-5 The growth of Roll-Your-Own (RYO) cigarettes in Ireland: exploring the experiences of young people and RYO tobacco.......210

RF-1230-5 'You do it through the grapevine'. A qualitative study of how young people in Europe access cigarettes ........................210

RF-1231-5 Youth who initiate tobacco use with e-cigarettes only differ

from those who start with cigarettes only or both products concomitantly... 
RF-1232-5 Smokefree sports grounds contribute to a smokefree generation in the Netherlands.

RF-1233-5 Universal coverage of comprehensive school-based tobacco control programs to reduce youth smoking in Seoul, Korea ........ 212

LB-1336-5 Prevalence and knowledge on e-cigarette among teenagers in Romania 2016 .........................................................212

LB-1341-5 Tobacco free duo - 25 years of experience from an evidence-based method to prevent tobacco use amongst young people...... 212

LB-1342-5 Active and passive tobacco smoking among students of the University of Yaoundé I, Cameroon...................................213

LB-1343-5 Tobacco use: the most influential risk factor on cannabis use among middle schoolchildren in Sousse, Tunisia ............. 213

LB-1344-5 Electronic cigarette use and conventional cigarette smoking initiation among youth, United States, 2015-2016............. 213

LB-1345-5 Still unhooking the "Hookah-ed" a comparative study of perceptions of Hookah amongst the young adults ................... 214

\section{Friday, 9 March 2018}

\subsection{Priorities and challenges for advancing the WHO FCTC}

FO-387-1 Smokeless tobacco in Central Asia: working towards an effective regulatory framework for nasvai in Tajikistan ..............214

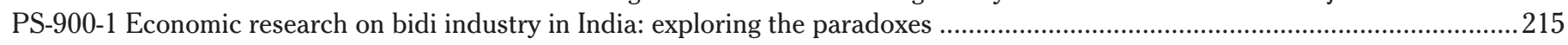

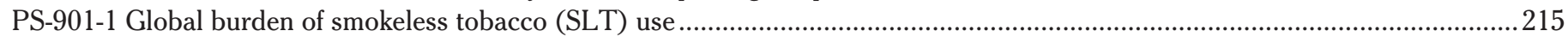

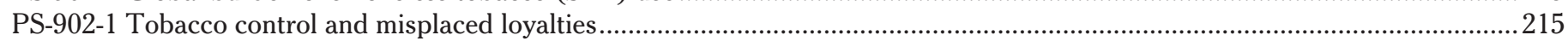

PS-903-1 Strengthening the implementation of the FCTC with a special focus to youth ...........................................................216

PS-904-1 Cigarette sale within 100 meters of school boundaries in metropolitan Manila, Philippines from June to August, 2016 .... 216 PS-905-1 Strategic prohibition of tobacco sale within 100 meters radius of health facilities

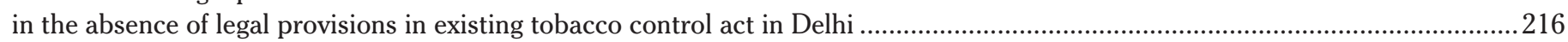

PS-906-1 Challenges and lessons learnt from a workplace based program to control tobacco use in Tunisia 2009-2014 .................217

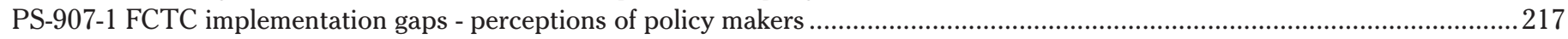

EP-268-1 Analyzing commitments under the WHO framework convention

on tobacco control using the TAPIC governance framework.

EP-274-1 The progress and challenges of implementation of the Framework Convention

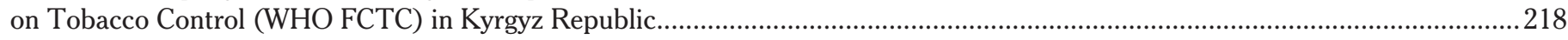

EP-275-1 Policy perspective on the global use of Smokeless Tobacco (ST): a literature review ...................................................218

EP-276-1 Global policy progress in Article 13 of World Health Organization's

Framework Convention on Tobacco Control (WHO FCTC) on Cigarettes (CIG) and Smokeless Tobacco (SLT).

\subsection{Integrating tobacco control into Health systems, global frameworks and strategies to reach WHO FCTC and SDG's targets.}

PS-908-1 Building momentum for adoption of UP-state policy on lines of WHO FCTC Article 5.3 .....

PS-909-1 Enhancing nursing competency to incorporate smoking cessation interventions

into standard nursing practice for patients with non-communicable diseases...

PS-910-1 Some cellular inflammatory characteristics in patients with chronic obstructive

pulmonary disease (COPD) with anterior tuberculosis compared to tobacco related COPD

PS-911-1 Create a public opinion on community based organization to implement Pictorial Health Warnings .................................2220

PS-912-1 Compability of Polish National Tobacco Control Program and cancer control strategy: results of a Delphi study.............220

PS-913-1 Practice of tobacco free environment among restaurant workers and owners in Kota Samarahan, Sarawak, Malaysia .......221

PS-914-1 Analysis of the effect of bidi regulations and its influence on tobacco control in India ...................................................221

PS-915-1 A dedicated implementation mechanism can eliminate the tobacco from the lives of human being ..................................221

PS-916-1 Exposure to the number of tobacco control policies and tobacco use cessation among adults in Sub-Saharan Africa ........222

EP-265-1 Effective way to prevent tobacco consumption of husbands through public health midwives ..........................................2222

EP-269-1 Ethiopian health care delivery system's responsiveness to smoking cessation therapy

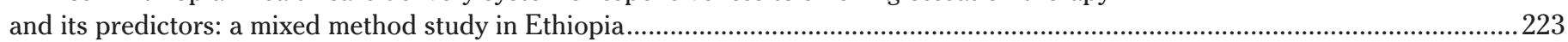

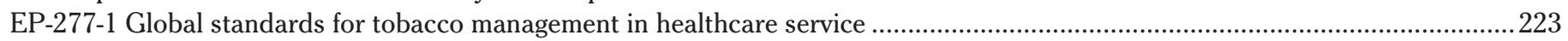

\subsection{Using data : Opportunities and gaps in measuring WHO FCTC impact on global health and development}

PS-917-1 Tobacco Control Scale website: a new tool for tobacco control advocates and researchers ...........................................2223

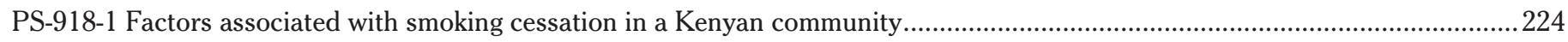

PS-919-1 Monitoring initiation of daily smoking as an indicator of tobacco control success: example of Ukraine............................224

PS-920-1 International law, national policies and youth smoking: an examination of the Framework Convention on Tobacco Control.......225

PS-921-1 China's smoking burden under business as usual and two replicable proven experiences from Taiwan and Hong Kong............225

EP-264-1 Impact of the reduction of smoking prevalence on the trend of lung cancer mortality in Brazil .......................................2225

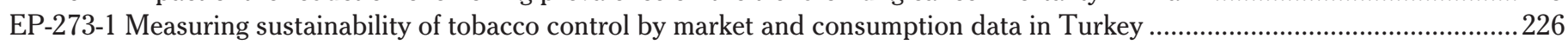

LB-1369-1 An empirical analysis of the impact of income change

and cigarette taxation in a price-tiered cigarette market of Bangladesh

\subsection{Setting the research agenda for translating science to policy and new tools for policy impact assessments}

PS-923-1 Knowledgebase of smokeless tobacco products and their chemicals .......................................................................227

PS-924-1 Consistency of self-report of smoking status among Korean young women....... 
PS-925-1 Prioritizing a global research and policy agenda for Betel Quid (BQ) and Areca Nut (AN)

\subsection{The End Game - Pathways to turning vision into reality.}

PS-926-1 Licensing tobacco vendors in the state of Himachal Pradesh in India - challenges,

opportunities and the way forward to implement the new legislation

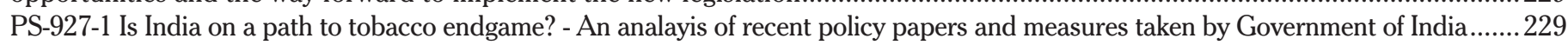

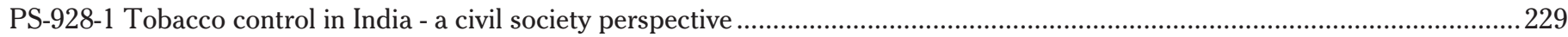

PS-929-1 Prohibition of importation, manufacturing and sale of Smokeless Tobacco (SLT) products: what is the Global Scenario? ........230

PS-930-1 Policy action for health professionals involvement in tobacco control...........................................................................2.231

PS-931-1 Understanding the tobacco retail environment; next frontier for UK tobacco control .....................................................231

PS-932-1 Critical analysis of hookah smoking samples to formulate public health strategy

to protect younger generations in Delhi, India

PS-933-1 Banning loose cigarette sale-a bold supply side measure by State Government

of Himachal Pradesh in India to decrease tobacco use.

PS-935-1 Sweden's pathway to Europe's lowest level of tobacco-related mortality …..............................................................232

EP-267-1 Leveraging public finance to achieve endgame for tobacco - possible national and global strategies .............................. 232

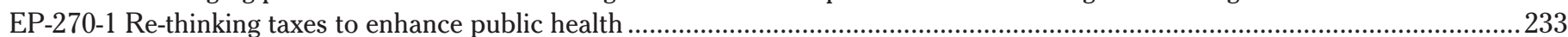

\subsection{Promoting transnational cooperation and Partnerships: models of Intergovernmental and nongovernmental collaboration across multiple sectors for high level commitments}

RF-1266-2 The role of social listening in promoting 100\% smoke-free law in Shanghai

RF-1267-2 Collaboration between government and civil society: engaging the public

in the implementation of Beijing's smoke-free law through integrated communications efforts.....

RF-1268-2 Bidi in demerit category (28\%) in Goods Service Tax (GST) was a big win in India

PS-936-2 Assess compliance to existing Tobacco Control Law among task force (TF)

committee members across 10 districts in Bangladesh

PS-937-2 The role of non-governmental organizations in tobacco control in Romania...............................................................2235

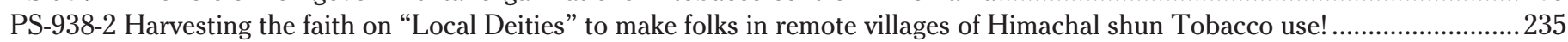

PS-939-2 Encouraging tobacco control using national multisectoral ministerial mandate and priorities in Indonesia.......................235

PS-940-2 Government's pro-public health policies and effective coordination is key

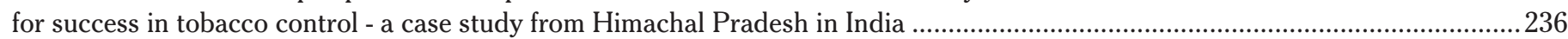

PS-941-2 South-south cooperation on tobacco control - Project Plan on Article 5.2. National Coordination Brasil - Colombia ........236

EP-190-2 Advocacy for a strong tobacco tax policy - experience and lessons of an INGO in Vietnam ...........................................237

EP-262-4 Shutting down of Shisha bars by the effective use of current legal provisions - a case series from Punjab, India ............... 237

\subsection{International tobacco trade and health diplomacy: understanding the nexus between the two and domestic international disputes}

PS-942-2 The Pacific Alliance, a threat to tobacco control? Analysis of an "under the radar" trade pact.

\subsection{Building leadership capacity and broader alliances for tobacco control policy advocacy}

RF-1258-2 Engaging new voices and key opinion leaders in support of smoke-free policies in China ..........................................238

RF-1259-2 Engage the business sector to promote smoke-free corporate culture through industry-oriented programmes.................238

RF-1260-2 Getting president's attention to reject tobacco bill through social media in Indonesia .................................................2239

RF-1261-2 Human rights based approach to tobacco control as an effective tool

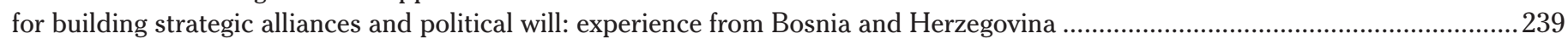

RF-1262-2 Role of media in disclosing tobacco industry interferences and building

policy pressure in implementation of $90 \%$ pictorial health warning ...

RF-1263-2 The North East of England successfully uniting as a region

behind the campaign for the implementation of standardised packaging..

RF-1264-2 International collaboration to build tobacco control capacity:

a case study of KOMPLY from the World Heart Federation Emerging Leaders program............................................................240

RF-1265-2 Using innovations to enable public monitoring and enhance enforcement of Beijing smoking control regulation ............240

PS-944-2 Advocacy for mainstreaming and sustainable implementation of Tobacco Control Law in Bangladesh ..............................241

PS-945-2 Correlates of MPOWER policies and tobacco smoking - an analysis of WHO South East Asia regional countries .............241

PS-946-2 From press conferences to media dialogues, Cameroon learned to be more effective in relaying tobacco control messages.........242

PS-947-2 Engaging health and non-health sectors to advance progress on tobacco control..........................................................242

PS-948-2 Investigation report on public attitude towards carpet smoking ban in ten cities of China ...............................................243

PS-949-2 Getting involved: Uganda cancer society's efforts to build strategic

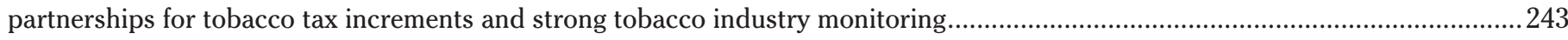

PS-950-2 Change in knowledge of tobacco use and secondhand smoke exposure among health workers in Uganda...........................244 
PS-951-2 Strengthening National Tobacco Control Program (NTCP) to advance tobacco control (TC) policy in Uttar Pradesh (UP) .... 244 PS-952-2 How we won the sin tax law: the Philippine sin tax story in social media messages

PS-953-2 Paving way for tobacco free schools towards building leadership

and broader alliances for implementing tobacco control policy.....

PS-954-2 Tobacco Free Network - the actions of tobacco users and survivors:

advocacy on enforcing tobacco control legislation and behavioural change in Tamil Nadu, India ...............................................245

PS-955-2 Contested evidence, contested policy? Evidence use in the development of Scottish e-cigarette policy .............................245

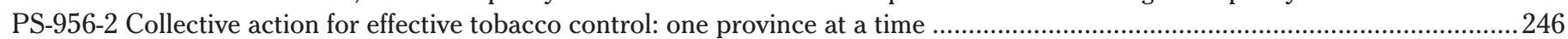

PS-957-2 Media as advocacy tool to prioritize tobacco control as component

of public health policy in a state with high penetration of other substances of abuse ..................................................................246

PS-958-2 Institutionalization of Indian Tobacco Control Programme at Grassroot level - experiences

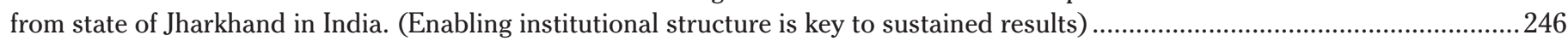

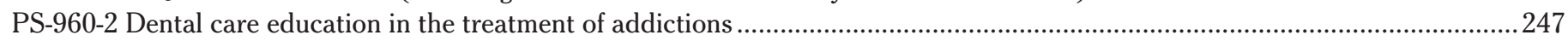

PS-961-2 Reflections on 50 years as an anti-smoking activist: the danger of the single narrative .................................................247

PS-962-2 Plaidoyer pour l'adoption d'une loi forte en Mauritanie par la mobilisation des communautés et des parlementaires........248

EP-194-2 Harnessing youth power in making Myanmar tobacco-free .......................................................................................248

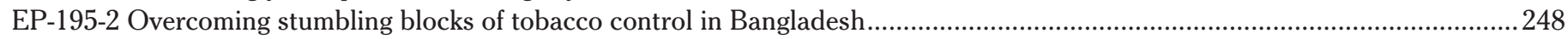

EP-196-2 Community of practice on tobacco control to health inspectors of Brazilian Sanitary Surveillance System:

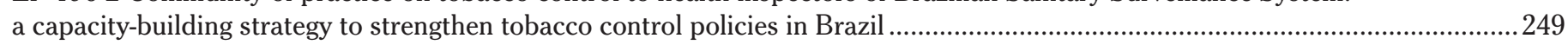

EP-197-2 Development of an omnibus policy for tobacco control in the Philippine department of health......................................249

EP-198-2 Systematic research capacity building program makes a big difference ...................................................................250

EP-199-2 Mobilizing public opinion for a political commitment for a Tobacco Endgame strategy - obstacles and possibilities .........250

EP-200-2 Tracking the health interest of the Sin Tax Reform Law: thru the development

and implementation of a Sin Tax Monitoring and Evaluation Framework .

EP-201-2 Mathiwos Wondu-YeEthiopia Cancer Society (MWECS) experiences

in involvement of Ethiopian Tobacco Control Policy Advocacy.......

EP-202-2 Dutch municipalities on their way towards a smokefree generation ...

EP-203-2 Investment and getting back the reward: training different

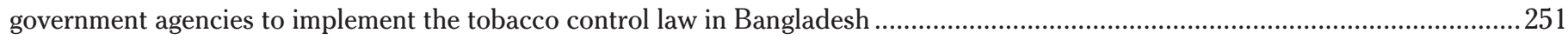

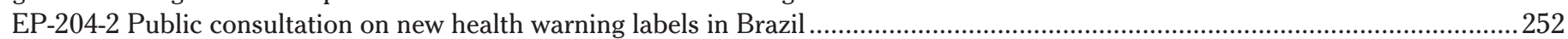

\subsection{Tobacco industry interference: New tactics and counter measures}

RF-1269-2 Protecting local Government Public Health Policy from vested interests.....

PS-963-2 Countering tobacco industry interference/tactics in implementation of $85 \%$

pictorial health warnings (PHWs) on all tobacco packs through policy and political advocacy ...................................................253

PS-964-2 The method used to control the tobacco consumption in the post war period in Sri Lanka ............................................253

PS-965-2 New companies act in India and Corporate Social Responsibility (CSR)

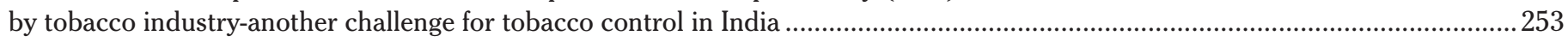

PS-966-2 Tobacco industry interference with the 2017 Thai Tobacco Product Control Act.......................................................254

PS-967-2 Tobacco industry interference challenging public health in India; time for the implementation of FCTC Article 5.3 …........254

PS-968-2 Print media study on communicate misleading information by tobacco industry..........................................................255

PS-969-2 Tobacco industry targeting Sri Lankan non-tobacco-growing farmers in corporate social investments............................255

PS-970-2 Monitoring and exposing tobacco industry tactics around Conference of Parties (COP 7) .............................................255

PS-971-2 Advocacy for a mobile court for ban on TAPS by Japan Tobacco International (JTI)

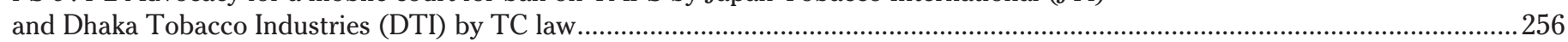

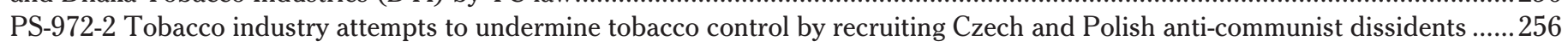

PS-974-2 Tobacco industry's use of litigation to stop tobacco control measures in Brazil ...............................................................256

PS-975-2 Declaring schools tobacco free: protecting young generation to save Nation

(multipronged coordinated interventions to declare 3517 schools tobacco free in Jharkhand state in India) ...................................257

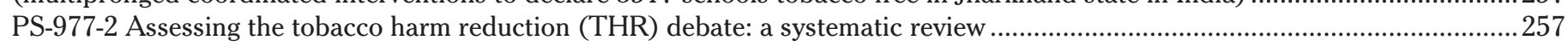

PS-978-2 Confronting the challenges associated with teaching tobacco harms to students

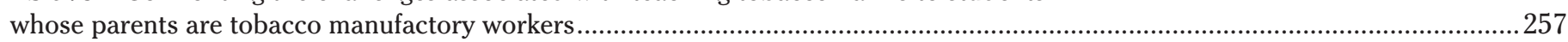

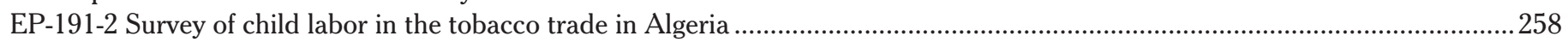

EP-192-2 Cartography monitoring of tobacco industries in Côte d'Ivoire ...................................................................................258

EP-193-2 The right of governments to enact public health laws - the Brazilian tobacco additives ban case …...................................259

LB-1361-2 Passing FCTC compliant tobacco control legislation amid conspicuous industry interference: the case of Senegal............259

B-1370-2 Brazil responses to the strategies used by the tobacco industry to resist Tobacco Control National Policy ………............259

\subsection{Implementing sustainable funding mechanisms for tobacco control}

PS-979-2 Tracking investments by financial institutions in tobacco companies

(2007-2016) - what tobacco control advocates need to know and do about it?.....

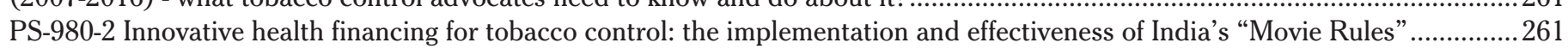

PS-981-2 Imposing 1\% health development surcharge (HDS) to protect

public health due to public demand through strong policy advocacy. 


\subsection{Pricing, Taxation and countering illicit trade: Innovations and reform strategies}

PS-983-3 Political battle in increasing tobacco tax to support Universal Health Coverage in Indonesia........................................262 PS-984-3 Indigenous and non-indigenous experiences and views of tobacco tax increases: findings from the ITC New Zealand Survey ...............263 PS-985-3 Proxy purchasing - a project to determine the awareness of the public in England of a new offence and improve practice .........263 PS-986-3 The prevalence of illicit cigarette consumption and related factors in Vietnam: results from GATS 2015 ........................264 PS-987-3 Expected cigarette price premium on willingness to quit among men smokers in Vietnam ............................................264

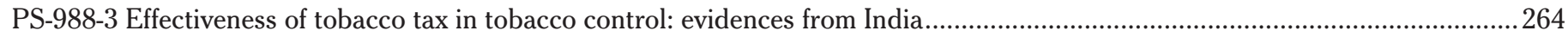
PS-989-3 Media advocacy for building support for taxation of all

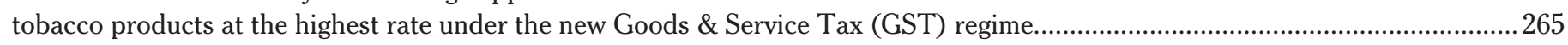
PS-990-3 Public support on increasing cigarette prices and excise to finance UHC in Indonesia ...................................................265 PS-991-3 Neoliberal policy impact: supply-side growth and emergence of duality in Turkish tobacco product market .....................265

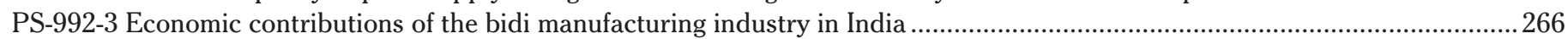

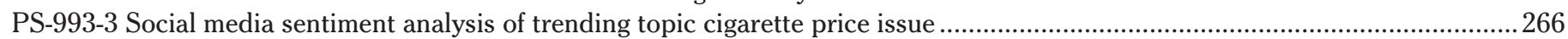
LB-1359-3 The economics of tobacco control in Nigeria: modelling the fiscal

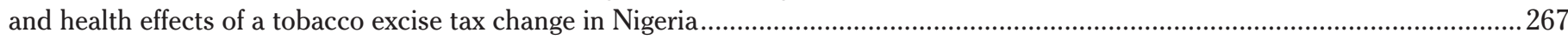
LB-1365-3 Burden of disease attributable to tobacco use and impact of different taxation schemes in Latin America ......................267 LB-1373-3 Affordability of cigarettes products in the WHO Report on the Global Tobacco Epidemic, 2017 ................................268

\subsection{Advances in protection from tobacco pollution, second- and third-hand smoke}

PS-1000-3 Achieving the very first successful smoke free city in Myanmar: a case study of Shan State ........................................268

PS-1001-3 Knowledge on harms of thirdhand smoking is associated with greater support on smokefree policies ............................268

PS-1002-3 Increasing smoke free public places \& creating tobacco free educational institutions .....................................................269

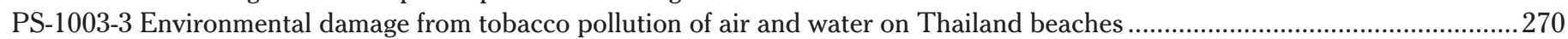

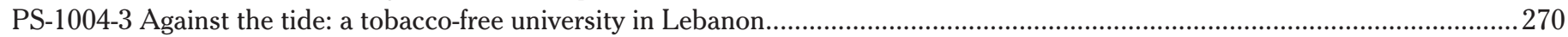

PS-1005-3 Implementing comprehensive tobacco control polices in medical

education institutions and associated teaching hospitals - a case study from a northern state in India .........................................270

PS-1006-3 Malaysian health promotion board (MySihat) smoke-free city initiative ..................................................................2. 271

PS-1007-3 Effective way to prevent tobacco consumption of husbands through pregnant mothers ...............................................2.271

PS-1008-3 Social inequalities in exposure to secondhand smoke in households with children under 12 in Spain...........................271

PS-1009-3 Outdoor hospitality venues: a real challenge for tobacco control policies ...................................................................2.272

PS-1010-3 Protection from exposure to second hand SLT use in public places - spitting a public health concern or a public nuisance? ....2272

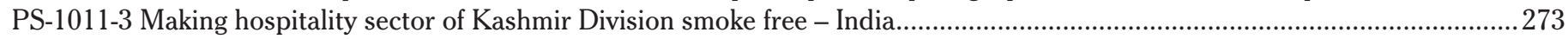

PS-1012-3 Multilevel intervention in reducing in-home secondhand smoke exposure

among pregnant woman in North Lombok district, Indonesia.

PS-1013-3 The association between the awareness of the effects of smoking/secondhand smoke and the desire to quit ...................273 PS-1014-3 Implementing smoke-free laws in low- and middle-income countries:

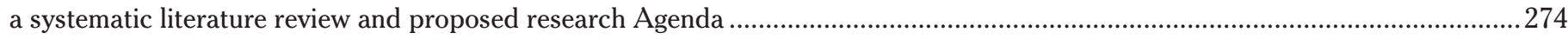

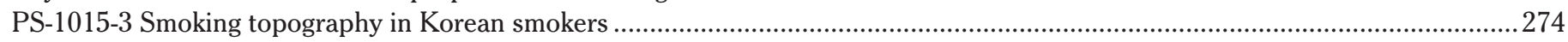

PS-994-3 Second-hand smoke exposure in private and public places in Italy: data from the TackSHS pan-European survey ...........274

PS-995-3 Passive exposure to electronic cigarette aerosol in Italy: data from the TackSHS pan-European survey ..........................275 PS-996-3 Knowledge and risk perceptions of israelis towards combustable cigarettes: the need for immediate remedial action........275 PS-997-3 Advancing towards tobacco-free state: institutionalizing tobacco control programme in Indian state of Bihar..................275

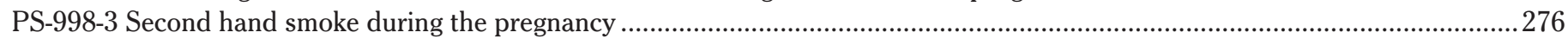
PS-999-3 Comparing particulate emissions between electronic nicotine delivery devices: context for smoke-free indoor air quality............276 EP-253-4 Commercial presentation of waterpipe tobacco poses fundamental impasse

for tobacco control: Web-based search results for Istanbul, 2017

\subsection{Mind the gaps: loopholes in the Ban on Sponsorships, Advertising and Promotions}

PS-1016-3 How compliant is Kolkata Metro to prohibition of tobacco advertisement, promotion and sponsorship legislation? A case study from India.....

PS-1017-3 Assessing tobacco marketing regulation implementation level in Georgia:

evidence from Non Communicable Disease Risk Factors STEPS Survey Georgia 2016 .............................................................277

PS-1019-3 Role of the judge in defending tobacco control measures in Colombia:

total ban on advertising, promotion and sponsorship of tobacco products case .....................................................................278

PS-1020-3 Assessing point of sale violations in Delhi for strategic advocacy on TAPS ban..........................................................278

PS-1021-3 Increase the media contribution to reduce tobacco promotion in Tamil media..............................................................2.278

PS-1022-3 Tobacco advertisements, promotion and sponsorships (TAPS):

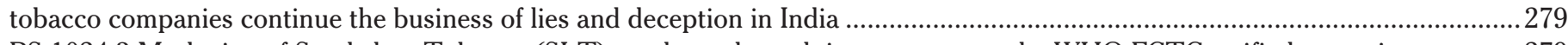

PS-1024-3 Marketing of Smokeless Tobacco (SLT) products through internet among the WHO FCTC ratified countries ...............279

PS-1025-3 Removing tobacco advertisements from all shops in Kashmir division, India .............................................................279

PS-1026-3 Enabling the way from policy to practice realizing the gaps and loopholes in ban on promotion ...................................280 
EP-206-3 Norway introduces standardised packaging on smokeless tobacco.

EP-208-3 Effectiveness of the ban on tobacco industry sponsorship in Brazil:

findings from the ITC Brazil Wave 1 to 3 Surveys (2009 to 2016 - 17).....

EP-209-3 Exposure to electronic cigarette advertising and intention to use electronic cigarettes in Hong Kong adolescents ............ 281

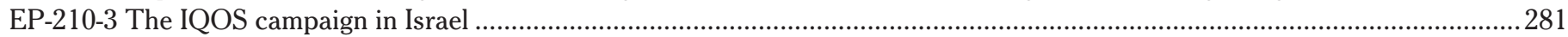

EP-212-3 Retail reform. How conditions of license can be applied to strengthen tobacco control in a range of local circumstances ..........281

EP-213-3 Scottish young people's perceptions of standardised packs - a qualitative study ..........................................................282

EP-214-3 Marketing to the selfie generation: a case study of "A" cigarette brand marketing communication in Indonesia.................282

EP-215-3 Misleading descriptors on cigarette packs in Indonesia, the Philippines

and Vietnam: before and after implementation of misleading packaging regulations....................................................................283

EP-216-3 A global scan of policies regulating e-cigarette advertising, promotion and sponsorship ...............................................283

EP-217-3 Identifying characteristics of cigarette displays at points of sale in Brazil ...................................................................283

EP-218-3 Marketing of flavored cigarettes at the point-of-sale (POS) near schools in 5 Latin American cities .............................. 284

\subsection{Innovations in media educational campaigns and health warning labels on cigarettes and other tobacco products}

PS-1027-3 Real smokers - helping us take on a killer through the media

PS-1028-3 Effect of visual attention according to the position of graphic health warning labels .......

PS-1029-3 Multimedia campaign aimed at increasing support among the Dutch population

for measures to protect children from the temptations of tobacco.

PS-1030-3 Effectiveness of pictorial health warnings on cigarette packs among adults in a rural Sri Lankan community .................285

PS-1031-3 International best practice adopted in China: evidence-based mass media

campaigns to support city-level $100 \%$ smoke free laws

PS-1032-3 Exposing tobacco industry tactics in implementation of 85\% GHW's through media advocacy ...................................286

PS-1033-3 Study on print media behavior at the time of implementing pictorial health warnings in Sri Lanka ..............................286

PS-1034-3 Fight for 85\% pictorial health warnings on tobacco product packages - a success story from India ............................... 287

PS-1035-3 Observational study to check the compliance of implementation of $85 \%$

graphic health warnings on tobacco products in India from April 1, 2016.

PS-1038-3 Building support for the implementation of 85\% GHW's through social media.....

PS-1039-3 Examining the insertion of pictorial health warning on cigarette packets in Tehran city ...............................................28

PS-1040-3 Pre-test of pictorial health warnings on tobacco products to test their effectiveness - study based in India ......................288

PS-1041-3 Celebrities' voice for pictorial health warnings on cigarette packets at 'television opera' award ceremonies ....................288

PS-1042-3 Impact of pictorial warning and advertisement among tobacco users in India.............................................................289

PS-1043-3 Driving behavioural change and building public opinion through effective

partnership and sustained media advocacy for tobacco demand reduction across Delhi-NCR

EP-220-3 Indigenous language as a tool for MPOWER component 4 .....

EP-221-3 Pictorial health warnings on waterpipe tobacco packs: rating of efficacy

in comparison to an alternative waterpipe-specific set.

EP-222-3 Larger picture health warnings on tobacco packages - overview of international momentum and innovative approaches ............290

EP-223-3 Does 85\% pictorial health warning on the tobacco products have the impact among tobacco users in India?

EP-224-3 Evidence of the continuing weak impact of China's health warnings:

longitudinal findings over nine years (2006 to 2013 - 15) from the ITC China project

EP-226-3 From anti-smoking activist to archivist and back again: how museum exhibitions

on the history of tobacco control are helping to educate a new generation.

EP-227-3 Effectiveness of 85\% graphic health warning on cigarette packs on smoking behaviours of Thai teenagers

EP-228-3 Tobacco packaging and labeling in the WHO African region:

progress after the adoption of WHO FCTC's Article 11 guidelines ........

EP-229-3 Exposing the inconvenient truth of tobacco in China: a nationwide exhibition of graphic pack warnings.

EP-230-3 Low knowledge among Zambian smokers and the need for large

pictorial health warnings: findings from the ITC Zambia Wave 2 survey......

EP-231-3 Using social media to mobilize grassroots advocates and raise awareness about tobacco harms in Indonesia .................. 293

EP-233-3 ASEAN: world's first geopolitical region to implement pictorial health warnings ...........................................................294

EP-256-4 Evaluation of health warnings for waterpipe tobacco smoking

among university students in the Eastern Mediterranean region.....

EP-258-4 An evidence based public campaign against waterpipe / hookah smoking in Turkey ...................................................295

EP-263-4 Effectiveness of pictorial health warnings on the waterpipe device and tobacco packs: a qualitative study .......................295

LB-1360-3 South African adult smokers perception of pictorial warnings on quitting behavior.

\subsection{Opportunities for regulating tobacco products' content and emissions to reduce harm and addiction potential}

PS-1044-3 Classification, labelling and packaging of the most popular e-cigarette refill liquids across 9 European countries ...........296

PS-1047-3 Evaluating compliance of labelling on tobacco packets in countries across the Middle East .........................................296

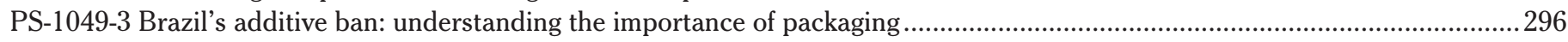

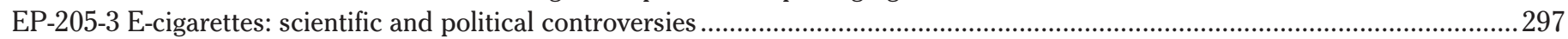

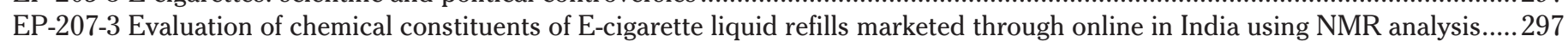




\subsection{Prevalence of tobacco and nicotine-related products' use: Traditional and novel products}

PS-1050-4 How effective is gutka (a smokeless tobacco) ban in West Bengal, India? A case study ...............................................298 PS-1051-4 Spatial pattern of risky health behavior in India: evidence from recent National Family Health Survey $2015-16 \ldots \ldots \ldots . . .299$ PS-1052-4 Trends in smoking prevalence in South Africa for 1998 - 2014 - overcoming the challenge of multiple data sources........299 PS-1054-4 Declining trend of tobacco use in a rural community of Bangladesh, 2006-2013 ......................................................299 PS-1055-4 Current tobacco use and its associated factors among adults in Georgia:

findings from Non-Communicable Disease Risk Factors STEPS Survey Georgia 2016.

PS-1058-4 Social determinants of tobacco smoking in Mexico stratified by sex and age.

Mexico, Global Adult Tobacco Survey (GATS) 2009 and 2015

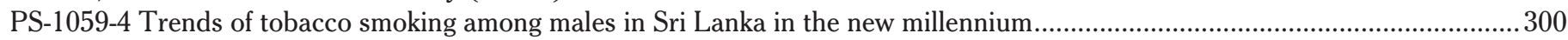

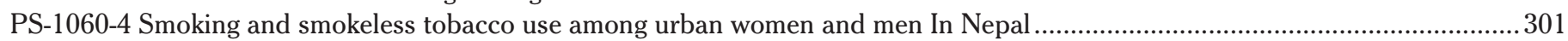

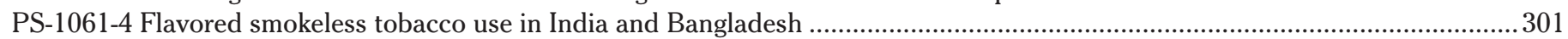

PS-1062-4 The ugly truth about tobacco: a permissive factor for periodontal disease among bus drivers ..........................................301

PS-1064-4 Effects of cigarette tax increase of smoking rate reduction according to income status and residential area.................... 302

PS-1065-4 Tobacco consumption among class III \&IV employees of health care setup in Gujarat: a cross sectional study ............... 302

PS-1066-4 Electronic Nicotine Delivery System (E-cigarettes) marketing,

sale and availability - an emerging challenge for tobacco control in India.....

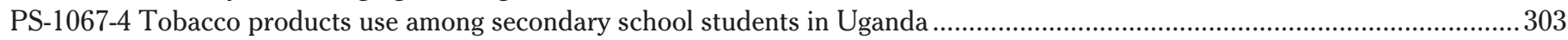

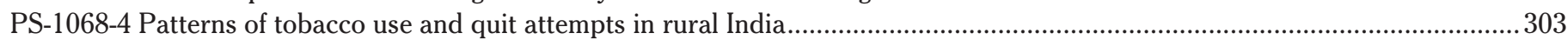

PS-1069-4 Tobacco use among young Qatari population: profile, prevalence and predictors........................................................303

PS-1070-4 The comparative analysis of Global Youth Tobacco Survey 2003, 2008, 2014 in the Kyrgyz Republic.......................... 304

PS-1071-4 Tobacco use and interest in quitting among patients hospitalized in Mumbai, India ....................................................304

PS-1072-4 Prevalence and correlates of tobacco smoking, smokeless tobacco

and passive smoking in Georgian adults: results of non-communicable disease risk factors STEPS survey Georgia 2016 ................ 304

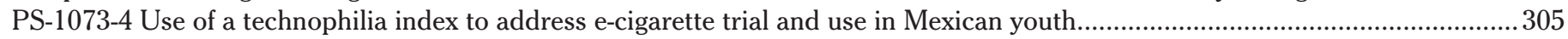

PS-1074-4 Nicotine dependence and readiness to quit tobacco among past and present

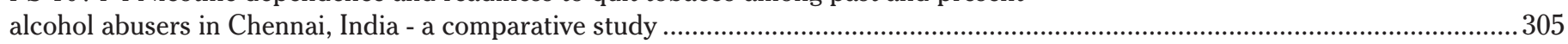

PS-1075-4 The prevalence of oral conditions and tobacco use among dental patients at princess Marina Mental Clinic, Botswana ...............305

PS-1076-4 E-cigarettes use behaviour, perceptions and barriers among Indian adults: pilot qualitative research study ................... 306

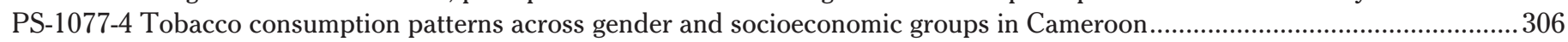

PS-1078-4 Demographic and socioeconomic determinants of smoking in the Biyem-Assi health district, Yaoundé, Cameroon......... 306

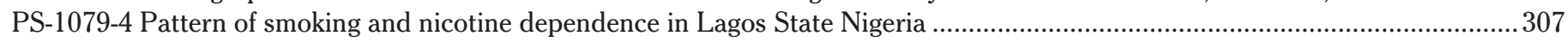

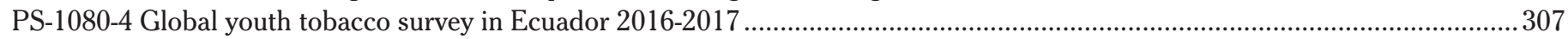

PS-1081-4 Comparison of concomitant use of water pipe and cigarette to exclusive cigarette smoking...........................................307

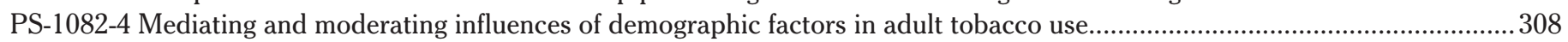

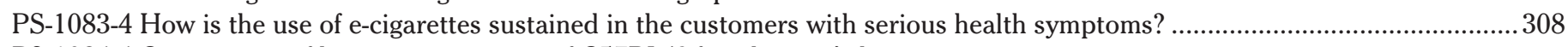

PS-1084-4 Comparison of long-term exposure of C57BL/6 female mice's lungs

to three different types of cigarette smoke available in Brazil

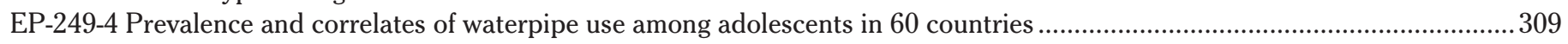

EP-252-4 Evaluation of concurrent use of tobacco products (water pipe and cigarettes) and associated factors in adults in Tehran ...........309

EP-254-4 Water-pipe tobacco (shisha) use among undergraduate health professional

students - College of Health Sciences, Nairobi University Kenya, $2014 .$.

EP-255-4 Reasons for use, potential use, or discontinued use of hookah among us young adult college students.............................310

EP-257-4 Waterpipe (Shisha) smoking: pattern and predictors of use among University of Ibadan undergraduate students ............. 310

LB-1367-4 Knowledge and perception of waterpipe harm among university students

in five Eastern Mediterranean countries: a cross sectional study .....

\subsection{Burden and Disparities in tobacco-related diseases: Non-communicable diseases (NCDs) and communicable diseases (TB and IIIV/AIDS)}

FO-390-4 Smoking and HIV in sub-Saharan Africa: a 25 country analysis of the demographic and health surveys ..

FO-391-4 Tuberculosis remains the leading cause of death attributed to smoking

in South Africa: results from the South African death certificate study.

FO-392-4 Estimation of the impact of tobacco smoking factor on the morphological,

molecular and genetic parameters of the lung cancer tumor.....

FO-393-4 Tobacco exposure in adolescence and the risk of nasopharyngeal carcinoma:

preliminary results from a multi-center case-control study in Hong Kong, China .....

FO-394-4 Differences in African American and White mortality caused by cigarette smoking in the U.S..................................... 313

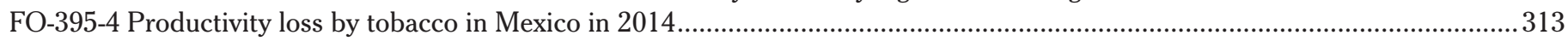

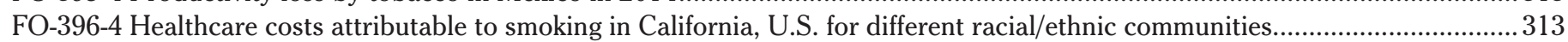

PS-1085-4 The association between tobacco use and lung cancer risk: difference caused by smoky coal use ................................... 314 
PS-1086-4 Tobacco and cancers - burden and sites of cancers associated with the use of tobacco in India .........

PS-1087-4 Association between smoking, tuberculosis and diabetes-TB: an analysis on Indian TB patients

PS-1088-4 Global Bridges Healthcare Alliance for Tobacco Dependence Treatment:

implementing evidence-based smoking cessation treatment in mental health and addiction care units in Brazil and Portugal.......... 315

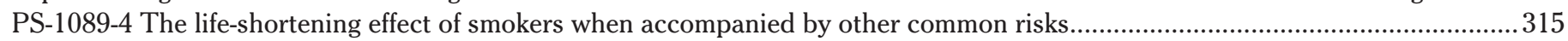

PS-1090-4 Smokers with a high normal heart rate $(80-99 / \mathrm{min})$ found their life span shortened by 13 years ................................... 316

PS-1091-4 Mind the gap: cigarette smoking rate is higher among adolescents and young adult males

with HIV than those without HIV in sub-Saharan Africa...

PS-1092-4 National framework on TB-Tobacco collaborative activities: most cost-effective way

to manage TB-Tobacco comorbidity and extend tobacco cessation services ......

PS-1093-4 Tobacco use and presumptive tuberculosis in Uganda: findings

from the Uganda National TB Prevalence Survey 2014 - 2016..

\subsection{New tools for monitoring policy impact and tobacco use surveillance}

PS-1094-4 Compliance with indoor and outdoor smoke-free ban in Catalan hospitals: what do patients think? .............................317

PS-1095-4 Interest in electronic cigarettes in 5 mayor Spanish speaking countries in Latin America ............................................318

PS-1096-4 A proportional mortality study on smoking and lung cancer using different causes of deaths for dead controls .............. 318

PS-1097-4 Online informatics system of key outcome indicators to support tobacco control planning, surveillance and reporting ............ 319

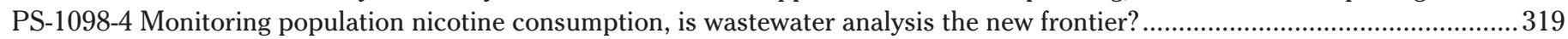

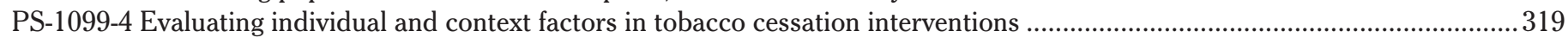

PS-1 100-4 Influence of recent tobacco control policies and campaigns on Quitline call volume in Korea ......................................3320

PS-1101-4 Tobacco retail and publicity at points of sale (PoS) around schools in three major cities in Mexico (2014-2016) ...........320

EP-251-4 Global reporting of waterpipe tobacco policy in online news articles in 2015: a cross-sectional analysis .......................... 321

\subsection{Tobacco dependence treatment and smoking cessation: Innovations in Practice and Opportunities for scaling}

FO-397-4 Sustaining a system wide intervention to promote increased smoking cessation rates among pregnant women...

FO-398-4 Impact of a tobacco cessation intervention on adherence

to tobacco use treatment guidelines among village health workers in Vietnam.

FO-399-4 Healthcare providers' concerns regarding smoking cessation

pharmacotherapies in pregnancy: calls to a teratology information service .....

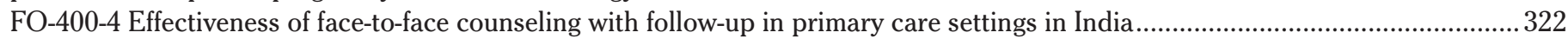

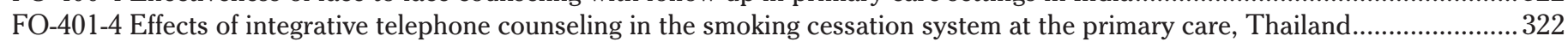

FO-402-4 Feasibility of an integrated complex intervention targeting tobacco

and alcohol cessation among patients with tuberculosis in three South African provinces

PS-1 102-4 Dental specialist trainees' competence in instituting tobacco cessation services in Nigeria........

PS-1 103-4 Tobacco Control Training Programme for health professionals

of the Catalan Network of Smoke-Free Hospitals: 11-year experience .....

PS-1104-4 Physical activity was associated with unassisted quitting:

cross-sectional and prospective findings from the Hong Kong Population Health Survey.

PS-1 105-4 Stoptober: an effective way for smokers to quit for 28 days, with five times more chance to stop permanently................ 324

PS-1 106-4 Inter professional initiative using service training to enable a fisher folk community to quit tobacco habits ..................... 325

PS-1107-4 Tobacco consumption prevalence among nursing students and their knowledge in tobacco addiction: ECTEC study .... 325

PS-1108-4 Effects of SMS to quit on quit rate and satisfaction

among smokers calling Thailand National Quitline (preliminary analysis)

PS-1109-4 A follow up of activity and clinical experience among Swedish tobacco cessation counsellors 1-12 years after training .............326

PS-1110-4 Could be applied genetic markers to smoking cessation treatment? ..........................................................................326

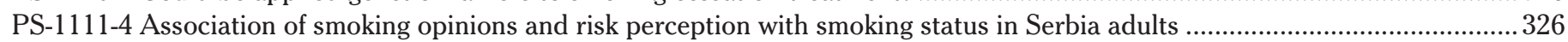

PS-1112-4 Impact of smoke-free home on smoking intensity in low- and middle-income countries................................................3 327

PS-1113-4 Incentive-based interventions for smoking cessation: early findings from the SMILE Trial in Thailand.......................... 327

PS-1114-4 A factor analysis of the Fagerstrom Test for Nicotine Dependence

for bidi smokers and smokeless tobacco users (FTND): findings from India.....

PS-1115-4 Systems approach in tobacco dependence treatment through hospitals - a one year study from Jaipur, India .................. 328

PS-1116-4 Characteristics of clients with nicotine dependence and short-term abstinence:

findings from the USM Tobacco Quitline Service in Malaysia ...

PS-1117-4 GB smoking cessation training for health care providers in LA:

midterm confidence in competencies and estimation of population impact....

PS-1118-4 Strengthening quit smoking services in Malaysia through Malaysia Quit (mQuit) Program.........................................329

PS-1119-4 Cessation attempts in dual users (smoking plus smokeless): findings from two states in India...................................... 330

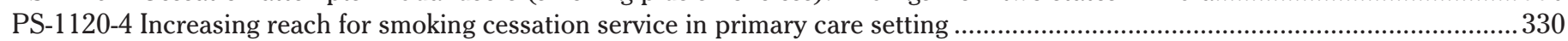

PS-1121-4 Introducing tobacco free workplace policy in workplaces in India - challenges and learning ..........................................330

PS-1122-4 Why are male Chinese smokers unwilling to quit? - A cross-sectional study

on smoking rationalization and intention to quit....

PS-1123-4 An examination of tobacco initiation and its implications for tobacco control strategies...

PS-1124-4 Development of an evaluation tool for smoking cessation clinics and services...... 
PS-1125-4 Explore to operating mode of smoking cessation clinic services in China.....

PS-1126-4 Effectiveness of a brief, self-determination intervention for smoking cessation

(immediate or progressive) among people attending emergency departments: a randomised controlled trial......

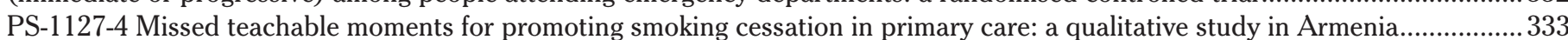

PS-1 128-4 The overlooked role of physical activity in mitigating the mortality risk in smokers and nonsmokers with COPD ........... 333

PS-1129-4 Social environmental factors as predictors of late relapse: prospective follow up results

among Quitline users in Republic of Korea....

PS-1130-4 Using synergised social media approaches to support tobacco control policy

and cessation related behaviours in Bangladesh......

PS-1 131-4 Results of one year monitoring of the National telephone quitline on smoking cessation in the Kyrgyz Republic ............ 334

PS-1132-4 Who should have the strongest quit smoking willingness and much easier to persuade?................................................335

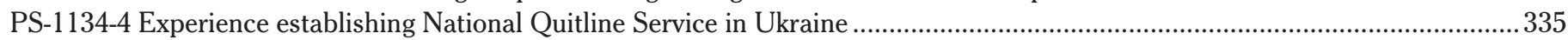

PS-1135-4 Child hospitalization due to respiratory illness: an opportunity for parental/family smoking intervention....................... 335

PS-1136-4 An innovative smoking cessation program using Facebook reaching 7'000 Swiss smokers ...........................................336

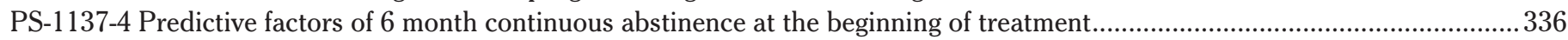

PS-1138-4 Exposure to secondhand smoke as a risk factor for severe tobacco smoking among young healthy men in South Africa .......... 336

PS-1139-4 Bridging the gap: challenging attitudes towards smoking in pregnancy among healthcare professionals ......................... 337

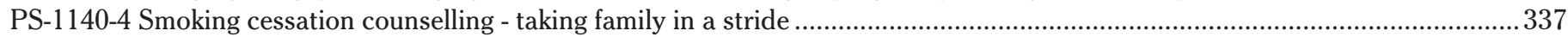

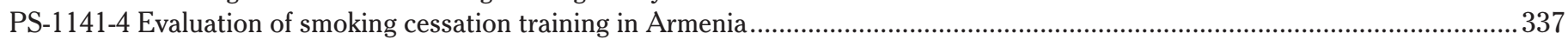

PS-1143-4 Material of support to community health agents for the tobacco approach of "family health" in Brazil...........................338

PS-1 144-4 Dental institutional model of a tobacco cessation clinic: six years of success story ......................................................338

PS-1 145-4 Tobacco cessation services by community pharmacists; a potential opportunity window …..........................................338

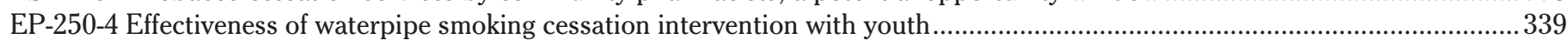

EP-259-4 Dependence and withdrawal symptoms among waterpipe tobacco smokers

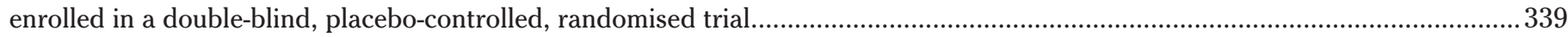

EP-260-4 A double-blind, placebo-controlled, randomized trial of varenicline in hookah tobacco smokers in Pakistan .....................340

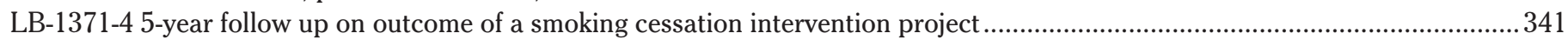

\subsection{Tobaceo and Women: The gendered nature of tobaceo}

PS-1 147-5 Smoking addiction among young women working at night at International call centres in India ......

PS-1148-5 Factors associated with short and long term successful quit

among adults female smokers: prospective follow up of Quitline users in Republic of Korea

PS-1151-5 Are poor women in the reproductive age group more prone to consume tobacco:

a study of the factors that lead to tobacco consumption in India......

PS-1152-5 Prevalence of violence against women and negative predictors

in female smokers attended in a smoking cessation unit in Buenos Aires city: a cross-sectional study

PS-1153-5 Attitudes of women from Kazakhstan regarding tobacco control policy as cancer prevention .......................................343

EP-261-4 Assessment of exclusive and dual cigarette and hookah smoking among a sample of pregnant women in Egypt ...............343

LB-1368-5 Gender disparity in hard-core-smoking: evidence from 14 GATS countries .....

\subsection{Tobacco in vulnerable populations (e.g. indigenous populations)}

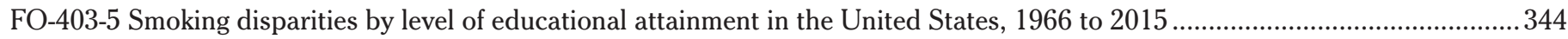

FO-404-5 Urban Indigenous commercial tobacco use in Canada: our health counts Toronto........................................................344

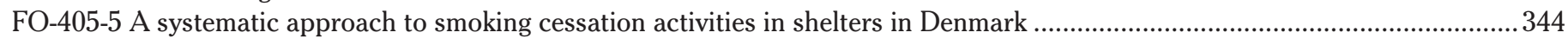

FO-406-5 Characteristics of individuals that complete treatment v. discontinue among criminal justice smokers ............................ 345

FO-408-5 Impact of social inclusion on tobacco use and wellbeing of street children: learning from field experiences in India.......... 345

FO-409-5 Tobacco control policy and smoking cessation and intensity in a longitudinal sample of US older adults (1992 - 2014) .. 345

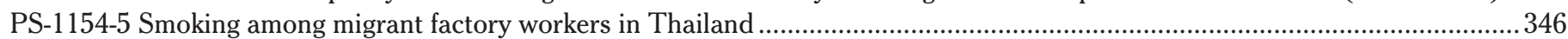

PS-1 155-5 Tobacco use and other problem behaviours among street youths in South Western Nigeria ........................................346

PS-1156-5 Tobacco addiction and it's consequences on oral health

of indigenous and rural people - a cross sectional study from central India.....

346

PS-1157-5 A national collaboration on reducing tobacco use in the behavioral health population in the United States ................... 347

PS-1158-5 Tackling tobacco: addressing high smoking prevalence among mental health consumers through systems change .......... 347

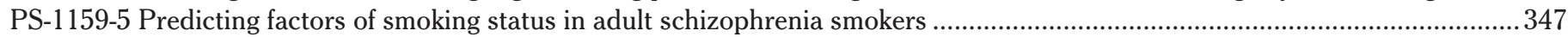

PS-1160-5 Evaluation of a targeted intervention to most at risk populations

of tobacco users through a tea-shops community signage program...

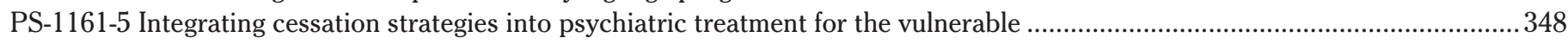

PS-1 163-5 Characterising smoking among cancer patients in Poland - a cross-sectional pilot survey .............................................349

PS-1165-5 Deciding protection or provocation: helping young Siddi women translating knowledge into action .............................. 349

PS-1166-5 Strategies in engaging special populations for Tobacco Cessation in South Florida, USA - success models ....................349

PS-1 167-5 Tobacco usage among tribal population of Nilgiris, Tamil Nadu - a cross sectional study ............................................350

PS-1168-5 How to approach the question of smoking in health units with a tuberculosis program in Brazil? ................................... 350

PS-1 169-5 Of Mice and Menthol: Rethinking Well-intentioned

but Insufficient Strategies to Counteract Tobacco Use and Promotion in Minority Populations 
EP-234-5 Afghan frontier: understanding tobacco practices among migrant population in India.

EP-237-5 Tobacco retail outlet density, local smoking norms and maternal smoking during pregnancy: a case crossover study ...... 351

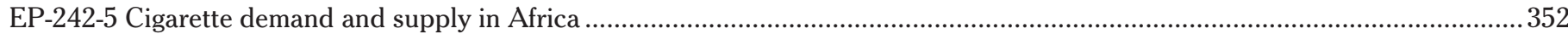

EP-243-5 Prevalence and correlates of ENDS use among adults being treated for chronic lung disease ........................................352

EP-244-5 Self-initiated pre-quit smoking reduction among community correction smokers .........................................................352

EP-245-5 National estimates and correlates of cigarette smoking among Hispanic/Latino construction workers in the US............... 353

EP-246-5 Secondhand smoke and its impact on nicotine dependence and smoking cessation among individuals with mental illnesses.......353

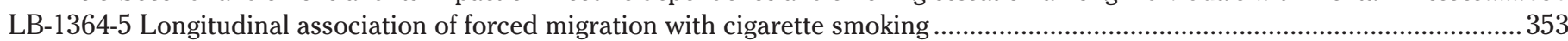

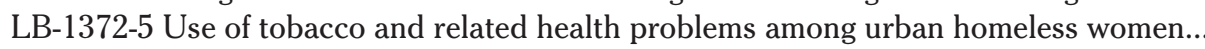

\subsection{Tobacco, inequality and poverty}

PS-1170-5 Family and school socioeconomic status as predictors of tobacco and e-cigarette use in adolescents: a study from a perspective of material, human, and social capital......

PS-1171-5 "Leaving no one behind": the vulnerability of children from low SES households

suffering from tobacco-related harm in light of human rights and social justice.

PS-1172-5 Socioeconomic differences in secondhand smoke exposure among adolescent

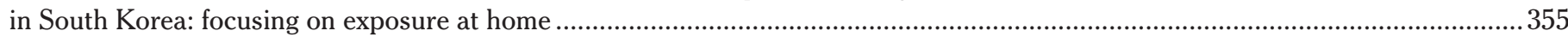

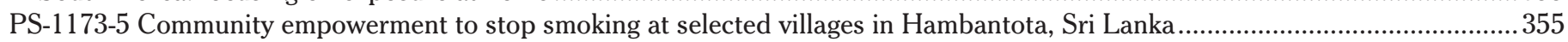

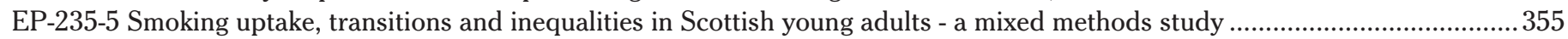

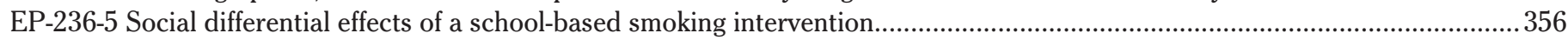

EP-238-5 Influence of cigarette prices and social environment smoking on allocation

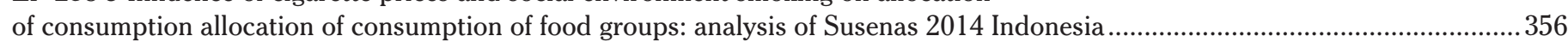

EP-239-5 Educational inequalities in smoking uptake and cessation: a birth cohort analysis of the German GEDA study ............... 356

EP-240-5 Smoking during pregnancy among primary health care clients in Argentina. Identification of resources and stressors ...... 357

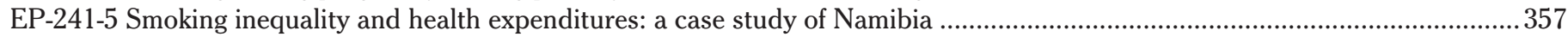

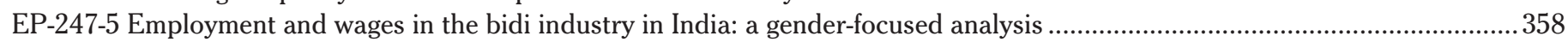

LB-1363-5 Do tobacco using households have greater risk of catastrophic healthcare

expenditure? Implication for tobacco control policy

\section{4. Tobacco farming: Opportunities and experiences in facilitating alternative livelihoods and preventing tobacco adverse environmental impact}

PS-1174-5 Moving to tobacco cultivation free livelihoods in Sri Lanka: lessons learned from India and Kenya ...............................358

PS-1175-5 Defeating attempt made to introduce tobacco cultivation for farmers

in Sri Lanka through sustainable agriculture development programme: a case study of Kandy district.

PS-1176-5 The plight of tobacco farming under hegemony of transnational

tobacco companies in Turkey: repercussions and remedies

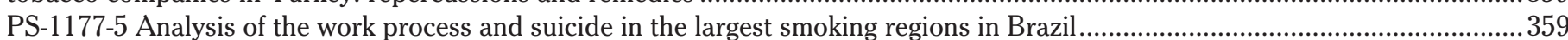

PS-1 178-5 Health education programs for tobacco workers: reflecting on principles for empowering farmers ................................ 360

PS-1179-5 Alternative crop for tobacco farming in Lebanon - an effective tool

to change and educate on tobacco environmental and economical impact ......

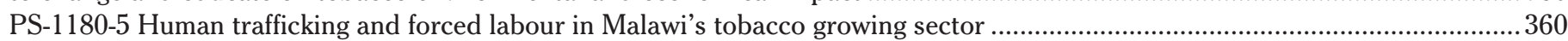

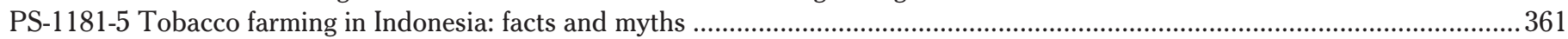

LB-1362-5 Alternative crops to tobacco: a gateway for tobacco farmers Ruvuma region, southern Tanzania.................................. 361

LB-1366-5 Successful experience with tobacco crop substitution in Yuxi, Yunnan, China ..........................................................362

\subsection{Youth tobacco experience}

PS-1 182-5 Flavor-related descriptors on economy-priced flavored cigarette packs in five Latin American countries........................ 362

PS-1 184-5 Cigarette smoking and reasons for leaving school among school dropouts in South Africa ..............................................3 362

PS-1 186-5 Attempt to quit smoking by electronic cigarette use among smoking adolescents in Korea ............................................3 363

PS-1 187-5 Second hand smoke: awareness and acceptability among school going adolescents In India ......................................3 363

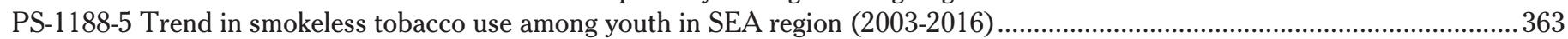

PS-1 190-5 Trends in adolescent and young adult smoking in India (2009-2017): patterns and implications ...................................364

PS-1191-5 Prevalence and correlates of smoking at home in adolescent smokers - a cross-sectional study .....................................364

PS-1192-5 How behavioral norm and social influence affect smoking in young adulthood: the experience of Korean young adults ........... 364

PS-1193-5 The association of electronic vapor product use and conventional cigarette smoking with Korean adolescents' oral health ........365

PS-1 194-5 Anti-smoking legislation in educational institutions in India: opinion of stakeholders and challenges in implementation ........... 365

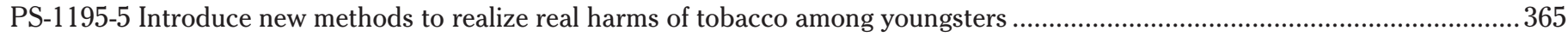

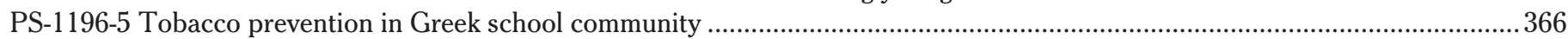

PS-1197-5 Impact on patterns of tobacco and its initiation among children

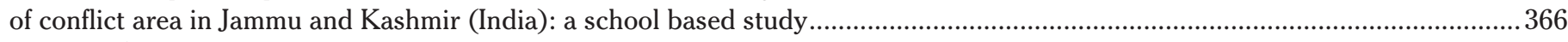

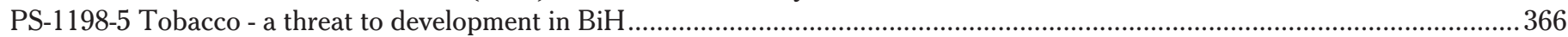

PS-1200-5 Socio-economic status as predictors of initiation and persistence of youth smoking in Mizoram ......................................3 367

PS-1201-5 Three year school based intervention for Tobacco prevention 
in the region of Sousse: quasi experimental design 2009-2014

PS-1202-5 Dare or dream: helping young people of Delhi slums to stop using tobacco

367

PS-1203-5 Youth response to pictorial warning on tobacco products in Chandigarh India. 368

PS-1205-5 Second hand smoking among schoolchildren in the region of Sousse 368

PS-1206-5 Waterpipe (Shisha) smoking: the experience and harm perception

as narrated by University of Ibadan Undergraduate Students

\section{Abbreviations}

PS: Poster Session

FO: Full Oral Session

EP: ePoster Session

RF: Rapid Fire Session

LB: Late Breaking Session

SUS: Symposium 


\section{Wednesday, 7 March 2018}

\subsection{Priorities and challenges for advancing the WHO FCTC}

\author{
$12: 30-14: 00$ \\ PS-500-1 A quantitative analysis of the 2015 \\ WHO MPOWER report \\ G Heydari
}

'WHO Collaborating Center on Tobacco Control in EMRO, Tehran, Iran, Islamic Rep. Of. E-mail: ghrheydari@yahoo.com Background: A report of the MPOWER activities of countries worldwide is published once every two years by the WHO. Our objective was to perform a quantitative analysis of $\mathrm{WHO}$ report on tobacco control program in countries and regions to make a simple view of global tobacco control programs and to find best parties on it. Methods: This was a cross-sectional study by filling out a validated check list from the $2015 \mathrm{WHO}$ report (MPOWER). All 10 measures were changed to indicators and getting scores. The scores were entered independently by two individuals and a third party compared the values and confirmed their accuracy. The scores were summed and presented in a descending order.

Results: Fifteen countries, which acquired the highest scores (85\% of total 37) included Panama and Turkey with 35, Brazil and Uruguay with 34, Ireland, United Kingdom, Iran, Brunei, Argentina and Costa Rica with 33 and Australia, Nepal, Thailand, Canada and Mauritius with 32 points.

Conclusions: These 15 countries may indicate as a best model for other parties in implementation and enforcement of tobacco control program. Comparison of scores of different countries in this respect can be beneficial since it creates a challenge for the health policy makers to find weakness of the tobacco control programs to work on it.

Tob. Induc. Dis. 2018;16(Suppl 1):A1

DOI: $10.18332 /$ tid/83763

\section{2:30-14:00}

PS-501-1 Attitude towards plain packaging of tobacco products among the adult population in Bangladesh: a mixed method approach

K Ahmed ${ }^{1}$, M Bukht ${ }^{2}$, S Hossain ${ }^{3}$, S Ahmad ${ }^{1}$

'Bangladesh University of Health Sciences, Dhaka, Bangladesh, ${ }^{2}$ BRAC Centre, Dhaka, Bangladesh, ${ }^{3}$ Jahangirnagar University, Savar, Dhaka, Bangladesh.E-mail: Iopa_birdem@yahoo.com Background: The aim of this study was to investigate the attitude and reactions towards plain tobacco pack among the adult population in Bangladesh.

Methods: This was a cross-sectional study with mixed method approach. Data was collected from 458 respondents through faceto-face interview for quantitative part and another 40 through indepth interview with 10 key informants' interview for qualitative part. In every cases of this study, dummy cigarette plain packet, which was developed in comparison with the existing graphic health warning pack,were used. To measure respondents' attitude on different aspect of plain tobacco pack, a five-point scale was used in this study. Response options for all items were: completely agree (5), slightly agree (4), neither agree nor disagree, that is neutral (3), slightly disagree (2) and completely disagree (1). Furthermore, reaction of the respondents observed by the researcher and it was noted under four subheadings likeAlertness, Unpleased, Concerned and Discomfort. Using a four point scale, response was taken and the options were: more (4), moderate (3), little (2) and negative answer to the statement (1). Thematic content analysis was performed and SPSS version 22.0 software was used for data analysis.

Results: Of the total respondents $96.5 \%$ were positive about the introduction of plain packaging of tobacco products in Bangladesh. About $94 \%$ respondents' perceived that it would reduce the attractiveness and appeal of tobacco products. Plain pack was believed to be able to prevent the tobacco advertisement and branding promotion, reduce youth experimentation, misleading consumers, youth tobacco initiation, and motivate tobacco users to quit by the majority of the participants.

Conclusions: The findings of this study provide baseline information to initiate debate with country policy makers to formulate plain packaging of tobacco products legislation in Bangladesh.

Tob. Induc. Dis. 2018;16(Suppl 1):A2

DOI:10.18332/tid/83885

\section{2:30-14:00}

PS-502-1 Policy progress of health warnings on tobacco products as per Article 11 of WHO FCTC: a global analysis

S Grover ${ }^{1}$, DN Sinha ${ }^{1}$, PC Gupta ${ }^{2}$, R Mehrotra ${ }^{3}$

${ }^{1}$ National Institute of Cancer Prevention and Research, WHO FCTC Global Knowledge Hub on Smokeless Tobacco, Noida, India, ${ }^{2}$ Healis - Sekhsaria Institute of Public Health, Navi Mumbai, India, ${ }^{3}$ National Institute of Cancer Prevention and Research, Noida, India. E-mail: shekhargrover84@gmail.com

Background: This paper evaluates the coverage and quality of global implementation of Article 11 of the WHO Framework Convention on Tobacco Control (WHO FCTC), for Cigarettes (CIG) and Smokeless Tobacco (SLT).

Methods: A cross-sectional database of policies with implementation dates in FCTC ratified countries $(n=179)$ was prepared. Information was validated by two sources. World Bank Income groups were used for categorizing countries. Notified warnings were scored according to topics specified at FCTC Warning database website. Data was analyzed using SPSS.

Results: By December 2016, of ratified countries, 94\% and 48\% population ( $\geq 15$ years) were covered with $\geq 30 \%$ Health warning (HW) on CIG and SLT respectively. Population coverage by HW on CIG packages in high income countries (HICs), middle-income countries (MICs) and low-income countries (LICs) were 98.6\%, $95 \%$ and $64 \%$ respectively, however, such coverage for SLT was < $50 \%$ population in all income-group countries. Global population coverage of Pictorial Health Warnings (PHW) ( $\geq 30 \%$ size) on $\mathrm{CIG}$ and SLT were $62 \%$ and $37 \%$ respectively. Global population coverage with 'high content' was $60 \%$ for CIG and only $5.2 \%$ for SLT. High content information on CIG covered $90 \%, 55 \%$ and $33 \%$ population in HIC, MIC and LIC respectively. However, this coverage for SLT in HIC, MIC and LIC was 4.6\% $4.5 \%$ and $12 \%$ respectively.

Conclusions: There is unequal attention on implementation of Article 11 on different tobacco products. Countries need to provide quality information with equal focus on all tobacco products.

Tob. Induc. Dis. 2018;16(Suppl 1):A3

DOI:10.18332/tid/83949

12:30-14:00

PS-503-1 Global Policy progress in Article 16 of WHO FCTC on cigarettes (CIG) and smokeless tobacco (SLT) 
S Grover ${ }^{1}$, DN Sinha1, A Yadav1 ${ }^{1}$ PC Gupta², R Mehrotra ${ }^{3}$ ${ }^{1}$ National Institute of Cancer Prevention and Research, WHO FCTC Global Knowledge Hub on Smokeless Tobacco, Noida, India, ${ }^{2}$ Healis - Sekhsaria Institute of Public Health, Navi Mumbai, India, ${ }^{3}$ National Institute of Cancer Prevention and Research, Noida, India.E-mail: shekhargrover84@gmail.com

Background: Article 16 of WHO FCTC focuses upon limiting the supply and exposure of tobacco products to Minors. Implementation of Article 16 has been researched by few local and regional studies, primarily focusing on Cigarettes. This paper evaluates global progress in the area of access of SLT products to minors and compares with cigarettes.

Methods: A cross-sectional database of notified policies in FCTC ratified countries $(n=179)$ was prepared for SLT and CIG. Indicators studied were: Ban on Sale to minors; Treaty requirements (under the Ban on Sale to Minor); ban on sale by minor, prohibition on distribution of free tobacco products and prohibition on sale of loose/individual tobacco products. Countries were categorized according to World Bank Income groups and WHO regions. Data was analyzed using SPSSv17.0. Results: Unequal implementation of provisions under Article 16 has been implemented for CIG and SLT products (nearly $79 \%$ Parties for CIG and $67 \%$ for SLT). Most countries have a partial (or none) policy in place (only 12\% Parties have notified all provisions under Article 16(1) for CIG, and 10\% for SLT). Implementation has been evaluated for CIG through indicators in GYTS, but not for SLT.

Conclusions: A comprehensive policy formulation on banning sale of tobacco to minors and its proper enforcement is required to prevent access and availability of tobacco including SLT to minors.

Tob. Induc. Dis. 2018;16(Suppl 1):A4

DOI: $10.18332 /$ tid/83950

\section{2:30-14:00}

PS-504-1 Awareness and implementation of cigarette and other tobacco products act in selected communities in Northern India

RS Rath ${ }^{1}$, A Krishnan 1 , B Nongkynrih¹, P Misra ${ }^{1}$

${ }^{1}$ All India Institute of Medical Sciences, Centre for Community Medicine, New Delhi, India. E-mail: rsr14585@gmail.com

Background and challenges to implementation: Legislative route is one of the important method to change the behavior. To decrease the consumption of tobacco India has enacted various laws at different times. Latest addition to this is Cigarette and Other Tobacco Products Act (COTPA), 2003. This study was conducted in 2013 to find compliance of the establishments selling tobacco COTPA and the knowledge of the people about to this law.

Intervention or response: Community audit was conducted in selected communities of district Faridabad Haryana to map establishments selling tobacco and look for tobacco related advertisements. All establishments selling tobacco were assessed for compliance to provisions of COTPA using a checklist. Thirty participants aged 18-65 years were selected from each cluster by modified EPI cluster sampling method and interviewed to assess their knowledge about COTPA.

Results and lessons learnt: Around 78\% of the establishments were selling tobacco products. In these establishments smoked tobacco products were the predominant type sold (52\%). Around $41 \%$ were selling tobacco products to minors and $10 \%$ were located with in 100 yards of educational institutes. No establishment was fully compliant to COTPA act. Only $5 \%$ of the people $(n=552)$ were fully aware of COTPA provisions. Although banned by COTPA participants admitted that they were exposed to tobacco advertisements.

Conclusions and key recommendations: Although tobacco control laws were enacted long ago the process of implementation was found to be poor.

Tob. Induc. Dis. 2018;16(Suppl 1):A5

DOI:10.18332/tid/83965

\section{2:30-14:00}

PS-505-1 Are strong tobacco control policies associated with bans on the retail sales of electronic nicotine delivery systems?

A Liber ${ }^{1,2}$, R Kennedy ${ }^{3}$

'University of Michigan, Health Management and Policy, Ann Arbor, MI, United States of America, ${ }^{2}$ American Cancer Society, Intramural Research, Atlanta, GA, United States of America, ${ }^{3}$ Johns Hopkins Bloomberg School of Public Health, Department of Health, Behavior \& Society, Baltimore, MD, United States of America. E-mail: alex.liber@cancer.org

Background: Globally, as of June 2017, 35 countries banned the retail sale of electronic nicotine delivery systems (ENDS), while 44 countries regulated these products. To understand what type of tobacco control regulatory frameworks are associated with adopting bans, we test whether countries with higher levels of tobacco control policy development are more likely to ban ENDS retail sales.

Methods: A dataset on the policy choices of 79 countries who have adopted formal policies regulating ENDS products was combined with data from the WHO 2015 Global Tobacco Control Report to create a weighted index of tobacco control policy development in a modified version of Joosen and Raw's Tobacco Control Scale (TCS) (2006). Logistic regression analysis on the final cross-sectional dataset was performed to test the hypothesis that countries with greater levels of overall tobacco control policy development and on subscales corresponding to the MPOWER letters were more likely to ban than regulate retail sales of ENDS. Results: Countries with higher TCS scores were not more likely to adopt bans on retail sales of ENDS $(\beta=-0.0004, p=0.977)$ compared to countries with lower scores. Particularly, countries with higher scores on the subscales of warning users of the dangers of tobacco smoke were more likely to ban ENDS retail sales $(\beta=0.251, p=0.002)$ while countries with higher scores on tobacco tax policy were more likely to allow retail sales of ENDS $(\beta=0.140, p=0.003)$.

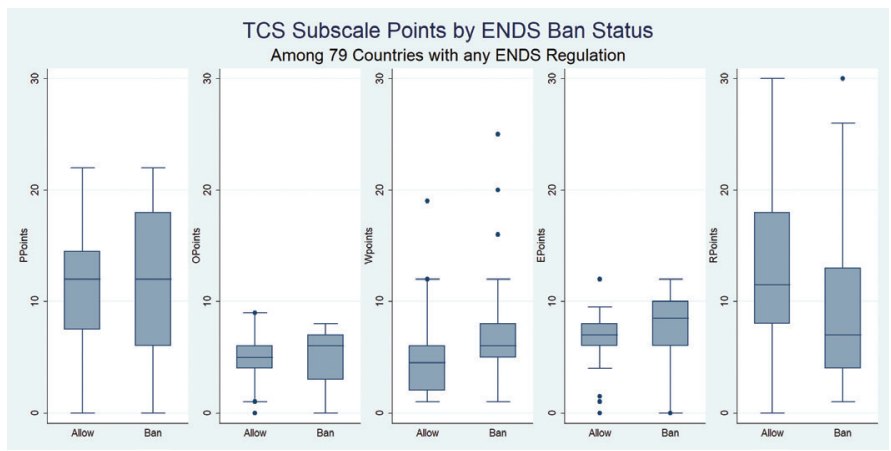

[TCS Subscale Points by ENDS Ban Status]

Conclusions: The likelihood of a country banning retail sales of ENDS has a complex association with tobacco control policy development, varying on important subscales in direction. The 
role of the European Union's Tobacco Product Directive appears to have played an outsized role in creating observed patterns, necessitating study of how this will play out going forward.

Tob. Induc. Dis. 2018;16(Suppl 1):A6

DOI:10.18332/tid/84007

\section{2:30-14:00}

PS-506-1 Global policy progress in Article 20 of World Health Organization's Framework Convention on Tobacco Control (WHO FCTC) on Smokeless Tobacco (SLT)

D Sinha ${ }^{1}$, A Kumar ${ }^{1}$, S Grover ${ }^{1}$, H Kaur ${ }^{1}$, PC Gupta ${ }^{1}$, R Mehrotra ${ }^{1}$ ${ }^{1}$ National Institute of Cancer Prevention and Research, Noida, Noida, India. E-mail: dhirendrasinha1@gmail.com

Background: Article 20 deals with research, surveillance and exchange of information on tobacco products. This paper mandates to update comprehensive information on appropriate scientific national research and established surveillance mechanisms as well as programs that address the magnitude, patterns, determinants, related social, economic and health indicators and consequences of Smokeless Tobacco (SLT) consumption.

Methods: The present study is a review of global SLT use prevalence among ratified countries. Nationally representative data per country has been collected and validated from respective Global Adult Tobacco Surveys (GATS); Global Youth Tobacco Surveys (GYTS); WHO report on Global Tobacco Epidemic - 2013 \& 2015; WHO STEPwsie approach to chronic disease surveillance (STEPS surveys); and Global Progress Report on implementation of the WHO FCTC - 2016.

Results: Comprehensive information on Global SLT use is limited. Data on SLT use among adults is available at national level in 140 Parties only. Periodic and recent data is available in 15 Parties only. Information of SLT use among adolescents at national level is available for 110 countries only. Periodic and recent data among adolescents is available in 20 Parties. In SEAR, SLT use among adolescents is higher than smoking, unlike other regions. Research on economics and health consequences is available for 25 and 10 Parties respectively.

Conclusions: Parties should periodically monitor and share the magnitude, patterns, determinants and related social, economic and health consequences of all tobacco products including SLT especially in SEAR.

Tob. Induc. Dis. 2018;16(Suppl 1):A7

DOI:10.18332/tid/84084

\subsection{Integrating tobacco control into Health systems, global frameworks and strategies to reach WHO FCTC and SDG's targets.}

11:00-12:30

SUS The United Nations and its agencies, and their relationship with tobacco industry - an analysis of positions, policy and practice

$P$ Lal $^{1}$

'International Union Against Tuberculosis and Lung Disease, New Delhi, India.E-mail: plal@theunion.org

Background and challenges to implementation: Since the early 1990s, UN agencies have been encouraged to generate resources from the private sector. Owing to a lack of exclusion criteria and standards for partnership and participation, companies largely responsible for causing the NCD epidemic have ironically become development partners with UN agencies. The tobacco industry has historically offered substantial financial assistance to public health initiatives, being cognizant of the significant political mileage these partnerships. In June 2012, at the Rio +25 , the UNDP conferred its highest prize to a tobacco company for environment protection. This set-off a strong backlash from tobacco control advocates after which the UNDP Administrator admitted to the oversight. Tobacco control advocates have since urged all UN agencies to exclude tobacco industry.

Intervention or response: This survey analyzes the position taken, policies proposed and practices undertaken by United Nations and its agencies since Rio +25 , with respect to the tobacco sector (farming, investment, manufacturing and marketing).

Results and lessons learnt: While most United Nations agencies have a stated policy to exclude tobacco industry, there are instances when local offices and partners of United Nations or those working through their grants continue to engage and partner with tobacco sector. Two agencies (FAO, ILO) have no stated position on tobacco and continue to engage with tobacco sector.

Conclusions and key recommendations: The respectability gained by that tobacco companies by associating with the UN system fundamentally distracts the focus that the global public health community needs to maintain on the growing epidemic of NCDs. Through the influence that the multilateral system exerts on national governments, tobacco companies effectively influence health policy at a global level while obfuscating the focus of their responsibility for aspects of the NCD epidemic and achieving the SDGs. Civil society needs to sustain its efforts to advocate for tobacco exclusion and alert of potential conflicts of interest.

Tob. Induc. Dis. 2018;16(Suppl 1):A8

DOI: $10.18332 /$ tid/84356

$12: 30-14: 00$

PS-508-1 Universities' role on tobacco control law support in Thailand

P Srimoragot ${ }^{1}$

${ }^{1}$ Mahidol University, Surgical Nursing Department, BangkokNoi,Thailand.E-mail:pongsri.sri@mahidol.edu

Background and challenges to implementation: In Thailand, Tobacco control laws had announced since 2535 B.E. So that tobacco product, marketing, and sponsorship and consuming of tobacco is advanced and not effective to control. As seen from the prevalence of tobacco smoking did not reduce and its trend to increase especially among youth and women. So Ministry of public health in Thailand was begin to renovate, improve, and readjust both tobacco control law. On the process of law revision, the main objective was set to protect all adolescent from starting to be the new comer and to be consistent with WHO FCTC.

Intervention or response: To review experiences of universities role on supporting the revision of tobacco control law. Mahidol University took action to be the leader to support the revision of tobacco control law. By declare via public media such as president interviewing to show scientific reason to support the benefit for youth and mankind from tobacco control law, sent advocacy letter to the parliament, lobby related stakeholders, many public health expertise try to educate, advocate the public on the impact of tobacco harmfulness on individual and public health, economic, social with various stakeholders, especially the prime minister, cabinet, and member of parliament. Mahidol University collaborated and coordinated with many sectors to support the law. These actions aim to make sure that the leaders, and public gain more understanding and awareness on main intention of 
revision of tobacco control law.

Results and lessons learnt: The revision of tobacco control in Thailand was passed the final vote of parliament, announced in government gazette and be formal enforcement. University is also the significant jig saw for related law revision, enforcement and regulation.

Conclusions and key recommendations: Except teaching, research, academic services and cultural conservation, university should show action on related law support, policy mobilization, law regulation and enforcement.

\section{Tob. Induc. Dis. 2018;16(Suppl 1):A9}

DOI:10.18332/tid/83759

\section{$12: 30-14: 00$}

PS-509-1 NCD prevention: a pathway to India's sustainable development

S John ${ }^{1}$

1Policy Analyst, Mumbai, India. E-mail: shobajohn@gmail.com Background: India's economy has been steadily growing, with a projected Gross Domestic Product growth rate of $7.5 \%$ for 2017 2018. While the country has made significant gains in reducing HIV/AIDS and poverty, socio economic inequalities, lack of access to basic needs and increasing burden of noncommunicable diseases (NCDs) deter its development.

Changing lifestyles and related NCDs are found to thwart India's development aspirations and global commitments. For instance, tobacco use and supply impede its efforts to achieve the targets of the UN Sustainable Development Goals (SDGs) and decelerate its implementation of the WHO the Framework Convention on Tobacco Control (FCTC).

Objectives: The research therefore aimed to:

1. Describe the ways in which NCD risk factors undermine India's development.

2. Discuss the relevance of tobacco control for India's implementation of the SDGs.

3. Explore the potential for integration of NCD prevention in national development programmes.

Methods: The research was based on review of published literature. Country specific research, augmented by global evidence, was analysed using the SDG framework to understand the linkages of NCD risk factors with India's development. Country's key development programmes were examined for their relevance to NCD prevention, such as for tobacco control.

Results: Evidence indicates that:

1. tobaccoisacauseand consequenceofIndia'sunderdevelopment.

2. its supply and use undermines health, economics, environment, national security and poverty alleviation efforts of households and the country.

3. tobacco control can potentially improve the outcomes of India's development programmes.

Conclusions: Action by stakeholders across sectors is critical to enable India in meetings its FCTC obligations and commitment to the SDGs. Political will, robust business case and effective multisectoral coordination for NCD prevention can help advance India's march to sustainable development.

Tob. Induc. Dis. 2018;16(Suppl 1):A10

DOI:10.18332/tid/83856

12:30-14:00

PS-510-1 Assessment of 2016 Taiwan tobacco control performance based on WHO MPOWER guidelines
H-Y Chung ${ }^{1}$, S-Y Lo ${ }^{1}$, Y-J Liu ${ }^{1}$, M-C Lin ${ }^{1}$, L-H Yu ${ }^{1}$, Y-W Wang ${ }^{1,2}$, Y-R Wang ${ }^{1}$

${ }^{1}$ Health Promotion Administration, Ministry of Health and Welfare, Taipei, China, ${ }^{2}$ School of Medicine, Tzu Chi University, Hualien, China. E-mail: yiren@hpa.gov.tw

Background: Smoking kills more than 27,000 people each year in Taiwan. This study aims to demonstrate the significant progress with Taiwan MPOWER performances and compare achievements with OECD and other Asian countries.

Methods: Assessment data from Taiwan's laws and regulations, a comprehensive health surveillance system, and research database and reports, with WHO MPOWER performance rating indicators. Results: The adult smoking rate declined from $21.9 \%$ in 2008 to $15.3 \%$ in 2016 , indicating a $30.3 \%$ decline in smoking rate and a decrease in the number of smokers by approximately 810,000 within 8 years. Taiwan has protected over $90 \%$ of the population from smoke-free environments by enforcing the law in eliminating second hand smoke in public areas, and promoted the recognition of tobacco hazards to youth especially via campus activities, official websites, Facebook, and YouTube. It's worth noting that Taiwan is the 2nd country to carry out the ordinance of smokefree parks and national parks around the world. Assisting over 110,000 smokers since 2012 in quitting smoking successfully by providing the Second Generation Smoking Cessation Program and other services such as Quitline, cessation classes and cessation counseling. Over $40 \%$ of smokers attempt to quit after Taiwan enacted health warning labels on tobacco packs. The Health Promotion Administration has worked with the National Communications Commission (NCC) to establish the guideline that anti-tobacco textual messages are required to add on Radio and TV programs, reminding people of tobacco harming.

Conclusions: Taiwan gains five 1st performance (Monitoring, Smoke-free environments, Cessation programmes, Anti-tobacco mass media campaigns, Bans on advertising, promotion and sponsorship) while compared to OECD and other Asian countries (New Zealand, Japan, Republic of Korea, Thailand, China, etc.), and ranks among the best with Singapore, Canada, United Kingdom, and Australia. Continued efforts are needed to raise the health warnings and taxations in the future.

Tob. Induc. Dis. 2018;16(Suppl 1):A11

DOI:10.18332/tid/83994

$12: 30-14: 00$

PS-511-1 Empowering librarians on effective tobacco demand reduction through public libraries in Sri Lanka

\section{S Dharmasena}

${ }^{1}$ Alcohol and Drug Information Center (ADIC), Library \& Information Programme, Colombo, Sri Lanka. E-mail: sasanka. dharmasena@yahoo.com

Background and challenges to implementation: Since 2010 Alcohol and Drug Information Centre (ADIC) has been maintaining a library network and it has been identified as one of the most effective and appropriate path to disseminate effective and evidence based information. Sri Lanka holds more than 1000 libraries under its local government authorities. In 2014 ADIC empower librarians to make community pressure on discouraging Tobacco usage.

Intervention or response: The first attempt was to ensure the availability of information on Tobacco prevention. ADIC organized trainings for librarians regarding effective methods of Tobacco and other drug prevention. As a result, librarians organized exhibitions, 
awareness programs in their respective villages. Further, in 2014 ADIC developed a module for librarians on making community pressure against cigarette sales.. The community was encouraged to participate in town campaigns regarding tobacco prevention organized by the librarians using human and financial assets of their local government authorities. Librarians have taken steps to acknowledge shop-owners who took active participation on this, display prevention messages and also made the general public aware through announcements around the villages.

Results and lessons learnt: Librarians have step forward from their general responsibilities to discourage and reduce Tobacco usage and cigarette sales in respective areas. From 2014 to 2017, librarians have conducted 60 town campaigns in their villages and 160 shops have stopped selling cigarettes during Wesak \& Poson weeks in Sri Lanka.

Conclusions and key recommendations: Community driven initiations are successful in addressing an issue in the grass-root level. Achievement of the objective was mostly based on the active and innovative participation of librarians and community members. Therefore, empowering librarians and developing libraries is an effective way of reduce the Tobacco demand and consumption in communities.

Tob. Induc. Dis. 2018;16(Suppl 1):A12

DOI:10.18332/tid/84099

\section{$12: 30-14: 00$}

PS-512-1 Upgrade needed: South African tobacco control policy and the WHO-framework convention on tobacco control

CO Egbe1, SA Bialous ${ }^{2,3}$, SA Glantz ${ }^{1,4}$

${ }^{1}$ University of California San Francisco, Canter for Tobacco Control Research and Education, San Francisco, CA, United States of America, ${ }^{2}$ University of California San Francisco, San Francisco, CA, United States of America, ${ }^{3}$ University of California San Francisco, Social and Behavioral Sciences Department, School of Nursing, San Francisco, CA, United States of America, ${ }^{4}$ University of California San Francisco, Department of Medicine (Cardiology), Cardiovascular Research Institute, Philip R. Lee Institute for Health Policy Studies, Helen Diller Family Comprehensive Cancer Center, San Francisco, CA, United States of America. E-mail: catherine.egbe@ucsf.edu Background: Tobacco control policy in South Africa was one of the strongest in the African continent before the WHO Framework Convention on Tobacco Control (FCTC) entry into force in 2005. South Africa ratified the FCTC in 2005 and the treaty came into force same year. However, with many African governments keying into implementation of the FCTC, South African tobacco control laws are lagging behind with regards to compliance with FCTC requirements. Methods: We reviewed South Africa's current tobacco control laws with the aim of identifying key areas including smoke-free public places, packing and labelling and tobacco advertising, promotion and sponsorship, which are in need of additional regulations or amendments to make the tobacco laws more FCTC-compliant.

Results: Under smoke-free regulations, there is need to have hospitals and patients' rooms $100 \%$ smoke-free and smokefree work place policies should be effectively implemented. The proportion of tobacco packages with health warning labels (HWL) need to be upgraded from 20\% to at least 30\%, although the evidence now supports much larger warnings and these must be considered. HWL should be in English and the predominant languages of South Africa to allow for a better understanding of these warnings by the people. On tobacco advertising, promotion and sponsorship, the South African law needs to be tightened around international and cross border advertisement of tobacco products, allowance of charitable financial contributions and points-of-sale advertising.

Conclusions: There is evidence supporting strengthening the existing legislation and to make the policy more aligned with the FCTC standard regulations. However, if tobacco industry opposition at the national level remains strong, the South African tobacco law allows for provinces to make stronger laws than the national law. This provision of the law can be exploited by tobacco control advocates to sensitize provincial governments to make tobacco control laws at the provincial level more FCTC-compliant.

Tob. Induc. Dis. 2018;16(Suppl 1):A13

DOI: $10.18332 /$ tid/84157

$12: 30-14: 00$

PS-513-1 Prevalence, perceptions and predictors of smokeless tobacco use in a rural community in South Eastern Nigeria

U Ofonakara ${ }^{1}$, T Kotey ${ }^{2}$, 0 Oluwakemi ${ }^{3}$

'Federal Teaching Hospital Abakaliki, Community Medicine, Abakaliki, Nigeria, '2uth Teaching Hospital, Department of Community Medicine, Lagos, Nigeria, ${ }^{3}$ University of Lagos, Community Health and Primary Care, College of Medicine, Lagos, Nigeria.E-mail: druzooffor@yahoo.co.uk

Background and challenges to implementation: Nationally representative prevalence studies have consistently reported higher rates of tobacco use in the South-Eastern parts of Nigeria but little is known about the pattern and perceptions and predictors of smokeless tobacco use in these parts of the country. The aim of this study was to determine the pattern and predictors of smokeless tobacco use, their health related knowledge and social attitudes towards its use among residents of Ukpo community of Dunukofia LGA of Anambra State, Nigeria.

Intervention or response: Across-sectional descriptive study was carried out among 490 residents of Ukpo using a two-stage sampling method. Data was collected using anonymous pre-tested interviewer administered questionnaires adapted from GATS. Odd ratio and $95 \%$ confidence intervals were computed and $\mathrm{P}$ values of $<0.05$ were considered statistically significant.

Results and lessons learnt: The results showed that respondents were mostly male 300(61.2\%), aged between 20 and 70 years, mean of $42.2 \pm 15.4$ years. Almost half of the respondents, 210 (42.9\%) were ever-users of smokeless tobacco with majority of them 188 (89.5\%) remaining as current users. Dry snuff 155 (73.8\%) was the most common form of smokeless tobacco used. The primary reasons for use were: to relieve stress $115(61.2 \%)$; to increase levels of alertness 106(56.4\%); for personal pleasure 105(55.9\%) and social acceptance 98 (52.1\%). Age ( $<<0.0001)$, male gender $(p<0.0001)$, being single $(p<0.0001)$ and lower educational attainment $(\mathrm{p}<0.0001)$ were associated with smokeless tobacco use. Only about half of the respondents were aware that smokeless tobacco is dangerous to human health and many $254(51 \%)$ people believed that that it is safer than cigarettes, a way of promoting friendship (65\%) and should be used within their community (73\%).

Conclusions and key recommendations: Efforts targeted at raising community awareness of the health effects of smokeless tobacco use are needed in rural communities. Programs should be directed at young males with lower levels of education.

Tob. Induc. Dis. 2018;16(Suppl 1):A14

DOI:10.18332/tid/84234 
12:30-14:00

PS-514-1 Tobacco control and sustainable development goals in Brazil

M Andreis ${ }^{1}$, A Monteiro ${ }^{2}$, P Johns ${ }^{3}$

${ }^{1}$ ACT Health Promotion, NGO Vice-Director, São Paulo, SP, Brazil, ${ }^{2}$ ACT Health Promotion, NGO Communications, Rio de Janeiro, RJ, Brazil, ${ }^{3}$ ACT Health Promotion, NGO Director, Rio de Janeiro, RJ,Brazil. E-mail: monica.andreis@actbr.org.br

Background and challenges to implementation: Brazil ratified FCTC and tobacco use decreased 55\% from 1990 to 2015. It is important to monitor and implement other policies to maintain this downward trend. Brazil is also committed with SDGs and presented its voluntary national report in 2017. Tobacco control was not mentioned on it. It is crucial to mobilize the government to include tobacco control and NCDs prevention in the agenda of SDGs. ACT is a Brazilian NGO working for FCTC implementation, healthy food policies and NCDs' prevention.

Intervention or response: A civil society work group was created to promote the Agenda 2030. It is composed by over 40 organizations from different areas. ACT is a member of the WG, which has been working on capacity building and governance, advocacy at Executive and Legislative Branches, communications strategies and participation in events. In 2017 the WG produced and disseminated a shadow report on SDGs implementation in Brazil.

Results and lessons learnt: The report titled "Spotlight synthesis report - the 2030 sustainable development agenda in Brazil" was launched in the High Level Political Forum on sustainable development with strong impact. It showed the gaps and provides recommendations for the achievement of the SDGs goals. It was also launched at national level, in 3 States so far. The inclusion of tobacco control and NCDs on the report was consequence of the ACT's involvement on it. The WG also collaborated for the creation of a National Commission, besides had key role on the creation of the Parliamentary Front for SDGs. A website was also created: https://brasilnaagenda2030.org/

Conclusions and key recommendations: It is crucial to promote tobacco control and NCDs prevention in the Agenda 2030 and the engagement with the broad group responsible for the SDGs implementation at national level. The Brazilian model of the civil society WG' strategies can be useful to other countries discussing SDGs priorities and means of implementation.

Tob. Induc. Dis. 2018;16(Suppl 1):A15

DOI:10.18332/tid/84258

\section{2:30-14:00}

PS-515-1 Effectiveness of tobacco cessation intervention among patients visiting primary care settings in India: a quasi-experimental study

R Panda', S Mahapatra ${ }^{2}$

'Public Health Foundation of India, Research and Policy, Gurgaon, India, ${ }^{2}$ Public Health Foundation of India, Research and Policy, Bhubaneswar, India. E-mail: raj.panda@phfi.org Background: Tobacco cessation interventions can substantially reduce tobacco-related morbidity. While we know $5 \mathrm{~A}$ is evidence based, its suitability for primary care (with minimum resources) has rarely been tested in low middle income countries. The present study aims to examine the effectiveness of tobacco cessation intervention on intention to quit among patients in primary care settings in two states India.

Methods: A quasi-experimental study was conducted among 1382 patients visiting primary care facilities in two states of India.
This study compared: the intervention arm comprising of intensive counseling (5As: Ask, Advice, Assess, Assist, Arrange) and the control arm comprising of routine advice by the physicians. Change in intention to quit in 30 days was the primary outcome measured at two time points (baseline and endline). Logistic regression model was applied using intention to treat principle with intention to quit as the primary outcome while adjusting for socio-demographic variables.

Results: About half of the patients were willing to quit tobacco in 30 days. An increase of $37 \%$ was observed from baseline to endline in intention to quit tobacco among patients in intervention units as compared to the $5 \%$ increase in control units. Patient who received tobacco cessation counseling were about three times more willing to quit tobacco use in intervention units as compared to those who received routine care in control units (intention to treat analysis; $\mathrm{OR}=2.85 ; \mathrm{CI}=1.78-3.8$; $\mathrm{p}$ value- 0.00 ).

Conclusions: The ' $5 \mathrm{~A}$ ' model used in the study for tobacco cessation can have a significant effect in motivating tobacco users to quit tobacco in primary care and may act as a viable option to be included in the routine care practices in primary care settings of India. More large scale studies and studies on the costeffectiveness of the intervention should also be taken up in future.

Tob. Induc. Dis. 2018;16(Suppl 1):A16

DOI:10.18332/tid/84269

\subsection{Using data: Opportunities and gaps in measuring WHO FCTC impact on global health and development}

\section{2:30-14:00}

PS-516-1 Usage of big data for global tobacco control: exploring the trends in quantitative research from 2006 to 2015

$\mathrm{H}$ Mongi ${ }^{1,2}$

'Tanzania Tobacco Control Forum, Dar es Salaam, Tanzania, United Rep., ${ }^{2}$ University of Dodoma, Department of Information Systems, Dodoma, Tanzania, United Rep... E-mail: hjmongi@ yahoo.com

Background: Measuring the impact of tobacco control initiatives like in other major themes of research relies on the data. Recently, there has been growing interest in the usability of big data which is characterized by large volumes, high computer processing speeds and huge storage networked databases. All these offer opportunity to make senses out of data through discovering new patterns and possibly creating new fields. This study tried to discover the usage trends of volumes of data in quantitative research to inform WHO FCTC impacts on tobacco control across the world between 2006 and 2015.

Methods: The systematic literature review and meta-analysis were adopted for data collection and analysis. The articles on quantitative research on tobacco control were collected from journals, books, as well as from abstracts and full texts presented at the World Conferences on Tobacco or Health (WCToH) during the period of 10 years from 2006 to 2015. Data collected were analysed by continents as well as at overall global level. Data were summarized by year, by continent, and by the objective of the research. Association between the number of yearly quantitative research articles on WHO FCFC impacts and the amount of data they employed and objective were determined using Pearson's Correlation Coefficients.

Results: Results indicated that there has been a growth in the amount of data in quantitative exploratory studies but limited 
in conclusive research which has a better chance to inform the WHO FCTC impacts. While the usage of volumes of data has been increasing over recent years, the increase varies according to geographical locations and economic status of the regions.

Conclusions: The study concludes that despite incremental data in impact-based quantitative research that demonstrates slowly response of tobacco control research ecosystem to emerging big data opportunities; there are still gaps in their massive usage across regions and continents.

Tob. Induc. Dis. 2018;16(Suppl 1):A17

DOI:10.18332/tid/83777

\section{$12: 30-14: 00$}

PS-517-1 Willingness to quit tobacco smoking and its correlates among Indian smokers - findings from GATS India, 2009 - 10

SS Kar ${ }^{1}$, MM Reddy ${ }^{1}$, S Kanungo ${ }^{1}$, BN Naik ${ }^{1}$

'JIPMER, Puducherry, Preventive and Social Medicine, Puducherry, India. E-mail: drsitanshukar@gmail.com

Background: In the behavioural change model for smoking cessation 'willingness to quit' forms the starting point. Identifying these sects of smokers who are willing to quit can lead to important policy development and program implementation in any country. To determine the prevalence and correlates of willingness to quit among smokers in India from Global Adult Tobacco Survey (GATS), 2009-10.

Methods: Secondary data analysis of GATS, 2009-10 was done to find the correlates of willingness to quit among smokers. All the socio-demographic variables, smoking related factors like frequency, previous attempt to quit and also effect of anti-tobacco messages delivered to various media were tested for association using multivariate analysis. Prevalence rate ratio with 95\% CI was used to report the risk.

Results: Of the 9627 current smokers analysed, 50.9\% (95\% CI: $49.9 \%$ to $51.9 \%$ ) were willing to quit smoking. Among these; $25.1 \%$ and $26.0 \%$ were willing to quit within the next month and within next 12 months respectively. Multivariate analysis showed that younger age, individuals who have their first smoke after 60 minutes of wakeup, those living in a house with smoking restriction, those who received advice to quit from doctor, those who attempted to quit in the past 12 months, having knowledge about illness caused due to smoking and those who have noticed anti-smoking messages in billboards / hoardings were willing to quit smoking compared to their counterparts.

Conclusions: About one in two smokers were willing to quit smoking and, among those who were willing to quit, half of them were willing to do so within the next one year. Targeted interventions can be planned in the sub-groups identified.

Tob. Induc. Dis. 2018;16(Suppl 1):A18

DOI: $10.18332 /$ tid/83881

\section{2:30-14:00}

PS-518-1 The Data on Alcohol and Tobacco in Africa (DATA) project

AV Darsamo', H Ross'1, L Woolfrey ${ }^{2}$

${ }^{1}$ University of Cape Town, School of Economics (Southern Africa Labour Development Research Unit), Cape Town, South Africa, ${ }^{2}$ University of Cape Town, School of Economics (DataFirst), Cape Town, South Africa. E-mail: darsamovanessa@gmail.com Background and challenges to implementation: Researchdriven tobacco and alcohol control policies need country-specific data. Although several institutions collect data on tobacco and alcohol, few tools exist for discovering and accessing these data in Africa. The goal of the Data on Alcohol and Tobacco in Africa (DATA) Project is to catalogue economic data on tobacco and alcohol in Africa and publish these data for research purposes. The pilot stage of the project focuses on Botswana, Kenya, Namibia, Senegal, and South Africa, with the goal of expanding across Africa.

Intervention or response: The DATA Project establishes relationships with data producers and obtains permission to share their data on the project's site. The Project documents our experience in securing data and identifies best practices for increasing open access to data.

The Project curates all secured data, shares these in researchready datasets, uses metadata to describe data available in external repositories and redirects users to these sites. It also collates time-series data from various publicly available sources into referenced data sheets. Our work saves researchers the time and effort involved in finding and collating data.

Results and lessons learnt: Currently 69 datasets are listed on the DATA Project's site. These include open access data as well as discovery metadata and links to relevant data available on other sites. Our findings reveal that despite the availability of relevant tobacco and alcohol data, data producers are not using the available tools to facilitate data visibility and access from their websites. We find that data collectors are still reluctant to make their data Open.

Conclusions and key recommendations: We hope to encourage Open access to data, by providing the essential infrastructure and expertise for data sharing. Additionally, we wish to advance the quality of these data, through data users' feedback. Our efforts should increase Open access data for alcohol and tobacco control in Africa.

Tob. Induc. Dis. 2018;16(Suppl 1):A19 DOI:10.18332/tid/83948

\section{$12: 30-14: 00$}

PS-519-1 Impact of tobacco control policies on smoking prevalence and quit ratios in 27 European Union countries from 2006 - 2014

A Feliu ${ }^{1,2,3}$, L Joossens $^{4}$, FT Filippidis ${ }^{5}$, GT Fong ${ }^{6,7}$, Cl Vardavas $^{8,9,10}$, Y Castellano ${ }^{1,2}$, C Martinez $z^{1,2,3}$, E Fernández $z^{1,2,3}$

'Institut Català d'Oncologia, Tobacco Control Unit, WHO Collaborating Centre for Tobacco Control, L'Hospitalet del Llobregat, Spain, ${ }^{2}$ Institut d'Investigació Biomèdica de BellvitgeIDIBELL, Cancer Prevention and Control Group, L'Hospitalet del Llobregat, Spain, ${ }^{3}$ University of Barcelona, Departament of Clinical Sciences, School of Medicine, Campus Bellvitge, L'Hospitalet del Llobregat, Spain, ${ }^{4}$ Association of European Cancer Leagues, Brussels, Belgium, 5 Imperial College London, Department of Primary Care and Public Health, School of Public Health, London, United Kingdom, ${ }^{6}$ University of Waterloo, Department of Psychology and School of Public Health and Health Systems, Waterloo, ON, Canada, 'Ontario Institute for Cancer Research, Toronto, ON, Canada, ${ }^{8}$ University of Crete, Clinic of Social and Family Medicine, School of Medicine, Heraklion, Greece, ${ }^{9}$ National and Kapodistrian University of Athens, Center for Health Services Research, School of Medicine, Athens, Greece, ${ }^{10}$ European Network for Smoking and Tobacco Prevention - ENSP, Brussels, Belgium. E-mail: afeliu@iconcologia.net

Background:Smokingis stillhighly prevalentinEurope. According to the $\mathrm{WHO}$, tobacco control policies vary substantially across countries. The Tobacco Control Scale (TCS) was developed 
to quantify the implementation of tobacco control policies at country level in Europe. The objective was to assess the impact of tobacco control policies (quantified by TCS scores) on smoking prevalence and quit ratios and their relative changes from 20062014 in 27 European Union (EU27) countries.

Methods: We conducted an ecological study at the country level. We used TCS scores in EU27 in 2007, and the prevalence of tobacco and quit ratios (No. ex-smokers / No. ever smokers) data from the Eurobarometer surveys (2006 and 2014 waves). We analysed the relationship between the TCS scores and smoking prevalence and quit ratios and their relative changes by means of scatter plots, Spearman rank-correlation coefficients (rsp), and a multiple linear regression model adjusted for all TCS components. Results: In EU27, the smoking prevalence decreased by $14 \%$ (95\%CI:7.3\%-20.6\%) (2006-2014) and varied from a relative decrease of $48.9 \%$ in Sweden to $0.4 \%$ in Bulgaria. The increase in the quit ratio in EU27 was 19.2\% (95\%CI:5.4\%-33.1\%) (2006-2014) and ranged from $125.8 \%$ in Sweden to $4.3 \%$ in Bulgaria. The correlation between TCS scores and smoking prevalence was negative ( $\mathrm{rsp}=-$ $0.444 ; \mathrm{p}=0.02$ ). A positive correlation was observed between TCS scores and quit ratios in $2014(\mathrm{rsp}=0.373 ; \mathrm{p}=0.06)$ and in the relative changes in smoking prevalence $(r s p=0.415 ; p=0.03)$. The percentage of smoking prevalence in 2014 explained by all TCS components in the regression model was $28.9 \%$

Conclusions: European countries with higher TCS scores, which indicates higher tobacco control efforts, have lower prevalence of smokers, higher quit ratios, and higher relative decreases in their smoking prevalence over the last decade. Funding: EC Horizon2020 HCO-6-2015 (EUREST-PLUS No. 681 109); Government of Spain \& European Regional Development Fund (RTICC RD12/0036/0053); Government of Catalonia (2014SGR999).

Tob. Induc. Dis. 2018;16(Suppl 1):A20

DOI: $10.18332 /$ tid/83951

\section{2:30-14:00}

PS-520-1 Trends in tobacco use among Indian adults 1998 - 99 to 2015 - 16: results from nationally representative data of the national family health surveys

GK Mini ${ }^{1}$, KR Thankappan ${ }^{2}$

${ }^{1}$ Amrita Institute of Medical Sciences, Public Health, Thiruvananthapuram, India, ${ }^{2}$ Sree Chitra Tirunal Institute for Medical Sciences and Technology, Achuth, Trivandrum, India. E-mail:gkmini.2014@gmail.com

Background: India ratified the framework convention on tobacco control (FCTC) in the year 2004. In order to understand the impact of the implementation of FCTC it is important to look at the trends in tobacco use. We studied the trends in tobacco use among Indian adults aged 15-49 years from 1998-99 to 2015-16. Methods: The data for the analysis were obtained from the three nationally representative population level cross-sectional surveys in India. We studied the National Family Health Survey-2 (NFHS-2) in 1998-99 (women:1,21,031; men:1,25,104), NFHS3 (women: 1,24,385; men: 69,751) in 2005-06 and NFHS-4 (women: $6,99,686$; men: $1,03,525)$ in 2015-16 in India. All the NFHS surveys included men and women in the age group of 15-49 years. Results: Any tobacco use by men increased from $46.5 \%$ in NFHS2 to $57 \%$ in NFHS-3 and decreased to $44.5 \%$ in NFHS- 4 . Any tobacco use by women reported consistent reduction in all the three NFHS surveys: $13.8 \%$ in NFHS-2, $10.8 \%$ in NFHS-3 and $6.8 \%$ in NFHS-4. Percentage reduction in tobacco use was higher in rural areas than in urban areas both among men (urban:50\% to $39 \%$; rural: $61 \%$ to $48 \%$ ) and women (urban: $7 \%$ to $4 \%$; rural: $13 \%$ to $8 \%$ ) from NFHS-3 to NFHS-4. Except the four states of Meghalaya, Andhra Pradesh, Manipur and Telangana, all other Indian states reported a reduction of any form of tobacco use from NFHS-3 to NFHS-4.

Conclusions: More efforts are required in the four states of Meghalaya, Andhra Pradesh, Manipur and Telangana to reduce tobacco use. In other states continued efforts are required to attain further reduction in tobacco use.

Tob. Induc. Dis. 2018;16(Suppl 1):A21

DOI:10.18332/tid/83974

\subsection{Setting the research agenda for translating science to policy and new tools for policy impact assessments}

16:00-17:30

SUS Cost-effectiveness and budget impact analysis of smoking cessation interventions in chronic obstructive pulmonary disease patients in Thailand R Kongsakon'

${ }^{1}$ Mahidol University, Psychiatry, Bangkok, Thailand. E-mail: ronnachaikong@gmail.com

Background: To determine the cost-effectiveness of different smoking cessation interventions in Thailand compared with unassisted cessation in chronic obstructive pulmonary disease (COPD) patients.

Methods: A cost-effectiveness study was undertaken using a societal perspective. Cohorts of smokers with COPD: male and female aged 35 years who regularly smoke at least 10 cigarettes per day were simulated in a markov model. Interventions were counseling with nortriptyline or bupropion or varenicline. All were compared to counselling in hospital. Incremental cost per quality-adjusted life years (QALYs) gained was calculated. One-way and probabilistic sensitivity analyses were also performed. Annually budget impact analyses were also estimated for five years.

Results: Based on Thai willingness-to-pay threshold (USD 4848), nortriptyline was the most cost-saving (USD 100) with QALY gained (0.07 QALY). Varenicline is cost-effective (USD 2181 per QALY) while bupropion was not cost-effectiveness (USD 4917 per QALY) when compared to counselling in hospital alone. Probabilistic sensitivity analysis revealed that nortriptyline has very high probabilities $(96 \%)$ of being cost-saving while varenicline and bupropion are $98 \%$ and $90 \%$. The most influential parameter is efficacy of counseling alone. Average annual budget for varenicline, bupropion and nortriptyline are $23 \mathrm{MB}, 25 \mathrm{MB}$ and $3 \mathrm{MB}$, respectively.

Conclusions: Adding pharmacology treatment to conventional counselling for smoking cessation resulted in cost-saving. The use of nortriptyline appeared to be the most cost-effective smoking cessation option for COPD patients. Promoting smoking cessation especially for patient with COPD will benefit for individual patient and societal perspective. Thai government may consider including smoking cessation program into national COPD clinic.

Tob. Induc. Dis. 2018;16(Suppl 1):A22

DOI: $10.18332 /$ tid/83780

\section{$12: 30-14: 00$}

PS-521-1 Restaurant smoking sections in South Africa and the perceived impact of the proposed smoke-free laws: evidence from a nationally representative survey 
M Little', C Van Walbeek

${ }^{1}$ University of Cape Town, School of Economics, Southern Africa Labour and Development Research Unit, Economics of Tobacco Control, Cape Town, South Africa. E-mail: firdaleconsulting@ gmail.com

Background: In 2016 the South African Minister of Health announced his intention to introduce tobacco control legislation to make all public areas completely smoke-free. As such, restaurant smoking sections, introduced in 2001, will become obsolete. This study aims to analyse current smoking policies of restaurants in South Africa, whether and how these policies have changed over the past decade, and restaurateurs' attitudes towards the proposed legislative changes.

Methods: From a population of nearly 12000 restaurants, derived from four websites, we sampled 2000 restaurants, stratifying by province and type (independent versus franchised) and disproportionately sampling small strata to ensure meaningful analysis. We successfully surveyed 741 restaurants, mostly telephonically. We also surveyed 60 franchisors from a population of 82 franchisors.

Results: Currently $45 \%$ of restaurants in South Africa do not have smoking sections, $44 \%$ have smoking sections outside and $11 \%$ have smoking sections inside. Smoking areas are more common amongst independent restaurants (62\%) than franchised restaurants (43\%). Of the restaurants with smoking sections, $33 \%$ of the smoking sections are busier than the non-smoking sections. $23 \%$ of restaurants have changed their smoking policies in the past ten years, mostly removing or reducing the size of the smoking sections. Customer requests (39\%), compliance with the law (35\%) and cost and revenue pressures (14\%) were the main reasons for changing smoking policies. $91 \%$ of the restaurant respondents support the current legislation, while $63 \%$ support the proposed legislative changes. $68 \%$ of respondents who are aware of the proposed legislation support it, compared to $58 \%$ of respondents who are not aware of the proposed legislation.

Conclusions: In contrast to the vehement opposition to the 1999 legislation, which resulted in restaurants going partially smokefree in 2001, there is limited opposition from restaurants to the proposed $100 \%$ smoke-free legislative changes. Support for the proposed legislation will probably increase as knowledge thereof grows.

Tob. Induc. Dis. 2018;16(Suppl 1):A23

DOI:10.18332/tid/83823

\section{2:30-14:00}

PS-522-1 Comparative jurisdictional analysis of municipal smoke-free policies in Canada

A Pederson ${ }^{1,2}$, CTC Okoli $^{3}$, W Rice ${ }^{4}$, JL Bottorff5,6

${ }^{1}$ BC Women's Hospital + Health Centre, Vancouver, BC, Canada, ${ }^{2}$ Simon Fraser University, Faculty of Health Sciences, Vancouver, BC, Canada, ${ }^{3}$ University of Kentucky, College of Nursing, Lexington, KY, United States of America, ${ }^{4}$ Centre of Excellence for Women's Health, Vancouver, BC, Canada, ${ }^{5}$ University of British Columbia's Okanagan Campus, Kelowna, BC, Canada, ${ }^{6}$ Institute for Healthy Living and Chronic Disease Prevention, Kelowna, BC, Canada.E-mail: ctokol1@uky.edu

Background: The growing adoption of outdoor smoke-free policies globally has endorsed a new area of tobacco control policy research. This study compares the development, adoption, and implementation of outdoor smoke-free bylaws in city parks and recreational areas within jurisdictions in British Columbia, Canada. This study combines theoretical policy analysis with data on policy implementation to inform discussions on alternative approaches to outdoor smoke-free policy.

Methods: This is a mixed methods comparative case study of three Canadian municipalities, informed by Kingdon's concept of policy spillover, Weimer's discussion of policy discourse, Sabatier's Advocacy Coalition Framework (ACF) and Mahoney at al.'s equity-focused health impact assessment. Key informant interviews $(n=20)$ and policy documents were used to generate a historical narrative of each jurisdictions' policy development process, and a thematic analysis of these data was conducted to identify dominant frames used to support the smoke-free policy in each setting. Particular attention was paid to discourses of evidence, equity and ethics--three theoretical constructs from the policy literature.

Results: Health, environmental and social concerns informed the introduction of the smoke-free policies, but the emphasis on each factor differed for historical, practical, and political reasons. Despite some uncertainty of the health effects of outdoor SHS exposure, health issues were the most salient. Equity concerns differed as well. Whereas there is little evidence equity issues were a concern in Vancouver, equity was a more prominent theme in the other jurisdictions.

Conclusions: This comparative policy analysis suggests that to be accepted, smoke-free policies should be tailored to the features of a particular setting. Documenting these variations can help guide tobacco control advocates to develop equitable policies, and inform the translation of tobacco control science into policy while using innovative equity focused tools to assess policy impact.

Tob. Induc. Dis. 2018;16(Suppl 1):A24

DOI:10.18332/tid/83969

12:30-14:00

PS-523-1 Gaps in tobacco control research in accordance with WHO FCTC guidelines: a systematic literature review in Thailand

S Benjakul', S Mongkolsomlit², C Phetphum ${ }^{3}$, K Sukornnun ${ }^{4}$ ${ }^{1}$ Mahidol University, Health Education and Health Promotion, Bangkok, Thailand, ${ }^{2}$ Thammasat University, Faculty of Public Health, Bangkok, Thailand, ${ }^{3}$ Naresuan University, Faculty of Public Health, Phitsanulok, Thailand, ${ }^{4}$ Mahasarakham University, Faculty of Public Health, Mahasarakham, Thailand. E-mail: dr.benjakul@gmail.com

Background: Evidence-based policy is a term often applied in various field of public health including tobacco control policy. Strengthening evidence -based tobacco control policy development should also base on reliable evidence, in particular research approach. The coverage of tobacco control research under WHO FCTC guidelines is crucial issues.

Methods: A systematic literature review was conducted for identifying the gaps in tobacco control research. The search for relevant publications was done by the search engine. The key inclusion and exclusion criteria were defined, focusing on published original article conducting in Thai context, both English and Thai language in the past 5 years. The quality of articles was assessed. The text of articles was analyzed based on WHO FCTC articles, at totally 15 articles as well as the 2nd National Strategic Plan for Tobacco Control 2015 - 2019.

Results: The initial search resulted in 1,048 abstracts. After applying the inclusion and exclusion criteria for two rounds, 176 original articles were selected for the review. In the article reviewed, the most of WHO FCTC articles applying into the research conceptual framework was article 20 (63.1\%) with the 
purpose of gain new knowledge on tobacco harms and effects on health and cross sectional survey design for surveillance tobacco use among various groups. The rest of articles were focused on research related WHO FCTC article 12 (31.0\%) and article 14 (19.3\%). Three articles did not conducted as a research framework, articles 5.3, 9 - 10 and 19. However, those resulted were implemented in specific area, setting and target groups that's not applicable for scaling up.

Conclusions: The successful transformation of research results to effective tobacco control policy under WHO FCTC depends on various issuers, especially time-based for conducting the research that sufficient to prove the project outcome and appropriate budget for comprehensive research design, coverage target area and population.

Tob. Induc. Dis. 2018;16(Suppl 1):A25

DOI:10.18332/tid/84040

\section{2:30-14:00}

PS-524-1 Developing synthetic birth cohortspecific smoking histories using Global Adult Tobacco Surveys - an example from India

D Sharma ${ }^{1}$, P Lal $^{2}$, A Pandey $^{3}$

${ }^{1}$ Government Medical College Chandigarh, Chandigarh, India, 'International Union Against Tuberculosis and Lung Disease, New Delhi, India, ${ }^{3}$ The Union SEA Office, Delhi, India. E-mail: drdeepakpgimer@gmail.com

Background: Nearly 111 million adults smoke in India, and a tenth (nearly 1.2 million) of these die from smoking attributable diseases every year. Predicting future trends based on current prevalence and other epidemiologic variables are essential for policymaking. However, given the paucity of reliable population level data, several high prevalence use countries especially in the developing world have been unable develop models that can inform policy process. We present the first such model for India based on GATS 2009 and 2017.

Methods: Using data from population-wide survey, we develop a synthetic cohort of smoking trends from 2010 to 2050 based on initiation, cessation, intensity, and prevalence patterns India between 2009 and 2017.

Results: We estimate that till 2037-2040, the number of smokers in India would be same as current rate of smoking prevalence, following which it begins to decline marginally (at about 0.8 to 1.2 percent on a year on year basis).

Conclusions: Policymakers need to take of this as smoking-related deaths will continue to rise beyond 2070 at current trends. This calls from stricter approaches for tobacco control in India like a "sinking lid" approach to reduce and eventually eliminate tobacco use in a given timeframe.

Tob. Induc. Dis. 2018;16(Suppl 1):A26

DOI: $10.18332 /$ tid/84336

\section{2:30-14:00}

PS-525-1 Chemical profiling of smokeless tobacco and their disease association

A Kumar ${ }^{1}$, D Bhartiya ${ }^{1}$, J Kaur², S Kumari ${ }^{1}$, A Sharma², H Singh ${ }^{2}$, D Sinha ${ }^{1}$, R Mehrotra

${ }^{1}$ National Institute of Cancer Prevention and Research (NICPR), Noida, India, ${ }^{2}$ Indian Council of Medical Research, New Delhi, India.E-mail: amitbioinfo@gmail.com

Background: Smokeless tobacco (SLT) products are consumed by millions of people in over 130 countries around the world. SLT products have a large impact on the health of individuals since the products cause several diseases including cancer.
Though the smokeless tobacco products have been found to be associated with a large number of health effects and diseases, the understanding of the functions of chemical compounds of the products and their toxicity is very limited. There is a complete lack of extensive analysis of the targets of the chemical compounds and their associated biological mechanisms remain unknown.

Methods: One hundred and one chemical compounds of SLT products were studied and similarity search algorithms were used to identify similar compounds along with their protein targets using ChEMBL. The protein targets were functionally annotated using PANTHER classification system and Kyoto Encyclopedia of Genes and Genomes (KEGG) to identify the associated Gene Ontologies and diseases respectively and the interaction network was studied. In addition, pharmacological and toxicological profiling ADMET (Absorption, Distribution, Metabolism, Excretion and Toxicity) of chemicals were also studied.

Results: The study shows that the chemical compounds from SLT products target more than 350 human proteins involved in a plethora of human biological processes and affect around 100 pathways. The study has identified associations of diseases such as neurodegenerative, immune and cardiac diseases which were not known previously along with already established diseases such as cancer.

Conclusions: We provide the first comprehensive report and systematic analyses of 101 chemical compounds from different SLT products used worldwide. The finding indicates far-reaching impact of SLT products on human health than already known and this finding needs further validations using epidemiological, in-vitro and in-vivo methodologies.

Tob. Induc. Dis. 2018;16(Suppl 1):A27

DOI:10.18332/tid/84371

\subsection{The End Game - Pathways to turning vision into reality.}

\section{4:30-16:00}

F0-335-1 Modelling how to achieve 5\% adult smoking prevalence by 2025: a regional approach A Rutter ${ }^{1}$, R West $^{2}$

${ }^{1}$ Fresh, Smokefree North East, Durham, United Kingdom, ${ }^{2}$ University College London, London, United Kingdom. E-mail: ailsa.rutter@freshne.com

Background: The North East of England (population 2.6 million) has a notable track record in tobacco control, and local government organisations have a vision to "Make Smoking History", with all 12 localities agreeing in 2014 to work towards 5\% adult smoking by 2025 . The target seems ambitious but a comprehensive subnational programme is in place to achieve it. With a baseline smoking prevalence of $18.7 \%$ in 2015 , work was undertaken in 2017 to model current trends in smoking prevalence, uptake and quitting in the North East, and identify key targets and policies for further improvements and to assess how realistic the goal is. Methods: Data from the Smoking Toolkit Study (www. smokinginengland.info - annual sample size national of $>20,000$ ) and the UK's Annual Population Survey (annual national samnple size 300,000 ) were used to model trends in key parameters (cigarette smoking prevalence; ever-smoking prevalence in young people; quit attempt prevalence; quit success rate; overall quit rate) and make forecasts under a range of different assumptions. Results: Smoking prevalence has declined in the North East slightly faster than the national average and this appears to be because of higher quit success rates. Increasing quit attempt rate 
to $45 \%$ per year, maintaining a quit success rate at $20 \%$, and reducing uptake to $0.3 \%$ per year could put the North East on a path to $5 \%$ adult smoking prevalence within 10 years. These are all realistic targets but will require the implementation of a package of policies and a sustained commitment to investment.

Conclusions: Modelling has shown that a bold ambition of all but eliminating smoking by 2025 can be achieved in an English region without unrealistic assumptions about the changes that need to be made in uptake, quit attempts and quit success rates. This modelling work has galvanised further action especially within the health system.

Tob. Induc. Dis. 2018;16(Suppl 1):A28

DOI:10.18332/tid/84018

\section{4:30-16:00}

\section{F0-336-1 Achieving an end to government tobacco} tax revenues: another challenge for the tobacco endgame?

SL Hogg ${ }^{1}$, G Thomson ${ }^{2}$, R Edwards ${ }^{2}$

${ }^{1}$ University of Edinburgh, College of Medicine and Veterinary Medicine, Edinburgh, United Kingdom, 2University of Otago, Wellington, New Zealand. E-mail:scott.hogg@doctors.net.uk Background: Tobacco taxation is an effective intervention to reduce tobacco consumption. Tobacco taxes also generate substantial government revenue, and this is often framed as an argument to support tobacco tax increases. Achieving the tobacco endgame would eliminate tobacco taxation revenue, and the implications of this for endgame progress are not currently understood. This study aimed to:

(1) Quantify changes to tobacco tax revenue in four endgamecommitted countries (New Zealand, Finland, Scotland, Ireland).

(2) Review how revenue in an endgame context is addressed in tobacco tax policy guidance published by health and financial institutions.

Methods: (1) Annual tobacco tax receipts (2008-2015, as available) were extracted from online government sources and tobacco revenue as a proportion of total government tax revenue calculated.

(2) Relevant published guidance was identified using dynamic Internet searches and reviewed with respect to our research question.

Results: In three countries, tobacco revenue increased in absolute terms and as a proportion of total government revenue over the study period: New Zealand ( $\$ 1001 \mathrm{~m}$ to $\$ 1562 \mathrm{~m}, 1.82 \%$ to $2.41 \%$ ), Finland ( $€ 622 \mathrm{~m}$ to $€ 874 \mathrm{~m}, 0.78 \%$ to $0.95 \%$ ) and Scotland ( $\$ 917 \mathrm{~m}$ to $£ 1224 \mathrm{~m}, 2.02 \%$ to $2.98 \%$ ). In Ireland, revenue decreased in absolute and proportional terms ( $€ 1171 \mathrm{~m}$ to $€ 1082 \mathrm{~m}, 2.05 \%$ to $1.73 \%$ ).

Published guidance of the World Health Organisation, National Cancer Institute, International Monetary Fund and World Bank included no specific reference to tax revenue in an endgame context. Conclusions: Increasing government tobacco tax revenues threaten to impede political will for continued endgame progress. Despite this, current policy guidance includes no recommendation regarding how governments may transition towards having no tobacco taxation revenue. Our findings suggest that Ireland have exceeded the point of 'critical elasticity': where increases to the tobacco tax rate are associated with declining revenue. This is an informative example of one way in which governments may progressively diminish tax revenue in preparation for the endgame.

Tob. Induc. Dis. 2018;16(Suppl 1):A29

DOI:10.18332/tid/84176

\section{4:30-16:00}

F0-337-1 The tobacco reduction targets act: a legislated phase out for combustible cigarettes C Callard', N Collishaw

'Physicians for a Smoke-Free Canada, Ottawa, ON, Canada. E-mail: ccallard@smoke-free.ca

Background and challenges to implementation: The recent widespread marketing of alternative nicotine devices and heated tobacco products is heralded by some as a viable harm reduction strategy, and feared by others as a means of perpetuating the smoking epidemic. Missing from the often heated debate are proposals for ways to ensure that combustible products are removed from the market as these 'reduced risk' products are introduced.

Intervention or response: In other markets involving such diverse consumer products as light bulbs, automobile fuel, refrigerators, the introduction of less harmful goods has been accompanied by requirements that the more harmful products are removed from the market. Such interventions have yet to be implemented for the manufactured cigarette or other combustible tobacco products. Regulatory approaches developed for these other goods are available as an ENDGAME tool for tobacco control, and prototype legislation developed for Canada illustrates one of many ways in which this can be done.

Results and lessons learnt: Much has been learned from how harmful products can be successfully phased out. The challenge remains to see how well these lessons can be applied to a proposed phase-out of combustible tobacco products.

Conclusions and key recommendations: Voluntary efforts by tobacco companies to remove combustible products from the market cannot be relied upon. Legislative approaches to remove the most harmful products from circulation can be developed as a next generation tobacco control measures.

Tob. Induc. Dis. 2018;16(Suppl 1):A30

DOI:10.18332/tid/84158

14:30-16:00

F0-338-1 How many cigarettes a day in an endgame?

M Chaiton ${ }^{1}$, E Guindon ${ }^{2}$

${ }^{1}$ University of Toronto, Toronto, ON, Canada, ${ }^{2}$ McMaster University, Hamilton, ON, Canada. E-mail: michael.chaiton@ utoronto.ca

Background: Around the world, countries are setting endgame targets that look to bring smoking prevalence below a certain rate. Canada, for instance, has set a target of $5 \%$ smoking by 2035. However, what numbers of cigarettes smoked per day should countries expect as they approach these scenarios? The information is particularly important for government to understand the impacts of endgames on revenue.

Methods: A population change model was developed to predict change in cigarettes per day under endgame scenarios for the Province of Ontario, Canada. Population predictions were obtained from Statistics Canada for the period 2014-2035 including expected births, deaths, and immigration by age group (12-19, 20 34, 35-44, 45-64, 65+). Smoking prevalence was obtained from the 2014 Canadian Community Health Survey. Change in cigarettes per day was modelled scenarios assuming that the effects on cigarettes per day was consistent with prevalence change.

Results: To reach the tobacco endgame by 2035 , it would be require for Ontario to go from 2.1 million smokers in 2014 to 
700,000 smokers in 2035 with expected prevalence rates ranging from $1.8 \%$ among $12-19$ year olds to $6.9 \%$ among $20-34$ year olds. Average number of cigarettes per day was expected to be 4.0 cigarettes smoked per day on average by 2035 down from 13.3 cigarettes a day in 2014.

Conclusions: In the province of Ontario, it would be expected that the average remaining smoker in an endgame scenario would smoke 4 cigarettes per day. This analysis uses the same assumptions used to estimate the effects of tax on prevalence, but the effect of prevalence change on cigarette consumption may differ with other interventions. However, this analysis presents a challenge to the hardcore hypothesis.

Tob. Induc. Dis. 2018;16(Suppl 1):A31

DOI: $10.18332 /$ tid/83941

\section{4:30-16:00}

F0-339-1 Should we pay all smokers to quit smoking? A mixed methods survey exploring New Zealand smokers' perceptions of financial incentives

L Robertson ${ }^{1}$, P Gendall2, J Hoek², L Marsh'1, R Mcgee

'University of Otago, Preventive and Social Medicine, Dunedin, New Zealand, '2University of Otago, Marketing, Dunedin, New Zealand. E-mail: I.robertson@otago.ac.nz

Background: Evidence suggests financial incentives can support smoking cessation among pregnant smokers and for employees in a workplace setting, yet low acceptability may limit the wider implementation of such schemes. The increasing adoption of endgame goals by numerous countries indicates a need for new and potentially controversial strategies that could bring about rapid declines in smoking prevalence. Limited research has examined how smokers themselves view the idea of financial incentive interventions for which the general population of smokers would be eligible.

Methods: A sample of 623 smokers was recruited from an internet panel to a survey assessing support for, and perceived effectiveness of, financial incentives for smoking cessation. We used descriptive statistics, plus logistic regression, to test associations between demographics and smoking, and support. We used qualitative content analysis to analyse open-ended responses to a question that invited respondents to comment on financial incentive schemes.

Results: Thirty-eight percent of smokers supported financial incentives to quit smoking; $42 \%$ did not. Support was higher among heavy (OR 3.96, CI 2.39 - 6.58) and moderate smokers (OR 1.68, CI 1.13 - 2.49), and those with a recent quit attempt (OR $1.47,1.04-2.07)$. Support was strongly associated with perceived effectiveness. Of those who did not oppose financial incentives: $45 \%$ preferred a Government-funded reward-only scheme, while $35 \%$ preferred a Government-funded deposit-based scheme; few respondents supported employer-funded schemes. Openended responses $(n=301)$ indicated three overarching themes in relation to opposition to financial incentives: smokers' individual responsibility for quitting, concerns about abuse of an incentive scheme, and concerns about unfairness.

Conclusions: Financial incentive schemes designed to reward smokers for quitting create controversy and attract low public support, despite growing evidence of their effectiveness. Media advocacy and health education could be used to increase public understanding of, and support for, financial incentives for smoking cessation.

Tob. Induc. Dis. 2018;16(Suppl 1):A32

DOI: $10.18332 /$ tid $/ 84068$

\section{4:30-16:00}

F0-340-1 A model positive licensing scheme for tobacco sellers

S Walsberger ${ }^{1}, \mathrm{M}$ Allen ${ }^{2}$, R Ireland ${ }^{1}$, J Carpenter ${ }^{2}$

${ }^{1}$ Cancer Council NSW, Cancer Prevention and Advocacy Division, Woolloomooloo, NSW, Australia, ${ }^{2}$ Allen and Clarke, Wellington, New Zealand. E-mail: scottw@nswcc.org.au

Background: Evidence shows retail availability of tobacco is ubiquitous and associated with higher smoking rates and relapse among attempting quitters. Few strategies to reduce availability are included in comprehensive tobacco control frameworks. This research aimed to develop a model licensing scheme to reduce the number of retailers and curb the supply of tobacco products. Methods: A literature review was conducted to identify international best practices in retail licensing. The components of existing schemes were compiled, alongside evidence of effectiveness and applicability within the Australian regulatory environment. Expert feedback was gathered at a forum of policymakers, researchers and international guest speakers from jurisdictions where licensing schemes had been successfully implemented. Recommendations following the forum were incorporated into the model.

Results: A model licensing scheme will be presented. This model is being promoted in Australia and potentially other developed and perhaps developing countries. A licensing scheme should cover all tobacco retailers, require an annual (re)application process and fees that ensure full cost recovery. It should include a range of measures for ensuring full compliance with retail controls, including sanctions and the ability to lose the licence for non-compliance with specific conditions or wider tobacco control laws. A licensing scheme can also be used as a vehicle for controls on density/number of retailers, and for securing useful data on the retail environment, as well as for education of customers.

Conclusions: A licensing scheme is a foundational step on which further regulatory steps may be required in order to reduce supply over time, such as caps on the number of licenses issued. In countries where the number of licences have been capped, smoking rates have decreased and compliance with tobacco control laws has increased. Reducing the retail availability of tobacco products must be a priority alongside other proven strategies in comprehensive tobacco control in the future.

Tob. Induc. Dis. 2018;16(Suppl 1):A33

DOI: $10.18332 /$ tid/84168

$14: 30-16: 00$

F0-341-1 How tobacco control advocates and organizational leaders in California view endgame policies

\section{E Smith ${ }^{1}$, P McDaniel ${ }^{1}$, R Malone $^{1}$}

${ }^{1}$ University of California, Social \& Behavioral Sciences, San Francisco, CA, United States of America. E-mail: ruth.malone@ ucsf.edu

Background: Tobacco control policy innovations often start at the local level. The U.S. state of California, and localities within it, have been tobacco control policy leaders. Currently, policies designed to end, rather than just reduce or mitigate the tobacco epidemic are being discussed around the world. This study explored California tobacco control leaders' and advocates' opinions about new policy concepts characterized as endgame ideas.

Methods: We conducted 7 focus groups with tobacco control advocates (professional and volunteer) in 5 California cities. In 
each group, participants were invited to comment on 4 briefly described proposed endgame policies (smoker licensing, banning sales, aging out smoking/tobacco-free generation (TFG), and gradual retailer reduction). We also interviewed leaders of 11 major tobacco control and health voluntary organizations and networks. Interview questions explored participants' knowledge of tobacco endgame discussions and ideas, and their reactions to the 4 policy proposals.

Results: Of the four proposals, the TFG and retailer reduction were the most popular, though some participants saw advantages and disadvantages to all of them. TFG's focus on youth and noninterference with current smokers were regarded positively; however, participants were concerned that continuing to allow sales to current smokers would limit its effectiveness and might exacerbate existing inequities. Retailer reduction was approved for being politically viable, addressing the problem more directly, and focusing on sales rather than users. Objections included doubt about its effectiveness, and concerns about equity if reductions were not distributed fairly. All proposed policies were thought to lead to black markets.

Conclusions: Advocates and leaders were concerned that endgame proposals be effective and equitable. Black markets were seen as a downside to all proposals. Novel proposals will need careful development and messaging to gain advocates' support.

Tob. Induc. Dis. 2018;16(Suppl 1):A34

\section{DOI: $10.18332 /$ tid/83833}

\section{2:30-14:00}

PS-526-1 California policymakers' perspectives on tobacco endgame approaches

P McDaniel $^{1}$, E Smith ${ }^{1}$, R Malone ${ }^{1}$

'University of California San Francisco, Department of Social and Behavioral Sciences, San Francisco, CA, United States of America. E-mail: ruth.malone@ucsf.edu

Background: California is uniquely positioned to achieve a tobacco endgame, due to the effectiveness of its state tobacco control program in changing public views of tobacco and of the tobacco industry, public support for stronger policy measures, and dedicated resources. However, no research has explored how California policymakers view the endgame goal and proposed options for achieving it.

Methods: We conducted interviews with 11 California state legislators and legislative aides. Questions focused on their reactions to four state-level endgame approaches: banning tobacco sales, reducing the number of retailers over a ten-year period, registering smokers, and prohibiting tobacco sales to all those born after a certain year ("tobacco-free generation").

Results: The tobacco sales ban was rejected by most interviewees on the grounds that it would lead to black markets. Opinion was divided regarding the appeal of the remaining endgame proposals, with each drawing some support. However, interviewees identified two proposals as the most politically feasible and least controversial: retailer reduction, which, in the words of one interviewee, represented a "long going out of business curve for the tobacco industry in California," and the tobacco free generation proposal, which could be framed as protecting youth while maintaining the status quo among current smokers. All interviewees agreed that the state legislature would be more inclined to act on one or more endgame proposals if similar action occurred at the local level first.

Conclusions: Endgame proposals that gradually eliminate tobacco sales are considered politically feasible by California policymakers. Action at the local level is likely a necessary precondition of state-level policymaking.

Tob. Induc. Dis. 2018;16(Suppl 1):A35

DOI: $10.18332 /$ tid/83794

\section{2:30-14:00}

PS-527-1 Ban on Gutka in India: symbolic Victory or actual end-game for smokeless tobacco. Largescale household survey in Delhi finds out!

G Kumar ${ }^{1}$, P Kumar ${ }^{1}$, Smokeless Tobacco Users, Gutka Users ${ }^{1}$ GMERS Medical College \&t Hospital, Community Medicine, Ahmedabad, India. E-mail: gauravkumar067@yahoo.com

\section{Background: Smokeless}

tobacco (ST) use by over 200 million is major public health challenge in India. Gutka and Khaini are most popular products. Beginning in 2012, most states in India banned gutka and panmasala containing tobacco. Ban on ST seemed like beginning of End-game. Almost 10 studies evaluated implementation or impact of ban. But, suffered from convenience sampling and reporting bias. Through first-of-its-type large-scale household survey in Delhi on Global Adult Tobacco Survey (GATS) model, we assess impact of ban on practices, patterns and quitting of tobacco by gutka users.

Methods: Male adults in Delhi were interviewed during Mar-Dec, 2016 on tobacco-use currently and before gutka-ban. Changes were made in questionnaire of GATS-India (2010), developed by CDC,WHO and Govt. of India, to accommodate retrospectivecohort study design. 1710 households were sampled using 3-step randomization. Inbuilt mechanisms in standardized questionnaire cross-validated self-report and minimized recall-bias. Data were entered into SPSS and statistically analyzed.

\section{Results:}

$95.2 \%$ of 1710 household visited agreed to participate. Delhi banned ALL ST products in 2015, but except pre-mixed gutka, all ST products are freely available. Interestingly, $97.5 \%$ respondents believed tobacco as very harmful(78.9\%) or somewhat harmful(18.6\%). However, only $18.84 \%$ gutka users attempted quitting after ban. $2.4 \%$ successfully quitted. $84.1 \%$ of pre-ban gutka-users switched to twin-sachet (pan-masala and chewingtobacco sold separately to circumvent law). Another major shift is in unit size from single-dose sachets earlier to multi-dose sachet. $10.1 \%$ switched to khaini or other ST products. $3.4 \%$ shifted to smoking. But $1.4 \%$ among them were former dual users.

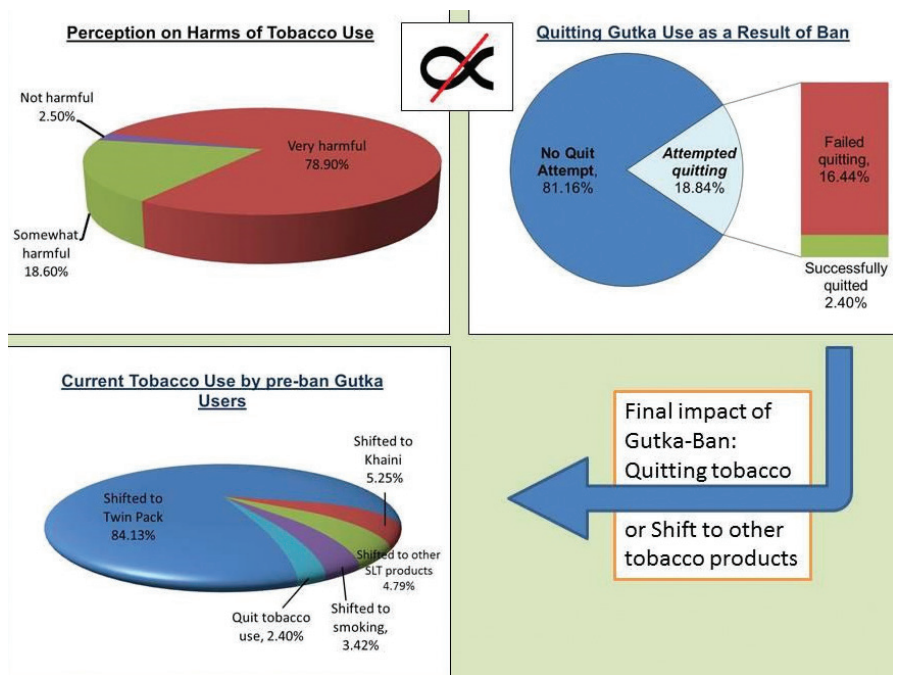

[Impact of gutka-ban on pattern of tobacco use and ] 
Conclusions: If selective ST products are banned, most users switch to another ST product. Though few try quitting, success rate is low. There is minimal tendency to initiate smoking. Hence, apprehension of increased smoking and second-hand smoke exposure isn't true. Only comprehensive ban on ALL SLT products coupled with strong quitting campaign can achieve End Game.

Tob. Induc. Dis. 2018;16(Suppl 1):A36

DOI:10.18332/tid/83876

\section{2:30-14:00}

PS-528-1 Redesigning government regulations towards a tobacco endgame strategy: a comparative law approach

LE Yarcia ${ }^{1,2}$, AC Nadate N $^{3,4}$

${ }^{1}$ Alliance for Improved Health Outcomes, Quezon, Philippines, ${ }^{2}$ University of the Philippines, College of Law, Diliman, Philippines, ${ }^{3}$ Health Justice Philippines, Quezon, Philippines, ${ }^{4}$ University of the Philippines-Diliman, College of Law, Quezon, Philippines. E-mail: Ipyarcia@up.edu.ph

Background and challenges to implementation: Developments in international law, after international tribunals' respective rulings in Philip Morris Asia Ltd. v. Australia (2017) and Philip Morris v. Uruguay (2016), have re-articulated and re-affirmed States' police power to carry out non-discriminatory regulation for the protection of public health in accordance with due process. This norm allows domestic legal systems to reframe tobacco industry regulation in many ways, one of which is towards nationalisation or public monopolisation, with the end-goal of decreasing supply vis-à-vis demand reduction through smoking cessation, denormalisation, and health promotion strategies.

Intervention or response: A critical review of sources of law (i.e., conventional and customary international law, and domestic constitutions and statutes) using a comparative law approach in several jurisdictions was done to define the norm described and set its scope and limits. Using the Philippines as a more in-depth case study considering its hybrid legal regime, which incorporates both civil and common legal traditions, a normative or law-making process designed for a tobacco endgame strategy has been formulated.

Results and lessons learnt: When incorporated in domestic jurisdictions and in consonance to treaty obligations under the Framework Convention on Tobacco Control, the norm described can potentially carve out an exception to general antimonopolistic measures seen in many jurisdictions. Legal analysis of the Philippine Constitution shows that public monopolisation of the tobacco industry was considered during the debates of the Constitutional Commission, therefore qualifying the anti-monopoly provisions of country's charter. Harmonising constitutional intent with international legal obligations allows the Legislature to craft statutes designed for a tobacco endgame strategy in the country. Conclusions and key recommendations: Incorporation of international law in domestic law allows States to formulate tobacco endgame strategies through legislative and/or executive actions. The formulation based on the Philippines could be a basis for other States to formulate their respective end game strategies, taking into consideration their unique political, cultural, economic, and epidemiological contexts.

Tob. Induc. Dis. 2018;16(Suppl 1):A37

DOI:10.18332/tid/83877

\section{$12: 30-14: 00$}

PS-529-1 Seeking patterns of countries banning sales of electronic nicotine delivery systems at different stages of the tobacco epidemic: an exploratory analysis

A Liber ${ }^{1,2}$, R Kennedy ${ }^{3}$

${ }^{1}$ University of Michigan, Health Management and Policy, Ann Arbor, MI, United States of America, ${ }^{2}$ American Cancer Society, Intramural Research, Atlanta, GA, United States of America, ${ }^{3}$ Johns Hopkins Bloomberg School of Public Health, Department of Health, Behavior \& Society, Baltimore, MD, United States of America. E-mail: alex.liber@cancer.org

Background: Emerging tobacco products including electronic nicotine delivery systems (ENDS) present an opportunity and a challenge with respect to charting a pathway to the demise of tobacco use. As of June 2017, 35 countries ban the retail sale of ENDS. The current study sought to understand the associations between countries banning sales of ENDS and the country's stage of the tobacco epidemic in the Lopez (1994) model.

Methods: Data on the policy choices of 186 countries to ban retail sales of ENDS was combined with daily cigarette smoking prevalence and death figures from tobacco smoking by country, sex, and year. Countries in 2015, were classified as being in stages 1 to 4 of the Lopez epidemic model, according to whether tobacco smoking prevalence and attributable deaths were rising or falling over the prior decade. Logistic regression analysis on the final cross-sectional dataset was performed to test the hypothesis that countries in the earlier stages of the tobacco epidemic and countries with relatively smaller populations of female smokers were more likely to ban the sale of ENDS.

Results: $18.8 \%$ of countries in the total sample banned the retail sale of ENDS. Countries in stages 1, 2, and 3 of the epidemic had odds ratios of $1.024,1.106$, and 0.633 relative to countries in the final stage of the epidemic of adopting a ban on retail sales of ENDS. Although statistically insignificant (all p>0.05), the proportions suggest that those jurisdictions at the height of the epidemic (stage 3 ) are slightly less likely to ban e-cigarettes.

\begin{tabular}{ccc|} 
Stage & Vot Bamned & Bamned \\
\hline 1 & 18 & 5 \\
2 & 10 & 3 \\
3 & 64 & 11 \\
4 & 59 & 16 \\
\hline
\end{tabular}

[Country ENDS Sale Ban by Stage of Tobacco Epidemic]

Conclusions: Countries in early stages of the tobacco epidemic may be less likely to ban e-cigarettes, because there may be little to no presence of the products. Countries in the latter stages of the tobacco epidemic may be more able to regulate e-cigarettes, rather than ban them.

Tob. Induc. Dis. 2018;16(Suppl 1):A38

DOI:10.18332/tid/84006

\section{$12: 30-14: 00$}

PS-530-1 Baseline and Endline study helped administration to declare 8 districts compliant to various sections of Indian Tobacco Control Act in a scientific manner

MK Sinha'

'M.P. Voluntary Health Association, Indore, India. E-mail: sinhamukesh63@gmail.com

Background: Section 4 of Indian Tobacco Control Act prohibits smoking at public places,section 5 prohibits advertisements of 
tobacco products, section 6 a prohibits sale of tobacco to minors, $6 \mathrm{~b}$ prohibits sale of tobacco within 100 yards of educational institution, section 7 demands mandatory 85 percent pictorial warnings on tobacco products. Madhya Pradesh Voluntary Health Association with support of The Union in collaboration with administration, and health department carried out various activities for implementation of Indian Tobacco control Act in 8 districts of Madhya Pradesh.The process was continued for 18 months and at the end an Endline compliance study was conducted.

Methods: Study was conducted in 8 districts, 7 types of public places like accommodation facilities, eateries, offices, educational institutes ,healthcare facilities , commonly visited places and public transport were observed with point of sale. The sample size was obtained by using The Union \& CTFK Compliance Assessment Guide and Open Epi software.Districts were divided into clusters and random sampling was done from each cluster. A total of 57 blocks of 8 districts with sample size of 6544 public places and 1143 Point of Sale were covered doing cross sectional study by trained field investigators.

Results: 4 districts Betul,Hoshangabad Sagar and Tikamgarh had more than 80 percent compliance of section 4,6a and 7 of Indian Tobacco control Act. 4 districts Harda,Panna,Damoh and Chhatarpur had more than 80 percent compliance on section $4,5,6 \mathrm{a}$ and 7 of Indian tobacco control act. Based on the findings of the study 8 District Magistrate declared their respective districts compliant to various sections of Indian Tobacco Control Act. Thereby protecting 15 million population.

Conclusions: Compliance survey are useful in understanding the status of implementation of tobacco control act and to make official declaration of the district compliant to various sections of the act by the local administration.

Tob. Induc. Dis. 2018;16(Suppl 1):A39

DOI:10.18332/tid/84048

\section{2:30-14:00}

PS-531-1 Achieving the tobacco endgame: the case for removing additives: findings from the ITC New Zealand Survey

R Edwards' ${ }^{1}$, A Waa ${ }^{1}$, J Stanley ${ }^{2}$, S Kaai ${ }^{3}$, A C.K.Quah ${ }^{3}$, G Fong ${ }^{3,4}$ ${ }^{1}$ University of Otago, Public Health, Wellington, New Zealand, 'University of Otago, Dean's Department, Wellington, New Zealand, ${ }^{3}$ University of Waterloo, Psychology, Waterloo, ON, Canada, ${ }^{4}$ Ontario Institute for Cancer Research, Toronto, ON, Canada. E-mail: richard.edwards@otago.ac.nz

Background: Tobacco products can contain a range of flavourings designed to reduce the harshness of tobacco and enhance its taste. These features can make it easier for new smokers to become addicted or existing smokers to maintain their addiction. A recently released national action plan identified removal of tobacco flavourings such as menthol as an important strategy for achieving New Zealand's (NZ) 2025 endgame goal. The present study explored smoker use of and support for banning tobacco flavourings.

Methods: Data were drawn from the first wave (Aug 2016-April 2017) of the International Tobacco Control (ITC) New Zealand Survey. Interviews were conducted using computer aided telephone interviewing. The sample comprised 1,023 smokers including 358 who identified as Māori (indigenous peoples of NZ).

Results: Almost two thirds of participants said taste was part of their decision for smoking their preferred brand. Around a fifth of smokers were using tobacco with any flavour including menthols, with over $10 \%$ reporting they smoked menthols, and a further $10 \%$ using tobacco with a flavour other than menthol. Females (18\%) were much more likely to smoke menthols than males (7\%). Males (13\%) were more likely than females (6\%) to smoke other types of flavoured tobacco. Only $11 \%$ of menthol cigarette users thought they were healthier than regular cigarettes. Among all menthol users around half said they would reduce the amount they smoked or quit smoking entirely if menthol flavoured tobacco was banned. Around half of participants supported banning flavours added to tobacco.

Conclusions: Tobacco flavour is an important factor in determining brand preference. A substantial proportion of NZ smokers smoked flavoured tobacco products. The finding that the removal of flavours like menthol would encourage some smokers to quit or cut down combined with evidence of support among many smokers indicates there is a strong case for removing tobacco flavourings.

Tob. Induc. Dis. 2018;16(Suppl 1):A40 DOI:10.18332/tid/84074

12:30-14:00

PS-532-1 Decreasing temptation: attitudes to tobacco sales by alcohol-licensed premises and policy implications

S Burton ${ }^{1}$, M Ludbrooke², K Williams², S Walsberger², S Egger ${ }^{2}$ ${ }^{1}$ Western Sydney University, Penrith, NSW, Australia, ${ }^{2}$ Cancer Council NSW, Sydney, NSW, Australia. E-mail: s.burton@ westernsydney.edu.au

Background: In response to evidence that tobacco retailers contribute to smoking by creating environmental cues to smoke and by making cigarettes accessible, multiple commentators have called for a review of tobacco retailing, and a decrease in the number of retailers. Among tobacco retailers, outlets licensed to sell alcohol are particularly problematic, due to the strong association between consuming alcohol and smoking. Yet policy action to decrease the number of tobacco retailers is rare, leaving voluntary cessation of selling tobacco as the most likely mechanism to decrease an observed over-supply of tobacco retailers. This study investigates the attitude of alcohol-licensed premises to selling tobacco, to inform potential policy options for stopping tobacco sales at such premises.

Methods: Data were collected by a telephone survey of 1,042 bars, pubs and packaged liquor outlets in NSW Australia. The response rate was $86.1 \%$. Qualitative and quantitative data were obtained. Logistic and linear regression were used to determine factors associated with the probability of selling and stopping selling, and the importance of cigarette sales.

Results: While there were differences in the reported importance of tobacco sales by different types of alcohol-licensed outlets, more outlets rated tobacco sales as 'unimportant' than 'important', and nearly a third considered that tobacco sales would be banned in alcohol-licensed premises within five years. Qualitative responses showed that many outlets thought that such a ban would have little effect on profits, and that smokers would adjust.

Conclusions: Despite claims by tobacco companies that tobacco sales are important for many Australian retailers, tobacco sales appear to be of very limited importance for alcohol-licensed premises. This means that opposition to stopping tobacco sales where alcohol is consumed may be less than expected, and a licensing scheme with an annual fee may provide a mechanism to encourage such outlets to cease tobacco sales.

Tob. Induc. Dis. 2018;16(Suppl 1):A41

DOI:10.18332/tid/84162 


\section{2:30-14:00}

PS-533-1 The effect of tobacco control policies on smoking prevalence using the SimSmoke Ireland model: influence of interventions 2010-2016 on reaching End Game 2025

S Li', D Levy', L Clancy'

${ }^{1}$ TobaccoFree Research Institute Ireland, Dublin, Ireland, ${ }^{2}$ Georgetown University, Washington, WA, United States of America.E-mail: shashali@tri.ie

Background: This study estimates the impact of tobacco control policies implemented between 1998 and 2016 on smoking prevalence reduction in Ireland by 2016. It also assesses if Ireland at 2016 has outperformed the projection from the previous model based on the data between 1998 and 2010. If yes, what contributed to the outperformance and how will it help Ireland to get closer to Tobacco Free Ireland (TFI) 2025 (End Game)?

Methods: SimSmoke, the dynamic simulation model of tobacco control policy, was adapted to examine the present impact of Irish tobacco control policies on smoking prevalence, through initiation and cessation, and smoking-attributable deaths and to make predictions for the future.

Results: The previous model projection for Ireland 2016 male smoking prevalence was $25 \%$ under Status Quo and $21 \%$ under all MPOWER policies. The new model predicts that the male smoking rate for ages 18 and older of $33.4 \%$ in 1998 falls to $19.7 \%$ in 2016 achieving a $41 \%$ reduction, which matches observed. The outperformance could be explained by price increase, stronger health warnings, improved youth 'access' enforcement and improved smoking cessation treatment between 2011 and 2016 .

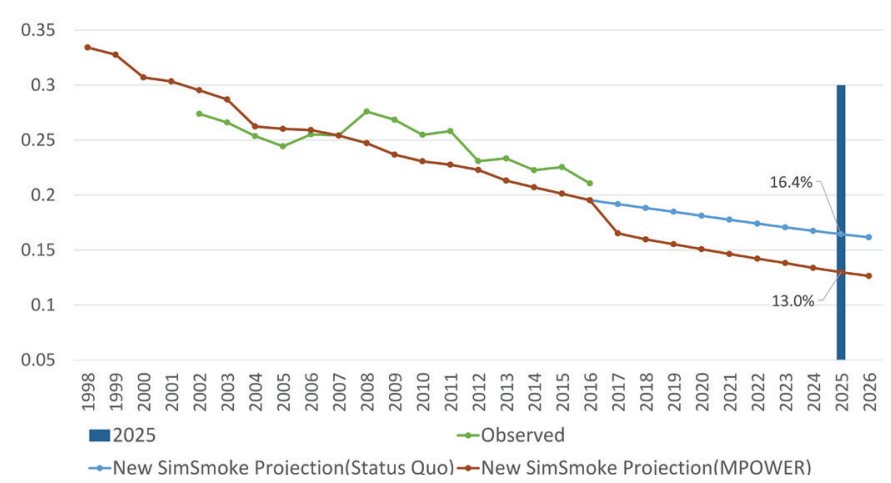

[New SimSmoke Ireland Model of Smoking Prevalence]

Conclusions: Ireland smoking prevalence in 2016 has outperformed the old model prediction under all MPOWER policies. Although between 2011 and 2016 Ireland has improved in the areas mentioned above, they did not reach the highest levels according to MPOWER standard, which implies two points. One is that there is more room for Ireland to further reduce smoking prevalence by fully applying policies according to MPOWER. Some policies are not fully accounted for by the model, which have been introduced in the period, such as the ban on smoking in cars where children are present and plain packaging legislation, but new policies are needed to achieve TFI.

Tob. Induc. Dis. 2018;16(Suppl 1):A42

DOI: $10.18332 / \mathrm{tid} / 84260$

$12: 30-14: 00$

PS-534-1 Regulating Shisha/ waterpipe smoking \& $\mathrm{E}$-cigarettes by regulating nicotine as poison: a success story from India
H Goswami

${ }^{1}$ Tobacco Free India Coalition/ Burning Brain Society, Chandigarh, India. E-mail: goswami@hemant.org

Background and challenges to implementation: In many jurisdictions across the world, Shisha smoking and E-Cigarettes are not regulated under the tobacco control legislation. Shisha and E-Cigarettes contain Nicotine as one of the chemical ingredient. Nicotine, in itself is a highly poisonous chemical. Even $30 \mathrm{mg}$ of Nicotine can be fatal. In 2007, when Chandigarh, a city in north India with a population of over a million, was being declared smoke-free, it was realized that there were no laws in place to prevent use of non-tobacco Nicotine based Shisha and E-Cigarettes. The author, through CSO "Burning Brain Society," filed a Writ-Petition in the High Court for shutting down all Shisha outlets, and use of Nicotine - a Poison.

Intervention or response: The Public Interest Litigation, CWP $14597 / 2007$ in the High Court was contested for six years by the State, Tobacco Companies and Shisha Bar owners. The author argued that even "The Insecticide Act" in India categorized Nicotine as an insecticide and the "Hazardous Chemical Rules" also label Nicotine as a hazardous and toxic chemical, thereby Nicotine must be treated as Poison. In 2012, the High Court passed judgement in favour and accepted that Nicotine was Poison and its use deserves penal action.

Results and lessons learnt: The Court passed a favourable order in November 2012, accepting Nicotine as a poisonous substance. Since then more than 232 criminal cases have been registered. A permanent task force to monitor use of Nicotine was also constituted. It took another 5 years for many other States of India to notify Nicotine as poison under the poisons Act.

Conclusions and key recommendations: By using an innovative strategy, the use of Shisha and E-Cigarettes was put to an end. It also resulted in a cascaded effect and many jurisdiction in India have now put Nicotine in the list of Poison. This strategy has the potential of being used as the End-Game strategy.

Tob. Induc. Dis. 2018;16(Suppl 1):A43

DOI: $10.18332 /$ tid/84263

\subsection{Promoting transnational cooperation and Partnerships: models of Intergovernmental and nongovernmental collaboration across multiple sectors for high level commitments}

12:30-14:00

PS-535-2 Policy and advocay in tobacco control in Romania of the Romanian Society of Pneumology F Mihaltan'

${ }^{1}$ National Institute of Pneumology, Pneumology, Bucharest, Romania. E-mail:mihaltan@starnets.ro

Background and challenges to implementation: Tobacco control in Romania has his own history. It's a history of development of partnerships of NGO with the Romanian Society of Pneumology which is the only medical association having a anti tobacco section in my country. In the same time it is a history of long debates and contacts with international NGO, international stakeholders and political leaders.

Intervention or response: I describe here all the steps made together with our partners in organising activities and meetings with the authorities, government, population at risk and mass media to prepare changing of view of the opinion leaders and Ministers of Health. In the same time I am underlining the impact of 
our activities in educating our young's, teenagers and journalists. In my presentation I give a bird eye view of the consequences in changes of tobacco control initiatives I am telling also the relations and collaborations with ENSP, Bloomberg Foundation, Tobacco Free Kids association and the modalities we choosed to fight with the tobacco industry.

Results and lessons learnt: The strengths of this local and international collaboration helped us to build the new anti tobacco Romanian law. It's a lesson about how we finalised this long process for obtaining this European new law.

Conclusions and key recommendations: The Romanian anti tobacco law was a long democratic process who brought more courage to implement future measures for tobacco control in our country and to continue to dream for the neutral Romanian package.

Tob. Induc. Dis. 2018;16(Suppl 1):A44

DOI: $10.18332 /$ tid/83751

\section{2:30-14:00}

PS-536-2 Smoking habits and anti-smoking acts awareness among general public in Gurgaon, Haryana, India

CJK Francis'

${ }^{1}$ Nestle India Limted, Finance / Social work, Gurgaon, India. E-mail: clement.k12@fms.edu

Background: India is the world's third largest tobacco-growing country. The Indian scenario as far as tobacco consumption is concerned is far worse because of the prevalence of the tobacco chewing habit which covers a wide spectrum of socioeconomic and ethnic groups and is spread over urbanized area as well as remote village.Despite the facts, that the harmful effects of tobacco chewing and smoking are widely known, many young people start smoking during adolescence, largely because they believe that smoking will boost their social acceptability and image. This study was contemplated with an aim to assess tobacco / smoking habits and awareness about anti-smoking act among general public in Gurgaon, Haryana, India.

Methods: A structured questionnaire consisting of 14 questions related to tobacco/smoking habits and awareness about antismoking act were asked to general public and their response was recorded.Random sampling method was used and data was collected from a cross-sectional survey.

Results: The study population consisted of total 430 individuals, male $364(84.65 \%)$ and females $66(15.34 \%)$. Then the questionnaires were asked and statistically analyzed. Around 286 (78.57\%) from 364 male were indulged in some form of tobacco usage $($ smoker $=32.86 \%$, tobacco chewer $=16.78 \%$, both $=11.18$ $\%$, alcohol +tobacco user $=21.67 \%$ ).

Conclusions: In the present study, most common cause of tobacco use was pleasure $40.5 \%$, inducing factor were friends $53.1 \%$ followed by parents and siblings. $36.20 \%$ patients used tobacco as second hand exposure in job places. $54.8 \%$ were aware about the anti-smoking act in public places, so only $8.6 \%$ people from all males enrolled, were smoking in public places.

Tob. Induc. Dis. 2018;16(Suppl 1):A45

DOI:10.18332/tid/83799

\section{2:30-14:00}

PS-537-2 Reconciling profits and pandemics? An analysis of attitudes to commercial sector engagement in health policy and research

J Collin'1, E Plotnikova', M Kandlik Eltanani², R Ralston', S Hill', K Smith ${ }^{1}$
'University of Edinburgh, Global Public Health Unit, Edinburgh, United Kingdom, ${ }^{2}$ University of Edinburgh, Sociology, Edinburgh, United Kingdom.E-mail: jeff.collin@ed.ac.uk

Background: Public health's terms of engagement with unhealthy commodity industries (alcohol, tobacco and ultra-processed food and drinks) have become increasingly contested across policy and research contexts. This has become particularly significant in the context of the Sustainable Development Goals' (SDGs) commitment to partnership with private sector actors, and efforts to promote coherence across NCD policy areas. We sought to identify approaches that could attract consensus support within and across policy domains.

Methods: Using snowball sampling, we undertook an online survey of 335 health researchers, advocates and policymakers, in 40 countries, assessing responses to stated principles, claims and recommendations for engaging with unhealthy commodity industries in relation to key policy and research initiatives.

Results: Most respondents identified a fundamental conflict between industry interests and public health objectives for all three industries, with agreement greatest in relation to tobacco and weakest for food. This pattern was replicated across diverse questions regarding potential forms of engagement, including rejecting voluntarism and partnership approaches to health policy. While awareness of tobacco industry tactics to influence policy and research was higher than for alcohol and food, most respondents rejected the view that the influence of the latter was less significant for public health. There was also substantial variation in attitudes to engagement with the emergent e-cigarette industry.

Conclusions: The strong consensus around restricting interactions with the tobacco industry supports increased implementation of the WHO Framework Convention on Tobacco Control's conflict of interest provisions. There is strong support for the extension of such practices to the alcohol industry, challenging current norms. More mixed responses indicate a need for greater clarity in defining the food industry, and for research analyzing links, similarities and differences across different types of unhealthy commodity producers.

Tob. Induc. Dis. 2018;16(Suppl 1):A46

DOI:10.18332/tid/83807

\section{2:30-14:00}

PS-538-2 Initiatives of Sirajganj district administration to tobacco control in Bangladesh MH Sarker ${ }^{1}$, Al Sujon ${ }^{2,3}$, OM Sohel Rana ${ }^{4}$

${ }^{1}$ Ministry of Public Administration, Sirajganj, Bangladesh, ${ }^{2}$ Work for a Better Bangladesh (WBB) Trust, Manager - Advocacy and Communication, Dhaka, Bangladesh, ${ }^{3}$ Swamaswar (anti tobacco newsletter), Bangladesh Anti Tobacco Alliance, Dhaka, Bangladesh, ${ }^{4}$ Development for Disadvantaged People (DDP), Sirajganj, Bangladesh. E-mail: hshashib@gmail.com

Background and challenges to implementation: Bangladesh passed 'Smoking and Tobacco Products Usage (Control) Act 2005 ' that further amended on 29 April 2013 for more befitting to FCTC, including Graphic Health Warnings (GHW) on $50 \%$ of tobacco packets, ban on all of Tobacco Advertisement, Promotion and Sponsorship (TAPS) and increases definition of public places etc. Amended law significantly increases fine for violation of law. Law implementation became big challenge due to interference of Tobacco Industries (TIs), lack of coordination among District Taskforce etc. Tobacco advertisements at Point of Sales (PoS), improper GHW and smoking in public places/transports were rampant. 
Intervention or response: District Administration (DA) of Sirajganj took various initiatives with the help of NGOs to ensure implementation of law, including seminars with stakeholders, disseminate law gazettes among owners of PoS and owners of public places. Based on the letter from district administration; all high schools organized seminar on tobacco control law.

DA conducts Mobile Courts (type of summary trial, conducted by Executive Magistrates at the place of offence by pronouncing spot punishment like imprisonment, fine etc.) for effective implementation of law at the $\mathrm{PoS}$, public places, and factories/ offices of TIs. Mobile courts removed tobacco advertisements from $\mathrm{PoS}$ and destroyed those materials publicly.

Results and lessons learnt: All of tobacco advertisements from PoS had been removed, smoke free environments at public places are ensured, TIs were fined and became careful to obey law. On the basis of tremendous work on law implementation, Sirajganj DA rewarded 'Best District in tobacco control' in 2015. This positive example has been transmitted into all other district in Rajshahi division through a letter from Divisional Head of Administration. Conclusions and key recommendations: As DA holds full legal power on law implementation at district level, so, if concern ministry instructed every DA, it will ensure proper implementation of law at all districts in Bangladesh.

Tob. Induc. Dis. 2018;16(Suppl 1):A47

DOI:10.18332/tid/84033

\section{2:30-14:00}

PS-539-2 Mobile app to identify gaps in implementation and provide evidences for effective policy change

S Cyril Alexander ${ }^{1}$, R Anthony ${ }^{1}$

'Mary Anne Charity Trust-MACT, Chennai, India.E-mail: cyril@ mactindia.org

Background and challenges to implementation: Cigarettes and Other Tobacco Products Act (COTPA), 2003 is a robust tobacco control act of India. However, with the presence of bureaucratic system, the general public does not have an effective tool to report tobacco control violations or acquire support to practice tobacco control in their lives or that of the community. Also there was no system in place which could provide real time field based on which the Public Health Care could investigate and advocate for policy change.

Intervention or response: In order the fill the gap between the General Public and the Government; and to also provide field based evidences to the Government; Mary Anne Charity Trust (MACT) developed the android based mobile application called Tobacco Monitor. It is an open source mobile application wherein a person can acquire information about the latest happenings related to tobacco eradications, regulations and cessation and also give complaints on tobacco related violations.

Any request for support or complaint is processed by the Tobacco Monitor Team.sent to the concerned Government Authority.

Results and lessons learnt:

- More than 500 individuals across 13 states of India are using this app

- Reviewed complaints have been reported to the respective government. The State Government of Tamil Nadu and Punjab have considered the complaints reported and directed the district authorities to take action on the complaints

- Used as an awareness tool for sensitizing public about the types of violations related to tobacco

The app works well in urban cities where there is a presence of Internet Services. The time taken to decentralize information is largely reduced. It is a success among the young adults since the process of reporting becomes easier. The complaints ensure that real time issue are brought to the Government's view.

Conclusions and key recommendations: Tobacco Control with the support of mobile technology becomes expedite, efficient and effective.

Tob. Induc. Dis. 2018;16(Suppl 1):A48

DOI:10.18332/tid/84181

$12: 45-14: 00$

EP-100-2 Dissuasion of nonsmoking doctors teachers and civil servants survey

Y Song 1

'Shenyang In China Institute for Health Education and Promotion, ShenYang, China. E-mail:ys_cdc@163.com

Background: One of the efficient ways of tobacco control is that people who don't smoke safeguard their rights. The job nature and social status of doctors, teachers and civil servants make them more representative in dissuading smoking.

Methods: The job nature and social status of doctors, teachers and civil servants could be influential on dissuading people from smoking. With 2457 questionnaires taken back, 2377 of them are valid. This survey's valid rate is $96.74 \%$.

Results:

1. $35 \%$ interviewees indicate that there are smokers in their home while $73.6 \%$ of these families have the limited smoking area. 52.5\% interviewees acknowledge dissuasion behavior. Besides, the most effective dissuasion reason is children.

2. $53.5 \%$ interviewees indicate that there are smokers in workplace. $28.2 \%$ nonsmokers would dissuade while $46.2 \%$ would leave and $25.6 \%$ would do nothing.

3. $9.5 \%$ nonsmokers would take the initiative to discourage smoking in public places while $79 \%$ would leave and $11.5 \%$ would do nothing. The most effective reason is no-smoking rules. $5.9 \%$ people consider the dissuasion effective every time while $51.9 \%$ people consider it effective in most of the time.

4. Among the three professions, doctor takes the highest percentage to dissuade smoking in public places, home and workplace.

Conclusions:

1. The rate of nonsmokers dissuading smoking is still low. Dissuasion at home happens more frequently than in public places and workplace. Dissuasion behavior more possibly happens between acquaintances.

2. Nonsmokers discourage smoking at home because they worry about the smokers' health while the reason turns into worrying about their own health during public place dissuasion and workplace dissuasion.

3. The most effective dissuasion reason at home is "do not smoke beside children" while the most effective dissuasion reason in public places is nonsmoking rules.

4. The doctors' rate of dissuasion is significantly higher than teachers and civil servants.

Tob. Induc. Dis. 2018;16(Suppl 1):A49

DOI:10.18332/tid/83796

$12: 45-14: 00$

EP-101-2 Is ban on smokeless tobacco products effective in controlling the menace: a case study from Punjab, India

R Gupta', V Roojam², A Singh ${ }^{3}$, G Singh ${ }^{1}$, R Kaur ${ }^{1}$

${ }^{1}$ State Tobacco Control Cell, Department of Health and Family Welfare, Chandigarh, India, ${ }^{2}$ Indian Administrative Services, 
Department of Health and Family Welfare, Chandigarh, India, ${ }^{3}$ Directorate Health and Family Welfare, Department of Health and Family Welfare, Chandigarh, India. E-mail: rakesh60. mahajan@gmail.com

Background and challenges to implementation: Oral cancers incidence in India is very high, which is attributable to the widespread habit of chewing tobacco. In 2011, Rule 2.3.4 under Food Safety and Standards Act (FSSAI), 2006 prohibited the use of tobacco and nicotine as ingredients in any food product. Though ban has been in place, there is a lack of evidence regarding enforcement \& implementation on ground level. The objective of the study was to assess the effectiveness of implementation of ban on smokeless tobacco products in Punjab.

Intervention or response: The present study was conducted in the period of June 2016 to Dec 2016 in Punjab. In 2012, Commissioner Food and Drug Administration imposed a ban on Smokeless tobacco (SLT) products in the entire Punjab State. In 2016, revised notification issued by Commissioner Food and Drug Administration, imposing a complete ban on Flavored/ Scented chewable tobacco. Every month it was mandatory to take five samples of Flavored/Scented chewable tobacco by the Food Safety Officers (FSOs). After that samples were tested in the state food lab and court cases were launched against the failed samples by the Food Safety Officers under FSSAI.

Results and lessons learnt: Total 204 samples of SLT products were taken by FSO's from June 2016 to December 2016. Out of which 92 samples failed in lab testing. 32-court case have been launched by Food Safety Officers against vendors from where these failed samples were collected under FSSAI

Conclusions and key recommendations: Strict implementation of ban on Smokeless tobacco products has yielded results in the form of decreased availability. A national ban should be there to stop the inter-state smuggling of smokeless tobacco products.

Tob. Induc. Dis. 2018;16(Suppl 1):A50

DOI:10.18332/tid/83772

\subsection{International tobacco trade and health diplomacy: understanding the nexus between the two and domestic international disputes}

\section{2:30-14:00}

SUS A "win-win situation overall": lessons from tobacco industry influence of the negotiation of the North American Free Trade Agreement

K Lee' ${ }^{1}$, E Eckhardt ${ }^{2}$

'Simon Fraser University, Faculty of Health Sciences, Burnaby, BC, Canada, ${ }^{2}$ University of York, Department of Politics, York, United Kingdom. E-mail: kelley_lee@sfu.ca

Background: NAFTA was the most expansive agreement of its kind when it came into force in 1994. Contemporary trade negotiations grapple with how to deal with tobacco, but there is limited assessment of industry influence of past agreements and how adopted measures have impacted tobacco control.

Methods: We reviewed internal documents dating from the 1980s to understand the industry's influence of NAFTA negotiations and implementation. We also analysed transnational tobacco companies' (TTCs) business strategies among NAFTA countries before and after the agreement using secondary sources, news articles, industry reports and policy documents. We compiled trade data using the UN Comtrade Database and Trade Map Databases. Data was organized by date and company, and iteratively reviewed to build a narrative related to the tobacco industry and NAFTA.

Results: TTCs actively lobbied policymakers to include tobacco under NAFTA and specific provisions related to taxation, rules of origin and tariff rate quotas. The industry publicly argued that NAFTA would yield economic benefits to the US and Canada by increasing exports and employment. Privately, TTCs were planning to restructure operations on a North American scale towards a regional business strategy. This strategy was then pursued once NAFTA came into effect, resulting in large-scale decline of US and Canadian operations, and buying up of Mexican companies. TTCs consequently realised greater economies of scale, cheaper inputs and new market access.

Conclusions: The tobacco industry has supported the inclusion of tobacco under trade and investment agreements on the grounds of economic benefits to signatory countries. NAFTA suggests the industry supports agreements to further regional and global business strategies. Far from serving the interests of local farmers and workers, NAFTA further enabled tobacco industry globalization. The findings provide evidence of the need to protect trade negotiations from industry influence, and reject industry efforts to leverage public health protections for promised economic gains.

Tob. Induc. Dis. 2018;16(Suppl 1):A51

DOI: $10.18332 /$ tid $/ 84023$

\section{2:30-14:00}

PS-540-2 Intellectual property arguments in tobacco industry legal challenges: lessons from recently concluded cases

S Zhou'

${ }^{1}$ Cancer Council Victoria, McCabe Centre for Law and Cancer, Melbourne, VIC, Australia. E-mail: suzanne.zhou@cancervic. org.au

Background and challenges to implementation: In recent years, intellectual property (IP) arguments have been central to legal challenges brought by the tobacco industry against measures implementing the WHO Framework Convention on Tobacco Control (FCTC). Many parties have faced IP-related litigation and threats under trade, investment, and domestic law, particularly in relation to measures to regulate tobacco packaging and labelling. These cases have raised novel legal issues that were previously the subject of academic commentary, but only limited case law.

Intervention or response: As these cases have concluded, a substantial body of case law has developed in the last five years on the relationship between WHO FCTC implementation and IP protections, affirming states' right to regulate for public health purposes. Five recent cases were analysed and lessons for WHO FCTC implementation were drawn out.

Results and lessons learnt: The cases confirm that tobacco companies have no right under international law to use their trademarks to market their product, that IP protections are not absolute but subject to state regulatory power, and that IP treaties recognise states' right to restrict trademark use for public health. As such, measures to implement WHO FCTC articles 11 and 13, including large graphic health warnings, plain packaging, and restrictions on brand variants, will not infringe international IP protections provided they do not affect registration rights or the negative right to prevent third parties from using trademarks. The case law also suggests actions states can take to strengthen their position in IP-related litigation, including explicitly preserving negative and registration rights, stating the public health purpose 
of a measure, and collating the best available evidence to support the measure.

Conclusions and key recommendations: A substantial body of jurisprudence now confirms that IP does not provide the scope of protection commonly claimed by the industry. Tobacco control practitioners faced with such arguments can be confident that they are unfounded.

Tob. Induc. Dis. 2018;16(Suppl 1):A52

DOI:10.18332/tid/83914

[Recent cases raising trademark issues]

\begin{tabular}{|c|c|c|c|c|c|}
\hline $\begin{array}{l}\text { Country } \\
\text { measure } \\
\text { jurisdiction }\end{array}$ & $\begin{array}{l}\text { Australia -plain } \\
\text { packaging } \\
\text { (WTO dispute } \\
\text { settlement } \\
\text { system) }\end{array}$ & $\begin{array}{l}\text { Australia } \\
\text { plain } \\
\text { packaging } \\
\text { (Iligh Court of } \\
\text { Australia) }\end{array}$ & $\begin{array}{l}\text { Australia - plain } \\
\text { packaging (investor } \\
\text { statc arbitration) }\end{array}$ & $\begin{array}{l}\text { Uruguay - restrictions } \\
\text { on brand variants and } \\
80 \% \text { graphic lneallh } \\
\text { warnings (investor } \\
\text { state arbitration) }\end{array}$ & $\begin{array}{l}\text { United Kingdom - } \\
\text { standardised packaging } \\
\text { (Court of Appeal of England } \\
\text { and Wales) }\end{array}$ \\
\hline IP issues & $\begin{array}{l}\text { Obligation to } \\
\text { provide certain } \\
\text { trademark } \\
\text { protections under } \\
\text { TRIPS }\end{array}$ & $\begin{array}{l}\text { Protection of } \\
\text { trademark as } \\
\text { property under } \\
\text { constitution }\end{array}$ & $\begin{array}{l}\text { Expropriation of } \\
\text { trademark as investment } \\
\text { under treaty; fair and } \\
\text { equitable treatment re } \\
\text { treatment of trademarks }\end{array}$ & $\begin{array}{l}\text { Expropriation of } \\
\text { trademark as investment } \\
\text { under treaty; fair and } \\
\text { equitable treatment re } \\
\text { treatment of trademarks }\end{array}$ & $\begin{array}{l}\text { Obligation to provide certain } \\
\text { trademark protections under } \\
\text { TRIPS and EU law; protection } \\
\text { of trademark as property under } \\
\text { European and UK law }\end{array}$ \\
\hline $\begin{array}{l}\text { Decided in } \\
\text { favour of }\end{array}$ & $\begin{array}{l}\text { Pending, } \\
\text { reportedly } \\
\text { Australia }\end{array}$ & Australia, 2012 & $\begin{array}{l}\text { Australia, } 2015 \\
\text { (dismissed at } \\
\text { jurisdictional stage) }\end{array}$ & Uruguay, 2016 & United Kingdom, 2016 \\
\hline $\begin{array}{l}\text { Positive } \\
\text { right to use } \\
\text { trademark? }\end{array}$ & $\begin{array}{l}\text { Pending, point } \\
\text { conceded as 'no' } \\
\text { by complainants }\end{array}$ & No & Not decided & No & No \\
\hline $\begin{array}{l}\text { Public health } \\
\text { justification }\end{array}$ & Pending & Not applicable & Not decided & Yes & Yes \\
\hline
\end{tabular}

\subsection{Building leadership capacity and broader alliances for tobacco control policy advocacy}

\section{$12: 30-14: 00$}

\section{PS-541-2 Second hand smoke "a threat to child's health and development" \\ S Omiyefa ${ }^{1}$, Children \\ 'Youth Action on Tobacco Control and Health (YATCH), Programs, Ibadan, Nigeria. E-mail: omiyefaseye@yahoo.com}

Background: According to the Global Youth Tobacco Survey 2008, 25.9\% students in Lagos Nigeria currently live in homes where others smoke in their presence, $43.1 \%$ are around others who smoke in places outside their home, $14.6 \%$ of the students currently use any tobacco product. $38.9 \%$ think smoke from others is harmful to them, $5.0 \%$ have one or more parents who smoke while $2.4 \%$ have most or all friends who smoke. It is evident that second-hand smoke causes many of the same diseases as direct smoking, including cardiovascular diseases, lung cancer, and respiratory diseases.

Methods: A descriptive cross sectional study was carried out in Ibadan, involving students who doesn't attend schools regularly in 10 schools in Ibadan Oyo State, Nigeria. A multistage cluster random sampling was employed to select 300 students in the schools. Data was collected using structured questionnaires by trained interviewers.

Results: The result revealed that 141 (47\%) were within the age group 15 to 17 years, 110 (36.7\%) were within the age group 12 to 14 years while $49(16.3 \%)$ of the respondents were within the age group 18 to 20 years. Ninety one (30.3\%) of the respondents live with both parents, 79 (26.3\%) live with relatives, 66 (22\%) live with father alone while $64(21.3 \%)$ also live with mother alone. A majority, 149 (49.7\%) have relatives, parents or siblings who smoke around them, 123 (41\%) have experienced sicknesses related to pneumonia and 90 (30\%) have experience asthma. The result further showed that about 268 (89.4\%) students not attending school regularly are attributed to second hand smoking related illnesses.

Conclusions: Since Second Hand Smoke affects a child's health, education and overall development in Ibadan, Oyo State Nigeria. The State government should intensify effort towards domestication of the National Tobacco Control Law in Oyo State.

Tob. Induc. Dis. 2018;16(Suppl 1):A53

DOI:10.18332/tid/83779

\section{$12: 30-14: 00$}

PS-542-2 Sensitization of Ministries, Departments and Agencies (MDAs) on tobacco control measures of the public health ACT 851 and WHO FCTC Article 5.3 in Ghana

DD Logo ${ }^{1}$, SA Kyei-Faried ${ }^{2}$, J Ansong ${ }^{3}$, L Musah ${ }^{4}$

${ }^{1}$ Ghana Health Service, Research and Development Division, Accra, Ghana, ${ }^{2}$ Ghana Health Service, Disease Prevention and Control Division, Accra, Ghana, ${ }^{3}$ World Health OrganizationAfro, Health Promotion Department, Accra, Ghana, ${ }^{4}$ Vision for Alternative Development, Accra, Ghana. E-mail: divine. logo@ghsmail.org

Background and challenges to implementation: Ghana on 11th July 2012 passed a Public Health- Act 851-2012), part six of which is Tobacco Control Measures. MDAs have the responsibility to harmonize and coordinate approach to capacity development in the public sector and also in the administration according to existing Government of Ghana systems including laws, regulations, procedures and operating systems, hence matters so much in the success of tobacco control policy implementations in Ghana.

Objective: To strengthen tobacco control governance systems and improve knowledge, specifically providing guidance for MDAs to prevent tobacco industry influence on policies.

Intervention or response: Materials used during the training were, Videos dubbed; "Don't Start" and "A Heavy Price" which show evidence based documentary of testimonies from persons with all forms of cancers, cardiovascular diseases and other diseases due to tobacco use and "Smoker's Body". Power Point 
Presentations on the topics; Health Effects of Smoking/Tobacco use, Global Burden of Tobacco related diseases, Framework Convention on Tobacco Control (FCTC), Ghana's Public Health Law, ACT 851 (Part Six Tobacco Control Measures) Tobacco Industry Interference/What to do to counteract them, Ghana's Performance Status by the FCTC provisions, Prevalence of tobacco use and related mortality in Ghana, Tobacco Control; Whose Responsibility and Role of MDAs. There were also open forum for questions and answers.

Results and lessons learnt: A total of 566 MDAs in six from the ten regions in Ghana namely; Ashanti, Eastern, Brong Ahafo, Western, Northern and Volta Regions were sensitized.

Conclusions and key recommendations: This exercise was in compliance with WHO FCTC Articles implementation. It offered education to MDAs and prepared them in position to handle Tobacco Industry influence on policy that will only advance their vested interest which is to maximize profit at the expense of people's health.

Tob. Induc. Dis. 2018;16(Suppl 1):A54

DOI: $10.18332 /$ tid/83870

\section{2:30-14:00}

PS-543-2 Story from Indonesia: the media advocacy strategy on restraining 'The Tobacco Bill Draft'

N Samidi ${ }^{1}$, L Pratiwi $^{1}$, N Luntungan ${ }^{1}$

${ }^{1}$ National Commission for Tobacco Control Indonesia, Jakarta Pusat, Indonesia. E-mail:midiasih@gmail.com

Background and challenges to implementation: Strong tobacco industry interference continued to influence the implementation of adequate tobacco control policy in Indonesia. Labours and farmers issues were overly used to mislead public and to challenge tobacco control policies. In 2009, Indonesia passed its Health Law. One of its translation is The Government Law on Tobacco Control, issued in 2012. This law is Indonesia's most comprehensive tobacco control regulation up-to-this date. Following that event, still in 2012, the People's Parliaments (DPR) initiated The Tobacco Bill Draft, which was loaded with tobacco industry's interest, solely aim to increase cigarette production. Since then, civil societies have been fighting to restrain DPR from passing the bill.

Intervention or response: The National Commission for Tobacco Control (NCTC) Indonesia continuously built strong and proactive communication strategies through media advocacy and open access to information. The efforts challenged manipulating facts and black campaigns that aim to mislead public opinion for industry's favour. NCTC closely monitored progress and setback about bill and informed media through press conferences, briefings, and fact sheets production. As a result, media coverage that support tobacco control increased significantly. In-depth investigative articles produced by national media emerged. Public messages about tobacco control shifted into positive tone.

Results and lessons learnt: In early 2017, The Tobacco Bill Draft was listed again by DPR as The Priority List for National Legislation (Prolegnas). In respond to this critical situation, NCTC Indonesia generated rigorous media advocacy efforts which resulted in 66 news articles and collected more than 25,000 online petition within two months (January to March 2017). In three consecutive days, three in-depth stories against The Tobacco Bill Draft were published at the front page of Indonesian newspaper largest chain.

Conclusions and key recommendations: This efforts contributed to the bill's expulsion from Prolegnas by the executive government in March 2017. This shows that effective and robust media campaign could shift political decision towards tobacco control.

Tob. Induc. Dis. 2018;16(Suppl 1):A55

DOI: $10.18332 /$ tid/83888

\section{2:30-14:00 \\ PS-544-2 Effectiveness of health warning on smoking, Cambodia \\ R Ray}

${ }^{1}$ National Center for Health Promotion (NCHP), Tobacco or Health,Phnom Penh, Cambodia.E-mail: rayrany@nchp.gov.kh

Background and challenges to implementation: Tobacco use is a major factor of many diseases such as lung diseases, cancers and cardiovascular diseases which are results in death in the world. Currently around 7.2 million of users and 900,000 nonusers die each year due to tobacco use and exposure to secondhand smoke In Cambodia, smoking prevalence among adult men remains high. About 1.6 million of adults currently smoke tobacco products. In $2016,1.5 \%$ of youth currently use any tobacco product while $0.6 \%$ currently smoke cigarettes.

Intervention or response: The sub-decree on $55 \%$ of Pictorial Health Warning on package of tobacco products in Cambodia was approved on 22nd October 2015 and it is to become effective from 22nd July 2016. The study aims to identify effectiveness of PHW on behavior changes of current smokers with specific focus as follow:

- Identify exposure, perceptions, attitudes of current smokers to PHW

- Examine the effect of PHW on behavioral changes of current smokers (consider quitting smoking \& quit)

Results and lessons learnt: Finding shows no participants have seen PHW on cigarette packs available in the market. More than $90 \%$ of the respondents exposed text health warning on cigarette pack. Most of the respondents believed that health warning on cigarette packs are effective at communicate the health effects of smoking to public, in motivating smokers/tobacco users to quit, prevent youth not to start smoking/using tobacco products, and ex -tobacco users to stay on quit. Nearly $90 \%$ of respondents supported government initiative to implement PHW on packs of tobacco products.

Conclusions and key recommendations: Enforcement of the fully implementation of the sub-decree on $55 \%$ pictorial health warning is essential. Tobacco industry is key player to ensure the compliance to the sub-decree, and this needs strong commitment from all tobacco stakeholders at national and sub-national levels actively participate to motivate tobacco industry, wholesalers, and retailers to effectively implement the sub-decree on 55\% PHW.

Tob. Induc. Dis. 2018;16(Suppl 1):A56

DOI:10.18332/tid/83897

12:30-14:00

PS-545-2 Asia Pacific Mayors Alliance for Tobacco Control (AP-CAT): a breakthrough to gain political will

S Karapan', T Singh Bam

'International Union against Tuberculosis and Lung Disease, Tobacco Control, Singapore, Singapore. E-mail: skarapan@ theunion.org

Background and challenges to implementation: Tobacco kills around 6 million people each year; that is one death roughly every six seconds. In many countries Asia Pacific, comprehensive tobacco control policies, plans and programs are severely 
lacking. Many countries in the region face similar roadblocks to comprehensive tobacco control. Mayors generally head the political and administrative parts of urban and therefore are able to make important decisions in the infrastructures and services that directly influence quality of life.

Intervention or response: The Asia Pacific Cities Alliance for Tobacco Control (AP-CAT) was formed joined by 12 cities from 8 countries with support from Ministry of Health Indonesia, Ministry of Health Cambodia, Ministry of Health Myanmar, Ministry of Health Lao PDR and the International Union Against Tuberculosis and Lung Disease (The Union). AP-CAT has two co-chairs: DR Bima Arya Sugiarto, Mayor of Bogor City, Indonesia and Francis Anthony S Garcia, Mayor of Balanga City, Philippines. The Union Asia Pacific Office in Singapore hosts the Secretariat of the AP-CAT.

Results and lessons learnt: AP-CAT members committed to the following tobacco control measures: 1 . To Create a smoke free city, 2.Implement TAPS Bans, 3. Support Ministry of Health to Increase/implement PHW size, 4. Raise taxes, 5. Establish NCD prevention programs.

Conclusions and key recommendations: Mayor alliances create political will and build ownership for the health of cities. The Alliance can be used to improve other public health interventions as well.

Tob. Induc. Dis. 2018;16(Suppl 1):A57

DOI:10.18332/tid/83903

\section{2:30-14:00}

PS-546-2 To encounter the challenges of tobacco industry: concerted community effort to advocate for policy change

A Kwong ${ }^{1}$, V Lai ${ }^{1}$

${ }^{1}$ Hong Kong Council on Smoking and Health, Hong Kong, Hong Kong. E-mail: lawrence_chu@cosh.org.hk

Background and challenges to implementation: The Hong Kong Government proposed to enhance pictorial health warnings (PHW), prominently increasing the size to 85\%, since May 2015. Opposition and interference of tobacco industry (TI) was fierce. They formed a strong opposition force by mobilizing front groups and stakeholders to lobby legislators and delayed the legislation for 2 years. TI' intimidations on policy change were resolved with concerted community effort.

Intervention or response: Tailor-made smoke-free promotion programmes were introduced by Hong Kong Council on Smoking and Health (COSH), targeting at all perspectives of society. Every year, education and community involvement programmes for specific target group, e.g. students, elderly, middle aged, women, medical and health, and business sector, etc were organized to build up smoke-free concept in the community and engage them as persistent working partners in tobacco control.

Results and lessons learnt: Since July 2015 to May 2017, over 220 organizations' representatives/individuals took part to support the 85\% PHW. Apart from health advocates, medical professionals and scholars, participants also included ex-smokers, students, teachers, social workers, community leaders, businessman, etc. It demonstrated to legislators that tobacco control is the whole community's concern and should be superior to TI's commercial interests. The 85\% PHW was finally passed in June 2017.

Lobbying of TI is ferocious and always with pseudo-science. It should note that $\mathrm{TI}$ also mobilized over 110 representatives, including smokers, marketing experts, lawyers, politicians, social activists and scholars. Challenges for advancing smoke-free legislation could be huger as we expected. Wider support and collaborations with the community should be solicited.

Conclusions and key recommendations: Nearly $90 \%$ of Hong Kong population are non-smokers and public support to tobacco control measures is generally high. It is critical to identify and engage these supportive but silent forces in the legislative process. Smoke-free seeds should be sowed broadly, deeply and sustainably in all sectors of community for the policy change.

Tob. Induc. Dis. 2018;16(Suppl 1):A58 DOI:10.18332/tid/83958

\section{2:30-14:00}

PS-547-2 JHSPH scholar's program: strengthening the next generation of global tobacco control leaders

N Kibria', S Tamplin'1, J Cohen ${ }^{1}$

'Johns Hopkins University, Johns Hopkins Bloomberg School of Public Health, Baltimore, MD, United States of America. E-mail:nkibria1@jhu.edu

Background and challenges to implementation: The Johns Hopkins Bloomberg School of Public Health (JHSPH) has supported a Global Tobacco Control Leadership Program and an academic Certificate Program for over a decade. These capacity building programs have reached approximately 1700 people in over 85 countries. Program evaluations and feedback from our Partners indicated a need for an advanced leadership program. In 2017, a pilot project to enhance the leadership skill set of selected JHSPH Program alumni from low- and middle-income countries was undertaken.

Intervention or response: Leadership and Certificate Program alumni were invited to submit a brief concept note addressing a tobacco control problem they would address as part of a project during the course of the year. Applicants were also evaluated on their statement of interest; commitment from their parent organization; their qualifications and experience; and letters of reference. Selected scholars are acquiring advanced leadership skills through a year-long curriculum focused on a systems thinking and design approach. The curriculum comprises of in-person components (lectures; group work etc.), online components (webinars), targeted mentoring by established leaders and implementation of the project. Program evaluation will include assessing of the extent to which the scholars were able to apply advanced leadership skills in the context of a systems approach in their work.

Results and lessons learnt: While recognizing leadership potential in individuals can be challenging from afar, in-depth queries, the strength of their organization's commitment, and the willingness of others to mentor and support an individual are expected to be indicative of the scholar's potential. A creative approach to curriculum design can help ensure that leadership value is added across a diverse scholar cohort.

Conclusions and key recommendations: The scholar's program provides a tailored approach to capacity building in low-and middle- income countries and provides an opportunity to advance the next generation of tobacco control leaders.

Tob. Induc. Dis. 2018;16(Suppl 1):A59

DOI:10.18332/tid/83968

12:30-14:00

PS-549-2 Balparishad - a health assembly of young leaders advocating for tobacco control around schools and in the communities 
N Lad ${ }^{1}$, V Shankar ${ }^{1}$, A Mehenndale ${ }^{1}$, R Kadam ${ }^{1}$

'Salaam Bombay Foundation, Tobacco Control \&t Advocacy, Mumbai, India. E-mail: tshering@salaambombay.org

Background and challenges to implementation: Tobacco use among school children is a serious problem in India. Lack of proper guidance, urge to imitate elders, peer pressure, misconceptions, availability of variety of tobacco products near schools and attractive advertisements of tobacco products attract the children to tobacco. Salaam Bombay Foundation (SBF), under its leadership program made an effort to create a team of young advocates and equipped the team with self confidence, refusal skills and advocacy concepts to work towards making their schools and communities tobacco free.

Intervention or response: The process for students' health assembly formation begins with an intensive training of students on harmful effects of tobacco, tobacco control law and tobacco free schools. A class level Balpanchayat is created involving eight students from each class. Then a school Balpanchayat is formed involving eight students selected from the class level Balpanchayats. The leaders of school Balpanchayats are further trained in Balparishad (Student's Health Assembly) to initiate and lead the advocacy activities to make their schools and communities tobacco free. The members of Balparishad sensitize the community, coordinate with stakeholders from all the concerned authorities and present their demands before concerned authorities.

Results and lessons learnt: Due to advocacy efforts of students' health assembly 153 shops in Mumbai stopped selling tobacco products. Municipal Corporation of Greater Mumbai (MCGM) issued a circular to ban surrogate tobacco advertising in Ganeshotsav pandals. MCGM included COTPA 2003 in Shops and Establishments Act and made compliance of its section 6 which prohibits sale of tobacco products to minors and within 100 yards of educational institutions. Tobacco advertisements were removed from 4,680 BEST public transport buses.

Conclusions and key recommendations: The results of intervention show that training and capacity building of youth is helpful in creating awareness on harms of tobacco products and motivating them to take steps to sensitize stakeholders to act against violations in sale and promotion of tobacco products.

Tob. Induc. Dis. 2018;16(Suppl 1):A60

DOI:10.18332/tid/84098

\section{2:30-14:00}

PS-550-2 Capacity building for female community health workers - an effective tool for tobacco control and empowerment

R Kadam¹, D Patil', A Pilankar ${ }^{1}$

'Salaam Bombay Foundation, Tobacco Control \& Advocacy, Mumbai, India. E-mail: tshering@salaambombay.org

Background and challenges to implementation: Tobacco is a significant public health concern in rural India. In Maharashtra, India's second largest state, $31.4 \%$ of adults report tobacco use (Global Adult Tobacco Survey India, 2009). In villages, tobacco use is endemic. Smokeless tobacco use is engrained in social and cultural norms. At the same time, poor rural health care infrastructure poses challenge to diagnosis and treatment of tobacco-related diseases.

Intervention or response: Under rural initiative 'Salaam Mumbai Foundation' (SMF) started by Salaam Bombay Foundation (SBF) in 2007, initial needs assessment was conducted in nine villages across Chandrapur district in Eastern Maharashtra. This assessment reported $45 \%$ of all villagers using tobacco which also included two years old children. After needs assessment, SBF initiated capacity building sessions with 60 female community health workers known as "sakhis" to empower them to become the primary implementers of tobacco control in villages they serve. Capacity building activities included sensitization workshops covering tobacco's harmful health effects, existing tobacco legislation; follow up sessions to assess progress, and specific guidance to address tobacco control issues in villages.

Results and lessons learnt: 60 trained female community health workers implemented various tobacco control activities at village level using methods like songs, street plays, puppet shows, antitobacco rallies, and incorporated anti-tobacco messages into their other health activities. Through these activities they reached to 28,000 residents in 60 villages. An evaluation conducted one year after the program revealed that awareness on harmful effects of tobacco use increased up to $90 \%$ among the residents of intervention villages and tobacco consumption had reduced.

Conclusions and key recommendations: Community health workers are uniquely situated to implement tobacco control programmes due to their access to community members, their role as trusted sources for health information, and their ability to understand the unique issues faced in their communities. Hence, they can be trained to implement tobacco control activities in villages and work with all the stakeholders.

Tob. Induc. Dis. 2018;16(Suppl 1):A61 DOI:10.18332/tid/84108

\section{2:30-14:00}

PS-551-2 Strengthening smokeless tobacco control in Myanmar: challenges and the way forward

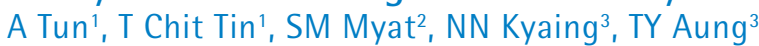
${ }^{1}$ Ministry of Health and Sports, National Tobacco Control Programme, Nay Pyi Taw, Myanmar, ${ }^{2}$ Ministry of Health and Sports, School Health Division, Department of Public Health, Nay Pyi Taw, Myanmar, ${ }^{3}$ Tun Khit Foundation, Tobacco Control, Yangon, Myanmar.E-mail: aungtunmm@gmail.com

Background and challenges to implementation: Smokeless tobacco (SLT) use is highly prevalent in Myanmar, particularly among women and youth. Ministry of Health and Sports has established and strengthened smokeless tobacco control programme at national and subnational level by adopting comprehensive policy of tobacco control since 2012, with the support of WHO and The UNION.

Intervention or response: These key policies are inclusion of SLT as an integral part of the national and local tobacco control frameworks, Raising public awareness on the adverse health and social consequences of SLT use, banning smokeless tobacco in public places, workplaces and public transport, Strengthening SLT Free Youth Programme, Inclusion of SLT use into regular Global Tobacco surveillance mechanisms and preventing tobacco industry interference on SLT.

Results and lessons learnt: Key policy achievements were made i)adopted a smoke free national regulation which bans smoking in all public places, work places and public transport; ii)adopted a pictorial health warning regulation that mandates tobacco industry to print at least $75 \%$ pictorial health warning on all tobacco pack including smokeless tobacco both in front and back on upper part. In addition we have built capacity of national and sub-national tobacco control cell and officials from health, NGOs and media.

Tobacco control Policy awareness was built among member of 
parliaments and inter-ministerial departments. According to 2016 Global Youth Tobacco Survey (GYTS), current smokeless tobacco use among students aged 13-15 years had declined from $9.8 \%$ to $5.7 \%$ between 2011 and 2016 .

Conclusions and key recommendations: Misconception of SLT use as less dangerous than smoking products is a big challenge to the tobacco control measures added by ease of purchase and low prices of SLT products. Efforts should be prioritized on law enforcement as well as amendment of existing national law to include the control of SLT products and illicit trade of tobacco in Myanmar.

Tob. Induc. Dis. 2018;16(Suppl 1):A62

DOI: $10.18332 /$ tid/84143

\section{2:30-14:00}

PS-552-2 Advocacy with police department for effective implementation of tobacco control polices N Lad ${ }^{1}$, D Patil', A Pilankar ${ }^{1}$, R Kadam ${ }^{1}$

'Salaam Bombay Foundation, Tobacco Control \&t Advocacy, Mumbai, India. E-mail: tshering@salaambombay.org

Background and challenges to implementation: Children and youth in India are vulnerable to tobacco initiation and tobacco industry uses tactics to target this age group violating section $6 \mathrm{a}$ of Cigarettes and Other Tobacco Products Act (COTPA 2003) which prohibits sale of tobacco products to minors. To curb this and to protect the children from tobacco addiction, Juvenile Justice Act (JJA) was amended in 2015 to include a jail term up to 7 years and fine of 1 LAKH Rupees and other provisions of COTPA 2003 related to tobacco products. Salaam Bombay Foundation took this opportunity to sensitize the Mumbai Police department to take action against the tobacco vendors violating the law.

Intervention or response: In 2015 Salaam Bombay Foundation conducted Sensitization Workshops on COTPA 2003 with Mumbai Police Department under the leadership of Mr.Pravin Kumar Patil, Deputy Commissioner of Police (Enforcement) Mumbai. Police officers and child Protection officers from all the 94 police stations were invited for these workshops.

Results and lessons learnt: Till date, 29000 policemen from Mumbai Police have attended the sensitization workshop.After the Policemen attending the workshop took action against 153 tobacco shops violating COTPA 2003. The workshop mobilized the government authorities and Assistant Police Inspector; Juvenile Action Police Unit of Mumbai Police (JAPU) received a letter from Health Department from Maharashtra state government to take action on Violations of section 6 a of COTPA act. The officials took guidance and assistance from Salaam Bombay Foundation representatives to take necessary actions.

Conclusions and key recommendations: Capacity building of governmental authorities would help to implement the existing laws on sale of tobacco products more effectively and efficiently. Non-governmental organizations can contribute in this by supporting governmental agencies.

Tob. Induc. Dis. 2018;16(Suppl 1):A63

DOI:10.18332/tid/84163

\section{2:30-14:00}

PS-553-2 Advocacy by children to remove surrogate products advertisements on the public transport buses in Mumbai

N Lad ${ }^{1}$, V Shankar ${ }^{1}$, D Patil ${ }^{1}$, R Kadam $^{1}$

'Salaam Bombay Foundation, Tobacco Control \&t Advocacy, Mumbai, India. E-mail: tshering@salaambombay.org
Background and challenges to implementation: Section 5 of Cigarettes and Other Tobacco Products Act (COTPA, 2003) of India prohibits direct and indirect advertisements of tobacco products. As a result, advertisements of surrogate products like 'pan masala' (a mouth freshener containing areca nut and slake lime) bearing the same brand name and packaging as tobacco products are had risen dramatically, particularly on the buses of Brihanmumbai Electric Supply and Transport (BEST) which runs nearly 3600 buses in neighbourhoods of Mumbai.

Intervention or response: Salaam Bombay Foundation (SBF) conducted a survey in May 2011 among 3000 Mumbai residents to build evidence on the impact of such surrogate advertisements at places like BEST buses. The respondents were shown a series of flash cards of three advertised 'pan masala' brands- 'Goa 1000', 'RMD' and 'Rajshree' and three popular non-tobacco brands. The results of survey were submitted to policy makers along with the demand charter from students participating in SBF's Super Army Leadership Program and advocacy meetings were conducted with Superintendent, License and Advertisement Department, Brihanmumbai Municipal Corporation (BMC) and the chairman of media agency controlling display of advertisements on BEST buses.

Results and lessons learnt: The survey results showed that $82 \%$ children and $84 \%$ adults identified 'pan masala' in the advertisements as 'gutkha' (a widely used smokeless tobacco product in India). After submission of report of the survey and advocacy meetings with BMC officials and advertising agency, $\mathrm{BMC}$ issued an official letter to all vendors to restrict display of surrogate advertisements for tobacco products, and, within a month all surrogate advertisements were removed from all the BEST buses.

Conclusions and key recommendations: Tobacco industry finds ways to circumvent tobacco control laws prohibiting product advertisements. Surrogate advertising have been effectively used to advertise tobacco brands. Targeted advocacy with stakeholders has proved effective in curbing surrogate advertisements at public transport systems. Thus, building evidence for advocacy to curb such advertising is important.

Tob. Induc. Dis. 2018;16(Suppl 1):A64

DOI:10.18332/tid/84164

\section{2:30-14:00}

PS-554-2 Evaluation of a training workshop on tobacco cessation: capacity building initiative in India

S Jhanjee', MK Varshney', UK Panda'1, GS Kaloiya', P Dayal', D Yadav

${ }^{1}$ All India Institute of Medical Sciences, National Drug Dependence Treatment Centre, AIIMS, New Delhi, India. E-mail: mohitvarshney.aiims@gmail.com

Background: The World Health Organization's (WHO) Tobacco Free Initiative highlights the role of health care professionals such as nurses, pharmacists, counselors and related support staff, in implementing smoking-cessation services. However, to be competent in providing smoking-cessation interventions, they need to establish and demonstrate knowledge, skills, and confidence in this field.

Methods: Pre-Post quasi-experimental design was used in this study to test the effectiveness of the educational training program. A total of 90 healthcare professionals, including nurses, nursing trainees, psychology trainees, social workers and trainees attended the workshop. Seventy six completed the pre-post assessment as 
a part of the one day workshop at All India Institute of Medical Sciences, New Delhi. The tool developed by the researchers included twenty questions evaluating the knowledge, attitude and skills related to Tobacco cessation activities. Each item in the questionnaire had a score of one for correct and zero for incorrect responses. Paired sample t-test was performed to compare the scores on above parameters. Ethical approval was obtained from Institute Ethics Committee.

Results: The participants were predominantly females (80.26\%) with professional experience of up to 3 years $($ Mean $=1.68$; $\mathrm{SD} \pm 0.84)$ in various healthcare related activities. There was a statistically significant improvement in total scores after the workshop as compared to before (Mean change: 2.86; $\mathrm{t}=8.71$, $\mathrm{p}<0.001)$. The improvement was statistically significant in both knowledge related $(\mathrm{t}=7.46, \mathrm{p}<0.001)$ and attitude/ skills related scores $(t=2.94, p=0.004)$. The level of professional experience and previous attending workshops could not statistically predict change in scores.

Conclusions: The workshop proved to be an effective approach in enhancing knowledge and imparting attitudinal changes in the healthcare professionals. Continued educational programs should be organized for capacity building in Tobacco cessation activities. Tob. Induc. Dis. 2018;16(Suppl 1):A65 DOI:10.18332/tid/84169

\section{$12: 30-14: 00$}

PS-555-2 Efforts made for Tobacco Control (NTCP) implementation in all health institution, Govt. offices and Districts Basti Tobacco Free State of Uttar Pradesh

AK Singh ${ }^{1}$, SK Tripathi ${ }^{2}, A B$ Singh ${ }^{3}$

${ }^{1}$ District Magistrate, U.P. Govt, Basti, India, ${ }^{2}$ State Tobacco Control Cell, Health, Lucknow, India, ${ }^{3}$ NCD - NHM, NHM U.P, Lucknow, India. E-mail: anuskain10@yahoo.co.in

Background and challenges to implementation: Uttar Pradesh State of India is the most populated along with high rate of tobacco consumption, mortality and disability. Implantation of NTCP and Cigarettes and Other Tobacco Product Act (COTPA) 2003 Noncompliance of domestic tobacco control laws can be seen all government departments even in Health department also. Since last two years as Director Administration in Department of health and family welfare and District Magistrate in Dist. Basti with consistent team efforts and support from district/state administrations has resulted in a remarkable progress in term of compliance of tobacco control law in the health institution and govt. offices.

Intervention or response: Establish a District level Coordination Committee involving representatives from different government departments including Civil Society Organizations, to provide technical support for improving implementation of COTPA 2003 in the District Co-ordination committee have taken important steps to warn people of the dangers of tobacco use. These measures have included the use of strong pictorial health warnings as well as comprehensive hard-hitting mass media campaigns.

Results and lessons learnt: In the last two years we have established around 6 District LeveTask Force Committee working in the districts a. inter-departmental circulars have been issued. More than 100 educational institutions, 80 government offices and In order to combat the CLEAN GREEN AND HEALTHY tobacco free Basti, As oath is taken on the eve of World No Tobacco Day, 31 May, 2017 to ensure district Basti Tobacco Free to continue all efforts that will create awareness to each \& every individual of society to quit tobacco and sustain the government \& public offices, public places, school colleges, hospitals as neat, clean, green and tobacco free zone.

Conclusions and key recommendations: Collaborative work; consistent support from district administration; and support from civil society partners created favorable environments and improved the compliance of tobacco control law in the District beyond our expectation.

Tob. Induc. Dis. 2018;16(Suppl 1):A66

DOI:10.18332/tid/84170

\section{$12: 30-14: 00$}

PS-556-2 Compliance COTPA 2003 and other supportive law for tobacco free district Kanpur Nagar in State of Uttar Pradesh

AK Srivastava ${ }^{1}$, SK Tripathi ${ }^{2}$

${ }^{1}$ Health Department, Lucknow, India, ${ }^{2}$ State Tobacco Control Cell, Lucknow, India. E-mail: tri80in@yahoo.co.in

Background: Kanpur nagar is big industrial city of India and hub for pan masala and tobacco manufacturers. Despite these challenges district administration along with health department has taken important steps to combat the control use of tobacco in mission mode. Under the umbrella of National Tobacco control Program the strict implementation of Sec- 4, 5, 6 \& 7 of COTPA Act 2003 is executed in District Kanpur Nagar both rural and urban areas.

Government of india enacted' (COTPA)' 2003, which prohibits smoking in public places.

Methods: It was Cross sectional study conducted in May 2015 in 6 urban and 2 rural blocks of Kanpur city. For compliance monitoring an observation checklist was used which had been developed by department of Community Medicine GSVM Medical college Kanpur, the minimum sample size was estimated to be 499 at the the estimated compliance of $50 \%$, confidence interval of $95 \%$ and at $5 \%$ margin of error. Other law used for Tobacco free offices, like Spitting law, MCD.

Results: "No Smoking/Tobacco free sinages"were displayed at $84.50 \%$, "No smoking/Tobacco free sinages were displayed either at entrance or conspicuous places at $98.14 \%$, Cigarette butts or bidi stubs at the time of visit were noticed at $15.33 \%$ public places. In none of the educational institute, point of sale was found inside the campus point of sale of tobacco products was within 100 yards of radius distance from main gate of 12 (15\%) educational institution.

Conclusions: In view of finding of the present survey across 574 sites in urban and rural Kanpur Nagar representing 7 different categories of public places the compliance to provisions of section 4 , section 5 and section 6 of COTPA is observed to be more than 80 Percent.

Tob. Induc. Dis. 2018;16(Suppl 1):A67 DOI:10.18332/tid/84194

\section{2:30-14:00}

PS-557-2 Brazilian Virtual Health Library of Prevention and Cancer Control: facebook fanpage and tobacco control

D Rangel', L Casado ${ }^{2}$

'Brazilian Virtual Health Library of Prevention and Cancer Control, Rio de Janeiro, RJ, Brazil, ${ }^{2}$ Brazilian National Cancer Institute, Rio de Janeiro, RJ, Brazil. E-mail: danielarangel@ hotmail.com

Background and challenges to implementation: As part of the Brazilian Virtual Health Library (BVS) of Prevention and Cancer 
Control communication plan, it was launched a facebook fanpage in 2013. Social media was chosen because nowadays it is an essential strategy for spreading information in a fast and dynamic way. The objective was simply to disseminate the Library and attract more people to use it.

Intervention or response: This case study shows that the BVS of Prevention and Cancer Control facebook fanpage became an important tool to engage partners to spread information about cancer and, specifically, tobacco control, since tobacco is one of its most current, and popular, subjects. Communication in health is about making people to acknowledge their role in promoting their own health and working in a collective way to promote healthy choices. Through the posts on the facebook fanpage it is possible to achieve these goals.

Results and lessons learnt: The fanpage of the BVS has about 3500 "likes", which makes it the sixth of its kind on facebook. Our public is looking for information based on scientific evidence. So, it is a person that is interested in the general information on the tobacco control area but it is, also, a person that has some knowledge in the field. Each post on the BVS Prevention and Cancer Control facebook fanpage has about 600 engaged people. The ones about tobacco control reach as much as 2100 people.

Conclusions and key recommendations: Social media is, nowadays, proved to be a communication tool very effective in engaging people on subjects as it has a wide range but with the possibility to direct the information to people who are willingly to search that subject. And, more important, in the case of the BVS of Prevention and Cancer Control fanpage, its posts are directed to people who work or research the area of cancer control.

Tob. Induc. Dis. 2018;16(Suppl 1):A68

DOI:10.18332/tid/84229

\section{$12: 30-14: 00$}

PS-558-2 Citizen Oversight Groups (Veedurías), a suitable instrument to advance in the tobacco control agenda, the Colombian experience

LA Avila Garcia ${ }^{1}$, E Baron ${ }^{2}$, B Llorente ${ }^{3}$, AM Claro $^{4}$, JH Arcila ${ }^{5}$

${ }^{1}$ Educar Consumidores, Bogota, Colombia, ${ }^{2}$ Liga Colombiana contra el Cáncer, Bogotá, Colombia, ${ }^{3}$ Fundación Anhas, Bogota, Colombia, ${ }^{4}$ Colectivo Aquí y Ahora, Bogotá, Colombia, ${ }^{5}$ Corporate International Accountability, Bogota, Colombia. E-mail: avilalilianaandrea@gmail.com

Background and challenges to implementation: Colombia's Constitution enshrines several instruments of citizen-participation that aim to foster government accountability and encourage democratic deliberation. Such constitutional principles materialize in the right of citizens to establish Citizen Oversight Groups (Veedurías). These organizations represent community interests, surveilling activities of public and private actors, including NGOs, regarding public policy issues, particularly those that involve publicly-owned resources. (Law 134,1994). Veedurías are a suitable instrument to address transparency issues, which is at the core of the challenges to advance in the tobacco control agenda in any country.

Intervention or response: Veeduría for Tobacco Control(VCCT) uses this surveillance tool to promote the implementation of the Framework Convention on Tobacco Control(FCTC), VCCT benefits from a multidisciplinary, multi-actor approach, advocating for: Acknowledgement of human, health, social and economic costs of tobacco consumption. Implementation of international commitments derived from FCTC. Protect tobacco control policies from tobacco industry interference Promote transparency, legality, and citizen participation. Promote opportunities for social transformation through education, and the protection of the right to information.

Results and lessons learnt: VCCT has offered guidance, promoted dissemination of scientific evidence (e.g. a tax policy brief, position statements on e-cigarettes, and illicit trade, organization of academic forums) multiplied impacts of those projects led by its member organizations. It has provided an efficient coordination mechanism to deploy a wide range of technical and social resources to promote legislation initiatives or counteract those that oppose FCTC guidelines, on topics such as health warnings, e-cigarettes, tax increases. VCCT implements education interventions to empower citizens to assert their rights. Conclusions and key recommendations: CVCT has became a relevant actor in the tobacco control agenda in Colombia due to, at least; three keys factors: Firstly, "united-we-stand"; VCCT works from a multidisciplinary, multi-actor approach. Secondly, this approach is always based in evidence and best practices; and finally, VCCT advocates for different issues than congregates different key actors.

Tob. Induc. Dis. 2018;16(Suppl 1):A69

DOI:10.18332/tid/84312

\section{2:30-14:00}

PS-559-2 True progress is tobacco-free campaign: engaging civil society to implement FCTC Article 5.3 in the Philippines

RE Degollacion

${ }^{1}$ HealthJustice Philippines, Quezon City, Philippines. E-mail: ralph.healthjustice@gmail.com

Background and challenges to implementation: The Philippines is a State Party to the World Health Organization Framework Convention on Tobacco Control. Article 5.3 of the Convention requires that "in setting and implementing their public health policies with respect to tobacco control, Parties shall act to protect these policies from commercial and other vested interests of the tobacco industry in accordance with national law."

This provision recognizes that there is a fundamental and irreconcilable conflict between the tobacco industry's interests and public health policy interests.

The tobacco industry has been very active in the Philippines, given that one in four of Filipino adults consume their product. They have, for example, actively campaigned against raising taxes on tobacco products among legislators. This interference in tobacco regulation continues.

If lives were to be saved against the deadly tobacco epidemic and to discharge the country's international obligation under Article 5.3 , public health advocates and community leaders should anticipate tobacco industry strategies and tactics and include plans to counter them in their campaigns.

Intervention or response: The campaign introduced stakeholders to the importance of FCTC Article 5.3 in fighting not only the tobacco epidemic but the threat of increasing NCDs in the country. The activities conducted include the following:

(1) civil society advocacy mapping,

(2) manifesto signing,

(3) press/media conferences, and

(4) capacity building workshops.

Results and lessons learnt: The campaign encouraged more than 50 civil society organizations to sign the manifesto of support rejecting tobacco industry donations. More than 100 health and non-health civil society groups have also participated in the 
various activities conducted under the campaign.

Conclusions and key recommendations: The campaign is not yet over. It's has taught us to be creative, flexible, and patient. Educating civil society about the true intentions of the tobacco industry is a fight worth pursuing. Sustained financial and technical support is needed in order to reach more groups in the country.

Tob. Induc. Dis. 2018;16(Suppl 1):A70

DOI:10.18332/tid/84319

\subsection{Tobacco industry interference: New tactics and counter measures}

$11: 00-12: 30$

F0-314-2 Building up echo chambers and framing messages: comparison of tobacco industry's think tank activities

S Lipponen ${ }^{1}$, T Tammi ${ }^{2}$

${ }^{1}$ Cancer Society of Finland, Strategy, Helsinki, Finland, ${ }^{2}$ National Institute for Health and Welfare, Helsinki, Finland. E-mail: satu.lipponen@cancer.fi

Background and challenges to implementation: Think tanks are a part of the tobacco industry's third party strategy. They help in building up the so-called 'echo chamber' described in industry documents. Think tanks are used to influence both media and public opinion. Industry-supported think tanks blur people's opinions with science. This is a challenge for policy makers, media and journalism. With its historical roots in the US, think tank advocacy is source of misinformation. In many countries, third party strategies are frequently used as Article 5.3 of the FCTC puts light on direct pressure from the industry.

Intervention or response: The aim was to identify existing and new schemes typical of the tobacco industry, with a special focus on how the tobacco industry tactic of discrediting science evolves. This was done by comparing the rhetoric used published reports and publicity operations in three cases in Europe in 2017.

Results and lessons learnt: The long-term goal of the tobacco industry is to influence public opinion. Basic rhetorical stratagems include criticism of regulation, strong support for freedom of choice, and the separation of state from free market economy mechanisms. In Europe, there seems to be an ongoing attack against civil society aimed at strengthening anti-science attitudes. The media does not adequately report linkages to the tobacco industry.

It is important to inform science journalists on how to identify how tobacco industry-supported think tanks operate. They should be better aware of industry tactics.

Conclusions and key recommendations: Tobacco control experts must maintain a critical perspective towards industry tactics in the media. It is important to collaborate with journalists of specific interest in research, scientific methods and medical topics. More coverage is needed of industry ties to think tanks, as the public and journalists are not aware of these connections.

Tob. Induc. Dis. 2018;16(Suppl 1):A71

DOI:10.18332/tid/84282

$11: 00-12: 30$

F0-315-2 Influencing decision makers - model of interference of the tobacco industry in Brazil D Silva', A Medeiros' ${ }^{1}$, S Turci' ${ }^{1}$, D Carvalho ${ }^{1}$

'Fundação Oswaldo Cruz, Escola Nacional de Saúde Pública, Rio de Janeiro, RJ, Brazil. E-mail: daniellebarata@gmail.com
Background: To monitor the strategies of the tobacco industry against public tobacco control policies in Brazil.

Methods: Documentary research with survey of documents available at the Observatory on Tobacco Industry Strategies, in Brazil, of the Oswaldo Cruz Foundation of Brazil's Ministry of Health. Documents refering to tobacco industry's financing to brazilian parlamentarians in 2014 elections campains were collected. The documents were analyzed based on the strategy Influencing Decision Makers, described in the WHO document entitled Tobacco Industry Interference with Tobacco Control.

Results: 88 documents were found. The analysis showed that of 35 political parties existing in Brazil, the tobacco industry financed 13 of several ideological segments. Of the parliamentarians contemplated, 88 are pro-tobacco, being that 79 of them received funding from the tobacco industry for election campaigns and have been opposing smoking control policies, preventing or hindering the progress of the guidelines. The value declared by the industry was $\mathrm{R} \$ 2,337,892.20$.

Conclusions: In Brazil there is industry interference in the government, which culminated in the creation of the Chamber of the Tobacco Production Chain, linked to the Ministry of Agriculture, Livestock and Supply, contributing to the strengthening of tobacco industries, promotion of their products and Politicians who have declared themselves against bills that address the following issues: prohibition of cigarette advertising, additives that confer flavor and aroma, standardization of cigarette packaging and traffic violation of smoking in vehicles with passengers under eighteen years of age, amongst other measures.

Unfortunately, the number of politicians who prevent the advance of public health in the fight against smoking is greater, which demonstrates how influential the tobacco industry is to make its interests prevail by strengthening an apparatus permeated by instruments and agents capable of erecting a real institutional blockade structure that works in favor of the companies in fields of political, economic and social action.

Tob. Induc. Dis. 2018;16(Suppl 1):A72

DOI:10.18332/tid/84209

$11: 00-12: 30$

FO-316-2 Local level action on FCTC Article 5.3 in Bangladesh

S Akter ${ }^{1}$, A Islam Sujon 1,2, SA Rahman', G Pearee ${ }^{3}$

${ }^{1}$ Work for a Better Bangladesh (WBB) Trust, Health Rights and Tobacco Control, Dhaka, Bangladesh, ${ }^{2}$ Bangladesh Anti Tobacco Alliance (BATA), Communication, Advocacy - Tobacco Control, Dhaka, Bangladesh, ${ }^{3}$ Work for a Better Bangladesh (WBB) Trust, Administration and Management, Dhaka, Bangladesh. E-mail: sharminarini@gmail.com

Background and challenges to implementation: Throughout Bangladesh, the tobacco industry regularly organizes events and parties to which it invites government officials and sponsors local events. These activities make it less likely that local officials will rein in the industry when it breaks the law. Meanwhile, research conducted in 2015 by Work for a Better Bangladesh Trust (WBB) found that just $10 \%$ of local officials knew nothing about the FCTC Article 5.3 on industry interference.

Intervention or response: During 2016-17, WBB conducted a series of local-level seminars jointly with government officials and NGOs. In the seminar we presented about FCTC Article 5.3 and the need to take strong action to prevent such interference in government policy. We also presented officials with a book we have written in Bengali on the topic. 
Results and lessons learnt: As a result of the seminars, government officials in five of the seven divisions of Bangladesh drafted and passed official orders on industry interference. This means that local officials from the divisional level down to the local level are now aware of the issue and have clear guidelines on avoiding it. We realized that a large part of the problem is that NGOs may remain silent on this issue, while the tobacco industry actively pursues the government officials. A fairly simple set of activities may be sufficient to strengthen the resolve of officials to avoid industry interference. These activities parallel work we have done on the national level, which is proceeding at a slower pace. Conclusions and key recommendations: Our experience sheds light on the possibility of making important progress on FCTC Article 5.3. If progress is slow on the national level, it may be useful to address the local level as well, where there are fewer obstacles to success.

Tob. Induc. Dis. 2018;16(Suppl 1):A73

DOI:10.18332/tid/83916

\section{$11: 00-12: 30$}

F0-317-2 Exposing interference of tobacco industry in Vietnam

HA Pham Thi', N Nguyen Hanh ${ }^{1}$, T Le Thi ${ }^{1}$

${ }^{1}$ HealthBridge Foundation of Canada, Vietnam Office, Hanoi, Viet Nam. E-mail: lethu@healthbridge.org.vn

Background: Vietnam ratified the FCTC in 2004 and passed a National Tobacco Control Law in 2012. However, due to strong interference of the tobacco industry (TI), the policy development/ implementation process has faced a lot of challenges, and the country has not yet implemented FCTC Article 5.3. The aim of this research was to investigate and expose TI tactics and violations in tobacco advertising, promotion and sponsorship.

Methods: Media surveillance was conducted from 1/2014 to $5 / 2016$ on a daily basis, including online media and the 56 most exposed printed newspapers. A screening mechanism was set up to identify all articles on tobacco and tobacco control and classified them by specific topics and by the "attitude" (positive or negative point of view). Information on TI was examined in depth to identify tactics and violations, and inform tobacco control networks and government authorities.

Results: 1,353 articles related to the TI were identified among a total of 3,908 tobacco-related articles. Promoting of TI corporate social responsibility (CSR) activities were the most common violation. The main kinds of CSR activities were charitable, living condition improvement, and poverty reduction. Key TI partners in performing CSR included local government, and socio-political organizations, and some newspapers. There were high levels of interaction between TI and government through rotation of officials between the TI and the Ministry of Trade and Industry, collaboration in smuggling control, active participation of the TI in policy development, and because of high appreciation of government for the TI's contribution. The TI interfered in tobacco tax policy development by providing misleading information on the burden of smuggling, and exaggerating the tobacco control environment in Vietnam.

Conclusions: There is increasing interference of the TI to undermine the impact of tobacco control efforts. The country needs to strengthen the implementation of FCTC Article 5.3 to reduce $\mathrm{TI}$ interference.

Tob. Induc. Dis. 2018;16(Suppl 1):A74

DOI:10.18332/tid/83999
$11: 00-12: 30$

FO-318-2 Is "hookah cafe" a tool to bypass smokefree law?

E Dagli ${ }^{1}$, P Ay ${ }^{1,2}$, T Gezer $^{1}, 0$ Elbek ${ }^{1}$, M Guner ${ }^{1}$, F Yildiz $^{1}$

${ }^{1}$ Health Institute Association, Istanbul, Turkey, ${ }^{2}$ Marmara University, Public Health, Istanbul, Turkey. E-mail: elifzdagli@ gmail.com

Background: In Turkey consumption of tobacco products in all indoor places are banned since 2009. Hookah and cigarettes which do not contain tobacco but used in a way to imitate the tobacco products are considered as tobacco products since 2013. The objective of this study was to determine hookah consumption in enclosed spaces of licensed hospitality establishments in Istanbul. Methods: This is a cross-sectional study. There are 428 hospitality establishments licensed to offer hookah tobacco products in Istanbul. Seven districts which encompass $51.2 \%$ of all the licensed establishments were selected as the study area. Sample size was calculated as 141 establishments assuming a violation rate of $50 \%$, margin of error of 0.05 , confidence level of $95 \%$ and a $10 \%$ non-response. Establishments were selected through simple random sampling. Data were collected through direct observation and presences of hookah use, smoking, signage display were noted. Observations were carried out after 3:00 PM for 10 minutes.

Results: Of the 141 establishments 128 were accessed (90.8\%). The majority were cafes (68.8\%) and $3.1 \%$ had kids menu. Only $57.0 \%$ had existence of open terrace and $68.8 \%$ had extensions with removable panels. Of the establishments $15.6 \%$ had $<100$ meters distance from formal education institutions. Among all $50.7 \%$ had no-smoking sign display, and of these only $61.5 \%$ were appropriate in terms of size and content. Violation for cigarette smoking and hookah use were $73.4 \%$ and $78.1 \%$, respectively. In only four establishments health warnings were present on hookah bowls and two were appropriate in terms of size and content.

Conclusions: The absence of open terraces, lack of no-smoking signage, health warnings on hookah bowls, proximity to educational institutions and violations indicate problems with licensing and inspection procedures. Hookah premises without open spaces render detection of cigarette smoking impossible. There is a need to revise the methods of enforcement.

Tob. Induc. Dis. 2018;16(Suppl 1):A75

DOI: $10.18332 /$ tid/84015

$11: 00-12: 30$

F0-319-2 Monitoring and exposing tobacco industry tactics in implementation of 85 percent pictorial health warnings

B Mathew

'Voluntary Health Association of India, Delhi, India. E-mail: binoymathew84@gmail.com

Background and challenges to implementation: Pictorial health warnings on packaging of tobacco products is legally mandated as per India's national tobacco control legislation Cigarettes \& Other Tobacco Products Act 2003. Pictorial health warnings were notified on 15th October, 2014 \& effective from 1st April, 2015 - pictorial warning to cover $85 \%$ area on both sides of tobacco packs. However the notification was kept in abeyance in March 2015, due to tobacco industry pressure.

Intervention or response: Right to Information Act (RTI) is a part of fundamental rights under Article 19(1) of the Constitution of India. This empowers citizens to question the Government, inspect their files. The RTI was used as a 
tool to get info on representations from the Tobacco Industry for delaying the implementation and exposing the industry interference.

Results and lessons learnt: The information received under RTI, revealed that several hundreds of written representations have been sent by tobacco industry as well as so-called independent and/or industry bodies opposing on various grounds in the implementation of the larger health warnings on tobacco product packs. This was exposed in the media and the stories created pressure and become a national debate. After a two-year battle, India implemented 85 percent graphic health warnings on tobacco products package from 1st April, 2016.

Conclusions and key recommendations: This helped to effectively strategise and mount a stringent campaign on tobacco control across the country, garner political support from select leaders, sensitize the media and seek general public support for compliance \& implementation of pictorial warnings.

Tob. Induc. Dis. 2018;16(Suppl 1):A76

DOI:10.18332/tid/84483

\section{$11: 00-12: 30$}

F0-320-2 Analysis of media reports to understand tobacco industry tactics in undermining tobacco tax policies in Bangladesh

S Kumar Singha

'Jamuna Television, Senior Reporter, Dhaka, Bangladesh. E-mail: sinhasmp@yahoo.com

Background: Mass media plays an important role in influencing tobacco control policies. The tobacco industry also uses the mass media to promote their products and undermine tobacco control policies, in particular tobacco tax policies. To understand the tactics of tobacco industry to prevent effective tobacco tax policies through analyzing media reports on tobacco tax issues during the budget discussion in Bangladesh.

Methods: The study analyses tobacco related reports published in more than twenty national newspapers in Bangladesh during April-May of 2015 and May-June of 2016. Both Quantitative and qualitative analysis focused on the contents and number of the stories.

Results: The study shows that 290 reports out of 1398 reports during this time focused on smuggling by tobacco industries to mislead the policy makers and public with the message that increasing tobacco taxes may lead to increase in the smuggling. While all the newspapers studied published stories on smuggling of tobacco products, the anti-tobacco news were not found in all those newspapers. Most of the anti-tobacco reports are event news and lack specific contents on tobacco tax related issues. Whereas the stories supporting tobacco industry position to prevent tax increase include very specific statistics to influence the tax policy makers. Only 58 stories focused on real tobacco taxation from tobacco control perspective, whereas 110 stories support the position of tobacco industry.

Conclusions: Accurate information and statistics need to be provided to the policy makers to counter the influence of stories on tobacco smuggling myths. The anti-tobacco events should have very clear and specific demands on effective tobacco tax policies and under understanding of tobacco control activists on tobacco tax issues needs to be enhanced. Understanding and skills of journalists for reporting on tobacco tax issues need to be sharpened.

Tob. Induc. Dis. 2018;16(Suppl 1):A77

DOI:10.18332/tid/83821

\section{2:30-14:00}

PS-560-2 Effective implementation of Article 5.3 of the WHO framework convention on tobacco control to curb tobacco industry interference

R Gupta', A Bhawra², V Roojam², R Bhalla ${ }^{3}$

'State Tobacco Control Cell, Department of Health and Family Welfare, Chandigarh, India, ${ }^{2}$ Indian Administrative Services, Department of Health and Family Welfare, Chandigarh, India, ${ }^{3}$ Directorate Health and Family Welfare, Department of Health and Family Welfare, Chandigarh, India. E-mail: rakesh60. mahajan@gmail.com

Background and challenges to implementation: The tobacco industry has historically employed a multitude of tactics to shape and influence tobacco control policy. In Punjab, Officials at various levels have been approached by representatives of Tobacco Industry for easing implementation of Cigarettes and Other Tobacco Products Act 2003 and withdrawing certain notifications to tackle Tobacco/Nicotine menace. Industry has used its economic power, lobbying and marketing machinery, and manipulation of the media to discredit scientific research and influence governments in order to propagate the sale and distribution of its deadly product.It goes to the credit of political leadership and bureaucracy that most of their attempts have been foiled. The objective of the study was to assess effective implementation of Article 5.3 of the WHO Framework Convention on Tobacco Control.

Intervention or response: The present study was conducted in the period of August 2015 to May 2017 in Punjab. An Empowered Committee was constituted to implement WHO Article 5.3 Framework Convention on Tobacco Control (FCTC) vides notification no. 539949/3 dated 17/7/15. A protocol was made that has to be followed while dealing with Tobacco Industry. Regular Meetings of Empowered Committee were conducted for effective implementation.

Results and lessons learnt: In Punjab, Officials at various levels have been approached by representatives of Tobacco Industry. After the formation of the empowered Committee, no one from the Tobacco industry contacted the department for any kind of partnerships/ events that are directly or indirectly related to tobacco control.

Conclusions and key recommendations: As the strategies and tactics used by the tobacco industry evolve constantly these guidelines should be reviewed and revised periodically to ensure that they continue to provide effective guidance to the parties on protecting their public health policies on tobacco control from tobacco industry interference.

Tob. Induc. Dis. 2018;16(Suppl 1):A78

DOI: $10.18332 /$ tid $/ 83773$

\section{2:30-14:00}

PS-561-2 Emotional attachment to personal tobacco packages might affect quitting behaviour. Results from a cross-sectional survey among tobacco users

I Lund ${ }^{1}$, J Scheffels ${ }^{1}$

${ }^{1}$ Norwegian Institute of Public Health, Drug Policy, Oslo, Norway. E-mail: ingeborg.lund@fhi.no

Background: Emotional attachment to your brand of cigarettes or snus might imply a lower tendency to want to quit smoking or using snus.

Aim: Investigate emotional attachment to the personal snus or cigarette pack, and its potential effect on quitting plans, among 
snus users and smokers.

Methods: A sample of 1625 smokers and 1626 snus users, aged 16-75, recruited from a web-panel, and weighted according to national statistics on smoking and snus use within gender, age and region. Mean age: 41 years, and $41 \%$ women. A principal component analysis showed that all positive feelings loaded on a single component, which was used in a multinomial adjusted regression, with a three-point quitting-plans variable as the dependent variable (no quitting plan, plan to quit during this month, plan to quit later). Adjustments were made for gender, age, education, tobacco product (cigarettes or snus), and last month quitting attempts.

Results: A relatively large minority (20-30\%) of smokers and snus users agreed fully or partly with statements expressing positive feelings related to buying and using their choice of tobacco brand. The exception was the statement "I like my current/regular pack", which was endorsed by $65 \%$ of smokers and $75 \%$ of snus users. Adjusted multinomial regression showed that those who had less positive feelings related to their pack were more likely to have plans to quit tobacco within the next month $(\mathrm{AOR}=1.5, \mathrm{p}<$ $0.001)$, or to have plans to quit at some later date $(\mathrm{AOR}=1.2$, $\mathrm{p}<0.001$ ), than to have no quitting plans (ref). Other significant factors were gender and earlier quit attempts.

Conclusions: Emotional attachment to cigarette and snus brands and packages reduces the likelihood of having plans to quit tobacco, which might in turn result in lower actual quitting behaviour. Regulatory actions that reduce emotional attachment, e.g. standardised packaging, may potentially affect quitting behaviour.

Tob. Induc. Dis. 2018;16(Suppl 1):A79

DOI:10.18332/tid/83801

\section{2:30-14:00}

PS-562-2 One unhealthy commodities industry? Understanding links across tobacco, alcohol and ultra-processed food manufacturers and their implications for tobacco control and the SDGS

J Collin', E Plotnikova', S Hill'

'University of Edinburgh, Global Public Health Unit, Edinburgh, United Kingdom. E-mail: jeff.collin@ed.ac.uk

Background: FCTC Article 5.3 requires protection against tobacco industry interference in policy-making. By contrast, manufacturers of alcohol and ultra-processed food and drink products are often identified as potential partners in multi-sectoral health initiatives, including via the Sustainable Development Goals (SDGs). This divergence has been questioned given evidence of strategic similarities across sectors, to which this presentation adds an examination of structural links and their implications for health policy. This focuses on an analysis of 'interlocking directorates', via which directors of one organisation also occupy positions on different boards, widely as the principal indicator of network ties across corporations.

Methods: Using data from corporate websites, annual reports and business databases, we employ UCINET social network analysis software to examine interlocks in the top six transnational companies of each sector within and across tobacco, alcohol and food companies, with political elites, and with health and development agencies.

Results: We present findings via (i) profiles of individual tobacco industry directors, highlighting strategically valuable links to other actors; (ii) a quantitative comparison of interlocks across the three sectors, with no direct links between tobacco and food companies but with alcohol companies providing several bridges between them, and with food companies more extensively linked to political elites and health agencies; (iii) a case study of the board of brewing giant SAB Miller at the time of its mega-merger with $A B$ InBev to highlight the significance to tobacco control of wider interactions enabled by interlocks.

Conclusions: This account of linkages across tobacco, alcohol and ultra-processed food companies calls into question regulatory approaches that treat the tobacco industry as an exceptional case. Neglecting conflicts of interest with other unhealthy commodity producers is potentially damaging to efforts to implement Article 5.3. This highlights important opportunities for efforts to promote policy coherence for NCDs and advancing the SDG agenda.

Tob. Induc. Dis. 2018;16(Suppl 1):A80 DOI: $10.18332 /$ tid/83806

12:30-14:00

PS-566-2 The filter fraud: debunking the myth of "Safer" as a key new strategy of tobacco control A Blum", T Novotny²

'University of Alabama, Center for the Study of Tobacco and Society, Tuscaloosa, AL, United States of America, ${ }^{2}$ San Diego State University, Biostatistics and Epidemiology, San Diego, CA, United States of America. E-mail: ablum@ua.edu

Background and challenges to implementation: Although efforts have been made to eliminate the use of misleading descriptors such as "low-tar," "lights," and "mild" from cigarette marketing, the elimination of the cellulose acetate filter--which is on $95 \%$ of cigarettes and which does not confer any reduced health risks to smokers--has been largely overlooked as a tobacco control strategy. While manufacturers have avoided making explicit health claims for filtered cigarettes (thus putting the burden of blame on consumers), there is an urgent need for improved education of smokers, health professionals, and regulatory agencies alike that filtered cigarettes are no less lethal than non-filtered ones. The 2014 Surgeon General's Report on the Health Consequences of Smoking and the 2001 NCI Monograph 13 report that the nearuniversal adoption by smokers of filtered cigarettes since their introduction in the 1950s has not reduced these consumers' risks for cancer and other diseases.

Intervention or response: A review of cigarette marketing practices reveals the deception perpetrated by tobacco companies about filters that has allayed consumers' concerns about the adverse health effects of smoking and has consequently made cigarettes more acceptable-and addictive-for new and continuing smokers. This presentation summarizes current filter research plus six decades of cigarette advertisements, tobacco industry documents and trade journals that have perpetuated the filter fraud.

Results and lessons learnt: This review further explodes the myth that filtered cigarettes are safer than unfiltered brands and provides evidence that filters should be eliminated from both cigarette manufacture and from the environment into which these non-biodegradable products are discarded.

Conclusions and key recommendations: Additional research on the potential health and environmental benefits of removing filters from the marketplace should be conducted, aimed at stricter product regulation. Meanwhile, rather than solely continuing to educate the public about the dangers of smoking, public health professionals and clinicians need to better communicate the message that filtered cigarettes do not confer any health protection whatsoever.

Tob. Induc. Dis. 2018;16(Suppl 1):A81

DOI: $10.18332 /$ tid/83988 
12:30-14:00

PS-567-2 Use of electronic games as tobacco cross boarder advertisement

D Carvalho', V Figueiredo ${ }^{1}$, VL da Costa e Silva ${ }^{2}$, S Rubano Turci' ${ }^{1}$ Fundaçao Oswaldo Cruz, Centro de Estudos Sobre Tabaco e Saúde/Escola Nacional de Saúde Pública, Rio de Janeiro, RJ, Brazil, ${ }^{2}$ FCTC Convention Secretariat, Geneva, Switzerland. E-mail:dcscarvalho86@gmail.com

Background: As years pass by, we see different ways in which the Tobacco industry interferes with media, to achieve its goals (specially in developing countries). Knowing that teenagers and youngsters are their target, they will use the best media to reach them. Since young people play electronic games (e-games), worldwide, it's a cross boarder media with great efficiency, and it is possible that they are interfering with computer games, in order to insert Tobacco images or other Tobacco related material.

Methods: The goal of the research is to bring out visibility on Tobacco industry's influence on e-games to create a link and influence young people throughout the world. A online research was done, using Google, YouTube and accessing different computer games by downloading or buying them, to find scenes and material that showed appearances of Tobacco. Researches on Lilacs and PubMed and Grey Literature were made, using the key words: "Tobacco and computer games", "Tobacco in video games", "Tobacco in e-games" and "Tobacco in games".

Results: No publications were found in PubMed or Lilacs, related to Tobacco insertions on computer games. However a article was found, on the British Medical Journal (BMJ) platform, related to Tobacco warnings on video games by the Entertainment Software Rating Board (ESRB), and articles referring to Tobacco and alcohol insertion in electronic games were found also on Grey Literature, showing a crescent concern of researchers.

Conclusions: E-games have to be observed, studied and adopted as a dangerous and viable media for Tobacco funding and marketing use, by the Tobacco industry. To do so, researches and investigation of Tobacco industry's E-games financing should be done, partnerships with different game producers and government should be made, in order to stop those insertions and punish them for transgressing the Art.13 of the Framework Convention on Tobacco Control guidelines.

Tob. Induc. Dis. 2018;16(Suppl 1):A82

DOI:10.18332/tid/84052

\section{2:30-14:00}

PS-568-2 Exposing the false statements disseminated by tobacco industry - media campaign

\section{A Fonseka}

'Alcohol \& Drug Information Centre, Media Programme, Colombo, Sri Lanka. E-mail: asithadharshana@gmail.com

Background and challenges to implementation: Sri Lanka is a country which tries its best to regulate the tobacco industry in the Asian region. Major government policies have been changed based on the FCTC recommendations. With this scenario, the tobacco industry has shown its influence through media where we observed more than 100 industry favorable articles being published before and after the new tobacco control policy adjustments. The industry tried to mislead public, policy makers and other related parties. Therefore it was necessary to reveal the true facts to the country.

Intervention or response: The media discussion was held under the support of Ministry of Mass Media and Center for Combatting Tobacco - a newly established body to monitor and report the tobacco industry efforts under the FCTC recommendation 5.3 with the presence of over 30 media stations. The facts were delivered by veterans of the field including economists, lecturers and doctors. A press release with the evidence based facts to counter the tobacco industry false statements was distributed among 100 media personnel and opinion leaders.

Results and lessons learnt: Two TV channels, six newspapers, and two web based news streams exposed the incorrect facts of tobacco industry. The media discussion created a platform for the journalists to raise their matters regarding the existing and planned tobacco control policies. The existing structures like Ministry of Mass media was utilized well to organize such an event which saved money, time and also gained the attention of the media.

Conclusions and key recommendations: A media discussion is a good method to expose the tobacco industry false statements and unethical media practices of the agencies to a mass audience based on evidence based, updated facts and details. The facts derived from credible sources and presented by the professionals who are actively engaged in tobacco prevention could help to build faith in people regarding the facts presented.

Tob. Induc. Dis. 2018;16(Suppl 1):A83

DOI:10.18332/tid/84054

\section{2:30-14:00}

PS-569-2 Widespread violations of point-of-sale advertisements of tobacco products law around schools in India

T Bhutia', A Mehendale', N Lad', R Kadam

'Salaam Bombay Foundation, Tobacco Control \&t Advocacy, Mumbai, India. E-mail: tshering@salaambombay.org

Background: In India, children and youth between the ages of 15 and 24 years are vulnerable to tobacco initiation. Global Youth Tobacco Survey (GYTS) India 2009 showed that 14.6\% youth aged 13 to 15 years were tobacco users. It is evident from tobacco industry's internal documents that children and youth are targeted in marketing strategies. The area around schools where students spend a significant amount of time offer tobacco companies a valuable marketing platform. In 2016, Salaam Bombay Foundation (SBF) conducted a survey to study the various marketing strategies of tobacco industry around schools. Methods: Student participants of SBF's School Leadership Programme were invited to participate in the survey. In November 2015, participants received orientation training. An area within 100 yards surrounding 40 schools in Mumbai was surveyed by these students. During survey, students also took photographs of marketing strategies observed.

Results: 368 tobacco shops were identified by students around schools. 199 (54.1\%) shops advertised tobacco products. 153 (41.6\%) shops used at least one advertisement using specific colour schemes, patterns and designs without actual brand names. 96 (26.1\%) shops had applied such stickers or adhesive panels below the counter directly at the eye level of children. 298 (81\%) shops positioned tobacco products in proximity to child friendly consumer goods like candy, biscuits, stationary etc. Masking the health warnings on tobacco products, use of dummy packages for display, hanging displays of smokeless tobacco products, selling surrogate products having brand names of tobacco products were the other marketing strategies observed to target children and youth.

Conclusions: The results of the survey suggest that the tobacco industry has developed the strategies to bypass the existing 
laws that limit the marketing of tobacco products with specific strategies to attract youth. In order to combat industry's efforts to target youth a strong and comprehensive law must be introduced.

Tob. Induc. Dis. 2018;16(Suppl 1):A84

DOI:10.18332/tid/84093

\section{2:30-14:00}

PS-570-2 Brazilian observatory to monitor the strategies of the tobacco industry: first year of results

S Turci'

'Brazilian Ministry of Health - Oswaldo Cruz Foundation, Center of Studies on Tobacco or Health, Rio de Janeiro, RJ, Brazil. E-mail: slvnrubanoturci@gmail.com

Background and challenges to implementation: It is well known that tobacco industry is as a vector in tobacco-related diseases epidemic. The Article 5.3 of the WHO-FCTC, recommend the Parties shall act to protect these policies "from commercial interests, considering that the tobacco industry has adopted strategies to commit any action for the development of policies that result in effective tobacco control". By ratifying the treated in 2005, Brazilian government has committed to monitor tobacco industry activities.

Intervention or response: The Center of Studies on Tobacco or Health is building an academic resource: "The Observatory to monitor the strategies of the tobacco industry", that explores how the tobacco industry influences policy and public health in Brazil and have as an objective the tobacco industry monitoring. Available in: http://cetab.ensp.fiocruz.br/index.php/Página_ principal

Results and lessons learnt: Since March, 2016, when it was launched, we reunited around 700 documents, divided in 6 strategies, such as: to maneuvered the politicians to hijack the political and legislative process; to exaggerate they economic importance; to manipulated public opinion to gain respectability; to fabricated support through front groups, to discredit with proven science to create doubt about the evidences, to intimidated governments with litigation or the threats of litigation and people linked with tobacco industries.

Conclusions and key recommendations: Is important and necessary all countries to establish a tobacco industry monitoring system, disseminated these information and promote academic studies/fact sheets/Bulletins about tobacco industries strategies.

Tob. Induc. Dis. 2018;16(Suppl 1):A85

DOI:10.18332/tid/84146

\section{2:30-14:00}

PS-572-2 Ongoing relationships between the tobacco industry and universities: an insidious obstacle to tobacco control

\section{A Blum ${ }^{1}$}

'University of Alabama, Center for the Study of Tobacco and Society, Tuscaloosa, AL, United States of America. E-mail: ablum@ua.edu

Background: Although more than 1750 colleges and universities in the US alone have become smokefree campuses over the past 20 years (including nearly 1500 that claim to have adopted entirely tobaccofree policies), progress in reducing cigarette, smokelsss tobacco, and hookah use among US university students has slowed. Prevalence may be as high as $25 \%$. Globally, reported smoking prevalence among university students ranges from $14 \%$ in Brazil to $60 \%$ in Bangladesh. A little-studied obstacle to reducing tobacco use among university students is the ongoing financial relationships between the tobacco industry and academia.

Methods: A review of tobacco company annual reports, teacher pension fund holdings, tobacco industry documents, campus career center websites, tobacco company websites, and tobacco industry trade publications over the past 25 years was conducted in order to gauge the extent of the relationships between tobacco companies and universities. Documentation was also compiled of the prominent presence of cigarette company recruiters at campus job fairs through attendance at more than 20 such events.

Results: Significant ongoing ties between the tobacco industry and academia include funding of research, investment in tobacco stocks by university endowments and faculty pension funds, involvement in business school curricula, and underwriting of lectureships, professorships, and career centers. The world's largest cigarette company, Philip Morris, continues to recruit students at career center job fairs on more than 35 US university campuses for internships and post-graduate positions as Marlboro territory sales managers. The company's recruitment slogan is "Can't Beat the Experience." Few university endowments have divested tobacco stocks. Nor has the largest college teacher pension fund, TIAA-CREF, which remains a major investor in Philip Morris.

Conclusions: Although progress has been made in reducing tobacco use on university campuses, coordinated strategies to diminish the influence of the tobacco industry in academia are lagging and require greater attention by tobacco control proponents.

Tob. Induc. Dis. 2018;16(Suppl 1):A86

DOI:10.18332/tid/84172

\section{2:30-14:00}

PS-573-2 Strategies and impacts of Japanese tobacco industry in shutting down domestic factories and setting up production plants in Taiwan SW Yau', CL Lin ${ }^{1}$

'John Tung Foundation, Taipei, China. E-mail: service108@ jtf.org.tw

Background and challenges to implementation: Since 2010, Japanese tobacco industry has shut down 4 tobacco factories and laid off 1600 staff workers in Japan. In order to reduce production costs, they have instead set up factories in Taiwan by promising the Taiwanese government that they would invest 300 million US dollars, create 300 more jobs, and sell all the tobacco products to other countries. The Ministry of Economic Affairs in Taiwan hence approved their investment plans, during which the Ministry of Health and Welfare did not render any opposition. The local government thereafter offered 7.6 hectares of lands for the Japanese tobacco industry to set up factories in 2015 and begin to sell low price cigarettes to youngsters at NT $\$ 50$ per pack since 2017.

Intervention or response: It is not only a blatant violation of Article 5.3 of the FCTC in allowing Japanese tobacco industry to set up manufacturing factories in Taiwan, but also a violation of Taiwan's Statute for Investment by Foreign Nationals. The Taiwanese government even used taxpayers' money to subsidize the tobacco industry, so much so that they could avert tariffs worth nearly NT $\$ 2$ billion. What is even worse is that because of those subsidies, Japanese tobacco industry are able to sell tobacco products at a much lower price to our future generations, thereby also inspire many other international tobacco companies to follow suit. 
Results and lessons learnt: Damages caused by tobacco industries:

$\begin{array}{ll}\text { Health } & \text { Each year, } 24000 \text { people die from tobacco-related } \\ \text { Impacts } & \text { illness in Taiwan. } \\ \text { Monetary } & \text { In Taiwan, national health expenditures on } \\ \text { Losses } & \text { treatment of tobacco-related illness amount to NT\$ } \\ & 50.7 \text { billion each year. }\end{array}$

The Taiwanese government will receive NT\$ 2 billion less for customs duties each year by allowing Japanese tobacco industry to set up manufacturing sites in Taiwan.

(Customs duties imposed upon Japanese tobacco products will be reduced from NT\$ 2.7 to NT\$ 0.2 each pack, and Japanese tobacco industry can sell more than 760 million packs of tobacco. Thus, NT\$ 2 billion will be saved each year by the Japanese tobacco industry.)

Tainan City offers 7.6 hectares of valuable lands and many other programs of reduction on taxes such as housing taxes, land taxes, excise taxes and profit taxes to Japanese tobacco industry.

$\begin{array}{ll}\text { Image } & \text { Taiwan became a new overseas stronghold for } \\ \text { Damages } & \text { Japanese tobacco industry. If the tobacco products } \\ \text { made by Japanese tobacco industry are sold to } \\ \text { neighboring countries in large quantities, Taiwan } \\ \text { will be considered responsible for such beggar-thy- } \\ \text { neighbor policy and become a new Tobacco Golden } \\ \text { Triangle. }\end{array}$

[Damages caused by tobacco industries]

Conclusions and key recommendations: International tobacco companies' aggressiveness is not a problem only for Taiwan. We hope all countries could closely collaborate with each other on this issue in preventing tobacco companies from expanding their killing fields.

\section{Tob. Induc. Dis. 2018;16(Suppl 1):A87 DOI:10.18332/tid/84184}

\section{2:30-14:00}

PS-574-2 Analysis of tobacco related articles in English newspapers

PR Vithanage ${ }^{1}$

${ }^{1}$ Alcohol and Drug Information Center (ADIC), Research \& Evaluation Programme, Colombo, Sri Lanka. E-mail: peshanirisna@gmail.com

Background: Direct Alcohol, tobacco and other drugs (ATO) advertisements are banned on print media in Sri Lanka by National Tobacco and Alcohol Authority (NATA) Act. But, indirect advertisements that can influence the reader are still seen in print media.

Objective: To analyze indirect tobacco advertisements in English medium newspapers.

Methods: Four highly circulating daily newspapers published on weekdays and six Sunday newspapers were selected purposively. Study was conducted from April to November in 2014 and total newspapers surveyed were 1046. The number of articles analyzed was 2210 using a code book developed by an expert team. Major themes in the code book were promotion, prevention and policy related. Promotions: promote tobacco expressed by words, social belief, surrogate advertising, unfair privileges and if unintentionally promote them through prevention mode. Prevention: any article, picture or any content published that express positive message for prevention attempts. Policy: any article published about exist policy implementations of the country. Most of time we would able to find articles that is regarding policy cases and national policies such as Pictorial Health Warnings and NATA act.

Results: Of the promoting articles, Ceylon Tobacco Company had the highest number of promotions $(n=77,58 \%)$. A number of articles had been written by famous individuals supporting and promoting the tobacco industry. Colors and symbols representing the industries and the product trademarks were used in the articles. Articles criticizing prevailing policies and regulations on Tobacco were also seen.

Conclusions: Tobacco industries are using various indirect strategies to promote their products through newspaper articles. Seasonal campaigns, articles by opinion leaders, politicians, famous individuals and writers are the commonly used strategies to promote industries views. National level policy amendments should be made to prevent indirect Tobacco advertisements in newspapers.

Tob. Induc. Dis. 2018;16(Suppl 1):A88

DOI:10.18332/tid/84192

$12: 30-14: 00$

PS-575-2 Smoking out the lies: documents research on tobacco tax policy in Kenya

R Kidiya', J Arinaitwe ${ }^{2}$, M Otanez $^{3}, \mathrm{~K}^{1}$ Namusisi ${ }^{4}$, E Wanyonyi ${ }^{5}$, D Kiptui ${ }^{6}$, J Muchangi ${ }^{7}$, A Kendagor ${ }^{8}$

IInternational Institute for Legislative Affairs, Research and Development, Nairobi, Kenya, ${ }^{2}$ Centre for Tobacco Control in Africa, Centre Manager, Kampala, Uganda, ${ }^{3}$ University of Colorado, Denver, CO, United States of America, ${ }^{4}$ Centre for Tobacco Control in Africa, Monitoring and Evaluation, Kampala, Uganda, ${ }^{5}$ International Institute for Legislative Affairs, Nairobi, Kenya, ${ }^{6}$ Ministry of Health, Kenya, Tobacco Control Unit, Nairobi, Kenya, ${ }^{7}$ The Star Newspaper, Nairobi, Kenya, ${ }^{8}$ Ministry of Health, Kenya, Nairobi, Kenya. E-mail: kidiyarodgers@gmail.com

Background: Tobacco Industry (TI) interference in policy development and implementation in Kenya has been widely reported in both local and international media outlets. A local newspaper, Daily Nation, dated 22nd November, 2004, reported that when the current tobacco control law was tabled in parliament, British American Tobacco Kenya (BATK) and Mastermind Tobacco Kenya (MTK) organized a weekend-long retreat in Chale Island, for 40 Members of Parliament (MPs) to lobby against the Bill. Despite the passage of the tobacco control law in 2007, the TI still had its way in influencing tobacco tax and price measures using different tactics. We carried out this project therefore, to expose and document these tactics employed by the TI in Kenya.

Methods: The Truth Tobacco Industry Documents Archive (http://legacy.library.ucsf.edu), a database compiled by the University of California in San Francisco in 2002, served as the primary source of information for the study. Key Informant Interviews were purposively sampled from relevant government ministries and agencies, civil society and other actors. The findings from the library and the KII, were then triangulated with statements from industry and government officials and other related articles in newspaper stories and publications.

Results: We established that the tobacco industry in Kenya has repeatedly used 6 main tactics to influence tobacco tax and price policies, they include: use of front groups, use of tobacco advertisements, promotion and sponsorship, influencing policy development and legislative processes, Illicit and Deceptive Trade Practices, Intimidation and Litigation, Phony statistics 
and researches.

Conclusions: The TI armed with their financial muscle and highpowered political connections, will go any distance to achieve their goal for profit. However, their tactics and strategies in interference has been similar over the years. This implies then, that awareness of these tactics by policymakers will go a long way in preventing future TI interference.

Tob. Induc. Dis. 2018;16(Suppl 1):A89

DOI:10.18332/tid/84204

\section{2:30-14:00}

PS-576-2 Tobacco industry tactics to retail vendors in Sri Lanka

\section{A Tenna}

1ADIC, Colombo, Sri Lanka. E-mail: tennaamaranath@gmail.com Background and challenges to implementation: Mainly Tobacco industry depends on retail tobacco vendors. Community groups and heath people are empowered to minimize the availability of cigarettes in respective areas. Hence, Tobacco Company has started to lobby retail vendors for continuing selling cigarettes.

Intervention or response: A study was carried out by ADIC with cigarette retail vendors in Hambantota city. A structured questioner and observation were used as data collection methods. Trained field officers interviewed 31 retail tobacco vendors. Questions were related to knowledge of harm done by cigarette and attitude on Tobacco Company. Observation was used to identify cigarette promotions inside retail shops.

Results and lessons learnt: Result reveled that the main reason (25 out of 31 responds) for cigarette selling is as an essential item of a shop. Even cigarette profit is comparative to low it helps to sell other goods. 8 Vendors stated that they had participated conferences organized by Tobacco Company. Tobacco company educated vendors on what benefit the business could got by selling cigarettes.

Another strategy has been identified during the study. Tobacco Company has been introduced award system for cigarette vendors. It is named as "Abisheka" (anointed vendors). Six cigarette vendors named as anointed vendors. Those vendors got certificates and privilege cards for business.

Attitude regarding cigarette business examined at the study. 18 vendors of 31 did justification of harm done by cigarette because they made argument that other product may harm. They did not believe tobacco industry do marketing to children majority of responds (77\%) said that smoking was adult choice.

Cigarette promotions could be identified in different ways because tobacco promotion was prohibited. We found that promotional item such as board was displayed as a vendor's message. Awarded shops got colourfull cashier point and showcase for cigarettes.

Conclusions and key recommendations: Tobacco industry cheats vendors as Trojans to continuing cigarette selling.

Tob. Induc. Dis. 2018;16(Suppl 1):A90

DOI:10.18332/tid/84290

\section{$12: 45-14: 00$}

EP-102-2 Tobacco and anti-tobacco advertisement in Poland, 1989-2000

M Zatonski ${ }^{1,2}$, M Gorsky $^{1}$, M Mckee $^{1}$

${ }^{1}$ London School of Hygiene \& Tropical Medicine, Public Health and Policy, London, United Kingdom, ${ }^{2}$ Health Promotion Foundation, Nadarzyn, Poland. E-mail: zatonski.mateusz@ gmail.com
Background: Smoking prevalence in communist Poland reached the highest level in the world in the 1980s. Despite the statesanctioned anti-tobacco campaigns less than half of all Poles believed that smoking was a serious hazard to health. In 1989, as communism collapsed, transnational tobacco companies (TTCs) entered Poland. TTCs spent US\$100 million annually on cigarette publicity, becoming the leading advertiser in the country. The TTCs predicted an increase in cigarette sales in Poland.

Instead, a rapid decline in smoking occurred in the 1990s. A very active health advocacy movement developed, attempting to counteract the marketing efforts of TTCs, also through antitobacco advertising. By the end of the century cultural norms shifted - a European survey showed that by the late 1990s Poland had the highest acceptance of anti-smoking measures in Europe. Methods: A thematic analysis of relevant advertising materials produced between 1989 and 2000 available in Polish media archives, collections of anti-tobacco advocacy groups, and tobacco industry websites, was conducted.

Results: After the collapse of communism both tobacco and antitobacco messaging in Poland focused around four major themes - the appeal of smoking to children, the use of historical and patriotic motifs, employing shock tactics, and the struggle over which lifestyle embodies 'western' values. Many of the antitobacco images were designed by Poland's leading poster artists and were characterised by high artistic value.

Conclusions: Polish anti-tobacco advocates successfully reclaimed many of the themes and values used by the TTCs in promoting smoking. They challenged the industry's attempts to portray smoking as a 'western' habit, employed patriotic tropes and shock tactics, and steered the debate towards the health effects of tobacco use.

Tob. Induc. Dis. 2018;16(Suppl 1):A91

DOI:10.18332/tid/84573

\section{$12: 45-14: 00$}

EP-103-2 Tobacco industry securing connections with political elites: a case study from Sri Lanka KMN Perera ${ }^{1,2,3}$, K Evans-Reeves ${ }^{3}$, PR Vithanage ${ }^{1,4}$, I Buddhika ${ }^{1,4}$, S Lakmal ${ }^{1,4}$, M Rajasuriya ${ }^{1}$, A Gilmore $^{3}$, Centre for Combating Tobacco (UCFM) \&t Tobacco Control Research Gr

${ }^{1}$ Centre for Combating Tobacco, Faculty of Medicine, University of Colombo, Colombo, Sri Lanka, ${ }^{2}$ Faculty of Medicine, University of Kelaniya, Public Health, Ragama, Sri Lanka, ${ }^{3}$ University of Bath, Tobacco Control Research Group, Bath, United Kingdom, ${ }^{4}$ Alcohol and Drug Information Center (ADIC) Sri Lanka, Colombo, Sri Lanka. E-mail: kmanujanp@gmail.com

Background: Ceylon Tobacco Company PLC (CTC), a British American Tobacco subsidiary, holds a de facto monopoly of the Sri Lankan cigarette market with a $99 \%$ market share. A CTC internal document dated 1985 justified recruitment of an ex-army official to its Board of Directors (Board) citing that the he had "close relationships with officials who now hold senior positions in the Government". This study aimed to explore the political and professional connections of Sri Lankans who served on the CTC Board from 2000 onwards and their transparency in reporting them.

Methods: CTC Annual reports were used to identify all locals who served on the Board. Media articles, interviews and personal profiles published in public domains were identified via the Google search engine and manually in print literature. Identified data were analysed using content analysis to explore other professional (positions held in other corporates/organisations), political 
(links with political parties and politicians) and family networks (famous/powerful relatives) and transparency of reporting their affiliations to CTC.

Results: During the period, 12 Sri Lankans served on the CTC Board, 10 were men. Four of these served as Chair, all men. Board composition of five Sri Lankans to zero expatriates in 2000 , gradually shifted to two to two in 2016. All held high rank positions either in government institutions, other private corporations or professional associations related to commerce and management. Five simultaneously held high-rank positions in government agencies in appointments made by the President or a Minister. Two came from famous families. The only two females served in the board were 'tax experts'.

Except for CTC Annual Reports, personal profiles and media articles reporting events directly related to CTC, their affiliation to CTC was rarely mentioned.

Conclusions: The local tobacco directors were well linked socially and politically. Their affiliation to CTC was not satisfactorily disclosed in public media.

Tob. Induc. Dis. 2018;16(Suppl 1):A92 DOI:10.18332/tid/84309

\section{$12: 45-14: 00$}

EP-104-2 Profile of characters involved in tobacco appearances in Brazilian TV programs

C Cortes ${ }^{1}$, H Carvalho ${ }^{1}$, M Reis ${ }^{1}$, S Turci ${ }^{1}$, LA Camacho², S Bialous ${ }^{3}$, VL da Costa e Silva ${ }^{4}$, V Figueiredo

${ }^{1}$ Fundação Oswaldo Cruz (Fiocruz)/ Escola Nacional de Saúde Publica, Centro de Estudos sobre Tabaco e Saúde (CETAB), Rio de Janeiro, RJ, Brazil, 'Fundação Oswaldo Cruz (Fiocruz)/ Escola Nacional de Saúde Publica, Rio de Janeiro, RJ, Brazil, ${ }^{3}$ University of California San Francisco School of Nursing, Social and Behavioral Sciences (SBS), San Francisco, CA, United States of America, ${ }^{4}$ Organização Mundial da Saúde, Secretariado da Convenção-Quadro para o Controle do Tabaco, Genebra, Switzerland. E-mail: krolcortes@hotmail.com

Background: Favorable images of smoking in the media have been pointed out as a potential motivator of the initiation to nicotine addiction. This study aimed to analyze the profile of the characters involved in tobacco appearances in Brazilian TV programs.

Methods: Descriptive analysis, with 400 hours of programming including soap operas, Brazilian series, reality shows and film sessions. For this study, characteristics of tobacco users, the place where people smoke and the general message perceived were analyzed. All analysis were obtained using Stata 12.0 software.

Results: A total of 164 programs portraying tobacco use were identified, representing 3 hours of visualization, with 388 appearances of characters involved with smoking. Of these, $75 \%$ were male, $60 \%$ appeared to be older than 30 years old, $48 \%$ were good, $22 \%$ were evil and in $30 \%$ of the cases, it was not possible to establish whether they were good/bad. These characters appeared smoking in $74 \%$ of the scenes, $18 \%$ contained only verbal message, $7.5 \%$ smoke-free contact and $0.5 \%$ body language. The most outstanding characteristics were: beautiful (46\%) and lean (41\%). The least mentioned characteristics were: supernatural and sick ( $1 \%$ each). In the programs, $43 \%$ of the characters were main characters. Smoking scenes overlap, being that $70 \%$ of them occur indoors, $67 \%$ outdoors, $82 \%$ close to nonsmoking adults, $6 \%$ in non-smoking areas, $3 \%$ around children and $2 \%$ around pregnant women/sick people. Cigarette smoking was the most prevalent type of tobacco use $(87.5 \%)$. When observing the general message portrayed by the programs, most cited were: Addiction/Habit (85\%) and Relaxation (63\%). Less cited messages were: Rebellion (5\%) and Loser (3\%).

Conclusions: This study allowed us to observe that television media still contributes to the exhibition of characters who smoke, which can influence the use of tobacco by children and adolescents, demonstrating a challenge for Tobacco Control Policies.

Tob. Induc. Dis. 2018;16(Suppl 1):A93

DOI:10.18332/tid/84225

$12: 45-14: 00$

EP-105-2 Hostage to fortune: an empirical study of the tobacco industry's business strategies since the advent of E-cigarettes

$\mathrm{M}$ de Andrade ${ }^{1,2}, \mathrm{~K}$ Angus $^{3,4}, \mathrm{G}$ Hastings $\mathrm{s}^{3,4}, \mathrm{~N}$ Angelova $^{5}$

${ }^{1}$ University of Edinburgh, School of Health in Social Science, Edinburgh, United Kingdom, ${ }^{2}$ UK Center for Tobacco and Alcohol Studies, Edinburgh, United Kingdom, ${ }^{3}$ University of Stirling, Stirling, United Kingdom, ${ }^{4}$ UK Center for Tobacco and Alcohol Studies, Stirling, United Kingdom, ${ }^{5}$ University of Edinburgh, Edinburgh, United Kingdom. E-mail:marisa.deandrade@ed.ac.uk Background: The tobacco market has been transformed by e-cigarettes and alternative nicotine delivery systems (ANDS). Despite speculation about the tobacco industry's (TI) business intentions regarding these developments, there is little empirical evidence. This study with industry stakeholders - tobacco and independents - addresses this.

Methods: Empirical data synthesis using strengths-weaknessesopportunities-threats framework. Primary data from a unique dataset of elite interviews with TI and independents $(n=28)$ triangulated against secondary data $(n=1,022)$ including company reports, investor/market research analyses, press releases, government consultations $(n=170)$.

Results: Tobacco multinationals were taken by surprise by e-cigarettes. Clarity provided by the fiduciary imperative has enabled them to turn a threat into profitable opportunities. Companies have calculated that ANDS play to their strengths - customer links, expertise in nicotine and, thanks to tobacco, immense financial resources. This enables multiple opportunities: diversification into broader product portfolios where combustible and alternative products coexist; new long-term strategic goals; scientific and regulatory stature developments; retrieving corporate reputations; collaboration with public health. ANDS are reducing the TI's existing weaknesses, especially poor standing among consumers and stakeholders.

Conclusions: Far from undermining companies, ANDS have made the TI stronger and ensured it will flourish. The arbiter of success will be the marketplace, not health ministry. If ANDS become more profitable than combustibles, they will prevail with reduction in health harms provided safe and effective. If combustibles retain an economic edge, they and their collateral will continue for many years. The latter is more likely in poorer countries where tobacco seems set to remain the most affordable option. The TI is gaining dominance over research and emerging evidence. Thus future judgements about relative merits of tobacco control products/approaches; about where harm is genuinely reduced or inadvertently increased, will largely depend on TI data/expertise. In this way tobacco control will become hostage to both the fortune and fidelity of the TI.

Tob. Induc. Dis. 2018;16(Suppl 1):A94

DOI:10.18332/tid/84451 
12:45-14:00

EP-106-2 Stopping Tobacco Industry Interference (TII): bottom-up approach to achieve national policy on FCTC Article 5.3 in India

R Sharma', P Lal' ${ }^{1}$, A Pandey', G Tripathi'

'The Union South-East Asia, Tobacco Control, New Delhi, India. E-mail:rsharma@theunion.org

Background and challenges to implementation: TII has been a major roadblock in implementing policies particularly since some policymakers have represented farmers constituencies and lobbies, and have direct and indirect links with tobacco companies as well. For the past ten years, India has observed many instances, including dilution and delaying of pictorial health warnings (PHWs) of TII. TII was seen openly during FCTC COP-7 held in New Delhi, India in November 2016, where the tobacco industry used farmers and front groups, and demanded that it be allow to participate in COP 7 and labelled their exclusion as "undemocratic". There is a felt need for a national law that complies with Article 5.3 of FCTC. Given that health is a state subject the efforts to advance Article 5.3 policy has to take place at the state and national level.

Intervention or response: This case study examines the evidence of TII at the state-level and presents it to policymakers for a development of a guideline for its prohibition in line with FCTC Article 5.3.

Results and lessons learnt: In 2015-2016 the states of Punjab and Mizoram, followed by Bihar and Himachal Pradesh have developed (in 2017) state policies which encompass constitution of an empowered committee and protocol to deal with tobacco industry. In 2017 three districts of Karnataka and two districts of West Bengal (Darjeeling and Howrah) have passed similar notifications. Few other states including Rajasthan, Kerala, Sikkim and Jharkhand have agreed in principal to enact such sub-national laws that will strengthen the argument to develop a national policy.

Conclusions and key recommendations: The advocacy efforts of civil societies through state-level coalitions and partnerships, judicial direction and media led advocacy are creating awareness for states to adopt sub-national policy to stop TII. This will gradually build generate evidences and build precedence for Government of India to develop and adopt a national policy.

Tob. Induc. Dis. 2018;16(Suppl 1):A95

DOI:10.18332/tid/84378

\section{$12: 45-14: 00$}

EP-107-2 Ending corporate giving to halt tobacco industry interference in the ASEAN region

W Jirathanapiwat

'Southeast Asia Tobacco Control Alliance, Bangkok, Thailand. E-mail: worrawan@seatca.org

Background and challenges to implementation: As the ASEAN region becomes more aware of the deadly effects of tobacco and governments step-up regulation of the industry, tobacco companies are resorting to more below-the-line tactics to promote their corporate name to reach consumers. Corporate Social Responsibility (CSR) activities is one of the key tactics used by the tobacco industry (TI) to enhance its image in the public and governments' eyes.

None of the governments in the ASEAN region require the TI to declare their expenditure on CSR activities, sponsorship, retailer incentives and lobbying. Hence the actual amount the companies spend on these activities is not quantified. FCTC Article 5.3 requires such disclosure. Three countries in the ASEAN region (Myanmar, Lao PDR and Thailand) have banned CSR activities. Intervention or response: Banning the publicity of CSR activities alone is insufficient to restrict the industry as experienced by Vietnam. The tobacco industry always finds a way to promote itself in the media and online. In Malaysia, Philippines and Indonesia, recipients of the tobacco industry grants, such as retailers associations and farmers groups are mobilized to fight tobacco control measures. The tobacco companies spend extensively on CSR in Indonesia and the Philippines and both countries continue to face intense opposition to tobacco control such as banning tobacco advertising.

Results and lessons learnt: In several countries the TI is latching on to the SDG Goals and talking-up "sustainable agriculture", "sustainable communities" and "sustainable environment". However the UNDP and ECOSOC have clear policies implementing Article 5.3 and ensuring their policies are protected from tobacco industry interference.

Conclusions and key recommendations: Tobacco related CSR activities should be banned. Government officials and UN agencies must rejects grants from and partnerships with the tobacco industry and publicise their official position on nonpartnership with the industry.

Tob. Induc. Dis. 2018;16(Suppl 1):A96

DOI: $10.18332 /$ tid $/ 83872$

\section{$12: 45-14: 00$}

EP-108-2 Tobacco plain packaging coverage in Australian newspapers 2008-2014

C Miller ${ }^{1,2}$, A Brownbill ${ }^{1,2}, K_{\text {K Ettridge }}^{1,2}$, J Dono $0^{1,2}$

${ }^{1}$ South Australian Health and Medical Research Institute, Adelaide, SA, Australia, ${ }^{2}$ University of Adelaide, Adelaide, SA, Australia.E-mail: caroline.miller@sahmri.com

Background: In 2012 Australia was the first country in the world to implement plain tobacco packaging. Both public health agencies and the tobacco industry advocated their position on the legislation in the news media. This study comprehensively documents print media coverage of the plain packaging initiative over the duration of the policy initiation and implementation.

Methods: Major Australian print newspaper $(n=17)$ articles on plain packaging were sourced through the Australian/New Zealand Reference Centre and Factiva online databases. Articles $(\mathrm{n}=701)$ were collected over the 7 year duration of the policy debate and pre- and post-implementation from January 2008 to December 2014. Content analysis regarding article type, topic and frame was undertaken.

Results: Print media coverage of plain packaging was initially low (2008-2009) and increased during the legislative process, and lead up to implementation. Print media coverage declined substantially post-implementation. News articles were the most common form of coverage (79.6\%), followed by editorials (14.3\%). Updates on progress of the policy were most commonly discussed (29.4\%) followed by the tobacco industry's response to the proposed legislation including 'nanny state' objections (18.3\%) and discussions of plain packaging as a tobacco control initiative $(17.0 \%)$. As well as reporting of policy announcements, policy rationale, implementation, legal challenges and their outcomes, there was also reporting of industry arguments which centred around predictions of unintended consequences including illicit trade, youth smoking and harm to small business, as well as legal arguments about acquisition of intellectual property.

Conclusions: Plain packaging received significant media coverage in Australia in the years leading up to its implementation, and far less post implementation. Subsequently disproven industry predictions did receive substantial coverage, however they were countered by articles on the public health rationale and the 
policy's successful progression. Policy makers should expect but not be deterred by coverage of industry arguments through media.

Tob. Induc. Dis. 2018;16(Suppl 1):A97 DOI:10.18332/tid/84354

\section{$12: 45-14: 00$}

EP-109-2 Tobacco control law written by JTI: a case study of flagrant industry interference in Kyrgyzstan K Shats' ${ }^{1}$ C Bekbasarova ${ }^{2}$, J Abrams'1 0 Knorre ${ }^{3}$

'Campaign for Tobacco Free Kids, Washington, DC, United States of America, ${ }^{2}$ Smoke-Free Kyrgyzstan, Bishkek, Kyrgyz Republic, ${ }^{3}$ Campaign for Tobacco Free Kids, Moscow, Russian Federation.E-mail: kshats@tobaccofreekids.org

Background and challenges to implementation: In 2017, all but one of 116 parliamentarians voted to introduce amendments to weaken the tobacco control law in Kyrgyzstan, including mandating smoking areas and other measures contrary to the Framework Convention on Tobacco Control (FCTC), which Kyrgyzstan has ratified. The law appears to have been drafted by Japan Tobacco International (JTI), as evidenced by metadata that lists JTI as the author of the file containing the official law on the Kyrgyz parliamentary website (see Figure 1 below).

The parliamentarian who introduced the bill had been working with JTI since 2012 as part of a corporate social responsibility (CSR) project for the Kyrgyz Society for the Blind and Deaf. An internal source informed us that JTI offered compensation to the Kyrgyz media not to report this incident.

Intervention or response: Legal and advocacy tactics are being employed to repeal and replace the law with FCTC compliant measures including a comprehensive smoke-free law, to educate government officials about the implications of tobacco industry dealings, and to engage the global public health community to help expose the industry and advocate for the enactment of FCTC Article 5.3 measures in the region.

Results and lessons learnt: Despite such overt JTI interference with the policy-making process in Kyrgyzstan, our analysis found no viable domestic or international legal avenues to address this conduct. Although there are international agreements on bribery and corruption, few apply or can be enforced under these circumstances, resulting in a significant and concerning regulatory gap.

Conclusions and key recommendations: We expect that neither these findings nor this conduct are particular to the region, and therefore significant law reform is required. This includes: implementation of Article 5.3 measures, particularly in vulnerable countries; enactment and enforcement of laws on bribery, corruption and lobbying transparency in countries where the tobacco industry is headquartered; and expanding and strengthening international agreements to hold industry and governments accountable.

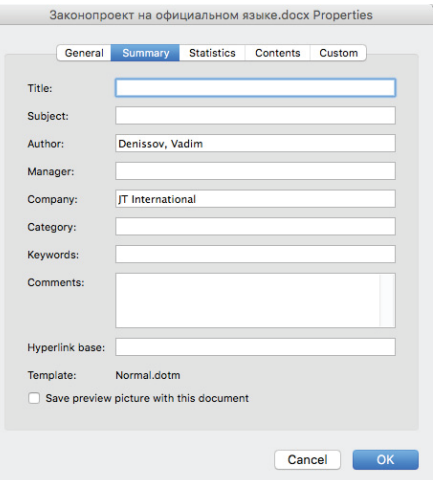

Tob. Induc. Dis. 2018;16(Suppl 1):A98

DOI:10.18332/tid/84292
12:45-14:00

EP-110-2 Philip Morris' litigation prevention program in Asia

S Lee', J-H Kim²

${ }^{1}$ Korean Association on Smoking or Health, Seoul, Korea, Republic of, ${ }^{2}$ Seoul National University, Seoul, Korea, Republic of. E-mail: wwwvince77@gmail.com

Background: Since the Master Settlement Agreement (MSA) in 1998, Philip Morris (PM) as a transnational tobacco company (TTC) has concerned about the tobacco litigation filed outside of the US and subsequent litigation expenses. We identified the strategies of $\mathrm{PM}$ and its affiliates in the intervention and prevention of litigation outside the US, especially in South Korea and other Asian countries.

Methods: Analysis of 193 documents obtained from the Truth Tobacco Industry Documents, and data from governments/ international organisation reports, media, and scholar articles.

Results: PM organised and operated the Litigation Prevention Program (LPP) to create legal environments making tobacco litigation difficult to begin, and resources such as networks with local lawyers, officials, media, and even competitors to effectively response to such litigation. PM developed the LPP based on its legal strategies in the US against tobacco litigation, and disseminated them all around the world including South Korea. In 1999, the first joint action against Korea Tobacco and Ginseng Cooperate, (KTGC) a state-owned tobacco company in Korea, and Korean government was begun. KTGC asked PM to support this tobacco litigation, and PM provided KTGC with its legal strategies through the LPP. In front of legal threats, tobacco companies, competitors in markets, jointly fought back the litigation in Korea.

Conclusions: Any litigation against a single local tobacco company may confront legal networks of transnational tobacco companies. Development of guidelines of Article 19 of the Framework Convention on Tobacco Control is needed to support tobacco litigation around the world. Without the legal support from international anti-tobacco community, it would be hard for local tobacco litigation to win.

Tob. Induc. Dis. 2018;16(Suppl 1):A99

DOI:10.18332/tid/83787

\section{$12: 45-14: 00$}

EP-111-2 Challenges in the implementation of the National Tobacco Control Law in Argentina

J Konfino'

${ }^{1}$ Centro de Estudios de Estado y Sociedad (CEDES), Quilmes, Argentina. E-mail: jkonfino@gmail.com

Background and challenges to implementation: In Argentina the National Tobacco Control Law sanctioned in 2011bans advertising with the exception of point of sales. The federal organization of the country, the lack of appropriate administrative resources and tobacco industry tactics contributed to fail in the implementation and enforcement of the ban.

Intervention or response: In 2013 several directorates and undersecretaries of the Ministry of Health involved in the enforcement of the national law were brought together by the head of the national tobacco control program to build an administrative and legal procedure assigning responsibilities and competences for the inspection and sanction of the law that until that moment were not among the roles of none of them.

Results and lessons learnt: After 6 months deliberating the best and legal approach for the enforcement of the law, the 
Ministerial Resolution 425/2014 was sanctioned by the Minister. The availability of a legal resolution that assigned concrete roles to the parties involved led to the sanction of more than 300 citations and fines during 2014. This new proceeding generated a reaction in the tobacco industry that firstly asked for audiences with authorities of the Ministry of Health and then started to impugn the fines. There was a political will of continuing with the enforcement and none of the industry claims were heard. Since then, tobacco industry had to recur to ordinary justice that rejected their claims too. The start-up of this proceeding generated a change in the pattern of tobacco industry advertising that migrated from full-color billboards to the display of cigarette packs only at point of sales.

Conclusions and key recommendations: While advocates should fight for improving the law and a complete ban on advertising in Argentina is necessary to take advantage of the resources we already have and guarantee the enforcement of current law.

Tob. Induc. Dis. 2018;16(Suppl 1):A100

DOI:10.18332/tid/83760

$12: 45-14: 00$

EP-112-2 Tobacco industry engaging agriculture ministers in Sri Lanka

SC Lakmal ${ }^{1,2}$, KMN Perera $^{1,3,4}$, I Buddhika ${ }^{1,2}$, CS Perera ${ }^{1}, \mathrm{H}$ Wijesooriya', M Rajasuriya', Centre for Combating Tobacco (UCFM)

${ }^{1}$ Centre for Combating Tobacco, Faculty of Medicine, University of Colombo, Colombo, Sri Lanka, ${ }^{2}$ Alcohol and Drug Information Center (ADIC) Sri Lanka, Colombo, Sri Lanka, ${ }^{3}$ Faculty of Medicine, University of Kelaniya, Public Health, Ragama, Sri Lanka, ${ }^{4}$ University of Bath, Tobacco Control Research Group, Bath, United Kingdom. E-mail:sameeralakmal111@gmail.com Background: In recent years, media reported joint activities of Ceylon Tobacco Company (CTC), the British American Tobacco (BAT) subsidiary holding cigarette manufacturing monopoly in Sri Lanka, and the Agriculture Ministers (Ministers). CTC is the only BAT subsidiary in the region that engages in a complete leaf to manufacture process in cigarette manufacturing. Tobacco needed in manufacturing was cultivated in Sri Lanka for the past decade. The Ministry of Agriculture is the government authority over agricultural activities and the Minister is the de facto decision maker on agricultural policy development and implementation. The study aimed to explore the engagement of Ministers of Agriculture in activities of CTC from 2000 onwards.

Methods: The study used a qualitative design on data obtained via participatory methods. Media reports and industry documents (reports, press releases and shareholder presentations) were identified using Ministers' names as key words. Photographs and other community level documentary evidence were obtained from the tobacco control activists via an open invitation. Data were analysed using a deductive content analysis on themes; government and political party, type of activity, the possible impact to the community and industry and extent and context of media coverage received.

Results: During the period, four individuals, all males, belonging to three political parties and four governments, served as Agriculture Ministers. All participated in activities organised by the CTC. All activities were under the label "Sustainable Agriculture Development Programme", the social investment initiative of CTC. The type of activities were visiting farmers, attending award ceremonies for farmers or their children and attending ceremonial openings of infrastructure developed under
CTC sponsorship. Most were conducted jointly with the Ministry or the local governments and all received positive media coverage. Conclusions: Ceylon Tobacco used corporate social investment activities to engage Agriculture Ministers and the ministry, receiving wide positive media coverage in the process.

Tob. Induc. Dis. 2018;16(Suppl 1):A101

DOI:10.18332/tid/84473

$12: 45-14: 00$

EP-113-2 Why do violations of FCTC Article 5.3 occur?: The case of tobacco industry incentives in Turkey

E Evrengil $^{1}$

${ }^{1}$ Turkish National Coalition on Tobacco or Health, Istanbul, Turkey.E-mail: efzagil@yahoo.com

Background: Aiming at eliminating tobacco industry interference, Article 5.3 forms the backbone of Framework Convention on Tobacco Control (FCTC). By way of analysis of investment and export incentives granted to tobacco industry in Turkey, this research intends to demonstrate that the task of realizing Article 5.3 provisions often entails political choice between conflicting legal frameworks of FCTC and neoliberal capitalism.

Methods: Data for incentives for tobacco manufacturing were compiled and analysed through keyword screening of lists of Investment Incentive Permits (IIP) for 2000-2016 and Inward and Outward Processing Permits (IPP and OPP) for 2015-2016 as published in the Official Gazette.

Results: Tobacco companies have received 30 IIPs, 39 IPPs and 2 OPPs in the periods researched. Granted pursuant to economic incentive regulations, but in stark contradiction to FCTC, and comprised of tax exemptions and financial aid, these incentives have increased over time, have been granted predominantly to transnational firms, creating vast new manufacturing capacities for cigarettes, waterpipe tobacco, and macarons (empty cigarette tubes). Production, consumption, and export figures have risen markedly in the same periods.

Conclusions: Incentives constitute a gross violation of FCTC Article 5.3, a key principle of which provides, “...the tobacco industry should not be granted incentives to establish or run their businesses". As the Turkish case shows, FCTC Article 5.3 may get violated and rendered completely obsolete, not necessarily because of political or bureaucratic weakness or inaptitude, but often on grounds of neoliberal policies and regulations. Political action is needed at national and international levels, to abolish tobacco industry incentives, strengthen effectiveness of FCTC implementation through supply-side measures which would allow Article 5.3 to take root, uphold FCTC as an overriding, powerful legal instrument, and operationalize and fortify its binding nature.

Tob. Induc. Dis. 2018;16(Suppl 1):A102

DOI:10.18332/tid/84506

\subsection{Implementing sustainable funding mechanisms for tobacco cont rol}

12:30-14:00

PS-577-2 Progress towards a health surcharge in Bangladesh

F Islam¹, A Islam Sujon ${ }^{1,2}$, G Pearee ${ }^{3}$, SA Rahman ${ }^{1}$

${ }^{1}$ Work for a Better Bangladesh (WBB) Trust, Health Rights and Tobacco Control, Dhaka, Bangladesh, ${ }^{2}$ Bangladesh Anti Tobacco Alliance (BATA), Communication and Advocacy - Tobacco Control, Dhaka, Bangladesh, ${ }^{3}$ Work for a Better 
Bangladesh (WBB) Trust, Administration and Management, Dhaka, Bangladesh. E-mail: fahmida.islam03@gmail.com

Background and challenges to implementation: While Bangladesh has made significant strides on tobacco control, we have been weak on pricing. Tobacco control advocates were less competent in this area than in policy areas such as smokefree places, TAPS, and packet warnings. Intensive industry lobbying contributed to blocking significant progress on taxation. As a result, the other policy areas have not had the anticipated success. Intervention or response: Work for a Better Bangladesh Trust has been advocating for higher tobacco taxes virtually since its inception and for a dedicated surcharge since 2005. We did international research on health promotion foundations. We wrote a short book on the issue, including specific suggestions on its use. We carried out a signature campaign. We met with policymakers to discuss the issue. We organized seminars and participated in international discussions on the issue. We found several high-level advocates to push for the issue. We also engaged our extensive network in promoting the surcharge idea.

Results and lessons learnt: In 2014-15, the Government of Bangladesh introduced a health surcharge on tobacco products of $1 \%$ of price. The government did not, however, make a decision at the time as to the use of the collected surcharge. As a result, it went into general revenue. More recently, the government has decided to allocate the surcharge to tobacco control in the following areas: prevention of non-communicable disease, policy implementation, function of the National Tobacco Control Cell, cessation, and treatment of tobacco-related disease. It is hoped that the allocation will begin within the next year.

Conclusions and key recommendations: Sustainable funding for tobacco control is necessary in order to maintain an effective response to the epidemic. A dedicated surcharge is the best source of sustainable funding. Advocates should not be disappointed if the process requires many years, as the benefits will continue for decades to come.

Tob. Induc. Dis. 2018;16(Suppl 1):A103

DOI: $10.18332 /$ tid/83915

\section{2:30-14:00}

PS-578-2 Pooling local public health budgets to achieve exceptional value for money: the case of the North East of England

A Rutter ${ }^{1}$, L Surtees $^{1}$, E Milne ${ }^{2}$, A Healy $^{3}$, A Lloyd $^{1}$, C Taylor $^{1}$, C Shevills ${ }^{4}$

${ }^{1}$ Fresh-Smoke Free North East, Durham, United Kingdom, ${ }^{2}$ Newcastle City Council, Newcastle upon Tyne, United Kingdom, ${ }^{3}$ Durham County Council, Public Health, Durham, United Kingdom, ${ }^{4}$ Balance - North East Alcohol Office, Durham, United Kingdom.E-mail: ailsa.rutter@freshne.com

Background and challenges to implementation: The North East of England (pop 2.6m) has significant health challenges, largely resulting from an historic industrial past, and subsequent high rates of tobacco use. The region united in pooling public health budgets in order to deliver key interventions at scale. Eight key strands of tobacco control have been delivered synergistically over the last decade including smoking cessation, advocacy and media, regulation, research, secondhand smoke protection.

Intervention or response: The overall annual budget of USD 915k is used cost-effectively and funds a small regional multidisciplinary team supporting the work of the localities providing expertise, strategic support, advocacy, mass media campaigns, coordinating practice sharing and industrializing system wide reform, and delivering work at scale on complex issues. The work is guided by international best practise and informed by national tobacco strategies including those from ASH UK, Department of Health and Public Health England.

Results and lessons learnt: There has been a $41 \%$ reduction in the smoker population - the biggest regional decline in the last decade. A social movement has grown with high levels of public support for future regulation. Making tobacco control 'everyone's business' has been important and having a clear vision for the future vital to maintain funding commitment and focus. It is important to constantly reinforce the rationale for this investment from a health inequalities perspective and the strong return on investment tobacco control gives.

Conclusions and key recommendations: Significant economies of scale have been achieved with high value for money. In the UK there is a need for effective tobacco action from the community grass roots level right up to the national and international stage. A similar approach is in place around alcohol denormalisation now through the regional programme "Balance" and the two programmes are working together with notable success. The North East England provides a useful model for others.

Tob. Induc. Dis. 2018;16(Suppl 1):A104

DOI:10.18332/tid/84022

\section{2:30-14:00 \\ PS-579-2 Vietnam tobacco control sustainable funding \\ H Phan \\ 'Vietnam Ministry of Health, Vienam Tobacco Control Fund, Hanoi, Viet Nam. E-mail: haivinacosh@yahoo.com}

Background and challenges to implementation: Vietnam is one of the 15 leading countries with highest smoking prevalence ( $45.3 \%$ in male and $1.1 \%$ female). Thanks to the strong political commitment, in 2012, Viet Nam passed a comprehensive tobacco control law. In which, a first ever Tobacco Control Fund using $1.5 \%$ of surcharge tax being compulsory contribution from tobacco industry is established.

Intervention or response: The Fund is multi-sectoral with engagement across sectors and of government and civil society in advocating for and supporting the Fund. The sstrategic to have the voice of non-health players - helps to convey that the importance and relevance of the Fund is universal and crosssectoral. A very clearly mechanism for funding, strong vision and objectives and function defined by Law. Strategic targeted focus in early days (high priority areas to reflect and address gaps in tobacco control; tight criteria for grants (proactive and critical also when move to open grants) and outcomes based funding from outsets and very detail outputs, indicators for both the Fund and its grantees. Code of conduct is strictly complied while capacity building, M\&E system developed from outset

Results and lessons learnt: After 3 years of operations, the unprecedented investment has made, spreading over 102 grantees from ministerial and mass organizations, local authority, hospitals. The series of programs are development of smoke free in different settings, communication, tobacco cessation consultancy service, capacity building, network expansion, research and evidence based program evaluation. Smoking rate among adult males has reduced marginally from 47.4 among males to $45.3 \%$. Secondhand tobacco smoke has reduced significantly by $18 \%, 23 \%$ and $43 \%$ at home, workplaces and public transport respectively. Prevalence among youth aged $13-15$ decreased from $3.3 \%$ to $2.5 \%$ between 2007 and 2014 (GATS, GYTS). 
Conclusions and key recommendations: Low tax and price, high rate of tobacco second hand smoke in hospitality settings, tobacco industry interference.

Tob. Induc. Dis. 2018;16(Suppl 1):A105

DOI:10.18332/tid/84032

\section{2:30-14:00}

PS-580-2 Member states of the FCTC can generate self-sustaining funding by applying the polluterpay principle to the tobacco industry

\section{N Collishaw ${ }^{1}, C^{2}$ Callard ${ }^{1}$}

'Physicians for a Smoke-Free Canada, Ottawa, ON, Canada. E-mail: ncollishaw@smoke-free.ca

Background and challenges to implementation: Compared to the size of the problem and the public health work to be done, the FCTC remains woefully underfunded. Past attempts to raise funds, whether through assessed contributions, voluntary contributions, Overseas Development Assistance or other means have not generated funds commensurate with the size of the problem.

Intervention or response: The FCTC contains mechanisms for establishing subisidiary bodies. One such body could be established to receive funds from a new levy on tobacco revenue. For most FCTC member states this levy would be on money about to leave the country, destined for the head offices of multinational tobacco companies. Member states could agree to send a portion of the money so raised to WHO for global tobacco control programming. The rest could be used for national and regional tobacco control work.

Results and lessons learnt: A 5\% levy, administered globally, would yield about USD one billion per year. If USD 200 million were designated for global tobacco control, Member States would collectively still have USD 800 million to spend on national and regional tobacco control programming.

Conclusions and key recommendations: At the next meeting of the Conference of the Parties, FCTC Member States should agree on a new globally coordinated tobacco levy, to be imposed by each Member State. At least USD 200 million should be designated for global tobacco control programming, to be administered by WHO, with the remainder of the proceeds from the levy to be spent on national and regional tobacco control programming.

Tob. Induc. Dis. 2018;16(Suppl 1):A106

DOI:10.18332/tid/84159

\section{2:45-14:00}

EP-144-3 Surcharge tax for tobacco and health promotion: measure for sustainable development MM Cho', B Ritthiphakdee ${ }^{2}$

'Southeast Asia Tobacco Control Alliance, Sustainable Financing, Bangkok, Thailand, ${ }^{2}$ Southeast Asia Tobacco Control Alliance, Bangkok, Thailand. E-mail: maychodr@gmail.com

Background and challenges to implementation: Funding for public health is a major problem in many countries especially in low income countries where the burden of disease is huge. In developing countries, while infectious diseases are still a problem, non-communicable diseases are soaring. With low health care budget especially on health promotion, to achieve the sustainable development goals especially in the area of NCDs are a long and winding road. Since the nature of health promotion is beyond the health care scope and needs multi-sectoral collaboration, it is essential to have a new financial mechanism which is sustainable for innovative health promotion projects.
Intervention or response: Among all the risk factors, tobacco use contributes to all four major NCDs. Therefore, taxing harmful product such as tobacco for public health is a sound measure which is recommended by the Article 5 general obligation and the article 26 of the Framework Convention on Tobacco Control (FCTC). More than 40 countries used earmarked tobacco tax for health globally. Experts are now discussing on earmarking tobacco tax for development.

Results and lessons learnt: In many countries health promotion projects were not sustainable due to lack of funding. For this reason, countries need to find local and sustainable financing mechanisms. Surcharge tax is recommended for countries because the revenue is predictable, sustainable and most importantly, it is paid by the industry causing the NCDs. Therefore, it is a winwin for both revenue and public health. In ASEAN, Singapore, Thailand, Malaysia, Vietnam and

Indonesia has local funding mechanism for tobacco control or health promotion. Different countries have different funding mechanisms.

Conclusions and key recommendations: Southeast Asia Tobacco Control Alliance (SEATCA) is working with ASEAN countries on tobacco taxation policy and mechanisms to earmark or surcharge tobacco taxes for tobacco control or health promotion. Experiences and lesson learned from countries will be discussed in this paper.

Tob. Induc. Dis. 2018;16(Suppl 1):A107

DOI:10.18332/tid/83746

\subsection{Pricing, Taxation and countering illicit trade: Innovations and reform strategies}

\section{4:30-16:00 \\ F0-321-3 Raising tobacco taxes, saving lives: evidence from Vietnam \\ NT Duyen ${ }^{1}$, HV Minh'}

'Hanoi University of Public Health, Center for Population Health Sciences, Hanoi, Viet Nam. E-mail: ntd@ huph.edu.vn Background: Regardless of good progress on implementing MPOWER package, Vietnam is still struggling with Raising tobacco tax intervention. Total taxes on tobacco products only account for $41.6 \%$ of retail price (WHO's recommendation rate is $65-85 \%$ ). The study aims to assess health impacts of different scenarios of tobacco taxes by the reduction in tobacco consumption and smoking attributable deaths.

Methods: We developed a simple static model based on previous works by Jha Prabhat et al. 2010 with several improvements. The scenarios on raising cigarette taxes were aligned with Vietnam Tobacco Tax Simulation Model developed by WHO \& Vietnam MOF. Each scenario calculated the reduction in initiation and current consumption and then estimating smoking attributable deaths averted and health cost saved based on existed evidence in low and middle income countries. Four smoking groups including current youth smokers, future youth smokers, current adult smokers and future adult smokers were evaluated separately.

Results: Four scenarios of raising tobacco taxes would prevent 278,380 - 289,268 premature deaths, equivalent to the mortality cost of US\$ 2,528 - 2,636 million. Estimated 4.5\% of smoking attributable deaths would be averted if the smoking mortality and quitting proportion are at $30 \%$. In the extreme scenario, raising total taxes by $116 \%$ comparing with figures of 2016 could lead to a tax share at $65 \%$ of retail price. This extreme scenario 
would prevent over 919,000 premature deaths and save up to 8,378 million US $\$ .14 .7 \%$ of smoking attributable deaths in total population would be avoided while this would made up to $52.38 \%$ among future youth smokers.

Conclusions: Raising cigarette tax and prices in Vietnam could lead to considerable reduction in deadly burden of tobacco use, further result in saving huge amount of money for society. Therefore, Vietnam should pay more attention on the implementation of tax and price policies as recommended by WHO.

Tob. Induc. Dis. 2018;16(Suppl 1):A108

DOI:10.18332/tid/83769

\section{4:30-16:00}

F0-322-3 Associations of cigarette price differentials with infant mortality in 23 European countries

F Filippidis', A Laverty ${ }^{1}$, T Hone ${ }^{1}$, J Been ${ }^{2,3}$, C Millett ${ }^{1}$

${ }^{1}$ Imperial College London, Public Health Policy Evaluation Unit, School of Public Health, London, United Kingdom, ${ }^{2}$ Erasmus University Medical Centre - Sophia Children's Hospital, Division of Neonatology, Department of Paediatrics and Department of Obstetrics \& Gynaecology, Rotterdam, Netherlands, ${ }^{3}$ The University of Edinburgh, Centre for Medical Informatics, Usher Institute of Population Health Sciences and Informatics, Edinburgh, United Kingdom. E-mail: f.filippidis@imperial.ac.uk

Background: Increasing cigarette prices has been associated with improved perinatal and child health outcomes. Transnational tobacco companies have adopted pricing strategies which maintain the availability of cheap cigarettes. We aimed to assess associations between median cigarette prices, cigarette price differentials and infant mortality in Europe.

Methods: We conducted a longitudinal ecological study of 276 regions in 23 European countries from 2004 to 2014. We obtained median cigarette prices and the differential between these and minimum cigarette prices from Euromonitor International. We calculated pricing differentials between minimum and median cigarette price as proportions of the median price. Prices were adjusted for inflation. Associations of median prices and price differentials with annual infant mortality rates were assessed using linear fixed-effect panel regression models adjusted for smoke-free policies; Gross Domestic Product; unemployment rate; education; maternal age; and underlining temporal trends. The analysis was conducted at a regional level.

Results: $A € 1$ per pack increase in the median cigarette price was associated with a decline of -0.23 infant deaths per 1,000 live births in the same year (95\% Confidence Interval [CI]: -0.37 to -0.09$)$ and -0.16 per 1,000 live births the following year (95\% CI: -0.30 to -0.03 ). An increase of $10 \%$ in the price differential between median and minimum priced cigarettes was associated with an increase of 0.07 infant deaths per 1,000 live births (95\% CI: 0.01 to 0.13 ) the following year. Cigarette price increases across 23 European countries between 2004 and 2014 were associated with 9,208 (95\% CI: 8,601 to 9,814) fewer infant deaths; an estimated 3,195 (95\% CI: 3,017 to 3,372 ) infant deaths could have been avoided had there been no differential between median and the minimum priced cigarettes during this period.

Conclusions: Combined with other evidence this research suggests that legislators should implement tobacco tax and price control measures which eliminate budget cigarettes.

Tob. Induc. Dis. 2018;16(Suppl 1):A109

DOI:10.18332/tid/83850
14:30-16:00

F0-323-3 Tobacco Tax Index 2017: assessing implementation of WHO FCTC Article 6 in ASEAN countries

S Ratanachena', U Dorotheo ${ }^{2}$

'Southeast Asia Tobacco Control Alliance, Tobacco Tax Program, Bangkok, Thailand, ${ }^{2}$ Southeast Asia Tobacco Control Alliance, Bangkok, Thailand. E-mail: sophapan@seatca.org

Background and challenges to implementation: Because tobacco is a threat to sustainable social development, tobacco tax policy will play a significant role in achieving the 2030 Sustainable Development Goals (SDGs) to end poverty, protect the planet, and ensure prosperity for all. Precisely because of the effectiveness of tobacco taxes in discouraging consumption, tobacco companies continue to lobby against effective taxation to protect their profits. Despite the known public health and fiscal benefits of taxing tobacco, many governments have not maximized their use of tobacco taxes. As a result, tobacco consumption rates remain high in Southeast Asia resulting in more tobacco-related diseases and increased healthcare costs required to treat them.

Intervention or response: The WHO FCTC Article 6 Guidelines provide clear guidance to governments on tobacco tax policies. SEATCA developed a tobacco tax policy[1] survey tool based on the WHO FCTC Article 6 Guidelines to assess current tobacco tax measures and provide ASEAN governments with recommendations for strengthening their tobacco tax policies. An initial assessment was done in 2015 and repeated in 2017.[1] See more detail at: http://seatca.org/?p=6281

Results and lessons learnt: Compared with 2015, the ASEAN region, as a whole, has seen limited progress over the past two years. Most ASEAN countries still do not have long-term tobacco tax policies with consistent verifiable fiscal and public health targets. Key obstacles limiting effectiveness include tobacco tax structures, weak tax policy, as well as the tobacco industry's interference in and obstruction of tax policy formulation and administration.

Conclusions and key recommendations: Tobacco Tax Index 2017 of SEATCA is the first civil society's report to urge all ASEAN government to effectively implement the WHO FCTC Article 6. It documented the best practices, challenges and lessons learned among ASEAN countries. Therefore, the tobacco tax policymakers are assisted with practical information to achieve in the WHO FCTC Article 6 implementation which contributing to the SDGs achievement as a whole.

Tob. Induc. Dis. 2018;16(Suppl 1):A110

DOI:10.18332/tid/83776

14:30-16:00

F0-324-3 The price sensitivity of choice and consumption of cigarette by brand categories in Bangladesh: findings from ITC Bangladesh Surveys AG Hussain', N Nargis ${ }^{2}$

'Dhaka University, Economics Department, Dhaka, Bangladesh, ${ }^{2}$ American Cancer Society, Economic \&t Health Policy Research, Washington, DC, United States of America.E-mail: ghussain@ econdu.ac.bd

Background: The cigarette market in Bangladesh is segmented into four brand categories-low, medium, high and premium. These brands are differentiated by flavor, mildness, filter, and packaging. Smokers choose brands based on their preference for these product characteristics that closely interact with the affordability of different cigarette brands. Despite wide variation in price and product 
characteristics, brands can be close substitutes due to their addictive nicotine content, a feature that can trigger switching of brands in response to change in market price and income of smokers.

Methods: We use nationally representative cohort data on smokers from four waves of International Tobacco Control (ITC) Policy Evaluation Survey conducted over 2009-2015. First, we specify a multinomial logit model to identify the determinants of brand choice. Second, we specify four conditional demand functions of daily cigarette consumption for four brand categories and estimate own- and cross-price elasticities and income elasticity of cigarettes in each category. Both models are estimated using pooled ordinary least squares and fixed effects methods.

Results: The results of estimation show that increase in own price decreases the probability of choosing a brand in a category. Price increase of other brands increases the probability of choosing in that brand category suggesting substitutability. The own-price elasticity is negative and statistically significant for medium and high brands and statistically insignificant for low and premium brands. The cross-price elasticities are positive, suggesting that brands are substitutes and that smokers have incentive to switch down to cheaper alternatives in the event of price increases, instead of quitting or cutting down consumption.

Conclusions: Increasing cigarette excise tax to induce price increases for cigarettes can prove ineffective in achieving the objective of reducing smoking under price and product differentiation, which need to be minimized through uniform specific excise system that tends to reduce the variability of price and product regulation.

Tob. Induc. Dis. 2018;16(Suppl 1):A111

DOI:10.18332/tid/84082

\section{4:30-16:00}

F0-325-3 Tobacco taxes: a win-win strategy for public health and government revenue in Kenya E Wanyonyi ${ }^{1}$, M Carmona ${ }^{2}$

${ }^{1}$ International Institute for Legislative Affairs, Nairobi, Kenya, ${ }^{2}$ Campaign for Tobacco Free Kids (CTFK), Washington, DC, United States of America. E-mail: ewanyonyi@ilakenya.org

Background and challenges to implementation: Kenya's excise tax system has changed over the years from specific taxes to advalorem, hybrid, tiered system and eventually a uniform specific rate for all tobacco products. Prior to 2015 the real price of cigarettes was on the decrease, increasing their affordability; cigarette tax revenues was decreasing even as tax rates and consumption was increasing and the excise tax share in cigarette price was low, compared to the WHO recommended $70 \%$. This impacted negatively on public health and fiscal goals of tobacco taxation in Kenya.

Intervention or response: From 2010, Kenyan TC advocates implemented a campaign to advocate for implementation of effective tobacco tax systems in Kenya. The campaign comprised of five key components; advocacy leadership by a national civil society organization; buy- in and commitment of the national treasury and the revenue authority; effective engagement and collaboration of key local and international partners who provided research, technical, financial, media and other support; effective countering of tobacco industry tax arguments and strategies with research and action; and strategic use of media to engage the public around tax issues and gain public support.

Results and lessons learnt: The main outcome of the this campaign was the enactment of the Excise Duty Act 2015 which simplified the structure to uniform specific and increased the rate from Ksh. 1200 per mille to KShs 2,500 per mille. It was estimated that this would result in a 3.4\% decline in consumption and $0.3 \%$ decline in adult smoking prevalence.

The success is attributed to strong macro- economic expertise in the local advocates, a good understanding of processes and role of different actors, local data for evidence based advocacy and monitoring, exposing and countering industry arguments.

Conclusions and key recommendations: An effective tobacco tax campaign is anchored on engaging non- traditional TC partners like the ministry of finance, revenue authorities and finance committees of national assemblies.

Tob. Induc. Dis. 2018;16(Suppl 1):A112 DOI:10.18332/tid/84443

\section{$16: 15-17: 45$}

F0-342-3 Exposing industry-driven retail incentive programs that undermine tobacco tax policies

H Rathjen ${ }^{1}$, F Doucas ${ }^{1}$

${ }^{1}$ Coalition Québécoise pour le Contrôle du Tabac, Montreal, QC, Canada. E-mail: hrathjen@cqct.qc.ca

Background and challenges to implementation: As of 2008, Canadian cigarette manufacturers established retailer incentive programs. These programs were largely unknown to the public and, despite numerous complaints from independent stores, retailer associations remained silent on this issue. In accordance with recommendations of the FCTC, the Quebec Coalition for Tobacco Control (CQCT) sought to ban them.

Intervention or response: The CQCT's first and most important step was to build a dialogue with a small number of retailers. Thanks to their testimony and the corporate documents they provided, the CQCT was able to determine how retail incentive programs undermined Quebec's tobacco taxation and promotion policies. Typically, programs force retailers to respect various pricing conditions, including price ceilings on already low priced cigarette brands, and meet sale volume targets. In exchange, retailers obtain substantial rebates on the wholesale price of these or other brands. In addition to putting pressure on retailers to sell more products than they would otherwise, these programs artificially lower the market price of various cigarette brands.

Results and lessons learnt: Following the CQCT efforts exposing to the media and to politicians the inner workings of such programs, the Quebec government decided to act. Since November 26th 2016 , the province formally prohibits manufacturers "from offering rebates, gratuities or any other form of benefit related to the sale or the retail price of tobacco products to operators of retail outlets, including their employees".

Conclusions and key recommendations: Retail incentive programs are complex and the tobacco industry tries to keep them secret. Health advocates, researchers and officials need to better understand how they work - especially the way they undermine tobacco tax policies - in order to ultimately ban them. A ban on industry retail programs will help protect vulnerable price-sensitive populations (namely youth and the economically disadvantaged) from industry manipulations that artificially lower cigarette market prices and drive retailers to push sales.

Tob. Induc. Dis. 2018;16(Suppl 1):A113

DOI:10.18332/tid/84664

\section{$16: 15-17: 45$}

F0-343-3 Understanding tobacco industry pricing strategy: evidence from China's 2015 Tax Reform R Zheng ${ }^{1}$, M Goodchild ${ }^{2}$

${ }^{1}$ University of International Business and Economics, WHO 
Collaborating Center on Tobacco and Economics, Beijing, China, ${ }^{2}$ World Health Organization, Prevention of Noncommunicable Diseases, Geneva, Switzerland. E-mail: zr3377@gmail.com

Background: Tax increases are the most effective means of reducing tobacco use, but their effectiveness also depends on tobacco industry pricing strategies - which determine the extent to which taxes are passed-onto consumers. Common strategies include the over-shifting or absorption (under-shifting) of taxes. China raised cigarette taxes in 2015, providing a unique opportunity to examine the pricing strategy of China's State Tobacco Monopoly Administration (STMA).

Methods: WHO TaXSiM China model was used to calculate the magnitude of the tax pass-through for each cigarette Class at the wholesaler, retailer, and consumer levels after China 2015 tobacco tax increase. The net effect across STMA's entire market was assessed using sales-weighted averages.

Results: China's tax increase was partly absorbed in the profit margins of wholesalers across all classes. However, on a weighted average basis, it was fully passed-on at the wholesale level. The tax increase was over-shifted at the retail level, with retail margins increasing except for Class III. The impact on consumers varied by Class, but with the net effect being an over-shifting of tax. Tax was over-shifted for Class V (the cheapest category) and under-shifted for Class III (the most popular and mid-price category).

Conclusions: The STMA employed a pricing strategy that was tailored to different Classes with the aim of managing the shape of the market in the manner consistent with its wider "key brand" strategy to promote mid and premium-priced brands.

Tob. Induc. Dis. 2018;16(Suppl 1):A114

DOI:10.18332/tid/83837

\section{$16: 15-17: 45$}

F0-344-3 Tobacco industry strategies to keep tobacco prices low: evidence from industry data A Gilm, 'University of Bath, School of Management, Bath, United Kingdom, ${ }^{3}$ King's College London, Addictions, Institute of Psychiatry, Psychology \& Neuroscience, London, United Kingdom.E-mail: a.gilmore@bath.ac.uk

Background: The tobacco industry (TI) can undermine the public health gains realised from tobacco taxation through its pricing strategies. This study aims to examine contemporary TI pricing strategies in the UK and the implications for tobacco tax policy.

Methods: Review of commercial and TI literature, and analysis of 2009 to 2015 Nielsen Scantrak electronic point of sale systems (EPOS) data on tobacco sales and price data. The study was set in a high income country with comprehensive tobacco control policies and high tobacco taxes (UK). The main outcome measures were: tobacco price segmentation; monthly prices, sales volumes of and net revenue from roll your own and factory made (RYO and FM) cigarettes by price segment; use of price-marking; and pack sizes. Results: The literature review and sales data concurred that both RYO and FM cigarettes were segmented by price into premium, mid-price and value segments, with an additional sub-value FM segment introduced in 2012. Despite regular tax increases, average real prices for RYO value and FM sub-value segments remained steady from 2013 whilst their volumes grew. The TI maintains low prices through reducing the number of cigarettes per pack, price-marking (forcing retailers to sell cheaper products at low prices) and absorbing tax increases. Each year, at the point the budget is implemented, the TI drops its revenue by up to 18 pence per pack, absorbing the tax increases (undershifting). Later in the year it gradually passes the tax increase onto consumers, effectively smoothing the price change consumers face across the year. Undershifting of taxes is most marked for the cheapest products.

Conclusions: The TI employs a variety of strategies to keep tobacco cheap and minimise the impact of tobacco tax increases. The implementation of standardised packaging can prevent small pack sizes and price-marking but further changes in tax policy are needed to minimise undershifting.

\section{Tob. Induc. Dis. 2018;16(Suppl 1):A115 DOI:10.18332/tid/84000}

\section{$16: 15-17: 45$}

F0-345-3 An evaluation of the cigarette tax increase impact on illicit trade in 5 Colombian cities N Maldonado ${ }^{1}$, JM Gallego², B Llorente ${ }^{3}$

${ }^{1}$ Fundación Anaás, Bogotá, Colombia, ${ }^{2}$ Universidad del Rosario, Bogotá, Colombia, ${ }^{3}$ Fundación Anáas, Bogota, Colombia. E-mail: juan.gallego@urosario.edu.co

Background: Few studies in middle income countries assess the effect of a sharp tax increase on observed consumption of illicit cigarettes. The 2016 tax reform in Colombia resulted in a $100 \%$ increase of the excise tariff for 2017. This study evaluates the changes in penetration of smuggled products associated with the tax increase nine months after it became effective. The policy of interest is the excise tax increase effective in January 2017 (from 23 to 48 cents per 20 cigarette pack), the first jump in a two-year adjustment period.

Methods: Non-participant group is defined as smokers in 2016, since the tax measure affects all smokers. Evaluation will use the results of a smoker survey in five Colombian cities in 2016 and a second one planned for September 2017, specifically designed to measure penetration of illicit trade. A matching technique is used considering three dimensions: spatial, age and gender.

This provides an adequate comparison. By comparing individuals in the same zone in each city of the same gender and the same age it can be established to what extent the price increases (attributable to new tax levels) are associated with consumption patterns.

Results: The 2016 survey estimated a $3,5 \%$ penetration of illicit cigarettes, with significant differences across cities. Price behaviour indicates that tax has not been fully transfered to consumers yet, with a $22 \%$ annual hike (first semester average) but it is getting closer to the expected result.

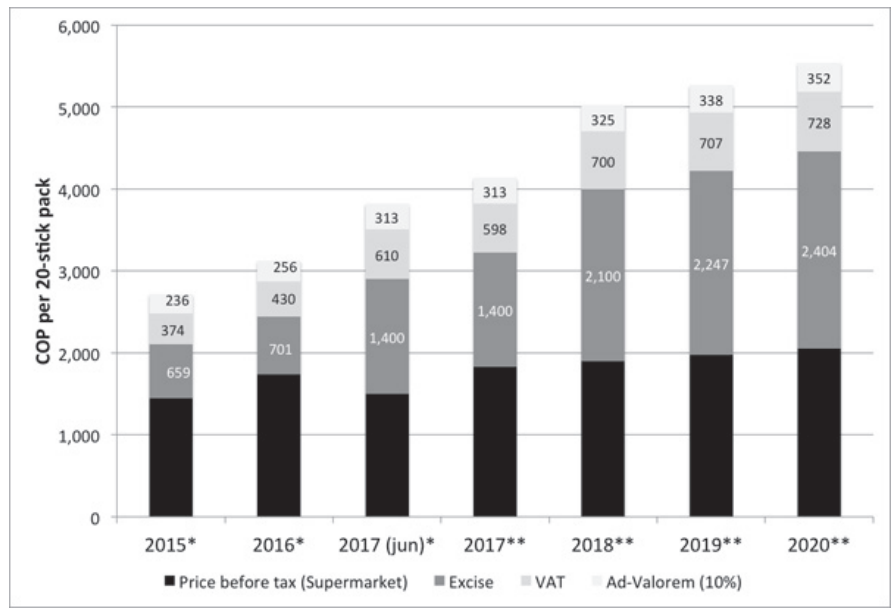

[Changes in price structure after tax reform]

Conclusions: First wave results suggest that tax levels are not the only driver of illicit trade, since cigarette taxes are the same for all 
the country. Cities closer to the border and with weaker institutional conditions are more prone to have consumers of smuggled cigarettes. This points at the need to dedicate efforts to target tax administration efficiency to protect revenues in those areas.

Tob. Induc. Dis. 2018;16(Suppl 1):A116

DOI:10.18332/tid/84330

\section{$16: 15-17: 45$}

F0-346-3 Consumption of illicit cigarettes in India: findings from a retail survey

RM John', H Ross ${ }^{2}$

${ }^{1}$ Health Policy Analyst, Ernakulam, India, ${ }^{2}$ University of Cape Town, Southern Africa Labour and Development Research Unit, Cape Town, South Africa. E-mail: rmjohn@gmail.com

Background: Tobacco industry estimates the share of illegal cigarettes in India at more than $20 \%$ of the total cigarette market and claims it has doubled over the past 10 years. However, there is a paucity of independent and scientifically verifiable estimates of illicit trade in cigarettes in India. This study, for the first time, intends to fill that void.

Methods: The study employed a modified approach to cigarette pack analysis. Empty cigarette packs from a single day's sale were collected at close of business directly from retail cigarette vendors in four metro cities and four smaller towns covering the length and breadth of India. Ten areas based on pin codes were randomly selected from each city/town and all shops selling cigarettes within one kilometer were surveyed. The study considered a cigarette pack to be illicit if it had at least one of the following attributes: (a) has a duty free sign; (b) has no graphic health warnings; (c) has no textual health warnings; or (d) has no mention of "price inclusive of all taxes" or similar text.

Results: A total of 11,063 empty cigarette packs were collected from 1,727 retailers and $2.73 \%$ of these cigarette packs were classified as illicit. The estimates varied substantially across locations with the highest prevalence of illicit packs being in Aizawl (36\%). The share of illicit was found to be much higher (13.77\%) among the cheapest cigarette brands. Illicit cigarettes are primarily distributed via more structured general stores compared to relatively informal tea/pan shops.

Conclusions: Our estimate of the illicit cigarette market share of $2.73 \%$ casts serious doubt on the tobacco industry estimates. It appears that any concerns of illicit cigarette trade in India should not hinder the government from raising tobacco taxes.

Tob. Induc. Dis. 2018;16(Suppl 1):A117

DOI:10.18332/tid/83789

\section{$16: 15-17: 45$}

F0-347-3 Converging combustible and electronic cigarette prices: evidence for Nielsen retail scanner data in the United States from 2011 to 2015 A Liber ${ }^{1,2}$, M Stoklosa ${ }^{1}$, J Drope ${ }^{1}$, J Huang ${ }^{3}$, F Chaloupka ${ }^{4}$ ${ }^{1}$ American Cancer Society, Intramural Research, Atlanta, GA, United States of America, ${ }^{2}$ University of Michigan, Health Management and Policy, Ann Arbor, MI, United States of America, ${ }^{3}$ Georgia State University, School of Public Health, Atlanta, GA, United States of America, ${ }^{4}$ University of Illinois at Chicago, Chicago, IL, United States of America. E-mail: alex.liber@cancer.org

Background: Very little is known about the prices of new noncombustible tobacco products such as electronic cigarettes. Moreover, because of this paucity of data, it is difficult to evaluate the cost for individuals to use e-cigarettes and similar products relative to combustible tobacco products. As governments consider economic policies such as taxation to address tobacco use, an understanding of the changing landscape of product prices will be crucial.

Methods: This study utilizes Nielsen retail scanner data from 53 designated market areas in the United States from the period of 2011 through 2015 to examine the trends in tobacco product prices over time and in relation to one another. Average prices for combustible cigarettes, rechargeable e-cigarettes, and e-liquid were calculated for each market and a payback period to recoup the investment in an e-cigarette was calculated in a similar manner to Liber et al., 2016 (Tobacco Control). Constant growth regression was employed to determine rates of change over time. Results: We find that prices for combustible cigarettes and e-cigarette refills increased during the period of 2011-2015, while prices for disposable and rechargeable e-cigarettes decreased. However, overall, combustible cigarette prices remained lower than disposable e-cigarette prices. The period to recoup the purchase of a rechargeable e-cigarette for a pack-a-day smoker who has switched from combustible cigarettes decreased from 12 to 2 days.

$\begin{array}{lcccccc} & 2011 & 2012 & 2013 & 2011 & 2015 & \text { A.PC } \\ \begin{array}{l}\text { Combustible } \\ \text { Cigarettes }\end{array} & 5.42 & 5.47 & 5.55 & 5.70 & 5.82 & 1.90 \% \\ \begin{array}{l}\text { E-cigarette } \\ \text { Starter Kit }\end{array} & 31.37 & 27.58 & 21.89 & 13.70 & 12.29 & -23.37 \% \\ \begin{array}{l}\text { E-cigarette } \\ \text { Refill }\end{array} & 2.16 & 3.03 & 3.14 & 3.09 & 3.01 & 6.70 \% \\ \begin{array}{l}\text { Payback Period } \\ \text { Paybo }\end{array} & 12.02 & 7.29 & 5.20 & 2.59 & 2.15 & -37.60 \%\end{array}$

[Mean Product Prices in 53 Nielsen DMAs, 2015 USD]

Conclusions: In the United States, the cost of e-cigarettes quickly approached parity with combustible cigarettes. Future observation of the effects of relative prices on consumption patterns will guide the development of enlightened tax and price policies around the world.

Tob. Induc. Dis. 2018;16(Suppl 1):A118

DOI: $10.18332 /$ tid/83902

$16: 15-17: 45$

F0-348-3 The demand for cigarettes: new evidence from South Africa

A Mukong

'University of Cape Town, Cape Town, South Africa. E-mail: mukalf83@gmail.com

Background: There is increasing body of evidence that tobacco excess tax is the most effective policy for reducing cigarette smoking. The effectiveness of taxes, however, are contingent on how the increase impact the retail price. As to whether the burden of the tax increase is equitable, depends on the responsiveness of different socioeconomic groups to price change. This paper provides an extensive analysis of the price and income elasticity of demand for cigarette. Specifically, the identify how individuals from different socioeconomic status response changes in cigarette retail price.

Methods: A unique large sample of repeated cross-sectional data from South Africa 2008 - 2014 allows reduced-form cigarette demand equations to be estimated by socioeconomic status, gender, race and age group. This data equally allows us to control smoking addiction and other individual characteristics. We employ the pooled ordinary least square and the random effect technique to estimate the price and income elasticity of demand for cigarette.

Results: We find a negative cigarette price and a positive income 
effect on smoking intensity. The price effects are stronger for low income individuals, women, Black Africans and young and old individuals. The income effects are higher for high income individuals, men, whites, and older adults.

Conclusions: The findings highlight the importance of this policy space. Tobacco price increases appear to reduce cigarette consumption more among the vulnerable population and in part may be a policy tool for reducing inequality health.

Tob. Induc. Dis. 2018;16(Suppl 1):A119

DOI:10.18332/tid/83930

\section{$12: 30-14: 00$}

PS-581-3 Taxation policy impact on tobacco sales in Argentina

J Konfino ${ }^{1}$

${ }^{1}$ Centro de Estudios de Estado y Sociedad (CEDES), Quilmes, Argentina.E-mail:jkonfino@gmail.com

Background: In the last 10 years the increase in purchasing power in Argentina without a strong tobacco taxation policy led to an increase in cigarettes affordability. But in April 2016 a presidential decree increased from $60 \%$ to $75 \%$ the tobacco internal tax (excise tax) that generated an increase in cigarettes prices by $50 \%$. The objective of this study was to describe the evolution of cigarettes sales during the last 10 years, particularly in 2016 after the price increase.

Methods: Official data from the Ministry of Agriculture on cigarettes sales was analyzed. Trends from 2006 to 2017 were considered for the analysis.

Results: In 20061,993 million of 20 cigarettes packs were sold reaching 2,031 million in 2015. During that period a peak of 2,188 million was reported in 2011 (when national tobacco control law was enacted). Since then, reductions of $0.7-2.4 \%$ were observed annually. In 2016, 1,794 million of 20 cigarettes packs were sold, representing a reduction of $11.7 \%$ compared with 2015. In 2016, an average of 149.5 million packs was sold monthly compare with a monthly average of 169 million in 2015. May 2016 (after the increase) was the month with fewer sales with 98.6 million.

Conclusions: Cigarette sales were affected by the tobacco tax increase in Argentina, showing responsiveness to price variations. The enactment of a taxation national law could add sustainability to this successful strategy.

Tob. Induc. Dis. 2018;16(Suppl 1):A120

DOI:10.18332/tid/83758

\section{2:30-14:00}

PS-582-3 Goods and service tax reform and estimated impact on tobacco products in India

RM John', E Dauchy², M Goodchild ${ }^{3}$

${ }^{1}$ Health Policy Analyst, Ernakulam, India, ${ }^{2}$ Campaign for Tobacco Free Kids, Washington, WA, United States of America, ${ }^{3}$ World Health Organization, Genva, Switzerland. E-mail: rmjohn@ gmail.com

Background: The indirect tax system in India is undergoing a major overhaul with a national Goods \& Services Tax (GST) expected to be rolled out in 2017. This paper analyzes the potential impact of the new tax rates on tobacco proposed under GST on consumption and tax revenues for different tobacco products in India.

Methods: We use the most recently available data on prices, tax rates, and tax revenue for the most consumed tobacco products in India, and forecast their tax burden in the fiscal year directly prior to the GST reform in 2017-18 using tobacco product specific inflation rates. From this benchmark scenario, we simulate the impact of the proposed tobacco tax reform options under GST on tobacco consumption, tax revenue, and affordability using own-price elasticities of demand, specific to different tobacco products and income tertiles.

Results: We find that the proposed GST rate of $28 \%$ plus cess would increase cigarette consumption by $1.7 \%$ and increase tax revenue by about 0.4 percentage. On the other hand, a $28 \%$ GST rate applied on bidis would result in a $1.5 \%$ reduction in consumption while increasing tax revenue by $33.14 \%$. For smokeless tobacco products, the proposed increase of $28 \%$ plus cess would result in a substantial increase in consumption by $7 \%$ while simultaneously decreasing revenue by $22 \%$.

Conclusions: The impact of the current GST proposals to reform the tobacco tax system is mixed, as it would decrease in the consumption of bidis but increase the consumption of both cigarettes and smokeless tobacco products. To more significantly reduce tobacco use and raise extra revenue, the government of India should continue to impose the existing excise in addition to the proposed GST.

Tob. Induc. Dis. 2018;16(Suppl 1):A121

DOI:10.18332/tid/83781

\section{2:30-14:00}

PS-583-3 Implementing a sub-national strategic framework to reduce the illicit tobacco market to support national strategies

A Rutter $^{1}$, C Taylor ${ }^{1}$, A Lloyd ${ }^{1}$, R Ferry $^{1}$

${ }^{1}$ Fresh, Durham, United Kingdom. E-mail: catherine.taylor@ freshne.com

Background and challenges to implementation: North East (NE) England has had the fastest drops in smoking prevalence in the country. The UK's illicit tobacco market has almost halved following successful national strategies but still remains a problem in some communities because price and availability increases accessibility for children and reduces smokers' motivation to quit. In 2009, 16\% of the NE tobacco market was illicit. Fresh, the NE's comprehensive tobacco control programme, has implemented an innovative and successful strategic framework to reduce the supply and demand. Intervention or response: Since 2007, Fresh has worked with health and regulatory partners to reduce the illicit tobacco market. Several waves of quantitative and qualitative research with the public and stakeholders have informed the development of a multi-component strategic framework based on international principles which enhances national strategies and can be implemented locally or sub-nationally. Its eight objectives are to develop partnerships, engage frontline workers, generate intelligence, deliver enforcement, deliver marketing and communications, work with businesses, protect policies from the tobacco industry and assess progress.

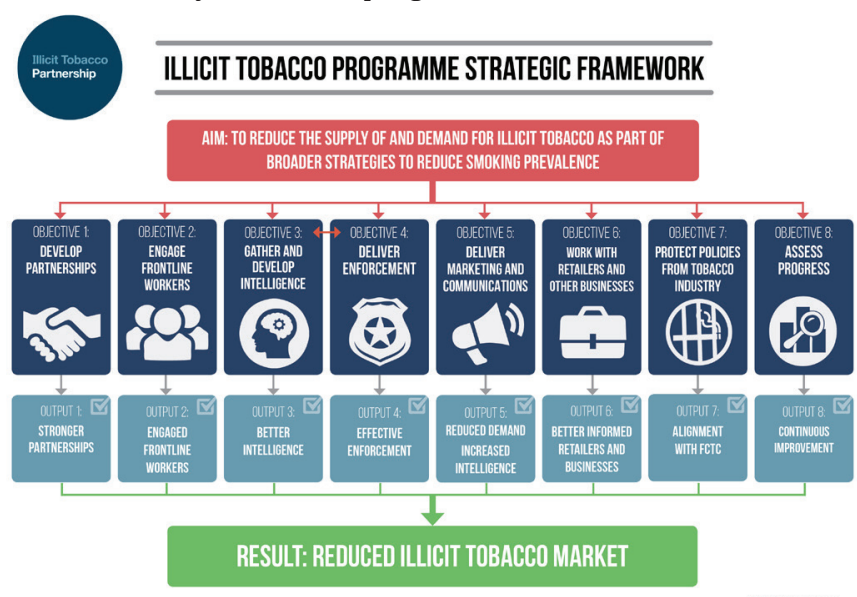

[Illicit Tobacco Programme Strategic Framework] 
Results and lessons learnt: The illicit tobacco market in the NE has declined from $16 \%$ in 2009 to $12 \%$ in 2017 . The proportion of smokers who buy illicit tobacco has reduced from $24 \%$ to $18 \%$ over the same timeframe. In-depth qualitative research has supported the development of effective, evidence-based demand reduction campaigns which have led to increases in intelligence and reductions in comfort levels with the illicit tobacco trade. Regulatory partners are prioritising illicit tobacco as part of their broader enforcement policies.

Conclusions and key recommendations: The success of this strategic framework demonstrates that activity can be coordinated locally or sub-nationally to support national strategies to reduce the illicit tobacco market. Activity should always be placed within the broader context of 'all tobacco kills' and should contain measures to reduce both supply and demand. Useful resources for other programmes and settings can be found at www.illicit-tobacco.co.uk.

Tob. Induc. Dis. 2018;16(Suppl 1):A122

DOI:10.18332/tid/83784

\section{$12: 30-14: 00$}

\section{PS-584-3 Ban of single stick cigarettes}

SD Peiris

'Thirasara Foundation, Nugegoda, Sri Lanka. E-mail: sujeewadilangana@yahoo.com

Background and challenges to implementation: Sri Lanka implemented FCTC's Article 11 at 80\% from June 2015. Thirasara Foundation in collaboration with Jeewaka Foundation initiated a study in 2016 to monitor the compliance with the PHW printed on tobacco packets in 18 out of the 26 health districts of the country.

The study established that the conformity with the law is 100 percent, but informal evidence suggests that few purchase cigarettes as packets. Self-evidently PHWs cannot be significantly impact in reduction of smoking. Why? Because the majority purchase cigarettes in singles.

Therefore banning single cigarette sale should be a moderating part of the country's tobacco control policy.

Single stick sales facilitate smoking among non-affluent youth and beginning smoking. Retailers are more likely to sell single cigarettes to minors than to adults, thus probably initiation of smoking.

Intervention or response: The first step was to gather information related to sale of single stick cigarettes locally by observation and internationally by a literature survey. Based on the information leaflets were prepared for propaganda purposes. The importance of banning single stick cigarette sales was communicated to policy makers including the President of the Republic of Sri Lanka and the Health Minister. (with a legal background) The documents for formally demanding the ban of single cigarettes and other tobacco demand reducing proposals were prepared and submitted to the influential GMOA for necessary action. The GMOA had a formal meeting with $\mathrm{HE}$, the President and the Health Minister for implementation of the proposals. Wide publicity was secured by the media, print, electronic and social.

Results and lessons learnt: The government, not only accepted the desirability of banning sale of single stick cigarettes, in order to promote reduction of tobacco use, but took practicable steps to implement the proposal.

Conclusions and key recommendations: Demand reduction policies for tobacco if clearly conceptualized and enthusiastically publicized can be made a reality.

Tob. Induc. Dis. 2018;16(Suppl 1):A123

DOI: $10.18332 /$ tid/83836

\section{2:30-14:00}

PS-585-3 Impact of increased tobacco tax on revenue and prices in Panama 2009 - 2016

VH Herrera Ballesteros ${ }^{1}, 2$ I Moreno' ${ }^{1}$, B Gómez'1, R Roa ${ }^{3}$

${ }^{1}$ Gorgas Memorial Institute for Studies of Health, Research and Evaluation of Sanitary Technology, Panama, Panama, ${ }^{2}$ University of Panama, Theory and Economic Development, Panama, Panama, ${ }^{3}$ Ministry of Health, Planification, Panama, Panama.E-mail: vherrera@gorgas.gob.pa

Background: To demonstrate the impact of the increase of the Selective Tax on the Consumption of Cigarettes and other tobacco products (ISC) in the tax collection and increases in the prices of cigarettes, after 2009.

Methods: The primary source of information is the database of the 2015 cigarette market survey conducted in the districts of Panama, Colón, David, Barú, San Miguelito and the indigenous districts of Guna Yala and Ngäbe-Buglé in July 2015. The fiscal collection data of the ISC of the Ministry of Economy and Finance were used as secondary sources and a linear trend analysis of the evolution of tax revenues derived from the ISC and linear regression of demand was made.

Results: The ISC increase by $100 \%$ increased collection by 2.3 times between 2009 and 2016, in addition to providing resources to the health sector. Having a USD floor of 1.50 per pack led the price dispersion to rise for both legal and illegal brands.

Conclusions: Anti-tobacco taxation policy has proven to be effective in increasing tax collection and prices for tobacco products in addition to helping to reduce the prevalence of tobacco consumption to $6.1 \%$ in smoked tobacco. The increase in taxes must be a continuous process to continue to maintain their effectiveness for what is proposed indexation.

Tob. Induc. Dis. 2018;16(Suppl 1):A124

DOI:10.18332/tid/83887

\section{2:30-14:00}

PS-586-3 Monitoring tobacco taxation in the Philippines at the TAP (Tobacco Action Patrol) of a finger

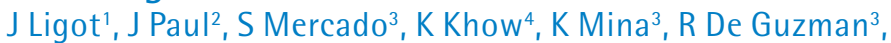
J-A Diosana ${ }^{5}$, M Aloria $^{5}$

${ }^{1}$ World Health Organization, Geneva, Switzerland, ${ }^{2}$ World Health Organization, Prevention of Noncommunicable Disease, Geneva, Switzerland, ${ }^{3}$ World Health Organization Regional Office for the Western Pacific, Division of NCDs and Health through the Lifecourse, Manila, Philippines, ${ }^{4}$ World Health Organization, Office of the Representative to the People's Republic of China, Beijing, China, ${ }^{5}$ Action for Economic Reform, Manila, Philippines. E-mail: ligotj@who.int

Background and challenges to implementation: In 2012, the Philippines passed the "sin tax law" restructuring its tobacco taxation scheme from a multi-tiered to a unitary system with annual increases in tobacco taxation planned from 2017 onwards. However, monitoring of implementation of the sin tax law was a challenge for government authorities due to limited resources and the country's geography (the Philippines is an archipelago of over 7000 islands).

In the Philippines, the population has a high penetration rate of mobile communication services with 115.8 mobile cellular subscriptions per 100 inhabitants. Leveraging the reach of mobile technology, a crowd-sourcing strategy was conceptualized jointly by $\mathrm{WHO}$, government and civil society to monitor tobacco products at point-of-sale. 
Intervention or response: A smart phone app was developed and youth leaders from all over the country were convened for a workshop on tobacco control and use of the app for price monitoring. Afterwards, these youth leaders trained and mobilized youth volunteers from their universities to monitor tobacco prices in the community. The app allowed users to input tobacco prices and other details (e.g. brand, variant, quantity, tax stamps) at point-of-sale. Data was then transmitted to a central server located at the Philippine Department of Finance.

Results and lessons learnt: Over a period of 1 month, over 6000 entries were submitted from all 17 regions, providing a snapshot national tobacco price and product survey. Data analysis provided market intelligence for government authorities and tobacco control advocates along with reports of violations to policies that prohibit tobacco sales within the perimeter of schools.

Conclusions and key recommendations: The pilot demonstrated the application of mobile communications technology and youth mobilization as strategies for strengthening monitoring of tobacco control initiatives whilst overcoming resource constraints.

Tob. Induc. Dis. 2018;16(Suppl 1):A125

DOI:10.18332/tid/83937

\section{$12: 30-14: 00$}

PS-587-3 Illicit cigarette packs found in urban retail locations

K Welding ${ }^{1}$, K Smith ${ }^{1}$, J Cohen

'Johns Hopkins Bloomberg School of Public Health, Institute for Global Tobacco Control, Department of Health, Behavior and Society, Baltimore, MD, United States of America. E-mail: kwelding@jhu.edu

Background: Understanding the illicit cigarette market is important for tobacco control. Tobacco companies have used illicit trade to introduce products to new markets and avoid taxation. The industry lobbies against tobacco control efforts by warning about lost tax revenue from increased illicit trade. This study investigates the change in the illicit cigarette prevalence in urban retailers between 2013 and 2015/2016 within nine countries (Bangladesh, Brazil, China, India, Indonesia, Philippines, Russia, Thailand, Vietnam) across four World Health Organization regions.

Methods: The Tobacco Pack Surveillance System (TPackSS) systematically collects unique cigarette packs sold in low- and middle-income countries with high tobacco use. In 2015-16, TPackSS returned to countries that changed their health warning regulations since a 2013 pack collection. In each country, one vendor in each of 12 low, middle, and high socioeconomic areas in 3 major cities (4 in India in 2016, 5 in China) was sampled and unique packs were purchased. The absence of country health warning labels was used to identify illicit packs.

Results: The 2013 sample of 2,034 packs found an illicit sample proportion that ranged from $71 \%$ in Bangladesh to $0 \%$ in Brazil and Indonesia. The 2015-16 sample of 2,356 packs again found a large illicit prevalence in Bangladesh and minimal illicit pack presence in Brazil's retail environment. A significant increase of 13 percentage points (pp) in the illicit prevalence was observed in Indonesia. A significant increase (11pp) was also found in China and significant decreases were found in Philippines (31pp) and Thailand (20pp). The proportion of illicit cigarettes found in the other five countries did not significantly change. We also report on the origin of the illicit packs.

Conclusions: Changes in the illicit prevalence of cigarette packs in legitimate urban retailers could be the result of changes in enforcement, brand availability, or consumer demand. Ongoing research is investigating potential causes.

Tob. Induc. Dis. 2018;16(Suppl 1):A126

DOI:10.18332/tid/83938

\section{$12: 30-14: 00$}

PS-588-3 Availability and price distribution of three popular brand variants

K Welding ${ }^{1}$, K Smith ${ }^{1}$, J Cohen ${ }^{1}$

'Johns Hopkins Bloomberg School of Public Health, Institute for Global Tobacco Control, Department of Health, Behavior and Society, Baltimore, MD, United States of America. E-mail: kwelding@jhu.edu

Background: Price is an important determinant of demand for tobacco products, with price increases being a highly effective tobacco control measure. This study investigates the availability and purchase prices of three brand variants within each of nine countries to better understand the distribution and pricing strategies of tobacco companies.

Methods: In 2015-16, three brand variants were systematically purchased in nine low- and middle-income countries (Bangladesh, Brazil, China, India, Indonesia, Philippines, Russia, Thailand, Vietnam). The brand variants included a popular national and international variant in each country, and Marlboro Reds in all countries. Brand variants were selected by combining information from Euromonitor, Global Adult Tobacco Surveys, and incountry partners. In each country, a brand variant purchase was attempted from one vendor in each of 12 low, middle, and high socioeconomic areas in 3 major cities (4 in India, 5 in China). Local prices were converted to US dollars.

Results: In every country, Marlboro Reds were readily available; a pack was found in every city and socioeconomic area visited. The other popular brand variants were less widely available. Samples in India and Philippines were too small for within country comparisons. The average price of Marlboro Reds was significantly higher than the other variants in five out of seven countries. In Brazil, Marlboro pricing was not significantly different from the national variant, and in Indonesia, Marlboros were more affordable than the other variants. On average, the price range in Marlboro Reds was $\$ 1.09$ compared to $\$ 0.36$ for the national variant and $\$ 0.23$ for the other international variant. The removal of foreign and duty free Marlboro Reds does not change the average price results, but decreases the price range to $\$ 0.48$.

Conclusions: Differences were found in availability and pricing at the country and brand variant levels. We will discuss possible explanations for the availability and price distribution findings.

Tob. Induc. Dis. 2018;16(Suppl 1):A127

DOI:10.18332/tid/83939

$12: 30-14: 00$

PS-589-3 The impact of a 2018 tax increase on illicit cigarette trade in Mongolia - baseline results T Batmunkh'1, H Ross ${ }^{2}$

'National Cancer Council of Mongolia, Ulaanbaatar, Mongolia, ${ }^{2}$ University of Cape Town, Cape Town, South Africa. E-mail: tsetsegsaikhan@yahoo.com

Background and challenges to implementation: Mongolia battles with high smoking prevalence - $49 \%$ of adult male and $5.3 \%$ of adult female smoked in 2013. An evidence based tobacco tax policy could substantially reduce this prevalence. A study found that a 9-fold increase in tobacco tax could help Mongolia to 
reach its Sustainable Development Goals target of 30\% prevalence reduction by 2025 .

Despite a lot of opposition to a tobacco tax increase citing a possible increase in illicit trade, the government of Mongolia increased tobacco excise tax by $10 \%$ effective Jan 1, 2018. This tax increase is insufficient to have a meaningful impact on the prevalence.

Intervention or response: We designed a study to measure the impact of the tax increase on any change in the size of illicit cigarette trade. It is based on collecting discarded cigarettes packs on the streets of Ulaanbaatar and another two provinces bordering Russia and China in three waves- April 2017, April 2018 and October 2018. We are recording the packs' features, such as the presence of a Mongolian tax stamp, the correct graphic and text health warnings, and the presence of duty-free signs. This method will identify any changes in illegal tax evasion and legal tax avoidance, both representing tax losses for the governments. Results and lessons learnt: We have collected the total of 7000 discarded packs in April 2017. We will present the results of this first wave of data collection in terms of share of packs that did not pay taxes in Mongolia.

Conclusions and key recommendations: We will also show the share of packs with special promotional features such as "Super Slim" or "Less Smoke Odour", and analyse the share of packs with different pictorial health warnings since the companies are aware that certain pictures are more unpleasant compared to others.

Tob. Induc. Dis. 2018;16(Suppl 1):A128

DOI:10.18332/tid/84047

\section{$12: 30-14: 00$}

PS-590-3 Assessing market competition in the Philippine cigarette industry

M Reganon ${ }^{1}$, MJ Aloria ${ }^{2}$

'Philippine Competition Commission / University of the Philippines School of Economics, Quezon, Philippines, ${ }^{2}$ Action for Economic Reforms, Quezon, Philippines.E-mail:mlreganon@ yahoo.com

Background: The recent passage of the Philippine Competition Act has caused many to rethink the market structure of Philippine industries. Foremost is the cigarette industry, whose structure bear important implications on the health of Filipinos.

A competitive cigarette industry may mean price wars and intensified advertising, disproportionately harming the young and the poor. On the other hand, a concentrated industry may mean a dominant player with ability to engage in predatory pricing. The latter will also likely possess power to lobby against tobacco control policies.

In this study, we assess the market competition in the Philippine cigarette industry, and its correlation with cigarette affordability in recent years.

Methods: Using retail volume data from Euromonitor International and financial reports from the Securities and Exchange Commission, we calculate for various measures of market concentration such as the Top 4 Concentration Ratio (C4), the Herfindahl-Hirschmann Index (HHI), and the Dominance Index (DI) over the period 2007 to 2016 . We then compare these measures against cigarette affordability trends.

Results: Across all measures, we find a highly concentrated cigarette industry. C4 ratios ranged from 97\%-99\%, HHI from 4754-8848, and DI from 7479-9973. In 2010 when Philip Morris acquired Fortune Tobacco, industry concentration peaked (HHI rose by $72 \%$ and DI by $33 \%$ ). In 2012 when the
Sin Tax Law was passed, competition slightly intensified with Mighty Corporation taking advantage of the transitionary dual tax structure.

Most significantly, fluctuations in market concentration did not affect cigarette affordability. A pack of cigarettes costed 7.4\%$8.4 \%$ of the daily minimum wage between $2006-2012$.

Conclusions: Assessing the market structure of the cigarette industry better informs the formulation of effective tobacco control regulations. For a concentrated cigarette industry such as in the Philippines, an effective tax policy must temper the power of companies to absorb taxes so that these are passed onto consumers to discourage smoking.

Tob. Induc. Dis. 2018;16(Suppl 1):A129

DOI:10.18332/tid/84049

\section{2:45-14:00}

EP-130-3 Gender analysis on drivers of cigarette consumption in Kenya: two-part model approach P Kipkorir ${ }^{1}$, V Ngeno ${ }^{2,3}$

${ }^{1}$ Moi University, Student, Department of Agricultural Economics and Resource Management, Eldoret, Kenya, ${ }^{2}$ Moi University, Senior Lecturer, Agricultural Economics and Resource Management, Eldoret, Kenya, ${ }^{3}$ Bomet University College, Dean, School of Business and Economics, Bomet, Kenya. E-mail: kipkorirp@ymail.com

Background: Objectively, this study conducted a gender demographic and psychosocial analysis on drivers of cigarette consumption in Kenya.

Methods: The study heavily relied on data from Kenya Global Adult Tobacco Survey 2014. The data was analyzed using the probit regression and OLS technique to determine the relationship between the predictor and response variables. The results were tested against the stated hypothesis at $1 \%$ and $5 \%$ level of significance.

Results: The study revealed that smoking policy at indoor workplaces had a significant $(\rho<0.050)$ and reducing $(\beta=-$ 0.3723 ) effect on the male smoking participation as compared to female smoking participation. However, opinion about increase in cigarette tax had a significant effect on the probability of both male $(\rho=0.000)$ and female $(\rho=0.000)$ smoking participation. The average consumption weighted price per pack, negatively $(\beta=-0.2046)$ and significantly $(\rho<$ $0.050)$ affected the probability of female smoking participation while it positively $(\beta=0.1248)$ and significantly $(\rho<0.010)$ affected the male smoking intensity. The study found that drivers such as age $(\beta=0.2360, \rho<0.050)$, years spent in education $(\beta=0.0527, \rho<0.050)$, presence and number of children $(\beta=-0.4543, \rho<0.010)$ and household size $(\beta=0.3284$, $\rho<0.050$ ) had significant influence on the general (male and female) smoking intensity. Male's opinion on cigarette tax adjustment had a significant $(\rho<0.050)$ and reducing $(\beta=-$ 0.2973 ) effect on their smoking intensity.

Conclusions: Thus, strengthening the adherence of smoking policy not only in indoor workplaces but also in open spaces and public utilities will help to reduce both smoking participation and smoking intensity among males and females. Cigarette tax adjustments have been proven to be the most effective way of reducing tobacco use among males and females. This is mainly achieved through price increase of cigarettes and thus resulting in reduction in Tobacco use among men and women.

Tob. Induc. Dis. 2018;16(Suppl 1):A130

DOI:10.18332/tid/84559 
12:45-14:00

EP-131-3 Implementation of the WHO FCTC article 6 - price and tax measures to reduce the demand for tobacco in Ghana

EK Wellington ${ }^{1,2}$

${ }^{1}$ Ghana Health Service, Research and Development Division, Accra, Ghana, ${ }^{2}$ Monash University, Global Public Health, Sch. of Medicine and Health Sciences, Kuala Lumpur, Malaysia. E-mail: edith.wellington@ghsmail.org

Background: Countries including Ghana have taken initiative to pass legislation on tobacco use and raise taxes in a bid to discourage tobacco consumption. The effect of high tobacco taxation is known to significantly result on reduction of tobacco use, among the youth and low income groups. Ghana's Excise tax as a percentage of cigarettes price was one of the lowest in the region and in an effort to reduce consumption of tobacco and related health hazards, there was need to increase excise duty on tobacco products to bring in tandem with the average for Africa. This study examined tobacco taxation and policies with overall aim of increasing taxes, reducing tobacco importation and consumption.

Methods: Using routine data from the revenue generating institutions i.e. Customs, Excise and Preventive Service, and Ministry of Finance and Economic Planning, the study gathered information on different taxes levied on tobacco, revenue generated, companies importing tobacco into Ghana.

Results: Parliament of Ghana approved an increase in tobacco taxes through the passage of the Excise Duty (Amendment) Bill, 2015 into Law. The Bill seeks to amend the first schedule of the Excise Duty Act, 2014 (Act 878) and proposes an increase the excise duty on cigarettes and cigars from 150 percent of the exfactory price to 170 percent.

Conclusions: Advocating for high excise taxes as well as review of import duty on tobacco will discourage consumption of tobacco in Ghana. This measure was in line with Ghana's obligations under Article 6 of the WHO FCTC. Ghana should continue to raise tobacco taxes, ensure tobacco taxes are adjusted periodically for inflation, strengthen tax administration and protect public health policies from tobacco industry interference. Funds generated from increased taxes could be used to implement and enforce tobacco control policies and programs.

Tob. Induc. Dis. 2018;16(Suppl 1):A131

DOI:10.18332/tid/84593

\section{$12: 45-14: 00$}

EP-132-3 Measuring illicit cigarette consumption with tobacco surveillance data: evidence from the Global Adult Tobacco Survey for Mexico

B Saenz de Miera Juarez', MA Rodriguez², LM Reynales

${ }^{1}$ London School of Economics and Political Science, London, United Kingdom, ${ }^{2}$ nstituto Nacional de Salud Pública, Cuernavaca, Mexico.E-mail: belensaenzdem@yahoo.com.mx Background: Illicit trade in cigarettes poses a threaten to tobacco control. Reliable measures of the extent of this problem are scarce, however. The objective of this study is to use tobacco surveillance data to measure illicit cigarette consumption in Mexico.

Methods: Data comes from the GATS Mexico 2009 and 2015. In particular, self-reported information of brands is employed to identify illicit cigarettes. Since the GATS 2015 collected images from packs in smokers' personal possession, we also use this information to validate self-reported data.

Results: Self-reported information indicates that illicit cigarette consumption increased from $0.5 \%$ to $2.7 \%$ between 2009 and 2015. For the subsample of smokers with both self-reported and cigarette pack information, the share of illicit cigarette consumption is lower if calculated with the former, but the difference is not statistically significant. Additionally, if we use cigarette pack information to adjust the total estimate for 2015, this increases by just 1.1 percentage point. Only $25 \%$ of the smokers had a pack at interview, however. This is related to marked differences in consumption patterns: being a daily smoker and having bought a pack at last purchase, as opposed to being occasional smokers and having bought a single cigarette, increases the odds of having a pack at interview. These characteristics are also associated with the likelihood of consuming illicit cigarettes.

Conclusions: Despite important increases in tobacco taxes during 2009-2015 in Mexico, surveillance data indicate that illicit cigarette consumption increased by only 2 percentage points and is currently below $5 \%$. This figure is much lower than the estimates of the tobacco industry, which is consistent with the findings of previous academic studies for other countries. Selfreported information was rather accurate for more heavy smokers, but future research should assess whether that is also the case for occasional smokers, who are more likely to buy single cigarettes.

Tob. Induc. Dis. 2018;16(Suppl 1):A132 DOI: $10.18332 /$ tid/84167

$12: 45-14: 00$

EP-133-3 Public health and fiscal impacts of radical decrease of ad valorem excise share in the mixed tobacco excise system

K Krasovsky'

'Ukrainian Institute for Strategic Studies of the Ministry of Health, Tobacco Control Unit, Kyiv, Ukraine. E-mail: krasovskyk@ gmail.com

Background: Guidelines for implementation of the FCTC Article 6 recommend implementing specific or mixed excise systems with a minimum specific tax floor. Over recent years, many EU countries decreased the share of ad valorem excise. The most radical changes have been undertaken in 2011-2013 in Sweden, Denmark and the Netherlands, where ad valorem excise rate was decreased to $1 \%$. The aim of the paper is to estimate public health and fiscal impacts of radical ad valorem excise share decrease in the mixed excise system.

Methods: Data on tobacco excise rates, revenues and sales were taken from the European Commission site. For average cigarette prices calculations, consumer price index for cigarettes was used. Net-of-tax cigarette price was calculated as: Average price Specific excise - Average price * (VAT + Excise) ad valorem rate. Results: After radical decrease of ad valorem excise share, Sweden, Denmark and the Netherlands experienced rather common trends:

(1) Tobacco sales substantially (by 10-20\%) decreased;

(2) Tobacco revenues stagnated;

(3) Tobacco industry increased net-of-tax cigarette price to much greater extent than the specific excise increase and inflation rate. As the ad valorem excise rate was substantially reduced, the tobacco industry could increase profits by increasing the industry's part of the price.

Conclusions: Radical decrease of ad valorem excise weight in the mixed excise system reduces tobacco consumption. However, such policy does not increase tobacco excise revenue, while tobacco industry apparently keeps its incomes as cigarette prices rise by more than the tax yield. Countries with mixed tobacco 
excise systems should be careful in changing their excise tax structures to keep tobacco taxation a "win-win" policy. Large increase in the specific excise rate is the most beneficial change in terms of public health, but ad valorem rates do not need to be much reduced.

Tob. Induc. Dis. 2018;16(Suppl 1):A133

DOI: $10.18332 /$ tid/83883

\section{2:45-14:00}

\section{EP-134-3 Collecting cigarette price data on a limited} budget

C van Walbeek ${ }^{1}, \mathrm{~K}$ van der Zee ${ }^{1}$

${ }^{1}$ University of Cape Town, Southern Africa Labour and Development Research Unit (SALDRU), Economics of Tobacco Control Project (ETCP), Cape Town, South Africa. E-mail: cwalbeek@gmail.com

Background: Data forms the backbone of any scientific research project. In many countries the statistical authorities collect information on cigarette prices but often the coverage of this data is restricted to large metropolitan areas or these databases are not made available. The Economics of Tobacco Control Project at the University of Cape Town (UCT) has used foreign based students to collect cigarette price data in a number of African countries on a very limited budget.

Methods: Students are recruited at UCT and are trained in data collection techniques and etiquette. When they return to their home countries on holiday they collect cigarette price data (packs and single sticks) from both formal and informal retail outlets. The quality of the information is verified as fieldworkers are required to take photographs of the cigarettes with their mobile phones. Students are paid on a piecework basis.

Results: Cigarette price data have been collected in Mauritius, Botswana, Namibia, Lesotho, Zimbabwe, Swaziland and rural areas of South Africa in three waves [January $2016(n=1025)$, July $2016(n=9301)$ and January $2017(n=10185)]$, with more waves planned for 2017 and 2018. The data allows one to investigate inter- and intra-country differences, and pack/single stick differences in prices. Besides the data that is generated, this data collection method makes students aware of the economics of tobacco control and attracts them to do further research in this area.

Conclusions: Using students as fieldworkers for collecting price data in their home towns and countries is an efficient and costeffective way of collecting price data. This method also generates interest about tobacco control issues among students. This approach can easily be applied in countries with limited resources to perform pricing surveys.

Tob. Induc. Dis. 2018;16(Suppl 1):A134

DOI: $10.18332 /$ tid/83919

\section{2:45-14:00}

EP-135-3 \# OneTobaccoTax: social media campaign for unified tobacco taxes under Goods and Services Tax in India

P Puri', V Mallik ${ }^{2}$, D Svenson ${ }^{3}$, S Hamill ${ }^{3}$, N Singh Negi ${ }^{2}$, S Mullin ${ }^{3}$, N Murukutla ${ }^{3}$

'Vital Strategies, Gurgaon, India, ${ }^{2}$ Vital Strategies, New Delhi, India, ${ }^{3}$ Vital Strategies, New York, NY, United States of America. E-mail:ppuri@vitalstrategies.org

Background and challenges to implementation: India introduced a Goods and Services Tax (GST), effective 1 July 2017. There was ambiguity on how tobacco would be taxed - cigarettes were known to be in the highest tax bracket, there was possibility bidi would fall under the 'essential commodities tax rate of 5\%' and there was no information about smokeless tobacco. Vital Strategies and partners were involved in advocating for a unified tax in the highest tax bracket for all tobacco.

Intervention or response: The campaign focussed on health harm messaging while calling for all tobacco products to be included in the highest bracket. Vital Strategies executed an intensive "\#OneTobaccoTax" campaign from October 2016 - July 2017. The social media campaign ran in multiple phases in sync with mass media campaigns, earned media activities and offline advocacy. It comprised a website and social media messages and an online Thunderclap campaign. The objective was to influence key decision makers by (1) Calling for high, unified taxes (2) A focus on Bidi, calling for their inclusion in the highest bracket.

Results and lessons learnt: Analytics suggested that \#OneTobaccoTax, reached out to approximately 1.3 million people. 1,474 tweets targeted Prime Minister Narendra Modi and Finance Minister Arun Jaitley, which were viewed 283,757 times. Two thunderclap campaigns acquired 747 supporters that were potentially seen by 7,12,239 people on Twitter and Facebook. Key opinion leaders tweeted using \#OneTobaccoTax.

Conclusions and key recommendations: This demonstrates how social media can be used to target messages to policy makers and key opinion leaders. People who agreed with our campaign lent their voice and shared our messages through their own social media, which showed support for higher tobacco taxes, and allowed supporters to target decision makers at national and subnational level. The campaign's objective was attained as the GST council decided to tax all tobacco products, including bidi, under the highest tax bracket.

Tob. Induc. Dis. 2018;16(Suppl 1):A135

DOI:10.18332/tid/84403

\section{$12: 45-14: 00$}

EP-136-3 The counterproductivity of a stagnant tobacco tax on national tobacco control efforts in Nigeria

O Ogunnika', A Ojo²

${ }^{1} T a B H e a l t h$ Initiative, Ibadan, Nigeria, ${ }^{2}$ University of Ibadan, Ibadan, Nigeria.E-mail: jogunnika@gmail.com

Background: Tobacco consumption is very injurious to the health and wellbeing of people. This is because both passive and active smokers bear disproportionate burden of smoking. Nigeria is the most populous nation in Africa with youths accounting for about $60 \%$ of the population. The rate of tobacco consumption in Nigeria is disturbing. An estimated 4.5 million people are addicted to tobacco in Nigeria. Nigerians spend about NGN 89 billion on tobacco annually. However, there are regional difference in the consumption of tobacco in Nigeria. South Eastern Nigeria has the highest incidence of smokeless tobacco usage while Northern Nigeria has the highest tobacco smokers.

Methods: Available statistics, reveal youths are the highest consumers of tobacco in Nigeria accounting for 15.4percent of national tobacco consumption. The Nigerian government recently formulated a policy to control consumption of tobacco in Nigeria. The policy states that citizens can only consume certain amount of cigarette otherwise it will not be sold to them.

Results: This policy will be counterproductive as it will expand consumption for tobacco consumers. Furthermore, tobacco tax is significantly lower in Nigeria than elsewhere. Therefore, tobacco companies in Africa post high profits even when other markets 
are shrinking due to the favourable conditions in Nigeria is not far-fetched.

Conclusions: This paper therefore recommends that government should be more stringent in the implementation of existing antitobacco legislations especially increasing tobacco tax.

Tob. Induc. Dis. 2018;16(Suppl 1):A136

DOI: $10.18332 /$ tid/84661

\section{2:45-14:00}

\section{EP-137-3 Characterization of cigarrete smuggling} distribution in Colombia

B Llorente1, D Díaz², LF de Angulo ${ }^{3}$, R Iglesias ${ }^{1}$, MC Ávila ${ }^{1}$

${ }^{1}$ Fundación Anaás, Bogotá, Colombia, ${ }^{2}$ Diversidad Rural, Bogotá, Colombia, ${ }^{3}$ CREER-IHRB Centro Regional de Empresas y Emprendimientos Responsables, Bogotá, Colombia. E-mail: bllorente@fundacionanaas.org

Background: The challenges associated with a historical pattern of interaction between the State and the territory, predominance of informal cigarette sales, corruption and well-organized mafias, create favorable conditions for illicit trade of cigarettes in Colombia. Guajira encompasses all this elements and it is the most traditional smuggling hub. The study also includes the cities of Cúcuta (close to the Venezuelan border with 23\% penetration of illicit cigarettes), and Bogotá (with the largest number of smokers) This study identifies actors in the illicit trade distribution chain (retail and wholesale), their behaviour, incentives and interactions.

Methods: Passive participant research (e.g. buying cigarettes), semi-structured interviews, and focus groups. Fieldwork performed between July - November 2017. Selected actors include authorities, smugglers, travelers in border areas, street vendors and owners of small businesses.

Results: Preliminary findings obtained from interviews reveal three significative changes in Guajira: military and police forces have rearranged priorities in the territory as a result of the dynamics of the peace process; communities acknowledge the need of State action to preserve order, but smuggling continues to be the exception (e.g. reports of violent incidents between communities and police after seizure operatives); traditional leaders' profile is undergoing transformations. Economic slowdown, and dererioriating environmental conditions are mentioned as driving forces for a renewed presence of smuggling activity in the region. Findings reveal presence of minors in smuggling activities. In Bogotá street vendors (the main distribution channel for cigarettes) after the tax increase are more reluctant to sell full packs.

Conclusions: Changes along the distribution chain are bringing new risks and opportunities for an effective control of illicit trade flows. It is necessary to explore interventions that address institutional weaknesses, and the circumstances of vulnerable actors to develop sustainable policies to eliminate illicit trade in Colombia.

Tob. Induc. Dis. 2018;16(Suppl 1):A137

DOI:10.18332/tid/84596

\section{$12: 45-14: 00$}

EP-138-3 Increase in tobacco tax does not lead to increase in illicit cigarette consumption in Hong Kong: findings from a top-down approach against tobacco industry-funded results

T-H Lam¹, YTD Cheung ${ }^{2}$, J Chen², A Kwong ${ }^{3}$, V Lai ${ }^{3}$

${ }^{1}$ The University of Hong Kong, School of Public Health, Hong Kong, China, ${ }^{2}$ The University of Hong Kong, School of Nursing, Hong Kong, China, ${ }^{3}$ Hong Kong Council on Smoking and Health, Hong Kong, China. E-mail: hrmrlth@hku.hk
Background: The tobacco industry consistently and persistently exaggerates that tobacco tax increase leads to increased illicit cigarette consumption (ICC). Using a top-down approach, we found that the proportion of ICC was about $11.9 \%$ in 2012 in Hong Kong (published in Tobacco Control), versus 35.9\% reported in "Asia Illicit Tobacco Indicator 2012" (funded by tobacco industry). Their updated report in 2015 showed that ICC decreased to $29.1 \%$ in Hong Kong. We re-assessed the ICC in Hong Kong, where the tobacco tax increased by $41.5 \%$ in 2011 and $11.8 \%$ in 2014.

Methods: The top-down approach was done by first estimating the total CC of local residents and visitors (top), which was based on the official data of smoking prevalence and daily CC. Then, ICC was calculated by subtracting the total $\mathrm{CC}$ by the legal domestic sales and the duty-free cigarettes imported personally (down). The legal domestic sales were estimated using official revenue data due to the excise duty. The legal import was estimated with three alternative scenarios of cigarettes brought in by local residents and visitors. The estimates in 2015 were validated by a population-based survey that estimated the proportion of local smokers who purchased illicit cigarettes or the cigarettes brought in from overseas personally.

Results: The mid-point estimate of absolute ICC declined from about 412 million sticks in 2012 to 139 million sticks in 2015. The mid-point proportion of ICC in 2012, 13,14 and 15 was $11.9 \%$ (8.2\%-15.4\%), 6.5\% (2.7\%-10.1\%), 7.0\% (3.2\%-10.7\%) and 3.9\% ( $0.1 \%-7.7 \%)$, respectively. The estimates of $3.7 \%-6.3 \%$ obtained from the 2015 population-based survey were consistent with those from our top-down approach.

Conclusions: The industry-funded study has largely inflated ICC in Hong Kong. ICC declined over the 4 years, supporting that increase in tobacco tax does not lead to increase in ICC.

Tob. Induc. Dis. 2018;16(Suppl 1):A138

DOI: $10.18332 /$ tid/84408

\section{$12: 45-14: 00$}

EP-140-3 Implementing effective pricing and taxation measures for prevention and control of smokeless tobacco

A Yadav ${ }^{1}$, RM John², DN Sinha' ${ }^{1}$ N Nargis ${ }^{3}$, R Mehrotra $^{1}$ ${ }^{1}$ National Institute of Cancer Prevention and Research (NICPR), WHO FCTC Global Knowledge Hub on Smokeless Tobacco, Noida, India, ${ }^{2}$ Independent Consultant, New Delhi, India, ${ }^{3}$ American Cancer Society, Washington, WA, United States of America. E-mail: advocateamit@msn.com

Background: There are approximately 346 million adult smokeless tobacco (SLT) users in the world dominated by the South-East Asian region accounting for nearly $86 \%$. India alone accounts for $67 \%$ of the SLT users in the world. While the literature on price and tax measures for smoked tobacco products like cigarettes are more systematic and widely available this is not the case with SLT products. This paper presents the global status of price and tax measures implemented on SLT products by the Parties to FCTC.

Methods: An analysis of the country reports from the FCTC reporting instrument and the MPOWER Report 2015 is presented including a comparative analysis of cigarettes and SLT products. A review of the existing literature on the price and tax measures along with gaps and recommendations is also presented.

Results: Taxes on SLTs vary considerably across countries, from no tax on SLTs in five countries to $80.63 \%$ in Madagascar. 
Similarly, there is larger variation in prices of SLT products across countries and within country, across products. Available estimates show that the price elasticity for various SLTs are largely less than one, and hence, responds to price increases. Estimates on affordability of SLTs indicate that these products have become more affordable over the years. The recent changes in SLT taxation under the GST reform in India have reduced the tax burden further on these products.

Conclusions: Parties should equally implement price and tax measures on SLT. Taxation on SLT should be such that it keeps up with inflation, at the same time, ensuring that their prices sufficiently go up, making SLTs less and less affordable. The tax rates should be standardised across the SLT products to prevent substitution with other tobacco products. Efforts should be made to establish a standardized unit for the purposes of pricing or taxing SLTs.

\section{Tob. Induc. Dis. 2018;16(Suppl 1):A139} DOI:10.18332/tid/84372

\section{2:45-14:00}

EP-141-3 Analyzing current and future levels of cigarette affordability under the Philippine Sin Tax Law (RA 10351)

MJ Aloria', J-A Diosana', F Sta. Ana ${ }^{1}$

'Action for Economic Reforms, Fiscal Policy Team, Quezon, Philippines. E-mail: madzaloria@gmail.com

Background: The five-year old Philippine Sin Tax Law (RA 10351) is recognized as a global model. With the upcoming Congressional review, which can be a venue to either weaken or proactively improve the law, it is crucial to analyze its current and future impact on cigarette affordability, or the strength of income in purchasing tobacco products. This paper therefore analyzes trends in nominal cigarette prices (average price jumped from P1.20 to P2.20/stick according to the Philippine Statistics Authority (PSA) data)) and income trends to determine the future trajectory of cigarette demand. The upward trend in both individual (3\% annual increase) and macro-economic level income (7\% annual increase) of Filipinos could eventually make smoking behavior affordable again.

Methods: Trends in (i) Gross Domestic Product per capita, (ii) household income from Family Income and Expenditure Survey, (iii) and national effective minimum wage calculated from the National Wages and Productivity Commission, are used to estimate measures of cigarette affordability. Price data were gathered from PSA and projected using Walbeek model.

Results: After the passage of Sin Tax Law, cigarette Relative Income Price increased from $2.2 \%$ in 2012 to $2.9 \%$ in $2017 \mathrm{Q} 1$. However, with strong projections on GDP growth and the relatively low increase in price starting 2018 (4\% increase in excise tax), RIP will lower to $2.8 \%$ in 2018 . This is consistent with the estimates that the growth of cigarette prices would be slower than daily income by 2018 , making smokers potentially adaptable again.

Conclusions: Moving forward, the paper proposes to sustain the declining trend in cigarette affordability through increasing tobacco excise taxes further. This is consistent with the call of various health experts including Department of Health call to reduce smokers by 1 million by 2022 . Other countries seeking to emulate Sin Tax Law are also recommended to consider income growth in estimating appropriate tax levels.

Tob. Induc. Dis. 2018;16(Suppl 1):A140

DOI:10.18332/tid/84266

\section{2:45-14:00}

EP-142-3 Social cost benefit analysis of tobacco control policies in the Netherlands

$\mathrm{R}$ de Kinderen ${ }^{1,2}$, T Rombouts ${ }^{3}$

${ }^{1}$ Dutch Alliance for a Smokefree Society, Utrecht, Netherlands, ${ }^{2}$ Trimbos Institute, Centre of Economic Evaluations, Utrecht, Netherlands, ${ }^{3}$ Dutch Cancer Society, Amsterdam, Netherlands. E-mail: reina.dekinderen@alliantienr.nl

Background: The objective of this study was to investigate all the societal costs and benefits of tobacco control policies in the Netherlands in order to tackle the economic and financial argument of the tobacco industry.

Methods: A social cost benefit analysis (SCBA) was used to calculate the costs and benefits of different tobacco control policies from now until 2050 in the Netherlands. Predicting models were used to calculate the impact of tobacco control scenarios on smoking prevalence, deaths, health care impact, consumer surplus, quality adjusted life years, productivity, government incomes, and other actors in society. The tobacco control scenarios we investigated were mass media campaigns, different levels of taxation and multi-policy package based on the WHO FCTC's MPOWER package. Furthermore we looked at the social costs and benefits of a scenario in which no-one starts smoking from 2017 onwards. These scenarios were compared with a reference scenario in which no changes were assumed in the government's current smoking-related policy for a period of 35 years.

Results: In the reference scenario by 2050 , the prevalence of smoking decreased by 2.3 percentage points, compared to between 4.8 and more than 14 percentage points in the alternative scenario's. All alternative scenarios result in positive net benefits between 2 and $>100$ billion Euros over the entire time horizon. The MPOWER scenarios in which a combination of tax increases with other policies are introduced, result in benefits for consumers, employers and government through tax incomes.

\section{Predicted smoking prevalence per policy scenario}

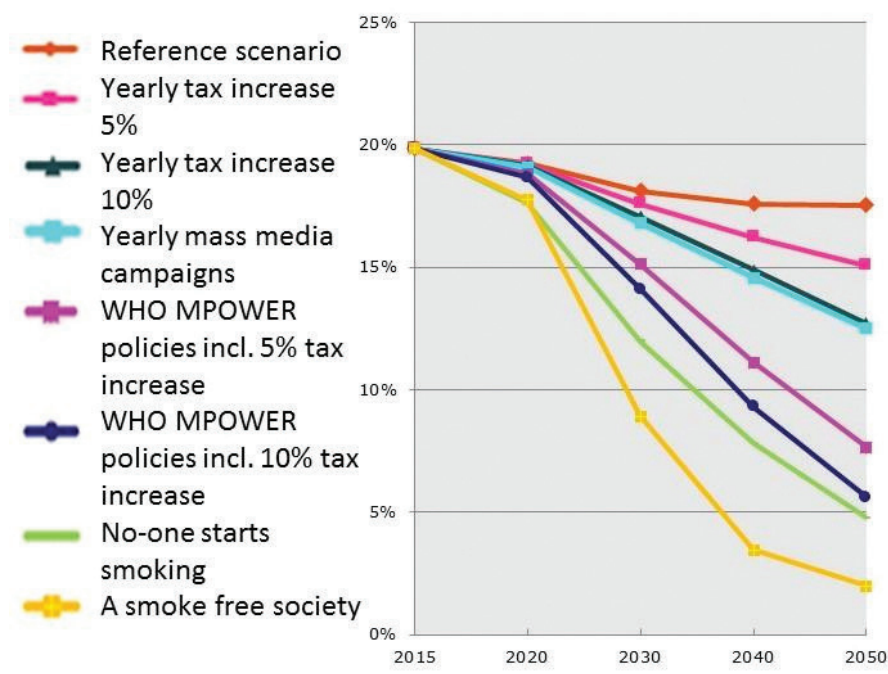

[Predicted smoking prevalence per policy scenario]

Conclusions: The health and economic burden of tobacco use is enormous. The intervention costs for the tobacco control policies are minimal, and investing in health is beneficial from both the public as the economic perspective. This study can act as an example/guide for other researchers and decision makers to 
investigate their own countries' social costs and benefits.

Tob. Induc. Dis. 2018;16(Suppl 1):A141

DOI:10.18332/tid/84034

$12: 45-14: 00$

EP-143-3 Cigarette affordability and impacts of 2014 tax reform in Vietnam

TT Tran'1, TNT Tran'1 TT Le², TS Dao ${ }^{3}$, TL Nguyen ${ }^{4}$, M Goodchild ${ }^{5}$

${ }^{1}$ Ministry of Finance, Department of Tax Policy, Hanoi, Viet Nam, ${ }^{2}$ HealthBridge Foundation of Canada, Vietnam Office, Hanoi, Viet Nam, ${ }^{3}$ Thuongmai University, Hanoi, Viet Nam, ${ }^{4}$ World Health Organisation, Country Representative Office, Hanoi, Viet Nam, ${ }^{5}$ World Health Organisation HO, Geneva, Switzerland. E-mail: lethu@healthbridge.org.vn

Background: Vietnam revised the Excise Tax Law in 2014 leading to an increase of the cigarette excise tax rate from $65 \%$ of ex-factory price to $70 \%$ in January 2016 , and to $75 \%$ in January 2019. The government plans to make other excise tax reforms in 2018. This study explored the impacts of 2014 Excise Tax Law on cigarette retail price, affordability, sale volumes and government tax revenue for 2016 and expected impacts for 2017-2019.

Methods: Relative Income Price (RIP) was used to measure the affordability of cigarettes. The retail price of three popular brands, between 1998 and 2016, was collected from government secondary data.

The WHO Tobacco Tax Simulation model was used to assess real and expected impacts of tobacco tax from 2016-2019

Results: The nominal prices of three cigarette brands have increased between 1998 and 2016, but the inflation-adjusted retail prices have decreased, which indicates an increase in cigarette affordability.

The average inflation-adjusted retail price of cigarettes increased by $11 \%$ in 2016 and will be about 14\% (1,500 Vietnam Dong) higher in 2019 compared to 2015.

Cigarette retail sale volumes decreased by $4 \%$ in 2016, but are projected to steadily increase from 2016 to 2019 by $2.3 \%$ ( 88 million packs).

Total inflation-adjusted tax revenue from cigarettes increased by $4 \%$ in 2016 , but will be $13 \%$ (1,988 billion Vietnam Dong) higher in 2019 compared to 2015.

Conclusions: Despite the increase in excise tax in the 2014 Excise Tax Law, cigarettes are becoming more affordable as a result of inflation and income growth. The real retail price and tax revenues increased only for the years of tax increase. The sales volume decreased slightly in 2016 but is expected to keep increasing from 2017-2019. Tobacco taxes should be increased at a higher rate and more frequently at least to keep up with inflation and income growth.

Tob. Induc. Dis. 2018;16(Suppl 1):A142

DOI: $10.18332 /$ tid/84523

\subsection{Advances in protection from tobaceo pollution, second- and third-hand smoke}

\section{6:00-17:30}

RF-1204-3 Exposure to secondhand smoke infiltration at home amongst Hong Kong residents: a population-based study

TT Luk', MP Wang', Y Wu', DYT Cheung ${ }^{1}$, SY Hoํ․ A Kwong ${ }^{3}$, V Lai ${ }^{3}$, TH Lam $^{2}$

${ }^{1}$ University of Hong Kong, School of Nursing, Hong Kong, Hong Kong, ${ }^{2}$ University of Hong Kong, School of Public Health, Hong
Kong, Hong Kong, ${ }^{3}$ Hong Kong Council on Smoking and Health, Hong Kong, Hong Kong. E-mail: luktt@hku.hk

Background: Secondhand smoke (SHS) contributes to significant morbidity and mortality worldwide including Hong Kong, a densely-populated city with the lowest daily smoking prevalence $(10.5 \%)$ in the developed world. Most Hong Kong citizens reside in multiunit housing where SHS can transmit from one unit to another because of the shared ventilation system and crowded living environment. We explored the prevalence of SHS infiltration and its sociodemographic correlates in the Hong Kong general population.

Methods: A cross-sectional sample of 5151 randomly selected Hong Kong residents aged $15+$ were interviewed by telephone (response rate $=85.8 \%$ ) for the Hong Kong Tobacco Control Policy-related Survey in 2016. Participants reported the number of days experiencing SHS infiltration from neighbour at home in the past 7 days, and their sociodemographic characteristics and smoking status. Data were weighted by the Hong Kong official data on age, gender and smoking status distributions. Chi-square tests and multivariable logistic regression examined the associations of home SHS infiltration with sociodemographic and smoking status.

Results: Overall, the prevalence $(95 \% \mathrm{CI})$ of ever and daily SHS infiltration at home in the past 7 days were $22.2 \%$ (20.3 to $24.2 \%$ ) and $5.8 \%$ (4.8 to $7.1 \%)$ respectively. SHS infiltration was individually associated with female $(P=0.002)$, younger age $(\mathrm{P}<0.001)$ and never smoking status $(\mathrm{P}<0.001)$ but not with indices of socioeconomic status including highest education level $(\mathrm{P}=0.07)$, family income $(\mathrm{P}=0.43)$, employment $(0.89)$ or immigrant $(\mathrm{P}=0.09)$ statuses. Multivariable regression analyses showed that current smokers were less likely to report home SHS infiltration (OR $=0.43,95 \%$ CI 0.32-0.58).

Conclusions: In Hong Kong, the prevalence of home SHS infiltration was very high given the low prevalence of smoking and did not vary with socioeconomic status. Implementation of smoke-free policy in multiunit housing is imperative to safeguard its residents from passive smoking.

Tob. Induc. Dis. 2018;16(Suppl 1):A143

DOI: $10.18332 /$ tid $/ 84086$

$16: 00-17: 30$

RF-1205-3 Implementation of smoke free policy in restaurant setting - experience of a NGO in Vietnam

THA Pham ${ }^{1}$, HN Nguyen ${ }^{1}$, TT Le', S Fitzgerald ${ }^{2}$

'HealthBridge Foundation of Canada, Vietnam Office, Hanoi, Viet Nam, ${ }^{2}$ HealthBridge Foundation of Canada, Ottawa, ON, Canada. E-mail: nguyen@healthbridge.org.vn

Background and challenges to implementation: The Vietnam Tobacco Control Law, effective since May 2013, requires all indoor public places including restaurants and cafeterias be $100 \%$ smoke-free. However, GATS 2015 findings showed the highest prevalence and the most modest reduction of second-hand smoke exposure in restaurants during $2010-2015$ (80.7\% and $84.9 \%$ respectively) compared to other settings. The challenges were low political commitment and reluctance of authorities to implement smoke-free policies in restaurants, the resistance of business owners due to concerns about losing profits and competitive edge, and high public acceptability of smoking.

Intervention or response: In 2015-2016, HealthBridge worked with the Hanoi city and districts' Health Departments and other partners on "Strengthening implementation of smoke-free 
restaurants (SFR) in Hanoi". The project's strategies were: i) Advocacy to add SFR implementation into the Hanoi city action plan; ii) Facilitate the role of local authorities at district level in managing, training, monitoring, and enforcing the implementation of SFR instead of focusing authorities at city level; iii) Peer education to engage managers of compliant SFR to share their experience to create the trust and motivation of restaurant owners; iv) Media campaigns to raise public awareness and support.

Results and lessons learnt: Managers of 600 restaurants were trained on SFR implementation. 400 of them signed a commitment to be smoke-free. Monitoring visits of authorities at district level was established for the first time and deployed regularly. This proved to be a sustainable enforcement mechanism because of its simple administrative procedure for setting up when compare to interdisciplinary team of authorities at city level. The rate of restaurants going smoke-free increased from $25 \%$ to $44 \%$ (prepost intervention survey). The knowledge and support from customers and restaurant owners on SFR policy also increased significantly.

Conclusions and key recommendations: The project's strategies have proven to be effective in improving the implementation of smoke-free policy in restaurants. These strategies should be scaled up to other areas.

Tob. Induc. Dis. 2018;16(Suppl 1):A144

DOI:10.18332/tid/83862

\section{$16: 00-17: 30$}

RF-1206-3 Smoke free policy in bars and restaurants in Kampala: a cross sectional study

C Aanyu' ${ }^{1}$, RR Apolot ${ }^{1}$, J Bagonza ${ }^{1}$

'Makerere University, Kampala, Uganda. E-mail:aanyuchristinee@ gmail.com

Background: Smoke Free Policies (SFPs) have been passed in different countries around the world to prevent exposure to Secondhand smoke. Uganda enacted a partial Smoke Free Policy in 2004 banning smoking in public places and allowing smoking only in designated smoking areas in public places like bars and restaurants. However, the extent of implementation of this policy has not been well studied in Uganda. This study therefore aimed to assess the prevalence of the "No Smoking" signage, compliance and enforcement of the Smoke Free Policy in bars and restaurants of Makindye division in Kampala district.

Methods: A cross sectional study was conducted among 154 randomly selected premises licensed as bar and restaurant after adjusting for finite population in 2013. The managers of the premises were purposively selected while participants were conveniently observed. Observation checklist and interviewer administered questionnaires were used for data collection. Three outcome variables; prevalence, compliance and enforcement of Smoke Free Policies were assessed. Analysis was done using SPSS (Version 16.0) and data summarized using frequencies.

Results: Of the 154 premises reached; $32.5 \%$ displayed "No Smoking" Signage with $68.0 \%$ having written Signage. $13.0 \%$ of the premises had designated smoking area with $30.0 \%$ of them clearly labeled. Only $11.7 \%$ of the designated smoking areas were enclosed rooms while $88.3 \%$ was an open area. $80.5 \%$ of the premises had no ash trays, $71.4 \%$ did not have individuals smoking in Non Designated Smoking areas, $64.3 \%$ had no cigarette butts and $69.5 \%$ had no cigarette packs. The average overall compliance to the Smoke Free Policy was, $63.8 \%$.

Conclusions: "No Smoking" Signage and compliance to the smoke free policy in Kampala bars and restaurants is still insufficient.
Sensitization of the managers on the requirements of the Smoke Free Policy and enforcement of the Smoke Free policy is vital if exposure to secondhand smoke is to be reduced.

Tob. Induc. Dis. 2018;16(Suppl 1):A145

DOI:10.18332/tid/84313

\section{$16: 00-17: 30$}

RF-1208-3 Advancing smoke-free public spaces: the challenge of water-pipe in the Canadian context L James ${ }^{1}$, H Kasi Vishwanathan

${ }^{1}$ Heart and Stroke Foundation of Canada, Health Policy, Ottawa, ON, Canada. E-mail: lesley.james@heartandstroke.ca

Background and challenges to implementation: Canada once led the way with smoke-free public spaces and as a result, most Canadians are protected from second-hand smoke through legislation at multiple levels of government. However water-pipe tobacco, which is growing in use, has presented a challenge in existing regulation. While some jurisdictions have revised legislation to restrict water-pipe use in public spaces, there has been much opposition to such from small business owners, retailers, middle-eastern community and tobacco control industry.

Intervention or response: As a result of concerted advocacy efforts, water-pipe tobacco has since been embedded within existing smoke-free public space legislation in a variety of settings. Advocates continue to strive for comprehensive legislation across Canada.

Results and lessons learnt: A variety of arguments and tactics were used on both sides to further each cause.

Water-pipe supporters:

The cultural lens was often applied from supporters of waterpipe. Experts on history or anthropology were leveraged to give credibility to cultural arguments. Small business owners from bars and restaurants pleaded on economic grounds, citing loss of revenue and jobs. Water-pipe products were portrayed to be tobacco-free and without risk.

Tobacco control community:

Protection of workers was a strong counter-frame. Heath experts and scientists put together a narrative demonstrating risk and trends. Public health experts highlighted tobacco content in waterpipe products, inaccurately labeled as tobacco-free. Advocates leveraged the frame of protecting youth to secure quick adoption. Conclusions and key recommendations: In recent years, Canada has made great strides in securing further protection with regard to water-pipe tobacco. The battle is not yet over, and large communities within Canada continue to be without regulation. Cases from across Canada can serve as useful lessons to make progress in the rest of the country and in the global context.

Tob. Induc. Dis. 2018;16(Suppl 1):A146

DOI:10.18332/tid/84642

\section{2:30-14:00}

PS-591-3 Parental perceptions of exposure to tobacco smoke: a new tool to shed light on parental smoking behaviour

V Myers Gamliel' ${ }^{1}$ S Shiloh², Laura (Leah) Rosen ${ }^{1}$

${ }^{1}$ Tel Aviv University, Health Promotion, School of Public Health, Tel Aviv, Israel, ${ }^{2}$ Tel Aviv University, School of Psychological Sciences, Tel Aviv, Israel. E-mail: vicki_myers@hotmail.com Background: Around $40 \%$ of children worldwide are exposed to tobacco smoke by their parents. Interventions to reduce exposure have limited success. 
Parental reports often do not match biomarker reports of children's exposure. We propose there may be a fundamental misunderstanding among smoking parents as to what exactly exposure is and in what circumstances exposure occurs.

We aimed to develop and validate a tool to assess and quantify parental perceptions of exposure (PPE) regarding children's exposure to tobacco smoke.

Methods: Qualitative research with smoking parents revealed common misperceptions: eg. exposure is avoided when smoking at the window, with a child in stroller, or in the car before collecting children. A model was developed and a questionnaire constructed using pictures and vignettes of different situations in which children are exposed to smoking. Parents rated exposure of a hypothetical child in each situation on a scale of 1-7.

Psychometric properties were assessed including exploratory factor analysis, test-retest reliability and Cronbach's alpha coefficient.

Results: 220 parents completed the questionnaire. Factor analysis produced 6 factors for parental perceptions of exposure (PPE), explaining a cumulative total variance of $76.3 \%$. Factors were named: 1)secondhand exposure; 2)thirdhand exposure; 3 ) knowledge/certainty; 4)sensory perceptions; 5)time; 6)distance. Test-retest showed high correlations of PPE: $r=0.856, p<0.001$. Correlation between PPE and parental risk perceptions (PPR) was significant $(0.766 \mathrm{p}<0.001)$. Smokers scored significantly lower than non-smokers on PPE and PPR signifying a narrower definition of exposure, definition of fewer situations as involving greater exposure, and attaching less risk to these situations $(\mathrm{p}<$ 0.001).

Conclusions: The PPE tool was demonstrated as valid and reliable. Smokers and non-smokers had different perceptions of exposure. This tool can shed light on parents' smoking behaviour around their children, may explain discrepancies between parental and objective reports of exposure and may ultimately help change parental smoking behaviour by altering parental perceptions of exposure.

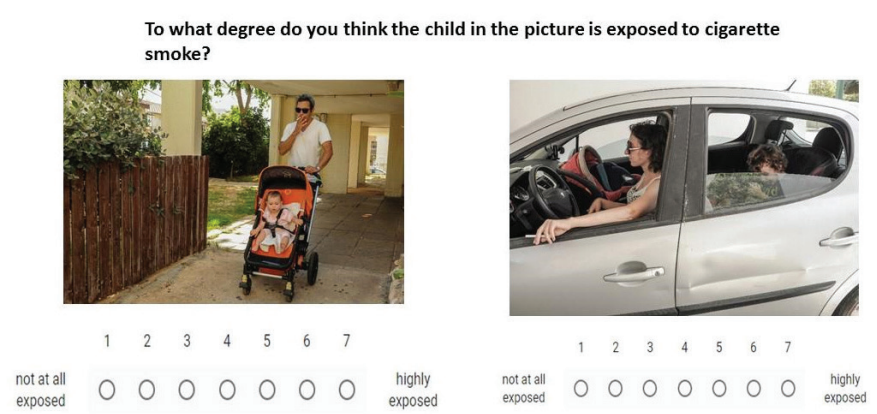

[Excerpt from Parental Perceptions of Exposure tool]

Tob. Induc. Dis. 2018;16(Suppl 1):A147
DOI:10.18332/tid/83778

12:30-14:00

PS-593-3 Exposure to second-hand smoke at work in the European Union, 2006 - 2014

B Cortini' ${ }^{1}$, G Carreras ${ }^{1}$, G Gorini ${ }^{1}$, S Verdi', E Chellini ${ }^{1}$

IISPO, Florence, Italy. E-mail:g.gorini@ispo.toscana.it

Background: After the classification of second-hand smoke (SHS) as a known human pulmonary carcinogen by International Agency for Research on Cancer (IARC), most Countries of the
European Union (EU) introduced smoking ban at workplaces and public places. Main aim of this study was to study trend of SHS exposure at work, 2006-2014.

Methods: Figures were collected in four cross-sectional surveys representative of population of each EU Country. Surveys were conducted in 2006, 2010, 2012, 2014 (Eurobarometer 66.2, $72.3,77.1,82.4$ ) in all 28 EU Countries.

The question on SHS exposure at work was recorded in bogh smokers and non-smokers: "How long are you exposed to tobacco smoke at your workplace?" Possible answers were: $<1$ hour a day; 1-5 hours; $>5$ hours a day; Never or almost never; don't know, In the 2012 and 2014 surveys a new category was added: "occasionally exposed".

Results: Prevalence of exposure to SHS at work decreased by $69 \%$ from 2006 to 2014 (Figure 1).

The drop was recorded since 2010 for exposures for $\geq 1$ hour per day, whereas the exposure to $<1$ hour per day dropped in the 2012 survey.

In 2012 and $201417 \%$ of participants reported to be exposed occasionally, not on a daily basis. Occasional exposure at work was not recorded in previous surveys.

Figures of SHS exposure for each EU Country will be presented reporting also the year of implementation of smoking ban per each Country.

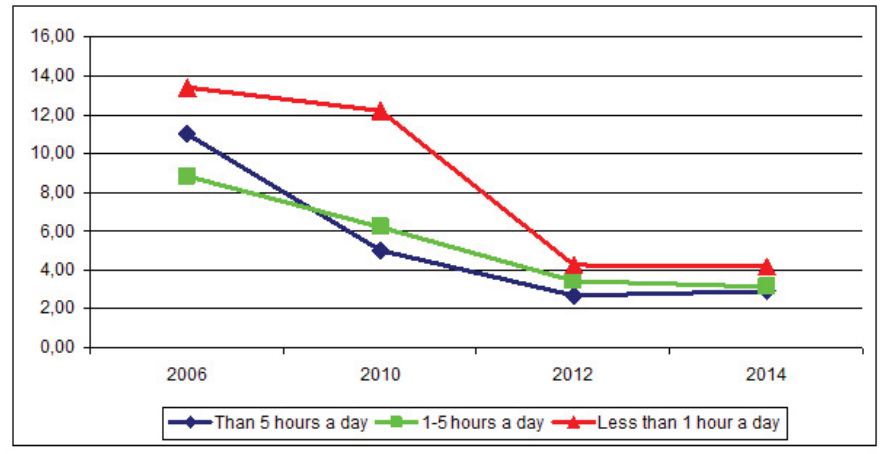

[SHS exposure $\geq 1$ hour/day ]

Conclusions: SHS exposure at work dropped in 28 EU Countries after the implementation of nationwide smoking bans.

Tob. Induc. Dis. 2018;16(Suppl 1):A148

DOI:10.18332/tid/83824

12:30-14:00

PS-594-3 Did local smoke free policy in Indonesia prevent youth from smoking?

W Septiono ${ }^{1}, \mathrm{~N} \mathrm{Ng}^{2}$, M Kuipers ${ }^{1,3}$, A Kunst ${ }^{1}$

${ }^{1}$ Academic Medical Center, University of Amsterdam, Department of Public Health, Amsterdam, Netherlands, ${ }^{2}$ Umeå University, Department of Public Health and Clinical Medicine, Umea, Sweden, ${ }^{3}$ University College London, Department of Epidemiology and Public Health, London, United Kingdom. E-mail: septiono.w@amc.uva.nl

Background: Although Indonesia has not ratified the WHO Framework Convention on Tobacco Control, it has developed a series of tobacco control policies at the local level. Strong presence of tobacco industry is marked with intensive marketing towards young people. This study assessed the impact of local smoke-free policy (SFP) on prevalence of daily and non-daily smoking among youth living in 33 provinces, 98 municipalities, and 412 regencies in Indonesia in 2007 and 2013.

Methods: Data on 239,170 adolescents (12-17 years old) was 
derived from the 2007 and 2013 basic health survey (RISKESDAS) performed by Indonesia Ministry of Health. Information on local tobacco control policies was derived from official documents from 84 districts and 8 provinces. District SFP and provincial SFP were distinguished. A multilevel logistic regression analysis compared the odds of daily and non-daily smoking between areas with and without SFP.

Results: Respondents who reported smoked daily was $4.6 \%$ and non-daily was $4.4 \%$ in 2007 . Smoking prevalence rates were high among males, in older adolescents, areas with high GDP per-capita, and rural areas. Different models consistently observed significantly reduction of daily smoking (OR:0.85; CI95\%:0.79-0.90) and non-daily smoking (OR:0.92; CI95\%:0.860.98 ) prevalence between 2007 and 2013. No difference in daily smoking and non-daily smoking was observed between districts with and without SFP. Intermediate compared with no provincial SFP was significantly associated to reduce the odds of non-daily (OR:0.82; CI95\%:0.69-0.98) smoking, while no association was found between no provincial SFP and high group.

Conclusions: Smoke-free policies at the municipal level seemed to have no effect to reduce smoking among youth in local settings of Indonesia while policies at the provincial level were associated with lower smoking rates. To have better result of the smoking prevention among youth in the future, we suggest enhancing provincial SFP and strengthening the implementation of local SFP as well.

Tob. Induc. Dis. 2018;16(Suppl 1):A149

DOI: $10.18332 /$ tid/83829

\section{$12: 30-14: 00$}

PS-595-3 A cross-country comparison of secondhand smoke exposure in public places among adults in five African countries - The Global Adult Tobacco Survey, 2012 - 2015

S Salandy ${ }^{1}, \mathrm{~L} \mathrm{Mbulo}^{2}, \mathrm{~K}$ Palipudi

${ }^{1}$ Centers for Disease Control and Prevention, Atlanta, GA, United States of America, ${ }^{2}$ Centers for Disease Control and Prevention, Atlanta, Georgia.E-mail: vtb0@cdc.gov

Background: Secondhand smoke (SHS) causes approximately 600,000 deaths annually worldwide. Timely surveillance can inform the development and enforcement of comprehensive smoke-free policies in indoor public places; yet, in many African countries, there is limited data on SHS exposure in this environment. This study examined self-reported SHS exposure in public places in five African countries.

Methods: Data from the Global Adult Tobacco Survey (GATS) were analyzed for Cameroon (2013), Kenya (2014), Nigeria (2012), Senegal (2015), and Uganda (2013). GATS is a standardized, nationally representative household survey of individuals aged $\geq 15$ years. Point prevalence estimates for past 30-day SHS exposure were assessed for the following environments: bars/nightclubs, restaurants, government buildings, public transportation, and healthcare facilities. Analyses were restricted to persons who reported visiting each environment. Estimates were calculated separately for all adults and nonsmokers; nonsmokers were defined as those who answered "not at all" to the question, "Do you currently smoke tobacco on a daily basis, less than daily, or not at all?" Data were weighted and analyzed using SPSS V.24.

Results: Among all adults who visited each environment, countryspecific SHS exposure ranged as follows: bars/nightclubs, $86.1 \%$ (Kenya) to $62.3 \%$ (Uganda); restaurants, $31.9 \%$ (Cameroon) to
16.0\% (Uganda); government buildings, 24.2\% (Senegal) to 5.7 \% (Uganda); public transportation, 22.9\% (Cameroon) to $7.8 \%$ (Uganda); and healthcare facilities, $10.2 \%$ (Senegal) to $4.5 \%$ (Uganda). SHS exposure among nonsmokers was as follows: bars/nightclubs, $85.6 \%$ (Kenya) to $60.9 \%$ (Uganda); restaurants, $32.0 \%$ (Cameroon) to $16.1 \%$ (Uganda); government buildings, $24.2 \%$ (Senegal) to $5.8 \%$ (Uganda); public transportation, $22.2 \%$ (Cameroon) to $7.7 \%$ (Uganda); and healthcare facilities, $9.9 \%$ (Senegal) to $4.5 \%$ (Uganda).

Conclusions: In the assessed African countries, SHS exposure was lowest in healthcare facilities and highest in bars/nightclubs. Smoke-free policies in indoor public places, consistent with the World Health Organization's MPOWER framework, are important to protect nonsmokers from SHS exposure.

Tob. Induc. Dis. 2018;16(Suppl 1):A150

DOI: $10.18332 /$ tid/83830

\section{2:30-14:00}

PS-596-3 Trends in SHS exposure and smokers' support for smoke-free laws in China: findings from the ITC China survey, 2007 - 15

Y Jiang ${ }^{1}$, G Sansone ${ }^{2}$, GT Fong ${ }^{2,3}$, M Yan ${ }^{2}$, L Craig $^{2}$, S Xu² ${ }^{2}$ ACK Quah $^{2}$, G Feng ${ }^{1}$

${ }^{1}$ Chinese Center for Disease Control and Prevention, Tobacco Control Office, Beijing, China, ${ }^{2}$ University of Waterloo, Psychology, Waterloo, ON, Canada, ${ }^{3}$ Ontario Institute for Cancer Research, Toronto, 0N, Canada.E-mail:jiangyuan88@ vip.sina.com

Background: Secondhand smoke (SHS) poses a major public health threat in China, causing over 100,000 deaths/year. China, however, has not yet implemented a national comprehensive smoke-free law, and the few subnational laws are only partial. This study presents trends in selected cities from 2007 to 2015 in: (1) exposure to SHS in key public places (workplaces, restaurants, bars), and (2) smokers' support for comprehensive smoke-free laws, comparing cities and rural areas in 2013-15.

Methods: Data are from Waves 2 to 5 of the International Tobacco Control (ITC) China Survey (2007-15), a face-to-face cohort survey of 800 smokers in each of five cities (Beijing, Guangzhou, Kunming, Shanghai, Shenyang), plus five rural areas at Wave 5 (2013-15) (total N=8000). GEE logistic regression models tested changes over time.

Results: At the latest wave (2013-15), the majority of respondents reported being exposed to SHS in public places. Smoking was most prevalent in bars, with little reduction over time across cities (from $93 \%$ overall in 2007 to $86 \%$ in 2013-15). Smoking in other venues decreased over time, but remained very high in $2013-15$ (from $94 \%$ to $67 \%$ for restaurants; and $75 \%$ to $51 \%$ for workplaces). In rural areas, smoking prevalence was higher in workplaces $(73 \%$ vs $56 \%, \mathrm{p}<.001)$ and lower in bars $(73 \%$ vs $88 \%, \mathrm{p}<.05)$ compared to cities; there was no difference in restaurants. Support for complete smoking bans among smokers in cities increased over time for each venue and is higher than nearly all other $20+$ ITC countries. Support did not differ between cities and rural locations.

Conclusions: Partial smoke-free laws in China are failing to protect both urban and rural residents from SHS, demonstrating the urgent need for a comprehensive national smoke-free law, as called for by the FCTC. Such a law would be supported by the majority of the Chinese public.

Tob. Induc. Dis. 2018;16(Suppl 1):A151

DOI:10.18332/tid/83849 
12:30-14:00

PS-597-3 Knowledge, attitude and practice regarding smoking among Police in Bangladesh MH Ahmed', J Ahmed ${ }^{2}$

${ }^{1}$ Mymensingh Medical College Hospital, ENT and Head Neck Surgery, Mymensingh, Bangladesh, ${ }^{2}$ National Heart Foundation Hospital \&t Research Institute, Dhaka, Bangladesh. E-mail: meheddi@gmail.com

Background: Smoking is not only a personal problem but it is a problem of community because not only smokers are affected but people around smokers are also suffering from harmful effect of second hand smoking. In developing countries like Bangladesh law enforcement members like police can play an important role in societal efforts to reduce smoking in public place. This study was aimed to elucidate the knowledge, attitude and practice of police towards smoking.

Methods: 656 male police working in different area of Mymensingh and Dhaka city were randomly selected for the study. The period of study was November 2016 to May 2017. Information regarding their smoking status, knowledge about second hand smoking, anti-tobacco strategies, and practice related to reduce smoking in public place was collected through pre designed questionnaire.

Results: Total number of the respondent was 656 . The mean age of the participant was 31.2 years. Among the respondent most of them are constable (57.4\%) and their education level was up to Secondary. Among the respondent $35.1 \%$ were smokers and $26.7 \%$ smoke in public place. About $79.4 \%$ know the harmful effect of second hand smoking to health. $95.8 \%$ of the respondent were aware of the anti-tobacco law and $52.5 \%$ inhibit smokers to smoke in public place. $66.3 \%$ told that increase awareness among people is the best way to reduce smoking in public place and $28.3 \%$ think that strict implementation of anti-tobacco laws will reduce the smoking rate in public place.

Conclusions: We found that the police was associated with an inhibition of secondhand tobacco smoking exposure in public places. However, the impact was limited. Our study suggests that effective smoking control program designed specifically for police need to be taken to utilize the effective role of police for smoking control in public place.

Tob. Induc. Dis. 2018;16(Suppl 1):A152

DOI:10.18332/tid/83855

\section{2:30-14:00}

PS-598-3 Beliefs and rules about vaping in home and smoke-free public places: findings from the ITC 4-country project

KM Cummings' ${ }^{1}$ D Braak', G El Nahas', B Heckman ${ }^{1}$, A Alberg ${ }^{1}$, H-H Yong' ${ }^{2}$, GT Fong ${ }^{3}$, SC Hitchman ${ }^{4}$

${ }^{1}$ Medical University of South Carolina, Psychiatry and Behavioral Sciences, Charleston, SC, United States of America, ${ }^{2}$ Cancer Council Victoria, Melbourne, VIC, Australia, ${ }^{3}$ University of Waterloo, Psychology, Waterloo, ON, Canada, ${ }^{4}$ King's College London, Addictions, London, United Kingdom. E-mail: cummingk@musc.edu

Background: This paper compares beliefs about the relative harms of exposure to secondhand electronic cigarette (EC) vapor compared to cigarette smoke, rules about smoking and vaping in the home, and adherence to rules restricting vaping in public places in Australia (AU) and Canada (CA) which have generally more restrictive policies on the marketing of ECs compared to England (EN) and the United States (US) which have less restrictive policies.
Methods: Data came from 12,411 adult (aged 18 years and older) current and former smokers and vapers who participated in the 2016 International Tobacco Control (ITC) Four Country Survey carried out in AU $(n=1491), C A(n=3801), E N(n=4339)$ and US $(n=2780)$. The web-based survey recruited participants from online panels in each country with selection criteria intended to generate representative samples of current and former smokers and vapers in each country.

Results: The majority of respondents in each country believed that exposure to secondhand EC vapor was less dangerous compared to exposure to smoke. Across all countries vapers were more likely than non-vapers to believe that exposure to secondhand EC vapor was less dangerous compared to exposure to smoke. The overwhelming majority of respondents in all countries did not allow smoking in their home, while most allowed vaping. Rules about whether smoking was permitted inside the home were more permissive among smokers whereas rules about vaping were more permissive among vapers. Nearly all vapers reported that they do not vape in public places when it is prohibited.

Conclusions: Being a vaper or smoker was more strongly associated than restrictiveness of EC regulations with beliefs about the dangers of secondhand EC vapor exposure, rules people have about allowing smoking and vaping in their homes, and adherence to rules restricting vaping in public places.

Tob. Induc. Dis. 2018;16(Suppl 1):A153 DOI: $10.18332 /$ tid/83861

$12: 30-14: 00$

PS-599-3 The development of local smoke free policy of Indonesia in $2004-2015$

W Septiono ${ }^{1}, \mathrm{~N} \mathrm{Ng}^{2}$, A Kunst $^{1}$

${ }^{1}$ Academic Medical Center, University of Amsterdam, Department of Public Health, Amsterdam, Netherlands, ${ }^{2}$ Umeå University, Department of Public Health and Clinical Medicine, Umea, Sweden. E-mail: septiono.w@amc.uva.nl

Background: Many low-income countries develop tobacco control policies not only national levels, but also at local levels. The dynamics underlying the development of local-level policies are still poorly understood. This study aimed to map and understand the development of local-level policies regarding smoke-free area, as the main tobacco control strategy in Indonesia, in 2004-2015.

Methods: We used an official government data base with data on smoke free policies for Indonesia's 33 provinces and 510 municipalities. The extent of smoke free policies was expressed in a summary scale derived from the Tobacco Control Scale (TCS) to map, and to relate these scores to provincial-level scores and to characteristics of municipalities.

Results: By 2015, about 30 percent of the municipalities had smoke-free policies. Many municipalities enacted such policies before these were introduced at province. Municipalities with stronger policies clustered within specific parts of the Indonesian archipelago. Municipalities within provinces with high TCS score were more likely to have smoke-free policies $(\mathrm{OR}=1.91$; CI95\%: 1.09-3.35) but no difference was found between low and intermediate group. Smoke-free policies were more common in municipalities with high urbanization rate $(\mathrm{OR}=2.65$; $\mathrm{CI} 95 \%$ : 1.46-4.84), high population density ( $\mathrm{OR}=4.35$; $\mathrm{CI} 95 \%$ : 2.298.22), and high GDP per-capita (OR=2.26; CI95\%: 1.27-4.02).

Conclusions: There was little evidence for a top-down development of tobacco control policy in Indonesia. Provincial tobacco control policy seemed to have stimulated local policies only if they were fully developed. Trends at local levels followed instead a diffusion 
pattern, with local tobacco control policies starting in clusters of areas with higher population density or economic development.

Tob. Induc. Dis. 2018;16(Suppl 1):A154 DOI: $10.18332 /$ tid/83871

\section{$12: 30-14: 00$}

PS-600-3 Small initiative, big success

H Ahmed $^{1,2}$, S Ahmed ${ }^{2,3}$, A Islam Sujon ${ }^{3}, 4$

1 Pratysha Anti Drug's Club, Tobacco Control, Dhaka, Bangladesh, ${ }^{2}$ Bangladesh Anti Tobacco Alliance (BATA), Policy Advocacy, Networking, Capacity Building, Dhaka, Bangladesh, ${ }^{3}$ Work for a Better Bangladesh (WBB) Trust, Health Rights and Tobacco Control, Dhaka, Bangladesh, ${ }^{4}$ Bangladesh Anti Tobacco Alliance (BATA), Communication and Policy Advocacy, Dhaka, Bangladesh. E-mail: pratyashaclub@gmail.com

Background and challenges to implementation: We often believe that tobacco control requires major resources. We certainly do need resources, but some important successes can be achieved even with very moderate resources, if we have the right combination of access, hard work, and strategic behaviour. In Bangladesh there are still major gaps to the implementation of the tobacco control law, specifically smoke-free places.

Intervention or response: Pratyasha Anti-Drug Club is a youthbased NGO with almost no funding but a large membership. We also have had a good relationship with the City Corporations of Bangladesh's capital city, Dhaka, with about 18 million inhabitants. We convinced the city corporations to give us permission to place smoke-free signboards at different major places visited by large numbers of people, such as the national zoo, national library, children parks, garden and a local fort/park. We also convinced restaurants throughout the city to place smoke-free signs on their premises and to remove all ashtrays.

Results and lessons learnt: A number of small and large venues have become smoke-free as a result of our initiative. With a population of about 15 million people, our initiative is likely to have reached over a million people. This is a huge success especially given the lack of funding behind it.

Conclusions and key recommendations: It is vital to the longterm sustainability of tobacco control that we succeed in gaining sustainable funding, particularly through a surcharge on tobacco products. But it is also important to remember that not all activities require major funding in order to succeed. We have shown that agile and strategic networking can achieve important successes.

Tob. Induc. Dis. 2018;16(Suppl 1):A155

DOI:10.18332/tid/83900

\section{2:30-14:00}

PS-601-3 What kind of factors influence the effective enforcement of smoke-free law in China? J Yang ${ }^{1}, \mathrm{Z} \mathrm{Xi}^{1}, \mathrm{X} \mathrm{Zhu}^{1}, \mathrm{X}$ Luo

'China CDC, Beijing, China. E-mail: bjyangjie@163.com

Background: As WHO FCTC took effect in China, more and more cities have legislated or amended laws against smoking in public places. There still existed many kinds of barriers and problems about the law enforcement. This study focused on the activities took by enforcers and owners of places to evaluate situations that influence the effect of laws and regulations against SHS.

Methods: This study combines methods of interviews and field observation. Study of compliance of net bars: 20 net bars at each city were observed.84 observations were accomplished. Invited 15 officers, 30 enforcers and 14 owners of net bar. Focus group and in-depth interviews were conducted at five cities.

\section{Results:}

1. Smoking behaviors were found in 69 net bars, attendants from only 2 bars discouraged smokers but failed. Smoking ban in bars didn't improve no-smoking environments in the bars.

2. City supervision and enforcement environments need to be improved. Comprehensive and partial smoke-free, penalty process and amounts bring about barriers in enforcement activities. The enforcement coordination mechanism and enforcement pattern is limited by authority, function, initiative and focus of enforcers.

3. Various factors influenced owners to take responsibility in the enforcement of law, et al, afraid of losing customers and incomes for owners; Lack of knowledge of hazards of SHS.

Conclusions: Not all of the net bars comply with the law. Power of the coordination, initiation and knowledge of hazards of SHS of the enforcers, all of these influence power of enforcement of net bars. Failure of creating social atmosphere of smoking ban before and after the law impairs effect of enforcement of the law.

Recommendation: Amending the law to achieve a comprehensive smoke in the indoor public places and develop a detailed enforcement guideline; building effective supervision mechanism, and mobilizing initiation of the enforcers; completing social supervision mechanism and motivating the public.

Tob. Induc. Dis. 2018;16(Suppl 1):A156

DOI: $10.18332 /$ tid/83918

\section{2:30-14:00}

PS-602-3 Tobacco control policies and perinatal and child health: a systematic review and meta-analysis T Faber ${ }^{1,2}$, A Kumar ${ }^{3}$, JP Mackenbach ${ }^{2}$, C Millett ${ }^{4}$, S Basu ${ }^{5}$, A Sheikh ${ }^{3,6,7}$, JV Been ${ }^{1,3,8}$

${ }^{1}$ Erasmus University Medical Centre - Sophia Children's Hospital, Division of Neonatology, Department of Paediatrics, Rotterdam, Netherlands, ${ }^{2}$ Erasmus University Medical Centre, Department of Public Health, Rotterdam, Netherlands, ${ }^{3}$ University of Edinburgh, Centre of Medical Informatics, Usher Institute of Population Health Sciences and Informatics, Edinburgh, United Kingdom, ${ }^{4}$ Imperial College London, Public Health Policy Evaluation Unit, School of Public Health, London, United Kingdom, ${ }^{5}$ Stanford University, Prevention Research Center, Stanford, CA, United States of America, ${ }^{6}$ Brigham and Women's Hospital/Harvard Medical School, Division of General Internal Medicine and Primary Care, Boston, MA, United States of America, ${ }^{7}$ Harvard Medical School, Department of Medicine, Boston, MA, United States of America, ${ }^{8}$ Erasmus University Medical Centre - Sophia Children's Hospital, Department of Obstetrics and Gynaecology, Rotterdam, Netherlands. E-mail: t.faber.1@erasmusmc.nl

Background: Tobacco smoking and smoke exposure during pregnancy and childhood cause considerable childhood morbidity and mortality. We aimed to determine whether implementation of the World Health Organization's recommended tobacco control policies (MPOWER) were of benefit to perinatal and child health. Methods: We searched 19 electronic databases, hand-searched references and citations, and consulted experts to identify (quasi-)experimental studies assessing the association between implementation of MPOWER policies and child health. Our primary outcomes of interest were: perinatal mortality, preterm birth, hospital attendance for asthma exacerbations, and hospital attendance for respiratory tract infections (RTIs). Where possible and appropriate, we combined data from different studies in random-effects meta-analyses.

Results: We identified 41 eligible studies that assessed (combinations of) MPOWER policies: smoke-free legislation 
( $\mathrm{n}=35)$, tobacco taxation $(\mathrm{n}=11)$, and smoking cessation services $(n=3)$. Following implementation of smoke-free legislation, rates of preterm birth decreased by $-3.77 \%$ (10 studies, 27,530,183 individuals; $95 \% \mathrm{CI}-6.37$ to -1.16 ), hospital attendance for asthma exacerbations decreased by $-9.83 \%$ (five studies, 684,826 events; 95\%CI-16.62 to-3.04), and hospital attendance for RTIs decreased by $-3.45 \%$ (two studies, 1,681,020 events; $95 \% \mathrm{CI}-4.64,-2.25$ ) for all RTIs, and by $-18.48 \%$ (three studies, 887,414 events; $95 \% \mathrm{CI}$ -32.79 to -4.17 ) for lower RTIs. Associations appeared to be stronger when comprehensive smoke-free laws were implemented. Among two studies assessing the association between smoke-free legislation and perinatal mortality, one demonstrated significant reductions in stillbirth and neonatal mortality. Meta-analysis of studies on other MPOWER policies was not possible; all four studies on increasing tobacco taxation and one of two on offering disadvantaged pregnant women help to quit smoking that reported on our primary outcomes had positive findings.

Conclusions: Smoke-free legislation is associated with substantial child health benefits. The majority of studies on other MPOWER policies also indicated positive impact. These findings provide strong support to implement such policies comprehensively across the globe.

Tob. Induc. Dis. 2018;16(Suppl 1):A157

DOI:10.18332/tid/83921

\section{$12: 30-14: 00$}

PS-603-3 Results from unannounced visits to check compliance with smoke-free regulations in three types of indoor places in Beijing

$\mathrm{S} \mathrm{Wu}{ }^{1}, \mathrm{H} \mathrm{Mei}^{1}, \mathrm{Z} \mathrm{Liu}{ }^{1}$

'Office of Beijing Patriotic Health Campaign Committee, Beijing, China.E-mail: b12071207@qq.com

Background: Beijing Tobocco control regulation come into force in 2015. According to the regulation, all indoor areas are smoke free. To assess compliance with the Beijing Regulations, identify weaknesses and problems, and provide scientific evidence for policy making by the government and other authorities.

Methods: on site, unannouncedvisits. Weconducted unannounced visits to a sample of 821 places in three types of places across the sixteen districts of Beijing, including medical and health facilities (including health and family planning commissions, CDCs, health inspection institutes and a variety of hospitals), colleges (including vocational schools) and taxis (including Shouqi Group vehicles booked online).

Results: Among medical and health facilities, all posted smokefree signage, and no cigarette end or smoking paraphernalia was found indoors, except in private hospitals, community health service centers and few maternal and child care centers. Among colleges, $96.9 \%$ of them posted smoke-free signage, cigarette end was found indoors in $9.9 \%$ of colleges, smell of smoking was detected indoors in $1.9 \%$ of colleges, and ashtray was found indoors in $0.6 \%$ of colleges. Among taxies, $6 \%$ of drivers of online booked vehicles permit passengers to smoke inside their car, and no smell of smoking, container of cigarette ends/ashes, or driver smoking was detected in these online booked vehicles. Among the conventional cruising taxis, $48.4 \%$ of taxi drivers permit passengers to smoke inside their car, smell of smoking was found in $9.6 \%$ of taxies, and $1.6 \%$ of taxi drivers smoke in their car.

Conclusions: Smoking in violation of the Regulations is serious in the taxi sector, and targeted stronger supervision and administration are needed.

Tob. Induc. Dis. 2018;16(Suppl 1):A158

DOI: $10.18332 /$ tid/83953
12:30-14:00

PS-604-3 A pre-post evaluation of and public support for smoke-free policies at the 2016 Rio Olympics: findings from the ITC Brazil survey, $2012-17$

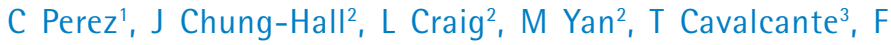
Mendes $^{3}$, A Szklo ${ }^{3}$, GT Fong ${ }^{2,4}$

${ }^{1}$ Fundação do Câncer, Rio de Janeiro, RJ, Brazil, ${ }^{2}$ University of Waterloo, Waterloo, ON, Canada, ${ }^{3}$ Brazilian National Cancer Institute, Rio de Janeiro, RJ, Brazil, ${ }^{4}$ Ontario Institute for Cancer Research, Toronto, ON, Canada.E-mail:cp772017@gmail.com Background: Large-scale international sporting events such as the Olympic Games provide opportunities for hosting nations to promote smoke-free environments. The Olympics have been tobacco-free since 1998, but there have been few studies on the effectiveness of smoke-free Olympic policies. This study evaluated the effectiveness of smoke-free policies at the 2016 Rio Olympics, which were supported by Brazil's 2014 comprehensive smoke-free law, and measured public support for smoke-free Olympic venues.

Methods: Data were from Waves 2-3 of the ITC Brazil Survey, a cohort survey of approximately 1200 adult smokers and 600 nonsmokers in Rio de Janeiro, São Paulo, and Porto Alegre. Wave 2 (2012-13), conducted before the 2014 smoke-free law and 2016 Olympics provided the pre-policy data, and Wave 3 (2016-17) provided the post-policy data. Analyses employed GEE logistic regression models.

Results: Of 1826 respondents surveyed in 2016-17, 116 (9.9\%) smokers and 57 (14.8\%) non-smokers attended the Olympics. The vast majority who attended at least one indoor event reported that smoking was banned inside venues (97.5\% smokers, $93.0 \%$ non-smokers); and noticed no-smoking signs inside venues (82.2\% smokers, $73.1 \%$ non-smokers). Only $10.7 \%$ of smokers and $7.2 \%$ of non-smokers noticed people smoking inside venues; $5.8 \%$ of smokers said they personally smoked at an indoor venue. Following the 2014 smoking ban and 2016 Olympics, support for the indoor smoking ban in Olympic venues increased among smokers $(79.2 \%$ to $93.3 \%, \mathrm{p}<.001)$ and non-smokers $(86.4 \%$ to $94.1 \%, \mathrm{p}<.001)$.

Conclusions: There was strong implementation of and high compliance with Brazil's comprehensive smoking ban at the 2016 Rio Olympics. Smoke-free Olympic venues were widely supported by the public before the Games, and increased after the Games. It would be beneficial for the upcoming 2020 Tokyo Olympics organizers to consult with Rio Olympics organizers to maximize the effectiveness of any efforts to make the Tokyo Olympics smoke-free.

Tob. Induc. Dis. 2018;16(Suppl 1):A159

DOI:10.18332/tid/83964

\section{2:30-14:00}

PS-605-3 Impact of the smoke-free legislation on the incidence and mortality of AMI and stroke in Tianjin China: analysis of routinely collected data $\mathrm{H} \mathrm{Xiao}^{1}$, G Yang ${ }^{2}$, X Wan ${ }^{2}$, Y Liư ${ }^{2}, \mathrm{M} \mathrm{Naghavi}{ }^{3}$

'University of Washington, Global Health Department, Seattle, WA, United States of America, ${ }^{2}$ Institute of Basic Medical Sciences, Chinese Academy of Medical Sciences and School of Basic Medicine, Peking Union Medical College, Beijing, China, ${ }^{3}$ Institute for Health Metrics and Evaluation, Seattle, WA, United States of America. E-mail: wanxiasnake@163.com

Background: Comprehensive smoke-free legislation is an effective way to protect the population from the harms of secondhand 
smoke (SHS) and has been implemented in many countries. Tianjin is one of the few cities in China that have passed smoke-free legislation. We investigated the impact of smoke-free legislation on acute myocardial infarction (AMI) and stroke in Tianjin.

Methods: An interrupted time series design adjusting for underlying secular trend, seasonal pattern, population size changes and meteorology factors was conducted to analyze the impact of the smoking free law on the weekly incidence and mortality of AMI and stroke. The study period was from January 1, 2007, to December 31, 2015 , with a post-ban follow-up of 3.5 years.

Results: Following the smoke-free law implementation, there was a decline in the annual proportional change for AMI and stroke mortality. A gradual 16\% (RR:0.84; 95\% CI: 0.83-0.85) decrease per year in AMI mortality among in population ages $\geq 35$ years and a gradual $4 \%$ (RR:0.96; 95\% CI: 0.95-0.98) decrease per year in stroke mortality among 35-64 age group in Tianjin was observed. Post-ban stroke incidence immediate reductions were found in in stroke in 35-64 age group (RR:0.96; 95\% CI: 0.950.98). AMI incidence immediate reductions were only found in females aged 35-64 (RR:0.71; 95\% CI: 0.65-0.78).

Conclusions: The smoke-free law in Tianjin was associated with gradual reductions in AMI and stroke mortality and immediate reductions in $\mathrm{AMI}$ and stroke incidence. The considerably modest and inconsistent effect on AMI and stroke incidence probably reflects the weak law enforcement and limitation of incidence surveillance data in Tianjin. This study reinforces the need for large-scale, effective and comprehensive smoke-free law in China. Funding: This research was supported by China Medical Board (15-208)

Tob. Induc. Dis. 2018;16(Suppl 1):A160

DOI:10.18332/tid/83971

\section{2:30-14:00}

PS-606-3 Strengthening tobacco-free worksite policies in India

S Bassi ${ }^{1}$, D Bahl ${ }^{1}, \mathrm{~N} \mathrm{Hundal}^{2}, \mathrm{H} \mathrm{Wipfli}^{2}, \mathrm{M} \mathrm{Arora}^{1}$

${ }^{1}$ Public Health Foundation of India, Health Promotion Division, Gurugram, India, ${ }^{2}$ University of South California, USC Institute for Global Health, Los Angeles, CA, United States of America. E-mail: shalini.bassi@phfi.org

Background: The International Labor Organization identifies tobacco use as workplace health hazard. The workplaces are key locations for implementing health promotion wellness initiatives. Tobacco-free workplace policies curtail tobacco use, prevent exposure to tobacco smoke, and increase cessation attempts among employees. The aim of this study (2016) was to generate evidence to inform corporate health and cancer prevention programmes.

Methods: The study was conducted with 10 worksites (manufacturing, technology and health care); size 250-5000 employees, purposively selected from 3 Indian cities (Delhi, Bangalore and Chennai). Semi-structured interviews were conducted with Human Resource Managers/Medical Officers. All interviews were digitally recorded, transcribed and coded using Dedoose.

Results: The focus of the programs was on disease management, nutrition, physical activity, stress management and smoking. Smoking was most prevalent form of tobacco use amongst employees and commonly used places for smoking were staircases/ parking areas. Almost all worksites had smoke-free policies and without provision of formal/informal designated smoking areas. Medical check-ups, talks on consequences of tobacco use, nosmoking runs, smoking cessation camps and counselling sessions represented some of the wellness activities offered by the worksites. Restriction on the use of smokeless tobacco (SLT) or support to quit SLT was not covered under any policies, despite a large number of SLT users. Almost all workplace did not have a cessation programs. One workplace had a counselor for smokers and two workplaces had cancer screening programs for women. Returns on investments was considered to be a motivation for the corporates for implementing these programs. Low levels of employees' participation were considered as a barrier.

Conclusions: The wellness program at worksites should be need-based and driven by data on consumption patterns. Increasing the knowledge about the health harms, providing cessation support and incentives could be the strategies for improving employees' participation.

Tob. Induc. Dis. 2018;16(Suppl 1):A161 DOI:10.18332/tid/83983

$12: 30-14: 00$

PS-607-3 The long road to smokefree bars in the Netherlands: findings from the ITC Netherlands Survey 2008-2016

G Nagelhout ${ }^{1,2}$, DJ van Mourik², K Hummel ${ }^{2}$, M Willemsen $^{2}, \mathrm{H}$ de Vries ${ }^{2}$, B van den Putte ${ }^{3,4}, \mathrm{G}$ Fong ${ }^{5,6}$

${ }^{1}$ IVO Addiction Research Institute, Rotterdam, Netherlands, ${ }^{2}$ Maastricht University, Maastricht, Netherlands, ${ }^{3}$ University of Amsterdam, Amsterdam, Netherlands, ${ }^{4}$ Trimbos Institute, Utrecht, Netherlands, ${ }^{5}$ University of Waterloo, Waterloo, $0 \mathrm{~N}$, Canada, ${ }^{6}$ Ontario Institute for Cancer Research, Toronto, ON, Canada.E-mail: gera.nagelhout@maastrichtuniversity.nl

Background: The Netherlands implemented smokefree legislation in July 2008. However, this legislation was reversed and reimplemented for small bars without employees several times. Since October 2014, the smokefree legislation applies to all bars again, but not all of them comply and designated smoking rooms are still allowed.

Methods: A nationally representative sample of adult smokers from the Netherlands was interviewed each year between 2008 and 2016 as part of the International Tobacco Control (ITC) Survey ( $n=1246-1773$ per year). Trends in perceptions of rules about smoking in bars they visited, seeing people smoke in bars during the last visit, support for smokefree bars, and social acceptance of smoking in bars were examined with GEE analyses. Results: There was a significant increase in smokers reporting that bars they visited had a total smoking ban (9\% in 2008 to $45 \%$ in 2016), which was more often reported by older and high income smokers. Between 10 and $18 \%$ of smokers reported that they did not know the rules about smoking in bars; this was more often reported by older smokers. Reports of having seen people smoking in bars during the last visit decreased from $93 \%$ to $19 \%$. Support for smokefree bars doubled (20\% to $43 \%$ ) and was higher among older and high educated smokers. Social acceptance of smoking in bars decreased (75\% to $41 \%$ ) and was higher among younger and low income smokers.

Conclusions: Eight years after the implementation of smokefree legislation in bars, smoking in bars has decreased but is far from eliminated in the Netherlands. Educational campaigns targeted at younger and lower socioeconomic status smokers are needed to inform these groups about the (need for) rules about smoking in bars. Now that only a minority of smokers think it is still acceptable to smoke in bars, the Dutch government should consider banning designated smoking rooms too.

Tob. Induc. Dis. 2018;16(Suppl 1):A162

DOI:10.18332/tid/83985 


\section{$12: 30-14: 00$}

PS-608-3 Promoting tobacco control law enforcement in China through applications of "Government Information Disclosure" and media advocacy

Z Wang ${ }^{1}, \mathrm{E} \mathrm{Li}{ }^{1}, \mathrm{X} \mathrm{Li}{ }^{2}, X \mathrm{Yin}^{3}, \mathrm{XYu^{2 }}$

${ }^{1}$ Beijing Impact Law Firm, Beijing, China, ${ }^{2}$ Campaign for TobaccoFree Kids, Beijing, China, ${ }^{3}$ Campaign for Tobacco-Free Kids, Washington, WA, United States of America. E-mail:wzy0594@ vip.sina.com

Background and challenges to implementation: China has not a national law on tobacco control. Since FCTC came into force in China, 18 cities have enacted smoke-free laws or policies and an increasing number of cities and even provinces are planning to adopt such policies. Strong enforcement is lacking in some of the cities for such reasons as lack of funds and incentives, or competing priorities of the local governments. Monitoring and promoting effective implementation of smokefree policies at the subnational level is important to enhance the smoke-free movement and decrease exposure to secondhand smoke in China.

Intervention or response: In 2015, Beijing Impact Law Firm (BILF) began to obtain details regarding enforcement of smoke-free policies from local governments, based on Regulations on the Openness of Government Information. Through analysis of the local smoke-free law or policy, BILF sent a list of questions regarding the enforcement mechanism, amount of fines collected, number of venues inspected, etc. This was the first time for the civil society in China to use "Government Information Disclosure" to strengthen tobacco control work. BILF developed a report ranking the performance of all these cities in enforcing the smoke-free policy along with suggestions to the local governments. BILF then released the report to the media, which has generated wide media coverage and pressured the local governments to improve their performance.

Results and lessons learnt: The release of the reports has generated public attention to the smoke-free work in their cities. The local governments do not want to be the bottom on the ranking list and have taken actions to improve their efforts in implementing the smoke-free policy.

Conclusions and keyrecommendations: Effectiveimplementation of smoke-free policies is important to create public health benefits and enhance the tobacco control movement. The civil society should make full use of existing policies or mechanisms such as "Government Information Disclosure" to monitor and promote tobacco control work.

Tob. Induc. Dis. 2018;16(Suppl 1):A163

DOI:10.18332/tid/83997

\section{2:30-14:00}

PS-609-3 Do European smokers opt for partial or total bans on smoking in homes and cars? Findings from the ITC 6 European Country Survey (EUREST-PLUS Project)

O Tigova ${ }^{1,2}$, Y Castellano ${ }^{1,2}$, M Fu ${ }^{1,2}$, GT Fong ${ }^{3,4}$, ACK Quah ${ }^{3}$, C Vardavas ${ }^{5,6}$, E Fernández ${ }^{1,2}$, on behalf of the EUREST-PLUS and ITC Projects

${ }^{1}$ Catalan Institute of Oncology (ICO), L'Hospitalet de Llobregat, Spain, ${ }^{2}$ Bellvitge Biomedical Research Institute (IDIBELL), L'Hospitalet de Llobregat, Spain, ${ }^{3}$ University of Waterloo, Waterloo, ON, Canada, ${ }^{4}$ Ontario Institute for Cancer Research, Toronto, ON, Canada, ${ }^{2}$ University of Crete, Rethimno, Greece, ${ }^{6}$ European Network for Smoking and Tobacco Prevention (ENSP), Brussels, Belgium. E-mail: otigova@iconcologia.net
Background: While smoke-free policies in most European countries are established for public places, private areas are mainly not regulated and may represent an important source of second-hand smoke exposure. We aimed to describe and characterise smoking rules in homes and cars of smokers from 6 European countries.

Methods: We used data from the first wave (2016) of the International Tobacco Control (ITC) 6 European Country Survey, part of the EUREST-PLUS Project, in representative national samples of adult (18+ years) smokers in Germany, Greece, Hungary, Poland, Romania and Spain (1000 in each country). We analysed questions about smoking rules in smokers' homes and cars with children and differentiated among: no rules, partial (allowed in some rooms or under special circumstances) and total ban (smoke-free homes and cars). We computed the prevalence (\%) of homes and cars according to the rules, and used Poisson regression models to identify factors associated with total smoking ban. We incorporated weights from the complex sample design.

Results: In homes, more smokers opted for partial ban rather than for a total ban. The highest and lowest proportions of smokefree homes were found in Hungary (35.5\%) and Spain (13.1\%), respectively, while partial ban prevalence ranged from $41.3 \%$ (Spain) to $49.9 \%$ (Greece). The proportion of smoke-free cars with children was overall higher compared to homes, ranging from $51.8 \%$ (Greece) to $67.7 \%$ (Germany). Having moderate or high level of education, presence of children, smoking less than 10 cigarettes per day and having previous attempts to quit smoking were positively associated with total smoking ban in homes and cars.

Conclusions: European smokers tend to opt for partial smoking ban at homes and for total ban in cars with children. Prevalence of smoke-free homes is relatively low and further legal and educational enforcement is needed to promote smoke-free environments.

Funding: EC Horizon2020 HCO-6-2015 (EUREST-PLUS No. 681109).

Tob. Induc. Dis. 2018;16(Suppl 1):A164 DOI: $10.18332 /$ tid/84004

$12: 30-14: 00$

PS-610-3 Togo smokefree compliance monitoring in public places after 3.5 years of implementation of the law (2017)

F Ebeh'1, K Walter ${ }^{2}$, K Odji1, S Allah'1, S Mourtala', S Uzzell ${ }^{3}$, B Camara $^{4}$, E Sebrie ${ }^{2}$

'Alliance Nationale des Consommateurs \&t de l'Environnement (ANCE-TOGO), Lome, Togo, ${ }^{2}$ Campaign for Tobacco-Free Kids, International Research, Washington, DC, United States of America, ${ }^{3}$ Campaign for Tobacco-Free Kids, International Grants, Washington, DC, United States of America, ${ }^{4}$ Campaign for Tobacco-Free Kids, International Advocacy, Washington, DC, United States of America. E-mail: esebrie@tobaccofreekids.org Background: Togo's 2010 tobacco control law bans smoking in indoor public places, workplaces, and means of public transport except in hotels, restaurants, bars, and public transport stations, where designated smoking areas (DSAs) are permitted. The objective of this study was to monitor the level of compliance with the smokefree law in four key public places after 3.5 years of implementation.

Methods: Monitoring was conducted in Lomé, Sokodé, and Atakpamé during February- March 2017. Data was collected from a convenience sample $(n=383)$ of four types of public places: bars, hotels, restaurants, and transport buildings. An 
observational checklist was used to collect data on the following law requirements: (1) no persons smoking any tobacco products indoors; (2) no indirect indicators of smoking (presence of ashtrays, cigarette butts) visible indoors; (3) presence of DSAs with all requirements; (4) presence of No Smoking signage (at entrance and inside the venues); and (5) presence of No Smoking signage with all requirements.

Results: The level of smokefree compliance (no person smoking any tobacco products indoors) in the public places was high overall (84\%). No indirect indicators of smoking were observed indoors in $81 \%$ of the venues. Only one venue had a DSA, which was not compliant with all requirements. The level of compliance with signage was very low. No Smoking signs were observed in only $6 \%$ of the venues at the entrance and $16 \%$ inside. Signage that met all law requirements was present in only $4 \%$ of the venues at the entrance and $9 \%$ inside. The level of compliance by type of venue is shown in the table.

Conclusions: Togo has made progress in implementing the smokefree provisions of their tobacco control law, especially in restaurants and hotels. However, authorities should actively monitor and enforce the law, particularly in transport buildings where the level of compliance was much lower.

\section{Tob. Induc. Dis. 2018;16(Suppl 1):A165}

DOI:10.18332/tid/84058

[Level of compliance with SF law, by venue]

\begin{tabular}{|c|c|c|c|c|c|}
\hline & BARS (n-185) & $\begin{array}{l}\text { RESTIURANTS } \\
\qquad(\text { (n-82) }\end{array}$ & IIOTELS $(n-98)$ & $\begin{array}{l}\text { TRA I NSPORT } \\
\text { BL II DINGS (n-18) }\end{array}$ & TOTAL (n-383) \\
\hline No smoking occurring indoors & $84.3 \%$ & $86.6 \%$ & $88.8 \%$ & $50.0 \%$ & $84.3 \%$ \\
\hline $\begin{array}{l}\text { No indirect indicators of smoking } \\
\text { (ashtrays, butts) }\end{array}$ & $76.2 \%$ & $86.6 \%$ & $91.8 \%$ & $50.0 \%$ & $81.2 \%$ \\
\hline $\begin{array}{l}\text { No Smoking sign at entrance with } \\
\text { all requirements }\end{array}$ & $1.1 \%$ & $4.9 \%$ & $8.2 \%$ & $5.6 \%$ & $3.9 \%$ \\
\hline No Smoking sign inside with all & $2.2 \%$ & $9.8 \%$ & $21.4 \%$ & $5.6 \%$ & $8.9 \%$ \\
\hline
\end{tabular}
requirements

\section{2:30-14:00}

PS-611-3 Smoking ban in public places in Japan - adverse legacy of the 2020 Olympic Paralympic Games?

\section{K Katanoda ${ }^{1}$, T Hirano ${ }^{1}$, M Nakamura ${ }^{2}$}

${ }^{1}$ National Cancer Center, Tokyo, Japan, ${ }^{2}$ Japan Association for Development of Community Medicine, Tokyo, Japan. E-mail: kkatanod@ncc.go.jp

Background: Although Japan is a nation where the world first epidemiological evidence on the health effect of secondhand smoke was produced, no indoor smoking ban was realized on a national level. In 2013, Tokyo was selected as the city of 2020 Olympic Paralympic Games, since when the Japanese Ministry of Health, Labour and Welfare (MHLW) and various concerned parties have been working to realize smoking ban in public places. However, as of July, 2017, the legislation has been unsuccessful. We chronologically reviewed the related events to clarify underlying obstacles to the legislation.

Methods: Events related to the movement of the national smoking ban in Japan were extracted from the internet and public available data sources. The concerned bodies were classified into five categories (academic/medical, administration, politics, industry, and advocacy), and chronological relations were analyzed.

Results: Academic/medical and administrative bodies closely collaborated and effectively released scientific rationale of smoking ban in 2016. The MHLW's draft policy released in Mar. 2017 included an exception of smoking ban for restaurants and bars (allowing designated smoking rooms). However, even this partial banning policy was faced with fierce objections from the allied industry including restaurant, hotel businesses and tobacco industry. Diet members in the ruling Liberal Democratic Party acted in a coordinated manner; reportedly $90 \%$ of the Diet members attending the party committee on health, labour and welfare objected to the MHLW's draft policy. The allied industry spread a signature collecting activity earlier and much more organizationally than academic/medical and advocacy groups.
The party committee of the LDP was held only twice. Although the MHLW proposed a temporary amendment, the committee failed to reach an agreement.

Conclusions: The series of events suggest that the tobacco lobbies are more powerful in the policy-making process in Japan than tobacco control advocates.

\section{Tob. Induc. Dis. 2018;16(Suppl 1):A166 DOI:10.18332/tid/84166}

\section{$12: 45-14: 00$}

EP-115-3 Youth exposure to second hand smoke: a study among secondary school students in public schools in an urban district in Lagos state Nigeria S Marinze1, 0 Ayankogbe², 0 Abiola², 0 Odukoya² ${ }^{1}$ College of Medicine, University of Lagos, Medicine and Surgery, Lagos, Nigeria, ${ }^{2}$ College of Medicine, University of Lagos, Community Health and Primary Care, Lagos, Nigeria. E-mail: marinzesheilaamanda@gmail.com

Background: Many non-smokers die annually as a result of exposure to second hand smoke. School going adolescents are often understudied but may be at increased risk.

Methods: A descriptive cross-sectional study was carried out among 353 students in four schools in Ajeromi Ifelodun an urban district in Lagos state, using a multi-stage sampling technique. Data was collected using a self administered questionnaire adapted from the global youth tobacco survey and analysed using Epi Info version 7.

Results: Of the respondents $51.8 \%$ were males and $48.2 \%$ females, their ages ranged from 12-20 years. More than half of the respondents (54.4\%) had good knowledge of the associated health risks of second hand smoke exposure. Majority were in favour of banning smoking in restaurants (64.6\%), clubs (64\%), and outdoor public places (62.6\%). Overall, $23.8 \%$ were exposed to second hand smoke at home, while $64.6 \%$ and $71.1 \%$ were exposed to second hand smoke in enclosed and outdoor public places respectively. 
Conclusions: Despite the passage of national and state wide anti smoking laws, the level of exposure of these students to second hand smoke was still high particularly in public places. This highlights the need for urgent efforts to enforce smoke free policies to protect young people from second hand smoke exposure.

Tob. Induc. Dis. 2018;16(Suppl 1):A167

DOI:10.18332/tid/84688

$12: 45-14: 00$

EP-116-3 Secondhand smoke exposure at home in China

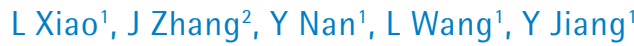

${ }^{1}$ China CDC, Tobacco Control Office, Beijing, China, ${ }^{2}$ Peking University, School of Public Health, Beijing, China. E-mail: xiaolin201304@126.com

Background: China took great efforts to protect people from secondhand smoke (SHS), including health education, smokefree environment creating and smoke-free law promotion. Now, more and more people support smoke-free in public places. This study aims to assess secondhand smoke (SHS) exposure at home and to examine factors associated with it in China.

Methods: A cross-sectional study was conducted among Chinese aged 15 years and above. Using a multistage complex survey design, 336 counties/districts (PSUs) were selected nationwide. In total, 15,095 individuals completed the survey and the response rate was $92.2 \%$. Data were weighted and analyzed by SPSS 20.0 complex survey data analysis program, and Logistic regression was used for exploring potential factors associated with SHS exposure at home.

Results: The proportion of nonsmokers exposed to SHS at home was $46.7 \%$ (37.4\% for male and $51.4 \%$ for female). Among female respondents, $41.9 \%$ reported being exposed to SHS every day in the last 30 days. SHS exposure rate was highest among primary (50.8\%) and middle school (53.4\%), followed by high school (46.8\%), college and above was the lowest (30.2\%). The rate also decreased as respondents' age goes up, nonsmokers in the age group 15-24, 25-44, 45-64, 65 years old and above had $55.8 \%, 45.5 \%, 44.0 \%$ and $38.8 \%$ SHS exposure rate respectively. Moreover, the rate is higher in rural area (57.5\%) than in urban area (36.9\%). Nonsmoker exposed to SHS at workplace had higher SHS exposure rate at home than those who did not exposed at workplace. $(\mathrm{OR}=1.40,95 \% \mathrm{CI}: 1.10-1.79)$.

Conclusions: SHS exposure at home is a serious public health problem, especially for women. Lower education, younger people, and those in rural area are more likely to be exposed to SHS at home. $100 \%$ smoke-free policy in workplaces might be helpful to reduce SHS exposure in home.

Tob. Induc. Dis. 2018;16(Suppl 1):A168

DOI: $10.18332 /$ tid/83935

\section{$12: 45-14: 00$}

EP-117-3 Exposure to secondhand smoke and intention to quit among South African smokers during 2007-2016: implications for policy

J Jakavula ${ }^{1}, 0$ Ayo-Yusuf ${ }^{1}$

'Sefako Makgatho Health Sciences University, Africa Center for Tobacco Industry Monitoring and Policy Research, Pretoria, South Africa. E-mail: jakavulaj@gmail.com

Background: Although smoke-free public places are an important population-wide intervention to protect non-smokers from the harm of smoking and to encourage smokers to quit, others suggest it may increase smoking in homes. This study therefore sought to determine exposure to secondhand smoke (SHS) at home and in public places before and after 2009 when an amendment to legislation that introduced significant increase in fines came into effect. The study also sought to explore the association between SHS exposure and smokers' intention to quit.

Methods: This cross-sectional study involved three nationally representative samples of South African adults aged $\geq 16$ years who participated in the $2007(n=2,907), 2010(n=3,112), 2016$ $(=3,063)$ South African Social Attitude Survey. Information obtained using interviewer-administered questionnaire included socio-demographic characteristics and self-reported exposure to tobacco smoking at home and public places (work, bars/clubs, and restaurants). The three datasets were merged (Pooled $\mathrm{N}=$ 9,082 ). Data analysis included chi-square statistics and multiple logistic regression analysis.

Results: During 2007-2016, the proportion of South Africans who reported exposure to SHS at home on any one day increased from $31.9 \%$ in 2007 to $36.6 \%$ in $2016(p=0.637)$, while exposure on any one day at any one of the various public places decreased significantly from $71.9 \%$ to $54.4 \%(\mathrm{p}<0.001)$, albeit significantly only between 2010 and 2016. Compared to smokers exposed to others' smoking, those not exposed were more likely to report planning to quit $(\mathrm{OR}=1.73 ; 95 \% \mathrm{CI}=1.07$ 2.79). However, after controlling for the number of cigarettes smoked daily, exposure to others' smoking in public was no longer significant, thus suggesting that the decreasing number of cigarettes smoked daily was the mediator of the effect of restricting public smoking.

Conclusions: While our findings suggest that the most recent legislative amendment related to public smoking seem to have reduced SHS exposure, further restrictions, which may motivate quitting, are still needed.

Tob. Induc. Dis. 2018;16(Suppl 1):A169

DOI:10.18332/tid/84689

\section{$12: 45-14: 00$}

EP-118-3 Building on the success of ten years of comprehensive smokefree legislation in England A Rutter ${ }^{1}$, A Lloyd ${ }^{1}$, C Taylor $^{1}$, L Surtees $^{1}$

'Fresh, Durham, United Kingdom. E-mail: catherine.taylor@ freshne.com

Background and challenges to implementation: England's comprehensive smokefree law (workplaces and enclosed public places), introduced in 2007, has been effective in protecting public health and has enjoyed increasing public support ( $86 \%$ in the $\mathrm{NE}$ in 2017) and consistent high compliance. The decade since has seen continued focus in the North East of England around protection from secondhand smoke, normalising smokefree spaces and building public support through a social movement around 'Making Smoking History'.

Intervention or response: Working with partners in local government and the health service, Fresh - the NE's regional comprehensive tobacco control programme - has delivered sustained activity to reinforce the importance of protection from secondhand smoke including training of front line staff and also voluntary policies around smokefree outdoor areas frequented by children, lobbied for further evidence based legislation whilst highlighting the ultimate aim of encouraging smokers to quit.

Results and lessons learnt: New regulations were introduced in 2015 to extend the smokefree law to cover private vehicles carrying children under 18 (91\% NE public support). With $40 \%$ 
of NE smokers reporting they still smoke in the home, the need for ongoing education is clear and in 2017 Fresh ran a "secondhand smoke is poison" campaign with a hard hitting message around taking smoking right outside the home and the importance of protecting children from exposure.

Conclusions and key recommendations: The success of the smokefree law has led the way in increasing public support for existing and potential tobacco control measures. It has provided the opportunity to go beyond the scope of the law by introducing new smokefree areas. Further legislation to make all private vehicles smokefree would be welcome. It has been important to place this work within an overall programme where quitting tobacco remains the goal. Overall smoking in the NE has declined by $41 \%$ in this decade.

\section{Tob. Induc. Dis. 2018;16(Suppl 1):A170 DOI: $10.18332 /$ tid/84122}

\section{2:45-14:00}

EP-119-3 Exposure to second-hand smoke and support for smoke-free policies in an urban district in Lagos, Nigeria

C0 Ikpe', T Olubodun², 00 Odukoya ${ }^{2}$

${ }^{1}$ College of Medicine of the University of Lagos, Medicine and Surgery, Lagos, Nigeria, ${ }^{2}$ College of Medicine of the University of Lagos, Community Health and Primary Care, Lagos, Nigeria. E-mail: amsyquin754@gmail.com

Background: Second hand smoke (SHS) is a danger to human health with no safe levels of exposure. With the recently enacted state-wide anti-smoking law, this study aimed to examine the selfreported exposure to SHS and assess the support for smoke-free policies among residents of Oriade Local Council Development Area, an urban district in Lagos.

Methods: This cross sectional descriptive study was carried out among 355 respondents in July 2016, two years after the enactment of the state-wide anti-smoking law. Multistage sampling method was used to select the respondents. Data was collected using interviewer-administered questionnaires adapted from the Global Adult Tobacco Survey (GATS) and analysed using EPI-INFO 7 statistical software.

Results: The mean age of the respondents was $33.9 \pm$ 11.0 years. Majority of the respondents (62.0\%) had good knowledge of the effects of SHS but $89.0 \%$ were unaware of the Lagos state anti-smoking law. Attitudes towards SHS were generally positive as $94.9 \%$ of respondents had little tolerance for SHS. Most (83.2\%) disallow smoking in their homes and over ninety percent agree adults should not smoke around children. About two-thirds support a law that prohibits smoking in all public places, over half (55.5\%) support a law prohibiting advertisements for tobacco products, and (73.3\%) agree that there should be a total ban on the production of tobacco products. Majority of the respondents reported exposure to second hand smoke at work (47.3\%), restaurants (54.7\%), markets $(59.4 \%)$, bars and clubs (58.6\%), roadside (86.8\%), bus stops (84.2\%) and motor parks (85.6\%). About seventy percent were non-smokers.

Conclusions: Knowledge and attitude to second-hand smoke was good, while knowledge of the anti-smoking law was poor. Exposure to SHS was high which calls for enforcement of the antismoking law, awareness and education campaigns on the effects of SHS in Lagos.

Tob. Induc. Dis. 2018;16(Suppl 1):A171

DOI:10.18332/tid/84545
$12: 45-14: 00$

EP-120-3 Children Learning About Second-hand Smoke (CLASS II): a large pilot cluster randomised controlled trial

K Siddiqi ${ }^{1}$, R Huque $^{2}$, M Kanaan ${ }^{3}$

${ }^{1}$ University of York, Health Sciences, York, United Kingdom, ${ }^{2}$ The ARK Foundation, Dhaka, Bangladesh, ${ }^{3}$ University of York, York, United Kingdom. E-mail: kamran.siddiqi@york.ac.uk

Background: Second-hand smoke exposure puts children's health at risk. Among other conditions, such children can acquire respiratory tract infections, asthma and tuberculosis. Our purpose was to pilot an educational intervention for primary school children - Smoke Free Intervention - and to assess the feasibility of recruiting, randomizing, and measuring outcomes in a definitive trial.

Methods: We conducted a large pilot cluster randomised controlled-trial in primary schools in Dhaka, Bangladesh. It recruited Year-5 children (10-12 years old) and randomly allocated eligible schools to either Smoke Free Intervention (intervention arm) or to normal lessons (control arm). Participating children, schoolteachers, and researchers could not be masked from the treatment allocation. In the intervention arm, schoolteachers delivered two 45-minute educational sessions and four 15-minute refresher sessions over four weeks. Our primary outcome was second-hand smoke exposure validated by salivary cotinine. Secondary outcomes included respiratory symptoms, lung function tests, school attendance, smoking uptake, and academic performance. We based our primary analysis on intention-to-treat. The trial registration number: ISRCTN68690577.

Results: Between April 01, 2015 and June 30, 2015, we approached 25 schools and recruited 12 . Out of 576 children in year-5 in these schools, 484 were present on the day of trial recruitment; 481 consented. Six schools (245 children) were allocated to the Smoke Free Intervention arm and six (236 children) to the control. All 12 schools were retained, however, 91.4\% children $(224 / 245)$ in the Smoke Free Intervention arm and $88.6 \%$ (209/236) completed their first follow-up. Twomonths after allocation, salivary cotinine at the cluster level was $0.53 \mathrm{ng} / \mathrm{ml}(\mathrm{SD} 0.36 \mathrm{ng} / \mathrm{ml})$ in the intervention arm and $1.84 \mathrm{ng} /$ $\mathrm{ml}(\mathrm{SD} 1.49 \mathrm{ng} / \mathrm{ml})$ in the control arm.

Conclusions: It is feasible to recruit, randomize and retain primary schools and children in a trial of this nature. The effectiveness of Smoke Free Intervention should be tested in a fully powered randomized controlled trial.

Tob. Induc. Dis. 2018;16(Suppl 1):A172

DOI:10.18332/tid/84044

$12: 45-14: 00$

EP-121-3 Second-hand smoke exposure among undergraduates and support for smoke free campuses in Lagos, Nigeria

Y Atiba ${ }^{1}, 00$ Odukoya ${ }^{1}$, T Olubodun ${ }^{2}$

${ }^{1}$ College of Medicine of the University of Lagos, Department of Community Health and Primary Care, Lagos, Nigeria, '2 Lagos University Teaching Hospital, Department of Community Health and Primary Care, Lagos, Nigeria. E-mail: oluboduntope@ gmail.com

Background: Smoke free policies are increasingly being enacted in many countries. Lagos state recently passed a state-wide smoke free law. However, campuses were excluded from the list of public places where smoking would be disallowed. This study aimed to assess the second-hand smoke (SHS) exposure among university 
undergraduates and assess their support for smoke free campuses. Methods: This cross sectional descriptive study was carried out among 421 university undergraduates in the University of Lagos, being the largest university in the state, and respondents were selected using a multi stage sampling method. Data collection was by pretested self administered questionnaires. EPI INFO 7 software was used to compute descriptive statistics and frequency tables.

Results: Majority (89.1\%) were aware of the dangers of SHS and agreed that SHS was harmful to health. Many (55.4\%) of the respondents agreed that students who are non-smokers have a right to inhale smoke free air on campuses and $57.1 \%$ felt irritated or angry (17.1\%) when exposed to SHS on campus. Majority (80.1\%) were in support of their university being smoke free and were in favor of banning smoking in any enclosed space on campus (79.6\%). Exposure to SHS on campus was high, as one in five respondents were exposed to SHS in their hostel room (19.9\%), in any other enclosed space on campus (19.7\%), or in outdoor campus spaces(44.9\%). Majority of the students exposed to SHS were non-smokers (92\%).

Conclusions: Most students are non-smokers but are exposed to SHS on campus. There is a huge support for smoke free campus policies among undergraduates, who are most affected by SHS on campuses. The state government should consider including campuses on the list of smoke free public spaces.

Tob. Induc. Dis. 2018;16(Suppl 1):A173

\section{DOI: $10.18332 /$ tid/84337}

\section{$12: 45-14: 00$}

EP-122-3 Smoking behaviour and second-hand smoke exposure inside vehicles in Uruguay

L Llambi', M Barros ${ }^{1}$, C Parodi ${ }^{1}$, A Pippo ${ }^{1}$, V Nunez ${ }^{1}$, M Colomar ${ }^{2}$, F Cavalleri $^{3}$, A Aleman ${ }^{2}$

'Universidad de la Republica Uruguay, Unidad de Tabaquismo, Dpto de Medicina, Hospital de Clinicas, Facultad de Medicina, Montevideo, Uruguay, ${ }^{2}$ Clinical Epidemiological Research Unit Montevideo, Montevideo, Uruguay, ${ }^{3}$ Universidad de la Republica Uruguay, Dpto de Metodos Cuantitativos, Montevideo, Uruguay. E-mail: Ilambil@hc.edu.uy

Background: Protection from second-hand smoke (SHS) is one of the fundamental principles of the World Health Organization Framework Convention for Tobacco Control. Scarce data are available on SHS exposure in private areas, such as vehicles. This study aimed to estimate prevalence of smoking inside vehicles and assess the consequent levels of exposure.

Methods: Levels of fine respirable particles with a diameter of 2.5 micrometres or less (PM2.5) were measured in five experimental models in smokers' and non-smokers' vehicles. Prevalence of smoking in vehicles was estimated in two steps: direct observation of vehicles in different socio economic status areas, and further correction for detectability.

Results: Median PM2.5 concentration was $181 \mu \mathrm{g} / \mathrm{m} 3$ in "smoking vehicles" and $0 \mu \mathrm{g} / \mathrm{m} 3$ in "non-smoking vehicles" $(\mathrm{p}<0.001)$. The highest concentration reached $2900 \mu \mathrm{g} / \mathrm{m} 3$ in a parked car with driver's window partially open. We observed 10,011 vehicles. In 219 (2.2\%; 95\% confidence interval, 1.91-2.49) of them, smoking was observed, and in $29.2 \%$ of these, another person was exposed to SHS. According to the multiplying factor we constructed, direct observation detected one of six to nine vehicles in which smoking occurred. The observed prevalence of smoking in vehicles $(2.2 \%)$ could reflect a real prevalence between $12 \%$ and $19 \%$.

Conclusions: Smoking was found to occur in $12 \%$ to $19 \%$ of vehicles, with involuntary exposure in one of three. Concentration of particular matter in vehicles reached very high levels, similar to those at certain sites in countries with weak tobacco control policies. These facts underscore a need for new public policies to eliminate SHS in vehicles to protect public health.

Tob. Induc. Dis. 2018;16(Suppl 1):A174

DOI:10.18332/tid/83786

$12: 45-14: 00$

EP-123-3 Support for ban on smoking in public places among adults in Sub-Saharan Africa

B Asare ${ }^{1}$ D Owusu², HM Mamudu ${ }^{3}, \mathrm{RM}$ John $^{4}$, A Ibrahim $^{5}, \mathrm{E}$ Brenya $^{6}$, AEO Ouma ${ }^{7}$

${ }^{1}$ University of Ghana, Political Science, Accra, Ghana, ${ }^{2}$ Georgia State University, School of Public Health, Atlanta, GA, United States of America, ${ }^{3}$ East Tennessee State University, Health Services Management and Policy, Johnson City, TN, United States of America, ${ }^{4}$ Indian Institute of Technology, Jodhpur, Rajasthan, India, ${ }^{5}$ University of Ghana, School of Public Health, Accra, Ghana, ${ }^{6}$ Kwame Nkrumah University of Science and Technology, Kumasi, Ghana, ${ }^{7}$ World Health Organization, Geneva, Switzerland. E-mail: bossasare@gmail.com

Background: Secondhand smoke (SHS) exposure continues to be a major problem across Sub-Saharan Africa (SSA). Studies have called for immediate steps to address SHS exposure in SSA. Although several SSA countries are implementing smokefree policies (SFPs), public support of these policies can affect their success. While support for SFPs has been studied in other countries, there is limited literature on SFPs in SSA. This study estimated the prevalence and factors associated with support for SPFs in four SSA countries.

Methods: Data from 27,952 participants of the Global Adult Tobacco Survey conducted in Nigeria (2012), Cameroon (2013), Uganda (2013) and Kenya (2014) were analyzed. Supports for smoking ban in eight public places were assessed. Descriptive analysis was conducted to estimate prevalence of support for SPF for the eight places and any support (defined as a support for SFP in at least one public place) for each country and pooled data. A multivariable logistic regression was used to examine factors associated with any support for SFPs. We report odds ratios and 95\% confidence intervals $(\mathrm{CI})$.

Results: Support for any SFP was $99.3 \%, 99.4 \%, 99.8 \%$, and 99.8\% in Kenya, Uganda, Cameroon and Nigeria, respectively. In the pooled data, support for SFPs ranged from $64.4 \%$ in restaurants to $98.8 \%$ in worship places. Adjusting for covariates, support for SFPs was significantly increased in participants who were aware of SHS harm, believed smoking causes serious illness, lived in homes where smoking is restricted, lived in smoke-free homes, resided in countries where three to five public places were completely smoke-free, and those aged $15-44$ years.

Conclusions: The results suggest that support for SFPs increases as the number of smoke-free public places in a country increases. Implementing complete SFPs as described in the WHO FCTC in the SSA countries will likely succeed due to the prevailing high support for these policies.

Tob. Induc. Dis. 2018;16(Suppl 1):A175

DOI:10.18332/tid/84627

\section{$12: 45-14: 00$}

EP-124-3 Awareness and perception of the state tobacco control law prohibiting smoking in public places among selected stakeholders in Ekiti State, Nigeria 
VA Onwu ${ }^{1,2}$, AM Adebayo $^{3}$, OA Adebiyi ${ }^{2}$

'State Ministry of Health, Public Health, Ado-Ekiti, Nigeria, ${ }^{2}$ University of Ibadan, Community Medicine, Ibadan, Nigeria, ${ }^{3}$ Univeristy of Ibadan, Community Medicine, Ibadan, Nigeria. E-mail: victoronwu@gmail.com

Background: Tobacco smoking in public places is prevalent in Nigeria despite global recognition of its deleterious effects. Ekiti State is one of the few states in Nigeria that has enacted the law prohibiting smoking in public places without decline in the practice. This study was conducted to assess awareness and perception of State tobacco control law prohibiting smoking in public places among selected stakeholders and the factors influencing adherence to the law in Ekiti State.

Methods: A descriptive cross-sectional study was conducted using a mixed method of quantitative and qualitative approach. Respondents were selected stakeholders who were directly involved with tobacco control law within the State. Respondents' perception and attitude towards the tobacco control law was assessed using standardized scales. The qualitative survey employed key informant interview (KII) using KII guide. Quantitative data were analyzed using descriptive statistics and Chi-square at $\mathrm{p}=0.05$ while qualitative data were analyzed using thematic approach.

Results: The mean age of respondents was $39.9 \pm 7.7$ years and $55.5 \%$ were males. Majority (73.0\%) were aware of the state tobacco control law, had good perception $(86.0 \%)$ of and positive attitude towards the law (93.4\%). More of the older age groups were aware of the law compared to the younger $(p=0.018)$. The qualitative findings revealed there was little or no compliance with the law prohibiting tobacco smoking in the public in the State. Some identified reasons for non-compliance by most respondents were poor level of information dissemination and awareness and lack of political will.

Conclusions: This study revealed high level of awareness, good perception and positive attitude but poor adherence to the law prohibiting tobacco smoking in public places among stakeholders in Ekiti State. Concerted efforts are required by the government to address identified bottlenecks against compliance for effective implementation and enforcement of the law in the State.

Tob. Induc. Dis. 2018;16(Suppl 1):A176

DOI:10.18332/tid/83910

\section{2:45-14:00}

\section{EP-125-3 Intervention for mothers during} pregnancy to reduce exposure to second-hand smoke (IMPRESS): a pilot randomized controlled trial in Bangladesh

R Huque $^{1,2}$, P Chandra $^{3}$, M Dherani ${ }^{4}$, V Satyanarayana ${ }^{3}$, C Jackson $^{5}$, K Siddiqi ${ }^{5}$, A Rahman ${ }^{4}$

${ }^{1}$ University of Dhaka, Department of Economics, Dhaka, Bangladesh, ${ }^{2}$ ARK Foundation, Dhaka, Bangladesh, ${ }^{3}$ NIMHANS, Bangalore, India, ${ }^{4}$ University of Liverpool, Liverpool, United Kingdom, ${ }^{5}$ University of York, York, United Kingdom. E-mail: rumanah14@yahoo.com

Background: Exposure to secondhand smoke (SHS) during pregnancy is associated with harmful health effects to the foetus and newborn baby. We piloted an intervention targeting pregnant women whose husbands smoked at home, explored intervention acceptability and trial feasibility.

Methods: The IMPRESS study was administered in Comilla District, Bangladesh. We recruited 48 pregnant women in their first and second trimester, and followed them up at 3 months and within 48 hours after delivery. Outcomes included foetal exposure to SHS measured by pregnant women's salivary cotinine levels, SHS knowledge and smoking behavior of husbands/family members. Interviews explored intervention acceptability. Trial feasibility was assessed by recruitment and retention rates.

Results: The recruitment target of 48 pregnant women (100\%) was achieved in 40 days. Among 96 pregnant women approached, 35 were non-eligible, and 13 declined to participate. Retention was $100 \%$ at 3 months, $62 \%$ within 48 hours after delivery. Mean cotinine level (intervention arm) declined from $0.43 \mathrm{ng} /$ $\mathrm{ml}(\mathrm{SD} \mathrm{0.42)}$ ) at baseline to $0.34 \mathrm{ng} / \mathrm{ml}(\mathrm{SD} \mathrm{0.6)}$ ) at first follow up. The difference between intervention and control arms was not statistically significant. Knowledge increased in both arms (+1.1 intervention, +0.6 control, NS). Smoking in the presence of pregnant women reduced by $50 \%$ (intervention) versus $17 \%$ (control) $(p=0.014)$. Men and women described good engagement with the intervention. Its perceived impact was attributed to new SHS knowledge, women gaining confidence in negotiating a smoke free home and men feeling guilty about smoking close to others.

Conclusions: Recruitment and retention rates suggest a trial with pregnant women at the community level in a rural setting is feasible in Bangladesh. The intervention appears acceptable and to have potential for change. Its effectiveness should be tested in a fully powered randomised controlled trial.

Tob. Induc. Dis. 2018;16(Suppl 1):A177 DOI:10.18332/tid/84394

$12: 45-14: 00$

EP-126-3 Self-reported smoking patterns and compliance of cigarette smokers after passage of the statewide smoking law in Lagos, Nigeria

F Nnadiwa', TF Kotey², 00 Odukoya

${ }^{1}$ College of Medicine, University of Lagos, Community Health and Primary Care, Lagos, Nigeria, ${ }^{2}$ Lagos University Teaching Hospital, Community Health, Lagos, Nigeria. E-mail: tolukotey@ yahoo.com

Background: Smoke free policies are an effective tobacco control strategy and can contribute towards the reduction of tobacco related morbidity and mortality when adequately enforced. Prior to the passage of a national law, in 2014, Lagos State, the commercial capital of Nigeria, passed a law to regulate smoking in public places. Since then, no study has assessed the effect of this law on the smoking patterns of cigarette smokers. This study aimed to assess the level of compliance with the law and its effect on smoking patterns among cigarette smokers in the state.

Methods: The study was a descriptive cross-sectional study conducted among 430 current cigarette smokers selected by convenience sampling from 29 hospitality centers in the state. An interviewer administered questionnaire was used to collect the data.

Results: The majority of the respondents (73.6\%) have never complied with the law, and they still smoke in places designated as "no-smoking areas". Among those who complied, the main reason for compliance was perceived fear of penalties attached to the law $(78.4 \%)$ and the desire for a safe environment (78.4\%). The enactment of the law did not affect smoking patterns. Majority of the respondents reported no effect on the quantity of cigarette smoked daily (64.4\%), number of times smoked daily (62.4\%), amount spent on cigarette monthly (63.1\%) and their decision to quit smoking (66.4\%). There was a statistically significant difference $(\mathrm{p}<0.05)$ between educational and employment status and self-reported compliance with the law. 
Conclusions: Despite the passage of the state wide smoking law, many cigarette smokers did not comply with its tenets. Similarly, the law did not seem to have any effects on the smoking habits of the respondents. Efforts to ensure adequate compliance need to be put in place to ensure the benefits of the smoking law are optimized.

Tob. Induc. Dis. 2018;16(Suppl 1):A178

DOI:10.18332/tid/84185

\section{$12: 45-14: 00$}

EP-127-3 City sustained free of hookah bars by applying different acts and continued monitoring by law enforcers

MK Sinha ${ }^{1}$

${ }^{1}$ M.P. Voluntary Health Association, Indore, India. E-mail: sinhamukesh63@gmail.com

Background and challenges to implementation: GYTS 2009 says around $14.6 \%$ (19\% boys and $8.3 \%$ girls) use tobacco products in India. In last 4-5 years more than 40 Hookah bars cropped-up and attracted adolescent and youths violating the norms in Indore city of India, which has students from different parts of the country.

Hookahs are water pipes that are use to smoke tobacco by groups, poses serious health risks to smokers and others exposed to the smoke. In order to protect adolescents and youths serious intervention were needed to make city free from Hookah bars.

Intervention or response: Madhya Pradesh Voluntary Health Association submitted evidences to District Magistrate cum Chairperson Tobacco Control Committee, orientation of Law enforcers done, directives issued, enforcement squad formed, warning announcement made and monitoring started.

Part success achieved, fine imposed but hookah bars continued their services violating the norms. Administration reviewed and decided to combine other strong acts with Indian Tobacco Control Act to completely close the hookah bars.

Administration passed order under section 144 of CRPC-public nuisance act section 268, 278 of Indian Penal Code 1860 with strict implementation of Indian Tobacco Control Act. Hookah bar operators were informed that further violation will debar them from their district for a definite period and license of their eateries and restaurants will be cancelled. Honorable High Court, reference W.P.No-8740/2015, also made decision in favor of District Magistrate.

Results and lessons learnt: All hookah bars in city completely closed and have stopped illegal practices. Innocent customer, adolescents, youths are protected now. Every single Act has some grey areas. Violators take advantages of that.

Conclusions and key recommendations: Planned approach, strong administrative will, combination of different acts with enforcement, systematic monitoring with support of social advocates are always helpful in sustaining the process.

Tob. Induc. Dis. 2018;16(Suppl 1):A179

DOI:10.18332/tid/84046

\section{$12: 45-14: 00$}

EP-128-3 Exposure to second hand smoke and community knowledge on its health effects in rural and urban settings in Unguja Island - Zanzibar FH Faki $^{1}$

${ }^{1}$ Ministry of Health, Planning and Pilicy, Zanzibar, Tanzania, United Rep.E-mail: hfakki1967@gmail.com

Background: Exposure to Second-hand smoke (SHS) is common in many countries but the magnitude of the problem is poorly described worldwide. Hence, there is less public awareness of its effects on health. This study aimed to assess the magnitude of exposure to SHS and public knowledge on its effects among adults in urban and rural settings in Zanzibar.

Methods: A cross sectional study was conducted in Unguja Island of Zanzibar, Tanzania. Four hundred and seventy five heads of households were randomly selected and ten key informants were purposefully selected and interviewed. Household exposure to tobacco smoke was assessed based on responses about current smoking history among household members at home, working places and public transport. Descriptive statistics using SPSS (version 16.0) was performed. The proportion of households that reported exposure from tobacco smoke was obtained. Chi-square test was used to compare sets of nominal data; P-value of less than 0.05 was considered not significant, while thematic analysis was used to generate report for qualitative data.

Results: Prevalence of daily exposure to tobacco smoke at work places was $19.6 \%$, while exposure at home was $15.6 \%$ and $9.9 \%$ were exposed in public transportation. The urban population were more exposed daily to tobacco smoke at home (16.2\%) than the rural (14.0\%). There was statistical difference between rural and urban residents' exposure to second hand smoke in indoor workplaces $(\mathrm{x} 2=2.32, \mathrm{df}=1, \mathrm{p}$-value $=0.12)$. Exposure to tobacco smoke in public transport was higher among rural residents (15.8\%) compared to urban $(8.0 \%)$. Rural residents were more aware that tobacco smoke from other people may cause health effect to someone else.

Conclusions: Exposure to SHS is a problem in Zanzibar. Policies prohibiting smoking in public and work places should be instituted, in order to protect employees and the general public from the effects of SHS.

Tob. Induc. Dis. 2018;16(Suppl 1):A180

DOI:10.18332/tid/84078

\section{$12: 45-14: 00$}

EP-129-3 Awareness and support for the statewide smoking law among cigarette smokers in Lagos State, Nigeria

F Nnadiwa', TF Kotey², 00 Odukoya

${ }^{1}$ College of Medicine, University of Lagos, Community Health and Primary Care, Lagos, Nigeria, '2Lagos University Teaching Hospital, Community Health, Lagos, Nigeria.E-mail: tolukotey@ yahoo.com

Background: Smoke free policies ensures the creation of a smoke free environment by eliminating smoking in a particular space or environment. Support for the legislation of these policies has a positive effect on controlling smoking. In 2014, Lagos State, the commercial capital of Nigeria, the most populous nation in Africa passed a law for the regulation of smoking in public places. This study aimed to assess the awareness and support for the smoking law among cigarette smokers in the state.

Methods: The study was a descriptive cross-sectional study conducted among 430 current cigarette smokers selected by convenience sampling from 29 hospitality centers in the state. An interviewer administered questionnaire was used to collect the data.

Results: Majority of the respondents (67\%) were not aware of the existence of the law. Among those who were aware of the law, $86 \%$ were aware of places where smoking is allowed. Only about $40 \%$ were aware that it is a punishable offence to smoke in the presence of a person less than 18years old. 
The majority (95\%) of respondents were supportive of the law as they believe its enactment would effectively reduce the exposure of non-smokers to second hand smoke. There was a statistically significant difference $(\mathrm{p}<0.05)$ between gender, age, educational and employment status and the awareness of the law.

Conclusions: The majority of cigarette smokers were not aware of the existence of the law two years after its passage but were supportive. Increased awareness and enlightenment campaigns should be done to increase the awareness and enforcement of the law.

Tob. Induc. Dis. 2018;16(Suppl 1):A181

DOI:10.18332/tid/84291

\subsection{Vind the gaps: loopholes in the Ban on Sponsorships, Advertising and Promotions}

\section{$14: 30-16: 00$}

F0-328-3 How tobacco companies in the United Kingdom responded to standardised packaging of cigarettes and rolling tobacco

C Moodie', D Mitchell', K Angus'

${ }^{1}$ University of Stirling, Institute for Social Marketing, Stirling, United Kingdom. E-mail: danielle.mitchell1@stir.ac.uk

Background: Tobacco packaging is a key promotional tool. The Standardised Packaging of Tobacco Products Regulations and Tobacco Products Directive requires all packs of cigarettes (factory-made and roll-your-own) in the United Kingdom to be drab brown and display pictorial health warnings on the principal display areas, and contain no less than 20 cigarettes or 30 grams of tobacco. The legislation was phased in between May 2016 and May 2017. Our objective was to monitor pack, brand and product changes pre- and post-implementation.

Methods: Our surveillance of the cigarette market comprised a review of the trade press, a monthly monitor of supermarket websites, and regular visits to retailers, from May 2015 to June 2017.

Results: Changes to the packaging in the run-up to standardised packaging included novel designs (e.g. limited edition packs) and innovations (e.g. re-sealable inner foil, re-usable tins), and changes since standardised packaging included newer cigarette pack sizes (e.g. 23 and 24 packs). Changes to the branding since standardised packaging included brand migrations (e.g. St. Moritz Menthol became Dunhill St. Moritz), and brand and/or variant name change (e.g. Carlton Smooth Blue became Carlton Bright Blue), with the inclusion of colour descriptors common (e.g. Richmond Menthol became Richmond Green). Product changes in the run-up to standardised packaging included the introduction of a range of novel filter designs (e.g. filters with two flavour-changing capsules, tube filters, firmer filters, and filters with granular additives). There appeared to be non-compliance with the legislation, with very slim packs (e.g. Alluvé Lilac, Vogue Original Green) on sale after standardised packaging was fully implemented; the depth of these packs $(12 \mathrm{~mm})$ was less than the minimum required depth $(20 \mathrm{~mm})$.

Conclusions: Our findings highlight the need to monitor developments in markets introducing standardised packaging, and for countries moving towards standardised packaging we provide an insight into how tobacco companies respond to this measure.

Tob. Induc. Dis. 2018;16(Suppl 1):A182

DOI: $10.18332 /$ tid/83986
14:30-16:00

F0-334-3 Tobacco packaging design for reducing tobacco use: cochrane systematic review

A McNeill'1, S Gravely², S Hitchman'1 , L Bauld³ ${ }^{3}$ D Hammond², J Hartmann-Boyce ${ }^{4}$

'King's College London, Addictions, London, United Kingdom, ${ }^{2}$ University of Waterloo, Waterloo, ON, Canada, ${ }^{3}$ University of Stirling, Stirling, United Kingdom, ${ }^{4}$ University of Oxford, Oxford, United Kingdom. E-mail: ann.mcneill@kcl.ac.uk

Background: Articles 11 and 13 of the WHO FCTC call for the elimination of logos, colours, brand images and promotional information on tobacco packaging that make tobacco products more attractive. We aimed to assess, using a systematic review, the effect of standardised tobacco packaging (STP) on tobacco attitudes and behaviours, including, for the first time, populationlevel outcomes from Australia where STP was implemented in 2012. The review was published in 2017 , but not yet presented at a conference.

Methods: Using standard Cochrane methods, we searched nine databases for peer-reviewed articles evaluating STP prior to January 2016, extracted relevant data and also assessed risk of bias.

Results: We identified 51 peer-reviewed studies, with over 800,000 participants, a variety of methods and designs, and assessing a range behavioural and non-behavioural outcomes; these were conducted predominantly in high-income countries. We found that overall, STP had a positive effect on behavioural and nonbehavioural outcomes; while we also found some null results, there were no negative effects. Australian evidence suggested that STP reduced smoking prevalence, but certainty this outcome was limited by the concurrent introduction of enhanced pictorial warnings. The impact on non-behavioural outcomes was clearer (e.g. STP consistently reduced the appeal of tobacco products), and provided plausible mechanisms of effect consistent with the finding on prevalence. The impact of STP was affected by the detail of the regulations (e.g. whether they banned descriptors and controlled pack shape).

Conclusions: STP reduced the promotional appeal of tobacco packs in line with regulatory objectives, and the available evidence also showed it may reduce smoking prevalence. There is an urgent need for research to be conducted among low and middle-income countries where $80 \%$ of the world's smokers live. Cochrane reviews are evolving documents, updated as other studies become available.

Tob. Induc. Dis. 2018;16(Suppl 1):A183

DOI: $10.18332 /$ tid/84186

\section{6:00-17:30}

RF-1200-3 Point of sale display ban in Scotland: retailers' views of the effects on customer transactions, sales, youth and feelings about selling tobacco

M Stead', D Eadie1, R Purves', S Haw'1 J Pearce², A Amos², C Best $^{1}$, A Ford ${ }^{1}$, DISPLAY Study Group

'University of Stirling, Stirling, United Kingdom, ${ }^{2}$ University of Edinburgh, Edinburgh, United Kingdom. E-mail: a.j.ford@ stir.ac.uk

Background: In April 2015, point of sale (POS) displays of tobacco products were prohibited in small retail outlets in Scotland. Retailers had several years to prepare for the ban. As part of the DISPLAY (Determining Impact of Smoking Point-ofSale Legislation Among Youth) study, retailers was interviewed annually for 5 years pre- and post-implementation of the ban to 
explore their perceptions of its anticipated and actual impact on customer transactions, sales and profitability, young people's interest in tobacco, and their own feelings about selling tobacco. Methods: A cohort sample of 24 small retailers in four communities in central Scotland was recruited and interviewed annually over a 5-year period (2013-2017). Interviews were conducted in-store and lasted between 30-60 minutes. Transcript data were coded in NVivo and themes analysed both at each wave and longitudinally. Results: Initial expectations regarding the implementation and impact of the ban were largely negative. However, actual implementation was straightforward for most of the retailers, compliance was high, and concerns about a negative impact on customer transactions were largely unfounded. Retailers were divided in their views of the ban's impact, with some feeling it made no difference and others that it had contributed to decreasing customer interest in premium brands and reduced sales. Some perceived that the ban also reduced young people's interest in tobacco. Some retailers experienced increased tobacco company contact and pressure after the ban, which fostered feelings of ambivalence regarding their role in selling tobacco.

Conclusions: Countries considering POS bans can benefit from studying retailer experiences in countries which have implemented bans. Display bans can be implemented with minimal retailer burden and do not, contrary to industry arguments, adversely affect transaction times. The implementation of such bans and corresponding tobacco industry pressure on retailers may increase retailers' willingness to reduce their reliance on tobacco.

Tob. Induc. Dis. 2018;16(Suppl 1):A184

DOI:10.18332/tid/84248

\section{$16: 00-17: 30$}

RF-1201-3 Effectiveness of TAPS bans and public support for point-of-sale (POS) bans in Brazil: findings from the ITC Brazil Survey, 2009-17

F Mendes ${ }^{1}$, J Chung-Hall ${ }^{2}$, M Yan ${ }^{2}$, G Li $^{2}$, T Cavalcante $^{1}$, A Szklo ${ }^{3}$, C Perez ${ }^{4}$, G Fong ${ }^{2}$

${ }^{1}$ National Cancer Institute, Executive Secretariat of National Comittee for FCTC Implementation, Rio de Janeiro, RJ, Brazil, 'University of Waterloo, Department of Psychology, ITC Project, Waterloo, ON, Canada, ${ }^{3}$ National Cancer Institute, Division of Epidemiology, Rio de Janeiro, RJ, Brazil, ${ }^{4}$ Cancer Foundation, Rio de Janeiro, RJ, Brazil. E-mail: fmendes@inca.gov.br

Background: Research in high-income countries shows that exposure to point-of-sale (POS) product displays encourages youth smoking, promotes impulse purchasing, and undermines quitting, and that there is strong public support for POS advertising bans. Brazil banned tobacco advertising, promotion, and sponsorship (TAPS) in most channels in 2000 and POS advertising in 2014. However, public awareness of tobacco promotion remains high in Brazil compared to other countries, and POS product displays are still permitted.This study evaluated the impact of the 2014 Brazil POS advertising ban, and assessed public support for complete bans on in-store advertising and POS displays.

Methods: Data were from Waves 1-3 of the ITC Brazil Survey, a cohort survey of approximately 1200 adult smokers and 600 non-smokers residing in Rio de Janeiro, São Paulo, and Porto Alegre. Waves 1 and 2 were conducted before the 2014 POS advertising ban (2009 and 2012-13), and Wave 3 was conducted after the ban (2016-17). Data were analyzed using GEE logistic regression models.

Results: From 2009 (pre-ban) to 2016-17 (post-ban), noticing things that promote smoking decreased among smokers (42.3\% to $34.4 \%$, $\mathrm{p}<.01$ ) and non-smokers (34.0\% to $25.8 \%, \mathrm{p}<.05$ ). Following the 2014 POS advertising ban, $19.9 \%$ of smokers and $20.0 \%$ of nonsmokers noticed signs/items with cigarette logos in stores; $61.3 \%$ of smokers and $58.5 \%$ of non-smokers noticed cigarette displays in stores. There was strong support for complete bans on in-store advertising (74.2\% smokers, $87.8 \%$ non-smokers) and POS cigarette displays (71.9\% smokers, $85.5 \%$ non-smokers); and generally no differences in support between cities.

Conclusions: TAPS bans in Brazil have reduced exposure to tobacco marketing, but POS advertising continues to be visible. These findings demonstrate that Brazil should implement a complete ban on in-store tobacco advertising, including POS displays, and that such a ban would be supported by the majority of smokers and non-smokers.

Tob. Induc. Dis. 2018;16(Suppl 1):A185 DOI:10.18332/tid/84011

\section{$16: 00-17: 30$}

RF-1202-3 Exposures and responses to pointof-sale tobacco displays and support for banning displays in Hong Kong: a population-based crosssectional survey

YTD Cheung ${ }^{1}$, MP Wang ${ }^{1}, \mathrm{SY} \mathrm{Ho}^{2}$, A Kwong ${ }^{3}$, V Lai ${ }^{3}$, TH Lam ${ }^{2}$ ${ }^{1}$ University of Hong Kong, School of Nursing, Hong Kong, Hong Kong, ${ }^{2}$ University of Hong Kong, School of Public Health, Hong Kong, Hong Kong, ${ }^{3}$ Hong Kong Council on Smoking and Health, Hong Kong, Hong Kong. E-mail: mpwang@hku.hk

Background: Point-of-sale (POS) tobacco displays can promote smoking and purchase of tobacco in current and former smokers, but no studies have examined such effect in never smoking adults. We investigated the correlates of frequent exposure to, and responses and attitudes towards POS tobacco displays in never smokers in Hong Kong, China.

Methods: We included 1,833 never smokers aged 15+ years from two random telephone surveys in $2015(\mathrm{n}=932)$ and 2016 $(n=901)$. All respondents reported how often they noticed POS tobacco displays in the past 30 days (often, sometimes, not noticing), whether they perceived displays as attractive, felt encouraged to smoke by them, perceived them as advertisements, and supported the banning displays. Risk ratio (RR) adjusting for socio-demographic characteristics was used to examine factors associated with often noticing the display and other responses.

Results: Young adults aged 15-29 years (vs 60+) were more likely to report often noticing POS displays $(\mathrm{RR}=2.39,95 \% \mathrm{CI}$ 1.47-3.91). Those with primary education or below (vs tertiary) reported less often noticing $(\mathrm{RR}=0.41,95 \% \mathrm{CI} 0.23-0.73)$, but more perceiving displays as attractive $(\mathrm{RR}=2.52,95 \% \mathrm{CI} 1.51$ 4.22). Often noticing displays (vs not noticing) was associated with perceiving them as attractive $(\mathrm{RR}=1.90,95 \% \mathrm{CI} 1.32-2.75)$, and being encouraged to smoke ( $\mathrm{RR}=4.05,95 \% \mathrm{CI} 0.98-16.85)$. Sometimes ( $R R=0.89,95 \%$ CI $0.80-0.997)$ or often noticing ( $\mathrm{RR}=0.86,95 \% \mathrm{CI} 0.77-0.97)$ displays were associated with lower support for banning displays.

Conclusions: In never smokers, younger adults were more exposed to POS tobacco displays and those with low education level were more attracted by them. Frequent exposure could induce never smokers to smoke, and reduce their support for banning displays. To eliminate the advertising and normalization of POS tobacco displays in the retail environment, the ban on tobacco advertisements should include those displays simultaneously.

Tob. Induc. Dis. 2018;16(Suppl 1):A186

DOI:10.18332/tid/84072 


\section{6:00-17:30}

RF-1203-3 Global evidence on the effect of pointof-sale display bans on smoking prevalence Y He $^{1}$, C Shang ${ }^{2}$, J Huang ${ }^{3}$, K-W Cheng ${ }^{4}$, FJ Chaloupka ${ }^{5}$ 'University of Illinois at Chicago, Chicago, IL, United States of America, ${ }^{2}$ IHRP, UIC, Health Policy Center, Chicago, IL, United States of America, ${ }^{3}$ Georgia State University, Tobacco Center of Regulatory Science, School of Public Health, Atlanta, GA, United States of America, ${ }^{4}$ University of Illinois at Chicago, Institute for Health Research and Policy, Chicago, IL, United States of America, ${ }^{5}$ University of Illinois at Chicago, Institute for Health Research and Policy; Health Policy, School of Public Health, Chiacgo, IL, United States of America. E-mail: cshang@ uic.edu

Background: Since Iceland became the first country to impose a ban on point-of-sale (POS) displays in 2001, there have been 20 countries in total in the world implementing POS display bans as of 2016.

Methods: This study examined the effect that POS display bans have on smoking prevalence to provide evidence on their effectiveness in tobacco control.The data were sourced from Euromonitor International and WHO MPOWER package during 2007-2014 from 77 countries worldwide. Generalized Linear Model with country and year fixed effects was implemented to analyze the effect of POS display bans on smoking prevalence.

Results: Having a POS display ban decreased overall adult smoking, male smoking, and female smoking by about $7 \%, 6 \%$, and $9 \%$, respectively.

Conclusions: Having a POS display ban is likely to reduce smoking prevalence. Adopting such policies has the potential to generate public health benefits. Countries currently without a POS display ban are encouraged to implement such policies to curb the smoking epidemic.

Tob. Induc. Dis. 2018;16(Suppl 1):A187

\section{DOI:10.18332/tid/84575}

\section{$16: 00-17: 30$}

RF-1207-3 Point of sale tobacco advertising compliance with legislation in 3 large cities in Argentina

\section{S Braun ${ }^{1}$, A Ángel ${ }^{1}$, A Pérez ${ }^{2}$, R Mejía ${ }^{1,2}$, J Thrasher $^{3}$}

'University of Buenos Aires, Internal Medicine, Buenos Aires, Argentina, ${ }^{2}$ Centro de Estudios de Estado y Sociedad (CEDES), Department of Health Economy, Buenos Aires, Argentina, ${ }^{3}$ University of South Carolina, Department of Health Promotion, Education, and Behavior, Columbia, SC, United States of America. E-mail: sandrabraun@fibertel.com.ar

Background: Since 2011, tobacco marketing in Argentina is banned through traditional channels. However, marketing at the point of sale $(\mathrm{PoS})$ is allowed with only few limitations. PoS are currently the primary venue where youth are exposed to marketing of tobacco products.

Our objective was to assess the compliance with the PoS legislation among retailers located near assess secondary schools in Buenos Aires, Córdoba and Tucumán.

Methods: During 2016 a survey was conducted among all the retailers within $300 \mathrm{~m}$ of a convenience sample of 33 secondary schools in Buenos Aires, Córdoba and Tucumán. We assessed the compliance with the seven points imposed by the Tobacco Control National Law regarding PoS. An index was created with these seven items and dichotomized into low compliance (4 or more violations) and high (3 violations or less). Store type, distance from the school, city, marketing of flavoured cigarettes and alcoholic beverages were also evaluated. The association between potential predictors of noncompliance was investigated by calculating crude and adjusted OR using a logistic regression controlled by covariates.

Results: Of the 340 stores surveyed, 190 (55.88\%) were kiosks, 84 (24.71\%) were small convenience stores, 46 (13.53\%) supermarket and 20 (5.88\%) others. Overall, 217 (63.82\%) of the PoS had low compliance. Most, $87.35 \%$, had at least one infraction. The three most common were the failure to display a "prohibition of sale to minors" sign, 290 (85.29\%), to have more than one advertising sign, 208 (61.18\%) and the sale single cigarette 167 (49.12\%). Buenos Aires (the capital city) was the place with more violations, $48.82 \%$.

The strongest predictors of low compliance were marketing of flavoured cigarettes $(\mathrm{OR}=3.78,95 \% \mathrm{IC}$ 1.97-7.24) and alcohols beverages $(\mathrm{OR}=1.25,95 \% \mathrm{IC} 1.12-1.51)$.

\begin{tabular}{|c|c|c|c|c|}
\hline & OR & $95^{\circ} \% I C$ & AOR & $95^{\circ} \%$ IC \\
\hline $\begin{array}{l}\text { City: Bs. } \\
\text { Aires } \\
\text { Córdoba } \\
\text { Tucumán }\end{array}$ & 9.520 .310 .23 & $\begin{array}{c}5.57-16.28 \\
0.18-0.52 \\
0.14-0.39\end{array}$ & $\begin{array}{c}10.13^{*} \\
0.28^{*}\end{array}$ & $\begin{array}{l}0.06-0.25 \\
0.14-0.58\end{array}$ \\
\hline $\begin{array}{l}\text { Alcohol } \\
\text { marketing }\end{array}$ & 1.16 & $1.09-1.29$ & $1.25^{*}$ & $1.12-1.51$ \\
\hline $\begin{array}{l}\text { Flavored } \\
\text { cigarette } \\
\text { marketing }\end{array}$ & 3.93 & $2.39-6.45$ & $3.78^{*}$ & $1.97-7.24$ \\
\hline
\end{tabular}

${ }^{*}<0,000 \quad \begin{gathered}\text { Adjusted by } \\ \text { distance and } \\ \text { type of store. }\end{gathered}$

[Predictors of low compliance with PoS regulations]

Conclusions: Most of the stores do not comply with the current legislation regarding the PoS. A higher enforcement of the National Tobacco Law is needed.

\section{Tob. Induc. Dis. 2018;16(Suppl 1):A188 \\ DOI:10.18332/tid/84284}

16:00-17:30

RF-1210-3 Monitoring of compliance with the national tobacco advertising law in 11 cities in China

$\mathrm{G} X \mathrm{u}^{1}$

${ }^{1}$ Chinese Association on Tobacco Control, Beijing, China. E-mail: xuguihua@catcprc.org.cn

Background: China's revised national Advertising Law went into effect on September 1, 2015. It prohibits tobacco advertising to minors in any form and comprehensively bans tobacco advertising in public places, public transportation and outdoor areas. To assess compliance with the advertising ban at points of sale (POS), in public places and outdoor areas.

Methods: During March to April, 2016, we conducted a survey in 11 cities in China (Beijing, Shanghai, Guangzhou, Shenzhen, Kunming, Xi'an, Hangzhou, Wuhan, Lanzhou, Haerbin and Zhangjiakou), and visited POS (tobacco specialty stores, supermarkets, convenience stores and newsstands), public places (bus stops, bus terminals, train station, airports, hospitals, restaurants, bars and shopping malls), and outdoor areas. An observation form was developed to record the number, type, brand, size, other relevant tobacco advertising and promotion 
information, and the presence of minors in the selected places. Results: POS $(n=1,236)$ : Tobacco advertising was observed in all 11 cities and in almost $47 \%$ of the observed places. The most prevalent was in tobacco specialty stores (78\%). The most common type of tobacco advertising was pasted advertising (34\%). $70 \%$ of the POS did not have the "No tobacco to Minors" sign and almost $18 \%$ stores had minors inside. POS tobacco promotion was found in 10 cities and in $4 \%$ of the POS. Public places $(n=1,453)$ : Tobacco advertising was found in 9 cities and in almost $4 \%$ of the observed places in these cities. Outdoor areas ( $n=149$ major blocks): Tobacco advertising was observed in 4 cities and from 19\% of the observed blocks in these cities.

Conclusions: Despite implementation of the revised national Advertising Law, tobacco advertising and promotion is still commonly found at tobacco POS in China. Law enforcement agencies should increase inspection and enforcement measures on tobacco advertising and promotion.

Tob. Induc. Dis. 2018;16(Suppl 1):A189

DOI:10.18332/tid/84028

\section{$16: 00-17: 30$}

RF-1211-3 Student advocacy efforts to remove tobacco advertising from their school environment, Jakarta \& Bandung, Indonesia

H Pradityas ${ }^{1}$, E Orlan², H Hendriyani ${ }^{3}$, L Sundari ${ }^{4}$

${ }^{1}$ Indonesia Smoke-Free Agents, Jakarta Pusat, Indonesia, 'University of North Carolina at Chapel Hill, Health Behavior, Chapel Hill, NC, United States of America, ${ }^{3}$ University of Indonesia, Depok, Indonesia, 'Lentera Anak Foundation (LAF), Jakarta, Indonesia.E-mail: hasnatyas@gmail.com

Background and challenges to implementation: By Indonesian law, schools are tobacco-free areas, where sales, advertisements, and use are banned. However, tobacco companies still advertise near schools, through billboards or branding of kiosks. We conducted surveys of school areas in 5 cities and found tobacco advertisements near 85 percent of areas surveyed. After these findings were captured, we approached schools in Jakarta and Bandung with these results.

Intervention or response: Eight schools mobilized to remove tobacco advertising in their school environments. This effort was spearheaded by school principals and other community stakeholders. At the same time, we worked to educate the students about the impact of tobacco advertising. The students assisted with the effort to remove the tobacco advertising, visiting their local government officials to explain these harms.

Results and lessons learnt: In 2016, advertisements were removed from 8 school areas, as a result of advocacy from the students and school administrators to local kiosk owners and officials. Although Jakarta has a smoke-free area law, we found that these laws are not always followed, and thus community education, followed by action is necessary to ensure that these communities remain ad-free. This project, in combination with other advocacy efforts, led to the adoption of a smoke-free law in Bandung. Further, these actions sparked attention from the media, due to the creative methods students and communities used to remove the advertising. Civilian grassroots efforts can help to spark change in communities, leading to wider acceptance and adoption of tobacco control laws.

Conclusions and key recommendations: While some Indonesian cities have regulations to ban outdoor advertisements near schools, not all school environments are protected by these regulations. This project shows the benefit of engaging non-traditional community stakeholders, from youth to school administrators to government officials, to ensure that TAPS bans are followed in places like Indonesia, where enforcement is weak, and in other contexts where policies are not thoroughly implemented.

Tob. Induc. Dis. 2018;16(Suppl 1):A190

\section{DOI:10.18332/tid/84241}

\section{2:30-14:00}

PS-613-3 Awareness of tobacco advertising, promotion and sponsorship in four states: findings from TCP India survey - Wave 1 and Wave 2

N Puntambekar ${ }^{1}$, M Pednekar ${ }^{1}$, K Adhikari $^{1}$, ACK Quah², GT Fong $^{3}$, D Pete ${ }^{2}$, PC Gupta

${ }^{1}$ Healis - Sekhsaria Institute of Public Health, Research, Navi Mumbai, India, 'University of Waterloo, Psychology, Waterloo, ON, Canada, ${ }^{3}$ University of Waterloo, Ontario Institute of Cancer Research, Toronto, ON, Canada. E-mail: puntambekarn@healis. org

Background: India's Cigarette and Other Tobacco Products Act (COTPA) of 2003 prescribes a complete ban on all forms of tobacco advertisements, promotions and sponsorships (TAPS) of events by tobacco companies across India. We examined the level of variation and predictors of awareness of TAPS in four Indian states.

Methods: We used data from the International Tobacco Control Policy (TCP) Survey India from Wavel (Aug 2010- Dec 2011) and Wave2 (Aug 2012-Dec 2013) consisting of 10,585 and 10,501 respondents, respectively, surveyed from Madhya Pradesh (MP), Bihar (BR), Maharashtra (MH) and West Bengal (WB). Bivariate analysis and multivariable logistic regression was used to investigate associations between sociodemographic factors, states and TAPS awareness. All analyses were performed using SPSS V. 20.0

Results: Noticing tobacco advertisements was highest in $\mathrm{MH}$ (78.5\%) and lowest in WB (50.1\%) in Wave 1 , while in Wave 2 , it was highest in MP (83.5\%) and lowest in WB (32.6\%). Noticing tobacco promotions and sponsorships was highest in MP (promotions: $35.7 \%$, sponsorships: 14.4\%) in Wave 1, while in Wave 2, it was highest in WB (promotions:15.0\%, sponsorships:10.8\%). Across waves, education was a predictor of noticing tobacco advertisements, promotions and sponsorships. In Wave 1 , but not Wave 2, noticing tobacco advertisements (OR:2.11) and promotions (OR:2.02) was highest among the youngest age group (15-17yrs) compared to corresponding oldest age group (55yrs + ). Noticing advertisements and sponsorships remained consistent in urban areas across both waves. In Wave 1 tobacco promotions were observed twice as frequently among rural population as compared urban population but the association was reversed in Wave 2.

Conclusions: Awareness of tobacco marketing varied across the four states of India to observe a greater impact of the tobacco control legislation. This study suggests that even though policies are the same in all states, a coordinated effort is required to implement the law on TAPS ban consistently.

Tob. Induc. Dis. 2018;16(Suppl 1):A191

DOI:10.18332/tid/83793

$12: 30-14: 00$

PS-614-3 Denormalising tobacco at a Danish music festival

MK Schjoerring ${ }^{1}$, A Knudsen ${ }^{2}$, NT Kjaer², M Groenbaek ${ }^{3}$

${ }^{1}$ National Institute of Public Health, Copenhagen, Denmark, ${ }^{2}$ The Danish Cancer Society, Copenhagen, Denmark, ${ }^{3}$ National Institute of Public Health, Copehagen, Denmark.E-mail: ask@ 


\section{cancer.dk}

Background and challenges to implementation: In Denmark, the tobacco advertising ban has exemptions, as e.g. a "neutral placement of tobacco" at point of sales is allowed. This loophole is utilized by the tobacco industry to promote tobacco at music festivals and similar arenas. It is a well-known and efficient strategy for the industry to encourage smoking uptake by children. Strøm is a Danish electronic music festival which each year attracts 15.000 people mainly aged $15-30$ years old. Until recently like many other music festivals Strøm received cigarettes from tobacco companies free of charge for resale to the audience. In this setting festival artists, volunteers, staff and audience frequently were depicted smoking in social media posts and promotional material. Intervention or response: In 2014 Strøm initiated a partnership with The Danish Cancer Society and the Municipality of Copenhagen. The aim of the partnership was to end the promotion of tobacco at the festival and to start denormalising tobacco in the Danish music scene. One intervention aimed at reducing the visibility of tobacco by not accepting tobacco for resale at the festival and by development of a non-smoking code for promotional material, social media and by employees at the festival.

Results and lessons learnt: With the code, tobacco is no longer sold at the festival. The code increased the awareness among the festival employees of how their visible smoking may influence adolescents and they were increasingly conscious of not smoking in the context of the festival.

Conclusions and key recommendations: We found that it was feasible to run a music festival without the direct and indirect tobacco promotion. This awareness can be used to disseminate the no-tobacco code to other music festivals and cultural settings. The inside knowledge of the tobacco industry tactics can be an eye-opener in youth campaigns and help build support among the public and policy makers for a comprehensive advertising ban.

Tob. Induc. Dis. 2018;16(Suppl 1):A192

DOI:10.18332/tid/83839

\section{$12: 30-14: 00$}

PS-616-3 Removing tar information from cigarette packages may reduce South Korean smokers' misconceptions about low-tar cigarettes

H-J Paek ${ }^{1}$, T Hove ${ }^{1}$

${ }^{1}$ Hanyang University, Department of Advertising \& Public Relations, Ansan, Korea, Republic of. E-mail: hjpaek@gmail.com Background: Many smokers still have misconceptions about "light" or "low tar" cigarettes. In South Korea, low-tar (<3 mg) cigarette sales have increased sharply from $1.8 \%$ in 2002 to $49.2 \%$ in 2015 . Although government regulations forbid cigarette packages from displaying messages such as "mild," "low-tar," and "light," numbers indicating tar amounts are still permitted. This study examines whether removing tar information from packaging altogether reduces people's misconceptions about low tar cigarettes.

Methods: An online experiment was conducted among 531 smokers who were randomly assigned to one of two conditions. In the "tar" condition, 271 participants were shown in random order three cigarette packages for three major brands (Esse, The One, Marlboro) with different tar amounts. In the "no-tar" condition, 260 participants were shown the same packages without tar information. Next, participants evaluated which type of cigarette was mildest, least harmful, easier for nonsmokers to start smoking, and easier for smokers to quit. After descriptive statistics were checked, twelve sets of chi-square tests were performed.

Results: Average age of the participants was 26.22 (14 - 62 years); $53.5 \%$ were male. All 12 chi-square tests were statistically significant. Participants in the tar condition judged the lowest-tar cigarette to be mildest, least harmful, easier to start, and easier to quit. In the no-tar condition, for the Korean brands Esse and The One, most respondents evaluated all cigarette types to be the same only for harm, ease of starting, and ease of quitting; for Marlboro, judgments were the same as those in the tar condition except that "easier to quit" was judged to be the same across the three types.

Conclusions: Banning tar information from cigarette packages may help reduce smokers' misconceptions about low-tar cigarettes. People have inconsistent judgments about differently packaged cigarettes when tar information is absent.

Tob. Induc. Dis. 2018;16(Suppl 1):A193

DOI: $10.18332 /$ tid/83970

\section{$12: 30-14: 00$}

PS-617-3 Collaboration between stakeholders: TAPS free model rail station

MA Razzak ${ }^{1,2}$, S Ahmed ${ }^{3,4}$, A Islam Sujon ${ }^{3,5}$

'Social Advancement Forum - SAF, Tobacco Control, Kushtia, Bangladesh, ${ }^{2}$ Bangladesh Anti Tobacco Alliance (BATA), Local Level Advocacy, Dhaka, Bangladesh, ${ }^{3}$ Work for a Better Bangladesh (WBB) Trust, Health Rights and Tobacco Control, Dhaka, Bangladesh, ${ }^{4}$ Bangladesh Anti Tobacco Alliance (BATA), Tobacco Control, Dhaka, Bangladesh, ${ }^{5}$ Bangladesh Anti Tobacco Alliance (BATA), Communication and Advocacy, Dhaka, Bangladesh.E-mail:saf.razzak@gmail.com

Background and challenges to implementation: Smoking and Tobacco Products Uses (Control) Act 2005 (amended in 2013) is provide opportunities for non-government organizations to ensure implementation of this law. This law has banned all sorts of Tobacco Advertisement, Promotions and Sponsorships (TAPS), but Tobacco Industries (TIs) violates this law across the country through various ways. Display tobacco products, decorated boxes, competition among smokers, free sample distribution at the point of sales (PoS's) are most common forms of violation of law. PoS's takes little space, have no control by any authority and $\mathrm{PoS}$ is available at all areas, in front of schools, hospitals, bus stand, rail stations etc.

Intervention or response: Kushtia based voluntary organization Social Advancement Forum (SAF) is member of the Bangladesh Anti Tobacco Alliance (BATA), a nationwide civil society platform and district Task Force for tobacco control law implementation headed by deputy commissioner (DC). SAF learned about law implementation from national level organizations and work with all local government officials, people representatives at various local governments, shop owners, authority of rail station, law enforcing agencies (Police) etc. SAF organized various public awareness campaigns, meetings and disseminate promotional materials of law with gazette. SAF succeed to convince all concerned people to make a model rail station that free from tobacco advertisement and promotion. SAF regularly monitor this place in which more than 10,000 people visit every day.

Results and lessons learnt: As a result, we have made 100\% compliance of law with removing all advertisement, display, colored boxes and promotional activities. Poradaha rail station is also smoke free. Efforts on law implementation to create model rail station can be replicate any other rail station in any country, where tobacco advertisement is banned. 
Conclusions and key recommendations: Remove PoS advertisements became big challenge around the country. But $\mathrm{SAF}$ in Kusthia successful to create PoS ads free zone through strong collaboration among all concern sectors.

Tob. Induc. Dis. 2018;16(Suppl 1):A194

DOI:10.18332/tid/84009

\section{$12: 30-14: 00$}

PS-618-3 Effects of 85\% pictorial health warnings on manufactured cigarette package: a crosssectional survey at retail shops

S Benjakul', S Nakju²

${ }^{1}$ Mahidol University, Department of Health Education and Behavioral Sciences, Faculty of Public Health, Ratchathewi, Thailand, ${ }^{2}$ Ramkhamhaeng University, Sukhothai Campus, Faculty of Public Health, Sukhothai, Thailand. E-mail: dr.benjakul@ gmail.com

Background: The trend for larger, picture health warnings is growing and unstoppable. Thailand have now finalized full color picture health warnings (PHWs) requirements, an increase from the $50 \%$ of the cigarette package front and back to $85 \%$ that had implemented by September 23, 2014. Even though a larger size are more likely to be noticed, it can be communicate the health risk of tobacco use through desire to quit smoking among current smokers and not want to smoke for those who non-smokers.

Methods: A cross sectional survey was conducted to determine the effects of $85 \%$ PHWs level for both non-smokers and current cigarette smokers. Those were selected by an accidental sampling when they come to purchase anything including cigarette for themselves at the retail stores after the enforcement of $85 \% \mathrm{PHWs}$ for 6 th weeks. A single-stage cluster sampling and a systematic random sampling were adopted to select 260 retail stores at 7 target areas over country. Statistical analysis was performed by using descriptive and inferential statistics.

Results: A total of 541 customers were interviewed. $48.4 \%$ of those who were non-smokers, $11.3 \%$ were former smokers, and $40.3 \%$ currently smoking manufactured cigarettes. After noticed 85\% PHWs, $99.2 \%$ of non-smokers did not want to start smoking and $100.0 \%$ of former smokers did not want to return smoking, relapsing. $53.7 \%$ of current smokers desired to quit smoking. As compare the intensity of desire to quit smoking between noticing $85 \% \mathrm{PHWs}$ and $55 \% \mathrm{PHW}$, the survey found that $13.5 \%$ of current smokers who did not want to stop smoking when noticing $55 \%$ PHWs desired to quit after noting $85 \%$ of PHWs.

Conclusions: The more increase the sizes of PHWs, the more increase the health risk of tobacco use and then follow by the increasing rate of intention to quit smoking.

Tob. Induc. Dis. 2018;16(Suppl 1):A195

DOI:10.18332/tid/84027

\section{2:30-14:00}

PS-619-3 "Pro-tobacco propaganda": a case study of tobacco industry-sponsored elementary schools in China

\section{J Fang ${ }^{1}$, X Wan ${ }^{2}$, G Yang ${ }^{2}$}

'Simon Fraser University, Faculty of Health Sciences, Burnaby, BC, Canada, ${ }^{2}$ Institute of Basic Medical Sciences, Chinese Academy of Medical Sciences and School of Basic Medicine, Peking Union Medical College, Beijing, China.E-mail:jennifer_fang@sfu.ca Background: China is the largest producer and consumer of tobacco products worldwide, with $16.5 \%$ of all deaths attributed to tobacco use in 2010. While direct marketing and advertisement of tobacco products has been restricted, indirect marketing still exists under the guise of sponsorship and corporate social responsibility (CSR). This case study is focused on tobacco industry-sponsored elementary schools in Chinese rural areas and discusses the success story of a major tobacco control campaign to remove tobacco-related branding and logos from school buildings. Methods: Field visits were undertaken to 3 tobacco industrysponsored elementary schools in Yunnan in 2012, and semistructured interviews were conducted with students, teachers, school principals and parents to understand their perceptions of the tobacco industry and its sponsorship of schools. In 2016, semi-structured interviews with tobacco control activists were conducted in Beijing, where respondents discussed the outcome of those field visits and the resulting work in petitioning government sectors to remove tobacco-related branding from school buildings. Interview data was transcribed and coded, and key themes were developed using thematic analysis.

Results: While health consequences of smoking were generally known, attitudes towards the tobacco industry and its CSR activities remained positive among the general public. Educators and parents did not perceive any impacts on schoolchildren from exposure to "pro-tobacco propaganda" created by the industry's CSR activities. Attitudes among tobacco control activists were drastically different, with consensus that CSR activities constitute indirect marketing attempts that should be banned.

Conclusions: The successful removal of branding from tobacco industry-sponsored schools shows that meaningful action is possible even in the absence of relevant legislation, and can be achieved through the mobilization of stakeholders, including academics and civil society organizations. National tobacco control legislation banning all forms of indirect marketing including sponsorship and CSR activities is needed in order to protect the health of the future generations.

Tob. Induc. Dis. 2018;16(Suppl 1):A196

DOI:10.18332/tid/84053

$12: 30-14: 00$

PS-620-3 Monitoring of tobacco advertising and promotion in Lagos, Nigeria: observational assessment to inform proposed regulations to the National Tobacco Control Act

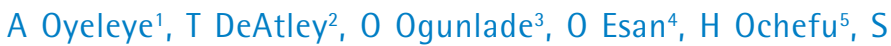
Uzzell $^{6}$, B Camara ${ }^{7}$, E Sebrie $^{2}$

${ }^{1}$ Ekiti State University, Mechanical Engineering, Ado-Ekiti, Nigeria, ${ }^{2}$ Campaign for Tobacco-Free Kids, International Research, Washington, DC, United States of America, ${ }^{3}$ Ekiti State University, Geology, Ado-Ekiti, Nigeria, ${ }^{4}$ Ekiti State University, Physics, Ado-Ekiti, Nigeria, ${ }^{5}$ Campaign for Tobacco-Free Kids, International Advocacy, Lagos, Nigeria, ${ }^{6}$ Campaign for TobaccoFree Kids, International Grants, Washington, DC, United States of America, ${ }^{7}$ Campaign for Tobacco-Free Kids, International Advocacy, Washington, DC, United States of America. E-mail: esebrie@tobaccofreekids.org

Background: In 2015, Nigeria approved the National Tobacco Control Act, banning tobacco advertising and promotion with the exception of advertising to "consenting" adults, which was not defined in the law. As of July 2017, implementing Regulations have not been issued by the Ministry of Health. The objective of this study was to assess the level of tobacco advertising and promotion in public places before implementation of the law.

Methods: In June 2017, monitoring was conducted in five Local Government Areas in Lagos, Nigeria: Kosofe, Surulere, Ikeja, 
Agege, and Apapa. Data was collected from a convenience sample $(n=250)$ of public places, some of which sold tobacco products. Public places were categorized into 9 types: kiosks, roadside tobacco sellers, bars, convenience stores/lock up shops, hawker $\&$ mobile shops, supermarkets, motor parks/tricycle garages, open markets and other. An observational checklist was used to collect data on indoor and outdoor forms of tobacco advertising and promotion.

Results: Among all public places visited, $64 \%$ sold tobacco including cigarettes, cigars, shisha and smokeless tobacco. All of the public places had some form of tobacco advertising or promotion located inside or outside. The most frequent forms of advertising or promotion were: display of tobacco products $(n=101)$, physical structures such as umbrellas $(n=80)$, poster/ signage $(n=38)$, mix of one or more forms $(n=27)$, stickers $(n=2)$ and free gifts $(n=2)$. Almost all forms of advertising and promotion were for cigarettes.

Conclusions: As the provisions are developed for the proposed Regulations of the National Tobacco Control Act, Nigerian authorities should consider closing existing loopholes in the law following recommendations of Article 13 of the Framework Convention on Tobacco Control. This includes banning the display of all tobacco products and any other form of tobacco advertising and promotion at tobacco retailers and all other public places.

\section{Tob. Induc. Dis. 2018;16(Suppl 1):A197 DOI:10.18332/tid/84059}

\section{2:30-14:00}

PS-621-3 Monitoring compliance with the ban on tobacco advertising and promotion at the point of sale in Bangladesh (2017)

S Choudhury ${ }^{1}$, K Walter², A Al-Mamun', S Jubayer ${ }^{1}$, M Bhuiyan ${ }^{3}$, L Candler $^{4}$, V Shah ${ }^{5}$, E Sebrie ${ }^{2}$

${ }^{1}$ National Heart Foundation Hospital and Research Institute, Epidemiology and Research, Dhaka, Bangladesh, ${ }^{2}$ Campaign for Tobacco-Free Kids, International Research, Washington, DC, United States of America, ${ }^{3}$ Campaign for Tobacco-Free Kids, International Advocacy, Dhaka, Bangladesh, ${ }^{4}$ Campaign for Tobacco-Free Kids, International Legal Consortium, Washington, DC, United States of America, ${ }^{5}$ Campaign for Tobacco-Free Kids, International Advocacy, Washington, DC, United States of America. E-mail: esebrie@tobaccofreekids.org

Background: Bangladesh amended its comprehensive tobacco control law in 2013, banning all direct and indirect tobacco advertising and promotion at points of sale (POS). While the law does not explicitly ban POS tobacco product display, legal interpretation considers it to be covered as a form of advertising. The objective of this study is to monitor compliance with the ban on tobacco POS advertising and promotion, including product display in Bangladesh.

Methods: Monitoring was conducted in Dhaka in May 2017 as a pilot study. Data was collected from a convenience sample $(n=46)$ of POS including supermarkets, groceries, general stores, tobacco shops, roadside sellers, mobile vendors, tea shops, and restaurants, located in high and low-income areas. An observational checklist was used to collect data on the presence of: (1) different types of advertising and promotion, and (2) tobacco product displays.

Results: Among all POS visited, 85\% sold cigarettes, 50\% sold smokeless tobacco, and $20 \%$ sold bidis. Nearly all POS (87\%) had at least one type of tobacco advertising, promotion, or product display. Almost half (48\%) of POS had advertising (including posters, stickers, signs, and mockup packs), and few (13\%) had promotions (including discounts and free gifts with purchase of tobacco products). Slightly over three quarters of POS (76\%) displayed any tobacco products. Tobacco products were displayed mostly in organized shelves or by laying them out on a tray or table, and a few had illuminated displays, hanging displays, or power walls (organized shelves of cigarettes on the wall behind the cash register).

Conclusions: Findings of this pilot study showed high levels of violations of Bangladesh's ban on tobacco POS advertising, promotion, and product display in Dhaka. Authorities should actively monitor and strictly enforce the ban, and should consider amending the law to explicitly ban POS tobacco product display for greater clarity.

Tob. Induc. Dis. 2018;16(Suppl 1):A198

DOI:10.18332/tid/84060

\section{2:30-14:00}

PS-622-3 Menace of surrogate advertising of Gutka-tobacco brand advertisement and resultant rampant selling bypassing Gutka ban in State of U.P A Kumar

'U.P. Health Services, Lucknow, India. E-mail: omrudrayanamah@ gmail.com

Background and challenges to implementation: Till $2012 \mathrm{Gutka}$ was selling as different brands like Dabang, Sudh Plus, Shikhar, Kamala Pasand and many more.On 1st April 2012 U.P. govt. FDA department banned Gutka, after that manufacturers came out twin packs of the same brands mentioned above, one having Pan Masala and other one tobacco-chotu thus circumventing the Legislation. In the absence of vendor licensing and also makeshift type of Pan masala and chewable tobacco sellers, it is very difficult to stop selling of twin pack at same outlet, which consumers use either mixing both or separately.

Intervention or response: Crackdown against surrogate advertisement at prominent traffic intersections, large shopping arcades, in the vicinity of educational establishments and around Govt. Offices/health facilities was done by Anti tobacco committee constituted in each 75 district with the help of Flying squad comprising departments of Health, FDA, Education, Excise, Labour, Police. Advertisement Materials removed by enforcement squad. Also surrogate selling by retailers were targeted specially around Educational institutions, Health facilities and Govt. Offices.

Results and lessons learnt: Resulted in marked decrease in availability of twin sachet of pan masala along with tobacco sachet. Advertisement and hoarding removed from the prominent places. It is inferred, since Pan masala is also a health hazard and a menace to cleanliness also an environmental hazard, need of hour Effective legislation required for ban on availability of pan masala containing many ingredients including arecanut etc.

Conclusions and key recommendations: There should be strict legislation for surrogate advertising and selling of twin sachet.

Section-4 of COTPA is ineffective in controlling chew able tobacco menace, requires amendment likewise.

Some effective legislation required to crackdown on surrogate advertising and resultant selling of twin sachets. It is perceived that some vendor licencing mechanism promulgated and enacted for retailers would be helpful in identification and regulation of indiscriminate and ambulatory retailers.

Tob. Induc. Dis. 2018;16(Suppl 1):A199

DOI:10.18332/tid/84081 


\section{2:30-14:00}

PS-623-3 E-cigarette marketing online: characteristics of websites serving the Indian market

N Lad' ${ }^{1}$, A Mehendale' ${ }^{1}$ V Shankar', R Kadam ${ }^{1}$

'Salaam Bombay Foundation, Tobacco Control \&t Advocacy, Mumbai, India. E-mail: tshering@salaambombay.org

Background: Electronic nicotine delivery systems (ENDS), commonly known as e-cigarettes in India are an emerging and controversial product in tobacco control debates. Previous studies suggest that use of ENDS by youth in numerous countries is expanding, however very limited information on use and access is known for the Indian market.

Methods: A survey of online merchants selling e-cigarettes was conducted in May 2015 by student volunteers of the Salaam Bombay Foundation. The search terms "e-cigarette", "electronic nicotine delivery system" etc. were used to identify online vendors. All websites selling and delivering e-cigarettes in India were included.

Results: 21 online vendors providing delivery in India were found in the survey. 17 vendors (81.0\%) exclusively sold ENDS, while remaining shops sold products beyond ENDS. 62\% shops $(n=13)$ sold refillable and disposable products. Remaining $38 \%$ shops $(n=8)$ sold refillable ENDS exclusively. The ENDS with a lowest price was Rs. 219 (\$3.25 USD) and average lowest price was Rs. 905 (\$13.44 USD). 52\% online vendors $(\mathrm{n}=11)$ included a health warning, and two-thirds of vendors $(n=14)$ included a message indicating sales to those under 18 years of age were not permitted. Positive health messages indicating that ENDS are safe or safer than cigarettes, were found on the web pages of $57.1 \%(n=12)$ vendors. The most common positive health claims included were, ENDS are "safer" $(42.9 \%, \mathrm{n}=9)$, have "no smoke" $(28.6 \%, \mathrm{n}=6)$ and produce "no ash" $(19.0 \%, n=4) .90 \%$ online vendors $(n=19)$ included a link to social media platforms.

Conclusions: This study suggests that there are a variety of online vendors available to children and youth in India interested in purchasing ENDS at various price-points. Such online sales should be regulated to remove positive health claims and ensure mechanisms to prevent the sale of ENDS to minors. Tobacco control programs should include information on the potential harms of ENDS devices.

Tob. Induc. Dis. 2018;16(Suppl 1):A200

DOI:10.18332/tid/84111

\section{2:30-14:00}

PS-624-3 Study on violation of tobacco control law (Tobacco Advertisement, Promotion and Sponsorship) in TV drama

A Sikder', TAPS Violation in TV Drama Study Group

${ }^{1}$ Ambala Foundation, Non-Government Ogranization, Dhaka, Bangladesh.E-mail: sikder@ambalafoundation.org

Background: The major area of the 2013 amendment to the 'Smoking and Tobacco Products Usage (Control) Act, 2005' and the corresponding Rules is the comprehensive ban on tobacco advertisement, promotion and sponsorship (TAPS) that includes prohibition of depiction, display and description of tobacco use in all entertainment media including Television. Consequently, this all sort of tobacco and smoking scene broadcasting in television is completely banned. The logic behind this kind of restriction is any kind of demonstration (direct or indirect) of tobacco product or tobacco related material is considered as one kind of advertising.

In spite of having a strong law, TV channels are still violating the law and screening smoking scene in drama. In reality, there is no monitoring mechanism to observe these kinds of violation of law in TV channels regarding broadcasting tobacco and smoking scene. Ambala Foundation conducted two studies on TV drama published on leading channels of Bangladesh. The objectives of the studies were to observe tobacco and smoking scene in different TV channels. The study also scrutinized how strategically tobacco and smoking scene are broadcasted in drama.

Methods: Total 14 national TV Channels were selected for both of studies. A total 244 dramas for study- 1 and a total number of 187 drama for study-2 were observed during this study.

Results: About 23 dramas out of 431 contained smoking scenes. In total 65 times smoking scenes is screened. Among them 11 times in one drama and 7 in another drama. The studies found that the length of smoking scene is total 40 minutes 26 Seconds. The report was submitted to Bloomberg Philanthropies and Government authorities taking step immediately.

Conclusions: This report can be a valuable documentation in advocating for strengthening enforcement of ban on all types of advertisement and promotional activities in electronic media through policy advocacy at country level.

Tob. Induc. Dis. 2018;16(Suppl 1):A201

DOI:10.18332/tid/84113

\section{2:30-14:00}

PS-625-3 Big Tobacco, Tiny Targets: discrediting tobacco industry tactics targeting kids through ads by school communities in 5 cities in Indonesia G Achnas' ${ }^{1}$ L Sundari²

'Campaign for Tobacco Free Kids (CTFK), Advocacy, Jakarta, Indonesia, ${ }^{2}$ Lentera Anak Foundation (LAF), Advocacy, Jakarta, Indonesia.E-mail: gachnas@tobaccofreekids.org

Background and challenges to implementation: Lack of strong laws has led tobacco companies such as PMI, BAT, and Djarum to systematically target children in Indonesia to start smoking through aggressive marketing strategies, including placing cigarette ads around schools. Influenced by ads, $30 \%$ of children picked up a smoke before age of 10 . The LAF research in 2015 showed $85 \%$ of schools were surrounded by cigarette ads. Only six cities have issued bans on outdoor tobacco ads.

Intervention or response: LAF, supported by CTFK, trained 2,700 school children, teachers, and local leaders to do monitoring around 90 schools in 5 cities, and mobilise a grassroots campaign to discredit tobacco companies marketing to kids. About 60 cigarette brands put advertising next to schools during the groups' research between September 2016 and February 2017. The children removed tobacco ads near schools with help of local authorities, leaders, and kiosk owners; galvanized online campaign \#RefuseToBeATarget; spoke to media; met with health and education officials.

Results and lessons learnt: In February 2017, 300 school representatives held a rally to call on President Jokowi to protect kids from Big Tobacco tactics. The students laid 120 mainly PMI and BAT tobacco advertisements removed around schools and displayed them as a "quilt" in front of Palace. The report, "The Invasion of Cigarette Ads around Schools" is being used by students from more cities to appeal to President Jokowi and presented to Education Ministry. This Indonesian initiative has spearheaded a global CTFK campaign discrediting Big Tobacco tactics targeting kids in 10 countries in Africa, Latin America, and Asia. A Global Report will be launched in early 2018. 
Conclusions and key recommendations: The campaign got significant media coverage and attention of policy makers. LAF and young advocates are using this momentum to keep discrediting tobacco industry tactics. They will lobby government officials to scale up campaign in Child Friendly Cities program in 10 cities in 2018 .

Tob. Induc. Dis. 2018;16(Suppl 1):A202

DOI: $10.18332 /$ tid/84134

\subsection{Innovations in media educational campaigns and health warning labels on cigarettes and other tobacco products}

\section{4:30-16:00}

F0-329-3 Over-time changes in reactions to pictorial health warning labels and association with quitting behavior among adult smokers in Thailand: findings from ITC Thailand survey $(2005$ - 2012)

A Jampaklay', R Borland ${ }^{2}$, G Fong ${ }^{3}$, B Sirirassamee ${ }^{1}, \mathrm{~L} \mathrm{Li}^{2}, \mathrm{H}-\mathrm{H}$ Yong $^{2}$, ACK Quah ${ }^{3}$

${ }^{1}$ Mahidol University, Institute for Population and Social Research, Salaya, Thailand, ${ }^{2}$ Cancer Council Victoria, Melbourne, VIC, Australia, ${ }^{3}$ University of Waterloo, Department of Psychology, Waterloo, 0N, Canada.E-mail: aree.ude@mahidol.ac.th

Background: This presentation explores over-time changes in smokers' reactions to pictorial health warning labels (PWLs) and impact on subsequent quitting in Thailand where PWLs on tobacco packs were first implemented in 2005 and were revised in 2007, 2010, 2011, and 2014 .

Methods: Nationally representative longitudinal data from six waves of the ITC Thailand Survey (2005-2012) were analyzed, involving a cohort of 2,000 smokers aged 18 years and older at baseline. The primary outcome was subsequent quitting behavior, including intentions to quit, quit attempts, and successful quitting.

Results: Descriptive analyses show that the effectiveness of the PWLs increased over time, evidence of the positive benefits of periodic increases in PWL size and changes in content to minimize the over-time reductions in PWL effectiveness shown in other ITC countries (e.g., Canada, US, Mauritius, Australia) when warnings have not changed. Specifically, there were increases in the percentage of smokers who often/very often noticed the warning labels, read the warning labels closely, reported that the PWLs made them think of harm of smoking, reported that PWLs influenced them to think about quitting a lot, and reported that PWLs stopped them from smoking more than once. Preliminary bivariate analyses found that there was a significant association between these indicators of warning effectiveness and subsequent attempts to quit. Additional analyses will examine whether these indicators also predict subsequent intentions to quit, quit attempt, and successful quitting when controlling for other known predictors of quitting.

Conclusions: The new PWLs on tobacco products implemented in 2005 with periodic rotation appear to show increased effectiveness over time among adult smokers in Thailand. More research is needed to understand the extent to which the health warnings contribute to the public health objective of increasing cessation among smokers and decreasing uptake among nonsmoking youth.

Tob. Induc. Dis. 2018;16(Suppl 1):A203

DOI:10.18332/tid/83813
14:30-16:00

F0-330-3 Identifying credible attribution statements for sources on pictorial health warning labels in China RD Kennedy', J Hardesty' ', Z Zhu', JE Cohen

'Institute for Global Tobacco Control at the Johns Hopkins Bloomberg School of Public Health, Baltimore, MD, United States of America. E-mail: rdkennedy@jhu.edu

Background: China currently uses text-only health warning labels (HWLs) on cigarette packages. Improving the design and content of cigarette HWLs is important to improve labels' credibility and effectiveness. Some countries use an attribution statement which gives an identified source for the health warnings and messages on the cigarette packaging, such as a Ministry of Health, or a public health leader such as the Surgeon General. This study tested the perceived credibility and effectiveness of four sources on HWLs in China, including the Chinese Center for Disease Control and Prevention (China CDC), the World Health Organization (WHO), the government agency of China's tobacco monopoly (STMA), and China's First Lady, Liyuan Peng (LP).

Methods: A cross-sectional study was conducted in 2016 with adults in 4 cities in China, including: Beijing, Shanghai, Kunming, and Wuhan $(\mathrm{N}=1,999)$. Study participants were shown a HWL with one of the 4 sources and asked "Which warning label appears the most credible?"; and "Which warning label appears the most effective at making people quit?", and "Which warning label appears the most effective at preventing young people from starting to smoke?"

Results: More than one third of respondents (36\%) selected the $\mathrm{WHO}$ as the most credible source, followed by the China CDC (30\%), STMA (24\%), and LP (9\%). The China CDC was perceived to be the most effective source for supporting people to quit, chosen by $38 \%$ of participants, followed by WHO (35\%), STMA (18\%), and LP (9\%). When asked to select the most effective HWL to prevent young people from starting to smoke, $35 \%$ selected WHO, followed by China CDC (33\%), STMA (18\%), and LP (13\%).

Conclusions: Using national or international public health authorities as the source for HWLs in China may help HWLs be more credible and effective at supporting quitting behaviors, and preventing young people from initiating cigarette use.

Tob. Induc. Dis. 2018;16(Suppl 1):A204

DOI:10.18332/tid/84063

14:30-16:00

F0-331-3 Indonesian adolescents' reactions to different text and image types of pictorial health warnings for cigarette packages

D Anshari ${ }^{1}$, J Thrasher ${ }^{2}$

${ }^{1}$ Universitas Indonesia, Health Education and Behavioral Sciences, Depok, Indonesia, ${ }^{2}$ University of South Carolina, Health Promotion, Education \& Behavior, Columbia, SC, United States of America. E-mail: dienanshari@gmail.com

Background: Research is needed on which characteristics of pictorial health warning (PHW) are most likely to reduce tobacco use among Indonesian adolescents. We assessed the effects of different text and image types of PHWs on emotional, motivational and cognitive reactions across key subgroups of Indonesian adolescents.

Methods: This study used both between- (i.e., text type) and within-subject (i.e., image type) designs, collecting data from adolescents aged $15-18$ years $(n=280$ smokers; $n=313$ 
nonsmokers) in Jakarta city and Bogor district. The main independent variables assessed in this study were text type (didactic vs. testimonial) and image type (graphic vs. suffering vs. symbolic). Participants rated each warning for fear, message credibility, message relevance, increasing health concerns, and motivating not to smoke. Adjustment variables include smoking status, sensation seeking, gender, and health topics of the PHWs. To adjust for correlated data due to repeated measures, linear mixed effect models were estimated separately for each outcome, regressing them on warning characteristics and adjustment variables.

Results: Adolescents rated didactic PHWs higher than testimonials only for message credibility $(\beta=0.31, p=0.015)$ and increasing health concerns $(\beta=0.25, \mathrm{p}=0.048)$. Symbolic PHWs were rated lower than suffering PHWs on all outcomes $(\mathrm{p}<0.001)$ except for message relevance $(\beta=-0.05, p=0.537)$, while graphic PHWs were rated higher than suffering PHWs for all outcomes $(p<0.001)$. Smoking status has no significant effect on fear $(\beta=-$ $0.01, p=0.935)$, positive effect on message relevance $(\beta=1.45$, $\mathrm{p}<0.001)$, but negative effects on message credibility $(\beta=-0.37$, $\mathrm{p}=0.010)$, increasing health concerns $(\beta=-0.80, \mathrm{p}<0.001)$, and motivating not to smoke $(\beta=-1.52, p<0.001)$. Sensation seeking has significantly negative effects on all outcomes.

Conclusions: PHWs with graphic imagery and didactic text are likely to have the greatest impact across subgroups of adolescents in low- and middle-income countries like Indonesia. Future studies should explore PHW characteristics that may work better among adolescents with higher sensation seeking.

Tob. Induc. Dis. 2018;16(Suppl 1):A205

DOI:10.18332/tid/84456

\section{4:30-16:00}

\section{F0-332-3 Health warnings effects on the decision} to quit. GATS analysis from Argentina

B Casetta ${ }^{1,2}$, J Konfino $^{3}$, AJ Videla ${ }^{1}$

${ }^{1}$ Ministry of Health, National Tobacco Control Program, Buenos Aires, Argentina, ${ }^{2}$ Instituto Universitario CEMIC, Family Medicine, Argentina, ${ }^{3}$ Centro de Estudios de Estado y Sociedad (CEDES), Buenos Aires, Argentina. E-mail: brunilda.casetta@gmail.com Background: The WHO MPOWER strategy recommends the use of health warnings on cigarette packs about the impact of tobacco on health. Previous studies have shown the association of noticing warnings and making a quit attempt. Argentina introduced warnings as by the National Tobacco Control Law in 2012. This study aimed to measure the association of health warnings and attitudes towards quitting.

Methods: Secondary analysis from the Global Adult Tobacco Survey (GATS) done in Argentina in 2012, restricted to adult smokers of 18 years old or more. Results from a populationbased study were analyzed using multivariable logistic regression modelling for the association of noticing health warnings and having favorable attitudes (referring one quit attempt during last year, quit less than a year ago or reported intention of quitting during the next month or year).

Results: Our sample included 1,717 smokers and recent quitters representing $6,431,623$ in a population-weighted projection (mean age 40.21 years, $37.77 \%$ women). Favorable attitude was present in $26.15 \%$ 95\%CI 20.53-32.67 (mean age 41.41 years, $35.86 \%$ females). Most of them (83.55\%) referred noticing warnings during the last month and $40.09 \%$ (95\%CI 33.12-47.49) admitted warnings made them consider to quit. Multivariate analysis showed health warnings were strongly associated with favorable attitudes towards smoking cessation (OR 4.64 95\%CI 2.28-9.41, $\mathrm{p}=0.001$ ) after adjusting by age, gender, educational level and income.

Conclusions: Noticing health warnings on cigarette pack was associated to favorable attitudes toward cessation. They had a robust effect and were effective regardless of smoker demographics.

Tob. Induc. Dis. 2018;16(Suppl 1):A206

DOI:10.18332/tid/83977

\section{4:30-16:00}

F0-333-3 Using message adaptation and pretesting to achieve resource efficiencies for best practice lung health communication programs in low- and middle-income country settings

N Singh Negi', V Mallik' ${ }^{1}$ T Turk², P Puri' ${ }^{1}$, S Mullin³ ${ }^{3}$ N Murukutla3, Vital Strategies

Vital Strategies, Policy, Advocacy and Communication, New Delhi, India, ${ }^{2}$ Vital Strategies, Policy, Advocacy and Communication, Sydney, SA, Australia, ${ }^{3}$ Vital Strategies, Policy, Advocacy and Communication, New York, NY, United States of America. E-mail: nnegi@vitalstrategies.org

Background: Effective message design is essential if lung health communication programs are to be cost efficient as well as meeting behavioural objectives. Strong and scientific methodology of message testing precedes development of any mass media campaign. There is a need of a careful combination of scientific content and marketing expertise to make a campaign effective and impactful. The most important component of any effective media campaign is to allow target audience to view, engage and respond to the campaign. Hence, adaptation and pre-testing of messages becomes important to test the comprehension, understanding and potential effectiveness of the campaign.

Methods: Twelve scripts were adapted from successful tobacco control communication programs conducted in high-income countries. The scripts were adapted to address smoking, smokeless tobacco use, and second-hand smoke, following which they were developed into animatics (animated video and audio executions) for ease of interpretation, and pre-tested through 20 FGDs with tobacco users and non-users conducted in India. Qualitative discussion and quantitative assessment was conducted through self-administered questionnaires with thirteen standardised items and 5 point Likert scales.

Results: Quantitative findings identified that a number of concepts for smoking/smokeless tobacco, and second-hand smoke scored consistently highly against indicators including: message was easy to understand, taught me something new, is believable, is relevant to my life, and; l would be likely to talk to someone else about this message. Qualitative findings provided insights into diagnostic amendments for the concepts.

Conclusions: Following analysis of findings, highly rated concepts were modified and final television and radio public service announcements were produced to support government of India, Cigarettes and Other Tobacco Product Act (COTPA) communication programs (sample animatics and final PSAs to be shared during the presentation).

Tob. Induc. Dis. 2018;16(Suppl 1):A207

DOI:10.18332/tid/84423

$12: 30-14: 00$

PS-626-3 The dispute between health and market: a communicational analysis of the cigarette package in Argentina 


\section{B Konfino', J Konfino ${ }^{2}$}

${ }^{1}$ Universidad de Buenos Aires, Quilmes, Argentina, ${ }^{2}$ Centro de Estudios de Estado y Sociedad (CEDES), Buenos Aires, Argentina. E-mail: brendakonfino@hotmail.com

Background: Cigarette package is among the most effective communication tools, not only used by the tobacco industry to market their products but also maximized by governments to warn the population about tobacco use and exposure risks.

The objective of the was to analyze from a sociosemiotic perspective the discourse enunciated in the cigarette packages.

Methods: We analyzed cigarette package communicational features: colours, typography, dimensions, phrases, images present in the industry marketed side of the package and also in the health warnings. We selected a convenient sample of the 3 most popular brands in Argentina that contained the 10 current pictorial health warning implemented in 2016. Texts were analyzed from a lexical, syntactic, morphologic and social perspective and images also from their chromatist characteristics.

Results: Health warnings occupied the $50 \%$ of the front and back package surface (23.65 squared centimeters), accomplishing national regulations. Their text had a role in limiting the imaging power of the picture. Together configure the discourse emitted in the health warnings. Infinitive verbs were usually used in the absence of a specific subject addressing a wide audience. Tobacco industry uses the other $50 \%$ with each brand identity, segmenting the targeted population with different communicational features, appealing to legacy, exclusiveness, funny and esthetic designs pursuing consumers' attention. We observed a conflicting coexistence of two contradictory discourses (health vs. market)

Conclusions: An equal distribution of marketed and health warned sides of the packages could be confusing to the population and could undermine the real effectiveness of the public health intervention. Policymakers should also consider the communicational advantages to deliver the message of increasing the size of health warnings.

Tob. Induc. Dis. 2018;16(Suppl 1):A208

DOI: $10.18332 /$ tid/83878

\section{2:30-14:00}

PS-628-3 An innovative approach to improving implementation of pack warnings

SS Alam', A Islam Sujon ${ }^{2,3}$, SA Rahman²

${ }^{1}$ Work for a Better Bangladesh (WBB) Trust, Communication - Tobacco Control, Dhaka, Bangladesh, ${ }^{2}$ Work for a Better Bangladesh (WBB) Trust, Health Rights and Tobacco Control, Dhaka, Bangladesh, ${ }^{3}$ Bangladesh Anti Tobacco Alliance (BATA), Communication and Advocacy - Tobacco Control, Dhaka, Bangladesh.E-mail: shovan1209@yahoo.com

Background and challenges to implementation: In Bangladesh by law there are pictorial warnings on cigarette packs. The system is that all packs display the same warning for six months, then shift to another warning. This system makes it difficult to know if companies are complying with the law.

Intervention or response: We decided to try demanding that, in accordance with various laws, tobacco companies display the production and expiry dates on all tobacco products. This would allow us to know which warnings the packs should display. The court case dragged on at length. Tobacco interference in legal cases was demonstrated when British American Tobacco was able to bring three barristers to the final hearing, whereas we were only allowed to bring one.

Results and lessons learnt: Despite a number of favourable decisions and a final ruling that the companies must display these dates, in the end nothing has changed. Although ultimately the case did not achieve the desired result, it was instructive for us in a number of ways. Among others, we had clear evidence of the intervention of tobacco companies in legal cases.

Conclusions and key recommendations: There is something seriously wrong when governments are not able to stand up to the tobacco industry. By definition, governments should have more power than corporations. They should be able to set the rules by which companies operate in their country. When this is not the case, democracy is not fully functional. This case gave us an opportunity to view the dysfunctional nature of the court systems in microcosm and reminded us of the importance of the WHO FCTC Article 5.3, so as to liberate governments from the influence of tobacco corporations.

Tob. Induc. Dis. 2018;16(Suppl 1):A209

DOI:10.18332/tid/83917

12:30-14:00

PS-629-3 Impact of pictorial health warnings on Vietnamese male smokers' knowledge of smoking harms

TT Ngan ${ }^{1,2}$, LV Anh $^{3}$, NN Bich ${ }^{4}$

${ }^{1}$ Hanoi University of Public Health, Centre for Population Health Sciences, Hanoi, Viet Nam, ${ }^{2}$ International Union against Tuberculosis and Lung Disease, Hanoi, Viet Nam, ${ }^{3}$ Vietnam Public Health Association, Hanoi, Viet Nam, ${ }^{4}$ Hanoi University of Public Health, Faculty of Environmental and Occupational Health, Hanoi, Viet Nam. E-mail: ngantran.hsph@gmail.com Background: In 2012, Vietnam National Assembly approved the first ever Law on Prevention and Control of Tobacco Harms. Enacted by the Law, since May 2013, pictorial health warnings (PHWs) covered $50 \%$ of the cigarette pack was obliged to print on all cigarette products. This study aims to assess the impact of PHWs on smokers' knowledge of smoking harms.

Methods: Two rounds of a cross-sectional study were conducted in 6 provinces of Vietnam. The 1st round was in May 2014 which was before the deadline for manufacturers to print PHWs (July 2014). The 2 nd round was in May 2015. Around 1,500 male smokers aged 18 to 35 were interviewed in each round. Respondents were asked to give their level of agreement towards 6 statements described the health consequences of smoking (same as the messages in PHWs). Based on their responses, the knowledge score was calculated.

Results: Smokers' average knowledge score on smoking harms in round $2(\mathrm{M}=22.5, \mathrm{SD}=3.7)$ was 0.86 point higher than in round 1 of the study $(\mathrm{M}=23.4, \mathrm{SD}=3.7)$ which is statistically significant $(\mathrm{t}(2949)=6.3, \mathrm{p}<0.001)$. Although there is no difference on average knowledge score between urban and rural and among different age groups, the positive association between education level and average knowledge score is significant $(\mathrm{F}(5 ; 2495)=6.1, \mathrm{p}<0.001)$. Indeed, those with higher education are more likely to have higher average knowledge score. However, between the 1st and 2nd round, the increase of average knowledge score among those with lower level of education (high school and below) is significantly greater than those with higher level of education (university and above) $(\mathrm{t}(2875)=3.9, \mathrm{p}<0.001)$.

Conclusions: PHWs consist of pictures and accompanying health warning messages have a certain impact on raising smokers' knowledge on smoking harms. Besides, the PHWs also help reduce the health disparities between groups with different level 
of education.

Tob. Induc. Dis. 2018;16(Suppl 1):A210 DOI:10.18332/tid/83928

\section{$12: 30-14: 00$}

PS-630-3 Pictorial warning labels in Jordan: are we maximizing the benefit for smokers?

R Bader ${ }^{1}$, R Shihab ${ }^{1}$, D Al-Rimawi ${ }^{1}$, F Hawari $^{1}$

'King Hussein Cancer Center, Amman, Jordan. E-mail: rbader@ khcc.jo

Background: Pictorial warning labels (PWLs) motivate smokers to quit and support continued abstinence. In Jordan, where $70 \%$ of adult males smoke, mass media campaigns are non-existent making PWLs of additional importance in communicating the benefits of quitting and prompting quit attempts.

Methods: We conducted an evaluation of the PWLs used in Jordan after having them in circulation for 2.5 years. We surveyed a cross-sectional convenience sample of smokers aged 17-26 years $(n=309)$. Respondents were asked about: previous quit attempts, beliefs in the need for PWLs and for more information within PWLs, frequency of noticing PWLs and recall of PWL messages, and considering quitting in response to PWLs. Finally, they were prompted with individual PWLs, and requested to rate each on its ability to evoke fear, add information, and motivate quitting.

Results: Results indicate that:

- $49 \%$ (150/309) of the respondents have had a failed quit attempt in the 12 months preceding the survey

- $48 \%(149 / 309)$ of the respondents believe there is a need for PWLs, among which 54\% (81/149) believe that PWLs should provide more information

- $63 \%$ (195/309) of the respondents noticed PWLs frequently in the month preceding the survey, among which $86 \%(168 / 195)$ correctly recalled at least one PWL message

- $36 \%(71 / 195)$ of those reporting frequent notice of PWLs also report considering quitting in response to PWLs

Respondents' rating of the individual PWLs is depicted in Figure 1.

Figure 1: Respondents' rating of individual PWLs

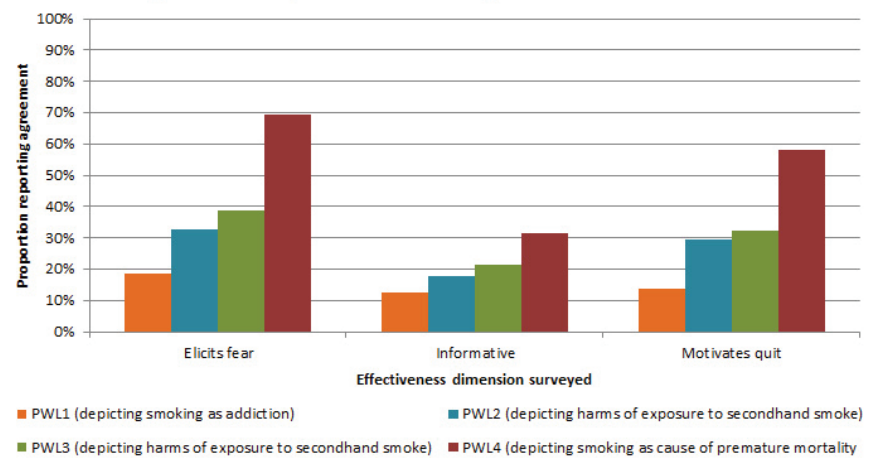

Conclusions: In Jordan, smokers seem to believe PWLs are necessary and seem to be affected by them to the extent of considering quitting. Yet, given the rate of failed quit attempts, and in light of the request for more information in PWLs, authorities in Jordan should consider supplementing PWLs with content to support self-efficacy to quit, such as including information on the available cessation services and behavioral tips for unaided quitting.

Tob. Induc. Dis. 2018;16(Suppl 1):A211

DOI:10.18332/tid/83936
$12: 30-14: 00$

PS-631-3 Evaluating the impact of health warnings in Brazil over 7 years (2009 - 2016): findings from the ITC Brazil Wave 1-3 surveys

C Perez ${ }^{1}$, SC Kaai ${ }^{2}$, T Cavalcante ${ }^{3}$, A Szklo ${ }^{4}$, L Craig ${ }^{2}$, G Li$^{2}$, ACK Quah $^{2}$, GT Fong', ${ }^{2}$

${ }^{1}$ Fundação do Câncer, Rio de Janeiro, RJ, Brazil, ${ }^{2}$ University of Waterloo, Waterloo, ON, Canada, ${ }^{3}$ Executive Secretariat of National Commission for Implementing WHO Framework Convention on Tobacco Control / Brazilian National Cancer Institute, Rio de Janeiro, RJ, Brazil, ${ }^{4}$ Brazilian National Cancer Institute, Epidemiology, Rio de Janeiro, RJ, Brazil, ${ }^{5}$ Ontario Institute for Cancer Research, Toronto, ON, Canada. E-mail: cp772017@gmail.com

Background: Studies demonstrate that large pictorial warnings (PHWs) on both sides of the pack have greater impact than textonly warnings. For over a decade, Brazil has had 100\% pictorial warnings but only on the back of pack. In 2009, Brazil introduced dramatic fear-arousing images. In 2016, Brazil finally added a front warning although text-only and $30 \%$. This study evaluated the 2009 and 2016 changes on key perceptual and behavioural indicators of warning impact.

Methods: Data were analyzed from Waves 1-3 (2009-2016) of the International Tobacco Control (ITC) Brazil Survey, a longitudinal cohort survey of representative samples of adult smokers in Rio de Janeiro, São Paulo, and Porto Alegre $(\mathrm{N}=1,719)$. Key outcomes were 6 validated indicators of warning impact. The 2009 warning revision was implemented 2 months after Wave 1 (2009) and 3 years before Wave 2 (2012-13). The 2016 warning revision (introducing front $30 \%$ text warnings) was implemented 3 years after Wave 2 (2012-13) and 8 months before Wave 3 (2016-17). Thus, pre-post evaluations of the two revisions were conducted by testing differences in the impact indicators across the three waves. Results: Three indicators of warning effectiveness increased significantly between W1 and W2 (showing greater effectiveness of introducing dramatic fear-arousing images of the 2009 revision) and declined at W3 (showing lack of effectiveness of introducing the front text-only warning): noticing (W1 $=45 \%$; $\mathrm{W} 2=51 \%$; $\mathrm{W} 3=49 \%)$, reading $(\mathrm{W} 1=34 \%$; $\mathrm{W} 2=41 \%$; $\mathrm{W} 3=36 \%$ ), avoiding labels $(\mathrm{W} 1=43 \%$; $\mathrm{W} 2=45 \%$; $33=34 \%)$. The remaining 3 indicators (forgoing smoking, thinking about risks, and thinking about quitting) declined at W2 and W3.

Conclusions: Introducing dramatic fear-arousing images (2009) increased warning effectiveness, but introducing $30 \%$ textonly front warnings (2016) did not. There was also evidence of "wearout" of warning impact. These findings demonstrate the importance of frequent warning revision, and the need for pictorial warnings on the FRONT of the pack, consistent with FCTC Article 11 Guidelines.

Tob. Induc. Dis. 2018;16(Suppl 1):A212

DOI:10.18332/tid/83942

\section{$12: 30-14: 00$}

\section{PS-632-3 Translational practice: Media Beacon} C Curell ${ }^{1}$

'Vital Strategies, Policy, Advocacy and Communications, New York, NY, United States of America. E-mail: ccurell@ vitalstrategies.org

Background and challenges to implementation: In high- and low-income countries alike, there is still a global lag between evidence and practice in tobacco control communications. Whether or not it is due to a lack of resources, proper 
application of health communication science, and use of effective communication material, are often missing from the mass media campaign development process.

Intervention or response: Vital Strategies' new Media Beacon resource addresses this by providing evidence-based materials that can be easily adapted and licensed from their original owners, at little or no cost, for outcome-oriented mass media campaigns. The site also provides practical guidance on campaign planning and development

Results and lessons learnt: Theresource ofvideomaterial appropriate for television PSAs is available in Arabic, Bahasa, Chinese, English, French, Hindi, Russian and Spanish. Beacon also holds a library of 92 images available for use on graphic pack warnings, with a special focus on images relevant to the AFRO region. The searchable, userfriendly online resource provides information and evidence-based materials likely to be useful in national-level mass media campaigns targeting behavior and policy change.

Conclusions and key recommendations: National governing bodies and tobacco control partners should utilize the Media Beacon resource when planning and developing mass media campaigns or implementing graphic health warnings on tobacco packaging, particularly in low- and middle-income countries where this can greatly reduce the cost of production.

Tob. Induc. Dis. 2018;16(Suppl 1):A213

DOI:10.18332/tid/83945

\section{2:30-14:00}

PS-633-3 Perceived effectiveness of larger graphic health warnings and plain packaging among urban and rural adolescents and adults of Delhi and Telangana, India

T Rawal ${ }^{1}$, GP Nazar ${ }^{1}$, S Ravishankar ${ }^{2}$, N Grills ${ }^{3}$, P Webster ${ }^{2}$, M Arora ${ }^{1}$

'Public Health Foundation of India, Health Promotion Division, Gurgaon, India, 'University of Oxford, Nuffield Department of Population Health, Oxford, United Kingdom, ${ }^{3}$ University of Melbourne, Nossal Institute for Global Health, Melbourne, VIC, Australia.E-mail: teena.rawal@gmail.com

Background: Plain packaging has been demonstrated to be effective in Australia, where it led to increased quit attempts among adult smokers. Graphic Health Warnings (GHWs) on tobacco packs in India have increased from $40 \%$ to $85 \%$. This qualitative study assesses perceptions of Indian adults and children about impact of larger GHWs and plain packaging of tobacco products.

Methods: Focus Group Discussions (FGDs), conducted with children aged 13-17 years and adults aged $17+$ years in the community settings. Separate FGDs were conducted with adults (male and female) and children (boys and girls) in selected urban and rural communities in Delhi and Telangana in 2016. Four dummy tobacco packs categories [A-40\% old GHWs, B-40\% new GHWs, C-85\% new GHWs; D-85\% new GHWs with plain pack] were shown. Data was coded and thematic analysis undertaken with using Atlas.ti 6.2.

Results: In total, fourteen (six in Delhi and eight in Telangana) FGDs were conducted. Participants highlighted the importance of larger GHWs on tobacco products in demonstrating the consequences of tobacco use and limit the pack's appeal. Participants opined, category $\mathrm{C}$ and $\mathrm{D}$ warnings were most effective to curb tobacco use. Category D was considered the most unattractive pack due to larger GHW, dull color and brand name not being prominent.

Conclusions: Larger GHWs and PP were perceived to be effective in reducing tobacco use. Plain packaging was further perceived to reduce the attractiveness of pack, enhance noticeability of the GHW, deter new users, and improve quitting among users.

Tob. Induc. Dis. 2018;16(Suppl 1):A214

DOI:10.18332/tid/83952

\section{2:30-14:00}

PS-634-3 Cancer foundation campaign for the 2017 World No Tobacco Day

C Gomes ${ }^{1}$, M Pinho ${ }^{1}$, AC Martins ${ }^{1}$, H Daher $^{1}$

'Fundação do Câncer, Rio de Janeiro, RJ, Brazil. E-mail: claudia. gomes@cancer.org.br

Background and challenges to implementation: The 2017 World No Tobacco Day (WNTD) theme was "Tobacco - a threat for development" because of the numerous damages that tobacco consumption and production causes to people, the environment, the individual and global economy.

Intervention or response: The Cancer Foundation (CF) adopted the theme and developed a one-week communication strategy with posts on social media Facebook, Instagram and Twitter. There were six-diary post accompanied by explanatory text addressing the damages related to the image of the day. An infographic was developed that in a synthetic way approached several aspects of the WNTD theme.

The campaign was also shared with partner institutions of the $\mathrm{CF}$ by email marketing.

\section{Tabaco: Uma ameaca ao desenvolvimento}

31 DE MAIO: DIA MUNDIAL SEM TABACO

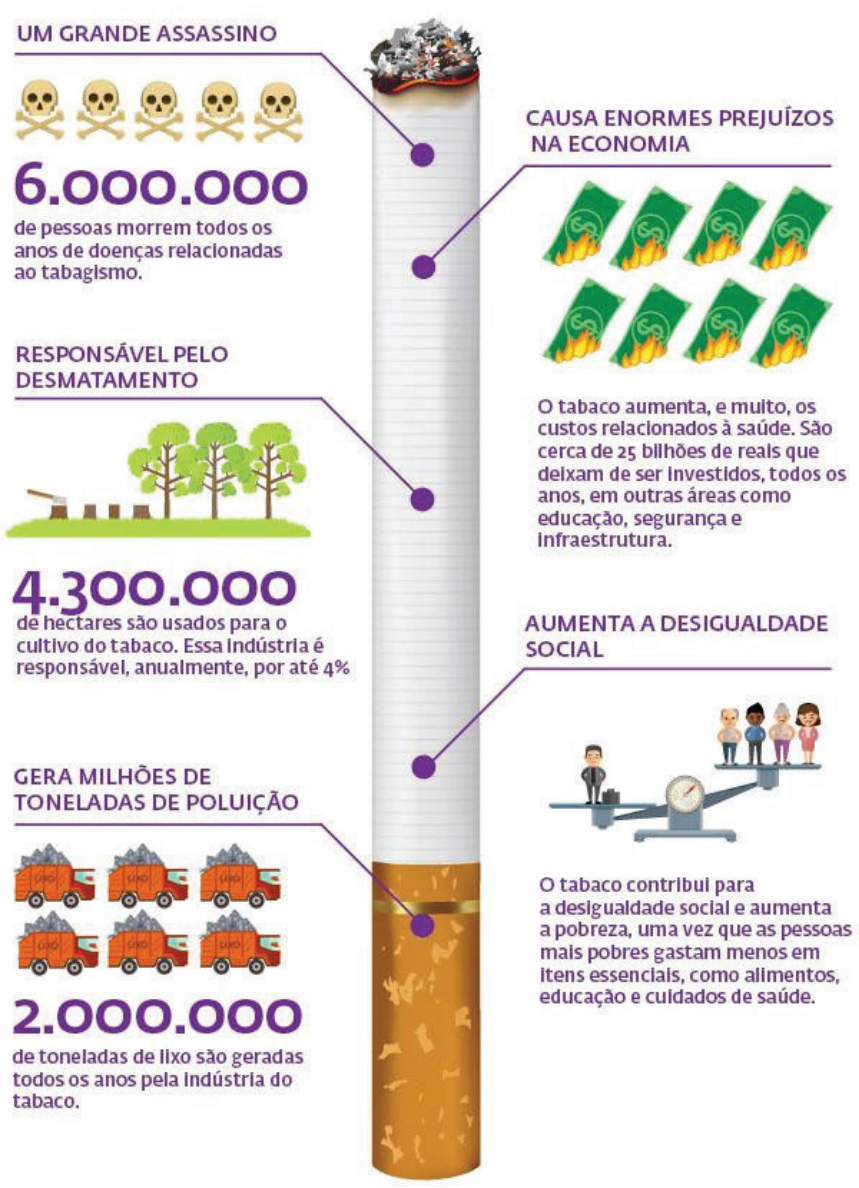

[Infographic] 
Results and lessons learnt: The CF Facebook's profile has more than $260 \mathrm{k}$ followers and the campaign reached more than $22.3 \mathrm{k}$ during the week, without any financial boost. the post with the longest reach was the infographic with more than $6.6 \mathrm{k}$ and 234 reactions, followed by the post of May 31 WNTD, with $5.6 \mathrm{k}$ and 228 reactions and May 29's with 3.3k and 144 reactions. On Instagram and Twitter, the range was lower. Another great campaign adopted by the institution is the UICC World Cancer Day and some posts were boosted, reaching up to $81 \mathrm{k}$ followers. However, unpaid campaign posts ranged from $2.5 \mathrm{~K}$ to $5.5 \mathrm{~K}$, similar to the reach of the 2017 WNTD adopted by CF.

Conclusions and key recommendations: The tobacco control campaign of 2017 was well received and had a similar scope when compared to other Foundation campaigns. The comments were satisfactory and the content elaborated could be used for other occasions during the year. However, boosting the posts would give a much greater scope to action and this will be reevaluated next year. By the way, this campaign is also timeless and can be used on other dates related to tobacco control.

Tob. Induc. Dis. 2018;16(Suppl 1):A215 DOI:10.18332/tid/83966

\section{2:30-14:00}

PS-635-3 Impact of textual framing and depictions of harm on indicators of cigarette pictorial health warning label effectiveness in China

J Hardesty', J Cohen', Z Zhu', R Kennedy'

'Johns Hopkins Bloomberg School of Public Health, Baltimore, MD, United States of America. E-mail: jhardesty@jhu.edu

Background: Cigarette pictorial health warning labels (HWLs) are more effective than text-only HWLs at increasing cessation intention. Measuring indicators of HWL effectiveness, such as salience of HWLs, thinking about the health risks of smoking, thinking about quitting smoking, avoidance of HWLs, perceived risk of smoking, and likelihood of forgoing next cigarette can help explain how viewing HWLs impacts cessation intention. Our aim was to conduct a pre-post analysis of these indicators to evaluate how pictorial HWL textual framing (factual- vs. personal-text) and depictions of harm (smoking- vs. secondhand smoke-related harms) encourage cessation intention.

Methods: A cross-sectional randomized experimental survey was conducted during 2016 in four Chinese cities: Beijing, Shanghai, Wuhan, and Kunming. Adult smokers $(n=1612)$ answered questions regarding indicators of effectiveness of the current text-only HWLs in China, were presented eight pictorial HWLs with factual- or personal-text and depictions of smokingor secondhand smoke-related harms, and answered questions regarding indicators of pictorial HWL effectiveness. T-tests were conducted to compare differences between current text-only and pictorial HWL indicators.

Results: Reported salience, thinking about health risks, thinking about quitting, avoidance, perceived risks, and likelihood of forgoing next cigarette significantly increased after viewing the personal pictorial HWLs (each test, $\mathrm{p}<0.01$ ). Similar results were found for factual pictorial HWLs; however, avoidance of factual pictorial HWLs with depictions of smoking- or secondhand smokerelated harms was unchanged ( $\mathrm{p}=0.1$ and $\mathrm{p}=0.6$, respectively).

Conclusions: All reported indicators of HWL effectiveness increased after viewing pictorial HWLs with the exception of avoidance of factual pictorial HWLs suggesting that both personal and factual pictorial HWLs depicting smoking or secondhand smoke-related harms are effective at encouraging cessation intention. Future studies can use these indicators as tools to identify the most effective and culturally relevant pictorial HWLs for Chinese smokers.

Tob. Induc. Dis. 2018;16(Suppl 1):A216

DOI: $10.18332 /$ tid $/ 83979$

\section{2:30-14:00}

PS-636-3 The role of digital and social media within mass media anti-smoking campaigns

A Lloyd ${ }^{1}$, J Mckendrick', A Rutter ${ }^{1}$

${ }^{1}$ Fresh, Smokefree North East, Durham, United Kingdom, ${ }^{2}$ Robson Brown, Newcastle upon Tyne, United Kingdom. E-mail: ailsa. rutter@freshne.com

Background and challenges to implementation: The evidence for mass media to raise awareness about the harm of smoking and benefits of quitting remains strong. However, pressures on budgets means TV is not always seen as an affordable option, while the emergence of digital media presents an attractive option to funders as a way of saving costs while maintaining responses. Digital and social media has provided us with learnings about the role of "push" (broadcast) and "pull" (direct responses) within campaigns and the balance of positive and negative messages.

Intervention or response: Fresh (a subnational tobacco control programme) has ran three hard hitting health campaigns on regional TV while using digital media including advertising and boosted Facebook posts as a supporting channel to prompt discussion and to generate responses to a campaign website. Content online has included real people's stories, including emotionally engaging videos of former smokers with COPD or cancer.

Results and lessons learnt: Our experience has found that as well as generating thousands of responses:

Social media is a new platform to share hard hitting real people's stories which convey the shock, emotion and life-limiting reality of smoking. The public are keen to engage on a discourse around how smoking has devastated families. People tag friends, share posts and tell their own stories about loved ones affected. This challenges assumptions by some that social media needs to be constantly positive to compete with commercial brands for attention and doubtless provides opportunities on other tobacco control topics.

Conclusions and key recommendations: Digital media plays an important role in driving direct responses to campaign websites that offer support, and in generating discussion. However, it is still TV that drives the conversation and response rates online; digital display performs best if it features campaign visuals, while TV advertising is often discussed in Facebook responses. We continue to make the case to fund TV advertising.

Tob. Induc. Dis. 2018;16(Suppl 1):A217

DOI:10.18332/tid/84020

\section{2:30-14:00}

PS-637-3 Implementation of graphic health warnings in Bangladesh - current status and challenges MB Rahman 1,2, SH Patwary², A Islam³ , F Zaman², M Mohiuddin² 'Dhaka International University, Department of Business Administration, Dhaka, Bangladesh, ${ }^{2}$ Dhaka International University, Tobacco Control and Research Cell, Dhaka, Bangladesh, ${ }^{3}$ Work for a Better Bangladesh (WBB) Trust, Health, Dhaka, Bangladesh.E-mail: sohel_tarafdar@yahoo.com

Background: Graphic Health Warning (GHW) in $50 \%$ of all tobacco packets/packages has been implemented from 19 March 
2016 following the tobacco control (TC) law that amendment in 2013 and rules notified in 2015. But, there are many small and large tobacco industries bypass and ignore the TC law in different ways. The study was conducted to identify and show the current GHW implementation and challenges in Bangladesh and the sample collection period of the study was January 2017 to April 2017.

Methods: Out of 64, survey was conducted in 25 Districts and both qualitative and quantitative techniques, semi structured questionnaire and observational methods used to conduct the study. GHWs in all types of tobacco products were observed. 3 retail shops and 3 wholesale shops selected from each district. A research team was developed and organized a workshop regarding tobacco control law and GHW implementation for effectiveness and quality of the study and Excel software was used to analyze the data.

Results: Total 5234 tobacco products was investigated, 1757 smoking tobacco (1497 Cigarette packets and 260 Bidi packets) and 3477 smokeless tobacco (3263 different packs of Jarda and $214 \mathrm{Gul})$. Study found that, 34\% Bidi, 25\% Jarda and $15 \% \mathrm{Gul}$ in market without GHW. 64\% bidi, 75\% Jarda, 85\% Gul provide GHWs, but among these, $50 \%$ bidi, $45 \%$ jarda and $30 \%$ gul doesn't follow law. It shows that, excluding cigarettes packets, bidi and smokeless tobacco vastly ignore about GHWs section of law. Absence of manufacturing date and different size/forms of smokeless tobacco and bidi packets, it is very difficult to measure GHWs implementation on all tobacco products.

Conclusions: Its necessary to ensure uniform packets for smokeless tobacco and bidi with date of manufacture and monitoring for effective implementation of GHW provisions in Bangladesh.

Tob. Induc. Dis. 2018;16(Suppl 1):A218

DOI: $10.18332 /$ tid/84039

\section{2:30-14:00}

PS-638-3 Urgent need for implementing media education campaigns and health warnings in Parties with high burden of SLT use

A Yadav' ${ }^{1}$ DN Sinha' ${ }^{1}$ K Chandan ${ }^{1}$, R Mehrotra ${ }^{1}$

${ }^{1}$ National Institute of Cancer Prevention and Research, WHO FCTC Global Knowledge Hub on Smokeless Tobacco, Noida, India.E-mail: advocateamit@msn.com

Background: The FCTC country reports indicate that $70 \%$ of the reporting parties have implemented Article 12 in their jurisdictions. The MPOWER Report 2015 also indicates that nearly $40 \%$ of the Parties conducted at least one anti-tobacco national mass-media campaign. However, both reports do not provide any specific information on implementation of Article 12 with respect to SLT products. There are 23 Parties with more than 1 million SLT users.

Methods: This paper presents an analysis of the FCTC country reports, MPOWER report, besides the relevant indicators under GYTS, GSPS, GHPS and GATS with respect to mass-media, education and training efforts undertaken and reported by the Parties with high SLT burden. The health warning regulations of these parties has been reviewed. The paper also looks at other sources of mass-media information on hazards of SLT use like YouTube.

Results: The analysis reveals that:

- Only a limited number of Parties have implemented national and sub-national mass-media campaign on SLT.

- India is the only country with dedicated national massmedia on anti-SLT awareness, besides tobacco free schools, communities and villages.

- Only nine Parties from high burden countries have implemented health warnings on SLT.

- Some countries have also used mass-media including YouTube for raising anti-SLT awareness besides earned media.

- School education have limited information on hazards of SLT use.

- Limited Parties have included prevention of tobacco use in school curriculum.

- Teachers are not trained nor do they have access to teaching materials for youth tobacco prevention.

Conclusions: Parties with high SLT burden should use the Global Tobacco Surveillance System to collect data on various aspects of Article 12 on SLT use. This will help in developing and implementing focused and comprehensive national media education and communication initiatives to prevent SLT use, especially among vulnerable population group. Social media campaigns can also be used as effective interventions for SLT control.

\section{Tob. Induc. Dis. 2018;16(Suppl 1):A219 DOI:10.18332/tid/84076}

\section{2:30-14:00}

PS-639-3 Connecting the region through infographics and social media in tobacco control campaigns

W Balderas'

'Southeast Asia Tobacco Control Alliance, Media and Communications, Bangkok, Thailand.E-mail:wendell@seatca.org

Background and challenges to implementation: Over 300 million people are active social media users in South East Asia ${ }^{1}$. Social media offers civil society and non-government organizations (NGOs) a chance to move from a one-way to a two-way type of communication that is interactive and engaging. It is an important and instinctive tool that NGOs can use in different advocacies to inform, educate and get people to act on a particular issue. Social media is an extremely efficient and affordable tool that should be part of every organization's communications strategy. Because people are flooded with so many information in social media, infographics can really grab your audience's attention.

${ }^{1}$ Digital in Southeast Asia in 2017 https://wearesocial.com/sg/ blog/2017/02/digital-southeast-asia-2017

Intervention or response: During the 2017 World No Tobacco Day, the Southeast Asia Tobacco Control Alliance (SEATCA) launched a 7-day social media campaign on Facebook and Twitter. With the theme: "Tobacco - A Threat to Development", SEATCA developed materials in the form of infographics that showed how tobacco poses a threat to the sustainable development of all countries and how these threats can be addressed by different tobacco control measures ${ }^{1}$. We encouraged partners from different parts of the region to localize the materials based on their country data and experience.

${ }^{1}$ SEATCA Infographics for World No Tobacco Day 2017: http:// bit.ly/2tPgSQX http://bit.ly/2uxdlit http://bit.ly/2uR0bl4

Results and lessons learnt: Studies also show that materials with visual elements like compelling images or graphics can generate more views than text only messages. Infographics can also be a truly effective way to increase awareness on your organization.

Conclusions and key recommendations: Combining your infographics with a well-written message is a powerful way to help your content cut through the information jungle. It is also 
important that messages in your social media materials are relatable to your followers.

Tob. Induc. Dis. 2018;16(Suppl 1):A220 DOI:10.18332/tid/84088

\section{2:30-14:00}

PS-641-3 Engaging youth in anti-tobacco awareness campaigns in India

R Kadam ${ }^{1}$, D Patil'1, N Lad ${ }^{1}$

'Salaam Bombay Foundation, Tobacco Control \&t Advocacy, Mumbai, India.E-mail: tshering@salaambombay.org

Background and challenges to implementation: Tobacco use among school children is a serious problem in India. Lack of proper guidance, urge to imitate elders, peer pressure, misconceptions, easy availability of variety of tobacco products and innovative advertisements attract the children to tobacco. Salaam Bombay Foundation (SBF), under its leadership program conducts tobacco control awareness campaigns engaging the school children.

Intervention or response: Since 2006 Salaam Bombay Foundation has launched various long term tobacco control awareness campaigns engaging the youth. The accompanying table has detailed information on the year/date of launch of awareness campaigns with their objectives and modes of implementation.

Results and lessons learnt: Quit Tobacco Movement campaign in now extended to every school to advocate for quitting consumption of tobacco among children, communities etc. 'Life Se Panga Mat Le Yaar' campaign reached out to 165,000 to 227,000 people through radio stations and got 2,581 youtube views. It also earned print media attention in Times of India and Hindustan Times. In 'Election Campaign' program 692 children from three municipal schools in Mumbai participated. 2290 campaign slogans were collected from participating schools. "Tambakhu ko Dishum" was an innovative online campaign to create awareness on tobacco which grabbed the attention of youth as well as media. After submission of demand charter during "Right to Tobacco Free School", government authorities discussed it with children and assured that official letters would be sent to all the schools in Mumbai to fulfill all the demands mentioned in demand charter.

Conclusions and key recommendations: Engagement of empowered youth in the tobacco awareness campaigns helps to create awareness on tobacco control among their peers, community. Such campaigns involving youth will prove beneficial in reducing the tobacco habits in future generations of India. But, the effectiveness of the campaigns rests in utilizing energy and innovative minds of youth appropriately.

Tob. Induc. Dis. 2018;16(Suppl 1):A221

DOI:10.18332/tid/84115

[Tobacco Control Campaigns of SBF]

Launch of Campaign
implemented every year in the schools having SBF's Super
Army Leadership Program
Life Se Panga Mat Le Yaar (2011) This campaign was
launched involving a celebrity from Indian film industry
to get the attention of youth on the important issue of
tobacco and its harmful effects. The campaign had a long
term impact due to media coverage.
Election Campaign (2014) This campaign was done during
the parliamentary elections in India in 2014. The similar
campaign will be conducted during the state and municipal
elections to create awareness on tobacco

"Tambakhu Ko Dhishum" (2015) It is a long term online campaign which makes the use of social media like Facebook where photographs clicked by children are posted on a continuous basis.

\begin{tabular}{|c|c|}
\hline Objective & Implementation \\
\hline $\begin{array}{l}\text { To promote } \\
\text { freedom from } \\
\text { tobacco }\end{array}$ & $\begin{array}{l}\text { Inspired by the Quit India Movement Used freedom struggle } \\
\text { imagery to promote freedom from tobacco. Street plays, } \\
\text { rallies, poster exhibitions at railway stations and public places. }\end{array}$ \\
\hline $\begin{array}{l}\text { To counteract } \\
\text { positive images } \\
\text { of tobacco in }\end{array}$ & $\begin{array}{l}\text { Launched using a celebrity ambassador Street plays, rallies } \\
\text { at public places. Audience engagement through radio, social } \\
\text { media. }\end{array}$ \\
\hline
\end{tabular}

popular cinema.

To encourage people to stop using tobacco as part of New Year's resolution.

To collect cases of violation of COTPA 2003 act
The students took on the role of campaigners and voters for a hypothetically created "Tambakhu Mukta Party". Automated voting machine was created to showcase various voting options to choose from various tobacco products.

Online campaign launched on 'World No Tobacco Day 2015' with NGOs in Mumbai. The campaign monitored the violations of the act related to sale and promotion of tobacco products.

\section{2:30-14:00}

PS-642-3 A study of gruesome graphical health warning labels among Georgian adults: for whom are they more effective?

C Lingwall ${ }^{1}$, M Topuridze ${ }^{2}, \mathrm{~N}$ Maglakelidze ${ }^{2}$, L Sturua ${ }^{2}, \mathrm{M}$ Shishniashvili², A Gamkrelidze ${ }^{2}$, C Berg $^{1}$

${ }^{1}$ Emory University, Atlanta, GA, United States of America, ${ }^{2}$ Natinal Center for Disease Control and Public Health, Tbilisi, Georgia. E-mail: cjberg@emory.edu

Background: Graphic health warning labels demonstrate effectiveness, particularly for low- and middle-income countries. This study examined six recommended health warning labels, three of which include gruesome imagery and three relatively benign imagery, in a sample of 1,163 adults in Georgia, a middleincome country with high smoking prevalence. We examined: 1) each of these graphical warning labels to text only as well as to one another in terms of "effectiveness in motivating smokers to quit or preventing people from starting smoking"; and 2) prevalence and correlates of rating gruesome (versus benign) images as more, effective, or equally effective.

Methods: We conducted a split-half experimental design in the context of a national survey, whereby participants were asked to rate two different sets of text and graphical warning labels.

Results: Gruesome images were rated as more effective than text and benign images (p's $<.001)$; only one benign image was rated as more effective than text $(\mathrm{p}=.003)$. While $43.8 \%$ rated gruesome images more effective on average, $43.4 \%$ rated gruesome and benign images equally effective (no difference), and $12.9 \%$ rated benign images as more effective. Nominal logistic regression indicated that, compared to those rating the images equally effective (referent group), those reporting benign images more effective had higher monthly income $(\mathrm{p}=.034)$ and fewer friends 
who smoked ( $\mathrm{p}=.042)$; no significant predictors of rating gruesome images as more effective were identified (R-squared $=.058$ ). Among current smokers, compared to those rating the images equally effective, those reporting benign images as more effective rated quitting smoking as more important $(\mathrm{p}=.006)$ and were older at age of initiation $(\mathrm{p}=.023)$; those reporting gruesome images more effective had lower household income $(p=.002)$, were less likely to have children $(p=.05)$, and were more likely to be unemployed ( $\mathrm{p}=.05$; R-squared $=.534$ ).

Conclusions: Gruesome imagery is not more effective for all people; understanding the effectiveness in different populations is critical.

Tob. Induc. Dis. 2018;16(Suppl 1):A222

DOI:10.18332/tid/84121

\subsection{Opportunities for regulating tobacco products' content and emissions to reduce harm and addiction potential}

\section{$12: 30-14: 00$}

PS-643-3 MADES study: evaluation plan of the new tobacco product directive 2014/40/UE in Italy

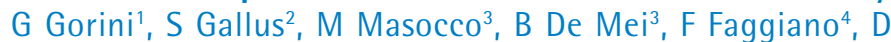
Galeone ${ }^{5}, \mathrm{P} \mathrm{Izzo}^{3}$, R Pacifici ${ }^{3}$, MADES Study Group

${ }^{1} I S P O$, Florence, Italy, ${ }^{2}$ RCCCS - Istituto di Ricerche Farmacologiche 'Mario Negri', Department of Epidemiology, Milan, Italy, ${ }^{3}$ National Institute of Health (ISS), Rome, Italy, ${ }^{4}$ Oriental Piedmont Unversity, Novara, Italy, ${ }^{5}$ talian Ministry of Health, Center for Disease Prevention and Control, Rome, Italy. E-mail: g.gorini@ispo.toscana.it

Background: The transposition of the New Tobacco Product Directive (TPD) 2014/40/UE in Italy entered into force in 2016. Main regulations were:

1. Reporting obligations, ban of some ingredients and of some products with characterising flavours

2. Pictorial warnings with Quitline number

3. Sale ban of 10-cigarette packages

4. Reporting obligations for new electronic-cigarettes (e-cigs); Advertising ban; child- and tamper-proof for refill containers.

The transposition was an occasion to add new measures :

1. Stricter enforcement of the sale ban to minors

2. Smoking ban in cars with minors and pregnant women

3. Smoking ban in outdoor areas of Pediatric and Gynecologic Departments

4. Sale ban of e-cig and refill containers to minors

Methods: Presentation of the evaluation plan of the impact of the new TPD in Italy. Presentation of preliminary results for the year 2017.

Results: The behavioural risk factor surveillance system PASSI, the ISTAT survey and the DOXA surveys on representative samples of Italians will allow us to study smoking and e-cigarette prevalence in adults; quitting attempts among smokers; the impact of pictorial warnings on smokers; second-hand smoke exposure in non-smokers; attitudes, perception, and behaviours of Italians after the coming into force of the new TPD;

The GYTS (Global Youth Tobacco Survey) will monitor smoking and e-cig prevalence, tobacco access and availability among Italian adolescents;

The National Institute of Health (ISS) will monitor the compliance with the smoking ban in cars, and the outdoor ban outside some Hospital Departments;

Temporal trend in Quitline calls will be studied. The impact of pictorial warnings will be also studied among smokers calling the
Quitline, or attending Italian Smoking Cessation Centers.

The online transmission of ingredients of tobacco products from tobacco companies will be followed at the ISS.

We will add preliminary results not yet produced.

Conclusions: The evaluation plan will allow to study all dimensions of the TPD impact.

Tob. Induc. Dis. 2018;16(Suppl 1):A223

DOI:10.18332/tid/83822

\section{2:30-14:00}

PS-644-3 An overview of the role of flavors in e-cigarette addiction

E Krüsemann ${ }^{1,2}$, S Boesveldt' ${ }^{2}$, K de Graaf ${ }^{2}$, R Talhout ${ }^{1}$

${ }^{1}$ National Institute for Public Health and the Environment (RIVM), Centre for Health Protection, Bilthoven, Netherlands, ${ }^{2}$ Wageningen University, Division of Human Nutrition, Wageningen, Netherlands. E-mail: erna.krusemann@rivm.nl Background: E-cigarettes are available in a wide variety of flavors, which increases sensory appeal and stimulates smoking initiation, especially among youth. To determine regulatory measures on flavors in e-cigarettes, e.g. restriction or prohibition, more insight should be obtained in the role of flavors in e-cigarette addiction. Core components of addiction are liking, learning, and wanting. We provide an overview of e-cigarette flavors related to these aspects of addiction, including differences between youth and adults, and smokers and non-smokers. In addition, we aim to identify e-liquid flavors that are representative for different flavor categories (e.g. sweet, fruit, tobacco).

Methods: A systematic literature review was performed in May 2017 using PubMed and EMBASE databases. Key words included terms to capture concepts associated with e-cigarettes, flavors, liking, learning, and wanting in articles published from database inception to the search date. Results were independently screened (0.92 Cohen's Kappa) and reviewed.

Results: Searches yielded 387 unique studies of which 32 were included. Research designs varied between cross-sectional, experimental, mixed-method, case study, and longitudinal. Flavors were described as an important reason for e-cigarette initiation. Youth mainly prefer fruit and sweet flavors, while tobacco is more popular among adults. Studies used different flavor categories such as sweet, fruit, tobacco, mint, candy and dessert. E-liquids representing these categories varied across study designs (e.g., vanilla represented the sweet, candy, as well as the dessert category). Nevertheless, results of different studies were comparable for each flavor category.

Conclusions: Published research mainly focused on flavors for e-cigarette liking. Research gaps exist on the learning and wanting components of addiction. Our review helps researchers developing study designs to investigate e-cigarette addiction. In addition, our overview will provide policy makers a first guideline towards regulating the amount of e-cigarette flavors available in order to prevent e-cigarette initiation among non-smoking youth.

Tob. Induc. Dis. 2018;16(Suppl 1):A224

DOI: $10.18332 /$ tid/84095

$12: 30-14: 00$

PS-645-3 Concentrations of hazardous chemicals in mainstream aerosol generated by heat-notburn tobacco

N Kunugita', K Bekki', Y Inaba', S Uchiyama'

${ }^{1}$ National Institute of Public Health, Department of Environmental Health, Wako, Japan. E-mail: kunugita@niph.go.jp 
Background: The Japanese government is trying to establish effective countermeasures for avoidance of secondhand smoke in indoor environments for tobacco-free Tokyo Olympic and Paralympic games 2020, as requested by the International Olympic Committee (IOC) and the World Health Organization (WHO). On the other hand, the tobacco industries have launched heat-not-burn (HNB) tobacco products which it claims is designed to produce less harmful components in Japan recently. Smokers strongly demand for products claiming or implying reduced health risks. There is little scientific data, however, of the hazards and toxicity of HNB tobacco.

Methods: In this study, we evaluated several harmful compounds (nicotine, tar, carbon monoxide (CO) and tobacco-specific nitrosamines (TSNAs)) in the mainstream aerosol and fillers of iQOS produced by Philip Morris International (PMI), and compared their concentrations with those from conventional combustion cigarettes.

Mainstream aerosol was collected under the conditions of 55 $\mathrm{ml}$ puff volume, $2 \mathrm{~s}$ puff duration, $30 \mathrm{~s}$ puff interval, and $100 \%$ blocking of the filter ventilation holes according to the Health Canada, Official Method T-115.

Results: The concentrations of nicotine in tobacco fillers and the mainstream aerosol of iQOS were almost the same as those of conventional combustion cigarettes, while the concentration of TSNAs was one fifth and CO was one hundredth of those of conventional combustion cigarettes.

Conclusions: "Tobacco companies continue reassuring health concerned smokers by offering with their new products the illusion of safety (WNTD 2006)." The market share of HNB tobaccos have been rapidly increased in Japan. One reason why HNB may be gaining market share is that nicotine-containing ENDS are prohibited for sale in Japan. PMI applied for US-FDA approval of the heat-notburn tobacco product "iQOS" as a modified risk tobacco product in 2016. Although it is low concentration in this study, toxic compounds are definitely included in the mainstream aerosol of iQOS.

Tob. Induc. Dis. 2018;16(Suppl 1):A225

DOI:10.18332/tid/84245

\section{2:30-14:00}

PS-646-3 Prevalence of lower harm perceptions of cigarette product characteristics: findings from 10 low-middle-income countries from the ITC project S Gravely', G Meng ${ }^{2}$, S Xu ${ }^{2}$ S Kaai ${ }^{2}, \mathrm{~F} \mathrm{Goma}^{3}$, JR Ong'ang' ${ }^{4}$, P Burhoo $^{5}$, GT Fong, on behalf of the ITC Project ${ }^{2}$
${ }^{1}$ University of Waterloo, International Tobacco Control Policy Evaluation Project (the ITC Project), Department of Psychology, Waterloo, ON, Canada, ${ }^{2}$ University of Waterloo, Waterloo, ON, Canada, ${ }^{3}$ University of Zambia, Lusaka, Zambia, ${ }^{4}$ Kenya Medical Research Institute, Nairobi, Kenya, ${ }^{5}$ Mauritius Institute of Health, Pamplemousses, Mauritius.E-mail: shannon.gravely@ uwaterloo.ca

Background: A major objective of FCTC Article 9, which calls for tobacco product regulation, is to eliminate or reduce tobacco industry product design strategies that have been shown to lead smokers to believe that some cigarettes are less harmful than others. However, nearly all of the studies documenting beliefs about harm perceptions have come from high-income countries; little is known whether the same misperceptions about harmfulness are present in low-middle income countries (LMICs) - where $80 \%$ of the world's smokers reside. This study measured the level of perceptions of harmfulness of light and menthol cigarettes among smokers from $10 \mathrm{LMICs}$ of the International Tobacco Control (ITC) Project.

Methods: Cross-sectional analyses of ITC surveys in Bangladesh, Brazil, China, India, Kenya, Malaysia, Mauritius, Mexico, Thailand, and Zambia were conducted using the country's most recent survey wave (ranging from 2011 to 2016). Adult smokers were asked whether each of three design features - (1) light/low tar, (2) filters, and (3) menthol-were less harmful.

Results: The percentage of smokers with erroneous beliefs was variable but substantial: 'light cigarettes are less harmful': $21 \%$ (Mexico) to $66 \%$ (China); 'low tar cigarettes are less harmful': $41 \%$ (Zambia) to $71 \%$ (China); 'menthol cigarettes are less harmful': $11 \%$ (Brazil) to 52\% (China); 'filters reduce harm': $36 \%$ (Mexico) to $82 \%$ (China); 'if a cigarette tastes lighter, it is less harmful': $39 \%$ (Zambia) to $71 \%$ (Kenya).

Conclusions: A substantial proportion of smokers in the 10 LMICs erroneously believe that light, low tar, and menthol cigarettes are less harmful, and that filters reduce harm. This is particularly disturbing in China, where prevalence of industryinduced misconceptions was the highest for 4 of the 5 measures. These findings point to the necessity of Article 9 regulations to restrict/ban product design features that mislead consumers about the harmfulness of tobacco products, particularly in LMICs.

Tob. Induc. Dis. 2018;16(Suppl 1):A226

DOI: $10.18332 /$ tid/84287

[POH 10 LMICs ITC]

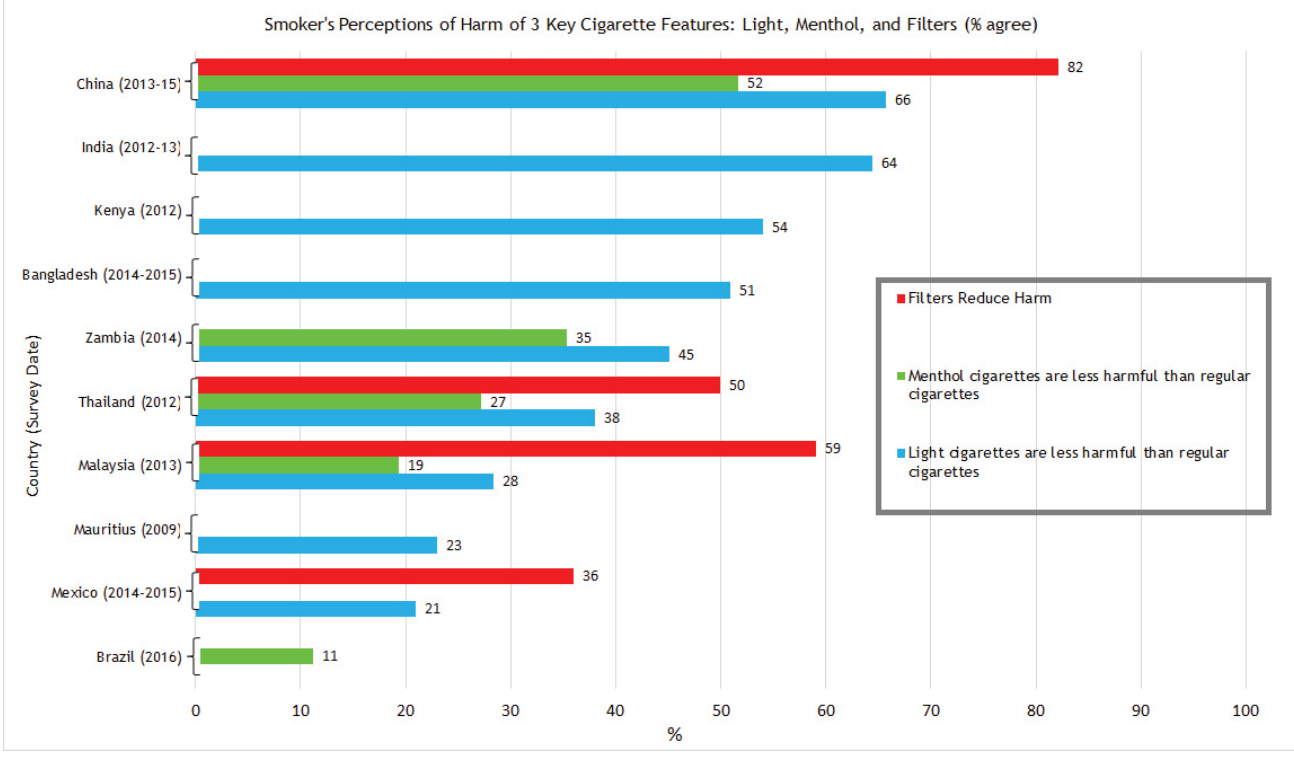




\section{2:30-14:00}

PS-647-3 E-liquid product labels: the good, the bad, and the ugly

S O'Connor', R Luk', R Schwartz', Ontario Tobacco Research Unit 'University of Toronto, Dalla Lana School of Public Health, Toronto, ON, Canada. E-mail: shawn.oconnor@camh.ca

Background: E-liquid products have exploded in Canada over the last number of years. Although current federal regulations apply to some aspects of these products (eg, Made in Canada designation, net quantity, French/English text), there are a number of other packaging elements that are not covered by existing regulations. The purpose of this research was to enumerate what choices are being made on e-liquid packaging by manufacturers, surveille compliance with existing regulations, and to inform governments about packaging elements that could benefit from strengthened regulations.

Methods: A sample of 90 e-liquid products were obtained from across 5 Canadian provinces, representing 49 unique manufacturers/distributors. This included 26 non-nicotine products and 64 nicotine products. All e-liquid containers were examined for type of information presented on product packaging including branding, promotional elements, claims, usage instructions, and warnings.

Results: Diverse labeling practices among manufacturers were observed. Of the 90 samples examined, 62 were highly legible, 21 were of moderate legibility and 7 were low. Only $62 \%$ of the products had English and French text, with English predominant. All but one sample included an ingredient list. However, $11 \%$ of samples listed quantity of nicotine as separate from the ingredient list. $19 \%$ listed nicotine quantity with a handwritten mark. $6 \%$ of sample had no text warning related to children (keep out of reach of children). $10 \%$ of samples did not include specific agerestrictive text. Only $72 \%$ of sample included a danger/caution image (skull, exclamation mark!).

Conclusions: Labeling practices among our sample of e-liquid products varied widely, with some manufacturers having strong practices, whereas others falling well short. Future labeling regulations that consider the main elements described in this study will have the potential to provide consumers of e-liquid products with pertinent information they need to make informed purchase decisions.

Tob. Induc. Dis. 2018;16(Suppl 1):A227

DOI:10.18332/tid/84357

\subsection{Prevalence of tobacco and nicotine related products' use: Traditional and novel products}

\section{2:30-14:00}

PS-666-4 Reasons for e-cigarette use and perceptions of harm in Brazil: findings from the ITC Brazil Wave 2 (2012-13) and 3 (2016-17) surveys

C Perez ${ }^{1}$, T Cavalcante 2 , F Mendes ${ }^{3}$, A Szklo ${ }^{4}$, GT Fong ${ }^{5,6}$, L Craig $^{5}, \mathrm{M} \mathrm{Yan^{5 }}, \mathrm{G} \mathrm{Li}{ }^{5}$

${ }^{1}$ Fundação do Câncer, Rio de Janeiro, RJ, Brazil, ${ }^{2}$ Executive Secretariat of National Commission for Implementing WHO Framework Convention on Tobacco Control/ Brazilian National Cancer Institute, Rio de Janeiro, RJ, Brazil, ${ }^{3}$ Brazilian National Cancer Institute, Rio de Janeiro, RJ, Brazil, ${ }^{4}$ Brazilian National Cancer Institute, Epidemiology, Rio de Janeiro, RJ, Brazil, ${ }^{5}$ University of Waterloo, Waterloo, ON, Canada, ${ }^{6}$ Ontario Institute for Cancer Research, Toronto, ON, Canada. E-mail: cp772017@gmail.com
Background: Little is known about the harmfulness perceptions of e-cigarettes (ECs) outside of high-income countries. This study examined reasons for EC use and harmfulness perceptions of ECs compared to regular cigarettes among smokers in Brazil, where $18.3 \%$ of smokers and $2.2 \%$ of non-smokers have tried ECs.

Methods: Data were from Waves 2 (2012-13) and 3 (2016-17) of the International Tobacco Control (ITC) Brazil Survey, a cohort survey of 1200 adult smokers and 600 non-smokers in Rio de Janeiro, São Paulo, and Porto Alegre. At Wave 3, EC users (those who used ECs daily, weekly, monthly, and less than monthly; total $\mathrm{n}=48$ ) were asked why they started and reasons for using ECs. At Waves 2 and 3, smokers who had ever heard of ECs ( $N=392$ at Wave 2; N=859 at Wave 3) were asked whether they thought ECs are more, less, or equally harmful as regular cigarettes.

Results: Among the 43 smokers who reported using ECs, the most common reasons for using e-cigarettes were: "using ECs is less harmful than smoking to other people" (69.3\%), "out of curiosity" (59.4\%), "they may not be as bad for your health" (59.0\%), and "as a way to help you quit" (57.5\%). Between 201213 and 2016-17, the percentage of smokers who "don't know" about the relative harmfulness of ECs decreased (41.6\% to 28.0\%; $\mathrm{p}<.001)$ and the percentage who believed that ECs are equally or more harmful than cigarettes increased (22.7\% to $35.2 \%$; $\mathrm{p}<$ $.001)$.

Conclusions: Smokers using ECs most commonly use ECs to reduce harm to others, reduce harm to their own health, and to help them to quit smoking. More smokers now have an opinion about the relative harmfulness of ECs, and a greater percentage of smokers now incorrectly believe that ECs are equally or more harmful than cigarettes.

Tob. Induc. Dis. 2018;16(Suppl 1):A228

DOI: $10.18332 /$ tid/83960

$11: 00-12: 30$

F0-307-4 Use of electronic cigarettes across 13 ITC countries with different regulatory environments S Gravely', SC Hitchman ${ }^{2}$, A McNeil ${ }^{3}$, KM Cummings ${ }^{4}$, R Borland ${ }^{5}$, H-H Yong ${ }^{5}$, D Hammond ${ }^{1}$, GT Fong ${ }^{1}$, on behalf of the ITC Project ${ }^{1}$ 'University of Waterloo, Waterloo, ON, Canada, ${ }^{2}$ King's College London, Psychology \&t Neuroscience, Addiction, London, United Kingdom, ${ }^{3}$ King's College London, London, United Kingdom, ${ }^{4}$ Medical University of South Carolina, Charleston, SC, United States of America, ${ }^{5}$ Cancer Council Victoria, Melbourne, VIC, Australia.E-mail: shannon.gravely@uwaterloo.ca

Background: Electronic cigarettes have become an international phenomenon, although few studies have compared e-cigarette use across countries. This paper presents prevalence estimates of self-reported e-cigarette awareness, trial (ever-tried), current use (daily/weekly/monthly), and daily use from 13 countries that vary on the strictness of e-cigarette policies/regulations.

Methods: Cross-sectional analyses of representative samples of adult ( $\geq 18$-years) current and former smokers participating in International Tobacco Control Project (ITC) surveys in 13 countries from the most recent ITC survey wave (2013-2017). Countries were categorized into those with no e-cigarette policies (NP), less restrictive policies (LRP), or more restrictive policies (MRP).

Results: Weighted prevalence estimates of four key measures were computed:

[1] Awareness: for NP countries: Zambia (2014): 3\%, Bangladesh (2014/15): 7\%, China (2013/15):59\%;LRP countries: Netherlands (2017): 92\%, Republic of Korea (2016): 94\%, United States (US) 
and England (2016): 99\%; MRP countries: Uruguay (2014): 52\%, Mexico (2014/15): 61\%, Brazil (2016): 73\%, Malaysia (2013/2014): 86\%, Australia and Canada (2016): 99\%.

[2] Trial: for NP countries: Zambia (0\%), Bangladesh (1\%), China (11\%); LRP countries: Netherlands (39\%), Korea (44\%), England (52\%). US (58\%); MRP countries: Uruguay (7\%), Mexico (10\%), Malaysia (38\%), Australia (45\%), Canada (49\%). [3] Daily/weekly/monthly use: for NP countries: Zambia and Bangladesh (< 1\%); China (1\%); LRP countries: Korea (6\%), Netherlands, US, England (7\%); MRP countries: Uruguay (0\%), Brazil (1\%), Mexico (2\%), Australia and Canada (6\%), Malaysia (12\%).

[4] Daily use: for NP countries: Zambia, Bangladesh, China (all 0\%); LRP countries: Korea (3\%), Netherlands (3\%), US (3\%), England (4\%); MRP countries: Uruguay and Brazil (0\%), Mexico (1\%), Canada (2\%), Australia (3\%), Malaysia (4\%).

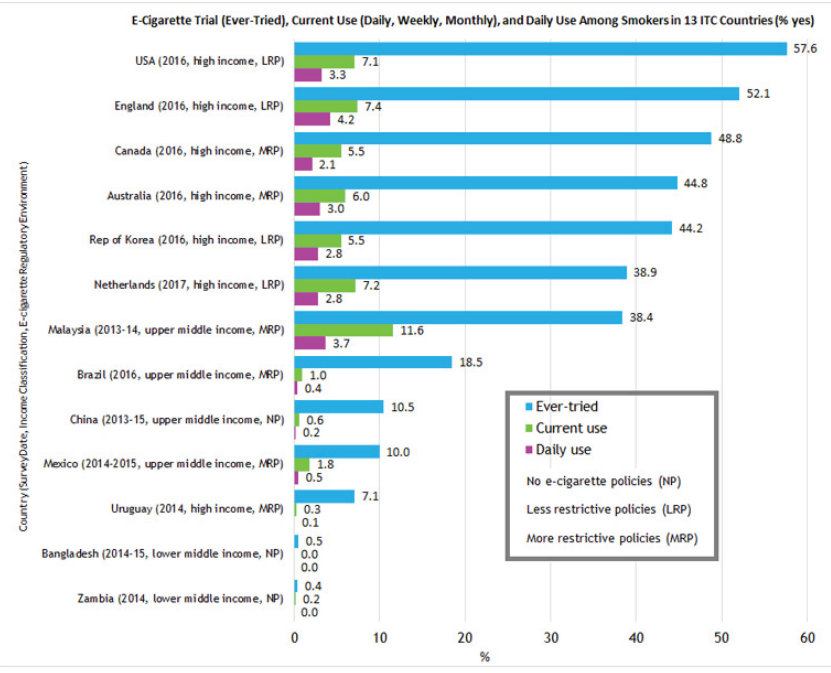

[E-cigarette Trial, Current Use, and Daily Use]

Conclusions: With minor exceptions (e.g. Australia), awareness, trial, and use of e-cigarettes across the 13 countries generally reflected the de facto environment rather than the statutory environment implied by the law(s). Country income classification and survey year also appear to be strongly associated with use.

Tob. Induc. Dis. 2018;16(Suppl 1):A229

DOI:10.18332/tid/84279

$11: 00-12: 30$

F0-308-4 Awareness and use of electronic cigarettes among youth: evidence from the Global Youth Tobacco Survey 2012 - 2015

KM Palipudi ${ }^{1}$, C Jones ${ }^{1,2}$, KA Lee ${ }^{1,3}$, E Pun ${ }^{1,4}$, R Arrazola ${ }^{1}$, I Ahluwalia

${ }^{1}$ CDC, Global Tobacco Control Branch, Atlanta, GA, United States of America, ${ }^{2}$ RTI International, Atlanta, GA, United States of America, ${ }^{3}$ Northrop Grumman Information Systems, Atlanta, GA, United States of America, ${ }^{4} \mathrm{DB}$ Consulting, Atlanta, GA, United States of America. E-mail:gou8@cdc.gov

Background: Globally, evidence on the awareness and use of electronic cigarettes (e-cigarettes) is limited. We examined the awareness and current use of e-cigarettes among male and female students aged 13-15 years old across 16 countries or regions that conducted the Global Youth Tobacco Survey (GYTS).

Methods: GYTS is a cross-sectional, nationally representative, school-based survey of students aged 13-15 years. Data from the 16 countries or regions were used to examine student awareness (ever heard of) and current use (in the past 30 days) of e-cigarettes. Sample sizes across the 16 countries or regions ranged from 962 in Georgia to 5,153 in Paraguay and response rates ranged from $75.4 \%$ in Georgia to $99.2 \%$ in Sudan. Weighted point estimates and $95 \%$ confidence intervals $(\mathrm{CI})$ were calculated and statistically significant differences $(\mathrm{p}<0.05)$ by gender were assessed using a two-sample t-test.

Results: Awareness of e-cigarettes ranged from 19.6\% (95\% CI: 16.1-23.6) in Peru to 93.0\% (95\% CI: 91.4-94.3) in the Moscow region of Russia. Across all sites, only Seychelles and United Nations Relief and Works Agency for Palestine Refugees in the Near East (UNRWA) had significantly higher awareness of e-cigarette among boys than girls. Current use of e-cigarettes ranged from $1.6 \%$ (95\% CI: 1.1-2.5) in Kazakhstan to $15.4 \%$ (95\% CI: 12.6-18.5) in UNRWA. In Albania, Belize, Macao, Seychelles, and UNRWA, boys had a significantly higher prevalence of current e-cigarette use than girls.

Conclusions: The findings show that awareness and use of e-cigarettes varies considerably across 16 countries/regions. GYTS is a viable platform to measure tobacco use among youth, including emerging products such as e-cigarettes.

Tob. Induc. Dis. 2018;16(Suppl 1):A230

DOI:10.18332/tid/83859

$11: 00-12: 30$

F0-309-4 Awareness, use, sales and interest of heat-not-burn tobacco products in Italy

A Lugo ${ }^{1}$, X Liu' 1 , R Pacifici ${ }^{2}$, L Spizzichino ${ }^{3}$, S Gallus ${ }^{4}$

'IRCCS - Istituto di Ricerche Farmacologiche 'Mario Negri', Milan, Italy, ${ }^{2}$ stituto Superiore di Sanità, Rome, Italy, ${ }^{3}$ Italian Ministry of Health, Rome, Italy, ${ }^{4}$ IRCCS - Istituto di Ricerche Farmacologiche 'Mario Negri', Department of Environmental Health Sciences, Milan, Italy. E-mail: silvano.gallus@marionegri. it

Background: Heat-not-burn tobacco products are cigarettes heated by an electronic device to generate an aerosol containing nicotine. iQOS is the brand name of such a product by Philip Morris International. It was launched in December 2014 in Italy as a pilot country for the European market. Currently, no information is available on iQOS awareness, use and sales in Italy.

Methods: We annually conduct a national-wide face-to-face survey on smoking. In order to investigate awareness and use of iQOS, we devoted to heat-not-burn tobacco product a specific section in our 2017 survey. The survey was conducted on a sample of 3086 individuals, who represented the Italian population aged $\geq 15$ years (52.4 million). Official quarter sales data on iQOS were obtained from the Italian Ministry of Health and the relative web search volumes in Italy were retrieved from "Google trends".

Results: Among the investigated subjects, $19.5 \%$ were aware of iQOS, and $1.4 \%$ have tried it. Overall, $1.0 \%$ of never, $0.8 \%$ of ex- and $3.1 \%$ of current smokers have tried iQOS. As regard to e-cigarettes, $2.9 \%$ of ex- and $7.7 \%$ of current users have tried iQOS. In just one year, iQOS quarter sales were boosted by 17 times from 4.4 tonnes in the early 2016 to 80.0 tonnes in the early 2017 , currently representing $0.4 \%$ of the whole tobacco market share; Google trends shows similar exponential increase in the relative web search volume.

Conclusions: Although the share of iQOS in the whole tobacco market is still limited in Italy, we have observed a growing interest in it. Our individual-level data suggest that more than 700 thousand Italians have already tried this tobacco product, 
including more than 340 thousand never and 50 thousand exsmokers. Attention should be paid on this emerging phenomenon, which may represent in the near future an impairing gateway to nicotine addiction.

\section{Tob. Induc. Dis. 2018;16(Suppl 1):A231 \\ DOI:10.18332/tid/84144}

\section{$11: 00-12: 30$}

F0-310-4 Does use of electronic nicotine delivery systems predict current cigarette use among young adults?

A Loukas ${ }^{1}$, CN Marti ${ }^{1}$, MR Creamer ${ }^{2}$, CL Perry ${ }^{2}$

${ }^{1}$ University of Texas-Austin, Austin, TX, United States of America, ${ }^{2}$ University of Texas School of Public Health Austin Regional Campus, Health Promotion \& Behavioral Sciences, Austin, TX, United States of America. E-mail: alexandra.loukas@austin. utexas.edu

Background: Studies indicate that use of electronic nicotine delivery systems (ENDS) predicts subsequent cigarette use, but these are limited by a focus primarily on adolescents or examination of cigarette initiation/onset. Little is known about the impact of ENDS use in current (past 30-day) cigarette use or in changes in current use across young adulthood. This study examined if 1) ENDS use predicted trajectories of young adult current cigarette use over a two-year period.

Methods: Data are from a five-wave 24-college study in Texas, USA. Wave 1 data were collected in Nov 2014-Feb 2015 and every six months thereafter. To examine the role of ENDS in current cigarette use, only students who reported never using cigarettes at Wave 1 were included, $\mathrm{N}=2577$. At Wave 1 , students were 18-25 year olds $(\mathrm{m}=19.7 ; \mathrm{s}=1.60) ; 67.7 \%$ female; $31.9 \%$; non-Hispanic white, $27.4 \%$ Hispanic, and $40.7 \%$ another race/ethnicity. Growth curve modeling was used to examine predictors of the current cigarette use trajectory across the five waves.

Results: Wave 1 ever ENDS use predicted a faster increase in the current cigarette use trajectory $(\mathrm{OR}=2.87[1.32,6.25])$ in the unconditional model (model with no covariates). However, this effect became non-significant when Wave 1 time-invariant covariates, including socio-demographics, family tobacco use, friend cigarette use, cigarette use susceptibility, and use of other tobacco (i.e., hookah, cigars, smokeless) products were added. Examination of the full model indicated that peer use $(\mathrm{OR}=1.44$ $[1.01,2.06])$, susceptibility to cigarette use $(\mathrm{OR}=4.49[2.84$, $7.10])$, and other tobacco use $(\mathrm{OR}=2.40[1.62,3.56])$ predicted an increasing cigarette use trajectory.

Conclusions: Findings are in contrast to research indicating the ENDS use predicts cigarette initiation, but also extend it by showing that young adults who are susceptible to cigarette use and who use other tobacco products (besides ENDS) are at elevated risk for current cigarette use.

Tob. Induc. Dis. 2018;16(Suppl 1):A232

DOI:10.18332/tid/84509

\section{$11: 00-12: 30$}

F0-311-4 Effect of seeing e-cigarettes in small shops on probability of e-cigarette experimentation by 1 year follow up in adolescents in Scotland, UK C Best ${ }^{1}$, F Haseen ${ }^{2}$, D Currie ${ }^{2}$, G Ozakinci ${ }^{3}$, AM Mackintosh ${ }^{4}$, S Haw ${ }^{1}$ ${ }^{1}$ University of Stirling, Faculty of Health Sciences and Sport, Stirling, United Kingdom, '2University of St Andrews, Child and Adolescent Research Unit (CAHRU), School of Medicine, St Andrews, United Kingdom, ${ }^{3}$ University of St Andrews, School of
Medicine, St Andrews, United Kingdom, ${ }^{4}$ University of Stirling, Institute for Social Marketing, Faculty of Health Sciences and Sport, Stirling, United Kingdom. E-mail: catherine.best2@ stir.ac.uk

Background: Previous research has found that small shops are frequently visited by young people and product exposures in this environment influence their health-related behaviours. Evidence from cross-sectional research suggests that recall of seeing e-cigarettes in small shops is associated with adolescent experimentation with e-cigarettes. This study is the first to explore these relationships with longitudinal follow up.

Methods: Data are from the Determining the Impact of Smoking Legislation among Youth (DISPLAY) study. A prospective cohort survey was conducted in 4 high schools in Scotland UK during spring 2015 ( $n=3807$ ) with follow up 1 year later. Analysis was restricted to young people who had never used an e-cigarette in $2015(\mathrm{n}=2839)$ and 2388 of these (84\%) provided their e-cigarette status in 2016.

Results: At baseline 636 (22.6\%) of young never e-cigarette users recalled seeing e-cigarettes for sale in small shops in the last 30 days. In a logistic regression the adjusted odds ratio for e-cigarette experimentation by follow up was 1.50 (95\% CI 1.08 to 2.08 ) for young people who recalled seeing e-cigarettes in small shops compared to those who did not. The model was adjusted for recall of e- cigarettes in supermarkets, recall of e-cigarettes on the internet, recall of e- cigarettes adverts on other media (TV, radio, newspapers), having friends who smoke, having family members that smoke, young person's smoking status, sex, age, family affluence scale and school.

Conclusions: Seeing e-cigarettes for sale in small shops may increase the likelihood that young people will experiment with them. The model was adjusted for noticing other forms of e-cigarette advertising so this corrects for the fact that young people who are already interested in e-cigarettes may be more likely to notice potential sources and information about e-cigarettes. The role of small retailers in normalizing novel nicotine product use in young people requires further investigation.

Tob. Induc. Dis. 2018;16(Suppl 1):A233

\section{DOI:10.18332/tid/84188}

\section{$11: 00-12: 30$}

F0-312-4 Use of flavored cigarettes in the first few puffs: a step toward smoking initiation and nicotine addiction? Data from a national survey among Brazilian adolescents

VC Figueiredo o, ${ }^{1,}$, KV Bloch $^{3}$, J Thrasher ${ }^{4}$, MC Kuschnir ${ }^{5,6}$, L Casado $^{7}$, ME Reichenheim ${ }^{8}, \mathrm{M} \mathrm{Szklo}^{9}$, Study of Cardiovascular Risks Among Adolescents (ERICA)

${ }^{1}$ National School of Public Health Sergio Arouca, Oswaldo Cruz Foundation, Center for Studies on Tobacco and Health, Rio de Janeiro, RJ, Brazil, ${ }^{2}$ University Estacio de Sá, Professional Master's in Family Health, Rio de Janeiro, RJ, Brazil, ${ }^{3}$ Federal University of Rio de Janeiro - UFRJ, Public Health Institute - IESC \& Faculty of Medicine, Rio de Janeiro, RJ, Brazil, ${ }^{4}$ University of South Carolina, Health Promotion, Education, and Behavior Arnold School of Public Health, Columbia, SC, United States of America, ${ }^{5}$ University of Rio de Janeiro State, Biomedical Center, School of Medical Sciences., Rio de Janeiro, RJ, Brazil, ${ }^{6}$ University Estacio de Sá, Board of Directors and Administration, Center for Biological and Health Sciences, Rio de Janeiro, RJ, Brazil, ${ }^{7}$ Brazilian National Cancer Institute, Prevention and Surveillance Coordination, Rio de Janeiro, RJ, Brazil, ${ }^{8}$ University of Rio de Janeiro State, Institute of Social Medicine, Rio de 
Janeiro, RJ, Brazil, ${ }^{9} J o h n s$ Hopkins Bloomberg School of Public Health, Epidemiology, Baltimore, MD, United States of America. E-mail: valeskacf@ensp.fiocruz.br

Background: Little is known about the importance of flavored cigarettes for initiation among youth in Brazil, which has amongst the greatest number of smokers in the world. This study aimed at analyzing the relationship between trying to smoke mainly mentholated and flavored cigarettes and initiation and intensity of smoking among Brazilian students.

Methods: A cross-sectional school-based survey that included a multistage probability sample of 12-17 years old students was conducted in cities with more than 100,000 inhabitants, who participated in the Study of Cardiovascular Risks Among Adolescents in 2013-2014 (ERICA) $(\mathrm{n}=70,589)$. Data was collected using self-administered questionnaires. Logistic models regressed current smoking (last 30 days) and days smoking (6 ore more vs 5 or fewer) on preference for flavored cigarettes, adjusting for gender, age, skin color, type of school (public vs private), family structure (with vs without two parents), having a paid job, time since experimentation, and geographic region.

Results: Among the $18.5 \%$ of adolescents who ever tried cigarettes, $26.4 \%$ (95\% confidence interval, CI 24.1, 28.9) of males and $28.8 \%$ (CI 26.4, 31.4) of females had flavored cigarettes as their first choice brand. Of the $5.7 \%$ who smoked in the past 30 days, flavored cigarettes were the first choice in $54.7 \%$ of males (CI $49.9,59.4$ ) and $60.6 \%$ (CI 55.6, 65.4) of females. In adjusted models, experimenters who preferred flavored cigarettes were more likely to have smoked in the past 30 days (adjusted Odds Ratio, adjOR $=1.62$, CI 1.36; 1.92), and to smoke 6 or more days (vs $1-5$ days) in the past 30 days (adjOR $=1.37$, CI 1.01; 1.86) than experimenters who preferred non-flavor cigarettes.

Conclusions: Preferences for flavored cigarettes among Brazilian adolescents is very high. Banning flavored cigarettes could reducing the palatability of cigarettes for youth and thereby reduce smoking initiation and prevalence.

Tob. Induc. Dis. 2018;16(Suppl 1):A234

DOI: $10.18332 /$ tid/84647

$11: 00-12: 30$

F0-313-4 Tobacco cigarette use versus electronic cigarette use: determinants of smoking and vaping behavior

K Romijnders ${ }^{1,2}$, M Beijaert ${ }^{1}$, L van Osch ${ }^{2}, \mathrm{H}_{\text {de }}$ Vries $^{2}$, R Talhout ${ }^{1}$ ${ }^{1}$ RIVM, Centre for Health Protection, Bilthoven, Netherlands, ${ }^{2}$ Maastricht University, Health Promotion, Maastricht, Netherlands. E-mail: reinskje.talhout@rivm.nl

Background: It is important to know why individuals use electronic cigarettes (e-cigarettes) compared to tobacco cigarettes. This comparison provides policy makers with opportunities to target different types of users. This study examined behavioral determinants associated with both tobacco and e-cigarette use. Differences between non-users (neither e-cigarette users nor smokers), smokers, e-cigarette users, and dual users were assessed for tobacco use versus e-cigarette use among adults ( $>18$ years). Methods: A cross-sectional survey $(\mathrm{n}=726)$ among 223 nonusers, 287 smokers, and 216 e-cigarette users was conducted in the Netherlands. The questionnaire assessed demographics, tobacco and e-cigarette behavior, knowledge, attitudes, social ties, risk perception, intention, and deliberation.

Results: Health benefits was the most frequently reported reason for e-cigarette use. Overall, $30.8 \%$ of the respondents were nonusers, $39.5 \%$ smokers, $29.7 \%$ e-cigarette users, and $15.4 \%$ dual users. Ever use of e-cigarettes was $29.6 \%$. Of the ever users, 45.6 $\%$ used e-cigarettes daily, $20.9 \%$ weekly, and $10.7 \%$ on a monthly basis. A low socio-economic status was associated with ever use of e-cigarettes, and older participants were less likely to have used e-cigarettes. Participants knew more about tobacco cigarettes than e-cigarettes. Furthermore, non-users perceived the social environment to be more negative towards e-cigarette and tobacco use. Additionally, non-users and smokers perceived e-cigarettes as more harmful than e-cigarette users. Smokers were significantly more likely to have an intention to start vaping compared to nonusers. Finally, e-cigarette users made a deliberate choice to vape. Conclusions: Determinants of smoking and vaping behavior differ between non-users, smokers, e-cigarette users, and dual users. Using the determinants of behavior assessed, we are able to predict e-cigarette use among non-users and smokers. Policy makers could use these determinants, such as e-cigarette attractiveness, to specifically target dual users, smokers, e-cigarette users or non-users. Tob. Induc. Dis. 2018;16(Suppl 1):A235 DOI:10.18332/tid/84216

\section{2:30-14:00}

PS-648-4 The use of flavour-changing capsule cigarettes among smokers in the United Kingdom C Moodie', S Hitchman², AM Mackintosh'1, A McNeill²

'University of Stirling, Institute for Social Marketing, Stirling, United Kingdom, ${ }^{2}$ King's College London, Institute of Psychiatry, Psychology and Neuroscience, London, United Kingdom. E-mail: c.s.moodie@stir.ac.uk

Background: Cigarettes with flavour-changing capsules in the filter have experienced phenomenal global growth in the last decade. However, very few studies have explored the use of these products. Our objective was to explore which smokers in the United Kingdom use these products, and why.

Methods: An online survey was conducted between April-May 2016 with a sample of 6234 factory-made (FM) and/or handrolled cigarette smokers. This analysis focused on the 3548 smokers (aged 18 years and over) of FM cigarettes. We explored socio-demographic and smoking-related factors associated with smoking capsule cigarettes, reasons for using them, and whether users burst the capsule.

Results: $13 \%$ of FM smokers ( $\mathrm{n}=454)$ smoked capsule cigarettes, with younger smokers more likely than older smokers to use them (30\% of $18-24$ year olds compared with $5 \%$ of those $55+$ ). Capsule use was significantly more common among those from other white backgrounds (22\%) than among white British (12\%), and among those planning to quit in the next six months (16\%) than those not planning to quit (11\%). Most capsule users crushed the capsule always or most of the time (70\%), with some never crushing it (9\%). The most common reasons for using capsule cigarettes were that they taste better (52\%), are smoother (41\%), provide a choice of flavours (32\%), the enjoyment of clicking the capsule (25\%) and that they are more interesting than regular cigarettes (21\%). For those who never crushed the capsule, almost half (49\%) smoked capsules because their brand only had capsule variants. Capsule and non-capsule smokers did not differ in their perceptions of the harmfulness of their brand relative to other brands, or their concern about the effect of smoking on their health.

Conclusions: Approximately one in eight FM cigarette smokers used capsule cigarettes, with the key drivers of use related to taste, having a choice of flavours and interactivity.

Tob. Induc. Dis. 2018;16(Suppl 1):A236

DOI:10.18332/tid/83749 


\section{2:30-14:00}

PS-650-4 Socioeconomic differences in smoking declining? Result from population-based surveys in 1978 - 2016 in Finland

0 Ruokolainen'1, A Heloma'1, P Jousilahti' , J Lahti², 0 PentalaNikulainen', 0 Rahkonen², P Puska'

${ }^{1}$ National Institute for Health and Welfare, Helsinki, Finland, ${ }^{2}$ University of Helsinki, Department of Public Health, Helsinki, Finland. E-mail: otto.ruokolainen@thl.fi

Background: Smoking is declining, but in most countries lower educated smoke more than higher educated. Our aim was to examine development with these differences in a country with strict tobacco control policies, Finland.

Methods: We used annual population-based random sample data of 25-64 year-olds from 1978-2016 ( $\mathrm{N}=104,317)$. Response rate varied between $84 \%$ and $40 \%$ during the study period. We used relative education as a measure for socioeconomic position. For each year education was stratified into tertiles, and low and high educated were included in further analysis. The outcome variable was daily smoking. Weights were used in order to enhance the population representation. Prevalence figures and logistic regression analysis were used.

Results: At the start of the study period, $40 \%$ of lower educated men and $33 \%$ of higher educated men smoked. For women, the corresponding proportions were $18 \%$ and $13 \%$, respectively. Smoking decreased in each educational group except lower educated women. Preliminary analyses suggest that, compared with the time period before 2009, all population groups except lower educated women were less likely to smoke after 2009. In the 2010s, educational differences in smoking slightly declined both among women and men due to a more prevalent decline in smoking among lower educated. In 2016 among men, $26 \%$ of lower educated smoked, while $6 \%$ of higher educated smoked. Among women corresponding proportions were $19 \%$ and $9 \%$.

Conclusions: Smoking has declined both among lower and higher educated and socioeconomic differences diminished in the 2010s in Finland. However, educational differences in smoking are still marked, which underlines the need for further actions reducing the overall public impact of these differences. Further analyses will test relative differences in smoking among socioeconomic groups and careful sensitivity analyses will be conducted to validate the results.

\section{Tob. Induc. Dis. 2018;16(Suppl 1):A237 DOI:10.18332/tid/83783}

\section{2:30-14:00}

PS-651-4 Dual use of combustible and electronic cigarettes: patterns and associations between products

T Brandon', U Martinez', V Simmons', L Meltzer', S Sutton', D Drobes', M Unrod', P Harrell ${ }^{2}$

${ }^{1} \mathrm{H}$. Lee Moffitt Cancer Center, Health Outcomes and Behavior, Tampa, FL, United States of America, ${ }^{2}$ Eastern Virginia Medical School, Pediatrics, Norfolk, VA, United States of America. E-mail: thomas.brandon@moffitt.org

Background: The use of electronic cigarettes (e-cigarettes) has increased dramatically over the past decade. Although the majority of users report initiating e-cigarette use (vaping) to quit or reduce use of combustible cigarettes (smoking), a substantial proportion become "dual users" of both. Little is known about the patterns of dual use of, and dependence on, these products. This study describes those patterns as well as associations between smoking with vaping variables.

Methods: During 2016-17, 2894 dual users were recruited into a 2-year longitudinal study testing nicotine cessation interventions. Inclusion criteria required at least weekly use of both products. This report uses baseline questionnaire data collected via mail or Internet before randomization. Descriptive and inferential statistics were calculated.

Results: Participants' mean age was 29.9 (SD 11.3) years, and $63 \%$ were male. They reported smoking a median of $16-20$ cigarettes per day (CPD) for a mean of 13 years before vaping, which was initiated a median of 13-24 months earlier. Smoking declined to 6-10 CPD currently, with 79\% reporting decreased and $3 \%$ reporting increased smoking. $69 \%$ reported daily vaping, with $57 \%$ reporting vaping "continuously" or at least 30 times/ day. CPD was inversely related to both weekly (days) and daily (uses) vaping frequency (ps $<.001)$, but positively associated with nicotine dosage of the e-cigarette solution $(\mathrm{p}<.001)$. Duration of vaping was not associated with cigarette use (CPD, time to first cigarette) or dependence, but was positively associated with a measure of e-cigarette dependence $(\mathrm{p}<.001)$. Additional results will be reported.

Conclusions: The current cross-sectional findings suggest that vaping may reduce and partially replace cigarette use. However, e-cigarette dependence may increase over time without further reduction in smoking among those who maintain dual use. Future results from this longitudinal study should clarify use patterns, with implications for both tobacco intervention and policy.

Tob. Induc. Dis. 2018;16(Suppl 1):A238

DOI: $10.18332 /$ tid $/ 83791$

\section{2:30-14:00}

PS-652-4 Varieties in vaper identitys in a Norwegian context

R Tokle

'Norwegian Institute of Public Health, Department of Substance Use, Oslo, Norway. E-mail: rikke.tokle@fhi.no

Background: In this qualitative study, we analyze how users of e-cigarettes (vapers) articulate and express the meaning of vaping and their identity as vapers. Particular attention are drawn to how they understand their use in a Norwegian context, where use of snus is widespread, combustible cigarettes are denormalized, and perspectives on harm reduction within the tobacco control community are conflicting.

Methods: Semi-structured personal interviews with 32 vapers, strategically recruited (age, sex, soci.ec.status) to ensure coverage of different perspectives, and systematic observations of a Norwegian online vaping forum ( $<12 \mathrm{~K}$ members), are conducted to explore the complexity of identity-making processes for users of e-cigarettes.

Results: Majority of interviewed vapers perceived their use of e-cigarettes as a less harmful substitute for smoking. Analyzing personal interviews and observations on a virtual vaping forum we observed that their identities as vapers varies, and can be classified into three main groups: (I) Users who vape to replace cigarette smoking. (II) Users who (also) have vaping as a hobby and participate in a vape oriented subculture. In addition, (III) recreational users who switch between tobacco products (snus+) and who value the novelty of e-cigarettes in some occasions, but also are ambivalent in regard to identifying themselves as vapers because of what they see as the social meaning of use of e-cigarettes (addiction, hobby, activists).

Conclusions: This study provides new insight into the varieties 
of vaper identities in a context were also snus have a substantial marked share. They portray their vaping in various ways, but hold a shared understanding of use of e-cigarettes as harm reduction. The results are relevant to policy makers in order to understand how to address users and potential new users of e-cigarettes.

Tob. Induc. Dis. 2018;16(Suppl 1):A239

DOI:10.18332/tid/83800

\section{$12: 30-14: 00$}

\section{PS-653-4 Smoking and snus use among Finnish} young males

M Danielsson'1, A Lammi², S Siitonen', L Pylkkänen³ , J Ollgren4, T Vasankari ${ }^{2}$

${ }^{1}$ The Finnish Defence Forces, Helsinki, Finland, ${ }^{2}$ Finnish Lung Health Association, Helsinki, Finland, ${ }^{3}$ Cancer Society of Finland, Helsinki, Finland, ${ }^{4}$ National Institute for Health and Wellfare, Helsinki, Finland. E-mail: maria.danielsson@helsinki.fi

Background: The aim of this study was to evaluate the prevalence of cigarette, snus (Swedish type of smokeless tobacco) and dual use as well as transition from one tobacco product to another among a representative population of young Finnish males. In addition, we evaluated the correlation between educational level and the use of tobacco.

Methods: A questionnaire-based survey was carried out among military conscripts during their first week of service $(n=1916$, mean age 19.4; response rate $95 \%$ ). The questionnaire consisted of 25 questions including age, gender, basic education, use of tobacco products and questions assessing nicotine dependency. The usage of electric cigarettes was asked but excluded from the analysis due to very low usage (1.4\%).

Results: In our survey, $34 \%(n=635)$ reported snus usage of which $42 \%$ used snus daily ( $n=237,14 \%$ of the whole study population). A quarter, $26 \%(n=486)$, of the study population smoked daily. A total of $40 \%(n=741)$ reported sporadic or regular cigarette usage. Every fifth, $21 \%(n=402)$, of all conscripts reported simultaneous usage of cigarette and snus. The probability of dual usage was higher among smokers. $35 \%$ of former smokers reported daily snus use and $43 \%$ of reported quitters in the snus-group were smoking daily. Smoking was common among study subjects with basic educational background of whom $57 \%$ smoked daily. A similar association between snus users and the level of education could not be demonstrated. Signs of electric cigarettes as a replacement for tobacco or snus could not be demonstrated.

Conclusions: Exclusive snus use as well as dual use of cigarette and snus were significantly higher than expected. Educational level did not correlate with exclusive snus use.The notably high prevalence of snus usage seems to reflect the aspiring change of trend among tobacco consumers.

Tob. Induc. Dis. 2018;16(Suppl 1):A240

DOI:10.18332/tid/83805

\section{$12: 30-14: 00$}

PS-654-4 Factors associated with ever-use of e-cigarette among college students in the Republic of Korea

AN Lee ${ }^{1}$, YS Yang ${ }^{2}$, JE Lee ${ }^{1}$, JH Kong ${ }^{1}$, PH Sun ${ }^{1}$, MY Kim¹ ${ }^{1}$ AH Park', JH Kim

'Korea Health Promotion Institute, National Tobacco Control Center, Seoul, Korea, Republic of, ${ }^{2}$ Korea Health Promotion Institute, Seoul, Korea, Republic of. E-mail: ahnnalee@khealth.or.kr

Background: Since e-cigarette was introduced into the global market, its use has become popular especially among young adults. But little is known about the prevalence and associated factors of e-cigarette use among young adults in Korea. The purpose of this study is to identify the factors associated with ever-use of e-cigarette among Korean college students.

Methods: A survey to collect the data for this study was distributed to 550 students in 5 universities in S city and $\mathrm{K}$ city from October to December 2016. Data from the survey which consists of self-reporting questionnaires were analyzed using binary logistic regression analysis. Ever-use of e-cigarette was assessed by asking "Have you ever used an e-cigarette?" (yes/no). The final sample for this study $(n=399)$ included those who had ever heard of e-cigarette.

Results: Among 399 students who had ever heard of e-cigarette, $68(17 \%)$ were e-cigarette ever-users. E-cigarette ever-use was higher among male students $(\mathrm{OR}=6.82,95 \% \mathrm{CI}=2.02,23.02, \mathrm{p}<$ $0.05)$, students living in dormitory $(\mathrm{OR}=10.19,95 \% \mathrm{CI}=1.16$, $89.49, \mathrm{p}<0.05)$, whose father is a smoker $(\mathrm{OR}=58.41,95 \%$ $\mathrm{CI}=8.70,392.39, \mathrm{p}<.001)$, who have the experience of past 2-week binge drinking $(\mathrm{OR}=43.78,95 \% \mathrm{CI}=5.61,341.93, \mathrm{p}<$ $.001)$ and past 30 -day cigarette smoking $(\mathrm{OR}=1.02,95 \% \mathrm{CI}=1.01$, $1.03, \mathrm{p}<.001)$.

Conclusions: This study showed significant association between e-cigarette ever-use and gender, residence type, father's smoking status, past 2-week binge drinking and past 30-day cigarette smoking. Associated factors of e-cigarette use should be considered for smoking prevention program for college students. To better understand the patterns and determinants of e-cigarette use among young adults in Korea, further studies are needed.

Tob. Induc. Dis. 2018;16(Suppl 1):A241

DOI:10.18332/tid/83812

12:30-14:00

PS-655-4 Electronic cigarette effectiveness to quit smoking in the representative Italian population PASSI survey, 2014 - 2016

G Gorini', G Ferrante ${ }^{2}$, V Minardi², M Masocco², P FatehMoghadam $^{3}$, S Campostrini ${ }^{4}$, P D'Argenio ${ }^{5}$, D Galeone ${ }^{6}$, PASSI Coordinating Group

${ }^{1}$ ISPO, Florence, Italy, ${ }^{2}$ National Institute of Health (ISS), Rome, Italy, ${ }^{3}$ Provincial Agency for Health Services, Health Education Service, Trento, Italy, ${ }^{4} \mathrm{Ca}$ ' Foscari University, Department of Statistics, Venice, Italy, ${ }^{5}$ PASSI Coordinating Group, Rome, Italy, ${ }^{6}$ Italian Ministry of Health, Center for Disease Prevention and Control, Rome, Italy. E-mail: g.gorini@ispo.toscana.it

Background: This study explored electronic cigarette (e-cigarette) use as an aid to quit smoking and compared abstinence rates for different quitting methods in a representative sample of the Italian population, 2014-2016.

Methods: In the 2014-2015 PASSI survey, the ongoing Italian behavioural risk factor surveillance system, 6,112 adults who smoked and made at least one quit attempt in the previous 12 months, were categorised into three groups according to the method used in their most recent quit attempt: ecigarette only, no aid, other quitting methods (medications; programmes delivered in smoking cessation services; other unspecified methods). The primary outcome was self-reported abstinencefor a period $\geq 6$ months, adjusted for potential confounders.

We will perform the same analysis for 2016 Passi survey.

Results: Eleven percent used e-cigarettes only, $86 \%$ no aid, 3\% other quitting methods. Smoking abstinence was reported among $9 \%$ of those using no aid; $8 \%$ of e-cigarette users; $15 \%$ of those using other methods. Compared with those reporting no aid to 
quit smoking, no statistically significant differences in abstinence were observed for e-cigarette users compared with those reporting no aid (adjusted Prevalence Ratio $[\mathrm{aPR}]=0.81 ; 95 \%$ Confidence Interval $(\mathrm{CI})=0.58-1.14)$, neither for those using other quitting methods $(\mathrm{aPR}=1.42 ; 95 \% \mathrm{CI}=0.95-2.13)$. Changing the reference group to e-cigarette users, users of other quitting methods were significantly more likely to report abstinence than e-cigarette users e-cigarette users $(\mathrm{aPR}=1.76 ; 95 \% \mathrm{CI}=1.07-2.88)$.

We will add results for the 2016 Passi survey.

Conclusions: One out of ten smokers who attempted to quit in 2014-2015 in Italy used e-cigarettes, a proportion three times higher than that recorded for other quitting methods. E-cigarettes users were no more as likely to report abstinence than as those using no aid, but were less likely to report abstinence than users of established quitting methods. Further studies are needed to understand the relationship between e-cigarette types used to quit and abstinence rates.

Tob. Induc. Dis. 2018;16(Suppl 1):A242

DOI:10.18332/tid/83814

\section{$12: 30-14: 00$}

PS-656-4 Perceptions, work environment, and job stress related with tobacco use among fishermen in remote Island, Rebun Japan

E Kanazawa

${ }^{1}$ Dosanko Global Health International, Akita, Japan. E-mail: kanazawa.eri@gmail.com

Background: In Japan, lung cancer is the highest leading cause of death. From the data of Japan Tobacco Incorporated (JT) in 2016, the smoking rate was $19.3 \%$ and $29.7 \%$ in male, and $9.7 \%$ in female. Hokkaido showed the highest smoking rate both in male(35.3\%) and female(18.1\%). Rebun Island located at north of Hokkaido prefecture in Japan and main income is fishing industry. Although studies about smoking among fishermen are rare, many studies showed the high prevalence of smoking among fishermen. This study was conducted to identify the situation of current smoker among fishermen and the factors related to smoking behavior.

Methods: A cross sectional study was conducted, and all of the questionnaire contents were developed based on the literature and theory reviews. In the questionnaire, general characteristics, perceptions based on Health Belief Model (perceived susceptibility, perceived severity, perceived benefits, perceived barriers, and cues to smoking), working environment, and job stress were included to collect data at fishermen's home. One hundred and six fishermen were selected by accidental sampling technique, and interview was performed one by one. Data were analyzed by descriptive statistics and analytical statistics for association analysis by Chi-square and Independent t-test at $95 \%$ confidence. Results: The result showed $45.3 \%$ were current smokers $(43.4 \%$ daily smoker, and $1.9 \%$ occasional smoker), and $54.7 \%$ (33.0\% exsmoker and $21.7 \%$ never smoker $)$ were non-smokers. Age $(r=0.070)$, marital status $(r=0.002)$, present of smoker in family $(r=0.030)$, having chronic diseases $(r=0.001)$, job experience $(r=0.002)$, perceptions (perceived susceptibility $(\mathrm{r}=0.033)$, perceived benefits $(\mathrm{r}=0.049)$, and perceived barriers $(\mathrm{r}<0.001)$ ) showed strong association with smoking behavior among fishermen in Rebun Island.

Conclusions: Health promotion program for fishermen is necessary in order to educate them for raising perception toward smoking and for understanding the importance of smoking cessation among fishermen in Rebun Island.

Tob. Induc. Dis. 2018;16(Suppl 1):A243

DOI:10.18332/tid/83835
12:30-14:00

PS-657-4 E-cigarettes or vaping: examining perceptions of use and associated harm among current users in Australia and Bangladesh

MA Rahman ${ }^{1,2}$, D Edvardsson ${ }^{3,4}$, C McDonald ${ }^{5,6}$, D Castle $^{7,8}$

${ }^{1}$ La Trobe University, Austin Clinical School of Nursing, Heidelberg, VIC, Australia, ${ }^{2}$ The University of Melbourne, Melbourne School of Population and Global Health, Parkville, VIC, Australia, ${ }^{3}$ La Trobe University, Austin and Northern Clinical School of Nursing, Heidelberg, VIC, Australia, ${ }^{4}$ Umeå University, Department of Nursing, Umeå, Sweden, ${ }^{5}$ Austin Hospital, Department of Respiratory and Sleep Medicine, Heidelberg, VIC, Australia, ${ }^{6}$ The University of Melbourne, Department of Medicine, Parkville, VIC, Australia, ${ }^{7}$ St Vincent's Health, Department of Psychiatry, East Melboourne, VIC, Australia, ${ }^{8}$ The University of Melbourne, Department of Psychiatry, Parkville, VIC, Australia. E-mail: aziz.rahman@y7mail.com

Background: E-cigarettes or vaping are currently increasing in popularity among smokers globally. This study aims to examine the perceptions of e-cigarette users regarding use and associated harm.

Methods: A cross sectional survey was conducted during July 2017 among members of different popular online forums in Australia and Bangladesh, who were current or ex-users of e-cigarettes. A structured questionnaire was used to collect data anonymously using Qualtrics.

Results: There were 452 study participants, mean age was $39( \pm 13.2)$ years and $80 \%(n=356)$ were men. Half of them $(n=223)$ resided in Australia and 32\% $(n=143)$ in Bangladesh. Three in four participants (77\%) lived in metropolitan areas, $47 \%$ were married, $33 \%$ had undergraduate level of education, a fifth of them were either professionals or employed. More than three quarters $(76 \%)$ of respondents were not current smokers and $40 \%$ of them quit smoking 1-5 years ago. Three quarters of the current smokers (76\%) tried to quit smoking cigarettes in the last 12 months. Almost all of the participants (96\%) were using e-cigarettes daily and $94 \%$ of them had nicotine in the e-liquid used. The average amount of e-liquid used, nicotine strengths and duration of use were $8.2( \pm 6.9) \mathrm{ml} / \mathrm{day}, 6.7( \pm 5.8) \mathrm{mg} / \mathrm{ml}$, and $25.2( \pm 23.3)$ months respectively. Reasons for using e-cigarettes were to reduce/quit cigarette smoking (91\%), good taste/flavor (50\%), low cost (41\%), safe to use (39\%) and can be used indoor/ smoke free areas (33\%). The majority of respondents $(81 \%)$ perceived e-cigarettes as less harmful than cigarettes and $65 \%$ perceived them as less addictive. The majority of respondents (88\%) did not try to stop using e-cigarettes, however, $75 \%$ of them had an intention to discontinue in the next five years.

Conclusions: E-cigarettes were primarily used for reducing/ quitting cigarettes, which supports prior evidence regarding the effectiveness of e-cigarettes for smoking cessation.

Tob. Induc. Dis. 2018;16(Suppl 1):A244

DOI:10.18332/tid/83852

12:30-14:00

PS-658-4 E-cigarettes or vaping: is there any difference in perceptions of use and associated harm among the current users between a developed and a developing country?

MA Rahman ${ }^{1,2}$, D Edvardsson $^{3},{ }^{4}$, C McDonald $^{5,6}$, D Castle $^{7},{ }^{8}$

${ }^{1}$ La Trobe University, Austin Clinical School of Nursing, Heidelberg, VIC, Australia, ${ }^{2}$ The University of Melbourne, Melbourne School of Population and Global Health, Parkville, VIC, Australia, ${ }^{3}$ La 
Trobe University, Austin and Northern Clinical School of Nursing, Heidelberg, VIC, Australia, ${ }^{4}$ Umeå University, Department of Nursing, Umeå, Sweden, ${ }^{5}$ Austin Hospital, Department of Respiratory and Sleep Medicine, Heidelberg, VIC, Australia, ${ }^{6}$ The University of Melbourne, Department of Medicine, Parkville, VIC, Australia, 'St Vincent's Health, Department of Psychiatry, East Melboourne, VIC, Australia, ${ }^{8}$ The University of Melbourne, Department of Psychiatry, Parkville, VIC, Australia. E-mail: aziz.rahman@y7mail.com

Background: E-cigarettes or vaping are currently increasing in popularity among smokers globally. This study aims to compare e-cigarette users between a developed country and a developing country to identify similarities/differences regarding perceptions of use and associated harm.

Methods: A cross sectional survey was conducted during July 2017 among members of different popular online forums in Australia and Bangladesh, who were current or ex-users of e-cigarettes. Data were collected anonymously using Qualtrics.

Results: There were 452 study participants, mean age was $39( \pm 13.2)$ years and $80 \%(n=356)$ were men. Daily or occasional smoking in the last 30 days was more frequent among the Bangladeshi participants than their Australian counterparts (38\% vs. $18 \%, \mathrm{p}<0.001$, ORs $2.85,95 \%$ CIs $1.76-4.62)$. Endeavour to quit smoking was also more common among the current smokers in Bangladesh (90\% vs. $72 \%, \mathrm{p}=0.013$, ORs $3.69,95 \% \mathrm{CIs} 1.16$ 11.7). Almost all of the participants in both countries were using e-cigarettes daily and had nicotine in the e-liquid. The average amount of e-liquid used, nicotine strengths and duration of use in Australia and Bangladesh were $9( \pm 7.9)$ vs. $5.9( \pm 3.5) \mathrm{ml} / \mathrm{day}$, $6.8( \pm 6.4)$ vs. $4.6( \pm 1.8) \mathrm{mg} / \mathrm{ml}$, and $22.9( \pm 22.3)$ vs. $15.9( \pm 12.8)$ months respectively. The most commonly cited reason for using e-cigarettes in both countries was to reduce/quit cigarette smoking, although there was a significant difference between Australia and Bangladesh (95\% vs. $83 \%, \mathrm{p}<0.001$, ORs $3.89,95 \%$ CIs 1.84 $8.21)$. More than three quarters of respondents in both countries perceived e-cigarettes as less harmful and more than two thirds perceived them as less addictive. The majority of respondents did not try to stop using e-cigarettes, however, intention to discontinue in the next five years was more in Bangladesh than Australia (85\% vs. $74 \%, \mathrm{p}=0.006$, ORs $1.99,95 \%$ CIs $1.15-3.46$ ).

Conclusions: E-cigarettes were primarily used for reducing/ quitting cigarettes in both countries, which supports prior evidence regarding the effectiveness of e-cigarettes for smoking cessation.

\section{Tob. Induc. Dis. 2018;16(Suppl 1):A245} DOI: $10.18332 /$ tid/83853

\section{2:30-14:00}

PS-659-4 Prevalence and characteristics of e-cigarette use among adults in Malaysia

J Ab Rahman', MH Nik Mohamed ${ }^{2}$, MF Mohd Yusoff ${ }^{3}$, NS Ab Rahman ${ }^{2}$, S Draman', M Kartiwi ${ }^{4}$, B Mahadir $^{3}$, T Aris $^{3}$

${ }^{1}$ International Islamic University Malaysia, Kulliyyah of Medicine, Kuantan, Malaysia, ${ }^{2}$ International Islamic University Malaysia, Kulliyyah of Pharmacy, Kuantan, Malaysia, ${ }^{3}$ Institute for Public Health, Bangsar, Malaysia, ${ }^{4}$ International Islamic University Malaysia, Kulliyyah of Information and Communication, Gombak, Malaysia.E-mail: arjamal@iium.edu.my

Background: E-cigarette (e-cig) use has become a worldwide phenomenon since 2010 . This study aims to determine the prevalence of e-cig use, distribution and characteristics among Malaysian adults. Malaysia is a typical Asian country. The results of this study will provide evidence for policy makers to formulate appropriate measures towards regulation of e-cig in Malaysia and can become a reference for other similar countries.

Methods: Complex sampling design was used to represent 19 million of Malaysian adult household. Samples were stratified by states and urbanity. Sampling units were districts, enumeration blocks and living quarters. All adults from the selected houses were invited to participate in this survey. Analysis was done using sampling weight and complex sampling analysis. Characteristics of current e-cig users were described unweighted.

Results: A total of 4,288 individual responded in this survey. Majority of the respondents were at 25-44 years of age group (44\%), Malay ethnicity (73\%), completed at least secondary level of education $(69 \%)$, working in private sectors $(26 \%)$, Muslim (79\%) and married (68\%). Male and female were equally represented. Based on weighted population, $72 \%$ of the respondents were from the urban residential area. The prevalence of current e-cig users in Malaysia was 3.2\% (95\%CI 2.5-4.1), involving an estimated population of about 600,000 household residence. The prevalence in urban and rural were $3.3 \%(95 \% \mathrm{CI}$ 2.4-4.4) and 2.8\% (95\%CI 2.1-3.8) respectively. E-cig users were common among young, male, Malay, highly educated, Muslim, single and those in urban area. Most of current e-cig users started smoking e-cig when they were 19 years and above. $48 \%$ used e-cig just to try, while $17 \%$ used it to quit cigarette smoking. $62 \%$ preferred nicotine in their e-cig liquid.

Conclusions: This study able to represent adults Malaysians and results can be used to guide healthcare managers to manage e-cigarette use countries with similar characteristics.

Tob. Induc. Dis. 2018;16(Suppl 1):A246

DOI: $10.18332 /$ tid/83854

\section{2:30-14:00}

PS-660-4 Prevalence of flavored cigarettes and e-cigarettes in Uruguay: findings from the Wave 5 of the ITC Uruguay survey

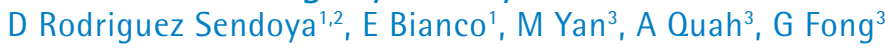
${ }^{1}$ Centro de Investigación para la Epidemia del Tabaquismo, Montevideo, Uruguay, ${ }^{2}$ Universidad de la Republica Uruguay, Sociología, Montevideo, Uruguay, ${ }^{3}$ University of Waterloo, Psychology, Waterloo, ON, Canada. E-mail: rodriguezsendoya@ gmail.com

Background: Uruguay has implemented strong tobacco control policies over the years which has resulted in decreased tobacco consumption. However, there is little information about flavored cigarettes and on electronic cigarettes (ECs). EC sales are banned in Uruguay. The purpose of this study is to evaluate consumption of these products.

Methods: Data is from the International Tobacco Control (ITC) Uruguay Wave 5 smokers aged 18 and older in five cities. The sample size was 1,428 smokers.

Results: The minimum age of onset of smoking was 5 years old and $96 \%$ of smokers started smoking before the age of 25 . Two out of three (68.6\%) smokers chose their cigarette brand because of the taste. $72.5 \%$ have tried flavored (menthol) cigarettes, and $1.3 \%$ currently smoking menthol brands. Younger age (94\% aged 18-24) and smokers with higher education (84\% high, 74\% medium, $61 \%$ low) have tried menthol cigarettes associated with. Awareness of ECs was $56 \%$ among the smokers, but it is higher among smokers aged 18-24 (62\%) and smokers with higher education (81\%). Among the smokers who had heard of e-cigarettes. $12.8 \% \mathrm{had}$ tried once (higher under age 24 (24\%) and higher among smokers 
with high education level (20\%)). Among the smokers, 1.3\% using EC daily and $1.9 \%$ using EC weekly.

Conclusions: Taste is a very important factor for Uruguayan smokers. Almost all young smokers have tried menthol cigarettes. ECs use is also on the rise, mainly among young peopleand people with higher education. There is a need for monitoring flavored cigarettes and EC consumptionto defining new regulations for them.

Tob. Induc. Dis. 2018;16(Suppl 1):A247 DOI:10.18332/tid/83879

\section{$12: 30-14: 00$}

PS-661-4 Contrasting trends of smoking cessation status: insights from the stages of change theory using data from the global adult tobacco survey L Mbulo $^{1}$, T Erguder ${ }^{2}$, H Van Minh ${ }^{3}$, LMR Shigematsu ${ }^{4}$, S Benjakul ${ }^{5}$, A Segarra ${ }^{6}$, KM Palipudi ${ }^{1}$, IB Ahluwalia ${ }^{1}$, Global Adult Tobacco Survey (GATS) Collaborative Group

${ }^{1} \mathrm{CDC}$, Office of Smoking and Health, Atlanta, GA, United States of America, ${ }^{2}$ World Health Organization, Ankara, Turkey, ${ }^{3}$ Hanoi Medical University, Institution for Preventative Medicine and Public Health, Hanoi, Viet Nam, ${ }^{4}$ Instituto Nacional de Salud Pública, Jefe Departamento de Investigación sobre Tabaco, Cuernavaca, Mexico, ${ }^{5}$ Mahidol University, Faculty of Public Health, Bangkok, Thailand, ${ }^{6}$ Department of Health, Manila, Philippines. E-mail:vyp7@cdc.gov

Background: Quitting tobacco use can reduce smokers' risks for disease and premature death. We used the trans-theoretical behavior change model to examine temporal differences in readiness to quit smoking among adults in five countries.

Methods: We analyzed two independent samples from the Global Adult Tobacco Survey, a standardized national adult household survey of persons aged $\geq 15$ years, for five countries: Mexico (2009, 2015), Philippines (2010, 2016), Thailand (2009, 2011), Turkey (2008, 2012), and Vietnam (2010, 2016). Response rates ranged $82.5 \%-96.3 \%$ and sample sizes ranged 8,996-20,606. Current smokers were defined as adults who smoked tobacco on a daily or non-daily basis. Using responses to questions assessing intention to quit cigarette smoking, we categorized current smokers into the following stages of change: pre-contemplation, contemplation, and preparation. Relative change was compared using z-tests $(\mathrm{p}<$ 0.05).

Results: No significant change in smoking prevalence between wave 1 and 2 occurred for Mexico (15.9\% to 16.4\%), Thailand (23.7\% to $24.0 \%$ ), or Vietnam (23.8\% to $22.5 \%$ ). In contrast, smoking prevalence declined for Philippines (28.2\% to $22.7 \%$ ) and Turkey (31.2\% to $27.1 \%$ ). There was an increase in the proportion of smokers in the pre-contemplation stage in Thailand (76.1\% to $85.4 \%)$ and Vietnam $(70.8 \%$ to $82.3 \%)$, while a decline occurred in Turkey (31.2\% to $27.1 \%$ ); no significant change occurred in Mexico or Philippines. The proportion of smokers in the contemplation stage declined in Thailand (17.6\% to $12.0 \%)$ and Vietnam (21.6\% to $14.1 \%)$, but increased in Turkey $(21.2 \%$ to 26.9\%); no significant change occurred in Mexico or Philippines. The proportion in the preparation stage declined in Thailand (6.3\% to $2.6 \%)$ and Vietnam (7.6\% to $3.6 \%)$; no significant changes occurred in, Mexico, Philippines, or Turkey.

Conclusions: Using the stages of change model, smokers' readiness to quit varies by country. Tailored interventions towards specific stages of cessation might help motivate quitting behavior.

Tob. Induc. Dis. 2018;16(Suppl 1):A248

DOI:10.18332/tid/83889

\section{2:30-14:00}

PS-662-4 Health care professional and cigarette cessation volunteers knowledge, attitude and practice on e-cigarettes

H Sharifi ${ }^{1}$

${ }^{1}$ Tobacco Prevention and Control Research Center, National Research Institute of Tuberculosis and Lung Diseases (NRITLD), Shahid Beheshti University of Medical Sciences, Tehran, Iran, Islamic Rep. Of. E-mail: drhooman2000@yahoo.com

Background: Electronic cigarettes (e-cigarette) are new phenomenon that has been widely accepted. E- Cigarettes are more popular that has become one of the preferable rout of smoking cessation in patients. Further researches are required for future advice on e-cigarette use.To determine Health Care Professional and Cigarette Cessation Volunteers Knowledge, Attitude and Practice on e-Cigarettes

Methods: In a cross-sectional description study, 147 medical professional and patients were enrolled in the study. After enrollment, a questionnaire with 23 questions designed for assessment of attendees' knowledge, attitude and practice. Seven questions were designed for knowledge, 7 for attitude and 7 for practice, and 2 for general assessment of e-cigarette acceptability as a method for quit smoking.

Results: Total numbers of 147 medical professional and patients with mean age of $41.05 \pm 6.28$ were enrolled in the study. Attitude of attendees about comparison of e-cigarette and standard cigarette harm to the health showed that $52.4 \%$ of patients believe that e-cigarette is less harmful compare to standard cigarette. About $49 \%$ of attendees believed that e-cigarettes need more research as a help to quit smoke and only $27.2 \%$ of patients answered yes to the question. Besides, $57.8 \%$ of attendees answered "yes" to the question on "does e-cigarette induce addiction?" , and $12.9 \%$ answered "No".

Conclusions: although e-cigarette does not produce tobacco smoke, they still contain nicotine and other potentially harmful chemicals and people believe they may induce addiction.

Tob. Induc. Dis. 2018;16(Suppl 1):A249 DOI:10.18332/tid/83894

$12: 30-14: 00$

PS-663-4 Smoking prevalence and smoking behavior change among the soldiers in the Korean military

J Kim', S Lee ${ }^{1}$

${ }^{1}$ Korean Association on Smoking or Health, Seoul, Korea, Republic of. E-mail: jy9651@gmail.com

Background: Republic of Korea has an obligatory military service. All Korean men in their 20s are obliged to serve the military for 21 months. The purpose of this study was to identify smoking prevalence among the Korean military and to understand how the soldiers change their smoking behavior during their obligated military service.

Methods: A tobacco use survey for the military was carried out by the Korean Association of Health Promotion on behalf of the Ministry of Health in July, 2016. The participants were asked to answer the questions regarding current and past experience of tobacco use, their smoking behavior change during the military service.

Results: The total number of participants in the survey were 3,800 soldiers. Among them, 44.9\% (1,707 soldiers) of the participants were current smokers. Marine Corps had the highest smoking prevalence with $51.7 \%$, following Army with 48.5\%, Navy with 
$39.1 \%$ and Air force with $32.7 \%$. The branches of the military were associated with soldiers' smoking prevalence $(\mathrm{p}<.001)$. There were 726 participants $(63.5 \%)$ who answered that their smoking frequency has increased in the military service. The reasons being; tough training or stress of the military life $(72.6 \%$, 527 soldiers). In addition, the participants answered that in order to have a break, they smoked more often. There was a large group of dual users (34.9\%) between cigarettes and electronic cigarettes. The rank was associated with dual use of cigarettes and electronic cigarettes $(\mathrm{p}=.007)$.

Conclusions: The smoking prevalence among soldiers were higher compared to that of the same age group in the population. The military life has affected the young soldiers' smoking behavior. Effective tobacco control intervention should be employed in the military and this will contribute to the decrease in the smoking prevalence among Korean males.

Tob. Induc. Dis. 2018;16(Suppl 1):A250

\section{DOI: $10.18332 /$ tid/83895}

\section{$12: 30-14: 00$}

PS-664-4 Association of tobacco control policies with cigarette smoking among school age youth 13 to 15 in the Philippines, $2000-2015$

RA Arrazola1, LM Dutra², IB Ahluwalia ${ }^{1}$, AB Seidenberg ${ }^{3}$, RTA De Guzman ${ }^{4}$, F Trinidad ${ }^{5}$

${ }^{1}$ Centers for Disease Control and Prevention, Office on Smoking and Health, Atlanta, GA, United States of America, ${ }^{2} \mathrm{RTI}$ International, Center for Health Policy Science and Tobacco Control Research, Berkeley, CA, United States of America, ${ }^{3}$ University of North Carolina at Chapel Hill, Department of Health Behavior, Gillings School of Global Public Health, Chapel Hill, NC, United States of America, ${ }^{4}$ World Health Organization Regional Office for the Western Pacific, Tobacco-Free Initiative, Manila, Philippines, ${ }^{5}$ World Health Organization, Office of the Representative in the Philippines, Tobacco-Free Initiative and Mental Health, Manila, Philippines. E-mail: fdy9@cdc.gov

Background: In 2003, the Philippines passed extensive tobacco control legislation, including minimum legal sales age (MLSA) laws, textual warning labels (TWL), and smoke-free public places (SFPP), and pricing strategies. This study examined the impact of these tobacco control policies on cigarette smoking among Filipino youth 13 to 15 years old who attended school.

Methods: Data from the Philippines Global Youth Tobacco Surveys were analyzed (2000, 2004, 2007, 2011, and 2015). Sample sizes ranged from 3,708 to 5,919 , and response rates from $79.5 \%$ to $84.8 \%$. Current cigarette smoking was defined as smoking cigarettes on $\geq 1$ day in the past 30 days. Current use of other tobacco products (OTP) was defined as using tobacco products other than cigarettes on $\geq 1$ day in the past 30 days. Annual average cigarette price was obtained for each year from Euromonitor data, and converted into 2015 Philippine pesos Tobacco control legislation (MLSA, TWL, and SFPP) was coded as all three components in effect (1) or none in effect ( 0 , reference). Logistic regression was used to model the association of tobacco control laws with current youth cigarette smoking, adjusting for cigarette price, age, sex, and current OTP use.

Results: Tobacco control legislation was associated with declines in current cigarette smoking among youth (AOR: 0.65, p< $0.0001)$. The price of cigarettes was not significantly associated with current cigarette smoking (AOR: 1.01, $\mathrm{p}=0.2895$ ], which is likely related to the timing and relatively limited magnitude of price increases during this period. Age, sex, and current OTP use were associated with current cigarette smoking $(\mathrm{p}<0.0001)$. Conclusions: The development and implementation of the 2003 tobacco control legislation was associated with decreases in current cigarette smoking among Filipino youth in school. Continued implementation and enforcement of comprehensive, evidence-based tobacco control policies can help reduce tobacco use among youths.

Tob. Induc. Dis. 2018;16(Suppl 1):A251

DOI:10.18332/tid/83901

12:30-14:00

PS-665-4 Prevalence and willingness to pay for home-made cigarettes (HC) and industry cigarettes (IC) of Thai men

S Grisurapong ${ }^{1}$

'Mahidol University, Faculty of Social Sciences \& Humanities, Nakhonpathom, Thailand. E-mail: siriwan.gri@mahidol.ac.th Background: Prevalence \& willingness to pay for tobacco consumption is in need for effective pricing of $\mathrm{HC}$ and IC. This study aimed to determine Thai prevalence of HC and IC consumption, its average expenditure and willingness to pay.

Methods: Roiet, a province with high cultivation rate of tobacco had been chosen as the site. A cluster random sampling was conducted by using Krejcie \& Morgan table to determine sample size. A survey method was employed to interview men who smoking. In total, 400 men were interviewed during August November 2016. Data were analyzed by descriptive and Chisquare technique.

Results: The results showed that participants smoking IC, HC, both types but more on IC and both types but more on $\mathrm{HC}$ were 37.3, 43.0, 12.0 and $7.8 \%$ respectively. Expenditure/ day of those smoking IC, HC, both types but more on IC and both types but more on $\mathrm{HC}$ were 1.02,0.17, 1.35 and $0.60 \mathrm{US} \$$. Expenditure for tobacco equaled to $16.1 \%$ of total expenditure. When expenditure was analyzed by income quintile, it was found that the lowest to highest quintile paid $0.42,0.55,0.70,0.85$ and $1.09 \mathrm{US} \$ /$ day for tobacco. Willingness to pay for tobacco was $0.83,0.95,1.22$, 1.43 and $1.66 \mathrm{US} \$$ /day. Number of cigarette smoked per day was $6.90,7.98,11.42,10.10$ and 10.61 . It was found that household cultivating tobacco had significantly less income than those cultivating other crops. Household with better economic status were likely to consume more tobacco, start smoking at younger age and had higher number of friends smoking together.

\begin{tabular}{|c|c|c|c|c|c|}
\hline $\begin{array}{l}\text { Income } \\
\text { Quintile }\end{array}$ & $\begin{array}{c}1 \\
\text { (poorest) }\end{array}$ & 2 & 3 & 1 & $\begin{array}{c}5 \\
\text { (Richest) }\end{array}$ \\
\hline $\begin{array}{l}\text { Average number } \\
\text { of cigarette } \\
\text { smoked / day }\end{array}$ & 6.90 & 7.98 & 11.42 & 10.10 & 10.61 \\
\hline $\begin{array}{l}\text { Average } \\
\text { expenditure for } \\
\text { tobacco /day } \\
\text { (US\$) }\end{array}$ & 0.42 & 0.55 & 0.70 & 0.85 & 1.09 \\
\hline $\begin{array}{l}\text { Willingness to } \\
\text { pay for tobacco / } \\
\text { day (US\$) }\end{array}$ & 0.83 & 0.95 & 1.22 & 1.43 & 1.66 \\
\hline $\begin{array}{l}\text { Percentage } \\
\text { of tobacco } \\
\text { expenditure to } \\
\text { total expenditure }\end{array}$ & 14.73 & 15.36 & 15.76 & 15.99 & 12.15 \\
\hline
\end{tabular}

[Details of tobacco consumption by income quintile] 
Conclusions: Thailand can increase price for cigarettes, particularly $\mathrm{HC}$, since willingness to pay for each item of cigarette is higher than their current expenditure for tobacco. People in higher income group pay more for cigarette than lower group although the number of cigarettes smoking may not different.

Tob. Induc. Dis. 2018;16(Suppl 1):A252

DOI:10.18332/tid/83934

\section{2:30-14:00}

PS-667-4 Characteristics and factors associated with tobacco use: findings of Kenya Global Adult Tobacco Survey, 2014

V Mwenda ${ }^{1}$, G Gathecha ${ }^{2}$, A Kendagor ${ }^{2}$, J Kibachio ${ }^{2}$, E Macharia ${ }^{3}$ ${ }^{1}$ Field Epidemiology and Laboratory Training Programme, Ministry of Health, Nairobi, Kenya, ${ }^{2}$ Non-Communicable Diseases Division, Ministry of Health, Nairobi, Kenya, ${ }^{3}$ International Livestock Research Institute, Statistics department, Nairobi, Kenya.E-mail:valmwenda@gmail.com

Background: Tobacco is a major risk factor for pulmonary and cardiovascular diseases. The Global Adult Tobacco Survey (GATS) has been conducted in 25 countries. Kenya conducted its first GATS in 2014. Our secondary analysis aimed to describe background characteristics and identify factors associated with adult tobacco use for Tobacco control planning within Kenya.

Methods: Kenya GATS was a multi-stage, clustered, nationally representative survey of persons above 15 years. We conducted secondary analysis of the dataset, with age, sex, education level, residence, occupation as exposure variables and tobacco use the outcome variable. Accounting for complex survey sampling, we calculated descriptive statistics for tobacco use. We used logistic regression to calculate prevalence odds ratios (POR) and confidence intervals $(\mathrm{CI})$ to identify factors independently associated with tobacco use.

Results: Of the 4408 persons surveyed, 2258 (51\%) were female. The mean age was 33.6 years ( \pm 16.0 years) while mean age at tobacco use initiation was 19 years ( \pm 3.2 years); $2867(65 \%)$ were rural residents and $2628(60 \%)$ had primary or no formal education. Among smokers, $60 \%$ reported initiating smoking within 30 minutes of waking up. Among non-smokers, $88 \%$ knew second-hand smoke causes illness, and $55 \%$ were aware of legal prohibition of smoking in public places. Sixty-five percent of smokers who had quit in the preceding 12 months did so because of health concerns, with $62 \%$ reporting no assistance. Forty-three percent of smokers would quit if cigarette price was doubled. Tobacco use was associated with being aged above 35 years (POR 2.95, 95\% CI 2.17, 4.0) and having primary or no formal education (POR 2.04, 95\% CI 1.50, 3.96).

Conclusions: Nicotine addiction and young initiation age may hamper cessation efforts. Advocacy on existing legal protections from tobacco harms, price increases, supporting users to quit and targeted campaigns among older, less educated individuals should constitute control planning.

Tob. Induc. Dis. 2018;16(Suppl 1):A253

DOI:10.18332/tid/83982

\section{2:30-14:00}

PS-668-4 Amendment of tobacco hazards prevention act in Taiwan: brought about a reduction of 810,000 smokers and cut health inequality pragmatically within eight years

Y-H Su ${ }^{1}$, H-Y Chung ${ }^{1}$, S-Y Lo ${ }^{1}$, Y-J Liu ${ }^{1}$, M-C Lin ${ }^{1}$, L-H Yu ${ }^{1}$, Y-W Wang $^{1,2}$, Y-R Wang ${ }^{1}$
${ }^{1}$ Health Promotion Administration, Ministry of Health and Welfare, Taipei, China, ${ }^{2}$ School of Medicine, Tzu Chi University, Hualien, China. E-mail: yiren@hpa.gov.tw

Background: Smoking kills more than 27,000 people each year. In 2009, amendment of the Tobacco Hazards Prevention Act came into effect with expanded ban of smoking including all public indoor places and some public outdoor places, prohibition of all types of promotion, and a raise in tobacco taxation. This study aims to evaluate the effectiveness of this amendment on smoking rate and health inequality.

Methods: Data were drawn from the Taiwan Adult Smoking Behavior Survey (TASBS) conducted under a nationally representative cross-sectional study with telephone interviews from 2004 to 2016. Probability proportional to size (PPS) sampling and post-stratification were used to better represent the characteristics of the population. The annual sample size is approximately 15,000-26,000 persons.

Results: The adult smoking rate declined from $21.9 \%$ in 2008 to $15.3 \%$ in 2016 , indicating a $30.3 \%$ decline in smoking rate and a reduction in the number of smokers by approximately 810,000 within 8 years. The speed of reduction in this period was more remarkable than that experienced in 4 years before the amendment. Among different subgroups, the reduction was more significant in males aged 18-29 and 65 or over than those aged 3064 , and people of lowest level of education (junior high or lower) than those of higher education.

Conclusions: Implementation of the amended Tobacco Hazards Prevention Act has managed to remarkably reduce both smoking rate itself and inequality in smoking rates. However, continued efforts are needed to achieve the goal of a $30 \%$ relative reduction in smoking rate by 2025 set by the World Health Organization (WHO), decreasing the adult smoking rate from $20 \%$ in 2010 to $14 \%$ in 2025 .

Tob. Induc. Dis. 2018;16(Suppl 1):A254

DOI: $10.18332 /$ tid/83993

\section{2:30-14:00}

PS-669-4 E-cigarette use in Germany from 2015 to 2017

K Schaller ${ }^{1}$, S Kahnert ${ }^{1}$, U Mons ${ }^{1}$

${ }^{1}$ German Cancer Research Center (DKFZ), Unit Cancer Prevention and WHO Collaborating Centre for Tobacco Control, Heidelberg, Germany. E-mail:k.schaller@dkfz.de

Background: Worldwide sales of e-cigarettes are increasing markedly as well as their use by adults and youths. The utility of e-cigarettes for smoking cessation is a matter of scientific debate. Increasing use among youths however - and especially among nonsmoking adolescents - is a matter of concern, as e-cigarette use is suspected to contribute to nicotine addiction and smoking initiation. Currently, for Germany, data on e-cigarette use are sparse.

Methods: The German Cancer Research Center conducted annual representative cross-sectional surveys from 2015 to 2017 , each comprising a sample of $n=2000$ persons aged 16 years and older. All respondents were asked whether they used e-cigarettes, smokers and former smokers were also asked about cessation methods they used in their last quit attempt. For February 2018, another survey is planned.

Results: Ever use of e-cigarettes increased from 2015 to 2017; in 2017 8.6\% of all respondents had ever used e-cigarettes. Ever use of e-cigarettes was highest in ages 16 - 19. Regular use was generally lower, but also markedly increased from 2015 to 2017; in $20172.5 \%$ of all respondents used e-cigarettes at least weekly. Regular use was highest in the age groups $20-29$ and 50+. More 
smokers $(4.3 \%)$ than former $(2.8 \%)$ or never $(1.0 \%)$ smokers used e-cigarettes regularly. The percentage of smokers who used e-cigarettes in an attempt to quit smoking decreased from $3.1 \%$ in 2015 to $2.5 \%$ in 2017 , but the percentage of former smokers who said that they successfully quit by using e-cigarettes increased from $0.5 \%$ in 2015 to $1.8 \%$ in 2017 .

Conclusions: In Germany, regular e-cigarette use is low, but increasing. E-cigarette experimentation is highest among youths, and regular use is highest among young adults. The percentage of former smokers who successfully stopped smoking by using e-cigarettes as cessation method is still low, but increasing.

Tob. Induc. Dis. 2018;16(Suppl 1):A255

DOI:10.18332/tid/84010

\section{2:30-14:00}

PS-670-4 "Chewing of tobacco laced areca-nuts" - is nothing but "Chewing of tiny-cancer-causingbombs in the mouth" and one more tobacco Hiroshima and Nagasaki is in waiting

R Venkatachalam Pillai ${ }^{1}$

'Volunteers against Smoking and Tobacco, Tiruchirapalli, India. E-mail:vregu@yahoo.co.in

Background and challenges to implementation: In India, Sweetened Suparis (Arecanut Granules, spices) and Gutkhas (Arecanut Granules, Tobacco, spicy compounds) - packed in small pouches - are freely marketed - which causes Oral Cancer. These products have an unrestricted reach among the youth, students, poor and downtrodden segments.

Intervention or response: To save the future society from Oral Cancer - urgent initiatives are required to douse these Supari \& Gutkha-Bombs.

Results and lessons learnt: A study - among the School/College Students and Low-Income Workers $(<1000)$ of Tiruchirapalli City (India) - reveals that usage of Suparis \& Gutkhas is largely-considered as "Socially-culturally-accepted-practice". Unaware of harmfuleffects of these Products, even some parents feed these products to their wards in many social-functions - making them to get hooked and addicted to this harmful habit at a very tender age of 5 or 6 .

It is found that more than $90 \%$ of the cigarette-smoking-students (boys) are using these products, initially as a smelly-shield, to get rid of their parent's attention and ire. However, in this process, within a very short time, they become addict to these deadly-powders and soon permanently get trapped to this chewing habit.

Around $70-80 \%$ of the Low-Income workers are using these products to suppress their appetite/hungry-feeling - without an iota of knowledge that these deadly-powders are causing cancers of vital organs (gums, mouth, throat, esophagus, lung, liver, stomach).

Conclusions and key recommendations: Unless the Indian Government BAN the manufacture of these highly harmful products in "Lock, Stock and Barrel", one more Hiroshima and Nagasaki is in waiting to happen, in the form of Oral Cancer. Let's positively focus our efforts to create a "Supari \& Gutkha-free Society" - a Society free from Oral cancer.

Tob. Induc. Dis. 2018;16(Suppl 1):A256

DOI:10.18332/tid/84016

\section{2:30-14:00}

PS-671-4 Passive exposure to e-cigarette emissions: irritation symptoms, severity and duration

A Tzortzi ${ }^{1,2}$, S Teloniatis ${ }^{1}$, G Mattiampa $^{1}$, G Bakellas ${ }^{1}$, V Vyzikidou ${ }^{1}$, Chara Tzavara $^{3}$, CVardavas ${ }^{1,2}$, E Fernandez Munoz ${ }^{4,5}$, P Behrakis $^{1,2,6}$ ${ }^{1}$ Hellenic Cancer Society, George D. Behrakis Research Lab, Athens,
Greece, ${ }^{2}$ American College of Greece, Institute of Public Health, Athens, Greece, ${ }^{3} \mathrm{National}$ and Kapodistrian University of Athens, Athens, Greece, ${ }^{4} \mathrm{WHO}$ Collaborating Center for Tobacco Control, Institut Català d'Oncologia - Institut d'Investigació Biomèdica de Bellvitge (ICO-IDIBELL), Barcelona, Spain, ${ }^{5}$ Universitat de Barcelona, Epidemiology and Public Health, Barcelona, Spain, ${ }^{6}$ Academy of Athens, Biomedical Research Foundation (BRFAA), Athens, Greece. E-mail: atzortzi@researchlab.gr

Background: The current study, part of the EU H2020 funded TackSHS project, aimed to test the hypothesis that passive exposure to e-cigarette emissions provokes systemic symptoms and to determine their severity and timing.

Methods: 30 nonsmokers, 18-35 years old, $\mathrm{BMI}<30$, with no significant medical history, no medications, normal physical examination and spirometry, were passively exposed in a $35 \mathrm{~m} 3$ room, during a 30-minute Control (no passive smoking) and Experimental (standardized e-cigarette smoking by a human smoker) session.

PM2.5 concentrations were $0.027 \mathrm{mg} / \mathrm{m} 3$ and $3.3 \mathrm{mg} / \mathrm{m} 3$ during the Control and Experimental sessions, respectively. Participants completed an irritation questionnaire, grading symptom severity at T0 (pre-exposure), T15 (midway), T30 (exposure endpoint) and T60 (30-minute post-exposure) in both sessions.

The questionnaire showed internal consistency (Cronbach's $a>0.70$ ). Scores 1-5 were generated for the environmental, ocular, nasal, airway and general complaints by adding symptoms per system. Analysis was performed using Wilcoxon-signed rank sum test and Spearman correlation $(\mathrm{p}<0.05)$.

Results: The most frequent and intense symptoms reported were mild eye burning, nasal and airway dryness.

Ocular irritation score gradually increased from $\mathrm{T} 0$ reaching a significant increase by T30 $(p=0.034)$. Nasal score increased significantly from T0 to T15 ( $\mathrm{p}=0.008)$ and remained significantly higher at T30. Airway irritation score increased significantly from T0 to T15 $(p=0.004)$ and furthermore from T15 to T30 $(p=0.018)$. All symptoms returned to T0 scores by T60 ( $>0.05)$. The increased scores for ocular, nasal and airway complaints were positively correlated with increased environmental scores at T15 and T30. General complaint scores showed a tendency to increase at T30 that was positively correlated with increased environmental scores at T30.

Conclusions: Short-term exposure of nonsmokers to e-cigarette emissions resulted in mild ocular, nasal and airway symptoms that persisted up to 30 minutes and were positively correlated with environmental indices. Further research is needed to investigate long-term health implications.

Tob. Induc. Dis. 2018;16(Suppl 1):A257

DOI:10.18332/tid/84038

\section{2:30-14:00}

PS-672-4 Cigarette brand preferences of adolescent and adult smokers in the United States

S Brink ${ }^{1}, \mathrm{G}$ Nahhas ${ }^{1}, \mathrm{KM}$ Cummings ${ }^{1}, \mathrm{M}$ Travers$^{2}$, R O'Connor², B Heckman ${ }^{1}$, A Alberg ${ }^{1}$

${ }^{1}$ Medical University of South Carolina, Charleston, SC, United States of America, ${ }^{2}$ Roswell Park Cancer Institute, Buffalo, NY, United States of America. E-mail: elnahas@musc.edu

Background: Despite restrictions on where and how cigarette companies can market their products, cigarettes remain a heavily advertised consumer product in America. Examining the brand preferences of smokers may provide clues to understanding how shifting consumer preferences and industry marketing strategies 
are influencing smoking related behaviors. This study presents estimates of cigarettes brand preferences of adolescents and adults in the United States.

Methods: Data for 10,509 adult (>18years old) and 283 adolescent (12-17 years old) current cigarette smokers were available from the Population Assessment of Tobacco and Health (PATH) wave 1 (2013-2014) survey. Only respondents who reported smoking in the past 30 days and purchasing their own cigarettes were included in this analysis.

Results: Overall, adult respondents reported 64 different cigarette brands and 330 sub-brands while youth respondents reported a total of 14 brands and 50 sub-brands. The three most popular brands purchased by adolescents were Marlboro (48.5\%), Newport (14.5\%), and Camel (12.6\%), accounting for over $75 \%$ of the total market share in that age group. Among adults, the 3 top brands Marlboro (40\%), Newport (15\%) and Camel (11\%) accounted for $66 \%$ of the market share. There was more diversity in the brand purchasing with increasing age, though Marlboro was the top selling brand in all age groups. Many of the top selling brands had different brand styles. Among adult smokers of Marlboro, over 40 different brand styles were reported; 20 styles were reported for Camel, and 14 for Pall Mall. Across all age groups brand market share did not differ for daily and nondaily smokers.

Conclusions: Marlboro was the top selling brand among both adolescents and adult smokers. The number and variety of brands and brand styles increased with age. The regular purchase of value priced brands was common in all age groups, but most popular with smokers over age 55 years.

Tob. Induc. Dis. 2018;16(Suppl 1):A258

DOI:10.18332/tid/84045

\section{2:30-14:00}

PS-673-4 "It Didn't Fill the Void": reasons for e-cigarette and tobacco dual use

L Robertson', J Hoek², M-L Blank², R Richards'

${ }^{1}$ University of Otago, Preventive and Social Medicine, Dunedin, New Zealand, ${ }^{2}$ University of Otago, Marketing, Dunedin, New Zealand. E-mail: I.robertson@otago.ac.nz

Background: Although moving from smoking to electronic nicotine delivery systems (ENDS) could reduce the harms smokers face, many ENDS users continue to smoke tobacco. We explored the contributing factors to tobacco use among ENDS users.

Methods: We conducted in-depth interviews with $n=20$ past 30 day ENDS and tobacco users (16 used ENDS daily; the median duration of ENDS use was 4.5 months). Interviews explored motivations for using ENDS, experiences of using ENDS and tobacco, and dual use patterns. We used qualitative thematic analysis to analyse interview data.

Results: Most, but not all, participants used ENDS to try and quit smoking entirely. Among those who reported trying to quit, social exposure to tobacco use and alcohol consumption cued cravings and undermined a full transition to vaping. Some participants found ENDS did not sufficiently alleviate stress or replicate the nicotine hit, mouth-feel or rituals they experienced with smoking. The convenience and perceived social acceptability of using ENDS indoors also facilitated dual use. Several participants cited financial reasons as the primary motivation for using ENDS; they reported alternating between ENDS and smoking as rationing their 'real' cigarettes helped manage the cost of tobacco. Younger, social tobacco smokers reported experiencing stigma when using ENDS, which inhibited their ENDS use in some social settings where tobacco use was normative. While many participants saw dual use as a transition towards becoming smokefree, others viewed their reduced smoking levels alongside ENDS use as a successful outcome. Some perceived ENDS as healthful, not merely less harmful than tobacco; this view appeared to offset dissonance about using tobacco and positioned cigarette smoking as a reward for being "good".

Conclusions: Understanding factors that facilitate and support dual use of ENDS and smoked tobacco may help inform interventions that support ENDS users to make a full transition from tobacco smoking to vaping.

Tob. Induc. Dis. 2018;16(Suppl 1):A259

DOI: $10.18332 /$ tid/84065

\section{2:30-14:00}

PS-674-4 Comparison of smoking prevalence and initiation between physician and general people of Bangladesh

MA Al Mamun ${ }^{1}$

${ }^{1}$ National Heart Foundation Hospital \& Research Institute, Epidemiology \&t Research, Dhaka, Bangladesh. E-mail: abdullah. mmcm26@gmail.com

Background: Despite the clear evidence of harmful effect of smoking on health and the leadership role taken by physicians to curb tobacco use, studies have shown that large number physicians still smoke. Global Adults Tobacco Survey (GATS) 2009 in Bangladesh showed that more than 44\% male smoke and average age at initiation of daily smoking was 19 years. Present study conducted to compare the prevalence and initiation of smoking among the physicians and general people of Bangladesh. Methods: A cross sectional study conducted between the periods of April to December 2013 in purposely selected one teaching hospital, one district hospital and one upazila health complex from each division of Bangladesh to find out the prevalence and initiation age of smoking among physicians. Ethical permission was taken from Bangladesh Medical Research Council (BMRC). Results of this study compared with the Global Adults Tobacco Survey (GATS) Bangladesh 2009.

Results: Pre-tested questionnaire sent to 1975 physicians and 1580 physicians returned the completed questionnaire. The response rate was $80.0 \%$. Mean \pm SD of age was $33.9 \pm 8.7$ with a range of 22 to 59 years. Among them $78.0 \%$ were male and $22.0 \%$ were female. As there is no female current smoker in this study we further analyse smoking among the male physicians. Among the respondents $29.2 \%$ were ever smoker. Among them $23.9 \%$ were current smoker and $5.3 \%$ were ex smoker. Average age at initiation daily smoking was 20 years.

Conclusions: Although smoking prevalence less in physicians and they initiate smoking in later age than general people in Bangladesh appropriate measures should be taken to prevent initiation of smoking during medical education. So they can be the role model for the general people.

Tob. Induc. Dis. 2018;16(Suppl 1):A260

DOI: $10.18332 /$ tid/84079

\section{2:30-14:00}

PS-675-4 Novel tobacco products in Japan - their risks and impacts to tobacco control policy and the society

Y Mochizuki ${ }^{1}$

1Japan Cancer Society, Tokyo, Japan. E-mail: mochizuki@jcancer.jp Background and challenges to implementation: Japan was 
chosen by tobacco industry as their first invented society for novel tobacco products (heat-not-burn tobacco, hereafter HNB). Since the government set its policy goal to reduce smoking prevalence from $19.5 \%$ to $12 \%$ by 2022 , smoking prevalence was kept steady contrary to the previous downward trend of smoking rates.

Intervention or response: HNB products were first introduced by Japan Tobacco (JT) in 2015, by Philip Morris International (PMI) in 2016 and finally by British American Tobacco (BAT) in 2017 , which obtained remarkable popularity very rapidly. This paper will discuss their risks and impacts to the policy and the society in Japan according to the MPOWER strategies of WHO. Results and lessons learnt: Monitor: Government surveys on smoking prevalence do not contain specific questions for HNB and their users consider or misconsider their smoking status how to respond the surveys. PMI recently announced that they obtained potential two million users of IQOS, which counts $10 \%$ of smoking population.

Protect: Smokefree legislation is under development except three local ordinance at local levels. National legislation is not prepared but it is hard for the government to restrict HNB products under its legislation.

Offer: Many smokers switch cigarettes to HNB to quit cigarette smoking. At cessation clinics, measurement of carbon monooxide is required but as $\mathrm{HNB}$ will not generate $\mathrm{CO}$.

Warn: Tobacco companies are promoting HNB as less harmful products using tricky wording as aerozol or vapor.

Enforcement: Information on HNB is widely distributed. Their appearance on the TV shows and dramas are prominant which induce the equivalent impact as advertisement and promotion.

Raise: HNB products are categorized in Japan as pipe tobacco whose tax rate is relatively lower than cigarettes. So, tax revenue to the goverment is harmed.

Conclusions and key recommendations: Careful monitoring of HNB is needed and Japan should establish a concrete principle to judge the HNB risks to the society.

Tob. Induc. Dis. 2018;16(Suppl 1):A261

DOI: $10.18332 /$ tid $/ 84080$

\section{2:30-14:00}

PS-676-4 Marketing tobacco at the point of sale: a survey of 675 tobacco shops in Mumbai, India T Bhutia', A Mehendale', N Lad'1 ${ }^{1}$ R Kadam ${ }^{1}$

'Salaam Bombay Foundation, Tobacco Control \&t Advocacy, Mumbai, India. E-mail: tshering@salaambombay.org

Background: Tobacco product advertisements at the point of sale (POS) are an important means for the tobacco industry to market to kids. POS advertisements make tobacco products accessible and desirable to children. Evidence shows that exposure to and promotion of tobacco advertisements is positively correlated with smoking among children. However, little research has explored the POS advertisement environment in Mumbai.

Methods: A survey was conducted to document POS advertisements at 675 tobacco shops in Mumbai in 2015. Information was collected on advertisements of tobacco brands, newer tactics of advertisements without brand name and placement of board about the ban on tobacco sales to minors facing away from the consumers.

Results: The survey found that $35.8 \%(n=242)$ shops had advertisements for tobacco products. More than half of shops with ads $(52.6 \%, \mathrm{n}=355)$ had advertisements from Indian Tobacco Company (ITC) and 21\% $(n=142)$ of shops had had advertisements by Godfrey Phillips. 42.6\% $(n=288)$ of tobacco shops reported tobacco advertisements without display of brand name. Only $12.4 \%(n=84)$ of tobacco shops posted the required board about the ban on sales to minors.

Conclusions: The display of point-of-sale advertisements in Mumbai is being driven by a limited number of brands and two national tobacco companies. Without these brands and the companies behind them, the tobacco advertisement market would look substantially different. The existing tobacco control law should be amended to curb the new tactics of these big tobacco companies targeting the youth.

Tob. Induc. Dis. 2018;16(Suppl 1):A262

DOI:10.18332/tid/84103

$12: 30-14: 00$

PS-677-4 Prevalence and factors that influence smokeless tobacco use among adults in pastoralist communities of Borena zone, Ethiopia: mixed method study

E Etu ${ }^{1,2}$, D Gemeda ${ }^{3}$, M Hussen $^{4}$

${ }^{1}$ Madda Walabu University, Goba Refferal Hospital, Goba, Ethiopia, ${ }^{2}$ Makerere University School of Public Health-Centre for Tobacco Control in Africa, Public Health, Kampala, Uganda, ${ }^{3}$ Jimma Univesrity, Epidemiology, Jimma, Ethiopia, ${ }^{4}$ Jimma University, Health Education and Behavioral Science, Jimma, Ethiopia.E-mail: esetu912008000@ymail.com

Background: Deaths due to tobacco consumption are on the rise, from 5.4 million in 2005 to 6.4 million in 2015 and 8.3 million in 2030 of which more than $80 \%$ will be in developing countries. Smokeless tobacco use is a significant cause of disease. Over 300 million people use smokeless tobacco worldwide. More than 250 million adult smokeless tobacco users are in low- and middleincome countries, the total burden of smokeless tobacco use is likely to be substantial. In Ethiopia, nationally representative data on the smokeless tobacco use is not available. Most studies conducted were focused on cigarette smoking.

Methods: A community based cross-sectional study using quantitative and qualitative approaches was conducted from September 14-29, 2015. The study was conducted among adults in pastoralist communities in Borena zone, Ethiopia. A total of 634 households were selected randomly for interview. An intervieweradministered questionnaire and in-depth interview guide was used to assess adults' practice, attitude, knowledge, and perception on Smokeless Tobacco use. Logistic regression was used to assess association between dependent and independent variables.

Results: Out of 634 participants, 287 (45.3\%) of them were current users of smokeless tobacco. Being Muslim ( $\mathrm{AOR}=.21$, 95\% CI: .13, .33), being Christian ( $\mathrm{AOR}=.38,95 \% \mathrm{CI}: .22, .67)$, and having good health risk perception toward smokeless tobacco use $(\mathrm{AOR}=.49,95 \% \mathrm{CI}: .34, .70)$ were protective factors for smokeless tobacco use, whereas favorable attitude $(\mathrm{AOR}=2.12$, 95\% CI: $1.48,3.04)$ and high social pressure towards smokeless tobacco use $(\mathrm{AOR}=1.73,95 \% \mathrm{CI}: 1.21,2.47)$ were factors independently associated with smokeless tobacco us.

Conclusions: This study concludes that smokeless tobacco use is very common in the selected districts of the Borena zone. The practice is strong linked to the lifestyle of the community.

Tob. Induc. Dis. 2018;16(Suppl 1):A263

DOI: $10.18332 /$ tid/84104

12:30-14:00

PS-678-4 Perceptions of risk and use of smoked tobacco products among youth 
T Bhutia', A Mehendale', N Lad', R Kadam

'Salaam Bombay Foundation, Tobacco Control \&t Advocacy, Mumbai, India. E-mail: tshering@salaambombay.org

Background: India is experiencing an epidemic of tobacco use among its youth. Evidence suggests that smoking tobacco is likely to increase due to tobacco industry's youth centered marketing strategies. However, little is known about patterns of use and attitudes towards smoked tobacco products among children in Mumbai.

Methods: A self administered survey was conducted among 1,558 students of 7 th, 8 th, and 9 th grades in 50 randomly selected government run schools in Mumbai in 2014. The survey covered demographic information, questions about tobacco product use, perception of harms and family use of tobacco products among children. The results were analyzed using SPSS.

Results: Cigarettes $(2.5 \%, \mathrm{n}=39)$ were the most commonly reported smoked tobacco product ever used, followed by bidis $(2.1 \%, \mathrm{n}=33)$ and hookah $(0.3 \%, \mathrm{n}=5)$. Majority of students identified cigarettes $(70.3 \%, \mathrm{n}=1095)$ and bidi $(57.6 \%, \mathrm{n}=897)$ harmful to health. However, only $8 \%(n=125)$ reported hookah as a harmful product. Boys were more likely to report ever using cigarettes and bidis as compared to girls (cigarettes: $3.4 \%$ vs $1.5 \%$; bidi: $2.5 \%$ vs $1.5 \%$ ). Students who smoked cigarettes were significantly less likely to believe they were harmful to health $(\mathrm{OR}=0.3 ; 95 \% \mathrm{CI}=0.1-0.9)$. Students whose father used tobacco were more likely to report ever use of cigarettes. $(\mathrm{OR}=0.4$; $95 \% \mathrm{CI}=0.2-0.8$ ).

Conclusions: While the overall reported use of smoked tobacco products was low among the sampled students, the low levels of knowledge and lack of perceived harm associated with these products is worrying and may lead to higher smoked tobacco use in future. School-based tobacco control programs are needed to ensure students understand the risks of smoked tobacco use. Since reported use is low among students in grades 7,8 and 9 , this may be the ideal time to intervene. Given the correlation between parental and child tobacco use, involvement of parents in tobacco control programs targeting youth will prove beneficial.

Tob. Induc. Dis. 2018;16(Suppl 1):A264

DOI:10.18332/tid/84109

\section{2:30-14:00}

\section{PS-679-4 The smoking situation in Hungary}

Z Cselkó1, G Kovács'1, I Horváth ${ }^{1}$

'National Korányi Institute of Pulmonology, Budapest, Hungary. E-mail:kovac@koranyi.hu

Background: Smoking and related illnesses signify major health and economic concern in Hungary. Accurate understanding of smoking habits is essential in combating smoking.

Methods: Analysis is based on the anonymized standard European Health Interview Survey (EHIS), conducted in 2009 and in 2014. Statistical analysis explored the associations of sociodemographic variables with daily smoking. Additional data from statistically supported health surveys carried out in 2000 and 2003 were used to determine smoking trends.

Results: Smoking prevalence in Hungary decreased from the peak $34 \%$ in 2003 to $28 \%$ in 2014 , with $34 \%$ of men and $22 \%$ of women reported smoking (Figure 1). Increased risk of smoking predominantly affects the 25-34 age group among men (41\%) and the 45-54 age group among women (31\%). The level of education is more pronounced among men than among women in determining daily smoking (men: $41 \%$ vs. $15 \%$ low vs. high level of education; women: $25 \%$ vs. $12 \%$ intermediate vs. high level of education).
Employment status, and among women income status less clearly influenced the likelihood of daily smoking. The likelihood of daily smoking however is higher among men facing economic hardship ( $45 \%$ vs. $24 \%$ lowest vs. highest household income quintile).

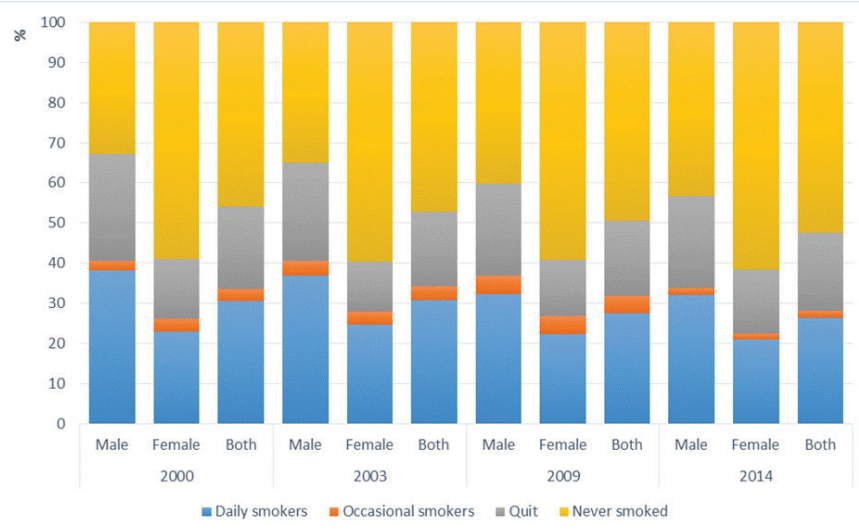

[Prevalence of smoking (\%)]

Conclusions: In recent years, Hungary introduced significant measures to curb smoking: smoking had been restricted in enclosed and in certain open public areas, the number of tobacco points of sale had been drastically reduced and the legally binding increase of excise tax has resulted rising prices. The decreasing smoking prevalence and cigarette consumption may be attributed to restrictive smoking measures. In order to further reduce rate of smoking, particular attention should be paid in the coming years to support and disseminate health promotion programs, specifically aimed at groups with increased risk of smoking, that combat habituation, as well as educate smokers about the harmful effects of smoking and cessation methods.

Tob. Induc. Dis. 2018;16(Suppl 1):A265 DOI:10.18332/tid/84120

\section{2:30-14:00}

PS-680-4 Prevalence and determinants of tobacco use among youth in Myanmar: review of findings from Global Youth Tobacco Survey 2016 Data

A Tun', T Chit Tin', ML Nwe', NNN Shein', S Kyaw Yin², PT Han ${ }^{3}$ ${ }^{1}$ Ministry of Health and Sports, National Tobacco Control Programme, Nay Pyi Taw, Myanmar, ${ }^{2}$ Ministry of Health and Sports, School Health Division, Nay Pyi Taw, Myanmar, ${ }^{3}$ Tun Khit Foundation, Tobacco Control, Yangon, Myanmar. E-mail: aungtunmm@gmail.com

Background: The study aims to reassess the health, knowledge and practice of students, (9-11 Grades) after the introduction of New National Tobacco Control program for 5 years. This study includes data on prevalence of cigarettes and other tobacco use as well as information on five determinants of tobacco use: access, exposure to secondhand smoke (SHS), cessation, media and advertising, and other indicators.

Methods: Myanmar GYTS uses a two-stage sample design with schools selected proportional to enrolment size. A total of 3633 eligible students in grades 9-11 completed the survey, of which 2621 were aged $13-15$ years

Results: Between 2011 and 2016, a reduction in the proportion of students currently use any tobacco products is observed (a fall from overall prevalence among $13-15$ year olds of $18.6 \%$ to $14 \%$ ). but currently smoked cigarettes had increased during the period from $6.8 \%$ to $8.3 \%$. 
Currently use any smokeless tobacco products had decreased (9.8\% to $5.7 \%$ ).74.5\% of current smokers tried to stop smoking in the past 12 months.33.2\% are exposed to tobacco smoke at home as well as $28.4 \%$ are exposed tobacco smoke inside any enclosed public place. $61 \%$ of current cigarette smokers bought cigarettes from a store, shop or street vendors. $42.3 \%$ of students noticed tobacco advertisements on point of sale. $65 \%$ of students thought other people's smoking is harmful to them.

Conclusions: Myanmar Youth Tobacco program should strengthen in all schools to be $100 \%$ tobacco-free and also incorporate training of school personnel on tobacco control, specifically youth-focused programs as joint efforts between Ministry of Health and Sports and Ministry of Education in collaboration with related ministries. Enforcement of the national legislation on tobacco control needs to be strengthened, and the National Tobacco Control Program needs to be more comprehensive incorporating measures to reduce smokeless tobacco use and other non-cigarette tobacco products.

Tob. Induc. Dis. 2018;16(Suppl 1):A266

DOI:10.18332/tid/84139

\section{2:30-14:00}

PS-681-4 Patterns of use of electronic cigarettes and use in public and private settings in Barcelona (Spain)

JM Martínez-Sáchez ${ }^{1,2,3}$, N Matilla-Santander ${ }^{1}, K_{\text {B }}$ Bunch ${ }^{1}$, M $\mathrm{Fu}^{2,3}, \mathrm{M}$ Ballbèㄹ, ${ }^{2,}$ C Lidón-Moyano ${ }^{1}$, JC Martín-Sánchez ${ }^{1}, \mathrm{E}$ Fernández ${ }^{2,3}$

'Universitat Internacional de Catalunya, Barcelona, Spain, Institut Català d'Oncologia, Barcelona, Spain, ${ }^{3}$ Institut d'Investigació Biomèdica de Bellvitge-IDIBELL, Barcelona, Spain.E-mail: jmmartinez@uic.es

Background: The objectives of this study are:

1) to describe the patterns of electronic cigarette (e-cigarette) use; and

2) to describe the prevalence of e-cigarette users who use in selected indoor public and workplaces, and private venues in Barcelona (Spain).

Methods: This is a cross-sectional study of adult $(>=18)$ current e-cigarette users and residents of Barcelona, Spain $(n=600)$, recruited in 2015. We conducted a face-to-face questionnaire. We calculated percentages and odds ratios (OR) with their $95 \%$ confidence intervals (CI).

Results: Of the total population, the most prevalent motivation for using e-cigarettes was to reduce tobacco smoking (48\%) and the most prevalent flavor used was tobacco (49\%). People younger than 25 years old $(\mathrm{OR}=4.36,95 \% \mathrm{CI} 1.99-9.57)$ and women $(\mathrm{OR}=1.87,95 \% \mathrm{CI} 1.02-3.43)$ were more likely to state their main motivation for use as to use where cigarettes were prohibited. Menthol and fruit flavors were more prevalent among women while fruit flavor was more frequent among the youngest age group. The highest proportion of participants who used e-cigarettes in public places was found at restaurants or bars (69.4\%) and nightclubs or pubs (55.4\%). Being current conventional tobacco smokers (dual users) was significantly associated with lower use of e-cigarettes in workplaces $(\mathrm{OR}=0.61,95 \% \mathrm{CI}: 0.41-0.91)$, restaurants $(\mathrm{OR}=0.66$, 95\%CI:0.45-0.97) and nightclubs (OR=0.56, 95\%CI:0.37- 0.86). $96.8 \%$ of e-cigarette users declared using the e-cigarette at home. Conclusions: Younger users of e-cigarettes are more likely than older individuals to use flavors other than tobacco and respond that the primary motivation for using is to be able to use where cigarettes are prohibited. Moreover, e-cigarette use in private settings and in public settings where their use is not regulated by law is high. Therefore, the public health administration should enact more expansive e-cigarette regulations.

Tob. Induc. Dis. 2018;16(Suppl 1):A267

DOI: $10.18332 /$ tid/84173

\section{$12: 45-14: 00$ \\ EP-160-4 Electronic cigarettes use and perception amongst medical students, a cross sectional survey from Sindh, Pakistan \\ N Iqbal', JA Khan' \\ ${ }^{1}$ Aga Khan University Hospital, Karachi, Pakistan. E-mail: nousheen.iqbal@aku.edu}

Background: The manufacturers of e-cigarettes are actively marketing their product by using electronic and social media. However there is scarcity of data regarding it use and perception among medical students of a developing country like Pakistan.

Methods: A Cross sectional survey was conducted from 1st July till 30th September 2016 in five medical universities of Sindh, Pakistan. A self-administered questionnaire was used, a total of 500 medical students consented to participate in the study

Results: The mean age was $21.5 \pm 1.7$ years and $58 \%$ were females. Over $65 \%$ had knowledge about e-cigarettes and majority were tobacco user $(87.6 \%, \mathrm{p}<0.001)$. The frequency of e-cigarette use was $6.2 \%$ while daily use was $1.2 \%$ and was found higher among tobacco user $13.9 \%$ and males $74 \%(\mathrm{p}<$ $0.001)$.E-Cigarettes were mostly perceived as less harmful than tobacco cigarettes(39\%), helpful in quitting smoking (27\%), associated with respiratory diseases (36\%), addictive (36\%) and not safe for pregnancy(44\%).E-cigarette users were found to be more indulged in other tobacco products including conventional cigarettes $(80.6 \%)$, shisha(83.9\%) and smokeless tobacco $(38.7 \%)$.

Conclusions: Although the use of e-cigarettes among medical students is low but most of the students who took on e-cigarettes ended up as a dual user rather than quitter.

Tob. Induc. Dis. 2018;16(Suppl 1):A268

DOI:10.18332/tid/84055

\section{$12: 45-14: 00$}

EP-161-4 Prevalence of tobacco consumption among Auto Rickshaw Drivers in Chennai City, Tamil Nadu, India

DL Francis ${ }^{1}$

${ }^{1}$ Tamil Nadu Dr MGR Medical University, Public Health, Chennai, India. E-mail: delfin_lovelina@yahoo.co.in

Background: Tobacco use is a major preventable cause of premature death and diseases. India is the second largest consumer of tobacco in the world. About $29 \%$ of adults use tobacco on a daily basis and an additional $5 \%$ use it occasionally. This study is contemplated with an aim to assess the prevalence of tobacco consumption and the associated factors involved in its consumption, as this group of the population is under constant pressure and account for the workforce of the country.

Methods: A Cross sectional descriptive study was conducted among Auto Rickshaw Drivers in Chennai City,who were working for more than two years and present on the day of examination and who were willing to participate in the study were included. Cluster random sampling technique was used. 400 samples were selected from 40 auto stands. Data was collected using a Survey Proforma which comprised of a Questionnaire which can assess the frequency of consumption, age of initiation, the amount of 
consumption, mental stress, economic factors, any past history of disease and most importantly the awareness towards oral cancer. Results: Prevalence among auto rickshaw drivers for consumption of tobacco products was very high (87\%). Auto rickshaw drivers were mostly used tobacco in the form of Gutkha (72\%) and bidi (40\%) in comparison to other products. It also shows that they use cheap tobacco products. Awareness level among auto rickshaw driver was high (70\%) but still uses tobacco products because of its addiction (66\%). In the opinion of auto rickshaw drivers increase in tax may reduce it consumption and the majority of drivers (70\%) think that tobacco must be banned.

Conclusions: Prevalence of tobacco use among auto rickshaw drivers was very high. Many were suffering from tobacco related diseases like cough, ulcer on mouth, lung disorder. They are in definite need of tobacco cessation activities.

Tob. Induc. Dis. 2018;16(Suppl 1):A269

DOI:10.18332/tid/83797

\section{$12: 45-14: 00$}

EP-162-4 Use of e-cigarettes among smokers and recent quitters: results from the ITC New Zealand Survey

R Edwards', A Waa ${ }^{1}$, J Stanley², S Kaai ${ }^{3}$, A C.K.Quah ${ }^{3}$, G Fong ${ }^{3}{ }^{4}$ ${ }^{1}$ University of Otago, Public Health, Wellington, New Zealand, 'University of Otago, Dean's Department, Wellington, New Zealand, ${ }^{3}$ University of Waterloo, Psychology, Waterloo, ON, Canada, ${ }^{4}$ Ontario Institute for Cancer Research, Toronto, ON, Canada.E-mail: richard.edwards@otago.ac.nz

Background: In New Zealand (NZ), nicotine-containing e-cigarettes (ECs) and e-liquids cannot be sold legally in shops, but can be imported via the internet. We describe patterns of EC use prior to a planned legislative change in 2018 permitting sales of nicotine-containing ECs.

Methods: We present data from CATI interviews with 820 current smokers and 252 recent quitters from Wave 1 (August 2016 to April 2017) of the ITC NZ Survey. The figures in the abstract are from preliminary analysis using unweighted data.

Results: Over a half (56\%) of participants had ever used an EC. A fifth (20\% ex-smokers, 19\% current smokers) were currently using ECs, of whom $32 \%$ were using ECs daily and $36 \%$ weekly or monthly. $17 \%$ had used a nicotine-containing EC in the last year. Of participants who had tried to quit in the last year, $29 \%$ used ECs in their latest quit attempt and $20 \%$ used a nicotine-containing EC (compared to 35\% who had used NRT and 19\% prescription cessation medications such as Zyban and Champix).

Among current EC users, common reasons for use among recent quitters included to quit smoking (86\%) and to help cut down on smoking (73\%). Similarly, current smokers (dual users) mostly reported using ECs to quit (74\%) or cut down (71\%). However, $51 \%$ of current smokers reported using ECs to replace some ordinary cigarettes so they didn't have to quit smoking.

Conclusions: Nicotine-containing EC use was surprisingly common in a setting where retail sales are prohibited. ECs were usually used to support quit attempts or cut down smoking. However, a high proportion of current smokers were using ECs as a susbtitute for cigarettes to avoid quitting. The findings suggest that ECs may contribute to achieving NZ's Smokefree 2025 goal, provided they are predominantly used as a quitting aid rather than a means to defer quitting.

Tob. Induc. Dis. 2018;16(Suppl 1):A270

DOI:10.18332/tid/84071
12:45-14:00

EP-163-4 Prevalence of e-cigarette in China: preliminary findings from two surveys

G Feng ${ }^{1}$, Y Nan ${ }^{1}$, Y Jiang ${ }^{1}$

${ }^{1}$ Chinese Center for Disease Control and Prevention, Tobacco Control Office, Beijing, China.E-mail: guozef@qq.com

Background: China is the world's largest tobacco producer and consumer, in recent years has become the world's largest producer of e-cigarette. According to media reports, China-made e-cigarette is mainly exported to foreign countries, and e-cigarette are not popular in China, but such conclusions need support from surveillance data.

Methods: Data of 2015 China Adult Tobacco Survey (CATS) and International Tobacco Control Policy Evaluation Project China Survey (ITC China Survey) were analyzed to describe rate of awareness and prevalence on e-cigarette. 2015 CATS is a nationally representative cross-sectional survey of adults aged 15 and over. ITC China survey is a cohort study designed to measure the impact of tobacco control policies on tobacco users over time. Between 2006 and 2015, five survey waves were conducted among a cohort of adult smokers and non-smokers in China.

Results: The CATS found that $40.5 \%$ of adults had heard of e-cigarette and $3.1 \%$ of adults had ever tried e-cigarette, these two rates are higher among male, urban, young and educated groups. Only $0.5 \%$ of adults reported using e-cigarette currently, but most of them are occasional users. ITC China survey revealed that the percentage of smokers who had ever heard of e-cigarette went up from $29 \%$ (wave 3,2009 ) to $60 \%$ (wave 5,2014 ). Similarly, percentage of smokers who had ever tried e-cigarette went up from $2 \%$ (wave 3,2009 ) to $11 \%$ (wave 5,2014 ). Among smokers who had tried e-cigarette at Wave 5 , only $8 \%$ reported they were using currently.

Conclusions: Although rate of current e-cigarette use is relatively low, attention should be paid to the fact that rate of awareness and ever tried use were going up among smokers. E-cigarette prevalence indicators should be included in tobacco use monitoring system, and more researches need be conducted to understand the characteristics of the population using e-cigarette.

Tob. Induc. Dis. 2018;16(Suppl 1):A271

DOI:10.18332/tid/84231

\section{$12: 45-14: 00$}

EP-164-4 Age, period and cohort trends in smoking in Mexico, 2000-2016

YK Lau', P Cao'1, J Jeon'1, R Meza ${ }^{1}$

${ }^{1}$ University of Michigan School of Public Health, Epidemiology, Ann Arbor, MI, United States of America. E-mail: yanlau@ umich.edu

Background: Mexico has one of the most substantial burden of tobacco-related illnesses in the world where smoking causes more than 60000 deaths per year in the country. Examining smoking patterns for different birth cohorts would provide valuable insight into the impact of tobacco control policies.

Methods: Using smoking data for adults (age $>=20$ ) from Encuesta Nacional de Salud (National Health Survey; 2000) and Encuesta Nacional de Salud y Nutricion (National Health and Nutrition Surveys: 2006, 2012, 2016), we evaluated smoking trends by gender through age-period-cohort models with restricted cubic splines. Specifically, temporal effects by age, period, and birth cohort (1905-1990) on the prevalence of ever and current smoking were estimated. In combination with these estimates, a methodology developed by the Cancer Intervention 
and Surveillance Modeling Network Lung group is used to reconstruct smoking histories by cohort and gender for Mexico population based on these cross-sectional surveys.

Results: As expected, levels of ever and current smoking prevalence are higher for men compared to women. The prevalence of ever smokers has been decreasing in both sexes across all birth cohorts, with a sharper rate of decrease for women. Current smoking prevalence has also been declining in both sexes across all birth cohorts. The rates of decrease for current smoking differ between men and women, however; the prevalence of current smokers in men decreased at a faster rate for cohorts born before 1960 compared to women. Moreover, current smoking has been decreasing across all ages in both men and women.

Conclusions: This is the first attempt to study smoking trends in Mexico using age-period-cohort models. The resulting estimates will help us reconstruct smoking histories, shedding light on patterns of smoking initiation and cessation in the population over time. Together, this information could inform tobacco control interventions to decrease the burden of tobacco-related illnesses in the country.

\section{Tob. Induc. Dis. 2018;16(Suppl 1):A272 DOI: $10.18332 /$ tid/84285}

\section{$12: 45-14: 00$}

EP-165-4 Increase of electronic cigarette use and awareness in Brazil: findings from a country that has strict regulatory requirements for electronic cigarette sales, import, and advertising

A Szklo ${ }^{1}$, C Perez ${ }^{2}$, T Cavalcante ${ }^{3}$, L Almeida ${ }^{4}$, L Craig $^{5}$, S Kaai ${ }^{5}$, A Quah ${ }^{5}$, G Fong ${ }^{5}$

${ }^{1}$ Brazilian National Cancer Institute, Epidemiology, Rio de Janeiro, RJ, Brazil, 2Fundaçao do Cancer, Rio de Janeiro, RJ, Brazil, ${ }^{3}$ Executive Secretariat of National Commission for Implementing WHO Framework Convention on Tobacco Control/ Brazilian National Cancer Institute, Rio de Janeiro, RJ, Brazil, 'Brazilian National Cancer Institute, Rio de Janeiro, RJ, Brazil, ${ }^{5}$ University ofWaterloo,Waterloo,ON, Canada.E-mail: aszklo@inca.gov.br Background: Because of the uncertainty about the health impact of Electronic Cigarettes (EC),Brazil established strict regulatory requirements in 2009 that prohibit the sale, import,and advertising of them until a manufacturer can demonstrate that the devices are safe and/or effective as cessation aid.This paper examined self-reported:(1) EC awareness and ever-use;(2) correlates of awareness and ever-use.

Methods: Data were analyzed from the 2012/2013 $(n=1,830)$ and 2016/2017 ( $n=1,828)$ waves of the International Tobacco Control Survey in Brazil.We calculated the differences over time in the proportion of individuals who have ever heard about ECs, and have ever tried an EC, stratified by smoking status.All estimates were adjusted for sampling weights and time in sample, taking also into consideration intraindividual correlation of data from participants observed at both waves. Moreover, a log-Binomial regression model was used to estimate the association between sociodemographic covariates and EC awareness (and ever-use).

Results: The proportion of EC awareness increased over time for both smokers (37.5\% to $71.9 \%)$ and non-smokers $(28.8 \%$ to $61.6 \%)$.The proportion of EC ever-use also increased over time for both smokers ( $7.8 \%$ to $18.3 \%)$ and non-smokers $(1.1 \%$ to $2.2 \%$ ). However, among EC users interviewed in 2016/2017 $(n=218)$, the proportion of recent/regular EC use was very low (daily use, $2.5 \%$; weekly use, $0.6 \%$, monthly use, $2.6 \%$; less than monthly, 20.7\%; not all, 73.6\%).Irrespective of the smoking status,EC awareness and ever-use were associated with younger ages, having higher income, and being bettereducated.

Conclusions: Despite strict EC regulatory requirements in Brazil (i.e.,EC are de facto banned), this was not sufficient to prevent EC awareness and consumption among Brazilians.Our findings may help in the discussion about what can happen to Brazil's strong reduction in the prevalence of ordinary cigarette smokers achieved in the last few years if the current EC regulatory regime is changed to allow smokers/non-smokers to have legal access to non-combusted sources of nicotine.

Tob. Induc. Dis. 2018;16(Suppl 1):A273

DOI: $10.18332 /$ tid $/ 83860$

\section{$12: 45-14: 00$}

EP-166-4 Use of roll-your-own tobacco among smokers in New Zealand: results from the ITC New Zealand Survey

R Edwards ${ }^{1}$, A Waa ${ }^{1}$, J Stanley ${ }^{2}$, S Kaai ${ }^{3}$, A C.K.Quah ${ }^{3}$, G Fong ${ }^{3,4}$ 'University of Otago, Public Health, Wellington, New Zealand, ${ }^{2}$ University of Otago, Dean's Department, Wellington, New Zealand, ${ }^{3}$ University of Waterloo, Psychology, Waterloo, ON, Canada, ${ }^{4}$ Ontario Institute for Cancer Research, Toronto, ON, Canada. E-mail: richard.edwards@otago.ac.nz

Background: In New Zealand (NZ), roll-your-own (RYO) cigarettes are commonly smoked. We explore patterns of use of RYO among NZ smokers.

Methods: We present data from CATI interviews with 910 current smokers from the Aug 2016-April 2017 (second cohort) of the current ITC NZ Survey. The findings are from preliminary analysis using unweighted data and they are compared with findings from the 2007/08 (first cohort) of the previous ITC NZ Survey.

Results: $32 \%$ of participants smoked RYO exclusively, and a further $29 \%$ smoked both RYO and factory-made (FM) cigarettes. The proportion (61\%) who smoked exclusively RYO or both RYO and FM increased from 2007/08 (52\%).

RYO smoking was most common among Māori (the indigenous people of NZ, 72.4\%, vs 55.5\% non-Māori) and younger smokers (71.7\% < $35 \mathrm{yrs}, 63.8 \% 35-54 \mathrm{yrs}, 46.6 \%>54 \mathrm{yrs})$. The proportion of Māori and younger smokers smoking RYO had increased since 2007/08.

Cheaper price was the most common reason (74\%) for smoking RYO. Other reasons included better taste (65\%) and RYO allowed participants to control how much they smoked (61\%). Belief that RYO cigarettes were less harmful than FM cigarettes was more common among exclusive RYO smokers (47.5\%) compared to both RYO and FM (32.4\%), and exclusive FM smokers (20.1\%). Exclusive RYO daily smokers were more likely than FM smokers to smoke their first cigarette within 15 minutes of waking, were less likely to have made a quit attempt in the last year, and were less likely to be planning to quit.

Conclusions: The increasing prevalence of RYO use, evidence of greater addiction and less quitting among exclusive RYO smokers, and greater RYO use among young and Māori smokers suggests that RYO may be undermining the achievement of a Smokefree NZ. Differential tax increases for RYO tobacco and communication strategies to correct mistaken beliefs about RYO cigarettes may be justified.

Tob. Induc. Dis. 2018;16(Suppl 1):A274

DOI:10.18332/tid/84067 
12:45-14:00

EP-167-4 E-cigarettes use among urban male tobacco smokers age 15 years or older in China

L Zhao', J Wang ${ }^{2}$

${ }^{1}$ Centers for Disease Control and Prevention (CDCF Fellow), Atlanta, GA, United States of America, ${ }^{2}$ China CDC, Beijing, China. E-mail: itz8@cdc.gov

Background: There is paucity of data regarding e-cigarette use in China. Monitoring e-cigarette use is important given the rapid increase of e-cigarette use in some countries. We investigated the prevalence and determinants of e-cigarette use among Chinese male urban tobacco smokers, as most Chinese urban residents who use e-cigarettes are male smokers ( $90 \%)$.

Methods: We analyzed data from China City Adult Tobacco Surveys, which were conducted in 14 major Chinese cities individually in $2013-14$ with 15,008 males aged $\geq 15$ years. Current e-cigarette users were defined as persons who reported using e-cigarette daily or less than daily at the time of the survey. We calculated the prevalence of e-cigarette use among male current tobacco smokers. Multivariate logistic regression was used to assess determinants of e-cigarette use, including age, education, quit attempts in the past 12 months, cigarettes smoked per day, and monthly expenditure on cigarettes.

Results: Among urban male current smokers in China, 3.6\% were current e-cigarette users. The likelihood of being current e-cigarette users was significantly higher among: smokers aged 15 $29(\mathrm{OR}=2.5)$ or $30-49(\mathrm{OR}=2.1)$ years than $50+$ years; those who attempted to quit than those who made no attempt $(\mathrm{OR}=4.5)$; those with $\geq$ college education than those with shigh school education $(\mathrm{OR}=2.6)$; and those who smoked $\geq 15$ cigarettes per day $(\mathrm{OR}=2.7)$ than those who smoked 1-14 cigarettes per day (all $\mathrm{p}<0.05$ ).

Conclusions: Among urban male tobacco smokers in China, current e-cigarette use was higher among those who were younger, had more education, attempted to quit smoking in the past year, and smoked more cigarettes per day. Given that e-cigarettes are not currently regulated and often marketed as healthier alternatives to cigarettes in China, continued monitoring of e-cigarette use is important to inform public health policy, planning, and practice.

Tob. Induc. Dis. 2018;16(Suppl 1):A275

DOI:10.18332/tid/83891

\section{$12: 45-14: 00$}

EP-168-4 Smoking prevalence among inpatients with drug resistant tuberculosis in KwaZulu-Natal, South Africa

\section{Z Shangase ${ }^{1}$, JM Tsoka-Gwegweni ${ }^{2}$, A 0kem ${ }^{3}$}

'University of KwaZulu-Natal, Durban, South Africa, ${ }^{2}$ University of KwaZulu-Natal, Public Health, Durban, South Africa, ${ }^{3}$ University of KwaZulu-Natal, Maurice Webb Race Relations Unit, Durban, South Africa. E-mail: shangasep@ukzn.ac.za

Background: Drug resistant tuberculosis (DR-TB) remains a significant cause of morbidity and mortality in South Africa. High smoking prevalence and the risk of adverse TB outcomes has been reported among TB patients in South Africa. This study investigated smoking-related prevalence and related behaviors of drug resistant tuberculosis (DR-TB) inpatients in KwaZulu-Natal (KZN), South Africa.

Methods: This study was conducted in three hospitals in KZN between September and December 2016. Eligible participants, who had DR-TB and self-reported as smokers were asked to complete a questionnaire which was administered by trained interviewers. The questionnaire, which was adapted from the global adult tobacco survey, included questions about smoking behaviour, types of cigarettes smoked and attempts at cessation. The questionnaire has been previously shown to be reliable. The study data were analysed using SPSS.

Results: The questionnaire was voluntarily completed by 211 DR-TB inpatients who self identified as smokers. Overall, 201 respondents (95.3\%) stated that they had ever smoked cigarettes, $104(49.3 \%)$ currently smoke cigarettes on a daily basis, $27(12.8 \%)$ on less than daily basis, $51(24.2 \%)$ do not currently smoke cigarettes at all. Smoking prevalence was significantly higher among males (88.6\%) than among females (11.4\%). The study revealed that $179(84.8 \%)$ respondents currently smoke manufactured cigarettes, 20 (9.5\%) reported that they also use smokeless tobacco, and 168 (79.6\%) had unsuccessfully tried to stop smoking during the past 12 months.

Conclusions: This study shows a high prevalence of smoking among DR-TB male inpatients in KZN. While most patients reported having made attempts to quit smoking, they have not been successful in their attempts. This study demonstrates that a range of actions to reduce smoking among patients with TB should be considered including restrictions on access and support for smoking cessation.

\section{Tob. Induc. Dis. 2018;16(Suppl 1):A276 \\ DOI:10.18332/tid/83790}

\section{2:45-14:00}

EP-169-4 Prevalence and patterns of use of smokeless tobacco in Mumbai schools

R Kadam', A Mehendale', D Patil ${ }^{1}$

'Salaam Bombay Foundation, Tobacco Control \&t Advocacy, Mumbai, India. E-mail: tshering@salaambombay.org

Background: Smokeless tobacco use among children is a serious problem in India. Smokeless tobacco products like gutkha, khaini, mawa and misheri and surrogate products like 'supari', 'pan masala' are commonly used by children in Mumbai. Maharashtra state government has banned the manufacture, storage, and sale of such products in the state. Salaam Bombay Foundation conducted a survey in 2014 in Mumbai schools to study the prevalence and patterns of use of tobacco products among children.

Methods: A cross-sectional survey was conducted among 1558 students of 7 th, 8 th and 9 th grades in 50 randomly selected government schools. Students were asked about smokeless tobacco use, perceptions of harm associated with these products and family use of tobacco products. The results were analyzed using SPSS.

Results: Mava 2.8\% ( $\mathrm{n}=44)$ was the most commonly reported form of smokeless tobacco ever used by students followed by mishri $2.2 \%(\mathrm{n}=34)$, gutkha $1.9 \%(\mathrm{n}=30)$ and khaini $1.2 \%$ $(\mathrm{n}=19)$. Male students were more likely to report both current and ever use of smokeless tobacco compared to female students $(\mathrm{p}=0.03) .(71.9 \%, \mathrm{n}=1120)$ students reported gutkha harmful to health. Students who used smokeless tobacco were significantly less likely to believe that smokeless tobacco are harmful $(\mathrm{p}=0.01)$. Students who used surrogate tobacco products (supari, pan masala) were more likely to report using smokeless tobacco products than their peers (supari: $\mathrm{OR}=0.2,95 \% \mathrm{CI}=0.1-0.3$ ) pan masala $(\mathrm{OR}=0.07,95 \% \mathrm{CI}=0.04-0.09)$. Students whose fathers used gutkha were more likely to report using gutkha $(\mathrm{OR}=2.8,95 \%$ $\mathrm{CI}=1.2-6.2$ ) compared to their peers.

Conclusions: Despite the ban, gutkha continues to be used by students in Mumbai schools along with other smokeless tobacco 
products like mava, mishri and khaini. Proposed efforts to enforce the ban and increase health warnings on tobacco packages in India should be supported to boost awareness of the health effects of these related products. School-based programs targeting kids should consider involving parents in campaigns.

Tob. Induc. Dis. 2018;16(Suppl 1):A277

DOI: $10.18332 /$ tid/84101

\section{$12: 45-14: 00$}

EP-170-4 E-cigarette perceptions, use frequency and susceptibility among young adults in Hong Kong

N Jiang ${ }^{1}$, C Cleland ${ }^{2}$, MP Wang ${ }^{3}$, A Kwong ${ }^{4}$, V Lai ${ }^{4}, \mathrm{TH} \mathrm{Lam}^{5}$

${ }^{1}$ New York University, Department of Population Health, New York, NY, United States of America, ${ }^{2}$ New York University, Rory Meyers College of Nursing, New York, NY, United States of America, ${ }^{3}$ The University of Hong Kong, School of Nursing, Hong Kong, Hong Kong, ${ }^{4}$ Hong Kong Council on Smoking and Health, Hong Kong, Hong Kong, ${ }^{5}$ The University of Hong Kong, School of Public Health, Hong Kong, Hong Kong. E-mail: nan. jiang@nyumc.org

Background: E-cigarettes have become increasingly popular. We examined perceptions of e-cigarettes, and their associations with e-cigarette use, frequency of use, and use susceptibility among young adults in Hong Kong.

Methods: An online survey was administered to 1,186 Hong Kong residents aged 18-35 in 2016-17. On a 0-10 visual scale, participants rated the perceived harm of e-cigarettes, perceived harm of secondhand exposure to e-cigarette aerosols, and perceived addictiveness. An overall perception score was calculated by averaging the values of non-missing perception items. Multinomial logistic regression examined the relationship of the overall perception score with former and current e-cigarette use relative to never use, controlling for age, gender, education, place of birth, current cigarette smoking, and perceived popularity of e-cigarettes among peers. Bivariate exact logistic regression assessed the association between perceptions and frequency of e-cigarette use (" $\geq 3$ days" vs. "1-2 days" in past 30 -day) among current users. Multivariate logistic regression assessed the relationship between perceptions and e-cigarette susceptibility among never users.

Results: $97.2 \%$ of participants were aware of e-cigarettes, and $16.1 \%$ had ever used e-cigarettes ( $11.3 \%$ former users; $4.8 \%$ current users). Of current e-cigarette users, $63.2 \%$ reported infrequent use. Among never users, 34.0\% were susceptible to e-cigarette use. E-cigarettes were perceived as less harmful and less addictive than cigarettes, particularly among current cigarette smokers. Low overall perception score was associated with former and current e-cigarette use, frequent use, and use susceptibility. Current cigarette smoking and perceived higher popularity of e-cigarettes were also related to e-cigarette use. Current cigarette smoking was associated with e-cigarette susceptibility.

Conclusions: Continued surveillance on e-cigarette use is needed. Interventions must address the conceptions about e-cigarettes. Health communication messages emphasizing the harmful and addictive nature of e-cigarette use and secondhand exposure to e-cigarette aerosols, and health warning labels on e-cigarette packages may help reduce e-cigarette use in young people.

Tob. Induc. Dis. 2018;16(Suppl 1):A278

DOI:10.18332/tid/84633
$12: 45-14: 00$

EP-171-4 Transition from experimentation with tobacco and nicotine products to regular smoking in Europe

F Filippidis', I Agaku², C Vardavas

'Imperial College London, Primary Care and Public Health, London, United Kingdom, ${ }^{2}$ Office on Smoking and Health, CDC, Atlanta, GA, United States of America, ${ }^{3}$ University of Crete, Clinic of Social and Family Medicine, School of Medicine, Heraklion, Greece. E-mail: f.filippidis@imperial.ac.uk

Background: Most of the tobacco-attributable deaths are from combustible tobacco products. We explored transitions from experimentation of different tobacco/nicotine products to regular use of combustible products in a European population.

Methods: We analysed a representative sample of persons aged $\geq 15$ years from 28 European Union member states (Eurobarometer wave 87.1; March 2017; n=27,901). Respondents who indicated ever trying tobacco/nicotine products, including combustible products (boxed and hand-rolled cigarettes; cigars/cigarillos; pipe; waterpipe); e-cigarettes; and smokeless products (oral/nasal/chewing tobacco), were asked which product they first tried. Questions on history of regular tobacco use ( $\geq 1$-time weekly) were further asked to determine whether ever tobacco users became established users at a point in their lifetime, regardless of their current use status. We used multilevel logistic regression to measure the association between first product tried and ever becoming an established smoker of combustible tobacco (ESCT), adjusting for age, sex, area of residence and financial difficulties We restricted analysis to those aged $<40$ years $(n=8,125)$ to reduce differential smoking-attributable mortality from different age cohorts.

Results: Overall, $58.7 \%$ of persons aged $<40$ years had ever tried a tobacco/nicotine product. Among these $79.7 \%$ first tried boxed cigarettes, $7.0 \%$ hand-rolled cigarettes, $6.2 \%$ waterpipe and $1.9 \%$ e-cigarettes. Compared to those who first tried e-cigarettes, the odds of ever becoming an ESCT were higher among those who first tried boxed (aOR=3.50; 95\%CI: 2.26-5.44) or hand-rolled cigarettes $(\mathrm{aOR}=4.70 ; 95 \% \mathrm{CI}: 2.74-8.04)$, but lower among those who first tried waterpipe ( $\mathrm{aOR}=0.30 ; 95 \% \mathrm{CI}: 0.18-0.51)$. Adjusted odds did not differ significantly among those who first tried pipe or smokeless tobacco compared to first trial with e-cigarettes.

Conclusions: About 4 in $5 \mathrm{EU}$ smokers first tried cigarettes, and these had increased likelihood of becoming established combustible tobacco smokers in their lifetime. Exploring reasons for experimenting with emerging products (e.g., e-cigarettes, hookahs) can inform public policy.

Tob. Induc. Dis. 2018;16(Suppl 1):A279

DOI:10.18332/tid/84024

\section{$12: 45-14: 00$}

EP-172-4 Dual use of tobacco and cannabis: significant change in use between Prince Edward Island (PEI) and Canadian students (2008/2009 to $2014 / 2015$ )

D Murnaghan ${ }^{1,2}$, J-A MacDonald ${ }^{2}$, M Munro-Bernard ${ }^{2}, T$ Cumming $^{3}, \mathrm{~V}$ Rynard $^{3}$

'Thompson Rivers University, School of Nursing, Kamloops, BC, Canada, ${ }^{2}$ University of Prince Edward Island, Faculty of Nursing, Charlottetown, PE, Canada, ${ }^{3}$ University of Waterloo, Waterloo, ON, Canada. E-mail: dmurnaghan@tru.ca

Background: Between 2012/2013 and 2014/2015, a significant increase in tobacco and cannabis use occurred among PEI students that did not align with patterns of use in other Canadian provinces. 
We have not identified why these rates are rising in PEI; however, there are growing concerns about health risks resulting from dual use, given the strong and consistent association between tobacco and cannabis.

Methods: From 2008/2009 to 2014/2015, the Canadian Student Tobacco Alcohol and Drugs Survey (CSTADS) (formerly the Youth Smoking Survey) collected biennial student substance use data across Canada. In 2014/2015, CSTADS data were collected from 42094 students in 336 Canadian schools (incl. $2256 \mathrm{PEI}$ students in 53 schools).

Results: PEI student tobacco use has risen significantly from 2012/2013 for three indicators (past 30 day use of menthol cigarettes [3.0 to 4.3\%]; little cigars or cigarillos [ 4.3 to $5.4 \%$ ]; and any tobacco product [13.8 to $16.1 \%]$ ). In 2014/2015, PEI student tobacco use rates (incl. current smoking, ever tried, and past 30 day use of any tobacco product) were all significantly higher than national rates. In addition, $44.5 \%$ of Canadian students (15+) reported ever using cannabis (incl. $>90 \%$ of current cigarette smokers). Provincially, prevalence of cannabis use (grades 7-12) varied significantly (PEI - $24.8 \%$ vs. Canada - $16.5 \%$ ). Access to cannabis was perceived 'easier' by students who reported smoking cigarettes.

Conclusions: This divergent pattern of tobacco and cannabis use in PEI raises alarms. Is there a possible "gateway effect," where use of one substance is increasing the likelihood of another, or, is the change the result of provincial policies/programs being focused on other student risk behaviours? A national call to action on dual use of tobacco and cannabis is needed, which sets clear targets for reducing use, documents the impacts of dual use on health, and creates policies to protect against harm.

Tob. Induc. Dis. 2018;16(Suppl 1):A280

DOI:10.18332/tid/84141

\section{2:45-14:00}

EP-173-4 Portrayal of orthodox and unorthodox uses of electronic cigarettes in YouTube videos

M Guy ${ }^{1,2}$, J Helt ${ }^{1,2}$, S Palafox ${ }^{3}$, E Soule ${ }^{2,4}$, S Maloney ${ }^{2,4}, \mathrm{~T}$ Eissenberg ${ }^{2,4}, \mathrm{P}$ Fagan ${ }^{2,5}$

'Virginia Commonwealth University, African American Studies, Richmond, VA, United States of America, ${ }^{2}$ Virginia Commonwealth University, Center for the Study of Tobacco Products, Richmond VA, United States of America, ${ }^{3}$ University of Hawaii, Honolulu, $\mathrm{HI}$, United States of America, ${ }^{4}$ Virginia Commonwealth University, Department of Psychology, Richmond, VA, United States of America, ${ }^{5}$ University of Arkansas for Medical Sciences, Health Behavior and Health Education, Little Rock, AR, United States of America.E-mail:pfagan@uams.edu

Background: Open electronic cigarette (e-cigarette) systems allow for "unorthodox" behaviors, that is, the use of the product not as intended by the manufacturer. Surveys provide valuable information on e-cigarette use prevalence, but limited information on the diverse ways in which e-cigarettes are used. This study used YouTube.com, the second most popular social media site in the world, to assess the popularity, prevalence, and characteristics of orthodox and unorthodox e-cigarette use.

Methods: Staff used search terms (e-cigarettes, vaping, and e-juice) to conduct monthly searches of YouTube videos by relevance and view count between June 30 and November 30, 2016. A customized scraper was used to collect static and dynamic video characteristics. After eliminating duplicates and videos that did not meet the inclusion criteria, 150 videos were viewed and coded independently by two staff. Staff conducted a content analysis to identify the category of e-cigarette behavior (orthodox versus unorthodox) and type of unorthodox use and document the popularity of videos (likes/dislikes). Descriptive statistics were used to calculate means and frequencies.

Results: Seventy-five percent of all videos showed unorthodox use of the e-cigarette device, liquid or both, with an average of $1,0441,005$ view counts compared to 838,966 for orthodox use. Staff identified 9 unique unorthodox behaviors and the most common observed were recreational use (75\%) (e.g., cloud chasing), modification of mechanical parts (57\%) (e.g., rebuilding), and substance use application (44.6\%) (e.g., dripping). Data on popularity showed that there was an average of 14,692 likes and 2,241 dislikes for videos coded as unorthodox compared to 4,549 likes and 736 dislikes for videos coded as orthodox.

Conclusions: The higher prevalence of unorthodox compared to orthodox e-cigarette use in videos suggests a need to further investigate the influence of YouTube videos on the uptake of these behaviors and harm associated with each type of unorthodox use.

Tob. Induc. Dis. 2018;16(Suppl 1):A281

DOI:10.18332/tid/84619

\subsection{Burden and Disparities in tobacco related diseases: Non-communicable diseases (NCDs) and communicable diseases (TB and IIIV/AIDS)}

\section{$16: 00-17: 30$}

SUS Correlates of tobacco use and disease burden - an analysis of the World Health Organisation South East Asian Countries

J Purushothama ${ }^{1,2}$, S Kumar ${ }^{3}$, V Rao U S ${ }^{4}$, M Dmello' ${ }^{1}$, P Rent ${ }^{3}$, Nitte Tobacco Control Group

${ }^{1}$ Nitte University, Public Health, Mangaluru, India, ${ }^{2}$ District Anti Tobacco Cell, Health \& Family Welfare, Mangalore, India, ${ }^{3}$ Nitte University, Public Health, Mangalore, India, ${ }^{4}$ High Power Committee, Health \& Family Welfare, Bangaluru, India. E-mail: jagannath.mph@gmail.com

Background: World Health Organisation's South-East Asia Region is home to about one fourth of the world's population. Of this, there are about 246 million smokers and 290 million smokeless tobacco users. In addition to the human toll, the effects of tobacco are felt significantly in the economy of a country. The World Bank has declared that, when effects of poverty due to loss of income, spending on tobacco and costs of illness are taken into account, tobacco use is a net loss to an economy.

Methods:

- Objective of the study: To determine the correlation of tobacco use with tobacco related disease burden and developmental indicators of South East Asian countries.

- Study method: Secondary data-based Cross-Sectional Study

- Data sources: Data on tobacco prevalence, cancer prevalence, human development index, tobacco economics and poverty indicators during the year 2014-2015 were collected from various sources such as WHO Report on Global Tobacco Epidemic 2015, World Bank data, Tobacco Atlas and World Life Expectancy 2014 reports.

- Data analysis: Data were analyzed using Pearsonn's Correlation Coefficient on SPSS version 16. Results were considered significant at $95 \%$ level of significance.

Results: The Study represented data of 11 Countries of the WHO South East Asia Region. Current smoking prevalence ranged between $2.80 \%$ and $39.80 \%$ with a mean prevalence of $20.7 \%$ 
( \pm 9.24). Both prevalence of smoking and number of cigarettes smoked per year per ca-pita in the 11 countries had a positive moderate correlation with the number of deaths per 100000 population due to lung cancer $(r=0.62 \& 0.76 ; \mathrm{P}=0.04 \& 0.01)$. Oral Cancer increased with prevalence of smokeless tobacco use $(\mathrm{r}=0.07 ; \mathrm{P}=0.07)$.

Conclusions: The study findings suggest that tobacco prevalence, tobacco related deaths and tobacco economics can be correlated with the health profile of countries in the region. These countries show positive correlation between tobacco use and related diseases.

Tob. Induc. Dis. 2018;16(Suppl 1):A282

DOI:10.18332/tid/84228

[Correlates between tobacco use and tobacco burden]

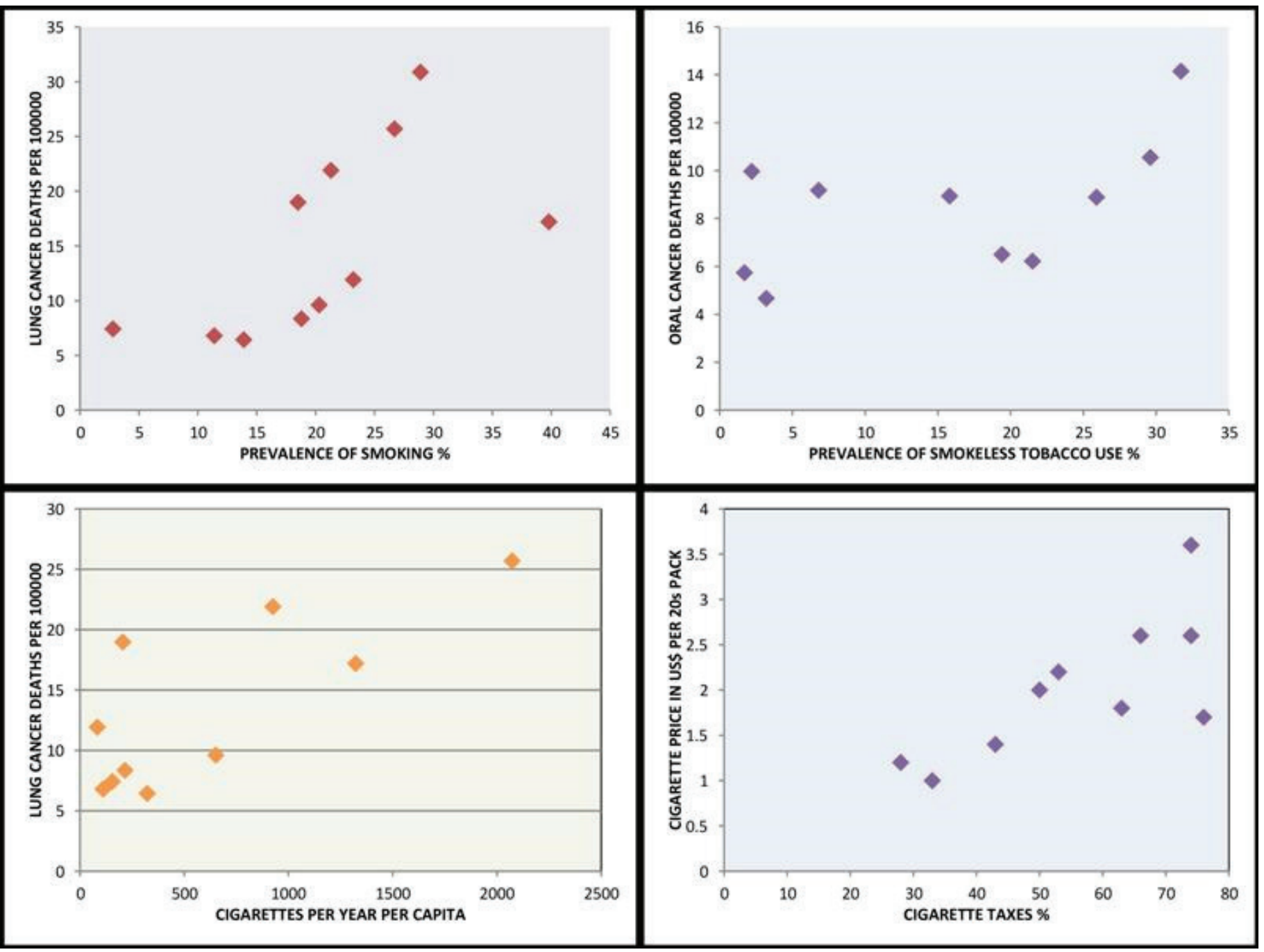

\section{6:00-17:30}

SUS Effect of elevated fasting serum glucose after smoking cessation on cardiovascular disease and mortality: an eight-year longitudinal study

S Choi ${ }^{1}$, K Kim ${ }^{1}$, J Chang ${ }^{2}$, SM Kim¹ ${ }^{1}$ MH Cho ${ }^{2}, \mathrm{~K} \mathrm{Lee}^{2,3}$, SM Park ${ }^{1,2}$

'Seoul National University College of Medicine, Department of Biomedical Sciences, Seoul, Korea, Republic of, ${ }^{2}$ Seoul National University, Seoul, Korea, Republic of, ${ }^{3}$ Seoul National University Bundang Hospital, Seongnam, Korea, Republic of. E-mail:seulggie@gmail.com

Background: While smoking cessation reduces the risk of cardiovascular disease (CVD), quitting also increases fasting serum glucose (FSG) levels. The effect of post-cessation FSG elevation on cardiovascular disease is unknown.

Methods: The study population consisted of 128,208 men without type 2 diabetes from the Korean National Health Insurance System - Health Screening Cohort database. Change in smoking habits and FSG was determined by the difference in smoking status and FSG levels from the first (2002 and 2003) and second (2004 and 2005) health examinations. Quitters were divided into those with FSG elevation of greater than $10.0 \mathrm{mg} / \mathrm{dL}$ and those without FSG elevation. The study participants were followed-up for cardiovascular disease and CVD-related death from 2006 to 2013.

Results: Compared to continual smokers, quitters without FSG elevation had decreased risk of cardiovascular disease (hazard ratio, $\mathrm{HR}, 0.77,95 \%$ confidence interval, $\mathrm{CI}, 0.69-0.86$ ), while quitters with FSG elevation did not (HR 0.86, 95\% CI 0.74-1.01). Similarly, quitters without FSG elevation had decreased risk of CVD-related death (HR 0.71, 95\% CI 0.51-0.99) while quitters with FSG elevation did not (HR 0.86, $95 \%$ CI 0.57-1.29). Both quitters with and without FSG elevation had decreased risk of myocardial infarction, while only quitters without FSG elevation had decreased risk of total stroke.

Conclusions: Post-cessation FSG elevation attenuated the beneficiary risk-reducing effects of quitting on cardiovascular disease and CVD-related mortality. Extra care should be given to controlling FSG levels among quitters in order to reduce the risk of cardiovascular disease and CVD-related death.

Tob. Induc. Dis. 2018;16(Suppl 1):A283

DOI: $10.18332 /$ tid/84310 
16:00-17:30

SUS Association between alpha1-antitrypsin (a1AT) levels, lipid profile and ventilatory pulmonary function in predicting the cardiovascular risk in simptomatic smokers

L Puiu', 0 Fira-Mladinescu², D Alexandrescu³, A Petrovan ${ }^{4}$, A Trofor $^{5}$

${ }^{1}$ Hospital of Pneumophthisiology, Pneumology, Baia Mare, Romania, ${ }^{2}$ Victor Babes' University of Medicine and Pharmacy, Pneumology, Timisoara, Romania, ${ }^{3}$ Pneumophthisiology Hospital, Pneumology, Brasov, Romania, ${ }^{4}$ University Hospital for Infectious Diseases, Infectious Diseases, Cluj-Napoca, Romania, ${ }^{5}$ Gr. T. Popa' University of Medicine and Pharmacy, Pneumology, lasi, Romania. E-mail: ligiapuiu676@yahoo.com

Background: Cigarette smoke oxidants can cause oxidative inactivation of methionine residues of a1-AT, leading to lipid binding, with dyslipidemia, inflammation and endothelial dysfunction, the early stage of atherosclerosis. The aim of the study was to assess the relationship between serum a1-AT levels, pulmonary function and lipids in predicting the risk of cardiovascular disease among symptomatic smokers adult subjects in an Out-patient Pulmonology Health Unit.

Methods: 100 respiratory symptomatic adults mean aged $53.34 \pm 9.69$ year-old, were grouped in smokers (current and former heavy smokers) $(n=64)$ and nonsmokers $(n=36)$. Demographic, anthropometric, pack-years smoking, serum a1-AT, plasma lipid profile $\{$ (total cholesterol-TC, low density lipoprotein cholesterolLDLc, high density lipoprotein cholesterol-HDLc, triglyceridesTG, nonhigh density lipoprotein cholesterol-nonHDLc, very low density lipoprotein cholesterol-VLDL), by which were calculated lipid indices\}, were performed. All subjects underwent spirometry according to the international recommendations. Lung function was expressed as forced vital capacity (FVC\% predicted), forced expiratory volume in one second (FEV1\% predicted), Forced Expiratory volume in 1 second/Forced Vital Capacity ratio (FEV1/ FVC\% predicted). Cardiovascular disease risk was evaluated through Framingham Risk Score (FRS). Statistical analysis included Spearman correlations tests and one-way ANOVA test. Results: $a 1-A T(p=0.008)$, FEV1 $(p=0.008)$, FVC $(p=0.03)$ was significantly lower, while LDLc/HDLc ratio $(p=0.01)$, Atherogenic Index of Plasma (AIP) $(p=0.04)$, FRS $(p=0.003)$ increased in smokers versus nonsmokers. In smokers, statistically significant correlationswerefound betweenal-AT andFVC $(\mathrm{r}=-0.26 ; \mathrm{p}=0.03)$; FEV1/FVC ratio $(\mathrm{r}=0.30 ; \mathrm{p}=0.01) ; \mathrm{TC}(\mathrm{r}=0.35 ; \mathrm{p}=0.003) ; \mathrm{LDLc}$ $(\mathrm{r}=0.27 ; \mathrm{p}=0.02)$; nonHDLc $(\mathrm{r}=0.26 ; \mathrm{p}=0.03)$.

Conclusions: a1-AT interacts with lipid oxidation pathways, decreasing in smokers. A better understanding of the relationships between smoking, oxidative modifications of al-AT, lung function and, lipid profile can better predict the risk of heart disease. Smoking is a modifiable health risk factor and tobacco cessation may prevent the occurence of cardiovascular diseases.

Tob. Induc. Dis. 2018;16(Suppl 1):A284

DOI:10.18332/tid/83869

\section{6:00-17:30}

SUS Tobacco use among urban residents with diagnosed major Non-communicable diseases in Bangladesh

MA Rahman 1,2, MA AI-Mamun², MR Bhuiyann ${ }^{1,2}$, F-T-N Malik ${ }^{3}$, SR Choudhury ${ }^{2}$

${ }^{1}$ Campaign for Tobacco-Free Kids (CTFK), International Advocacy, Washington, WA, United States of America, ${ }^{2}$ National Heart
Foundation Hospital \& Research Institute, Epidemiology and Research, Dhaka, Bangladesh, ${ }^{3}$ National Heart Foundation Hospital \& Research Institute, Cardiology, Dhaka, Bangladesh. E-mail:armasud80@yahoo.com

Background: Tobacco use is an important risk factor for noncommunicable diseases (NCD) and $43.3 \%$ adult use tobacco on regular basis in Bangladesh. Many patients continue to use tobacco even after diagnosis of NCDs indicating lack of awareness and appropriate cessation support. Present study was done to investigate the pattern of tobacco use among NCD patients in an urban population in Dhaka city, Bangladesh.

Methods: A cross sectional study was done in a middle class urban population of Dhaka city in 2013. Household survey was done to collect information on demography, lifestyle, personal habits, and disease history. Information on tobacco use of all adult residents aged 25 years or more were collected from household head by a structured questionnaire. Household members having history of ischemic heart disease (IHD), stroke, hypertension and diabetes were identified and corroborated by presence of physician's record.

Results: In total information of 43,727 individuals was collected and data of 23,731(54.3\%) respondents aged 25 and above were analyzed for the present study. Among the respondent's overall tobacco use were $29.6 \%$ (smoking $19.1 \%$, smokeless tobacco $12.3 \%)$. Smoking and smokeless tobacco user among male were $36.9 \%, 8.5 \%$ and among female were $0.3 \%, 16.4 \%$ respectively. Among the respondents $29.7 \%$ were diagnosed case of one of the major NCDs (21.9\% were hypertensive, $12.9 \%$ were diabetic, $6.4 \%$ had IHD and $2.7 \%$ had history of stroke). Among NCD patients $35.5 \%$ continued to use tobacco even after diagnosis. $35.3 \%$ hypertensive patients, $32.1 \%$ diabetic patients, $36.3 \%$ IHD patients and $34.5 \%$ stroke patients were tobacco user respectively. Conclusions: Tobacco use was highly prevalent in urban male population. Large number of hypertensive, diabetic, IHD and stroke patients of urban population continue to use tobacco. Measures should be taken for control of tobacco use at population level as well as NCD patients should be intervened effectively for tobacco cessation.

Tob. Induc. Dis. 2018;16(Suppl 1):A285

DOI: $10.18332 /$ tid $/ 83882$

\section{2:30-14:00}

PS-682-4 Tobacco related morbidity among patients hospitalized at Kenyatta National Referral Hospitals Kenya

G Gathecha', D Kiptui', A Kendagor', J Kibachio', S Amwayi ${ }^{2}$ ${ }^{1}$ Ministry of Health, Division of Non Communicable Diseases, Nairobi, Kenya, ${ }^{2}$ Ministry of Health, Nairobi, Kenya. E-mail: gladwellgathecah@gmail.com

Background: Tobacco use and exposure has been demonstrated to affect nearly all organs of the body. The Kenya Global Adult Tobacco Survey estimates that fifteen percent of men aged 15 and above and less than one percent of women currently smoke tobacco. The aim of the study was to determine the proportion of tobacco related illnesses (TRI) and pattern of tobacco consumption among the patients hospitalized at Kenyatta National Hospital.

Methods: A cross sectional study was conducted between January-June 2014 among adult patients. A TRI included; cancers (Oral, Pharyngeal, Oseophegeal, Colon, Rectal, Laryngeal, and lung and cervical), myocardial infarction, stroke, and chronic obstructive pulmonary disease COPD. Purposive sampling was used. An interviewer administered pre-tested questionnaire was 
used to collect data.

Results: Out of the 519 patients with TRI 442 (85\%) were interviewed. Majority of the respondents were female $(50.7 \%)$. Thirteen percent of the respondents were current smokers while $26 \%$ reported to be former smokers. Majority of the patients (76.5\%) were diagnosed with cancer, $14.9 \%$ had stroke, $5.4 \%$ had COPD and 3.2\% had Myocardial Infarction. Nearly half of all COPD patients had never smoked. Among the cancer cases, $12.4 \%$ and $27.4 \%$ were current and former tobacco smokers respectively. Thirty two percent of patients with a diagnosis of stroke were either current or former smokers. More than half of patients with MI had a history of smoking. Average number of cigarettes smoked per day was 9.1 .

Conclusions: Tobacco use and exposure remains a significant contributor to ill health in the Country. Magnitude of TRI provides important information on the need to institute structured initiatives such as cessation services and public health education to reduce this avoidable burden.

Tob. Induc. Dis. 2018;16(Suppl 1):A286

DOI:10.18332/tid/83815

\section{2:30-14:00}

PS-683-4 Reported health effects of passive smoking by bar workers in Ibadan, Nigeria M Onigbogi ${ }^{1}$, O Onigbogi ${ }^{2}$, Tobacco Cessation Study Group ${ }^{1}$ Initiative for Better Public Health, Community Health, Lagos, Nigeria, ${ }^{2}$ College of Medicine, University of Lagos, Community Health, Lagos, Nigeria.E-mail: dupefatokun@yahoo.com

Background: Workplace exposure as occurs in indoor bars and restaurants in Nigeria are the major areas where workers get exposed to such. This survey was conducted to compare the immediate reported health problems between a selected group of indoor bar workers and outdoor bar workers and evaluate their perceptions of about the dangers of passive tobacco smoke.

Methods: We selected 42 clubs in Ibadan using simple random sampling and recruited 195 bar workers. Interviewer-administered questionnaires were used for the study. The questionnaires had sections on knowledge about health effects of passive tobacco smoke and health problems of respondents as well as their attitude towards passive smoking. There was also a self-reported component of general health feeling, common ailments and the number of sick days off work.

Results: The median duration of exposure to passive smoking was 2.5 months (range 1-13 months) in the indoor group. Seventy-nine respondents in the indoor group (83\%) reported a poor health feeling as against 15 (15\%) in the outdoor group. Cough and nasal irritation were reported in $10(11 \%)$ of the workers in the indoor areas as against $8(8 \%)$ in the outdoor areas $(P=0.042)$. Skin irritation were reported in 7 (7.4\%) of those in the indoor areas as against $7(7.0 \%)$ in the outdoor areas $(P=0.11)$, while acute eye irritation and watery discharges were reported in 14 (15\%) of workers in the indoor areas as against 7 (7\%) in the outdoor areas $(\mathrm{P}=0.015)$, and nausea in $8(8.5 \%)$ and $2(2 \%)$ in indoor workers and outdoor workers respectively $(\mathrm{P}=0.03)$.

Conclusions: The reported health feeling and common ailments among this group of workers point to the fact that indoor exposure to passive smoking may have impacted on the health of the workers. Future studies could measure particulate matter and respiratory function.

Tob. Induc. Dis. 2018;16(Suppl 1):A287

DOI:10.18332/tid/83816
12:30-14:00

PS-684-4 Physical and psychological health problems associated with tobacco consumption among professional drivers in India

RK Soni ${ }^{1}$, N Kaur ${ }^{2}$, S Kaur ${ }^{2}$

'Dayanand Medical College and Hospital, Department of Community Medicine, Ludhiana, India, '2Dayanand Medical College and Hospital, Ludhiana, India.E-mail: rksonidmc@ yahoo.com

Background: Tobacco use is a global health issue and a leading preventable cause of morbidity and mortality. More than 25 diseases are known or strongly suspected because of tobacco use. Individuals with lower level of education and low socio economic status are found to have higher consumption of tobacco as compared to other job titles. Therefore the present study was planned to assess tobacco related physical and psychological health problems of professional drivers.

Methods: A descriptive research design was used to collect the data. A total of 521 professional drivers were contacted in Ludhiana district of India, of which 331 agreed to participate. Besides socio demographic profile, the data pertaining to duration of driving, history of illness, age of starting tobacco, duration of tobacco regularly used, form of tobacco, tobacco dependence, etc. were collected. The information was collected on structured questionnaire and Kessler scale was used to assess the psychological distress. The data was statistically analyzed by using SPSS 20.0 version.

Results: Out of 331 professional drivers,201( $60.7 \%$ ) were regularly consuming tobacco. $41.5 \%$ were in the age of $31-40$ years; $51.5 \%$ educated upto secondary level; $81.5 \%$ married and $60.5 \%$ residing in urban area. Majority (79.8\%) of subjects who consume tobacco reported various types of physical health problems- $25.8 \%$ had respiratory; $33.7 \%$ cardiovascular; $23.0 \%$ gastrointestinal and $8.5 \%$ urinary problems. Only $0.66 \%$ reported lung cancer. $36.5 \%$ had medium while $63.5 \%$ low psychological distress. There was significant positive correlation $(p=0.001)$ between level of dependence and psychological distress. Almost half (49.5\%) of the drivers wanted to quit while the other half refused to quit due to various reasons like taking tobacco is not harmful (27.5\%); impossible to quit (16\%) and to remain active (6.5\%).

Conclusions: Professional drivers consuming tobacco are found to suffer from various physical and psychological health problems. Their health need to be assessed regularly for improving their quality of life.

Tob. Induc. Dis. 2018;16(Suppl 1):A288

DOI:10.18332/tid/83818

12:30-14:00

PS-685-4 Risk of cancers associated with tobacco use in India: a systematic review and meta-analysis JB Prasad ${ }^{1}$, M Dhar $^{1}$

${ }^{1}$ International Institute for Population Sciences, Population Studies, Mumbai, India. E-mail: jbiips12@gmail.com

Background: Several studies on cancers associated with tobacco suggested that smokeless and smoking tobacco increases the risk of oral, lung, oropharynx, esophagus, larynx etc. No systematic review has been reported for evidence of consistent studies in India. Therefore, this study undertook a meta-analysis to quantify the overall risk of different cancer sites associated with various forms of tobacco use to investigate the risk variation in each site by different forms of tobacco in a systematic manner. 723

Methods: Analyses were carried out on 22 published studies 
with reported sample of cases and control among exposed and non-exposed with forms of tobacco. The pooled odds ratios for each cancer by forms of tobacco were calculated using random effects model. 724

Results: A significant association was found for oropharynx $(\mathrm{OR}=5.26 ; 95 \% \mathrm{CI}: 2.28-12.14)$, hypopharynx $(\mathrm{OR}=3.36 ; 95 \%$ CI: 1.95-5.79), esophagus (OR=2.67; 95\% CI: 2.06-3.47), larynx $(\mathrm{OR}=5.47 ; 95 \% \mathrm{CI}: 4.01-7.46)$, lung (OR=5.07; 95\% CI: 2.40 $10.71)$ and oral $(\mathrm{OR}=1.95 ; 95 \% \mathrm{CI}: 1.51-2.53)$ cancers among smoker, while among chewer, esophagus $(\mathrm{OR}=3.46 ; 95 \% \mathrm{CI}$ : 2.83-4.22) and oral (OR=6.59; 95\% CI: 5.18-8.39)cancer. Among bidi smoker, esophagus (OR=3.63; 95\% CI: 2.41-5.45), lung $(\mathrm{OR}=5.92 ; 95 \% \mathrm{CI}: 2.67-13.10)$ and oral $(\mathrm{OR}=2.85 ; 95 \% \mathrm{CI}$ : 1.52-5.36) cancer were significant, whereas lung $(\mathrm{OR}=2.15 ; 95 \%$ CI: $1.22-3.78)$ cancer was significantly associated with cigarettes smoking. There was considerable heterogeneity in the pooled odd ratios among all the cancer sites associated with forms of smoking and chewing tobacco. 725

Conclusions: This study clearly indicates that smoking tobacco increases the risk of oropharynx, esophagus, hypopharynx, oral, larynx and lung cancer while chewing tobacco increases the risk of oral and esophagus cancer. The detailed information on quantum of associated additional risk may be incorporated into tobacco prevention and termination efforts particularly among widely prevalent regions in India. 726

Tob. Induc. Dis. 2018;16(Suppl 1):A289

DOI:10.18332/tid/83906

\section{2:30-14:00}

PS-686-4 Projections of burden of tobacco related cancers: a new approach for measuring incidence cases for India and its states - Till 2025

JB Prasad ${ }^{1}$, M Dhar ${ }^{1}$

${ }^{1}$ International Institute for Population Sciences (IIPS), Population Studies, Mumbai, India. E-mail: jbiips12@gmail.com

Background: Cancer has emerged as an important public health problem in India also as a result of control of infectious diseases and resultant increase in life expectancy during second half of last century. Tobacco use is a measure contributor to the cancer burden which is preventable. It accounts for around 24 to $64 \%$ of cancers in males and 7 to $42 \%$ in females. Therefore, the objective of present study was to assess the burden of tobacco related cancers (TRCs) for India and its states for 2015-2025.

Methods: National Cancer Registry Programme (NCRP) of ICMR is the only source of reliable data on cancer in India. The cancer incidence rates generated by population based cancer registries under NCRP and population of India and states projected by Registrar General of India formed the sources of data. Best possible assessment of incidence rates for states using limited data available was worked out. The linear regression method was used to assess trend and project the rates for the study period 2015-2025.

Results: Overall burden of TRCs in India was estimated to be 366 thousand in 2015 and it was projected to increase to 508 thousand by 2025 , an increase of more than $38.9 \%$. Major portion of this burden was due to tobacco use in men (three fourth) and in rural males (one half). Detailed analysis indicated regional diversity in the burden of different types of TRCs.

Conclusions: In view of increasing burden of TRCs, there is urgent need to initiate focused tobacco prevention measures to combat the same.

Tob. Induc. Dis. 2018;16(Suppl 1):A290

DOI:10.18332/tid/83907

\section{2:30-14:00}

PS-687-4 Prevalence and correlates of dry nasal snuff use among HIV-infected adult women in South Africa

JL Elf1', L Lebina ${ }^{2}$, K Motlhaoleng ${ }^{2}$, N Gupte ${ }^{1}$, R Niaura $^{3}$, EVariava ${ }^{4}$, JE Golub ${ }^{1}$, N Martinson ${ }^{2}$

'Johns Hopkins University School of Medicine, Baltimore, MD, United States of America, ${ }^{2}$ University of Witwatersrand, Perinatal HIV Research Unit, SAMRC Soweto Matlosana Collaborating Centre for HIV/AIDS and TB, Soweto, South Africa, ${ }^{3}$ Schroeder Institute at Truth Initiative, Washington, DC, United States of America, ${ }^{4}$ Klerksdorp Tshepong Hospital Complex, Department of Internal Medicine, Department of Health North West Province, Klerksdorp, South Africa. E-mail: jelf1@jhu.edu

Background: Dry nasal snuff is a fine powdered smokeless tobacco product inhaled through the nose. People living with HIV (PLWH) in South Africa have a higher prevalence of smoking than their sero-negative peers, however little is known about the prevalence of snuff use or its health effects.

Methods: A cross-sectional survey was conducted among randomly selected adults with HIV infection attending 3 HIV treatment clinics in Klerksdorp, South Africa from April 2012 to August 2013. A questionnaire was administered to participants to assess snuff use and current smoking status, and urine for cotinine analysis was collected from each participant.

Results: We enrolled 1,130 HIV-infected adults, of whom 705 (62\%) were women. At the time of interview, 304 (43\%) women and 11 (3\%) men self-reported snuff use. Only 10 (3\%) women who used snuff also smoked cigarettes, thus we limited our analysis to the $n$ = 606 non-smoking women. Nearly all participants were receiving ART, and their median (IQR) CD4 count and BMI were 390 cells/ $\mathrm{mL}$ ( 236 - 564 cells $/ \mathrm{mL})$ and $25 \mathrm{~kg} / \mathrm{m} 2$ (21 - $30 \mathrm{~kg} / \mathrm{m} 2)$, respectively. Adjusted analysis showed odds of snuff use were lower among women completing grade 12 or higher (OR $0.3 ; 95 \% \mathrm{CI}: 0.2,0.6$ ). Odds of snuff use were higher among women who had ever had tuberculosis (TB) (OR 1.4; 95\% CI 1.0 - 2.1). A subset of participants was asked about alcohol use, which was positively associated with snuff use (OR 2.8; 95\% CI: 1.3 - 6.0). In the model including alcohol, association with TB lost statistical significance.

Conclusions: An extremely high proportion of women with HIV use snuff. Snuff prevention and cessation strategies are needed and should not be overlooked by tobacco control efforts, especially for women, in this population. Drivers of snuff use and the health consequences, particularly regarding TB, need further elucidation.

Tob. Induc. Dis. 2018;16(Suppl 1):A291

DOI:10.18332/tid/83940

\section{2:30-14:00}

PS-689-4 Physical activity level and its relationship with tobacco use among youth: a cross-sectional survey in North India

D Monga', S Goel', SK Padhy²

'PGIMER, School of Public Health, Chandigarh, India, ${ }^{2}$ PGIMER, Department of Psychiatry, Chandigarh, India. E-mail: divmonga3@gmail.com

Background: Tobacco use and physical inactivity are major risk factors for NCDs. Adequate physical activities keep one healthy but sedentary life style may contribute to other unhealthy practices. There is a need to identify if and how the two risk factors are interrelated so as to address them more strategically. Thus, the present study was undertaken to assess the level of physical activity and to identify its association with tobacco use 
among college going youth in Chandigarh.

Methods: Data on physical activity and tobacco use was collected from 500 students aged 18-24 years studying in different professional and nonprofessional colleges of Chandigarh during June- July 2015, through multistage simple random sampling using a self-administered questionnaire.

Results: The study findings indicated $12.8 \%(\mathrm{n}=64)$ were inactive, $10 \%(n=50)$ were active and $76.2 \%(n=381)$ were highly active out of the 500 individuals. Females (15.8\%) were more inactive as compared to males (12.1\%). Highly active respondents were seen more in $18-20$ years age group $(77.6 \%)$ as compared to 20 22 years age group (76.1\%). In regard to relationship between physical activity and tobacco use status of the respondents in study population, it was observed that odds of consuming tobacco is significantly 0.48 times lower in active respondents as compared to inactive respondents ( $\mathrm{p}$ value $<0.05$ ).

Conclusions: NCDs are emerging as a big threat among youth. Our findings indicate a significant relationship between physical activity and tobacco use among youth. $(\mathrm{OR}=0.48 ; \mathrm{p}<0.05)$ Thus, promoting physical activities may be a useful educational tool for reducing tobacco use.

Tob. Induc. Dis. 2018;16(Suppl 1):A292

DOI:10.18332/tid/84037

\section{$12: 30-14: 00$}

PS-690-4 Effect of smokeless tobacco on vascular changes following flap surgery - a doppler study

T Harikrishnan', K Deivanayagam², V Sampath ${ }^{3}$, Chronic Periodontitis Study Group

'Sri Ramachandra University, Oral Pathology and Microbiology, Chennai, India, ${ }^{2}$ Sri Ramachandra University, Conservative Dentistry and Endodontics, Chennai, India, ${ }^{3}$ Sri Ramachandra University, Public Health Dentistry, Chennai, India. E-mail: hthamizh@gmail.com

Background: Very little attention has been paid to the association of smokeless tobacco and the health of gingival and periodontal tissues. Recent studies suggests the role of tobacco chewing in deterioration of gingival and periodontal tissues. Capillary units in the crestal gingiva are affected by inflammation and gingival blood flow may serve as a prognostic marker for inflammation. The study aims to evaluate the effectiveness of vascular flow following periodontal surgery amongst smokeless tobacco chewers and non tobacco chewers using ultrasound doppler flowmetry.

Methods: Based on an inclusion and exclusion criteria, two group of subjects with chronic periodontitis were randomly selected for the study. The control group consisted of subjects without tobacco chewing habit while the test group consisted of subjects with the habit of tobacco chewing. Necessary blood and radiographic investigations were taken and subjects were treated with flap surgeries in both the group of patients. Following the surgery, vascular blood flow was analysed on the second and seventh post operative days using laser doppler flowmetry.

Results: Smokeless tobacco chewers demonstrated decreased vascular flow following periodontal flap surgery compared to non chewers.

Conclusions: A positive correlation was observed between Laser Doppler Flowmetry readings and clinical signs of inflammation. After surgery, there was a decrease in blood flow, among tobacco chewers, when compared with non chewers, on second \& seventh post - operative days in doppler study.

Tob. Induc. Dis. 2018;16(Suppl 1):A293

DOI:10.18332/tid/84137

\subsection{New tools for monitoring policy impact and tobacco use surveillance}

\author{
12:30-14:00
}

PS-691-4 How to monitor WHO-MPOWER policies implementation at sub national level in low resource settings? Experience from the state of Himachal Pradesh in India

G Chauhan ${ }^{1}$

'Directorate Health and Family Welfare, National Health Mission, Shimla, India.E-mail:drgopal7475@yahoo.co.in

Background and challenges to implementation: MPOWER are the WHO recommended policies for reducing demand and supply of tobacco under Framework Convention (FCTC). Tobacco surveillance is important for policy development and action but due to huge cost it is difficult to use MPOWER toolkit in poor resource settings. The state of Himachal Pradesh in India have successfully demonstrated an effective mechanism for tobacco surveillance by customising MPOWER toolkit since 2010 .

Intervention or response: Key indicators have been developed to monitor the MPOWER compliance on monthly basis as a part of routine reporting mechanism as:

$\mathrm{M}$ - a) Number of the tobacco related violations observed

b) Number of violators panelised

$P$ - a) Number of public places where active smoking observed

b) Number of violators panelised

$\mathrm{O}$ - a) Percentage of health institutions having tobacco cessation facilities

b) Percentage of tobacco users utilising the cessation services

W - a) Number of violations reported for selling tobacco without pack warnings

b) Number of prosecutions launched in the courts

E - a) Number of violations reported related to tobacco advertisement and promotion

b) Number of prosecutions launched in the courts.

Results and lessons learnt: The monthly progress review is leading to a healthy competition among the districts to perform better. The validation of the progress is done by academic institutes also. Himachal has successfully sustained smoke free status since 2013. The community based tobacco cessation services have been started in addition to indoor facilities. The compliance of specified pack warnings on tobacco products is quit high and most of the places do not have any outdoor advertisement of tobacco. There is a constant rise in tobacco taxes since 2010.

Conclusions and key recommendations: Cost effective tools to monitor MPOWER compliance is the way forward for optimum allocation and utilisation of resources in low income settings.

Tob. Induc. Dis. 2018;16(Suppl 1):A294

DOI:10.18332/tid/83811

12:30-14:00

PS-692-4 Impact of India's National Tobacco Control Programme on bidi and cigarette consumption: a difference-in-differences analysis

GP Nazar ${ }^{1,2}$, S Srivastava ${ }^{3}$, K Chang $^{4}$, N Pearce ${ }^{2}$, A Karan $^{5}$, C Millett ${ }^{4,6}$

'Public Health Foundation of India, Health Promotion Division, Gurugram, India, ${ }^{2}$ London School of Hygiene \&t Tropical Medicine, Non-Communicable Disease Epidemiology, London, United Kingdom, ${ }^{3}$ Johns Hopkins Bloomberg School of Public Health, Department of International Health, Baltimore, MD, United States of America, ${ }^{4}$ Imperial College London, Public Health 
Policy Evaluation Unit, School of Public Health, London, United Kingdom, ${ }^{5}$ Indian Institute of Public Health, Gurugram, India, ${ }^{6}$ Public Health Foundation of India, Gurugram, India. E-mail: gaurang.nazar@phfi.org

Background: Notwithstanding the importance of decreasing tobacco use to achieve Sustainable Development Goals' mortality reduction targets, evaluations of tobacco control programmes in low- and middle-income settings are scarce. We studied the impact of India's National Tobacco Control Programme (NTCP) on household-reported consumption of bidis and cigarettes using a Difference-in-differences (DID) analysis, typically used in econometrics for programme impact evaluation.

Methods: Secondary analyses were conducted on three crosssectional datasets from nationally representative Household Consumer Expenditure Surveys (CES) (1999-2000; 2004-05 and 2011-12). Their sample sizes varied between 100,000-125,000 households. The CES collected information on consumption and expenditure of 350 food and non-food items. Outcomes were: any bidi/cigarette consumption in the household during the past 30 days; and monthly consumption of bidi/cigarette sticks per person. DID two-part models were used to compare changes in outcomes over time and between districts with NTCP and without NTCP implemented, adjusting for socio-demographic characteristics and time-based heterogeneity.

Results: There were no significant reductions in the proportion of households reporting bidi (adjusted odds ratio [AOR] 1.04, 95\% confidence interval $[\mathrm{CI}] 0 \cdot 84-1 \cdot 28$ ) or cigarette consumption (AOR: $1 \cdot 01,95 \%$ CI 0.82-1.26) in NTCP districts compared with non-NTCP districts. Among households that reported any bidi/ cigarette consumption, we observed an overall reduction in the monthly per person consumption of bidi/cigarette sticks over time. However, these reductions were not significantly different between NTCP and non-NTCP districts (adjusted coefficients were: 0.07 bidi sticks, $95 \%$ CI $-0 \cdot 13-0 \cdot 28$ bidi sticks; and -0.002 cigarette sticks, $95 \%$ CI -0.26-0.26 cigarette sticks).

Conclusions: Although the consumption of bidis and cigarettes had reduced in India between 1999-2000 and 2011-12, these reductions were not significantly different between NTCP and nonNTCP districts. Strengthening implementation and enforcement of tobacco control policies is vitally important to reduce the future burden of tobacco use in the country.

Tob. Induc. Dis. 2018;16(Suppl 1):A295

\section{DOI: $10.18332 /$ tid/83825}

\section{2:30-14:00}

PS-693-4 Classification and regression tree for characterising smoking patterns among adults: evidence from global adult tobacco survey, Bangladesh

MA Kabir

'Jahangirnagar University, Savar, Dept. of Statistics, Savar, Dhaka,Bangladesh. E-mail: alamgirfa_juniv@yahoo.com

Background: Tobacco consumption is a preventable public health problem. Many tobacco related studies have employed logistic regression in their analysis and they mostly analyzed categorical variables with dichotomous outcomes. In comparison to logistic regressions, classification and regression tree (CART), a data mining technique have not been widely applied for tobacco related research though this technique has enormous benefits over other methods. Therefore, this study examines the smoking patterns among adults by CART method and to compare findings with other traditional techniques.
Methods: Dataset covered a nationally representative sample of 9,629 respondents extracted from Global Adult Tobacco Survey, Bangladesh and used CART techniques for its suitability than others such as, binary logistic regression, multinomial logistic regression, chi-squared automatic interaction detector, quick unbiased statistical test.

Results: CART was used to characterize the cigarette smoking behaviour among adults aged 15 years and above in Bangladesh. The algorithm builds a tree model to classify "average number of cigarettes smoked per day" using some attributes as predictors. CART was found easy to understand compared to other data mining techniques. Logistic regression model requires the parametric assumption (PA) of the dependent variable. However, this PA often restricts when data "A mixture of categorical and continuous variables". Therefore, CART is appropriate because: (i) Purely non-parametric and is independent of distribution assumptions (ii) Can handle both continuous and categorical data (iii) Can use skewed or multi-modal data without requiring the independent variables to be normally distributed (iv) Can handle missing data (v) Relatively automatic 'machine learning (vi) Less input is needed for analysis and (vii) Visualization character and its results are simple to interpret even for non-statisticians.

Conclusions: Among the different techniques so far used in characterizing smoking patterns among adults, CART is the best in terms of all aspects and suggested for future research.

Tob. Induc. Dis. 2018;16(Suppl 1):A296

DOI:10.18332/tid/83962

\section{2:30-14:00}

PS-694-4 Computerized tablet based versus traditional paper - based survey methods: results from adolescent's health research in schools of Maharashtra, India

N Agarwal ${ }^{1}$, B Paswan ${ }^{2}$, PH Fulpagare ${ }^{2}$, DN Sinha ${ }^{3}$, T Thamarangsi ${ }^{1}$, M Rani ${ }^{1}$

'World Health Organization, Noncommunicable and Tobacco Surveillance, New Delhi, India, ${ }^{2}$ International Institute for Population Sciences, Department of Extra Mural Studies and Distance Education, Mumbai, India, ${ }^{3}$ School of Preventive Oncology, Patna, India. E-mail: agarwaln@who.int

Background and challenges to implementation: Technological advancement is growing very fast in India and majority of young population is handling electronic devices often during leisure as well as at work. This study indicates that electronic tablets are less time consuming and improves survey response rate over the traditional paper-pencil survey method.

Intervention or response: An Android-based Global Schoolbased Health Survey (GSHS) questionnaire was used with the help of an Open Data Kit to assess adolescent's health. Effectiveness of tablet survey was tested in 15 public schools of Mumbai, India over traditional paper-pencil survey method. The questionnaire was administered in three different languages. Tablet and Paper surveys were simultaneously and alternatively used i.e. first tablet followed by paper pencil in one school and in next school, first paper and then tablet to balance uniformity in the survey method. Results and lessons learnt: A total of 600 students aged 13-17 years were covered in the survey for this experimental study. Interestingly all sampled students participated in the survey. The overall response rate using tablet survey was higher than the paper based survey method. Average time taken to fill in the tablet survey was lesser as compared to the paper based survey. It is remarkable to state that missing values on paper for most 
variables were more as compared to the tablet. Most of the students favoured tablet method of data collection as they found it very user friendly, convenient, less human error while filling the answers and less time taking.

Conclusions and key recommendations: Use of electronic tablets for conducting public health surveys suggests an improved response rate and time saving as compared to traditional paper based survey methods. Thus, the present study strongly recommends that tablet method of data collection may be the best method and can be used as effective, economic and quick survey method in future.

Tob. Induc. Dis. 2018;16(Suppl 1):A297

DOI:10.18332/tid/83998

\section{2:30-14:00}

PS-695-4 Mapping of tobacco use among people visiting mass gathering (Kumbh Mela) in India K Oswal ${ }^{1}$, S Balsari ${ }^{2}$, R Merchant $^{3}$

${ }^{1}$ Indian Dental Association, Mumbai, India, ${ }^{2}$ Harvard T.H. Chan School Of Public Health, Boston, MD, United States of America, ${ }^{3}$ K.B.H. Dental College and Hospital, Nashik, India. E-mail: oswal.kunal@gmail.com

Background: Organizers of most mass gatherings offer basic health services to attendees, and this is especially true of events of longer duration like the Hajj, the Olympics, or the Kumbh Melas in India. In 2015, at the world's largest mass gathering, the 35-day Kumbh Mela in Nashik, the government offered basic primary care to the estimated 10 million visiting pilgrims Prevalent studies to report tobacco use in mass gathering is not cited in the literature. Hence a study was planned to provide useful insights in the rising trends of diseases among the public during the mass gathering like Kumbh Mela.

Methods: The study was planned to understand the pattern of tobacco use, among people visiting Kumbh Mela. A convenience sampling was undertaken at the temporary clinic established by the public health department at Nashik and Tribakeshwar. A total of 5302 visitors voluntarily opted for screening services offered for 13 days at Tapovan hospital in Nashik and for three days at the Rural Hospital in Trimbakeshwar.

Results: The different types of tobacco consumption reported were in the form of smoking (24.6\%), smokeless $(28.4 \%)$ and both smoking and smokeless (2.4\%). Tobacco use was found to be more in males $(79.6 \%)$ as compared to females (20.4) The participants who were consuming both smoking and smokeless tobacco had $85.3 \%$ of premalignant lesions as compared to smoking (78.7\%) and smokeless (68.3\%) alone. Among different pre-malignant lesions examined, majority were smokers palate $(22.4 \%)$, tobacco pouch (16.6\%), leukoplakia (11.8\%) and erythroplakia (10\%).

Conclusions: Mass gathering offers important public health surveillance for monitoring disease trends among the masses. The burden of undetected (and untreated) NCDs in India remains economically crippling. Until such time that the effective primary care is widespread and accessible, active surveillance efforts remain critical to combating NCDs.

Tob. Induc. Dis. 2018;16(Suppl 1):A298

DOI:10.18332/tid/84035

\section{$12: 30-14: 00$}

PS-696-4 A sudden surge in outpatient volume of the smoking cessation clinic after the implementation of Beijing tobacco control regulation

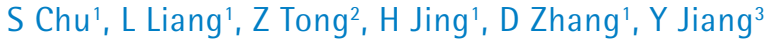

${ }^{1}$ Beijing Chaoyang Hospital, Capital Medical University, Beijing Respiratory Medicine Institute, Clinical Epidemiology \& Tobacco Dependence Treatment Department, Beijing, China, ${ }^{2}$ Beijing Chaoyang Hospital, Capital Medical University, Beijing Respiratory Medicine Institute, Department of Respiratory and Critical Care Medicine, Beijing, China, ${ }^{3}$ Chinese Center for Disease Control and Prevention, Tobacco Control Office, Beijing, China. E-mail: Ilrcruie@sohu.com

Background: Smoke-free policy could promote the smokers to quit. From June 1st 2015, Beijing Tobacco Control Regulation, which was the most strict tobacco control regulation in China mainland, was implemented. This study was to investigate the impact of implementation of Beijing Tobacco Control Regulation on outpatient volume of smoking cessation clinic and the seasonal variations of the outpatients volume of smoking cessation clinic,in order to provide evidence for decision-making in increasing the outpatient volume of smoking cessation clinic in China mainland.

Methods: Before and after the implementation of Beijing Tobacco Control Regulation (June 1st, 2015), the outpatient volume of smoking cessation clinic in Beijing Chaoyang Hospital, which was the first smoking cessation clinic set up in 1996 and has hold the largest out-patient consultations annually in China mainland, was analyzed. Time series seasonal index method was used to analyze seasonal variations in the outpatient volume.

Results: From 2012 to 2016, the outpatient volume of smoking cessation clinic in Beijing Chaoyang Hospital was gradually increasing every year. The largest increase was found in 2016 (1405 outpatients), in which the outpatient volume rose 82.1 percent from 2015, and reached 3.1 times of that in 2012. There were significant differences both in outpatient volume and in return visit ratio between before and after the implementation (all $\mathrm{P}<0.001)$. In average, the peaks of outpatient volume appeared in March, June, August, September, and December, whereas the troughs appeared in January, February, April, May, July, and November.

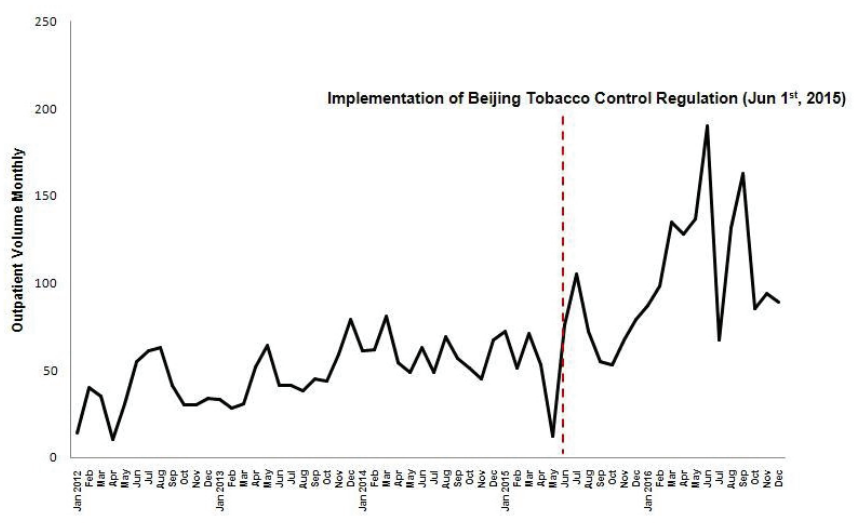

[Monthly variation of outpatient volume of smoking ]

Conclusions: Since Beijing Tobacco Control Regulation implemented, the number of outpatients and return visit of the smoking cessation clinic have increased significantly. With regard to the seasonal variation in the outpatient volume, the government should strengthen publicity of smoking cessation in winter and spring to avoid the decline in the outpatient volume.

Tob. Induc. Dis. 2018;16(Suppl 1):A299

DOI:10.18332/tid/84066 


\section{2:30-14:00}

PS-697-4 Implementing section - 6b of Cigarettes and Other Tobacco Products Act 2004, prohibiting the sale around educational institutions using technology based software - safe zone

E Vidhubala ${ }^{1,2}$, D Parthasarathy ${ }^{3}$

${ }^{1}$ Indian Institute of Sports Medicine, Chennai, India, ${ }^{2}$ Cancer Institute, Resource Center for Tobacco Control, Chennai, India, ${ }^{3}$ VAYAS Technology Solutions, Chennai, India. E-mail: vidhubalae@gmail.com

Background and challenges to implementation: Section $6 \mathrm{~b}$ of Cigarettes and Other Tobacco Products Act (COTPA)-2004 and Article 16 of Framework Convention on Tobacco Control (FCTC) insists on restricting the access and availability of tobacco products to children as one of the supply reduction measures. Several countries have taken initiatives restricting the sale of tobacco products near Educational Institutions (EIs) and in India it is prohibited around 100 meter radius. However, the implementation has become a major challenge as it is difficult to map the distance between EIs and tobacco outlets, and to prove the shop keepers that they are evading the law. To address this issue, an android based application called Safe Zone is designed. Intervention or response: Safe Zone is a Software tool that helps to identify tobacco outlets in and around EI. The technology used was Google MAP API, Google Maps, HTML5 / CSS3 / Java Script, GPS - Global Positioning System and GPRS - Data connection, Geo-coordinates algorithm. The details of the software are given as Figure 1.

Results and lessons learnt: This software gets your current GPS location and finds all EIs within 100 meter radius nearby and flags us along with the distance. It can show us the precise DISPLACEMENT between given shop and given EI locations. This software works in almost all smart phones that have GPS and GPRS - data connection.

Conclusions and key recommendations: This can be customized and used in any country based on their respective country law. It will help the Social activists, Government officials and Law Enforcement officers, who wants to enforce the Law of the Land in creating safe zone to take evidence based actions to curtail the sale of tobacco in the EI zones and General Public who wants to check whether their kid's school/ home is located in a tobacco free zone or not.

Tob. Induc. Dis. 2018;16(Suppl 1):A300

DOI:10.18332/tid/84102

[The internals of the software]

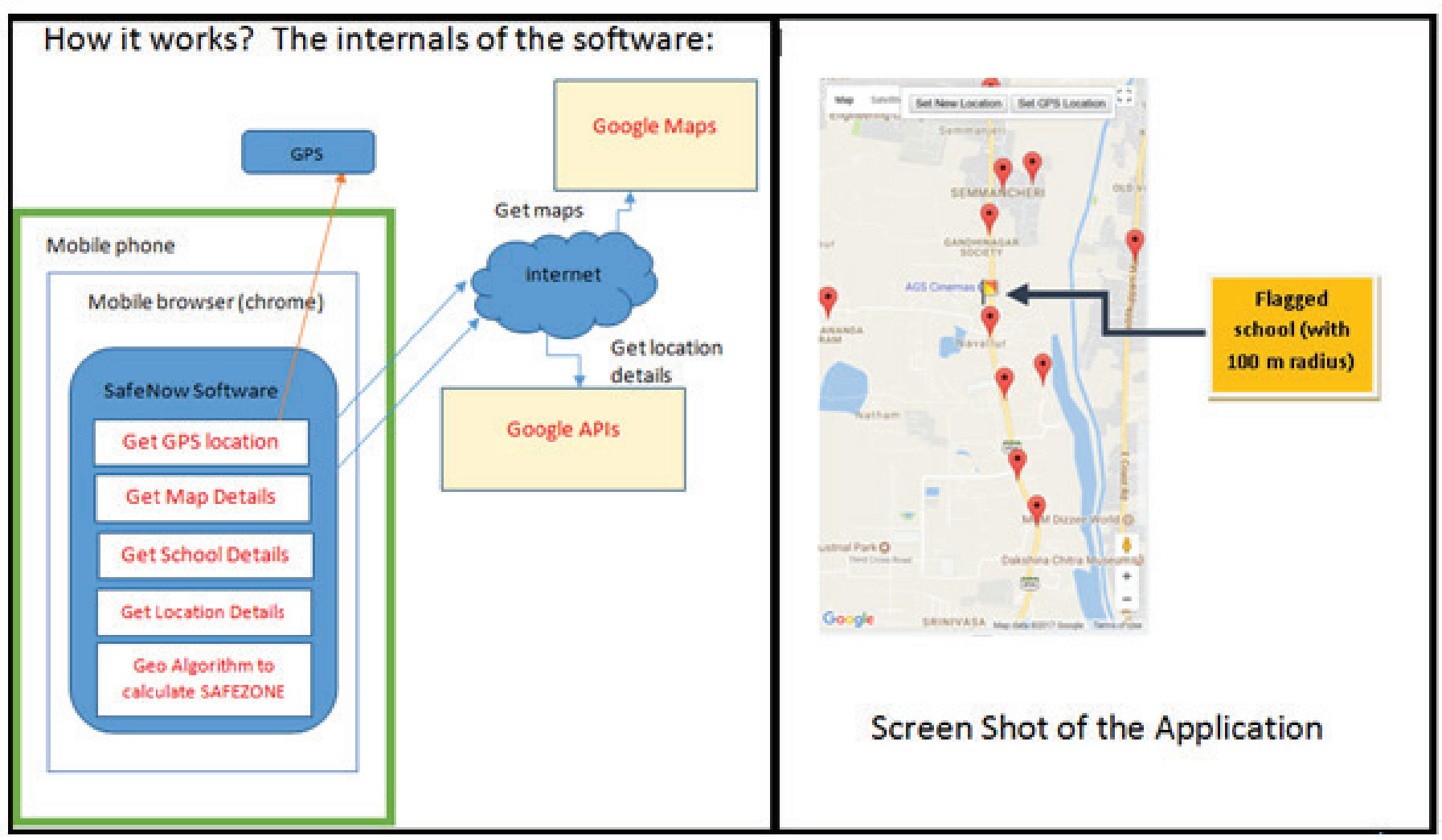

\section{2:30-14:00}

PS-698-4 Building capacity for tobacco-free schools in rural Maharashtra: the Salaam Mumbai model R Kadam¹, D Patil', A Pilankar' ${ }^{1}$

'Salaam Bombay Foundation, Tobacco Control \&t Advocacy, Mumbai, India. E-mail: tshering@salaambombay.org

Background and challenges to implementation: Tobacco is a significant public health risk faced by youth in rural India. In rural Maharashtra, up to $45.4 \%$ of youth use tobacco. India's tobacco control law, the Cigarettes and Other Tobacco Products Act (2003) prohibits the sale of tobacco within 100 yards of school grounds. Still, sale of tobacco is commonly seen around schools across the state.

Intervention or response: In 2008, Salaam Mumbai Foundation (SMF) began a tobacco-free schools initiative in rural Maharashtra. Awareness meetings were held with the Direct of State Education and District Education Officers to build motivation to promote the tobacco-free schools programme. District-level workshops were held with Master Trainers for the Education Ministry. Master Trainers included training on tobacco-free schools as part of their regular training curriculum. Once trained, teachers conducted tobacco control initiatives with students. SMF rewards schools 
for reaching levels of compliance with the 11 criteria created in accordance with an education board. Bronze-level awards are given to schools reaching 4 criteria, silver-level awards to schools reaching 8 criteria and Gold-level awards to school reaching all 11 criteria.

Results and lessons learnt: Yavatmal district has 2,137 government run schools. In 2016-17 all these schools attained the tobacco free status by fulfilling all 11 criteria. Celebrating 31 st December 2015 as ‘Tobacco Free Life Resolution Day' 406,384 people at 2,109 Government run schools across Yavatmal took the oath for Tobacco Free Life. This has been recorded in the 'Limca book of Records' in 2016.

Conclusions and key recommendations: Using the existing infrastructure, Maharashtra's rural teacher training programme has been a successful and efficient strategy to make schools in the state tobacco free. Using a graded system to reward schools provides an incentive for schools to expand compliance and serves as a useful tool for measuring increases in compliance over time. Maharashtra State Government recognized SMF's work and felicitated the organization with Mahatma Gandhi Vyasanmukti Award.

Tob. Induc. Dis. 2018;16(Suppl 1):A301

DOI:10.18332/tid/84105

\subsection{Tobacco dependence treatment and smoking cessation: Innovations in Practice and Opportunities for scaling}

\section{$12: 30-14: 00$}

PS-699-4 Tobacco cesssation - as we want it! An interview study with young people

I Edvardsson Aurin ${ }^{1,2}$, L Lendahls ${ }^{2,3}$, K Haraldsson $^{4}$

'Lunds University, Department of Clinical Sciences Malmö, Lund, Sweden, ${ }^{2}$ Kronoberg County Council, Department of Research and Development, Växjö, Sweden, ${ }^{3}$ Linneaus University, Kalmar, Sweden, 'Department of Research and Development, Halmstad, Sweden. E-mail: ingrid.edvardsson@kronoberg.se Background: Smoking among teenagers and young people is still common and about every third young smoker states that they want to quit smoking. There are no obvious evidence-based methods for tobacco cessation for young people, and therefore, the same methods are used for young people as well as adults. The aim was to study young people's views about what support they would like for quit smoking, with the intention of developing a model for tobacco cessation for young people through interviews with the target groups.

Methods: A qualitative study using a descriptive and exploratory design was conducted. Young smokers 16 - 29 years old $(n=25)$ were interviewed in five focus groups in southern Sweden. Data were analyzed by content analysis.

Results: The results illuminate young people's suggestions to develop a model for tobacco cessation. Four main categories emerged; Existing support rejected - existing apps, support- by SMS, and self-help brochures did not appeal to young people. Agreement between friends - To sign an agreement between two friends which included a fee on relapse. Adult support - To organize the model with duo groups and give adapted support. Celebrate and competition - Lift the positive with the freedom of smoke and create the opportunity to win simple prizes to celebrate the achieved interim goals.

Conclusions: The young people suggested that the model for tobacco cessation would contain adult support and involves an agreement between friends and has elements of celebration and competition. The challenge will be to implement this model into environments where young people live.

Tob. Induc. Dis. 2018;16(Suppl 1):A302

DOI: $10.18332 /$ tid/83756

\section{2:30-14:00 \\ PS-700-4 Third times the C.H.A.R.M.S: a socioecological thirdhand smoke cessation pilot study \\ J Quinlan', Behavioral Health \\ ${ }^{1}$ Catholic Family Center, Rochester, NY, United States of America. E-mail: janinequinlan@outlook.com}

Background and challenges to implementation: Research shows that Passive Tobacco Exposure (PTE), via Thirdhand Smoke (THS), is cytotoxic. 16 million Americans are affected by tobacco. Smoking bans show interest in reducing exposure of PTE yet literature remains scarce. Public smoking bans, raising tobacco taxes, and tobacco litigation demonstrate change in Smoke Cessation (SC) but a new approach is needed to effect societal smoking as well as global smoke cessation. The study investigates if education on the effect of THSe harms increases contemplation to stop smoking. Primary Challenges to implementation are Addiction, Big Tobacco influences and societal beliefs and views on PTE.

Intervention or response: The pilot study used a quasiexperimental, pretest-posttest design and an educational intervention Pre and posttest measures utilized the Contemplation Ladder for readiness to quit smoking. Education on Firsthand, Secondhand and Thirdhand Smoke harms in a Motivational Interviewing milieu was provided.

Results and lessons learnt: Paired t-tests show statistical significance for desire to quit smoking between three groups and as one group, $n=34$. Results indicate that education on the harms of THSe to passive recipients may increase the contemplation to quit smoking and desire to reduce PTE.

Conclusions and key recommendations: The dangers of smoking have been studied to determine effect on public health, animals and the environment. The usefulness of MI in smoke cessation groups in a variety of settings has some mixed results. The study expands the existing body of knowledge on motivation to quit smoking in a socioecological context.

Organizational stakeholders of health centers, non-profits, small practices, veterinarians and community action agencies will find these results useful when considering SC methods and in creating environments that are free of THSe. Practitioners may widen the scope of SC by including teaching of harms to therapy or domestic animals and informing persons on the financial consequences of THS. Collaboration with a variety of disciplines for SC is recommended.

Tob. Induc. Dis. 2018;16(Suppl 1):A303

DOI:10.18332/tid/83757

\section{2:30-14:00}

PS-701-4 Empowering smoke-free environment through self-verbalization and cognitive re-appraisal psychotherapies among university participants in a smoke-stoppage programme

J Osiki ${ }^{1}$, Category ${ }^{1}$ Scientific Research

'University of Ibadan, Guidance and Counselling, Ibadan, Nigeria. E-mail:jonathanosiki@yahoo.co.uk

Background: Over the years, the attempt to encourage the stoppage of smoking behaviour has not been easy. While different 
reasons are offered on why people smoke, the environmental effect, aside from the danger of smoking on both self and the family is staggering (Fact Sheet-Smoking Statistics, 2010). Cigarette/tobacco smoking poses irreversible health hazards that lead to series of unquantified health implications to the health of smokers, the health of side-stream (second hand and/or passive) smokers as well as the smokers' significant persons, especially children. Despite the health hazards that smoking poses to everyone including the smokers, the continued benefits for applying psychotherapeutic packages for both its reduction and or stoppage are not exhaustively the vogue in Sub-Saharan Africa. Methods: It screened and selected 21 volunteer smokers using the number of cigarette smoked per day within the frequency index for eligibility in a seven month time frame. With the use of the frequency counts subsumed within smoking personal motivation scale the ANCOVAR statistical methods at 0.05 alpha was applied in data analysis.

Results: Overall, the findings showed that the treatment outcome that utilized both self-verbalization and cognitive reappraisal psychotherapies were effective $(p<0.05)$ for reducing the frequencies of cigarette smoked and eventual stoppage for participants. Though initial effectiveness was $61.6 \%$ success, the follow-up monitoring activities indicated that at seven months expiration period, the treatment had recorded $86.5 \%$ success.

Conclusions: The regular application of the suggested psychotherapies are germane to a healthy smoke-free environment while more efforts should be directed to understand the personal and fate beliefs of individuals sustaining continuous smoking despite its deleterious health effects on the people.

Tob. Induc. Dis. 2018;16(Suppl 1):A304

DOI:10.18332/tid/83762

\section{2:30-14:00}

PS-702-4 Experiences of young smokers in quitting smoking in twin cities of Pakistan: a phenomenological study

K Shaheen ${ }^{1}, \mathrm{H}_{\text {Masud }}{ }^{1}$

${ }^{1}$ Al Shifa School of Public Health, Rawalpindi, Pakistan. E-mail: haleemamasud@yahoo.com

Background: Smoking is highly prevalent in Pakistan claiming lives of over 100,000 individuals every year. A significant proportion of smokers makes quit attempt each year but $97.4 \%$ fails to quit successfully. Little is known about the reasons of failed quit attempts.

Methods: A qualitative study was carried out using phenomenological approach in Rawalpindi and Islamabad to document experience of smokers during quitting. Eleven male participants were interviewed. All study participants had made at least one quit attempt. The study participants were a mix of smokers who failed to quit smoking, intermittent smokers and successful quitters. Streubert's (1991) method of phenomenology was followed during data analysis.

Results: The journey of quitting smoking is deeply embedded in smoking phase experiences of smokers. Smoking phase experiences consist of three stages: getting in contact with stimuli to smoke, journey from first puff to enjoying smoking and then finally smoking becomes part of life. However, the quitting phase is not as smooth and simple as is the case with smoking phase. Instead, smokers get trapped into three overlapping vicious circles of smoking and quit attempts: smoking \& forced quitting, smoking \& intentional quitting, and smoking \& intermittent smoking before successful quitting at young age. Breaking the vicious circle is not easy in the presence of trapping factors (addiction, high availability, easy affordability, conducive social setup and low perceived risks of smoking). Three factors play a major role in breaking these circles which are strong will power, continuous peer support and avoidance of smokers' company.

Conclusions: A young smoker, during his experience of quitting smoking gets entrapped into several overlapping vicious circles of smoking \& quit attempts before successful quitting. There are known entrapping factors as well as factors which help in breaking these circles. Targeted interventions are needed to facilitate smoking cessation among young smokers in Pakistan.

Tob. Induc. Dis. 2018;16(Suppl 1):A305

DOI: $10.18332 /$ tid/83764

\section{$12: 30-14: 00$}

PS-705-4 ABC in ED results of the "ASSET" study: outcomes of "Brief Interventions" to stop smoking given in the emergency department of a central city hospital (2016)

KJ Stevens'1, RJ Hopkins², RP Young ${ }^{2}$

${ }^{1}$ Auckland District Health Board, Smokefree Services, Auckland, New Zealand, ${ }^{2}$ University of Auckland, Auckland, New Zealand. E-mail: kstevens24c@gmail.com

Background: Studies indicate that at any one time, about $70 \%$ of smokers want to quit smoking. Asking about smoking behaviour (A), giving brief advice to stop (B), and making an offer of support to do so (C), by all healthcare practitioners, this $\mathrm{ABC}$ approach is integral to New Zealand reaching its Smokefree 2025 aspirational goal.

Methods: Retrospectively the names and basic demographic details of all current smokers discharged from ED in each of 6 months were supplied for the project and the data was scrutinized to ensure that documented

evidence existed to show that brief advice had been given by professional departmental staff. Criteria for study inclusion were:

- discharged home from the Emergency Department

- given brief advice to stop smoking

- data were clearly documented in the hospital record

- candidate aged over 18 years

A telephone based questionnaire was developed and used for this study.

Results: Over the 6 month period, 500 patients met our eligibility, the current analysis is based on 195 completed participant responses of which:

- $30 \%$ attempted to stop smoking (20\% of subjects had 1 quit attempt)

- $21 \%$ attempted to quit "cold turkey"

- $20 \%$ used some form of smoking cessation "medication" (8\% used NRT) -these are not mutually exclusive

- $3 \%$ were still quit at the time of phone interview

- $50 \%$ smoked less than usual (with $15 \%$ reducing their daily intake by 10 cigarettes or more per day and $14 \%$ reducing by 5-9 cigarettes per day).

Conclusions: The majority of participants agreed that being asked about their smoking behaviour was acceptable in an ED setting.

There were no features that predicted who might have the greatest benefit from receiving brief advice.

Therefore the $\mathrm{ABC}$ approach should be incorporated into all ED assessments.

Funding: Funding provided by the NZ MoH, Tobacco Control Department.

Tob. Induc. Dis. 2018;16(Suppl 1):A306

DOI: $10.18332 /$ tid $/ 83788$ 


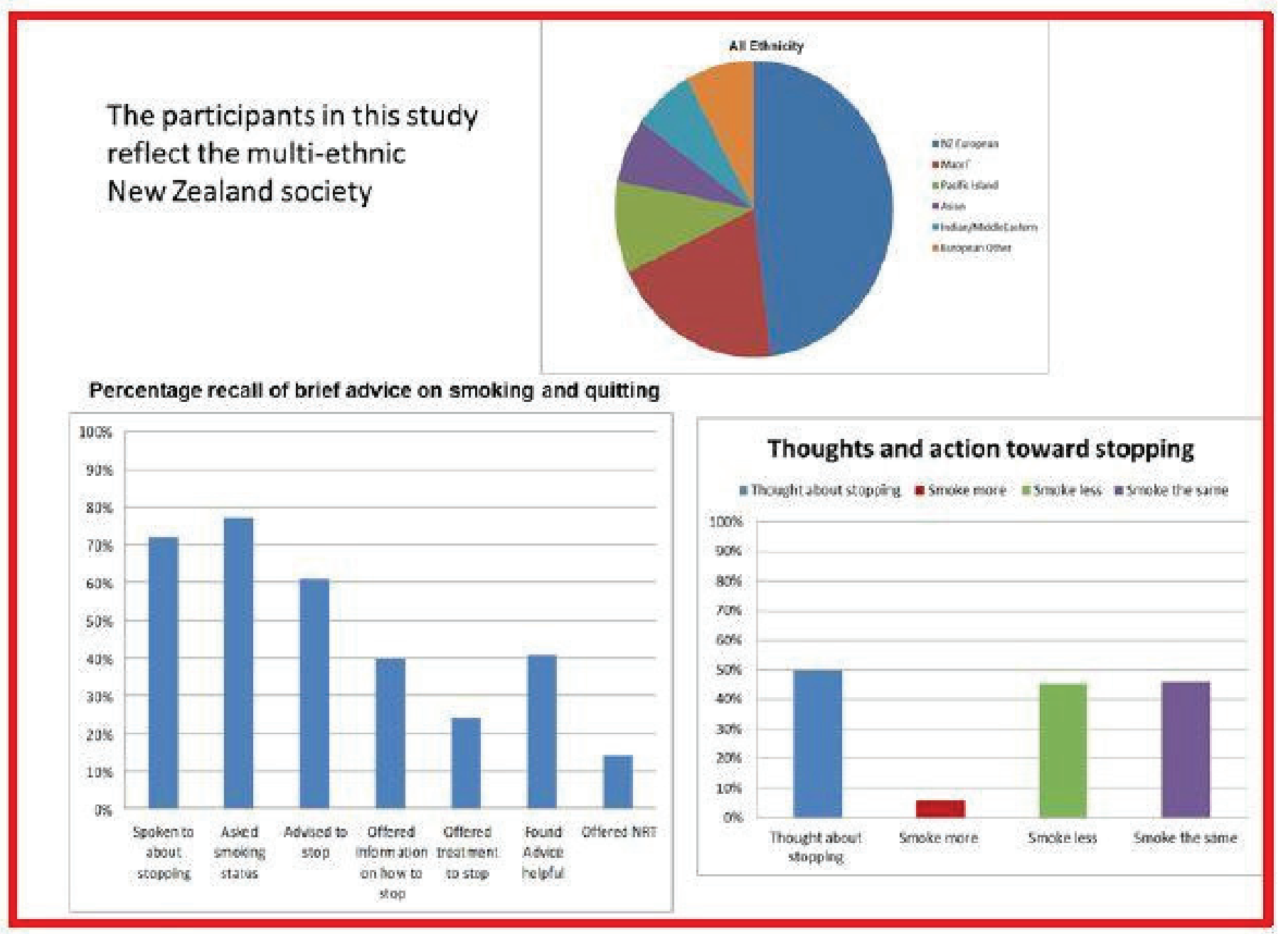

\section{2:30-14:00}

PS-706-4 Development of a guided imagery tobacco cessation intervention to be delivered by quitline and website

J Gordon', J Armin'1, M Bell', P Giacobbi², U Nair', G Povis' 'University of Arizona, Tucson, AZ, United States of America, ${ }^{2}$ West Virginia University, Morgantown, WV, United States of America.E-mail: judithg@email.arizona.edu

Background: Telephone quitlines are an effective and highly scalable way to help individuals quit smoking. However quitlines are an underutilized resource, and do not reach certain segments of the population, including males and racial/ethnic minorities. Guided imagery is a form of mind-body therapy that involves controlled visualization of specific mental images. Studies have shown that imagery training results in significantly increased smoking abstinence rates. Imagery is used by a significant number of racially diverse male and female athletes. We hypothesize that guided mental imagery delivered using the quitline "coaching model" could be an effective and disseminable intervention strategy. In addition, this model may be more readily accepted by underrepresented smokers, and may increase the reach and effectiveness of telephone quitlines. The objective of this study is to develop and test the feasibility of a telephone plus website imagery intervention for smoking cessation.

Methods: We will develop the intervention and companion website with input from quitline personnel, a community advisory board, expert consultants, focus groups, and user groups of raciallyand ethnically-diverse smokers in preparation for a randomized clinical trial.

Results: We will present findings on our community-based development process and how we adapt the program content and materials for use with a wide range of smokers. We will share lessons learned and provide an overview of the guided imagery intervention protocol and program materials.

Conclusions: The results of this study have the potential to improve public health through increased reach of telephone quitlines. If successful, this model could be expanded to address other health behaviors such as diet, physical activity and other substance use.

Tob. Induc. Dis. 2018;16(Suppl 1):A307

DOI: $10.18332 /$ tid/83795

\section{2:30-14:00}

PS-707-4 Integration of a personalized approach for tobacco use and dependence in a dentistry setting-preliminary results

\section{A Trofor ${ }^{1}$, IA Grosu $^{2}, \mathrm{C} \mathrm{Vicol}^{3}$, L Moldovan ${ }^{3}$}

'University of Medicine and Pharmacy 'Grigore T.Popa', Clinic of Pulmonary Diseases, Iasi, Romania, ${ }^{2}$ University of Medicine and Pharmacy 'Grigore T.Popa', Iasi, Romania, ${ }^{3} \mathrm{Clinical}$ Hospital of Pulmonary Diseases, Pulmonology ', Iasi, Romania. E-mail: atrofor@yahoo.com

Background: Tobacco smoking is a major risk factor for many oral cavity disorders, amongst which periodontal disease and oral cancer - the most frequent conditions in current dentistry practice. Oral pathology clinics lack their own smoking cessation services, 
and a personalized approach of tobacco consumers addressing dentistry settings would improve their oral health.

Aim: To design a personalized smoke-free approach in a dentistry department and to preliminary evaluate the effectiveness of smoking cessation counseling in a pilot group of smokers with oral cancer.

Methods: Screening for oral health and smoking status of 270 patients admitted in an oral pathology university department for periodontal disease and oral malignancies. All patients answered a personalized oral health and tobacco use questionnaire. 59 smokers with oral cancers underwent a specially designed 3 sessions smoking cessation counseling program (personalized enrollment questionnaire, brief advice and counseling) with initial and in 8 weeks follow-up clinical evaluation of both smoking and oral status

Results: Periodontal disease was more frequent in smokers (60\%) with poor oral hygiene and diet. Majority of smokers with oral cancers were men, age average 57.2 (SD: 4.6) and 49.2 smoked $>20$ cigs/day. Most frequently, oral malignancy affected the lips and the jowl. All 59 smokers with oral cancers answered the personalized questionnaire and attended the brief advice session, whilst 46 (78\%) agreed to try to quit smoking, with a $52.5 \%$ abstinence rate at 8 weeks follow up and a significant oral status improvement identified in all quitters.

Conclusions: There is a great need to design a personalized smoke-free approach for dental patients, by routinely adding screening and treatment of tobacco use to overall oral health evaluation. Smoking cessation proved beneficial in the oral cancers pilot group.

Tob. Induc. Dis. 2018;16(Suppl 1):A308

DOI:10.18332/tid/83840

\section{2:30-14:00}

PS-708-4 "Lung age" - a motivational smoking cessation tool in smokers with chronic obstructive pulmonary disease

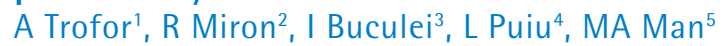

'University of Medicine and Pharmacy 'Grigore T.Popa', Clinic of Pulmonary Diseases, Iasi, Romania, ${ }^{2}$ Hospital of Pulmonary Diseases lasi, Pulmonology ${ }^{3}$, Iasi, Romania, ${ }^{3}$ Hospital of Pulmonary Diseases lasi, Pulmonology ${ }^{1}$, Iasi, Romania, ${ }^{4}$ Pneumophthisiology Hospital Baia-Mare, Baia-Mare, Romania, ${ }^{5}$ University of Medicine and Pharmacy 'Iuliu Hatieganu', Cluj-Napoca, Romania. E-mail: atrofor@yahoo.com

Background: Smoking cessation is the most efficient treatment for chronic obstructive pulmonary disease (COPD). The educational and motivational role of the "lung age" concept, used together with lung function testing and biochemical validation of tobacco exposure, deserves thorough attention for driving intention to quit.

Aim: To demonstrate the motivational role of "lung age" measurement towards stopping smoking in COPD patients. To assess effects of continuing versus stopping smoking on "lung age", on exhaled carbon monoxide ( $\mathrm{CO}$ ) and on lung function, and to determine correlations between these parameters, in such patients.

Methods: 100 COPD patients naive to any smoking cessation intervention, were invited to attend a "lung age" measurement study. Only 61 patients (30 smokers and 31 ex-smokers) agreed a full evaluation for demographics, smoking characteristics (packs-years -PY, nicotine dependence score), COPD status: lung function (FVC,FEV1,FEV1/FVC ratio), mMRC dyspnea,
COPD assessment test (CAT), exhaled CO and "lung age", in a prospective study, with statistic SPSS Inc pack analysis.

Results: All participants acknowledged the motivational value of "lung age" for stopping smoking. Mean "lung age" was 118.3 years in smokers (mean 41.63 PY) and 119.58 years in ex- smokers (mean $36.32 \mathrm{PY}$ ). Lung age was statistically significant correlated with FVC $\%(p=0.03)$, with FEV1\% $(p<0.001)$ and respectively with mMRC dyspnea $(\mathrm{p}<0,026)$. CAT scores ranged from 20.9 (smokers) to 22.7 (ex-smokers). Significant statistic differences were found in exhaled $\mathrm{CO}$ concentrations of smokers (9.73 ppm) compared to ex-smokers (0.58 ppm) (ps 0.001). Exhaled CO was statistically correlated with FEV1/FVC ratio $(p=0.05)$.

Conclusions: "Lung age" measurement, used together with lung function and exhaled $\mathrm{CO}$ testing, adds value to proving impact of tobacco exposure on COPD patients and helps increasing motivation to quit smoking in this difficult to treat category of patients.

Tob. Induc. Dis. 2018;16(Suppl 1):A309

DOI: $10.18332 /$ tid/83843

\section{2:30-14:00}

PS-709-4 Self-perceived ability to cope with stress without smoking predicts successful smoking cessation 12 months later in a quitline setting: a randomized trial

E Nohlert' ${ }^{1}$, Ö Örvik ${ }^{1,2}$, ÁR Helgason ${ }^{3,4}$

${ }^{1}$ Centre for Clinical Research, Uppsala University, Västerås, Sweden, ${ }^{2}$ Karolinska Institutet, Department of Medicine, Stockholm, Sweden, ${ }^{3}$ Karolinska Institutet, Department of Public Health Sciences, Social Medicine, Stockholm, Sweden, ${ }^{4}$ Reykjavik University, Reykjavik, Sweden. E-mail: eva.nohlert@ regionvastmanland.se

Background: Telephone-based smoking cessation services ('quitlines') are both effective and cost-effective. Knowledge of modifiable baseline factors in real-life settings with heterogeneous participants is essential for the development and improvement of treatment protocols to assist in telephone-based smoking cessation. The aim was to assess self-efficacy as a predictor for abstinence at the 12-month follow-up at the Swedish National Tobacco Quitline (SNTQ).

Methods: The data were collected from a randomized controlled trial comparing the effectiveness of proactive and reactive service at the SNTQ. Included were 612 clients calling the SNTQ between February 2009 and September 2010. Outcome measures were self-reported point prevalence and 6-month continuous abstinence at the 12-month follow-up. Plausible predictors of smoking cessation were assessed at the first call and in a baseline questionnaire. Self-efficacy was measured by three questions: (1) the likelihood of being smoking free in 1 year; (2) the ability to handle stress and depressive mood without smoking; and (3) the likelihood of using medication against craving if necessary. The associations between predictors and outcome were subjected to logistic regression analysis.

Results: Of the three self-efficacy predictors for abstinence at month 12 , only the perceived ability to handle stress and depressive mood without smoking remained significant in the adjusted analyses (odds ratio, OR, 1.1 for point prevalence and 1.2 for 6 -month continuous abstinence). The overall strongest predictor in the adjusted analyses was smoking status in the week before baseline (OR 2.7 for point prevalence and 3.1 for 6-month continuous abstinence).

Conclusions: The perceived ability to handle stress and 
depressive mood without smoking at baseline predicted the subjects' abstinence at the 12-month follow-up. An assessment of/adjustment for stress and depressive mood may be appropriate in future smoking cessation research.

Tob. Induc. Dis. 2018;16(Suppl 1):A310

DOI:10.18332/tid/83845

\section{$12: 30-14: 00$}

PS-710-4 Scaling up the availability of tobacco dependence treatment training: leveraging the experience of King Hussein Cancer Center to create new training hubs

R Bader ${ }^{1}$, A Hatoqai ${ }^{1}$, I Ghonimat ${ }^{1}$, N Obeidat ${ }^{1}$, F Hawari $^{1}$

${ }^{1}$ King Hussein Cancer Center, Amman, Jordan. E-mail: rbader@ khcc.jo

Background and challenges to implementation: Despite their proven value, tobacco dependence treatment (TDT) services are short in the Eastern Mediterranean Region (EMR) partly due to the lack of expertise among healthcare providers (HCPs). Since training is a proven strategy to avail TDT, King Hussein Cancer Center (KHCC) trained 2,000 HCPs in EMR (between 2011 and 2016). However, centralization meant limited training capacity, calling for scaling up.

Intervention or response: KHCC collaborated with institutions in Oman, Egypt, Tunisia, and Morocco to build sustainable evidence-based local training hubs. The collaborating institutions host and manage the hubs. (1) In each country, needs were assessed to inform identification of target audiences, program design, and selection of HCPs to serve as trainers; and relevant system-level barriers and facilitators were scanned. (2) KHCC shared its evidence-based training curriculum with hubs for translation and necessary customization. (3) KHCC held training of trainers (ToT) workshops, observed trainers in action, and provided feedback. The ToT workshops provided an opportunity for the group of trainers to reflect on barriers and plan action for a TDT-supporting environment.

Results and lessons learnt: $84 \mathrm{HCPs}$ were engaged as trainers (all non-smokers and mostly clinicians). Results from two rounds of semi-annual post-ToT online follow up indicate trainer engagement (Table 1). Respondents report the need for strengthened TDT clinical experience as a barrier to engaging in training, and administrative issues and shortage of medications as barriers to practice.

Within the first year of launching, the hubs offered 8 training workshops through their local trainers (almost double KHCC's historical capacity of 4.5 workshops). Hubs also report miscellaneous TDT awareness activities, and TDT advocacy work.

Conclusions and key recommendations: Our program succeeded in expanding the availability of TDT training and in building a network of trainers/advocates. Yet, hubs need to strengthen their advocacy role to address barriers holding them from achieving full potential, such as those preventing some trainers from engaging in training.

Tob. Induc. Dis. 2018;16(Suppl 1):A311

DOI: $10.18332 /$ tid/83875

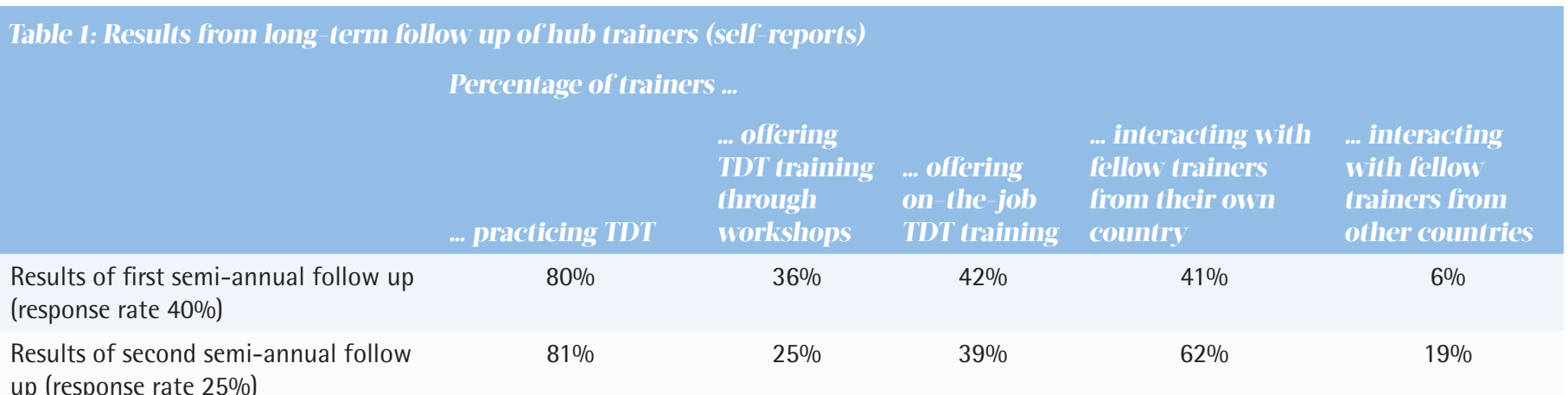

\section{2:30-14:00}

PS-711-4 Effect of community based tobacco cessation intervention in a rural community of Bangladesh

S Jubayer ${ }^{1}$, SR Choudhury ${ }^{1}$, MA Al Mamun ${ }^{1}$, J Ahmed ${ }^{2}$, MM Zaman $^{3}$

${ }^{1}$ National Heart Foundation Hospital and Research Institute, Epidemiology and Research, Dhaka, Bangladesh, ${ }^{2}$ Ekhlaspur Center of Health (ECOH), Chandpur, Bangladesh, ${ }^{3}$ World Health Organization, Research \& Publication, Dhaka, Bangladesh. E-mail: bith74@yahoo.com

Background and challenges to implementation: Bangladesh is one of the high tobacco consumption countries of the world. Global Adult Tobacco Survey (GATS), Bangladesh 2009 shows that $44.7 \%$ of men and $1.5 \%$ of women smoke, while $26.4 \%$ of men and $27.9 \%$ of women use smokeless tobacco. According to GATS, $68 \%$ of smokers and $48 \%$ of smokeless tobacco user wanted or planned to quit tobacco in past 12 months. Clinic based tobacco cessation service is available only in few urban settings.A community based tobacco cessation program utilizing community health workers (CHW) has been initiated in a rural setting from 2014.

Intervention or response: Two $\mathrm{CHWs}$ of a non-government (NGO) health care provider ECOH were trained to deliver cessation advice during courtyard meetings as a part of their regular health promotion activities in a village. Courtyard meetings were conducted involving 20 to 25 members of several households in regular intervals covering the whole village in a month. CHWs delivered the messages regarding the ill effects of tobacco use and encouraged identified users to quit. Subsequently, those who agreed or showed an intention to quit were followed through a mobile phone call after two weeks and subsequently after two and six months.

Results and lessons learnt: During the program period of 18 months, 196 (Men 92, Women 104) tobacco users were registered from 26 courtyard meetings. Mean $( \pm \mathrm{SD})$ age of the participants was $42 \pm 17.6$. Among them smoking tobacco (ST) user were 78 (40\%, all men), while smokeless tobacco (SLT) user were 118 (60\%, men 17, women 101). At six-month follow-up, overall tobacco quit rate was $8.1 \%$. Number (\%) of SLT quitter 13 (11\%), 
among them 9 (8\%) were women and 4 (23\%) were men. For ST $3(3.8 \%)$ men were quitted.

Conclusions and key recommendations: Brief advice through $\mathrm{CHW}$ in courtyard meeting can be an effective cessation method of quitting SLT for the rural community in Bangladesh.

Tob. Induc. Dis. 2018;16(Suppl 1):A312

DOI:10.18332/tid/83884

\section{2:30-14:00}

PS-712-4 Long-term effectiveness, safety, perception, satisfaction and cost of electronic cigarette among single and dual users in Malaysia

MH Nik Mohamed ${ }^{1}$, A Rahman ${ }^{1}$, S Jamshed ${ }^{1}$

${ }^{1}$ International Islamic University Malaysia, Pharmacy Practice, Kuantan, Malaysia.E-mail: haniki@iium.edu.my

Background: More information is needed regarding long-term electronic cigarette (e-cig) effectiveness and safety for smoking cessation and reduction among single vs. dual users (SU vs. DU). This study assessed complete nicotine abstinence rate and safety of e-cig among both groups. Additionally, cigarette smoking abstinence and reduction, perception, satisfaction and cost were evaluated.

Methods: A one-year observational study conducted among 220 e-cig users $>18$ to 65 years old recruited via convenience and snowball sampling from Kuantan and Pekan, Pahang, Malaysia. Follow-up visits were at week 4, 24, and 52. Validated questionnaires captured relevant information, including exhaled carbon monoxide and salivary nicotine levels, adverse events, perception, satisfaction and related cost. All subjects signed informed consent. Study approved by institutional research ethics committee. Analyses via SPSS (version 21 ), $\mathrm{p}<0.05$ considered as significant.

Results: At week $52,15.9 \%$ of SU ( $n=11)$ vs. $6.8 \%$ of DU $(n=10)$ quit both tobacco and e-cig, OR: 2.57; $\mathrm{p}=0.048 .34 .8 \%$ of $\mathrm{SU}$ $(\mathrm{n}=24)$ vs. $17.1 \%$ of DU $(\mathrm{n}=25)$ quit only tobacco, OR: $2.58 ; \mathrm{p}$ $=0.005$. Among the DU, $23.3 \%(n=34)$ reduced $\geq 50 \%$ tobacco smoking vs. $21.7 \%$ of $\mathrm{SU}(\mathrm{n}=15)$, OR: $1.092 ; \mathrm{p}=0.863$. SU perceived less e-cig adverse events and withdrawal symptoms vs. DU. Subjects mainly reported coughing, breathing problems and craving for tobacco smoking. 33\% of subjects were satisfied and rated e-cig as an effective smoking cessation aid. E-cig total monthly average cost was USD27 (MYR132.45).

Conclusions: E-cig is effective and safe for long-term nicotine abstinence and tobacco smoking reduction. Subjects generally have good perception and satisfaction towards e-cig, especially among single users. Monthly expenditure on e-cig is less as compared to tobacco smokers.

Tob. Induc. Dis. 2018;16(Suppl 1):A313

DOI:10.18332/tid/83886

\section{2:30-14:00}

PS-713-4 Evaluation of a national tobacco dependence treatment program in Uruguay 2017 E Esteves', G Saona', M Baldizzoni', I Wald ${ }^{1}$, N Rey ${ }^{1}$

${ }^{1}$ National Fund of Resources, Montevideo, Uruguay. E-mail: eesteves@fnr.gub.uy

Background: Tobacco Control is State Policy in Uruguay since 2005, since that time more than 200.000 people quit smoking. In addition to population measures, was essential provide effective dependence treatment. The intervention of the National Fund of Resources (FNR) enabled accessible and affordable help. Since 2004, FNR has managed a network of Smoking Units (SU) free or with very low cost, currently are 105 working throughout the country. Until now have been included 33.308 patients. To evaluate program operation and outcomes we study patients admitted between April 2014 and March 2015.

Methods: Descriptive study and prospective follow-up of a randomized sample stratified by sex, health coverage, number of controls and use of medications.

Results: 1509 patients were studied. Sample 426 individuals, 336 were contacted (79\%). Female $56.1 \%$, mean age 47.6 , from the capital city $45.3 \%$, with coverage in the Public Health $47.8 \%$. Mean age of onset 16.4 years, mean of consumption $20.3 \mathrm{cpd} .57 .1 \%$ had fixed work and $25.4 \%$ had primary education. Performance indicators: 1509 patients with initial interview, 73.2\% (1104) had follow-up, of which 59.3\% (655) reached 3 visits. Average follow-up period 48.1 days (S.D 53.8). $76.4 \%$ of the patients (844) received pharmacological treatment. $40.0 \%$ of those who had follow-up achieve punctual abstinence (7 days). $30.4 \%$ of the patients in the sample were abstinent (7 days) at the followup at 15 months of admission. $24.9 \%$ and $19.8 \%$ achieved 6 and 12-month sustained smoking cessation, respectively. Regarding patients with 3 visits or more, punctual abstinence at 15 months was $38.2 \%$, sustained abstinence for 6 months $32.5 \%$ and for 12 months $27.3 \%$.

Conclusions: The operation of the program as a whole meets the standards required for a good quality of assistance and the outcomes obtained are comparable to those published by various programs with similar characteristics.

Tob. Induc. Dis. 2018;16(Suppl 1):A314

DOI:10.18332/tid/83892

\section{$12: 30-14: 00$}

PS-715-4 Readiness to quit addiction! A study among patients attending tertiary care hospital in Western India

K Mehta ${ }^{1}$, C Pandya ${ }^{1}$, D Solanki ${ }^{1}$, P Chavda ${ }^{1}$

${ }^{1}$ GMERS Medical College \&t Hospital, Gotri, Community Medicine, Vadodara, India.E-mail: kedar_mehta20@yahoo.co.in

Background: Tobacco use has been an important public health problem in India. Tobacco cessation is considered a cost effective intervention in controlling the tobacco epidemic in terms of preventable morbidity and mortality. Readiness to quit is one of the key steps in the process towards tobacco cessation. However, it is important to know the addiction pattern in the particular region so as to initiate de-addiction initiatives. So the current study was carried out with an objective to find the addiction habits and readiness to quit addiction among patients visiting GMERS Gotri General Hospital, a tertiary care hospital attached to Medical College in Gujarat, India.

Methods: All the patients at the registration desk of the hospital were randomly selected during May 2015. A total of 626 patients having any form of addiction were enrolled in this cross sectional study after taking written informed consent. They were interviewed in vernacular language using a validated semistructured questionnaire. After counseling them for quitting tobacco use, their readiness to quit was enquired and those willing were referred to de-addiction clinic in Psychiatry department. Data was entered and analysed using Epi Info Software.

Results: Out of 626 addictive patients registered, $97 \%$ were males, $50.8 \%$ in the middle age group and $59.3 \%$ from rural areas. The most common form of addiction was tobacco chewing $(66.4 \%)$ followed by smoking (32.7\%) and alcohol (19.4\%). Among them $428(68.4 \%)$ showed readiness to quit. Out of those ready to quit 
198 (46.2\%) were actually registered at the de-addiction clinic. Conclusions: Majority of patients were ready to quit addiction, so there is a need to have an ongoing program for awareness among patients about the de-addiction services already available within the hospital to improve quit rate. An awareness kiosk at the outpatient department can be explored in further studies.

Tob. Induc. Dis. 2018;16(Suppl 1):A315

DOI: 10.18332/tid/83920

\section{2:30-14:00}

PS-716-4 Pregnant smokers' views on babyClear ${ }^{\odot}$ : a package of measures including universal carbon monoxide monitoring and opt-out referral to support their quit

S Jones ${ }^{1}$, R Bell², V Araújo-Soares², E Milne ${ }^{3}$, A Rutter $^{4}, \mathrm{M}$ White $^{5}$, M Willmore $^{6}$, S Hamilton ${ }^{1}$

${ }^{1}$ Teesside University, School of Health and Social Care, Middlesbrough, United Kingdom, ${ }^{2}$ Newcastle University, Institute of Health and Society, Newcastle upon Tyne, United Kingdom, ${ }^{3}$ Newcastle City Council, Newcastle upon Tyne, United Kingdom, ${ }^{4}$ Fresh North East, Durham, United Kingdom, ${ }^{5}$ University of Cambridge, MRC Epidemiology Unit, Cambridge, United Kingdom, ${ }^{6}$ NHS England, Newcastle upon Tyne, United Kingdom. E-mail: ruth.bell@newcastle.ac.uk

Background and challenges to implementation: An intervention to encourage and support pregnant women to stop smoking by implementing National Institute of Health and Clinical Excellence guidance (2010) was introduced across north east England, a region with almost 30000 births per year.

Intervention or response: Comprised provision of equipment for universal, routine carbon monoxide (CO) monitoring, opt-out referral and enhanced follow-up. Positive impact was reported in Bell et al (2017). The current paper reports findings from qualitative interviews with pregnant smokers which aimed to ascertain the acceptability of the intervention. Women from the four areas where full implementation had taken place were interviewed individually face-to-face, initially at around 16 weeks of pregnancy $(n=17)$ and again $4-10$ weeks later $(n=8)$ or postpartum $(n=3)$. Interviews were recorded, transcribed then analysed thematically. Results and lessons learnt: Universal CO monitoring and opt-out referral appeared acceptable and the former motivated women to quit. When linked to a professional discourse of caring and concern, the intervention prompted women to take action. Where women continued to smoke or failed to attend smoking cessation services (SCS) appointments, they received a risk perception intervention (RPI), visually demonstrating the immediate, negative effects of $\mathrm{CO}$ on the foetus. Most women accepted the need for this hard-hitting approach, and although distressing, they expressed gratitude for it. Having this risk message personalised raised awareness and sometimes led to setting a quit date; however some resented the approach. Women generally found the RPI and enhanced follow up supportive and also benefited from greater family inclusion. Where maternity services were either less focused on prioritising the smoking cessation message, or less well integrated with SCS or where maternity staff were not as adept at delivering the RPI women found the intervention less acceptable.

Conclusions and key recommendations: The intervention proved to be acceptable to pregnant women smokers interviewed during and shortly after pregnancy. Wider application is recommended.

Tob. Induc. Dis. 2018;16(Suppl 1):A316

DOI:10.18332/tid/83923
12:30-14:00

PS-717-4 Analysis on current status and the evaluation criteria of smoking cessation clinics in China

Y Yang

${ }^{1}$ China CDC, Tobacco Control Office, Beijing, China. E-mail: yangyan8288@hotmail.com

Background: To understand the current situation of Chinese smoking cessation clinics and the situation of smokers who went to the clinic.

To develop a grading sheet for the evaluation criteria for smoking cessation clinic.

Methods: The data were collected by the registration and regular follow-up questionnaires which were finished by medical workers from smoking cessation clinics. Combine the questionnaire and interview results, as well as the discussing results from experts, the initial evaluation index for smoking cessation clinic could be formed. Third, Delphi method was used to revise the system and obtain score of each index in the system.

Results:

1. Among the 55 smoking cessation clinics which reported the one month follow-up data, there are only 7 clinics (12.73\%) reached the goal that registers are more than 200. The onemonth continuous quitting rate was $19.11 \%$ and three-month continuous quitting rate was $8.16 \%$.

2. The qualitative interviews study constructed a three-level evaluation index system of the smoking cessation clinic which is built on indicators of support, implement, and outcome. Then the system was revised by Delphi method. It contained three indicators of first-level, twenty-two indicators of second level and twenty-six indicators of third-level, then the weighting of each index is calculated. The experts participated in conducting this study had high activity and authority in their aspects. The indicators score of support, implement, and outcome are 0.86 , 0.87 and 0.84 respectively. And all of them are higher than 0.80 . The evaluation index is proved reasonable.

Conclusions: Currently, our country's smoking cessation clinics are still in the primary stage. The attention from responsible persons at all levels, the support of policy and funding.

The construction of the three-level evaluation criteria system of the smoking cessation clinic which is built on indicators of support, implement, and outcome is reasonable.

Tob. Induc. Dis. 2018;16(Suppl 1):A317

DOI:10.18332/tid/83933

\section{2:30-14:00}

PS-719-4 Depression symptoms and quitting among a nationally representative sample of smokers from Africa

F Goma', SC Kaai², J Ong'ang'o ${ }^{3}$, L Ikamari ${ }^{4}$, G Meng ${ }^{2}$, ACK Quah², GT Fong 2,5

${ }^{1}$ University of Zambia, School of Medicine, Centre for Primary Care Research, Lusaka, Zambia, ${ }^{2}$ University of Waterloo, Waterloo, ON, Canada, ${ }^{3}$ Kenya Medical Research Institute, Nairobi, Kenya, ${ }^{4}$ University of Nairobi, Nairobi, Kenya, ${ }^{5}$ Ontario Institute for Cancer Research, Toronto, ON, Canada. E-mail: fg882017@gmail.com

Background: There are mixed reports as to whether depression is associated with quitting among smokers, but almost all of these studies have been conducted in high income countries (HICs); nothing is known about this association in low- and middleincome countries (LMICs) including African countries. This is the 
first population-based study in Africa to examine the relationship between depression symptoms and key cessation variables-quit intentions and quit attempts.

Methods: Data were analyzed from the International Tobacco Control (ITC) Kenya Wave 1 (2012) and Zambia Wave 2 (2014) surveys of nationally representative samples of adult smokers $(\mathrm{N}=2,055)$. This study examined the relation between five symptoms of depression and quit intentions and quit attempts. Logistic regression analyses adjusted for sex, age, location, smoking status, and time-in-sample.

Results: $40 \%$ of Kenyan smokers and $51 \%$ of Zambian smokers had "ever" tried to quit smoking; $17 \%$ of Kenyan and 27\% of Zambian smokers planned to quit smoking within the next 6 months. Quit attempts were positively associated with 4 depressive symptoms: having a poor appetite $(\mathrm{OR}=2.43 ; 95 \%$ CI 1.61-3.67), not being able to control important things of life $(\mathrm{OR}=1.69 ; 95 \% \mathrm{CI} 1.15$ 2.48), feeling sad ( $\mathrm{OR}=1.59 ; 95 \% \mathrm{CI} 1.15-2.19)$, and feeling that people disliked them $(\mathrm{OR}=1.39 ; 95 \% \mathrm{CI} 1.05-2.85)$. Intending to quit was positively associated with 1 symptom-having a poor appetite (OR=1.76; 95\% CI 1.22-2.54). Moreover, being hopeful about the future was positively associated with both quit attempts $(\mathrm{OR}=1.50 ; 95 \% \mathrm{CI} 1.17-1.90)$ and intending to quit $(\mathrm{OR}=1.41$; 95\% CI 1.06-1.87). There were no significant differences between Zambia and Kenya.

Conclusions: In both Kenya and Zambia, depression symptoms were positively associated with quit attempts and quit intentions, consistent with findings from high-income ITC countries (Canada, United Kingdom, USA, Australia), suggesting that this association between depression and quit attempts/intentions is considerably broader than being limited to HICs.

Tob. Induc. Dis. 2018;16(Suppl 1):A318

DOI: $10.18332 /$ tid/83961

\section{2:30-14:00}

PS-720-4 Effect of healthcare practitioners' advice on the decision to quit. GATS analysis from Argentina

B Casetta ${ }^{1,2}$, J Konfino $^{3}$, AJ Videla

${ }^{1}$ Ministry of Health, National Tobacco Control Program, Buenos Aires, Argentina, ${ }^{2}$ Instituto Universitario CEMIC, Family Medicine, Buenos Aires, Argentina, ${ }^{3}$ Centro de Estudios de Estado y Sociedad (CEDES), Buenos Aires, Argentina. E-mail: brunilda. casetta@gmail.com

Background: Physicians have been paramount to lead the change in tobacco consumption worldwide. Healthcare team's advice on quitting has shown a small-sized but relevant effect. Argentine physicians have been found to smoke like the general population and adhere poorly to smoke-free rules in hospitals. The evidencebased National Guideline for Tobacco Cessation recommends doctors and nurses to give a short and strong advice to stop smoking. This study aimed to measure the quitting advice effect in real-life on the decision to do it.

Methods: Secondary analysis from the Global Adult Tobacco Survey (GATS) done in Argentina in 2012, restricted to adult smokers of 18 years old or more. Results from a populationbased study were analyzed using multivariable logistic regression modelling for the association of healthcare advice and a favorable attitude towards quitting (referring one quit attempt during last year, quitted less than a year ago or reported intention of quitting during the next month or year).

Results: Our sample included 1,717 smokers and recent quitters representing 6,431,623 (mean age 40.21 years, $37.77 \%$ women).
Favorable attitude was present in 26.15\% 95\%CI 20.53-32.67 (mean age 41.41 years, $35.86 \%$ females). During the last year, $46.82 \%$ consulted the healthcare system (51.06\% females). Smoking status were asked in $80.29 \%$ of cases and $76.51 \%$ received advice. Most of them referred 1 to 2 visits (59.58\%), 18.81\% 3 to 5 visits and $21.58 \% 6$ or more times. Multivariate analysis showed medical advice was strongly associated with a favorable attitude towards smoking cessation (OR 6.25 CI 95\% 2.91-13.42) after adjusting by age, gender, educational level and income.

Conclusions: In Argentina, healthcare practitioner's advice is strongly effective for promoting favorable attitude to quit regardless of age, gender, educational level and income. Advice was not systematically offered even after frequent visits. Additional strategies should be implemented to increase its delivery.

Tob. Induc. Dis. 2018;16(Suppl 1):A319

DOI:10.18332/tid/83975

\section{2:30-14:00}

PS-721-4 Impact of behavioural intervention to the predictors of quit success among smokers attending quit smoking services in Malaysia: 1month, 3-months and 6-months follow-up

LH Wee ${ }^{1}, B^{2} H_{0}^{2}$, A Ahmad ${ }^{3}$, A Mahdi $^{4}, K_{\text {K Perialathan }}^{5}$, AB Abdul Rahman $^{5}$, S Jagsingh ${ }^{6}$, MH Nik Mohammed ${ }^{7}$

${ }^{1}$ National University of Malaysia, Faculty of Health Sciences, Kuala Lumpur, Malaysia, ${ }^{2}$ Ministry of Health, Selangor, Malaysia, ${ }^{3}$ Ministry of Health, Perak, Malaysia, ${ }^{4}$ Ministry of Health, Sarawak, Malaysia, ${ }^{5}$ Ministry of Health, Kuala Lumpur, Malaysia, ${ }^{6}$ National Cancer Society of Malaysia, Kuala Lumpur, Malaysia, 7 International Islamic University Malaysia, Kuantan Pahang, Malaysia.E-mail: weeleihum@gmail.com

Background: Worldwide, success rates for quitting smoking have been stagnant for decades despite advancement in pharmacotherapy. This study aims to test the effectiveness of a current smoking cessation treatment programme which implemented the modified UK Standard Treatment Plan based on the NHS Centre for Smoking Cessation and Training (NCSCT). Methods: We conducted a double-arm, cluster-randomised controlled trial with 1-month, 3-months and 6-months follow-ups among newly registered smokers seeking quit smoking services from 19 public hospitals in Malaysia. Smokers were assigned to either NCSCT behaviour modification (treatment group; $\mathrm{n}=$ 330 ) or usual care (control group; $n=172$ ). The intervention was administered by dedicated health staff trained in the NCSCT. Data was collected through self-administered questionnaires, with validation conducted using expired air carbon monoxide measurement. Intention-to-treat analysis was adopted, with smokers who did not return for follow-ups considered smoking. Multiple logistic regression was conducted using a backward elimination model to assess the repeated measures treatment effect of quitting for each point of follow-up.

Results: No significant difference was discernible between the intervention and control group other than: first cigarette of the day $(p=0.05)$, urge to smoke $(p=0.020)$, method of quitting $(p=0.018)$ and COppm level $(p=0.0234)$ at baseline. Compared to the control group, individuals from the intervention group were more likely to quit successfully at 1-month, 3-months and 6-months followup $(\mathrm{OR}=1.698, \mathrm{OR}=3.395$, and $\mathrm{OR}=3.506$ respectively $)$. Older individuals were also more likely to abstain at 1-month, 3-months and 6-months follow-up $(\mathrm{OR}=1.015, \mathrm{OR}=1.022$ and $\mathrm{OR}=1.032$ respectively). Individuals who smoked at home were less likely to abstain at 1-month, 3-months and 6-months follow-up (OR= 
$0.637, \mathrm{OR}=0.592$ and $\mathrm{OR}=0.385$ respectively).

Conclusions: The modified Standard Treatment Programme appears superior to routine care practice in maintaining follow up sessions among smokers due to its structured and organized sessions.

Tob. Induc. Dis. 2018;16(Suppl 1):A320

DOI: $10.18332 /$ tid/83991

\section{2:30-14:00}

PS-722-4 Effects of promoting smoking cessation program in drug dependence disorder patients at the national institute on drug abuse treatment in Thailand

W Chaisalee', S Benjakul2 ${ }^{2}$ T Kengganpanich ${ }^{3}$, M Kengganpanich ${ }^{3}$ ${ }^{1}$ Princess Mother National Institute on Drug Abuse Treatment, Department of Medical Services, Ministry of Public Health, Muang Songkhla, Thailand, ${ }^{2}$ Mahidol University, Health Education and Health Promotion, Bangkok, Thailand, ${ }^{3}$ Mahidol University, Department of Health Education and Behavioral Sciences, Faculty of Public Health, Ratchathewi, Thailand. E-mail:dr.benjakul@gmail.com

Background: Drug dependence disorder patients are strongly associated with a high rate of smoking. Hospitalization aiming to drug abstinence will increase chance of quitting successfully. Moreover, smoking cessation intervention providing during hospitalization will also associated with the increased rate of long-term abstinence from any drug abuse.

Methods: This quasi-experimental research aimed to examine the effects of promoting smoking cessation program in drug dependence disorder patients at the national institute on drug abuse treatment in southern Thailand applying the Social Cognitive Theory. The samples comprised of 23 current smokers with drug dependence who were admitted at rehabilitation unit, at the institute. This program was conducted over a period of 8 weeks and composed of 6 activities including self-observation, judgment process, self-reaction, motivation, individual counseling and assigned the smoke-free environments in accordance with the laws. Data were collected by interviews at pre and post intervention on the 4th and 8th week. Statistics analysis was performed by using descriptive statistics and inferential statistics, i.e. Paired Sample t-test, Cochran's Q test and Friedman test.

Results: The results showed that the samples had significantly higher mean scores of perceived self-efficacy to quit smoking and outcome expectation of smoking cessation at post intervention on the 4th week ( $\mathrm{p} 0.05)$. In addition, 5 in 23 current smokers (21.7\%) and 9 in 23 current smokers (39.1\%) were able to quit smoking, at post intervention on the 4 th and 8 th week, respectively. Conclusions: The smoking cessation program can help smokers with drug dependence to stop smoking so it should further apply to any similar health service facilities.

Tob. Induc. Dis. 2018;16(Suppl 1):A321

DOI:10.18332/tid/84025

\section{$12: 30-14: 00$}

PS-723-4 Efficacy of short message service (SMS) in smoking cessation program among police personnel in Chennai city - an interventional study

P lyapparaja', PD Madan Kumar², I Nandabalan²

${ }^{1}$ Ragas Dental College, Public Health Dentistry, Chennai, India, ${ }^{2}$ Tamil Nadu Dr MGR Medical University, Public Health Dentistry, Chennai, India. E-mail: dr.iyapparaj@gmail.com

Background: Tobacco use is estimated to cause 800,000 deaths by 2015 in India annually.With changing patterns of lifestyle, smoking is falsely interpreted as a means to cope with negative emotions and to relieve mental stress. Research has shown that Policemen may consume tobacco at rates higher than the general population.

Text messaging is a means of reinforcement to tobacco cessation counseling which may favor smoking abstinence among police personnel and would provide an attractive, cost-effective method of intervention and follow ups. With no existing literature done in Indian population a randomized control study was conducted, aimed to assess the efficacy of tobacco cessation counseling with and without reinforcement through text messaging among policemen in Chennai city.

Methods: A concurrent parallel randomized control trial. A total of 100 Police personnel working within the jurisdiction of Chennai City were recruited using simple random sampling methodology. Two subzones were randomly selected from each zone. Participants in one arm were given intervention in the form of SMS text message in addition to regular tobacco cessation counseling and control arm were given regular tobacco cessation intervention in the form of counseling. Addiction level was measured by the 6 items scale of Fagerström Test for Nicotine Dependence (FTND).

Results: In the intervention group 32 participants expressed abstinence at baseline which decreased to 14 participants. As of the control group 29 participants abstained from smoking cigarettes at baseline which decreased to 5 participants at the end of the third month. Significant difference after one month $(p=0.008)$ and after 3 months $(p=0.02)$ was observed between the groups.

Conclusions: Intervention by SMS is considerably inexpensive, most people already possess mobile phone, and the messages were delivered spontaneously into real-life situations and have been proved as an effective way to deal with smoking dependence among police personnel in Chennai city.

Tob. Induc. Dis. 2018;16(Suppl 1):A322

DOI: $10.18332 /$ tid/84031

\section{2:30-14:00}

PS-724-4 The association between antitobacco advertising and calls to a tobacco Russian Quitline 0 Sukhovskaya ${ }^{1}$, P Yablonskiy ${ }^{2}$

'St Petersburg Research Institute of Phthisiopulmonology, Advisory Call Center for Smoking Cessation (Russian Quitline), St Petersburg, Russian Federation, ${ }^{2}$ St Petersburg Research Institute of Phthisiopulmonology, St Petersburg, Russian Federation.E-mail: ktcniif@mail.ru

Background: Russian Quitline was organized in 2011, National Tobacco Control mass media campaigns are held with 2012 to 2016, National Tobacco Control Law was passed in 2013. This study examines the effects of media campaign on telephone quitline for cessation services from May 2012 through May 2017 Methods: The campaign was primarily intended to encourage adult smokers aged 18-70 years to quit by making them aware of the health damage caused by smoking and letting them know that they could call the telephone if they needed free help to quit. The campaign included advertising on national and local cable television, local radio, billboards, print media. To determine the effects of the campaign on weekly quitline call volume were analyzed call immediately before, during, and immediately after the campaign period and compared them with data without antitobacco campaign periods.

Results: TV antitobacco National advertising during 1 month 
increased 6-7 times calls (from 3626 to 19515). The cumulative impact on Quitline calls remained significant for 6 months. Local TV antitobacco advertising increased the number of calls in 4 times. One anti-smoking program of National TV increased the number of appeal in 5 time during 2 day, Local TV - in 2,5 time in broadcast day and in 1,4 time in next day. Billboards with the slogan "Time to quit smoking" in the streets of St. Petersburg increased the number of initial applications in 3,2 times compared to the previous month and in 5 time - the same period of the previous year. Printed publications had less impact on the number of calls.

Conclusions: These results indicate that many smokers are interested in quitting and learning more about cessation assistance, and will respond to motivational messages that include an offer of help.

Tob. Induc. Dis. 2018;16(Suppl 1):A323

DOI:10.18332/tid/84036

\section{2:30-14:00}

PS-725-4 Effect of nurse-based brief intervention for tobacco cessation among admitted cardiac patients in a tertiary care hospital of Dhaka City, Bangladesh

S Choudhury ${ }^{1}$, S Jubayer ${ }^{1}$, A Al-Mamun ${ }^{1}$, F Malik $^{2}$

${ }^{1}$ National Heart Foundation Hospital and Research Institute, Dept. of Epidemiology and Research, Dhaka, Bangladesh, ${ }^{2}$ National Heart Foundation Hospital and Research Institute, Dept. of Cardiology, Dhaka, Bangladesh. E-mail: sohel_r_ choudhury@hotmail.com

Background and challenges to implementation: Global Adult Tobacco Survey (GATS), Bangladesh 2009 shows that $44.7 \%$ of men and $1.5 \%$ of women smoke, while $26.4 \%$ of men and $27.9 \%$ of women use smokeless tobacco. Only about $50 \%$ of smokers who visited physicians for consultation had advice for tobacco cessation in Bangladesh. Many cardiac patients continue to use tobacco even after hospital admission. A nurse based tobacco cessation service was introduced in National Heart Foundation Hospital for the first time in Bangladesh from 2015.

Intervention or response: 359 nurses were trained to deliver brief advice to tobacco user patients before discharge Tobacco use habit of all admitted patients were systematically documented in patient record form and all tobacco users were identified for a brief advice by nurse in the respective wards. All patients who received cessation advice were followed up over telephone after two and six months by nurses.

Results and lessons learnt: During the study period (Nov 2015 to Feb 2017), a total of 492 men and 30 women received cessation advice from nurses. Among them 374 were smoker and 118 were the smokeless tobacco user. Whether patients received lifestyle related advice from consulting physicians were not explored. At six-month follow-up 199 (39.9\%) patients reported that they have quitted. Among smoking tobacco user, 132 (35.3\%) patients quitted and among smokeless tobacco user, 65 (55\%) patients quitted tobacco.

Conclusions and key recommendations: A large proportion of patients who received brief tobacco cessation advice remained tobacco free at six months after discharge from hospital. As people are more receptive to advice by health care providers during hospitalization period, a nurse based systematic cessation intervention may have a good impact on quitting tobacco.

Tob. Induc. Dis. 2018;16(Suppl 1):A324

DOI:10.18332/tid/84043
12:30-14:00

PS-726-4 Integrating worksite smoking cessation services into the construction trade: opportunities and challenges

T Asfar', A Caban-Martinez', LA McClure', Y Xuan', G Jr, Clark', K D. Ward'2, NA Dietz ${ }^{1,3}$, D J. Lee ${ }^{1}$

'University of Miami, Miller School of Medicine, Miami, FL, United States of America, ${ }^{2}$ University of Memphis, Memphis, TN, United States of America, ${ }^{3}$ Broward Health, Fort Lauderdale, FL, United States of America. E-mail: tasfar@med.miami.edu Background: Almost 39\% of US construction workers (CWs) smoke. Hispanic/Latino CWs represent $23 \%$ of the US CWs, and they have lower access to cessation services mainly due to their transient and lack of culturally-sensitive intervention. One potential strategy is the provision of a work-based culturallytailored smoking cessation services. This study aimed to gain insight into the construction site management's current practices related to anti-smoking policies and smoking cessation services. It also sought views on "ideal" workplace cessation services, implementation commitment, and anticipated challenges.

Methods: We conducted semi-structured, 45-minute interviews with 25 key personnel at five construction sites in South Florida. Interviews were recorded, transcribed and analyzed thematically using Braun's six phases approach.

Results: Participants were all males and represented a range of professions including construction executive, senior/safety manager, and contractor/subcontractor. Smoking was prohibited only inside the construction buildings and participants perceived most workers as adherent to the policy. Although employers were concerned about the effects of smoking on productivity loss, safety, and insurance costs, nothing was provided to help smokers quit smoking. Only one-quarter of participants believed that worksite management would support implementing smoking cessation services. The majority considered distributing selfhelp materials with free medications as the most appropriate service and recommended providing the service in Spanish during lunch/breakfast breaks. Challenges to integrate services were smokers' low interest in quitting, time restriction, and cost. Recommendations to support implementing services were local or state government mandate, obtaining company approval, involving safety managers and subcontractors in the process, and providing services during employment orientation training.

Conclusions: Tailoring sustainable onsite smoking cessation services in the construction sector presents unique, but not insurmountable challenges. Involvement of key stakeholder groups including policymakers as well as construction management, supervisors, and subcontractors is necessary to support integrating smoking cessation services in this setting.

Tob. Induc. Dis. 2018;16(Suppl 1):A325

DOI:10.18332/tid/84050

\section{2:30-14:00}

PS-727-4 Tobacco treatment education for health care professionals in behavioral health settings in Kansas, USA

B Faseru'1, A Grodie ${ }^{1}$, K Richter $^{1}$

'University of Kansas Medical Center, Preventive Medicine and Public Health, Kansas City, KS, United States of America. E-mail: bfaseru@kumc.edu

Background and challenges to implementation: Prevalence of tobacco use and mortality from tobacco-related diseases are disproportionately higher among individuals with mental health 
and substance use disorders compared to the general population. In the U.S., people with serious mental illnesses die on average 25 years younger than other Americans, and much of this mortality is due to tobacco use. Some mental health care providers are reluctant to treat and some lack the training on how to effectively treat tobacco dependence, and how to use available resources to get smokers with mental illness and substance use disorders the longitudinal counselling, medications, and follow-up they need to be tobacco free.

Intervention or response: We provided 1) an accredited Tobacco Treatment Specialist (TTS) Training that includes an 8-hour online training and a $3 \frac{1}{2}$ day in-person core training that covers brief intervention, intensive counselling, cessation medications, follow up, and clinic systems change 2) Post-training monthly conference calls to work with TTSs on challenging cases and share current information. Additionally, all TTSs received 18-months paid membership of the Association for the Treatment of Tobacco Use and Dependence (ATTUD).

Results and lessons learnt: To date, thirty-nine participants with an average of 11 years of experience working in behavioral health programs are enrolled in the program. Less than half of the participants (16) held a supervisory role. Eleven of the participants were males and 28 were females. The median pretest treatment knowledge score was $60 \%$; following training the median posttest score was $80 \%$. Final comprehensive exams were taken within 6 weeks of the training and the average score was $86 \%$. Over $98 \%$ of the participants were very satisfied with the course and $98 \%$ of participants indicated their ability to deliver effective tobacco treatment significantly increased.

Conclusions and key recommendations: Portable accredited training program, on-going professional support and connection of trainees to a professional link serve are essential for dissemination and adoption of best practices.

Tob. Induc. Dis. 2018;16(Suppl 1):A326

DOI: $10.18332 /$ tid/84061

\section{2:30-14:00}

PS-728-4 Reducing financial barrier of smoking cessation treatment can effectively enhance prolonged abstinence: an experience from Taiwan C Lai ${ }^{1,2}$, B Chen ${ }^{2}$, M-D Liu², Office for Smoking Cessation Service, Taiwan

'Taipei Veterans General Hospital, Department of Family Medicine, Taipei, China, ${ }^{2}$ Office for Smoking Cessation Service, Taipei, China. E-mail: chihkuan.lai@gmail.com

Background and challenges to implementation: Taiwan has established nationwide smoking cessation treatment service which was founded by the revenue of cigarette taxation since 2002. The government reimburses physician counseling and subsidizes pharmacotherapy for adult smokers attempting to quit. The initial subsidy, 250 Taiwan dollars per week, accounted for only $20-35 \%$ cost of effective treatment. Lack of financial incentive was commonly recognized as a major barrier for smokers to use service.

Intervention or response: In March 2011, the government introduced a new policy to reduce financial barrier to cessation service. Smoker was responsible for $20 \%$ of drug cost, with a ceiling copayment of 200 Taiwan dollars for each prescription (1- to 4 -week ). This observational study aims to evaluate the effectiveness of new financing policy on service utilization and cessation outcome among adult smokers. We compare the selfreported 7-day point abstinence and 30-day prolonged abstinence by telephone interview among participants from 2008-2014.

Results and lessons learnt: A total of 582,281 smokers enrolled in cessation service from 2008-2014. Of them, 174,844 received telephone interview and 107,358 (61.4\%) completed the interview. After introducing new financing policy, the number of smokers enrolling in cessation treatment service increased 42.3\% between Mar-Dec 2011 and Mar-Dec 2012. The 30-day prolonged abstinence rates at 6 months significantly increased from $21.3 \%$ to $25.4 \%$ (difference $=4.1 \%, 95 \%$ CI $3.5 \%-4.6 \%$ ) after implementing new policy, with an adjusted $\mathrm{OR}=1.26(95 \% \mathrm{CI}$ 1.21-1.30). The 7-day point abstinence increased from $23.0 \%$ to $27.0 \%$ (difference $=4.0 \%, 95 \%$ CI $3.4 \%-4.6 \%$ ), with an adjusted $\mathrm{OR}=1.24$ (95\% CI 1.20-1.28).

Conclusions and key recommendations: Cessation treatment is an effective intervention for addictive smokers. Access to treatment and cessation outcome can be significantly promoted by reducing financing barrier. Smoker's affordability and willingness-to-pay should be evaluated in offering cessation service.

Tob. Induc. Dis. 2018;16(Suppl 1):A327

DOI: $10.18332 /$ tid/84087

$12: 30-14: 00$

PS-729-4 Reduction of tobacco consumption in a rural area with a community based tobacco control program in Bangladesh

J Ahmed ${ }^{1}$, SR Choudhury', M Zaman²

${ }^{1}$ National Heart Foundation Hospital and Research Institute, Dhaka, Bangladesh, ${ }^{2}$ Ekhlaspur Center of Health (ECOH), Chandpur, Bangladesh.E-mail: ahmedjasim1@gmail.com

Background: Bangladesh has very high tobacco use rate. Various efforts by the Government and non-government organizations are being made to reduce the tobacco consumption both at national and local levels. However, information is scare about the impact of these efforts on tobacco consumption in Bangladesh.

Methods: Ekhlaspur Center of Health (ECOH), non-government health service provider in a village has been conducting antitobacco campaigns for awareness raising and tobacco cessation program through community health workers since 2008 . Two cross-sectional surveys in 2011 and 2013 with similar methods were done to see the changes in tobacco use in the village.

Field interviewers visited all households of the village and randomly selected one adult aged 20 years or more from each household as respondents. Both smoking and smokeless tobacco use habit were obtained by an interviewer administered questionnaire.

Results: Over all prevalence of tobacco use were $47.7 \%$ and $34.6 \%$ in 2011 and 2013 respectively. Prevalence of smoking declined to $12.7 \%$ in 2013 from $22.7 \%$ in 2011. Smokeless tobacco uses also declined to $23.9 \%$ in 2013 from $30.0 \%$ in 2011 . In men smoking prevalence reduced to $24.0 \%$ in 2013 from $36.1 \%$ in 2011 . In men smokeless tobacco uses increased to $19.5 \%$ in 2013 from $14.6 \%$ in 2011 where as in female reduced to $28.1 \%$ in 2013 from $29.4 \%$ in 2011 .

Conclusions: Tobacco use decreased markedly in this rural community through aggressive anti-tobacco campaigns. Changes are likely to reflect the impact of continuous anti-tobacco campaign by local NGO as well as national government efforts.

Tob. Induc. Dis. 2018;16(Suppl 1):A328

DOI:10.18332/tid/84091

12:30-14:00

PS-730-4 Moving towards a tobacco free workplace at a cement manufacturing plant in Chandrapur, Maharashtra 


\section{G Mandal' ${ }^{1}$ H Gupte ${ }^{2}$, V Thawal' ${ }^{2}$, L Chaudhuri²}

${ }^{1}$ Salaam Bombay Foundation, Mumbai, India, ${ }^{2}$ Narotam Sekhsaria Foundation, Mumbai, India. E-mail: gauri.mandal@salaambombay. org

Background: According to Global Adult Tobacco Survey -2, 267 million adults consume tobacco in some form in India and $75 \%$ of them use smokeless tobacco. Smoking is banned in India in public places including indoor workplaces under the Cigarettes and Other Tobacco Products Act (COTPA). However, there are no regulations for smokeless tobacco use, the most prevalent form. Workplaces offer a unique opportunity to address employees' health and influence their tobacco use behavior.

LifeFirst tobacco cessation service was provided at a cement plant with about 2000 employees in Chandrapur, Maharashtra with the objective of promoting and aiding quit attempts of tobacco users. Methods: An awareness talk about tobacco and its ill effects and benefits of quitting was provided in groups to 1764 employees (all males). Employees voluntarily registered for the cessation service which included a detailed face to face counseling session followed by four follow up sessions over six months. Tobacco use status was recorded at each session. In addition to this, trainings for "Anti-tobacco champions" and the Medical Team were conducted for incorporating the initiative into the company's Occupational Health activities.

An announcement was made by the plant head to make the plant tobacco free within a year.

Results: 648 employees voluntarily registered for the cessation service. 607 (94\%) of these were smokeless tobacco users, majority (77\%) of them using the local tobacco product "kharra" (mixture of tobacco and areca nut). 552(85\%) attended the follow up session at 6 months and $438(68 \%)$ of all registered users reported not using any form of tobacco, $50(8 \%)$ had reduced use, $60(9 \%)$ had made a quit attempt but relapsed.

Conclusions: By providing tobacco cessation activities within a workplace, employees using smokeless tobacco as well as smoking can be helped to quit their tobacco habit and create an environment conducive to a tobacco free workplace.

Tob. Induc. Dis. 2018;16(Suppl 1):A329

\section{DOI:10.18332/tid/84094}

\section{2:30-14:00}

PS-731-4 Tobacco in my speciality - a pocket guide for specialists

H Gilljam', A Landgren², G Boëthius ${ }^{1}$

${ }^{1}$ Karolinska Institutet, Stockholm, Sweden, ${ }^{2}$ University of Gothenburg, Sahlgrenska, Göteborg, Sweden. E-mail: hans. gilljam@ki.se

Background and challenges to implementation: In spite of the enormous disease burden caused by tobacco use most doctors fail to inspire or help their smoking/snus using patients to stop.

Even when agreed that stopping smoking is the only way to ease symptoms, stop disease progression or decrease mortality, smoking cessation is not the first choice in the clinical toolbox. The reasons for this ineptitude may range from lack of knowledge about disease causality to lack of training or tools of helping patients to stop using tobacco.

Intervention or response: We developed a pocket guide with condensed updated knowledge of what is known about tobacco use (mainly smoking) in 6 different specialities. Our first guides were written for general practice, orthopaedics, ophthalmology, rheumatology, dermatology and gastro-intestinal diseases. The text varies between disciplines according to the strength of the available evidence. The guides zoom in on the role of tobacco and are sectioned as follows: a short introduction, causality, current awareness of tobacco-related causality, evidence of causality, current treatment strategies, effects of cessation, references. Less than half of the available space is text (7-800 words) and the rest shows the distinctive front, illustrations, logotypes etc. The complete content can be downloaded and printed double sided on a standard A4 sheet.

Results and lessons learnt: The Swedish Medical Association sponsored and distributed the first batch of pocket guides and The Swedish Society of Medicine and the NGO Doctors Against Tobacco have since supported the project. National specialist organisations have endorsed the project. A pilot study will be reported.

Conclusions and key recommendations: A very compact and easy to read pocket folder may serve as a reminder for clinicians of the role of tobacco in their speciality and initiate cessation.

Tob. Induc. Dis. 2018;16(Suppl 1):A330

DOI: $10.18332 /$ tid/84100

\section{$12: 30-14: 00$}

PS-732-4 Impact of an online training program for brief intervention on smoking cessation for health care workers in Bolivia, Guatemala and Paraguay

C Martinez ${ }^{1}$, M Margalef ${ }^{1}$, MA Arrien² ${ }^{2}$ C Sánchez ${ }^{3}$, P Caceres ${ }^{4}$, J Barnoya ${ }^{4}$, Y Castellano' ${ }^{1}$ E Fernández', Group of hospital coordinators in the Fruitful study

${ }^{1}$ Catalan Institute of Oncology (ICO), Bellvitge Biomedical Research Institute (IDIBELL), Tobacco Control Unit, L'Hospitalet del Llobregat, Spain, ${ }^{2}$ Instituto Oncologico del Oriente Boliviano de Santa Cruz de la Sierra, Santa Cruz de la Sierra, Bolivia, ${ }^{3}$ Ministerio de Salud y Pública y Bienestar Social, Public Health Department, Asunción, Paraguay, ${ }^{4}$ nstituto de Cancerología y Hospital Dr. Bernardo del Valle, Radiation Oncology Department, Guatemala, Guatemala. E-mail: cmartinez@iconcologia.net

Background: Tobacco cessation training programs are scarce in Spanish speaking low-income countries. Based on a previous program developed in Spain, the Fruitful Study adapted, implemented and evaluated the effectiveness of an online brief intervention smoking cessation training program addressed to hospital workers from Bolivia, Guatemala and Paraguay. The aim of this study is to examine the degree of implementation of the $5 \mathrm{~A}$ brief intervention model before and after the training and identify changes in cognitive, behavioral factors and in the perception of organizational support.

Methods: Pre-post evaluation through a questionnaire that evaluated 43 items previously identified in the literature. The questionnaires were completed immediately before and six months after the course. To examine pre-post changes in scores, the non-parametric test for paired data (Wilcoxon) was used.

Results: 202 professionals completed the questionnaires before and after the course. For this analysis, only those with clinical tasks $(\mathrm{n}=154)$ were selected $(57.1 \%$ were Doctors, $31.2 \%$ were Nurses, $11.7 \%$ were Other professionals). Overall, significant increases were achieved in all components of the brief intervention [Ask (5.5 to 7.6); Advise (5.9 to 8.0); Assess (5.0 to 7.1); Assist (2.7 to 5.8); and Arrange a follow up (1.8 to 5.1); $\mathrm{p}<0.001$ for all components]. Doctors and Other Health professionals obtained higher scores compared to Nurses. By country, the health workers from Paraguay obtained higher scores. Overall, the perception of the degree of preparation, preparedness in the management of 
medication, level of competence and, familiarity with resources such as quitlines, Internet, etc, increased $(\mathrm{p}<0.001)$.

Conclusions: The online training had a positive impact on the implementation of the brief intervention in the three countries. Online education in tobacco cessation is feasible and effective to improve evidence-based treatment for tobacco dependence in these countries

Tob. Induc. Dis. 2018;16(Suppl 1):A331

DOI:10.18332/tid/84106

\section{2:30-14:00}

PS-733-4 Smoking cessation therapy in Ethiopia: responsiveness and the predictors

N Dereje $e^{1,2}$

'Wachemo University, Public Health, Hosanna, Ethiopia, ${ }^{2}$ Nigist Elleni M/M/Hospital, Hosanna, Ethiopia.E-mail:neba.jahovy@ gmail.com

Background: Tobacco is one of the leading public health burdens globally. The health system and healthcare providers can play a major role in promoting smoking cessation to their patients. However, the Ethiopian health system's responsiveness towards smoking cessation therapy is not well understood. Therefore study was conducted to assess the health care delivery system's responsiveness to smoking cessation services and its associated factors among health care providers in Ethiopia.

Methods: A cross sectional study employing both quantitative and qualitative methods was conducted to determine the health worker's knowledge, attitude, and practice towards smoking cessation therapy. For these, 323 participants were selected from five health professional groups: medical doctors, dentists, health officers, nurses, and midwives. A two-stage stratified sampling technique was employed to select the districts and the health workers. Data was collected by using self-administered questionnaires and descriptive statistics, bivarate and multivariate analysis were done.

Results: Majority of the health care providers had poor knowledge (70.4\%) and had negative attitude (86\%) towards smoking cessation intervention. Moreover, almost all of the health care providers 306(97.5\%) had below average level of practicing smoking cessation intervention. In the multivariate analysis; being female, receiving training, having good knowledge score, having positive attitude were associated with the above average practice level of smoking cessation intervention.

Conclusions: The Ethiopian health delivery system's responsiveness for the provision smoking cessation therapy is premature. The health care providers (HCPs) were with poor knowledge, negative attitude and below average practice level. The HCPs had no access to training and organizational supports. Therefore, the ministry of health should give due emphasis to smoking cessation intervention particularly the tobacco dependence treatments (pharmacological therapy) should be introduced to the health care system and the existing behavioral intervention should be strengthened by providing training for the health care providers.

Tob. Induc. Dis. 2018;16(Suppl 1):A332

DOI:10.18332/tid/84110

\section{2:30-14:00}

PS-734-4 Predictors of intention to quit smokeless tobacco among adults in pastoral communities of Borena Zone, South Ethiopia

E Etu $^{1,2}$, M Hussen $^{3}$

${ }^{1}$ Madda Walabu University, Goba Refferal Hospital, Goba,
Ethiopia, ${ }^{2}$ Makerere University School of Public Health-Centre for Tobacco Control in Africa, Public Health, Kampala, Uganda, 3Jimma University, Health Education and Behavioral Science, Jimma, Ethiopia.E-mail: esetu912008000@ymail.com

Background: Smokeless tobacco (SLT) covers various types of tobacco products used in un-burnt form mainly orally or nasally worldwide. Smokeless tobacco products contain nicotine, which is addictive and contains over 30 carcinogens. Thus, its use associated with increased risk of multiple health problems. Assessing readiness to quit and its predictors provides reliable data to tobacco control and prevention efforts. Ethiopia is a party of the framework convention on tobacco control (FCTC). However, there were no studies that assessed intention to quit SLT use. Thus, this study assessed intention to quit SLT use and associated factors among adults.

Methods: A community based cross sectional study was conducted among 810 randomly selected adults in three districts of Borena Zone from January 1 to 20/2016 The data were collected using interviewer administered structured questionnaire. Multivariable logistic regression analyses were employed to identify factors associated with intention to quit smokeless tobacco use.

Results: Among 810 adults, $33.5 \%$ had intention to quit smokeless tobacco use. Age 65 and above $[\mathrm{AOR}=0.35,95 \% \mathrm{CI}: 0.14$, $0.89]$, past quit attempt [AOR=3.16, 95\% CI: $2.21,4.53]$, health professionals advise to quit smokeless tobacco use $[\mathrm{AOR}=1$. 61, 95\%CI: 1.13, 2.30], and health effects awareness [AOR= $1.29,95 \% \mathrm{CI}: 1.14,1.46]$ were associated with intention to quit smokeless tobacco use.

Conclusions: Moderate number of adults had intention to quit smokeless tobacco use. This study depicts the need to raise adults' health effect awareness and encouraging health professionals to give advice to their smokeless tobacco users.

Tob. Induc. Dis. 2018;16(Suppl 1):A333

DOI:10.18332/tid/84119

\section{2:30-14:00}

PS-735-4 Does free or lower cost smoking cessation medication stimulate quitting? Findings from the International Tobacco Control (ITC) Netherlands and United Kingdom Surveys

FA Van den Brand', GE Nagelhout t, ${ }^{2,}$ K Hummel $^{4}$, MC Willemsen ${ }^{4}$, A McNeill', CP Van Schayck'

${ }^{1}$ Maastricht University, Family Medicine, Maastricht, Netherlands, ${ }^{2}$ Maastricht University, Health Promotion/Family Medicine, Maastricht, Netherlands, ${ }^{3}$ IVO Addiction Research Institute, Rotterdam, Netherlands, ${ }^{4}$ Maastricht University, Health Promotion, Maastricht, Netherlands, ${ }^{5}$ King's College London, Addictions Department, London, United Kingdom. E-mail: f.vandenbrand@maastrichtuniversity.nl

Background: Free or lower cost cessation medication is reported more often as a trigger to think about quitting by smokers with low education and/or income, but it is not clear whether this leads to actual quitting of smoking. The aim of this study was to investigate whether reporting free medication as trigger is related to medication use, reported quit attempts and quit success and whether this association is modified by education and income.

Methods: Data were derived from the 2013 and 2014 surveys of the International Tobacco Control (ITC) Netherlands $(n=1164)$ and United Kingdom ( $n=768)$ cohort. From the 2013 survey, current smokers were selected who also participated in the 2014 follow-up survey. Logistic regression analyses were used to assess associations between free medication as a trigger to think about 
quitting in 2013 and the use of smoking cessation medication, quit attempts and smoking cessation in 2014.

Results: $37.0 \%$ of smokers in the UK and $24.9 \%$ of smokers in the Netherlands reported free or lower cost medication as trigger to think about quitting. Smokers who reported this trigger were more likely to have used cessation medication during a quit attempt both in the $\mathrm{UK}(\mathrm{OR}=4.19, \mathrm{p}<0.001)$ and in the Netherlands $(\mathrm{OR}=2.14, \mathrm{p}=$ 0.033). The association between free medication as trigger to think about quitting and quit attempts was significant in the UK $(\mathrm{OR}=$ $1.45, \mathrm{p}=0.030)$, but not in the Netherlands $(\mathrm{OR}=1.10, \mathrm{p}=0.587)$. There was no significant association with quit success. Associations did not differ across income and education groups.

Conclusions: Free or lower cost smoking cessation medication may stimulate quit attempts and increase the use of cessation medication among smokers with low, moderate, and high socioeconomic status.

\section{Tob. Induc. Dis. 2018;16(Suppl 1):A334}

DOI:10.18332/tid/84125

\section{2:30-14:00}

PS-736-4 Efficacy and safety of V. cinerea (L.) less. for smoking cessation: a systematic review and meta-analysis of randomized controlled trials P Puttarak ${ }^{1}$, K Bunditanukul ${ }^{1}$, Panupong Puttarak ${ }^{1 *}$, Patarachai Pornpanyanukul ${ }^{1}$, Thunyaluk Meetam² ${ }^{2}$ Katha Bunditanukul ${ }^{3}$, Nathorn Chaiyakunapruk 4,5,6,7*

'Prince of Songkla University, Department of Pharmacognosy and Pharmaceutical Botany, Hat Yai, Thailand.E-mail: bkkatha.s@ gmail.com

Background: To determine the efficacy and safety of Vernonia cinerea (L.) Less. for smoking cessation using a systematic review and meta-analysis approach.

Methods: Data sources: Nine databases were searched through April 2017.

Study selection: Randomized controlled trials that reported the clinical effect of V. cinerea. for smoking cessation were included. Data extraction: Important data and outcomes were extracted by two independent researchers. Study quality was assessed using the Cochrane risk of bias tool and JADAD score.

Results: Data synthesis: Five trials with 347 active smokers were included. Two trials were rated as low risk of bias in all domains while others were rated as some concerns. V. cinerea treatment group was associated with significant cessation rate higher than that in the control group with no evidence of heterogeneity for both continuous abstinence rate (CAR) (week 8 risk ratio (RR): 1.69, 95\% CI [1.00, 2.86]; week 12 RR: $2.18,95 \%$ CI [1.17, 4.04]) and 7-day point prevalence abstinence rate (PAR) (week $8 \mathrm{RR}$ : $1.51,95 \%$ CI [1.01, 2.27]; week 12 RR: $1.93,95 \%$ CI [1.24, 2.99]) at week 8 and 12 , respectively. There was no significant difference of all adverse events between treatment and control groups.

Conclusions: This comprehensive systematic review and metaanalysis suggests that V. cinerea might be considered as a potential alternative for smoking cessation treatment. Further welldesign RCTs of standardized V. cinerea compared with standard treatment should be conducted to strengthen this evidence.

Tob. Induc. Dis. 2018;16(Suppl 1):A335

DOI:10.18332/tid/84129

12:30-14:00

PS-737-4 Process and outcome evaluation of a novel online-only Tobacco Treatment Specialist (TTS) training program

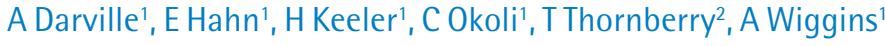
${ }^{1}$ University of Kentucky, College of Nursing, Lexington, KY, United States of America, ${ }^{2}$ Morehead State University, Psychology, Morehead, KY, United States of America. E-mail: audrey.darville@uky.edu

Background: Competency standards for tobacco treatment specialists and training have been formalized by the Association for Treating Tobacco Use and Dependence (ATTUD). To increase access to formalized TTS training in remote regions, we developed a novel online-only format incorporating video technologies. The purposes of the study were to: 1) evaluate and compare knowledge and skill acquisition using synchronous video-conferencing versus uploaded video content reviewed asynchronously; and 2) assess the fidelity of evaluating tobacco treatment specialist trainee knowledge and skills using standardized case presentations.

Methods: An observational pilot study design was used to evaluate the effectiveness of synchronous versus asynchronous online evaluation strategies. Twenty trainees were randomly assigned to synchronous (Zoom; $\mathrm{n}=11$ ) and asynchronous (YouTube; $\mathrm{n}=9$ ) groups to demonstrate skills using a standardized case scenario. All trainees then completed a written case study. Two independent raters evaluated the groups using a rubric and checklist.

Results: There were no significant differences in total mean scores between the two groups using the rubric. Intra-class correlation coefficient (ICC) between raters for total rubric scores were 0.68 in the synchronous group and 0.33 in the asynchronous group. For the checklist, trainees in the synchronous group had higher mean scores (19.1) than the asynchronous group (16.6; $\mathrm{p}=.03$ ). Checklist ICC scores differed significantly between groups; 0.80 for synchronous and 0.41 for asynchronous. For the written case, there was a larger, but non-significant, increase in scores for the asynchronous group (1.5 versus 0.3 points). All participants achieved a passing written case score.

Conclusions: There was moderate to strong agreement between raters when using the synchronous method while the asynchronous method yielded weak agreement. Synchronous (Zoom) evaluation yielded higher mean assessment skill scores than asynchronous (YouTube) assessment. The synchronous evaluation trended toward lower variability in scores when compared to the written case; the standard evaluation used for all participants.

Tob. Induc. Dis. 2018;16(Suppl 1):A336

DOI: $10.18332 /$ tid/84131

\section{2:30-14:00}

PS-740-4 Potential for sustainability of strategies for implementing tobacco use treatment guidelines in the Vietnam public healthcare system: qualitative post intervention assessment

N Van Devanter', M Vu' ${ }^{2}$ N Nguyen ${ }^{3}$, T Nguyen ${ }^{3}$, D Shelley ${ }^{4}$ ${ }^{1}$ New York University, Rory Meyers College of Nursing, New York, NY, United States of America, ${ }^{2}$ Emory University, Behavioral Sciences and Health Education, Atlanta, GA, United States of America, ${ }^{3}$ Institute of Social and Medical Studies, Hanoi, Viet Nam, ${ }^{4}$ New York University School of Medicine, Population Health, New York, NY, United States of America. E-mail: nvd2@ nyu.edu

Background: Tobacco cessation services are not widely available in low middle-income countries (LMICs) where smoking rates remain high. In Vietnam $45 \%$ of males are current smokers. We recently completed a two-arm randomized control trial comparing the effectiveness of two strategies for implementing tobacco use treatment (TUT) guidelines in community health centers (CHCs) 
in Vietnam. All sites received training and patient education materials. Intervention sites received a system for referring smokers to a village health worker (VHW) for multisession counseling. Guided by the Consolidated Framework for Implementation Research (CFIR), we conducted a post-intervention qualitative analysis of factors that facilitate or challenge implementation and sustainability of screening and treatment of TU in CHCs in Vietnam.

Methods: Semi-structured interviews $(\mathrm{N}=42)$ were conducted with healthcare staff and VHWs in $18 \mathrm{CHCs}$. Interviews were transcribed verbatim and translated. Two investigators analyzed transcripts using an inductive and deductive approach to develop codes and identify themes relevant to the study research questions.

Results: Interviews explored five domains of the CFIR (Intervention characteristics, Outer and Inner settings, Characteristics of individuals, and Process). Facilitators of program implementation included perceived importance of and need for smoking cessation services, ease of integrating the TUT intervention into routine care, and a high level of satisfaction with the quality of intervention components. Challenges to implementation included patient barriers (e.g., not ready to quit) and lack of a referral resource in comparison sites. The main challenge to sustainability is competing Ministry of Health $(\mathrm{MOH})$ priorities that result in a relative lack of resources for TUT (e.g., training/materials) compared with other health programs.

Conclusions: A robust planning process resulted in a good fit between current practice and the implementation strategies used to increase adoption of TUT guidelines in CHCs. Sustainability will depend on $\mathrm{MOH}$ commitment of resources, including ongoing training and integration with other programs.

Tob. Induc. Dis. 2018;16(Suppl 1):A337

DOI:10.18332/tid/84150

\section{2:30-14:00}

PS-741-4 Smoking cessation intervention programme in four secondary boys' schools in Malaysia

SJ Jag Singh', M Abdul Razak', W Lei Hum², MH Nik Mohamed', S Lee ${ }^{1}$, S Somasundaram ${ }^{1}$

${ }^{1} T h e$ National Cancer Society Malaysia, Kuala Lumpur, Malaysia, ${ }^{2}$ National University of Malaysia, Faculty of Health Sciences, Kuala Lumpur, Malaysia, ${ }^{3}$ International Islamic University Malaysia, Kuantan, Malaysia.E-mail:swinder@cancer.org.my Background and challenges to implementation: Smoking among Malaysian secondary school students is on the rise especially among boys between 13-15 years old. There are no proven sustainable school-based intervention programmes to tackle this issue. Hence, a 6 -month smoking cessation programme at 4 boys' schools in urban Kuala Lumpur was conducted. The initial challenges faced during the implementation of this programme include lack of cooperation from school personnel, inadequate time, high absenteeism among students and inability to prescribe pharmacotherapy. The recruitment process consists of a talk and screening using the CO breath analyzer. 143 identified smokers were offered to voluntarily participate with 128 non-smoking support buddies recruited. Weekly visits were scheduled during the first month, followed by once a fortnight for the 2nd and 3rd months, and once a month for the last 3 months. Group counselling was conducted during each visit with interactive activities including quizzes, video case studies, essay writing and games. Smokers with CO level $>10 \mathrm{ppm}$ and unable to quit after the 6 th counselling session were counselled individually.
Intervention or response: The team worked with the Ministry of Health to train school counsellors in smoking cessation. WhatsApp groups were created to provide off-site support, reminders and problem solving. Vitamin $\mathrm{C}$ tablets were given instead of pharmacotherapy and special attention was given to the heavy smokers. The team worked closely with the school counsellors, discipline teachers and buddies in making informed decisions.

Results and lessons learnt: $\mathrm{CO}$-verified smoking cessation rate at 6-month follow-up was $64.0 \%$. Four students had transferred. Those who continued to smoke were recruited into the 2017 programme. Frequent school visits, face-to-face support, utilizing social media, involvement of the counsellors and rewards were supportive factors of the programme's success rate.

Conclusions and key recommendations: High smoking cessation rate can be achieved via an integrated programme.

Tob. Induc. Dis. 2018;16(Suppl 1):A338

DOI: $10.18332 /$ tid/84174

$12: 30-14: 00$

PS-742-4 The effectiveness of the smoking cessation telephone quitline service in Clalit Health Services, Israel

D Levin-Zamir ${ }^{1}$, A Yom-Tov¹, S Gan-Noy¹, G Verber², C Kay ${ }^{3}$ ${ }^{1}$ Clalit Health Services, Health Promotion and Education, Tel Aviv, Israel, ${ }^{2}$ Clalit Health Services, Department of Medicine, Tel Aviv, Israel, ${ }^{3}$ Clalit Health Services, Nursing Department, Tel Aviv, Israel. E-mail: anatyo1@clalit.org.il

Background and challenges to implementation: Smoking cessation workshops were added to the Israel Basket of Health Services in 2010, however a quitline service wasn't included. In 2013 Clalit Health Services established a pilot project testing a proactive counseling quitline. The service includes six individual counseling provided by cessation counselors, offered in five languages, tailored and cultural adapted. The service is free of charge, participants are given instructions about cessation medication and a tool kit is send by mail.

Intervention or response:

Goals: To evaluate the effectiveness of the program, in terms of smoking cessation and maintenance; understand the barriers to smoking cessation and the reasons for dropping out before the end of the process; understand the factors that drive success for improvement and adaptation of the quitline, according to a needs assessment.

Methods: Telephone interviews were conducted among 400 participants who received counseling, using a structured questionnaire, one year following counseling.

Results and lessons learnt: Counseling was provided mainly in Hebrew (75\%), Russian and Arabic. Of the participants who completed the counseling- $77.7 \%$ quit smoking and with a one-year continuous abstinence rate of $39.2 \%$. A significant correlation was found between the duration of participation in telephone counseling and the rate of smoking following one year $(p<0.001)$. Cessation was associated with education and income ( $p=0.02$ and $p=0.03$, respectively). Among those who relapsed, $50 \%$ relapsed within 3 months, due to stress, tension and life/ family problems (55\%). Telephone consultation was noted as the leading factor for cessation and maintenance (74\%). About $90 \%$ expressed "satisfaction" to "high-satisfaction" with the quitline. Conclusions and key recommendations: This service provides an effective method for cessation and complements the workshops. The results suggest the need to examine reasons for dropout 
and relapse prevention, such as adding periodical support. In addition, special populations were identified as in need of more intensive support. It's important to include quitline in the Israel basket of health services.

Tob. Induc. Dis. 2018;16(Suppl 1):A339

DOI:10.18332/tid/84177

\section{2:30-14:00}

PS-744-4 Perceptions of permanent and contractual employees about a tobacco free workplace policy among four workplaces in Maharashtra, India

H Gupte ${ }^{1}$, G Mandal ${ }^{2}$, V Thawal' ${ }^{1}$, L Chaudhuri ${ }^{1}$

${ }^{1}$ Narotam Sekhsaria Foundation, Mumbai, India, ${ }^{2}$ Salaam Bombay Foundation, Mumbai, India. E-mail: himanshu@nsfoundation. co.in

Background: Cigarettes and Other Tobacco Products Act, (COTPA) prohibits smoking in public places including indoor workplaces. Despite this, few workplaces in India have documented tobacco free workplace policies being implemented. The policy influences employees about their tobacco use behavior. However, before implementing such a policy, it is important to assess perceptions and readiness of employees.

A situational analysis survey was conducted among employees of four organisations to know their readiness about having a policy, their self-reported tobacco use and the need of counseling support to quit tobacco.

Methods: A structured questionnaire was administered online to 447 permanent staff while face-to-face interviews were conducted among 281 contractual workers from August 2016 to March 2017. Results: Majority of permanent and contractual workers agreed that the organization should have a tobacco free policy and that it will have a positive impact on the employees' health. Equal proportion of permanent (55\%) and contractual staff (54\%) were aware of the existing rules and among them, $84 \%$ and $86 \%$ respectively were satisfied with them. $26 \%$ of permanent and $56 \%$ of contractual staff self-reported ever using some form of tobacco while $9 \%$ and $38 \%$ respectively reported current tobacco use (last 30 days). Fewer contractual (79\%) as compared to permanent staff (89\%) felt that some action should be taken against violation of the policy. Monetary fine and written warnings were suggested as action by majority from both groups. More contractual (98\%) than permanent staff (84\%) reported that there should be counseling service at the workplace for tobacco users.

Conclusions: Both contractual and permanent employees endorsed the need for a tobacco free workplace policy with penal action for violations. However, the differences in tobacco use and perceptions towards the policy of these two groups have to be considered while implementing the policy and offering cessation services.

Tob. Induc. Dis. 2018;16(Suppl 1):A340

DOI:10.18332/tid/84200

\section{$12: 45-14: 00$}

EP-175-4 Salivary thiocyanate as a biomarker for tobacco exposure - implications in diagnosis and tobacco cessation

A D'Cruz', A Benny ${ }^{1}$

${ }^{1}$ A.B. Shetty Memorial Institute of Dental Sciences, Nitte University, Public Health Dentistry, Mangalore, India. E-mail: audreydcruz@yahoo.co.in

Background: Tobacco use continues to be the single most leading cause of preventable deaths globally causing more than 5 million deaths/ year. The tobacco use status declared by patients must be objectively validated by biochemical tests. Salivary thiocyanate has been shown to be a suitable indicator of habitual smoking. However, data on its levels among tobacco chewers and passive smokers is lacking. The objective of the present study was to estimate and compare the salivary thiocyanate levels among smokers, passive smokers, tobacco chewers, non-tobacco users in Mangalore, Karnataka, India.

Methods: A self-administered, pre-tested questionnaire was distributed to one hundred hospital outpatients in Mangalore, Karnataka, India. Based on the responses obtained, the participants were categorized into Smokers (25), Smokeless tobacco users (25), Passive smokers (25) and Non-Users (25). Saliva was collected from the subjects in sterile plastic containers and sent for biochemical analysis to estimate thiocyanate levels. The results obtained were tabulated in Microsoft Excel for Windows and subjected to statistical analysis using SPSS statistical package 17.0. Kruskal Wallis test was used to compare the data among the various groups and the difference was considered as statistically significant if $\mathrm{P}<0.05$. Pairwise comparison between groups was done using the Mann Whitney U test.

Results: The mean salivary thiocyanate levels were $79.46+7.80$ $\mathrm{mMol} / \mathrm{L}, 50.16+13.83 \mathrm{mMol} / \mathrm{L}, 50.59+6.87$ and $36.61+5.84$ among the smokers, tobacco chewers, passive smokers and nonusers respectively which was statistically significant. Pairwise comparison of the groups showed that there was significant statistical difference among all pairs except between the passive smokers and smokeless tobacco users.

Conclusions: Salivary thiocyanate can be used as a reliable diagnostic marker for differentiation of tobacco users from nontobacco users. Salivary thiocyanate levels may also be a valuable indicator in tobacco cessation clinics for assessing the prognosis of tobacco abusers in their attempt to quit the habit.

Tob. Induc. Dis. 2018;16(Suppl 1):A341 DOI:10.18332/tid/84274

\section{$12: 45-14: 00$}

EP-176-4 Impact of weight change after quitting cigarettes on all-cause and cause-specific mortality in middle-aged male smokers: national health screening cohort study

K Kim¹, S Choi ${ }^{2}, \mathrm{MH} \mathrm{Cho}^{3}$, JH Jun ${ }^{4}$ J Chang ${ }^{5}$, SM Kim², K Lee', SM Park ${ }^{3}$

'Seoul National University, Seoul, Korea, Republic of, ${ }^{2}$ Seoul National University, Department of Biomedical Sciences, Seoul, Korea, Republic of, ${ }^{3}$ Seoul National University Hospital, Department of Family Medicine, Seoul, Korea, Republic of, ${ }^{4}$ Seoul National University Bundang Hospital, Department of Family Medicine, Seongnamsi, Korea, Republic of, ${ }^{5}$ Seoul National University, College of Medicine, Seoul, Korea, Republic of, ${ }^{6}$ Seoul National University Bundang Hospital, Department of Family Medicine, Seoul, Korea, Republic of. E-mail: kwkim16@ snu.ac.kr

Background: We aimed to investigate the association between weight change after smoking cessation and the risk of all-cause and cause-specific mortality among middle-aged male smokers.

Methods: We conducted a prospective cohort study using the National Health Insurance Service National Health Screening Cohort (NHIS-HealS) database. Male Participants $(n=102,403)$ without critical conditions aged between 40 and 79 at baseline who underwent biennial health examination were included in this study. Participants were categorized into continued smokers, 
recent quitters (within 4 years), long-term quitters (more than 4 years), and never-smokers based on the self-reported smoking status. Weight change was determined by the change of Body Mass Index (BMI) between the first (2002-2003) and second (2004-2005) health examination records. We followed patients from January 1, 2006 to December, 31, 2013. To assess the risk of all-cause, cancer, cardiovascular disease (CVD) mortality, and non-cancer, non-CVD mortality according to smoking cessation and weight change, we computed Hazard Ratio (HR) and 95\% Confidence Interval (95\% CI) using Cox proportional hazard models.

Results: Severity of weight gain was more prevalent among recent quitters compared to long-term quitters. After adjusting for covariates and weight change, both recent quitters (HR:0.74; 95\% CI: 0.63-0.87) and long-term quitters (HR:0.53; 95\% CI:0.450.61 ) had decreased risk of all-cause mortality compared to continued smokers. Similarly, both recent and long-term quitters had decreased risk of cancer, CVD, non-cancer, and non-CVD death regardless of weight change following smoking cessation. Compared to continued smokers, non-smokers also had a lower risk of overall and cause-specific death.

Conclusions: Post-cessation weight change did not modify the protective association of smoking cessation with reduced risk of allcause and cause-specific death. From a public health perspective, smoking cessation program may contribute to reducing risk of death in middle-aged male smokers despite the concern on weight change after quitting smoking.

Tob. Induc. Dis. 2018;16(Suppl 1):A342

DOI:10.18332/tid/84073

\section{$12: 45-14: 00$}

EP-177-4 Factors associated with quit attempts and smoking cessation in Brazil: findings from the International Tobacco Control Brazil Survey

L Casado ${ }^{1}$, JF Thrasher ${ }^{2}$, C Perez ${ }^{3}$, LC Thuler ${ }^{4}$, GT Fong ${ }^{5,6}$

${ }^{1}$ Brazilian National Cancer Institute, Prevention and Surveillance Coordination, Rio de Janeiro, RJ, Brazil, '2University of South Carolina, Columbia, U.S.A., Department of Health Promotion, Education \& Behavior, South Carolina, DC, United States of America, ${ }^{3}$ Cancer Foundation, Health Promotion, Rio de Janeiro, RJ, Brazil, ${ }^{4}$ Brazilian National Cancer Institute, Clinical Research Division and Stricto Sensu Post-Graduation Program in Oncology, Rio de Janeiro, RJ, Brazil, ${ }^{5}$ University of Waterloo, Department of Psychology and School of Public Health and Health Systems, Waterloo, ON, Canada, ${ }^{6}$ Ontario Institute for Cancer Research, Toronto, ON, Canada.E-mail: leticiac@inca.gov.br

Background: In Brazil, the treatment of tobacco dependence is available at no cost. This study aimed to identify factors associated with attempting to quit and of successful smoking cessation in a population-based sample of Brazilian smokers.

Methods: Data came from the first two waves of the International Tobacco Control (ITC) Brazil Survey, conducted in 2009 and 2012/2013 in three cities: Rio de Janeiro, São Paulo, and Porto Alegre. Prospective cohort data were collected from 488 adults ( $\geq 18$ years) who smoked at Wave 1 who were resurveyed at Wave 2. Crude and adjusted relative risks (RR) for two outcomes (making a quit attempt between Wave 1 and Wave 2 and successfully quitting by Wave 2) were estimated. Multivariable multilevel logistic regression models were used, whereby variables were added to the models in a series of blocks.

Results: Nearly two-thirds (65.6\%) of smokers attempted to quit between waves, and $23.4 \%$ had quit at Wave 2 . Intention to quit smoking at Wave 1 was the only variable associated with attempt to quit by Wave $2(\mathrm{OR}=2.85 ; 95 \% \mathrm{CI} 1.64-4.94$; $\mathrm{p}<0.001$ ). Smokers of higher socioeconomic status (ORhigh versus low $=1.80 ; 95 \% \mathrm{CI} 1.05-3.10 ; \mathrm{p}=0.03)$ and lower nicotine dependence (ORlow HSI versus high $\mathrm{HSI}=1.94$; 95\%CI 1.10$3.43 ; \mathrm{p}=0.02$ ) were more likely to successfully quit. The presence of another adult smoker at home was negatively related to successful quitting $(\mathrm{OR}=0.50 ; 95 \% \mathrm{CI} 0.26-0.94 ; \mathrm{p}=$ 0.03).

Conclusions: These results are generally consistent with prior research and have potential to inform governmental interventions to promote tobacco cessation, particularly among disadvantaged groups.

Tob. Induc. Dis. 2018;16(Suppl 1):A343 DOI:10.18332/tid/84484

12:45-14:00

EP-178-4 Systematically scaling up cessation services provider referrals through electronic medical records to improve patient acceptance rates S Karn ${ }^{1,2}$, X Li $^{1}$, B Sharp ${ }^{3}$

${ }^{1}$ University of Texas-Austin, Austin, TX, United States of America, ${ }^{2}$ Walden University, Minneapolis, MN, United States of America, ${ }^{3}$ Texas Department of State Health Services, Austin, TX, United States of America. E-mail: shelleykarn@austin.utexas.edu

Background and challenges to implementation: We know that providers are more willing to refer patients if an automatic referral is imbedded into the Electronic Medical Record (EHR). Extensive education to improve knowledge for healthcare providers to refer tobacco using patients to cessation services will help the patient better understand the services in which they are being referred and could improve overall acceptance rates.

Intervention or response: The intervention included monthly referral numbers from Quitline data for a 29 month period from January 2015 to May 2017. Our research questions included an increasing trend of number of referral by EHR, and whether EHR is a significant factor for the rate of acceptance by comparing this to paper-based fax referrals to the same service. Two analysis were conducted to answer the research questions: 1) a time series analysis looking at the trend of number of referral by EHR; 2) A MANOVA testing whether EHR is a significant factor for number of referrals and rate of acceptance.

Results and lessons learnt: The average acceptance rates were $7.0 \%$ for EHR referrals and $4.2 \%$ for fax referrals. Based on timeseries models, there was a seasonally increasing trend of referral by $\mathrm{EHR}(\mathrm{B}=.13, \mathrm{R} 2=.62)$. Both the average numbers of Quitline referrals and the rate of acceptance were statistically significantly different between EHR and fax referral $(p<.001)$. Although compared to paper-based referrals, number of Quitline referrals by EHR was lower, the acceptance rates was higher if referred by EHR.

Conclusions and key recommendations: Integrating an electronic referral for cessation services provides favorable results in provider adherence to referring patients to services and the patients' willingness to accepting services to quit tobacco. The key finding and recommendation is to continue to work in the field of integrating a systematic approach to referring patients to cessation services through the EHR. Patient acceptance of services significantly improved with a systematic approach in place.

Tob. Induc. Dis. 2018;16(Suppl 1):A344

DOI:10.18332/tid/84214 
$12: 45-14: 00$

EP-179-4 2017 National Survey of Tobacco Cessation Clinics in China: evidence from the combination of nationwidegovernment and clinic on-line survey

H Lin ${ }^{1,2,3}$, Z Liu ${ }^{1,2,3}$, O Shi ${ }^{1,2,3}$, D Xiao ${ }^{1,2,3}$, C Wang ${ }^{2,3,4}$

${ }^{1}$ China-Japan Friendship Hospital, Tobacco Medicine and Tobacco Cessation Center, Beijing, China, ${ }^{2}$ WHO Collaborating Centre for Tobacco Cessation and Respiratory Diseases Prevention, Beijing, China, ${ }^{3}$ China-Japan Friendship Hospital, Center for Respiratory Diseases, Beijing, China, ${ }^{4}$ China-Japan Friendship Hospital, Department of Pulmonary and Critical Care Medicine, Beijing, China. E-mail: linhaoxiang0115@163.com

Background: Accessible tobacco cessation network provide china one of the most effective measures to avoid disability and premature death.The purpose of thisstudywas to analyze the current status of tobacco cessation clinics in China and identify barriers to the development of cessation treatment, with the goal of strengthening the national tobacco cessation network in China. Methods: This was a nationwideon-line survey. All 31 Provincial Health and Family Planning Commission (provincial HFPC) and 366 health institutions with tobacco cessation clinics were involved. Government support and cessation clinics status were evaluated by government and clinic questionnaire respectively. Subsequently, we divided the clinics by if they had staffs ever participated Smoking Cessation Training and Research Programme. A comparative analysis was made between the participated institution vs. non-participant institution (PI vs. $\mathrm{NPI}$ ), using the proportion comparison and odds ratios (OR) with $95 \%$ confidence intervals.

Results: $87.1 \%$ provincial HFPC published the notice toward general tobacco control, only $41.9 \%$ provincial HFPC transmitted "Guideline on China Clinical Smoking Cessation". Among all 366 health institutions that reported had cessation clinic, most $72.7 \%$ were general hospitals and set in respiratory department(230, $62.8 \%$ ). PI group more likely to provide medication for smokers $(\mathrm{P}<0.05, \mathrm{OR}=1.86)$ and had higher possibility of using micro $\mathrm{CO}$ monitor $(\mathrm{P}<0.05, \mathrm{OR}=1.92)$. In addition, although insignificant, the $\mathrm{PI}$ group conducted better first visit registration $(\mathrm{P}>0.05$, $\mathrm{OR}=1.18$ ), more likely to arrange more than 6 times follow-up visit within 6 month $(\mathrm{P}>0.05, \mathrm{OR}=1.76)$.

Conclusions: Most provincial HFPC more focused on general tobacco control policy, lack effective support to promote cessation clinics development. The number of hospitals that established cessation clinic were increased with the national support, however cessation resources were imbalance distribution and tobacco cessation treatment had not been integrated into primary care system. Cessation treatment was still facing challenges, Smoking Cessation Training and Research Programme standardized cessation treatment at hospital level.

Tob. Induc. Dis. 2018;16(Suppl 1):A345

\section{DOI:10.18332/tid/84220}

\section{$12: 45-14: 00$}

EP-180-4 A hypothesis for an alternative treatment for tobacco addiction: phenelzine, a monoamine oxidase inhibitor

CA Curran ${ }^{1,2}$, RA Boshes ${ }^{1,3}$, DY Lee ${ }^{1,3}$, PM Coratti Curran ${ }^{4}$, B Hott $^{4}$ 'John Corrigan Community Mental Health Center, Laboratory of Clinical and Experimental Psychopathology, Fall River, MA, United States of America, ${ }^{2}$ Claude A. Curran MD, General Adult Psychiatry, Fall River, MA, United States of America,
${ }^{3}$ Harvard Medical School, Dept. of Psychiatry, Beth Israel Deaconess Medical Center, Boston, MA, United States of America, ${ }^{4}$ Miriam Hospital, Women's Medicine Collaborative at Lifespan, Providence, RI, United States of America. E-mail: curranmdca@aol.com

Background: Current smoking cessation methods are primarily based on the theory that nicotine is the principal addicting compound overlooking the contribution of the monoamine oxidase inhibitors present in tobacco. Our study tests the hypothesis that the MAOI phenelzine can successfully mimic and replace the MAOI effects of tobacco's harmala alkaloids and aid in the reduction and cessation of tobacco use.

Methods: Current smokers were treated with the MAOI phenelzine. All patients were given information regarding the risk of interactions between MAOIs and other medications and certain tyramine-containing foods. Those who had previously been treated with SSRIs, pain medications or other potentially reactive medications were told to stop taking those medications for a wash-out period of 2 weeks prior to starting the MAOI. The patients were titrated up from an initial dose of approximately $1.875 \mathrm{mg}$ (one-eighth of a $15 \mathrm{mg}$ tablet) every 2 hours to a maximum of $15 \mathrm{mg}$ TID.

Results: Both of the patients described here had failed previous treatment with NRTs (nicotine gum, nicotine lozenges, and nicotine transdermal patches). Following treatment with phenelzine each demonstrated a significant improvement in scoring on the Fagerstrom Test for Nicotine Dependence (FTND) and the Quality of Life and Satisfaction Questionnaire-Short form (Q-LES-Q-SF).

Conclusions: These preliminary findings, which have been duplicated in approximately $35 \%$ subjects treated with phenelzine, suggest a potent new treatment for tobacco addiction. We are currently collecting more data on smokers receiving phenelzine to fortify our argument that tobacco addiction is about more than nicotine dependence. Multiple components in tobacco, their combustion products, and their metabolized by-products may contribute to tobacco addiction. Phenelzine, a non-selective monoamine oxidase inhibitor, appears be useful in the treatment of tobacco addiction, a scourge which kills or cripples millions of people throughout the world as well as drains financial resources from those chained to this highly addictive compound.

Tob. Induc. Dis. 2018;16(Suppl 1):A346

DOI: $10.18332 /$ tid/83981

$12: 45-14: 00$

EP-181-4 Factors associated with intention to quit among tobacco users in India: findings from TCP India survey - Wave 1 and Wave 2

K Adhikari', M Pednekar', N Puntambekar', ACK Quah², GT Fong ${ }^{2,3}$, P Driezen ${ }^{2}$, PC Gupta

${ }^{1}$ Healis - Sekhsaria Institute of Public Health, Navi Mumbai, India, ${ }^{2}$ University of Waterloo, Department of Psychology, Waterloo, ON, Canada, ${ }^{3}$ Ontario Institute for Cancer Research, Toronto, ON, Canada.E-mail: adhikarik@healis.org

Background: In India, it is important to understand the factors that promote intention to quit as quitting is rather uncommon. Data from International Tobacco Control Policy (TCP) Project conducted in four states (Maharashtra, Bihar, Madhya Pradesh and West Bengal) were used to investigate the factors associated with intention to quit tobacco.

Methods: Data from Wave 1 (Aug 2010-Dec 2011) comprising 8,051 tobacco users and Wave 2 (Aug 2012-Dec 2013) comprising 
7,401 users were analysed. Respondents reporting planning to quit using tobacco in the next month, in the next six months, or sometime in the future, were categorized as having an intention to quit. Bivariate analysis and multivariable logistic regression was used to study the relationship between intention to quit and factors associated with intention to quit among tobacco users. All analyses were performed using SPSS V.20.0.

Results: Intention to quit decreased from $19.6 \%$ in Wave 1 to $13.5 \%$ in Wave 2. In both waves, education, advice from doctors to quit and exposure to warning messages on tobacco packages were associated with intention to quit tobacco. Exposure to antitobacco messages on public transportation vehicles $(\mathrm{OR}=2.13$, $\mathrm{CI}=1.49-3.08)$, in restaurants $(\mathrm{OR}=1.63, \mathrm{CI}=1.11-2.40)$, in bars $(\mathrm{OR}=1.81, \mathrm{CI}=1.07-3.06)$ and at workplaces $(\mathrm{OR}=1.73, \mathrm{CI}=1.23$ 2.44 ) were associated with intention to quit in Wave 1 . However, these relationships were not significant in Wave 2.

Conclusions: Perceptions of individuals leading to intention to quit are rapidly changing over the years. Future tobacco control efforts could emphasize on making warnings more effective that depict the harm of tobacco, taking into consideration, education of individuals. Also, involving doctors to advise patients to quit might increase motivation to quit tobacco use.

Tob. Induc. Dis. 2018;16(Suppl 1):A347

DOI: $10.18332 /$ tid/83913

\section{2:45-14:00}

EP-182-4 Personal tobacco use and attitudes towards cessation among undergraduate health professional students in South Africa

G Perez ${ }^{1}$, Y Saloojee ${ }^{2}, 0$ Omole $^{3,4}$, L Baldwin-Ragaven ${ }^{3,5}$

${ }^{1}$ University of Cape Town, Faculty of Health Sciences, Cape Town, South Africa, ${ }^{2}$ National Council Against Smoking, South Africa, Johannesburg, South Africa, ${ }^{3}$ University of the Witwatersrand, Johannesburg, South Africa, ${ }^{4}$ Gauteng Department of Health, Sedibeng, South Africa, ${ }^{5}$ Gauteng Department of Health, Johannesburg, South Africa. E-mail: gonda.perez@uct.ac.za Background: Advice from health professionals helps tobacco users to quit. Despite evidence that even simple advice about quitting during routine clinical care increases the likelihood of success, it is unclear whether these skills are taught and mastered during health professional training. What impact does personal tobacco use have on students' abilities to appropriately counsel; and, how well-equipped do South African health professional students feel to effectively assist tobacco users to quit?

Methods: A modified Global Health Professional Student Survey (GHPSS) was administered to all undergraduate health sciences students at two South African universities (University of the Witwatersrand - Wits and University of Cape Town - UCT) in 2012-13. In addition to personal tobacco use, students were asked whether they had been taught about the dangers of tobacco, reasons why people smoke, smoking cessation approaches and counselling preparedness.

Results: Of 469 Wits and 573 UCT respondents, $56 \%$ and 52\% respectively had tried smoking at least once; $14 \%$ of respondents from Wits and $10 \%$ from UCT were active smokers at the time of the study. $89 \%$ of students at Wits and $79 \%$ at UCT believed that specific training on tobacco cessation techniques is necessary. They acknowledged receiving training on the dangers of smoking (74\% UCT and 94\% Wits) and discussing the reasons why people smoke (55\% UCT and $76 \%$ Wits). However, only $31 \%$ at UCT and $44 \%$ at Wits said that they received formal training in smoking cessation approaches to use with patients.
On confidence to counsel patients about the dangers of smoking, $43 \%$ of the respondents from Wits and $26 \%$ from UCT felt they were equipped to do so.

Conclusions: This research highlights gaps in the curricula of health sciences students. There is an urgent need address personal smoking cessation among students and improve their competence in clinical settings to become more effective advocates.

Tob. Induc. Dis. 2018;16(Suppl 1):A348

\section{DOI:10.18332/tid/84646}

12:45-14:00

EP-183-4 Every try counts: collaborating to motivate smokers to keep trying to quit

A Brubach ${ }^{1}$, E Augustson ${ }^{2}$

'U.S. FDA, Center for Tobacco Products, Silver Spring, MD, United States of America, ${ }^{2}$ U.S. National Cancer Institute, Tobacco Control Research Branch, Bethesda, MD, United States of America. E-mail: april.brubach@fda.hhs.gov

Background and challenges to implementation: Research has shown that increasing the frequency of quit attempts, shortens the time to achieving cessation. However, smokers often take months or years between quit attempts, and few resources are available to improve engagement in quit attempts among uncommitted smokers. Intervention or response: With this in mind, the U.S. FDA Center for Tobacco Products (CTP) and the National Cancer Institute (NCI) collaborated to develop Every Try Counts. This national education and intervention campaign uses gain-framed messaging to increase motivation to make quit attempts among smokers who have recently been unsuccessful at quitting and are ambivalent about trying again. To reach the audience, Every Try Counts utilizes paid media tactics in and around the pointof-sale environment to (1) reframe quitting as a process in which progress is made regardless of outcome and (2) to decrease the time between quit attempts by encouraging "practicing the quit" without commitment to long-term cessation.

CTP developed the campaign strategy and completed extensive formative testing on motivational enhancement messaging. NCI lead development and adaptation of digital tools to engage populations with various readiness to attempt to quit. Campaign resources include a mobile-optimized website featuring resources to help users develop confidence, skills, and behavioral momentum toward quit attempts, such as text message programs, daily challenges, a smartphone app, real-time online chat with a cessation coach, links to quitlines, and information normalizing slips during the quit process.

Results and lessons learnt: CTP will launch an evaluation of Every Try Counts in conjunction with the campaign in early 2018. Initial data of receptivity, information-seeking, and metrics of use patterns will be presented.

Conclusions and key recommendations: This case study provides an opportunity to learn about the potential benefits and challenges of large-scale, inter-agency collaborations on cessation campaigns, especially of those targeting unique tobacco use populations.

Tob. Induc. Dis. 2018;16(Suppl 1):A349

DOI: $10.18332 /$ tid $/ 84511$

12:45-14:00

EP-184-4 Understanding the correlates of successful abstinence in a randomized double blind placebo controlled trial of varenicline for smokeless tobacco dependence in India 
S Jhanjee ${ }^{1}$, R Jain ${ }^{1}$, V Jain², T Gupta ${ }^{1}$, M Varshney ${ }^{1}$, R Schnoll ${ }^{3}$ ${ }^{1}$ All India Institute of Medical Sciences, Psychiatry (NDDTC), Delhi, India, ${ }^{2}$ All India Institute of Medical Sciences, Center for Dental Education and Research, Delhi, India, ${ }^{3}$ University of Pennsylvania, Department of Psychiatry, Philadelphia, PA, United States of America. E-mail:sonali_arj@hotmail.com

Background: Rates of smokeless tobacco(ST) use in India are very high and associated with grave health consequences. Research on effective ST cessation approaches is a priority. In a randomized double blind placebo controlled trial of varenicline for ST dependence in AIIMS, India, biochemically confirmed end-of-treatment(EOT) abstinence was greater for varenicline versus placebo( $25.2 \%$ vs. $19.5 \%)$, but this was not statistically different $(\mathrm{AOR}=1.6,95 \% \mathrm{CI}=0.84-3.1, \mathrm{p}=.15)$. Here, we studied the correlates of successful abstinence in this trial as such an evidence-base is crucial to optimize treatment strategies.

Methods: 237 ST users who were randomized to receive either varenicline(12 weeks, $1 \mathrm{mg}$, twice per day) or placebo were included in this analysis. Socio-demographics, ST use parameters (age of initiation, duration, chews per day, and FTND-ST scores), abstinence related parameters, baseline urinary cotinine levels, and treatment adherence were analyzed. Multivariate analysis, using biochemically confirmed point prevalence abstinence as the dependent variable, was done to predict the odds of quitting at EOT.

Results: Participants were predominantly males (97\%) and mean age was $34.2(\mathrm{SD} \pm 9)$ yrs. Using logistic regression on the whole group, baseline urinary cotinine and number of 24 hour quit attempts in the past significantly $(\mathrm{p}<0.05)$ predicted cessation at EOT. The odds of abstinence increased with unit decrease in urinary cotinine levels $(\operatorname{Exp}(B)=0.86)$ and unit increase in number of 24 hour quit attempts $(\operatorname{Exp}(B)=0.66)$. In the varenicline arm average chews per day $(\operatorname{Exp}(B)=0.91)$ and baseline depression scores using HAM-D also significantly predicted successful quitting. Greater adherence increased EOT cessation rates for varenicline (39\% vs. $18 \%, \mathrm{p}=.003)$ but not for placebo ( $28 \%$ vs. $14 \%, \mathrm{p}=.06)$.

Conclusions: This has implications for optimizing treatment planning at a program level, especially in resource poor countries. Strategies to enhance treatment adherence and address depression would be important.

Tob. Induc. Dis. 2018;16(Suppl 1):A350

DOI:10.18332/tid/84161

\section{$12: 45-14: 00$}

EP-185-4 Comparing 2 different intensities of active referral to smoking cessation services: a cluster randomized controlled trial

MP Wang ${ }^{1}$, TY Cheung ${ }^{1}$, WHC Li ${ }^{1}$, YN Suen ${ }^{2}$, A Kwong $^{3}, \mathrm{VLai}^{3}$, SSC Chan ${ }^{1}$, TH Lam ${ }^{4}$

'University of Hong Kong, School of Nursing, Hong Kong, Hong Kong, ${ }^{2}$ University of Hong Kong, Department of Psychiatry, Hong Kong, Hong Kong, ${ }^{3}$ Hong Kong Council on Smoking and Health, Hong Kong, Hong Kong, ${ }^{4}$ University of Hong Kong, School of Public Health, Hong Kong, Hong Kong. E-mail: mpwang@hku.hk

Background and challenges to implementation: Studies found that actively referring smokers to smoking cessation (SC) services increased quitting. We compared 2 different intensities of SC active referral for community smokers in Hong Kong.

Intervention or response: A single-blinded, parallel threearmed cluster randomized controlled trial was conducted among smokers who joined 2016 "Quit-to-Win" Contest organized by the Hong Kong Council on Smoking and Health. Biochemically validated $(\mathrm{CO}>4 \mathrm{ppm})$ daily smokers were proactively recruited and randomly assigned to onsite SC active referral (Group A) $(n=395)$, text messaging on promoting and encouraging smokers to attend SC services (Group B) $(n=385)$ and control group on general brief SC advice (Group C) $(n=383)$. Group A and B also received model guided (AWARD) brief $\mathrm{SC}$ advice with a warning leaflet and a referral card at baseline and telephone brief advice at 1-month and 2-month. Telephone survey at 3-month (retention rate $63.4 \%$ ) to assess primary outcome: self-reported 7-day point prevalence abstinence (PPA) and secondary outcomes: smoking reduction rate (SRR, daily cigarette consumption reduced by $\geq 50 \%$; excluding quitters) and biochemically validated quit rate $(\mathrm{CO}<4 \mathrm{ppm})$. Analyses were done by intention to treat.

Results and lessons learnt: Group A, B and C had self-reported PPA: $14.4 \%, 13.0 \%$ and $8.6 \%$ (A vs. C; B vs. C, p <0.05) and validated quit rates: $6.8 \%, 6.0 \%$ and $4.7 \%$ (all $\mathrm{p}>0.05$ ), respectively. Corresponding SRRs were $18.7 \%, 16.9 \%$ and $16.2 \%$ (all p>0.05). Compared with Group C, Group A had a higher odds ratio (OR) of 1.65 (95\% CI 1.08-2.52) and Group B smokers had a higher OR of 1.56 (95\% CI 1.02 - 2.04) for self-reported PPA, adjusting for baseline intention to quit and clustering effect.

Conclusions and key recommendations: Proactive intervention with AWARD guided brief SC advice and onsite active referral or text messaging may be effective in increasing quitting at 3-month compared with general brief SC advice in community smokers.

Tob. Induc. Dis. 2018;16(Suppl 1):A351

DOI:10.18332/tid/84183

$12: 45-14: 00$

EP-186-4 Health-care provider intervention and utilization of cessation assistance in low- and middle-income countries

D Owusu ${ }^{1}$, K-S Wang², M Quinn², J Aibangbee ${ }^{3}$, RM John ${ }^{4}$, HM Mamudu ${ }^{5}$

${ }^{1}$ Georgia State University, School of Public Health, Atlanta, GA, United States of America, ${ }^{2}$ East Tennessee State University, Biostatistics and Epidemiology, Johnson City, TN, United States of America, ${ }^{3}$ East Tennessee State University, Johnson City, TN, United States of America, ${ }^{4}$ Indian Institute of Technology, Jodhpur, Rajasthan, India, ${ }^{5}$ East Tennessee State University, Health Services Management and Policy, Johnson City, TN, United States of America. E-mail: mamudu@mail.etsu.edu

Background: The psychological and physiological addictive nature of tobacco smoking makes it difficult for some smokers to quit without assistance. Tobacco cessation and utilization of cessation assistance rates are low in low-and-middle income countries (LMICs). It is not clear if health care provider tobacco screening and quit advice promote utilization of assistance to quit tobacco. This study examined the relationship between health care provider intervention and utilization of cessation assistance in 12 LMICs.

Methods: Data from 13967 participants of the Global Adults Tobacco Survey (GATS) in 12 LMICs were analyzed. Outcome variables were utilization of counseling/cessation clinic, prescription medication, quit line and any cessation assistance. Health care provider intervention ('no intervention', 'tobacco screening', 'quit advice') was the independent variable. Four multiple logistic regression models were fit to evaluate the relationship between the independent variable and each outcome, 
adjusting for covariates. All analyses were conducted using SAS version 9.4. Adjusted odds ratios (ORs) with 95\% confidence intervals (CIs) were estimated.

Results: Approximately 52\%, $8 \%$, and $40 \%$ of participants received no intervention, tobacco screening, and advice to quit, respectively. Overall, $0.4 \%, 1.9 \%, 3.0 \%$ and $4.5 \%$ used quit line, counseling/cessation clinic, prescription medication, and any cessation assistance, respectively. Compared to no intervention, quit advice was associated with increased utilization of counseling/ cessation clinic $(\mathrm{OR}=4.41,95 \% \mathrm{CI}=3.2-6.1)$, prescription medication $(\mathrm{OR}=1.67,95 \% \mathrm{CI}=1.2-2.3)$ and any assistance $(\mathrm{OR}=2.80,95 \% \mathrm{CI}=2.2-3.6)$.

Conclusions: A comprehensive tobacco control program, with frequent tobacco screening and quit advice by health care providers may improve utilization of cessation assistance in LMICs.

Tob. Induc. Dis. 2018;16(Suppl 1):A352

DOI:10.18332/tid/84289

\section{$12: 45-14: 00$}

EP-187-4 Comparison of two approaches in achieving smoking abstinence among patients in an outpatient clinic: a phase 2 randomized controlled trial

KY Ho ${ }^{1}$, WHC Li ${ }^{1}$, MP Wang ${ }^{1}$, SSC Chan ${ }^{1}$, TH Lam²

${ }^{1}$ The University of Hong Kong, School of Nursing, Hong Kong, Hong Kong, ${ }^{2}$ The University of Hong Kong, School of Public Health, Hong Kong, Hong Kong. E-mail: devilbb2@hku.hk

Background: Having a disease and requiring medical attention present an excellent 'teachable moment' for smoking cessation interventions. However, nicotine is addictive and quitting is difficult, with a high rate of relapse, particularly among chronic smokers. Our previous smoking cessation projects in the community have revealed that many smokers who are reluctant to quit are interested in reducing the number of cigarettes they smoke per day. Therefore, smoking reduction may be an important alternative strategy for promoting smoking cessation. This Phase 2 randomized controlled trial of smokers who had medical followups in an outpatient clinic compared the effectiveness of two approaches to smoking cessation: quitting immediately and cutting down to quit.

Methods: A total of 100 subjects were randomized into two groups, 50 in the quit immediately group, who received an intervention on abruptly quitting, and 50 in the cut down to quit group, who received an intervention on gradual reduction. All subjects were followed up at 6 and 12 months via telephone, and their smoking status was assessed. The primary outcomes were self-reported 7-day point prevalence of abstinence at 6 and 12 months. Intention-to-treat analysis was employed.

Results: On average, subjects had smoked 11.31 cigarettes per day over 37 years and $96 \%$ had mild nicotine dependence. At the 6-month follow-up, the self-reported quit rate of subjects in the quit immediately group was significantly higher than in the cut down to quit group $(18.0 \%$ vs. $4.0 \%, \mathrm{p}=0.04)$. However, this difference was not significant at the 12-month follow-up $(12.0 \%$ vs. $4.0 \%, \mathrm{p}=0.16)$.

Conclusions: These data suggest that quitting immediately might be more effective than cutting down to quit in smokers who need to quit sooner, such as those with diseases requiring medical attention. Nevertheless, the effectiveness of the cut down to quit approach requires further testing.

Tob. Induc. Dis. 2018;16(Suppl 1):A353

DOI:10.18332/tid/84434
$12: 45-14: 00$

EP-188-4 The characteristics, conseling service and effectiveness of China Quitline

H Jing', L Liang' ', Z Tong ${ }^{1}$, S Chu' ${ }^{1}$, D Zhang ${ }^{1}$, D Hu ${ }^{2}$, X Yin ${ }^{2}$

'Beijing Chaoyang Hospital, Clinical Epidemiology \& Tobacco Dependence Treatment Department, Beijing, China, ${ }^{2}$ Chinese Association on Tobacco Control, Beijing, China.E-mail: Irrcruie@ sohu.com

Background: In 2004, the first quitline (010-65089393) was set up by Beijing Chaoyang Hospital in China mainland, which was upgraded to free quitline (400-888-5531) in 2009, only provided passive counseling. In May 2016, the Chinese Association on Tobacco Control has established partnerships with Beijing Chaoyang Hospital in co-manage this quitline and renamed "China Quitline". This study was to analyze the characteristics of the callers and to estimate the effectiveness of active counseling intervention provided by this quitline.

Methods: Callers to "China Quitline" from 27 May 2016 to 31 May 2017 were included. Active counseling service was provided to smoking callers who needed it, included 1 registration call and 4 cessation counseling calls. The demographic characteristics, tobacco dependence assessment, readiness to quit and the cessation outcomes were analyzed.

Results: During one year, China Quitline had served 3521 callers from 31 provinces, of which $85.7 \%$ were male, $62.7 \%$ were middle aged, and $71.7 \%$ were smokers. The smoking callers were more dependent on nicotine (85.3\% FTND score $\geq 4)$, more likely to make a quit attempt (77.1\%) and quit by self-help (94.6\%). In addition, $53.7 \%$ of smoking callers were ready to quit in 30 days, and $41.5 \%$ were willing to use active counseling intervention. In callers received active counseling service, the 7 -day-point quit rates in day 7 and day 30 were $21.6 \%$ and $9.6 \%$, respectively. Smokers who are older than 40 years (OR,2.04;95\% CI,1.253.35 ), with tobacco related diseases (OR,9.65;95\% CI,5.38-17.33), counseling cessation methods (OR,3.73;95\% CI,2.45-5.68), were associated with readiness to quit in 30 days, and that counseling smoking cessation medications(OR, 0.35; 95\% CI, 0.21-0.60), and smoking cessation clinic (OR, 0.38; 95\% CI, 0.44-0.64), were less willing to receive active conseling intervention.

Conclusions: China Quitline has provided cessation conseling service to a relatively represent active smokers in China mainland and the active conseling service provided was effective.

Tob. Induc. Dis. 2018;16(Suppl 1):A354

DOI:10.18332/tid/84362

\subsection{Tobaceo and Women: The gendered nature of tobacco}

$11: 00-12: 30$

F0-300-5 Smoking cessation in Argentina: a gender-based perspective from GATS results

B Casetta ${ }^{1,2}$

${ }^{1}$ Ministry of Health, National Tobacco Control Program, Buenos Aires, Argentina, ${ }^{2}$ Instituto Universitario CEMIC, Family Medicine, Buenos Aires, Argentina. E-mail: brunilda.casetta@gmail.com Background: It is a common belief that men are more successful in quitting than women. However, results from the evidence are controversial.

While some studies do not show differences in number of quit attempts and abstinence rates by gender, other sources find abstinence is more difficult for women. In Argentina, more girls than boys smoke, but men smoke more than women. This study 
aimed to measure the quitting effect evaluating the characteristics of current and former smoker from a gender perspective.

Methods: Secondary analysis from the Global Adult Tobacco Survey (GATS) done in Argentina in 2012, restricted to adult smokers of 18 years old or more. Results from a populationbased study were analyzed using multivariate logistic regression modelling for the association of socio-demographic, attitudinal, dependence and environmental characteristics and the status of former smoker vs smokers.

Results: The analysis includes 3,038 subjects representing in a population-weighted projection $12,017,116$, mean age 47.47 years (95\%CI 45.37-49.57). Former smokers were 50.46\% (95\%CI 44.72-56) $42.21 \%$ women (95\%CI 33.89-51). There were no differences of elapsed time since quitting by gender $(p=0.053)$. For current smokers ( $n=1,610$, women $37.44 \%$ 95\%IC 30.73-44.68), there was a significant difference in smoking years (females 20.75 years 95\%IC 18.14-23.35, males 25.12 years 95\%IC 21.92-28.32, $\mathrm{p}=0.039)$. In multivariable analysis former smoker status was strongly associated to female gender (OR 1.53 95\%CI 1.06-2.19), age (OR 1.11 95\%CI 1.02-1.2 every 5 years), smoke-free homes (OR 2.92 95\%CI 1.78-4.78), support to raise tobacco taxes (OR 2.32 95\%CI 1.52-3.56), and non-daily consumption (OR 2.06 95\%CI 1.25-3.39), independently of educational or income level. Conclusions: Results from GATS Argentina show women were more likely to quit than men among other factors associated with quitting. These results challenge the common assumption that women find it harder to quit. Tailored gender-specific strategies could enhance smoking cessation.

Tob. Induc. Dis. 2018;16(Suppl 1):A355

DOI:10.18332/tid/83978

\section{$11: 00-12: 30$}

F0-301-5 Attitudes, policy and behaviour change: the effect of attitudes towards smoke-free laws on quit attempts among smokers

Y Sheng ${ }^{1}$

'Virginia Commonwealth University, Health Behavior and Policy, Richmond, VA, United States of America. E-mail:yaou.sheng@ vcuhealth.org

Background: A growing number of studies have examined the effects of smoke-free laws on behavioral change among active smokers. However, few studies have evaluated whether the effect of policies differ depending on attitudes towards laws. The aims of this study were to disentangle the effects of smoking bans at bars and smokers' attitudes towards such laws on quit attempts, in addition to exploring how these effects might vary by gender. Methods: Data from two panels (2006-2007 and 2010-2011) of the Current Population Survey, Tobacco Use Supplements (CPSTUS) were used to obtain information on smokers' attitudes towards laws that ban smoking at bars and other smoking-related behaviors. Step-wise empirical models were estimated to measure the effect of smoke-free laws and attitudes on quit attempts among smokers. The analyses included state fixed effects and controlled for a series of time-varying characteristics at individual and state level. Stratified analyses were performed to evaluate effects by gender.

Results: Favorable attitudes towards the smoke-free laws were significantly associated with quit attempts among active smokers (OR $=1.12, \mathrm{p}<0.001)$. However, association between implementation of laws and quitting was insignificantly. Stratified analyses by gender indicated that attitudes were more likely to affect female smokers in their smoking cessation process (OR = $1.16, \mathrm{p}=0.001)$, relative to male smokers $(\mathrm{OR}=1.09, \mathrm{P}=0.07)$.
Conclusions: This study provides insights on how attitudes towards smoke-free laws influence individual self-control and contribute to behavioral change among smokers, which supports economic self-control theory. Attitudes yield differential effects by gender, emphasizing potential gender disparities in smoking cessation processes. Thus, addressing gender specificity in tobacco control policies is necessary in future research.

Tob. Induc. Dis. 2018;16(Suppl 1):A356

DOI:10.18332/tid/83761

$11: 00-12: 30$

F0-302-5 A qualitative study exploring women's journeys to becoming smokers in the social context of urban India

D Salvi ${ }^{1,2}$, A Nagarkar ${ }^{1}$

'Savitribai Phule Pune University, Pune, India, ${ }^{2}$ Boston University, School of Public Health, Boston, MA, United States of America. E-mail: devashri.salvi@gmail.com

Background: In India, the prevalence of smoking among women is increasing, and the reasons behind this are unclear. We aimed to study the factors leading to initiation and maintenance of the smoking habit in women in Pune, India.

Methods: Twenty-seven urban women smokers, ranging from 21 to 60 years of age $(31.96 \pm 10.70$ years $)$, were interviewed between September 2015 and February 2016. The in-depth interviews consisted of questions on pre-decided categories, including initiation, motivation to continue smoking, and risk perception. They were asked to describe their first smoking experience, factors that trigger them to smoke and the benefits that they seek from smoking.

Results: Thematic analysis revealed that peer pressure, curiosity, fascination, experimentation, and belonging to a group were factors that led to initiation, while lack of alternatives for stress relief, work environments, and lack of leisure time activities provided circumstances to continue smoking. Participants recognized a sense of liberation and independence from smoking cigarettes and perceived health risks as minor and distant.

Conclusions: Social factors, apart from peer influence, were important for initiation of smoking among this study's participants, while individual or personal factors, such as stress and habit, determined motivation to continue. Low awareness of health risks, perceived emotional benefits and misconceptions of being in control of the habit led to further addiction. These factors should be kept in mind when designing smoking prevention and cessation interventions.

Tob. Induc. Dis. 2018;16(Suppl 1):A357

DOI:10.18332/tid/83753

$11: 00-12: 30$

F0-306-5 The Vietnam Women's Union Testimonial Campaign - "Women create smoke-free homes" T Carroll', CT Dang2, LA Tran³, TH Doan ${ }^{3}$, MH Nguyen ${ }^{3}$, TP Do ${ }^{4}$, C Curell $^{5}$, N Singh Negi ${ }^{6}$

'Vital Strategies, Policy, Advocacy and Communication, Coogee, NSW, Australia, ${ }^{2}$ Vietnam Women's Union, Hanoi, Viet Nam, ${ }^{3}$ Danson Media, Hanoi, Viet Nam, ${ }^{4}$ Vital Strategies, Policy, Advocacy and Communication, Hanoi, Viet Nam, ${ }^{5}$ Vital Strategies, Policy, Advocacy and Communication, New York, NY, United States of America, ${ }^{6}$ Vital Strategies, Policy, Advocacy and Communication, New Delhi, India. E-mail: tcarroll@ vitalstrategies.org

Background and challenges to implementation: Almost 16 million Vietnamese adults currently smoke tobacco, including 
$45.3 \%$ of men and $1.1 \%$ of women. In Vietnam more women die from tobacco-related disease $(9.5 \%)$ than smoke cigarettes, suggesting that women suffer disproportionately greater illness and premature death from exposure to secondhand smoke (SHS). Intervention or response: The Vietnam Women's Union developed the "Women create smoke-free homes" national initiative to help protect women and children from exposure to SHS throughout Vietnam. Supported by Vital Strategies and Danson Media, a campaign was designed for the initiative, featuring the personal story of a 41 year-old, non-smoking victim of lung cancer, Ms Nguyen Thi Huong, with a call to action to "Protect yourselves and your loved ones". Pre-testing research was conducted on initial testimonial edits to strengthen communication of $\mathrm{Ms}$ Huong's story. The resulting three 30 -second ads were launched by the Women's Union and disseminated from December 2016 to February 2017 through Facebook, with accompanying Facebook posts, radio spots and public relations activities in print, online, radio and television media.

Results and lessons learnt: The testimonial ads received 4,152,937 views, with Facebook comments mostly from women tagging male partners. A national tobacco campaign evaluation study $(n=2022)$ found $8 \%$ of respondents, including $10 \%$ of female non-smoker respondents, recalled the testimonial ads unprompted (representing approximately 4.75 million people aged $15-55$ years). Of these, $74 \%$ of female non-smokers (and $75 \%$ of male smokers) reported trying to make their home 'smokefree' and $77 \%$ reported trying to persuade others to quit smoking as a result of seeing the ads, while $67 \%$ of male smokers reported making a quit attempt after seeing the ads.

Conclusions and key recommendations: The campaign demonstrates effectiveness of a national Women's Organization communicating personal stories of tobacco-related harm to women. This supports previous evidence of the power of testimonial approaches and points to further potential opportunities for tobacco control communication in Vietnam and other countries.

Tob. Induc. Dis. 2018;16(Suppl 1):A358

DOI:10.18332/tid/84196

\section{2:30-14:00}

PS-745-5 Designing effective cigarette health warning labels for women in China - findings from a 4 city study

\section{R Kennedy ${ }^{1}$, J Hardesty ${ }^{1}$, JE Cohen ${ }^{1}$}

'Institute for Global Tobacco Control at the Johns Hopkins Bloomberg School of Public Health, Baltimore, MD, United States of America. E-mail: rdkennedy@jhu.edu

Background: It is estimated that less than 3\% of Chinese women smoke cigarettes, compared to $37 \%$ of Chinese men. Despite this relatively low prevalence, China has the second greatest population of women who smoke. Pictorial health warning labels (HWLs) on cigarette packages is an important tobacco control policy. Currently China uses text-only HWLs. The following study assessed perceived effectiveness of pictorial HWLs to support smoking cessation (smokers) or the prevention of smoking initiation (non-smokers).

Methods:Adult women non-smokers $(\mathrm{n}=39)$ and smokers $(\mathrm{n}=165)$ were recruited from 4 cities in China (Beijing, Shanghai, Kunming, and Wuhan) in 2016. Participants were randomly assigned to one of four HWLs based on design features including: depictions of harm (to the smoker OR to "others" from secondhand smoke), and textual framing (factual, as in 'smoking causes lung cancer', or personal as in 'smoking caused my lung cancer'). HWLs were rated effectiveness of the label at discouraging smoking initiation (non-smokers) or supporting smoking cessation (smokers); a 10 -point scale was used, with 10 being the most effective.

Results: Survey respondents who were smokers rated the HWL with impacts on "others" using personal text the highest (mean rating 7.4), and rated HWLs with health impacts on smokers using personal text the lowest (mean rating 6.7). Survey respondents who were non-smokers rated the HWL with depictions of harm to smokers, using personal texts the highest rating (mean rating 8.1), and rated the HWL with depictions of harm to "others" using personal text the lowest (mean rating 7.5).

Conclusions: The evidence from this study indicates that pictorial HWL content is important to improve the effectiveness of encouraging smoking prevention and cessation among women in China. Having a variety of labels in rotation with a range of depictions of harm and different textual framing will help improve effectiveness among women who are smokers and non-smoker.

Tob. Induc. Dis. 2018;16(Suppl 1):A359

DOI:10.18332/tid/84064

\section{$12: 30-14: 00$}

PS-746-5 Most vulnerable victim of tobacco use and the key target of tobacco industry's aggressive marketing globally - the double trap of tobacco for women

N Yadav ${ }^{1}$, A Yadav ${ }^{1}$

IIndependent Consultant, Noida, India. E-mail: advocatenishayadav@gmail.com

Background: Globally women face the problem of tobacco both as user and non-user. She face the greatest indirect burden including the health, economic and social cost of tobacco use by a family member while the tobacco industry continue to target women globally to increase their profits by recruiting more female tobacco users. This is increasingly complex with multiple products and multiple factors at play. Though in the developed countries tobacco use among females is declining, it is still very high and in places increasing among the low SES. In LMICS the gender gap is further narrowed while in some cases girls being ahead of boys.

Methods: The paper undertakes extensive review of available literature on girls and women with respect to tobacco. The information is reviewed for a gender analysis on prevalence, pattern and tobacco industry tactics for promoting tobacco use.

Results: Women are exposed to tobacco smoke at home. They suffer greater impact when pregnant. In case of tobacco use by family women are the ones who bear the indirect cost of such tobacco use by family. On the other hand they are targeted by tobacco industry and lured to using tobacco at an early age. In societies where smoking by women has been considered a taboo for long, the number of girls and women smokers is increasing. They are also using all kinds of smokeless tobacco products easily available in such societies, especially in South East Asia.

Conclusions: Tobacco control efforts globally should become gender responsive. Policy makers should recognise the importance of taking into consideration the gender aspects of tobacco use. It is important to understand the social and health issues challenging girl's and women's both due to their own tobacco use and tobacco use by a family member. We need more female leaders in the fight against tobacco control globally.

Tob. Induc. Dis. 2018;16(Suppl 1):A360

DOI:10.18332/tid/84124 


\section{2:30-14:00}

PS-747-5 Evaluation of smokeless tobacco cessation program - an in vivo study

S Gokhale ${ }^{1}$

'Bharati Vidyapeeth Dental College and Hospital, Endodontics, Pune, India. E-mail: shantanu.gokhale@yahoo.com

Background and challenges to implementation: In India, smokeless tobacco is more prevelant, specially in lower socioeconomic strata, including women, and this fact is often underemphasized by the dental community.

The prime reason for visiting a dental practitioner is tooth pain; many times the patient is counseled to the specific cause of the pain. For example, an endodontist focuses on the root canal therapy.

Intervention or response: This is a study undertaken by an endodontist, in which a sample of 150 female patients reporting with toothpain were questioned not just about the pain, but also about the usage of smokeless tobacco. It was revealed that 64 were using smokeless tobacco (mishri, khaini, mawa etc.)

These users were administered a questionnaire which included several key factors like personal information, details of tobacco use, family history of tobacco use, physical health problems. Behavioral counselling was done by the endodontist. This was done, as female patients showed reluctance to approach cessation clinics.

Results and lessons learnt: The Fagerstrom test was used for scoring the levels of nicotine dependence. Followup was taken at regular intervals of two weeks, one month, three months and six months.

The findings revealed that $67 \%$ of the patients did not report back even for the first followup. Only 4 patients of the patients followed up right upto six month and were successful in substantial reduction or cessation of tobacco.

Conclusions and key recommendations: Dental practitioners should make it a regular practice to counsel patients at their individual levels taking the consideration the paucity of cessation clinics in India. Women are reluctant and fear the stigma of approaching cessation clinics. Proactive measures must be used to motivate women to go through the cessation follow up.

Tob. Induc. Dis. 2018;16(Suppl 1):A361

DOI:10.18332/tid/84135

\section{2:30-14:00}

PS-748-5 Supporting young women to have smokefree pregnancies - BabyBe Smokefree

H Wareing ${ }^{1}$, J Pullen ${ }^{2}$, P Hooper

${ }^{1}$ Improving Performance in Practice (iPiP), Warwick, United Kingdom, ${ }^{2}$ Revealing People, St Austell, United Kingdom. E-mail: Inaylor@ipip.co.uk

Background and challenges to implementation: Given the damage that tobacco smoke can have on the unborn child and the high associated costs, it is critical that rates of smoking in pregnancy are reduced.

Current pathways for supporting pregnant smokers are not meeting the needs of some of the youngest and most vulnerable women as evidenced by the low rate of uptake of support and the high rate of young women who smoke throughout their pregnancy. Intervention or response: Two insight-driven projects were undertaken in three diverse areas across England. The intervention was to facilitate a better understanding of young women drivers and barriers to changing behaviour and develop asset-based approaches which encourage and enable young women aged between 16 and 24 to have pregnancies that are free from tobacco use.

Results and lessons learnt: The projects provided a better understanding of:

- Their experiences of being pregnant and of smoking during pregnancy.

- The real barriers to quitting.

- What or who they would turn to if they wanted support and how they want to quit.

- Their expectations of health professionals.

- What information they want, how and from whom.

- Trusted messengers - who they want to trust and when/why they can't.

- Influences on choice.

- View to risk and how it's measured.

- Clear thoughts on what would work and outline of the delivered intervention.

Conclusions and key recommendations: An insights driven approach can:

- Positively impact on the commissioning and provision of evidence based support for young vulnerable women.

- Influence the design of interventions and professional practice to better meet the needs of target populations.

Tob. Induc. Dis. 2018;16(Suppl 1):A362

DOI:10.18332/tid/84230

$12: 30-14: 00$

PS-749-5 Gender differences in tobacco use disorder phenotypes among smokers in the largest metropolitan area of South America

JM Castaldelli-Maia'1, S Martins², L Andrade' ${ }^{1}$

'University of São Paulo, Psychiatry, São Paulo, SP, Brazil, ${ }^{2}$ Columbia University Mailman School of Public Health, Epidemiology, New York, NY, United States of America. E-mail: jmcmaia2@gmail.com

Background: Given the gender differences that permeate Tobacco Use Disorders (TUD), we aimed to identify phenotypes of TUD in female and male in a representative sample of smokers in a developing country.

Methods: Data came from lifetime weekly smokers ages 18 and older taking part in the São Paulo Megacity Mental Health Survey collected between 2005-2007 $(\mathrm{n}=1,386)$. Latent class analysis (LCA) was performed on nine TUD criteria stratified by gender. Logistic regression models explored the association between latent classes and socio-demographic and psychiatric variables. All analysis were performed using Mplus taking into account sampling weights and complex survey design features.

Results: The best fitting LCA model had three classes within a severity continuum in both genders: a "non-symptomatic class"(Women[W]:30.2\%, Men [M]: 44.4\%), a "moderate symptomatic class"(W:45.5\% , M:30.7\%), and a "high-moderate symptomatic class"(W: $24.3 \%$, M: $24.7 \%)$. Respondents in the "moderate symptomatic class" were more likely to have higher household income among female, and high-average education among male than those in the "non-symptomatic class". Both women and men in the "high-moderate symptomatic class" were more likely to have past-year anxiety, but only men were more likely to have pastyear insomnia than those in the "non-symptomatic class".

Conclusions: Both men and women smokers are divided into three TUD phenotypes, with approximately a quarter being highly dependent, which is associated with psychiatric comorbidity. However, women are more vulnerable to experiencing lifetime TUD symptomatology: $70 \%$, in contrast to $56 \%$ of men. The 
intermediate symptomatic phenotype in women was associated with high income, which may show an important differential in the smoking behaviors paradigm in this developing country.

Tob. Induc. Dis. 2018;16(Suppl 1):A363

\section{DOI:10.18332/tid/84238}

\section{2:30-14:00}

PS-750-5 Second hand smoke exposure among pregnant women in Argentina and Uruguay P Morello', M Berrueta', L Llambi ${ }^{2}$, A Aleman ${ }^{3}$, F Althabe ${ }^{1}$ 'Institute for Clinical Effectiveness and Health Policy, Caba, Argentina, ${ }^{2}$ Universidad de la Republica Uruguay, Unidad de Tabaquismo, Dpto de Medicina, Hospital de Clinicas, Facultad de Medicina, Montevideo, Uruguay, ${ }^{3}$ UNICEM, Montevideo, Uruguay. E-mail: paolamorello@hotmail.com

Background: Secondhand smoke (SHS) exposure during pregnancy is associated with complications for the pregnant women, the fetus and the newborn that can be very severe. Uruguay and Argentina still have a high prevalence of smoking and national surveys show a high prevalence of SHS exposure in the general population in spite of current legislation. Our objective was to assess SHS exposure among non smoking pregnant women in Argentina and Uruguay.

Methods: Data were collected from October 2011 to November 2013 as part two-arm parallel cluster randomized trial with baseline and follow-up cross sectional measurements conducted in 10 clusters in Buenos Aires and 10 in Montevideo. During the first 48 hours after delivery, we surveyed pregnant women using a validated questionnaire about tobacco use and SHS exposure at home, at work and indoors in general during pregnancy.

Results: We surveyed 3039 women in Uruguay and 3799 in Argentina. Overall, 1877 (61.7\%) in Uruguay and 2786 (73.3\% in Argentina) were non smokers. Among non smoking pregnant women, $37.0 \%$ in Argentina and $36.7 \%$ in Uruguay had a partner who smoked. Also, 44.4\% in Argentina and 47.1\% in Uruguay lived with at least one smoker. Overall, $47.7 \%$ in Argentina and $48.8 \%$ in Uruguay were exposed to secondhand smoke at home (live with at least one smoker/has a partner who smoke and do not have smoke free home rule). Among those who had a job, $13.8 \%$ in Argentina and 9.3\% in Uruguay were exposed to SHS at work. Conclusions: SHS exposure in Argentina and Uruguay is very high during pregnancy, particularly at home. As legislation cannot impose smoke free homes, health care professionals should take advantage of the prenatal care visits to routinely ask about SHS exposure and recommend women to avoid exposure and to implement $100 \%$ smoke free homes.

Tob. Induc. Dis. 2018;16(Suppl 1):A364

DOI:10.18332/tid/84305

\section{2:30-14:00}

PS-751-5 Gender differences in unassisted cessation attempts by South Australian smokers: 1998-2014

K Martin', J Bowden ${ }^{1}$, C Miller ${ }^{1,2}$

'South Australian Health and Medical Research Institute, SAHMRI Population Health Research, Adelaide, SA, Australia, 2University of Adelaide, School of Public Health, Adelaide, SA, Australia.E-mail: kimberley.martin@sahmri.com

Background: Unassisted cessation is the most common method of quitting successfully among both men and women, yet women have lower quit rates overall. It is important to examine characteristics of unassisted cessation attempts with regard to gender.
Methods: Household surveys of approximately 3000 South Australians (aged 15+) were conducted each year from 1998 to 2014 , yielding an overall sample size of 50100, including 4662 female smokers and 5866 male smokers. The survey assessed attitudes and behaviours regarding smoking and quitting.

Results: The proportion of cessation attempts that were unassisted decreased significantly over time, from $63.5 \%$ to $39.3 \%$ among males and $58.7 \%$ to $30.9 \%$ among females (logistic regressions, both $\mathrm{p}<.001)$. Females had lower average nicotine dependence than their male counterparts among assisted and unassisted attempts ( $\mathrm{t}$ tests, $\mathrm{p}<.001$ ).

For unassisted cessation attempts resulting in abstinence of up to one year, females had a longer average period of abstinence (49.8 days) compared to males (37.6 days) and females that used assistance (37.2 days) (t-tests, both $\mathrm{p}<.001)$. There were no gender differences in the length of abstinence from unassisted attempts when only accounting for attempts lasting less than 3 months (89\% of all attempts).

Conclusions: The high rate of using assistance used by female smokers despite having lower average nicotine dependence may be reflective of lower self-efficacy to quit, especially given that females do not have superior quitting outcomes resulting from their higher use of support.

Females should not be discouraged from attempting to quit unassisted if they wish to do so as this can lead to lengthy periods of abstinence in excess of that achieved from assisted attempts.

Trends in the use (and non-use) of cessation supports by female smokers should continue to be monitored given the potential for overuse of cessation support on non-successful attempts to ultimately damage self-efficacy to quit.

Tob. Induc. Dis. 2018;16(Suppl 1):A365 DOI:10.18332/tid/84339

\section{$12: 45-14: 00$}

EP-145-5 Age of initiation of cigarette smoking and quit attempts among young women in India evidence from Global Adult Tobacco Survey 2009 and 2017

P Lal' ${ }^{1}$, M Aghi², D Sharma ${ }^{1}$, A Pandey

'International Union Against Tuberculosis and Lung Disease, New Delhi, India, ${ }^{2}$ Independent Consultant, New Delhi, India. E-mail:plal@theunion.org

Background and challenges to implementation: We estimate the age at initiation of occasional and regular cigarette smoking among young women (15-24) and estimate the likelihood of quitting smoking through age 35 years.

Intervention or response: We use disaggregated data from Global Adult Tobacco Survey 2009 and 2017 and use socioeconomic variables (age, place of residence, educational attainment and income) to compare trends between this period.

Results and lessons learnt: Women younger than 30 years were more likely to be past or current smokers, began smoking earlier (median age $<20$ years), and smoked more cigarettes per day than older women, yet attempted to quit smoking more often before age 35 (log-rank $\mathrm{P}<.001$ ).

Conclusions and key recommendations: Young female smokers have a higher propensity to initiate and quit smoking compared with older women. Supporting young women smokers to quit and averting initiations may be an important strategy to reduce smoking prevalence in the future among women.

Tob. Induc. Dis. 2018;16(Suppl 1):A366

DOI:10.18332/tid/84333 
12:45-14:00

EP-147-5 Revaluating the triad of women, tobacco and violence in Nigeria: findings from the 2013 NDHS survey

0 Popoola' ${ }^{1}, 0$ Ige $^{2}$, A Adebiyi $^{1}$

${ }^{1}$ College of Medicine, University of Ibadan, Department of Community Medicine, Ibadan, Nigeria, ${ }^{2}$ United Methodist Missions, Global Health, Atlanta, GA, United States of America. E-mail:drpopee2001@yahoo.com

Background: Tobacco use is an unambiguous health threat but the discourse on its effects and use predictors is more often focused on physical rather than psychological factors. The authors of this paper previously examined associations between domestic violence and tobacco use in women. In line with the MPOWER focus of monitoring tobacco use and predictors, we re-evaluate these relationships in the latest DHS survey of women in Nigeria. Methods: Data from the 2013 DHS survey in Nigeria was analysed. The dataset examined tobacco use in a nationally representative sample of 38,740 women and violence experience in a subset of 27,634 women aged 15 - 49 years. Logistic regression models were fitted to derive adjusted odds of association between tobacco use and violence experience or perpetration in various domains, controlling for the effect of geographic location and womens' education.

Results: The prevalence of tobacco use among women was $0.4 \%$ (95\% CI 0.3-0.6), a 55\% reduction from 2008 values. $0.2 \%$ smoked cigarettes, $0.1 \%$ used snuff or chewing tobacco respectively. Tobacco use showed a consistent association with experience of severe physical violence in both the 2008 and 2013 surveys with a $60 \%$ increase in tobacco users violence odds. Experiencing sexual violence and perpetrating physical violence were not associated with tobacco use in 2013 counter to findings in 2008. Similarly experiencing emotional violence was a predictor of tobacco use in 2013 a change from 2008 findings

$\begin{array}{lcc}\text { Violence domains } & \begin{array}{c}\text { Idjusted } \\ \text { odds }\left(95^{\circ} \circ\right. \\ \text { CI } 2008\end{array} & \begin{array}{c}\text { Adjusted } \\ \text { oddils }\left(95^{\circ}\right.\end{array} \\ \text { CII) } 2013\end{array}$

[Logistic odds of violence and tobacco use]

Conclusions: Despite the absolute reduction in tobacco use prevalence in this report, tobacco use and violence maintain a worrying co-occurrence in Nigerian women. Tobacco control efforts must identify vulnerable sub populations like women in domestic violence situations as priority for intervention. National programmes should explore opportunities for synergy between tobacco control and violence prevention programmes for women.

Tob. Induc. Dis. 2018;16(Suppl 1):A367

DOI: $10.18332 /$ tid/84574

\section{2:45-14:00}

EP-149-5 Exposure to tobacco during pregnancy among mothers with low birth weight newborns in a rural area, Arua district, Uganda - 2016: a case control study

A Asiimwe ${ }^{1,2}$

'Makerere University School of Public Health / Uganda Peoples'
Defence Forces, Epidemiology, Kampala, Uganda, ${ }^{2}$ Uganda Peoples' Defence Forces, Medical, Kaampala, Uganda. E-mail: alexasiimwek@yahoo.com

Background: Exposure to tobacco during pregnancy is an important risk factor for the low birth weight of the newborns. Arua district has a high prevalence of low birth weight (10.6\%). Also, the prevalence of tobacco use in the district is the highest in Uganda being $33.7 \%$ and $4.7 \%$ among men and women respectively. We aimed at establishing the relationship between low birth weight and exposure to tobacco during pregnancy in Arua district.

Methods: A facility based un-matched case control study. Cases were mothers that delivered newborns with birth weight less than $2500 \mathrm{~g}$ during the study period whereas controls were mothers that delivered newborns with birth weight equal to or greater than $2500 \mathrm{~g}$ during the study period. Two controls were selected per case. A semi-structured questionnaire was used to collect information. Stratified analysis was performed and Odds ratios (OR) used as the measure of association.

Results: Exposure to tobacco during pregnancy was significantly associated with low birth weight $(\mathrm{AOR}=2.21,95 \% \mathrm{CI}=1.31-3.74)$. The OR for the association between active tobacco use during pregnancy and low birth weight was $8.2(95 \% \mathrm{CI}=3.46-21.45)$. After adjusting for age of the mother, alcohol use during pregnancy and mother's nutritional status, the Adjusted Odds Ratios(AOR) were $11.95(95 \% \mathrm{CI}=4.89-29.16), 6.69(95 \% \mathrm{CI}=2.92-15.37)$, and $5.16(95 \% \mathrm{CI}=2.24-11.86)$ respectively. Tobacco farming and processing was also found to be significantly associated with low birth weight $(\mathrm{AOR}=2.98,95 \% \mathrm{CI}=1.54-5.79)$. Exposure to Second Hand Smoke at home was significantly associated with low birth weight $(\mathrm{AOR}=1.85,95 \% \mathrm{CI}=1.15-3.00)$.

Conclusions: Exposure to tobacco during pregnancy was associated with low birth weight in Arua district. We recommend intensive health education of mothers by the Ministry of Health against exposure to tobacco during pregnancy.

Tob. Induc. Dis. 2018;16(Suppl 1):A368

DOI: $10.18332 /$ tid/83767

\subsection{Tobacco in vulnerable populations (e.g. indigenous populations)}

$11: 00-12: 30$

F0-303-5 Modeling smoking and depression comorbidity in the U.S

J Tam¹, G Taylor', K Zivin'1, R Meza ${ }^{3}$

${ }^{1}$ University of Michigan, Health Management and Policy, Ann Arbor, MI, United States of America, ${ }^{2}$ University of Bristol, Bristol, United Kingdom, ${ }^{3}$ University of Michigan, Epidemiology, Ann Arbor, MI, United States of America. E-mail: jamietam@ umich.edu

Background: Evidence shows that the relationship between depression and smoking may be bi-directional, with smoking increasing the risk for subsequent depression, and vice versatranslating into significantly higher smoking rates among those with depression compared to the general population. Computational models can be used to assess trends in smoking but have not been utilized to examine populations with behavioral health comorbidities. We develop the first joint model of smoking and depression, projecting future smoking patterns under a 'status quo' scenario.

Methods: We use a compartmental model to simulate individuals transitioning across smoking and depressive states from birth to 
age 99 or death. The model is calibrated to reproduce smoking and depression data from the 2005-2014 U.S. National Survey on Drug Use and Health. Current smokers report smoking within the past year and smoked at least 100 cigarettes in their lifetime while those with depression report a major depressive episode in the past year. Parameters for the effects of smoking and depression on each other were drawn from existing literature.

Results:

From 2015 to 2050 , smoking prevalence among women with depression is projected to decline by $43.5 \%$ (27.6\% to $15.6 \%$ ), compared to $46.9 \%$ (17.9\% to $9.5 \%$ ) for women who report never having had a depressive episode. Among men with depression, smoking prevalence is expected to decline by $32.0 \%$ (34.4\% to $23.4 \%$ ), compared to $39.0 \%$ (24.1\% to $14.7 \%$ ) for men who report never being depressed. The likelihood of smoking among those with depression is projected to increase.

\section{Conclusions:}

This model can be used to examine trends and identify optimal interventions to address smoking disparities by depressive status. Smoking prevalence among people with depression is expected to decline, though not as rapidly as for those without depression. In the absence of population-level strategies, smoking and tobaccorelated diseases will continue to further disproportionately burden people with depression.

Tob. Induc. Dis. 2018;16(Suppl 1):A369

DOI:10.18332/tid/83864

[Smoking and depression model diagram]

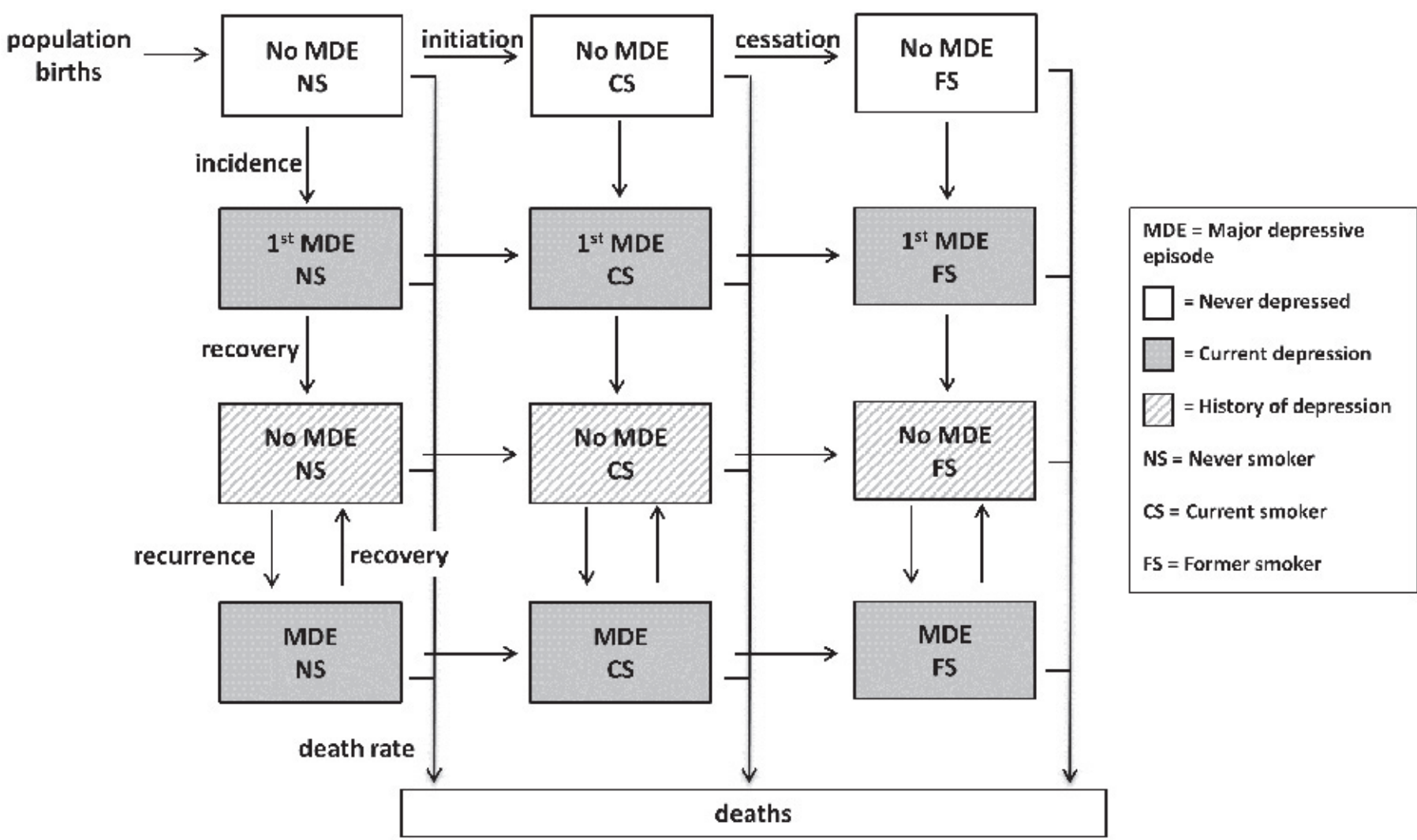

12:30-14:00

PS-752-5 Usages du tabac chez les travailleuses de sexe à Yaoundé. Perceptions, facteurs associés et risques sanitaires

MMM Nsangou Mbouemboue $e^{1,2}$

${ }^{1} \mathrm{CDBPH}$, Yaoundé, Cameroon, ${ }^{2}$ Université de Yaoundé I, Sociologie, Yaoundé, Cameroon. E-mail: jordynsangou@yahoo.fr

Background: Au Cameroun, l'usage du tabac est un problème social qui se pose avec acuité dans le milieu de la prostitution. Plusieurs programmes de santé interviennent dans ce milieu pour les activités de prévention des maladies comme les infections sexuellement transmissibles et le VIH. Nous avons mené une étude sur les travailleuses de sexe dans la ville de Yaoundé pour documenter leurs perceptions, les facteurs associés et leurs connaissances sur les risques sanitaires de l'usage du tabac.

Methods: Une étude mixte (quantitative et qualitative) a été menée entre mai et juin 2016. 355 travailleuses de sexe ont participé au volet quantitatif en remplissant un questionnaire et 30 autres ont été sélectionnées pour participer à des entretiens semi-structurés. Des observations directes ont été faites sur 20 participantes.

Results: Dans la ville de Yaoundé, 89,0\% de travailleuses de sexe consomment du tabac fumé. D'autres formes d'utilisation du tabac sont: tabac non fumé et le tabac injecté (drogue). Les perceptions de l'usage du tabac dans ce groupe sont diverses: outil de combat du froid; outil de gestion du stress et de la peur; outil de marquage social et de respectabilité et outil de rétrécissement du vagin. Concernant les connaissances des risques sanitaires de l'usage du tabac, toutes sont conscientes que le tabac peut nuire à leur santé. Cependant, elles ont des connaissances limitées sur les maladies que peut engendrer le tabac.

Conclusions: La plupart des travailleuses de sexe ont une connaissance limitée des risques liés à l'usage du tabac. D'où la nécessité de concevoir des programmes et de les sensibiliser sur les risques sanitaires liés à l'usage du tabac.

Tob. Induc. Dis. 2018;16(Suppl 1):A370

DOI:10.18332/tid/83754 
12:30-14:00

PS-753-5 Tobacco use, awareness and cessation among Malayali tribes, Yelagiri Hills, Tamil Nadu, India

DL Francis

${ }^{1}$ Dr MGR Medical University, Public Health, Chennai, India. E-mail: delfin_lovelina@yahoo.co.in

Background: Health is a state of complete wellbeing free from any discomfort and pain. Despite remarkable world-wide progress in the field of diagnostic, curative and preventive medicine. India has the second largest tribal population of the world next to the African countries. About half of the world's autochthonous people live in India, thus making India home to many tribes which have an interesting and varied history of origins, customs and social practices. The present study was conducted to assess the tobacco use, awarness and its effect on health among Malayali tribes, Yelagiri Hills, Tamil nadu, India.

Methods: The inhabitants of the 14 villages of the Yelagiri hills, who have completed 18years and residing for more than 15years present on the day of examination and who were willing to participate in the study were included. Data was collected from a cross-sectional survey, using a Survey Proforma, clinical examination and a pre-tested questionnaire which included Demographic data, tobacco habits. An intra-oral examination was carried out by a single examiner to assess the Oral Health Status using WHO Oral Health Surveys - Basic Methods Proforma (1997).SPSS version 15 was used for statistical analysis.

Results: Results showed that among 660 study population, $381(57.7 \%)$ had no formal education. Among the study population $75 \%)$ had the habit of alcohol consumption. Of those who had the habit of smoking, $26 \%$ smoked beedi, $10.9 \%$ smoked cigarette, $65 \%$ chewed raw tobacco, $18 \%$ chewed Hans and $28 \%$ had a combination of smoking and smokeless tobacco usage. Prevalence of oral mucosal lesions was very high.

Conclusions: From the results of this study it may be concluded that the Malayali tribes were characterized by a lack of awareness about oral health, deep rooted dental beliefs, high prevalence of tobacco use and limited access to health services.

Tob. Induc. Dis. 2018;16(Suppl 1):A371

DOI:10.18332/tid/83755

\section{2:30-14:00}

PS-755-5 An assessment of oral health status, tobacco use and cancer awareness among tea plantation workers (Irula tribes), Nilgiri Hills, Tamilnadu, India

DL Francis

'Dr MGR Medical University, Public Health, Chennai, India. E-mail: delfin_lovelina@yahoo.co.in

Background: Tea is an important agro-industry of India, which contributes immensely to the countries economy. Tea garden population constitutes approximately $1 / 12$ th of tea growing state's population. Poor socio-economic conditions, ignorance due to illiteracy, over-crowded and unhygienic living conditions in the residential colonies make tea garden population vulnerable to various communicable diseases and malnutrition. Hence this study was contemplated with an aim to assess the oral health status, tobacco use and cancer awareness among tea plantation workers, Nilgiri Hills, Tamil Nadu, India.

Methods: A cross-sectional descriptive study was conducted to assess the tobacco use and cancer awareness among tea plantation workers, Nilgiri Hills. Data was collected using a pretested Questionnaire, which included Demographic data, tobacco habits, its frequency and form. The data collected was analysed using SPSS version 15.

Results: Results showed that among 900 study population, showed $57 \%$ had no formal education, $34.5 \%$ had not visited dentist before. $64.5 \%$ had indigenous brushing habits. $52 \%$ of oral mucosal lesions and $6 \%$ malignant oral tumors were observed. A very high prevalence of periodontal disease, tobacco chewing, deep rooted beliefs and customs regarding dentition and dental treatment was observed in this community. Prevalence of oral mucosal lesions in the study population was due to tobacco usage and lack of awareness regarding the deleterious effects of the products used.

Conclusions: The dangers from smoking and chewing tobacco are well documented within the literature but the public's lack of knowledge of the risks is a concern. Health professionals are encouraged to ensure that the public is made aware of these risks, especially those within high-risk groups.

\section{Tob. Induc. Dis. 2018;16(Suppl 1):A372}

\section{DOI:10.18332/tid/83798}

\section{2:30-14:00}

PS-756-5 Tailor-made smoke-free programme to clear the myths of smoking among Hong Kong elder people

A Kwong ${ }^{1}$, V Lai $^{1}$

${ }^{1}$ Hong Kong Council on Smoking and Health, Hong Kong, Hong Kong.E-mail: lawrence_chu@cosh.org.hk

Background and challenges to implementation: In Hong Kong, over $20 \%$ of the 641,300 daily smokers aged 60 years or above. Public's awareness on smoking hazards has increased in recent years. But elder people are misled by the misconceptions on smoking and underestimate the health risks. Elder smokers have lower intention and knowledge on the smoking cessation.

Intervention or response: In order to inform the tobacco hazards, clarify misconceptions and encourage elder smokers to quit, Hong Kong Council on Smoking and Health (COSH) launched the "Elderly Smoking Cessation Promotion Project" in 2012. Health talks and community smoke-free activities are organized. Nonsmoking elderly are also engaged as smoke-free agents.

Results and lessons learnt: Since 2012, COSH has delivered over 140 sessions of health talk and reached over 10,000 elderly. To spread out the smoke-free messages, radio promotions were produced and broadcasted in the channels targeted at elderly.

Various smoke-free promotion activities were organized, including centre visits, pledge ceremony, street promotions, photo exhibition and workshops, to increase elder smokers' motivation to quit and arouse public's awareness and support to smoking cessation. The Project has successfully trained about 60 elder volunteers as ambassadors, encouraged over 200 smokers to pledge to quit smoking and engaged 900 elderly to promote smoke-free lifestyle. Mass public education and promotion may not able to reach the elder population. They hold stronger misconceptions on smoking cessation and are more reluctant to quit. Tailor-made and reachout intervention programmes should be developed to build up smoke-free concepts among elderly. Collaborations with elderly service organizations could add benefit to the interventions.

Conclusions and key recommendations: Elder smokers have comparatively low awareness on harms of smoking and intention to quit. To address the specific need of elder population, COSH's tailor-made Elderly Smoking Cessation Promotion Project strengthened their knowledge on smoking hazards, cleared their 
myths of quitting and mobilized elder smokers to quit, through an interactive approach and community engagement.

\section{Tob. Induc. Dis. 2018;16(Suppl 1):A373 DOI:10.18332/tid/83828}

\section{$12: 30-14: 00$}

PS-757-5 The prevalence of secondhand smoke exposure among women and children in the deep South of Thailand

S Nima ${ }^{1}$, V Chongsuvivatwong ${ }^{2}$

'Prince of Songkla University, Health System Management Institute, Hatyai, Thailand, ${ }^{2}$ Prince of Songkla University, Epidemiology Unit, Faculty of Medicine, Hat Yai, Thailand. E-mail: sophienima@gmail.com

Background: In lower-middle income countries, women and children are vulnerable populations that are regularly susceptible to secondhand smoke (SHS) exposure in their own private residences. In Thailand, the highest smoking rate has consistently demonstrated for two decades in the Deep South that related to cultural-specific of tobacco product use. The purpose of this survey was to describe the prevalence of exposure to SHS among women and children at homes in the 5 southernmost provinces of Thailand.

Methods: A descriptive cross-sectional household survey using 2-stage random sampling was conducted in April-May, 2015 to those among people aged 12 years and over in Satun, Songkhla, Pattani Yala, and Narathiwas province. Both self-report and face to face questionnaires containing socio-demographic characteristics, household characteristics and smoking status at home were used to evaluate the SHS on 4,500 households and 4,515 individual samples. Results: The prevalence of current tobacco use and SHS in their homes were $33.38 \%$ and $73.40 \%$, respectively. All of tobacco smokers were male that commonly smoked at homes in every day (35.34\%), every other day (5.19\%) and twice a week (2.69\%). On the other hands, non-tobacco smoke persons living with those smokers in the same houses were mainly women and children that exposed to SHS every day (31.05\%). In addition, both groups were exposed to SHS by community members and smoking neighbors who always came to smoke inside their homes (29.89\%).

Conclusions: This study indicates that women and children are at highly increased of health risks from SHS exposure at their own homes. This finding will be contributed to motivate the development of tobacco control policy advocacy and specific properly interventions for smoke-free homes in accordance with socio-cultural context in the Deep South of Thailand.

Tob. Induc. Dis. 2018;16(Suppl 1):A374

DOI:10.18332/tid/83896

\section{2:30-14:00}

PS-758-5 Tobacco smoking among HIV infected people using antiretroviral therapy

D Bhatta ${ }^{1,2}$

${ }^{1}$ University of California, Cardio Vascular Research Institute, San Francisco, CA, United States of America, ${ }^{2}$ Tribhuvan University, Peoples' Dental College, Community Medicine and Public Health, Kathmandu, Nepal. E-mail: dnbhatta@yahoo.com

Background: Life expectancy was found decreased among HIV infected people who developed habit of tobacco smoking and alcohol drinking than those infected ones who did not smoke tobacco and drink alcohol. We aimed to determine the proportion and the associated factors related to tobacco smoking among HIV infected people.
Methods: A cross-sectional study was conducted at an ART clinic in Kathmandu, Nepal between September and December 2014. The data was collected from a sample of 132 HIV infected individuals using random sampling technique and face-to-face meeting. Binary logistic regression was used to estimate the factors associated with the current tobacco smoking.

Results: Among the HIV infected people, the proportion of current tobacco smoking was 26.5\% (95\% Confidence Interval [CI]; 18.9, 34.1); and alcohol drinking was $22.7 \%$ (95\% CI; 15.5 , 30.0) among the HIV infected people. The respondents, who were infected after the sexual contact with sex workers, were more likely to have tobacco smoking $(\mathrm{OR}=15.2)$ and alcohol drinking $(\mathrm{OR}=4.50)$ than those who were infected by other than sex workers. The HIV infected individuals, who ever forgot to take ART medication, were three times more likely $(\mathrm{OR}=3.17)$ to alcohol drinking than those who did not forget to take ART medication.

Conclusions: The findings show that tobacco smoking and alcohol drinking are common among the HIV infected individuals in Nepal. There is an urgent need to develop instant, sustainable and efficient programs to control tobacco smoking and alcohol drinking trajectories among vulnerable populations in low and middle income countries like Nepal.

Tob. Induc. Dis. 2018;16(Suppl 1):A375

DOI: $10.18332 /$ tid/83946

\section{2:30-14:00}

PS-759-5 Awareness about anti-smoking related laws and legislation in healthcare facilities of Delhi, India

T Anand ${ }^{1}$, N Sharma ${ }^{2}$, S Grover ${ }^{3}$

${ }^{1}$ North Delhi Municipal Corporation Medical College, Community Medicine, Delhi, India, ${ }^{2}$ Maulana Azad Medical College, Delhi, India, ${ }^{3}$ National Institute of Cancer Prevention and Research, Noida, India.E-mail: drtanu.anand@gmail.com

Background: Healthcare facilities are vital to effective implementation of a anti-smoking law. Healthcare institutions play an integral role in educating and modeling health behaviors of our communities. It is therefore important to assess their opinion and ensure compliance. Therefore, the present study was undertaken to assess the awareness amongst people in healthcare institutions regarding the initiative years after intensive implementation.

Methods: It was a cross-sectional study conducted in the participants selected by multi-stage sampling from the health institutions in 6 districts of Delhi using a pre-tested semi-structured questionnaire. A total of 594 people were interviewed.

Results: Out of the total, $35.2 \%(n=204)$ reported to be current smokers. Only $31.5 \%(n=182)$ of the participants were aware about the name of COTPA. Awareness about organization implementing the COTPA was also very low $(n=46 ; 4.5 \%)$ among the respondents. Even though they had less awareness about COTPA by name (as stated above), they were aware about some of the provisions of the act. Majority of the participants $(n=512 ; 86.7 \%)$ perceived that smoke free zones are the places where smoking forms of tobacco are banned. Regarding the awareness about the places designated as "smoke free zones", $86.7 \%(n=515)$ of the respondents named healthcare institutions. About $74 \%$ of the people interviewed reported to have seen people smoking at public places on the day of interview while only $24.1 \%$ reported to have seen any one getting punished/fined for smoking at public places.

Conclusions: The awareness about anti-smoking law and smoke free initiative was low among the study group. Further, action 
against the violators of the law was not being. Thus, there is a need for stricter implementation of the law and increased spreading of awareness among the people in healthcare institutions about the anti-smoking legislation.

\section{Tob. Induc. Dis. 2018;16(Suppl 1):A376}

DOI:10.18332/tid/83956

\section{2:30-14:00}

PS-760-5 Tobacco chewing habits and risk of precancerous oral lesions among Paniya tribes of Wayanad, India - a cross sectional study

S Palliyal ${ }^{1}$

'DM Wayanad Institute of Medical Sciences, Dentistry, Kalpetta, India.E-mail:dr.shanavasp@dmwims.com

Background: Oral cancer is a major public health problem among Paniya tribes, a marginalized tribal group in Kerala state, South India. Previous studies have documented a high prevalence of tobacco chewing habit among Paniya tribals in Wayanad. Precancerous lesions of oral mucosa, known as potentially malignant disorders are consists of a group of diseases, which should be diagnosed in the early stage. The aim of this study was to evaluate the risks imposed by tobacco chewing in the development of oral cancer.

Methods: A cross-sectional descriptive study was conducted to assess tobacco chewing and precancerous oral lesions prevalence among 600 Paniya tribes in the Wayanad District,India. Information on tobacco chewing status, type, quantity and duration of chewing was collected from cases and controls using a questionnaire. Inhabitants of the villages aged 18 to 65 years who were residing for more than 10 years were included. All the examinations were performed by a single examiner. Data was collected using a survey proforma which comprised of a questionnaire and WHO Oral Health Surveys (1997). The collected data was subjected to statistical analysis.

Results: In this study precancerous lesions were found to be far more prevalent among chewing Paniya tribes than among the controls $(\mathrm{P}<0.0001)$. The prevalence of precancerous oral lesions were higher among tobacco chewers (18.9\%,95\%,CI 16.5-20.9). This was much higher than found among the non chewer $(0.8 \%, 95$ CI 0.6-1.3). Among the tobacco chewers a statistically significant relationship was observed between precancerous lesions and poor access to health care $(\mathrm{P}<0.001)$.

Conclusions: Prevalence of precancerous oral lesions in the study population was due to tobacco usage and lack of awareness regarding the deleterious effects of the products used. Regular oral examination by dental professionals, dental health education and motivation to maintain oral hygiene should be insisted to improve the oral health status of this community.

Tob. Induc. Dis. 2018;16(Suppl 1):A377

DOI:10.18332/tid/83963

\section{2:30-14:00}

PS-761-5 Assessment of smoking/tobacco smoke exposure and provision of smoking cessation advice/assistance by healthcare professionals at a women's and children's hospital - practices and barriers

OH Teoh'1, AH Teo ${ }^{1}$, SY Tan' ${ }^{1}$, SXA Chiang ${ }^{1}$, ENA Goh ${ }^{1}$, OM Chay ${ }^{1}$ ${ }^{1}$ KK Women's and Children's Hospital, Department of Paediatrics, Singapore, Singapore. E-mail: teo.ai.huay@kkh.com.sg

Background: Children and pregnant women are vulnerable to the harms of tobacco smoke. All healthcare professionals (HCPs) have a responsibility to protect them from tobacco smoke. This study aims to identify the gaps between this knowledge and real world practice in a clinical setting.

Methods: Self administered questionnaires were distributed to 2436 HCPs at a women's and children's hospital. The HCPs were asked about their practice of smoking/tobacco smoke exposure (TSE) assessment and provision of smoking cessation advice/ assistance in their clinical work, and the barriers they face in carrying out these activities.

Results: The response rate was $74.6 \%$ (1817 respondents), from 276 (15.2\%) doctors, 1498 (82.4\%) nurses and 43 pharmacists (2.4\%). Only $20 \%$ of HCPs would always ask about smoking/TSE, while $19.5 \%$ do not at all. Only $15.1 \%$ of HCPs would always provide smoking cessation advice/assistance to patient smokers or smoker parents/caregivers, while $28.4 \%$ do not at all. Only $7.9 \%$ of Paediatrics HCPs would always ask about smoking/TSE, while $38.7 \%$ of O\&G HCPs would always do so $(p=0.000)$. The reasons/barriers for not always assessing smoking/TSE or providing smoking cessation advice/assistance are listed in Table 1.

\section{$\begin{array}{ll}\text { Vot always assessing for } & \text { Vot always providing } \\ \text { smoking TSE } & \text { assistance }\end{array}$ \\ Smoking/TSE is not a common/ significant issue (35.6\%) \\ Smoking/TSE is not a common/ significant issue (23.4\%) \\ Not my direct responsibility (21.7\%) \\ Not my direct responsibility (20.9\%), \\ Smoking/TSE is not related to the Smoking/TSE is not related to patient's disease (19.4\%) the patient's disease (17.0\%) \\ Fear of negative reaction from smoker (14.5\%) \\ Lack of time $(7.5 \%)$ \\ Lack of counseling skills (16.5\%) \\ Fear of negative reaction from smoker (15.7\%)}

Inadequate resource personnel to Lack of time (14.6\%) assist (3.2\%)

[Reasons and Barriers]

The most common interventions for patient smokers and smoker parents/caregivers are provision of general advice for the smoker to quit smoking (36.5\%), assessment of smoker willingness to quit $(15.7 \%)$, and provision of reading materials $(9.9 \%)$. The suggestions from HCPs on how to overcome the barriers identified are relevant training courses for all HCPs (62.2\%), setting up of smoking cessation clinics (42.4\%), and training more smoking cessation counselors (30.6\%).

Conclusions: There is room for improvement in the real world practice of HCPs in protecting children and women from tobacco smoke. Awareness and mindset issues are barriers to be addressed. Fear of negative reaction and lack of counseling skills limit HCPs' confidence but the majority are receptive to receiving training.

Tob. Induc. Dis. 2018;16(Suppl 1):A378

DOI:10.18332/tid/83987

12:30-14:00

PS-762-5 Tobacco packaging in India: assessing compliance with Health Warning Label (HWL) laws and marketing appeals for cigarettes, bidis and smokeless products

K Smith ${ }^{1,2}$, K Welding $^{1}$, S Saraf ${ }^{1}$, C Washington ${ }^{1}$, M lacobelli ${ }^{1}$, J Cohen ${ }^{1,2}$ 
'Johns Hopkins Bloomberg School of Public Health, Institute for Global Tobacco Control, Baltimore, MD, United States of America, ${ }^{2}$ Johns Hopkins Bloomberg School of Public Health, Health, Behavior and Society, Baltimore, MD, United States of America. E-mail: katecsmith@jhu.edu

Background: Over 100 million Indian adults use tobacco. India enhanced tobacco warning label requirements as of 2016; a HWL should now cover $85 \%$ of the 2 principal displays. Smokers make up a smaller proportion of Indian tobacco users; smokeless users tend to be less educated and live in more rural areas. We assess HWL compliance and product appeals conveyed on packs of cigarettes, bidis and various forms of smokeless tobacco.

Methods: We purchased tobacco products (cigarettes, bidis and smokeless) according to a systematic protocol in four cities (New Delhi, Mumbai, Chennai and Bangalore) during NovemberDecember 2016. Within each city, we constructed a purposive sample of 12 diverse neighborhoods and data collectors followed a walking protocol to select sales outlets, from which they purchased one of each unique pack (all products). Two independent coders assessed each pack using product and country specific HWL law codebooks, and a standardized codebook for product appeals.

Results: We collected 226 unique packs; 94 cigarettes, 47 bidis and 85 smokeless. For cigarettes, 59\% $(\mathrm{N}=55)$ had a current HWL; of these, $53 \%(n=29)$ were compliant with size, placement and warning label components. For bidis, $91 \%(\mathrm{~N}=43)$ displayed the current HWL, but none were compliant with all aspects of the law. For smokeless products, 85 packs were collected of which $64 \%(n=54)$ displayed the correct HWL, but only 1 was compliant by our indicators. We discuss challenges in assessing HWL compliance for non-cigarette products and compare marketing appeals between products, including cultural and religious symbolism, and depiction of individual manufacturers.

Conclusions: India has a progressive health warning label law, but compliant implementation differs between tobacco products. Our discussion of HWL compliance and appeals on packs suggest areas for attention to ensure that India continues to see rates of tobacco use decline and protect all Indians from harm from all forms of tobacco.

Tob. Induc. Dis. 2018;16(Suppl 1):A379

DOI:10.18332/tid/84002

\section{2:30-14:00}

PS-763-5 Use of puppet shows for promoting smoke-free home and training children about the harmful of smoke and care for themselves, in Tehran's kindergartens

F Matinkhah

'Iranian Anti Tobacco Association, Tehran, Iran, Islamic Rep. Of.E-mail: e.matinkhah@gmail.com

Background and challenges to implementation: By selecting ten kindergartens in Tehran and using a questionnaire, Clean Breath Center measured the rate of smoking in the families. About $54 \%$ of parents reported tobacco (cigarette or hookah) smoking in their home; while in 49 percent of cases, tobacco use was in the presence of children. The center, in a new move, prepared several entertaining puppet shows for children and performed them annually in at least sixty kindergartens.

Intervention or response: In these shows, the effects of cigarette smoke on children were explained, and the puppets displayed strategies to avoid tobacco smoke. In fact, children learned not only about the disadvantages of tobacco smoking, but also about talking with their parents and encouraging them to quit. Children also learned to not sitting next to smokers. Moreover, educational pamphlets on the effects of tobacco use and its smoke on the body of children were distributed among parents. A few months later, interview with about 200 children, a questionnaire was distributed among parents to examine the outcomes of the program.

Results and lessons learnt: The children were happy with the shows and had conveyed anti-tobacco messages to their parents. Also, if someone did not listen to them and smoked next to them, they left the place. Parents also were happy with the training, and 38 percent of them said that the exposure of children to tobacco smoke in home had been reduced, while 5 percent of parents had quit smoking.

Conclusions and key recommendations: Childhood is an important stage for shaping the attitudes of children, and they must be provided with age-appropriate education in a cheerful and attractive way. Children can play an important role in tobacco control and encouraging people to quit. Through these activities, children learn that the clean air is their actual right and they must defend their rights and their health in the community.

Tob. Induc. Dis. 2018;16(Suppl 1):A380

DOI: $10.18332 /$ tid/84077

12:30-14:00

PS-764-5 Perception of vulnerable populations of label warnings in Global Adult Tobacco Survey (GATS) - Panama

HK Quintana' ${ }^{1}$ I Moreno Velasquez'1, V Herrera', R Roa², B Gomez' ${ }^{1}$ Gorgas Memorial Institute for Health Studies, Health Technology Assessment, Panama, Panama, ${ }^{2}$ Panama Health Ministry, Panama, Panama. E-mail: beatrizg@gorgas.gob.pa

Background: Tobacco products consumption is responsible of 148.6 million DALYS and 6.4 million deaths around the World in 2015, due to a large array associated medical conditions. Tobacco products are required to illustrate label warnings with information about such conditions to reduce their consumption in some countries, such as Panama. We aim to assess the proportion of opinions against exhibiting these label warnings according to sex, age and geographical area.

Methods: Study population derived from the Global Adult Tobacco Survey (GATS) conducted in Panama in 2013 $(n=16,962)$. We described the proportion of opinions against label warnings on tobacco products according to sex, age and geographical area (urban, rural and indigenous). Comparisons of the proportion of negative opinions within strata were assessed using chi square test.

Results: In the study population, 14,746 people replied to the question regarding their opinion in favor or against label warnings on tobacco products. Opinions against label warnings were more frequently stated by men $(6.2 \%)$ than women $(5.3 \%)$, p-value < 0.05 . People 60 years or older had a higher proportion of opinions against label warnings (7.4\%) than people younger than 15 years old $(3.4 \%)$, p-value $<0.05$. People living in urban and rural areas had similar proportion of opinions against label warnings (5.5\%, $\mathrm{p}$-value $>0.05)$. However, people living in indigenous areas had a higher proportion of opinions against label warnings than those living in urban and rural $(10.5 \%$, p-value $<0.05$ compared to urban and rural areas, respectively).

Conclusions: Opinions against label warnings are more frequent in indigenous people, older people and men. These groups are the most vulnerable to be target of advertisement of tobacco companies.

Tob. Induc. Dis. 2018;16(Suppl 1):A381

DOI:10.18332/tid/84140 


\section{2:30-14:00}

PS-765-5 Antenatal tobacco use and iron deficiency anemia: formative research to integrate tobacco cessation into antenatal care for low-income women in Mumbai, India

R Mistry ${ }^{1}$, A Jones², M Pednekar ${ }^{3}$, G Dhumal ${ }^{3}$, A Dasika ${ }^{4}$, U Kulkarni ${ }^{4}$, M Gamore ${ }^{5}$, P Gupta ${ }^{3}$

'University of Michigan School of Public Health, Ann Arbor, CA, United States of America, ${ }^{2}$ University of Michigan, Ann Arbor, $\mathrm{MI}$, United States of America, ${ }^{3} \mathrm{Healis}$ - Sekhsaria Institute of Public Health, Mumbai, India, ${ }^{4}$ University of Michigan School of Public Health, Ann Arbor, MI, United States of America, ${ }^{5}$ Municipal Corporation of Greater Mumbai, Mumbai, India. E-mail: riteshm@umich.edu

Background: A formative study was conducted at governmental antenatal clinics serving low-income women in Mumbai, India to measure the association of tobacco use with iron deficiency anemia, consumption of iron-rich foods and household food insecurity; and to identify opportunities to integrate tobacco cessation services with existing antenatal care for iron deficiency and anemia control.

Methods: We administered a structured questionnaire to a sample of 100 pregnant patients at 5 governmental antenatal care clinics that serve low-income communities. Blood tests were done for serum cotinine, hemoglobin and ferritin. We also conducted 10 key informant interviews with physicians and auxiliary nurse midwives, and 5 focus group discussions with community health workers to better understand the services offered during antenatal clinic and home visits.

Results: Blood test revealed that $16 \%, 72 \%$ and $41 \%$ of women used tobacco, were anemic and had iron deficiency anemia (IDA), respectively. Tobacco use was independently associated with IDA $(\mathrm{OR}=14.3 ; 95 \% \mathrm{CI}=2.6,77.9)$. Tobacco use and IDA were not associated with household food insecurity. Clinics had a system for screening and follow-up care for anemia and iron-deficiency, but not for tobacco use. Antenatal care providers were interested in including services to screen for tobacco use and provide cessation guidance. Patients wanted help to quit.

Conclusions: It may be worthwhile to assess the feasibility of integrating tobacco use screening and cessation services with antenatal services for anemia and iron deficiency at governmental clinics serving low-income populations in India.

Tob. Induc. Dis. 2018;16(Suppl 1):A382

DOI:10.18332/tid/84152

\section{2:30-14:00}

PS-766-5 Generativity, self-rated health and smoking behavior of older people in Sri Lanka B Perera ${ }^{1}$, B Wickramarachchi $^{2}$

'University of Ruhuna, Community Medicine, Galle, Sri Lanka, ${ }^{2}$ University of Ruhuna, Nursing Degree Program, Galle, Sri Lanka. E-mail: pperera@indiana.edu

Background: Sri Lanka has one of the fastest aging populations in Asia. Smoking may increase older peoples' risk of developing chronic diseases including cognitive impairments and depression. This study investigated the associations between smoking habits, self-rated psychological health and generativity concern (desire to nurture next generations) in a sample of older people in Galle, Sri Lanka.

Methods: A sample of older people (60 years and above) were surveyed using an interviewer-administered questionnaire. Generativity was measured using Loyola Generative Scale (LGS). Higher scores of LGS indicate higher generative concern. Self-rated health of the participants was measured using 5 point likert scale.
Results: A Total of 208 elders ( $40 \%$ were men) were surveyed. The mean age was $70.7(\mathrm{SD}=7.6)$ and $13.4 \%$ of men and $1.6 \%$ women were current smokers $(\mathrm{p}<.01)$. The mean values of LGS scores were $47.5(\mathrm{SD}=7.1)$ for men and $47.3(\mathrm{SD}=7.5)$ for women. Mean LGS score of current smokers (smoke at least once a month) is lower than that of others (43.2 versus $47.7, \mathrm{p}=0.03$ ). Self-rated psychological health in current smokers was lower than that of non-smokers $(p<.05)$. LGS score was positively associated with self-rated psychological health $(r=0.34, \mathrm{p}<0.01)$.

Conclusions: Concern about and having more interactions with younger people would probably help older people to control or quit smoking. In communities where generative concern is highly valued, the construct could be used as an effective health promotion strategy to control smoking behavior in older people.

Tob. Induc. Dis. 2018;16(Suppl 1):A383

DOI:10.18332/tid/84175

\section{2:30-14:00}

PS-767-5 Prevalence of tobacco use among street children in India

V Dogra', I Dwivedi

'Save the Children, Knowledge Management, Zirakpur, India, ${ }^{2}$ Punjab University, Biotechnology, Chandigarh, India. E-mail: vani1825@gmail.com

Background: Street children constitute one of the most vulnerable groups in India. Among marginalized children, they are at higher risk of developing early tobacco use and thus lifelong addiction. However, literature on the tobacco use among this segment of population is scarce in India. This study estimates the prevalence of tobacco use and associated health problems among street children in India.

Methods: A cross-sectional survey was conducted from May 2015 to September 2015. We purposively selected 40 street children (Age, 6-18 years) under each of the randomly sampled ten NGOs working on rehabilitation of the street children in New Delhi. Data on key variables such as sociodemographic profile, health status, and tobacco use were captured using pretested semi-structured questionnaire. STATA version 13.1 was used for data analysis.

Results: In total, 400 street children were surveyed (mean age 13.8 years). Majorities were males $(80 \%)$ and fewer were females (20\%). Almost all (92\%) of the street children belonged to socially disadvantaged classes. Nearly one third of them were suffering from skin (32\%), eye (18\%) ear infections (12\%) and cough (9\%). Thirty seven percent (148) of the street children reported ever use of tobacco products; largely bidi (54\%) and pan masala (45\%). Among ever tobacco users, nearly a third reported tobacco use on daily basis (38\%), once a week (26\%), once in two weeks $(21 \%)$, once in a month $(15 \%)$. The mean age of tobacco product initiation was 11.2 years (boys) and 12.1 years (girls).

Conclusions: Tobacco use among street children in India is high. Such data need to be collected from different parts of the country to develop anti-tobacco campaigns and supporting evidence based policy decisions for young and vulnerable population.

Tob. Induc. Dis. 2018;16(Suppl 1):A384

DOI:10.18332/tid/84178

\section{2:30-14:00}

PS-768-5 Oral malignancies and tobacco related habits among Aranadar tribals in Kerala, India: a population based study

F Pullishery ${ }^{1}$

'Batterjee Medical College, Public Health, Jeddah, Saudi Arabia. E-mail:drfawazp@gmail.com 
Background: Aranadar tribals also known as Eranadar are tribal people living in the Nilambur forests of Malabar Kerala. The aim of the study was to assess the prevalence of oral malignancies and also to assess the practices on tobacco and alcohol related habits. Methods: The study was conducted during the time period of 2014-2015. A cross sectional study design was done by selecting different age groups and gender. The Oral health Status were measured using WHO format and data on practices related to tobacco and alcohol practices by were collected using a pre-tested Questionnaire. Dietary patterns were recorded in a dietary chart. Results: We examined a total of 3685 people from all the age groups and gender. The study population consisted of $67 \%$ of total Aranadar tribals in the Nilambur forest region. The prevalence of Oral Malignancies was found to be $27 \%$ in the study population. Cancer of tongue (45.5\%) and buccal mucosa (39\%) was found to be more common in these population. Pre-malignant lesions like leukoplakia(18\%), erythroplakia(7\%) and lichen planus(3.2\%) were also reported among these tribal people. $68 \%$ of the $10-15$ year old male children were found using tobacco products in one or the other forms. $34.7 \%$ of the women reported the use of tobacco. The most common cancers among the youngest age group are those of tongue and buccal mucosa (51.26\%). Alcohol use were reported in $57 \%$ of the study population. There was strong association with tobacco and oral cancer in tribal people and it was statistically significant $(\mathrm{p}<0.05)$.

Conclusions: The prevalence of oral cancer was found to very high in these tribals people compared to other semi-urban and urban populations. The study reveals that awareness regarding the harmful effects of use tobacco and local arrack among these Aranadar tribals were very less.

Tob. Induc. Dis. 2018;16(Suppl 1):A385

DOI:10.18332/tid/84195

\subsection{Tobacco, inequality and poverty}

\section{4:30-16:00}

F0-326-3 Poverty and smoking: assessing the financial impact of tobacco purchases on households' budgets in Argentina

S Melitsko ${ }^{1}$

${ }^{1}$ National Administration of Social Security, Ciudad Autónoma de Buenos Aires, Argentina. E-mail: silvana.melitsko@gmail.com Background: Opportunity costs of resources spent on tobacco are higher for low income households. This study assesses the financial impact of tobacco consumption on households' budgets in Argentina. First, it examines budget allocation among tobacco and other goods and services using a nationally representative household survey. Second, it explores the impact of tobacco consumption on the probability of having out-of-pocket expenditures on healthcare.

Methods: We perform statistical analysis on the latest Household Expenditure Survey carried out by the National Institute of Statistics of Argentina in 2012/2013. This probabilistic, stratified, multistage sample contains information on consumer expenditures and socioeconomic characteristics of 20.960 urban households. Descriptive analysis of budget allocation by income quintile is performed first. Second, the impact of tobacco consumption on healthcare spending is assessed by a probit model. The dependent variable takes the value of 1 if the household had out-of-pocket health expenditures, and zero otherwise. Regressors include standard socioeconomic determinants of healthcare spending, such as presence of minor and senior members in the household, family income, medical insurance, sex, age, occupational status, and education.

Results: Purchases of tobacco products represent $2.9 \%$ of total spending in households where at least one member consumes tobacco products. The proportion is higher in low-income households: it averages $3.3 \%$ in the bottom income quintile, and $2.2 \%$ in the top income quintile. Furthermore, the probit model shows that, other factors being held equal, having a smoker in the household increases the probability of out-of-pocket healthcare expenditures by $8.72 \%$ (95\% CI: 4.87\%-12.57\%).

Conclusions: Smoking imposes significant financial constraints on households' budgets. These constraints are more severe on lowincome households, and may prevent them from escaping poverty by reducing resources available for long-term investments. This study provides evidence that an effective tobacco control policy may reduce disparities among socioeconomic groups beyond its well-known effects on health outcomes.

Tob. Induc. Dis. 2018;16(Suppl 1):A386

DOI:10.18332/tid/84631

\section{$14: 30-16: 00$}

F0-327-3 Lived experience of tobacco tax increase among socioeconomically disadvantaged smokers M Chan Sun ${ }^{1}$, R Vincent ${ }^{1}$

'University of Mauritius, Medicine, Reduit, Mauritius. E-mail: lan.sun@uom.ac.mu

Background: Tobacco tax increase reduces tobacco use, but smokers especially socioeconomically disadvantaged who do not quit smoking may experience financial stress (Lantz et al. 2005; Siahpush et al. 2007; Krueger et al. 2008; Pampel et al. 2010). This study was carried out to explore the lived experience of socioeconomically disadvantaged smokers with respect to tobacco tax increase. The study objectives were to look into their spending mechanism, to examine their smoking behaviour and to capture their cognition to quit smoking.

Methods: This qualitative study with phenomenological approach took place in a smoking cessation clinic of a regional hospital in Mauritius. Apurposive sample of socioeconomically disadvantaged smokers was constituted until theoretical saturation was reached. A semi-structured guide was used to collect data through face-toface interviews which were recorded and transcribed verbatim. Data was analysed using Thematic Framework Analysis in order to establish the emerging themes. Ethical clearance was obtained from the relevant research ethics committee.

Results: The main themes which emerged included the following: Tobacco-related expenses as priority, Large amount of monthly income for tobacco-related expenses, Need to satisfy cravings, Exacerbation of financial stress, Scepticism about tobacco tax effectiveness, Strategies to maintain smoking, Need for complete ban of cigarettes in the country and Need for government to provide assistance for smoking cessation. The findings from this study constitute new qualitative data pertaining to the lived experience of a group of socioeconomically disadvantaged smokers.

Conclusions: This study shows that tobacco taxation contributes to the socioeconomic burden of smokers who maintain smoking. In order to address tobacco-related poverty and inequality, there is need for targeted intervention by health authorities to provide assistance for smoking cessation among socioeconomically disadvantaged smokers, especially during the aftermath of tobacco tax increase policy of the country.

Tob. Induc. Dis. 2018;16(Suppl 1):A387

DOI:10.18332/tid/83972 


\section{6:00-17:30}

RF-1209-3 Smoke-free legislation and socioeconomic inequalities in smoking-related morbidity and mortality among adults: a systematic review

M Jamaludin ${ }^{1,2}$, GP Nazar ${ }^{3,4}$, R Palladino ${ }^{5}$, G Tsakos ${ }^{2}$, RG Watt ${ }^{2}$, C Millett ${ }^{5,6}$

${ }^{1}$ University of Malaya, Department of Community Dentistry and Clinical Prevention, Kuala Lumpur, Malaysia, ${ }^{2}$ University College London, Department of Epidemiology and Public Health, London, United Kingdom, ${ }^{3}$ Public Health Foundation of India, Health Promotion Division, Gurugram, India, ${ }^{4}$ London School of Hygiene \&t Tropical Medicine, Non-Communicable Disease Epidemiology, London, United Kingdom, ${ }^{5}$ mperial College London, Public Health Policy Evaluation Unit, School of Public Health, London, United Kingdom, ${ }^{6}$ Public Health Foundation of India, Gurugram, India. E-mail: gaurang.nazar@phfi.org

Background: Previous systematic reviews examining the impact of smoke-free legislation (SFL) suggest more beneficial impacts in higher socioeconomic status (SES) groups. However, these have focused on associated changes in smoking behaviour due to a paucity of studies examining health outcomes. This review includes more recent evidence to assess the impact of comprehensive and partial SFL on socioeconomic inequalities in smoking-related: a) disease-specific morbidity and mortality and b) all-cause mortality among adults.

Methods: We searched Medline and fourteen other databases up to September 2016. Studies in English, reporting the differential impacts by SES of SFL on smoking-related morbidity and mortality among adults ( $\geq 18$ years) were eligible. Following data extraction, quality and risk of bias among the included studies were assessed using the Cochrane EPOC and EPHPP criteria. Harvest plot and narrative methods were used to illustrate either i)neutral effect of SFL on inequalities, ii) positive effect (greater benefits among lower SES groups, iii) negative effect (greater benefits among higher SES groups) or iv) mixed/unclear effect.

Results: Eight studies (six interrupted time-series and two before-after studies), all from high-income countries were included; five studies examined the impact of comprehensive SFL and three the impact of partial SFL. Implementation of comprehensive SFL led to either a positive effect (three studies) or no effect (two studies) on socioeconomic inequalities in health outcomes; partial SFL led to either no effect (two studies) or unclear effect (one study). The choice of the socioeconomic indicator may have had an important influence on findings. Except for the before-after studies, most studies were judged to be high quality with a suitable study design.

Conclusions: Comprehensive SFL potentially reduces inequalities in smoking-related health outcomes, partial SFL may not. All countries should implement comprehensive SFL to achieve the Sustainable Development Goal targets to reduce premature NCD mortality and inequalities.

Tob. Induc. Dis. 2018;16(Suppl 1):A388

DOI:10.18332/tid/84127

12:30-14:00

PS-772-5 Completely given up sale of cigarette at a village in Sri Lanka

A Tenna

'ADIC, Colombo, Sri Lanka. E-mail: tennaamaranath@gmail.com Background and challenges to implementation: "Katthapahuwa" is a village with 1341 population locates in Kurunegala district. Among the social issues, cigarette consumption was high; at baseline server reveled that $3 / 4$ (341) of adult male consume cigarettes. There were 21 cigarette vendors scatted all over the village. Nearly 1550 cigarettes were sold per day its value was USD 504.16.

Intervention or response: Interventions started in late March 2016, with developing a youth club at the village in collaboration with Sanasa Rural Development Bank. ADIC had trained 16 village youth leaders to overcome cigarette issue and they had identified one of the strategy was to reduce the cigarette availability at the village.

Firstly, youth leaders met the vendors' association (VA) and emphasized regarding how Tobacco Company cheated vendors for small profit and explained that how much money they have stolen from our village. Future educated VA on their children also will be trapped by the tobacco industry.

The main argument was made by the VA that if vendors stop selling cigarette, selling of other goods may reduce because customers move to other shops. We explained them that majority of the customers come not for cigarette but Tobacco Company taught you that business runs lost if not sell cigarettes.

Finally the VA decided to stop sell cigarettes. Vendors needed one month period because they already purchased cigarettes.

Mid Jun of 2016, all vendors stopped cigarette selling totally. The youth team organized an event to appreciate service of vendors. Interactions continually maintain with vendors by the youth group today too.

Results and lessons learnt: 21 vendors totally stopped cigarette selling, nearly USD 15124.92 money served for month. End year server reveled that significantly decreasing of cigarette use by $43 \%$ (147) and 13 persons quitted use.

Conclusions and key recommendations: The decline of cigarette consumption in a village can have a big effect on the stop sale of cigarettes.

Tob. Induc. Dis. 2018;16(Suppl 1):A389

DOI: $10.18332 /$ tid/83908

\subsection{Tobacco farming: Opportunities and experiences in facilitating alternative livelihoods and preventing tobacco adverse environmental impact}

12:30-14:00

PS-773-5 Financing alternatives to tobacco growing: Philippine experience in implementing Art. 17 and 18

JL Reyes'

'Southeast Asia Tobacco Control Alliance, Bangkok, Thailand. E-mail: jennie@seatca.org

Background and challenges to implementation: The tobacco industry has perpetuated the myth that alternatives to tobacco are not economically viable or are non-existent. However, increasing global evidence showing the harmful effects of tobacco among workers, their families and communities, the economy, and the environment are compelling even the world's top tobacco producers to find alternatives to tobacco growing.

FCTC Articles 17 and 18 obligate Parties to provide support for economically viable alternative livelihood to tobacco growers and workers. Policy options and recommendations on economically sustainable alternatives to tobacco growing were adopted at the sixth session of the Conference of the Parties in 2012.

This presentation will highlight the Philippine's experience in using the Sin Tax Law's provision of earmarked revenues 
specifically on implementation of alternative livelihood to tobacco farming. Key issues and challenges hindering implementation will be identified, while policy recommendations will be proposed to ensure that viable alternatives are implemented effectively.

Intervention or response: The Philippine's Sin Tax Law enacted in 2012 restructured the excise taxes of tobacco and alcohol products. The law was both a fiscal measure to increase government revenues, and a health measure that discourages tobacco consumption and increases allotment for healthcare. The law also amended existing laws that allocated incremental revenues from tobacco excise taxes for programs to promote economically viable alternatives for tobacco farmers and workers in areas where they are cultivated.

Results and lessons learnt: Vegetables and corn are suitable alternatives in areas where tobacco is cultivated. Cost returns analysis shows that cultivating vegetables gave 1.5 to 5 times higher net income while corn is at par in terms of revenue.

Conclusions and key recommendations: By providing funding for implementation of alternative livelihood, the Philippines has started complying with FCTC Article 17 and 18. Government efforts should be expanded to ensure that programs aimed at promoting alternative livelihoods to tobacco farming are implemented effectively.

Tob. Induc. Dis. 2018; 16(Suppl 1):A390

DOI:10.18332/tid/83857

\section{2:30-14:00}

PS-774-5 Policy recommendations on tacing and reducing program mismatch and perverse incentives present in earmarking sin tax to tobacco growing areas

MJ Aloria', M Reganon ${ }^{2}$, JJ Chavez ${ }^{3}$

${ }^{1}$ Action for Economic Reforms, Fiscal Policy Team, Quezon, Philippines, ${ }^{2}$ Philippine Competition Commission / University of the Philippines School of Economics, Mandaluyong, Philippines, ${ }^{3}$ Action for Economic Reforms, Industrial Policy Team, Quezon, Philippines. E-mail:madzaloria@gmail.com

Background: Recognizing that the Philippine 1991 tobacco tax sharing law favoured the development of tobacco, the Sin Tax Law, a separate law on restructuring cigarette tax, expanded the use of such to also cover shifting farmers to viable alternative livelihood. Aside from health-driven supply reduction objectives, this is crucial as evidence shows that tobacco production is continuously declining starting decades ago, requiring to actively shift farmers.

Methods: Data on tobacco excise tax earmarking and utilization, farmers' production and shifting behaviours, labor improvement and poverty alleviation indicators, and LGU capacity were analysed to determine potential program mismatches and perverse incentives. Supporting qualitative data were used to identify policy and structural gaps to address these.

Results: Financing livelihood projects becomes the least priority (only $6 \%$ average share i funds), as a result of LGU's final allocation being determined by their share in total tobacco leaf production, which actually put pressure on their farmers to increase production volume. Meanwhile, infrastructure continue to get bulk of sin tax earmarking and are linked to its political benefits. Last, the provision of cooperative and agro-industrial projects in selected areas were shifting behaviour is heavy can still be improved.

Conclusions: As the two tobacco tax sharing laws fund both programs to develop tobacco and to shift tobacco farmers to other livelihood, a schizophrenic management exists. Key structural and policy ingredients have to be present to reverse this. First is the need to establish institutional support to manage alternative livelihood funds, in order to balance the powers of National Tobacco Administration over tobacco growing areas. Allocation should not be based on production volume but rather on a systemic or comprehensive welfare assessment of shifted farmers. As livelihood programs are most commonly coursed through civil society organizations and cooperatives, trainings on local budget engagement, tobacco interference, and enterpreneurial skills, have to be offered.

Tob. Induc. Dis. 2018;16(Suppl 1):A391

DOI:10.18332/tid/83874

\section{2:30-14:00}

PS-775-5 Evaluating results of the program for tobacco leaf diversification in Brazil

F Mendes ${ }^{1}$

${ }^{1}$ National Cancer Institute, Executive Secretariat of National Comittee for FCTC Implementation, Rio de Janeiro, RJ, Brazil. E-mail:fmendes@inca.gov.br

Background: The FC TC took special care to the most fragile link of tobacco production chain: the tobacco leaf producer. Articles 17 and 18 require countries to adopt specific policies to identify more profitable and healthy product diversification mechanisms.

Brazil is the third largest producer in the world and the largest exporter of tobacco leaf, with 144,000 producer families distributed in the South region. Of the 590,000 tons of tobacco produced in $2016,85 \%$ was exported, which means that the continuity of this activity depends on the world's demand, impacted by the accelerated FCTC implementation.

The Program was created in 2005 and its objective is to articulate a set of public policies and programs (credit, guaranteed purchase, distribution, etc.) to reduce farmers' dependence on tobacco leaf production. The Program is operationalized through public grants, for which local organizations and cooperatives submit projects to provide technical assistance to farmers. By 2017, 11 thousand families were covered.

Methods: Interviews were conducted by Ministry of Agrarian Development in 2011 and 2013 with 250 families assisted by the Program, with questions on family and production profiles, health issues, productivity, income, etc. The research allowed evaluating the impacts of the work developed by the organizations benefited by the public grants.

Results:

- Households not producing tobacco anymore (2011-2013): $6,5 \%$ to $10,2 \%$

- Income grows: $\mathrm{R} \$ 1.8$ thousand to $\mathrm{R} \$ 2.3$ thousand

- $76 \%$ of family farmers wish to leave tobacco growing. Why? $-23.2 \%$ hard work

$-22.7 \%$ low financial income

$-20.8 \%$ health problems

- $13.5 \%$ use of agrochemicals

Conclusions: The data shows that the Diversification Program had a positive impact on the family incomes. However, the lack of marketing networks and the lack of articulation with public marketing policies are still an obstacle, as the purchase of tobacco leaf is guaranteed by the tobacco industry.

Tob. Induc. Dis. 2018;16(Suppl 1):A392

DOI:10.18332/tid/84012 
12:30-14:00

PS-776-5 Instructing people about the effects of tobacco on the environment by adolescents in Tehran

F Matinkhah', The Clean Breath Study Group

'Iranian Anti Tobacco Association, Tehran, Iran, Islamic Rep.

Of.E-mail: e.matinkhah@gmail.com

Background and challenges to implementation: Public attention has recently been drawn to the harmful effects of tobacco on the environment. Cigarette butts are toxic materials that pollute the nature and organisms, and leaving them in nature causes serious damage to the life cycle. The Clean Breath Center which is active in the area of tobacco prevention in children and adolescents has recently focused a part of its agenda on the theme of environmental effects of tobacco products.

Intervention or response: According to the plan, the volunteer adolescents were encouraged to collect cigarette butts found in parks and various urban areas and to demonstrate the butts to the people and instructing them about harmful effects of cigarette butts on the environment. Distribution of informative pamphlets, including images of nature and injured animals, was another part of the program. The project was conducted in the last two years concurrent with various occasions, such as the Health Week, the National No-Tobacco Week, and the Clean Air Week.

Results and lessons learnt: About hundred adolescents aged 10 to 16 participated in the project and, as a part of it, collected a large amount of cigarette butts from the urban areas. About 98 percent of the people interviewed did not know about the effects of tobacco use on the environment. In addition, the adolescents' peers became familiar with the destructive effects of smoking through them.

Conclusions and key recommendations: The involvement of adolescents in tobacco control programs and encouraging them to lead such activities can play an important role in the enhancement of community health. Unfortunately, the government's cooperation in this regard and the current public training and information dissemination are not satisfactory. Also, the government can impose fines for contaminating the environment by smokers and the companionship of the youngsters and their concern for the future generations can be useful in reducing the harmful effects of tobacco.

Tob. Induc. Dis. 2018;16(Suppl 1):A393

DOI:10.18332/tid/84042

\section{2:30-14:00}

PS-777-5 The reality of tobacco farmers exploitation in a region in Nigeria

E Cadmus', T Ajayi', T Iken', P Japkor'ㄹ, A Oluwafemi², A Adebiyi' 'University of Ibadan, Community Medicine, Ibadan, Nigeria, 2ERA/FoEN, Lagos, Nigeria.E-mail: eniyolacadmus@gmail.com

Background: In recent times, the production of tobacco leaves has shifted from high-income countries to developing countries, especially those in Africa. In these countries, the governments support tobacco farming as a method of poverty alleviation. In addition, the Tobacco Industry (TI) actively promotes the alleged benefits of tobacco farming to the farmers and country, and these supposed benefits have been used to obstruct tobacco control policies. There is limited data regarding effects of tobacco farming from the view point of the growers themselves. This study set out to document the experience of tobacco farmers regarding the processes and profit gained through tobacco farming.

Methods: A cross sectional study using qualitative method of enquiry was carried out. Two agrarian communities with huge acreage ownership by the British American Tobacco Company
Nigeria for tobacco farming were purposively selected for the study. Key informant interviews were conducted among the farmers and data obtained were transcribed and analysed though thematic analysis.

Results: Findings from the study show that although the tobacco farmers received support from the Industry to grow tobacco, they were often forced to sell their products with minimal margins of profit to the tobacco companies since it cannot be sold on open markets in Nigeria. Furthermore, there is a market monopoly as only one company buys the produce further reducing their bargaining power. Furthermore, the labour intensive nature of the tobacco farming process necessitates recruitment and of their children and other family members leading to hazardous exposure. Conclusions: This study provides evidence which refutes claim by the Tobacco Industry about promises of prosperity to all involved in tobacco cultivation. Interactions with the farmers, did not indicate that tobacco farmers were wealthier than the non-tobacco farmers. This information will assist the drive for tobacco control policies.

Tob. Induc. Dis. 2018;16(Suppl 1):A394

DOI: $10.18332 /$ tid/84202

\section{2:30-14:00}

PS-778-5 Carbon footprint of the cigarette industry - an analysis from India

P Lal ${ }^{1}$

'International Union Against Tuberculosis and Lung Disease, New Delhi, India. E-mail: plal@theunion.org

Background and challenges to implementation: When accounted for the entire lifecycle for its production, cigarettes are devastating for global and local environment - from clearing of forests (reduces carbon capture potential of soils, food security, erosion of biodiversity and soils, impact of water table etc..), cultivation (use of pesticides, soil erosion), curing (high use of wood and emissions), storage (use of fumigants like methyl bromide), packaging (using virgin pulp and paper) and disposal of packaging and cigarette.

Intervention or response: This study uses "the whole of the lifecycle" approach to measure carbon emissions of an average cigarette (from energy used for farming to energy consumed to make raw materials and manufacturing of the product, right through to its use, reuse and disposal or recycling) and compare this with other commonly used products. We using the open source software developed by UNFCC. Data was procured from open accessed data from the 2009 sustainability report of India's leading cigarette manufacturer (Indian Tobacco Company, ITC). Results and lessons learnt: Deriving the carbon produced to make and dispose one cigarette, this study conservatively estimates that in 2010, India which produced 100,000 million cigarettes would have emitted nearly $67500 \mathrm{tCO} 2 \mathrm{e}$ to produce these (limited lifecycle, no historical emission and mitigation ability is considered, leaf for export not considered).

Conclusions and key recommendations: Given that manufacturing cigarette has a deep and significant environmental footprint makes for a strong case on environmental grounds to cease production of tobacco and production of tobacco products. To do this, tobacco control advocates need to reach out to environmental organisations, many of whom receive financial support from tobacco companies, which is an inherent conflict of interest. Environmental organisations are oblivious of this paradox.

Tob. Induc. Dis. 2018;16(Suppl 1):A395

DOI:10.18332/tid/84366 
12:30-14:00

PS-779-5 Development of Indonesia's tobacco farming and business in the era of FCTC

Y Syaukat ${ }^{1}$

'Bogor Agricultural University (Institut Pertanian Bogor), Resource and Environmental Economics, Bogor, Indonesia. E-mail:ysyaukat@gmail.com

Background and challenges to implementation: Indonesia is one of the biggest producers and consumers of tobacco products (mainly cigarettes) and also one of the largest producers of tobacco. Indonesia is one of eight countries who has not ratified the WHO FCTC. Currently, Indonesia is at a crossroad in controlling production and consumption of tobacco and tobacco products. The government is reluctant to effectively implement the existing regulations on controlling production and consumption of cigarettes and tobacco since he receives a large amount of tax incomes from tobacco products, and tobacco farming also absorb a large amount of agricultural labors.

Intervention or response: The existence of regulation that explicitly and comprehensively control production and consumption of tobacco and tobacco product is a necessary condition to Indonesia. Indonesia's policy should be adapted to be inline with the situations in FCTC era. Strategies and programs in development of tobacco farming and business need to develop to support tobacco farmers in meeting the local tobacco demands and at the same time increase farmers' welfare.

Results and lessons learnt: Most of the farmers are poor, since they operate at low area of land, low productivity, high production costs, and low price of output. In addition, under the current policy regime, Indonesia imports a large amount of tobacco, ranging from 45 to 75 percent of total domestic tobacco production, thus has negative consequences on the prices of tobacco and the incomes of farmers.

Conclusions and key recommendations: The affirmative programs in developing tobacco business, including: development of alternative crop to tobacco, provision of support programs to the farmers in implementing this crop conversion, reducing tobacco import level by promoting the use of domestic tobacco, and improvement in tobacco marketing are needed in the future. These programs have to be firmly and consistently implemented to optimize local tobacco production, increase farmers' incomes and at the same time reducing the poverty level.

Tob. Induc. Dis. 2018;16(Suppl 1):A396 DOI:10.18332/tid/84429

\section{2:30-14:00}

PS-780-5 Elimination of tobacco growing is possible: a case study from Karnataka India

\section{P Prabhakara ${ }^{1}$, AK Pandey ${ }^{2}$, P Lal ${ }^{2}$, V Suvarna ${ }^{3}$}

'Department of Health and Family Welfare, Government of Karnataka, State Anti Tobacco Cell, Department of Health and Family Welfare, Bangalore, India, ${ }^{2}$ The Union South East Asia Office, New Delhi, Tobacco Control, New Delhi, India, ${ }^{3}$ Government of Karnataka, Agriculture Department, Mysore, India.E-mail: biprabhakar.kar@gmail.com

Background: Article 17 and 18 of WHO's Framework Convention on Tobacco Control mandates Parties to adopt strategies for alternative farming for tobacco growers. The Indian state of Karnataka has shown its commitment in tobacco control. However Karnataka is also the second largest tobacco growing state India. The registered tobacco growers in the state have more than doubled has grown by $222.59 \%$ (from 18751 to 41737 ) and the area under tobacco cultivation has increased by $269.72 \%$ (from 29852 hectares to 80516 hectares) from in 2001 and 2016. Methods: Since 2015, Karnataka has adopted supply reduction measures of tobacco crop in a phased manner. The Department of Agriculture and Horticulture in collaboration with The Tobacco Board has piloted a project to help shift tobacco growers to alternative crops and adopt new cropping patterns. It has undertaken sensitization of farmers to adopt alternative cropping, provided access to soft loans from banks, free soil testing, drip irrigation, subsidized fertilizer, among other incentives; and have linked them to Horticultural Producers' Co-operative Marketing and Processing Society Ltd. (HOPCOMS) to ensure market for grown products.

Results: In Hunsur block of Mysore 1000 tobacco growers have voluntarily stopped tobacco farming and have adopted alternate crops. Given the early success of the pilot project, The Government has scaled up the intervention from current 250 hectares to 4500 hectares, covering other four blocks in three tobacco growing districts.

Conclusions: Tobacco farmers are ready to shift to other crops or cropping patterns but need guidance and support from Government. Farmers realize that there is limited future in tobacco cultivation due to variability in rain and declining fertility of soil which they attribute to monocropping of tobacco; and more importantly an increasing awareness among the public (and growers) on the harms of tobacco use.

Tob. Induc. Dis. 2018;16(Suppl 1):A397

DOI:10.18332/tid/84437

\subsection{Youth tobacco experience}

\section{2:30-14:00}

PS-782-5 Smoking facts and evidence among medical students from „Carol Davila" University of Medicine and Pharmacy, Bucharest, Romania F Mihaltan', S Costache ${ }^{2}$

${ }^{1}$ National Institute of Pneumology, Pneumology, Bucharest, Romania, ${ }^{2}$ UMF C. Davila, Pneumology, Bucharest, Romania. E-mail:mihaltan@starnets.ro

Background: The purpose of this study is to identify the main reasons why medical students from "Carol Davila" University of Medicine and Pharmacy, Bucharest, Romania are smoking, how many of them are using cigarettes, what is their level of addiction, and moreover, what is their attitude towards smoking.

Methods: A questionnaire was distributed to the medical students of the "Carol Davila" University of Medicine and Pharmacy, Bucharest, Romania, from February 14 to March 18, 2016. 969 medical students answered the questionnaire. The questionnaire was composed of 30 questions and Fagerstrom test. Data analysis was performed using descriptive statistics.

Results: A total of 969 (277 males and 692 females) completed and returned the questionnaire, From a total of 308 first year students, 144 (46.8\%) have been smoking (37.5\% males and $62.5 \%$ females); The mean age a student begins smoking was 15.8 years. The most important reasons for cigarette smoking were: decrease of anxiety (51.4\%), pleasure (48.8\%) and habitude (43.7\%). One hundred and forty six (34\%) need to light up a new cigarette after 1-3 hours from the previous one. The study shows that one hundred ninety-one (44.2\%) of current smokers want to stop smoking and two hundred forty $(56 \%)$ have already tried it, but they failed. Eighty (18.5\%) medical students quit smoking and the main reason was because smoking is harmful to human health 
(62.7\%). According to the Fagerstrom test, 59\% were evaluated to have low dependence, $33 \%$ moderate dependence and $8 \%$ high dependence.

Conclusions: The results of current study indicate a need to provide comprehensive tobacco control programs among medical students, and smoking cessation programs. An unceasing effort is needed by all concerned to reduce the number of smokers between medical students.

Tob. Induc. Dis. 2018;16(Suppl 1):A398

DOI:10.18332/tid/83750

\section{2:30-14:00}

PS-783-5 Photoaging smartphone app to reduce smoking prevalence in secondary schools: the smokerface randomized trial

\section{TJ Brinker}

${ }^{1}$ University Hospital of Essen, Essen, Germany. E-mail: titus. brinker@uk-essen.de

Background and challenges to implementation: School-based tobacco prevention is often costly and does not harness the broad usage of smartphones in adolescence.

Intervention or response: We took advantage of the widespread availability of mobile phones and adolescents' interest in appearance to develop a free selfie photoaging app "Smokerface" which is promoted via a poster campaign in secondary schools. This study involving 10,000 students in 126 German secondary schools aims to evaluate its effectiveness regarding smoking prevalence and students' attitudes towards smoking at two years follow-up.

Results and lessons learnt: After one year of follow-up, preliminary results show good adhesion of the schools to the posters (110 schools of 126 fully comply with the study protocol), only seven dropped out. This is the first national study to show that current e-cigarette and cigarette smoking prevalence in grades 6 and 7 of secondary schools are the same (4.7\% use cigarettes and $4.6 \%$ use e-cigarettes).

Conclusions and key recommendations: Our research has the potential to pave the way for a new form of low-cost and broadly available school-based tobacco prevention in the form of poster campaigns promoting a free app. Our baseline analysis shows good comparability between the groups at baseline after randomisation and provides new insights into the prevalence of smoking and the use of e-cigarettes among pupils in the 6th and 7th grades in Germany.

Tob. Induc. Dis. 2018;16(Suppl 1):A399

DOI:10.18332/tid/83775

\section{2:30-14:00}

PS-784-5 The ASSIST smoking prevention programme in adolescent social networks beyond the school setting: an exploratory study

F Dobbie', L Bauld', A Amos'2, S Haw ${ }^{1}$

'University of Stirling, Stirling, United Kingdom, ${ }^{2}$ University of Edinburgh, Edinburgh, United Kingdom. E-mail: fiona.dobbie@ stir.ac.uk

Background: ASSIST (A Stop Smoking in Schools Trial) is a peerled, smoking prevention, social network intervention developed in the UK. ASSIST trains students (aged 11-13) to work as peer supporters to disseminate non-smoking norms in their year. To date, evaluation of ASSIST has focused on delivery within schools only. This study sought to address this gap by using qualitative social network research to map the social networks of peer supporters to explore the extent, nature, content and perceived impact of message diffusion beyond the school year.

Methods: Qualitative, face to face interviews conducted in school with 16 young people aged 12-13 incorporating the creation of 'egocentric sociograms' to collect network data. Sociograms were then used to create a qualitative narrative to explore the extent, and perceived impact, of message diffusion in the wider social networks of peer supporters. Computer packages UCINET and NVivo were used to conduct analysis.

Results: Peer supporter networks were fairly large with a total of 155 people. Peer supporters recalled having conversations about smoking with 102 network members, two thirds $(n=63)$ of which were with people out with their school year (e.g. parents, siblings, other family members, out of school friends, neighbours). Thematic analysis of conversation content revealed three types of conversation: protecting non-smokers from starting to smoke; encouraging smokers to stop; and protecting people in wider social networks. Perceived impact was noted for 37 people in peer supporter networks, with examples of positive and negative impact, focusing on the dynamics of a child speaking to an adult. Conclusions: Smoking prevention message diffusion was not limited to school year, reaching in to the wider networks of peer supporters. This creates learning opportunities for the future delivery of ASSIST or other similar peer-led interventions for smoking prevention, and the role of social network interventions in prevention.

Tob. Induc. Dis. 2018;16(Suppl 1):A400

DOI:10.18332/tid/83792

\section{2:30-14:00}

PS-785-5 Does level of physical activity influence tobacco use behaviour among youth? A crosssectional survey from India

S Goel', D Monga'

'PGIMER, School of Public Health, Chandigarh, India. E-mail: sonugoel007@yahoo.co.in

Background: Tobacco use and physical inactivity are major risk factors for Non Communicable Diseases (NCDs). Adequate physical activities keep one healthy but sedentary life style may contribute to other unhealthy practices like indulging in tobacco use. There is a need to identify if and how the two risk factors are interrelated so as to address them more strategically. The present study was undertaken to assess the level of physical activity and to identify its association with tobacco use among college going youth in a jurisdiction of North India.

Methods: Data on physical activity and tobacco use was collected from 500 students aged 18-24 years studying in different professional and nonprofessional colleges of a Union Territory of Chandigarh, India through multistage simple random sampling using self-administered standardized questionnaires (Global Adult Tobacco Survey, Fagerstorm Addiction Scale, Global Physical Activity Questionnaire).

Results: Around $12.8 \%(\mathrm{n}=64)$ were physically inactive, $10 \%$ $(n=50)$ were active and $76.2 \%(n=381)$ were highly active. Females (15.8\%) were more inactive as compared to males (12.1\%). Highly active respondents were seen more in 18-20 years age group (77.6\%) as compared to $20-22$ years age group (76.1\%). The prevalence of tobacco use was $20.2 \%$. It was observed that odds of consuming tobacco was significantly lower in physically active respondents as compared to inactive respondents ( $\mathrm{p}$ value $<0.05)$. Further, higher odds of smoking was observed among younger age groups, females and non-professionals as compared 
to their counterparts.

Conclusions: NCDs are emerging as a big threat among youth. Our findings indicate a significant relationship between physical activity and tobacco use among youth thus underscoring the need of promoting physical activity among youth to curb tobacco use. Regular physical activity and counselling sessions for discouraging tobacco use should be integrated with academic curriculum so as to achieve Goal-3 of Sustainable Development Goals.

Tob. Induc. Dis. 2018;16(Suppl 1):A401 DOI:10.18332/tid/83808

\section{2:30-14:00}

\section{PS-786-5 Youths and tobacco in the Gambia}

\section{K Conteh ${ }^{1}$}

'African Network for Information and Action Against Drug (RAID-The Gambia), Banjul, Gambia. E-mail: karamosbc@ gmail.com

Background and challenges to implementation: Studies have shown that, the use of tobacco is still on the increase in the Gambia especially among the youth folk of the populace. Several studies were conducted over the years to determine the prevalence of tobacco use in different contexts showed interesting revelations. Tobacco use prevalence: Male 34\%, Female 1.5\% (WHO 2010) youth aged 13-15 years 24\% (GYTS 2008); no data on smokeless tobacco.

Even though, Gambia has made significant achievements over the past years on tobacco control, there's no proper implementation or effective enforcement plan for tobacco control legislation that will minimize the use of tobacco among young people in the Gambia. Intervention or response: The Gambia has developed a tobacco control strategy and enforcement of various tobacco control legislation, particularly the Prohibition of Smoking (in public places) and Ban on advertisement of tobacco products in 2003, packaging and labeling of tobacco product in October 2010 and the recent comprehensive tobacco control law in December 30th 2016.

Results and lessons learnt:

1. Coordination is very powerful in tobacco control

2. The connection between government institutions and civil society is indeed crucial for tobacco control

3. The involvement of youths, women's group and religious leaders is essential in tobacco control

4. Advocacy, sensitization and awareness creation on tobacco among the different age cohort is crucial

5. The involvement of media fraternity in tobacco control is needed

Conclusions and key recommendations:

1. Strengthen coordination between the civil society tobacco control advocates, multi-sectoral working group and law enforcement body for effective and efficient control and implementation of the comprehensive tobacco control law in the Gambia

2. Build the capacity of tobacco control advocates particularly the CSO, journalist/ or Health journalist at country level to effectively monitor and control tobacco

3. Provide Evidence through research and expertise on TC

Tob. Induc. Dis. 2018;16(Suppl 1):A402

DOI:10.18332/tid/83809

$12: 30-14: 00$

PS-787-5 Youth initiatives in promoting tobacco free campuses in Rwanda
0 Nimbabazi ${ }^{1,2,3}$, Smoking Youth Study Group

${ }^{1} \mathrm{AB}$ Global Health Initiative, Kigali, Rwanda, ${ }^{2}$ Rwanda Pharmaceutical Students Association, Huye, Rwanda, ${ }^{3}$ Young Proffesionals for Chronic Disease Network, Research, Kigali, Rwanda. E-mail: onimbabazi@gmail.com

Background and challenges to implementation: Global youth tobacco use is widespread throughout the world and this is a problem that will results in continuation in adulthood, early cardiovascular and lung diseases, nicotine dependence and also premature death from smoking, being a youth issues the problem become that it's easy to spread by peer pressure, in Rwanda the case is the same and a big number of those young smokers they learn from others in boarding schools and campuses, it's from here youth led organizations took initiatives to teach their colleagues the harmfulness of smoking.

Intervention or response: Youth initiatives of peer education about the harm of tobacco has been initiated by healthcare students led organizations in different campuses that motivate their colleagues quit smoking and goes with advocacy in stopping smoking in schools area, banning tobacco sales in schools, putting poster with messages around the campus and messages in different gatherings like sport competitions all these are for promoting smoke free campuses and these students will be the intellectuals that will teach their respective home communities.

Results and lessons learnt: More than 10000 youth have been reached by these initiatives in campuses and around campuses areas where they had got different messages on tobacco harm, strategies to quit smoking and all info that can help youth to promote a smoke free generation starting from campuses. Some important concerns and challenges met have been reported to universities and biomedical centre for further policy and decision making.

Conclusions and key recommendations: Educating youth is key to have a sustainable smoke free generation, and they are interested with that and still these initiatives need more support to reach many people. Universities and governments have to support these initiatives as they are cheap interventions in reducing burden of smoking in country. And more researches can be done to know more info of what can help youth quit smoking.

Tob. Induc. Dis. 2018;16(Suppl 1):A403

DOI:10.18332/tid/83810

$12: 30-14: 00$

PS-788-5 Social differences in implementation of a school based smoking preventive intervention LS Bast ${ }^{1}$, P Due $^{1}$, A Andersen ${ }^{1}$

${ }^{1}$ University of Southern Denmark, National Institute of Public Health, Copenhagen, Denmark.E-mail: Ioni@niph.dk

Background: If school based preventive initiatives are well implemented they have the possibility to reach almost all adolescents, thereby helping to reduce the social inequality in health. However, if implementation is different according to social position, there is a risk of widening the social inequality instead. Generally, school based smoking preventive initiatives are most effective if they target adolescent smoking from multiple sides simultaneously, i.e. structural as well as individual initiatives. However, there is a risk that especially individually oriented initiatives may be implemented different according to social position. Here we examined whether the individual level initiative of a smoking preventive intervention - a smokefree contract and dialogue - was implemented differently according to social position.

Methods: The smoking preventive intervention - X:IT - was 
implemented at 51 Danish elementary schools. Main intervention components were smokefree school grounds, curricular activities, and parental involvement comprising smoke free contracts and dialogues between parents and kids.

We used questionnaire data from 2.146 pupils aged 13 years (85.0\% of eligible pupils), collected after first year of intervention. Results: 1.793 of pupils (83.6\%) had signed a smokefree contract. Here $30.0 \%$ was from high, $41.7 \%$ from middle and $13.6 \%$ from low social position ( $14.8 \%$ was unclassifiable). Of pupils without a contract $25.0 \%$ were from high, $36.3 \%$ from middle and $16.2 \%$ from low social position ( $25.7 \%$ unclassifiable) $(p=0.002)$.

1.717 pupils $(80.0 \%)$ had the smokefree dialogue (high $31.2 \%$, middle $41.9 \%$, low $12.2 \%$, unclassifiable $14.4 \%$ ). Pupils without dialogue; high $21.0 \%$, middle $36.6 \%$, low $19.8 \%, 22.6 \%$ unclassifiable $(\mathrm{p}<.0001)$.

Conclusions: The smokefree contracts and dialogues were implemented to at higher extent among kids of high social position. Parents from this social group may possess more resources to involve in school and health activities, which is an important barrier to overcome in future school based interventions.

Tob. Induc. Dis. 2018;16(Suppl 1):A404

DOI:10.18332/tid/83842

\section{2:30-14:00}

PS-789-5 Are school tobacco policies effective at reducing smoking among young people? A study on vocational schools in Denmark

S Andersen ${ }^{1}$, V Pisinger ${ }^{1}$, M Hulvej Rod ${ }^{2}$, J Tolstrup ${ }^{1}$

${ }^{1}$ University of Southern Denmark, National Institute of Public Health, Copenhagen, Denmark, ${ }^{2}$ National Research Centre for Disadvantaged Children and Young People, Copenhagen, Denmark. E-mail: sua@niph.dk

Background: In Danish vocational schools the prevalence of daily smoking is particularly high (nearly 40\%). Schools are increasingly implementing school tobacco policies (STP). However, studies on the effects of STP are inconclusive, and not all elements of STP may be equally effective. Our objectives were to describe STP characteristics and practices, and examining the associations between STP and students' smoking behaviour.

Methods: This study used data from Danish National Youth Study 2014. Participants were 5,013 vocational school students (median age $=19.1 ; 76 \%$ male) at 40 campuses. Implementation of STP was measured by principal questionnaires and field observations. Multinomial logistic regression models assessed whether STP characteristics and practices were associated with students' daily and occasional smoking compared to non-current smoking. Negative binominal regression models assessed cigarettes per day among daily smokers.

Results: All schools had an official tobacco policy. Fourteen (35\%) campuses had complete smoking ban. The majority (87\%) had implemented sanctions for breaking smoking rules; however, student smoking was observed on $78 \%$ of campuses. Students attending schools with sanctions were less likely to smoke daily (odds ratio $=0.82,95 \%$ confidence interval: $0.70,0.97$ ) and fewer cigarettes were reported among daily smokers (14.9 versus 16.0 , $\mathrm{p}<.05$ ). Occasional smoking was lower (odds ratio $=0.72,95 \%$ confidence interval: $0.55,0.92$ ) for students attending schools with comprehensive focus on tobacco restriction and prevention programmes compared to those without comprehensive STP. Conversely, occasional smoking was 1.3 times (95\% confidence interval: 1.1, 1.6) higher for students attending schools with visible student smoking. These associations were not found for daily smoking. There were no significant findings for sale of cigarettes on campus.

Conclusions: Dimensions of STP were related to smoking behaviour in different ways. These findings support the need for additional research focusing on how elements and practice of STP impact on various stages of smoking progression among young people.

Tob. Induc. Dis. 2018;16(Suppl 1):A405

DOI:10.18332/tid/83847

\section{2:30-14:00}

PS-790-5 Knowledge, attitude and practice regarding use of tobacco among medical students of a medical college of Eastern region Nepal

\section{R Shrestha}

${ }^{1}$ Chitwan Medical College, School of Nursing, Chitwan, Nepal. E-mail: rosyshrestha2005@yahoo.com

Background: Tobacco use is one of the leading preventable causes of premature death, disease and disability around the world. It is one of the risk factors for six out of eight leading causes of death worldwide. Medical students, the future health professionals, who should be the role model, are indulging in the tobacco use which may flow the wrong information to the public. The study was conducted with the objective to assess the Knowledge, Attitude and Practice regarding use of tobacco among medical students of a Medical College, Dharan.

Methods: A descriptive, cross-sectional study design was adopted with total of 100 sample size in this study. Stratified proportionate random sampling technique was used. Pre-designed, pretested, selfadministered research instrument was used for the study. Descriptive and inferential statistics were used appropriately to analyze the data. Results: The median percentage score of knowledge of the respondents was $66.67 \%$. More than half (51\%) of the respondents had adequate knowledge regarding use of tobacco. Almost all (93\%) had positive attitude against use of tobacco. It was found that $24 \%$ of the respondents were tobacco users either smoking or smokeless (chewing tobacco). There was significant association of knowledge regarding use of tobacco with residence (0.012). Attitude regarding use of tobacco was also significantly associated with age (0.022). Study findings also depicted that there was no relationship between knowledge and attitude. There was no significant association of knowledge with practice but attitude was found to be significantly associated with practice $(0.01)$.

Conclusions: Knowledge was adequate in more than half of the respondents and almost all had positive attitude. Despite this, there was still practice of use of tobacco in one fourth of the respondents. This symbolizes that the awareness regarding use of tobacco, its harmful effects and different tobacco cessation programs needs to be implemented in the medical students.

Tob. Induc. Dis. 2018;16(Suppl 1):A406

DOI:10.18332/tid/83904

\section{$12: 30-14: 00$}

PS-791-5 Effect of school based smoking prevention program in Korea

J Chung $^{1}$

${ }^{1}$ Korea Health Promotion Institute, Tobacco Control Program Team, Seoul, Korea, Republic of. E-mail: claire@khealth.or.kr Background and challenges to implementation: Tobacco use is a leading cause of preventable death, and is estimated to cause about 60,000 deaths a year in Korea. Smoking in adolescents causes more physical damage than adults.

The younger age of starting smoking has high potential to become 
lifelong smokers, the more difficult it becomes to quit. The school is the place where students spend the most time and has the best conditions for smoking prevention. Until 2014 , only $10 \%$ of schools participated the program nationwide.

The most outstanding policy was about $80 \%$ increase of tobacco price in 2015 for the first time in 10 years. We expanded implementation of smoking prevention education in all elementary, middle and high schools nationwide with increased budget.

The aim of the project is to reduce the smoking rate of adolescents by operating a school-based program that prevents students from accessing tobacco in advance and encourages smoking cessation. Intervention or response: We are improving the efficiency of our projects that matches the characteristics of each school. Types of programs include non-smoking declaration, smoking prevention education and activities, smoking cessation, education for faculty and parents, training, campaign, and specialized program throughout the year.

Results and lessons learnt: Smoking rate in adolescents has the lowest rate ever recorded during 10 years due to project expansion and raising tobacco price. We believe this has consistently influence decreased smoking rate among youth. Also, results of the school based project survey in 2015, Of the 65,428 students, $83.4 \%$ answered that they would not smoke in the future, and $38.7 \%$ of the smoking students reduce or quit smoking after education, and $74.9 \%$ of smoking students can refuse smoking after education.

Conclusions and key recommendations: Through continuous school based projects, we will create a smoke free school environment to support the healthy growth of students and to become the foundation for spreading the culture of smoke free community.

Tob. Induc. Dis. 2018;16(Suppl 1):A407

DOI:10.18332/tid/83912

\section{2:30-14:00}

PS-792-5 Is tobacco use as a gateway behavioral risk factor? Clustering of health compromising behaviours among urban Indian adolescents $\mathbb{E}$ associated inequalities

M Mathur ${ }^{1}$, R Watt ${ }^{2}$, G Tsakos², P Parmar ${ }^{3}$, A Singh ${ }^{4}$

${ }^{1}$ Public Health Foundation of India, New Delhi, India, ${ }^{2}$ University College London, London, United Kingdom, ${ }^{3}$ University of Oulu, Oulu, Finland, ${ }^{4}$ University of Adelaide, Adelaide, SA, Australia. E-mail:manurajmathur@gmail.com

Background: Non Communicable Diseases (NCDs) are majorly associated with four common health compromising behaviours tobacco use, physical inactivity, unhealthy diet and harmful use of alcohol. These health compromising behaviours do not occur in isolation and often tend to co-occur as clusters. The aim of this study was to explore the prevalence and clustering of four behavioural risk factors for NCDs among adolescents and to assess if socioeconomic inequalities exist in these clustering patterns.

Methods: A cross sectional study was undertaken among 1,085 adolescents (15-19 year old) in the city of New Delhi, India. An interviewer administered questionnaire was used to assess health compromising behaviours (tobacco use, alcohol use, unhealthy diet and physical inactivity), and socio-demographic data. Clustering was assessed using pairwise correlations, counts of clustering of health compromising behaviours and comparison of observed/ expected ratios. Multivariable logistic regressions were used to assess the relationship between variations in clustering by self-reported wealth and education of adolescents.

Results: Two major clusters of health behaviours emerged; tobacco and alcohol use and tobacco and unhealthy diet, with tobacco use emerging as a gateway health compromising behaviour. Pronounced clustering of health compromising behaviours were observed with lower levels of education and wealth. In the multi-variable regression models, the crude estimates showed that poorer adolescents were at 2.6 times $(95 \% \mathrm{CI} 1.6,4.2)$ and 3.4 times $(95 \%$ CI $1.9,6.2)$ higher odds of having the clustering between tobacco and alcohol and tobacco and unhealthy diet respectively in comparison to richer adolescents.

Conclusions: Tobacco use was the gateway risk behaviour which significantly clustered with other NCD risk behaviours. Clustering of health behaviours was socio-economically patterned. Area of residence and age modified the associations between clustering of behaviours and socio-economic position. Targeting interventions to promote positive health behaviours especially for tobacco control amongst adolescents should be a key public health priority.

Tob. Induc. Dis. 2018;16(Suppl 1):A408 DOI:10.18332/tid/83947

\section{2:30-14:00}

PS-793-5 Sale of tobacco products among minors in a satellite township near Kuala Lumpur, Malaysia. An observational study

DD Dicksit ${ }^{1}$, S Sulugodu Ramachandra

'SEGi University, Faculty of Dentistry, Petaling Jaya, Malaysia. E-mail:danieldev83@gmail.com

Background: Prevalence of smoking among minors

is $18.2 \%$ in Malaysia. The legal age to buy tobacco products in Malaysia is 18 years and the Government intends to raise the permissible age to 21 years. The aim of the study was to assess the environment of the shops selling tobacco products about its sale among minors.

The objective also included structured interviews of the sales personal regarding their knowledge and attitude towards the sale of tobacco products among minors.

Methods: Two researchers observed 31 shops/restaurants selling tobacco products (cigarettes/shisha). Shops within 500 meters of schools and universities were studied. Observations included presence of signages regarding ban on sales of tobacco products to minors and also the ban on sale of single sticks/loose cigarettes. Sales person of these shops willing to be interviewed were administered with a questionnaire and data was collected. Collected data was analysed using descriptive statistics.

Results: Out of 31 places, 5 were restaurants catering shisha and 26 were selling cigarettes. Two restaurants were catering shisha smoking to minors. Among the 26 shops selling cigarettes only $42.3 \%(n=11)$ had a clear signage regarding ban on sales of tobacco products to minors. Loose cigarettes were sold in $35 \%(n=9)$ of the shops. Out of 26 shops, only 7 sales person were willing to be interviewed by the researcher (DDD). All interviewed individuals, were aware about the adverse health effects of smoking and the legal age to buy tobacco products in Malaysia. Five sales person agreed that they had sold tobacco products to minors.

\begin{tabular}{|l|c|c|c|c|}
\hline \multirow{2}{*}{ Type Of Shop } & \multicolumn{2}{|c|}{$\begin{array}{c}\text { Signage of Legal age to buy } \\
\text { tobacco products }\end{array}$} & \multicolumn{2}{|c|}{ Selling Single Sticks } \\
\cline { 2 - 5 } & Present & Absent & Yes & No \\
\hline General Store & 6 & 15 & 9 & 12 \\
\hline Petrol Station & 3 & 0 & 0 & 3 \\
\hline Restaurant & 2 & 5 & 0 & 7 \\
\hline Total & 11 & 20 & 9 & 22 \\
\hline
\end{tabular}

[Descriptive Statistics Showing Signages and sales] 
Conclusions: Minors would be tempted to try tobacco products if strict ban is not enforced. Even though current laws exist to address the ban of tobacco products among minors, implementation seems to be weak in the studied locality. The authorities should monitor sale of tobacco products in future.

Tob. Induc. Dis. 2018;16(Suppl 1):A409
DOI:10.18332/tid/83984

\section{$12: 30-14: 00$}

PS-794-5 Assessing the predicting validity of the tobacco marketing receptivity among youth

S Braun ${ }^{1,2}$, EN Abad-Vivero ${ }^{3}$, R Mejía ${ }^{4,5}$, I Inti Barrientos ${ }^{6}$, J Sargent ${ }^{7}$, J Thrasher ${ }^{8}$

${ }^{1}$ University of Buenos Aires, Internal Medicine, Buenos Aires, Argentina, ${ }^{2}$ Centro de Estudios de Estado y Sociedad (CEDES), Buenos Aires, Argentina, ${ }^{3}$ National Institute of Public Health, Department of Tobacco Research, Center for Population Health Research, Cuernavaca, Mexico, ${ }^{4}$ Universidad de Buenos Aires, Internal Medicine, Buenos Aires, Argentina, ${ }^{5}$ Centro de Estudios de Estado y Sociedad (CEDES), Department of Health Economy, Buenos Aires, Argentina, ${ }^{6}$ National Institute of Public Health, Department of Tobacco Research, Center for Population Health Research, Cuernava, Mexico, ${ }^{7}$ Dartmouth Medical School, Department of Pediatrics, Hanover, NH, United States of America, ${ }^{8}$ University of South Carolina, Department of Health Promotion, Education, and Behavior, Columbia, SC, United States of America. E-mail: sandrabraun@fibertel.com.ar

Background: In a previous cross-sectional study, we developed a marketing receptivity index (MRI), which had independent, positive associations with positive smoking expectancies, smoking susceptibility and current smoking behavior in a sample of early adolescents in Argentina. The current study aimed to assess the predictive validity of the MRI.

Methods: Data come from a longitudinal, school-based survey conducted in 33 secondary schools in Argentina. We included students who had never smoked at baseline and were successfully followed up approximately 17 months later $(n=1700)$. Marketing receptivity was assessed with questions in three domains: frequency of going to stores that sell tobacco; cued recall of brand names for 3 cigarette packages with brand name removed; and ownership of branded merchandise. A four-level MRI was derived (low PoS marketing exposure only; high PoS exposure or recall of 1 brand; recall of 2 or more brands; and ownership of branded merchandise). Self-report of having tried to smoke at follow-up (i.e., initiation) was the primary outcome assessed. Logistic models regressed smoking initiation on the MRI, the MRI components (considered separately), and willingness to try one of the brands shown in the cued recall task, adjusting for sociodemographics, social influences and sensation seeking.

Results: The 4 level MRI had independent positive associations with smoking initiation (AOR2 vs. $1=1.42,95 \% \mathrm{CI}=1.12-1.79$; $\mathrm{AOR} 3$ vs $1=2.04,95 \% \mathrm{CI}=1.38-3.00$; $\mathrm{AOR} 4$ vs. $1=2.16,95 \%$ $\mathrm{CI}=1.21-3.82$ ). The index components, analyzed separately, were associated with outcomes except for ownership of branded merchandise. Willingness to try one of the brands shown in the recall task was also associated with initiation $(\mathrm{AOR}=1.73,95 \%$ $\mathrm{CI}=1.31-2.28)$.

\begin{tabular}{|c|c|c|c|c|}
\hline $\begin{array}{l}\text { Varketing } \\
\text { Receptivity Index } \\
\text { (VIRI) }\end{array}$ & $\begin{array}{c}\text { OR } \\
\left(95^{\circ} \% \mathrm{CI}\right)\end{array}$ & p & $\begin{array}{c}\text { AOR } \\
\left(95^{\circ} \circ \mathrm{CI}\right)\end{array}$ & p \\
\hline $\begin{array}{l}\text { 1-Low PoS, } 0 \text { brands } \\
\text { recalled }\end{array}$ & 1 & & & \\
\hline $\begin{array}{l}\text { 2-High PoS } \\
\text { exposure or } 1 \text { brand } \\
\text { recalled }\end{array}$ & $\begin{array}{l}1.12 \\
(0.91-1.39)\end{array}$ & NS & $\begin{array}{l}1.42 \\
(1.12-1.79)\end{array}$ & $<0.01$ \\
\hline $\begin{array}{l}3-2 \text { or more brand } \\
\text { recalled }\end{array}$ & $\begin{array}{l}1.75 \\
(1.32-2.31)\end{array}$ & $<0.001$ & $\begin{array}{l}2.04 \\
(1.38-3.00)\end{array}$ & $<0.001$ \\
\hline $\begin{array}{l}\text { 4-Own branded } \\
\text { object }\end{array}$ & $\begin{array}{l}1.95 \\
(1.16-3.29)\end{array}$ & $<0.05$ & $\begin{array}{l}2.16 \\
(1.21-3.82)\end{array}$ & $<0.01$ \\
\hline
\end{tabular}

[Predictors of smoking initiation among secondary s]

Conclusions: The marketing receptivity index and its component were associated with cigarette trial, suggesting its predictive validity and utility for future studies. Ownership of branded merchandize may be more useful for studying smoking progression amongst those who have already tried smoking.

Tob. Induc. Dis. 2018;16(Suppl 1):A410

DOI:10.18332/tid/83989

\section{2:30-14:00}

PS-795-5 Use of e-cigarettes rising among junior and senior high school students in Taiwan

H-Y Chung ${ }^{1}$, Y-H Su${ }^{1}$, T-L Lee ${ }^{1}$, S-Y Lo ${ }^{1}$, Y-J Liu ${ }^{1}$, C-S Chang ${ }^{1}$, L-H Yu' ${ }^{1}$, Y-W Wang ${ }^{1,2}$

${ }^{1}$ Health Promotion Administration, Ministry of Health and Welfare, Taipei, China, ${ }^{2}$ School of Medicine, Tzu Chi University, Hualien, China. E-mail: Isy@hpa.gov.tw

Background: According to the World Health Organization (WHO) report, e-cigarette use is doubling from 2008 to 2012 among adolescents. In Taiwan, e-cigarettes contain nicotine are controlled under Pharmaceutical Affairs Act since 2009, but it will violate Tobacco Hazards Prevention Act if e-cigarettes are in the form of tobacco products. This study aims to demonstrate the prevalence of e-cigarette use and reasons for trying e-cigarettes among adolescents.

Methods: Data were drawn from the Taiwan Global youth tobacco survey (Taiwan GYTS) conducted under a school-based and cross-sectional study from 2014 to 2016 . The annual sample size is approximately 45,000 persons.

Results: Although tobacco use by adolescents has declined substantially over the past few years, the prevalence of e-cigarette use by junior and senior high school students has risen from $2.0 \%$ and $2.1 \%$ in 2014 to $3.7 \%$ and $4.8 \%$ in 2016 respectively, both nearly doubling within this three years. $44.1 \%$ of junior high school students and $29.4 \%$ of senior high school students who have never used any tobacco product have tried e-cigarettes. "Friends are using them" and "The flavor or smell is better" are the two major reasons for trying e-cigarettes among adolescents. Our findings also show that e-cigarette use by adolescents is influenced by the views on conventional tobacco use from parents and friends.

Conclusions: Facing the rapid development of e-cigarettes, Taiwan's governments have cooperated with each other to comprehensively block hazards from e-cigarettes by border seizure and inspection, source tracking, channel inspection, monitoring and management, education broadcasting and cessation guidance, etc. Taiwan's Tobacco Hazards Prevention Act is now in the process of amending to regulate e-cigarettes under two laws - Pharmaceutical Affairs Act 
and Tobacco Hazards Prevention Act - with the prohibitions on import, manufacture, and sale, persons under the age of eighteen shall not use e-cigarettes is also prohibited.

Tob. Induc. Dis. 2018;16(Suppl 1):A411

DOI:10.18332/tid/83995

12:30-14:00

PS-797-5 Tobacco and areca nut cessation programme for adolescent school students in Mumbai, India

G Mandal' ${ }^{1}$, H Gupte ${ }^{2}$, V Thawal ${ }^{2}$, L Chaudhuri ${ }^{2}$

'Salaam Bombay Foundation, Mumbai, India, ${ }^{2}$ Narotam Sekhsaria Foundation, Mumbai, India.E-mail:gauri.mandal@ salaambombay.org

Background: The prevalence of tobacco use among children aged $13-15$ years is $14.6 \%$ and $15.5 \%$ of non-users intended to start smoking in the next year. School going children also consume areca nut ("supari"), which is an easily available carcinogenic, psychoactive substance, acting as a gateway product to tobacco use. This current and intended use will exacerbate the burden of tobacco related morbidity and mortality. LifeFirst program was implemented in 15 schools catering to lower socioeconomic population in slum areas of Mumbai in the academic year 2016-17 for helping students quit their tobacco and supari use.

Methods: Orientation sessions about harmful effects of tobacco and areca nut were conducted using audio-visual aids for 2379 students of the 7th, 8th and 9th grades. Students were informed about the availability of a cessation service within the school and encouraged to register voluntarily. The registered students were divided into groups of 10-15 students each and six group-sessions involving videos, games, role plays and activities were conducted over six months. The sessions were theme based; covering topics like rapport building, ill-effects of tobacco, coping mechanisms, refusal skills etc. The self-reported status of tobacco use was recorded individually during each session. Extended 4-month post-program follow-up was conducted.

Results: Of the 492 students ( $84 \%$ boys) registered for the program, $88 \%$ were only supari users, $10 \%$ used supari and tobacco, $2 \%$ only smokeless and less than $1 \%$ only smoked. $67 \%$ were daily users. The mean age of initiation was 11.7 years and $79 \%$ were introduced to the product by their peers. $71 \%$ reported as not using tobacco and supari at the end of the programme with $12 \%$ relapse recorded during extended follow up.

Conclusions: Providing structured cessation services with positive peer influence facilitated by trained counselors encourages and aides tobacco and areca nut users to stop their habit.

Tob. Induc. Dis. 2018;16(Suppl 1):A412

DOI: $10.18332 /$ tid/84030

\section{2:30-14:00}

PS-798-5 Youth involvement on tobacco control law implementation: success story

M Maharjan ${ }^{1,2}$, A Islam Sujon ${ }^{3,4}$, SL Mulmi ${ }^{5,6}$

'Resource Centre for Primary Health Care (RECPHEC), Kathmandu, Nepal, ${ }^{2}$ Health Rights and Tobacco Control Network, Kathmandu, Nepal, ${ }^{3}$ Bangladesh Anti Tobacco Alliance (BATA), Dhaka, Bangladesh, ${ }^{4}$ National Girl Child Advocacy Forum, Dhaka, Bangladesh, ${ }^{5}$ National Pressure Group against Tobacco, Kathmandu, Nepal, ${ }^{6}$ Nepal NCD Alliance, Kathmandu, Nepal. E-mail: manjushree012@gmail.com

Background and challenges to implementation: It is prohibited to retail sale sticks of cigarettes, bidi or cigar to the person age under 18 years old. Due to lack of awareness, monitoring, reporting to the concerned officials, law was not well-enforced. In particular, teens are openly buying and consuming tobacco products. So that, Health Rights and Tobacco Control (HRTC) district networks start monitoring the law in their areas through Youth Watch Groups (YWG). This group is dedicated on antitobacco campaigns in 58 districts out of 75 districts of Nepal.

Intervention or response: YWG volunteers regularly monitor illegal selling, distribution of tobacco products and report to the police and take necessary action for law enforcement. YWG is protecting such illegal transactions in the grassroots level. Youth knows the youth better than others age groups. So, in order to prevent youths in consuming tobacco, the YWG volunteers taken actual action in right time and right place. Youth gatherings for the awareness on law as well as health risks due to tobacco addiction and campaigns are organized regularly in which more youth motivated towards tobacco control, and volunteer to YWG. This group work hard to aware people about smoke free public place and transports and increase graphic health warnings (GHW) as well.

Results and lessons learnt: YWG intervention helps to enforce tobacco control law in line with section to prohibit selling tobacco to teens and prevent smoking among youth. YWG initiatives also involving more and more youth as lifelong anti tobacco activists on tobacco control. Nepal is providing world largest GHW (90\%) in all tobacco products.

Conclusions and key recommendations: Youth is not only leader of future; they have potentials to lead for betterment of society at present. Engage youth into tobacco control, it will benefit nation for long time.

Tob. Induc. Dis. 2018;16(Suppl 1):A413

DOI: $10.18332 / \mathrm{tid} / 84090$

\section{2:30-14:00}

PS-799-5 Trend of easiness to purchase cigarettes among Korean adolescents: the Korea Youth Risk Behavior Web-based Survey 2005-2016

SY Kang ${ }^{1}$, H-J Cho

'Asan Medical Center, University of Ulsan College of Medicine, Family Medicine, Seoul, Korea, Republic of. E-mail: hjcho@ amc.seoul.kr

Background: According to the Juvenile Protection Act in Korea, tobacco is regarded as juvenile harmful substance, and no one is allowed to sell, rent or distribute tobacco products to adolescents. Furthermore, Framework Convention on Tobacco Control Article 16 prohibits the sales of tobacco products to minors. In this study, we investigated the tread and associated factors of easiness to purchase cigarettes among Korean adolescents from 2005 to 2016.

Methods: The analyses were based on the data of the Korea Youth Risk Behavior Web-based Survey. We estimated the trend of easiness to purchase cigarettes from 2005 to 2016 and evaluated associated factors by multivariate logistic regression analyses after adjusting for year, sex, grade, geographic area, self-perceived socioeconomic status, problematic drinking in last one year, quit attempt in last one year, current smokers within family members, current smokers within close friends, pocket money, and average amount of cigarettes smoking.

Results: Easiness to purchase cigarettes decreased from $83.9 \%$ in 2005 to $71.4 \%$ in 2016 , but still in very high level. The odds ratios of easiness to purchase cigarettes greater in adolescents with higher grade, those who smoke higher amount of cigarette 
smoking in a day, those who live in metropolitan and big cities, those who had problematic drinking in last one year, and those who have current smokers within close friends.

Conclusions: Although easiness to purchase cigarettes among Korean adolescents decreased, more than $70 \%$ of Korean adolescents can purchase cigarettes without an effort. Low enforcement must be strengthened in order to prevent adolescent smoking.

\section{Tob. Induc. Dis. 2018;16(Suppl 1):A414 DOI: $10.18332 /$ tid/84107}

\section{2:30-14:00}

PS-800-5 The hidden burden of tobacco use among school adolescents in Ethiopia: call for action

\section{N Dereje ${ }^{1}$}

'Wachemo University, Public Health, Hosanna, Ethiopia. E-mail: neba.jahovy@gmail.com

Background: Tobacco use is one of the leading public health threats in the world and the spread of is growing at fast among adolescents. However, little information is known about the magnitude and predictors of tobacco use in Ethiopia. Hence study was conducted to assess the prevalence and predictors of tobacco use among school adolescents of Ethiopia.

Methods: A cross sectional survey was employed among school adolescents in the age category of 13 - 19 years. The study was carried out to determine the prevalence of current (within the last 30 days) tobacco use among adolescents. Thus, 1704 students were selected from a total of 12 public and private schools in Ethiopia. A two-stage stratified sampling technique was employed to select the schools and the final sampled students were enrolled using systematic random sampling. Data were collected by using self-administered global youth tobacco survey questionnaires and descriptive statistics, bivariate and multivariate analysis were done.

Results: The prevalence of cigarette and shisha smoking were found to be $17.2 \%$ and $12.6 \%$ respectively. In addition, more than half (60.8\%) of adolescents were exposed to environmental tobacco smoke. In the multivariate analysis sex, alcohol use, parent smoking, peer smoking, exposure to movie with actors smoking, not being exposed to anti-smoking media messages, not discussing in the class about danger of smoking, and having perception that smoking is not dangerous to health were seen significantly associated with current cigarette smoking among adolescents.

Conclusions: The burden of tobacco use in any form among adolescents is unacceptably high and exposure to environmental tobacco exposure is widespread. Therefore the government of Ethiopia should legislate, enact, and enforce laws that control tobacco spread in any form. Moreover adolescents of the country should be enriched with the knowledge on the dangers of tobacco use.

Tob. Induc. Dis. 2018;16(Suppl 1):A415

DOI: $10.18332 /$ tid/84117

\section{2:30-14:00}

PS-801-5 Exposure to advertising of tobacco products at points of sale and consumption of tobacco in adolescents from 3 large cities in Argentina. Cross-sectional study

A Ángel ${ }^{1}$, S Braun ${ }^{1}$, A Pérez ${ }^{2,3}$, R Méjía ${ }^{1,2}$, J Thrasher ${ }^{4,5}$, J Sargent ${ }^{6}$ ${ }^{1}$ University of Buenos Aires, Internal Medicine, Buenos Aires, Argentina, ${ }^{2}$ Centro de Estudios de Estado y Sociedad (CEDES),
Department of Health Economy, Buenos Aires, Argentina, ${ }^{3}$ Universidad de Buenos Aires, Buenos Aires, Argentina, ${ }^{4}$ University of South Carolina, Department of Health Promotion, Education, and Behavior, Columbia, SC, United States of America, ${ }^{5}$ National Institute of Public Health, Department of Tobacco Research, Center for Population Health Research, Cuernava, Mexico, ${ }^{6}$ Dartmouth Medical School, Department of Pediatrics, Hanover, NH, United States of America. E-mail: adrianaaangel@icloud.com

Background: Several researches have demonstrated a strong association between exposure tobacco advertising at point of sale (PoS) and tobacco susceptibility, experimentation and adolescent uptake. However, there are no local studies demonstrating this association. Our objective was to explore the association between exposure to tobacco advertising in PoS located near secondary schools and smoking in early adolescents in 3 Argentinean cities: Córdoba, Tucumán and Buenos Aires

Methods: This is a cross-sectional study with two descriptive sources: information about tobacco consumption was obtained through a survey of adolescents from 33 secondary schools in Buenos Aires, Córdoba and Tucumán. In addition, data on cigarette advertising in the PoS were relayed within 300 meters of each selected school. An exposition index was derived by multiplying the frequency of going to tobacco shops and a marketing index of each PoS (obtained by adding the number of signs, visibility from outside and displaying tobacco less than $1 \mathrm{~m}$ from children's products). We explored the association between exposure to $\mathrm{PoS}$ advertising and smoking at individual and school level through multilevel logistic regression, adjusting for sociodemographics, social network smoking, and sensation seeking.

Results: The questionnaire was completed by 1680 students, 158 (9.4\%) smokers, 895 (53\%) no susceptible non-smokers, 389 (23\%) susceptible non-smokers and 274 (16.3\%) experimenters. We surveyed $340 \mathrm{PoS}, 88$ (25.88\%) in Tucumán, 80 (23.53\%) in Córdoba and 170 (50\%) in Buenos Aires. Exposure to PoS advertising was significantly associated with being an experimenter $(\mathrm{OR}=1.58,95 \% \mathrm{CI} 1.16-2.14)$ and susceptible $(\mathrm{OR}=1.65,95 \%$ CI 1.2-2.25) in the highest quartile of exposure.

\begin{tabular}{llll} 
& $\begin{array}{l}\text { Smokers OR } \\
\left(95^{\circ}, I C\right)\end{array}$ & $\begin{array}{l}\text { Avperimenters } \\
\text { OR }\left(95^{\circ}, I C\right)\end{array}$ & $\begin{array}{l}\text { Susceptibles } \\
\text { OR }\left(95^{\circ}, I C\right)\end{array}$ \\
\hline Quartile 2 & 1.19 & 1.16 & 1.33 \\
& $(0.838-1.685)$ & $(0.873-1.534)$ & $(0.983-1.792)$ \\
Quartile 3 & 1.32 & 1.32 & 1.15 \\
& $(0.930-1.874)$ & $(0.996-1.754)$ & $(0.839-1.584)$ \\
Quartile 4 & 1.22 & 1.58 & 1.65 \\
& $(0.848-1.758)$ & $(1.160-2.147) \S$ & $(1.207-2.250) \S$
\end{tabular}

$\mathrm{OR}=$ Odds Adjusted for age,

Ratio $\S<$ sex, sensation seeking, having parents, siblings or friends that smoke.

[Exposure to advertising and tobacco consumption. ]

Conclusions: Our findings suggests an association between exposure advertising of tobacco product at PoS and experimentation and susceptibility. PoS could be a critical factor in the onset of this addiction in young people.

Tob. Induc. Dis. 2018;16(Suppl 1):A416

DOI: $10.18332 /$ tid/84155 
12:30-14:00

PS-802-5 Assessing knowledge and practice of tobacco retailers around primary and secondary schools, on selling tobacco products to and by minors in Kigali Rwanda

MR Nyirakamana' ${ }^{1}$, M Nzayirambaho' ${ }^{1}, \mathrm{~K}^{2}$ Namusisi ${ }^{2}$

'University of Rwanda, College of Medicine and Health Sciences, Kigali, Rwanda, ${ }^{2}$ Center for Tobacco Control in Africa, Uganda, Kampala, Uganda. E-mail: marierosenyirakamana@gmail.com Background: Tobacco is a major public health problem since decades. Given the current pattern of tobacco use globally it is estimated 250 million who are alive today, would die prematurely because of tobacco and mostly in developing countries such as Rwanda. While most tobacco control laws prohibit retail sales to minors, these restrictions are often not enforced. Failing to enforce minimum age laws not only wastes a constructive opportunity to reduce youth smoking but also tells kids that the laws need not be taken seriously, which undermines other tobacco-reduction efforts in the media, schools, and communities. This study aims to provide an assessment on the knowledge and practice on selling tobacco products to and by minors around schools.

Methods: This study was a descriptive cross-sectional study among tobacco retailers surrounding the primary and secondary schools and policy implementers in Nyarugenge District Kigali Rwanda. A total number of 268 retailer outlets located within 1000 meters from the schools were sampled. This was augumented with quantitative assessments with policy makers.

Results: The majority (78.2 \%) of them has a little knowledge of the existing tobacco control law in Rwanda, specifically the law on selling tobacco products to and by minors. Knowledge was equally low among the policy makers, on the article, citing lack of regulations and guidelines to enfore the Tobacco Control Act. Regarding practice, $42.5 \%$ of the retailers reported to be selling tobacco products to minors and $3 \%$ retailers were using minors to sell tobacco products.

Conclusions: This study revealed a failure to enforce the current tobacco control law in Rwanda in general and specifically with regard to selling tobacco products to and by minors. There are no regulations to guide implementation of the Act. In addition, knowledge and practice on selling tobacco products to and by minors is still very low.

Tob. Induc. Dis. 2018;16(Suppl 1):A417

DOI:10.18332/tid/84156

\section{$12: 45-14: 00$}

EP-150-5 Did the Irish 2004 smokefree legislation contribute to the sharp decline in youth smoking?

S Li', S Keogan ${ }^{1}$, L Clancy ${ }^{1}$

'TobaccoFree Research Institute Ireland, Dublin, Ireland. E-mail: shashali@tri.ie

Background: While the smoke-free workplaces legislation introduced in 2004 in Ireland has reduced adult smoking prevalence, its impact on youth prevalence is less clear. A particularly large reduction was observed in youth smoking prevalence between 2003 and 2007 . This study sets out to assess if the smoking ban was effective in reducing youth smoking in Ireland and to see if it could explain the large fall in prevalence between 2003 and 2007.

Methods: Data is from the European School Survey Project on Alcohol and Other Drugs (ESPAD) Ireland, which took place every four years from 1995 to 2015. A logistic regression model on grouped data was used. Dependent variable is whether a student was a smoker in last-30 day. Independent variables are time, gender and policy indicators, including workplace ban indicator and Point-of-Sale (POS) ban indicator.

Results: Smoking prevalence among youths in Ireland has dropped from $41 \%$ in 1995 to $13 \%$ in 2015 , an average annual reduction of $1.4 \%$ (95\% confidence interval $1.3 \%-1.5 \%)$. The workplace ban had strongly significant impacts on reducing youth smoking, while the contribution of POS ban was negligible. In particular, a significantly improved fit was obtained by the model which included term for workplace ban with a $4.3 \%$ (1.2\%$7.5 \%)$ reduction in prevalence, in addition to an average annual reduction of $1.1 \%(0.8 \%-1.3 \%)$.

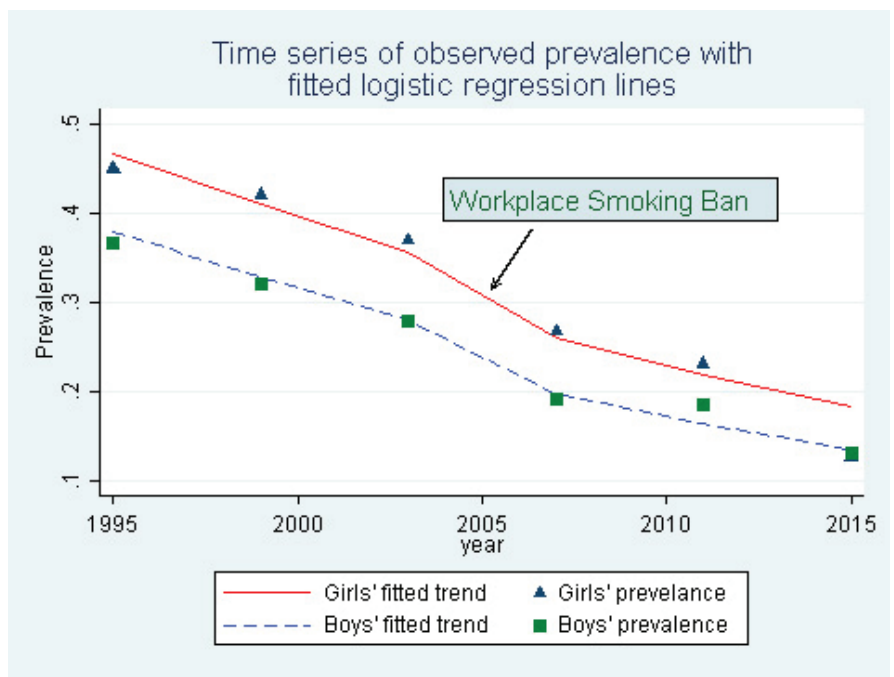

[Prevalence and fitted regression lines]

Conclusions: The workplace ban introduced in 2004 has significantly helped to explain the out-of-trend reduction in youth smoking prevalence, although the legislation was not particularly targeting youths. While removal of point of sale tobacco promotion was to reduce awareness of smoking among young people, its impact on prevalence was insignificant. The significance of time variable may capture the impact of other tobacco control policies implemented, particularly tax increase every year. There were no school-specific policies introduced during this period.

Tob. Induc. Dis. 2018;16(Suppl 1):A418

DOI:10.18332/tid/83959

\section{2:45-14:00}

EP-151-5 Current and frequent cigarette smoking trends in South African learners

R Sewpaul', P Reddy', A Ellahebokus ${ }^{2}$

${ }^{1}$ Human Science Research Council, Population Health, Health Systems and Innovation, Cape Town, South Africa, ${ }^{2} \mathrm{ARCH}$ Actuarial Consulting, Cape Town, South Africa. E-mail: rsewpaul@hsrc.ac.za

Background: South Africa (SA) has enforced comprehensive tobacco control measures aimed at reducing tobacco use among the population. Tobacco control is especially important for young people because a lifelong addiction to nicotine is usually established in early teenage years. The impact of these tobacco control measures on trends in prevalence and frequency of cigarette smoking among SA youth have not been fully examined. 
Methods: Data was analysed from the four Global Youth Tobacco Surveys (GYTSs) conducted in 1999, 2002, 2008 and 2011. We assessed the prevalence and trends of current and frequent (smoked $>=20$ days in the preceding month) cigarette smoking by gender for grades 8 - 10 school learners. Logistic regression analysis with orthogonal polynomials was used to determine linear and non-linear trends from 1999 to 2011, controlling for age and race.

Results: In boys, there was a significant linear decrease in current smoking from $28.8 \%$ (95\% CI: 24.5-33.1) to $21.7 \%$ (18.5-25.0) across the four surveys $(\mathrm{p}=0.004)$. Frequent smoking in boys showed a significant linear decrease from $13.2 \%$ (9.8-17.5) to $7.8 \%(6.3-9.6)(\mathrm{p}=0.015)$. In girls, there was a non-linear decrease in current smoking $(\mathrm{p}=0.028)$ from $17.5 \%(13.1-21.9)$ to $12.1 \%$ (9.9-14.4) and a significant nonlinear decrease in frequent smoking from $7.2 \%(5.2-10.0)$ to $1.9 \%(1.4-2.8)(\mathrm{p}=0.004)$.

Conclusions: In both boys and girls, the prevalence and frequency of cigarette smoking has showed significant declines. However, given that $16 \%$ of learners still smoke, increased efforts are required to address tobacco use among learners.

Tob. Induc. Dis. 2018;16(Suppl 1):A419

DOI:10.18332/tid/84551

\section{$12: 45-14: 00$}

EP-152-5 Age-period-cohort effect of adolescent smoking in Korea: from 2006-2016

H Kang ${ }^{1}$, SY Kim ${ }^{1}$, S-I Cho ${ }^{1,2}$

${ }^{1}$ Department of Public Health Science, Graduate School of Public Health, Seoul National University, Seoul, Korea, Republic of, ${ }^{2}$ Institute of Health and Environment, Seoul National University, Seoul, Korea, Republic of. E-mail: hiw0301@snu.ac.kr

Background: Achievement of a tobacco-free generation depends on the use on tobacco among adolescents. Targeting to reduce smoking from adolescence can be effective because experimentation of tobacco smoking in adolescence is a strong predictor of tobacco use throughout the later life-course. With governmental efforts to reduce tobacco-use among adolescents since 1999, smoking prevalence of Korean adolescents decreased from $12.8 \%$ in 2006 to $6.3 \%$ in 2016 . However, the ultimate goal is to reach smoking prevalence of $0 \%$ among adolescents. In order to achieve such goal, continuous monitoring of smoking prevalence is indispensable.

Methods: Data were acquired from the Korea Youth Risk Behavior Risk Survey, which has been conducted every year since 2005. Data from the first survey which was conducted in 2005, were excluded because the third grades of high schools were not surveyed due to a scheduled national college examination. Measures such as age, sex and tobacco use j165

(ever/current) were used. This study applied age-period-cohort analysis on time trends of adolescent smoking to examine the effect of chronological age, period and birth cohort. All statistical analyses were performed using $\mathrm{R}$.

Results: Current smoking was positively associated with age in both sexes. Ever smoking also increased as age advanced among boys, but decreased at the age of 18 among girls. The cohort effect suggests that there was not much difference in current smoking for newborns between 1988 and 1997. However, for newborns from 1998, current smoking was negatively associated with birth year. Ever smoking showed similar patterns. Period effect was dramatic as current and ever smoking decreased as time advanced. However, the effect was less striking among boys.

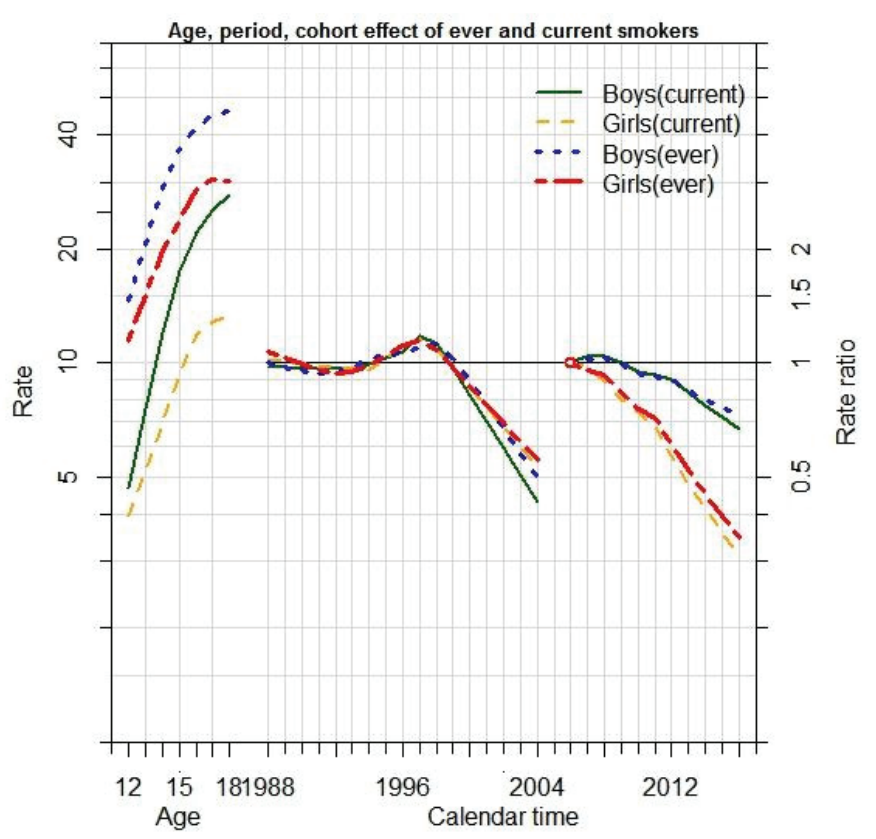

[Age, period, cohort effect in adolescent smokers]

Conclusions: Efforts to reduce tobacco-use among adolescents appears to be playing a substantial role in reducing current smoking and ever smoking prevalence. Ongoing surveillance for trends in adolescent cigarette smoking is essential to implement effective tobacco control programs.

Tob. Induc. Dis. 2018;16(Suppl 1):A420

DOI:10.18332/tid/84265

$12: 45-14: 00$

EP-153-5 Assessing youth appeals on cigarette packs in 6 low and middle income countries

K Smith ${ }^{1,2}, K_{\text {Welding }}^{2}$, C Washington ${ }^{2}$, M lacobelli², J Cohen ${ }^{1,2}$ 'Johns Hopkins Bloomberg School of Public Health, Health, Behavior and Society, Baltimore, MD, United States of America, ${ }^{2}$ Johns Hopkins Bloomberg School of Public Health, Institute for Global Tobacco Control, Baltimore, MD, United States of America. E-mail: katecsmith@jhu.edu

Background: Cigarette marketing impacts brand awareness of very young children in low- and middle-income countries (LMICs). Exposure to tobacco marketing during childhood is associated with favorable attitudes towards smoking and early engagement in tobacco use. With more restrictions on tobacco marketing, the pack is becoming an increasingly important platform for branding. To what extent and how is branding on tobacco packs designed in such a way as to appeal to youth in LMICs?

Methods: We purchased cigarette packs from 6 LMICs (Brazil, Indonesia, Philippines, Russia, Vietnam, and Thailand) in 20152016. Packs were purchased from 12 diverse neighborhoods in three diverse cities in each country and were coded by 2 independent coders; youth appeal was measured using 2 of more than 70 standardized codes applied to all packs.

Results: From our sample of 1,351 packs, 21 (<1\%) were coded for explicit youth appeal-these packs tended to reference sports or depict popular music. A subsequent, more inductive review of all packs identified numerous other elements that are potentially relevant but more difficult to assess objectively as appealing primarily/exclusively to youth. These include the appeal of certain colors (e.g. purple), fonts, patterns and holograms. Depictions of animals (e.g. butterflies and cats) and animal prints may attract young people's attention, as might fruits or flowers. References to 
sustainability and natural products may appeal. Special edition packs may also tap into young people's tendency to collect things (e.g sports cards or stickers). Finally, we will discuss the need to consider the appeal of a specific pack to a specific audience (such as youth) in relation to other packs within the same brand or brand variant.

Conclusions: There is value to a common coding approach to assessing pack appeals across numerous settings, but cultural specificity and artistic quality complicate accurate interpretation and application of more subtle or nuanced codes.

Tob. Induc. Dis. 2018;16(Suppl 1):A421

DOI:10.18332/tid/84003

\section{$12: 45-14: 00$}

EP-154-5 The variation of tobacco use behavior among Beijing college students and middle school students from 2008 to 2014

JL Duan ${ }^{1}$, XP Zeng ${ }^{1}$, Y Sun ${ }^{1}$

${ }^{1}$ Beijing Center for Disease Prevention and Control, Beijing, China.E-mail:cdcdjl@126.com

Background: Understanding the changes in smoking rate and try smoking rate among Beijing college students and middle school students from 2008 to 2014 , and preliminarily evaluate the school tobacco control work efficiency.

Methods: Using multi-stage stratified cluster random sampling method. Throughout the city, Select 63 junior high schools, 102 senior high schools and vocational and technical schools, 12 universities, as the monitoring schools. In 2014 and 2008,using the "Chinese adolescent health related behavior questionnaire" undertake the students' collective self-filling anonymous questionnaire investigation, compare the changes of students' tobacco use behavior.

Results: Among college students and middle school students in Beijing, try smoking rate in 2008 is $30.05 \%$,then fall to $24.30 \%$ in 2014; have smoked a whole cigarette report rate in 2008 is $23.46 \%$, then fall to $18.19 \%$ in 2014 ; current-smoking report rate in 2008 is $14.41 \%$, then reduce to $10.89 \%$ in 2014 , the differences are statistical significance; among the current-smoking students, heavy-smoking rate in 2014 is $5.0 \%$, higher than 2008 is $4.1 \%$, and the differences are statistical significance; the rate of smoking frequency $\geq 20$ day/month in 2008 is $41.07 \%$ and in 2014 is $42.13 \%$, the difference was not statistically significant.

Conclusions: The over all launched tobacco control work in Beijing have made some progress, the tobacco use behavior of college students and middle school students decline sharply. however, the smoking severity of students who have become a smoker makes little improvement. So, reduce try smoking, not to become smokers is the first step to prevent adolescents from the tobacco hazard.

Tob. Induc. Dis. 2018;16(Suppl 1):A422

DOI:10.18332/tid/83785

\section{2:45-14:00}

EP-155-5 A Stop Smoking in Schools Trial (ASSIST) a decade on: insights from a mixed method process evaluation

F Dobbie $^{1}$, R Purves ${ }^{1}$, J McKell ${ }^{1}$, J White ${ }^{2}$, R Campbell $^{3}$, A Amos $^{4}$, L Moore $^{5}$, L Bauld

${ }^{1}$ University of Stirling, Stirling, United Kingdom, ${ }^{2}$ Cardiff University, Cardiff, United Kingdom, ${ }^{3}$ University of Bristol, Bristol, United Kingdom, ${ }^{4}$ University of Edinburgh, Edinburgh, United Kingdom, ${ }^{5}$ University of Glasgow, Glasgow, United Kingdom.E-mail: fiona.dobbie@stir.ac.uk
Background: ASSSIT (A Stop Smoking in Schools Trial) is a UK peer led, school based, smoking prevention programme that encourages the dissemination of non-smoking norms among 11-13 year olds. It is based on strong evidence, with results from a large cluster randomised trial showing a reduction in smoking uptake. However, data for this trial was collected in 2002-2004 and since then implementation of tobacco control policy in the UK has resulted in a further decline in adolescent smoking prevalence. This presentation will present data from an evaluation of ASSIST in Scotland, the first national evaluation of ASSIST since the original RCT.

Methods: Mixed method study involving a before and after school survey ( $\mathrm{n}=2166$ at follow-up), in-depth interviews, paired interviews, mini focus groups, observation with school staff, trainers, students, policy and commissioning leads $(n=101)$.

Results: Three different delivery models were piloted. This did not impact on fidelity or acceptability which was rated highly. Partnership working, from the onset, was viewed as being key to successful delivery and securing school participation. Feedback was overwhelmingly positive regarding the wider benefits of taking part in ASSIST for peer supporters (i.e. personal and communication skills) but also for the school and communities. There was less certainty regarding the extent of message diffusion and the impact this may have an adolescence smoking. Student survey results showed no significant change in self-reported smoking between baseline (2.5\%) and follow-up (3\%) and conversation recall with a peer supporter was low at $9 \%$.

Conclusions: ASSIST is a well delivered, popular programme with additional benefit for students, their wider social network, school and community. Yet, there is uncertainty regarding the extent of message diffusion within the school year which raises questions around the continued contribution of the programme to lowering the adolescent smoking rates. Further research is needed to update the existing evidence-base.

Tob. Induc. Dis. 2018;16(Suppl 1):A423

DOI:10.18332/tid/83804

\section{$12: 45-14: 00$}

EP-156-5 Decentralised public education campaigns for tobacco control by Malaysian youth tobacco control advocates towards smoke-free generation RY Ho1 ${ }^{1}$ AT Miao Thong ${ }^{2}$, AS Ping ${ }^{2}$

${ }^{1}$ Malaysian Green Lung Association, Bukit Mertajam, Malaysia, ${ }^{2}$ USM Green Lung Secretariat, Penang, Malaysia. E-mail: alan. tan55@gmail.com

Background and challenges to implementation: The scientific evidence shows that public education campaigns prevent the initiation of tobacco use among youths and reduce the smoking prevalence. However In Malaysia, it is observed that the tobacco control campaign activities are passive and less appealing to young population and thus less cost-effective.

Intervention or response: Malaysian Green Lung Association (MGLA), as the only youth-run civil society organisation for tobacco control in Malaysia, actively works together with Ministry of Health Malaysia and state health departments to promote and disseminate anti-tobacco messages to the general public via inexpensive and active way. In year 2015, MGLA pilot tested an idea during the state-level celebration of World No Tobacco Day in the World Heritage Site of George Town, Penang. One centralised exhibition site was set up by the Penang State Health Department at the Penang Youth Centre, involving canopy setup and exhibition booths. Meanwhile MGLA set up five mobile 
exhibition spots by the walkways in front of the shop houses and tourist attractions around the Penang Youth Centre, which involved two advocates and one informative poster per spot. Station games were introduced to actively involve general public especially youngsters and families to find clues and check in all five exhibition spots.

Results and lessons learnt: It is observed that instead of organising the exhibition at one central setting, decentralising public education campaign by setting up multiple exhibition touch points at different parts of the area does not only increase the coverage of the campaign, but also encourage active participation from the general public and local stakeholders. Besides, such approach greatly saved cost especially the infrastructures setup and rental cost to attain the similar outcomes as centralised campaign.

Conclusions and key recommendations: Decentralised public education campaign was preliminarily shown to be more costeffective in engaging the general public actively. This approach could be duplicated under limited financial resources to achieve the similar outcomes.

\section{Tob. Induc. Dis. 2018;16(Suppl 1):A424 DOI:10.18332/tid/84526}

\section{$12: 45-14: 00$}

\section{EP-157-5 Tobacco use behaviour among minors} in India

A Gupta ${ }^{1}$

${ }^{1}$ Tata Institute of Social Sciences, Mumbai, India. E-mail: amritagupta7@gmail.com

Background: Tobacco use is one of the major preventable causes of morbidity and morbidity worldwide. India is the third largest producer and consumer of tobacco in the world. Tobacco use among minors in India is a matter of serious concern as early age of tobacco use among the young population might lead to a number of health problems. The study brings out the prevalence of tobacco use behaviour among minors (15-18 years) in India.

Methods: The study is based on Global Adult Tobacco Survey India (GATS-India) 2009-10 data. The study includes population between 15-18 years of age. Suitable bi-variate and multivariate techniques have been used.

Results: The tobacco use is high among minors in India. An alarming $11 \%$ of the minors are current tobacco users in India and among $15 \%$ males and $7 \%$ females. Among the current tobacco users $8 \%$ minors are daily tobacco users and among them $11 \%$ are males and $5 \%$ are females. The prevalence of smokeless tobacco $(10.5 \%)$ is higher among the minors as compared to smoking (2\%). The prevalence of smokeless tobacco use is double among males (14\%) and compares to females (7\%). The most commonly used tobacco produt among males was gutkha (tobacco, lime and areca nut mixture), followed by kahini (tobacco lime mixture), betel quid with tobacco, cigarette and bidi. Among female oral use of tobacco was highest followed by gutkha, khaini, betel quid with tobacco, cigarette and bidi. Interestingly awareness about of the ill effects of tobacco was almost universal among minors.

Conclusions: The high prevalence of tobacco use among minors is a matter of grave policy concern requiring immediate attention from government, policy makers and NGO's. Tobacco use at an early age might make the minors susceptible to a number of health ailments. Proper counselling and treatment are required to deaddict the minors from tobacco use.

Tob. Induc. Dis. 2018;16(Suppl 1):A425

DOI:10.18332/tid/84679

[Tobacco use status among minors in India (2009-10)]

\begin{tabular}{|c|c|c|c|c|c|c|c|c|c|}
\hline \multirow[b]{2}{*}{ Status of use } & \multicolumn{3}{|c|}{ Tobaceo users } & \multicolumn{3}{|c|}{ Smokers } & \multicolumn{3}{|c|}{ Smokeless tobacco users } \\
\hline & Total & Male & Female & Total & Male & Female & Total & Male & Female \\
\hline Current users & 11.3 & 15.2 & 7.1 & 2.1 & 3.9 & 0.2 & 10.5 & 13.6 & 7.1 \\
\hline Daily user & 7.9 & 10.5 & 5.0 & 0.9 & 1.7 & 0.1 & 7.2 & 9.3 & 5.0 \\
\hline Occasional user & 3.5 & 4.7 & 2.1 & 1.2 & 2.1 & 0.1 & 3.3 & 4.4 & 2.1 \\
\hline
\end{tabular}

\section{$12: 45-14: 00$}

EP-158-5 Smoking status and cognitive performance among vocational school students in Beijing, China H Pengjuan ${ }^{1}$, H Lili², Z Shuang ${ }^{1}$, X Dan ${ }^{1}$

${ }^{1}$ China-Japan Friendship Hospital, Tobacco Medicine and Tobacco Cessation Center, Beijing, China, ${ }^{2}$ Beijing Polytechnic, Beijing, China. E-mail: hupengjuan163@163.com

Background: Studies of differences in cognitive function between smokers and non-smokers have yielded inconsistent results. However, most of studies have focused on middle-aged and elderly adults. The aim of our study was to examine the links between smoking status and cognitive function among vocational school students in Beijing, China.

Methods: A total of 213 students aged 16- 20 (98 smokers and 115 non-smokers) were recruited from three vocational schools in Beijing. Participants completed three subtests of Wechsler Adult Intelligence Scale (WAIS) (information, arithmetic, digit span) and Dysexecutive Questionnaire (DEX). Participants who were smokers also completed a cigarette smoking questionnaire and Fagerstrom test for nicotine dependence (FTND).

Results: Non-smokers performed better than smokers on the tests of arithmetic and digit span forward $(\mathrm{P}<0.05)$. They also had a lower total score of DEX and lower score on its three subtests (inhibition, knowing-doing dissociation and social regulation) $(\mathrm{P}<0.05)$.Smokers with mild nicotine dependence scored lower than smokers with moderate to severe nicotine dependence in the total score of DEX and in-resistance (one of subtests of DEX) $(\mathrm{P}<$ 0.05). A positive but weak correlation exists between digit span backward and the age at start of smoking $(\mathrm{r}=0.262, \mathrm{P}<0.05)$.

Conclusions: Smokers performed worse on some cognitive function (mental arithmetic reasoning ability, short-term attention and executive function) than non-smokers. It is not clear whether smoking exerts an adverse effect on cognitive function, or whether students with poorer cognitive skills are more likely to smoke.

Tob. Induc. Dis. 2018;16(Suppl 1):A426

DOI: $10.18332 /$ tid/84210

\section{$12: 45-14: 00$}

EP-159-5 Factors promoting initiation of cigarette smoking among adolescents in rural and urban areas in Oyo state, Nigeria

A Olumide ${ }^{1}$, A Shmueli², 0 Omotade ${ }^{1}$ 
'University of Ibadan/ University College Hospital, Institute of Child Health, Ibadan, Nigeria, ${ }^{2}$ The Hebrew University of Jerusalem, Department of Health Management and Economics, Jerusalem, Israel. E-mail: daisyolu@yahoo.co.uk

Background: Nigeria is a signatory to the WHO Framework Convention on Tobacco Control (FCTC) and the Tobacco Control Bill was signed in 2015. In spite of these, there are indications that many adolescents still smoke cigarettes. Our aim was to determine factors associated with initiation of cigarette smoking among adolescents in rural and urban locations in Oyo state, Nigeria.

Methods: This cross-sectional study utilized a mixed-methods approach. The quantitative survey involved 1142 adolescents selected by multi-stage sampling. The qualitative enquiry employed life history interviews conducted among 64 purposively-selected adolescents. Descriptive and analytic statistics were conducted. Qualitative data was analyzed using ATLAS.ti.

Results: The adolescents' mean age was $16 \pm 1$.8years, $56.0 \%$ were male and $51.1 \%$ were currently schooling. Forty-nine $(4.3 \%)$ had ever initiated smoking (i.e. smoked a whole cigarette). Mean age at smoking initiation was $15 \pm 2.2$ years. About $82 \%$ of adolescents typically purchased single sticks of cigarettes which usually cost N5.00-N100 (USD0.01 - USD0.28) each. Factors predictive of initiation of cigarette smoking were urban $(6.5 \%)$ versus rural (2.1\%) residence [OR=2.62, CI:1.22 - 5.62]; living with friends (33.3\%) versus living with parents $(2.5 \%)$; [OR=8.49; CI:1.92 - 37.51] and currently being out-of-school (8.1\%) versus being in-school (0.7\%); [OR=4.68 CI:1.52 - 14.39]. Inadequate parental supervision, especially in childhood was also contributory. Community level factors promoting initiation of cigarette smoking were ready access of cigarettes to minors in terms of low cost, uninhibited sale of sticks of cigarette and sales to minors.

Conclusions: Factors promoting initiation of cigarette smoking by adolescents abound in the study area. Efforts to enhance implementation of the FCTC and the tobacco control bill at community levels are urgently needed. Interventions targeting parents and interventions to encourage adolescents to remain in school could also be beneficial in reducing initiation of cigarette smoking and ensuing problems among adolescents in the study area.

Tob. Induc. Dis. 2018;16(Suppl 1):A427

DOI:10.18332/tid/84654

\section{Thursday, 8 March 2018}

\subsection{Priorities and challenges for advancing the WHO FCTC}

$11: 00-12: 30$

RF-1212-1 Adoption of the Tobacco Control Regulations - Legislative Instrument (LI) 2247 to reduce the burden of NCDs and to advance WHO FCTC implementation in Ghana

\section{Ali ${ }^{1}$}

'Vision for Alternative Development, Accra, Ghana. E-mail: issahwonder@gmail.com

Background and challenges to implementation: The Ghana's Public Health Act 851 of 2012 of which part six is dedicated to demonstrate commitment towards the domestication of the WHO-FCTC. Progress has been made towards the reduction of the NCDs burden by enacting laws and further recognizing its implementation in Ghana's development plan. To operationalize the Tobacco Control (TC) Measures, there was the need for the adoption of the TC Regulations. This will provide details on how to effectively implement the Law. Policy makers and enforcement agencies were not able to effectively implement key provisions such the ban on smoking in public places, registration of retailers, implementing Graphic Health Warnings, etc. due to lack of TC Regulations. The tobacco industry (TI) interferences delayed the adoption of the regulations.

Intervention or response: Strategic meetings with Civil Society Organisations were held. Community member were engaged and mobilize to pressure government to adopt the Tobacco Control Regulations.

The media/social media were used to generate discussions and drew government attention on the delay and exposed the TI. There were courtesy calls to Parliament, WHO Ghana to intervene.

Participants at public forum demanded reasons for the delay. Campaign materials advocating for the adoption were circulated to the public. VALD supported the development of a draft TC Regulations.

Results and lessons learnt: The programmes caught the attention of the government and policy makers which led to the submission of the draft Tobacco Control Regulations to Parliament and the Tobacco Control Regulation 2247 was adopted and passed into law on 4th January, 2017.

Conclusions and key recommendations: The Tobacco Control Regulations LI 2247 has outlined specifics implementation laws on the ban on Smoking, No-Smoking warning signs and Graphic Health Warnings. Ban on advertisement and promotion, and prohibition of tobacco industry interference. Regulation 2247 will empower Food and Drugs Authority to enforce the tobacco law and achieve the Health Goals of the Sustainable Development Goals.

Tob. Induc. Dis. 2018;16(Suppl 1):A428

DOI: $10.18332 /$ tid/84145

\section{$11: 00-12: 30$}

RF-1213-1 Perception of the adult population on packaging and labelling of cigarettes. Panama. Year: 2015

R Roa ${ }^{1,2}$, F Bajura ${ }^{2}$, V Herrera ${ }^{2}$, C Niño ${ }^{2}$, Tobacco Control Study Group

${ }^{1}$ Ministry of Health, Tobacco Control - National Planning Direction, Panama, Panama, ${ }^{2}$ Instituto Conmemorativo Gorgas de Estudios de Salud, Panama, Panama. E-mail: reinaro5906@ gmail.com

Background: Packaging and labelling of tobacco products are the first contact between the tobacco industry and the smoker. It's design works as an image for the brand, to attract potential clients. Other studies make in Panama, focused this investigation, on advertising, promotion and sponsorship of tobacco products, to uncover the perception about marketing characteristics used by the tobacco industry in the packaging of its products. The results were useful to implement plain packed and increase the amount of the warnings up to 80 to $85 \%$ in Panama.

Methods: The sample size was of 890 subjects selected to convenience, was applied an electronic observational guide, with questions about design, location, content, dimensions, shape, use of misleading terminology, and sanitary warnings on the boxes. We included cigarette boxes of different sizes with warnings and a standardized cigarette box on a software to validate and collect the data.

Results: Most reported characteristics: Brilliant colors: 50; appealing logo: 22.5\%; sanitary messages in text: $11.7 \%$, soft/ 
light product: $6.4 \%$

Times that the sanitary warning was noticed: frequent to very frequent: $62.4 \%$

Times that the image with the sanitary messages regarding negative effects on health was noticed: frequent to very frequent: $71.9 \%$

Times they read or paid attention to the sanitary messages: frequent to very frequent: $50 \% ; 9.0 \%$ of smokers had never read them.

Times the messages on tobacco products that make them consider the damage smoking causes: frequent to very frequent: $79.3 \%$ Colors on the cigarette box: White, gold and silver: Soft/ultrasoft; Color blue: Fresh 23.3\%;

Color green: menthol 51.9\%;

Color black and strong red: $80 \%$

Simple packaging: Not attractive. They are indifferent to it and reported they would not buy it: $68.1 \%$

Conclusions: The population supports increasing the size of warnings and plain packaging.

Tob. Induc. Dis. 2018;16(Suppl 1): A429

DOI:10.18332/tid/83976

\section{$11: 00-12: 30$}

RF-1217-1 Why does tobacco consumption increase in a MPOWER-compliant country?

T Gezer ${ }^{1}$, E Dagli' ${ }^{1}$, FYildiz'1, P Ay², 0 Elbek¹, M Ceyhan'1, M Güner ${ }^{1}$ ${ }^{1}$ Health Institute Association, Istanbul, Turkey, ${ }^{2}$ Marmara University, Public Health, Istanbul, Turkey. E-mail: elifzdagli@ gmail.com

Background: Turkey is praised by the global health sector as an exemplar for tobacco control being the first country in the world to pass all policies into law. Tobacco use that decreased in 20092013, increased again after 2013 along with respiratory-related mortality rates. This study is carried out to detect the challenges to effective implementation, to analyze the weaknesses and develop recommendations for securing tobacco control sustainability.

Methods: Tobacco production and sales data, tobacco industry activities, legislative and litigation process, civil and public infrastructure for tobacco control was investigated between 20092016 by media monitorization, political mapping and website surveys. Results: Domestic cigarette sales decreased by 15.8 billion sticks between 2009 -2013 and increased by 13\% between 2014- 2016 . Similar trends were recorded in other tobacco products. Waterpipe tobacco sales which increased by $18 \%$, and 'roll-your-own' tobacco by $126 \%$. The factors which elevated this reversal include; tobacco industry interference and corporate social responsibility (CSR) activities, government incentives to the industry, the legal challenges instigated by the industry that have delayed the passage of new tobacco control laws in particular on labeling, weakened implementation and enforcement of tobacco policies in bureaucracy and at local level. Burnout experienced by the public and tobacco control stakeholders contributed to the trend.

Conclusions: The implementation of financial and structural strategies such as having a portion of tobacco excise taxation dedicated to a health promotion fund, a national focal point for tobacco control, ensuring civil society representation on national advisory committees, regular data collection for tobacco related mortality, morbidity and social costs, and having a national evaluation framework can assist in reducing tobacco use in Turkey. Key underpinning policies may include; enacting FCTC Article 5.3 as a cross-governmental policy, and establishing a policy to prevent tobacco industry CSR activities.

Tob. Induc. Dis. 2018;16(Suppl 1):A430

DOI:10.18332/tid/84057
$11: 00-12: 30$

RF-1218-1 Compliance with the Framework Convention on Tobacco Control: a 9-country study M lacobelli', K Welding', K Smith', J Cohen

'Johns Hopkins Bloomberg School of Public Health, Department of Health, Behavior and Society, Institute for Global Tobacco Control, Baltimore, MD, United States of America. E-mail: miacobe1@jhu.edu

Background: Of the 14 low- and middle-income countries with the greatest number of smokers, nine countries implemented improved packaging laws between 2015 and 2017. Using the Framework Convention on Tobacco Control (FCTC) as a global benchmark, we compared in-country legislation from nine countries with Article 11 recommendations, assessed unique cigarette packs against the FCTC, and identified areas for improvement in country level tobacco packaging legislation.

Methods: We purchased unique cigarette packs according to a systematic protocol from 12 economically diverse neighborhoods within three of the largest cities within each of the countries. Country specific Health Warning Label (HWL) codebooks were created. Coding was completed by two independent coders. FCTC Article 11 was analyzed with respect to in-country legislation, with recommendations for tobacco packaging being identified. Four recommendations were used to create indicators by which HWL legislation was scored: HWL coverage $>=50 \%$; HWL positioned at the top of the principal display area; presence of graphic HWL; and qualitative statements on constituents and emissions.

Results: Overall cigarette pack HWL compliance with in-country legislation ranged from $53 \%$ in India to $95 \%$ in Russia. In-country legislation was scored against FCTC recommendations from $0-4$. HWL compliance had a strong negative correlation $(-0.77)$ with FCTC score. In general, HWL compliance was higher for countries with lower FCTC scores. We will report detailed FCTC analysis by country.

Conclusions: Although these nine countries have improved their HWL requirements, most still do not meet the floor set by the FCTC. We will discuss how HWL comprehensiveness, as measured by FCTC impacts overall HWL compliance. Our discussion suggests there are still multiple areas of improvement fourteen years after the FCTC was adopted. It is incumbent on governments to continue to improve HWL legislation, above and beyond the FCTC. Future legislation must be clear, comprehensive and easily implemented to ensure enforcement.

Tob. Induc. Dis. 2018;16(Suppl 1):A431

DOI:10.18332/tid/84212

\section{$11: 00-12: 30$}

RF-1221-1 Achieving organization sustainability: recommendations from the literature

J Hardesty ${ }^{1}$, J Cohen ${ }^{1}$

'Johns Hopkins Bloomberg School of Public Health, Baltimore, MD, United States of America.E-mail: jhardesty@jhu.edu

Background: To continue implementing the WHO FCTC, it is important for tobacco control organizations to remain sustainable, i.e., vibrant, with sufficient infrastructure and operations that allow continuation of day-to-day work despite future uncertainties. The purpose of this review was to synthesize the literature regarding tools to assist organizations in remaining sustainable.

Methods: A review of the literature was conducted during 2016 to identify sustainability-related reports and tools. Our review included 12 search terms, including non-profit sustainability, program sustainability, organization sustainability, partnership 
sustainability, initiative sustainability, and philanthropy exit strategies. The final review included 16 sustainability reports and tools.

Results: The Speakman Management Consulting's "Nonprofit Organizational Life Cycle" and "Keys to Life Cycle Transitions" are tools that categorize the maturity level of organization infrastructure and operations and provide strategies for reaching the next stage of maturity or maintaining maturity. Strategies may differ depending on the life stage of an organization, which include invention; start-up; growth; sustainability; stagnationand-renewal; and decline-and-shutdown. The U.S. Department of Health and Human Services' "Building Sustainable Programs: The Resource Guide" is a self-reflective tool that assesses an organization's sustainability readiness on eight key factors: creation of an action strategy; assessment of the environment; adaptability; community support; integration into community infrastructure; leadership team; strategic partners; and diverse financial opportunities. The guide provides direction on implementing strategies to address low readiness scores. Other reports suggest planning for future donor exits by leveraging donor support to implement sustainability strategies and by anticipating the impact of losing a donor's brand.

Conclusions: Tobacco control organizations may utilize several resources to conduct self-evaluations of their current infrastructure and operations and to develop and implement plans to improve organization sustainability. Organizations can also attempt to leverage donor support to achieve organization sustainability and plan for future brand loss.

Tob. Induc. Dis. 2018;16(Suppl 1):A432

DOI:10.18332/tid/83929

\section{6:30-18:00}

RF-1234-4 Article 14 of WHO FCTC: gaps in implementation \& recommendations

S Nethan'1, D Sinha', K Chandan'1, R Mehrotra'

${ }^{1}$ National Institute of Cancer Prevention and Research, Noida, India.E-mail: suzanne.nethan@gmail.com

Background: Article 14 of the WHO Framework Convention on Tobacco Control (FCTC) deals with demand reduction measures concerning tobacco dependence and cessation. This paper identifies the gaps in its implementation globally, with appropriate recommendations to fulfill the same.

Methods: Information regarding tobacco cessation support among the WHO-FCTC member parties was obtained from the MPower 2017 database and from surveys like Global Adult Tobacco Survey (GATS), the Global Health Professions Student Survey and Global School Personnel Survey. In addition, meta-analysis of the results of the globally available randomized trials and cohort studies on smokeless tobacco (SLT) cessation interventions was performed, to determine the efficacy of the same.

Results: Tobacco cessation support is available at various health care facilities in $<20 \%$ Parties; National Quitlines in $31 \%$ Parties while Nicotine Replacement Therapy in $70 \%$ parties (mostly in high resource and European countries). Very few parties provide full cost coverage of the same. Health care professionals do not equally care for SLT users and smokers. There is a lack of formal training on tobacco cessation among health professionals, health professional students and school personnel. Many countries have experience in smoking cessation but only $3 \%$ Parties have experience in SLT cessation. Meta-analysis has shown that behavioral intervention alone has $60 \%$ more chance of enabling quitting and is the most effective way of intervention both for low and high resource set ups.

Conclusions: Tobacco cessation support and surveillance need to be strengthened especially in the low resource and high SLT burden countries. Sensitization and training on cessation to professional group is the critical issue. Effective SLT prevention $\&$ cessation programs must be encouraged at school level itself.

Tob. Induc. Dis. 2018;16(Suppl 1):A433

DOI:10.18332/tid/84092

$18: 15-19: 15$

LB-1300-1 Compliance with tobacco control laws before and after the enactment of a national Tobacco Control Act in Ghana

W Agbenyikey ${ }^{1}$, EK Wellington ${ }^{2}, \mathrm{~K}$ Asante-Nkrobea $\mathrm{Jnr}^{3}, \mathrm{H}$ Mamudu ${ }^{4}$, P Subedi ${ }^{4}$, A Ouma ${ }^{5}$, MA Duah ${ }^{6}, \mathrm{~J} \mathrm{Rijo}^{7}$

${ }^{1}$ Martin Luther Health Training School - Kintampo Campus, Kimntampo, Ghana, ${ }^{2}$ Monash University Sunway, School of Medicine and Health Sciences, Malaysia, Malaysia, ${ }^{3}$ University of Ghana, School of Nursing, Accra, Ghana, ${ }^{4}$ East Tennessee State University, Tennessee, TN, United States of America, ${ }^{5} \mathrm{WHO}$, Accra, Ghana, ${ }^{6}$ Chamberlain University, College of Nursing \&t Health Professions, Downers Grove, IL, United States of America, ${ }^{7}$ India Institute of Technology, Johpur, India. E-mail: wilfred.agbenyikey@aya.yale.edu

Background: Tobacco smoke contains around 70 known toxins and carcinogens. Worldwide, billions of nonsmokers, including children are exposed to secondhand tobacco smoke (SHS), contributing to over 600,000 annual deaths. Public smoking ban is an effective policy to protect nonsmokers from SHS exposure, yet, only 7 of 47 countries in Sub-Saharan Africa (SSA), have comprehensive smoke-free policies. In 2012, Ghana enacted and implemented a national Tobacco Control Act, the Public Health Act 851 , which prohibited smoking in all public places. The study aimed to evaluate the impact of the public smoking ban on SHS exposure in hospitality venues.

Methods: Public recreational places $(\mathrm{N}=137)$ in towns across Ghana, including Accra, were surveyed (2007: $\mathrm{n}=88,2015: \mathrm{n}=49)$. Research assistants recorded indoor PM2.5 concentrations along with information on services, infrastructure and smoke-free policy of the selected facilities. At least 30 minutes was spent in each facility to record PM2.5 concentrations using Sidepak monitor. Wilcoxon rank sum test and Mann-Whitney analysis was used to compare the PM2.5 concentrations in the facilities surveyed before and after the enactment of the Tobacco Control Act using SAS 9.4.

Results: Indoor smoking was observed in $86 \%$ and $56 \%$ of the facilities surveyed pre- and post-legislation, respectively. The average PM2.5 concentrations was median 553 [IQR 259-1038] at all venues that smoking was allowed (75) in 2007 and median 439 [IQR 234-576] in 2015. The average PM2.5 concentrations was significantly lower post-legislation compared to pre-legislation ( $\mathrm{p}$-value $=<.0001)$. Thus, there is no statistically significant difference between pre-legislation period in 2007 and post-legislation period in 2015 ( $\mathrm{z}=-1.660$, $\mathrm{p}=0.0969)$.

Conclusions: The introduction of the smoke-free policy in Ghana did not reduce the SHS exposure in hospitality venues, suggesting lack of compliance to the policy and the need for extension of smoke-free policies.

Tob. Induc. Dis. 2018;16(Suppl 1):A434

DOI:10.18332/tid/84733 


\section{8:15-19:15}

LB-1302-1 Role of WHO FCTC global knowledge hub on smokeless tobacco in smokeless tobacco control

A Chandra', R Mehrotra'

'ICMR-National Institute of Cancer Prevention and Research, Noida, India. E-mail: anshikac6@gmail.com

Background and challenges to implementation: An estimated 350 million users of Smokeless Tobacco (SLT) are spread worldwide, across 133 countries. The South-East Asian Region (SEAR) has a major epidemic of SLT use, with largest number of users residing in India and Bangladesh. Although prevalence of SLT use has also been reported in high income countries including USA and in Europe, the major chunk of SLT users reside in low and medium income countries. Knowledge about SLT products is minimal.

Intervention or response: Considering this epidemic of SLT in the SEAR, the WHO-Framework of Convention of Tobacco Control (WHO-FCTC) established a Global Knowledge Hub on SLT (GKH SLT) at the Indian Council of Medical Research - National Institute of Cancer Prevention and Research, (ICMRNICPR) at Noida India. The mandate of the hub included:

a) Generate knowledge, share expertise and information on SLT through a web portal.

b) Assist Parties of the Convention to develop programs and policies.

c) Support the Convention Secretariat in its work to promote control of SLT use globally.

Results and lessons learnt: The work of the hub has succeeded in generating expertise, information and knowledge through factsheets, guidelines and recommendations as well as a newly developed searchable web portal (http://untobaccocontrol.org/kh/ smokeless-tobacco/),thus disseminating the requisite information encompassing health effects and other aspects of prevention and control of SLT as well as social media communications.In the last year, the GKH-SLT has conducted an "Inter Country Meeting on SLT Control Policies" and two training workshops for health professionals. Lessons learnt included need for wider dissemination and developing capacity worldwide.

Conclusions and key recommendations: Recommendations for the future includework on profiling of new SLT products, coordination with testing laboratories, collaborative projects and information dissemination through workshops. The hub should be developed as a global nerve centre for SLT control and strengthened by all stakeholders.

Tob. Induc. Dis. 2018;16(Suppl 1):A435

DOI:10.18332/tid/84742

\section{8:15-19:15}

\section{LB-1303-1 Smoking rooms undermine FCTC agenda}

in Kazakstan

J Sadykova', Z Kalmatayeva ${ }^{2}$

'National Coalition 'For smokefree Kazakstan', NGO, Almaty, Kazakhstan, ${ }^{2} \mathrm{Al}$-Farabi Kazakh National University, Public Health and Medicine, Almaty, Kazakhstan. E-mail: nosmoke_kaz@ mail.ru

Background: The fundamental human rights and principles of Article 8 of the WHO FCTC requires implementation of a $100 \%$ smoke free environment by means of total elimination of smoking in public places. In Kazakstan, these principles have been only partially addressed by the Health Act (2009) which allows designated smoking rooms in places of "public eating" where food is served whilst other public places must to be smokefree. Methods: Our objective was to evaluate smokefree environment by obtaining and analyzing published and unpublished data on second hand smoking (SHS) exposure in public places in Kazakstan. Few survey have been analyzed: officially published GATS $(\mathrm{N}=4425)$ and GYTS $(\mathrm{N}=2083)$ conducted in 2014 by the National Healthy lifestyle center (HLSC) and the unpublished GYTS $2013(\mathrm{~N}=2085)$ and 2015 $(\mathrm{N}=2085)$ were conducted among Almaty teenagers within "Smokefree Almaty" program.

Results: Country wide GATS showed that $27.6 \%$ of adults are exposed to SHS in public eating places (bars, restaurants, café) and $19 \%$ are exposed in enclosed public places. Partially published GYTS 2014 data provided by HLSC showed high SHS exposure in teenagers in open public places (29.3\%) and enclosed public places (26.5\%) followed by less exposure in homes (18.2\%). GYTS unpublished surveys among Almaty teenagers who demonstrates the highest smoking rate (9\%) also suggest that most SHS exposure happens in open public places (25.4\% in 2013 and $26.6 \%$ in 2015). Almost equal SHS exposure to teenagers revealed at home (16.4\% in 2013 and $16.7 \%$ in 2015) and enclosed public places (14\% in 2013 and $12.5 \%$ in 2015) of Almaty.

Conclusions: Nearly $30 \%$ of Kazakstani adults and teenagers are exposed to SHS in open public places, $20 \%$ at home and $12,5 \%$ - 26,5\% in enclosed public places. It demonstrates that partial smoking ban has not eliminated from SHS which undermines implementation of $100 \%$ smokefree environment and FCTC country agenda.

\section{Tob. Induc. Dis. 2018;16(Suppl 1):A436} DOI: $10.18332 /$ tid $/ 84713$

\section{8:15-19:15}

LB-1304-1 How MPOWER-ed are we towards effective implementation of WHO FCTC?

A Islam'

'National Tobacco Control Cell, Tobacco Control, Dhaka, Bangladesh.E-mail: aisujon@yahoo.com

Background and challenges to implementation: Evaluation of national success on implementation of the WHO MPOWER package was lacking in Bangladesh until recently. In order to plan future work, it was important to gain an understanding of the advances and gaps in comprehensive tobacco control work.

Intervention or response: With support from The Union, Work for a Better Bangladesh (WBBT) carried out a six month long project on MPOWER implementation in Bangladesh. WBBT gathered background documents policy documents, and materials related to tobacco control that were printed by different organizations and discussed with various stakeholders through various national events. WBBT also carried out a series of key informant interviews with professionals working on tobacco control for several years, including staff of local- and national-level NGOs and officials of WHO.

Results and lessons learnt: We identified several gaps in MPOWER implementation, particularly in terms of monitoring, cessation, and taxation. Bangladesh still lacks a tax policy or specific guidelines. We found gaps in implementation of existing policy in terms of smokefree places. While the law exists, there are significant gaps in its implementation. There are gaps in terms of law implementation on warnings. With a wide variety of tobacco products available, some of them with minimal packaging, a significant portion of tobacco products do not carry the mandated warnings. There are also weaknesses in terms of point of sale advertising.

Conclusions and key recommendations: For a country to be properly MPOWER-ed to tackle the tobacco epidemic, we must 
be aware of how far we have progressed and what significant gaps remain. Occasional survey on specific matter is not enough to give us an overall sense of where we are and where we need to be stronger. An MPOWER study can be conducted fairly easily by combining limited observation with a number of key informant interviews to arrive at a fairly accurate assessment of the MPOWER implementation situation.

Tob. Induc. Dis. 2018;16(Suppl 1):A437

DOI:10.18332/tid/84745

\subsection{Integrating tobacco control into Health systems, global frameworks and strategies to reach WIO FCTC and SDG's targets.}

\section{1:00-12:30}

RF-1215-1 Promoting effective tobacco control through advancement of public health systems in Nigeria

O Anya ${ }^{1}$, A Erameh ${ }^{2}, \mathrm{H}$ Mamadu ${ }^{3}$

${ }^{1}$ Civil Society Legislative Advocacy Centre (CISLAC), Programs, Abuja, Nigeria, ${ }^{2}$ Civil Society Legislative Advocacy Centre (CISLAC), Programs, Federal Capital Territory (FCT), Nigeria, ${ }^{3}$ East Tennessee State University, Health Services, Management and Policy, Johnson City, TN, United States of America. E-mail: okekea@atim.co.za

Background and challenges to implementation: Nigeria's estimated population of 170 million with a large youth segment provides a veritable market for the Tobacco Industry.

Nigeria ratified the WHO Framework Convention on Tobacco Control (WHO) in 2005, and subsequently enacted the National Tobacco Control Act (NTCA) in 2015, with the Federal Ministry of Health $(\mathrm{FMoH})$ as the focal coordinating agency. Nigeria made attempts towards domesticating the convention through a national law in 2011 which failed but this was fortunately passed into a National Tobacco Control Act in 2015.

However, the law is yet to take traction amongst a wide range of Public Health Institutions (PHIs). This project aims at understanding the innate challenges in selected PHIs towards bridging the much required capacity gaps and to improve understanding and advocacy for mainstreaming Tobacco Control (TC) into a broad TC agenda in Nigeria.

Intervention or response: Since the enactment of the NTCA, advances in implementation and other broad TC initiatives in Nigeria can largely be attributable to activities of Non-State Actors (NSAs). Government's financial support to tobacco control has been very weak.

Results and lessons learnt: Notwithstanding the poor financial support to TC concerns, CSOs' interventions such as advocacy, media outreaches and general awareness for the populace have continued to give strength to tackling the Tobacco Industry tactics. Furthermore, the gaps witnessed underscore the need for enhanced coordination by the relevant government agencies which has the official and political mandate to deal with TC issues. Conclusions and key recommendations: The implementation of the NTCA in Nigeria has stalled because the law has not gained traction within PHIs. This suggests that enactment of NTCA does not necessarily translate into actionable policies and programs without the buy-in of key PHIs. Hence, implementing NTC policies that are consistent with the WHO FCTC requires the engagement of PHIs in the entire policy process.

Tob. Induc. Dis. 2018;16(Suppl 1):A438

DOI: $10.18332 /$ tid/84471
$11: 00-12: 30$

RF-1216-1 Ten years of India's National Tobacco Control Programme: achievements, challenges and the way forward

AK Pandey', RJ Singh', P Lal', B Gopalan'1, GK Tripathi', R Sharma' 'International Union Against Tuberculosis and Lung Disease, Tobacco Control, New Delhi, India. E-mail: apandey@theunion. org

Background and challenges to implementation: In India, more than 275 million adults use tobacco, which kills 1.2 million of these every year. More than one-quarter of India's youth begin tobacco use before they are 16 years old and $85 \%$ of all users begin before they are 18, the legal age of tobacco use. A diversity of tobacco products spurs India's hidden epidemic. In May 2003, the Indian Parliament passed a landmark tobacco control legislation - the Cigarettes and other Tobacco Products Act (COTPA). Following this the Government of India launched the National Tobacco Control Programme (NTCP) in 2007, with the aim to protect children and youth and to enforce the tobacco control legislation. Intervention or response: We assess the progress of tobacco control efforts in the states by analysing the compliance towards provisions of COTPA and achieving goals of The NTCP. We use third party compliance studies, analyses done by civil societies, orders and action taken reports of state governments and district administration, and media report from states. We prepare a semistructured report card based on compliance towards criteria specified under NTCP and COTPA.

Results and lessons learnt: The achievement in tobacco control have been mixed. We will present a state-wise, updated assessment of performance of tobacco control efforts in India, and identify drivers for their success and reasons for their limited progress. We find that political and administrative commitment and leadership are critical to get tobacco control started at sub-national level.

Conclusions and key recommendations: Sustainable enforcement and adoption of evidence-based strategies, investing in developing skilled human resources, and financial commitments (especially co-investments from sub-national sources) are key factors in achieving goals under National Tobacco Control Programme and ensuring long-term gains that advance tobacco control.

Tob. Induc. Dis. 2018;16(Suppl 1):A439

DOI:10.18332/tid/84485

\section{8:15-19:15}

LB-1305-1 The impact of anti-smoking policies of the 2010-2014 Hungarian government - a comprehensive evaluation

T Joó ${ }^{1}$, M Szócska' ${ }^{1}$ Z Z Vokó ${ }^{2}$, T Demjén ${ }^{3}$, J Bodrogi ${ }^{4}$, P Gaál ${ }^{1}$, KL Foley ${ }^{5}$ 'Semmelweis University, Budapest, Hungary, ${ }^{2}$ Eötvös Loránd University, Department of Health Policy and Health Economics, Budapest, Hungary, ${ }^{3}$ Ministry of Human Capacities, Focal Point for Tobacco Control, Budapest, Hungary, ${ }^{4} \mathrm{Health}$ Economist, Budapest, Hungary, ${ }^{5}$ Wake Forest School of Medicine, Department of Social Sciences and Health Policy, Winston-Salem, NC, United States of America. E-mail: joo.tamas@emk.sote.hu

Background and challenges to implementation: Hungary produced the greatest progress on the Tobacco Control Scale among 34 European countries in recent years, due to its tobacco control policies. Most strikingly, these measures were introduced with exceptional speed during the struggle with the world economic crisis and under constant pressure from the tobacco lobby threatening with the fall of employment, decline in tax 
revenues and the collapse of the hospitality industry.

Leading the charts in smoking-related diseases shows that smoking is one the most important health-risks in Hungary. According to a study of the National Institute of Health Development, 20,470 people died in smoking-related diseases in 2010. The economic burden of smoking was estimated to be HUF 441 billion, while tax revenues amounted to 360 billion HUF in 2010.

Intervention or response: The above figures explain why the fight against smoking became one of the most important public health priorities in Hungary during the $2010-2014$ period. The measures implemented in this period ranged from tax increase and the introduction of combined health warnings through the restriction of smoking in public places to the reorganization and control of tobacco retail sale.

Results and lessons learnt: These anti-smoking measures have resulted in decrease in the prevalence of smoking (2009: $38 \%$ vs. 2014: 30\%) and tobacco consumption (2012: 19,54bn vs. 2014: $14,87 \mathrm{bn}$ pieces) and in hospital admissions for acute coronary syndromes, and also had a positive impact on the hospitality industry and the state budget.

Conclusions and key recommendations: Reducing tobacco use plays a major role in Hungarian efforts to achieve target 3.a of SDG 3 which refers particularly to strengthening the implementation of the WHO Framework Convention on Tobacco Control (WHO FCTC). Politically, and tactically well-organized interventions took the form of a successful "76 days tobacco blitz" and in a short period, the government caught up with a more-than-decadelong deficit in meaningful actions in this important public health domain.

Tob. Induc. Dis. 2018;16(Suppl 1):A440

DOI: $10.18332 /$ tid/84756

\subsection{Setting the research agenda for translating science to policy and new tools for policy impact assessments}

\section{1:00-12:30}

RF-1214-1 Tobacco consumption change in the WHO European Region in 2008-2015

K Krasovsky ${ }^{1}$

${ }^{1}$ Ukrainian Institute for Strategic Studies of the Ministry of Health, Tobacco Control Unit, Kyiv, Ukraine.E-mail:krasovskyk@ gmail.com

Background: The aim of the paper is to estimate trends of tobacco consumption in 2008-2015 in the European Region as a whole and in individual countries.

Methods: Data on legal tobacco sales in the EU countries were taken from the European Commission. For other European countries, sales were estimated as cigarette turnover (= production + imports - exports), which was calculated from national statistics data and other sources. For those countries, where consumption of smoking tobacco (other than cigarettes) is high enough, tobacco sales estimates include other tobacco products in cigarette equivalents. Estimates of illicit tobacco trade volumes were taken from governmental reports; data from reports funded by the tobacco industry were cautiously considered.

Results: In 2008-2015, most European countries experienced a decline in tobacco turnover. For those countries, where an increase in tobacco sales was observed over these years (Belgium, Belarus, Georgia, Kyrgyzstan, Macedonia, Uzbekistan), the tobacco smuggling was the main factor of sales increase, but not the smuggling into these countries, but out of them.
In 50 countries of the WHO European Region combined, legal tobacco sales decreased from 1,572 billion cigarettes in 2008 to 1,221 billion cigarettes in 2015 or by $22 \%$. The reduction in Eastern Europe was greater.

Total illicit cigarette trade decreased in the European Region: both in most countries with high tobacco taxes (inflow smuggling) and in most countries with low tobacco taxes (outflow smuggling). Most contraband cigarettes in Europe were first legally manufactured in some other European country. So, the total legal (taxable) cigarette turnover in all countries of the European Region combined is rather close to the total cigarette consumption in the Region.

Conclusions: Total tobacco consumption in the European Region declined by at least $20 \%$ in $2008-2015$ and so tobacco control policies conducted in the European countries were effective.

Tob. Induc. Dis. 2018;16(Suppl 1):A441

DOI:10.18332/tid/84257

\section{$11: 00-12: 30$}

RF-1219-1 Tobacco marketing trends at the pointof-sale (POS) near schools in 9 middle and highincome countries

A Grant ${ }^{1}$, C Weiger ${ }^{1}$, J Brown ${ }^{1}$, J Cohen

${ }^{1}$ Johns Hopkins Bloomberg School of Public Health Institute for Global Tobacco Control, Health, Behavior and Society, Baltimore, MD, United States of America. E-mail: ashleyigtc@jhu.edu

Background: An observational study was conducted to document tobacco advertising, promotion, and sponsorship (TAPS) strategies in retail outlets in order to assess compliance with the law, and to identify tobacco industry strategies that circumvent restrictions, exploit loopholes in the language of the law, or that appeal to children.

Methods: Trained observers recorded information and photographs about the characteristics of tobacco product display and advertising signage, and offers for special promotions. Data collectors completed customized checklists that were inputted to mobile phones that captured the geographic coordinates for each location and uploaded data from the field, for real-time access and review. Data were collected from 3,964 retail outlets located within 100-250 meters of 766 primary and secondary schools in 7 middle-income countries (Argentina, Bangladesh, Bolivia, Bosnia and Herzegovina, Georgia, Nicaragua, and Ukraine) and 2 highincome countries (Slovenia and Switzerland). Retail outlets were identified by following a map to walk all streets located within the designated radius surrounding each school.

Results: Tobacco products were for sale in $74.8 \%$ of retail outlets $(n=2,964), 86.5 \%(n=2,563)$ of which displayed tobacco products. Among tobacco retailers, products were typically displayed near sweets $(61.8 \%, \mathrm{n}=1,831)$, in the cashier zone $(49.3 \%, \mathrm{n}=1,461)$, behind the cashier zone $(39.0 \%, \mathrm{n}=1,157)$, and 1 -meter or less from the floor $(27.7 \%, \mathrm{n}=821)$, which is considered to be the eye-level of children. Nearly half of tobacco retailers also used advertising signage to market tobacco products $(48.9 \%, \mathrm{n}=1,448)$. Conclusions: These data highlight the significance of the display of tobacco packs in prominent locations or near other products that attract the attention of children. POS advertising signage is another prevalent tactic that has been shown to have the same effect as traditional marketing media. Locally-customized TAPS monitoring is a useful approach to gather data that reveal such strategies and can inform effective policy development and implementation.

Tob. Induc. Dis. 2018;16(Suppl 1):A442

DOI:10.18332/tid/84648 
11:00-12:30

RF-1220-1 Spatial distribution of tobacco outlets and related regulation within a restricted market framework

R Valiente ${ }^{1,2}$, X Sureda $^{2}$, U Bilal ${ }^{2,3}$, A Navas-Acién ${ }^{4}$, J Pearce $^{5}$, M Franco ${ }^{2,6}$, F Escobar $^{1,2}$

'University of Alcalá, Department of Geology, Geography and Environmental Sciences, Alcalá de Henares, Spain, ${ }^{2}$ University of Alcalá, Social and Cardiovascular Epidemiology Research Group, School of Medicine, Alcalá de Henares, Spain, ${ }^{3}$ Drexel Dornsife School of Public Health, Urban Health Collaborative, Philadelphia, PA, United States of America, ${ }^{4}$ Columbia University Mailman School of Public Health, Department of Environmental Health Sciences, New York, NY, United States of America, ${ }^{5}$ University of Edinburgh, Centre for Research on Environment, Society and Health, School of GeoSciences, Edinburgh, United Kingdom, ${ }^{6}$ Johns Hopkins Bloomberg School of Public Health, Department of Epidemiology, Baltimore, MD, United States of America.E-mail: roberto.valiente@uah.es

Background: Restricting tobacco availability is likely to be central to the next generation of international tobacco control policies. In Spain, tobacco sales are limited to tobacco exclusive outlets and associated vending machines. Laws regulating the geographical concentration of tobacco outlets demand a minimum distance of 150-metres between them, unless they exceed an established legal sales-threshold to justify their closeness. Distances to other public facilities (e.g. schools, playgrounds, or health-centres) are not regulated. We aimed to evaluate the implementation of tobacco outlets' minimum distances, and to examine their proximity to schools, playgrounds and health-centres.

Methods: Our study area was Madrid municipality. Performing a proximity-analysis with Geographic Information Systems, we identified outlets that were less than 150-metres between them. We checked whether these outlets exceeded the legal salesthreshold, (established in three-times the average of sales of whole tobacco outlets within the municipality). Accessibility from schools, playgrounds, and health-centres to tobacco outlets were estimated. Sociodemographic data were used to characterize areas where tobacco outlets are closer than 150-meters to other retailers and, to schools, playgrounds and health-centres.

Results: We geocoded 638 tobacco outlets, of which 34 were within a 150-metres of another tobacco outlet. From those, 24 registered a sales volume lower than the legal sales-threshold, located in central neighbourhoods, with high density of leisure venues and high-educational level. Results also showed that $29 \%$, $20 \%$ and $6 \%$ of tobacco outlets were closer than 150 -metres to schools, playgrounds, and health-centres, respectively.

Conclusions: This study examines tobacco outlets distribution under a restricted market framework. Results showed little examples of over provision of tobacco retailing, empathising that the evaluation of Spanish policies could provide keys to improve regulations on tobacco sales restriction in other countries. However, we identified a significant number of stores close to schools, playgrounds and health-centres that suggest the need to enforce minimum distances to these facilities.

Tob. Induc. Dis. 2018;16(Suppl 1):A443

DOI:10.18332/tid/84239

\section{8:15-19:15}

LB-1301-1 Network analysis to detect gaps in research on smokeless tobacco: implications for future policy
H Kaur ${ }^{1}$, H Singh ${ }^{2}$, A Singh ${ }^{3}$, AS Dhama ${ }^{2}$, R Gupta ${ }^{3}$, A Sharma², DN Sinha ${ }^{4}$, R Mehrotra $^{3}$

IICMR-National Institute of Cancer Prevention and Research, WHO FCTC Global Knowledge Hub on Smokeless Tobacco, Noida, India, ${ }^{2}$ Indian Council of Medical Research, New Delhi, India, ${ }^{3}$ ICMR-National Institute of Cancer Prevention and Research, Noida, India, ${ }^{4}$ School of Preventive Oncology, Patna, India. E-mail: harleenkr.92@gmail.com

Background: Smokeless Tobacco (SLT) is increasingly becoming a global menace, though the greatest burden of its use is borne by South-East Asia region. Though extensive research has been conducted on various aspects of SLT use and its effects, no effort has yet been undertaken to consolidate this data and identify the still-under researched areas in this field.

This study was aimed at identification of the degree of collaboration between scientists involved in SLT research and the areas receiving thrust of scientific interest so far. The final aim was to identify the research gaps for better policy impact assessments Methods: Data about research in SLT were collected from PubMed database using inbuilt query and MESH Terms. Data was extracted in XML format and parsed into database using Hypertext Preprocessor (PHP). Networks were designed where node represented unique authors and edges represented degree of collaborations between them. Also, quantum of research in various aspects of SLT was identified using MESH terms.

Results: Data of 2094 records was analyzed. The highest number of paired collaborative research using author identifier was found in Pakistan (4) followed by United States of America (3).

Maximum number of articles were devoted to epidemiology of SLT use (925) and adverse effects of these products (1017) while least research has been conducted on economics of SLT (47). Trend analysis revealed that epidemiological research on SLT gained momentum significantly after 2005 .

Conclusions: The present analysis, first of its kind in the field of SLT, highlights the paucity of research studies on important topic like economic costs and alternative strategies are vital for policy makers to implement SLT control. We would like to suggest formation of core research groups in high-burden countries dedicated to important aspects of SLT.

Tob. Induc. Dis. 2018;16(Suppl 1):A444

DOI:10.18332/tid/84734

\subsection{Promoting transnational cooperation and Partnerships: models of Intergovernmental and nongovernmental collaboration across multiple sectors for high level commitments}

\section{$11: 00-12: 30$}

F0-357-2 Multilateral development banks and bilateral donors and their responsibility towards excluding tobacco investments and participation P Lal'

'International Union Against Tuberculosis and Lung Disease, New Delhi, India. E-mail: plal@theunion.org

Background and challenges to implementation: In 1991, The World Bank, world's largest lender, pledged that it would no longer support tobacco related projects. It was expected that other financial investors would follow, but most did not responded to this call. As a result, several financial institutions continue to invest in tobacco and fuel an epidemic to an unprecedented scale. Using tobacco as a case in point, this review highlights the 
continuing investment among financial institutions which conform to 'socially responsible investments' and calls for monitoring and reporting such unethical practices. There is a need to harmonise the numerous criteria, principles and voluntary codes that govern socially responsible investing. Monitoring of these can be mutually beneficial for all social sectors.

Intervention or response: We analyse annual reports of the seven major MDBs (like the World Bank Group, Regional Development Banks, Trade bloc banks etc..) and bilateral donors from 2010 to 2017 and estimate size and nature of investments or board-level participation by or in tobacco industry.

Results and lessons learnt: Most MDBs and bilateral have state tobacco as an exclusion criteria, yet we find discrepancies in investments, collaborations and partnerships (with financial and non-financial implications) across all donors.

Conclusions and key recommendations: Tobacco control advocates need to monitor the behaviour of MDBs and bilateral that have committed to exclude tobacco from their portfolio. Those MDBs and bilateral donors that still do not support exclusion of tobacco, must be advocated to do so.

Tob. Induc. Dis. 2018;16(Suppl 1):A445

DOI:10.18332/tid/84351

\subsection{Building leadership capacity and broader alliances for tobacco control policy advocacy}

$11: 00-12: 30$

F0-355-5 Generating local evidence on tobacco industry tactics to advance tobacco control objectives: a holistic approach embracing new technology

T DeAtley', E Green², A Grant ${ }^{3}$, C Renzulli', D Rosen ${ }^{5}$, M Carmona', J Cohen ${ }^{6}$, Y Richardson

${ }^{1}$ Campaign for Tobacco-Free Kids (CTFK), International Research, Washington, DC, United States of America, ${ }^{2}$ University of North Carolina at Chapel Hill, Health Behavior, Chapel Hill, NC, United States of America, ${ }^{3}$ Johns Hopkins Bloomberg School of Public Health, Baltimore, MD, United States of America, ${ }^{4}$ Campaign for Tobacco-Free Kids (CTFK), International Communications, Washington, DC, United States of America, ${ }^{5}$ Campaign for Tobacco-Free Kids (CTFK), Tobacco Industry Accountability, Washington, DC, United States of America, ${ }^{6}$ Johns Hopkins Bloomberg School of Public Health, Health Behavior and Society, Baltimore, MD, United States of America. E-mail: drosen@ tobaccofreekids.org

Background and challenges to implementation: As civil society organizations (CSOs) work to overcome barriers in tobacco control, tobacco industry monitoring is a powerful mechanism to advance these efforts. "Big Tobacco, Tiny Targets" is a global project that seeks to expose the tobacco industry's varied tactics used to market tobacco products to kids. Data were collected in 21 countries, among them South Africa, Indonesia, Argentina and Nigeria. Over the evolution of this project, two Bloomberg Initiative partners, Campaign for Tobacco-Free Kids (CTFK) and Johns Hopkins University (JHU) developed a unique toolkit that is fully adaptable across partners, countries and organizations with differing levels of research capacity.

Intervention or response: CSOs identified and documented how the tobacco industry targets children around schools, with training and technical assistance from CTFK. Capacity building activities included CTFK-led in-person and virtual trainings, development and dissemination of an advocacy survey, campaign toolkits, media training and photography guide.

This model evolved as more rigorous research was needed in 15 countries. JHU developed customized observation checklists, conducted in-person trainings for advocates and data collectors to systematically record observations using a mobile data collection platform and walking protocol, and prepared technical reports.

The synergy of these two approaches implemented together serves as a feasible and transferable capacity building approach for enabling CSOs to develop the evidence base for future advocacy campaigns.

Results and lessons learnt: The launch of in-country Tiny Targets campaigns served to discredit the global cigarette industry while advancing policy objectives, including spurring vigorous enforcement of advertising bans in Jakarta, Indonesia, where the campaign was first piloted. The continued use of this platform to document industry tactics can further expose and discredit the industry among policymakers and the public and build greater support for tobacco control.

Conclusions and key recommendations: Integrated capacity building for both research and advocacy work bolsters CSO's capabilities to discredit the tobacco industry and overcome barriers in the policy realm.

Tob. Induc. Dis. 2018;16(Suppl 1):A446 DOI:10.18332/tid/84017

$11: 00-12: 30$

F0-360-2 Devolving tobacco control legislation to local governments for enhanced capacity in tobacco control advocacy and implementation C Awuor ${ }^{1}$, E Wanyonyi'

'International Institute for Legislative Affairs, Nairobi, Kenya. E-mail: cawuor@ilakenya.org

Background and challenges to implementation: Kenya inaugurated a new constitution in 2010 that created a devolved system of government comprising of a National Government and 47 County Governments. This altered the governance structure in all sectors including health, consequently affecting implementation of Tobacco Control (TC). Even though Kenya has Tobacco Control Act, 2007, certain provisions of the Act have to be implemented at the county level. However, the counties had limited awareness and capacity to perform the new roles in TC implementation. Furthermore, Tobacco Industry targeted counties as the next frontier for killing TC. The challenges necessitated technical support and capacity in TC for the counties to develop local legislation.

Intervention or response: We carried out desk assessment on TC legislation framework to identify gaps to be addressed. Two research studies were done in selected counties to inform advocacy activities. A model county TC Bill benchmarked with the Framework Convention on Tobacco Control (FCTC) was developed for the counties to adapt to their local situations. Key stakeholders were identified- county health executives, assembly members and Civil Society Organisations (CSOs) who were trained on TC legislation development and advocacy.

Results and lessons learnt: The model law presented to counties how effective TC legislation should be structured. Different approaches worked in different counties; either presenting the law to the County Assembly as a private member's bill or using county health executives to front the bill. Through the project, awareness on TC at the counties increased, capacities enhanced and interest on the topic generated that made the process demand-driven by county stakeholders. 
Conclusions and key recommendations: TC legislation established at the county level, local capacities on TC built and TC implementation devolved to the local level. Such an approach can be applied in other areas of public health, where local governments are given power to develop and enforce laws suiting their situations.

Tob. Induc. Dis. 2018;16(Suppl 1):A447

DOI: $10.18332 /$ tid/84571

\section{8:15-19:15}

LB-1312-2 To assess control measures for tobacco consumption in Zambia between 2014 and 2017. What are the gaps?

D Kalunga ${ }^{1}, \mathrm{~N} \mathrm{Mbulo}^{2}$, JC Zulu ${ }^{3}$, EM Sinkamba ${ }^{4}, \mathrm{M} \mathrm{Chisenga}^{5}$, D Kaponda ${ }^{6}$

${ }^{1}$ Kasama General Hospital, Laboratory, Kasama, Zambia, ${ }^{2}$ Kasama General Hospital, I CT, Kasama, Zambia, ${ }^{3}$ Kasama General Hospital, Clinical Care, Kasama, Zambia, ${ }^{4}$ Provincial Health Office, Clinical Care, Kasama, Zambia, ${ }^{5}$ skills training institute, Education, kasama, Zambia, ${ }^{6}$ Northern Biomedical Sciences College, Education, Kasama, Zambia. E-mail: k_chisenga@ hotmail.com

Background: Cigarette smoking is a leading cause of global morbidity and mortality, in developing countries interest in smoking prevalence has been growing since 1999. Many factors are known to influence smoking prevalence and trends in prevalence, from individual level factors such as education level, to country-level factors such as national economic development and implementation of tobacco control policies, therefore there is need to assess the control measures for tobacco smoking in Zambia between 2014 and 2017 and determine the gaps in policy implementation in tobacco consumption in Zambia.

Methods: This was a retrospective study using policy documents from central statistical office.

Results: The following gaps were identified in which there was no legislation: concerning Institutions and mechanisms to provide for a funding mechanism, Public education requiring mass public education campaigns to change public attitudes regarding tobacco and tobacco control, Advertising, promotion and sponsorship as a comprehensive ban involves a ban on all forms of direct and indirect advertisements, promotion and sponsorship, Price and tax measures as an effective means of reducing tobacco consumption, especially among young people. Monitoring- No known data or no recent data or data that are not both recent and representative (WHO report on the global tobacco epidemic, 2017).

Conclusions: The absence of legislation in these areas of institutions and mechanisms, public education, advertising, promotion and sponsorship and the price and tax measures have far reaching consequences in tobacco control in Zambia.

Tob. Induc. Dis. 2018;16(Suppl 1):A448

DOI:10.18332/tid/84702

\section{$18: 15-19: 15$}

LB-1315-2 Understanding tobacco control policy at the national level: bridging the gap between public policy and tobacco control advocacy

MC Willemsen ${ }^{1,2}$

${ }^{1}$ Maastricht University, Health Promotion, Maastricht, Netherlands, ${ }^{2}$ Trimbos Institute, Utrecht, Netherlands. E-mail: marc.willemsen@maastrichtuniversity.nl

Background: While some countries have advanced tobacco control policies, other countries struggle to adopt and implement
FCTC's measures. This presentation uncovers the main factors that explain such variations, taking insights from public policy and political science as a starting point for a case study.

Methods: A case study of tobacco control policy making in the Netherlands, covering the period from the 1960s until the present. The study consisted of a systematic search and analysis of documents and proceedings of parliamentary debates on tobacco policy, supplemented with 22 interviews with key informants from the government, health organisations, politicians, and the tobacco industry. In addition, documents from the Truth Tobacco Industry Documents database, pertaining to the influence of the tobacco industry on Dutch policy making, were analysed.

Results: The Dutch government started relatively late to regulate tobacco. The choices in tobacco control policy making at the national level and the tempo in which they are made are explained by the interaction of the five main elements of the tobacco control policy making process:

1. Relatively stable context factors (constitutional structures, 'rules of the policy making game', national cultural values)

2. Relatively dynamic context factors (regime changes, EU regulation and FCTC guidelines, changing social norms, public support)

3. Transfer of ideas (availability and interpretation of scientific evidence)

4. Pro and anti-tobacco control networks and coalitions (their organisational and lobby strength)

5. Agenda-setting (changes in problem definition, issue framing, media advocacy)

Conclusions: Despite worldwide convergence of tobacco control policies, accelerated by the ratification of the FCTC treaty by most nations, governments develop approaches to tobacco control in line with cultural values, ideological preferences and specific national institutional arrangements. There is no one-size-fits-all approach.

The findings will be published in an upcoming book, entitled: Tobacco Control Policy in the Netherlands: Between Economy, Public Health, and Ideology.

Tob. Induc. Dis. 2018;16(Suppl 1):A449

DOI:10.18332/tid/84704

\section{$18: 15-19: 15$}

\section{LB-1317-2 The landscape of tobacco control in} Africa

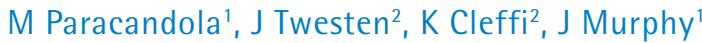

${ }^{1}$ National Cancer Institute, Rockville, MD, United States of America, ${ }^{2}$ The Bizzell Group, Lanham, MD, United States of America.E-mail: paramark@nih.gov

Background: Tobacco use prevalence in Africa is projected to rise over the next 15 years without stronger tobacco control measures. However, little research details the scope of tobacco control research being conducted in Africa. A systematic literature review was conducted to better understand the landscape of tobacco control efforts in Africa.

Methods: A literature search of tobacco research conducted in all African countries from 1996 to 2016 was performed in PubMed, Embase, and African Index Medicus. Published abstracts meeting the inclusion criteria of focusing on nicotine or tobacco product(s) and having been conducted in one or more African countries were selected for full coding and analysis. The authors coded on study characteristics such as type of research, tobacco product, and country. Three coders double-coded $5 \%$ of the articles reviewed to ensure agreement. 
Results: This review found 645 relevant articles, in French and English, representing 52 African countries. South Africa was the focus of the greatest proportion of these published tobacco control research articles (23\%), followed by Nigeria (17\%), Egypt (13\%), and Tunisia (12\%). Reporting the prevalence of tobacco use was the focus of $51 \%$ of these articles. Other areas of research included the potential determinants of tobacco use (28\%); knowledge, attitudes, and beliefs about tobacco products or policies (26\%); and biological consequences of tobacco use (25\%). Most studies (63\%) discussed cigarettes, and 15\% studied smokeless tobacco. Youth-targeted studies comprised 25\% of all research in Africa. Publications per year increased between 1996 and 2015, quadrupling in number by 2015 .

Conclusions: A comprehensive review of the literature provides a baseline understanding of the tobacco control landscape and the increased attention countries are showing to tobacco and tobacco control. This research may inform opportunities for further research and for strengthening networks and thereby the capacity to counteract the tobacco industry's advances in Africa. Tob. Induc. Dis. 2018;16(Suppl 1):A450 DOI:10.18332/tid/84758

\section{8:15-19:15}

LB-1319-2 The struggle towards FCTC-compliant legislation in Burundi: an inspiring advocacy experience

R Ciza ${ }^{1,2}$

${ }^{1}$ Health \& Healing Network Burundi-HHNB, Bujumbura, Burundi, ${ }^{2}$ University of the Witwatersrand, MSc Epidemiology \& Biostatistics/Implementation Science, Johannesburg, South Africa. E-mail: rogersciza@gmail.com

Background and challenges to implementation: Burundi ratified the FCTC in 2005 but 12 years later, the FCTC-related legislation is not adopted. Burundi became a target of tobacco industry which has deployed invasive strategies including unlawful bribes targeting key policymakers like the FCTC representative. The tobacco use among youth shifted from 5.4\% in 2008 (GYTS) to $19.3 \%$ in 2015 (WHO). MoH drafted a number of documents which have not been translated into FCTC-compliant TC laws. Following the 15th WCTOH in 2012, a youth-led CSO was created to implement conference recommendations and advocate for implementation of conventions ratified by the government.

Intervention or response: From 2014, a robust advocacy campaign to accelerate adoption of a national FCTC-compliant TC law and advocate for tax increase nationally was initiated. $\mathrm{MoH}$, National Assembly, CSOs, journalists, CTCA, CTFK are some of the stakeholders. For national partners, it is inclusive but strategically emphasizes more on key stakeholders and MPs, directly involved in law-making process.

Results and lessons learnt: Apart an inter-ministerial network, a strong partnership with the National Assembly is established and 38 MPs trained on FCTC-compliant laws. The partnership aims to ensure adoption of strong FCTC-compliant legalisation and high uptake from policymakers. MPs are members of the Health commission (17) and 3 from each of the 7 more commissions. They welcomed and accepted to defend in plenary amendments proposed by the juridical team in response to a weak law given to them by $\mathrm{MoH}$. Public and confidential lobbying sessions with other relevant influential stakeholders are organized in order to ensure all is done before 2017 ends.

Conclusions and key recommendations: The process to get a FCTC-compliant legislation is now going to the legislature and it is anticipated that law will be voted between 11th-15th December 2017; promulgation in the last week of December 2017. Advocacy for TC policies should use strategies that guarantee uptake and translation of TC law into practices.

Tob. Induc. Dis. 2018;16(Suppl 1):A451

DOI:10.18332/tid/84709

\section{$18: 15-19: 15$}

LB-1323-2 The role of mayor/regent alliance on tobacco control

S Rahma

'International Union Against Tuberculosis and Lung Disease, Jakarta Barat, Indonesia. E-mail: siti.rahma@theunion.org

Background and challenges to implementation: Indonesia is an archipelago country with 415 districts and 93 cities in 34 provinces with total population of 262 million people live in this country. Ministry of Health of Indonesia with the support of International Union Against Tuberculosis and Lungs Disease (The Union) has initiate Indonesia Mayor/Regent Alliance on tobacco control and non-communicable Disease. This alliance was led by a Regent from Kulonprogo district that has influenced and advocated other leaders to protect people from the harm of tobacco use through implementing smoke free policy.

Intervention or response: Gaining commitment from the local leader through one on one advocacy under the leadership of Regent Kulonprogo has been worked very effective. Furthermore, build the capacity and giving technical facilitation to the local team such as health district officer, law biro and civil society in developing policy and strategy of Smoke free.

Results and lessons learnt: Currently there are 292 smoke free regulations in Indonesia. Direct advocacy from other leader who has implemented smoke free policy is very effective. The experience regent has become a mentor for other regent or mayor in implementing smoke free policy. They able to learn the strategy based on best practices. Follow up the commitment from the Regent/Mayor and working with local team to develop the regulation. The alliance also has power in advocating national policy. The network of mayor/ regent alliance not only in Indonesia, Ministry of Health and The Union expand the network into Asia Pacific.

Conclusions and key recommendations: A strong leadership and commitment from the chair of Regent/Mayor Alliance on tobacco control and non-communicable disease has act as a mentor and influence other leader to implement smoke free policy. The alliance also has significant role in influence national policy especially in tobacco control.

Tob. Induc. Dis. 2018;16(Suppl 1):A452

DOI:10.18332/tid/84743

\subsection{Tobacco industry interference: New tactics and counter measures}

\section{$11: 00-12: 30$}

F0-356-2 The Philip Morris Genome Project: a guide to tracking alliances of the world's largest cigarette company

A Blum 1

'University of Alabama, Center for the Study of Tobacco and Society, Tuscaloosa, AL, United States of America. E-mail: ablum@ua.edu

Background: Beginning in the 1980s, tobacco control efforts shifted from school-based and clinic-based education about the dangers of smoking to direct confrontation of the tobacco 
industry. But the industry also evolved by developing innovative promotional campaigns, adopting new corporate identities, and forging alliances with other industries and charitable organizations.

Methods: To document the extent of the commercial, civic, political, academic, military, and cultural ties cultivated by the world's largest cigarette manufacturer, Philip Morris, a continuous, 35-year ethnographic analysis was conducted, involving daily monitoring of the global business press; monthly review of tobacco industry trade publications; scrutiny of annual company reports; and attendance at 20 shareholders' meetings, four international tobacco industry trade expositions, and over 200 sports, arts, and ethnic minority events sponsored by the company.

Results: 48 Philip Morris manufacturing facilities in 32 countries produce more than 800 billion cigarettes a year for 180 markets..A pioneer in sponsoring popular programs on the new medium of television in the 1950s; the company circumvented the 1971 ban on TV cigarette advertising by creating sporting events that were internationally televised. By acquiring beer and food companies In the 1980s, Philip Morris retained influence over the mass media covetous of advertising revenue. In 2002 the company changed its name to Altria to diminish the tobacco stigma. It makes the world's top cigarette brand, Marlboro, and holds $28 \%$ of the global market excluding China. The company has forged an extensive network of alliances with agricultural, marketing, chemical, pharmaceutical, financial, packaging, entertainment, shipping, and technology companies, as well as ties to hundreds of museums, arts organizations, universities, libraries, and charities combating problems such as domestic violence, hunger, pollution, illiteracy, and AIDS.

Conclusions: Greater recognition by health professionals and the public alike of the depth and breadth of the alliances in the Philip Morris genome is essential to progress in tobacco control.

Tob. Induc. Dis. 2018;16(Suppl 1):A453

DOI:10.18332/tid/84182

\section{$11: 00-12: 30$}

F0-358-2 The capsule trap - how tobacco companies communicate about flavor capsules in cigarette filters on the pack

C Washington ${ }^{1}$, K Smith ${ }^{1}$, K Welding ${ }^{1}$, J Cohen ${ }^{1}$

${ }^{1}$ Johns Hopkins Bloomberg School of Public Health - Institute for Global Tobacco Control, Health Behavior and Society, Baltimore, MD, United States of America.E-mail: cwashin8@jhu.edu

Background: Even in the context of ever more restrictive packaging requirements, tobacco companies are able to communicate about capsule technology on cigarette packs.

Methods: From 2013-2017 we purchased unique cigarette pack presentations using a systematic protocol in 14 low- and middleincome countries. Two independent coders examined the packaging and the stick from 4,510 cigarette packs for imagery and words marketing capsule activation. Additional content analysis was conducted to better describe how capsules are presented on packs.

Results: 142 cigarette packs were coded as having a capsule. We identified three features consistently used to communicate that the product offered a capsule: a ball and/or power button symbol (92\%); blue or green color for the capsule imagery (90\%); and suggestive capsule terms (e.g. "activate", "pop it”, "switch") (96\%). All capsule packs used at least two of these features and $78 \%$ used all three. We also identified that tobacco companies educated consumers and reinforced the capsule appeal through repeated exposure to a capsule image: tobacco companies used the pack panels, under the lid, foil, flip-top, cellophane, and the stick to market capsules. Most packs (88\%) displayed a capsule image on the front panel, and had a capsule image in two or more places on the packaging (85\%). All capsule packs used a capsule image on the stick itself. Companies frequently gave instruction on how to use the capsule: $61 \%$ of the packs had an image and/or statement related to activation.

Conclusions: Our analysis shows that strategic and consistent methods are used to communicate capsules to consumers. Industry reports have indicated that the market share for flavor capsule cigarettes is growing. It is important for public health regulators to recognize these marketing patterns; plain packaging would help to reduce the attractiveness of new cigarette features such as capsules.

Tob. Induc. Dis. 2018;16(Suppl 1):A454

DOI: $10.18332 /$ tid/84604

$11: 00-12: 30$

F0-359-2 Promoting compliance and enforcement of the tobacco advertising ban in China through legal actions and media advocacy by public interest lawyers

Z Wang ${ }^{1}, E \mathrm{Li}^{1}, \mathrm{X} \mathrm{Li}{ }^{2}, \mathrm{X} \mathrm{Yin}^{3}, \mathrm{X} \mathrm{Y \textrm {u } ^ { 2 }}$

${ }^{1}$ Beijing Impact Law Firm, Beijing, China, ${ }^{2}$ Campaign for TobaccoFree Kids, Beijing, China, ${ }^{3}$ Campaign for Tobacco-Free Kids, Washington, WA, United States of America. E-mail:wzy0594@ vip.sina.com

Background and challenges to implementation: The Amended Advertising Law took effect in September 2015. This national law prohibits tobacco advertising in mass media and in public places. It also bans any kind of tobacco advertising targeting minors. The Chinese tobacco industry has tried to circumvent the law by advertising tobacco products indirectly. The enforcement agency, State Administration for Industry and Commerce and its provincial and local bureaus, do not have enforcement guidelines, lack funds and incentives to enforce the law. Violations, particularly at points of sales, are common.

Intervention or response: Beijing Impact Law Firm (BILF) built a public Interest lawyers alliance for tobacco control covering 15 cities in China. Through training workshops, frequent communications and experience sharing on social media, BILF facilitated the communications among these lawyers and supported them in taking actions in their cities to promote compliance and enforcement of the Revised Advertising Law. These actions included monitoring, collecting evidence, taking legal actions such as litigation and filing complaints, and media advocacy. Results and lessons learnt: From 2013 to 2016, the network of public interest lawyers took 52 legal actions, 36 received official response, and 31 advertisements were removed as a result. Two successful examples have demonstrated that the monitoring and actions taken by lawyers resulted in enforcement and compliance. The media advocacy efforts during the legal actions resulted in wide media coverage, which effectively promoted public understanding of the law and the wrong-doing of the tobacco industry. Legal actions by public interest lawyers coupled with media advocacy can effectively promote enforcement and compliance. The alliance of public interest lawyers has served as a platform for the lawyers to exchange experience and share lessons learns.

Conclusions and key recommendations: Lawyers and lawyer networks are an important ally for tobacco control in China. More lawyers should be mobilized to monitor and improve implementation of tobacco control policies.

Tob. Induc. Dis. 2018;16(Suppl 1):A455

DOI:10.18332/tid/83911 


\section{$11: 00-12: 30$}

F0-361-2 Towards understanding the features of the waterpipe tobacco industry: findings from two consecutive visits to the International Hookah Fair M Jawad ${ }^{1}$, T Lotfi ${ }^{2}$, A Darzi ${ }^{3}$, N Singh ${ }^{4}$, B Hawkins ${ }^{5}$, R Nakkash ${ }^{2}$, $\mathrm{E} \mathrm{Akl}{ }^{3}$

${ }^{1}$ Imperial College London, Public Health Policy Evaluation Unit, London, United Kingdom, ${ }^{2}$ American University of Beirut, Faculty of Health Sciences, Beirut, Lebanon, ${ }^{3}$ American University of Beirut, Clinical Research Institute, Beirut, Lebanon, ${ }^{4}$ Brighton and Sussex Medical School, Brighton, United Kingdom, ${ }^{5}$ London School of Hygiene \&t Tropical Medicine, London, United Kingdom. E-mail: mohammed.jawad06@imperial.ac.uk

Background: Understanding the internal structures of the tobacco industry is vital in the development of effective tobacco policy. Currently, little is known about the waterpipe tobacco industry. We aimed to understand more about product development, marketing methods, compliance with legislation and features of the waterpipe tobacco industry.

Methods: We attended two annual waterpipe tobacco industry exhibitions held in Frankfurt, Germany, in 2014 and 2015. We collected information on the types of products on display, analysed marketing material, assessed compliance with health warning and labelling requirements, and questioned exhibitors to gather information about the features of the industry, including supply chains, cross-industry collaborations, customer loyalty, and lobby group membership.

Results: Despiteits presentation as a waterpipetradeexhibition, the fair was dominated by electronic cigarette (e-cigarette) companies and e-cigarette-like technology in waterpipe apparatuses. Waterpipe tobacco companies appeared distinct from those marketing e-cigarette-like waterpipe products. Marketing material across waterpipe tobacco products and accessories included claims of reduced harm (compared to cigarette smoking), safety, and quality. None of the 15 tobacco products collected complied with health warning and labelling requirements of FCTC Article 11 , and over half of charcoal products contained misleading descriptors of safety and quality. Discussions with exhibitors identified a globalised but decentralised and industry with growing ties to transnational tobacco companies. Most waterpipe tobacco companies are family-run businesses with an absence of strategic partnerships such as lobby group membership. Deliberate breaches of tobacco policy, such as the use of high levels of glycerine in the tobacco manufacturing process, were confirmed by company representatives.

Conclusions: Our observations add insight into key features of the waterpipe tobacco industry and can guide policy dialogue. We call for a waterpipe-specific policy framework to address unique aspects of the industry and the regulatory challenges it poses.

\section{Tob. Induc. Dis. 2018;16(Suppl 1):A456}

\section{DOI:10.18332/tid/84427}

\section{8:15-19:15}

LB-1313-2 Implementation of 85\% health warning labels in India: advocacy success factors

C Weiger', C Hoe' ${ }^{2}$ J Cohen

'Johns Hopkins Bloomberg School of Public Health, Health, Behavior and Society, Baltimore, MD, United States of America, ${ }^{2} J o h n s$ Hopkins Bloomberg School of Public Health, International Health, Baltimore, MD, United States of America. E-mail: cweiger2@jhmi.edu

Background: Tobacco control policies are critical to tackling the global tobacco epidemic. Unfortunately, little research has documented the factors that facilitate the implementation of tobacco control policies in low- and middle-income countries. In this study, we explored tactics and arguments used in India to advocate for the implementation of the 2014 rules increasing the size of pictorial health warning labels (HWLs) on tobacco products from $20 \%$ to $85 \%$ of the principle display area.

Methods: A case study approach was used. Key informant interviews $(\mathrm{N}=22)$ were conducted between June and September 2017 with individuals who were purposively selected based on their significant role in the fight for implementation of the 2014 HWL rules. Sixty-eight documents were purposively selected for review. Documents and interviews were analyzed using inductive and deductive coding in HyperRESEARCH, then triangulated.

Results: Litigation, media advocacy using personal anecdotes, sensitization of key political figures, scientific evidence of tobaccocaused disease, and lobbying emerged as the most impactful tactics. Collaboration between anti-tobacco organizations facilitated the presentation of a consistent message to the media and to policy makers, and also ensured that efforts were not duplicated and each organization could prioritize tactics that utilized their expertise. Arguments countering opponents' claims regarding economic impact of the law, anecdotes from individuals with tobacco-caused diseases, and scientific evidence were used to persuade key decision makers and judges to support HWL implementation.

Conclusions: The use of multiple, complementary tactics facilitated the success of the advocacy campaign to increase the size of HWLs in India. Arguing from both a scientific evidence base as well as a more humanizing standpoint using anecdotes through lobbying, litigation, and media advocacy was successful in the case of implementing larger HWLs in India. Advocates in other countries hoping to pass and implement tobacco control legislation may find these arguments and tactics helpful in their own countries.

Tob. Induc. Dis. 2018;16(Suppl 1):A457

DOI:10.18332/tid/84715

$18: 15-19: 15$

LB-1314-2 'Alternative fact' narrative influencing tobacco control: a case study of tobacco industry interference in print media

A Guruge'1, N Fernando

'Verité Research, Economics, Colombo, Sri Lanka. E-mail: fernandonilangika@gmail.com

Background: Sri Lanka saw a rise in misleading and false information on tobacco statistics in 2017. Vernacular press reported on government revenue decreasing, beedi smokers rising and illegal tobacco products reporting high growth without verifiable sources. Such articles were repeatedly published.

The rise of false information followed the government increasing cigarette taxes between $20-80 \%$ across five length categories. The increase in taxes was implemented to reconcile a gap created by over a decade of poor tax policies. While the increase was welcome by the public, media reported the tax increase would result in reducing government revenue and livelihood of tobacco farmers; neither of which were verified. It was evident that the tobacco industry which was concentrating on influencing taxation now was changing the narrative to change public opinion against tobacco control through misinformation.

This study aims to identify the loopholes in the Sri Lanka's laws and regulations (National Authority for Tobacco (NATA) Act 
and media Code of Conduct) and identify thematic areas that are targeted by the tobacco industry in spreading misinformation.

Methods: The period of analysis was September 2016 to October 2017 and all articles related to tobacco was firstly checked against the laws and media ethic guidelines. It then followed a case study method to thematically group the key narrative of selected articles. Results: Findings reveal the articles violated three codes under the media Code of Conduct and vague description on 'tobacco advertisement' as an article in the NATA Act caused confusion on its legal standing. The case study also revealed four thematic areas with false information.

Conclusions: The study draws attention to tobacco industry interference in using media to change the narrative on tobacco control. It also provides highlights the significance of a national tobacco data platform and engaging with journalists to combat the tobacco epidemic.

\section{Tob. Induc. Dis. 2018;16(Suppl 1):A458}

DOI: $10.18332 /$ tid/84741

\section{$18: 15-19: 15$}

LB-1318-2 Countering tobacco industry interference: role of civil society

TP Timilsina

${ }^{1}$ Action Nepal (Nepal), Admin/Finance, Kathmandu, Nepal. E-mail: ttulprasad@gmail.com

Background and challenges to implementation: Tobacco is one of the major public health challenge in Nepal. It kills more than 15000 people each year. To combat this epidemic, Nepal has adopted a comprehensive tobacco control law in 2011 with provision of $90 \%$ pictorial health warning(PHW) in directives. Enforcement of $90 \%$ PHW was not optimum. Tobacco Industry (TI) interference was at the highest level in decision making. There was sustained lobbying from US Chamber of Commerce and Phillip Morris International.TI litigation was at the Supreme Court of Nepal.

Intervention or response: To implement $90 \%$ PHW we carried out different techniques such as i) diagnose and document tobacco industry interference with policy maker ii) organize round table discussion iii) one to one meeting with government official iv) conduct a survey on effectiveness of the PHW. More importantly, we, Action Nepal, had registered the case in Supreme Court against the government for immediate enforcement of $90 \%$ PHW. We seriously urged to Supreme Court against the tobacco interference and its implication on health of general public. Eventually, Supreme Court verdict was on the favor of Action Nepal and instructed government to enforce the $90 \%$ PHW on tobacco products.

Results and lessons learnt: Conducting the series of workshop and policy meeting with enforcement agency, we convinced them to implement the PHW as per the Tobacco Control Act and directives. It was very challenging for us to counter the Tobacco Industry's arguments. Making a strong partnership with government,enforcement agencies includes Ministry of Health,Ministry of Industry and Nepal police and Media, we succeed on the project Implementation of 90\% PHW in Nepal.

Conclusions and key recommendations: Civil society can make a solid team of government official, doctors,media and public to counter the tobacco industry interference and so such team should be sustained and more dynamic in days ahead to implement such projects for tobacco free society.

Tob. Induc. Dis. 2018;16(Suppl 1):A459

DOI:10.18332/tid/84699
18:15-19:15

LB-1320-2 Possible consequences of relatively decreasing cigarette price in China from 1999 to 2015

W Jijiang ${ }^{1}$

${ }^{1}$ Chinese Center for Disease Control and Prevention, Beijing, China. E-mail: jjwang_cn@yahoo.com

Background: Cigarette price is an important factor affecting smokers' behavior. While raising price is considered to be a most cost-effective approach to confront the tobacco epidemic, a pricing stratagem could also be used as a weapon to interfere tobacco control efforts by the tobacco industry. The study examined the change of cigarette price from 1999 to 2015 in China, where inhabits a smoking population of more than 300 million, to explore its possible effects on population smoking behavior.

Methods: National Consumption Price Index (CPI), Tobacco Product Consumption Price Index (TCPI), tobacco consumption data were collected from China Statistical Yearbooks and China Tobacco Yearbooks. Tobacco use and cost data were acquired from two national tobacco surveys in 2010 and 2015.

Results: The TCPI in China increased 5.6\% from 1999 to 2015, while at the same time the national CPI increased $42.3 \%$, which meant the price for cigarettes relatively decreased $25.8 \%$ comparing to other commodities. Meanwhile, the annual number of cigarettes sold in China increased from 1.45 trillion to 2.49 trillion, raising $71.7 \%$ since 1999. Though the price of cigarettes changed little, the average cost per pack of cigarettes bought by smokers increased $42.9 \%$ (from RMB $¥ 7.0$ to $¥ 10.0$ ) in urban area and $59.2 \%$ (from RMB $¥ 4.9$ to $¥ 7.8$ ) in rural area from 2010 to 2015 , which was a result of shift to more expensive cigarette brands, regarding to the dramatic increase in the average resident income (climbing $93.0 \%$ in rural, and $63.2 \%$ in urban during 2010-2015 in China).

Conclusions: The cigarette price in China barely changed during a sixteen year period from 1999 to 2015, made cigarettes more and more affordable in China. Combining with the increase in income, it contributed to the huge increase in tobacco consumption and brand shift by smokers in China.

Tob. Induc. Dis. 2018;16(Suppl 1):A460

DOI: $10.18332 /$ tid/84710

\section{8:15-19:15}

LB-1322-2 Understanding tobacco industry tactics: a content analysis of newspaper coverage of tobacco issues in Pakistan

SA Rana ${ }^{1,2}, \mathrm{~N}_{\text {Iqbal }}{ }^{1}$

'TheNetwork for Consumer Protection in Pakistan, Islamabad, Pakistan, ${ }^{2}$ Health Services Academy, Public Health, Islamabad, Pakistan. E-mail: sanaz.rana.1@gmail.com

Background: Each year more than 110,000 people die because of tobacco use in Pakistan. There are approximately 23.6 million (age 15-45 years) tobacco users in Pakistan. GYTS-2014 revealed that $13.3 \%$ of boys and $6.6 \%$ of girls (age $13-15$ years) used tobacco products. Pakistan is the signatory of FCTC since 2004. Although efforts are being made but $\mathrm{TI}$ is continuously using its tactics targeting youth. The aim of this study is to examine the claims by tobacco industry as well as the counter-arguments by tobacco control advocates in the year 2016-17 in Pakistan to show patterns of arguments.

Methods: The present study took a qualitative approach to examine the articles about tobacco (cigarettes) in English Pakistan newspapers from October, 2016, to October, 2017. All tobacco related articles were chosen. The selected seventy articles were 
likely to represent most of the tobacco related news coverage in top five national newspapers during this period. Content analysis was done to understand the main messages conveyed by the articles and different themes were generated.

Results: Three major players were identified which included the government, civil society and the tobacco industry. Main stories generated were either focused on Tobacco Taxation, Graphical Health Warnings or Tobacco Control in general. The analysis showed that to reduce levies on cigarettes, TI worked in two phases. Firstly it created a smokescreen that counterfeit cigarettes have taken over the legitimate cigarette market. Secondly, TI related it to the loss of government revenues.

Conclusions: The findings indicate that tobacco Industry revolves around the similar patterns of tactics throughout the world taking a step by step approach. Tobacco advocates should study cases of other countries as well to better understand TI tactics and counter ways.

Tob. Induc. Dis. 2018;16(Suppl 1):A461

DOI:10.18332/tid/84712

\subsection{Pricing, Taxation and countering illicit trade: Innovations and reform strategies}

\section{8:15-19:15}

LB-1308-3 An assessment of metrics for progress in tobacco taxation in the WHO Global Tobacco Control Reports

R Cherukupalli

'Johns Hopkins University, New York, NY, United States of America. E-mail: rcheruku@jhu.edu

Background: Measuring global progress in taxation (R) is an objective, tractable exercise but is challenging in comparison to progress on other measures of the WHO MPOWER package. Countries are grouped into four groups based on the share of total taxes in cigarette price: $0-25 \%, 25-50 \%, 50-75 \%$ and $75-100 \%$ ). One challenge in this approach is that excise taxes are subsumed within total taxes. A second challenge is to explain whether progress in tax shares is driven by actual tax policy improvement. Methods: A ranking of countries by cigarette excise tax share was performed for each of the reports between 2008 and 2016 and this was compared with the published WHO rankings of total tax shares. Data on report-by-report changes in tax shares was used to compute transition probabilities for the published WHO $\mathrm{R}$ groupings to examine the likelihood of tax shares increasing or declining, both unconditionally and conditional to WHO regions and income-groupings.

Results: Changes in country rankings of excise tax shares closely track changes in total tax shares ranking, providing credence to the use of the current tax share measure. Unlike the "POWE" groupings where retrogression in tobacco control progress is uncommon, countries' tobacco tax shares are nearly as likely to fall as they are to rise. Between 2014 and 2016, total tax shares rose, was unchanged, and fell, in 96,24 and 75 countries respectively. When transition probabilities are examined, however, countries are significantly likely to stay within their tax grouping from year to year, with movement across groups driven by substantive tax policy changes.

Conclusions: Total tax shares and groupings in the WHO GTCR largely capture excise tax policy differences over time. In 2016, 36 countries had total tax share between $65 \%$ and $75 \%$ in 2016 , pointing to many countries being at the threshold of making the highest R category.

Tob. Induc. Dis. 2018;16(Suppl 1):A462

DOI:10.18332/tid/84763
18:15-19:15

LB-1337-3 Spatial and economic proximity of cigarette sales to school children in Mongolia

P Singh1, B Purvedorj², E Suh'1, J Job ${ }^{1}$, J Banta ${ }^{3}$, P Enkhtuya ${ }^{4}$

${ }^{1}$ Loma Linda University, Center for Health Research, Loma Linda, CA, United States of America, ${ }^{2}$ Mongolia National University of Medical Sciences, School of Public Health, Ulaanbatar, Mongolia, ${ }^{3}$ Loma Linda University, Center for Leadership and Health Systems, Loma Linda, CA, United States of America, ${ }^{4}$ Public Health Institute, Ulaanbatar, Mongolia.E-mail: psingh@ Ilu.edu

Background: The Western Pacific Region has the highest rate of cigarette smoking in the world. In this region, Mongolia has ratified the WHO FCTC treaty and, as part of treaty implementation, monitored school children using the 2014 Global Youth Tobacco Survey (GYTS). Our objective is to examine spatial and economic factors associated with cigarette use in school children of Mongolia. Methods: The 2014 GYTS is a cross-sectional, stratified, multistage cluster survey of 7,298 school children (ages 13-15) from 63 schools sampled from all urban and rural regions of Mongolia. We conducted logistic regression modelling to examine whether spatial (proximity of cigarette sales to schools), economic (pocket money available to school children), and other environmental/ contextual factors were predictors of cigarette use in school children.

Results: We found that only $11 \%$ of rural students and $23 \%$ of urban students had adequate amounts of pocket money to purchase a cigarette pack (average price of the pack: 1.8 USD) and have money remaining for the week. Our model indicated that those students given $>2$ USD of weekly pocket money were two times more likely (OR=2.2 95\% CI $[1.20,4.21])$ to be current cigarette users than those with no weekly pocket money. Current pricing of the cigarette pack may not be preventive in students since we found that, among those who smoked cigarettes, $37.5 \%$ smoked single cigarettes, and that this increased to $47.5 \%$ when cigarette vendors were near the schools. Our models indicated that the presence of cigarette vendors near schools increased the likelihood of cigarette smoking $(\mathrm{OR}=1.37 ; 95 \% \mathrm{CI}$ [1.03-1.81]) and nearly doubled the likelihood of single cigarette smoking $(\mathrm{OR}=1.76 ; 95 \% \mathrm{CI}$ [1.09-2.83]).

Conclusions: Our findings from Mongolia indicate that despite the higher pricing of cigarette packs (relative to the region), illicit sales of single cigarettes are targeting school children near their schools.

Tob. Induc. Dis. 2018;16(Suppl 1):A463

DOI:10.18332/tid/84735

\subsection{Advances in protection from tobacco pollution, second- and third-hand smoke}

$18: 15-19: 15$

LB-1306-3 A community led tobacco free city

ND Galmangoda Guruge', MN Perera², SK Kithalawaarachi', SH Piyasumana ${ }^{4}$

'Faculty of Applies Sciences, Rajarata Universirty of Sri Lanka, Health Promotion, Anuradapura, Sri Lanka, ${ }^{2}$ University of Kelaniya, Department of Public Health, Faculty of Medicine, Kalaniya, Sri Lanka, ${ }^{3}$ Presidential Task Force on Drug Prevention, Sri Lanka, Colombo, Sri Lanka, ${ }^{4}$ Medical Officer of Health, Office, Wellawaya, Sri Lanka. E-mail: dumindaguruge@gmail.com

Background and challenges to implementation: Framework Convention for Tobacco Control advocates for smoke free cities 
as an effective strategy. A 'tobacco-free city' advocated by this project is not only a city that protects exposure to tobacco smoke, but has reduced demand and availability of cigarettes as well.

Aim was to evaluate the effectiveness and challenges of reducing availability of cigarettes in a semi-urban area in Sri Lanka by a community led process.

Intervention or response: A community group led the process. Collective actions such as discussions at different community settings, informal campaigns in public transport, poster campaigns and protests against industrial influence were implemented to improve awareness on effects on health and economy and change norms and attitudes. Evaluation was conducted using participatory mechanisms. Pre and post assessments and the comparison with a control area to assess long term outcomes used mixed methods.

Results and lessons learnt: In the pre-test, $91 \%$ of shops were selling cigarettes. In one year, daily cigarette sales reduced by 10,415 sticks (86\%). Percentage of shops selling cigarettes reduced from $91 \%$ to $25 \%$ in one year. Among the community, a majority showed a good level of knowledge on health effects and legislations and negative attitudes related to smoking after a one year. Of the participants $63.0 \%$ : $95 \%$ CI $(57.2,68.8)$ stated they perceived smoking as a "stupid and a meaningless habit". The prevalence of smoking $(9.09 \%$ \& $28.0 \%)$ and exposure to tobacco smoke ( $11.7 \% \& 65.0 \%)$ was low compared to the control area in 18 months $(\mathrm{p}<0.001)$.

Conclusions and key recommendations: Community led initiatives based on Health Promotion approach are effective in establishing 'Tobacco-free' cities. The community empowerment processes linked with such approaches can reduce the exposure to passive smoking, generating "smoke free cities" as well. Continuous vigilance at ground level and supportive actions from the national level are needed to sustain the results and enhance long term outcomes.

Tob. Induc. Dis. 2018;16(Suppl 1):A464

DOI:10.18332/tid/84694

\section{$18: 15-19: 15$}

LB-1307-3 Formative research to support the transition of multi-unit rental housing to smokefree

A Klassen ${ }^{1,2}$, N Lee $^{3}$, J Lopez ${ }^{1}$, M Tefferi $^{4}$, R Coffman $^{4}$, C Bettigole ${ }^{4}$ ${ }^{1}$ Drexel University Dornsife School of Public Health, Community Health and Prevention, Philadelphia, PA, United States of America, ${ }^{2}$ Johns Hopkins Bloomberg School of Public Health, Health, Behavior and Society, Baltimore, MD, United States of America, ${ }^{3}$ Drexel University Dornsife School of Public Health, Epidemiology and Biostatistics, Philadelphia, PA, United States of America, ${ }^{4}$ Philadelphia Department of Public Health, Division of Chronic Disease Prevention, Philadelphia, PA, United States of America.E-mail: ack57@drexel.edu

Background: Rented homes house $37 \%$ of US households, and $65 \%$ of those are multi-unit, translating into $>60$ million Americans who rely on housing policies to protect their health from environmental exposures, including secondhand smoke (SHS) incursion from adjoining units. As tobacco addiction becomes concentrated among low income, ethnic and racial minority, chronically ill, and other vulnerable populations, who are more likely to reside in multi-unit housing (MUH), controlling MUH SHS is a key tobacco control disparities priority. Although the US Department of Housing and Urban Development (HUD) and many municipalities promote policies to increase the proportion of MUH that is smoke-free, less is known about how to support landlords with smaller portfolios serving low-income tenants.

Methods: In Philadelphia, which has both the highest tobacco use and poverty rates of the 10 largest US cities, we conducted an online needs assessment survey in 2017 of members of the City's largest landlord association. Survey items measured current smoke-free portfolio, attitudes and experiences with tenants' tobacco use, and knowledge of Philadelphia's 2016 mandatory smoking disclosure law for MUH leases. Respondents were also offered technical assistance on transitioning to, or maintaining smoke-free rentals.

Results: Of 226 respondents, $67 \%$ were unfamiliar with Philadelphia's disclosure ordinance, $40 \%$ allowed smoking in all or some units, and only $3 \%$ believed it was illegal to prohibit smoking in leases, but $34 \%$ were unsure, and only $61 \%$ knew it was legal. Respondents reported large (41\%) or some (40\%) demand for smoke-free properties, and most recognized benefits, including lower costs, inter-resident tension, and health risks. Drawbacks included resources required to transition properties and for enforcement. Over half of landlords with and without current smoke-free portfolios requested technical assistance.

Conclusions: Results suggest that MUH initiatives such as disclosure ordinances could be enhanced by technical assistance to increase landlord adoption of smoke-free MUH.

\section{Tob. Induc. Dis. 2018;16(Suppl 1):A465 DOI:10.18332/tid/84711}

\section{8:15-19:15}

LB-1310-3 Empowering decision makers in Latin America: potential impact of three interventions for tobacco control

A Alcaraz¹, MB Rodríguez¹, A Hernández Vásquez¹, N Soto', S García Martí1, A Bardach ${ }^{1}$, A Palacios ${ }^{1}$, A Pichón-Riviere ${ }^{1}$ Institute for Clinical Effectiveness and Health Policy, Buenos Aires, Argentina. E-mail: aalcaraz@iecs.org.ar

Background: The burden of disease attributable to tobacco use in Latin America is very high. The objective of this study was to evaluate the potential impact of implementing cigarette plain packaging (PP), 100\% smoke-free environments (SFE) and complete ban on advertising, promotion and sponsorship of tobacco (BAPS) in Argentina, Bolivia, Brazil, Chile, Colombia, Mexico and Peru.

Methods: A mathematical model was used to estimate the chances people have to get sick or die because of 17 smoking-related conditions. A systematic review was carried out to identify the epidemiological and cost data relevant to each country, as well as the effectiveness of the interventions. The model considered each country's legislation and level of compliance for each intervention. Then, we modeled the expected impact on health and costs of different potential scenarios for these interventions.

Results: We estimated the following benefits for all seven countries: PP could avoid 204073 deaths, 212867 cardiovascular events, 26456 cancer diagnoses, 99784 COPD cases, and 23739 USD on direct medical costs in the next ten years. $100 \%$ SFE could lead to avoid 150361 deaths, 247171 cardiovascular events, 77400 cancers and 311129 COPD cases, and 15413 USD on direct medical costs in the next ten years. $100 \%$ BAPS could save 86911 deaths, 327390 cardiovascular events, 38883 cancers, 142104 COPD cases, and 10505 USD on direct medical cost to the health system in the next ten years. Different scenarios were modeled for each country. An example-the case for Colombia- is shown in the table. 
Conclusions: The potential impact of fully implementing PP, $100 \%$ SFE and BAPS could avoid a large burden of disease and costs for the health systems in Latin America. This information could be useful for decision makers to promote these policies.
Tob. Induc. Dis. 2018;16(Suppl 1):A466

DOI:10.18332/tid/84748

[Potential impact of different scenarios, Colombia]

\begin{tabular}{|c|c|c|c|c|c|c|c|}
\hline COLOVIBIA & $\begin{array}{l}\text { Vedium } \\
\text { warnings }\end{array}$ & $\begin{array}{l}\text { Strong } \\
\text { warrnings }\end{array}$ & $\begin{array}{l}\text { Plain } \\
\text { packaging }\end{array}$ & $\begin{array}{l}100 \% \text { SFE } \\
(\text { LOC: } 50 \%)\end{array}$ & $\begin{array}{l}100 \% \text { SFE } \\
(\mathrm{LOC}: 100 \%)\end{array}$ & $\begin{array}{l}\text { Complete } \\
\text { BAPS } \\
\left(\text { LoC: } 75^{\circ}\right)\end{array}$ & $\begin{array}{l}\text { Complete } \\
\text { BAPS } \\
\text { (LoC: } 100 \%)\end{array}$ \\
\hline Avoided deaths & 3465 & 7038 & 24086 & 11035 & 18151 & 7797 & 10584 \\
\hline Avoided events & 22775 & 46256 & 158300 & 72524 & 119293 & 60961 & 69561 \\
\hline Avoided costs (USD millions) & 196 & 399 & 1365 & 625 & 1029 & 442 & 600 \\
\hline
\end{tabular}

USD, United States dollars. 1 USD = 2743,39 Colombian pesos (December, 2015). SFE, Smoke-Free Environment. BAPS, Ban on Advertising, Promotion and Sponsorship of tobacco. LoC, Level of Compliance.

\section{8:15-19:15}

LB-1311-3 The relationships between secondhand smoke exposure and blood cadmium, lead and mercury concentration in the Korean population: findings from the 2008-2012 KNHANES

Y-A Lee ${ }^{1}$, S-W Song

${ }^{1}$ The Catholic University of Korea, St. Vincent's Hospital, Family Medicine, Suwon, Korea, Republic of. E-mail: sswkoj@unitel. co.kr

Background: We examined relationships between secondhand smoke exposure and blood cadmium, lead and mercury concentration among South Korean adults aged $\geq 19$ years.

Methods: The analysis used data from the Korean National Health and Nutrition Examination Survey (KNHANES), a crosssectional survey of Korean civilians, conducted from 2008 to 2012. In this study, a sample of 5,304 participants was analysed. Results: As urinary cotinine levels increased, the adjusted mean level of blood mercury increased. The adjusted mean level of blood lead was higher in the people who were exposed to secondhand smoke (SHS) than in those who were not. The duration of SHS exposure at work and home, was related to blood cadmium concentration ( $p=0.05$ for at work; $p=0.05$ for at home). Furthermore, as the duration of SHS exposure at work increased, the adjusted mean levels of blood lead and mercury increased.

Conclusions: This study showed that there were significant associations between SHS exposure and blood cadmium levels at work and home. Therefore, social efforts to reduce the SHS exposure at work and home are needed to promote a healthier environment.

Tob. Induc. Dis. 2018;16(Suppl 1):A467

DOI:10.18332/tid/84727

\section{8:15-19:15}

LB-1338-3 Leaders for little lungs - children as advocates for clean air in the Western Cape province in South Africa

A Westwood ${ }^{1,2}$

'Department of Health, Western Cape Province, Child Health, Cape Town, South Africa, ${ }^{2}$ University of Cape Town, Paediatrics, Cape Town, South Africa. E-mail: anthony.westwood@ westerncape.gov.za

Background and challenges to implementation: Passive smoking affects fetal and childhood lung growth and development. It is also responsible for aggravation of symptoms in many childhood respiratory disorders. High levels of adult smoking and associated passive smoking among pregnant women, fetuses and children persist in the Western Cape province in South Africa. Efforts to reduce the prevalence of smoking have had limited effect.

Intervention or response: Empowering children to be advocates for their own lung health, especially in relation to reducing exposure to tobacco smoke was seen as a novel approach to altering society's attitude to active and passive smoking. This led us to conceptualise the 'Leaders for Little Lungs' programme with the slogan 'Look after Little Lungs'.

Results and lessons learnt: The programme is to have three elements: a booklet of facts for children on lungs and the deleterious effects of smoking; educational group work at community and health service levels for children to become knowledgeable advocates; and campaign materials such as T-shirts, pamphlets and stickers. The development of T-shirt for health workers and children has produced positive responses from health workers, students, parents and children. The booklet is in the development and testing phase. Roll out of the programme is due to occur in the first winter on 2018, coinciding with the annual surge in pneumonia in the Western Cape.

Conclusions and key recommendations: This novel programme has the potential to change attitudes to passive and active smoking in high-prevalence communities, and to reduce smoking-associated lung diseases among children and adults in the Western Cape province.

Tob. Induc. Dis. 2018;16(Suppl 1):A468

DOI: $10.18332 /$ tid/84703

\subsection{Mind the gaps :loopholes in the Ban on Sponsorships, Advertising and Promotions}

$14: 45-16: 15$

F0-373-3 (Mis)perceptions related to Electronic Nicotine Delivery Systems (ENDS) and hookah: making a case for policy strengthening through a multi-stakeholder qualitative study from New Delhi, India

R Shrivastav ${ }^{1}$, P Kathuria ${ }^{1}, M$ Arora ${ }^{1,2}$, VG Munish ${ }^{3}$, P Sinha ${ }^{3}$, FT Tullu ${ }^{3}$

${ }^{1}$ HRIDAY, New Delhi, India, 2 Public Health Foundation of India, Gurugram, India, ${ }^{3}$ World Health Organization Country Office for India, New Delhi, India. E-mail: radhika@hriday-shan.org Background: Electronic Nicotine Delivery Systems (ENDS) e-cigarettes and e-hookahs and traditional products like hookah, revamped in attractive forms, have garnered wide popularity in India, particularly among young people. Without a national 
legislation to regulate these products, the tobacco industry has spearheaded surreptitious promotional campaigns to market these products, exploiting gullible consumers. This qualitative study aimed to gauge perceptions of multiple stakeholders (students, parents and teachers) on ENDS and hookah, underscoring the urgent need for regulating these products, which are circumventing the current inadequate regulatory framework.

Methods: Eleven Focus Group Discussions (FGDs) were conducted with 102 respondents [school students, 12-15 years (19), teachers (15), parents (37) and college students, 18-25 years (31)], using a standardised FGD guide. The intent was to gauge perceptions/knowledge about use of and access to ENDS and hookah, among youth. Thematic issues explored included: information and knowledge gaps around constituents/harmful effects; accessibility and affordability; characteristics; mis-leading claims ['non-tobacco'/herbal (hookah)]; marketing/advertising; possible regulatory mechanisms etc. FGDs were audio-recorded and transcribed for qualitative analysis using ATLAS.Ti (version 6.2).

Results: Gross mis-information and myths existed about ENDS and regular hookahs, being non-tobacco or non-nicotine products, which are safe or less hazardous/addictive than regular tobacco products, particularly cigarettes. High popularity, social acceptance, convenient availability and easy affordability were reported, particularly by students. College students were most updated about recent trends, followed by school students, teachers and parents (least aware). All schools visited, reported cases of ENDS confiscation/search. Widespread exposure to promotional campaigns was reported, maximally on e-commerce sites. All respondents stressed on the need of a comprehensive and nation-wide regulation on ENDS and hookah, similar to other tobacco products.

Conclusions: There is an urgent need for progressive regulation/ prohibition on ENDS and hookah under the Indian tobacco control law/other applicable legislation, for strengthening the implementation of the Framework Convention on Tobacco Control (FCTC), in India.

Tob. Induc. Dis. 2018;16(Suppl 1):A469

DOI:10.18332/tid/84580

\section{6:30-18:00}

RF-1246-3 Regulating promotion of tobacco through films - a pioneering endeavor of India

R Singh ${ }^{1}$, AK Pandey ${ }^{2}$

${ }^{1}$ Ministry of Health and Family Welfare, Government of India, National Tobacco Control Cell, Delhi, India, ${ }^{2}$ International Union Against Tuberculosis and Lung Disease, Tobacco Control, New Delhi, India.E-mail:singhranjit617@gmail.com

Background and challenges to implementation: Cinema influences children of young impressionable mind. A LANCET publication revealed that viewing smoking scenes in movies promotes smoking initiation among adolescent. The findings indicated that the effect of exposure to movie smoking was stronger on the adolescent than parental smoking.

The Government of India and the WHO supported study on "tobacco in movies and impact on youth" also revealed that after the ban on all forms of Tobacco Advertisement Promotion and Sponsorship in 2003, by India there was nearly 50\% increase in smoking scene by lead character and more than $50 \%$ increase in tobacco products/brands placement.

Considering the influence of movies on youths in India, the Government of India in 2005, banned the depiction tobacco usage in Films.

Subsequently, The Tobacco-free Film Rules were legally challenged by the film industry for alleged violation of freedom of speech and expression. There was also difference of opinion between the Health Ministry and Information \& Broadcasting Ministry. This resulted in deferment in implementation of Rules. Intervention or response: The two Ministries through interministerial consultations reached on an agreement have an effective law to curb TAPS through films. The Government of India also responded strongly while defending the new warnings in the court cases.

Finally with the intervention of the Supreme Court of India the Film Rules in its modified form were implemented from 2 nd October 2012.

Results and lessons learnt: The Film rules mandate that to depict tobacco product or its usage in any film, an editorial justification will be given by the producer explaining the necessity of such scenes.

Also a anti-tobacco disclaimer and health spots in the beginning and middle of the films and display of anti-tobacco health message during such scenes have been made mandatory.

Conclusions and key recommendations: Through strong political commitment and coordinated advocacy and legal efforts, its is possible to restrict depiction of tobacco use in films.

Tob. Induc. Dis. 2018;16(Suppl 1):A470

DOI:10.18332/tid/84452

$16: 30-18: 00$

RF-1247-3 Exposure of smoking scenes in popular movies among Koreans from 2006-2015

S Lee ${ }^{1}$, J Kim ${ }^{1}$, Y-J Paek ${ }^{2}$

${ }^{1}$ Korean Association on Smoking or Health, Seoul, Korea, Republic of, ${ }^{2}$ Hallym University, College of Medicine, Seoul, Korea, Republic of. E-mail: wwwvince77@gmail.com

Background: To identify a trend of smoking scene exposures in popular movies among the Koreans and to investigate where and when the smoking scenes were appeared.

Methods: One hundred commercially successful movies from 2006 to 2015 were selected from Korea Film Council's Database. Ten monitoring teams watched the selected movies to collect data following the structured questionnaire which was developed by the authors.

Results: There were 5 G, 47 PG-13, $37 \mathrm{R}$ and $11 \mathrm{NC}-17$ rated movies among the selected movies. The total smoking scene exposures in 100 movies during the past 10 years was 618 . Almost $45 \%$ (276 scenes) of the smoking scenes was exposed in the $\mathrm{R}$ rated movies, while $20.6 \%$ (127) was exposed in the PG-13 rated movies. There were on average 2.7 smoking scene exposures in a PG-13 rated movie and 7.5 smoking scene exposures in $\mathrm{R}$ rated movies. Smoking scene exposures in the movies that youth could watch (PG-13 and $\mathrm{R}$ rated movies) has increased almost $50 \%$ from 42 times in 2006 to 64 times in 2015. One third of the smoking scenes (123 out of 372 ) was shown without any relevance to the storyline of the movie, while almost $17 \%$ of the smoking scenes were when actors faced stress, death of a loved one or divorce. Worry, violation, break time or drinking alcohol were other situations where smoking scenes were shown. Thirtyfive percent of smoking scenes (135 out of 379) showed actors smoking in non-smoking areas, including schools, restaurants or public places, while $15.6 \%$ and $8.7 \%$ of the smoking scenes took place in the home and street, respectively.

Conclusions: Global and domestic efforts to achieve de- 
normalization of tobacco use in real life can be undermined by smoking scene exposures in movies. Monitoring and protecting youths from smoking scenes in PG-13 and R rated movies are needed.

\section{Tob. Induc. Dis. 2018;16(Suppl 1):A471 \\ DOI: $10.18332 /$ tid $/ 83873$}

\section{6:30-18:00}

RF-1248-3 Exposure to tobacco in video games and smoking in Argentina

R Mejia ${ }^{1,2}$, A Perez $^{3}, N_{\text {Cabrera }}{ }^{1}$, S Forsyth ${ }^{4}$, JD Sargent ${ }^{5}$, JFR Thrashe $^{6}$

${ }^{1}$ Centro de Estudios de Estado y Sociedad (CEDES), Buenos Aires, Argentina, ${ }^{2}$ Universidad de Buenos Aires, Hospital de Clinicas, Buenos Aires, Argentina, ${ }^{3}$ Universidad de Buenos Aires, Facultad de Ciencias Exactas y Naturales, Buenos Aires, Argentina, ${ }^{4}$ University of aplied California, San Francisco, CA, United States of America, ${ }^{5}$ Department of Pediatrics, Geisel School of Medicine at Dartmouth, Hanover, NH, United States of America, ${ }^{6}$ University of South Carolina, Columbia, SC, United States of America. E-mail: raulmejia@cedes.org

Background: Our objective was to assess whether exposure to tobacco in videogames is associated with smoking in adolescents from Argentina.

Methods: We performed a cross-sectional study with 3,646 s middle school students from public and private schools in Argentina. Tobacco content in video games was estimated using previously validated methods and adolescent's tobacco exposure was assessed by multiplying tobacco content by the hours per day played (range 0 to 5). Outcome measure was current smoking among video game players. Logistic regression models with random intercepts for school were used to regress current smoking on tobacco exposure and study variables multivariate models were adjusted for age, sex, parental education, rebelliouness, sensation seeking, parenting style of mother and father, tecnophilia and rules about the use of video games.

Results: 3113 students responded to the survey (response rate 85.4\%), 1802 boys and 1312 girls; 1658 (92\%) boys and 737 $(56.2 \%)$ girls were gamers and were included in the analytic sample $(\mathrm{n}=2442)$. Mean age was 14.3 years $(\mathrm{SD}=1.1)$, and the prevalence of smoking was 13.8 among boys and $22.0 \%$ among girls; $74.5 \%$ of boys played more than 1 hour per day compared with $47.7 \%$ of girls. High exposure to tobacco as compared to no exposure to tobacco in video games was independently associated with current smoking among girls $(\mathrm{OR}=1.78 ; 95 \% \mathrm{CI}=1,02$ 3,09 ) but not among boys ( $\mathrm{OR}=0.98 ; 95 \% \mathrm{CI}=0,64-1,51$ ).

Conclusions: Higher levels of exposure to tobacco in videogames were associated with higher likelihood of smoking in girls from Argentina.

Tob. Induc. Dis. 2018;16(Suppl 1):A472

DOI:10.18332/tid/83831

\section{6:30-18:00}

RF-1249-3 Pro-smoking apps for smartphones in the Republic of Korea: prevalence and potential risks

JH Kong ${ }^{1}$, JE Lee ${ }^{1}$, AN Lee ${ }^{1}$, AR Kong ${ }^{1}$

${ }^{1}$ Korea Health Promotion Institute, National Tobacco Control Center, Seoul, Korea, Republic of. E-mail:kjh3191@khealth.or.kr

Background: Smartphone has become a main part of our daily lives, and its impact on human behavior has been extensively studied. In Korea, 8 out of 10 people have their own smartphone and download 48 applications on average to get and share information. Recently, tobacco marketing prevails in the smartphone application but there is limited research on this area. This study investigated the prevalence of pro-smoking applications (apps) in Korea in order to understand the current status and potential risks.

Methods: From mid-June to early July 2017 (for 3 weeks), this study searched the Google Play store for pro-smoking apps. 8 keywords (cigarette, e-cigarette, nicotine, cigar, hookah, etc) were used to identify tobacco related apps. Apps achieved a top 100 ranking for each keyword then classified and analyzed based on apps name, retailer category, content category, number of downloads, publisher names, and content rating.

Results: Among a total of 675 apps identified for 8 keywords, 511 were analyzed for this study to avoid duplication. Although some anti-smoking apps $(n=119,23.3 \%)$ provided information on health effects of smoking or smoking cessation, majority were classified as pro-smoking apps $(\mathrm{n}=392,76.7 \%)$ containing information about how to make tobacco, advertisement on tobacco products and shops, places where smoking is allowed, game to simulate smoking behavior or to run tobacco company by selling cigarette, and smoking advocacy. More importantly, $99.7 \%$ of these prosmoking apps were rated as minors under 18 year-old can use them without any restrictions.

Conclusions: Pro-smoking apps prevail in the market while there is no restriction on access, install and use. Considering its crossborder characteristics, this would pose great challenge against tobacco control policies where smartphone use is increasing. Strategic approach to deal with novel tobacco marketing tactics needs to be developed with special attention to youth population.

Tob. Induc. Dis. 2018;16(Suppl 1):A473 DOI:10.18332/tid/83820

16:30-18:00

RF-1250-3 The new age of tobacco marketing: Imagery on social media

L James $^{1}, \mathrm{H}$ Kasi Vishwanathan

${ }^{1}$ Heart and Stroke Foundation of Canada, Health Policy, Ottawa, ON, Canada.E-mail: lesley.james@heartandstroke.ca

Background: While many areas of tobacco marketing have been restricted, the rise of digital and social media has presented an opportunity for new marketing avenues. This study looked at celebrity social media culture and its contribution to tobacco marketing.

Objectives:

- Assess the prevalence of such marketing.

- Assess who is being targeted, which audience segments.

- Determine what types of tobacco products are being marketed.

- Analyze themes or frames used to make these products appealing. Methods: Qualitative and quantitative methods were used to assess the celebrity social media setting. The top 10 global celebrity personalities were included for assessment. Only Instagram accounts were included as sources for data collection. Instagram posts were reviewed for imagery of tobacco products, content, and audience targets. From this data a thematic analysis was conducted to assess frames and narratives used within marketing tactics. Review of social media posts was limited to a 3 year time frame starting October 1, 2014 and ending September 30, 2017.

Results: Tobacco related marketing remains present on social media within the celebrity culture. Many of the top 10 followed celebrities had portrayals of tobacco embedded within their profile and/or posts. Young females are overtly targeted and traditional themes such as fashion, glamour, fun, sex appeal and free-will 
continue to be used as marketing frames.

Conclusions: These findings demonstrate the need for intervention to monitor and restrict tobacco related marketing on social media. While it's difficult to determine the source of tobacco marketing social media (industry placed or without paid placement) it warrants a discussion about influence of the celebrity and digital cultures on health behaviours. In the absence of regulation, celebrities should be considerate and responsible for the harmful imagery shared over social media.

\section{Tob. Induc. Dis. 2018;16(Suppl 1):A474 \\ DOI:10.18332/tid/84639}

\section{$16: 30-18: 00$}

RF-1255-3 Use of social media to circumvent sales and advertising ban of electronic cigarettes

M Güner ${ }^{1}$, U Pece Sonmez ${ }^{1}$, E Dagli ${ }^{1}$, T Gezer $^{1}$, F Yildiz ${ }^{1}, 0$ Elbek ${ }^{1}, \mathrm{P} \mathrm{Ay}{ }^{2}, \mathrm{M}$ Ceyhan ${ }^{1}$

${ }^{1}$ Health Institute Association, Istanbul, Turkey, ${ }^{2}$ Marmara University, Public Health, Istanbul, Turkey. E-mail: mguner@ superonline.com

Background: There are no legal electronic cigarette products available in Turkey. According to the law whether including tobacco or not products that resemble tobacco products can not be advertised, promoted and sold through internet. However the use of electronic cigarettes are frequently observed. This study was carried out in order to find out the sales outlet and advertising media of the publicly available products.

Methods: Internet search of the key words "e-cigarettes and electronic cigarettes" in Turkish language was carried out . The survey of the forum and websites identified were further analyzed to detect the presence of advertising, promotion and trading elements. Results: There were 94 websites and one social forum with 69,354 members on electronic cigarettes in Turkish language. Of the websites $95 \%$ had advertising and facilitated online sales. Social media forum had a section for beginners which was accessible by non-member, containing information about the device, liquids and usage. Under the heading" starter kit for beginners" 143 brands were promoted. The suppliers were introduced under 74 headings and 44774 messages. 25 suppliers sold e-cigarettes through the forum by 221 various advertising. Online shopping section was consisted of 1990 topics and 179.100 messages. The forum also facilitated exchange of products between members, as well as international sales without customs. All the websites had Turkish domain names and used regular shipping companies for the delivery of the products. The social media forum had an international hosting. Advertising contained false claims about the safety of the products referring to reputable international organizations. Social media forum provided home-made recipes and the names and adresses of chemical supplier companies.

Conclusions: Social media was extensively used for the sale and advertising of electronic cigarettes that were not licenced in Turkey. Laws need to be enforced not only in real life but also at the virtual media.

Tob. Induc. Dis. 2018;16(Suppl 1):A475 DOI:10.18332/tid/84130

\section{6:30-18:00}

RF-1256-3 Use of tobacco in Brazilian TV programs: status and potential influence on the low income population

HN Carvalho ${ }^{1}$, CC Moreira ${ }^{1}$, AP Natividade ${ }^{1}$, SR Turci ${ }^{1}$, LAB Camacho $^{2}$, S Bialous ${ }^{3}$, VL da Costa e Silva ${ }^{4}$, VC Figueiredo
'Fundação Oswaldo Cruz, Centro de Estudos sobre Tabaco e Saúde, Rio de Janeiro, RJ, Brazil, ${ }^{2}$ Fundação Oswaldo Cruz, Escola Nacional de Saúde Pública, Rio de Janeiro, RJ, Brazil, ${ }^{3}$ University of California San Francisco School of Nursing, UCSF School of Nursing/ Social Behavioral Sciences, San Francisco, CA, United States of America, ${ }^{4}$ World Health Organization, WHO FCTC Secretariat, Genebra, Switzerland. E-mail: hannah_ nascimento@hotmail.com

Background: Tobacco companies advertising and promotions affect behavior related to smoking. In line with WHO-FCTC's Article 13, Brazil has banned direct advertising, promotion and sponsorship in all type of media since 2000. This study aimed to analyze and describe the characteristics of indirect tobacco advertising in Brazilian TV programs.

Methods: Descriptive study, content analysis of 345 hours of programming in 2014, including novels, Brazilian series, reality shows and films with the highest audience for the population with medium and low income in free-to-air channels in Brazil. With an adaptation of the Breath California's "Thumbs Up Thumbs Down" method of Sacrament Emigrants Trails, tobacco appearances were encoded in which an incident is any on-screen product appearance (actual use, implicit use, verbal or gestural message, or atmosphere of smoke). Each program was viewed by two reviewers independently. Data were processed and analyzed using CSPro and Stata12 programs.

Results: Out of the 470 analyzed programs (345hs), 35\% presented incidents / messages related to tobacco (3hs). Out of the programs that portrayed tobacco, use, Reality Shows represented the majority $(44,5 \%)$, followed by films $(21,3 \%)$ and novels and series (17,1\% each). $63 \%$ of the programs were considered suitable for 12 year olds, $16 \%$ for 14 year olds, $15 \%$ for 10 year olds, $6 \%$ for 16 year olds and above and $27 \%$ presented 21 or more incidents. 609 tobacco-related scenes were identified, $76.7 \%$ of which were cigarettes, apparitions of cigars (15\%), pipe (4.5\%) and narguile (3.8\%) were also observed. $95.6 \%$ of the scenes contained pro-tobacco messages.

Conclusions: This research made it possible to measure the frequency of "indirect advertising" in large audience programs aimed at the lower classes of the Brazilian population, which is one of the tobacco marketing alternatives, even with the prohibition of tobacco advertising throughout the National territory.

Tob. Induc. Dis. 2018;16(Suppl 1):A476

DOI:10.18332/tid/84128

16:30-18:00

RF-1257-3 Indian television channels become vehicle for tobacco advertisement, promotion and sponsorship (TAPS) violations in India - results of a sub-national survey in a northern Indian city R Chand ${ }^{1}$

'Indira Gandhi Medical College, Hospital Administration, Shimla, India. E-mail: dr.rameshigmc@gmail.com

Background: Indian tobacco control legislation (Section 5, subsequent rules dated October 2nd, 2012 of COTPA, 2003) puts complete ban on Tobacco Advertisement Promotion and Sponsorships (TAPS), but industry is circumventing the law to carry the bussiness. Rules also mandate that, if there are tobacco use scenes in a movie or television program, a health spot, an audio visual disclaimer and health warning must be displayed during the telecast. However, there are gaps in the implementation. It is important for law enforcers to understand the nature and types of TAPS violations being carried out through television channel to 
better prepared for taking action.

Methods: Total 32 television channels telecasted between January-March 2017 in Shimla city in Northern India selected through stratified random sampling were observed during prime time (19:00 PM-22:00 PM) for their compliance to the provisions of Indian cinema and television rules, 2012. The TV programs including serials and movies and the advertisements in between the programs were assessed as per the pre-tested checklist.

Results: Direct advertisements were not found in any of the channel. In near one fourth of television channels, TAPS was carried out as surrogate advertisements in the form of mouth freshners and paan masala and brand stretching/trademark diversification. Atleast one smoking scene was found in 9 television channels playing the movie, however, specified health spot, audio-video disclaimer and health warning could be observed in eight channels. News channels and regional channels had comparatively more surrogate advertisments and smoking violations as compared to other channels.

Conclusions: Cinema and television rules under Section 5 of COTPA are not strictly implemented in Indian television channels. TAPS are being carried out as surrogate advertisments, brand stretching and trademark diversification. Statuary requirements recommended under the rules for scenes showing tobacco uses are not followed. A stronger action from authorities is needed against the violators.

Tob. Induc. Dis. 2018;16(Suppl 1):A477

DOI:10.18332/tid/84424

\subsection{Innovations in media educational campaigns and health warning labels on cigarettes and other tobacco products}

\section{$14: 45-16: 15$}

F0-372-3 Do pictorial health warnings on waterpipe tobacco packs matter? Recall effectiveness among waterpipe smokers \& non-smokers

A Mostafa ${ }^{1}$, HT Mohammed ${ }^{1,2}$, RS Hussein ${ }^{1}$, A Aboul Fotouh ${ }^{1}$ ${ }^{1}$ Faculty of Medicine, Ain Shams University, Department of Community, Environmental, and Occupational Medicine, Cairo, Egypt, ${ }^{2}$ School of Pharmacy, University of Waterloo, Waterloo, ON, Canada. E-mail: aya.kamaleldin@med.asu.edu.eg

Background: Only a few countries have adopted pictorial health warnings (PHWs) on waterpipe tobacco (WT) packs, despite the global increase in WT smoking. Moreover, scarce information is available on their post-marketing effectiveness. We measured the recall effectiveness of the existing PHWs on WT packs in the Egyptian market among WT smokers and non-smokers.

Methods: We conducted a population-based survey including a convenience sample of 1490 adult WT smokers and 524 nonsmokers of both genders in Cairo and a rural village in Egypt. Participants who reported ever noticing PHWs on WT packs were asked to answer questions through memory recall about PHWs' effect on salience, depth of processing, behavioral control and quit attempts. Descriptive and univariate statistical analyses were performed.

Results: Salience: About two-thirds of WT smokers (63.8\%) and non-smokers (59.2\%) acknowledged the presence of PHWs on WT packs, most of whom correctly recalled their position $(98.7 \%$ versus $97.4 \%$, respectively). The information on the packs' side were the least recalled (1.1\%) (Table 1). Depth of processing: $77.6 \%$ of all participants who ever noticed PHW on WT packs understood them. PHW significantly increased information on
WT smoking health hazards in non-smokers than WT smokers ( $80.3 \%$ versus $74.3 \%, \mathrm{p}=0.033$ ). Non-smokers were significantly more likely to discuss PHW with others (86.4\% versus $72.4 \%$, $\mathrm{p}<0.001$ ), while WT smokers avoided looking at the PHWs (74.6\%) significantly more than non-smokers (60.0\%) $(p=0.002)$ (Table 1). Behavioral control and quit intentions: PHWs on WT packs encouraged $64.5 \%$ of WT smokers to reduce the number of smoked hagars, made $42.2 \%$ forgo a smoke, and $58.5 \%$ more likely to quit. They also made $38.4 \%$ of former WT smokers actually quit and discouraged $59.0 \%$ of non-smokers to start WT smoking. Conclusions: Findings suggest that inserting PHWs on WT packs is an effective WT labelling policy. More countries should consider adopting it within a comprehensive regulatory framework.

\begin{tabular}{|c|c|c|c|c|}
\hline & $\begin{array}{l}\text { Total } \\
\text { N-2014 } \\
(\%)\end{array}$ & $\begin{array}{l}\text { W/PS - } \\
\begin{array}{l}\text { W } 1190 \\
(\%)\end{array}\end{array}$ & $\begin{array}{l}\text { NS } \\
\text { N } 521 \\
(\%)\end{array}$ & p value \\
\hline \multirow[t]{2}{*}{$\begin{array}{l}\text { 1. Salience 1a. Know of } \\
\text { PHWs on WTPs: Yes }\end{array}$} & $\begin{array}{l}1261 \\
(62.6)\end{array}$ & $\begin{array}{l}951 \\
(63.8)\end{array}$ & $\begin{array}{l}310 \\
(59.2)\end{array}$ & 0.810 \\
\hline & $\begin{array}{l}N=1261 \\
(\%)\end{array}$ & $\begin{array}{l}N=951 \\
(\%)\end{array}$ & $\begin{array}{l}N=310 \\
(\%)\end{array}$ & $p$-value \\
\hline $\begin{array}{l}\text { 1b. Different from } \\
\text { cigarette pack PHWs: No }\end{array}$ & $\begin{array}{l}1029 \\
(81.6)\end{array}$ & $\begin{array}{l}791 \\
(83.2)\end{array}$ & $\begin{array}{l}238 \\
(76.8)\end{array}$ & $<0.001$ \\
\hline $\begin{array}{l}\text { 1c. Contains a hot line } \\
\text { number for cessation: Yes }\end{array}$ & $\begin{array}{l}1118 \\
(88.7)\end{array}$ & $\begin{array}{l}854 \\
(89.8)\end{array}$ & $\begin{array}{l}264 \\
(85.2)\end{array}$ & 0.005 \\
\hline $\begin{array}{l}\text { 1d. PHW position on WTP } \\
\text { recalled correctly: Yes }\end{array}$ & $\begin{array}{l}1232 \\
(97.7)\end{array}$ & $\begin{array}{l}926 \\
(97.4)\end{array}$ & $\begin{array}{l}306 \\
(98.7)\end{array}$ & 0.131 \\
\hline $\begin{array}{l}\text { 1e. Know information } \\
\text { on WTP sides: Yes }\end{array}$ & $14(1.1)$ & $12(1.3)$ & $2(0.6)$ & 0.368 \\
\hline $\begin{array}{l}\text { 2. Depth of processing } \\
\text { 2a. Looked closely at } \\
\text { PHW on WTP: Yes }\end{array}$ & $\begin{array}{l}1029 \\
(81.6)\end{array}$ & $\begin{array}{l}770 \\
(81.0)\end{array}$ & $\begin{array}{l}259 \\
(83.5)\end{array}$ & 0.353 \\
\hline $\begin{array}{l}\text { 2b. PHW contents were } \\
\text { understandable: Yes }\end{array}$ & $\begin{array}{l}980 \\
(77.7)\end{array}$ & $\begin{array}{l}723 \\
(76.0)\end{array}$ & $\begin{array}{l}257 \\
(82.9)\end{array}$ & 0.006 \\
\hline $\begin{array}{l}\text { 2c. PHW made you } \\
\text { think about health } \\
\text { hazards: Yes }\end{array}$ & $\begin{array}{l}1053 \\
(83.5)\end{array}$ & $\begin{array}{l}782 \\
(82.2)\end{array}$ & $\begin{array}{l}271 \\
(87.4)\end{array}$ & 0.034 \\
\hline
\end{tabular}

[Recall effectiveness of Pictorial Health ]

Tob. Induc. Dis. 2018;16(Suppl 1):A478 DOI:10.18332/tid/84617

16:30-18:00

RF-1251-3 Evaluation of the national tobacco control mass media campaign in China

$X$ Jin $^{1}$, J Song ${ }^{1}$, Y Zhang ${ }^{1}$

${ }^{1}$ Chinese Center for Health Education, Beijing, China. E-mail: yaya22nd@hotmail.com

Background and challenges to implementation: Tobacco use is the leading preventable cause of morbidity and mortality worldwide, yet the usage rate continues to increase in low- and middle-income countries such as China.Of over 310 million smokers lives in China, which accounts for $27.7 \%$ of the general population.

Intervention or response: In 2014, the BI collaborated with the Chinese Center for Health Education to implement the national tobacco control mass media campaign in China. The campaign was expected to improve knowledge about health effects of smoking and SHS exposure and shape attitudes and behaviors toward tobacco control policies. 
The campaign was adapted from materials which depicts a office environment with SHS to show how it affects everyone. It was broadcast on the national Television channels and six provincial satellite networks, meanwile on social media and mini-site for a four-week period.

Results and lessons learnt: To evaluate the effectiveness of this campaign, a total of 2400 participants were selected to complete interviews through a stratified random sampling. The participants consisted of $828(34.5 \%)$ smokers, and 1,572(65.5\%) nonsmokers. The campaign reached about $24 \%$ of the Chinese population and increased smokers' knowledge about the harmful effects of SHS exposure(73\% vs. $85 \%, \mathrm{p}<0.05)$. Both smokers and nonsmokers increased their knowledge that smoking lead to diseases other than lung cancer. Although smokers exposed to the campaign were more likely to take actions to reduce smoking than those not exposed(13\% vs. $7 \%, \mathrm{p}<0.05)$, the percentage was still low. The support for bans on smoking in public places increased after the campaign, especially among nonsmokers and females. However, the support for ban on smoking in bars (48\%) and restaurants(62\%) were lower than that in hospitals(95\%) and schools(94\%).

Conclusions and key recommendations: The mass media campaign reinforced people's knowledge and attitudes about harmful health effects of smoking and SHS exposure, increased people's desire to quit, and improved people's support for smoking bans in public places.

Tob. Induc. Dis. 2018;16(Suppl 1):A479

DOI:10.18332/tid/84435

\section{$16: 30-18: 00$}

RF-1252-3 The effectiveness and cost-effectiveness of tobacco control TV mass media campaigns broadcast in Scotland (2003 - 2012)

S Haw ${ }^{1}$, H Haghpanahan², D Mackay², K Boyd², T Langley³, E Mcintosh ${ }^{2}$, J Pell ${ }^{2}$

'University of Stirling, Stirling, United Kingdom, ${ }^{2}$ University of Glasgow, Glasgow, United Kingdom, ${ }^{3}$ University of Nottingham, Nottingham, United Kingdom. E-mail: s.j.haw@stir.ac.uk

Background: Tobacco control (TC) mass media campaigns (MMCs) are an important component of tobacco control strategies and are effective in reducing smoking prevalence. However, with increasing pressure on public health budgets, there has been a shift in some countries from MMCs to much cheaper community-based initiatives. Methods: Using multivariate time series analyses we examined the relationships between exposure to TV MMCs (adult TV viewer ratings (TVRs)); and quitting behaviour (calls to Smokeline, prescriptions for NRT), and reduction in numbers of smokers. In the economic analysis, we compared the costs and effects of TV MMCs against a 'No TV/do-nothing' alternative, from a public sector payer perspective (Scottish Government and NHS).

Results: TV MMCs increased calls to Smokeline by 385.9 calls (95\% CI, 171.0 - 600.7) per 1 SD TVRs (194). This was sustained for 6 months. Adverts that evoked positive feelings generated the largest increase in calls $(69.7 \%: 46.1,93.0)$. The increase in NRT prescriptions was insignificant (£1,361.4: -£9,138 - £11,860.9). An increase of one TVR/month reduced the number of smokers by 41 (-40.9: -102.07, 20.24). Although, statistically insignificant, our economic analysis indicates that TV MMCs generated an additional 116,885 quit attempts and 7,305 sustained 52-week quits per annum compared with no TV MMCs. The incremental cost per 52-week quitter was $£ 102$, which is highly cost-effective. With the very low annual cost of TV MMCs of $£ 0.66$ per smoker and potential lifetime savings in terms of avoiding cost of smoking related diseases, the lifetime model resulted in overall cost savings (-£319:-£974,-£31) and QALY gains (0.016: 0.0017,0.048) that were significant and demonstrated little uncertainty.

Conclusions: This study provides convincing evidence that tobacco control TV MMCs can increase quit attempts and are both effective and cost-effective in reducing the number of smokers. Their continued use, as part of a comprehensive TC strategy, is strongly recommended.

Tob. Induc. Dis. 2018;16(Suppl 1):A480

DOI:10.18332/tid/84455

\section{$16: 30-18: 00$}

RF-1253-3 "Little Lungs" - using digital platforms, innovative creative, and audience passion points to prevent smoking among U.S. youth

A Brubach

${ }^{1}$ U.S. FDA, Center for Tobacco Products, Silver Spring, MD, United States of America. E-mail: april.brubach@fda.hhs.gov Background and challenges to implementation: Nearly 9 out of 10 U.S. adult daily smokers smoke their first cigarette by age 18 , making youth prevention a critical interception point. U.S. FDA's The Real Cost campaign is a nationwide public education effort targeting the nearly 10 million at-risk youth ages $12-17$ to prevent and reduce tobacco use. The campaign's challenge is to catch the attention of teens and get them to engage with tobacco-prevention messaging--something they have limited interest in. To succeed, the campaign requires innovative advertising that speaks to teen passion points. Because teens don't think they'll become addicted and ignore the long-term health consequences of smoking, the campaign speaks to consequences they care about in the hereand-now, such as stunted lungs and cosmetic health effects.

Intervention or response: In response to this challenge, U.S. FDA developed, "Little Lungs," a digital campaign based on audience insights that teens like snarky humor. A series of 15-30 second ads were developed to tell the story of the main character "Little Lungs," who smoked as a teen, never grew to normal size, and now can't keep up in the activities he loves. This leads to his "tragicomic" demise. The video series launched on digital platforms (https://www.youtube.com/watch?v=nEjrjTsVLY4). Paid digital ads drove traffic, relevant hashtags were used to insert the campaign into teens interest areas.

Results and lessons learnt: "Little Lungs" was viewed over 17 million times on YouTube, 8 million times on Facebook and Instagram, and the content received over 8.2 million social engagements with a $17 \%$ social engagement rate.

Conclusions and key recommendations: Teens are already deeply submerged in the digital world--inserting "Little Lungs" in that space presented an opportunity to deliver an impactful message that aligned with passion points to spark initial interest and then seamlessly tie in a tobacco prevention message.

Tob. Induc. Dis. 2018;16(Suppl 1):A481

DOI:10.18332/tid/84281

$16: 30-18: 00$

RF-1254-3 Can Facebook help reduce smoking? A qualitative study to investigate how to use social media for tobacco control among Australian Indigenous people

M Hefler ${ }^{1}$, V Kerrigan', DP Thomas ${ }^{1}$

${ }^{1}$ Menzies School of Health Research, Casuarina, NT, Australia. E-mail: marita.hefler@menzies.edu.au

Background: The tobacco industry was quick to exploit social media 
to subvert tobacco advertising and promotion bans, and directly connect with consumers. Social media offers similar opportunities for tobacco control, however evidence for how to effectively use it to influence behaviour is limited. The aim of this study was to understand how traditional mass media and peer influence approaches may need to be adapted for Facebook-based strategies.

Methods: Community-based Indigenous peer researchers who were active Facebook users were recruited to share tobacco control content weekly from January to June 2017. Peer researchers were given three content options each week, and required to share at least one. They documented reasons for their choice and recorded both online and offline interactions it generated. Contacts from within the peer researchers' networks were interviewed faceto-face to understand the real world impact of the content, particularly posts which generated no online interaction. Data was inductively analysed using grounded theory methodology.

Results: Messages which were direct, positive, specific and relatable for Aboriginal people's values and social context, and which had practical information were favoured. Content that leveraged people's care and concern for children was most likely to be shared, even if the content was international. The impact on peer researchers' Facebook contacts varied significantly. Some reported never seeing the content; others were directly impacted - including quitting smoking as a direct result of posts.

Conclusions: The popularity of a Facebook post, as measured by visible reactions such as likes, shares and comments, does not necessarily equate with its real world impact. Shocking and graphic tobacco control messages used for traditional mass media campaigns are often bypassed or ignored by both smokers and non-smokers when sharing content on Facebook. Further research is required around the impact of tobacco control campaigns which are strengths-based and target Indigenous people.

Tob. Induc. Dis. 2018;16(Suppl 1):A482

DOI:10.18332/tid/84165

\section{$18: 15-19: 15$}

LB-1339-3 Mobilizing school children in "No Smoking, Be Charming!" media campaign in Taiwan TT-L Chen', CL Lin', T-I Lu'

'John Tung Foundation, Taipei, China. E-mail: service108@ jtf.org.tw

Background and challenges to implementation: In Taiwan, government's generous reimbursement to hospitals and clinics for providing smoking cessation treatments, plus a very successful "Quit \& Win" nationwide campaign, have helped bring down adult smoking rate recently. However, a great concern is rising as youth smoking rate has not been decreased. With influx of e-cigarettes and other cheap tobacco products, as well very aggressive tobacco industries' promotion strategies targeting at youth, smoking among Taiwan youth has become a threat. The urgent need to help youth to resist pressure of smoking is apparent.

Intervention or response: In light of this, John Tung Foundation developed a media campaign initiative aimed at engaging school children on building capacity to resist smoking. This proposal promptly received support from Taiwan government and NGOs. At the initiation phase, a very popular actress, Ning Chang, was recruited to play role of "Smoke-Free Dancer" in a video that messaging "No Smoking, Be Charming!". The beautifully made video were then widely shown in television and circulated through website, Facebook, and blogs. Thereafter, videos, pamphlets, placates, posters were distributed to schools and health stations. Billboards were displayed in over 4,600 places in cities and towns.
Schools were engaged to run smoking education programs to raise health literacy on label reading and inoculate no-smoking capacity. Classes were encouraged to post creative anti-smoking campaign photos on Facebook Fan Page. Students from 416 classes, 153 schools in over 20 cities participated.

Results and lessons learnt: A national sample of 4,642 K-12 and college students were surveyed that showed $95 \%$ approval rating. This project showed very promising results in mobilizing students to be no-smoking campaign crusaders.

Conclusions and key recommendations: The project seems useful to lead youth to temper their smoking intention and help created a smoke-free atmosphere in schools.

Tob. Induc. Dis. 2018;16(Suppl 1):A483

DOI: $10.18332 /$ tid/84714

\subsection{Opportunities for regulating tobacco products' content and emissions to reduce harm and addiction potential}

\section{$14: 45-16: 15$}

F0-369-3 Knowledge, attitudes and practices of Shisha smoking among youths in Kampala, Uganda C Aanyu ${ }^{1}$, JB Ddamulira ${ }^{1}$, K Nyamurungi $^{2}$, M Ediau $^{1}$, W Bazeyo ${ }^{1}$ ${ }^{1}$ Makerere University, Kampala, Uganda, ${ }^{2}$ Center for Tobacco Control in Africa (CTCA), Kampala, Uganda. E-mail: aanyuchristinee@gmail.com

Background: Globally tobacco use kills nearly 6 million people annually; a figure expected to increase to 8 million deaths every year by 2030 . Though perceived to be safe, shisha smoking is reported to have the same or worse health effects as cigarette smoking. Yet, this practice has gained popularity especially among youths in Kampala. The extent to which this form of tobacco consumption is practiced by the youths, their attitudes and knowledge regarding the health risks in Uganda is unknown. This study therefore aimed to establish knowledge, attitudes, practices and factors associated with shisha smoking among youths.

Methods: A cross-sectional study was conducted among 530 systematically selected youths aged between 18-30 years found in bars in the two divisions of Kampala between April and May 2014. Youths who are incapacitated were excluded. Three outcome variables; knowledge, attitudes and practice were assessed. Data was collected using a semi structured interviewer administered questionnaire. Descriptive statistics of key variables were generated at univariate analysis. Multivariable logistic regression analysis with Odds Ratios and corresponding 95\% Confidence Intervals were used to measure associations. STATA version 12.0 was used for analysis. Results: Of 530 participants, $86.4 \%$ had low knowledge, $69.2 \%$ had negative attitudes and $36.4 \%$ smoked Shisha. $75.7 \%$ smoked flavored and sweetened tobacco, $37.6 \%$ smoked on weekly basis, $92.8 \%$ smoked in the company of friends, $91.2 \%$ shared shisha pipes and $93.8 \%$ smoked in bars. A further $62.7 \%$ smoked shisha with cigarettes. Factors associated with shisha smoking included; smoking cigarettes (Adjusted Odds Ratio (AOR): 6.33, 95\% CI: 4.13-9.58); positive attitude (AOR: 4.13, 95\% CI: 2.65-6.44); Age (25-30 years) (AOR: 1.72, 95\% CI: 1.13-2.62) and no knowledge (AOR: 0.50, 95\% CI: 0.29-0.88).

Conclusions: Shisha smoking among youths attending bars is high with three in every ten youths smoking shisha. Targeted social mobilization would increase awareness regarding adverse health effects of shisha smoking.

Tob. Induc. Dis. 2018;16(Suppl 1):A484

DOI: $10.18332 /$ tid/84307 
$14: 45-16: 15$

F0-370-3 Effects of a real world menthol ban: changes in smoking behaviour and cigarette branding

M Chaiton ${ }^{1}$, R Schwartz ${ }^{1,2}$, T Eissenberg ${ }^{3}$, E Soule ${ }^{3}$, J Cohen ${ }^{4}$ ${ }^{1}$ Ontario Tobacco Research Unit, Toronto, ON, Canada, ${ }^{2}$ University of Toronto, Toronto, ON, Canada, ${ }^{3}$ Virginia Commonwealth University, Richmond, VA, United States of America, ${ }^{4}$ Johns Hopkins Bloomberg School of Public Health - Institute for Global Tobacco Control, Baltimore, MD, United States of America. E-mail:michael.chaiton@utoronto.ca

Background: The province of Ontario, Canada implemented a ban on menthol in all forms of tobacco products that came into effect January 1st, 2017. This presentation examines changes in behaviour among a cohort of menthol smokers as part of the first evaluation of a real world menthol ban.

Methods: 1003 past year menthol smokers (16+, living in Ontario) were surveyed by telephone $(n=329)$ or online $(n=674)$ between September and December 2016. Participants were re-contacted in February 2017. Logistic regressions, adjusted for demographic and smoking characteristics, were used to assess characteristics of participants who reported post ban a) past month menthol cigarette use, b) use of alternative tobacco or flavoured products after the ban, or c) quit attempts. A pack purchase of menthol and menthol replacement products was conducted.

Results: At follow up after the ban, 33\% (95\% CI: 30\%, 37\%) reported smoking a menthol cigarette, while $39 \%$ (95\% CI: $36 \%$, $43 \%$ ) were using an alternative tobacco or flavoured product, and $42 \%$ (95\% CI: $39 \%, 46 \%)$ had either made a quit attempt or had quit smoking. Predictors of smoking menthol after the ban included regularity of use before the ban and lack of support for the ban. Following the ban, participants were more likely to expect to quit because of the ban, and less likely to expect to use other tobacco or nicotine products as substitutes, than they were before the ban. Menthol replacement packs were found using a variety of different strategies including blue or green colouring, "choice" filters, and non menthol capsule.

Conclusions: Short term effects of the menthol ban suggest that the ban encouraged significant quitting behaviour as well as attempts by smokers to seek out other sources of menthol or alternative substitutes.

Tob. Induc. Dis. 2018;16(Suppl 1):A485

DOI: $10.18332 / \mathrm{tid} / 84323$

\section{4:45-16:15}

F0-371-3 The use of and beliefs about menthol cigarettes among Brazilian smokers: findings from Wave 3 (2016-17) of the ITC Brazil Survey

L Craig', V Figueiredo², C Perez ${ }^{3}$, GT Fong ${ }^{4,5}$, M Yan' ${ }^{1}$ S Kaai ${ }^{1}$, ACK Quah', JF Thrasher, ${ }^{6,7}$

'University of Waterloo, Waterloo, ON, Canada, ${ }^{2}$ Oswaldo Cruz Foundation, Rio de Janeiro, RJ, Brazil, ${ }^{3}$ Fundaçao do Cancer, Rio de Janeiro, RJ, Brazil, ${ }^{4}$ University of Waterloo, Dept of Psychology and School of Public Health and Health Systems, Waterloo, ON, Canada, ${ }^{5}$ Ontario Institute for Cancer Research, Toronto, ON, Canada, ${ }^{6}$ University of South Carolina, Arnold School of Public Health, Columbia, SC, United States of America, ${ }^{7}$ National Institute of Public Health, Cuernavaca, Mexico.E-mail: Ivcraig@uwaterloo.ca

Background: Menthol and other flavorings are appealing to young people. They mask the adverse effects of cigarette smoke, such as throat irritation, and are marketed as less harmful to appeal to health-concerned smokers. In March 2012, Brazil banned all flavors in tobacco products, including menthol, but the ban has not been implemented due to industry legal challenges. This is the first study in Brazil to examine menthol use and beliefs about menthols (particularly harm perceptions). Such research can increase an understanding of menthol use to inform/support regulatory action in Brazil and other countries.

Methods: Data were from Wave 3 (2016-17) of the International Tobacco Control (ITC) Brazil Survey, a cohort survey of adult smokers and non-smokers in Rio de Janeiro, São Paulo, and Porto Alegre. This study analyzed responses from 1216 smokers on prevalence and predictors of menthol use, beliefs about harmfulness, and support for banning additives.

Results: In 2016-17, 7.8\% of smokers with a regular cigarette brand reported that they smoked menthols (lowest of 10 ITC LMICs). $12.5 \%$ of smokers erroneously believed that menthol cigarettes are less harmful than regular cigarettes (lowest of 7 ITC middle-income countries); $33.4 \%$ believed menthols are smoother on the throat and chest (lowest of 4 LMICs). $59.6 \%$ of smokers support a complete ban on all additives including flavorings (3rd highest among 14 ITC countries).

Conclusions: These findings support Brazil's effort to ban all additives including menthol: menthol prevalence is comparatively low, erroneous beliefs that menthols are less harmful is low, and support for an additive ban is high. Brazilian media reporting of the risks of additives in the context of the tobacco industry injunction may have raised public awareness of the harms of additives and support for the ban. Also, the 2014 ban on advertising at points of sale may have reduced exposure to marketing of menthols.

\section{Tob. Induc. Dis. 2018;16(Suppl 1):A486}

DOI: $10.18332 /$ tid $/ 84513$

\section{$14: 45-16: 15$}

F0-375-3 Prevalence, perceptions and predictors of menthol cigarettes among African smokers: findings from the ITC Kenya and Zambia Surveys SC Kaai', J Ong'ang'o ${ }^{2}$, L Craig ${ }^{1}$, F Goma ${ }^{3}$, G Meng ${ }^{1}$, L Ikamari ${ }^{4}$, TE Marshall ${ }^{5}$, GT Fong ${ }^{1,6}$

${ }^{1}$ University of Waterloo, Waterloo, ON, Canada, ${ }^{2}$ Kenya Medical Research Institute, Nairobi, Kenya, ${ }^{3}$ University of Zambia, Lusaka, Zambia, ${ }^{4}$ University of Nairobi, Nairobi, Kenya, ${ }^{5}$ Centre for Addiction and Mental Health, London, ON, Canada, ${ }^{6}$ Ontario Institute for Cancer Research, Toronto, ON, Canada. E-mail: skaai@uwaterloo.ca

Background: Research in high-income countries (HICs) has raised awareness of the harms of menthol cigarettes, providing the foundation for a growing movement in HICs to ban menthol. Menthol masks the harshness of smoke, making it easier for youth to start smoking, and encouraging health-concerned smokers to switch to menthols, incorrectly believing that menthols are less harmful. There are few studies of menthols in LMICs, and almost none in Africa. We conducted the first population study in Africa to assess the prevalence of menthol cigarettes, beliefs about harmfulness of menthols, and predictors of menthol use.

Methods: Data were from the International Tobacco Control (ITC) Kenya Wave 1 (2012) and Zambia Wave 2 Survey (2014). These are longitudinal surveys of nationally representative samples of adults in each country. This study focuses on 1,449 smokers who answered questions on use, choice, and perceptions of menthol cigarettes (Zambia only).

Results: Prevalence of menthols was high in Zambia (43\%, highest of 24 ITC countries) and Kenya (21\%, third-highest). In 
Zambia, the erroneous belief that menthols are less harmful was high (36\%—second-highest), and most common among younger (69\%), medium-income (36\%), and non-daily smokers (42\%). In Zambia, menthol use was associated with: belief that menthol is less harmful $(\mathrm{OR}=3.68, \mathrm{p}=0.01)$, choosing brand because of taste $(\mathrm{OR}=2.46, \mathrm{p}=0.01)$, health concerns $(\mathrm{OR}=3.32, \mathrm{p}=0.0001)$, and price $(\mathrm{OR}=0.31, \mathrm{p}=0.04)$. In Kenya, menthol use was associated with: choosing brand because of taste $(\mathrm{OR}=3.49, \mathrm{p}=0.0002)$, price $(\mathrm{OR}=1.73, \mathrm{p}=0.05)$; health concerns $(\mathrm{OR}=1.57, \mathrm{p}=0.04)$; and perceiving that quitting is beneficial $(\mathrm{OR}=1.78, \mathrm{p}=0.04)$.

Conclusions: Menthol use was high in Zambia and Kenya, and predicted by health concerns. This is alarming because many smokers (in Zambia) incorrectly believe that menthols are less harmful. These findings support the need for African countries to raise awareness of the myth of menthols and to ban menthol and other flavorings to reduce initiation among youth and to encourage smokers to quit.

Tob. Induc. Dis. 2018;16(Suppl 1):A487

DOI: $10.18332 /$ tid/84123

\subsection{Opportunities for regulating tobacco products' content and emissions to reduce harm and addiction potential}

\section{$18: 15-19: 15$}

LB-1309-3 Flavor capsule cigarette use, user profiles and perceptions in South Korea

YJ Cho ${ }^{1}$, JF Thrasher ${ }^{1,2}$

'University of South Carolina, Health Promotion, Education, \& Behavior, Columbia, SC, United States of America, ${ }^{2}$ National Institute of Public Health, Department of Tobacco Research, Cuernavaca, Mexico. E-mail: ycho@email.sc.edu

Background: Industry reports celebrate market growth from the recent cigarette design innovation of flavor capsules in the filter, which smokers crush to release a liquid that flavors the smoke. However, limited information exists regarding flavor capsule cigarette smokers, including in South Korea, where this market segment has rapidly grown. This study assessed (1) the prevalence, correlates, and perceptions of smokers having flavor capsule cigarette brands as their regular brands in Korea, as well as (2) the recency of brand adoption and the reason for choosing the brand.

Methods: Data come from a nationally representative sample of 1,940 adult smokers who participated in the 2016 International Tobacco Control Project (ITC) Korea survey.

Results: The prevalence of smokers who preferred flavor capsule brand varieties was $18 \%$. Flavor capsule cigarette smokers were more likely than regular cigarette smokers to be younger (e.g. AOR [Adjusted odds ratio] 29-38 vs. 19-28: 0.38, p < . 001), female (AOR: 1.51, p <.05), and report lower nicotine dependence (AOR: 0.87, $\mathrm{p}<.01$ ). Flavor capsule cigarette smokers were also more likely than regular cigarette smokers to report that their cigarette varieties are lighter in taste (b: $0.18, \mathrm{p}$ $<.01$ ) and smoother on throat (b: $0.12, \mathrm{p}<.05$ ), which promotes misperceptions of reduced harm. They were more likely than regular cigarette smokers to report that they had smoked the current usual brand less than 1 year (AOR: $4.81, \mathrm{p}<.001$ ) and that they chose their brand because of taste (AOR: $4.55, \mathrm{p}<.05$ ). Conclusions: Preference for flavor capsule cigarettes is high in Korea, especially among vulnerable populations such as young adults and females. Given that flavors mask the harshness of tobacco smoke and promote addiction, regulators should ban this newly introduced cigarette design innovation.

Tob. Induc. Dis. 2018;16(Suppl 1):A488

\section{DOI:10.18332/tid/84716 \\ 4.1 Prevalence of tobacco and nicotine related products' use: Traditional and novel products}

\author{
$16: 30-18: 00$
}

FO-376-4 A real-world assessment of indoor air quality (ultrafine particles) following e-cigarette use in two e-cigarette shops

D Salvi', C Sennett ${ }^{1}$, A Fotedar ${ }^{1}$, J Levy ${ }^{1}$

'Boston University, School of Public Health, Boston, MA, United States of America. E-mail: devashri.salvi@gmail.com

Background: E-cigarettes are becoming increasingly popular, yet, it is not clear what effect they may have on human health. In particular, there is not much research on the subject of secondhand exposure to vapor from e-cigarettes within indoor settings. Ultrafine particulates (UFPs) are associated with e-cigarette use as well as a myriad of adverse health outcomes. The objectives of this study are to characterize indoor air concentrations of UFPs in two commercial locations that allow the use of e-cigarettes (vaping) indoors, to determine to what extent vaping activities contribute to increased numbers of UFPs and to assess the time taken for UFP numbers to decline.

Methods: We measured air quality (UFPs) in 2 shops for a portion of their regular business day (168-212 minutes). We also measured temperature, humidity and carbon dioxide. We conducted three separate sampling sessions, two in Shop A (1000ft2) and one in Shop B, (400ft2). In Shop A we could set up two monitors, one near the vaping activity and one set was kept $\sim 50$ meters away. For all sessions, we recorded the number of vapers and took air measurements on a minute-by-minute basis.

Results: Results suggest that vaping activities result in increases in indoor concentrations of UFPs. Concentrations of UFPs ranged from 10124 to 87940 particles $/ \mathrm{cm} 3$. However, there were significant differences in concentrations between Shop A and Shop B. The number of persons vaping influenced the rate of decline of measured UFP numbers.

Conclusions: Room size, distance from the vaping activity, air filtration systems and the number of persons vaping influence the number of UFPs measured during e-cigarette use. High UFP numbers suggest a possibility of increased health risk for exposed bystanders and employees in these shops. Similar studies conducted in real-world settings are required to make scientifically sound conclusions about effects of vaping on indoor air quality.

Tob. Induc. Dis. 2018;16(Suppl 1):A489

DOI: $10.18332 /$ tid/83768

$16: 30-18: 00$

F0-377-4 Serum cotinine by socioeconomic status and exposure to second hand smoke in a national sample of South Africans

R Sewpaul', P Reddy ${ }^{1}$, R Desai ${ }^{1}$

'Human Science Research Council, Population Health, Health Systems and Innovation, Cape Town, South Africa. E-mail: rsewpaul@hsrc.ac.za

Background: Cotinine is a widely-used biomarker of exposure to tobacco for both active and second-hand smoke (SHS). Given South Africa's tobacco control laws restricting smoking in public places in the last decade, we assessed the prevalence and extent of exposure to tobacco by socio-demographic characteristics, smoking status, and exposure to SHS in the home.

Methods: Data was analysed from the South African National 
Health and Nutrition Examination Survey. Interview and serum cotinine biomarker data were collected. A cotinine cut-off point of $10 \mathrm{ng} / \mathrm{ml}$ for no tobacco exposure was used in the South Africa context, and values over 300 indicated heavy tobacco smoking. Weighted multiple logistic and linear regressions were used to determine the factors associated with the presence and level of cotinine.

Results: Cotinine was detected in $63.3 \%$ of individuals aged $\geq 15$ years $(\mathrm{n}=4621)$, where $28.4 \%$ had values of $\varsigma 10 \mathrm{ng} / \mathrm{ml}, 22 \%$ had $10-300 \mathrm{ng} / \mathrm{ml}$, and $12.9 \%$ above $300 \mathrm{ng} / \mathrm{ml}$. Mean cotinine was 252.6 [235.7-269.5] for current smokers and 237.9 [197.8-278.0] for smokeless tobacco users. Among current smokers, mixed race ethnicity and being male were significantly associated with higher levels of cotinine whereas mean cotinine did not vary by exposure to SHS at home. Tobacco exposure $(>10 \mathrm{ng} / \mathrm{ml})$ was found in $18.7 \%$ of never users of any tobacco product. Among never users, the odds of tobacco exposure were significantly $(\mathrm{p}<$ $0.05)$ higher for those exposed to SHS in their homes ( $\mathrm{OR}=1.59)$, for males (1.56), African (2.16) and mixed race (2.23) ethnic groups, older ages, those residing in rural formal areas (2.57), and in low income (2.27) and intermediate income (1.66) households. Conclusions: The findings provide insight into the factors associated with tobacco exposure. A substantial number of never users had cotinine in their blood, which calls for increased tobacco control efforts to protect people from the harmful effects of tobacco smoke.

Tob. Induc. Dis. 2018;16(Suppl 1):A490

DOI:10.18332/tid/84400

\section{$16: 30-18: 00$}

F0-382-4 Secondhand smoke exposure, perceived risks and knowledge of the national tobacco law among non-smoking adults in outdoor motor parks in an urban area, Lagos, Nigeria

E Ezekiel $^{1}$, S Tomini ${ }^{2}, 0$ Odukoya ${ }^{3}$

${ }^{1}$ Lagos University Teaching Hospital, Department of Community Health, Lagos, Nigeria, ${ }^{2}$ University of Liverpool, Liverpool, United Kingdom, ${ }^{3}$ University of Lagos, Department of Community Health and Primaary Care, College of Medicine, Lagos, Nigeria. E-mail: evbu.ezekiel@gmail.com

Background: Secondhand smoke (SHS) exposure is a global public health concern, to which no safe limits exist. Exposure in outdoor public places poses great health risks. Smoking occurs illicitly in motor parks despite a national tobacco law in place. This study identified the prevalence of SHS exposure among nonsmoking adults in Eti-Osa Local Government Area (LGA) and the relationship between knowledge of the tobacco law and perceived risks of SHS exposure.

Methods: A cross-sectional study of 399 non-smoking adults (18yrs and over) was conducted by systematic sampling. Interviewer administered modified GATS questionnaire was used to obtain responses from drivers, conductors, traders and passengers on socio-demographics, SHS exposure, perceived risk of SHS exposure and knowledge of the national tobacco law. Data was analyzed using SPSS version 21. Bivariate and multivariate analyses were conducted with statistical significance at $95 \%$ confidence interval.

Results: The prevalence of SHS exposure in motor parks was $54.6 \%$, more in middle aged males with low level of education. Frequent use of the motor parks was associated with SHS exposure. The perceived risks of being SHS exposed was good (69.8\%) and there was a positive relationship with level of education and health status. There was poor knowledge of the national tobacco law (44.2\%) despite good perceived risk of SHS exposure. Following logistic regression, higher level of education was associated with good perceived risks of SHS exposure $(\mathrm{OR}=0.31 ; 95 \% \mathrm{CI}=$ $0.12-0.79)$ and good knowledge was negatively associated with perceived risks $(\mathrm{OR}=1.81 ; 95 \% \mathrm{CI}=1.17-2.79)$.

Conclusions: More than half of the participants were exposed to SHS and despite good perceived risks of SHS exposure, knowledge of the national tobacco law was poor. These findings warrant increased public health awareness and calls for policy makers and local authorities to ensure implementation of the law in the public places.

\section{Tob. Induc. Dis. 2018;16(Suppl 1):A491}

DOI: $10.18332 /$ tid/84641

\section{8:15-19:15}

LB-1328-4 Pattern of smokeless tobacco use and cigarette smoking among adult South Africans during 2007-2016

C Ezeh 1 , OA Ayo-Yusuf ${ }^{1}$

'Sefako Makgatho Health Sciences University, Medunsa, Pretoria, South Africa. E-mail: gozieze@yahoo.com

Background: Alternative tobacco products including smokeless tobacco have been marketed as harm reduction products particularly after 2010 when Phillip Morris International (PMI) bought Smokeless tobacco (SLT) business from Swedish Match. However, little information is available on tobacco use pattern. This study therefore sought to report the pattern of cigarette smoking and smokeless tobacco (SLT) use in South Africa.

Methods: This study involved a nationally representative sample of South African adults aged $\geq 16$ years who participated in the South African Social Attitude Surveys (SASAS). The SASAS used a face-to-face interviewer-administered questionnaire to obtain information on participants' sociodemographic characteristics and tobacco use behaviour. Data obtained from the $2007(\mathrm{n}=2907)$, $2011(n=3004)$ and $2016(n=3063)$ surveys were merged and the resultant dataset was used in the analysis. All statistical test took account of the complex sample design used in the SASAS.

Results: Exclusive smoking was estimated at 20.3\% (95\%CI $=18.1$ $22.5)$ in $2007,18.8 \%(95 \% \mathrm{CI}=16.8-21.0)$ in 2011 , increased to $19.3 \%(95 \% \mathrm{CI}=17.1-21.6)$ in 2016 . Similarly, exclusive snuff use reduced from $4.5 \%(95 \% \mathrm{CI}=3.4-6.0)$ in $2007,1.7 \%$ $(95 \% \mathrm{CI}=1.2-2.3)$ in 2011 , but then increased significantly to $3.2 \%$ $(95 \% \mathrm{CI}=2.4-4.2)$ in 2016. Dual use during 2007, 2011 and 2016 were $0.5 \%, 0.5 \%$ and $0.8 \%$ respectively. Unlike smoking, Snuff use was highest among females (6\%), black Africans (4.5\%), rural residents $(5.1 \%)$ and those with $<$ Grade 12 education (5.6\%). Among smokers, current snuff use was not associated with significantly greater odds of a quit attempt in the past 12 months $(\mathrm{OR}=2.58 ; 95 \% \mathrm{CI}=0.94-7.10)$.

Conclusions: The promotion of snuff as harm reduction alternative products in South Africa does not appear to have been associated with significant reduction in smoking, but might have resulted in significant increase in snuff use in recent times.

Tob. Induc. Dis. 2018;16(Suppl 1):A492

DOI:10.18332/tid/84759

\section{$18: 15-19: 15$}

LB-1331-4 Prevalence of Midwakh tobacco smoking in trend-setting Lebanon: an indicator of potential spread across the Arab world?

R Afifi ${ }^{1}$, L Ghandour ${ }^{2}$, N El Salibi², R Nakkash ${ }^{3}$, A Rady ${ }^{4}$, S 


\section{Sherman ${ }^{5}$}

${ }^{1}$ University of Iowa College of Public Health, Department of Community and Behavioral Health, lowa City, IA, United States of America, ${ }^{2}$ American University of Beirut Faculty of Health Sciences, Epidemiology and Population Health, Beirut, Lebanon, ${ }^{3}$ American University of Beirut Faculty of Health Sciences, Health Promotion and Community Health, Beirut, Lebanon, ${ }^{4}$ World Health Organisation - Lebanon, Beirut, Lebanon, ${ }^{5} \mathrm{New}$ York University School of Medicine, New York, NY, United States of America. E-mail: rema-afifi@uiowa.edu

Background: Alternative tobacco product (ATP) use is increasing in Lebanon. Dokha (or 'diziness') is an ATP smoked in a pipe called a midwakh. Research evidence suggests high nicotine content, with resultant physiologic effects. The objective of this study was to assess prevalence and correlates of midwakh tobacco use among 7th-12th grade students in Lebanon.

Methods: The analyses is based on one question included in the 2016 Lebanon Global School-based Student Health Survey (GSHS) that assessed past 30-day (current) use of midwakh in a representative sample of 5708 7th-12th graders sampled from public and private schools. All analyses were weighted accounting for the multi-stage cluster sampling design.

Results: Overall, $4.6 \%$ of students were current midwakh users, varying between $3.9 \%-5.4 \%$ across grade levels. A significantly higher percentage of males than females currently smoked midwakh $(6.7 \%$ versus $2.7 \%$ respectively). Current use was higher in public versus private school students (6.3\% vs. $3.6 \%$ respectively) but this difference was not statistically significant. Midwakh use was significantly associated with current smoking of cigarettes [OR: $16.61 ; 95 \% \mathrm{CI}=12.21-22.58]$, as well as initiating cigarette smoking prior to 14 years of age among cigarette smokers [OR: $2.31 ; 95 \% \mathrm{CI}=1.53-3.49]$. Midwakh smoking was also statistically significantly related to ever smoking of waterpipe [OR: $9.89 ; 95 \% \mathrm{CI}=6.78-14.45]$, current use of any tobacco products other than cigarettes [OR: $14.29 ; 95 \% \mathrm{CI}=8.53-23.94]$, parental smoking [OR: $1.61 ; 95 \% \mathrm{CI}=1.13-2.2]$, and with secondhand smoking [OR: $1.73 ; 95 \% \mathrm{CI}=1.11-2.70]$.

Conclusions: This study is one of the few that has provided prevalence rates and determinants of midwakh use outside of the Arabian Gulf, where it has been mostly confined. Although prevalence remains low, its consistency across grade levels is concerning. In addition, the pattern of associations is in line with evidence on other tobacco products. A trans-disciplinary research agenda for midwakh is urgently needed to understand use and effects, and contain its spread.

Tob. Induc. Dis. 2018;16(Suppl 1):A493

DOI:10.18332/tid/84722
$18: 15-19: 15$

LB-1332-4 Antenatal tobacco smoke exposure: impact on infant birth outcomes and lower respiratory tract infection in a South African birth cohort

A Vanker ${ }^{1}$, PM Nduru ${ }^{1}, \mathrm{RP} \mathrm{Gie}^{2}$, HJ Zar ${ }^{1}$, Drakenstein Child Health Study

${ }^{1}$ University of Cape Town and MRC unit on child and adolescent health, Paediatrics and Child Health, Cape Town, South Africa, ${ }^{2}$ Stellenbosch University, Paediatrics and Child Health, Cape Town, South Africa. E-mail: aneesa.vanker@uct.ac.za

Background: The impact of antenatal tobacco smoke exposure, on child health outcomes has not been well studied in African children.

This study investigated the prevalence of antenatal tobacco smoke exposure and the associations with infant birth outcomes and lower respiratory tract infection (LRTI) in the Drakenstein Child Health Study (DCHS), a South African birth cohort.

Methods: Consenting pregnant women were enrolled antenatally and mother-infant pairs followed from birth through the first year of life. Self-reported questionnaires assessed maternal and household smoking and maternal urine cotinine collected antenatally and at birth was measured. Birth outcomes including weight-for-age (WfA) z scores, low birth weight (LBW) and small for gestational age (SGA) were recorded. LRTI defined using WHO criteria was documented by trained study staff during the first year of life. Linear (WfA z-score), logistic (LBW, SGA) and Poisson (LRTI) regressions were used to investigate associations with tobacco smoke exposure.

Results: 1137 women were enrolled with 1143 live births. Urine cotinine measures classified 352/1093 (32\%) as active and $479 / 1093$ (44\%) as passive smokers. Median birth weight was 3,085 (IQR2,710 - 3,420) kg; 524 episodes of LRTI occurred. Infants of active smokers had a 0.32 (95\%CI 0.10 - 0.53) lower WfA z score and almost doubled risk of being SGA [OR 1.71 (95\%CI 1.11 - 2.64)]. Maternal smoking was associated with an increased risk of LRTI [IR 1.56 (95\%CI1.25 - 1.93)].

Conclusions: There was a high prevalence of antenatal maternal smoking and tobacco smoke exposure which significantly impacted on infant birth outcomes and LRTI incidence in infants. Urgent and effective interventions to reduce antenatal tobacco smoke exposure are required to improve child health.

Funding: Bill \& Melinda Gates Foundation (OPP1017641), Discovery Foundation, South African Thoracic Society AstraZeneca Respiratory Fellowship, CIDRI Clinical Fellowship, Medical Research Council, South Africa, National Research Foundation, South Africa

Tob. Induc. Dis. 2018;16(Suppl 1):A494

DOI:10.18332/tid/84757

[Tobacco smoke exposure, birth outcomes and LRTI] $18: 15-19: 15$

\begin{tabular}{|c|c|c|c|c|c|c|c|c|}
\hline Variable & $\begin{array}{l}\text { WRA Z score } \\
\text { (regression } \\
\text { co-efficients) }\end{array}$ & $\begin{array}{l}\text { Wra Z score } \\
\text { (regression } \\
\text { co efficients) }\end{array}$ & $\begin{array}{l}\text { Low birth } \\
\text { weight } \\
\text { (OR) }\end{array}$ & $\begin{array}{l}\text { Low birth } \\
\text { weight } \\
\text { (OR) }\end{array}$ & $\begin{array}{l}\text { Simall for } \\
\text { Gestational } \\
\text { age (OR) }\end{array}$ & $\begin{array}{l}\text { Small for } \\
\text { Gestational } \\
\text { age (OR) }\end{array}$ & LRTI (IRR) & LRTI (IRR) \\
\hline $\begin{array}{l}\text { Antepartum } \\
\text { smoking }\end{array}$ & Crude & Adjusted & Crude & Adjusted & Crude & Adjusted & Crude & Adjusted \\
\hline Active & $\begin{array}{l}-0.44 \\
(-0.62-0.26)\end{array}$ & $\begin{array}{l}-0.32 \\
(-0.53--0.10)\end{array}$ & $\begin{array}{l}1.91 \\
(1.21-3.05)\end{array}$ & $\begin{array}{l}1.40 \\
(0.80-2.47)\end{array}$ & $\begin{array}{l}1.83 \\
(1.28-2.63)\end{array}$ & $\begin{array}{l}1.71 \\
(1.11-2.64)\end{array}$ & $\begin{array}{l}1.40 \\
(1.16-1.69)\end{array}$ & $\begin{array}{l}1.56 \\
(1.25-1.93)\end{array}$ \\
\hline Passive & $\begin{array}{l}-0.01 \\
(-0.18-0.16)\end{array}$ & $\begin{array}{l}0.05 \\
(-0.13-0.23)\end{array}$ & $\begin{array}{l}1.13 \\
(0.71-1.80)\end{array}$ & $\begin{array}{l}1.05 \\
(0.63-1.75)\end{array}$ & $\begin{array}{l}0.80 \\
(0.55-1.16)\end{array}$ & $\begin{array}{l}0.79 \\
(0.53-1.17)\end{array}$ & $\begin{array}{l}1.09 \\
(0.91-1.32)\end{array}$ & $\begin{array}{l}1.11 \\
(0.91-1.35)\end{array}$ \\
\hline
\end{tabular}


LB-1333-4 Use of electronic nicotine delivery systems (ENDS) among Chinese adults: evidence from citywide representative surveys from 12 cities in China (2014 to 2016)

J Huang', P Redmon', Z Duan'1, Y Duan'1, Y Jiang'², M Eriksen ${ }^{1}$ 'Georgia State University, School of Public Health, Atlanta, GA, United States of America, ${ }^{2}$ China CDC, Beijing, China. E-mail: predmon@gsu.edu

Background: Tobacco is the leading cause of preventable death in China. Any comprehensive strategies to reduce tobacco use in China need to increase smoking cessation among current smokers. While the evidence is limited, ENDS may reduce the health risks for current smokers if they switch completely to ENDS. ENDS have the potential of reducing harms of tobacco smoking if Chinese smokers who do not intent to quit can switch completely to ENDS. To systematically assess the awareness and use of ENDS among Chinese adults, Tobacco Questions for Surveys (TQS) was conducted in twelve Chinese cities from 2014 to 2016. Methods: Citywide representative surveys were conducted in 12 Chinese cities between 2014 and 2016. The target population was noninstitutionalized urban adults age 15 and above. Multistage cluster sampling was applied to select 2,500 individuals from each city. SAS and R were used to obtain point estimates and adjusted associations accounting for the complex sample design features.

Results: Awareness of ENDS was above $40 \%$ in all 12 cities, the percent of respondents who have heard about ENDS ranging from 41.3\% (Qingdao) to $63.2 \%$ (Xi'an). The overall rate of ever use ENDS was $2.9 \%$, and the overall rate of current use was $0.9 \%$. In addition, across most cities, awareness of ENDS were statistically significantly associated with current smoking, being male, aging 25-34, and having higher education.

Conclusions: Significant variations existed in awareness and use of ENDS among urban Chinese adults. Tailored interventions, as part of a comprehensive tobacco control and cessation strategy, which may include ENDS in assisting smokers in achieving complete cessation, may have a positive public health impact in China. In addition, nation-wide surveillance is needed to monitor the use of ENDS among both adult smokers and among adolescents and young adults, with an emphasis on the complete abstinence from any nicotine product among adolescents.

Tob. Induc. Dis. 2018;16(Suppl 1):A495

DOI:10.18332/tid/84700

\section{8:15-19:15}

LB-1334-4 Exposure to second-hand aerosol produced by electronic cigarettes: a systematic review

E Fernández'1 M Fu², JM Martínez-Sánchez ${ }^{3}$, 0 Tigova² ${ }^{2}$ A Peruga ${ }^{4}$, TackSHS Project Investigators

'Catalan Institute of Oncology // Bellvitge Biomedical Research Institute (IDIBELL), Tobacco Control Unit, L'Hospitalet de Llobregat (Barcelona), Spain, ${ }^{2}$ Catalan Institute of Oncology // Bellvitge Biomedical Research Institute (IDIBELL), Barcelona, Spain, ${ }^{3}$ Catalan Institute of Oncology (ICO), L'Hospitalet de Llobregat (Barcelona), Spain, ${ }^{4}$ Universidad del Desarrollo, Santiago de Chile, Chile.E-mail: efernandez@iconcologia.net Background: Since 2007, the use of electronic cigarettes has grown rapidly around the world. This trend raises concerns about the potential exposure of bystanders to aerosols from electronic cigarettes, particularly considering that its use in public workplaces and other enclosed places is not regulated in most countries. This study aimed to review systematically the evidence on the content of, and exposure to, second-hand aerosols (SHA) from electronic cigarettes and other smoking-proxy electronic inhaling systems (SEIS).

Methods: A systematic review of original articles published up to August 2016 was performed using PubMed and Web of Science databases. The search strategy combined the topics "SEIS", "aerosol", and "second-hand exposure". The studies included in the selected articles were described according to whether they focused on aerosols produced by humans (SHA) or generated by smoking machines (first-hand aerosol, FHA), including aerosol composition and the exposure to, or effects of, the aerosol in humans.

Results: Some articles included more than one study, thus 62 studies from 58 publications were included into the final review. Twenty studies focused on SHA (18 analyzed its composition and 2 assessed human exposure) and 45 studies focused on FHA (43 analyzed its composition and 2 assessed human exposure). The content and concentration of toxic chemicals emitted from SEIS are highly variable, and include propylene glycol, particulate matter, volatile organic compounds, heavy metals, and nicotine. These chemicals could be taken into bystanders' bodies, as evidenced by cotinine measurements. The levels of some metals, such as nickel and chromium, are higher in SHA from SEIS than in second-hand tobacco smoke and certainly in background air.

Conclusions: SHA is a new air contamination source for toxic chemicals. Their concentration is higher than background levels and the associated health risks are still empirically unknown.

Tob. Induc. Dis. 2018;16(Suppl 1):A496

DOI: $10.18332 /$ tid $/ 84730$

\subsection{Burden and Disparities in tobacco related diseases: Non communicable diseases (NCDs) and communicable diseases (TB and IIIV/AIDS)}

\section{$16: 30-18: 00$}

F0-378-4 Healthcare costs attributable to secondhand smoke exposure at home for U.S. adults T Yao' ${ }^{1}$ H-Y Sung ${ }^{1}$, Y Wang ${ }^{1}$, J Lightwood ${ }^{1}$, W Max

'University of California San Francisco, San Francisco, CA, United States of America. E-mail: tingting.yao@ucsf.edu

Background: Secondhand smoke (SHS) exposure has been linked to numerous health conditions. The harmful health effects of SHS exposure have resulted in excess economic costs. National-level studies on the economic impact of SHS exposure are limited in the U.S. To provide the latest information on the national-level costs of SHS exposure in U.S., this study estimated healthcare costs attributable to SHS exposure at home among nonsmoking adults (18+) in the U.S. in 2000, 2005, and 2010.

Methods: We analyzed nonsmoking adults $(\mathrm{N}=67,735)$ using data from the 2000, 2005, and 2010 (the latest avaiable data on SHS exposure) U.S. National Health Interview Surveys. We examined hospital nights, home health care visits, doctor visits, and emergency room (ER) visits. For each, we analyzed the association of SHS exposure at home with healthcare utilization with a Zero-Inflated Poisson regression model controlling for socio-demographic and other risk characteristics (including age, gender, race and ethnicity, education, poverty status, and number of months without health insurance). Excess healthcare utilization attributable to SHS exposure was determined and multiplied by unit costs derived from the 2014 Medical Expenditures Panel Survey to determine SHS- 
attributable healthcare costs.

Results: SHS exposure was positively associated with hospital nights and ER visits, but was not statistically associated with home health care visits and doctor visits. The total annual SHSattributable healthcare costs in 2000, 2005 and 2010 were $\$ 4.6$ billion (including $\$ 3.8$ billion for hospital nights and $\$ 0.8$ billion for ER), $\$ 2.1$ billion (including $\$ 1.8$ billion for hospital nights and $\$ 0.3$ billion for ER), and $\$ 1.9$ billion (including $\$ 1.6$ billion for hospital nights and $\$ 0.4$ billion for ER), respectively.

Conclusions: SHS-attributable costs remain high, but have fallen over time. Tobacco control efforts are needed to further reduce SHS exposure and associated healthcare costs.

Tob. Induc. Dis. 2018;16(Suppl 1):A497

DOI:10.18332/tid/84564

\section{6:30-18:00}

F0-379-4 Association of household tobacco exposure with recent respiratory symptoms and medical services utilisation in Hong Kong young children under 2 years old

S Dai', KCC Chan ${ }^{1}$

${ }^{1}$ Chinese University of Hong Kong, Department of Paediatrics, Faculty of Medicine, Hong Kong, Hong Kong. E-mail: daisiyu@ link.cuhk.edu.hk

Background: This study aimed to explore the association between household tobacco exposure and recent respiratory symptoms and medical service utilisations in Hong Kong young children.

Methods: Analysis was performed on data obtained from a community-based cross-sectional pneumococcal carriage surveillance study of healthy children aged under 2 years. Information on demographics, household tobacco exposure status (any household smokers), household tobacco exposure level (total number of household smokers, total number of cigarettes consumed by household smokers per day), children's recent respiratory symptoms in 3 days and 1 month as well as children's recent medical services utilisation in 3 months were obtained by parent-reported questionnaires.

Results: 1541 subjects (mean age: 11.2 months, male: $50.7 \%$ ) were recruited from June 2013 to June 2014. The prevalence of household tobacco exposure was $31.5 \%$, prevalence of prenatal and postnatal maternal smokingwere $1.6 \%$ and $3.5 \%$ respectively. After adjustment for potential confounders, children's household tobacco exposure ( $\mathrm{AOR}=1.33,95 \% \mathrm{CI}: 1.03-1.70)$ and postnatal maternal smoking exposure $(\mathrm{AOR}=2.30,95 \%$ CI: 1.09-4.85) were significantly and independently associated with all-cause doctor consultation in recent 3 months; postnatal maternal smoking exposure $(\mathrm{AOR}=2.70,95 \% \mathrm{CI}: 1.16-6.27)$ was significantly and independently associated with all-cause hospitalisation in recent 3 months. In addition, children with household smokers consuming more than 20 cigarettes/day $(\mathrm{AOR}=3.47,95 \% \mathrm{CI}: 1.94-10.08)$ had adjusted higher risk of having recent respiratory symptoms in 1 month compared with those non exposed.

Conclusions: Household tobacco exposure was associated with recent medical service utilisation and respiratory symptoms in Hong Kong young children. As home is the most significant source of environmental tobacco exposure for young children, efforts for reducing such exposure are essential.

Tob. Induc. Dis. 2018;16(Suppl 1):A498

DOI:10.18332/tid/83924

16:30-18:00
FO-380-4 Morbidity attributable to secondhand smoke exposure in children under 5 years old in Spain, 2015

X Continente ${ }^{1,2}$, MJ López ${ }^{1,2}, T$ Arechavala ${ }^{1,2}$, M Pérez-Ríos ${ }^{3}, \mathrm{E}$ Fernánde $z^{4}$, A Schiaffino ${ }^{5}$

${ }^{1}$ Public Health Agency of Barcelona, Barcelona, Spain, ${ }^{2}$ CIBERESP, Madrid, Spain, ${ }^{3}$ Galician Directorate for Public Health, Galician Health Authority, Xunta de Galicia, Santiago de Compostela, Spain, ${ }^{4}$ Catalan Institute of Oncology (ICO), Bellvitge Biomedical Research Institute (IDIBELL), Barcelona, Spain, ${ }^{5}$ Generalitat de Catalunya, Departament de Salut, Barcelona, Spain. E-mail: efernandez@iconcologia.net

Background: Children are especially vulnerable to the detrimental health effects of secondhand smoke (SHS), which has been widely linked to asthma and otitis media (OM), among other diseases. This study aimed to estimate hospital admissions and disabilityadjusted life years (DALY) attributable to SHS exposure in terms of asthma and OM among children under 5 years old in Spain during the year 2015 .

Methods: The prevalence of SHS exposure in children under 5 was estimated through a telephone survey performed in 2016 in a representative sample of Spanish households with children. The Rate risks for asthma and otitis media were selected from different published international meta-analysis. The number of hospital admissions were obtained for each disease from the Minimum Basic Data Set provided by the Ministry of Health of Spain. Information about DALYs were obtained from estimations made by the World Health Organization. Both hospital admissions and DALYs attributable to SHS exposure were calculated using the population attributable fraction (PAF).

Results: $25.7 \%$ of children under 5 are exposed to SHS at home (any household member reported smoking either inside or on balconies or terraces). According to the literature, SHS exposure increases the risk of both asthma and OM by 1.32 . The estimated percentage of children presenting each disease attributable to SHS exposure at home is $7.6 \%$. In 2015, the number of children with at least one hospital admission for asthma $(n=2,451)$ and OM $(n=1,226)$ attributable to SHS exposure was 186 and 93, respectively. Likewise, attributable DALYs were 273 for asthma and 159 for OM.

Conclusions: $7.6 \%$ of burden disease for asthma and OM among young children is due to SHS exposure at home. This figure highlights the need of implementing smoke-free home interventions to reduce hospital admissions and DALYs for asthma and OM.

Tob. Induc. Dis. 2018;16(Suppl 1):A499

DOI:10.18332/tid/84399

\section{8:15-19:15}

LB-1326-4 Patterns of lung cancer mortality in Russia over a 16-year period, 2000-2015

I Zarubina', Y Neumark'

${ }^{1}$ Hebrew University, Braun School of Public Health, Jerusalem, Israel.E-mail: yehudan@ekmd.huji.ac.il

Background: Tobacco smoking has been historically high in Russian men, and steadily increased in Russian women following the Soviet Union's collapse. Much has been published about alcohol consumption and alcohol-related mortality in Russia, however, few studies have assessed regional tobacco-related mortality. This study describes regional and gender patterns of lung cancer mortality, as a marker for tobacco-related mortality, in Russia during the period 2000-2015.

Methods: Age-adjusted lung cancer mortality rates by gender and 
for eight federal districts and 83 regions were calculated based on mortality and population data extracted from the Russian Fertility and Mortality Database. Annual percentage changes in age-adjusted lung cancer mortality on the national and federal district levels were estimated, and average age-adjusted lung cancer mortality rates were mapped. Two-way ANOVA was applied to assess time-district interaction effects on lung cancer mortality.

Results: Age-adjusted lung cancer mortality rates were consistently higher in eastern and central regions of Russia. At the national level, a monotonic decline in lung cancer mortality during the 16-year period was noted among men (annual \% change $=1.9 \%$ ), while among women, national lung cancer mortality rates remained unchanged for most years. Differences in age-adjusted lung cancer mortality across federal districts and regions over time, as well as between the sexes, were noted. A strong time-district interaction for age-adjusted lung cancer mortality $(\mathrm{F}=1.810, \mathrm{p}<0.028)$, and age-adjusted lung cancer mortality male-to-female ratio $(\mathrm{F}=3.267$, $\mathrm{p}<0.001$ ) were found.

Conclusions: Given the significant increase in tobacco smoking prevalence among Russian women that began the 1990s, female lung cancer mortality will continue to rise in the coming decades, with a disproportionate burden on those regions with insufficient health services and located far from the federal centers. Additional work is needed to identify factors underlying the regional and sex differences.

Tob. Induc. Dis. 2018;16(Suppl 1):A500

DOI:10.18332/tid/84726

\section{$18: 15-19: 15$}

LB-1327-4 Tobacco use among tuberculosis patients across 30 districts in India in 2013 and 2017

K Sagili ${ }^{1}$, S Chadha ${ }^{1}$, S Satyanarayana ${ }^{1}$

${ }^{1}$ The Union South East Asia Office, New Delhi, India. E-mail: ksagili@theunion.org

Background: In 2016, India estimated about 2.8 million tuberculosis (TB) cases (Global TB Report 2017) and about 267 million adults (>15years) in India using tobacco in any form (GATS 2017). Tobacco is one of the important drivers of TB epidemic. The Union South East Asia office just concluded the Endline Knowledge, Attitude and Practice (KAP) survey on TB across 30 districts in India among various stakeholders including TB patients. As part of the survey, TB patient's tobacco use was also captured.

Methods: The Endline KAP survey methodology was similar to the previous surveys (midline in 2013 and baseline in 2011) except for data captured digitally in endline and paper based in previous surveys. Briefly, the survey was conducted as part of a large Global Fund supported TB project "Axshya" being implemented across 300 districts. 30 districts were selected using systematic random sampling and from each district 10 primary sampling units were selected randomly. The data was collected during October-November 2017. The survey identified about 307 self-reported TB patients. Pre-tested questionnaires were used after obtaining patient consent.

Results: The overall tobacco users in Endline were $34 \%$ $(\mathrm{N}=103 / 307)$ and in Midline 30\% $(\mathrm{N}=135 / 496)$. Among males, the proportion remained same about $43 \%$ in both the surveys, however among women this increased marginally to $14 \%$ from $12 \%$. Proportion of those who smoke decreased from $37 \%$ in midline to $31 \%$ in endline. And the proportion of those who use smokeless tobacco increased from $55 \%$ to $59 \%$ respectively. Interestingly, proportion of men using smokeless tobacco increased and that of women decreased. However these differences are statistically not significant.

Conclusions: The preliminary analysis shows proportion of tobacco users among TB patients is marginally increased. Though the overall tobacco users in India decreased, tobacco use among TB patients seems unchanged, indicating nothing significant has been done for tobacco cessation among TB patients.

Figure 1: Graph comparing the overall proportions of tobacco use and type of tobacco use in 2017 (Endline survey) and 2012-13 (Midline survey)

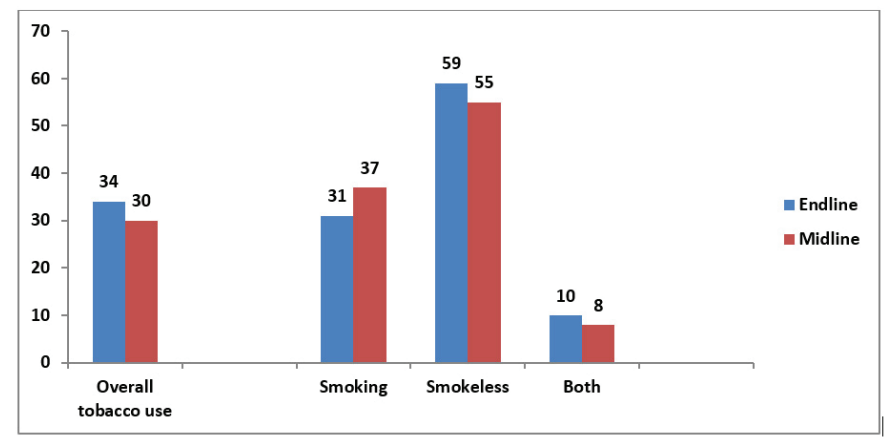

Endline survey N=103/307; Midline survey N=135/496

Tob. Induc. Dis. 2018;16(Suppl 1):A501 DOI:10.18332/tid/84747

\subsection{New tools for monitoring policy impact and tobacco use surveillance}

$14: 45-16: 15$

F0-362-4 Smokeless tobacco use among adults in ten countries of SEA region

S Satpathy'

${ }^{1}$ KIIT School of Public Health, Public Health, Bhubaneswar, India. E-mail: sudhirksatpathy@gmail.com

Background: Smokeless tobacco has gained more importance following its increased use in the post-smoking ban era. This has more significance in SEA region as it is the hub of smokeless tobacco manufacturing and use. However its assessment among adults from national surveys like global adult tobacco survey (GATS) is limited. This study compiles the NCD risk factor surveillance data as well as GATS data to have a complete picture of SLT use among adults in SEA region.

Methods: Both the GATS and the NCD risk factor surveillance data during last decade were compiled and SLT uses among adults were compared. NCD RF surveillance data from Bhutan, Maldives, Myanmar, Nepal, Sri Lanka, and Timor Leste, as well as GATS data from Bangladesh, India and Thailand were analyzed. Results: The prevalence of SLT use among adults varied from nil in DPR Korea (2008) to 43.2\% in Myanmar (2014). Only Maldives and Thailand had shown prevalence of SLT use less than $5 \%$ and other countries had higher prevalence (>15\%). India and Bangladesh had prevalence of $24.3 \%$ and $27.2 \%$ respectively. Decreasing trend in SLT use in India, Thailand, Nepal etc. were observed. Myanmar had shown increase in use of SLTs. Except Timor Leste in all other nine countries men chewed more SLTs than their women counterparts.

Conclusions: There is wide variation in SLT use in SEA region. Countries showing higher prevalence and increasing trend of SLT 
use should be prioritized for tobacco control. Complementing tobacco use surveillance tools should always be compared or triangulated for a comprehensive picture of tobacco use burden. Higher use of SLTs among women in Timor Leste needs further exploration.

Tob. Induc. Dis. 2018;16(Suppl 1):A502

DOI: $10.18332 /$ tid/84563

\section{$14: 45-16: 15$}

F0-363-4 Tobacco surveillance through electronic data collection on the Android operating system - evidence from the Global Adult Tobacco Survey E Rainey ${ }^{1,2}$, J Morton ${ }^{1,2}$, S Litavecz ${ }^{3}$, D Plotner ${ }^{3}$, P Suchita $^{3}$, K Pallipudi

${ }^{1}$ CDC, Office on Smoking and Health, Atlanta, GA, United States of America, ${ }^{2} \mathrm{CDC}$ Foundation, Atlanta, GA, United States of America, ${ }^{3}$ RTI International, Research Triangle Park, NC, United States of America. E-mail: vdf2@cdc.gov

Background: The Global Adult Tobacco Survey (GATS), a nationally representative household survey launched in 2007, could benefit from a cost-effective and easy to use platform that included support for multiple-languages, the ability to field surveys in countries with limited resources, and the integration of diverse data management methodologies. To fill these gaps, a new Android-supported General Survey System (GSS), based on the previous Windows Mobile GSS platform, was developed for GATS.

Methods: The GSS for Android system was modeled after the previously developed Windows Mobile-based system. It is a suite of software tools for survey design, implementation, and reporting. It includes various data management models that were developed to work in diverse conditions: SIM based tablet or Wi-Fi transmission with cloud integration, File Transfer Protocol (FTP), migration of data over SD cards or USB cables, and use of Wi-Fi capable external drives.

Results: Since 2014, the GSS for Android system has been implemented in 15 countries that have fielded GATS, using Android hardware. Over 1900 tablets have been programmed in 40 languages, and more than 220,000 households have been surveyed with over 175,000 individuals interviewed. Due to a robust data management plan, effective quality control processes, and rigorous training methodology, no data loss has occurred. Currently, more than 660 million tobacco related data points have been captured, which will inform the publishing of 15 fact sheets and country reports.

Conclusions: GSS for Android has been a successful new tool for tobacco surveillance. The system architecture and variety of hardware platforms have shown adaptability across countries, irrespective of resource availability, internet and telecom capabilities, hardware requirements, and differing physical environments.

Tob. Induc. Dis. 2018;16(Suppl 1):A503

DOI:10.18332/tid/83846

\section{$14: 45-16: 15$}

F0-364-4 Using discrete choice experiments to characterize demand for waterpipe tobacco smoking among university students in the Eastern Mediterranean region

R Salloum ${ }^{1}$, N Abu-Rmeileh², Y Al-Farsi ${ }^{3}$, R Hamadeh $^{4}, \mathrm{~K}$ Kheirallah ${ }^{5}$, A Mostafa ${ }^{6}$, A Yusufali ${ }^{7}$, R Nakkash $^{8}$

${ }^{1}$ University of Florida, Health Outcomes and Policy, Gainesville, $\mathrm{FL}$, United States of America, ${ }^{2}$ Birzeit University, Birzeit, State of Palestine, ${ }^{3}$ Sultan Qaboos University, Muscat, Oman, ${ }^{4}$ Arabian Gulf University, Manama, Bahrain, ${ }^{5}$ Jordan University of Science and Technology, Irbid, Jordan, ${ }^{6}$ Ain Shams University, Cairo, Egypt, ${ }^{7}$ Dubai Medical College, Dubai, United Arab Emirates, ${ }^{8}$ American University of Beirut, Beirut, Lebanon. E-mail: rsalloum@ufl.edu

Background: Waterpipe smoking originated in the Eastern Mediterranean region. It is highly prevalent among university students, and has been increasing in popularity despite mounting evidence showing it is harmful to health. The aim of this study was to measure preferences for waterpipe smoking and determine which product characteristics are most important to smokers.

Methods: Participants in 5 countries (Egypt, Jordan, Oman, Palestine, and UAE) completed an Internet-based discrete choice experiment to reveal their preferences for, and trade-offs between, the attributes of hypothetical waterpipe smoking sessions. Participants were presented with waterpipe lounge menus, each with 6 fruit-flavored options and 1 tobacco flavored option, in addition to an opt out option. Participants were randomized to either receive menus with a pictorial+text health-warning message or no message. Multinomial and nested logit models were used to estimate the impact on consumer choice of attributes and between-subject assignment of health warnings respectively. Results: On average, participants preferred fruit-flavored varieties to tobacco flavor. They were averse to options labelled with higher nicotine content. Females and non-smokers of cigarettes were more likely than their counterparts to prefer flavored and nicotine-free varieties. Participants exposed to a health warning were more likely to opt out.

Conclusions: Fruit-flavored tobacco and lower nicotine content labels, two strategies widely used by the industry, increase the demand for waterpipe smoking among young adults. Waterpipespecific regulation should limit the availability of flavored waterpipe tobacco and require accurate labelling of constituents. Waterpipespecific tobacco control regulation, along with research to inform policy, is required to curb this emerging public health threat.

Tob. Induc. Dis. 2018;16(Suppl 1):A504 DOI:10.18332/tid/84026

\section{$14: 45-16: 15$}

FO-365-4 Changes over time in illicit cigarette use in Brazil, overall and by educational level: proposed novel approach and results

A Szklo1, R Iglesias², M de Souza1, M Szklo ${ }^{3}, \mathrm{~L}$ de Almeida ${ }^{4}$

${ }^{1}$ Brazilian National Cancer Institute, Rio de Janeiro, RJ, Brazil, ${ }^{2}$ Center of Studies on Integration and Development (CINDES), Rio de Janeiro, RJ, Brazil, ${ }^{3}$ The Johns Hopkins Bloomberg School of Public Health, Epidemiology, Baltimore, MD, United States of America, ${ }^{4}$ Brazilian National Cancer Institute, Epidemiology, Rio de Janeiro,RJ, Brazil. E-mail: aszklo@inca.gov.br

Background: The strategy of raising taxes has reduced smoking prevalence in Brazil in the last few years. However, given the presence of illicit cigarette products in Brazil, smokers may have migrated to cheaper illicit cigarettes in order to save money, thus undermining efforts to further reduce smoking prevalence, mostly among underprivileged smokers. The aim of the present study was to estimate the proportions of illicit cigarette consumption in Brazil from 2012 to 2016.

Methods: We used an approach based on legal sales provided by the Secretariat of Federal Revenues and self-reported consumption data from a phone survey conducted annually (VIGITEL) to estimate the changes over time in illegal cigarette use in Brazil, 
overall and by educational level. For that purpose, we also used available information on the proportion of illegal cigarette consumption from a nationwide household survey conducted in 2013 to calculate a constant proportion of under-reporting from VIGITEL in relation to total sales in Brazil.

Results: There was an increase in the estimated proportion of illicit cigarette use from 2012 to $2013(+12.9 \%)$, then a decrease from 2013 to 2014 (-10.8\%), and then a sustained trend of increase from 2014 to 2016 ( $+26.7 \%$, from 2014 to $2015 ;+17.3 \%$, from 2015 to 2016). The same pattern was observed for both high and low educational level smokers. For each year, the latter had higher baseline proportions of illegal cigarette use.

Conclusions: Novel and feasible approaches to estimate the changes over time in illegal market are important to help in the effective implementation of tobacco excise tax policy.

Tob. Induc. Dis. 2018;16(Suppl 1):A505

DOI:10.18332/tid/83752

\section{$14: 45-16: 15$}

F0-366-4 Flavor capsule cigarettes in six countries: availability by brand, variant and flavor

J Brown ${ }^{1,2}$, J Cohen ${ }^{1,2}, \mathrm{~K}^{2}$ Smith $^{1,2}$

'Johns Hopkins Bloomberg School of Public Health, Health, Behavior \&t Society, Baltimore, MD, United States of America, ${ }^{2}$ Institute for Global Tobacco Control at the Johns Hopkins Bloomberg School of Public Health, Baltimore, MD, United States of America. E-mail: jbrow212@jhu.edu

Background: Flavor capsule cigarettes, containing capsules inside the filters that smokers can press to release a liquid that flavors smoke, are growing in popularity. Flavor capsule variants (FCVs) may appeal to youth and result in misperceptions of relative harm. We examined the FCVs on the market in six countries and describe their availability by variant and brand between 2013 and 2015/2016, as well as available flavors in 2015/2016.

Methods: A census of unique cigarette packs was purchased using a systematic protocol in 2013 and 2015/ 2016 in Brazil, Indonesia, the Philippines, Russia, Thailand, and Vietnam. Legal cigarette packs $(\mathrm{n}=2301)$ were coded for brand, brand owner, capsule presence, and flavor.

Results: In 2013, across six countries, we purchased 18 FCVs under 10 brands sold by four brand owners. In 2015/2016, there were $34 \mathrm{FCV}$ s under 19 brands from five brand owners. The number of FCVs purchased during 2015/2016 compared to 2013 stayed the same in Russia and increased in all other countries. In the Philippines, the number of FCV increased from three (2013) to 16 (2016). Of the $34 \mathrm{FCV}$ s sold across six countries in 2015/2016, 11 contained menthol or mint, four touted capsules that release a "fresh" flavor, and five incorporated mint and "purple" (e.g. Winston Purple Mint). Other flavors included lime $(\mathrm{n}=1)$, orange $(\mathrm{n}=1)$, and "Ibiza sunset" $(\mathrm{n}=1)$. The flavors of a further $11 \mathrm{FCVs}$ were not clear but the packaging and/or capsules incorporated blue and green colors, potentially indicating menthol flavoring.

Conclusions: The number of FCVs available on the market is growing and potentially fueling flavor capsule cigarette market share growth. Many FCVs contain menthol or mint capsules, however many are not described as characterizing flavors. These findings can inform advocacy efforts that call for stronger regulation of flavored cigarettes and inform research examining marketing and consumer perceptions of flavor capsule cigarettes.

Tob. Induc. Dis. 2018;16(Suppl 1):A506

DOI:10.18332/tid/83926

$14: 45-16: 15$
FO-368-4 Helping and harming: evaluating school tobacco programs and policies after 4 years in the COMPASS study

A Cole', S Aleyan'1, W Qian'1, S Leatherdale

'University of Waterloo, School of Public Health and Health Systems, Waterloo, ON, Canada. E-mail: agcole@uwaterloo.ca Background: The school environment is an equitable avenue to change youth health behaviours; however, there is little guidance for school-based programming. Specifically, the types of interventions schools use to prevent smoking uptake and progression are unknown. This analysis explored changes in school-based tobacco control programs and policies and the impact on smoking status.

Methods: COMPASS is a prospective cohort study that collects hierarchical longitudinal data from students and the schools they attend in Canada. Two cohorts of grade 12 students from 38 schools were identified at baseline (Y1) and three years later (Y4). The prevalence of susceptibility to future smoking, ever smoking, and current smoking among cohorts were compared in $\mathrm{Y} 1$ and $\mathrm{Y} 4$ in control (no change in tobacco control programing or policies) and intervention schools (reported a change in tobacco control programming or policies at any time during the study).

Results: Between Y1 and Y4, 33/38 schools reported changing their tobacco control programming or policies; 19 schools made a single change over the study period. Changes to enforcement activities and cessation programming were most common and usually involved external support. Few interventions significantly impacted the prevalence of susceptible never-smokers or ever smokers. The prevalence of grade 12 current smokers significantly increased at 12 schools and decreased at 3 schools. Thirteen interventions resulted in significant increases in students' perceptions of support to resist or quit tobacco.

Conclusions: Many schools implemented new tobacco control programming over three years with support from external organizations; however, few interventions significantly improved the smoking status of students over the long-term. Nevertheless, schools increased awareness of the issue of smoking by making a change to tobacco control programs or policies, which may support the behaviour of future students. The COMPASS study provides the infrastructure to continually monitor and evaluate school-based activities designed to advance youth health.

Tob. Induc. Dis. 2018;16(Suppl 1):A507

DOI: $10.18332 /$ tid $/ 84553$

$18: 15-19: 15$

LB-1324-4 The Tobacco Control Policy (TCP) Tool: a web-based interface to simulate the effects of tobacco policies in the United States

J Tam', D Levy², R Meza ${ }^{3}$

${ }^{1}$ University of Michigan, Health Management and Policy, Ann Arbor, MI, United States of America, ${ }^{2}$ Georgetown University, Washington, DC, United States of America, ${ }^{3}$ University of Michigan, Epidemiology, Ann Arbor, MI, United States of America.E-mail: jamietam@umich.edu

Background: Computational models of tobacco control policies that project their estimated impact on smoking and population health can be used to inform public health decision-making. To date, such models have not been made widely accessible for exploration by the general public or policymakers. We describe the iterative development process for extending a microsimulation model to evaluate the effects of tobacco control policies. The results from these simulations have been integrated into an 
interactive website for use by decision-makers and the public. Methods: We adapt the Cancer Intervention and Surveillance Modeling Network (CISNET) microsimulation model to estimate the population health effects of four tobacco control interventions in the United States: 1) cigarette taxes, 2) smoke-free air laws, 3) tobacco control program expenditures, and 4) the minimum age of legal access to tobacco. The results of these simulations were pregenerated and then integrated into the Tobacco Control Policy (TCP) tool, a dynamic, interactive web-based user interface that allows the user to specify their own proposed policy conditions and explore the estimated effects of that policy on population health outcomes in the US through 2060. Three rounds of usability testing were conducted with experts in tobacco control during web development to improve the interface and ensure that it was responsive to user needs.

Results: Model outcomes for each user-specified policy scenario include projections of future smoking prevalence, the number of deaths avoided, and the number of life-years gained. To increase its utility to state-level decision-makers, the model was extended to estimate these outcomes for each of the 50 states and Washington DC. The TCP tool website is available at http://www. tobaccopolicyeffects.org.

Conclusions: The TCP tool supports public health decisionmaking by allowing users to access the results of a complex microsimulation model of smoking and explore the potential health benefits of implementing tobacco control policies in their jurisdictions.

Tob. Induc. Dis. 2018;16(Suppl 1):A508

DOI: $10.18332 /$ tid/84717

[TCP tool estimates of select policy scenarios]

$18: 15-19: 15$

\begin{tabular}{|c|c|c|c|c|}
\hline Tobacco control policy & Simulated policy scenario & $\begin{array}{l}\text { Deaths avoided } \\
\text { by } 2060\end{array}$ & $\begin{array}{l}\text { Life years } \\
\text { gained by } 2060\end{array}$ & $\begin{array}{l}\text { Reduction in adult smoking } \\
\text { prevalence by } 2060\end{array}$ \\
\hline Cigarette taxes & $\begin{array}{l}\text { Increase the price per pack of cigarettes } \\
\text { by } \$ 1.00 \text { at national level }\end{array}$ & 267,070 & $4,930,389$ & 0.38 percentage points \\
\hline Smoke-free air laws & $\begin{array}{l}\text { Increase smoke-free air law coverage to } \\
100 \% \text { of workplaces, restaurants, bars } \\
\text { across all states }\end{array}$ & 101,877 & $1,808,233$ & 0.14 percentage points \\
\hline $\begin{array}{l}\text { Tobacco control } \\
\text { expenditures }\end{array}$ & $\begin{array}{l}\text { Fund all state tobacco control programs } \\
\text { at } 100 \% \text { of CDC recommendations }\end{array}$ & 91,584 & $1,792,281$ & 0.73 percentage points \\
\hline $\begin{array}{l}\text { Minimum age of legal } \\
\text { access (MLA) }\end{array}$ & $\begin{array}{l}\text { Raise the MLA from } 18 \text { to } 21 \text { for all } \\
\text { states }\end{array}$ & 14,138 & 552,082 & 0.71 percentage points \\
\hline
\end{tabular}

LB-1325-4 Does change in perception of tobacco risk affect smokers' behaviour? - An empirical analysis

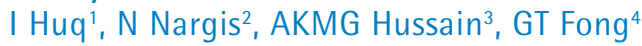

'East West University, Department of Economics, Dhaka, Bangladesh, ${ }^{2}$ American Cancer Society, Economic and Health Policy Research Unit, Atlanta, GA, United States of America, ${ }^{3}$ University of Dhaka, Department of Economics, Dhaka, Bangladesh, ${ }^{4}$ University of Waterloo, Department of Psychology, Waterloo, 0N, Canada.E-mail: iftekharulhuq.12@gmail.com Background: Information dissemination on harmful effects of tobacco has always been a major tool for reducing tobacco consumption in all countries. This paper studies how a change in the Perception of Tobacco Risk (PTR) affects the attitude of smokers and non-smokers towards smoking. First, this paper wants to observe how PTR varies among different types of smokers and non-smokers across different socio-demographic backgrounds. Second, it examines among the cigarette smokers across different socio-demographic groups how changing PTR over the years influences their behavior towards smoking.

Methods: A panel dataset from the ITC Bangladesh surveys is used for the analysis. Using the panel dataset, a Perception of Tobacco Risk Index (PTRI) is developed for all smokers and nonsmokers. OLS and Probit regressions are used on different groups based on income, education, age, residence to assess the effects of knowledge and income on smoking behaviour.

Results: Results show that among all different types of smokers, quitters have the highest PTRI whereas bidi (cheap local alternative to cigarettes) smokers have the lowest. Among the different socio-demographic groups the higher income, more educated, and those living in urban areas display a higher PTRI than their respective counterparts. However, it is also observed that the change PTRI is bigger among the lower income, less educated and those living in rural areas. Analysis of panel data reveals that the change in PTRI is positively correlated with the probability to quit smoking for most socio-demographic groups. However, increase in PTRI does not significantly affect initiation of smoking and reduces cigarette consumption per day only for the more educated group.

Conclusions: Results of this paper hold strong policy implications. First, they show that changing PTR holds promising implications for controlling tobacco consumption concerning raising quitting probability. Second, they bring into light, specific sociodemographic groups where policies to change PTR should be targeted.

Tob. Induc. Dis. 2018;16(Suppl 1):A509

DOI:10.18332/tid/84692

\subsection{Tobacco dependence treatment and smoking cessation: Innovations in Practice and Opportunities for scaling}

\section{6:30-18:00}

RF-1235-4 Who is more likely to have a quit intention in Brazil's major cities? Findings from the ITC Brazil Wave 3 Survey

L Casado', SC Kaai², C Perez³, M Yan², L Craig², ACK Quah², GT Fong 2,4

'Brazilian National Cancer Institute, Prevention and Surveillance Coordination, Rio de Janeiro, RJ, Brazil, ${ }^{2}$ University of Waterloo, Waterloo, ON, Canada, ${ }^{3}$ Fundaçao do Cancer, Rio de Janeiro, RJ, Brazil, ${ }^{4}$ Ontario Institute for Cancer Research, Toronto, ON, 


\section{Canada.E-mail: Ic662017@gmail.com}

Background: Intentions to quit is the strongest predictor of a future quit attempt. Therefore, identifying factors that are positively or negatively related to quit intentions is important to health care providers and researchers to develop effective smoking session services. Brazil has been a global leader in many tobacco control domains but has recognized the need to strengthen its cessation services. This study examined the factors associated with quit intentions among Brazilian smokers.

Methods: Data were analyzed from Wave 3 (2016-17) of the International Tobacco Control (ITC) Brazil Survey, a longitudinal cohort survey of representative samples of adult smokers in Rio de Janeiro, São Paulo, and Porto Alegre ( $\mathrm{N}=1,216)$. Main outcome was having a quit intention.

Results: Having a quit intention within the next 6 months in Brazil was $49 \%$, the highest of 25 ITC countries. $43 \%$ had made quit attempts in the past year. The following variables were positively associated with intentions to quit: being older (55+years; $\mathrm{OR}=3.07, \mathrm{p}=0.04)$, having low $(\mathrm{OR}=2.51, \mathrm{p}=0.03)$ or moderate $(\mathrm{OR}=2.53, \mathrm{p}=0.01)$ income, attempting to quit in the past year $(\mathrm{OR}=3.20, \mathrm{p}<.0001)$, believing that quitting is beneficial $(\mathrm{OR}=5.09, \mathrm{p}<.0001)$, worrying about future health consequences of smoking $(\mathrm{OR}=3.26, \mathrm{p}=0.01)$, and reporting that smoking is not enjoyable $(\mathrm{OR}=1.98, \mathrm{p}=0.001)$. Gender, education, and nicotine dependence were unrelated to quit intentions. $67 \%$ of smokers who had seen a health care professional in the past year reported being given advice to quit. $79 \%$ of smokers wanted the government to do more to help smokers quit.

Conclusions: Quit intentions are high in Brazil, and predictors of intentions are similar to those of many ITC countries (highincome and low-and middle-income countries). The vast majority of Brazilian smokers want the government to do more to help them quit. These findings support the need to strengthen cessation services to meet the growing demand of smokers who want to quit.

Tob. Induc. Dis. 2018;16(Suppl 1):A510

DOI:10.18332/tid/84217

\section{6:30-18:00}

\section{RF-1236-4 Self-efficacy predicts the outcome of} smoking cessation attempts

V Hayrumyan', A Harutyunyan'1, T Harutyunyan ${ }^{1}$

${ }^{1}$ American University of Armenia, Gerald and Patricia Turpanjian School of Public Health, Zvart Avedisian Onanian Center for Health Services Research and Development, Yerevan, Armenia. E-mail:vhayrumyan@aua.am

Background: Despite well-established advantages of smoking cessation in the prevention and treatment of myocardial infarction (MI) many patients continue smoking after a diagnosis of MI. Recent evidence suggests that higher self-efficacy (SE), or confidence in one's ability to abstain from smoking, is positively associated with successful smoking cessation attempts. This study aimed to investigate the association between SE and smoking cessation outcome at 6 to 12 months after MI in Armenia.

Methods: Cross-sectional survey was conducted among smoker adult MI patients who were hospitalized at the largest cardiac hospital in Armenia (Nork-Marash Medical Center). Data collection was done at 6 to 12 months after MI through medical chart review and interviewer administered telephone survey. SE at the time of MI was measured through widely used and validated Self-Efficacy Questionnaire (SEQ-12). SEQ-12 consists of two sixitem subscales which measure confidence in aptitude to abstain from smoking when facing internal stimuli (e.g. feeling nervous) and external stimuli (e.g. being with a smoker). Logistic regression analysis was performed to reveal the association between $\mathrm{SE}$ and quitting outcome.

Results: About half (54.37\%) of 103 surveyed participants quitted smoking after MI. The mean SE score was 33.55 (SD: 16.49) (out of possible 60) which was significantly higher among quitters compared to non-quitters (45.55 vs. 19.26, $\mathrm{p}<0.001)$. Likewise, the difference between was significant in terms of both internal and external subscale scores. Adjusted logistic regression elucidated that each unit increase in SE score was associated with 1.3 times higher odds of quitting (95\% CI: 1.17-1.44; $\mathrm{p}<0.001)$.

Conclusions: The results illustrated that $\mathrm{SE}$ is an independent predictor of smoking cessation after MI suggesting that increase in patients' confidence in ability to abstain from smoking will contribute to successful cessation outcomes. This emphasized the importance of behavioral interventions in encouraging and assisting smoking cessation attempts among MI patients.

\section{Tob. Induc. Dis. 2018;16(Suppl 1):A511}

DOI:10.18332/tid/84431

\section{$16: 30-18: 00$}

RF-1238-4 Feasibility and acceptability of e-cigarettes as an aid to quitting smoking among lung cancer patients: a pilot study

A Ford ${ }^{1,2}$, L Sinclair ${ }^{1}$, J Mckell ${ }^{1}$, S Harrow ${ }^{3}$, J Macphee $^{3}$, A Morrison $^{4}$, L Bauld ${ }^{1,2}$

${ }^{1}$ University of Stirling, Institute for Social Marketing, Stirling, United Kingdom, ${ }^{2}$ UK Centre for Tobacco and Alcohol Studies, University of Stirling, United Kingdom, ${ }^{3}$ Beatson West of Scotland Cancer Centre, Glasgow, United Kingdom, ${ }^{4} \mathrm{New}$ Nicotine Alliance, London, United Kingdom. E-mail: a.j.ford@ stir.ac.uk

Background: Many patients diagnosed with lung cancer continue to smoke even though this can make their treatment less effective and increase side effects. E-cigarettes form part of the UK's tobacco harm reduction policy landscape and are, by far, smokers' most popular quit attempt method. This pilot study explores feasibility and acceptability of e-cigarettes to aid smoking cessation among lung cancer patients undergoing chemotherapy.

Methods: 27 smokers with stage IV lung cancer were recruited from one NHS site in Scotland between May-16 and June-17. They were provided with a 2 nd generation e-cigarette kit at a baseline home visit conducted by a researcher and a volunteer who was an experienced e-cigarette user. Participants were followed-up weekly for four weeks and at 16 weeks. Participants' response to, and use of, e-cigarettes was explored along with cessation outcomes (self-reported and $\mathrm{CO}$ verified). In-depth qualitative interviews were conducted with health professionals $(n=8)$ engaged with lung cancer patients to obtain their views on the study.

Results: Overall, participants were motivated to stop smoking and took easily to using e-cigarettes. Minor issues arose around choice of flavour, and some side effects were noted, although participants reported difficulty in distinguishing these from treatment side effects. Seven participants were lost to follow-up. Preliminary findings show that at 4 -week follow-up: average $\mathrm{CO}$ reading had reduced from 14 (range 3-37) to 8 (range 1-29), and 70\% of participants reported daily e-cigarette use, however, use was dependent on individuals' day-to-day health. Health professionals interviewed were generally supportive of e-cigarettes as a tool for quitting, and suggested future efforts should concentrate on patients with curable cancer. 
Conclusions: E-cigarettes have a potential role to play for lung cancer patients. Future smoking cessation research should take account of the impact of cancer treatment on emotional and physical health and explore e-cigarette use among patients with earlier stage lung cancer.

Tob. Induc. Dis. 2018;16(Suppl 1):A512

DOI:10.18332/tid/84428

\section{$16: 30-18: 00$}

RF-1239-4 Creating the demand for tobacco cessation - an innovative approach to attract tobacco users contemplating to quit

D Subramani ${ }^{1}$, V Elangovan ${ }^{1}$, S Verraiah ${ }^{1}$, S Chidambaram ${ }^{1}$ ${ }^{1}$ Cancer Institute (WIA), Psycho oncology, Chennai, India. E-mail: divyarajprabhakar@gmail.com

Background: According to GATS 2, of the $28.6 \%$ tobacco users, $55 \%$ smokers and $50 \%$ smokeless tobacco users were contemplating to quit. Despite taking various measures, the number of tobacco users approaching tobacco cessation clinics is negligible in India. Moreover, the higher dropout and low quit rate was a concern. Therefore, an innovative concept named 'Dine with a Star' to attract the tobacco users contemplating to quit, was designed and its outcome is presented.

Methods: A video message by three celebrities inviting tobacco users willing to quit, to register through missed call was developed and circulated through social media. An opportunity to dine with celebrities was announced for those who quit. The same message was placed as hoardings in four prominent places in Chennai city. The users who gave missed calls $(n=115)$ were contacted and their demographic details including tobacco usage history were collected. A set quit date was announced and they were instructed to stay abstained from the day. The clients were motivated through text messages and real-time counselling over telephone, based on the need.

Results: Of the registered users, 56 (48\%) had reported to have quit. Of them, 21 (37.5\%) relapsed and 35 (62.5\%) had quit. The quit status was confirmed using urine cotinine test. The successful quitters were invited to dine with celebrities. Although, the attractive message motivated them to register, they reported that the regular support offered over phone (self-help tips and motivational text messages) helped to maintain their quit status.

Conclusions: This innovative cost effective approach achieved a highest quit rate when compared to the routine behavioral and pharmacological interventions as reported in the literature. In low resource countries like India, considering the large number of tobacco users, innovative approaches like this would bring results in reducing the tobacco related mortality and morbidity.

Tob. Induc. Dis. 2018;16(Suppl 1):A513

DOI: $10.18332 /$ tid/84189

\section{$16: 30-18: 00$}

RF-1240-4 What keeps healthcare professionals from advising their patients who smoke to quit? A large-scale cross-sectional study

E Meijer $^{1}$, R Van der Kleij ${ }^{1}, N_{\text {Chavannes }}^{1}$

'Leiden University Medical Center, Public Health and Primary Care, Leiden, Netherlands.E-mail: e.meijer@lumc.nl

Background: Quit-advice provided by healthcare professionals effectively increases quit rates. Tobacco dependence treatment guidelines therefore recommend providing quit-advice to all patients who smoke (PWS), however, many healthcare professionals do not advise PWS to quit. This results in worse patient outcomes and higher healthcare costs. We examined determinants of providing quit-advice among a large sample of healthcare professionals from different fields, most of which have not been included in research on tobacco discouragement before.

Methods: Online survey among addiction specialists $(n=13)$, anaesthesiologists $(n=61)$, cardiologists $(n=23)$, dental hygienists $(n=74)$, dentists $(n=40)$, general practitioners $(n=149)$, internists $(n=79)$, midwives $(n=82)$, neurologists $(n=29)$, ophthalmologist $(n=25)$, paediatricians $(n=42)$, pulmonologists $(n=121)$, surgeons $(n=65)$, youth specialists $(n=78)$ and other physicians $(n=74)$. Provision of quit-advice, and socio-cognitive determinants of and environmental/patient barriers to using the Dutch Tobaccodependence-guideline were assessed (entire sample), as well as perceptions of smoking (subsample).

Results: Most participants (27\%) advised the majority of PWS to quit, but only $16 \%$ advised all of them to quit and $18 \%$ advised none to quit (16\% half, $24 \%$ minority). Midwives were most likely to advise all PWS to quit (42\%). Multivariate logistic regression analysis $(n=760)$ showed that providing quit-advice (to all/majority vs. half/minority/none of PWS) was significantly associated with stronger intentions to use the guideline, stronger role perceptions, and sufficient training in smoking-cessationcare. Those who mentioned lack of reimbursement as a barrier to providing smoking-cessation-care were more likely to provide quit-advice, possibly because particularly those who are more involved in tobacco treatment experience reimbursement as a barrier. Furthermore, participants who perceived continuing smokers to lack willpower were less likely to provide quit-advice $(\mathrm{n}=446)$.

Conclusions:Quit-adviceis providedless often than recommended. This may be improved by training, and by making healthcare professionals more aware of their role in tobacco discouragement, and the role of addiction (vs. willpower) in continued smoking.

Tob. Induc. Dis. 2018;16(Suppl 1):A514

DOI:10.18332/tid/83858

\section{6:30-18:00}

RF-1241-4 Encouraging physical activity for smokers: benefits beyond expectation

JPM Wai', M-K Tsai ${ }^{2}$, J-H Lee ${ }^{2}$, P-J Lu², C-W Kao ${ }^{3}$, C-P Wen ${ }^{2,4}$, C-L Lin

${ }^{1}$ National Taiwan Sport University, Institute of Sport Science, Taoyuan, China, ${ }^{2}$ National Health Research Institutes, Institute of Population Science, Miaoli, China, ${ }^{3}$ Taipei Medical University, Master Program in Global Health and Development, Taipei, China, ${ }^{4}$ China Medical University Hospital, Taichung, China, 5John Tung Foundation, Taipei, China. E-mail: cwengood@ nhri.org.tw

Background: Guideline for smoking cessation often mentioned physical activity as a desirable behavior but has never considered it essential. This study aims to quantity the short-term and longterm benefits of leisure-time physical activity (LTPA) among smokers, including its impact on quitting behavior and on mortality outcome.

Methods: This cohort, consisting of 434,189 individuals (23.2\% smokers and $6.1 \%$ ex-smokers), went through standard medical surveillance program(s) from 1996 to 2008. History for smoking and physical activity was collected in the initial visit and subsequent visits. Exercise volume, expressed in MET·hour/week at each visit, classified each individual into inactive $(<3.75)$, low-active (3.75-7.49), or fully active ( $\geq 7.50)$ group. Fully active 
individuals met the current recommendation of 30 minute/day for 5 days or more per week. Hazard ratios (HR) were calculated with Cox model for mortality, adjusted for 10 confounders. Life expectancy was calculated using life table method.

Results: One fifth of smokers (22.9\%) and one third of former smokers (37.4\%) were active, an under-appreciated phenomenon favoring exercisers for success in cessation. Smokers who were fully active increased quit rate by $55 \%-81 \%$. Furthermore, active smokers had up to $30 \%$ less relapse rate when compared to less active individuals. Smokers who remained physically active had significant reduction in mortality for all-cause, all-cancers and cardiovascular diseases, by $23 \%, 14 \%$ and $34 \%$, respectively. Moreover, they gained 3.7 years in life expectancy. Smokers who quit and stayed active reduced mortality by $43 \%$ and gained 5.6 years in life expectancy.

\section{Level of exercise on all cause mortality by smoking status.}

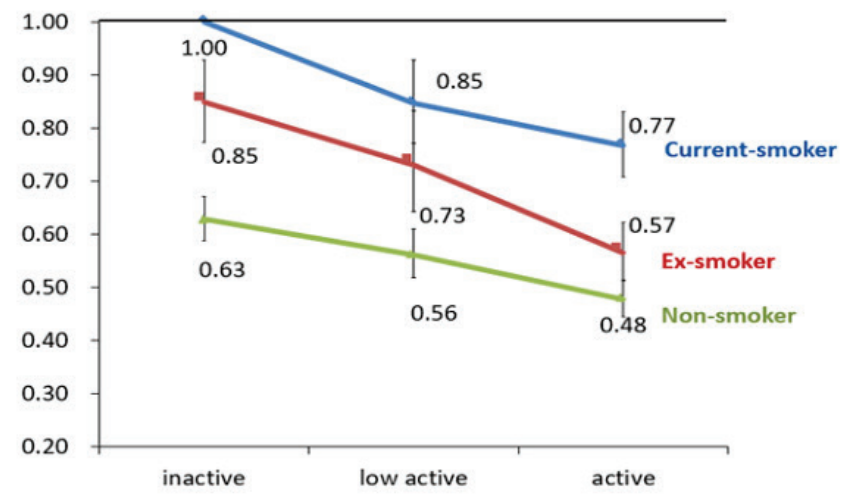

[Level of exercise on allcause mortality by smoking]

Conclusions: To engage in physical activity has unexpected benefits for smokers. It improved the success rate for smoking cessation, reduced relapse rates and extended life expectancy. Physical activity should be included as an essential aspect in the current guideline for smoking cessation.

Tob. Induc. Dis. 2018;16(Suppl 1):A515

DOI:10.18332/tid/84322

\section{$16: 30-18: 00$}

RF-1242-4 The role of behavioral therapy in treating waterpipe addiction

K Shishani', T Odom-Maryon', J Roll'

${ }^{1}$ Washington State University, Spokane, WA, United States of America.E-mail: kawkab.shishani@gmail.com

Background: To date, very few smoking cessation interventions targeting waterpipe tobacco smoking have been reported worldwide.

Methods: Thirty-one adults completed the study. Participants were adults 18 years of age and older who smoked tobacco using a waterpipe three times or more per week for the past one year, did not smoke cigarettes, and were not planning on quitting waterpipe tobacco smoking.

Design: A two-group, repeated measures (10 study visits on Mondays and Thursdays) design was used. Participants were randomly assigned to either the contingent $(n=19)$ or noncontingent ( $\mathrm{n}=20)$ study arms.

Intervention: Contingent participants received monetary incentives based on negative salivary cotinine results. Earning incentives started at $\$ 14$ and increased by $\$ 0.50$ for a maximum $\$ 192.50$.
Non-contingent participants earned incentives independent of salivary cotinine results. Participants in both study arms had the opportunity to earn the same amount of money. The primary study outcomes were biochemically verified prolonged abstinence and 7-day point prevalence.

Results: The prolonged abstinence rate in the contingent and non-contingent groups were $61 \%$ and $6 \%$, respectively, $(\mathrm{p}<$ $0.001)$. The 7 -day point prevalence in the contingent and noncontingent were $47 \%$ and $5 \%$, respectively, $(p=0.003)$. The odds of abstinence were $17.10(95 \% \mathrm{CI}=1.89,154.84)$ times greater for the contingent group than for the non-contingent group.

Conclusions: The preliminary results support further examination of contingency management as a strategy for initially motivating unwilling waterpipe smokers to quit.

Tob. Induc. Dis. 2018;16(Suppl 1):A516

DOI:10.18332/tid/84358

16:30-18:00

RF-1243-4 Roles of community pharmacists in providing smoking cessation services: a national survey in Thailand

S Chinwong ${ }^{1}$, D Chinwong

${ }^{1}$ Chiang Mai University, Department of Pharmaceutical Care, Faculty of Pharmacy, Muang Chiang Mai, Thailand. E-mail: surarong@gmail.com

Background: Thai community pharmacists play a vital role in helping smokers to quit smoking. This study aimed to examine the roles of community pharmacists across Thailand regarding smoking cessation services according to the 5 A's approach: ask, advise, assess, assist, and arrange.

Methods: This is a cross-sectional study using a selfadministered questionnaire via post, to collect information from the participants across Thailand. The participants were community pharmacists who provided smoking cessation services in their community pharmacies. Main outcomes were 5 A's activities provided by the community pharmacists. The participants were asked about their performing 5 A's in smoking cessation services in which they were asked to rate their frequency of $5 \mathrm{~A}$ 's practices in one of 4 categories: all the time, often, rarely, and never. Descriptive statistics were carried out for data analysis using STATA software.

Results: Of 152 respondents who completed and returned the questionnaire, $61 \%$ were women, $76 \%$ had an assistant pharmacist; $49 \%$ had been working as pharmacists of less than 10 years; $61 \%$ were members of the Thai Pharmacy Network for Tobacco Control. Ninety-five percent claimed that they asked their patients/customers about tobacco use status however only $38 \%$ asked all the time. About $97 \%$ advised to quit smoking but $30 \%$ advising all the time. About $93 \%$ assessed willingness to quit with $28 \%$ assessing all the time. Unfortunately, only $39 \%$ and $9 \%$ assisted smokers to quit and arranged for follow-up after providing the services all the time, respectively.

Conclusions: The study showed that more than $90 \%$ of community pharmacists did follow 5 A's for their practices in smoking cessation; however, they did not practice 5 A's all the time. Roles of community pharmacists in smoking cessation should be promoted and supported to encourage them to fully service all the time to help smokers to quit.

Tob. Induc. Dis. 2018;16(Suppl 1):A517

DOI:10.18332/tid/84569

16:30-18:00 
RF-1244-4 The impact of nicotine metabolite ratio on pharmacotherapy effectiveness in real-world settings: a longitudinal analysis

L Shahab ${ }^{1}$, L Bauld ${ }^{2}$, A McNeill ${ }^{3}$, R Tyndale ${ }^{4}$

'University College London, London, United Kingdom, ${ }^{2}$ University of Stirling, Stirling, United Kingdom, ${ }^{3}$ King's College London, London, United Kingdom, ${ }^{4}$ University of Toronto, Toronto, ON, Canada.E-mail: Iion.shahab@ucl.ac.uk

Background: Future developments in tobacco control are likely to involve personalisation of treatment to improve efficiency. Pharmacotherapy provision based on Nicotine Metabolite Ratio (NMR) status has been shown to improve outcomes in clinical trials: normal relative to slow metabolisers benefit more from varenicline than nicotine replacement therapy (NRT) treatment. However, it is unknown whether this effect translates to other settings.

Methods: Data come from 1,563 participants attending English Stop Smoking Services in 2012/13. Sociodemographic, mental/ physical health, smoking (dependence, determination to quit) and treatment characteristics (varenicline vs. NRT; group vs. one-to-one behavioural support) were assessed at baseline. Baseline salivary nicotine metabolites were measured and NMR (3-hydroxycotinine/cotinine) status computed, characterising smokers as slow $(\mathrm{NMR}<0.31, \mathrm{~N}=454)$ or normal $(\mathrm{NMR} \geq 0.31$, $\mathrm{N}=1,109)$ metabolisers. Carbon-monoxide verified 2-week and 50 -week continuous abstinence was assessed at 4-week and 52 week follow-up post-quit, respectively. The association between abstinence rates and predictor variables was determined using log-binomial generalised linear models that included a pharmacotherapy by NMR status interaction term and controlled for all covariates. All analyses were intention-to-treat.

Results: Of participants, $44.2 \%$ (95\% confidence interval (CI) $41.8-46.7 \%)$ and $8.0 \%(95 \%$ CI $6.8-9.5 \%)$ were continuously abstinent at 4-week and 52-week follow-up, respectively. There was no evidence of a pharmacotherapy by NMR status interaction on either outcome in adjusted analysis (see Figure). Higher abstinence rates were independently associated with higher socioeconomic group, treatment with varenicline, lower dependence and greater age at both follow-ups and with greater determination to quit and group support at 4-week follow-up, only.

Figure: Abstinent rates by pharmacotherapy type and NMR status

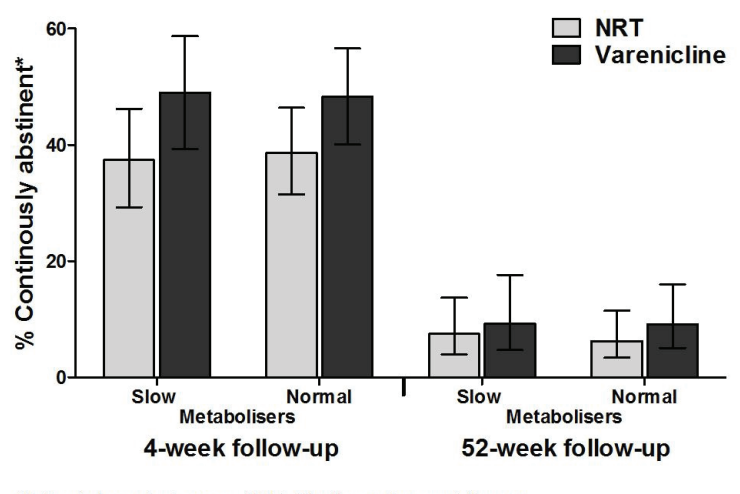

Conclusions: Our study did not replicate clinical trial data, indicating that NMR status does not substantially contribute to differential pharmacotherapy effectiveness in Stop Smoking Services. As this was an observational study, self-selection or unmeasured confounders may have influenced results. However, this suggests the effect of NMR in real-world settings is likely to be small, context-dependent and therefore may not translate globally.

Tob. Induc. Dis. 2018;16(Suppl 1):A518

DOI:10.18332/tid/84540

\section{$16: 30-18: 00$}

RF-1245-4 Advice from healthcare providers and cessation aid utilization among current smokers from 28 countries: findings from the Global Adult Tobacco Survey (GATS), 2008 - 2015

A Tripp', T Smith²

${ }^{1}$ Centers for Disease Control and Prevention, ORISE Fellow, Office on Smoking and Health, Atlanta, GA, United States of America, ${ }^{2}$ CDC Foundation, Office of Smoking and Health, Centers for Disease Control and Prevention, Atlanta, GA, United States of America. E-mail: kre7@cdc.gov

Background: The WHO Framework Convention on Tobacco Control calls for measures to reduce tobacco dependence, including the accessibility of cessation services. However, information on utilization of cessation services, including behavioral and pharmacotherapy among current smokers, is limited in several countries. We performed a cross-country comparison of quit behavior and use of clinical cessation aids among current smokers in 28 countries.

Methods: Nationally-representative data for individuals aged $\geq 15$ years were obtained from the 2008-2015 Global Adult Tobacco Survey. Data were analyzed for 28 countries from all six World Health Organization regions. Current smokers were defined as persons who smoked a tobacco product daily or nondaily at the time of the survey. Current smokers were asked if in the past 12 months they had been advised to quit smoking by a healthcare provider, if they had made a quit attempt, and if they had used a cessation aid (i.e., counseling, medications, or quit line). Countryspecific point estimates of quit attempts, healthcare provider advice, and use of cessation aids were assessed.

Results: Among current smokers, the proportion of those who made a quit attempt in the last 12 months ranged from $16.4 \%$ (Greece) to $53.9 \%$ (Senegal). The proportion who were advised to quit by a healthcare provider ranged from $41.9 \%$ (Ukraine) to $88.5 \%$ (Greece). Among current smokers who made a quit attempt in the past 12 months, use of any cessation aid ranged from $5.5 \%$ (Egypt) to $31.7 \%$ (Indonesia).

Conclusions: Variations in quit attempts and receipt of cessation assistance exist across countries. Opportunities exist for healthcare providers to advise smokers to quit smoking and to enhance the provision of cessation aids.

Tob. Induc. Dis. 2018;16(Suppl 1):A519

DOI:10.18332/tid/83866

18:15-19:15

LB-1329-4 Systematic delivery of a smoking cessation intervention after discharge from a secondary care setting

RL Murray', R Thorley', S Lewis', M Opazo Breton', B Nyakutsikwa', K Cheema', J Ablewhite', J Britton' ${ }^{1}$

'University of Nottingham, Epidemiology and Public Health, Nottingham, United Kingdom. E-mail: rachael.murray@ nottingham.ac.uk

Background: Hospital admission provides an ideal opportunity to promote smoking cessation. Clinical guidelines recommend delivery of cessation interventions for all admissions but no 
research to date has focused on supporting smokers who successfully abstain from smoking whilst in hospital to maintain abstinence after discharge.

We therefore designed a trial to test the effectiveness of an intensive home support intervention to maintain abstinence from smoking for newly abstinent smokers leaving hospital

Methods: Participants were recruited from 18 medical wards in one large teaching hospital in the UK and were individually randomised to either intervention or usual care using concealed allocation. During inpatient stay both treatment groups received smoking cessation support as recommended in NICE PH48 guidance. At discharge, patients randomised to usual care were offered a referral to a community Stop Smoking Service (SSS). Patients randomised to the intervention group were offered a home visit (or telephone call if a home visit is refused), as soon as practicable after discharge and typically within 48 hours, to deliver a multi-component intervention which included personalised behavioural support and nicotine replacement therapies (NRT). All smokers were asked to give consent to be contacted at fourweeks and three-months after discharge to assess smoking status and use of cessation support.

Results: 404 patients were randomised in total. 200 to intervention and 204 to usual care. Primary outcome data were available at four-weeks for 149 and 134 patients, respectively. Preliminary results demonstrate no statistically significant difference in quit rates between treatment groups at either 4 (21.00\% intervention, $19.12 \%$ usual care) or 12 weeks (19.50\% Intervention and $16.18 \%$ Usual Care) following discharge.

Conclusions: Adding a home visit to deliver additional smoking cessation support to patients who have remained abstinent during their hospital stay and treated according to NICE PH48 guidance does not increase quit rates.

Tob. Induc. Dis. 2018;16(Suppl 1):A520

DOI:10.18332/tid/84739

\section{8:15-19:15}

LB-1330-4 Introducing SmokeBeat ${ }^{\mathrm{TM}}$ - an innovative cigarette smoking behavior monitoring solution R Dar ${ }^{1}$, I Berlin²

${ }^{1}$ Tel Aviv University, School of Psychological Sciences, Tel Aviv, Israel, ${ }^{2}$ Univerisite P\&M Curie-Pitie-Salpetriere Hospital, Paris, France. E-mail: ivan.berlin@aphp.fr

Background: "SmokeBeat," developed by SomatixTM, a big data analytics software company, is a body motion detection application. It detects and records real-time hand-to-mouth movements, and differentiates with high precision smoking a cigarette from other hand-to-mouth gestures such as eating, drinking, shaving, teeth brushing etc. In addition to its ability to detect smoking episodes, SmokeBeat has a potential as a smoking cessation app.

Methods: Automated detection of smoking behavior not requiring smokers to cooperate: hand gestures of smoking are detected by a wearable, and data are transferred to the individual's smartphone and from there to the BMD ${ }^{\text {TM }}$ (Body Motion Data) platform. The smoking behavior of a given individual is followed in real time by a researcher or a health care provider. SmokeBeat can provide feedback to the individual smoker about his/her current smoking behavior and can utilize cognitive-behavior therapy techniques (coping tactics, reminders, real time cost of smoking, etc) to help smokers quit.

Results: 40 smokers who expressed a wish to reduce or quit smoking were randomly assigned to use the SmokeBeat app for 30 days or to a wait-list control group. Smokers in the experimental condition were notified whenever the SmokeBeat system detected a smoking episode and were asked to confirm or deny it. The SmokeBeat algorithm correctly detected over $80 \%$ of the smoking episodes and produced very few false alarms. According to both self-report and detection of smoking episodes by the SmokeBeat system, smokers in the experimental condition showed a significant decline in smoking rate over the 30 -day trial $(\mathrm{p}<.001)$. There was no change in the smoking rate of the control group.

Conclusions: The real-time automatic monitoring and notification feature of SmokeBeat may follow up real time cigarette use and potentially facilitate smoking reduction or even cessation in motivated smokers.

Tob. Induc. Dis. 2018;16(Suppl 1):A521

DOI:10.18332/tid/84706

\section{8:15-19:15}

LB-1335-4 Roll-your-own smoking and quitting behaviour among South African adults during 2007 - 2016

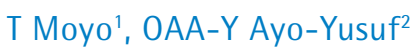

${ }^{1}$ Makoka and Associates, Development Research, Lilongwe, Malawi, ${ }^{2}$ SefakoMakgatho Health Sciences University, Pretoria, South Africa. E-mail: takondwamoyo68@gmail.com

Background: Roll-Your-Own (RYO) smoking is considered a cheaper and healthier alternative to smoking. However, only limited information is available on the prevalence and trends in the African region. The study aimed to determine the most current prevalence and trends in RYO smoking.

Methods: This study involved a secondary analysis of merged data obtained from adults $>15$ years, who participated in the South African Social Attitude Survey (SASAS) during $2007(\mathrm{n}=2907)$, $2011(n=3004)$ and $2016(n=3063)$. Current RYO smoker and current factory-manufactured cigarette (FMC) smokers are those who indicated daily or non-daily use of RYO cigarettes and FMC respectively. Participants were also asked if they had attempted to quit in the last 12 months. All statistical analysis took into account the complex sample design used in the SASAS.

Results: Current smoking was estimated at 20.8\%, 19.4\%, 20.1\% during 2007, 2011 and 2016 respectively. Among the general population, the prevalence of current RYO smoking was 4.2\%, 5.3\% and 5.3\% during 2007, 2011 and 2016 respectively. The prevalence of use of RYO among factory-manufactured cigarette (FMC) smokers significantly increased from $14 \%$ in 2007 to $24.1 \%$ in 2016 (p for trend $=0.09$ ). Over the study period, RYO smoking was most prevalent among male (8.8\%), coloureds (6.7\%), rural residents $(6.2 \%)$ and with $<$ Grade 12 education (6.4\%). Among smokers, RYO smoking was associated with a lower odds of reporting a quit attempt in the last 12 months $(\mathrm{OR}=0.60 ; 95 \% \mathrm{CI}=0.40-0.91)$.

Conclusions: RYO smoking remain most common among those of lower socioeconomic status and concurrent use of RYO with FMC has increased. RYO smoking may be associated with lower odds of quitting smoking, thus the need for targeted interventions.

Tob. Induc. Dis. 2018;16(Suppl 1):A522

DOI: $10.18332 /$ tid $/ 84755$

\subsection{Tobacco and Women: The gendered nature of tobacco}

$18: 15-19: 15$

LB-1350-5 Women's health, equality and empowerment in tobacco farming - findings from two counties in Yunnan, China

W Jie ${ }^{1}, \mathrm{H} \mathrm{Cao}^{1}, \mathrm{X} \mathrm{Li}^{1}, \mathrm{~J} \mathrm{Zhao}^{2}, \mathrm{XXiao^{3 }}, \mathrm{T}-\mathrm{W} \mathrm{Hu}{ }^{4}$ 
${ }^{1}$ Yunnan Pioneers for Health Consultancy Centre, Kunming, China, ${ }^{2}$ Yunnan Academy of Social Science, Gender and Development Research Centre, Kunming, China, ${ }^{3}$ Kunming Medical University, School of Pulic Health, Kunming, China, ${ }^{4}$ Center for International Tobacco Control Research at the Public Health Institute, Oakland, CA, United States of America. E-mail: caohong@pioneersforhealth.org

Background: Research takes place in Yunnan, the most important tobacco producing province in China, to gather empirical evidence of women's role in tobacco farming.

Methods: Research adopts mixed methods. Quantitative data was collected with structured questionnaire while qualitative data was obtained through individual interviews and focus group discussions. Participatory research method was employed as well in order to better understand the workload of women.

Due to sensitivity of tobacco control in Yunnan, respondents had to be reached through personal contacts. Thus a convenience sampling method was used. The information was collected from a sample of 436 female tobacco farmers in two counties in October - November 2015.

Results: Women spend $50 \%$ of working hours on tobacco farming. In tobacco farming season, women work $16+$ hours a day and on average they spend 2-3 hours more than their husbands on tobacco farming. $50.1 \%$ of respondents had to hire temporary help and $58.0 \%$ had to exchange labour (without pay) with neighbours / relatives for tobacco farming. $62.4 \%$ of respondents borrowed money for tobacco farming. The average income from tobacco farming is US $\$ 1,490$, accounting for about $26-35 \%$ of the household income.

Respondents experienced discomforts while growing and picking tobacco leaf, but did not associate these discomforts with tobacco farming. $50.5 \%$ of respondents were not aware of negative health effect of tobacco farming.

There is no mechanism or entity representing tobacco farmers to bargain with the tobacco corporation. Instead, farmers lost autonomy over farming activity under the pressure of both government and tobacco corporation. Women are even more vulnerable because they rarely participate in decision making at community and above levels although they have significant power over family finance and household farming activity..

Conclusions: Being at the bottom of exploitation chain, women lack awarness, knowledge, resource and mechanism to address their health and economic concerns in tobacco farming.

Tob. Induc. Dis. 2018;16(Suppl 1):A523

DOI:10.18332/tid/84697

\section{8:15-19:15}

LB-1351-5 Labor input of women tobacco farmers in Tabora, Tanzania

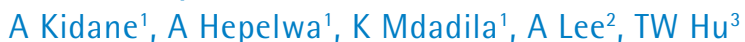

'University of Dar es Salaam, Economics, Dar es Salaam, Tanzania, United Rep., ${ }^{2}$ Public Health Institute, Oakland, CA, United States of America, ${ }^{3}$ University of California, Health Economics, Berkeley, CA, United States of America. E-mail: asmeromkidane2000@yahoo.com

Background: Literature on African women tobacco farmers in many countries suggests they are the most overworked members of their economies. The study presented here attempts to quantify the amount of labor women tobacco farmers exert in tobacco, maize and a third crop.

Methods: Stratified sampling, cross-classified tables, multiple linear regression were applied.
A sample of 408 women tobacco farmers from the Tabora region of Tanzania were selected and interviewed. Women labor input was measured in man-days and was compared with the man-days of adult males and children's labor input.

Results: The results show that 251 man-days per year are spent on farming activities; 42 percent of these are undertaken by women farmers. 78 percent of time devoted to farming is earmarked for tobacco farming. Out of the 198 days devoted to tobacco farming, 43 percent is undertaken by women. The contribution to labor input by children is only 5 percent. When classified by type of agricultural activities, men spend more time in land clearing and planting, while women spend more time in weeding and harvesting. When classified by age, older women spend more time than younger women.

Using a multiple linear regression, the determinants of the magnitude of women labor input were identified. The results show that women who spend more time in tobacco farming are relatively old; they get less or no remittance; they reside far from major towns; they do not have much wealth, and they have little access to alternative employment.

Conclusions: These variables indicate that women tobacco farmers are poor. Further, the amount of time women spend in tobacco farming appears to be very high; this is likely to lead to tobacco related health hazards.

\section{Tob. Induc. Dis. 2018;16(Suppl 1):A524}

DOI:10.18332/tid/84698

$18: 15-19: 15$

LB-1352-5 Smokeless tobacco, associated risk factors and women health: the study of garment workers in urban Mumbai, India

P Kurlikar ${ }^{1}$

${ }^{1}$ International Institute for Population Sciences (IIPS), Public Health and Mortality Studies, Mumbai, India. E-mail: prash. kurlikar@gmail.com

Background: India is one of the world's largest producers and consumers of tobacco. Smoking is rare among Indian women, but research shows that Indian women's use of smokeless tobacco products is increasing, with negative consequences on reproductive health. In India, smokeless tobacco consumption is highest in the labor classes and among those from a low socioeconomic status, less educated or illiterate, and the poor and marginalized groups. This workers lack proper knowledge and risk associated with the use of smokeless tobacco. These women consume cheap tobaccos that have negative impact on both their general and reproductive health. Therefore, there is need to understand the pattern of tobacco use, age of initiation of habit, reason for initiation, frequency of consumption in a vulnerable population, i.e. garment worker

Methods: Mixed method approach has chosen. A sample of 150 female garment workers were chosen in Mumbai partly by random and partly by snowball sampling. Ten in-depth interviews conducted using interview guideline. Bivariate analysis carried out on quantitative data.

Results: Total 150 adolescent girls and women garment worker were included in the study. The average age of the participant is about 23.04 years with $\mathrm{SD} \pm 2$ years. No women in our study reported smoking cigarettes. The estimated prevalence of SLT use in this population of urban, low-income, married women is high. Of these, more than one third reported use of more than one SLT daily. Women reported the use of five main types of smokeless tobacco: pan with tobacco, mishri, gutkha, chewed tobacco. 
Conclusions: It is clear from the study that tobacco consumption is highly prevalent among garment workers. Immediate intervention programs warranted to reduce the future burden of tobacco userelated morbidity among these workers who are exposed to the high pollution levels in garment factories.

Tob. Induc. Dis. 2018;16(Suppl 1):A525

DOI:10.18332/tid/84751

\section{$18: 15-19: 15$}

LB-1355-5 Women's awareness about toxic effect of tobacco consumption on health in India: Are they intending to quit too?

R Chowdhary

${ }^{1}$ Tata Institute of Social Sciencesl, School of Development Studies, Mumbai, India.E-mail: chowdhary1191@gmail.com Background: Tobacco needs special attention in the South-East Asia region owing to its high prevalence and up swinging use, including India. India is the second largest consumer of tobacco in the world. Tobacco leads to 1 million deaths each year - 9 $\%$ of all non-communicable diseases compared with $2 \%$ of all communicable disease related deaths. If current trends continue tobacco will account for $13 \%$ of all deaths by 2020 . This study aims to investigate the awareness level among women about impact of tobacco use on health and their intention to quit tobacco.

Methods: Data from the Global Adult Tobacco Survey, India (2009-2010) has been used for analysis purpose. We examined the prevalence of tobacco use among females in India by sociodemographic characteristics and factors associated with awareness level and intention to quit tobacco. Statistical techniques such as bi-variate analysis and binary logistic regression were used.

Results: Results shows that more than one-fifth of the women in India are currently consuming tobacco and more than one fourth of female starting tobacco use before the age of 18. Rural women $(38.1 \%)$ were more likely to notice health warning on tobacco products than urban women. Majority of women (90\%) believed that smoking cause lung cancer, stroke and heart attack however only $3 \%$ of them are intended to quit tobacco. About 5 $\%$ of poor women tobacco users thought of quitting the tobacco use as compared to just $1.6 \%$.of rich women.

Conclusions: The use of tobacco among the rural and the illiterate women is higher as compared to the urban women, keeping this in views, the government must draft some special focus policies and programmes so that, they can provide targeted intervention to these group. Enhancing government intervention on motivational campaign could be helpful in curbing the tobacco use among women.

Tob. Induc. Dis. 2018;16(Suppl 1):A526

DOI:10.18332/tid/84744

\subsection{Tobacco in vulnerable populations (e.g. indigenous populations)}

\section{$16: 30-18: 00$}

RF-1237-4 Smoking cessation practices among ethnic minorities. A case for ethnically-tailored smoking cessation services in Lagos State, Nigeria 0 Odukoya', M Olaleye', T Kotey ${ }^{2}$

'College of Medicine, University of Lagos, Department of Community Health and Primary Care, Lagos, Nigeria, ${ }^{2}$ Lagos University Teaching Hospital, Department of Community Health, Lagos, Nigeria. E-mail: drolukemiodukoya@yahoo.com

Background: In Nigeria, smoking rates are higher among ethnic groups in Northern Nigeria. Lagos state is the commercial nerve center of the most populous nation in Africa, and draws people of different ethnicities seeking economic gain. There is a large group of Hausa people in Lagos state. Some studies have cited higher smoking rates among ethnic minorities, however cessation efforts targeted at ethnic minorities in Nigeria are suboptimal. This study aimed at assessing cessation practices and preferences among smokers in the Hausa community of Idi-Araba in Mushin local government, Lagos State.

Methods: The was a descriptive cross-sectional communitybased study that used a snowball sampling technique to select 157 current cigarette smokers interviewed using pretested Modified version of the Global Adult Tobacco Survey tool.

Results: All the respondents were of the Hausa ethnic group and practiced Islam, $24.1 \%$ had either no formal education or Islamic education only and $38 \%$ could not read and write. More than half (52.9\%) had ever tried quitting and were ready to quit, primarily for health and economic reasons. Knowledge of the harmful effects of tobacco use was poor and significantly associated with intention to quit. ( $\mathrm{p}<0.05$ ). Only $6.8 \%$ had ever received professional help with quitting. Many were more willing to receive and pay for help from a traditional healer rather than a trained health professional. Conclusions: Ethnically tailored smoking cessation services are needed for this group of smokers. Training of traditional healers to identify and offer ethnically tailored smoking cessation services may be considered.

Tob. Induc. Dis. 2018;16(Suppl 1):A527

DOI:10.18332/tid/84275

\section{$18: 15-19: 15$}

LB-1346-5 Attitudes towards implementing tobacco-free-schools in vocational schools in Denmark: an important health promotion setting in youth

CD Klinker ${ }^{1}$, AV Hansen ${ }^{1}$, C Heinze' ${ }^{1}$ PD Jensen², S Rask², HT Maindal ${ }^{1,3}$

'Steno Diabetes Center Copenhagen, Health Promotion, Diabetes Prevention Research, Gentofte, Denmark, ${ }^{2}$ Danish Heart Foundation, Copenhagen, Denmark, ${ }^{3}$ Aarhus University, Public Health, Aarhus, Denmark. E-mail: charlotte.demant. klinker@regionh.dk

Background: The prevalence of daily smoking among youth at Danish vocational schools is $37 \%$, while the prevalence among high-school students is $12 \%$. Vocational schools are an important setting for health promotion to target inequality in health. Implementing Tobacco-Free-Schools (TFS) may reduce smoking among both students and staff. Smoking among students and staff has been found to be an important setting for socializing and bonding and the schools are reluctant to enforce TFS. The objective of this study was to investigate attitudes towards TFS among managers and staff across all Danish vocational schools, and determine characteristics of schools with a positive attitude towards TFS opposed to schools with a more negative attitude.

Methods: A total of 72 out of 87 (83\%) vocational schools in Denmark participated in an electronic survey on health promotion activities and implementation capacity with a least one respondent. Overall a total of 185 respondents from management and 345 teaching staff participated $(n=530)$. Data was analyzed in SPSS using univariate and multilevel modelling to detect differences between managers and staff in attitudes towards TFS and to identify characteristics of progressive TFS schools (28 schools) compared to non-progressive schools (44).

Results: Staff were more positive than managers towards future 
implementation of TFS $(\mathrm{p}<0,05)$. Size of school (increasing size), gender (having a female manager), school type (health and teaching) and school health promotion profile (more initiatives and policies in general) were associated with being more positive towards TFS at the school level.

Conclusions: This study is one of the first to address attitudes towards TFS and characteristics of being a progressive TFS school in a national representative sample. Vocational schools are an important setting to target smoking among youth, and this study highlights the need to address implementation of TFS on multiple levels.

Tob. Induc. Dis. 2018;16(Suppl 1):A528

DOI:10.18332/tid/84737

\section{$18: 15-19: 15$}

LB-1347-5 Smoking status predicts cancer patients' quality of life over time

U Martinez1, KO Brandon'1, TH Brandon', LR Meltzer'1, VN Simmons ${ }^{1}$

${ }^{1} \mathrm{H}$. Lee Moffitt Cancer Center, Health Outcomes and Behavior, Tampa, FL, United States of America. E-mail: ursula.martinez@ moffitt.org

Background: Previous studies indicate that quitting smoking significantly improves health-related quality of life (QOL) in patients with lung and head and neck cancer. However, few prospective studies have investigated the role of smoking status on QOL across patients diagnosed with a wide range of cancers. The aim of the present study was to examine quality of life (depression, pain, and fatigue) changes over time as a function of smoking status.

Methods: Participants were 351 cancer patients (e.g., gynecological, breast, thoracic, head and neck, genitourinary, hematological, cutaneous) who reported smoking abstinence within the previous 120 days. Smoking status and QOL (depression, pain severity, fatigue severity, and fatigue interference) were assessed at baseline, 2, 6, and 12 month follow-ups. Within a Structural Equation Modeling (SEM) framework, growth curve models with smoking status as a time varying covariate were employed to examine the effect of smoking status change in QOL measures over time. Baseline demographics (e.g., sex, income) and smoking history (e.g., nicotine dependence) were controlled.

Results: Overall, models with both time-varying covariates (smoking status) and time-invariant covariates (demographics, nicotine dependence) were good-to-excellent fits with the data. Smoking status had a main effect and an interaction with time upon depression and pain severity. For fatigue severity and fatigue interference, smoking status interacted with time, i.e., smoking abstinence at the 6 and 12 months follow-ups were associated with lower fatigue scores across the estimated growth curve.

Conclusions: These results extend previous findings showing that QOL improves in cancer patients who quit smoking. Specifically, patients who quit smoking experience a greater reduction in depression and pain levels at all time points, and the reduction increases over time. In the case of fatigue, the results suggest that patients experience the greatest improvement with longer ( $\geq 4$ months) abstinence.

\section{Tob. Induc. Dis. 2018;16(Suppl 1):A529 \\ DOI:10.18332/tid/84732}

\section{$18: 15-19: 15$}

LB-1349-5 Withdrawal symptoms upon a short motivational 26-hour smoking abstinence program in psychiatric patients

I Keizer ${ }^{1,2}$, C Wahl $^{3}$, M Gex-Fabry ${ }^{1,2}$, P Croquette ${ }^{1}$, J-P Humair ${ }^{3,4}$, J-M Aubry, ${ }^{1,2}$ AN Khan ${ }^{1}$

${ }^{1}$ HUG University Hospitals of Geneva, Department of Mental Health and Psychiatry, Geneva, Switzerland, ${ }^{2}$ University of Geneva, Geneva, Switzerland, ${ }^{3}$ CIPRET-Genève / Carrefour Addictions, Geneva, Switzerland, ${ }^{4} \mathrm{HUG}$ University Hospitals of Geneva, Division of Primary Care Medicine, Geneva, Switzerland. E-mail: corinne.wahl@cipret.ch

Background: Concerns about withdrawal effects and worsening of mental health conditions are barriers to proposing smoking cessation to psychiatric patients.

Methods: The study included 156 smokers who attended public psychiatric services and participated in a motivational intervention that included a 26-hour tobacco abstinence period and other components*. Carbon monoxide levels and withdrawal symptoms (MNWS) were evaluated at pre- (3 days before), during (9 hr and $26 \mathrm{hr}$ abstinence) and post-intervention (7 days after).

Results: Irritability, anxiety, depressed mood and concentration difficulties significantly decreased during the intervention (pre- and $26 \mathrm{hr}$ comparison), both for patients who did and did not succeed with smoking abstinence. Whereas craving decreased for those who succeeded, it remained elevated for those not succeeding.

Conclusions: Results are at odds with usual observations of increased discomfort associated with smoke deprivation and suggest that mental health patients may tolerate short abstinence periods, without worsening of their mental health condition. Although not systematically observed, craving may remain an obstacle for a subgroup of patients. We hypothesize that behavioural associations, habits and environmental factors could be more important obstacles when stopping smoking than usual withdrawal effects.

*Keizer, I., Gex-Fabry, M., Croquette, P. and Khan, A. N. (2016). A Short Motivational Program Based on Temporary Smoking Abstinence: Towards Increased Self-Efficacy to Quit in Psychiatric Inpatients. Journal of Addiction Research \& Therapy, 7:4.

Tob. Induc. Dis. 2018;16(Suppl 1):A530

DOI:10.18332/tid/84719

[MNWS scores (0-4)]

18:15-19:15

18:15-19:15 LB-1349-5 Withdrawal symptoms upon a short motivational 26-hour smoking abstinence program in psychiatric patients
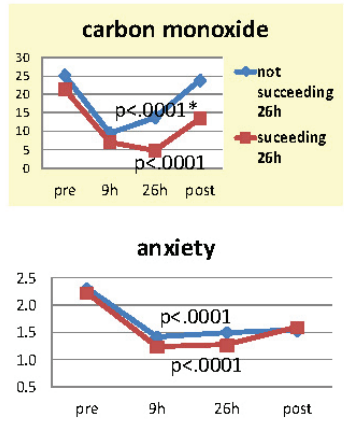

EARLY WITHDRAWAL SYMPTOMS

* Comparison pre-evaluation / after 26 h intervention (abstinence trial): Pairedsamples T Test

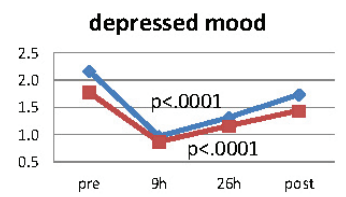

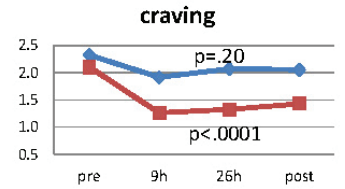

difficulty concentrating

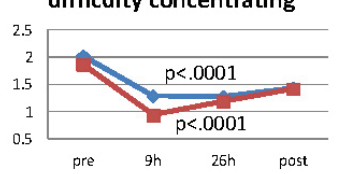

irritability / anger

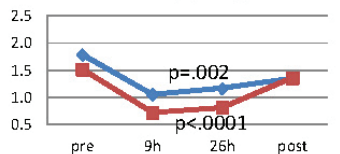

increased appetite

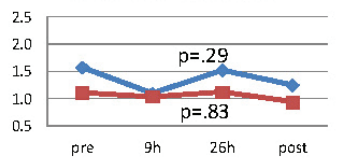

restlessness

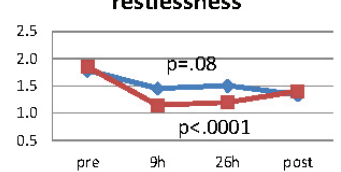

sleep disturbance

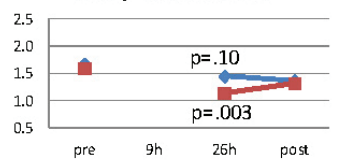




\section{LB-1353-5 Smokeless tobacco use among floating} people in Dhaka City

S Marandy', HMM Mahmud ${ }^{2}$, MK Ahmed ${ }^{3}$, MMA Talukder ${ }^{4}$

'Shanto-Mariam University of Creative Technology, Sociology and Anthropology Department, Dhaka, Bangladesh, ${ }^{2}$ Bangladesh Center for Communication Program, Dhaka, Bangladesh, ${ }^{3}$ Bangladesh Center for Communication Programs (BCCP), Dhaka, Bangladesh, ${ }^{4}$ Bangladesh University of Engineering and Technology (BUET), Dhaka, Bangladesh. E-mail: miraz584@ gmail.com

Background: In South Asia, the use of smokeless tobacco is common and this use raises various concerns. Increasing use has been reported not only among men, but also among such vulnerable groups as teenagers, women, and floating migrants. Dhaka city has a huge and bulging population and they are shanty, pavement dwellers or sheer vagabonds and smokeless tobacco using rate is increasing among them. The objective of the study was to investigate the existing patterns of smokeless tobacco among the floating people and to make policy recommendations to protect them from its devastating effects.

Methods: A descriptive study using a quantitative approach deployed to get information among the floating population in Dhaka city. Through purposive sampling technique, information from 500 respondents was collected through face-to-face interviews using structured questionnaire. Descriptive statistics were used for all variables test was carried out to explore the relationship between the use of smokeless tobacco products and socioeconomic status of the respondents.

Results: About three-fifths of the respondents were found to be current smokeless tobacco (SLT) users. The factors that influenced a person to become addicted to SLT were easy availability, social acceptance, peer influence, lower price of SLT products compared to cigarettes, after cessation of smoking, relief from toothache, etc. Although a majority of the respondents had knowledge about the harmful effects of SLT use, the current use of SLT among them was pronounced. Current SLT use was significantly associated with floating women $(\mathrm{p}<0.001)$ compared to their male and other counterparts.

Conclusions: This study provided a picture of smokeless tobacco use among floating people of Dhaka City. Multistage, effective behavioral change intervention programs may be implemented to overcome this health hazard.

Tob. Induc. Dis. 2018;16(Suppl 1):A531

DOI:10.18332/tid/84731

\section{$18: 15-19: 15$}

LB-1354-5 Investigating the practices of tobacco smoking in HIV-infected patients attending HIV health centers in the Western Cape Province, South Africa: patterns and associated risk factors

M Buwa ${ }^{1}$, A deVilliers², AP Kengne ${ }^{2}$

'South African Medical Research Council, Non-Communicable Diseases Research Unit, Tygerberg, South Africa, ${ }^{2}$ South African Medical Research Council, Non-Communicable Diseases Research Unit, Cape Town, South Africa. E-mail: muyunda.mutemwa@ mrc.ac.za

Background: Smoking is one of the major preventable causes of disease and premature death globally. Emerging findings have highlighted the intersection of cigarette smoking and HIV/AIDS as a significant public health concern. For adults with HIV, smoking increases both HIV-related and non-related outcomes. There is scarcity of information on the incidence and effects of cigarette smoking as well as its management in the HIV-infected population in South Africa. This study therefore aimed to determine the occurrence of smoking in HIV positive patients attending public healthcare facilities in the Western Cape Province of South Africa. Methods: An overall of 751 patients living with HIV randomly selected and screened from 17 different HIV/AIDS public facilities in the Western Cape at both primary and secondary level were included for this study between 2014 and 2015. This article focuses on the self-reported tobacco-using behavior of adults aged $>18$ years. The survey instrument included questions on the respondents' history of smoking tobacco, current use of other tobacco products, frequency and duration of use, and attempts to stop smoking tobacco or using other tobacco products.

Results: Of the $751 \mathrm{HIV}$ positive patients receiving medical care at public healthcare facilities, an overall of $20.7 \%(95 \% \mathrm{CI}, 17.3 \%$ to $24.1 \%$ ) were current smokers, $14.5 \%$ (CI, $11.6 \%$ to $17.4 \%$ ) were former smokers, while $65.3 \%$ (CI, $54.6 \%$ to $68.9 \%$ ) had never smoked. Out of these the current smokers, $18.9 \%(95 \%$ CI, $12.3 \%$ to $25.5 \%$ ) were men while $20.8 \%$ (95\% CI, $16.9 \%$ to $24.7 \%$ ) were women. Among the patients living with HIV, factors independently associated with greater smoking prevalence were gender, age, unemployment, household smoking, and excessive alcohol use.

Conclusions: We found a high prevalence of hypertension among patients receiving routine care for HIV infection across public HIV clinics in this setting.

Tob. Induc. Dis. 2018;16(Suppl 1):A532

DOI: $10.18332 /$ tid/84705

\subsection{Tobacco, inequality and poverty}

\section{8:15-19:15}

LB-1348-5 Individual cost of smoking in a study population

J Jensen', B Lindgren², D Hatsukami ${ }^{3}$

'University of Minnesota, Masonic Cancer Center, Minneapolis, MN, United States of America, ${ }^{2}$ University of Minnesota, Biostatistics Masonic Cancer Center, Minneapolis, MN, United States of America, ${ }^{3}$ University of Minnesota, Department of Psychiatry, Minneapolis, MN, United States of America. E-mail: jense010@umn.edu

Background: The rising price of cigarettes has prompted many smokers to quit. Those who continue to smoke are now concentrated in lower income populations and an increasing amount of their income is dedicated to tobacco. This analysis looks at the percentage of annual income spent on tobacco by smokers in a multi-site research study.

Methods: Subjects $(\mathrm{n}=605)$ screened for a study involving switching smokers to alternative nicotine products were asked demographics, annual income, purchase price per pack of cigarettes, and cigarettes smoked per day to estimate annual tobacco costs. Wilcoxon rank sum test compared the percent of income spent on cigarettes across several demographic variables.

Results: Subjects were an average of 43.4 years old $(\mathrm{SD}=13.1)$, smoking 15.6 (7.4) cigarettes per day for 10 years; $56.3 \%$ were white, $35.2 \%$ black and $8.6 \%$ other; and mostly male (58\%). Nearly a third of the subjects were unemployed (31.6\%) and an additional $5.5 \%$ were disabled; $46.6 \%$ reported either full/part time or casual work. The average amount spent on tobacco was US $\$ 1,743$ across all 3 sites or $10 \%$ of annual income. Percent of income spent across sites was 11.4 in Minnesota, 9.4 in Ohio, 
and 8.2 in New York. As expected, unemployed and disabled spent a significantly higher percent of their income compared to employed subjects ( 13.1 vs $8.0 ; \mathrm{p}<0.001)$. Gender or age were not related to cigarette spending. Over a quarter of the subjects reported an annual income of less than $\$ 10,000$ (28.6\%). Those reporting annual incomes of $<\$ 10,000$, well below the poverty line compared to those above this line, spent a significantly higher percent on cigarettes (17.2 and 7.2 respectively; $\mathrm{p}<0.001$ ).

Conclusions: Nicotine addiction is an economic burden for those smokers already living in poverty. Cessation treatment must be provided to this population especially when increased taxes are used for tobacco control.

Tob. Induc. Dis. 2018;16(Suppl 1):A533

DOI: $10.18332 /$ tid/84752

\section{8:15-19:15}

LB-1358-5 Perceptions of plain tobacco packaging among adolescents and adults: DePICT, a French national survey

F El Khoury' ${ }^{1}$ C Bolze ${ }^{1}$, M Melchior ${ }^{1}$

${ }^{1}$ INSERM UMR_S 1136, Pierre Louis Institute of Epidemiology and Public Health, Department of Social Epidemiology, Paris, France. E-mail: fabienne.khoury@inserm.fr

Background: Social inequalities in the consumption of tobacco in France are at all-time highs, with adults in lower socio-economic groups having higher rates of smoking than those who are more privileged. France was the second country in the world to introduce plain tobacco packaging, with all tobacco products sold starting January 1st 2017 having plain packaging. We sought to examine if perceptions of the change in tobacco packaging differs according to socio-economic position in adolescents as well as in adults.

Methods: DePICT is a two waves cross-sectional national telephone survey of residents of Metropolitan France, whose first wave was completed on November 15th 2016 and included more than 6,000 participants aged 12 to 64 years. Using multivariate logistic regression models, we examined the association between participants' educational attainment and their perceptions of plain tobacco packaging. Analyses were conducted separately among adults ( $\geq 18$ years, $\mathrm{n}=4,342)$ and adolescents $(\mathrm{n}=2,042)$, and were adjusted for multiple potential confounders such as socio-demographic characteristics, variables related to health warnings' salience and perceptions of tobacco smoking. All analyses were weighted to be representative of the French population.

Results: $77 \%$ of adults and $28 \%$ of adolescents had heard of plain packaging; respectively $38 \%$ of adults and $42 \%$ of adolescents had a favourable opinion of this policy. Higher education level was associated with a positive perception of plain packaging among adults, and schooling in a technical/vocation setting was associated with negative perceptions among adolescents. Smokers - adult and adolescent - were also more likely to have a negative perception of plain packaging.

Conclusions: A negative perception of plain packaging is associated with a lower educational level among adolescents and adults. Thus, the impact of this policy change on patterns of smoking could differ depending on individuals' socio-economic position.

Tob. Induc. Dis. 2018;16(Suppl 1):A534

DOI: $10.18332 /$ tid/84723

\subsection{Tobacco farming: Opportunities and}

\section{experiences in facilitating alternative livelihoods and preventing tobacco adverse environmental impact}

\section{8:15-19:15}

LB-1321-5 An investigative journalism perspective on tobacco control in Tanzania

MB Mgeta

${ }^{1}$ Association of Tanzania Health Journalists, Management, Dar es Salaam,Tanzania, United Rep.E-mail:mgeta2000@yahoo.com Background and challenges to implementation: The presentation is based on a investigative journalism study conducted in two districts, Urambo and Kaliua in Tabora region. The main goal of the study was to establish the reality about how tobacco farming contributing to deforestation and increased poverty to tobacco farmers, and propose better ways to control tobacco and its products.

Intervention or response: Tobacco farming regions in Tanzania were mostly affected by deforestation, while its population remained poor for the decades.

This presentation is based on a investigative journalism study conducted in two districts, Urambo and Kaliua in Tabora region. The main goal of the study was to establish the reality about how tobacco farming contributing to deforestation and increased poverty to tobacco farmers, and propose better ways to control tobacco and its products.

Results and lessons learnt: Overall this study found a larger tree cutting for curing tobacco and preparation for the tobacco farms. For example, in 2015/16 a total of 18,285 acres in Urambo district, Tabora region were used for tobacco farming, attracting 182,850 cubic meters of firewood in curing tobacco. This is equivalent to cut trees at the area of between 8,296 and 12,190 acres per year. However, effect linked to tobacco farming was drainage of waters sources which feed Igombe River and others pass through Maragalasi-Kigosi - Moyowosi Game Reserve to Lake Tanganyika which is the second oldest fresh water and second deepest lake in the world.

Poverty: Tobacco farmers live in an extremely poverty as the value chain not on their side, favors tobacco companies.

Conclusions and key recommendations:

1. Great need to fast truck the tobacco policy and national law.

2. Partners should team up, facilitate/support the current/new Income Generating Activities (IGAs) and improvement of alternative crops to attract more farmers to shift from tobacco farming.

3. Media advocacy and campaign on tobacco control should be of on- going perspective.

Tob. Induc. Dis. 2018;16(Suppl 1):A535

DOI:10.18332/tid/84754

\section{$18: 15-19: 15$}

LB-1356-5 "I know the harm of growing this deadly crop but I am forced to continue" - Tobacco farmers" perspective from India

H Shaikh'1, K Mogulluru²

${ }^{1}$ Rome Business School, Health Care Management, Noida, India, ${ }^{2}$ Lunds University, Sweden, CRC, Hyderabad, India. E-mail: drfheena@gmail.com

Background: India has been a Party of the World Health Organization Framework Convention on Tobacco Control since more than a Decade, National Tobacco-Control Program (NTCP), of India is in place. Though there were several tobacco control 
policies introduced in the country, the production side of tobacco menace, alternative crops to tobacco still need to strengthen further. Current study is based on a mixed methods observations study to explore various perspectives of tobacco farmers on their knowledge/awareness about health and tobacco, alternative crops options, and feasibility issues.

Methods: A mixed method study conducted to explore the farmers perspectives on tobacco and health, tobacco cultivation and alternative crops. A structured self-administered Quantitative questionnaire used for Tobacco farmers $(\mathrm{N}=150)$ of 6 districts, Andhra Pradesh state, India. Qualitative guide was used to conduct Focus Group Discussions (FGDs) for 36 tobacco farmers of 3 districts from Andhra Pradesh and Karnataka state.

Results: Awareness of tobacco related health hazards are, Tobacco crop is always profitable, previously the crop was profitable, always on losses $8 \%, 45 \%, 40 \%$.and $72 \% \%$ mentioned alternative crops are essential. $88 \%$ believed the tobacco causes various diseases, $4 \%$ consider the tobacco as a profitable crop rather public health concern. About $4 \%$ farmers have cancer patients in the family. About $28 \%$ said seek various forms of support from Govt, and $22 \%$ ask for financial benefits to quit tobacco cultivation. Only $8 \%$ said alternative crops not required. Qualitative findings revealed very interesting factors about why Farmers forced to grow tobacco though they know its deadly crop. Conclusions: This shows the role of National tobacco control program must address the alternative crops alongside of efforts to reduce Tobacco consumption. Farmers also addressed tobacco industries monopoly in controlling tobacco price and taking over maximum benefit out of tobacco crop than the farmer.

Tob. Induc. Dis. 2018;16(Suppl 1):A536

DOI: $10.18332 /$ tid/84749

\section{8:15-19:15}

LB-1357-5 Where do tobacco farmers live: association between Kenyan tobacco farmers' livelihood selection decision and their accessibility to nearby economic centers

\section{Q Li', P Magati², J Drope ${ }^{1}$}

${ }^{1}$ American Cancer Society, Atlanta, GA, United States of America, 2University of Nairobi, Nairobi, Kenya. E-mail: qing.li@cancer.org Background: Prosperous economic livelihood described by industry is hallucination in Kenya, smallholder tobacco farmers are making small or no profits [1-4]. World Health Organization Framework Convention on Tobacco Control (WHO FCTC) Article 17 encourages viable alternative livelihoods for tobacco farmers. However, little evidence on how tobacco farmers make livelihood selection decisions exists. [5] Therefore, we examined tobacco farmers' livelihood selection decisions under different accessibility levels to nearby economic centers.

Methods: 527 smallholder tobacco farmers in Kenya from three main tobacco-growing regions participated in our 2016-2017 wave economic livelihood survey, among whom 345 participants are current growers and 287 have a partnership contract. Geo-economic data was matched to surveyed farmers' Global Positioning System (GPS) coordinates to estimate each farmer's objective access to nearby economic centers. We examined the association between access to nearby economic centers and farmers' choice to grow tobacco and to grow tobacco under contract. Multivariate logistic regressions were adopted for models while controlling for social-economic status and selfreported activity in nearby economic centers. We also examined these models with regional variables fixed.

Results: Tobacco farmers rarely live within 10 kilometers range of an economic center. Farmers living 15-19.99 kilometers away from economic centers are $80.4 \%$ to $81.3 \%$ less likely to grow tobacco compared to those who live 10-14.99 kilometers away, and farmers living more than 20 kilometers away are $56.5 \%$ to $89.1 \%$ less likely to grow. Tobacco farmers living more than 20 kilometers away are 4.2 to 18.5 times more likely to grow under contract.

Conclusions: Farmers living further away from an economic center is less likely to grow tobacco, but if they grow tobacco, they are much more likely to grow under contract. The identification of geographic heterogeneity not only reveals the spatial economic dynamics, but also helps policy makers tailoring policies at different regional level.

Tob. Induc. Dis. 2018;16(Suppl 1):A537

DOI:10.18332/tid/84761

\subsection{Youth tobacco experience}

\section{$11: 00-12: 30$}

F0-349-5 Perceptions of cigarette and snus packages among youths and young adults in Norway J Scheffels' I Lund

${ }^{1}$ Norwegian Institute of Public Health, Drug Policy, Oslo, Norway. E-mail: janne.scheffels@fhi.no

Background: The aim of plain packaging is to take away the tobacco package's visual identity and appeal as an advertisement for the product and to reinforce the influence of health warnings. We studied perceptions of cigarette and snus packaging among youths and young adults in Norway before the implementation of plain tobacco packaging legislation (including both cigarettes and snus) on July 1st 2017.

Methods: We collected data in a web survey among 1200 youths aged 16-21 (55\% female). The participants answered questions about how often they had seen tobacco products and health warnings on packages, perceptions of package appeal, potential of harm and differentiation between products.

Results: Preliminary analyses showed that nine out of ten respondents reported having seen cigarette and snus packages often during the last six months. $50 \%$ reported having noticed health warnings on cigarette packages during the same period, while $23 \%$ had seen warnings on snus packages. A minority of the respondents evaluated cigarette packages as cool (4\%) or exciting to look at (6\%), while a larger share agreed that packages were ugly (48\%) or disgusting (40\%). Correspondingly, 15\% agreed to a statement that snus packages were cool and $20 \%$ to that they were exciting to look at, while $34 / 38 \%$ agreed that snus packages were ugly or disgusting. The participants evaluated cigarette and snus packages similarly as regards differentiation between packages based upon appearance or harmfulness.

Conclusions: The participants evaluated snus packages somewhat more positively compared to cigarette packages, while their perceptions of difference between products were rather similar for snus and cigarettes. New data will be collected to study to what extent the implementation of the plain packaging legislation will influence perceptions of cigarette and snus packages among youths in Norway.

Tob. Induc. Dis. 2018;16(Suppl 1):A538

DOI:10.18332/tid/83890

$11: 00-12: 30$ 
F0-350-5 The impact of a tobacco point-of-sale display ban on youth in the United Kingdom: findings from a repeat cross-sectional survey pre-, mid-and post-implementation

A Ford ${ }^{1,2}$, AM Mackintosh ${ }^{1,2}$, C Moodie $^{1,2}$, G Hastings $^{1,2}$, L Bauld $^{1,2}$ ${ }^{1}$ University of Stirling, Institute for Social Marketing, Stirling, United Kingdom, ${ }^{2}$ UK Centre for Tobacco and Alcohol Studies, University of Stirling, United Kingdom. E-mail: a.j.ford@stir. ac.uk

Background: The display of tobacco products at the point-of-sale (POS) allows tobacco companies to showcase their products and communicate with consumers. Evidence suggests that exposure to POS tobacco displays is associated with impulse tobacco purchasing among adult smokers and smoking susceptibility among never smoking youth. In the United Kingdom (UK) a ban on the open display of tobacco products was phased in between 2012 and 2015. Three waves of the Youth Tobacco Policy Survey were used to examine the impact of the ban on youth pre-, midand post-implementation.

Methods: A repeat cross-sectional survey was conducted with 11-16 year olds across the UK. Data was collected via an in-home face-to-face interview and self-completion questionnaire at three time points: pre- $(2011, \mathrm{n}=1373)$, mid- $(2014, \mathrm{n}=1205)$, and post-display ban $(2016, \mathrm{n}=1213)$. We examined whether the salience of displays was associated with smoking susceptibility among never smokers. Measures also explored cigarette brand awareness, attractiveness of displays and support for a display ban.

Results: In 2011, pre-ban, susceptibility to smoke was positively associated with noticing cigarettes displayed at POS and higher brand awareness. Susceptibility to smoke decreased from $28 \%$ in 2011 (pre-ban) to $18 \%$ in 2016 (post-ban), with the mean number of brands recalled falling from $1.56(\mathrm{SD}=1.88)$ in 2011 to 1.01 $(\mathrm{SD}=1.42)$ in 2016. With respect to support for the ban, in 2016 the vast majority of our sample ( $87 \%$ ) indicated that shops should have to keep cigarettes behind closed shutters. They also felt that having them behind closed shutters made them seem unappealing (73\%) and made them think that it's not ok to smoke (83\%).

Conclusions: That smoking susceptibility was lower following the ban suggests that placing tobacco out of sight helps safeguard young people and justifies this policy approach in the UK and elsewhere.

Tob. Induc. Dis. 2018;16(Suppl 1):A539 DOI: $10.18332 /$ tid/84517

\section{$11: 00-12: 30$}

F0-352-5 Tobacco 21: prevention of nicotine addiction by increasing the minimum legal access age, US and international experience

R Crane ${ }^{1,2}$, M Berman $^{3,4}$, M Myers $^{5}$

'Ohio State University, Family Medicine, Dublin, $\mathrm{OH}$, United States of America, ${ }^{2}$ Preventing Tobacco Addiction Foundation, Dublin, $\mathrm{OH}$, United States of America, ${ }^{3} \mathrm{O}$ hio State University, Moritz College of Law, Columbus, $\mathrm{OH}$, United States of America, ${ }^{4}$ Ohio State University, College of Public Health, Columbus, $\mathrm{OH}$, United States of America, ${ }^{5} \mathrm{Campaign}$ for Tobacco-Free Kids, Washington D.C., WA, United States of America. E-mail: mmyers@tobaccofreekids.org

Background and challenges to implementation: In accordance with the World Health Organization Framework Convention on Tobacco Control, almost all countries set a minimum legal access age (MLA) for tobacco products. Most countries have established their minimum at age 18, but the MLAs vary from 14 to 21 . Although the effectiveness of youth access restrictions has been mixed in the past, emerging evidence indicates the promise of increasing the MLA to 21.

Intervention or response: So far, over 250 local U.S. jurisdictions and three states-covering $22 \%$ of the U.S. population-have raised their MLA to 21. In addition, six countries: Honduras, Kuwait, Samoa, Singapore, Sri Lanka and Uganda have established an MLA of 21, and Japan's access age is 20. Success in enforcing these laws, however, has been variable, and adult smoking rates, especially among men, remain stubbornly high. In addition, tobacco manufacturers and tobacco retailers are increasingly mobilizing to stymie laws raising the MLA to 21

Results and lessons learnt: In the U.S., the prestigious Institute of Medicine assembled an expert panel to study the potential effects of higher MLAs. This panel compiled an extensive, evidencebased report, published in 2015. It projected that raising the MLA to 21 would prevent 4.2 million years of life lost in youth currently alive today, reduce youth tobacco use rates by $25 \%$, and reduce adult prevalence by $12 \%$, among other health benefits. Based on this report and other evidence, there is considerable interest in the U.S. in raising the MLA to 21.

Conclusions and key recommendations: The authors, respectively professors of medicine and law, have extensive academic and political experience in youth access issues including MLA 21, policy advocacy, and tobacco control legal issues. This presentation will review best practices for crafting clear and enforceable policy language designed to raise the MLA, and frame the scientific, social, and legal arguments necessary to win public and legislative support.

Tob. Induc. Dis. 2018;16(Suppl 1):A540 DOI: $10.18332 /$ tid/84153

$11: 00-12: 30$

F0-353-5 Children and youth exposure to tobacco products In Ghana

H Musah

'Vision for Alternative Development (VALD), Greater Accra, Ghana. E-mail: hajaramusah@valdghana.org

Background and challenges to implementation: Ghana Global Tobacco Youth Survey (2009) showed that $8.9 \%$ of students had ever smoked cigarettes and $12.5 \%$ currently use any tobacco product. Public Health Act 2012 bans tobacco advertisement close to school and children playing ground and sales to and by minors. The law has been violated. Cigarettes are sold closed to schools, children play ground and children buy and sell. Retailers are not aware of the laws. SDGs will not be achieved if children are not protected.

The project sought to draw attention to the violations of the law and to ensure that children are protected from tobacco.

Intervention or response: Data were collected in some selected communities in Greater Accra targeting aged 15-18 years to ascertain the level of compliance to cigarette sales to and by minor and sales close to schools as enshrined in the law. Retailers and community members were interviewed. The findings of this exercise was shared with stakeholders.

Results and lessons learnt: Out of 10 retail shops, 7 indicated were not aware of the laws banning sales to and by minors and 3 knew about the law. 5 out of the 10 shops sells to children but enquires who sent them, 3 sell to children without enquiring and 2 do not sell to children. 7 out of the 10 shops sell close to schools and 3 do not. 6 out of 10 displays cigarette on the counter visible 
to children.

Conclusions and key recommendations: Community members were not aware of the law on tobacco control. School going children are exposed to tobacco. Retailers and community member have been sensitized on the tobacco law.

Effort must be made to intensify awareness on the provisions of the law. Set-up taskforce to monitor compliance of the law. Conduct further research and findings on the children exposure to tobacco.

Tob. Induc. Dis. 2018;16(Suppl 1):A541

DOI: $10.18332 /$ tid/83848

\section{$11: 00-12: 30$}

F0-354-5 Tobacco-free generation campus initiative: a program to increase the number of tobacco-free colleges in the United States

B Sinha ${ }^{1}$, CE Douglas ${ }^{1,2}$

${ }^{1}$ American Cancer Society, Atlanta, GA, United States of America, ${ }^{2}$ University of Michigan School of Public Health, Ann Arbor, MI, United States of America. E-mail: bidisha.sinha@cancer.org Background and challenges to implementation: There are 20 million college and university students in the United States. More than 1 million of these young adults are projected to die from cigarette smoking. Progress has been made in protecting campus populations against tobacco. There are now 1,913 smoke-free college and university campuses, of which 1,611 are fully tobacco-free. Yet, there are 4,500 higher education institutions in the US, and many still lack comprehensive smoke- and/or tobacco-free policies due to inertia, competing needs and opposition from tobacco supporters and those expressing libertarian objections.

Intervention or response: In response, the American Cancer Society launched the Tobacco-Free Generation Campus Initiative (TFGCI) in 2016, which targets students, faculty, staff and visitors at a variety of academic institutions - from small community colleges to large research universities - across the country. TFGCI provides financial support to colleges and universities to pursue campaigns to adopt and implement comprehensive tobacco-free policies and is supported by the CVS Health Foundation. Grantees also receive technical assistance and materials. TFGCI advises campus leaders and students on advocacy best practices, policy adoption, implementation and enforcement issues, cessation strategies and communications, and includes an evaluation component.

Results and lessons learnt: Seven of the 20 institutions in Cohort 1 approved tobacco-free policies during the first year, a high level of success. Each institution confronted unique challenges, including opposition from skeptics - sometimes including the institution's leadership - but most effectively advanced knowledge and built support. Cohort 2 commences work in Fall 2017, and includes an additional 42 institutions. TFGCI will eventually support 125 institutions.

Conclusions and key recommendations: Cohorts 1 and 2 cover 930,000 students, plus many faculty, staff and visitors. TFGCI has demonstrated that, with sufficient resources and application of best practices, these campaigns can succeed. Recommendations include the utilization of evidence-based resources, effective communication and education, and application of best practices in all campus policy campaigns.

Tob. Induc. Dis. 2018;16(Suppl 1):A542

DOI:10.18332/tid/84306

14:30-16:00
RF-1222-5 Differences in rates of tobacco product use among U.S. Hispanic youth

M Moran' ${ }^{1}$, T Alexander², R Kennedy ${ }^{1}$, C Weiger ${ }^{1}$

JJohns Hopkins Bloomberg School of Public Health, Health, Behavior \& Society, Baltimore, MD, United States of America, ${ }^{2}$ Battelle, Public Health Research \& Translational Science, Baltimore, MD, United States of America. E-mail:mmoran22@ jhu.edu

Background: Hispanics are the fastest growing ethnic group in the United States, and projected to grow by $86 \%$ by 2050 . Hispanics are also a diverse ethnic group, consisting of individuals with heritage from many areas, including Mexico, South America, Cuba and Puerto Rico. Little is known about variations in tobacco use and susceptibility among such Hispanic sub-groups.

Methods: We analyzed Wave 2 youth data from the Population Assessment of Tobacco and Health (PATH) study. PATH is a nationally representative survey of 12,172 youth aged 12-17 and adults, living in the United States. This dataset provides information on whether a participant is Mexican, Cuban, Puerto Rican, or of another Hispanic heritage. We examined differences in susceptibility to use and ever use of four tobacco products (cigarettes, e-cigarettes, cigarillos and hookah) among Hispanic youth. We also examined correlates of susceptibility and use.

Results: Mexican, Cuban, Puerto Rican and other Hispanic youth were more likely than non-Hispanic youth to be susceptible to hookah use (ORs=1.7, 2.5, 1.5 and 1.6, respectively), while Mexican American and other Hispanic youth were more likely than non-Hispanic youth to be susceptible to e-cigarette use $(\mathrm{ORs}=1.3$ and 1.2 , respectively) and cigarillo use (ORs $=1.4$ and 1.4, respectively). Mexican, Cuban, and other Hispanic youth were more likely to have tried smoking hookah (ORs=1.5, 4.1 and 1.65 , respectively). Parental education, friends' tobacco use, and beliefs that tobacco would calm them down were consistent predictors of tobacco use. Specific differences in correlates of susceptibility and use of each product, and by Hispanic subgroup, will be discussed.

Conclusions: It is critical to take into account the diversity among the U.S. Hispanic population when examining rates of tobacco use. Failing to do so can mask disparities in tobacco use among specific sub-groups. Tobacco prevention campaigns and policies should similarly consider the diversity of this population.

Tob. Induc. Dis. 2018;16(Suppl 1):A543

DOI:10.18332/tid/84541

14:30-16:00

RF-1223-5 Multilevel analysis of the individual and contextual factors of tobacco use among Mexican adolescents using the National Addiction Survey (NAS), 2011

L Zavala Arciniega ${ }^{1}$, LM Reynales Shigematsu', N Fleischer ${ }^{2}$ ${ }^{1}$ National Institute of Public Health in Mexico, Department of Tobacco Research, Cuernavaca, Mexico, ${ }^{2}$ University of Michigan, Detroit, MI, United States of America. E-mail: luis.zavala@ insp.mx

Background: The tobacco industry has targeted low and middleincome countries and vulnerable groups such as women and young people. In Mexico we observe a slow decrease in tobacco consumption among adults, while in adolescents the trend in tobacco use shows an increase. Previous research in Mexico do not measure determinants at neighborhood level. The aim of the study is evaluate whether the contextual and individual factors are associated with tobacco use among Mexican adolescents. 
Methods: Data from this study came from NAS 2011 and National Census 2010. Descriptive statistics were estimated after adjusting for sampling weights. The study had two levels: individual (adolescents between 12-17 years) and contextual (neighborhoods). Multilevel logistic regression stratified by sex was conducted.

Results: The final sample includes 2785 adolescents from 364 neighborhoods. Prevalence of 30 days smoking in women adolescents was $3.8 \%$ and in male adolescents was $10.3 \%$. Female adolescents exposed to second hand smoke at home were more likely to be a current smoker (OR $2.87 \mathrm{CI}: 1.37,6.04)$ and in those who have an employment (OR $4.35 \mathrm{CI}$ : 1.03,18.3). School attendance was inversely associated with tobacco use (OR 0.38 CI: 0.16,0.89). In Male adolescents the likelihood of being a current smoker increase according to age (OR $4.35 \mathrm{CI}$ : $1.51,12.4$ for $15-17$ vs $12-14$ ) and in those exposed to second hand smoke at home (OR 5.03 CI 2.38,10.6), while school attendance decrease the likelihood of being a current smoker (OR 0.14 CI: 0.05,0.39).

Conclusions: This study suggest that school attendance is a protective factor for smoking. Second hand smoke at home is an important factor of tobacco use. Have an employment increase the likelihood of being a current smoker only in female adolescents. Implement a successful tobacco control policy in Mexico needs to take into account educational, economics, social and gender determinants.

Tob. Induc. Dis. 2018;16(Suppl 1):A544

DOI: $10.18332 /$ tid/84628

\section{4:30-16:00}

RF-1224-5 Youth attitudes and beliefs towards cigarette and waterpipe use in nine Middle Eastern Countries

H Mehrtash', M Parascandola²

${ }^{1}$ National Cancer Institute, Center for Global Health, Rockville, MD, United States of America, ${ }^{2}$ National Cancer Institute, Division of Cancer Control and Population Sciences, Rockville, MD, United States of America. E-mail: hedieh.mehrtash@nih.gov Background: The Eastern Mediterranean Region (EMR) is one of only two WHO regions project to increase tobacco use prevalence by 2025. Evidence shows initiation occurs at an early age, so understanding factors that influence youth tobacco initiation is a high priority. The purpose of this analysis to understand the factors that contribute to youth waterpipe use in the region.

Methods: This study uses Global Youth Tobacco Survey (GYTS) data from Egypt (2009), Jordan (2009), Kuwait (2009), Lebanon (2010), Morocco (2011), Oman (2010), Saudi Arabia (2010), Tunisia (2010) and Turkey (2012). GYTS is a nationally representative survey of 13-15-yearolds using a standardized protocol. Current cigarette smoking status is defined as having smoked at least once in the last 30 days preceding the survey. Current waterpipe smoking status is defined as use of at least one waterpipe rock) in the last 30 days preceding the survey.

Results: Of the total sample population, $7.65 \%$ were current cigarette smokers, $9.05 \%$ current waterpipe smokers, $22.0 \%$ experimented with cigarettes, and $26.3 \%$ experimented with waterpipe. Across all four countries, girls were more likely than boys to have negative opinions about tobacco use. Male gender and parental smoking were strong predictors of experimentation with both cigarettes and waterpipe when controlling for other variables. In addition, those who believed that waterpipe smoke was not harmful were more likely to experiment with waterpipe $(\mathrm{OR}=2.32,95 \mathrm{CI} \%$ : 1.8-2.9). But no association was seen between smoking behavior and perceptions of harm from cigarette smoke. Apart from Lebanon, over 30\% of smokers in each country stated that waterpipe smoking is "not harmful to health".

Conclusions: Low perceptions of harm from waterpipe smoking may be an important contributor to youth tobacco use in the EMRO region. Better understanding of attitudes, knowledge and risk behaviors around youth waterpipe smoking can inform effective interventions in these countries.

Tob. Induc. Dis. 2018;16(Suppl 1):A545

DOI: $10.18332 /$ tid/84154

\section{4:30-16:00}

RF-1225-5 Testing the efficacy of a schoolbased tobacco and supari cessation intervention in Mumbai, India

H Gupte', G Mandal'2, T Bhutia², L Chaudhuri', N Chatterjee $^{1}$ ${ }^{1}$ Narotam Sekhsaria Foundation, Mumbai, India, ${ }^{2}$ Salaam Bombay Foundation, Mumbai, India. E-mail: himanshu@nsfoundation. co.in

Background: Tobacco and supari (areca nut or betel nut) use has been reported with a high prevalence among students in secondary schools in Mumbai, India. Supari, which is classified as carcinogenic, is one of the most widely consumed addictive substances in India after nicotine, ethanol and caffeine.

A study was conducted to evaluate the efficacy of a school-based psychosocial intervention for all forms of tobacco and supari cessation among students.

Methods: A quasi-experimental trial with three measurements at baseline, Post-test 1 and Post-test 2 was conducted in 12 schools serving students with similar socioeconomic profile in 2015-16. Six schools were randomly selected for the cessation intervention and 6 matching schools were selected based on location and medium of instruction in comparison group. In the interventions schools, students who self-reported tobacco or supari use at baseline were assigned to receive a 6 -session group cessation intervention designed specifically for adolescents and school settings. All schools receive a life-skills and tobacco prevention program called Super-Army.

Results: 1313 students completed the baseline in intervention schools; 212 self-reported tobacco/supari use, of which 133 enrolled and received both Super-Army in classroom and 6 psychosocial cessation sessions in group setting. In comparison schools, 1320 students completed baseline; of which 191 reported tobacco/supari use and received only the Super-Army prevention messages in classroom.

At Post-test 1, conducted 4 weeks after sessions ended, reported use in intervention schools dropped by $30 \%$ and in comparison schools by $28 \%$. At Post-test 2, conducted 16 weeks later, in intervention schools self-reported use dropped further by $45 \%$. This was significantly different from comparison schools where users dropped by $13 \%$.

Conclusions: Although classroom-based life-skills curricula have an immediate impact on tobacco/supari use, the addition of a specialized cessation intervention, even if psychosocial in nature without replacement therapy, seems to have a durable effect on reducing use.

Tob. Induc. Dis. 2018;16(Suppl 1):A546

DOI:10.18332/tid/84096

14:30-16:00 
RF-1226-5 Tobacco use among high schoolchildren: social and behavioral determinants in the Indian context

R Shenoy', Tobacco use study group, prevalence study group 'Yenepoya University, Public Health Dentistry, Mangalore, India. E-mail: merekhap@yahoo.co.in

Background: Growing influence of tobacco among youth in developing countries has necessitated evaluation of social and behavioral factors responsible for the phenomenon. Mangalore taluk is a reputed health care center in Karnataka state, India, with high levels of urbanization and literacy. This investigation evaluated social and behavioral determinants of tobacco use among children of public and private high schools in urban and rural areas of Mangalore taluk.

Methods: Study participants were enrollees of ten high schools selected by stratified cluster sampling. After obtaining ethical clearance, permission from concerned authorities and assent from participants, a pre-tested questionnaire was used to collect data on current use, reason for initiation, funding for habit, source of tobacco, difficulty in procurement, changes in lifestyle and health, knowledge about ill-effects, etc. Data were analyzed using the Chi squared test. Results: Current tobacco use was prevalent among $12.8 \%$ of the 1340 participants aged between 11-18 years. Evaluation of responses revealed no significant differences based on type of school or location; however, gender differences among participants were pronounced. Among users, girls reported higher use of smokeless tobacco, higher frequency of daily use, and use by family members as primary reason for initiation into habit. A significant number of users had initiated use in the past year, $35.7 \%$ stated use by family as reason for initiation, a majority reported funding of habit by allowances from family, and 73.7\% reported no difficulty in procurement from vendors near public facilities. While $88.9 \%$ considered the habit dangerous and $31.6 \%$ wanted to stop use, $20 \%$ reported an increased self-esteem. Among non-users, $84.1 \%$ reported availability of tobacco for use. Among both categories, predominant source of knowledge on illeffects of use was the print media.

Conclusions: Rising tobacco consumption among girls and easy availability of tobacco for use among adolescents are a cause for concern.

Tob. Induc. Dis. 2018;16(Suppl 1):A547

DOI:10.18332/tid/83802

\section{4:30-16:00}

RF-1227-5 Identifying misleading propaganda of Electronic Nicotine Delivery Systems (ENDS) and hookah in New Delhi, India: building youth self-efficacy skills to resist new industry tactics P Kathuria ${ }^{1}$, R Shrivastav ${ }^{1}$, M Arora ${ }^{1,2}$, P Sinha ${ }^{3}$, V Gill Munish ${ }^{3}$, F Tullu ${ }^{3}$

${ }^{1}$ HRIDAY, New Delhi, India, ${ }^{2}$ Public Health Foundation of India, Gurugram, India, ${ }^{3}$ WHO Country Office for India, New Delhi, India.E-mail: prachi@hriday-shan.org

Background and challenges to implementation: The Indian tobacco control law (COTPA) prohibits tobacco advertising, promotion and sponsorship. However, emerging products like e-cigarettes and e-hookahs (ENDS) remain unregulated and traditional hookahs are regulated inadequately. Rampant promotional propaganda for these products has created a general mis-perception that they are safe/less harmful than regular tobacco products, resulting in increasing popularity among adolescents and youth.

Intervention or response: A youth-led monitoring exercise was conducted to build their capacity for identifying mis-leading and false ENDS and hookah advertisements and promotional campaigns, at multiple Points of Sale (PoS), at New Delhi, India.

Sensitisation sessions were conducted for 104 school and college students, (13-23 years) from 6 schools and 5 colleges, on the hazards of ENDS and hookah usage; deceptive strategies of the tobacco industry to take advantage of policy loopholes. These empowered youth monitored $79 \mathrm{PoS}$ (64 physical and 15 e-commerce sites), using standardised monitoring protocols/tools.

Results and lessons learnt: Approximately $31 \%$ of physical sites were within 100 yards of an educational institution (prohibited under COTPA). Warning boards against sale to minors were missing in more than half of the PoS. ENDS were commonly being promoted as: safe, herbal, as cessation devices and also as 'trendy' tobacco products. At $47 \%$ of PoS, hookah flavours were arranged as power walls. A range of e-commerce sites were selling ENDS and hookahs, without asking for any age proof or only selfdeclaration of age, which can be mis-reported. These websites offered attractive discounts and 'free home delivery' to boost sale. Conclusions and key recommendations: Youth-targeted promotion and sale of ENDS and hookah is rampant. E-commerce sites are particularly lucrative PoS. The youth had many false notions about ENDS and hookah. There is an urgent need to regulate these products through national and global policy measures, in order to prevent the tobacco industry from exploiting this grey area in tobacco control policy.

Tob. Induc. Dis. 2018;16(Suppl 1):A548

DOI:10.18332/tid/84568

[Youth led monitoring_Key Findings]

$14: 30-16: 00$

Youth-led monitoring of Electronic Nicotine Delivery Systems (ENDS) and hookah sale and promotion in New Delhi, India: Key Findings

Total Points of Sale (PoS) monitored: 79 (64 physical and 15 e-commerce sites)

Total PoS monitored for ENDS: 56*

Total PoS monitored for Hookah: 52*

*Some sites were common for ENDS and hookah

ENDS ( $n=56$ PoS)

\begin{tabular}{|l|l|l|l|l|l|l|l|}
\hline $\begin{array}{l}\text { No age } \\
\text { proof asked }\end{array}$ & Accessible & $\begin{array}{l}\text { Demonstration } \\
\text { of use }\end{array}$ & $\begin{array}{l}\text { Attractive } \\
\text { display }\end{array}$ & $\begin{array}{l}\text { Advertised } \\
\text { flavours }\end{array}$ & $\begin{array}{l}\text { No warning } \\
\text { (sale to } \\
\text { minors) }\end{array}$ & $\begin{array}{l}\text { Brands } \\
\text { identified }\end{array}$ & $\begin{array}{l}\text { Displayed the price of ENDS through } \\
\text { boards/posters }\end{array}$ \\
\hline $41 \%(n=23)$ & $36 \%(n=21)$ & $45 \%(n=26)$ & $33 \%(n=19)$ & $34 \%(n=19)$ & $53 \%(n=30)$ & 24 & $56 \%(n=32)$ \\
\hline
\end{tabular}

Continued 


\section{Continued}

\begin{tabular}{|l|l|l|l|l|l|l|l|}
\hline \multicolumn{2}{|l|}{ Hookah (n=52 PoS) } \\
\hline $\begin{array}{l}\text { No age } \\
\text { proof asked }\end{array}$ & Accessible & $\begin{array}{l}\text { Demonstration } \\
\text { of use }\end{array}$ & $\begin{array}{l}\text { Attractive } \\
\text { display }\end{array}$ & $\begin{array}{l}\text { Advertised } \\
\text { flavours }\end{array}$ & $\begin{array}{l}\text { No } \\
\text { warning } \\
\text { (sale to } \\
\text { minors) }\end{array}$ & $\begin{array}{l}\text { Brands } \\
\text { identified }\end{array}$ & $\begin{array}{l}\text { Youth noticed hookah } \\
\text { sponsorship/promotional offers }\end{array}$ \\
\hline $41 \%(n=21)$ & $38 \%(n=20)$ & $43 \%(n=22)$ & $53 \%(n=28)$ & $57 \%(n=30)$ & $61 \%(32)$ & 17 & $67 \%(n=35)$ \\
\hline
\end{tabular}

RF-1228-5 New approach to decrease smoking among young adult bar patrons

P Ling', N Lisha', T Neilands', J Jordan ${ }^{2}$

${ }^{1}$ University of California San Francisco, Medicine, San Francisco, CA, United States of America, ${ }^{2}$ Rescue Agency, San Diego, CA, United States of America. E-mail: pamela.ling@ucsf.edu Background: Young adulthood is critical both for tobacco initiation and cessation. This study evaluated the effectiveness of an innovative Social Branding intervention to decrease smoking among high risk "Hipster" young adults in bars and nightclubs. The intervention included an anti-tobacco Social Brand to compete with cigarette brands, sponsored social events in bars/ clubs, anti-tobacco messaging created by local young adult artists, branded clothing and promotional items, website, social media and direct mail promotions.

Methods: Quasi-experimental controlled study of young adult bar patrons in two intervention cities (San Diego and San Francisco, CA) and one comparison community (Los Angeles, CA), surveyed at baseline and two years follow-up (approximately 1200 each time point per city) between January, 2012, and October, 2016. Cross-sectional surveys were collected using randomized timelocation sampling of venues in each city. The outcome was current (past 30 day) smoking. Logistic regressions controlling for age, sex and race/ethnicity with interactions between location and time were conducted.

Results: Current smoking prevalence among bar patrons was $41-50 \%$, higher than the general California smoking prevalence of $12 \%$. In San Diego, where the intervention was established for several years, cigarette smoking was significantly lower $(42 \%$ baseline, 38\% follow up) than in Los Angeles (LA, 45\% baseline and $45 \%$ follow up) throughout the study. In San Francisco, where the intervention was new, cigarette smoking decreased significantly (51\% baseline, $44 \%$ follow up), compared to LA where there was no change.

Conclusions: An innovative Social Branding intervention was associated with a sustained lower smoking prevalence in San Diego and a significantly decreased smoking prevalence in San Francisco, compared to the control community. While these data are limited to California, this segmentation strategy can be used across the US and among young adults in many other countries. This approach could also build a global anti-tobacco brand for young adults.

Tob. Induc. Dis. 2018;16(Suppl 1):A549

DOI: $10.18332 / \mathrm{tid} / 84271$

14:30-16:00

RF-1229-5 The growth of Roll-Your-Own (RYO) cigarettes in Ireland: exploring the experiences of young people and RYO tobacco

E Breslin'1, J Hanafin', L Clancy'

${ }^{1}$ TobaccoFree Research Institute Ireland (TFRI), Dublin, Ireland. E-mail: ebreslin@tri.ie

Background: The current smoking prevalence rate in Ireland is 18.6\% (HSE, 2016). Ireland aims to be tobacco free by 2025 (prevalence rate of less than 5\%). Previously the TFRI found high prevalence rates of RYO use among young smokers (16-17 years) with this group being more likely to exclusively smoke RYO (32\%) compared to factory manufactured (FM) cigarettes (28\%) (Babineau et al., 2015). In adults RYO use has increased from $4.4 \%$ in 2006 to $29.7 \%$ in 2016 (HSE, 2016) and first time smokers under 25 years are more likely to smoke RYO cigarettes (50.6\%) compared to factory manufactured cigarettes (49.4\%; HSE, 2017).

Methods: This study explores the increasing popularity of RYO products and looks for possible policy interventions that can be recommended. Semi-structured interviews and focus groups were conducted with two groups aged 16-22 years, with one group comprising of university students and the other with young people attending youth organisations for early-school leavers.

Results: Preliminary findings indicate two overarching themes; facilitative and inhibitory factors. Intrinsic aspects of the products such as the lower cost of RYO tobacco in comparison to FM cigarettes often prompted users to begin smoking RYO, with peer influences and the presence of rituals surrounding the use of RYO products contributing positively to RYO users' experiences. Inhibitory factors associated with RYO include family disapproval, difficulty in concealment, time needed, weather conditions and embarrassment. Conclusions: Low cost of RYO products is the main incentive for their use and demands the equalisation of the prices of $\mathrm{RYO}$ and cigarettes by raising taxes on RYO tobacco products. Recommendations focused on the further denormalisation of smoking such as targeted programmes working with youth smokers, recognition of the role of family influences and particularly the introduction and enforcement of smoke-free third level campuses are warranted.

Tob. Induc. Dis. 2018;16(Suppl 1):A550

DOI:10.18332/tid/84126

\section{4:30-16:00}

RF-1230-5 'You do it through the grapevine'. A qualitative study of how young people in Europe access cigarettes

R Hewer' ${ }^{1}$ S Hill2 ${ }^{2}$ A Amos ${ }^{1}$

'University of Edinburgh, Centre for Population Health Sciences, 
Edinburgh, United Kingdom, ${ }^{2}$ University of Edinburgh, Global Public Health Unit, Edinburgh, United Kingdom. E-mail: s.e.hill@ ed.ac.uk

Background: Reducing youth smoking uptake is a key goal of tobacco control. Article 16 of the FCTC requires governments to prohibit tobacco sales to minors, yet young people continue to access cigarettes despite widely adopted legislation on minimum age of sale. This qualitative study explores the experiences of young people in Europe in accessing cigarettes.

Methods: Over 50 focus groups were carried out with 15-17 year olds in seven countries across Europe (Belgium, Finland, Germany, Ireland, Italy, the Netherlands, Portugal). Most participants were 15 , and all were below the legal age of sale in their country. Focus groups were conducted in the dominant national language and recorded, transcribed and translated into English. Data were analysed to identify key themes in young people's accounts of how they and their peers source cigarettes.

Results: Ability to access cigarettes was reported in all focus groups. Sources included direct purchase (from shops, vending machines, bars/cafes); proxy purchase (where an older person buys on their behalf); and reciprocal peer sharing arrangements. Participants' accounts indicate that social capital is an important resource in accessing cigarettes across all countries, with young people frequently asking older acquaintances or siblings to purchase for them. Social and cultural capital are also important in the (less common) use of strangers, which required a sophisticated understanding of how to recognise potential proxy buyers. Commercial sources of cigarettes varied by country, with shops (particularly small retailers) more important in some countries (eg Ireland), while vending machines were more commonly used in others (eg Portugal).

Conclusions: The effectiveness of tobacco sale age restrictions is influenced by broader aspects of the tobacco environment, including social norms and material access to less regulated outlets. Under-age smokers draw on different forms of capital to circumnavigate these legal restrictions, highlighting the importance of a multi-dimensional approach to youth smoking prevention.

\section{Tob. Induc. Dis. 2018;16(Suppl 1):A551 DOI:10.18332/tid/83774}

\section{4:30-16:00}

RF-1231-5 Youth who initiate tobacco use with e-cigarettes only differ from those who start with cigarettes only or both products concomitantly

M Harrell', C Jackson' ${ }^{1}$, J Delk', S Opara ${ }^{1}$, C Perry ${ }^{1}$

'UT Health School of Public Health, Austin, TX, United States of America. E-mail: melissa.b.harrell@uth.tmc.edu

Background: Few studies have compared the psychosocial risk profiles for youth who begin using tobacco with e-cigarettes only, cigarettes only, or both of these products.

Methods: More than 40 known psychosocial risk factors for youth tobacco use, including intra-personal (e.g., sensation seeking), inter-personal (e.g., peer influence), and environmental (e.g., tobacco marketing) factors were compared between those who started with e-cigarettes only ( $\mathrm{n}=156, \mathrm{~N}=14550)$, cigarettes only $(\mathrm{n}=59, \mathrm{~N}=7325)$, or both of these products $(\mathrm{n}=35, \mathrm{~N}=3510)$. Psychosocial risk factors were measured at baseline (2014-15) and tobacco use behaviors at 6,12 , and 18 month follow-ups (2015-16) in a population-based sample (n) of 3,116 students in 4 large urban areas of Texas that generalizes back to 353,552 students $(\mathrm{N})$ who were in the 6 th, 8 th, and 10 th grades and had never used tobacco at baseline.

Results: Youth who started with cigarettes only or both, compared to youth who started with e-cigarettes only, were more likely to have a family member that used cigarettes or e-cigarettes $(\mathrm{p}<$ $0.01)$ and be past 30-day marijuana users $(p=0.01)$ at baseline. Youth who started with e-cigarettes only, compared with the others, reported a higher positive affect $(\mathrm{p}=0.01)$ and lower negative affect at baseline $(\mathrm{p}=0.01)$ and were more likely to have positive outcome expectations about tobacco use (e.g., it helps me concentrate; $p=0.05)$ and be past 30-day alcohol users $(p=0.05)$ at baseline. Youth who started with both products, compared to one exclusively, were more likely to have engaged with tobaccorelated content on digital media (e.g., posted pictures, blogged) $(p<0.05)$ and less likely to report seeing displays for cigarettes at retail point-of-sale $(p<0.01)$ at baseline. No other differences in known risk factors (e.g., sensation seeking) were observed.

Conclusions: Psychosocial risk profiles do vary significantly by starter tobacco products. Implications for intervention will be discussed.

\section{Tob. Induc. Dis. 2018;16(Suppl 1):A552 DOI:10.18332/tid/84666}

\section{4:30-16:00}

RF-1232-5 Smokefree sports grounds contribute to a smokefree generation in the Netherlands

M van Dooremaal', R Haandrikman ${ }^{1}, \mathrm{MB}^{1}$ Bos $^{1}$

'Dutch Heart Foundation, Den Haag, Netherlands. E-mail: m.vandooremaal@hartstichting.nl

Background and challenges to implementation: Children spend substantial amount of time at sports clubs. Legislation prohibits indoor smoking at sports clubs. Outside, children are still frequently exposed to smoking. Children who see others smoking are more likely to start smoking when they get older. The Dutch Heart Foundation, co-initiator of the 'Smokefree Generation movement', developed a program to motivate and facilitate sports clubs in making sports grounds smokefree.

Intervention or response: Early 2016 the first sports clubs were recruited to implement a smokefree agreement. We developed a support package, including a step-by-step plan and smokefree signs. Early adopters were used as inspiration to others. Free publicity and campaigns brought smokefree sports to the attention of general public and sports clubs. Furthermore, we lobbied important stakeholders for their contribution to smokefree sports: sports associations, professional sports clubs and municipalities.

Results and lessons learnt: Smokefree sports is a fairly new subject. To increase awareness is crucial. The positive argument of protecting children against tobacco is well accepted.

Results:

The number of sports clubs with a (partly) smokefree sports ground increased from just a few in January 2016 to 135 half 2017 and we expect 200 at the end of 2017.

Partnerships with the Royal Dutch Football, Hockey and Korfball Association.

Baseline data shows that about $30 \%$ of managers from football, korfball and tennis clubs are willing to take smokefree steps. High level of social support: 9 out of 10 adults and children believe that sports grounds, were children are playing, should be smokefree.

Conclusions and key recommendations: Increasing the number of smokefree sports grounds will denormalise tobacco in society. Especially because it is part of a bigger movement towards a Smokefree Generation. Joining forces with important stakeholders 
will speed up the number of smokefree sports grounds. The use of a positive tone of voice is a key to social acceptance.

\section{Tob. Induc. Dis. 2018;16(Suppl 1):A553 DOI: $10.18332 /$ tid/84197}

\section{4:30-16:00}

RF-1233-5 Universal coverage of comprehensive school-based tobacco control programs to reduce youth smoking in Seoul, Korea

H Kang ${ }^{1}$, SY Kim ${ }^{1}$, M Jang ${ }^{2}$, S Yoo ${ }^{1,2}$, JY Chung ${ }^{3}$, S-I Cho ${ }^{1,2}$

'Department of Public Health Science, Graduate School of Public Health, Seoul National University, Seoul, Korea, Republic of, ${ }^{2}$ Institute of Health and Environment, Seoul National University, Seoul, Korea, Republic of, ${ }^{3}$ Korea Health Promotion Institute, Seoul, Korea, Republic of. E-mail: hiw0301@snu.ac.kr

Background: This study aims to examine the effects of comprehensive school-based tobacco control programs universally provided in schools of Seoul City, Korea.

Methods: To investigate the current status of school-based tobacco control programs, data were acquired from The Seoul Metropolitan Office of Education. To assess the capacity to implement tobacco control programs, the "Five-P's" matrix was used. The matrix consists of 5 domains: Policies, Program, People, Provision of funds, and Partnerships. To measure smoking behavior changes, we analyzed data from the 2015-2016 Korea Youth Risk Behavior Web-based Survey of adolescents (aged 1218 years) in Seoul. Measures included smoking prevalence, age at smoking initiation, and proportion of quit attempts.

Results: Since 1999, Korean government has promoted schoolbased tobacco control programs. Less than $10 \%$ of schools had offered the programs until 2014. With the increase of tobacco tax in $2015,100 \%$ schools started to provide comprehensive tobacco control programs including tobacco-free policies, anti-tobacco education and activities. Each school designated at least one teacher in charge. In 2016, a total of 3.9 million USD was invested in Seoul, with more than $70 \%$ of the funds allocated to a total of 1,300 schools, covering 976,000 students. Partnerships to enforce programs were also developed with community health centers. The prevalence of smoking among adolescents in Seoul was $7.0 \%$ in 2015 and $5.8 \%$ in 2016 , a $17 \%$ reduction in one year. Smoking initiation age increased slightly from 12.7 to 12.9 years. Proportion of quit attempts continued to be as high as $73.5 \%$ in 2015 and 2016.

Conclusions: Universal coverage of school-based tobacco control programs appears to result in a significant reduction of youth smoking. Previously decreasing trend of smoking prevalence in Seoul adolescents has accelerated with the expansion of school-based tobacco control programs. The effectiveness of the programs needs to be monitored and continuously enhanced to achieve a tobacco-free generation.

Tob. Induc. Dis. 2018;16(Suppl 1):A554

\section{DOI:10.18332/tid/84267}

\section{$18: 15-19: 15$}

LB-1336-5 Prevalence and knowledge on e-cigarette among teenagers in Romania 2016

I Munteanu', F Mihaltan'1, A Trofor ${ }^{2}$, D Todea ${ }^{3}$, M Marc $^{4}$, C Marginean ${ }^{5}$, M Trenchea $^{6}, 0$ Arghir $^{6}$

'Marius Nasta Institute of Pneumology, Bucharest, Romania, ${ }^{2}$ Pneumology Hospital lasi, lasi, Romania, ${ }^{3}$ Pneumology Hospital Cluj, Cluj, Romania, ${ }^{4}$ Pneumology Hospital Timisoara, Timisoara, Romania, ${ }^{5}$ Pneumology Hospital Tg. Mures, Tg. Mures, Romania,
${ }^{6}$ Pneumology Hospital Constanta, Constanta, Romania. E-mail: ioana.munteanu2015@yahoo.ro

Background: The prevalence of smoking for adolescents in Romania is growing, especially due to girls. According to the study GATS 2011 most of daily smokers started smoking at age $17-19$ years (43.1\%); $17 \%$ started smoking even before 15 years, mostly in rural areas Identifying factors that favor its use will allow better management of the phenomenon. Worrying is the fact that they use the device for inhalation of other psychoactive substances. The number of users among young people the electronic cigarette tends to be a in an ascendant trend among teenagers and especially girls.

Methods: The main objectives were to establish the prevalence of using, condition of using and knowledge about e-cigarette among them A number of 2000 original questionnaires was directly distribute to teenagers for high schools from 6 important towns from Romania.

Results: A number of 776 (457 girls and 319 boys ) adolescents from different parts of the country where involved. The main objectives were to establish the prevalence of using, condition of using and knowledge about e-cigarette among them. 324 (42\%) had used the device 177 girls and 147 boys. Majority of users were smokers but a number of 61 (21\%) were nonsmokers and they used it. Although the majority said they use it occasionally they are able to present the advantages and disadvantages of the device. The one who never used it related no advantages and the users mentioned : less toxicity, money save, you can smoke it everywhere . Regarding disadvantages the option were more variety like: battery power, price, utility and so on.

Conclusions: In conclusion, in the absence of specific legislative measures for Romania we can expect an increasing number of users among young people the electronic cigarette tends to be a in an ascendant trend among teenagers and especially girls.

\section{Tob. Induc. Dis. 2018;16(Suppl 1):A555 DOI:10.18332/tid/84690}

\section{8:15-19:15}

LB-1341-5 Tobacco free duo - 25 years of experience from an evidence-based method to prevent tobacco use amongst young people Y Viklund ${ }^{1}$

${ }^{1}$ County Council of Västerbotten, The Public Health Unit, Umeå, Sweden.E-mail: ywonne.wiklund@vll.se

Background and challenges to implementation: Tobacco Free Duo is a long-term school-based intervention. It started 1993 with the aim to prevent cancer by reducing Tobacco-use. The program has developed over the years led by the Health Unit, Västerbotten County Council in Sweden, in collaboration with youth and local communities in the county. After an evaluation in 2009, the method has spread to other parts of Sweden with support from the government, and is implemented in $25 \%$ of Swedish municipalities.

Intervention or response: The name Tobacco Free Duo originates from an idea that pupils in grade 6 are given an opportunity to team up with a tobacco free adult, and form a pair - a Duo. This is formalized by signing a mutual agreement that last until the pupil leaves secondary school, grade 9 . The agreement has an annual follow up confirming being tobacco free. The programme has some key components; class-information to increase the knowledge and awareness of tobacco related issues, strengthen self-confidence, giving positive reinforcement by different awards, creating a positive non-smoking influence through social support 
and influence from friends, parents and local society. Groups of pupils are educated to be Tobacco Free Duo ambassadors at the schools. They are role models and plan for activities to strengthen the tobacco-free environment.

Results and lessons learnt: Every year $80 \%$ of the pupils aged 11 12 , sign the agreement and the second generation of members in Tobacco free Duo is a fact. The first evaluation of the programme showed a $50 \%$ decrease in youth smoking, and that $25 \%$ of the adults were tobacco users quitting tobacco to become a Duopartner. Västerbotten has the lowest prevalence of adult smoking in Sweden.

Conclusions and key recommendations: Tobacco Free Duo has lasted, developed and spread during the past 25 years, Examples of key activities and factors of success will be presented.

Tob. Induc. Dis. 2018;16(Suppl 1):A556

DOI:10.18332/tid/84728

\section{8:15-19:15}

LB-1342-5 Active and passive tobacco smoking among students of the University of Yaoundé I, Cameroon

JR Nansseu ${ }^{1,2}$, FK Assah'1, S-J Petnga ${ }^{3}$, BS Kameni $^{4}$, JJ Noubiap ${ }^{5}$, J Kamgno

'University of Yaoundé I, Department of Public Health, Faculty of Medicine and Biomedical Sciences, Yaoundé, Cameroon, ${ }^{2}$ Ministry of Public Health, Department for the Control of Diseases, Epidemics and Pandemics, Yaoundé, Cameroon, ${ }^{3}$ University of Yaoundé I, Faculty of Medicine and Biomedical Sciences, Yaoundé, Cameroon, ${ }^{4}$ Ngaoundéré Regional Hospital and Regional Technical Group for the fight against HIV/AIDS, HIV Care Unit, Ngaoundéré, Cameroon, ${ }^{5}$ University of Cape Town and Groote Schuur Hospital, Cape Town, Department of Medicine, Cape Town, South Africa. E-mail: jobertrichie_ nansseu@yahoo.fr

Background: Tobacco smoking is a leading cause of preventable deaths globally. Although tobacco-induced burden has started decreasing in developed countries, it is continuously increasing in developing countries with young populations being mostly affected. This study aimed to determine the prevalence of active and passive smoking among youths in Cameroon.

Methods: This was a cross-sectional study carried-out from May-July 2017 at the University of Yaoundé I, Cameroon. We included any student registered at the University, found at one of the campuses when the investigators visited, and volunteering to participate. Smoking status was classified as never, former (last smoked more than 12 month ago) or current (smoked regularly in the last 12 months). Passive smoking was defined as a regular exposure to tobacco smoke since the last twelve months.

Results: Overall, 949 (54\% males) were included in this study, with a median age of 23 years (interquartile range (IQR) 21-26). The prevalence of former, current and passive smoking was $3.4 \%$ (95\% confidence interval (CI): 2.3-4.6), 0.8\% (95\%CI: 0.2-1.4), and $27.7 \%$ (95\%CI: 24.9-30.6), respectively. Exposure to other people's smoke ranged from 1 to 50 hours per week, with a median of 1 hour/week (IQR 1-2). Those who smoked were more likely to be males (odds ratio (OR) 2.05, 95\%CI: 1.03-4.08; $\mathrm{p}=0.037$ ) and/ or hazardous alcohol drinkers (OR 4.93, 95\% CI: 2.55-9.52; $\mathrm{p}<$ 0.001 ). Similarly, males (OR 1.57, 95\%CI: 1.18-2.11; $\mathrm{p}=0.002$ ) and/or hazardous alcohol drinkers (OR 2.10, 95\%CI: 1.55-2.86; $\mathrm{p}<0.001)$ were more at risk of passive smoking.

Conclusions: Although the prevalence of active smoking seemed low in this population, almost one-third of students were passive smokers, reflecting a high burden of tobacco smoking in the general population. Therefore, regulations towards tobacco consumption should be reinforced in our country. Specifically, special laws banning and severely punishing smoking in public places should be voted and adopted.

Tob. Induc. Dis. 2018;16(Suppl 1):A557

DOI:10.18332/tid/84695

$18: 15-19: 15$

LB-1343-5 Tobacco use: the most influential risk factor on cannabis use among middle schoolchildren in Sousse, Tunisia

N Zammit', J Maatoug', F Radaoui', R Ghammam', S Bhiri', H Ghannem'

'Farhat Hached University Hospital, Epidemiology, Sousse, Tunisia. E-mail: nawelzommit@gmail.com

\section{Background:}

- Early adolescence is a critical period usually associated with substances experimentation. In Tunisia, some studies were carried out recently to evaluate tobacco and cannabis use in the colleges and high schools. However, few studies were led in the middle schools. While onset of substances use usually occur during early adolescence.

- Aims: To evaluate the prevalence of tobacco and cannabis use and to determine if there is an association between their use among the young adolescents of Sousse.

Methods: A cross-sectional study was conducted in the region of Sousse, Tunisia during the 2013/2014 school year among a proportional and stratified sample of 4272 schoolchildren randomly selected in 16 public middle schools from the region of Sousse. Data about sociodemographic characteristics, tobacco products and cannabis use among the schoolchildren, their parents, their siblings and their friends were collected using a self administrated questionnaire.

Results: Females represented 50.5\% (2157) of participants. The mean age of schoolchildren was $13.3 \pm 1.2$ years. Among participants, 12.9\% (CI95\%: 11.9\%-13.9\%) reported lifetime tobacco use, $4.5 \%$ (CI95\%: 3.9\%-5.1\%) were current cigarette smokers, 3.2\% (CI95\%: 2.7\%-3.7\%) were current water pipe users and $1.9 \%$ (CI95\%: 1.5\%-2.3\%) reported lifetime cannabis use. The age of tobacco use onset $(11.2 \pm 2.7$ years $)$ was significantly inferior to the age of cannabis use onset $(13.1 \pm 2$ years $)(p<0.001)$. The most influential risk factor on cannabis use was current cigarette smoking with an adjusted odds ratio of 7.3 (CI95\%: 4.2-12.5).

Conclusions: National school-based substances use prevention program is required in Tunisia. Tobacco use prevention deserves more efforts as it is a gateway to other substances use such cannabis. Tob. Induc. Dis. 2018;16(Suppl 1):A558 DOI:10.18332/tid/84720

18:15-19:15

LB-1344-5 Electronic cigarette use and conventional cigarette smoking initiation among youth, United States, 2015-2016

S Odani', K Marynak', B Armour', I Agaku'

${ }^{1}$ Centers for Disease Control and Prevention, Office on Smoking and Health, Atlanta, GA, United States of America. E-mail: Ipu7@cdc.gov

Background: Electronic cigarettes (e-cigarettes) are the most commonly used tobacco product among U.S youth. We used cross-sectional, nationally representative data of U.S. middle and high school students to examine the association between 
e-cigarette use and cigarette smoking initiation.

Methods: Data were from the 2015 and 2016 National Youth Tobacco Surveys, a school-based survey of U.S. 6th-12th graders (pooled $\mathrm{N}=38,386$ ). Questions on current age and age at initiation of different tobacco products were used to assess temporality. The study included 35,775 students who had never smoked conventional cigarettes five years before the survey (i.e., baseline), including never-smokers and those who first smoked < 5 years ago. Baseline never smokers were classified by e-cigarette use status into: (1) those who ever used e-cigarettes on/before or without ever smoking cigarettes; or (2) those who had never used e-cigarettes, or started only after initiating cigarette smoking. The outcome variables were cigarette smoking at pre-determined periods: any time within the past 5 years; past 1 year; past 6 months; past 30 days; and past 7 days. Adjusted odds ratios (AOR) were calculated using multivariable logistic regression. The models controlled for socio-demographic characteristics and use of smokeless tobacco, cigars, and hookah on/before cigarette smoking initiation.

Results: Among baseline never cigarette smokers, $17.4 \%$ used e-cigarettes, and $16.7 \%$ initiated cigarette smoking within the past 5 years. Those who used e-cigarettes on/before ever smoking cigarettes had higher odds of smoking cigarettes than those who did not at all periods assessed: any time within the past 5 years $(\mathrm{AOR}=2.61)$; past 1 year $(\mathrm{AOR}=3.18)$, past 6 months $(\mathrm{AOR}=2.59)$, past 30 days $(\mathrm{AOR}=1.75)$, and past 7 days $(\mathrm{AOR}=1.38)($ all $\mathrm{p}<0.05)$.

Conclusions: These cross-sectional findings reveal that e-cigarette use was associated with conventional cigarette smoking initiation among U.S. youth. Comprehensive efforts are important to prevent and reduce all forms of youth tobacco product use.

Tob. Induc. Dis. 2018;16(Suppl 1):A559

DOI: $10.18332 /$ tid/84740

\section{8:15-19:15}

LB-1345-5 Still unhooking the "Hookah-ed" a comparative study of perceptions of Hookah amongst the young adults

\section{R Gokhale}

'Brihan Maharashtra College of Commerce, Business Practices, Pune, India.E-mail:rgokhale69@gmail.com

Background: Hookah is popular with the young adults and is perceived as a great socialization avenue and bonding tool.. The objective of this study was to understand if there was a significant difference in the level of awareness of harm of hookah from 2011 to 2017 in Pune city which is considered as the Educational Hub of India.

Methods: This survey was conducted by students of an educational institute in Pune wherein a questionnaire was administered to 100 young adults from different educational institutes with the objectives.

1. To understand why youth are attracted to Hookah smoking.

2. To understand if the Hookah users are aware of the health aspects of the Hookah.

Results: The study revealed that the numbers of Hookah Parlors were fewer in number in Pune city as compared to 2011 due to stricter regulations. However the young adults are now indulging in the same at home or at private places on regular basis.

The most significant observation is the fact that the awareness levels of the harm of hookah are almost similar in both the surveys and the young adults [ An overwhelming majority of $85 \%$ ]still continue to perceive Hookah smoking as safe option due to the water and flavor attributes.

Another noteworthy observation was the reasons cited by the young adults for this perception. Less mass media campaigns of harm of Hookah even in the deaddiction campaigns, lack of warnings on the hookah shops and the blatant projection of the same in films and student theatre groups.

Conclusions: This study suggests that policies definitely play an important role in curtailing consumption of Hookah at Public places but there is more need for civil engagement in creating awareness of the harm of hookah to reduce consumption of Hookah amongst young adults.

Tob. Induc. Dis. 2018;16(Suppl 1):A560

DOI:10.18332/tid/84738

\section{Friday, 9 March 2018}

\subsection{Priorities and challenges for advancing the WHO FCTC}

\section{$11: 00-12: 30$}

F0-387-1 Smokeless tobacco in Central Asia: working towards an effective regulatory framework for nasvai in Tajikistan

K Shats ${ }^{1}$, E Kravchenko² ${ }^{2}$ B Khabibov ${ }^{2}$, H Elbanhawi ${ }^{3}$, J Abrams ${ }^{4}$, E Sebrie $^{3}$

${ }^{1}$ Campaign for Tobacco Free Kids, International Legal Consortium, Washington, DC, United States of America, ${ }^{2}$ Consumers Union of Tajikistan, Dushanbe, Tajikistan, ${ }^{3}$ Campaign for Tobacco Free Kids, International Research, Washington, DC, United States of America, ${ }^{4}$ Campaign for Tobacco Free Kids, International Advocacy, Washington, DC, United States of America. E-mail: kshats@tobaccofreekids.org

Background and challenges to implementation: Inadequate attention has been paid to the significant harms arising from smokeless tobacco in Central Asia, both from a public health and regulatory perspective. Nasvai or nas is a finely ground, moistened dipping tobacco product found in Tajikistan, Kazakhstan, Kyrgyzstan, Uzbekistan, Pakistan, Afghanistan, Iran and India. Studies show that nasvai contains high- and varyinglevels of nicotine and tobacco specific nitrosamines among other toxic constituents and carcinogens, and increases risks of cancer and cardiovascular disease. In Tajikistan, although cigarette prevalence is low (under 10\%), nasvai use is significantly higher (up to $60 \%$ among certain populations). As far as we are aware, there are no examples of effective regulatory measures.

Intervention or response: For the first time, a comprehensive tobacco control law that addresses nasvai use is being developed through a collaborative effort between civil society and the Tajikistan government. The initial draft law proposed regulating nasvai alongside other tobacco products. However, our legal analysis showed that measures developed to address combustible tobacco in the Framework Convention on Tobacco Control (FCTC) may not apply to nasvai regulation.

Results and lessons learnt: Although some FCTC measures may be effective, we identified significant challenges that necessitate a novel regulatory approach. These include: lack of awareness that nasvai is harmful, or a tobacco product; cultural acceptance of nasvai use; lack of a formal market or industry; and the structure of the cottage industry. We assessed potential risks that could arise from regulation, including inadvertently attracting or creating a formal industry, and of nasvai users switching to combustible 
products.

Conclusions and key recommendations: Further research into nasvai content, use and health harms is needed not only to enact much needed regulation, but also to educate target audiences (government, consumers, retailers, health professionals) and to develop cessation services. Our findings will support the development of policies that better address smokeless tobacco use in the region and beyond.

Tob. Induc. Dis. 2018;16(Suppl 1):A561 DOI:10.18332/tid/83865

\section{2:30-14:00}

PS-900-1 Economic research on bidi industry in India: exploring the paradoxes

\section{S Kumar}

'Institute for Studies in Industrial Development (ISID), Health Economics, New Delhi, India. E-mail: skhooda.jnu@gmail.com Background: India, similar to the several other countries, is facing a constant dilemma of contradictory policies of tobacco control and tobacco promotion. Such paradoxes are related with the fiscal instruments. Policy makers one side speak about the imposition/increase tax on tobacco to reduce its consumption, while on the other hand government provides institutional support/incentives and exemption for tobacco production/ growing and manufacturing. This study deals with the paradox relating to taxation on smoking tobacco product especially in the bidi manufacturing in India which hold position in manufacturing and consumption. Bidi predominantly outside the domain of tax regulation and manufacturers producing less than 2 million sticks a year without machines (handmade) get exemption from excise duty. Such paradoxes needs to be addressed. This study provides evidence and background to advocate for higher tax on bidi tobacco and remove exemption.

Methods: Study explored unit level data of various rounds of National Sample Survey (NSS) (for informal/unorganised bidi manufacturing) and Annual Survey of Industry (for formal/ organised manufacturing) and Unemployment round of NSS. Simple statistics and econometrics tools are used.

Results: This study has brought out evidences on what economic contribution the bidi industry has actually made, which type and nature of employment it provides, working conditions and compensation to workers (like wages, social security benefit, etc.) in comparison to the overall tobacco as well as overall manufacturing industry in the country? What have been the consequences, especially in respect of taxes, manufacturing and work conditions, of extending the exemption of excise duty to bidi manufacturers who produce less than 2 million sticks a year?

Conclusions: This study provides enough justification and rationale to increase tax and abolish the exemption on bidi manufacturing currently applicable to those producing less than 2 million sticks a year without machines.

Tob. Induc. Dis. 2018;16(Suppl 1):A562

DOI:10.18332/tid/84350

\section{2:30-14:00}

PS-901-1 Global burden of smokeless tobacco (SLT) use

D Sinha ${ }^{1}$, PC Gupta ${ }^{2}$, M Parascondola ${ }^{3}$, A Kumar $^{4}$, R Mehrotra ${ }^{4}$ ${ }^{1}$ National Institute of Cancer Prevention and Research, Noida, WHO FCTC Global Knowledge Hub on Smokeless Tobacco, Noida, India, ${ }^{2}$ Healis-Sekhsaria Institute for Public Health, Navi Mumbai, India, ${ }^{3}$ National Cancer Institute, Bethesda, MD, United States of
America, ${ }^{4}$ National Institute of Cancer Prevention and Research, Noida, Noida, India. E-mail: dhirendrasinha1@gmail.com

Background: Global burden of smokeless tobacco use among adults and adolescents has not been updated after NCI report 2013.

Methods: Most recent prevalence of smokeless tobacco use among adults was collected from standard surveillance system. Number of SLT users were calculated by corresponding age group population from UN statistics. Most recent prevalence of smokeless tobacco use among adolescent (13-15 years) was collected from Global Youth Tobacco Survey and numbers were calculated in the same way for adolescents also.

Results: One in 10 adults use smokeless tobacco in one or another form. Prevalence of SLT use is higher among males (10.8\%) as compared to females (5.7\%).Prevalence of smokeless tobacco use among in SEAR is higher than smoking. In 129 (72\% of the Parties) countries 357 million adult SLT users live. Nearly $93 \%$ of adult SLT burden is borne by Low resource countries (Low Middle Income Countries (LMIC) and Low Income Countries (LIC). Over 80\% burden is in South East Asia Region (SEAR). In 103 countries 12.2 million adolescent (13-15 years) use SLT. Of these $82.5 \%$ live in LIC and LMICs. Nearly $60 \%$ of adolescent users live in SEAR, 13\% in African Region and 12\% in Eastern Mediterranean region (EMR).Prevalence of current smoking was higher than current SLT use in all regions except SEAR where prevalence of SLT use was higher than smoking. Prevalence of SLT use varied from $1.2 \%$ in Western Pacific region to $8.2 \%$ in SEAR among adolescent aged 13-15 years.

Conclusions: The epicenter of smokeless tobacco use burden among adult and adolescent is low resource countries (LMIC and LIC) and especially countries in SEAR. Policy interventions in such countries should include SLT.

Tob. Induc. Dis. 2018;16(Suppl 1):A563

DOI:10.18332/tid/84444

\section{2:30-14:00 \\ PS-902-1 Tobacco control and misplaced loyalties MLKS Magtibay' \\ 'Taguig City Hall, Taguig, Philippines. E-mail: liasmagtibay@ gmail.com}

Background and challenges to implementation: Smoke-free policies have been on the rise since President Duterte won the in June 2016. After he issued Executive Order No.26 (EO26), or "Providing for the Establishment of Smoke-free Environments in Public and Enclosed Places" in May 2017, national government agencies(NGAs) and local government units(LGUs) have been rushing to adopt the EO.

However, a closer look at EO26 shows that it is not fully FCTCcompliant. While the EO, and hence all policies born out of it, seem to acknowledge the role of the FCTC, these issuances reveal that the FCTC is recognized but not necessarily acknowledged. Therefore, despite renewed efforts at a supposedly "smoke-free" Philippines, the reality of things show that tobacco control may not be headed towards the right direction.

Intervention or response: It must be impressed upon LGUs and NGAs that the Philippines ratified the FCTC in 2005. Thus, it has the force and effect of national law and should be complied with by GAs. The government, as a clear and legal obligation on all Parties found in Article 8, should protect the populace from tobacco smoke by adopting effective legislative, executive, administrative and other measures to reduce exposure.

The Constitution also grants LGUs local autonomy, so LGUs can adopt local laws applicable specifically within their jurisdiction. 
Results and lessons learnt: While the country is really taking its time at giving full recognition of the FCTC, tobacco control efforts are not futile. At the moment, at least 5 LGUs have FCTCcompliant ordinances. While the pace is slower than ideal, this shows effective tobacco control is possible.

Conclusions and key recommendations: Government agencies which fully support the FCTC should ceaselessly work together to ensure that the little result that was produced continues to grow and cause a snowball effect throughout the country. NGAs and LGUs should not indiscriminately issue smoke-free policies, but should turn to the provisions of the FCTC.

Tob. Induc. Dis. 2018;16(Suppl 1):A564

DOI:10.18332/tid/84465

\section{2:30-14:00}

PS-903-1 Strengthening the implementation of the FCTC with a special focus to youth

P Altan ${ }^{1}$, S Polat ${ }^{1}, \mathrm{G}$ Erkmen Yildirim ${ }^{1}$

${ }^{1} \mathrm{MOH}$, Tobacco Control Dep., Ankara, Turkey. E-mail: peymanaltan@gmail.com

Background and challenges to implementation: Tobacco industry (TI) still target youth despite the FCTC. With novel products and flavours to appeal to first-time smokers, TI seems to aim youth smokers. Turkey ratified the FCTC in 2004 and has been pertinaciously and successfully implemented through National Tobacco Control Programs (NTCP) and Action Plans since 2008. As stated in the last three WHO Global Tobacco Epidemic Reports, Turkey is among the countries implementing MPOWER measures at the highest level.

However despite the mentioned success, further preventive activities with a special focus to youth were needed considering tobacco use prevalence among youth at $6,9 \%, 8.4 \%$ and $10.4 \%$ respectively (2003, 2009, 2012).

\section{Intervention or response:}

1 - Young people from NGOs through collaboration with $\mathrm{MOH}$ have voluntarily contributed to the following:

- Two national workshops in 2017 to determine the aim, objectives and strategies for the control activities targeting youth have been organized.

- NTCP targeting the youth with a peer led tobacco prevention point of view has been launched in the World No Tobacco Day of 2017.

2 - Menthol is a prominent design feature used by TI to attract and retain new, younger smokers. Therefore, in 2015, Turkey became the first country to implement a ban on menthol as well as menthol derivatives, as an additive at any level in cigarettes and hand-rolled tobacco. The ban takes effect January 2019, at the manufacturer level, and May 2020, at the retail level.

Results and lessons learnt: Through the above mentioned activities, youth will be prevented from encountering tobacco products and smoking prevalence will significantly decline. Adolescent peers will be better advocates of tobacco control.

Conclusions and key recommendations: Comprehensive implementation of the FCTC with contributory activities on youth are essential. Moreover, the implementation of plain packaging and regular GYTS as well as revealing the scientific information on the harmful effects of new tobacco products like iQOS undermining the FCTC are crucial.

Tob. Induc. Dis. 2018;16(Suppl 1):A565

DOI:10.18332/tid/84501

12:30-14:00

PS-904-1 Cigarette sale within 100 meters of school boundaries in metropolitan Manila, Philippines from June to August, 2016

ML Alzona' ${ }^{1}$ J Lagahit ${ }^{2}$

${ }^{1}$ Metropolitan Manila Development Authority, Health and Public Safety, Makati City, Philippines, ${ }^{2}$ Metropolitan Manila Development Authority, MMDA The Union Project, Makati City, Philippines. E-mail:drlalzona@yahoo.com.ph

Background and challenges to implementation: The sale of cigarettes within 100 meters of schools, playgrounds and facilities frequented by minors is prohibited as stated in Section 10 of the Philippines' Tobacco Regulation Act.

The Metropolitan Manila Development Authority, supported by a grant from Bloomberg Philanthropies through The Union, monitors cigarette sale around schools in 17 cities of Metropolitan Manila. Results are forwarded to the mayors for information and appropriate action. The mayors issue license / business permits for operation of these stores. Violation on access restriction policy can result in revocation of permits and closure of business.

Intervention or response: To determine the effect of monitoring visits, when store owners are informed of access restriction policy around schools, MMDA environmental enforcers randomly re inspected 102 out of 1021 stores previously visited from June to August, 2016. Following were noted: display of tobacco products, presence of advertisement and posting of warning signs against smoking.

Results and lessons learnt: Results show 33\% of 102 re inspected stores continued to display cigarettes in full view of minors, $26 \%$ persisted to maintain advertising as store signage and only $20 \%$ of the stores retained warning signs posted by enforcers. Store owners, although provided with information on tobacco regulation particularly on the prohibition of cigarette sale around 100 meters of schools did not find it necessary to comply with the law. Local government units are not enforcing tobacco regulation so stores continue with their business despite the violation.

Conclusions and key recommendations: Despite providing information on access restriction policy, violations exist because there is no community enforcement. MMDA began advocating for passage of WHO - FCTC compliant ordinances at local level to ensure implementation of tobacco control regulation. Four out of 17 cities have passed comprehensive tobacco control ordinances. Implementation of the tobacco access restriction policy by community enforcers will improve efforts to protect minors from being initiated into smoking.

Tob. Induc. Dis. 2018;16(Suppl 1):A566

DOI:10.18332/tid/84507

\section{2:30-14:00}

PS-905-1 Strategic prohibition of tobacco sale within 100 meters radius of health facilities in the absence of legal provisions in existing tobacco control act in Delhi

S Arora' ${ }^{1}$, P Kumar' $^{1}$

'Directorate General of Health Services, Govt. of Delhi, Public Health Wing - 1, DGHS, New Delhi, India.E-mail: aroradrsk7@ yahoo.com

Background and challenges to implementation: In India section 6 of Cigarette and Other Tobacco Products Act (COTPA) prohibits the sale of Tobacco products within the radius of 100 yards/ 100meters as per Delhi Act 1996 from any educational institute to protect the younger generation from tobacco habits, But at the same time large number of children and pregnant 
women with unborn children are also visiting health facilities including hospitals. Some tobacco vendors sitting outside walls of health facilities are a great threat for the visiting children and pregnant females which are to be protected from tobacco habits as well as from second hand smoke.

Intervention or response: Meeting held with all Chief District Medical officers(CDMOs), District Nodal Officers under Director Health Services \&Sect Health. It was convinced that prohibition of sale of Tobacco is required within 100 meters radius of health facilities to protect children and pregnant ladies. In the absence of law, it was decided to implement this through an administrative order by each Chief District Medical Offices for respective district Health centres (dispensaries, polyclininc, mohalla clinic) and Medical superintendent of respective hospitals.awareness activity conducted \& informed in community

Results and lessons learnt: All the CDMOs of 10 out of 11 districts issued administrative order in public health interest. These are applicable for nearly 300 health facilities. Orders are displayed at gates and notice boards . Incharges of health facilities nominated as nodal/implementing officers. Tobacco free zone boards \& Nosmoking signage displayed in all dispensaries, polyclininc, mohalla clinic \& in 21 out of 32 hospitals. The nodal officers are enforcing in public health interest with some resistance at few places which is being tackled strategically.

Conclusions and key recommendations: In the absence of legal provisions, if administration desires can implement public health measures strategically. Govt should consider to extend the provision of prohibition of tobacco sale under section 6 to health facilities also.

Tob. Induc. Dis. 2018;16(Suppl 1):A567

DOI: $10.18332 /$ tid/84543

\section{2:30-14:00}

PS-906-1 Challenges and lessons learnt from a workplace based program to control tobacco use in Tunisia 2009-2014

S Bhiri ${ }^{1}$, F Ouni', J Maatoug ${ }^{1}$, R Ghammam ${ }^{1}$, N Zammit ${ }^{1}$, H Ghannem

'University Hospital Farhat Hached, Epidemiology Department, Sousse, Tunisia. E-mail: bhirisana@yahoo.com

Background: Tobacco use has become the leading preventable cause of death worldwide.

Therefore, Tunisia has ratified the WHO Framework Convention on Tobacco Control in 2010.

Objectve: We aimed to outline an experience of implementing a workplace based program of tobacco control in Sousse, Tunisia (2009-2014) in order to identify barriers and recommendations for future interventions in low and middle income countries.

Methods: We conducted a quasi-experimental study among a representative sample of employees in Sousse, Tunisia with two groups, intervention and control. Our three years workplace program consisted of a collective intervention (Smoking free workplace, open sensitization day : films and workshops on tobacco harmful effects) and an individual one (Free clinics for tobacco cessation offering free nicotine replacement therapy products). Participants enrolled at the pre intervention and post intervention assessments belonged to two independant samples in each group.

Results: Overall, the intervention showed positive effets in improving the knowledge of the participants on the harmful effects of active and passive smoking and on light cigarettes.
The intervention was associated to a significant increase of the employers involvment in tobacco control efforts. Regarding the smoking behaviors, in the intervention group, the prevalence of tobacco use decreased from $39.2 \%$ at the pre-assessment to $37.5 \%$ at the post assessment $(\mathrm{p}=0.43)$ with no substantial difference in the number of cigarettes consumed.

Conclusions: Despite the absence of positive effect of this project on smoking behavior, severall lessons may be deducted and it would be therefore considered as a corner stone. The fight against tobacco use is dependent on deep environmental changes. The role the employer is crucial but not sufficient. A multisectoral approach garanteeing an actual involvement of all the stakeholders and topped by a political will is strongly needed.

Tob. Induc. Dis. 2018;16(Suppl 1):A568 DOI:10.18332/tid/84589

12:30-14:00

PS-907-1 FCTC implementation gaps - perceptions of policy makers

EK Wellington ${ }^{1,2}$

${ }^{1}$ Ghana Health Service, Research and Development Division, Accra, Ghana, ${ }^{2}$ Monash University, Global Public Health, Sch. of Medicine and Health Sciences, Kuala Lumpur, Malaysia. E-mail: edith.wellington@ghsmail.org

Background: The WHO FCTC is one of the most rapid embracing international treaties in history. Ghana ratified the FCTC in 2004 among the first 40 countries. The COP called on developing Parties and Parties with economies in transition to conduct needs assessments in line with its obligations under the Convention. This study assessed the status, challenges and potential needs from the implementation report. Review information from available sources of information; analysis of the obligations; progress made; gaps that existed and possible actions and recommendations for achieving full implementation.

Methods: The Needs assessment was jointly conducted by Ghana Government and the Secretariat. It was developed through a consultative process that began with a developing a list of agencies and organizations; pre -briefing to key stakeholders; national conference and consultations with key stakeholders. It provides a detailed article by article analysis of obligations; progress made; gaps that existed and possible actions and recommendations for achieving full implementation.

Results: A National Tobacco Control Steering Committee has been established to support policy development, advocacy, and advice on effective intervention strategies. Parliament of Ghana approved an increase in tobacco taxes through the passage of the Excise Duty (Amendment) Bill, 2015 into Law. The Bill seeks to amend the first schedule of the Excise Duty Act, 2014 (Act 878) and proposed an increase of the excise duty on cigarettes and cigars. Ghana now has a tobacco control law, a component of the Public Health Act, 2012 (Act 851).

Conclusions: Establishment of multi-sectoralnational coordinating mechanism with a formal mandate from the government. The need to raise public awareness about both the existence and rationale of the Public Health Act, 2012 (Act 851) to support enforcement. It is urgent for Ghana to set up a funding mechanism for implementing the comprehensive Tobacco Control law in line with the Convention.

Tob. Induc. Dis. 2018;16(Suppl 1):A569

DOI:10.18332/tid/84624

$12: 45-14: 00$

EP-268-1 Analyzing commitments under the WHO 
framework convention on tobacco control using the TAPIC governance framework

H Jarman ${ }^{1}$

${ }^{1}$ University of Michigan School of Public Health, Ann Arbor, MI, United States of America. E-mail: hjarman@umich.edu

Background: The TAPIC governance framework (Transparency, Accountability, Participation, Integrity, Capacity) was recently developed by a team of researchers on behalf of WHO Europe and the European Observatory on Health Systems and Policies as a tool for assessing and improving health governance in $\mathrm{WHO}$ member states. TAPIC draws on several decades of research on governance across many policy areas, placing insights from these studies in health contexts. This presentation applies TAPIC to commitments made by parties to the Framework Convention on Tobacco Control (FCTC) and its related guidelines. How well do commitments made under the FCTC align with good governance principles as outlined in the TAPIC framework? To what extent does the FCTC promote good governance, and can we do better? Methods: Beginning with primary documentation (FCTC text, guidelines, reporting by Parties) and considering recent research literature, the main structures of the FCTC were examined to assess their alignment with the TAPIC framework and identify areas of opportunity.

Results: To the extent that it requires increased transparency from Parties, supports integrity within the policymaking process and promotes policymaking capacity, the FCTC is broadly likely to improve tobacco control governance in most countries. The FCTC itself could also be modified to further improve the governance strategies it contains. Further gains could be made by incorporating greater peer review, requiring Parties to codify rules around access to tobacco control policymaking and by identifying additional measures that capture governance procedures in additional to outcome variables.

Conclusions: Using the TAPIC framework to evaluate FCTC Commitments highlights ways in which the agreement can promote better governance and ultimately could increase the effectiveness of international efforts to combat tobacco.

Tob. Induc. Dis. 2018;16(Suppl 1):A570

\section{DOI:10.18332/tid/84606}

\section{2:45-14:00}

EP-274-1 The progress and challenges of implementation of the Framework Convention on Tobacco Control (WHO FCTC) in Kyrgyz Republic

C Bekbasarova

${ }^{1}$ Ministry of Health of the Kyrgyz Republic, Bishkek, Kyrgyz Republic. E-mail: chbekbasarova@gmail.com

Background and challenges to implementation: The Kyrgyz Republic is Party of the WHO FCTC since August 23, 2006. This abstract analyzes progress and challenges during 10 years of implementation of WHO's FCTC

Intervention or response: National Tobacco Control (TC) Law was adopted on August 21, 2006, entered into force on December 19, 2006 and was amended and supplemented during 10 years 2 times. TC measures were included, as one of main priorities, in the National Program on Health Reform for 2012-2018; the National program on noncommunicable diseases control and Prevention 2013-2020; the National Health Strategy "Health 2020" and etc. Results and lessons learnt: Currently tobacco smoking is completely prohibited in governmental, educational, medical, cultural and sports buildings, in entrances of residential buildings, public transports, except: trains, restaurants and bars.
12 Pictorial Health Warnings was adopted in December 2014, which is included the telephone quitline and website. Since 2016 the quitline was established. All types of tobacco advertising, promotion and sponsorship were banned since 2009. During last 10 years Kyrgyzstan has increased tobacco excise rates for: filtered cigarettes by $670 \%$; unfiltered cigarettes by $5000 \%$; other tobacco products - up to 330 times. However, nasvay (a form of smokeless tobacco) is not taxed at all. This makes tax increases less effective, since smokers can switch to cheaper tobacco products. As results, tobacco use prevalence in adult men decreased only by $7 \%$ due to nasvay use, which got increased by $5 \%$ in 2005 versus $11 \%$ in 2013. The 2003, 2008, 2014 (13-15 years) GYTS results show that cigarette smoking decreased by 2-times, however, prevalence of nasvay use increased by more than 3 times.

Conclusions and key recommendations: The above situation shows that Kyrgyz party should change tobacco control policy, adopt and implement a comprehensive tobacco control legislation (both smokeless and smoked tobacco products etc) for the effective implementation FCTC and more effective decrease tobacco use

Tob. Induc. Dis. 2018;16(Suppl 1):A571 DOI:10.18332/tid/84226

12:45-14:00

EP-275-1 Policy perspective on the global use of Smokeless Tobacco (ST): a literature review

K Siddiqi ${ }^{1}$, A Vidyasagaran ${ }^{2}$, A Readshaw ${ }^{2}$

'University of York, Health Sciences, York, United Kingdom, ${ }^{2}$ University of York, York, United Kingdom. E-mail: kamran. siddiqi@york.ac.uk

Background: Globally, Smokeless Tobacco (ST) is consumed by over 300 million people. ST is addictive and most products cause cancers of the oral cavity, pharynx and oesophagus. The aim of this review was to identify knowledge gaps in the implementation of key demand-reduction measures with regard to ST.

Methods: A series of focused literature reviews was conducted and findings were synthesized by relevant Articles (6 - 14) of the World Health Organization Framework Convention on Tobacco Control (WHO FCTC).

Results: We found sufficient evidence to support implementation of key demand-reduction measures for ST. For example, raising taxes can reduce ST consumption, with price elasticity on a par with, or sometimes higher than, cigarettes. Similarly, graphic pictorial warnings on ST packaging are far more effective than text-only warning labels. But despite supportive evidence, countries have either not implemented measures or have kept the bar much lower than that for cigarettes. For example, very few countries regulate the contents of ST products. Some have raised ST taxes but not to the extent of cigarette taxes, risking product substitution. Similarly, health-warning requirements for ST are less strict than those for cigarettes. Even where strong policies exist, compliance has been poor. For example, despite bans on ST advertising, most suppliers continue to promote products, especially at points of sale and via print and digital media. Consequently, ST demand-reduction measures have had limited impact.

Conclusions: The full implementation of FCTC Articles 6-14 is likely to reduce the global demand for ST, but their current state of implementation lags behind that for cigarettes. The existing policy gaps can be addressed through better knowledge of ST products, setting unambiguous standards, innovative systems for tax administration, building capacity in testing ST products and undertaking targeted research. 
Tob. Induc. Dis. 2018;16(Suppl 1):A572 DOI:10.18332/tid/83990

$12: 45-14: 00$

EP-276-1 Global policy progress in Article 13 of World Health Organization's Framework Convention on Tobacco Control (WHO FCTC) on Cigarettes (CIG) and Smokeless Tobacco (SLT)

DN Sinha ${ }^{1}$, S Grover ${ }^{1}$, H Kaur ${ }^{1}$, K Chandan ${ }^{1}$, PC Gupta ${ }^{2}$, R Mehrotra ${ }^{1}$

${ }^{1}$ National Institute of Cancer Prevention and Research, Noida, India, ${ }^{2}$ Healis - Sekhsaria Institute of Public Health, Navi Mumbai, India.E-mail: dhirendrasinha1@gmail.com

Background: Article 13 of the WHOFCTC provides guidelines for successful enforcement and implementation of effective measures against tobacco advertising, promotion and sponsorship (TAPS). Evidence suggests that TAPS ban reduces tobacco use, especially among young people, while partial bans provide opportunities for tobacco companies to find new marketing ways. Current paper provides an insight on global progress on implementation of Article 13 for Smokeless Tobacco (SLT) products, and compares it with cigarettes (CIG).

Methods: The current paper has evaluated global progress in ban on direct and indirect advertisement, promotion and sponsorship on SLT products. Exposure to advertisement and promotion was also assessed.

Results: There is unequal implementation of provisions under Article 13 on CIG and SLT products (direct advertisement in 'National TV and Radio', 'National Print media' and 'Billboards' is banned in $60 \%$ parties for SLT, and in $70 \%$ for CIG). Less than half of the Parties have banned Promotions and Sponsorship for SLT and less than $60 \%$ have done the same for CIG. Less than one-fifth of Parties have framed comprehensive policies for CIG and only one-eighth Parties have them on SLT. Core questions under TAPS have been investigated only for CIG, and a limited number of Parties (notably India and Bangladesh) have additionally investigated SLT, reporting nearly 50\% adult's response rate to exposure of SLT product advertisements.

Conclusions: A comprehensive ban of Tobacco Advertising, Promotion and Sponsorship (TAPS) should be developed and implemented for all tobacco products in all Parties.

Tob. Induc. Dis. 2018;16(Suppl 1):A573

DOI:10.18332/tid/84083

\subsection{Integrating tobacco control into Health systems, global frameworks and strategies to reach WIIO FCTC and SDG's targets.}

\section{$12: 30-14: 00$}

PS-908-1 Building momentum for adoption of UP-state policy on lines of WHO FCTC Article 5.3 RK Dwivedi', B Ramakant ${ }^{2}$, S Tripathi ${ }^{3}$, S Shukla ${ }^{4}$, DR Kant ${ }^{5}$ ${ }^{1}$ Citizen News Service, Health and Communication, Lucknow, India, ${ }^{2}$ Citizen News Service, Policy and Programme, Lucknow, India, ${ }^{3}$ Director General of Health and Medical Services, State Tobacco Control Cell, Lucknow, India, ${ }^{4}$ Citizen News Service, Editor, Lucknow, India, ${ }^{5}$ Piles To Smile Clinic, Medical Health, Lucknow, India. E-mail: rahul@citizen-news.org

Background and challenges to implementation: Since India ratified the WHO FCTC, it is obligated to follow WHO FCTC Article 5.3 as well. Indian court too has recognized obligations of India to not violate Article 5.3 of FCTC for instance. But Indian domestic tobacco control law doesn't have a policy on lines of FCTC Article 5.3. There are other laws and policies in Indian legal framework which help to check conflict of interest or hold corporations to account but more robust policy framework is needed for effective action.

Intervention or response: In September 2013, Vote For Health campaign hosted by CNS and Asha Parivar, joined hands with UP State Tobacco Control Cell, Network for Accountability of Tobacco Transnationals (NATT), Health Justice Philippines, to organise the first state training for CSOs on WHO FCTC Article 5.3. CNS signed another Memorandum of Understanding (MoU) with the UP state government to seek input from corporate accountability experts globally on a draft policy for UP on lines with FCTC Article 5.3 and support the implementation of the same on an ongoing basis. The draft policy for UP state was delivered to the government for further action.

Results and lessons learnt: UP state government promise to form a State level empowered committee for monitoring of tobacco industry interference; and consider adoption of the draft policy on lines with WHO FCTC Article 5.3.

Conclusions and key recommendations: It is important for the districts, states and nations to have stronger domestic laws and legal and policy frameworks to effectively check tobacco industry interference in health policy on lines with WHO FCTC Article 5.3. We also need to utilize or build upon existing legal and policy frameworks for holding corporations to account on human rights abuses and other harms they cause to climate, society and humanity.

Tob. Induc. Dis. 2018;16(Suppl 1):A574

DOI:10.18332/tid/84401

\section{2:30-14:00}

PS-909-1 Enhancing nursing competency to incorporate smoking cessation interventions into standard nursing practice for patients with noncommunicable diseases

\section{Panpakdee}

${ }^{1}$ Nurse Network on Tobacco Control of Thailand, Bangkok, Thailand.E-mail: orasa.pan@mahidol.ac.th

Background and challenges to implementation: Tobacco use is a major risk factor for non-communicable diseases (NCD). Nurses are in a good position to provide smoking cessation interventions to reduce the smoking rate. However, the barrier of nurses' workload and lack of competency lead to limitation of patient accessibility to smoking cessation services. This training project for nurses aims to increase smoking cessation interventions as part of standard nursing practice of Thai nurses.

Intervention or response: Project Activity consisted of:

1) providing funding to 10 hospitals to train nursing skills and implement smoking cessation guidelines at outpatient department,

2) providing knowledge and skill training about 5 As mode,

3) allocating responsibility among nurses at the OPD and smoking cessation clinic,

4) creating a multidisciplinary team for providing smoking cessation treatment, and

5) recording screening and smoking cessation outcomes.

Results and lessons learnt: The majority (90\%) of 250 nurses who participated in the smoking cessation training were satisfied with and had positive attitudes for providing smoking cessation service in their routine work. After completing one year of the project, 1394 patients of general and NCD clinics were detected 
as current smokers, $33.7 \%$ of them were referred to smoking cessation clinics. The quitting rate at 6 months was $24.4 \%$. We found that enhancing nurses' knowledge and skills about smoking cessation and having a nurse-led smoking cessation clinic increase the rate of access to smoking cessation services and rate of quit smoking. Motivation skills to help patients stop smoking and improving the referral system to smoking cessation clinics are needed for increasing the quit rate.

Conclusions and key recommendations: OPD Nurses can help NCD patients quit smoking as part of their routine work when they recognize the importance of their roles in smoking cessation and create a system to support their work.

Tob. Induc. Dis. 2018;16(Suppl 1):A575

DOI:10.18332/tid/84402

\section{2:30-14:00}

PS-910-1 Some cellular inflammatory characteristics in patients with chronic obstructive pulmonary disease (COPD) with anterior tuberculosis compared to tobacco related COPD

E Guiedem', MC Okomo Assoumou', G Ikomey Mondinde', EW Pefura Yone ${ }^{1}$, C Nkenfou $^{1}$

${ }^{1}$ University of Yaounde 1, Yaounde, Cameroon. E-mail: guiedemelise86@yahoo.fr

Background: Although smoking is the main cause of the chronic obstructive pulmonary disease (COPD), previous tuberculosis (TB) infection can also induce the disease. While the management of COPD is mainly performed with anti-inflammatory molecules, inflammatory profile of post tuberculosis obstructive disease is not yet known. The purpose of this study was to compare certain inflammatory cells of post tuberculosis COPD to that of post tobacco COPD.

Methods: This cross-sectional study conducted at the Yaoundé Jamot hospital consisted of 92 participants comprising 22 post tobacco COPD patients (COPD/tobacco), 24 post tuberculosis COPD (COPD/TB) and 46 healthy individuals constituting the control group. Sputum and blood were collected for cells counts. Results: In sputum, the mean count of neutrophils, lymphocytes and monocytes was statistically higher in COPD patients compared to the control group with p-values respectively of $0.0001,0.0001$ and 0.0001. Comparison of the two COPD groups showed that, neutrophils cells count is higher in COPD/tobacco than in COPD/ TB patients $(p=0.04)$. Monocytes and lymphocytes counts were similar between the two groups of patients with COPD with p-value of 0.052 and 0.91 respectivelly.

In blood, the rate of CD4 cells was higher in COPD patients compared to controls with a significant p-value of 0.0006 . The blood CD8 cell count was not statistically different between COPD patients and the controls group $(\mathrm{p}=0.6)$. Comparing the two COPD groups together, we had a blood CD8 rate higher in COPD/tobacco than COPD/TB ( $p=0.0043)$, and blood CD4 rate were not statistically different between the two COPD groups.

Conclusions: Neutrophils, monocytes and lymphocytes are involved both in the COPD/tobacco and COPD/TB, with high levels of sputum neutrophiles and blood CD8 cells in COPD/ tobacco patients. In blood TCD8 and CD4 lymphocytes may be involved in the pathogenesis of COPD.

Tob. Induc. Dis. 2018;16(Suppl 1):A576

DOI:10.18332/tid/84422

12:30-14:00

PS-911-1 Create a public opinion on community based organization to implement Pictorial Health Warnings

N Gamage ${ }^{1}$

${ }^{1}$ Alcohol \& Drug Information Centre, Social Mobilization, Colombo, Sri Lanka.E-mail: nisansalagamage78@gmail.com Background and challenges to implementation: In 2012, Health Minster of Sri Lanka proposed $80 \%$ pictorial Health warning on cigarettes packs as follows the FCTC recommendations. The tobacco industry did not allow implementing and filed a case on court. the PHW implementations has been delayed. Drug prevention practitioners all over the country used to respond the way of supporting PHW applying and convey the public interest on it. ADIC and Women Development Federation, Hambantota started awareness raising campaigns among the women.

Intervention or response: A silent protest was conducted front of the high courts on the court date by the staff members of WDF. Approximately 50,000 women employed in WDF at Hambantota District signed a petition and sent to the Health Minister requesting to implement the pictorial health warning. They spread the message through banners, posters, leaflets and also through protests. All stakeholders were addressed about industry influences on delay the PHW's and this opportunity was effectively used by community based organization.

Results and lessons learnt: WDF was able to show the public interest on implementing PHW's. As an community based organization able to create public pressure on politicians to support PHW's. The pictorial health warning was successfully implemented covering $80 \%$ of the Cigarette pack.

Conclusions and key recommendations: Tobacco industry was trying to undermine the Health Minister's stand on PHW's applying. Without various public support on this setting health minister would be a victim of tobacco industry. the public support is must at the whatever level policy formulation. it would be more sustainable.

Tob. Induc. Dis. 2018;16(Suppl 1):A577 DOI: $10.18332 /$ tid/84468

$12: 30-14: 00$

PS-912-1 Compability of Polish National Tobacco Control Program and cancer control strategy: results of a Delphi study

J Lobaszewski ${ }^{1}$, P Koczkodaj ${ }^{1}$, K Przewozniak $^{1}$

${ }^{1}$ Maria Sklodowska-Curie Memorial Cancer Center and Institute, Cancer Epidemiology and Prevention Division, a WHO Collaborating Centre for Tobacco Control, Warsaw, Poland. E-mail:jakub.lobaszewski@coi.pl

Background: Polish national Tobacco Control Program adopted for years 2014-2018 is a most important tool for reducing the harm related with tobacco use and monitoring the development of tobacco epidemic in Poland. Cancer Control Strategy adpoted for years 2015-2024, prepared by reowned Polish specialists in oncology and public health is a major strategic document describing the vision of development of cancer control in Poland, including tobacco control activities.

Methods: A 2-round modified Delphi survey sought opinions from Polish experts in the field of tobacco and cancer control, including representatives of Government, local authorities, medical universities and research institutions. In the first round, respondents were asked to assess the compability of goals, activities and solutions proposed both documents and share their opinions on issues and challenges that should be addressed in the future in order to improve tobacco control in Poland. In the second round, experts were asked to rate key concepts developed 
in the first round of the study and reach the consenus on the most important challenges that should be addressed in both documents. Results: 28 experts participated in the first round of the study. Experts have agreed that both documents are compatible in terms of propsed goals and main tasks. At the same time, they need to be updated with tasks and solutions concerning new challenges such as new tobacco products and use of e-cigarettes. Respondents noted that the problem of secondhand smoke is not perceived as an important tobacco control issue in the Cancer Control Strategy. The second round of the study is currently in progress and final results should be available by late August 2017 .

Conclusions: Study participants agreed that both analysed documents, although generally compatible, need to be updated in order to effectively address future challenges in tobacco control.

Tob. Induc. Dis. 2018;16(Suppl 1):A578

DOI:10.18332/tid/84477

\section{2:30-14:00}

PS-913-1 Practice of tobacco free environment among restaurant workers and owners in Kota Samarahan, Sarawak, Malaysia

MMR Mizanur ${ }^{1}$, B Felix¹, DN Hayati ${ }^{1}$, A Zahri ${ }^{1}$, C Yee En ${ }^{1}$ ${ }^{1}$ Universiti Malaysia Sarawak, Community Medicine and Public Health, Kota Samarahan, Malaysia. E-mail:rmmizanur@unimas. my

Background and challenges to implementation: Second-Hand Smoking (SHS) exposure is common in restaurants and other enclosed places where smoking is permitted as some people failed to notice that restaurant is a public place. Tobacco free environment policy is one of the way to reduce the SHS exposure. This study aimed to determine the level of knowledge, attitude and practice towards tobacco free environment among restaurant workers and owners and the factors affecting thereof.

Intervention or response: This was a cross-sectional study conducted among the restaurant workers and owners irrespective of gender. A total of 301 respondents had participated in this study. Data was collected by assisted self-administered questionnaires. Data on current tobacco control law, preventive knowledge and attitude, support and implementation of tobacco free restaurant were collected. Data entry and analysis were done by using SPSS version 22.0.

Results and lessons learnt: The mean (SD) age of the restaurant workers and owners was 26.0(8.9) years with minimum age 15 and maximum 74 years old. Most of the respondents (47.2\%) had good level of knowledge on Tobacco Control Law in Malaysia and 36.2\% of them had excellent knowledge on SHS. Besides, $30.2 \%$ of them had good attitude towards tobacco free restaurants. Moreover, less than two-fifth (14.6\%) of them were implementing $100 \%$ tobacco free restaurant. About half of the respondents (48.6\%) had good support towards tobacco free restaurants. The support towards tobacco free restaurants was highest among Iban, respondent with secondary educational level non-smokers, having good knowledge on tobacco control law and SHS and having excellent level of attitude towards tobacco free restaurants $(\mathrm{p}<0.05)$.

Conclusions and key recommendations: Although the results did not depict national scenarios, however, the findings could be important in helping the effort on implementation of tobacco free restaurants. The findings of the results could also be utilised by policy makers for planning the implementation of tobacco free policy in restaurants in Malaysia.

Tob. Induc. Dis. 2018;16(Suppl 1):A579

DOI:10.18332/tid/84512

12:30-14:00
PS-914-1 Analysis of the effect of bidi regulations and its influence on tobacco control in India

H A.Lando ${ }^{1}$, Z Kabir ${ }^{2}$, P Mohan ${ }^{3}$, S Panneer ${ }^{4}$

${ }^{1}$ Division of Epidemiology and Community Health, University of Minnesota, Minneapolis, MN, United States of America, ${ }^{2}$ University College Cork, Epidemiology and Community Health, Cork, Ireland, United Kingdom, ${ }^{3}$ Mohan Dental Clinic, Bangalore, India, ${ }^{4}$ Central University of Tamil Nadu, Social Work, Thiruvarur, India.E-mail: sdrpriya@gmail.com

Background and challenges to implementation: Limited regulations on bidis has multifaceted role in exacerbating the poverty and is a major barrier in the Tobacco control and achievement of SDGs $1 \& 3$ in India. Moreover bidis used domestically are expanding internationally, increasing global consumer base and threatening the tobacco control efforts undertaken via FCTC, WHO. This systematic review assesses the economic and disease burden imposed by poor regulations, and their surrogate role in preventing tobacco control targets of National Health Policy 2017, India.

Intervention or response: Systematic review of literature was carried out by Digital search of published data using key words with inclusion and exclusion criteria.

Results and lessons learnt: In India, $53 \%$ of the smokers consumed bidi. Low cost has influenced consumption among the poor and students. Carbon monoxide and nicotine are 3 times and tar is 5 times higher than cigarettes. Apart from NCDs was associated with Tuberculosis, Nutritional disorders and Candidiasis were. Bidis are manufactured as home based instead of mechanized to elude the rights of bidi workers and also evade taxes. Also promotes child labour and early initiation of tobacco consumption. Workers develop dual burden of tobacco associated diseases and occupational hazards. Tobacco exports have increased, especially to developed countries in the disguise of natural herbal product and being less hazardous.

Conclusions and key recommendations: Contrary to smoking trends elsewhere, bidi is the highest form of smoked tobacco among the poor. Absolute number of smokers between 15-24 is increasing. Being least expensive students are driven for early initiation and continuation. Further increase in taxation on cigarettes in 2017, possibility of reversal of consumption pattern and relative boost to bidi industry. Uniformity in policies, more specifically taxation, on all tobacco products, will aid in effective tobacco control, prevent product switching and realize the goals of poverty alleviation and good health via tobacco control.

Tob. Induc. Dis. 2018;16(Suppl 1):A580

DOI:10.18332/tid/84535

$12: 30-14: 00$

PS-915-1 A dedicated implementation mechanism can eliminate the tobacco from the lives of human being

GK Tripathi', R J Singh'

${ }^{1}$ The International Union Against Tuberculosis and Lung Disease, Tobacco Control, Delhi, India. E-mail: gtripathi@theunion.org Background and challenges to implementation: India launched its National Tobacco Control Programme (NTCP) in 2007-08 in 9 states.We made intervention in 53 districts of 29 states. The Union Team provided technical and financial support in 40 districts of 20 states with the objective to build supportive political and administrative environment, establish and strengthen the system, institutionalise enforcement of provisions of National Legislation. An analysis conducted to understand that how effectively Tobacco 
can be eliminated from the lives of Human being.

Intervention or response: Analysis conducted of 8 state level,20 district level,11 law enforcement, 5 municipal corporations,38 piggy back, 18 WNTD, 68 state and district level training and review workshops. In all 168 training/reviews conducted and trained 2550 officials and stakeholders during Oct 2013 to Mar 2015. Efforts shown good results and engagement of community and CSOs. The core parameters of impact evaluation were: establishment of system, availability of IEC material, establishment of enforcement mechanism, convergence with other stakeholders, Active involvement for issuance of directives/policy notifications, increased level of engagement of Media and civil society organisations.

Results and lessons learnt: Tobacco control infrastructure established at $80 \%$ jurisdictions, availability of awareness related material found at $70 \%$ places. Enforcement mechanism is established at $70 \%$ places. Multi stakeholders engagement initiated at $60 \%$ places of intervention areas. Issuance of directives/ policy notifications increased at $75 \%$ places. Increased level of engagement of Media, community and civil society organisations at $75 \%$ places of the all intervention areas.

Conclusions and key recommendations: Above results are just the glimpse of successful results, the same can be replicated in entire territory under NTCP. The analysis shows that dedicated efforts and strengthened system can save the lives of human being from harmful effects of Tobacco consumption.

Tob. Induc. Dis. 2018;16(Suppl 1):A581

DOI:10.18332/tid/84611

\section{2:30-14:00}

PS-916-1 Exposure to the number of tobacco control policies and tobacco use cessation among adults in Sub-Saharan Africa

H Mamudu ${ }^{1}$, L Wang ${ }^{2}$, D Owusu ${ }^{3}$, R John ${ }^{4}$, B Asare $^{5}$, A Ibrahim $^{5}$, E Brenya $^{6}$, A Ouma $^{7}$

${ }^{1}$ East Tennessee State University, Health Services Management and Policy, Johnson City, TN, United States of America, ${ }^{2}$ East Tennessee State University, Johnson City, TN, United States of America, ${ }^{3}$ Georgia State University, Atlanta, GA, United States of America, ${ }^{4}$ Independent Consultant, Johpur, India, ${ }^{5}$ University of Ghana, Legon-Accra, Ghana, ${ }^{6}$ Kwame Nkrumah University of Science and Technology, Kumasi, Ghana, ${ }^{7}$ World Health Organisation HO, Geneva, Switzerland. E-mail: mamudu@ etsu.edu

Background: Tobacco use and tobacco-induced diseases are rapidly increasing in Sub-Saharan Africa (SSA). Addressing this problem requires both tobacco use prevention and cessation. This study examined the rate of smoking cessation and assessed effects of tobacco control policies (TCPs) on cessation among adults in SSA.

Methods: During 2012 and 2014, Global Adult Tobacco Survey data were collected in Cameroon, Kenya, Nigeria, and Uganda and analyzed, with smoking cessation as the outcome. The exposure variable consisted of a composite index of the number of TCPs that participants have been exposed to. Covariates included participants' characteristic, knowledge of tobacco harm, exposure to anti-smoking messages, and tobacco industry promotions. Descriptive analyses were conducted for prevalence of smokers who had quit in the past year (i.e., ex-smokers) for each country and pooled data. A multivariable logistic regression was used to examine whether the number of TCPs a participant has been exposed to increase the likelihood of cessation. The odds ratios and associated 95\% confidence intervals were ascertained.

Results: Of 27,952 participants, 48\%, 32.1\%, 17.5\%, $81.2 \%$ had been exposed to anti-smoking media messages, health warning labels, smoke-free workplace, and smoke-free homes, respectively. Approximately $14.1 \%$ of the participants were ex-smokers, of which $7.1 \%, 6.1 \%, 5.0 \%$, and $10.6 \%$ had been exposed to anti-smoking media messages, health warning labels, and smoke-free workplace, and smoke-free homes, respectively. Preliminary logistic regression analyses suggest that likelihood of smoking cessation increases with increase in exposure to TCPs.

Conclusions: As of 2014, only 41 countries had comprehensive tobacco control programs, with $<10$ of the 47 SSA countries having national tobacco control laws. This study suggests that the number of TCPs in a country facilitates smoking cessation, suggesting that achieving the WHO FCTC goal of reduced tobacco use requires adoption of many TCPs, even in absence of a national law.

\section{Tob. Induc. Dis. 2018;16(Suppl 1):A582 DOI:10.18332/tid/84651}

$12: 45-14: 00$

EP-265-1 Effective way to prevent tobacco consumption of husbands through public health midwives

C Herath

${ }^{1}$ Alcohol and Drug Information Center (ADIC) Sri Lanka, Social Mobilization, Colombo, Sri Lanka.E-mail: wasanthi.chandi@ gmail.com

Background and challenges to implementation: During the pregnancy period the husband is a lot concerned about his child. Therefore he is very flexible to change towards the goodness during this period. Therefore we got this opportunity to influence the husbands through the pregnant mothers; to encourage their husband's to quit their tobacco consumption and to make a tobacco free environment.

Alcohol and Drug Information Centre (ADIC) implemented an intervention to make a tobacco free environment for pregnant mothers and help their husbands to quit smoking by conducting trainings for 221 public health midwives ( PHM ) in 06 districts. Intervention or response: ADIC trained PHM in order to implement prevention activities among the target community. They used materials such as hand bills, stickers distributed by ADIC. PHMs conducted short sessions during clinics and parents meetings, empowering women's groups in the community. Follow up's were done by field visits, at the places of measuring the weight of child, in the breast feeding and nutrition training programmes conducted for pregnant mothers.

We introduced a new monitoring tool to the Medical officer of health $(\mathrm{MOH})$ office to review the progress of the interventions. The result chart which has to be filled by the PHM when they come to main office for the special meeting conducted once a month.

Results and lessons learnt: Significantly 665 houses converted to a smoke free environment out of the 3000 pregnant mother's houses. 857 husbands reduced the number of times they smoked and 236 quit smoking.

The total expenditure is Rs 75,000.00 for PHMs trainings per year in one district. 388 person have reduced/quit smoking. Therefore the cost per person to help them to stop/quit their smoking habit is only Rs193.29.

Conclusions and key recommendations: Addressing a family thorough PHMs is a very effective and sustainable way of reducing 
the use of tobacco in a community.

Tob. Induc. Dis. 2018;16(Suppl 1):A583

DOI:10.18332/tid/84414

$12: 45-14: 00$

EP-269-1 Ethiopian health care delivery system's responsiveness to smoking cessation therapy and its predictors: a mixed method study in Ethiopia T Tamirat ${ }^{1}$

'Wachemo University, Epidemiology, Hossana, Ethiopia. E-mail: temesgentamirat58@yahoo.com

Background: Tobacco is one of the leading public health burdens, killing six million people globally each year. The health system and healthcare providers can play a major role in promoting smoking cessation by providing either behavioral and/or pharmacological smoking cessation interventions to their patients. However, the level of responsiveness of the Ethiopian health system and the knowledge, attitude and practice of health professionals towards smoking cessation therapy is not well understood. This study was aimed to assess health care delivery system's responsiveness to smoking cessation services and its associated factors among health care providers in Ethiopia.

Methods: Across sectional study was employed both quantitative and qualitative methods. The quantitative study was used to determine knowledge, attitude, and practice towards smoking cessation therapy of the health workers. For these 323 participants were selected from five health professional groups. Self-administered questionnaires were used for data collection and descriptive statistics, bivarate and multivariate analysis were done in SPSS.

In the qualitative study twelve Key informants from central, regional, zonal and district levels were selected to respond on health systems responsiveness. Then the qualitative data was transcribed, translated and thematized.

Results: Majority 306(97.5\%) health care providers had below average level of practicing smoking cessation intervention, $70.4 \%$ and $86 \%$ had poor knowledge and negative attitude towards smoke cessation therapy respectively.

In the multivariate analysis; being female, receiving training, having good knowledge score and having positive attitude were associated with the above average practicelevel of smoking cessation intervention. During key informant interviews, absence of well-organized and established system in smoking cessation intervention in Ethiopia was observed towards cessation intervention.

Conclusions: The Ethiopian health delivery system's responsiveness for the provision smoking cessation intervention is premature. There is no well-organized system throughout. The health care providers (HCPs) were with poor knowledge, negative attitude and below average practice level.

Tob. Induc. Dis. 2018;16(Suppl 1):A584

DOI: $10.18332 /$ tid/84001

\section{2:45-14:00}

EP-277-1 Global standards for tobacco management in healthcare service

A O'Riordan', R Gasser ${ }^{2}$

'Global Network for Tobacco-free Healthcare Services, Administrative Centre, Dublin, Ireland, ${ }^{2}$ Barwon Health, Occupational Health, Geelong, VIC, Australia. E-mail: enshglobal@gmail.com

Background and challenges to implementation: Tobacco use is highly addictive and harms every organ in the body. Tobacco users are therefore likely high among healthcare users and look to health professionals first for help and support.

To effectively delivery on this high expectation health professionals need to work in supportive environments.

Hospital and healthcare services have an obligation to reduce the use of tobacco in all its forms and its deleterious health effects. This obligation should be seen in a larger dimension of tobacco control, tobacco cessation and prevention activities in hospitals and healthcare facilities and should also include the participation in tobacco prevention activities at community level to create a tobacco free environment for all.

Intervention or response: By networking and using diffusion of innovation theory strategies to support sharing and learning, the Global Network for Tobacco-free Healthcare Services (Global Network) has developed an effective implementation concept for tobacco management that is validated already in 22 countries globally.

The concept is centered on 8 Standards, supported by a monitoring and quality improvement self-audit tool and a peer review recognition process for high level implementation of the 8 Standards.

The Global 8 Standards integrate FCTC guidelines and other key aspects for tobacco control into a comprehensive, whole organisation and systematic approach.

Results and lessons learnt: Since its inception in 2000, the Global Network has grown from 7 European members to 39 registered members in 22 countries worldwide. Many members are in themselves networks of multiple individual healthcare services.

High level implementation has been verified, validated and given global recognition in 5 Recognition GOLD Forum Processes to 30 healthcare services in 7 countries.

Conclusions and key recommendations: By joining healthcare service globally together, the Global Network (www.ensh.org) believes that high level implementation in all healthcare services of the 8 standards will contribute significantly to the delivery on FCTC on a global level.

Tob. Induc. Dis. 2018;16(Suppl 1):A585

DOI:10.18332/tid/83955

\subsection{Using data : Opportunities and gaps in measuring WHO FCTC impact on global health and development}

$12: 30-14: 00$

PS-917-1 Tobacco Control Scale website: a new tool for tobacco control advocates and researchers A Feliu' ${ }^{1,2}$, A Baena ${ }^{1,2}$, W Yared $^{3}$, S Lipponen ${ }^{4}$, E Saltó ${ }^{5}$, L Joossens ${ }^{3}$, E Fernández $1,2,6$

'Institut Català d'Oncologia, Tobacco Control Unit, WHO Collaborating Centre for Tobacco Control, L'Hospitalet del Llobregat, Spain, ${ }^{2}$ Institut d'Investigació Biomèdica de BellvitgeIDIBELL, Cancer Prevention and Control Group, L'Hospitalet del Llobregat, Spain, ${ }^{3}$ Association of European Cancer Leagues, Brussels, Belgium, ${ }^{4}$ Cancer Society of Finland, Helsinki, Finland, ${ }^{5}$ Agència de Salut Pública de Catalunya, Departament de Salut de la Generalitat de Catalunya, Barcelona, Spain, ${ }^{6}$ University of Barcelona, Departament of Clinical Sciences, School of Medicine, Campus Bellvitge, L'Hospitalet del Llobregat, Spain. E-mail: afeliu@iconcologia.net

Background: The Tobacco Control Scale (TCS) was first published in 2006 to monitor the implementation of tobacco control policies at country-level in Europe and, since then, every three years a new report $(2007,2010,2013$ and 2016) has been 
published. Our objective was to develop a website to gather and structure the information included in the TCS reports, to provide friendly dynamic graphics for professionals and the public, as well as, to compile other documents and publications derived from the scale.

Methods: The original TCS reports were used to bring together all the information and tables included in order to be able to access to all the information from one site. The researchers systematically reviewed all published articles that include the TCS, as main dependent or independent variable and other materials (seminars, photos, etc.) related to the scale. The researchers also generated dynamic graphics for visitors to explore how their countries' scores fluctuate across years and compare with other European countries. The website has been adapted to mobile devices.

Results: The website (www.tobaccocontrolscale.org) was launched at the 7th ECTOH in March 2017 in Portugal. It has had 1,556 visits (July 2017): $55.9 \%$ direct, $21 \%$ referral, $12.9 \%$ social (55.7\% via Twitter) and $10.2 \%$ others; the $73 \%$ have been new and $27 \%$ returning visitors. By country, $19.7 \%$ visitors were from Germany, 8.4\% from Switzerland, 8.4\% from Spain and 8.2\% from the UK. The $17.5 \%$ have accessed to the website using either a mobile phone or a tablet.

Conclusions: The website has been well received by the tobacco control community with almost 1,600 visits from all over the world; however, more diffusion has to be made to stimulate its use and reach a larger audience in the future that could probably extend the use of the scale for tobacco control purposes.

\section{Tob. Induc. Dis. 2018;16(Suppl 1):A586}

\section{DOI:10.18332/tid/84085}

\section{$12: 30-14: 00$}

PS-918-1 Factors associated with smoking cessation in a Kenyan community

D Magu' ${ }^{1,2}, \mathrm{~J} \mathrm{Mutai}^{2}$

${ }^{1} J$ kuat, Public Health, Nairobi, Kenya, ${ }^{2}$ Kemri, CPHR, Nairobi, Kenya. E-mail: magudennis@gmail.com

Background: The community members in any country play a major role in behavioral aspects of smoking cessation. Many smokers do not acknowledge that they are addicted to tobacco. Through this majority of individuals do not quit smoking and are left without intervention. The risk to experiment lead to development of a regular smoking pattern due to addictive nature of nicotine.

Methods: This was a cross sectional study carried out among community members in Kenya. Three hundred and ten study participants were selected to participate in the study. A convenience sampling technique was adopted during recruitment of the study participants. This was achieved through administering questionnaires, data was entered in Microsoft access database and later exported to SPSS for analysis. Descriptive statistics was done to report frequencies of variables and inferential statistics for categorical variables. The Chi square test of association was used to establish association between dependent and independent outcome variables.

Results: A total of 310 participants were recruited while $70 \%$ had inadequate knowledge on smoking cessation. Majority(72\%) of the respondents had not received information on smoking cessation. Majority (75\%) of the respondents felt it was difficult to quit smoking due to addiction. There was an association between peers, friends and the number of cigar $<0.05$. There were concerns on insufficient information and low knowledge level towards smoking cessation among the subjects. There was a significant association between smoking, level of income and education level $\mathrm{p}$ value $<0.05$

Conclusions: There is need to improve community's knowledge on smoking cessation through gatherings and local barazas to handle stress, low self esteem

Tob. Induc. Dis. 2018;16(Suppl 1):A587

DOI:10.18332/tid/84223

\section{$12: 30-14: 00$}

PS-919-1 Monitoring initiation of daily smoking as an indicator of tobacco control success: example of Ukraine

\section{T Andreeva $^{1}$}

'Babeș-Bolyai University, Cluj-Napoca, Romania, Department of Public Health, Kyiv, Ukraine. E-mail: tatianandreeva@gmail.com Background: While tobacco control

measures aim to both prevent smoking initiation and facilitate quitting smoking, these processes do not always occur in parallel. While the prevalence of daily smoking declined in Ukraine from $37 \%$ in 2005 to less than $25 \%$ more recently, it changes among young people much more clearly than among older groups. We explore approaches to consider data on smoking initiation.

Methods: Initiation of daily smoking was analyzed across 5 -year birth cohorts among the participants of two nationally representative surveys conducted in Ukraine in 2005 and 2010. The proportion of men and women who initiated daily smoking before age 20 was compared across genders and birth cohorts.

Results: Among men, about $50 \%$ were becoming daily smokers by age 20 since the 1950s. Among women, daily smoking remained negligible till about 1970s. From the 1990s, an increase in initiation of smoking became obvious among both genders and reached $70 \%$ of men and $20 \%$ of women initiating smoking by 20 years of age. However, after first tobacco control measures have been launched in Ukraine since 2005, youngest cohorts demonstrated reduced smoking initiation. Still, responses of older cohorts in men were consistent with data from earlier survey while women tended to underreport their past smoking initiation.

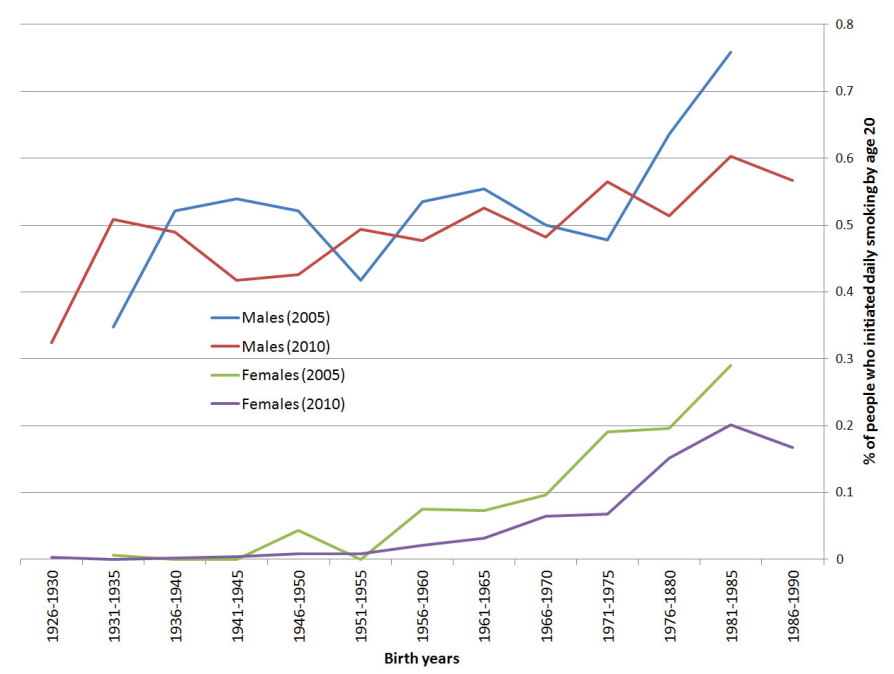

[Initiation of daily smoking by age 20 in Ukraine]

Conclusions: Smoking initiation data can reliably be collected from survey participants including that from older birth cohorts. The level of smoking initiation reflects both the periods when the tobacco industry was booming and the successes of tobacco control. Reduction in smoking initiation becomes an 
earlier outcome of tobacco control than stopping smoking and accumulation of former smokers.

\section{Tob. Induc. Dis. 2018;16(Suppl 1):A588 DOI:10.18332/tid/84299}

\section{2:30-14:00}

PS-920-1 International law, national policies and youth smoking: an examination of the Framework Convention on Tobacco Control

\section{GE Guindon', H Wipfli}

${ }^{1}$ McMaster University, Hamilton, ON, Canada, ${ }^{2}$ University of Southern California, Los Angeles, CA, United States of America. E-mail: emmanuel.guindon@mcmaster.ca

Background: The Framework Convention on Tobacco Control (FCTC) addresses a wide range of issues including protection from exposure to secondhand smoke and advertising, promotion and sponsorship. The FCTC represents a minimum set of tobacco control policies, although the treaty explicitly encourages countries to go above and beyond these measures. Despite its lack of mandatory provisions. FCTC ratification and its timing may be good proxy for a country's commitment to tobacco control. Our first objective is to assess the impact of the FCTC on global youth tobacco use. Our second objective is to explore two areas that are amenable to policy change: secondhand smoke and advertising.

Methods: We used pooled repeated-cross-sectional data from youth surveys conducted between 1999 and 2015 in more than 150 low- and middle-income countries and multilevel analyses to account for the nesting of students in schools and schools in countries. First, we examined the association between three outcome variables (smoking susceptibility, defined as the absence of a firm decision not to smoke, current smoking defined as 30-day smoking prevalence and a five-point scale of smoking uptake) and various indicators of the FCTC implementation. Second, we examined the association between exposure to secondhand smoke and advertising and indicators of the FCTC implementation.

Results: We found considerable heterogeneity in the association between different measures of youth smoking and indicators of the FCTC implementation. On the whole, we found clearer associations between exposure to secondhand smoke and advertising and indicators of the FCTC implementation.

Conclusions: A number of studies have examined changes in the implementation of tobacco control measures since the ratification of the FCTC but few studies have examined the effect of the FCTC on tobacco use; fewer still have used pre- and post-FCTC data. More research that use such data is needed.

Tob. Induc. Dis. 2018;16(Suppl 1):A589

\section{DOI:10.18332/tid/84565}

\section{2:30-14:00}

PS-921-1 China's smoking burden under business as usual and two replicable proven experiences from Taiwan and Hong Kong

W Gao'

'Taipei Medical University, Global Health and Development, Taipei, China. E-mail:waynegao@tmu.edu.tw

Background: Cigarettes kill more than one million Chinese each year and the number is expected to reach three million by 2030 . Total smoking attributable deaths (SADs) is expected to reach 200 million in China in 21 century under business as usual scenario. Methods: Taiwan and Hong Kong's historical transitions in male smoking endemic were used as references to project China smoking attributable deaths if it follows Taiwan and Hong Kong's authentic historical scenarios from 2000 to 2015 instead of its current trend.

Results: Male smoking was halved from $60 \%$ in early 1980 's to $28.6 \%$ in 2016 in Taiwan. Hong Kong has seen a similar $50 \%$ reduction to reach below $19 \%$. Taiwan's moderate policy enactment and implementation resulted in correspondingly, a flat decline before 2000 and was accelerated in line with the WHO FCTC implementation after 2005. China's daunting SDA could be largely reduced by 35 to $50 \%$ If decision makers would simply follow Hong Kong or Taiwan's proven tobacco control polices that leads to favorable declining scenarios.

Conclusions: Price affordability appears to be the biggest distinguishing factor. Cigarettes are still readily affordable to the public in China. The front-runner Hong Kong has implemented major tobacco control policies that have led to rapid reduction in smoking in the last few decades. Hong Kong and Taiwan's favorable transition in smoking endemic demonstrated valuable lessons as well as a culturally comparable reference for China. Taiwan hasn't implemented fully the WHO Mpower strategies and the price of cigarette remains too affordable. Further amendment of the tobacco control law is needed to continue this favorable decline trend. Had China implemented tobacco successful tobacco control polices a significant and accelerated decline in tobacco use similar to HK and Taiwan can be reasonably be expected.

\section{Tob. Induc. Dis. 2018;16(Suppl 1):A590 DOI:10.18332/tid/84673}

\section{2:45-14:00}

EP-264-1 Impact of the reduction of smoking prevalence on the trend of lung cancer mortality in Brazil

M Souza', A Szklo'1, LM de Almeida'

'Brazilian National Cancer Institute, Epidemiology Division, Rio de Janeiro, RJ, Brazil.E-mail: miriancs@inca.gov.br

Background: In Brazil the National Program for Tobacco Control is working for more than 30 years, and as consequence we can observe the reduction of smokers. The aim of this study is to evaluate the temporal trends in lung cancer mortality rates in Brazil observed from 1980 to 2015, and predicted estimates by 2040, by gender.

Methods: A time series study was carried out, using data from the Mortality Information System and information on the resident population obtained from the Brazilian Institute for Geography and Statistics. Crude, specific and age-standardized mortality rates per 100,000 men (and women) were calculated for each year between 1980 and 2015. Mortality predictions were made for the period 2016-2040 utilizing Nordpred program based on the period of 1980-2015, using the APC (age-period-cohort) model.

Results: While the decline in age-standardized lung cancer mortality rates has been most clearly observed for men since 2006, for women we will still experience a period of growth of these rates (2016-2025) followed by a period of stagnation (2026-2040) (Figure 1). Projections indicate that women aged 55-59 or younger will begin to experience a reduction in especific lung cancer mortality rates from 2021 onwards, but for women aged 75 or over these rates will continue to increase until 2040 . 


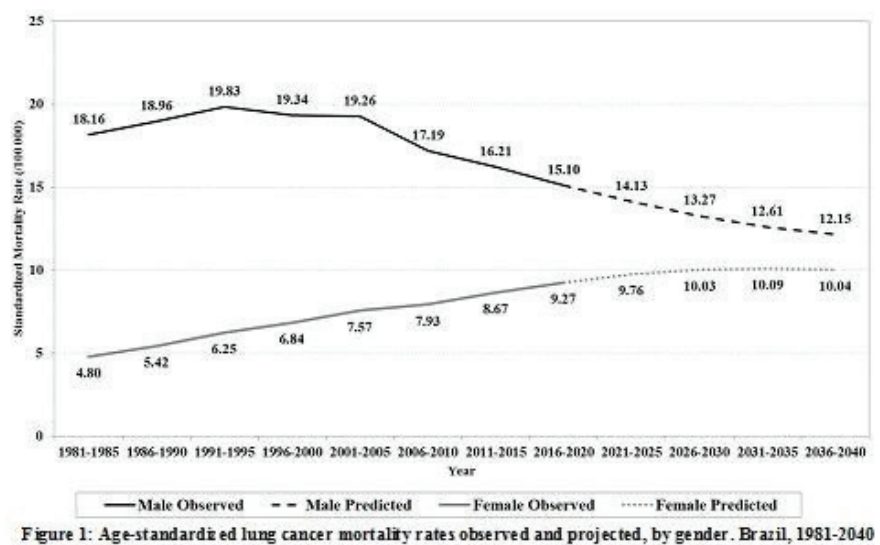

Conclusions: The decline in lung cancer mortality among men since 2006, but not among women, reflects not only the different stages in the tobacco epidemic by gender, i.e., women started smoking after men, but also the successful implementation of a set of tobacco control actions in Brazil in the last 30 years. The last contributed to a strong reduction in the prevalence of smokers and, as a consequence, to a decline in current (and future) smoking-related morbidity and mortality.

Tob. Induc. Dis. 2018;16(Suppl 1):A591

DOI:10.18332/tid/84021

\section{$12: 45-14: 00$}

EP-273-1 Measuring sustainability of tobacco control by market and consumption data in Turkey 0 Elbek'1 , E Dagli'1 , F Yildiz'1, T Gezer ${ }^{1}$, M Güner ${ }^{1}$

${ }^{1}$ Health Institute Association, Istanbul, Turkey. E-mail: elifzdagli@gmail.com

Background: Turkey has been recognized as a leader in tobacco control, implementing all MPOWER policies. However, reports have indicated challenges in the sustainability of the success.
This study aims to investigate the change in dynamics of tobacco manufacturing, marketing and consumption in the country as a measure of efficacy in policy implementation.

Methods: The data were obtained from Surveys of Turkish Statistics Institute and Tobacco and Alcohol Regulatory Authority, Global Tobacco Adult and Youth Surveys, Euromonitor reports.

Results: Turkish tobacco market is controlled by 5 transnational companies. Tobacco production increased by $40 \%$ between 2011 2016 in contrast to the reduction of $23 \%$ in $2008-2011$ period. Non-cigarette tobacco product manufacturing has increased by $82 \%$ in the last decade. Tobacco production per capita increased from $1.11 \mathrm{~kg}$ in 2011 , to $1.46 \mathrm{~kg}$ in 2016 . Annual domestic cigarette sales of 91 billion sticks in 2011 soared up to 105 billion sticks in 2016. Cigarette exportation increased by $290 \%$ between 2003 2016, while tobacco industry revenue due to domestic cigarette sales and exporation increased by $354 \%$ and $244 \%$ respectively. Between 2010-2015 increase in cigarette sales were reported as $12 \%$ in volume and $85 \%$ in revenue. The number of smokers reached to 15.7 million in 2015. By $201640 \%$ of men and $\% 13$ women were smoking cigarettes (in total $26.5 \%$ ). Current smokers in 13-15 age group increased from $6.9 \%$ in 2003 to $8.4 \%$ in 2009 and to $10.4 \%$ in 2012 . Tobacco related mortality was unchanged between 2011-2015, 124.8/100.00 and 123.7/100.000 respectively.

Conclusions: Starting from 2011 tobacco control has shown a downhill trend in Turkey, all gained seems to be lost by 2016. The reasons for this change must be investigated in order to restore sucess not only for Turkey but for all the countries which may follow the same path.

Tob. Induc. Dis. 2018;16(Suppl 1):A592

DOI:10.18332/tid/84056

[Tobacco production in Turkey]

\section{TOBACCO PRODUCTION}

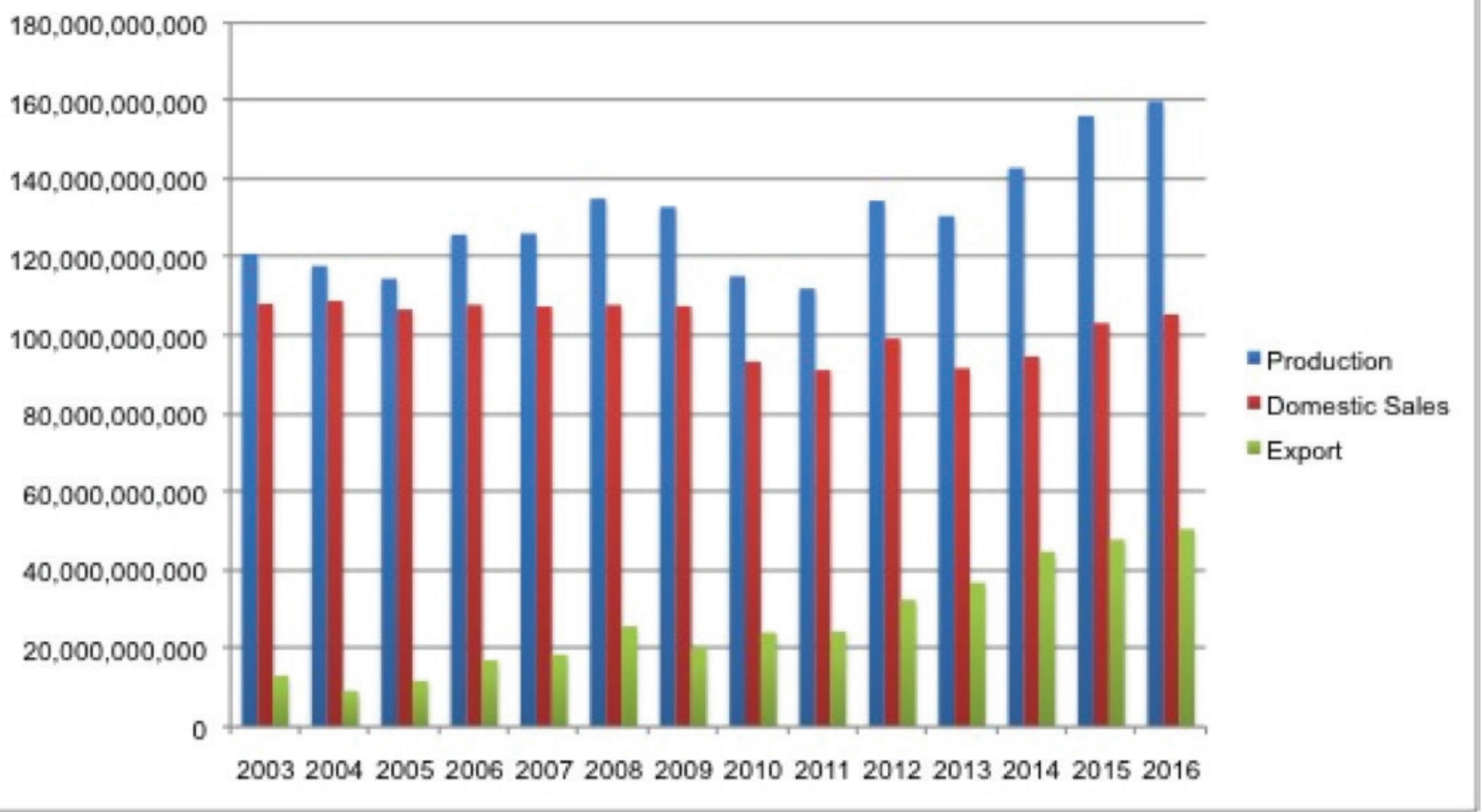




\section{5:45-16:45}

LB-1369-1 An empirical analysis of the impact of income change and cigarette taxation in a pricetiered cigarette market of Bangladesh

I Huq ${ }^{1}$, N Nargis'2, AKMG Hussain ${ }^{3}$, GT Fong ${ }^{4}$

${ }^{1}$ East West University, Department of Economics, Dhaka, Bangladesh, ${ }^{2}$ American Cancer Society, Economic and Health Policy Research Unit, Atlanta, GA, United States of America, 3University of Dhaka, Department of Economics, Dhaka, Bangladesh, ${ }^{4}$ University of Waterloo, Department of Psychology, Waterloo, ON, Canada.E-mail: iftekharulhuq.12@gmail.com Background: Taxing tobacco is among the most effective measures of tobacco control. However, in a tiered market structure where multiple tiers of taxes co-exist the anticipated impact of tobacco taxes on consumption is complex. This paper investigates changing smoking behavior in lieu of rising prices and changing income. The objective of the paper is to evaluate the effectiveness of change in prices (through taxes) and change in income in a price-tiered cigarette market. Do taxes successfully lead smokers to reduce consumption or simply move to a different price tier? How do income change affect smoking behavior?

Methods: A panel dataset from the ITC Bangladesh surveys is used for the analysis. The nature of dataset allowed for observing the impact of tax and income change on the smoking behavior of the same individual over a length of three years. Probit regressions are used to identify the effects of changes in prices and changes in income along with other control variables.

Results: Transition matrices show significant movement of smokers across price tiers from one wave to another. Regression results show when smokers face higher cigarette prices, their probability to down-trade increases and probability to uptrade decreases. Interestingly higher taxes do not increase the probability to quit. Also, higher income raises the probability to up-trade and decreases the probability to quit smoking.

Conclusions: It is evident from the results that a price-tiered market provides smokers more flexibility to accommodate their smoking behavior when faced with price and income change. Therefore, tiered structure of the tax system should be replaced with uniform taxes so that all price tiers are taxed with equal aggression. Results also reveal the need for overall cigarette taxes to be raised to an extent so that it off-sets any positive effects of income growth.

Tob. Induc. Dis. 2018;16(Suppl 1):A593

DOI:10.18332/tid/84691

\subsection{Setting the research agenda for translating science to policy and new tools for policy impact assessments}

\section{2:30-14:00}

PS-923-1 Knowledgebase of smokeless tobacco products and their chemicals

H Singh', J Kaur', A Sharma' ', A Kumar'2, S Kumari², D Bhartiya², D Sinha ${ }^{2}$, R Mehrotra ${ }^{2}$

${ }^{1}$ Indian Council of Medical Research, New Delhi, India, ${ }^{2}$ National Institute of Cancer Prevention and Research (NICPR), Noida, India. E-mail: hsingh@bmi.icmr.org.in

Background: Smokeless tobacco (SLT) products vary in appearance and in their composition of tobacco and non-tobacco chemical constituents thus differing in the mode of consumption. They may be consumed orally (chewed, sucked, dipped, held in the mouth, etc.) or nasally resulting in absorption of nicotine and other chemicals across mucus membranes. It is estimated that tobacco contains roughly 4,000 chemical constituents which undergo various chemical changes till the final SLT product is formed. Some of these are identified as potent carcinogens and are believed to play a crucial role in causing the negative health effects associated with SLT use.

Methods: List of chemical compounds present in various SLT products was extracted by exhaustive searching the peer-reviewed research articles, IARC monographs, published books and reports using various combinations of keywords. Chemical composition, $\mathrm{pH}$, moisture and free nicotine content along with brandwise and country-wise information of each SLT product were recorded. Physicochemical properties and target information of SLT chemicals was extracted from PubChem, ChemSpider and ChEMBL databases.

Results: All the data related to chemical composition of different smokeless tobacco products were compiled in a freely available and user-friendly database. This database contains information about 173 chemicals present in 161 different SLT products. Information regarding types of smokeless tobacco products, their trade or brand names and geographical locations and origin are also provided in this database. It also contains data related to variation of TNSAs (Tobacco-specific nitrosamines), Nicotine, metals, $\mathrm{pH}$, moisture and free nicotine content of various SLT products which determines the amount of nicotine available for absorption.

Conclusions: This database was developed to organize the data on SLT chemicals and their related content for better visualization and retrieval. This is the only one stop, open source, user friendly database that provides comprehensive information about the chemical composition of various smokeless tobacco products.

Tob. Induc. Dis. 2018;16(Suppl 1):A594

DOI: $10.18332 /$ tid/84381

\section{2:30-14:00}

PS-924-1 Consistency of self-report of smoking status among Korean young women

S Kim ${ }^{1,2}$, DW Shin ${ }^{3}$, SH Kim ${ }^{4}$, SK Hahm ${ }^{5}$, ES Lee ${ }^{6}$, YS Joo ${ }^{7}$, DH Lee $^{8}$, HS Kim ${ }^{9}$

${ }^{1}$ Korea University Ansan Hospital, Department of Family Medicine, Ansan, Korea, Republic of, ${ }^{2}$ Graduate School, Yonsei University, Department of Medicine, Seoul, Korea, Republic of, ${ }^{3}$ Samsung Medical Center, Seoul, Korea, Republic of, ${ }^{4}$ Seoul National University Bundang Hospital, Seongnam, Korea, Republic of, ${ }^{5}$ Hanil General Hospital, Seoul, Korea, Republic of, ${ }^{6}$ Inje University, Goyang, Korea, Republic of, ${ }^{7}$ Hallym University Medical Center, Anyang, Korea, Republic of, ${ }^{8}$ National Cancer Center, Goyang, Korea, Republic of, ${ }^{9} \mathrm{C}$ llege of Nursing, Shinhan University, Uijeongbu, Korea, Republic of. E-mail: september7777@hanmail.net

Background and challenges to implementation: Accurate estimation of smoking prevalence is important in a smoking cessation policy. Previous study using the Korean National Health and Nutrition Examination Survey (KNHANES) data compared women's self-report and urinary cotinine, and found that smoking prevalence is underestimated by self-report suggesting smoking concealment by female smokers due to stigma and cultural influence. We aimed to investigate whether female smokers report their smoking status consistently and related it to their smoking patterns (daily vs. intermittent).

Intervention or response: A cross-sectional, online panel survey was conducted with 2,080 women aged 19 to 39 . We first administered 
standard smoking status questions of KNHANES, and positioned unrelated health questions in between, and repeatedly asked about their smoking status and patterns. Then we cross-tabulated them to determine consistency of their self-report.

Results and lessons learnt: By initial self-reports, 366 (17.6\%) were current smoker, 120 (5.8\%) were former smoker, and 1594 (76.6\%) were never smoker. At second question, 102 (6.3\%) and 223 (14.0\%) women initially declared themselves as non-smoker answered that they are current smoker and former smoker. Current and former smokers at first smokers consistently reported their status at second questions. Among 256 daily smoker at second questions, 251 (98.0\%) reported they are smoker at first question. Among 210 intermittent smokers, only 115 (54.8\%) reported they are smokers at first questions.

Conclusions and key recommendations: At least $22 \%$ of current smokers $(102 / 468)$ reported they are not smoking at first standard KNHANES smoking status question. Most of them were intermittent smoker. Survey methods to reduce underestimation of female smoking prevalence are needed.

Tob. Induc. Dis. 2018;16(Suppl 1):A595 DOI:10.18332/tid/84524

\section{$12: 30-14: 00$}

PS-925-1 Prioritizing a global research and policy agenda for Betel Quid (BO) and Areca Nut (AN) M Parascandola ${ }^{1}, \mathrm{H}_{\text {Mehrtash }}{ }^{2}$, A Ndumele $^{3}, \mathrm{~K}_{\text {Duncan }}{ }^{3}$ ${ }^{1}$ National Cancer Institute, Division of Cancer Control and Population Sciences, Rockville, MD, United States of America, ${ }^{2}$ Leidos Biomedical Research Institute, Center for Global Health, National Cancer Institute, Rockville, MD, United States of America, ${ }^{3}$ National Cancer Institute, Center for Global Health, Rockville, MD, United States of America. E-mail: paramark@ mail.nih.gov

Background and challenges to implementation: $B Q / A N$ use is prevalent among approximately 600 million people globally and, is a risk factor for oral and esophageal cancers. It has also shown effects on the cardiovascular, nervous, GI and metabolic, respiratory, and reproductive systems. Research is needed to understand and implement effective prevention and control of $\mathrm{BQ} / \mathrm{AN}$, screening and treatment for related cancers, policy, and economic impacts.

Intervention or response: In 2016, the US National Cancer Institute and US National Institute of Dental and Craniofacial Research in collaboration with academic and government institutions based in U.S, Malaysia, Taiwan convened a group of experts to gain a greater understanding of the impact of $B Q / A N$ use, both with and without tobacco. Consequently, a literature review is being conducted to further inform global research initiatives around $\mathrm{BQ} / \mathrm{AN}$ policies and implementation strategies. Results and lessons learnt: The specific recommendations from the meeting are: 1) Expand global epidemiology of BQ/ AN use (incl. understanding of the cultural and social norms) 2) Establish prevention and cessation programs for BQ/AN users 3) Create evidence-based screening and early diagnosis oral cancer programs 4) Carry out policy interventions around Trade, Bans including use, manufacture, sales and agriculture, Health service provision, Crop substitution, and Public Awareness Outreach. There are also lessons learned from tobacco control evidence that can inform interventions in prevention and policy to address the burden. The review will build on these findings by systemically emphasizing both research strengths and gaps to provide recommendations for future research and policy agendas.
Conclusions and key recommendations: The evidence shows that $\mathrm{BQ} / \mathrm{AN}$ are widely used globally. There are major differences between tobacco and $\mathrm{BQ} / \mathrm{AN}$ use which impede governments and communities from taking actions. To reduce the use of $\mathrm{BQ} / \mathrm{AN}$ and associated adverse health effects, it is imperative to address the research gaps, with a focus on the translation of that evidence to effective policy and programs.

Tob. Induc. Dis. 2018;16(Suppl 1):A596

DOI:10.18332/tid/84612

12:45-14:00

EP-266-1 Is health warning label compliance a country or manufacturer issue: a 9-country multiyear study

M lacobelli1 ${ }^{1}$ K Welding ${ }^{1}$, C Washington ${ }^{1}$, K Smith ${ }^{1}$, J Cohen ${ }^{1}$ 'Johns Hopkins Bloomberg School of Public Health, Department of Health, Behavior and Society, Institute for Global Tobacco Control, Baltimore, MD, United States of America. E-mail: miacobe1@jhu.edu

Background: Two agents are responsible for producing effective cigarette health warning labels (HWLs): governments ensure HWL legislation is comprehensive with clear implementing rules, and tobacco companies are responsible for implementation. We determined what factors contribute to noncompliance with HWL legislation in nine countries that improved their HWL requirements over a three-year period.

Methods: Unique cigarette packs were purchased in 2013 $(\mathrm{N}=1164)$ and again in 2015-2017 $(\mathrm{N}=1413)$ across nine low- and middle-income countries (Bangladesh, Brazil, China, India, Indonesia, Philippines, Russia, Thailand, and Vietnam) that improved their HWL legislation. Country-specific HWL compliance codebooks were created to assess up to four HWL compliance indicators: location, label size (coverage), label elements (e.g., text or background color, borders), and text size. Results: HWL compliance in 2013 ranged from 17-90\%; compliance in 2015-2017 ranged from $33-95 \%$. Of the four indicators, HWL size (label coverage too small) and text size (text height too short or text and graphic content ratio incorrect) were the largest contributors to noncompliance. When the HWL size requirement was removed, compliance increased from $24-100 \%$ in 2013, and $84-100 \%$ in 2015-2017. Similarly, when HWL text size was removed, compliance increased from $45-90 \%$ in 2013 , and 53-95\% in 2015-2017.

Conclusions: We found that text size requirements ranged from non-existent, to simple (i.e., font size or text height requirements), to complex (i.e., specific text and graphic content ratios). The more complex requirements were oftentimes poorly specified and thus were difficult to assess for compliance. Governments could simplify text size requirements by: (1) providing minimum font or height measurements or incorporating text within the HWL as a template; and, (2) not specifying text/graphic ratios. We also found that tobacco companies are shirking their responsibility to ensure that the HWL is the minimum size required by governments. Tobacco companies should be held accountable for complying with HWL requirements.

Tob. Induc. Dis. 2018;16(Suppl 1):A597

DOI:10.18332/tid/84211

$12: 45-14: 00$

EP-271-1 Are statutory and pictorial health warning helping people consuming smokeless tobacco products to quit? 
L Rajpurohit', Control of Tobacco Product

'Dr D Y Patil Dental College and Hospital, Public Health Dentistry, Pune, India. E-mail: ladusinghr1@gmail.com

Background: India was among the first few countries to adopt WHO the Framework Convention on Tobacco Control (WHO FCTC) in 2004. India also has its own policy on Cigarettes and Other Tobacco Products Act passed in 2003 to reduce the sale of tobacco products, although there is decline in smokers according to WHO report (2015), there is no drop in smokeless tobacco consumers. Hence the objective of the study was to assess the effect of statutory and pictorial health warning among individuals consuming smokeless tobacco product.

Methods: The questionnaire study was conducted among smokeless tobacco users near tobacco seller outlets in Pune, India. 203 consumers formed the sample size of the study through census.12 closed ended questions were asked by interview method. The responses were collected, tabulated and analyzed using descriptive statistics.

Results: Mean age of the study participants was $37.67 \pm 8.14$ ranging from 23-65. 198(97.5) of the study participants were aware of the pictorial warning. Though 157(77.3) wanted to quit tobacco, 33(18.2) were not able to understand pictorial warning. Conclusions: It was concluded that though there was awareness about pictorial and statutory warnings which are found to be effective tools to help consumers quit tobacco. Poor quality of packaging and absence of warnings on few of smokeless tobacco did not serve the purpose.

Tob. Induc. Dis. 2018;16(Suppl 1):A598

DOI:10.18332/tid/83996

\subsection{The End Game - Pathways to turning vision into reality.}

12:30-14:00

PS-926-1 Licensing tobacco vendors in the state of Himachal Pradesh in India - challenges, opportunities and the way forward to implement the new legislation

G Chauhan'

'Directorate Health and Family Welfare, National Health Mission, Shimla, India. E-mail: drgopal7475@yahoo.co.in

Background and challenges to implementation: Himachal Pradesh having a population of 7 million is the first Smoke Free among all large 21 states in India. Since 2013 Himachal has achieved substantial progress in implementing all components of MPOWER. They are mainly demand reduction strategies. Tobacco control leaders are advocating for licensing retailers to restrict the easy availability of tobacco products. Many countries in the world have already made strict provisions for licensing tobacco vendors. Indian tobacco control laws prohibits sale of tobacco to minors and near educational institutes but its implementation remains a huge challenge.

Intervention or response: Keeping in view the high smoking prevalence and the huge purchase of loose cigarette $(>70 \%)$ in the state, a new law named Himachal Pradesh Prohibition of Sales of Loose Cigarettes and Biddies and Regulation of Retail Business of Cigarettes and Other Tobacco Products Act has been enacted in 2016 . The new law provides strict provisions for banning sale of loose tobacco /single sticks of cigarettes and to licence tobacco vendors. The license to sell tobacco shall be given only to those who are adhering to all the existing regulations.

Results and lessons learnt: Most of the tobacco vendors were selling tobacco with other confectionaries, grocery and daily need items. The new provisions provide licence to vendors either to sell tobacco only or other items. The fear of losing business is guiding the vendors for alternate business. Many have changed their business from tobacco and many are trying. There is a steep decline in sale of tobacco in the state in last one year.

Conclusions and key recommendations: Easy availability of tobacco products promotes its use and demand. Licensing tobacco vendors is a cost effective tool for reducing tobacco supplies. However the licensing process shall be strict and the numbers of vendors shall be restricted.

Tob. Induc. Dis. 2018;16(Suppl 1):A599

DOI:10.18332/tid/84278

\section{2:30-14:00}

PS-927-1 Is India on a path to tobacco endgame? - An analayis of recent policy papers and measures taken by Government of India

R Kumar ${ }^{1}, P$ Lal $^{2}$, RJ Singh ${ }^{2}$

'Directorate of Health Service, Public Health, Shimla, India, ${ }^{2}$ The Union SEA Office, Tobacco Control \& NCD, New Delhi, India.E-mail: ravindermph@gmail.com

Background and challenges to implementation: GATS India 2009:10 estimated that India is a home to over 275 million tobacco users. During initial few years, implementation of national legislation for tobacco control (COTPA 2003) was suboptimal. Post-2010, Ministry of Health Government of India ( $\mathrm{Gol}$ ) took few bold steps to advance the tobacco control agenda and demonstrated commitment to reduce tobacco use and demonstrated early commitment to towards a tobacco endgame. We analysed recent policy papers and measures taken by Government of India towards joining the global call for tobacco endgame.

Intervention or response: Ban on Gutkha (SLT) and display of specified pictorial health warning (PHWs) covering $85 \%$ of the principal display is among the boldest measures by the Government of India. Major focus was on law enforcement especially TAPS ban, smokefree rules, prohibition of sale to and by minors and banning outlets near educational institutions; introducing a toll-free helpline and raise in tobacco taxes and undertaking mass media campaigns.

Results and lessons learnt: The prevalence of tobacco use has decreased from $34.6 \%$ in GATS -I in 2009-10 to $28.6 \%$ in GATS -II in 2016-17. The number of tobacco users has reduced by about 81 lakh. The prevalence of tobacco use among the young population has reduced from $18.4 \%$ in GATS-I to $12.4 \%$ in GATS -II which is a $33 \%$ relative reduction. As anticipated, industry challenged the Gutkha ban both inside and out of court, opposed TAPS ban and display of PHWs of tobacco products in India; however public health wins at the end of the day.

Conclusions and key recommendations: India is in fairly advanced stage of implementing existing measures of MPOWER policies and national legislation; and is moving steadily towards a tobacco endgame. However, this may be the right time for India to think over the concept of tobacco free generation, regulating nicotine content and imposing a 'sinking lid' on tobacco supply.

Tob. Induc. Dis. 2018;16(Suppl 1):A600

DOI:10.18332/tid/84367
12:30-14:00
PS-928-1 Tobacco control in India - a civil society perspective
S Veeraiah', R Sudhakar'2, V Elangovan ${ }^{1}$, A Jacob ${ }^{3}$, A Siddique ${ }^{4}$ ${ }^{1}$ Cancer Institute (WIA), Psychooncology and Resource Centre 
for Tobacco Control (RCTC), Chennai, India, ${ }^{2}$ Cancer Institute (WIA), Psychooncology, Chennai, India, ${ }^{3}$ Cancer Institute (WIA), Resource Centre for Tobacco Control, Chennai, India, ${ }^{4}$ St.Georges University, Pre Medicine, Grenada, Grenada. E-mail: suren. psy@gmail.com

Background: Considering the magnitude of the tobacco menace, India ratified the FCTC in 2004, becoming the seventh country to do so. Following this, Tobacco control has been implemented in India for more than a decade, through comprehensive tobacco control legislation like Cigarette and Other Tobacco Products Act (COTPA). Implementation is being ensured by the extensive lobby by civil society or judicial interventions. There has been a delay in achieving the outcomes as obligated by FCTC. Therefore, it is crucial to understand and document civil society perspectives on the status of tobacco control in India, to accelerate the process, which is explored in the present study.

Methods: This was an exploratory study conducted using purposive sampling. Pioneering experts $(n=9)$ who were actively working in tobacco control in the state of Tamil Nadu, India for more than a decade, were interviewed with their consent, using a semi-structured interview schedule. The interview was audio- recorded, transcribed and analysed using thematic analysis.

Results: The activists report that unlike other major public health issues, tobacco control is given least priority across the country, thus resulting in poor enforcement of tobacco control policies. Five major themes emerged as challenges to tobacco control; enforcement, industry interference, awareness, cessation services and media. Though the experts perceive that tobacco control is possible, industry interference on the judiciary, executive and legislative level is a major hindrance at present. The results also highlight that lack of interdepartmental co-ordination among different departments responsible for enforcement and their ignorance about their role in tobacco control is also a deterrent. Need for establishing cessation facilities were felt crucial.

Conclusions: Tobacco industry interference is a big challenge for tobacco control in India. However, the experts are optimistic about making tobacco control a reality.

\section{Tob. Induc. Dis. 2018;16(Suppl 1):A601 \\ DOI: $10.18332 /$ tid/84440}

[Perspectives of Tobacco Control Experts]

\begin{tabular}{|c|c|c|c|c|c|}
\hline \multirow{2}{*}{$\begin{array}{l}\text { Themes } \\
\text { Sub } \\
\text { Themes }\end{array}$} & \multicolumn{2}{|c|}{ Enforcement } & $\begin{array}{l}\text { Industry } \\
\text { Interference }\end{array}$ & \multicolumn{2}{|c|}{ Awareness } \\
\hline & Lack of enforcement & $\begin{array}{l}\text { Need for effective } \\
\text { enforcement }\end{array}$ & $\begin{array}{l}\text { Lobbying of tobacco } \\
\text { industry }\end{array}$ & Lack of awareness & $\begin{array}{l}\text { Integration of } \\
\text { awareness into school } \\
\text { curriculum }\end{array}$ \\
\hline $\begin{array}{l}\text { Sample } \\
\text { Quotes }\end{array}$ & $\begin{array}{l}\text { "There is no particular } \\
\text { agency to provide } \\
\text { enforcement. The } \\
\text { government mechanism } \\
\text { is not functioning very } \\
\text { well." "So many sections } \\
\text { under the COTPA act } \\
\text { have not been notified } \\
\text { and enforced till now. } \\
\text { The implementation is } \\
\text { very poor." }\end{array}$ & $\begin{array}{l}\text { "I feel COTPA has to be } \\
\text { enforced more strictly } \\
\text { and this can increase its } \\
\text { effectiveness." }\end{array}$ & $\begin{array}{l}\text { "Tobacco industries } \\
\text { employ surrogate } \\
\text { advertising that has } \\
\text { adverse effects." } \\
\text { "Tobacco industries are } \\
\text { keeping close contacts } \\
\text { with politicians and } \\
\text { policy makers. They } \\
\text { try to influence them } \\
\text { and this is a major } \\
\text { challenge." }\end{array}$ & $\begin{array}{l}\text { "Awareness is highly } \\
\text { necessary in the rural } \\
\text { areas more than the } \\
\text { urban areas." "The } \\
\text { concerned authorities } \\
\text { themselves are unaware } \\
\text { of COTPA and the } \\
\text { various sections under } \\
\text { it in detail." } \\
\text { "There are still a lot of } \\
\text { misconceptions relating } \\
\text { to tobacco and its } \\
\text { related products" }\end{array}$ & $\begin{array}{l}\text { "Early initiation can } \\
\text { be curbed by giving } \\
\text { interventions at } \\
\text { schools." } \\
\text { "Early intervention } \\
\text { and awareness } \\
\text { through lessons will } \\
\text { encourage and promote } \\
\text { awareness amongst the } \\
\text { youngsters" }\end{array}$ \\
\hline
\end{tabular}

\begin{tabular}{|c|c|c|c|c|}
\hline Themes & Cessatic & Services & & dia \\
\hline $\begin{array}{l}\text { Sub } \\
\text { Themes }\end{array}$ & Inadequate cessation services & $\begin{array}{l}\text { Strengthening of cessation } \\
\text { services }\end{array}$ & $\begin{array}{l}\text { Paradoxical projection of } \\
\text { tobacco }\end{array}$ & $\begin{array}{l}\text { Media as an ambassador for } \\
\text { tobacco control }\end{array}$ \\
\hline $\begin{array}{l}\text { Sample } \\
\text { Quotes }\end{array}$ & $\begin{array}{l}\text { "The practitioners providing } \\
\text { cessation services themselves } \\
\text { are not aware of the correct } \\
\text { methodology for tobacco } \\
\text { cessation" }\end{array}$ & $\begin{array}{l}\text { "Every health personnel in the } \\
\text { country has to be educated } \\
\text { on the tobacco cessation } \\
\text { methodologies" }\end{array}$ & $\begin{array}{l}\text { Contradictory messages are } \\
\text { being projected through films } \\
\text { with regard to the use of } \\
\text { tobacco products." } \\
\text { "Section } 5 \text { (COTPA) violation } \\
\text { by the film industry" }\end{array}$ & $\begin{array}{l}\text { "Articles relating to ill effects } \\
\text { of tobacco usages so that } \\
\text { will help in awareness of the } \\
\text { same." } \\
\text { "Showcasing advertisement } \\
\text { featuring the ill effects of } \\
\text { tobacco use in cinema theatres } \\
\text { is very effective." }\end{array}$ \\
\hline
\end{tabular}

\section{2:30-14:00}

PS-929-1 Prohibition of importation, manufacturing and sale of Smokeless Tobacco (SLT) products: what is the Global Scenario?

D Saraf ${ }^{1}$

${ }^{1}$ National Institute of Cancer Prevention and Research(ICMR), Epidemiology \&t Biostatistics, Noida, India. E-mail: drdeepika. aiims@gmail.com

Background: Over the past 10 years, the WHO FCTC has served as a powerful tool to initiate, support, and advance national and global tobacco control efforts. However success of FCTC, but must not be allowed to conceal the fact that the job of tobacco control is far from done. This paper examines the tobacco legislations of various countries ratifying WHO FCTC with an objective to ascertain the status of prohibition of importation, sale and manufacturing of smokeless tobacco products. In addition, experiences of some countries that prohibit the sale, import or manufacture of these products were also studied. 
Methods: A literature search was conducted using PubMed, Google scholar, tobaccocontrollaws.org databases for all articles from January 1st, 2005 to May 2017 with the following search terms in the title, abstract, or keywords: 'smokeless tobacco', 'snuff', 'chewing tobacco', 'dip tobacco', 'tobacco regulation', 'SLT ban', tobacco', or 'SLT import ban', 'SLT sale ban', 'SLT manufacturing'. In the first stage of screening, an internal protocol was used to screen the titles and abstracts for all records according to a pre-defined selection criteria. In the second stage of search the WHO FCTC reports, previous $\mathrm{CoP}$ reports, $\mathrm{WHO}$ expert consultation reports were scrutinized to obtain desired information.

Results: The sale of smokeless tobacco products is prohibited in 42 Parties of the world that have ratified WHO FCTC and their laws are available in English language. Most of these bans are partial. Ten countries have prohibited manufacturing SLT products and five countries have imposed ban on its importation. The impact of these bans has been different for different countries. Conclusions: A comprehensive tobacco control strategy with effective tobacco cessation programme needs to be formulated that assists SLT users in quitting. In addition, multi-sectoral efforts are needed for effective implementation of the bans imposed by Governments.

Tob. Induc. Dis. 2018;16(Suppl 1):A602

DOI:10.18332/tid/84474

\section{2:30-14:00}

\section{PS-930-1 Policy action for health professionals} involvement in tobacco control

H A.Lando ${ }^{1}$, P Mohan $^{2}$, S Panneer ${ }^{3}$

'Division of Epidemiology and Community Health, University of Minnesota, Minneapolis, MN, United States of America, ${ }^{2}$ Mohan Dental Clinic, Bangalore, India, ${ }^{3}$ Central University of Tamil Nadu, Social Work, Thiruvarur, India. E-mail: sdrpriya@ gmail.com

Background and challenges to implementation: World Health Organization has declared tobacco addiction as a disease. Quitters need systematic professional cessation services. Healthcare providers have unique opportunity to identify and interact with tobacco users on individual basis, about the adverse effects, disease sequence, and also provide cessation counselling medication and follow up. Their involvement will enhance tobacco control.

Intervention or response: The aim was to assess the quitting capacity of tobacco users, their need for health professional care. GATS -1 and 2 reports regarding quitting and involvement of healthcare professionals in tobacco control was assessed and compared. Then digital search was carried out and scooping of the published data was done to examine the role of healthcare professionals in tobacco control in India and need based recommendations were drawn for policy action.

Results and lessons learnt: In GATS 1 and 2, 38\% tobacco consumers reported quit attempt. Those who planned to quit had increased from $46.6 \%$ to $55.4 \%$ in GATS -2 . There is marginal increase in smokers advised to quit by healthcare provider. Involvement of healthcare professionals and cessation services by them are not mandated by policies. National Health Policy 2017, has provision to implement specialist cessation services by trained professionals, but engagement of existing workforce remains untapped. Some have continued consumption, inspite of professional advice. Studies have shown that some health professionals perceive not all forms of tobacco is harmful. There is need for prevention or cessation training in medical and dental curriculum or the production of training programmes aimed at current medical and dental practitioners.

Conclusions and key recommendations: Considering the increasing number of potential quitters, and the growing need for professional assistance, compulsory training programmes for healthcare professionals in tobacco control needs to be conducted. Networking governance by public private partnership at Government and corporate hospitals be made as a mandatory primary healthcare service.

Tob. Induc. Dis. 2018;16(Suppl 1):A603

DOI: $10.18332 /$ tid/84488

\section{2:30-14:00}

\section{PS-931-1 Understanding the tobacco retail} environment; next frontier for UK tobacco control D Arnott ${ }^{1}, \mathrm{H}$ Cheeseman'1, S Hitchman ${ }^{2}$

${ }^{1}$ Action on Smoking and Health (UK), London, United Kingdom, ${ }^{2}$ King's College London, Institute of Psychiatry, Psychology and Neuroscience (IoPPN), London, United Kingdom. E-mail: deborah.arnott@ash.org.uk

Background and challenges to implementation: The UK is now a 'dark market' as such the supply chain and availability of tobacco has become a more important part of the marketing mix for tobacco companies.

A key tactic of tobacco companies has been to seek to persuade small tobacco retailers that their interests align with those of tobacco manufacturers and to encourage them to oppose tobacco regulations and participate in the promotion of products.

This project sought to better understand the extent to which tobacco retailers and tobacco manufacturers share interests and identify where they diverge.

Intervention or response: A report and infographic was produced using three sources:

- Analysis of retail trade publications to assess the claims being made by tobacco companies regarding the retail environment.

- Analysis of point of sale data from independent tobacco retailers testing the claims of tobacco companies regarding the importance of tobacco sales to small retailers.

- Survey of independent tobacco retailers in Britain. This sought to better understand the existing relationship between retailers and manufacturers, attitudes of retailers towards tobacco control policies and the extent to which they agreed with the positions taken by tobacco manufacturers regarding the retail environment.

Results and lessons learnt: While tobacco sales accounted for $25 \%$ of total income, margins were around $6 \%$ compared to an average $24 \%$ for the other products. As such average weekly profit was only $1.6 \%$ of income. Little evidence to support manufacturers claims that tobacco drives footfall or that retailers should stock full range of brand variants.

Conclusions and key recommendations: We demonstrated that the existing and future interests of small retailers and manufacturers are not aligned. We identified opportunities to encourage a more responsible approach to tobacco retailing aligning with public health interests.

Tob. Induc. Dis. 2018;16(Suppl 1):A604

DOI:10.18332/tid/84516

\section{2:30-14:00}

PS-932-1 Critical analysis of hookah smoking samples to formulate public health strategy to protect younger generations in Delhi, India

S Arora 
'Directorate General of Health Services, Govt. of Delhi, Public Health Wing - 1, DGHS, New Delhi, India. E-mail: aroradrsk7@ yahoo.com

Background: Hookah smoking is a traditional smoking in rural areas of India. But these days the hookah / pipe has entered urban metropolitan cities \& is seen in restaurants \& hotels with the hidden name of herbal hookah. Hookah owners claim that the hookah material does not contain tobacco or nicotine to get away from the clutches of legal provisions of cigarette \& other tobacco control act. This is actually a new strategy of tobacco industry to remain in market \& attract younger generation including females. Methods: Multiple raids conducted in different areas \& samples of hookah materials collected for analysis even though all the samples were claimed to be herbal \& herbal samples were shown to us during raids. The restaurant / hotel owners were challaned under section 4 for smoking in public places. The duly sealed samples sent to Govt of India labs for analysis for presence of tobacco / nicotine/ other hazardous chemicals. The samples tested at designated tobacco testing laboratory.

Results: Out of 18 samples, 3 could not be tested in view of insufficient quantity. Out of rest 15 samples all contained significant quantity of nicotine though all were claimed to be herbal. This clarifies that the restaurants / hotels are adding nicotine also inside the herbal samples in hidden manner with / without informing the customer.

Conclusions: It proves that most of the hookah smoking samples also contain nicotine though claimed to be herbal $\&$ are violations of Tobacco control act. It is difficult to conduct raids again \& again \& pick up samples for analysis. Therefore Govt should ban all kinds of hookah smoking to protect youth from the menace of tobaccos.

Tob. Induc. Dis. 2018;16(Suppl 1):A605 DOI:10.18332/tid/84579

\section{2:30-14:00}

PS-933-1 Banning loose cigarette sale-a bold supply side measure by State Government of Himachal Pradesh in India to decrease tobacco use

R Kumar', G Chauhan ${ }^{2}$

${ }^{1}$ Directorate of Health Service, Shimla, India, ${ }^{2}$ National Health Mission, NCD, Shimla, India. E-mail: ravindermph@gmail.com Background and challenges to implementation: Sale and use of loose cigarettes (also called singles or loosies) is widely acknowledged as a driver of the smoking epidemic in India. In Himachal Pradesh (HP) state (Population: 7 million) in India, more than $70 \%$ of cigarettes were sold as loosies. Following a high level of compliance to smokefree rules, State was declared smoke free in 2013, but an easy access, affordability and availability of cigarette as singles was a challenge for smoke free public places. Besides this, loose cigarette market neutralises four important tobacco control strategies-protecting minors, pictorial warnings, support quitting and effective taxation.

Intervention or response: Tobacco control advocates presented the facts to health department to ban sale of loose cigarettes and beedies in larger public health interest. Public support was garnered through a opinion poll and media coverage. Major advocacy efforts were done with senior government officials, legislators and state Health Minister.

Results and lessons learnt: State legislative assembly in October 2016 passed the draft bill "The Himachal Pradesh Prohibition of Sale of Loose Cigarettes and Beedies Act, 2016" and HP became the first state in India to ban sale of singles through legislation. Enforcement officials also notified. Legislation has a provision of major penalty in case of violations by the vendors. As anticipated, tobacco industry opposed the legislation from road to civil secretariat though tobacco vendors who acted their frontal groups. Government overlooked their opposition and issued directions to authorities to ensure implementation. Although this is half battle won.Cigarettes are still sold as singles by few vendors due to sub-optimal law enforcement.

Conclusions and key recommendations: State took a bold step to ban the sale of cigarette and beedies as singles; this will have long lasting impact on reduction in tobacco use. However, it is high time for the state to activate the enforcement mechanism to ensure $100 \%$ compliance.

\section{Tob. Induc. Dis. 2018;16(Suppl 1):A606}

DOI:10.18332/tid/84581

\section{2:30-14:00}

PS-935-1 Sweden's pathway to Europe's lowest level of tobacco-related mortality

\section{Ramström}

'Institute for Tobacco Studies, Täby, Sweden. E-mail: Iars. ramstrom@tobaccostudies.com

Background: Tobacco use patterns in Sweden have undergone dramatic changes during the last 50 years resulting in tobaccorelated mortality in men being the lowest in all European Union countries. The objective of this study is to describe this development and analyse the incentives and impediments that have influenced the course of the changes.

Methods: Timelines of tobacco use prevalence and tobacco control measures including tax policies were retrieved from various official sources of statistics. Details of initiation and cessation practices were retrieved from large national surveys in Sweden. These pieces of evidence were combined to analyse the development of tobacco use in Sweden from the 1970s to today.

Results: The major feature of the Swedish development is the transition from cigarettes to snus (the Swedish kind of low-toxicity oral tobacco) as dominating tobacco product among men, and this has been shown to be a major reason behind the record-low tobacco-related mortality. In 1976 the prevalence of daily use was $46 \%$ for smoking and $9 \%$ for snus use, in 2016 the figures were $8 \%$ respectively $18 \%$. The transition started in the late 1960 s. The main incentive came from the scientific reports that had created a wide awareness of the health risks of smoking (RCP 1962, US SG 1964). Snus was a nearby alternative and transition to snus was further encouraged by a beneficial price difference. Up till the early 2000 s the transition was gaining momentum in spite of shrinking price benefits. In 2006 there was a sudden "chock increase" of tax on snus but not on cigarettes. As a result the next following years showed a drop in snus use and an absence of continued drop in smoking.

Conclusions: Tobacco control policies should encourage transition from cigarettes to low-toxicity alternatives.

Tob. Induc. Dis. 2018;16(Suppl 1):A607

DOI: $10.18332 /$ tid $/ 84681$

$12: 45-14: 00$

EP-267-1 Leveraging public finance to achieve endgame for tobacco - possible national and global strategies

P Lal'

'International Union Against Tuberculosis and Lung Disease, New Delhi, India.E-mail: plal@theunion.org

Background: Calls for institutional investors to divest (sell off) tobacco stocks threaten the industry's share values, publicise 
its bad behaviour, and label it as a politically unacceptable ally. The rise of the socially responsible investment movement, increasing litigation against major tobacco companies, and an increasing emphasis on tobacco industry delegitimisation as a tobacco control strategy created a climate within which tobacco divestment was open for serious discussion.

Methods: This survey enquired from 19 environment (including groups working against GHGs, asbestos and Big Oil), social and development sectors (groups working on IFC exclusion criteria) on the role of public finance, and the arguments to support divestment from "sin" or exclusion sectors.

Results: In general the respondents to this survey presented the the following arguments which support divestment:

- Global obligations to the WHO's Framework Convention on Tobacco Control, and the other global treaties like the SDG.

- Ethical argument: Governments and their agencies that hold public funds can no longer support tobacco control and tobacco industry.

- Fiscal argument: high costs of national and global litigations make companies, public funded organisations and governments prone to heavy litigation costs and potential costs for damages

- Public health argument: Because tobacco industry continue to hold important policy positions, they block and interfere tobacco control efforts.

Conclusions: To begin a campaign for divestment would require preliminary work by civil society institutions which includes:

- Tracking and monitoring institutions and investments

- Collaborating with organisations which advocate for ethical or socially responsible investments.

- Developing a policy research and advocacy roadmap with stakeholders towards and end date for divestment

Tob. Induc. Dis. 2018;16(Suppl 1):A608

DOI: $10.18332 /$ tid/84345

\section{2:45-14:00}

EP-270-1 Re-thinking taxes to enhance public health

JR Branston', AB Gilmore ${ }^{2}$

'University of Bath, School of Management, Bath, United Kingdom, ${ }^{2}$ University of Bath, Department for Health, Bath, United Kingdom. E-mail: j.r.branston@bath.ac.uk

Background: Tobacco, like many other harm inducing products such as alcohol and unhealthy/sugary food, is mainly manufactured and supplied by profit seeking private enterprise. Such companies have a strong incentive to expand existing markets to increase profits at the expense of public health. Traditionally the objective of market interventions is to correct perceived market failures, such as the externalities created like second-hand smoking, or informational gaps regarding risk. Excise duty, the most effective intervention, has been viewed as a way of both raising revenue and deterring consumption through higher prices. Yet despite multiple market interventions global epidemics associated with smoking and other harm inducing products continue to create high levels of mortality and morbidity, and their associated costs. Methods: A narrative review of the impact of public health related market interventions on both the demand and supply side of the markets for tobacco, alcohol, and food. A set of criteria on the impacts of taxation on markets is developed.

Results: Existing interventions, including tobacco/alcohol duty, are identified as primarily changing the demand side, while there are very few supply focussed interventions. Complementary approaches to supply side taxation are identified which address corporate profits and the ability of shareholders to access that profitability, thereby changing the incentives faced by profit seeking companies.

Conclusions: A new systems approach is needed to re-examine and understand the wider impact and potential of taxation. Adopting such a holistic approach would help moves towards a tobacco endgame and moves to address other company related epidemics such as obesity and growing levels of diabetes. Companies could not only be directed towards healthier products (such as e-cigarettes or reduced sugar cereals), but also made to pay a greater contribution towards the costs to society they help to create.

Tob. Induc. Dis. 2018;16(Suppl 1):A609

DOI:10.18332/tid/84495

\subsection{Promoting transnational cooperation and Partnerships: models of Intergovernmental and nongovernmental collaboration across multiple sectors for high level commitments}

\author{
14:00-15:30 \\ RF-1266-2 The role of social listening in promoting \\ $100 \%$ smoke-free law in Shanghai \\ S Wen ${ }^{1}$, W Zhao ${ }^{2}$ \\ ${ }^{1}$ Campaign for Tobacco Free Kids (China Office), Beijing, China, \\ ${ }^{2}$ Peking University, Center of Social Media Research, Beijing, \\ China.E-mail: swen@tobaccofreekids.org
}

Background and challenges to implementation: The Shanghai Regulation on Smoking Control in Public Places was enacted in 2009, but DSRs were allowed in some indoor places, making the protection for Shanghai's 24 million residents in Shanghai ineffective. Opportunities to amend the law arose in 2016, however, there was no consensus among policymakers in terms of DSRs. Public awareness about the ineffectiveness of DSRs in preventing secondhand smoke exposure was low, and the public was generally indifferent about this issue.

Intervention or response: To increase public support for $100 \%$ smoke-free law, Campaign for Tobacco-Free Kids and the Center for Social Media Research of Peking University conducted social media monitoring from March to October 2016, listening to what Shanghai residents cared about, how they talked about issues that were important to them, whom they listened to, and what their knowledge and attitudes were regarding secondhand smoke. Based on the listening, CTFK together with tobacco control partners designed and implemented social media strategies and tactics, such as a Shanghai-style E-poster campaign to instill pride among Shanghai residents, a running event to appeal to health-conscious people, and a flash mob featuring a local favorite singing group to reach young people.

Results and lessons learnt: These tactics resonated with the Shanghai residents and won their support for the 100\% smokefree policy. The topic of Shanghai smoke-free law generated 15 million views and 13,000 comments. Public support increased from $40 \%$ in March of 2016 to $73 \%$ in October of 2016.

Conclusions and key recommendations: Supportive public opinion is important for the adoption and implementation of tobacco control measures. In order to reach the right people at the right time with the most insightful contents in the most relevant style, social listening was essential. Future tobacco control advocacy efforts in China and other countries can learn from the experience in Shanghai.

Tob. Induc. Dis. 2018;16(Suppl 1):A610

DOI: 10.18332/tid/83834 


\section{4:00-15:30}

RF-1267-2 Collaboration between government and civil society: engaging the public in the implementation of Beijing's smoke-free law through integrated communications efforts

$X$ Yin $^{1}$, S Wen ${ }^{2}$

${ }^{1}$ Campaign for Tobacco Free Kids, Washington, WA, United States of America, ${ }^{2}$ Campaign for Tobacco Free Kids (China Office), Beijing, China. E-mail: xyin@tobaccofreekids.org

Background and challenges to implementation: The Beijing Smoking Control Regulation was adopted in November 2014 and took effect in June 2015. The Regulations includes a provision requiring all indoor public places, work places and public transports to be smoke-free. Lawmakers were concerned that the law may be overly restrictive and difficult to enforce due to a variety of reasons, such as a small enforcement team, a large population (21 million), low awareness of smoking harms, the deeply rooted "smoking culture", etc.

Intervention or response: Civil society organizations and the government designed and implemented innovative and integrated communications strategies composed of a variety of tactics and materials to raise public awareness of the law and maximize compliance. These tactics and materials were delivered through a combination of traditional media, social media, community outreach, and other formats and channels. Examples included a "No Smoking" gesture campaign, a high-profile launch event at Beijing's iconic building, the Bird Nest, a smoke-free Beijing song and accompanying square dance, posters and postcards featuring celebrities, count-down events, TV and radio programs, production and dissemination of a short video on social media, A Letter to the Beijing Resident, a "Thank You for Not Smoking" campaign, among others.

Results and lessons learnt: Public knowledge of the law reached $81.3 \%$ in the first 3 months since the law took effect, public support for the law reached $93.83 \%$ and the compliance rate was $71 \%$ after 1 year since the implementation. With their respective unique advantages, the government and civil society groups worked closely to reach Beijing residents broadly and effectively.

Conclusions and key recommendations: The effective communications as a result of the collaboration between government and NGOs in Beijing was a key in achieving relatively high compliance rate in a short period of time. This model can be applied to other cities and in other tobacco control or public health policies.

Tob. Induc. Dis. 2018;16(Suppl 1):A611

DOI:10.18332/tid/83880

\section{4:00-15:30}

RF-1268-2 Bidi in demerit category (28\%) in Goods Service Tax (GST) was a big win in India

R Kumar ${ }^{1}$, A Sarin ${ }^{1}$, DS Rastogi ${ }^{1}$

'Sambandh Health Foundation, Gurgaon, India. E-mail: rajsen07@gmail.com

Background and challenges to implementation: Bidi is consumed on a large scale in India, especially in rural and low income regions One of the major reasons behind this is the price of bidi. Out of the 29 states in India only 6 have negligible taxes on Bidi with no other taxes being imposed on it. To equalize the price of products throughout the country the of India abolished more than 500 different types of taxes and planned implementation of one nation one tax system. This has been labelled as GST.

As the tax on Bidi was low, it was being expected that it will be kept in the lower tax bracket of GST.Bidi sale in India is 8 times more than cigarettes. More than $9.2 \%$ of smokers in the age group of more than 15 years smoke. The Indian formed a council to pass the GST bill.

Intervention or response: Various organisations conducted campaigns on a national scale to include Bidi in a higher tax bracket under the GST. Campaigns pertaining to the above were initiated in various parts of the country via print, news agencies, electronic media and social media.

The campaign received 87164 likes with the news of the campaign reaching more than 1.3 million people. 1474 people tweeted on this issue to Prime Minister with the tweets being viewed more than 283757 times.hastag (\#bidi, \#onetobaccotax, \#GST, \#PMMODI, \#Onetobaccotax, \#Tobaccokills, \#बीड़ी) created for one tobacco tax under GST which was seen by and agreed upon by 712239 people. The print media published more than a 1000 articles on this issue. the campaign.

Results and lessons learnt: The campaign to include Bidi in a higher tax slab(28\%) caused the govt \& council to include it in the higher tax category.

Conclusions and key recommendations: This shows the key role played by campaigns in various aspects in raising an issue if public interest to put pressure on the government.

Tob. Induc. Dis. 2018;16(Suppl 1):A612

DOI: $10.18332 /$ tid/84687

\section{$12: 30-14: 00$}

PS-936-2 Assess compliance to existing Tobacco Control Law among task force (TF) committee members across 10 districts in Bangladesh

B Rahman ${ }^{1,2}$, SH Patwary ${ }^{1}$, F Hossain ${ }^{3}$, A Islam ${ }^{4}$, F Zaman¹, M Mohiuddin ${ }^{1}$

'Dhaka International University, Tobacco Control and Research Cell, Dhaka, Bangladesh, '2Dhaka International University, Department of Business Administration, Dhaka, Bangladesh, ${ }^{3}$ Comilla University, Department of Statistics, Comilla, Bangladesh, ${ }^{4}$ Work for a Better Bangladesh (WBB) Trust, Health, Dhaka,Bangladesh. E-mail: sohel_tarafdar@yahoo.com

Background: Bangladesh is the first signatory countries of WHO FCTC. Bangladesh signed on 16 June 2003 and ratified on 14 June 2004. The government of Bangladesh has taken several initiatives to reduce tobacco use. Bangladesh government has enacted tobacco control law in 2005 and amended in 2013 along with notification of the revised rules in 2015 .

Methods: Cross sectional study design, quantitative and qualitative approaches, purposive sampling method and semistructured questionnaire and oral interview used to conduct the survey. The objectives of the study was to assess the knowledge and attitudes of Task Force (TF) regarding Tobacco Control (TC) Law. SPSS 21, Microsoft Excel used to analysis the data.

Results: $85 \%$ staff know about TC law among the visited office and still now people smoke into the office area in $25 \%$ offices. But $83 \%$ office display No Smoking Signage. In terms of Task Force (TF) committee meeting, 67\% TF members said that district TF meeting hold regularly. In terms of enforcement of tobacco control law, $85 \%$ respondents argue that mobile court conduct regularly but $57 \%$ said that they have lack of logistic support for conducting mobile court. Very interesting is that $59 \% \mathrm{TF}$ members do not know about FCTC article 5.3, which is known as safeguard for tobacco control.

Conclusions: It can be said that a noticeable change have been seen on tobacco control issues. But tobacco companies are still trying 
to increase their sales among the young as potential customer by different illegal promotional campaign. So that awareness building programs should be conducted, logistic support should be delivered and social pressure should be created as people can be aware of their strategy and can able to protect it. So, government should take initiative immediately to implement the graphic health warning on all tobacco products to protect people as well as society.

\section{Tob. Induc. Dis. 2018;16(Suppl 1):A613 DOI:10.18332/tid/84193}

\section{2:30-14:00}

PS-937-2 The role of non-governmental organizations in tobacco control in Romania

M Eremia', C Radu-Loghin², LM Lotrean ${ }^{3}$

${ }^{1}$ Aer Pur Romania, Bucharest, Romania, ${ }^{2}$ European Network for Smoking and Tobacco Prevention - ENSP, Bruxelles, Belgium, ${ }^{3}$ University of Medicine and Pharmacy 'Iuliu Hatieganu' Cluj, Cluj-Napoca, Romania.E-mail: marius.eremia@gmail.com

Background: World Health Organization calls for comprehensive measures for tobacco control which includes legislative, socioeconomical and educational measures. This presentation has two objectives. First it gives an overview of the tobacco control in Romania. Second it gives examples which illustrate the role plaid by non-governmental organizations in establishing activities and cooperation for long term strategies in this field.

Methods: The results are based on data from literature as well as personal experience of the authors. The presentation underlines the strengths and weakness of tobacco control in Romania, giving a special attention to the role plaid by non-governmental organizations. Results: The strengths of the tobacco control in Romania includes the legislation which limits the exposure to tobacco control advertisement and exposure to passive smoking in public places, the presence of pictogram on tobacco packages, increasing prices for tobacco products, development of some educational programs, including a smoking cessation program using the information technology. The weaknesses include the limited funding for educational activities for tobacco prevention and support for quitting smoking, poor reinforcement of legislation which prohibits the access of adolescents to tobacco products. The non-governmental organizations plaid an important role in developing national and international cooperation which stimulated the development of educational activities for tobacco prevention and cessation, training and capacity building as well as advocacy for adopting and implementation of legislation for tobacco control.

Conclusions: Non-governmental organizations play an important role in enhancing tobacco control in Romania through a wide cooperation with national and international partners, which several times stimulated the implication and commitment of several organizations in this field.

Tob. Induc. Dis. 2018;16(Suppl 1):A614

DOI:10.18332/tid/84242

$12: 30-14: 00$

PS-938-2 Harvesting the faith on "Local Deities" to make folks in remote villages of Himachal shun Tobacco use!

A Phull' ${ }^{1}, 0$ Kumar ${ }^{2}$

${ }^{1}$ The Staesman, Editorial, Shimla, India, ${ }^{2}$ Government of Himachal Pradesh, Health, Shimla, India. E-mail: archanaphul252@gmail. com

Background: Over 21 percent of adults in state of Himachal,
India, use some form of tobacco. The state cancer hospital receives about 6000 cases every year, with tobacco related mouth cancers at the top. The state of Himachal has banned tobacco products including Gutkha (Chewable smokelessTobacco) and has recently notified the ban on sale of loose cigarettes and bidis. The law enforcement by government on one side, the state, which is known as the land of Gods, is advantaged by the supreme faith of people on local deities in certain interior villages, as for as health related issues are concerned.

Methods: A study of cultural faiths of people in Hurang village of interior Chuhar valley of Mandi district and Tuin and Jathani villages in Lug valley of Kullu district was done by taking interviews of a cross section of local residents so as to assess the influence and role of local deities in decision making by the people in their day to day life.

Results: It was found that hill villages have their own deities, which have a great influence on the life of local people. The local deities has a say in village matters through their 'kardars', (priests). The people have day to day psycho-socio-cultural dependence on them. That is why in these villages, the local deity's diktat against smoking and any kind of tobacco use like smoking, chewing Gutkha, selling or bringing any tobacco product is completely prohibited by local deity's order. Unaware of ban on tobacco the deity's order is sacred for them and any violation can invite deity's wrath for them.

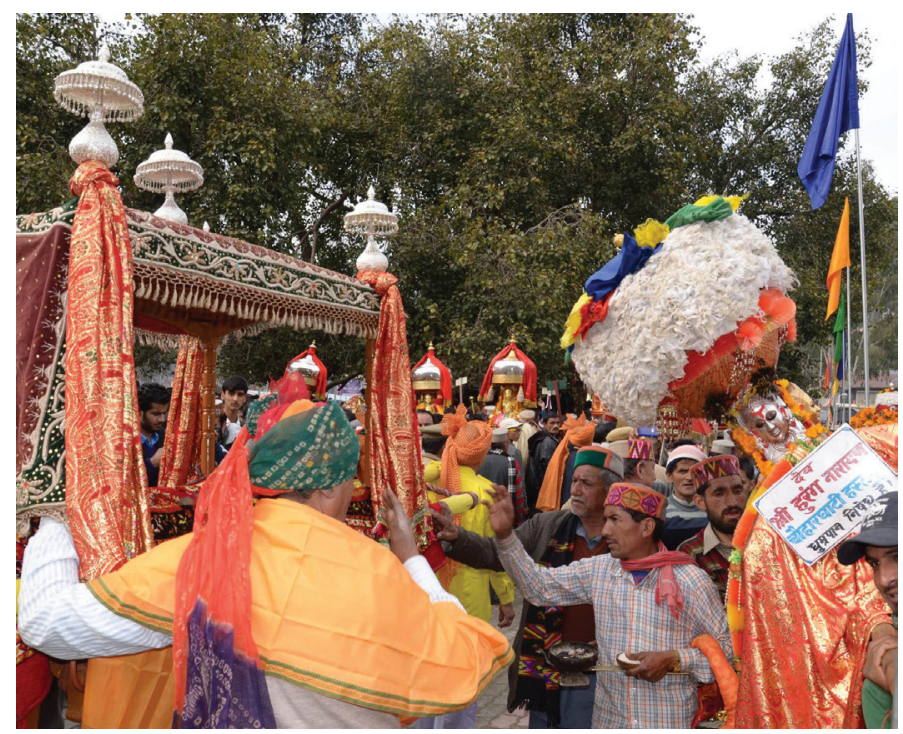

[Hurang Deity giving message of no smoking]

Conclusions: The cultural faiths should be re-enforced and integrated with tobacco control programme for greater influence by making the local symbols of faith, the 'Kardars' as brand ambassadors for spreading the message of shunning all tobacco products and drugs!

Tob. Induc. Dis. 2018;16(Suppl 1):A615

DOI: $10.18332 /$ tid/84252

12:30-14:00

PS-939-2 Encouraging tobacco control using national multisectoral ministerial mandate and priorities in Indonesia

F Qureshi', D Kania ${ }^{1}$

${ }^{1}$ WHO Department for Prevention of Noncommunicable Diseases, Jakarta, Indonesia. E-mail: qureshif@who.int

Background and challenges to implementation: Indonesia 
remains as one of the highest tobacco consuming countries. While there is intention by Ministry of Health to bring tobacco control as national multi-sectoral priority; still there is a need to find strategic platforms within the non-health sectors that can provide policy shifts for tobacco control ownership within the mandates of these Ministries.

Intervention or response: During the period 2014 to 2017 , WHO Indonesia have collaborated with various national Ministries, including Ministry of Human Rights and Law, Coordinating Ministry of Human Development and Culture, Ministry of Finance, Ministry of Women empowerment and Child Protection, and Ministry of National Planning. This strategic cohesion opened doors to discuss tobacco control as a policy measure beyond the conventional domains of health; also bringing tobacco control in the broader non-health paradigm such as demographic transition, Human Rights, taxation reforms, International trade and Health. Results and lessons learnt: This initial collaboration has resulted in improving perception of tobacco control as a multisectoral issue. The Ministries have understood the need to include tobacco control under their mandated policy frameworks; e.g. Ministry of Law and Human Rights now already considering to put tobacco control as part of annual National Action Plan on Human Rights; Ministry of Finance considering taxation on tobacco as part of longer term reform agenda; Ministry of Planning has started thinking on the lines of developing a multisectoral strategy for tobacco control.

Conclusions and key recommendations: In countries having strong tobacco industry influence, tobacco control issue needs to be brought forward within larger policy mandates of non-health sector ministries, using their national priorities.

Intergovernmental organizations as well as other partners and organizations working on tobacco control should expand reach out to sectors beyond health, establish and encourage dialogue; and help develop ownership of these sectors on specific policy interventions that directly or indirectly support implementation of key policy measures for tobacco control.

Tob. Induc. Dis. 2018;16(Suppl 1):A616 DOI:10.18332/tid/84256

\section{2:30-14:00}

PS-940-2 Government's pro-public health policies and effective coordination is key for success in tobacco control - a case study from Himachal Pradesh in India

R Chand', G Chauhan²

${ }^{1}$ Indira Gandhi Medical College, Hospital Administration, Shimla, India, ${ }^{2}$ National Health Mission, NCD, Shimla, India. E-mail: dr.rameshigmc@gmail.com

Background and challenges to implementation: Himachal Pradesh (Pop: 7 million) is a state in northern part of India. More than one-fifth of adult population in the State is tobacco user. State is not covered under India's National Tobacco Control Programme; as a result the state had major resource constraints in terms of manpower, finance and technical expertise. However, an effective collaboration between the state government, network of local NGOs (HPVHA) and The Union- an International organization worked very well and state became a model for tobacco control in India.

Intervention or response: HPVHA generated massive awareness among general public about the issue, strategically carried out political advocacy with policy makers. The Union provided funding and technical assistance. Government issued relevant circulars, notified squads and simplified the enforcement procedures.

Results and lessons learnt: Collaborated efforts resulted in setting up of an institutional framework. Stringent enforcement was carried out across the state. Till May 2017, more than 100,000 violations has been reported and near Rs 11 million has been collected as fine amount which is further utilized for tobacco control activities. TAPS violations at points of sale are nearly rooted out from the state. First conviction in the country under for TAPS violations, pictorial health warnings on tobacco packs and gutkha ban was carried out in the State. Based upon the findings of compliance survey on an International protocol, the state of Himachal Pradesh was declared "smoke free" in July 2013 by Health Minister. WHO awarded HPVHA and State government in the year 2011 and 2012 respectively for implementing tobacco control policies effectively.

Conclusions and key recommendations: State Government's pro-public health policies formulation and implementation and strategic collaboration between Government and NGOs are always vital for successful implementation of any public health initiative as demonstrated in State of Himachal Pradesh. This model can be replicated in other states in India or other developing countries with similar settings.

\section{Tob. Induc. Dis. 2018;16(Suppl 1):A617}

DOI:10.18332/tid/84599

\section{2:30-14:00}

PS-941-2 South-south cooperation on tobacco control - Project Plan on Article 5.2. National Coordination Brasil - Colombia

D Alves ${ }^{1}$, LV Calderón Pinzón ${ }^{2}$

${ }^{1}$ Ministry of Health of Brazil, Brasilia, DF, Brazil, ${ }^{2}$ Ministry of Health and Social Protection Colombia, Noncommunicable Diseases Office, Bogotá, Colombia. E-mail:dhtalves@gmail.com Background and challenges to implementation: Based on Colombian demands to meet its obligations under the WHO FCTC and face the challenges on strengthening and accelerating tobacco control programs, both countries set a Cooperation Project aimed to raise the topic on governmental agenda and ensure smooth and coordinated tobacco control multisectoral response at different levels of governance.

Intervention or response: Brazil will organize a virtual meeting to launch the project and make a presentation of its own governance context and structure. Provider to share with recipients the methodology for carrying out the mapping exercise Brazilian experience, lessons learned, current activities.

Recipient country carry out a mapping activity of key stakeholders working in the tobacco control area.

Understanding stakeholders role and how they impact on the good functioning of the National Coordinating Mechanisms in order to avoid possible overlapping responsibilities. Identify potential "opinion leader"/ focal point for tobacco control within each country Based on the information collected from each country and on the diagnosis, develop a general "Guidelines for smart approach", which will contain methodology related to stakeholders mapping and involvement.

Results and lessons learnt: Guidelines implementation. Workshops will be organized in the recipient countries, including simulation exercises that brings together key stakeholders.

Training the smart approach with opinion leader and possible focal points for tobacco control and set a plan for the approach; Implement the smart approach with stakeholders by opinion 
leader or national focal points to institutionalize well-functioning and reliable financed tobacco control focal points and National Coordinating Mechanisms (NCMs);

Implement the smart approach with stakeholders to allocate human and financial resources for planning and implementing of tobacco control activities by opinion leader or national focal points.

Conclusions and key recommendations: South South Cooperation is a tool that enables the exchange of information and knowledge between countries, recognizing the strengths and weaknesses of each and enhancing the capacity development in both supplier and recipient countries.

Tob. Induc. Dis. 2018;16(Suppl 1):A618

DOI:10.18332/tid/84600

\section{2:45-14:00}

EP-190-2 Advocacy for a strong tobacco tax policy - experience and lessons of an INGO in Vietnam THA Pham', TT Le', HN Nguyen', S Fitzgerald ${ }^{2}$

${ }^{1}$ HealthBridge Foundation of Canada, Vietnam Office, Hanoi, Viet Nam, ${ }^{2}$ HealthBridge Foundation of Canada, Ottawa, ON, Canada.E-mail:phanh@healthbridge.org.vn

Background and challenges to implementation: Vietnam ranks fifteenth in the world by the number of smokers. The government aims to reduce smoking prevalence among men from 47\% (2010) to $39 \%$ by 2020 but is reluctant to adopt a strong tobacco tax policy. The main challenges are low awareness of the benefits of increased taxes, and misconception among decision makers of the impacts of tax and price policy, led by the tobacco industry (TI). Intervention or response: HealthBridge works with the WHO, government and civil society in this campaign. Our approaches are: 1) Generate evidence on the benefits and best practices of tobacco taxation by conducting literature reviews and research; ii) Deliver evidence to policy makers, media, and people through workshops, articles and IEC; iii) Build capacity through technical workshops, involving government officers, and study tours; iv) Provide critical comments on the draft Excise Tax Law and guiding documents.

Results and lessons learnt: The 2014 Excise Tax Law was passed with an increased tax rate to $70 \%$ (from 65\%) on Jan 1st 2016, and to be increased to $75 \%$ on Jan 1st 2019; this led to an increase of retail price, revenue and some decrease of consumption in 2016, however the affordability has continued to rise due to inflation. The higher tax base revised in 2016 with improved definition of the payees in the distribution system is expected to reduce the risk of price transfer and the loss of government revenue.

There is improved awareness among policy makers and the media on the positive impacts of higher tobacco tax.

Conclusions and key recommendations: 2014 \& 2016 tax revision has positive impacts on retail price, revenue and consumption, but did not reduce affordability. Vietnam needs further tax reform.

The implementation of FCTC article 5.3 by the country is urgent to reduce tobacco industry interference in tobacco control.

Tob. Induc. Dis. 2018;16(Suppl 1):A619

DOI:10.18332/tid/84051

12:45-14:00

EP-262-4 Shutting down of Shisha bars by the effective use of current legal provisions - a case series from Punjab, India

R Gupta ${ }^{1}$, V Roojam², G Singh¹, G Kaur ${ }^{1}$
'State Tobacco Control Cell, Department of Health and Family Welfare, Chandigarh, India, ${ }^{2}$ Indian Administrative Services, Department of Health and Family Welfare, Chandigarh, India. E-mail: rakesh60.mahajan@gmail.com

Background and challenges to implementation: Tobacco in Shisha / Hookah is known to be carcinogenic. Health risks of smoking Shisha / Hookah include exposure to many toxic chemicals. Presently there is no provision under any law in India to ban Shisha / Hookah. But running a Shisha / Hookah Bar violate many provisions of other legislations of India like The Poisons Act, 1919, Section 4, 5, 6 \& 7 of COTPA, 2003, Food safety and Standards Act, 2006, Drugs \& Cosmetics Act, 1940, Juvenile Justice (Care \& Protection of Children) Act, 2015 (In case minors are present in Hookah/Shisha Bars) and Section 144 if promulgated. The objective of the study was to assess the effectiveness of current legal provisions in shutting down of Shisha bars.

Intervention or response: The present study was conducted in the period of April 2011 to March 2017 in Punjab. In 2013 Punjab Government had issued a Demi-Official letter to the Deputy Commissioners of all the districts to promulgate section 144 of $\mathrm{CrPC}$ against all the Shisha / Hookah bars. Health Advisory in year 2013 was issued regarding Shisha / Hookah by whatever names it is called. Regular enforcement drives and IEC activities were done at State, district to create awareness regarding the ill effects of Shisha / Hookah

Results and lessons learnt: Total 15 Shisha / Hookah bars have been raided in various districts of Punjab. FIRs have been launched against Shisha / Hookah bar owners by the Police department under section 144 of $\mathrm{CrPC}$ and cases have been launched under FSSAI, India. No complaint regarding a functional Shisha / Hookah bar has been received anywhere in Punjab.

Conclusions and key recommendations: The strict measures undertaken by the Punjab government against Shisha / Hookah bars was detrimental in functioning of Shisha / Hookah bars in the state. Strong interdepartmental co-ordination, awareness activities and regular enforcements drives are the re-enforcers in shutting down of Shisha/ Hookah bars.

Tob. Induc. Dis. 2018;16(Suppl 1):A620

DOI:10.18332/tid/83771

\subsection{International tobacco trade and health diplomacy: understanding the nexus between the two and domestic international disputes}

\section{$12: 30-14: 00$}

PS-942-2 The Pacific Alliance, a threat to tobacco control? Analysis of an "under the radar" trade pact S Bialous', E Crosbie ${ }^{2}$

'University of California San Francisco, School of Nursing, San Francisco, CA, United States of America, ${ }^{2}$ University of California San Francisco, Center for Tobacco Control Research and Education, San Francisco, CA, United States of America. E-mail: stella.bialous@ucsf.edu

Background: There has been robust debates about the impact of trade agreements, specifically the Trans-Pacific Partnership (TPP), on tobacco control. However, the potential impact on tobacco control of a multi-lateral trade agreement to increase trade in the Pacific region, the Pacific Alliance (PA), established in 2011 and with 52 observer countries, has not been discussed. Methods: Analysis of publicly available written records on the 
PA and news media archives, triangulated with key informant interviews.

Results: The PA (currently PA countries are Chile, Colombia, Mexico and Peru), representing a market of over 200 million people, is actively engaged in exploring trade partnerships with TPP countries (e.g. PA trade agreement with New Zealand). This engagement became stronger as the TPP countries are evaluating the impact of the US's departure. This expansion includes a formal advisory business council, composed of business representatives, including a former tobacco industry executive. In light of the uncertainties surrounding the TPP, media reports indicate tobacco companies are quickly considering the PA as an opportunity to consolidate business in Latin America to gain access to Asia Pacific markets. Meanwhile health-related discussions within PA have been minimal. Similar to the TPP the PA has an investor-state dispute settlement (ISDS) mechanism, which allows corporations to directly challenge public health regulations. However, unlike the TPP there are no exemptions for tobacco and no clarity on tobacco control, raising concerns of the impact of trade agreements on tobacco control.

Conclusions: Tobacco control community needs to take the offensive in monitoring the developments of the PA and their potential impact on tobacco control. There are opportunities to engage in dialogue and advocacy with the 4 founding countries, encouraging a whole of government approach that considers trade and health, ensuring that the PA does not have a negative impact on public health.

Tob. Induc. Dis. 2018;16(Suppl 1):A621

DOI:10.18332/tid/84160

\subsection{Building leadership capacity and broader alliances for tobacco control policy advocacy}

\section{4:00-15:30}

RF-1258-2 Engaging new voices and key opinion leaders in support of smoke-free policies in China KCH Khow', S Wen'르. Y Cui', J Sun ${ }^{1}$

${ }^{1}$ World Health Organization - China, Beijing, China, ${ }^{2}$ Campaign for Tobacco Free Kids(China Office), Beijing, China. E-mail: khowk@who.int

Background and challenges to implementation: One million people die a tobacco-related death every year in China, and this number will continue to grow if China does not act fast to rectify the problem. Rates of exposure to second-hand smoke are also extraordinarily high.

But the winds of change are blowing. Three major cities in China - its capital Beijing, Shenzhen and Shanghai have since passed $100 \%$ smoke-free laws which are being implemented very successfully.

The adoption of these comprehensive smoke-free laws was a genuine breakthrough for tobacco control in China. The laws set a new benchmark for smoke-free laws in other cities, and has generated a real sense of momentum for the adoption of a national smoke-free law.

Intervention or response: Given the widespread use and influence of social media in China, one of the key strategies of the \#RUFREE campaign is to engage with celebrities and key opinion leaders to give support to the smoke-free movement in China.

Results and lessons learnt: Results from two recent campaigns launched in 2017 - the WHO Smoke-Free Next Generation campaign (involving three of China's biggest young stars and celebrity photographer) and the Open Letter by Mothers campaign by Campaign for Tobacco-Free Kids involving high profile Chinese celebrities and key opinion leaders were hugely successful and generated a tremendous amount of social media buzz. The Smoke-Free Next Generation campaign went viral (the campaign hashtag set a new record by going from zero to over 130 million reads within a matter of days). The open letter campaign from one celebrity mother's social media post reached over 100,000 reads alone.

Conclusions and key recommendations: Engaging fresh and new voices with massive social media following has proven to be successful in helping raise the public awareness and support for smoke-free policies in China.

Tob. Induc. Dis. 2018;16(Suppl 1):A622

DOI: $10.18332 / \mathrm{tid} / 84406$

$14: 00-15: 30$

RF-1259-2 Engage the business sector to promote smoke-free corporate culture through industryoriented programmes

A Kwong ${ }^{1}$, V Lai $^{1}$

${ }^{1}$ Hong Kong Council on Smoking and Health, Hong Kong, Hong Kong.E-mail: lawrence_chu@cosh.org.hk

Background and challenges to implementation: The labour force participation rate is over 60 percent in Hong Kong and most of the employees spend 8 to 10 hours daily in their workplaces. "Most friends/ colleagues were smokers" was one of the most commonly cited reasons for failing to give up smoking. Smoking causes HK\$5.3 billion economic loss per year in Hong Kong, including loss of productivity due to premature death, costs of sick leaves, health care costs and nursing costs. However, corporates seldom put tobacco control or smoke-free promotion at the top of their agenda.

Intervention or response: Hong Kong Council on Smoking and Health urged the business sector to help promote smoke-free corporate culture through the Hong Kong Smoke-free Leading Company Awards and industry-oriented programmes which assisted corporates in setting up smoke-free policies and measures to promote smoking cessation among their stakeholders to foster an all-win situation for employees, employers and the society.

Results and lessons learnt: The Awards in 2011, 2013 and 2016 solicited support from over 80 commerce chambers and industry associations and mobilized around 1,000 corporates from a variety of industries to join, including large companies, small and medium enterprises, government departments and nongovernmental organizations, benefiting over 175,000 employees and other stakeholders, including the public.

Four major construction industry associations supported and referred around 40 companies to join the industry-oriented smoke-free programme launched in 2016, reaching over 16,000 industry workers whose smoking prevalence was over 40 percent. Outstanding companies were commemorated for their effective smoke-free measures and set as industry role models.

Conclusions and key recommendations: It is important to engage and empower different sectors of the community to maximize the impact and effectiveness of smoke-free promotion and tobacco control. Support from industry associations was crucial to raise awareness and boost participation of the business sector. Guidelines and industry-specific promotion collaterals shall be provided to facilitate companies in planning and implementing smoke-free policies.

Tob. Induc. Dis. 2018;16(Suppl 1):A623 


\section{DOI:10.18332/tid/83957}

14:00-15:30

RF-1260-2 Getting president's attention to reject tobacco bill through social media in Indonesia

R Suwardi

${ }^{1}$ Campaign for Tobacco Free Kids (CTFK), International Communications, Washington, DC, United States of America. E-mail: rsuwardi@tobaccofreekids.org

Background and challenges to implementation: Indonesia has a high smoking prevalence at $36.1 \%$ and is the only WHO member state in Southeast Asia that has not ratified the Framework Convention on Tobacco Control. With limited tobacco control regulations, Indonesia is a playground for the tobacco industry. To further weaken regulations and to protect their sales and profits, the tobacco industry has been supporting parliament members and academics to promote a Tobacco Bill since 2014. Tobacco control advocates have been fighting tirelessly to block this bill.

Intervention or response: Legislative process in Indonesia involves both Government and Parliament, it has to be approved by the President. Analysis of President's actions indicated that he responds to voices of citizens. The Campaign for TobaccoFree Kids, along with other tobacco control partners in Indonesia designed and implemented a strategic social media campaign to reach the President before he was due to convene a cabinet meeting to decide on the fate of the tobacco bill. Key messages and social media contents were developed, opinion leaders were mobilized, and an online petition was organized and promoted. Platforms such as Vlog, Twitter, and Facebook were selected to convey messages at specific times to maximize reach and visibility in order to get President's attention.

Results and lessons learnt: Our hashtag "Drop the Tobacco Bill" became a trending topic, picked up by the mainstream media who broadly covered the call for the President to reject the Tobacco Bill. After an extensive social media campaign, in March 2017, the President instructed his cabinet to reject tobacco bill. This endeavor showed that an effective social media campaign can influence policy decisions.

Conclusions and key recommendations: Indonesia has been regarded as the social media capital of the world with extremely high penetrations and some of the most active users. Tobacco control advocates should take advantage of this popular means of communications and networking to promote tobacco control policies.

Tob. Induc. Dis. 2018;16(Suppl 1):A624

DOI:10.18332/tid/84342

\section{4:00-15:30}

RF-1261-2 Human rights based approach to tobacco control as an effective tool for building strategic alliances and political will: experience from Bosnia and Herzegovina

U Bakh¹, A Sahacic ${ }^{1}$

${ }^{1}$ Association PROI, Sarajevo, Bosnia and Herzegovina. E-mail: ubakh@proi.ba

Background and challenges to implementation: Most countries in the world, including Bosnia and Herzegovina (BIH), are parties to the human rights conventions which are relevant to health and well-being, therefore to tobacco control. Reconceptualization of tobacco control through human rights perspective enables a holistic approach which at the center places a wide range of socioeconomic rights, consumers' rights, rights of children and women threatened by second-hand smoke, tobacco products' marketing and tobacco industry interferences.

Intervention or response: This approach from 2015 is applied by Association PROI for advocating for a new comprehensive tobacco control policies in BIH and establishing of partnership with the stakeholders from different sectors besides public health. Using human rights language, the strong tobacco control message was sent to decision makers and the public through different channels and tools - trainings, public events, media releases and informational materials. This PROI's concept was supported and promoted by the European Union within European Instrument for Democracy and Human Rights in BIH.

Results and lessons learnt: The human rights framework helped to push the boundaries of tobacco control and broaden the access to a wider group than "traditional" tobacco control advocates. Thus, women groups, children rights groups, human rights organizations, youth NGOs, environmental groups, and Ombudsmen became active participants of tobacco control movement.

The framework provides a powerful channel for communication with decision makers on FCTC's regulations in the way which is more familiar to them than conventional public health messages. Therefore, a clear and strong message was generated to the government calling for a concrete action on the protection of people's rights to clean air and healthy environment, thereby fulfilling their international commitments. This considerably speeded up decision-making processes of adoption of comprehensive tobacco control policies.

Conclusions and key recommendations: Human rights based approach in $\mathrm{BiH}$ confirmed itself as a powerful tool for tobacco control policy advocacy and for the establishment of effective broader partnerships.

Tob. Induc. Dis. 2018;16(Suppl 1):A625

DOI:10.18332/tid/84041

\section{$14: 00-15: 30$}

RF-1262-2 Role of media in disclosing tobacco industry interferences and building policy pressure in implementation of $90 \%$ pictorial health warning RP Neupane ${ }^{1}$

${ }^{1}$ www.nepalihealth.com, Editorial, Kathmandu, Nepal. E-mail: rpalpali@gmail.com

Background: The Nepal Tobacco (Control and Regulatory) Act of 2010 mandates government enforcement of at least $75 \%$ pictorial health warning (PHW) on tobacco packaging with a provision of the yearly rotation of the pictures and a required size. The enforcement and implementation of the $75 \%$ PHW was established successfully in 2014, which prompted the Ministry of Health and Population of Nepal to increase the size requirement to $90 \%$ in October 2014 with an enforcement date of 15th May 2015. However, enforcement of the $90 \%$ pictorial health warning still a challenge across the variety of tobacco products.

Methods: To build policy pressure and awareness, we applied various methods;

i) One to one interview with senior policy makers;

ii) interaction programs with senior government officials;

iii) market monitoring;

iv) disclosing tobacco industry interference.

Results: Implementation of the $90 \%$ pictorial health has been improved. Commitments of Ministry of Health and Ministry of Industry have been built. The Minister of Industry directed the industry to comply the law and regulation of tobacco control. Almost all media have been engaged in tobacco control with 
especial focus on implementation of pictorial health warning. More than 50 news articles related to pictorial health warning published in last six months with views of senior government officials and related stakeholders. They included Minister of Health, Minister of Industry, MPs, and senior officials and representatives from civil society and professional organizations. Pro-tobacco news were discouraged in most of the media. Industries meeting with health minister and other senior officials were disclosed.

Conclusions: The media's efforts significantly contributed in policy development and implementation of tobacco control in Nepal. These efforts need to be sustained.

Tob. Induc. Dis. 2018;16(Suppl 1):A626

DOI:10.18332/tid/84537

\section{4:00-15:30}

RF-1263-2 The North East of England successfully uniting as a region behind the campaign for the implementation of standardised packaging

A Rutter ${ }^{1}$, A Lloyd ${ }^{1}$, C Taylor ${ }^{1}$, L Surtees ${ }^{1}$

'Fresh, Durham, United Kingdom. E-mail: ailsa.rutter@freshne. com

Background and challenges to implementation: With tobacco advertising, sponsorship and promotion (2003) and point of sale displays (2015) prohibited, tobacco packaging had become the main source of promotion in the UK. Evidence shows that branding helps to recruit young people to a lifetime of addiction. A concerted national advocacy campaign on standardised packaging was undertaken from 2011 through the Smokefree Action Coalition (SFAC) with strong support by Fresh (North East England) and local authorities in the region.

Intervention or response: Fresh, along with colleagues in the NE, supported the campaign from its inception and responded in force to two governmental consultations with submissions from local authorities and in particular regulatory teams, the health service, the police and the voluntary sector. All NE authorities had robust policies in place to counter the efforts by the tobacco industry to derail progress towards implementation of the measure.

Results and lessons learnt: In 2015, the UK became the second country in the world to pass legislation on standardised packs with full compliance achieved by May 2017. The tobacco industry issued legal challenges and appeals to both the UK and the European Union, but they lost. Public support for the measure was $60 \%$ by February 2017. Early results from NE research shows that the measure is having an impact on smokers who had seen the packs, with $60 \%$ thinking they looked less attractive and $51 \%$ noticing the health warnings more. There is also no evidence to suggest that standard packs will lead to an increase in the illicit tobacco market.

Conclusions and key recommendations: Working through the SFAC ensured that there was a huge ground swell of support in the NE and scores of organisations were able to unite around an effective campaign. Collaboration internationally was also pivotal including the leadership from Australia. The introduction of standardised packaging is another key milestone in the NE ambition to Make Smoking History.

Tob. Induc. Dis. 2018;16(Suppl 1):A627

DOI:10.18332/tid/84247

$14: 00-15: 30$

RF-1264-2 International collaboration to build tobacco control capacity: a case study of KOMPLY from the World Heart Federation Emerging Leaders program
K Namusisi Nyamurungi', EN Achiri², A Oginni ${ }^{3}$, L Robertson ${ }^{4}$, S Gravely ${ }^{5}$, JC Rusatira ${ }^{6}$, M Huffman $^{7}$, S Yusuf ${ }^{8}$

${ }^{1}$ Center for Tobacco Control Africa, Kampala, Uganda, ${ }^{2}$ Framework Convention Alliance, Yaounde, Cameroon, ${ }^{3}$ Nigerian Heart Foundation, Lago, Nigeria, ${ }^{4}$ University of Otago, Dunedin, New Zealand, ${ }^{5}$ Waterloo University, Waterloo, ON, Canada, ${ }^{6} \mathrm{Healthy}$ People Rwanda, Kigali, Rwanda, ${ }^{7}$ Northwestern University, Chicago, IL, United States of America, ${ }^{8}$ McMaster University, Hamilton, ON, Canada. E-mail: nyamurungik@ctc-africa.org Background and challenges to implementation: Article 22 of the Framework Convention on Tobacco Control calls for collaboration among the Parties and international organizations to facilitate the development, transfer and acquisition of knowledge, skills, capacity and expertise related to tobacco control. International collaborations are especially important to help counter the tobacco epidemic in low and middle-income countries and will also contribute to UN Sustainable Development Goals. We will summarise the World Heart Federation (WHF) Emerging Leaders program, using the case study of the KOMPLY collaboration which focused on evaluating and supporting compliance with Uganda's newly implemented smoke-free legislation.

Intervention or response: As part of WHF's goal to reduce cardiovascular disease by $25 \%$ by 2025 , an Emerging Leaders program was initiated of which the 2016 focus was tobacco control. Twenty-five Emerging Leaders from across the globe were selected and attended a WHF think-tank. Participants received education, training, mentoring and the opportunity to apply for seed funding, to facilitate leadership and the development of a new collaborative tobacco control project.

Results and lessons learnt: In the 18 months following the thinktank, the KOMPLY team collected evidence that showed poor compliance with the smoke-free legislation in Ugandan hospitality venues (e.g. designated smoking areas were present, no-smoking signage was absent, hazardous levels of tobacco particulate matter in venues that allowed smoking). This evidence is being used by the Ugandan government to defend the 2015 Tobacco Control Act in response to litigation by British American Tobacco. Outputs produced include a factsheet and technical report for Ugandan stakeholders, academic articles and conference presentations. Team members established working relationships with individuals from key international tobacco control organisations, developed academic outputs, acquired new skills and opportunities for further professional development.

Conclusions and key recommendations: Initiatives such as WHF's Emerging Leaders program can make a substantial contribution to building capacity for tobacco control, through fostering international collaborations to increase leadership, research and advocacy efforts in LMICs.

Tob. Induc. Dis. 2018;16(Suppl 1):A628

DOI:10.18332/tid/84301

\section{4:00-15:30}

RF-1265-2 Using innovations to enable public monitoring and enhance enforcement of Beijing smoking control regulation

J Zhang ${ }^{1}, X$ Cui $^{1}, X$ Yin $^{2}, X \mathrm{Li}^{3},{ }^{3} \mathrm{Yu}^{3}$

${ }^{1}$ Beijing Tobacco Control Association, Beijing, China, ${ }^{2}$ Campaign for Tobacco-Free Kids, Washington, WA, United States of America, ${ }^{3}$ Campaign for Tobacco-Free Kids, Beijing, China. E-mail:1677828920@qq.com

Background and challenges to implementation: Beijing Smoking Control Regulation took effect in 2015. The regulation 
bans smoking in all indoor public places, work places and public transportations. Its unique feature is it encourages "social governance", enabling the public to play active roles in its implementation. The implementation has been particularly challenging in small- or middle-sized restaurants and office buildings. With over 21 million residents and only 2,000 enforcement officers in Beijing, the public needs to be engaged to ensure successful implementation.

Intervention or response: Beijing Tobacco Control Association (BTCA) developed Beijing Tobacco Control Map to enable public monitoring and timely reporting of violations. This GPS-enabled interactive map can be accessed from Beijing government's website and from WeChat, the most popular Chinese social media platform. The public can report violations through WeChat. The reports result in lights in different colors at a specific location on the Map based on the number of the reports. Volunteers can use the information to help the venue managers to comply and health inspectors can follow the lights to do targeted inspections.

Results and lessons learnt: Beijing Tobacco Control Map is a highlight in implementing the Beijing Tobacco Control Regulation. Using crowdsourcing technology and popular social media, this innovative approach provides a convenient channel for the public to participate in the implementation; it organizes volunteers to support venues in compliance and relieve enforcement officers' workload; it serves as a useful tool for the enforcement officers to be more effective and efficient.

Conclusions and key recommendations: Beijing Tobacco Control Map can be used in other cities and countries to promote effective implementation of tobacco control laws. Crowdsourcing and innovative technologies should be explored to enhance tobacco control efforts and other public health interventions. It can also strengthen the collaboration between the government, the civil society and the private sector as in Beijing.

Tob. Induc. Dis. 2018;16(Suppl 1):A629

\section{DOI: $10.18332 /$ tid/83893}

\section{2:30-14:00}

PS-944-2 Advocacy for mainstreaming and sustainable implementation of Tobacco Control Law in Bangladesh I Masud ${ }^{1}$

'Dhaka Ahsania Mission, Health, Dhaka, Bangladesh. E-mail: iq.masud@gmail.com

Background and challenges to implementation: The Government of Bangladesh enacted 'Smoking and Tobacco Products Usage (Control) Act, 2005' and formulated necessary rules in 2006. In 2013 enacted amendment to the law and formulated rules in 2015 .

Government has been tried to makes a comprehensive tobacco control (TC) law and almost cover all FCTC compliance issues beyond designated smoking area (DSA) at public places.

Police is the core law enforcement agency under the Ministry of Home Affairs. They have 17 training institutes. So mainstreaming and institutionalization of Tobacco Control Law will help to run the tobacco control program smoothly and bring good result with the involvement of Law Enforcement Agencies .

Intervention or response: Dhaka Ahsania Mission (DAM) as a BI grantee working with other BI grantees and government agencies. DAM organized different activity like- develop and finalize the different sectors policies through organize advocacy workshop with relevant authority. DAM also undertake extensive media campaigns different advocacy materials has developed and distributed.
Results and lessons learnt: Through different training and process of doing by learning has created enough rooms for development technical expertise for conducting result base advocacy, policy formulation, and smoke free guidelines development.

DAM intends to mainstream and sustain the Tobacco control law in different sectors through developing different implementation strategies and adaptation policies and build capacity. Peoples participation on tobacco control movement have been increased through organizing various campaign programs.

Conclusions and key recommendations: Some proactive policy makers and govt. officials in Dhaka were found who are actively participating different tobacco control activities and have taken initiatives for tobacco control. So we can use this opportunity for smoke free educational institutes. In future these coordination and collaboration among tobacco control organizations and other agencies will maintained for effective implementation of any tobacco control initiatives particularly for mainstreaming the tobacco control law in different sectors and campaign against TI tactics targeting the youth and students.

Tob. Induc. Dis. 2018;16(Suppl 1):A630

DOI: $10.18332 /$ tid/84360

12:30-14:00

PS-945-2 Correlates of MPOWER policies and tobacco smoking - an analysis of WHO South East Asia regional countries

S Kumar ${ }^{1}$, J Purushotham ${ }^{1}$, Nitte Tobacco Control Group ${ }^{1}$ Nitte University, Division of Public Health, Mangalore, India. E-mail:dr.sudeepkumar@nitte.edu.in

Background: One of the greatest threat to global health is the epidemic of tobacco. One third of world's population use tobacco and almost half of whom die prematurely. The WHO Framework Convention on Tobacco Control (WHO FCTC) brought out MPOWER package of interventions country-level implementation of effective measures to reduce the tobacco demand.

Methods: This study has en undertaken to determine the correlation between prevalence of tobacco smoking and existing tobacco control policy initiatives of selected South East Asia Regional Countries.A secondary data based correlation study where data on tobacco smoking prevalence and nations' compliance to tobacco control policies during the year 2013 were obtained from Tobacco Atlas 2013, 5th Edition. Data were analyzed using Pearson's Correlation Coefficient on SPSS version 16 . Results were considered significant at $95 \%$ level of significance.

Results: The study represented eleven countries of the WHO - South East Asia Region. Current smoking prevalence ranged between $2.8 \%$ and $39.8 \%$ with a mean prevalence of $20.5 \%$ ( \pm 9.67). As smoking prevalence among boys increased in these eleven countries, prevalence increased among adult males as well with a positive moderate correlation ( $\mathrm{r}=0.67$; $\mathrm{P}$ c0.05). Advertisement ban compliance negatively correlated with the prevalence of male smoking in the region ( $\mathrm{r}=-0.69$; $\mathrm{P} \varsigma 0.05)$. Total advertisement ban compliance correlated strongly with the indirect advertisement ban $(\mathrm{r}=0.78$; $\mathrm{P}$ 0.01$)$. Protection from smoke policies had a reduced effect on the male smoking prevalence ( $\mathrm{r}=-0.67$; $\mathrm{P} 0.05)$ in the region.

Conclusions: In this study, prevalence of tobacco smoking had a relationship with the major MPOWER policies such as total ban on tobacco advertisements and protection from smoke helps in reducing the prevalence. Hence, MPOWER policies contribute to reduce smoking prevalence in the WHO SEAR region. 


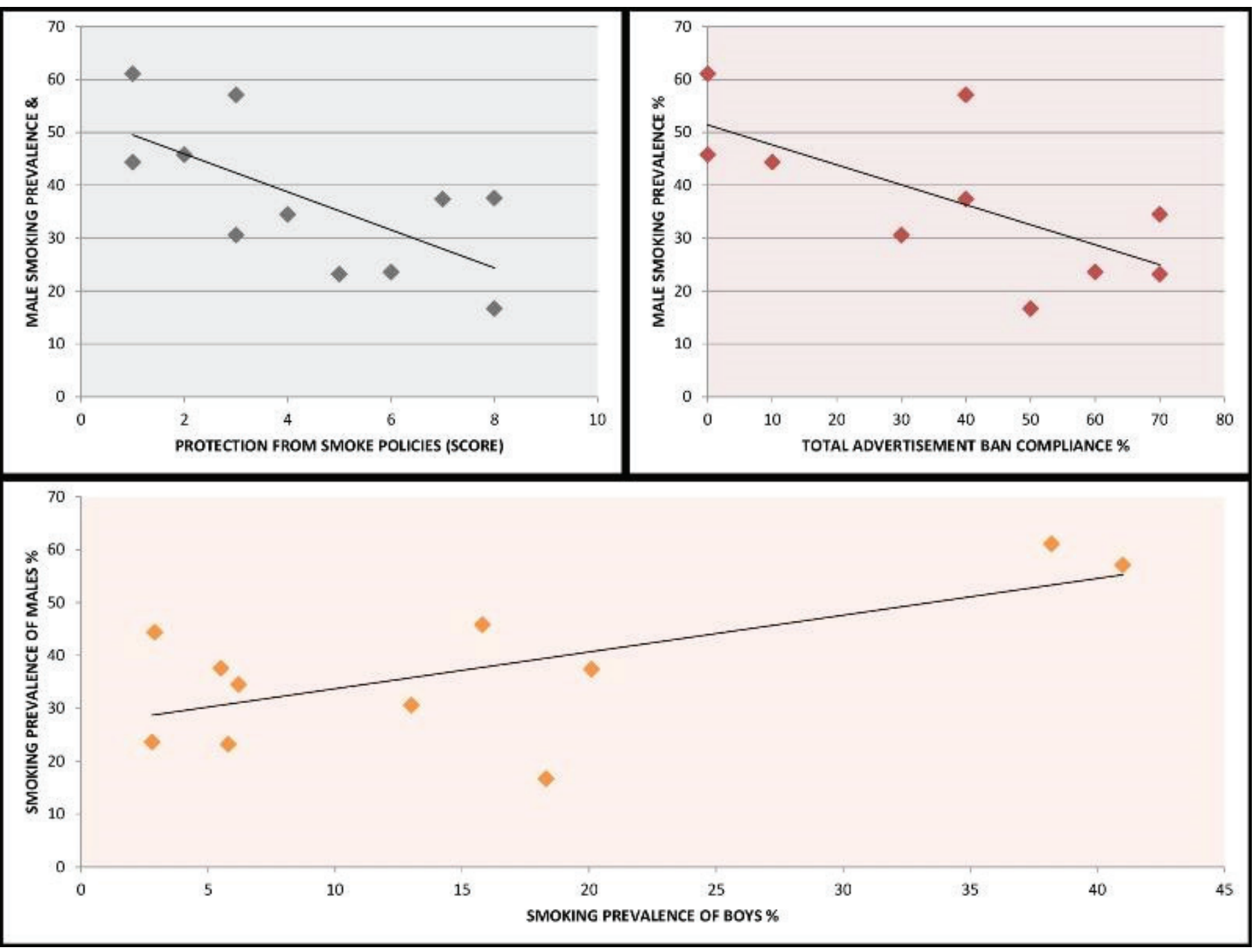

Tob. Induc. Dis. 2018;16(Suppl 1):A631

DOI: $10.18332 /$ tid/84374

[Tobacco Smoking Prevalence and MPOWER Policies]

\section{$12: 30-14: 00$}

PS-946-2 From press conferences to media dialogues, Cameroon learned to be more effective in relaying tobacco control messages

C Ayong ${ }^{1}$

${ }^{1}$ Cameroonian Coalition for Tobacco Control $\left(\mathrm{C}^{3} \mathrm{~T}\right)$, Yaounde, Cameroon.E-mail: ayongcaleb@gmail.com

Background and challenges to implementation: Cameroon ratified the World Health Organisation Framework Convention on Tobacco Control (FCTC) in 2006. Eleven years later, the country has still not adopted a national tobacco control bill, something the FCTC recommends be done three years into ratification.

Knowing how powerful the media is in relaying information to the general public and to decision makers, the Cameroonian Coalition for Tobacco Control (C3T) has over the past years equipped journalists with information that can lead to the adoption of better regulation and legislation of tobacco and its products in Cameroon.

Initially, C3T interacted with the media through Press Conferences. With time however, the coalition learned that it will communicate much more effectively if it transformed its Press Conferences to Media Dialogues. With such dialogues, there is the opportunity for both parties to discuss issues, contrary to Press Conferences where the journalists only receive information as it is given to them.

Intervention or response: C3T eventually switched from Press
Conferences to Media Dialogues. Instead of having a formal event full of protocol, journalists were introduced to a much more relaxed atmosphere where they could freely discuss tobacco control issues and crack a few jokes in the process.

Results and lessons learnt: The principal result of this approach is more engagement shown in tobacco control activities by the journalists. When they are given the opportunity to be part of the discussion of the problem, they themselves come up with innovative solutions; solutions that are not only very effective, but also feasible vis-a-vis the hurdles a journalist will normally face while discharging his/her duties.

Conclusions and key recommendations: It is absolutely necessary to engage the media in tobacco control discussions. If they are given the chance to actively participate in such discussions, they get more engaged in the subject and relay information (to decision makers and the general public) much more effectively.

Tob. Induc. Dis. 2018;16(Suppl 1):A632

DOI: $10.18332 /$ tid/84380

12:30-14:00

PS-947-2 Engaging health and non-health sectors to advance progress on tobacco control

RK Dwivedi', B Ramakant ${ }^{2}$, S Shukla ${ }^{3}$, DR Kant ${ }^{4}$

${ }^{1}$ Citizen News Service, Health and Communication, Lucknow, India, ${ }^{2}$ Citizen News Service, Policy and Programme, Lucknow, India, ${ }^{3}$ Citizen News Service, Editor, Lucknow, India, ${ }^{4}$ Piles To Smile Clinic, Medical Health, Lucknow, India. E-mail: rahul@ citizen-news.org 
Background and challenges to implementation: Despite towering disease and death burden attributed to tobacco use, and severe socio-economic implication thwarting progress on sustainable development, sectors other than tobacco control, seldom join hands for endgame of tobacco. We aspired to increase engagement of different health movements and groups as well as non-health groups to advance progress on tobacco control.

Intervention or response: Vote For Health campaign hosted by CNS and Asha Parivar, collaborated with government health agencies such as UP State Tobacco Control Cell, National Health Mission, National Programme for Prevention and Control of Cancer, Diabetes, Cardiovascular Diseases and Stroke (NPCDCS), Mental Health and initiatives of Skills and Entrepreneurship Ministry of Government of India; and NGOs such as Family Planning Association of India (FPA India), Hope Initiative, Society for Social Regeneration and Equity, National Alliance of People's Movements (NAPM), among others, to conduct a series of heath interventions like Rights and Responsibilities Trainings Programme, webinars, media dialogues, CSO meetings, media trainings, and sensitization programme at schools, paramedical institutions, nursing institutions, hospitals, workers at construction sites, slums and other public places etc; each month and during specific days such as World No Tobacco Day, World Health Day, World Heart Day, Word AIDS Day, World Pneumonia Day, World Tuberculosis Day etc.

Results and lessons learnt: Over 200 CSOs have extended their support to tobacco control initiatives facilitated by Vote For Health campaign. Several people's movements in other South Asian, South East Asian, African nations have also endorsed tobacco control initiatives hosted by us. We have generated more the 2000 news clippings in vernacular media reaching out to almost 6 million population.

Conclusions and key recommendations: United we stand and divided we fall - there is no other way to progress on tobacco control and SDGs but collectively. Partnerships are key to delivering on Agenda 2030.

Tob. Induc. Dis. 2018;16(Suppl 1):A633

DOI:10.18332/tid/84446

\section{2:30-14:00}

PS-948-2 Investigation report on public attitude towards carpet smoking ban in ten cities of China D Hu' ${ }^{1}, \mathrm{Y} \mathrm{Gao}^{1}$

${ }^{1}$ Chinese Association of Tobacco Control, Beijing, China. E-mail: dayi.hu@cacpr.org

Background and challenges to implementation: In November 24, 2014, the legal website of the China State Council published "the regulations on smoking control in public places (Draft)", and made public comments on the issue. The content of the manuscript is comprehensive and basically close to the FCTC's requirements. It is a relatively perfect tobacco control law and has been well obtained by domestic and oversea. However, as of 2016, the Regulations had not yet been promulgated. In order to understand the attitude of the public smoking ban, the "Regulations" to provide a complete ban on smoking in indoor public places, indoor workplaces, public transport, China Tobacco Control Association selected randomly 10000 people of the public and ask for investigation with questionnaire in ten provinces. CTCA provide these results of this investigation report as a reference to the Legal Affairs Office of the State Council, for constitute and promulgate the "Regulations".

Intervention or response: Ten cities which have been investigated was selected from ten provinces of 7 administrative regions. We investigate around 1000 people of the public in each city. Gender, age, educational background, smoking, and whether secondhand smoke is harmful to health, attitudes towards total smoking ban, satisfaction of smoke-free environment in public places, workplaces, public transport, etc..

Results and lessons learnt: We have surveyed 11523 people in 10 cities. The public awareness rate of harmful of the second-hand smoke was $95.2 \%$, support for a total ban on smoking in indoor public places, indoor workplaces and public transport rate of $91.9 \%$, the current smoke-free environment satisfaction of indoor public places, workplaces and public transport are 49.3\%, 64.5\% and $69 \%$ respectively.

Conclusions and key recommendations: The Chinese people has a strong awareness of the dangers of second-hand smoke, and has high support for total smoking ban of indoor public places, workplaces and public transport. China's comprehensive smoking ban in public places is ripe for settlement.

Tob. Induc. Dis. 2018;16(Suppl 1):A634

DOI:10.18332/tid/84462

\section{2:30-14:00}

PS-949-2 Getting involved: Uganda cancer society's efforts to build strategic partnerships for tobacco tax increments and strong tobacco industry monitoring E Angiji', P Ebusu'

1Uganda Cancer Society, Kampala, Uganda. E-mail: ebusup@ ugandacancersociety.org

Background and challenges to implementation: Uganda Cancer Society's (UCS) purpose is to coordinate civil society efforts for a systematic contribution towards effective cancer control in Uganda. UCS is moving towards fully integrating tobacco control in it activities. Globally tobacco is responsible for $20 \%$ of all cancer deaths. UCS recognizes that Uganda's Tobacco Control Act does not include tobacco taxation in its provisions yet high tobacco tax is proven as the most effective measure for demand reduction. UCS is aware that tobacco industry is heavily interfering with tobacco control in Uganda and has taken government to court challenging the Tobacco Control Act. Therefore, UCS is building strategic partnerships to foster local efforts in trying to push for higher tobacco taxes and stronger industry monitoring. UCS has joined the Prevent 20 global coalition of cancer organisations (wecanprevent20.org) and built partnership with Africa Centre for Tobacco Industry Monitoring and Policy Research (ATIM - www.atim.co.za).

Intervention or response: UCS approaches its advocacy through the Power Prism framework utilizing the six power tools. UCS media advocacy looks at engaging the public through opinion pieces published on local media and posted on social media platforms as a way to get government attention. UCS currently monitors industry interference through media surveillance geared towards understanding the nature and focus of tobacco related content.

Results and lessons learnt: UCS recent opinion piece on New Vision - "Tobacco's cost to our country", as of 21st July, 2017 reached 10,057 views, generating over 30 reactions including shares, and over 200 clicks. Preliminary assessment of media posts indicates that a majority of media content favors the tobacco industry and most of it is in line with legislative interference.

Conclusions and key recommendations: UCS believes that its efforts will push government to adopt higher tobacco taxes and establish mechanisms such as a strict code of conduct to deter government officials from working with tobacco industry. 
Tob. Induc. Dis. 2018; 16(Suppl 1):A635

DOI: $10.18332 /$ tid $/ 84466$

12:30-14:00

PS-950-2 Change in knowledge of tobacco use and secondhand smoke exposure among health workers in Uganda

H Mamudu ${ }^{1}$, K Namusisi ${ }^{2}$, W Bazeyo ${ }^{3}$, Y Olando ${ }^{4}$, J Surabhi ${ }^{5}$, F Makumbi $^{3}$, R Pack$^{1}$, E Rutebemberwa $^{3}$

'East Tennessee State University, Johnson City, TN, United States of America, ${ }^{2}$ Center for Tobacco Control in Africa (CTCA), Kampala, Uganda, ${ }^{3}$ Makerere University School of Public Health, Kampala, Uganda, ${ }^{4}$ World Health Organisation - AFRO, Brazzaville, Congo, ${ }^{5}$ World Health Organisation HO, Geneva, Switzerland. E-mail: mamudu@etsu.edu

Background: Tobacco use exacerbates diseases, including tuberculosis (TB) and interferes with recovery from such outcomes. However, there is sparse research on the integration of tobacco cessation into TB programs. Moreover, there is limited evidence on how mHealth solutions for tobacco can enhance cessation among TB patients. This study aimed to assess the impact of a training program to integrate tobacco cessation in TB program on the knowledge of health workers.

Methods: In June 2017, a 5-day training about tobacco use and control and the use of mHealth solutions to improve tobacco cessation and enhance adherence to TB treatment was conducted in Uganda. A comparison of percent of participants reporting knowledge on selected health outcomes of tobacco use and secondhand tobacco smoke (SHS) exposure was conducted. Knowledge was assessed on a 21-outcome-item before and after training. A non-parametric test, signrank for comparison of paired observations was conducted. The changes were considered statistically significant if the p-value was less than $5 \%$.

Results: Twenty three trainees from across the country attended (13 females, 10 males), with median age of the trainees was 39 years. Pre-training knowledge about tobacco use (66.6\%) was higher than SHS exposure (45\%). Following the training, both sets of knowledge significantly improved (median 100\%). Pre-training knowledge about health effects of tobacco use was particularly low for diabetes (27\%), meningitis (9.5\%), ear infection (43\%), impotence $(47.6 \%)$, and fibrosis (30\%). Except heart attack (76\%), lung illness among children (91\%), lung cancer (81\%), and chronic lung disease $(81 \%)$, pre-training knowledge about SHS was low for all other disease outcomes.

Conclusions: Healthcare providers play critical role in preventing and reducing tobacco use. The low pre-training knowledge of the TB health workers suggests the critical need for training health providers in Uganda and elsewhere in Africa in order to curtail the increasing trend in usage.

Tob. Induc. Dis. 2018;16(Suppl 1):A636

DOI:10.18332/tid/84518

\section{2:30-14:00}

PS-951-2 Strengthening National Tobacco Control Program (NTCP) to advance tobacco control (TC) policy in Uttar Pradesh (UP)

R Ranjan1, S Jain²

'Voluntary Health Association of India, New Delhi, India, ${ }^{2}$ Voluntary Health Association of India, Delhi, India. E-mail: rranjan@vhai.org

Background and challenges to implementation: Government of India launched NTCP in 2007-08 to create awareness about ill-effects of tobacco, enforcement of Cigarette and other Tobacco
Product Act (COTPA) and regulate tobacco consumption. It was launched in two districts of U.P. in 2011 and has gradually been increased to cover all the 75 districts.

The State Tobacco Control Cell (STCC) is responsible for overall planning, implementation and monitoring of different activities, and achievement of physical and financial targets planned in State programme.

Voluntary Health Association of India (VHAI) initiated work in tandem with STCC to remove bottlenecks, namely :

Low priority of TC

Lack of awareness on COTPA amongst bureaucrats of key departments

Inactive Inter-departmental Coordination

Ineffective and inadequate mass media campaign

Intervention or response: To initiate inter-departmental dialogue and coordination VHAI team facilitated constitution and meeting of a Working Group under the Chairmanship of Director GeneralHealth with representatives from departments like Food\&Drug, Police, Education, Municipal Corporation, Panchayati Raj, NGOs, Medical Colleges etc. VHAI worked in tandem with STCC and provided technical support in training of stakeholders, including District Staff of NTCP (District Coordinators, Social Worker and Psychologist). Technical expertise was also extended to the District Tobacco Control Cell of 6 cities for their on-going and upcoming activities. A mass media strategy was designed for NTCP and sample designs of Resource Kit, Poster, COTPA booklet etc. were shared. High level commitment was sought from political leaders as well as media houses.

Results and lessons learnt: An inter-departmental working group is now active that coordinates and streamlines enforcement of COTPA. IEC material designed by VHAI has been appreciated and adopted by the state and STCC has recognized VHAI as a technical support agency for future.

Conclusions and key recommendations: Providing technical assistance to strengthen NTCP and address gap areas of STCC has improved impact of TC initiatives of Health and key departments.

Tob. Induc. Dis. 2018;16(Suppl 1):A637

DOI:10.18332/tid/84527

12:30-14:00

PS-952-2 How we won the sin tax law: the Philippine sin tax story in social media messages

JJ Yparraguirre

${ }^{1}$ Action for Economic Reforms, Quezon City, Philippines. E-mail: jjyparraguirre@gmail.com

Background: Recognizing social media as a potent tool in lobbying for tobacco control policies, this paper analyzes the Philippines' success in enacting the 2012 Sin Tax Reform Law and the social media strategies that accompanied its passage.

The study aims to demonstrate successful messaging strategies that ultimately pushed the Philippine Sin Tax Reform to be passed into law. Specifically, this paper extracted core messages from original digital advocacy materials (social media posts, videos, etc) and compared them against

(1) information picked up by traditional media (e.g. news articles) and

(2) salient arguments in favor of the sin tax reform during legislative sessions.

Methods: Employing thematic analysis aided by qualitative research software Atlas.ti, the study analyzes social media messages of the Bawas Bisyo Facebook page (the main social media outlet of the Philippine sin tax advocacy) from October 
2012 to January 2013. This is the period during and immediately after the intensive online campaign for the passage of House Bill (HB) 5727. The core messages are then compared against top news articles captured by Google News with the keywords "Philippine sin tax law" within the same time frame.

Results: To date, the salient messages are:

(1) clamor for universal healthcare funded by tobacco tax,

(2) exposing tobacco industry interference through fabricated claims by pro-tobacco legislators and journalists,

(3) government experiencing increased revenue due to higher tobacco tax, and

(4) demand for irrefutable proof that a tobacco tax reform will ensure lowered smoking prevalence.

Conclusions: The aforementioned messages are the parts of a communication strategy that was successfully captured by traditional media and the legislative sphere. For a country able to hurdle reforms against a deeply-entrenched tobacco lobby, the Philippine example could serve as a reference to other groups seeking to replicate the same success with their own tobacco tax legislation.

Tob. Induc. Dis. 2018;16(Suppl 1):A638

DOI:10.18332/tid/84532

\section{$12: 30-14: 00$}

PS-953-2 Paving way for tobacco free schools towards building leadership and broader alliances for implementing tobacco control policy

A Rathinam ${ }^{1}$, A Savariyar ${ }^{1}$

'Pasumai Thaayagam Foundation, Chennai, India. E-mail: mailtopt@gmail.com

Background: Strong empirical evidence indicates that students are easily lured to the addiction of tobacco product use. FCTC Article 16 requires for implementation measures prohibiting sales of tobacco products to minors, display indicators about the prohibition at the point of sale and no visible display of tobacco products. Indian law COTPA (The Cigarettes and Other Tobacco Products Act, 2003) Section 6 prescribes ban on sale to and by minors, and bans sale of tobacco products within a radius of 100 yards of educational institutions. This is violated and tobacco products are sold near educational institutions.

Methods: Pasumai Thaayagam Foundation conducted a field study in March 2017, on compliance/violation of COTPA Section 6 in Chennai. Six wards were selected as random samples. The authors shared field tested questionnaire and gave orientation given to the field study team. 60 samples were included for the study in the three different areas. The study looked into the aspects of compliance of COTPA Section 6 and also patterns of violations were compiled with evidences.

Results: Result revealed that there was no display of mandatory warning board stating" "sale of tobacco product prohibited here". Tobacco products in violation of COTPA Section 6 were being sold near 58 education institutions out of the studied 60 . There was no display of statutory warning board that "smoking here is an offence." Both chewing form of tobacco and smoking form of tobacco products were available near those institutions. They were placed lucratively and conspicuously that could easily attract the children. Findings were used for informational briefing and advocacy with authorities of those institutions, and with health and education departments.

Conclusions: For ensuring stringent implementation of tobacco control policies broader alliance building is essential. For which tapping and documenting the lapses need to be scientific and evidence based.
Tob. Induc. Dis. 2018;16(Suppl 1):A639
DOI:10.18332/tid/84547

12:30-14:00

PS-954-2 Tobacco Free Network - the actions of tobacco users and survivors: advocacy on enforcing tobacco control legislation and behavioural change in Tamil Nadu, India

J Jasper ${ }^{1}$, Tobacco Free Network

TTobacco Free Network, Virudhunagar, India.E-mail:johnjasper@ gmail.com

Background and challenges to implementation: Tobacco use is a major public health challenge in India with 275 million adults consuming different tobacco products, of which 28,064,400 people above the age of $15 \mathrm{y} / \mathrm{o}$ are in Tamil Nadu. Chewable tobacco is the most prevalent - India carries the highest amount of mouth cancer cases in the world and it is more common among women. India passed a ban on chewable tobacco in September 2016 but the implementation lacks enforcement. $90,3 \%$ of smokeless tobacco consumers reported no difficulty in purchasing banned chewable tobacco in Tamil Nadu. The Government of India has passed legislation for tobacco control but it is not rigorously implemented. This is attributed to a lack of awareness and penalties. For this reason advocacy, community-based action and social mobilisation are required.

Intervention or response: We have mobilised tobacco users and survivors throughout Tamil Nadu and formed a strong network for tobacco control. We equipped them with the skills for advocacy at the top level for sensitizing authorities and government officials. This was also enforced through advocacy in the media.

Results and lessons learnt: From January'16 until now we have mobilised 645 members. They are creating a domino-effect in their communities and at the higher level by spreading awareness about tobacco control legislation and the need for proper implementation. Organisations from the public and private sectors, as well as the media, are used as advocacy leverage because they provide a leeway into government officials which we can influence enforce the tobacco control legislation.

Conclusions and key recommendations: The network of tobacco users and survivors is a powerful means to sensitize health authorities and government officials and to advocate for the enforcement tobacco control legislation at the local, state and national level.

Tob. Induc. Dis. 2018;16(Suppl 1):A640

DOI:10.18332/tid/84554

12:30-14:00

PS-955-2 Contested evidence, contested policy? Evidence use in the development of Scottish e-cigarette policy

T Ikegwuonu', S Hilton', H Weishaar², K Smith ${ }^{3}$, J Collin ${ }^{4}$ 'University of Glasgow, Glasgow, United Kingdom, ${ }^{2}$ Hertie School of Governance, Berlin, Germany, ${ }^{3}$ University of Edinburgh, Edinburgh, United Kingdom, ${ }^{4}$ University of Edinburgh, School of Social and Political Science, Edinburgh, United Kingdom. E-mail: jeff.collin@ed.ac.uk

Background: Electronic cigarettes (e-cigarettes) have become subject to highly contested public and political debates regarding approaches to regulation and marketing, which relate to the lack of evidence regarding their potential benefits and harms. This is a common scenario for new technologies, though one in which the evolving role of the tobacco industry has increased anxiety. Whereas evidence is still unclear, proponents of e-cigarettes see them as a unique way of helping smokers to quit, while opponents 
underline the dangers in re-normalisation and e-cigarettes being a gateway into the use of traditional cigarettes.

Methods: Taking the Scottish context as a case study, this project aimed to increase understanding of how different actors' are responding to the uncertainties and evidence gaps in regulatory debates about e-cigarettes. The broader project employed a mixedmethod approach to investigate organisational actors' position on, and claims about, the benefits and harms of e-cigarettes, combining thematic analysis of policy documents $(\mathrm{N}=157)$ and semi-structured interview data $(\mathrm{N}=25)$.

Results: Focusing mainly on the interview data, we examined how actors taking differing positions in debates about e-cigarette regulation each work to promote the validity of evidence/ interpretations underpinning their positions while discrediting more challenging evidence/interpretations, highlighting the translation between research findings and media engagement as a critical moment of contestation. We argue that, contrary to several participants' apparent surprise at the absence of 'reasoned debate', the deep division that has emerged around e-cigarette regulation in tobacco control research represents a core part of 'science in the making' (Latour, 1988). As such, the presentation sheds light on why research-based discussions about e-cigarettes have become so divisive and outlines likely future scenarios.

Conclusions: In this paper, we focus specifically on how actors presented available evidence and evidence-gaps and how this appeared to both inform, and be informed by, the conclusions they reached regarding the regulation of e-cigarettes.

Tob. Induc. Dis. 2018;16(Suppl 1):A641

DOI:10.18332/tid/84555

\section{$12: 30-14: 00$}

PS-956-2 Collective action for effective tobacco control: one province at a time

S Dhage ${ }^{1}$

'Government of Karnataka, Police, Bengaluru, India. E-mail: ssdhage2738@gmail.com

Background and challenges to implementation: Daily, nearly 5500 adolescents experiment with tobacco use in India. Tobacco use leads to many ailments including psychological/emotional distress and higher risk of addiction into other drug use (alcohol, marijuana, cocaine). Being the Superintendent of Police of a province called Gadag in a southern Indian state of Karnataka I felt it was my responsibility to ensure a safe and healthy province. Intervention or response: Tobacco control requires coordination among several stakeholders. The following steps were followed:

Letters sent to 64 government departments in Gadag about initiating a campaign to make Gadag the first COTPA (Cigarettes and other tobacco products act) compliant province in India

- Letter to all major private associations in the province like hotels, bars, tobacco retailers, transport companies etc.

Joint meeting of all stakeholders and sensitization about the law

Setting timelines and providing stakeholders an opportunity to comply with the law

Public notices via print and electronic media

Enforcement drives to penalize non-compliant establishments/ individuals after completion of deadlines

Review and compilation of department implementation details Incorporation into monthly reporting and review mechanisms Results and lessons learnt: This intensive campaign lasted for 3 months resulting in significant decline in sales of tobacco products and a very high level of awareness throughout Gadag on the harms of tobacco usage. The sales data from the retailers showed a drop to one third of the pre-campaign levels. The lessons learnt include:

A province is the best suited unit to initiate tobacco control activities Intersectoral action and coordination is possible and is a must for effective tobacco control

Conclusions and key recommendations: What started with one province Gadag has now been replicated in 23 other provinces throughout Karnataka. COTPA compliance or comprehensive tobacco control is achievable, replicable and will aid in building momentum for endgame of tobacco in India

Tob. Induc. Dis. 2018;16(Suppl 1):A642

DOI:10.18332/tid/84562

12:30-14:00

PS-957-2 Media as advocacy tool to prioritize tobacco control as component of public health policy in a state with high penetration of other substances of abuse

P Duggal ${ }^{1,2}$, A Dhawan $^{3}$, RS Bhullar ${ }^{3}$

'Swift Hospital, ENT \& Head Neck Surgery, Amritsar, India, ${ }^{2}$ Voice of Tobacco Victims, Patron Punjab, Amritsar, India, ${ }^{3}$ Sri Guru Ram Das Dental Institute, Oral \& Maxillo-Facial Surgery, Amritsar, India.E-mail: duggalprahlad@yahoo.co.in

Background and challenges to implementation: India is a country with large population and varying social structure wherein the perceived importance of tobacco control amongst public health policy makers is quite variable in different states. North Indian state of Punjab with a population of $>27$ million is facing an 'epidemic' of drug abuse with 836 drug users per 100,000 which is three times the national average. Till few years back, tobacco control was a non-issue for the policy makers in Punjab who were ignoring importance of tobacco as gateway substance and need to curb its use.

Intervention or response: Tobacco control (TC) was an unimportant thing in public health policy in Punjab till VoTV campaign stated in 2012. Late 2013, drastic cut in VAT (Value Added Tax) on Cigarettes by Punjab government provided VoTV a window to sensitize media about importance of TC in the state. VoTV effectively used all forms of media in naming and shaming government of the day which proved crucial as is clear from difference in number of policy decisions for TC taken by Punjab government in pre \& post VoTV eras.

Results and lessons learnt: A quantitative assessment of the tobacco related media reports and timeline for the number of Key Events and Good Practices Notifications for effective TC in the state from 2012 onwards which shows statistically significant year on year increase in such reports, events and notifications in Punjab (Statistically significant). WHO regional director's appreciation award for Punjab in 2015 is a testimonial for robust TC machinery in Punjab.

Conclusions and key recommendations: VoTV campaign in Punjab has demonstrated effectiveness of media as a tool in prioritizing tobacco control amongst public health policy makers despite rampant drug abuse being so rampant in the state thereby putting forward a first of its kind model of tobacco control advocacy in populations with high penetration of other substances of abuse.

Tob. Induc. Dis. 2018;16(Suppl 1):A643

DOI: $10.18332 /$ tid/84577

$12: 30-14: 00$

PS-958-2 Institutionalization of Indian Tobacco Control Programme at Grassroot level - experiences 
from state of Jharkhand in India. (Enabling institutional structure is key to sustained results) M Ranjan', DK Mishra ${ }^{2}$, A Pandey ${ }^{3}$

${ }^{1}$ Government of Jharkhand, Khunti, India, ${ }^{2}$ Socio Economic and Educational Development Society (SEEDS), New Delhi, India, ${ }^{3}$ International Union Against Tuberculosis and Lung Disease, Tobacco Control, New Delhi, India. E-mail: manishranjanias@ gmail.com

Background and challenges to implementation: In Jharkhand, prevalence of tobacco is a serious concern as its $50.01 \%(n=19.5$ million) of adults are edicted to tobacco as against national average of $34.6 \%$ as per GATS 2010. Government of India launched National Tobacco Control Programme (NTCP) IN 2010-11 in two districts of Jharkhand i.e. Dhanbad \& East Singhbhum as pilot basis. Enforcement of Indian tobacco control law, capacity building, awareness generation, school health programs and cessation are main components of NTCP.

Currently Jharkhand State Tobacco Control Cell in technical collaboration with SEEDS and The Union is implementing a project on tobacco control in 6 districts of state.

Intervention or response: Several effective strategic interventions were undertaken. All primarily revolved around institutionalization of efforts, value systems, management practices and administrative structures. Institutional framework was developed through four pronged strategies i.e. intense advocacy, capacity building \& follow up with government officials, effective monitoring at state and district level and consistent media mobilization without losing out to capture even a smaller event related to tobacco control issues.

Collaborative and systematic efforts were made. Meetings / workshops / trainings and brainstorming sessions were conducted in collaboration with the District Administration.

Results and lessons learnt: These resultes in sensitisation of program managers, law enforcers and media resulting in noticablely high commitment to the cause. Innovatively, formation of antitobacco squads, inter-departmental State and Districts Tobacco Control Coordination Committees' meetings, enforcement reviews in monthly crime review meetings were conducted. This provided distinctive outcomes:

- Appointment of nodal officers at- state level

- Appointment of three nodal officers in each district,

- Synergy between efforts of health, police and general administration,

- Review by Deputy Commissioners in their monthly meetings of all departments

Conclusions and key recommendations: It is established that successful enforcement and implementation is achieved only through well-established institutionalization of tobacco control within existing system. Enabling Institutional Structure is indeed Key to Sustained Results.

Tob. Induc. Dis. 2018;16(Suppl 1):A644

DOI:10.18332/tid/84584

\section{$12: 30-14: 00$}

PS-960-2 Dental care education in the treatment of addictions

S Marques', M Santos', I Silva', E Jorge', Grupo Programa Estadual Controle Tabagismo -SP

'Secretaria Estadual de Saúde, CRATOD, São Paulo, SP, Brazil. E-mail: drasandramarques@gmail.com

Background and challenges to implementation: Aim: To describe the implementation continuing education course program in oral health care in Substance Use Disorders (SUD). Intervention or response:

The program is based on:

1. How to properly integrate knowledge on oral health care addictions treatment in primary and secondary care;

2. Improvements in clinical practice through the identification and adequate treatment oral pathologies related to SUD.

The trainings are not restricted to dentists that work in public services. Lessons are made available through videos, and the program usually initiates in September 2017. It comprises a total of 8 hours of study, divided across 4 modules of 2 hours each. The modules are divided in:

1. The neurobiology of addictions, and the main characteristics of the substances used;

2. Oral health care and psychiatric comorbidities in SUD;

3. Anamnesis in SUD, possible improvements in preventive care, and the basis of pharmacotherapy of SUD;

4. Motivational interviewing and patient follow-up.

Results and lessons learnt: It is available to more than 86,000 dental surgeons in the State of Sao Paulo. These represent a third of the Brazilian dentists. In addition, more than 30,000 other oral health professionals benefit from the program, including technicians and auxiliary personnel.

Conclusions and key recommendations: The continuing education course program in oral health care in SUD is intended to increase the knowledge of SUD amongst the oral health care professionals in both primary and secondary care. The adequate implementation of the program will now enable further studies to evaluate the impact of this educational intervention in public health. We hypothesize that the program will enable not only improvements in oral health care, but also in the adherence to treatment and in the general outcomes.

Tob. Induc. Dis. 2018;16(Suppl 1):A645

DOI: $10.18332 /$ tid/84657

\section{2:30-14:00}

PS-961-2 Reflections on 50 years as an antismoking activist: the danger of the single narrative A Blum'

'University of Alabama, Center from the Study of Tobacco and Society, Tuscaloosa, AL, United States of America. E-mail: ablum@ua.edu

Background and challenges to implementation: Since writing his first article on smoking as a high school newspaper editor in 1964, Dr. Blum has presented over 2000 invited lectures on tobacco in all 50 US states and 18 other countries, including 45 presentations at 11 world conferences. In 1977, he founded the first physicians' anti-smoking activist organization DOC (Doctors Ought to Care), which pioneered in purchasing counteradvertising space in the mass media that ridiculed tobacco companies and cigarette brands. In the 1980s as editor of the New York State Journal of Medicine and the Medical Journal of Australia, he produced the first three theme issues on the world tobacco pandemic at any journal.

Intervention or response: Although DOC closed its national office in 2002, several of its 80 chapters remain at medical schools as an extracurricular activity. At its peak, DOC had 5000 physician and medical student members, many of whom have become leaders in tobacco control. Entirely dues-supported, DOC was not dependent on government or foundation funding. This independence enabled DOC to confront the tobacco industry and its allies in ways that government and voluntary health agencies 
alike were fearful of doing.

Results and lessons learnt: Tobacco control has become formulaic and increasingly reliant on government-led efforts that tend to be overly cautious. Even half a century after the landmark scientific reports confirming cigarette smoking's irredeemable harmfulness, the bulk of funding for tobacco control is devoted to research on the health consequences of smoking. There is insufficient prioritization, division of responsibilities, and accountability. Above all, there is a static herd mentality in tobacco control in contrast to the dynamic of the tobacco industry.

Conclusions and key recommendations: The single narrative of tobacco control, wherein research-based policy leading to legislation and regulation, contrasts with the grassroots activist approach that laid the foundation for today's generation of tobacco control professionals. Such activism needs to be studied and revived.

Tob. Induc. Dis. 2018;16(Suppl 1):A646

DOI:10.18332/tid/84676

\section{$12: 30-14: 00$}

PS-962-2 Plaidoyer pour l'adoption d'une loi forte en Mauritanie par la mobilisation des communautés et des parlementaires

S Boly', Membres de I' association Mauritanienne de lutte contre la Tuberculose, le Tabac et le SIDA (AMALUTS) et L'OMS/ Mauritanie

'ONG/AMALUTS, KSAR, Nouakchott, Mauritania. E-mail: sinnabolly@yahoo.fr

Background and challenges to implementation: II. Prévalence Population générale L'enquête STEP Wise sur les maladies non transmissibles de 2006 en Mauritanie montre:

$17,8 \%$ des hommes et femmes sont des fumeurs quotidiens . $32 \%$ d'hommes et $4,8 \%$ de femmes.

$16,8 \%$ (15-24 ans) et

$22 \%$ (25 et 34 ans)

Prévalence Population Jeune: Les L'Enquête des jeunes en milieu scolaire (GYTS) en 2002 a mis en exergue un taux de prévalence de 64\%(l'un des plus élevé en Afrique). Le GYTS en 2007 Exposés à la fumée des autres dans les lieux publics :50.9 (46.2 - 55.6) Pourcentage de sujets pensant que fumer devrait être interdit dans les lieux publics 58.7 (55.3 - 62.0)

Intervention or response:

- Réalisation d'une enquête pour doter les structures intervenant dans la lutte contre le tabagisme d 'une base de données fiables sur les circuits de commercialisation du tabac sous toutes ses formes et de permettre en plus du taux de prévalence élevé chez les jeunes de faire un bon plaidoyer Mobilisation des communautés par le renforcement des capacités des acteurs de la société civile, des journalistes

Mobilisation des parlementaires et des leaders religieux

- Sensibilisation des décideurs sur les méfaits delaconsommation du tabac en utilisant les données des enquêtes existantes.

Results and lessons learnt:

- Ratification de la CCLAT en 2005

- Mise en place d'un Programme National de lutte contre le tabagisme

- Adoption du projet de loi antitabac par le gouvernement en 2012

- Existence d'un réseau de parlementaire antitabac dynamique Existence d'un réseau de journaliste antitabac

Conclusions and key recommendations:

Intensifiér le plaidoyer Elargir la sensibilisation à toute la population Renouveler les enquêtes qui sont devenues obsolètes
Tob. Induc. Dis. 2018;16(Suppl 1):A647

DOI:10.18332/tid/84685

12:45-14:00

EP-194-2 Harnessing youth power in making Myanmar tobacco-free

MM Cho'

'Southeast Asia Tobacco Control Alliance, Sustainable Financing, Bangkok, Thailand. E-mail: maychodr@gmail.com

Background and challenges to implementation: Tobacco consumption in Myanmar is high, with an alarming $44 \%$ of adult men and $8 \%$ of women smoking. Smokeless tobacco use among both men and women in Myanmar is also the highest among ASEAN countries. Despite the adoption of The Control of Smoking and Consumption of Tobacco Product Law in May 2006 which bans tobacco advertising and sponsorship, compliance is still low. This presentation will highlight the activities of a youth network, 'Mnet' in working towards a tobacco free generation in Myanmar.

Intervention or response: Myanmar's University of Public Health, in collaboration with Southeast Asia Tobacco Control Alliance, established a youth network called MNet in 2016. Initially, the network consists of students from medical universities, public health and community health faculties with the aim to expand to other youths from all over Myanmar. This youth network aims to monitor and expose tobacco industry violations of the law, advocate strong enforcement mechanisms and promote the youth's voice for a tobacco free generation.

Results and lessons learnt: MNET found the tobacco industry, particularly international companies, were found to circumvent the national tobacco control law, especially the ban on tobacco advertising, promotion and sponsorship. Violations, such as promotion girls, were more prevalent in the urban areas especially in the capital city, Yangon. Mnet was able to draw youth form various parts to monitor the evidence and pass them on to policy makers for enforcement action. Since there are many youths over a wide area, they can devise mechanisms of monitoring and conduct effective advocacy for efficient enforcement.

Conclusions and key recommendations: Tobacco companies contravene the law by taking advantage of a developing country with limited resources in enforcement. Civil society, especially the youth, when organized into a network can play a crucial role in exposing the industry and assisting the government towards achieving a smoke-free society.

Tob. Induc. Dis. 2018;16(Suppl 1):A648

DOI:10.18332/tid/83868

\section{$12: 45-14: 00$}

EP-195-2 Overcoming stumbling blocks of tobacco control in Bangladesh

SM Mahbubus Sobhan'1, R Quader'1, M Ruhul Quddus', SM Alam², I Chowdhury ${ }^{3}$

${ }^{1}$ Ministry of Health and Family Welfare, Government of Bangladesh, National Tobacco Control Cell, Dhaka, Bangladesh, ${ }^{2}$ The Union South-East Asia, Tobacco Control, Dhaka, Bangladesh, ${ }^{3}$ Ministry of Civil Aviation and Tourism, Government of Bangladesh, Dhaka, Bangladesh. E-mail: smms_lincoln@ yahoo.com

Background and challenges to implementation: Tobacco leads to grave detriments on health. Bangladesh is among top ten countries of the world where tobacco use is most prevalent. It is facing tobacco epidemic, having dual burden of high production and consumption. It is a cumbersome task to curb the epidemic 
in a country like us. Having an excellent tobacco control law, lack of execution, makes it utterly insufficient. The deep concern is exposure to tobacco causing public health disaster for citizens, who have a universal right to governmental protection.

Intervention or response: National Tobacco Control Cell (NTCC) of Ministry of Health and Family Welfare (MOHFW) worked with NGOs, civil society, religious and community leaders in concerted effort for awareness on hazards of tobacco, Tobacco Control law and implementation of law by awareness campaign, engagement of print, electronic and social media, use of IEC materials and advocacy. Repeated directions sent from MOHFW to field administrations to activate taskforces for tobacco control. Various government agencies including Executive Magistrates, NGO and other stakeholders were trained on law and mobile court drives were operated across the country for exemplary punitive action. To decrease secondhand smoke, mangers of workplace, public place and transports were made accountable. Tax raised on all tobacco products. Banks gave soft loans for tobacco farmers to switchover to other crops. Tobacco advertisement, promotion and sponsorship were banned.

Results and lessons learnt: Exemplary penalty made people law abiding. Mobile court drives received tremendous support from the people from all walks of life and mass media which contributed in creating awareness. Coverage of clean-indoor-air law is increased. Tobacco control coalition developed from grass root to highest level. Increasing tax on tobacco found effective to discourage smoking initiation, moreover, stopped illicit trade. Advocacy through different media including internet found very useful.

Conclusions and key recommendations: More novel approaches are needed like tobacco cessation. Emphasis required for monitoring the activities of taskforces.

Tob. Induc. Dis. 2018;16(Suppl 1):A649

DOI:10.18332/tid/84609

\section{$12: 45-14: 00$}

EP-196-2 Community of practice on tobacco control to health inspectors of Brazilian Sanitary Surveillance System: a capacity-building strategy to strengthen tobacco control policies in Brazil

M Moreno dos Reis ${ }^{1}$, S Turci ${ }^{1}$, D Barata

${ }^{1}$ Oswaldo Cruz Foundation - Fiocruz, Center for Studies on Tobacco or Health, National School of Public Health Sergio Arouca, Rio de Janeiro,RJ, Brazil.E-mail:mmoreno@fiocruz.br Background and challenges to implementation: Brazil ratified the World Health Organization Framework Convention on Tobacco Control in 2005. Regulation of tobacco products, promotion of smoke-free environments (SFE), and actions related to advertising, promotion and sponsorship (TAPS) of tobacco products are under Brazilian Sanitary Surveillance System (SNVS) responsibilities. Since Brazil has a large territorial extension, including 5,570 municipalities and heterogeneous structure in local health surveillance services, the need to strengthen knowledge and improve health inspectors' skills for the verification and monitoring of regulations related to tobacco control is a challenge.

Intervention or response: A community of practice in the form of distance learning was identified as a strategy capable of strengthening tobacco control policies in Brazil. Community of practice is a learning space that facilitates the sharing of experiences among participants on topics of their interests. The virtual platform was developed by Oswaldo Cruz Foundation, in partnership with Brazilian Health Regulatory Agency and with the support of The Union. It was structured in 5 modules: Tobacco
Epidemic; Inspection routine; TAPS, and SFE.

Results and lessons learnt: Training program was offered three times during December 2015 and December 2016. It was attended by 777 health inspectors from three levels of SNVS (federal, state and municipal). The evaluation process showed that $64 \%$ of the participants completed at least one module. According to the knowledge gain assessment, $94 \%$ of participants stated that they had sufficient knowledge about the tobacco control policies in force in Brazil to carry out their work activities. Before attending training, 55\% made this statement. After attending training, 86\% health inspectors stated to feel themselves able to carry out health surveillance actions related to tobacco control in all its dimensions (tobacco products regulation, TAPS, and SFE). Before training, $44 \%$ made this statement.

Conclusions and key recommendations: This training strategy showed its high potential to strengthen tobacco control policies in large countries, covering many professionals in short time.

Tob. Induc. Dis. 2018;16(Suppl 1):A650

DOI:10.18332/tid/84534

\section{$12: 45-14: 00$}

EP-197-2 Development of an omnibus policy for tobacco control in the Philippine department of health

LE Yarcia ${ }^{1,2}$, AC Nadate Na, $^{2,3}$

${ }^{1}$ Alliance for Improved Health Outcomes, Quezon City, Philippines, ${ }^{2}$ University of the Philippines-Diliman, College of Law, Quezon City, Philippines, ${ }^{3}$ HealthJustice Philippines, Quezon City, Philippines. E-mail: Ipyarcia@up.edu.ph

Background and challenges to implementation: It is the primary mandate of the Department of Health (DOH) to formulate policies and programs in the field of health and to advocate for health promotion and healthy lifestyles for the general population in the Philippines. In leading the country's tobacco control effort, it has issued various administrative issuances throughout several decades, which led to regulatory regime complexes, regulatory gaps, and disparate operational mechanisms. With evolving tobacco control norms, as well as changes in inter-agency implementing set-ups, policies continue to be amended without a rational structure that integrates or harmonises past issuances.

Intervention or response: Comprehensive legal review of all tobacco control laws/administrative issuances, as well as stakeholder consultations through key informant interviews with the DOH's National Tobacco Control Program and members of civil society were conducted. Fourteen (14) Administrative Orders from 1993 to 2014 were surveyed, together with several Department Orders, Memorandum Circulars, and inter-agency issuances.

Results and lessons learnt: An Omnibus Policy on Tobacco Control was developed, rationalising, integrating, and harmonising the implementation of all extant $\mathrm{DOH}$ administrative issuances. An analytical tool was designed to rationalise issuances across wide implementation timeframes, utilising and simplifying methods of statutory construction. Horizon scanning guided by the Framework Convention on Tobacco Control (FCTC), the WHO's Health Systems Building Blocks framework, the Sustainable Development Goals, and overarching national economic and public health sector plans set out the fundamental strategies for a 2017-2030 Policy Development Plan on Tobacco Control.

Conclusions and key recommendations: Tobacco control regime complexes and regulatory gaps result from the difficulty of determining issuance provisions that have been impliedly 
repealed. The analytical tool developed threshed out repealed provisions, which would allow actors and stakeholders to fulfil individual mandates. Both Omnibus Policy on Tobacco Control and 2017-2030 Policy Development Plan on Tobacco Control would guide the $\mathrm{DOH}$ in formulating policies that strengthen the Philippines' compliance with the FCTC.

Tob. Induc. Dis. 2018;16(Suppl 1):A651

DOI:10.18332/tid/84419

\section{$12: 45-14: 00$}

EP-198-2 Systematic research capacity building program makes a big difference

MS Islam¹, M Shahjahan², DS Haque

${ }^{1}$ Bangladesh Center for Communication Programs (BCCP), Tobacco Control, Dhaka, Bangladesh, ${ }^{2}$ Bangladesh Center for Communication Programs (BCCP), Dhaka, Bangladesh. E-mail: sislam@bangladesh-ccp.org

Background and challenges to implementation: Historically, tobacco control has been considered a low-priority issue compared to other health-related programs in Bangladesh. In addition, local research and data around tobacco use, including the contextual factors that influence tobacco use, were inadequate to plan effective tobacco control program.

Intervention or response: Bangladesh Center for Communication Programs (BCCP) in collaboration with the Johns Hopkins Bloomberg School of Public Health has been offering the competitive Tobacco Control Research Grant Program since 2013 to create local data and build the research capacity of the young researchers of different universities and institutions. The programs organizes research capacity building mentoring workshops covering the whole spectrum of research process with an emphasis on engaging young and experienced researchers to promote peer learning. BCCP research team also nurtures the grantees by providing one-to-one mentorship in every step of research works. Besides, the research grantees are provided mentorship to prepare manuscripts for journal publication immediately after the research grants are over.

Results and lessons learnt: The program has produced tremendous results to build the research capacity of the young researchers as well as create local data. As of 2016, sixty young researchers from different universities and institutions were awarded with the research grants. The findings of the research studies were disseminated among the relevant stakeholders. The data of the research studies are being utilized to design various anti-tobacco awareness and advocacy programs in the country. A number of research studies have already been published in international journals and presented at international conferences. Bangladesh Tobacco Control Research Network (BTCRN) has been established and made operational to sustain the ongoing tobacco control research activities in Bangladesh.

Conclusions and key recommendations: The Government of Bangladesh and different universities have expressed interest to collaborate with BTCRN to carry forward its mandate. A systematically developed program can make a big difference for tobacco control.

Tob. Induc. Dis. 2018;16(Suppl 1):A652

DOI:10.18332/tid/84070

\section{$12: 45-14: 00$}

EP-199-2 Mobilizing public opinion for a political commitment for a Tobacco Endgame strategy obstacles and possibilities
G Boëthius ${ }^{1}$, L Sjöberg², E Thörnqvist ${ }^{1}$

${ }^{1}$ Tobaksfakta - Independent Tobacco Think Tank, Stockholm, Sweden, ${ }^{2}$ Health Professionals Against Tobacco, Stockholm, Sweden. E-mail: boethius@tobaksfakta.se

Background and challenges to implementation: A political commitment to a continuous Tobacco Endgame strategy was first demanded in 2012 by NGO tobacco control organizations in Sweden. The suggested goal to be reached in 2025 is less than five per cent daily smokers in the population - to be achieved through the implementation of the FCTC measures.

Intervention or response: Such a political decision, however, demands a broad and sustainable popular support. This has also been achieved mainly by NGOs on national, regional and local levels, resulting (in July 2017) in more than 160 organizations expressing their support to the endgame strategy. Most remarkably, all regional politically governed health care providers, realizing that they carry a substantial burden caused by smoking, are in favour of a strengthened strategy.

Results and lessons learnt: The Public Health Minister has expressed his support to the endgame idea - and the goal (less than five per cent daily smokers) is indeed included in a 2016-2020 national strategy for the prevention of drug, alcohol and tobacco use, the supervisor of which is the National Public Health Agency. But a concrete government decision is still missing, including a firm action plan and an adequate financial investment.

Conclusions and key recommendations: Since the present minority government, despite substantial pro-health ambitions, has not been successful enough our advocacy focus will now be more directly extended to parliamentarians running for a seat in the 2018 general election. An interparty long-term agreement is a key factor in order to reach the endgame goal in Sweden.

Tob. Induc. Dis. 2018;16(Suppl 1):A653

DOI:10.18332/tid/84297

$12: 45-14: 00$

EP-200-2 Tracking the health interest of the Sin Tax Reform Law: thru the development and implementation of a Sin Tax Monitoring and Evaluation Framework

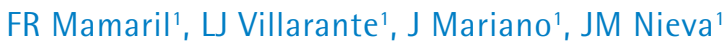

${ }^{1}$ Department of Health, Health Policy Development and Planning Bureau, Manila, Philippines. E-mail: fmamaril.dohhpdpb@ gmail.com

Background and challenges to implementation: The Sin Tax Monitoring and Evaluation (M\&E) Framework was developed in compliance with the Philippine Sin Tax Reform Law that was enacted to reform the existing law which has allowed the tobacco industry in the country to flourish. The reform law revised the multi-tiered taxation to a unitary scheme with adjusted rates and inflationary increase from 2018 onwards. The Sin Tax M\&E follows a logical framework tracking the input of the budget earmarked for health, output or the commensurate product of the investment, intermediate outcomes which is decreased smoking prevalence and the final health outcomes which are decreased morbidity and mortality and financial risk protection.

Intervention or response: The challenge was to ensure an accurate data per results chain. The Department of Health and the Department of Budget and Management agreed on the manner by which input additions were tagged as contributed by STL. This results to tagging of new and expanded health services as Sin Tax contributed outputs correlated the intermediate outcomes of the Sin Tax Law. However, special studies on decreased morbidity and mortality are still needed to support its association to STL. 
Results and lessons learnt: The $\mathrm{DOH}$ was able to publish on an annual basis the results of the M\&E as basis for reporting to the Philippine Congress which ensured stakeholders that the intention of the STL for health were attained with the following effects:

(1) increased health budget,

(2) increased health service coverage,

(3) availability of new health services, and

(4) decreased smoking prevalence.

Conclusions and key recommendations: In a highly political environment with a strong tobacco lobby, having a clear $M \& E$ framework guides the government in tracking the law's health interest. We recommend countries who will pursue FCTC Article VI (Price and Tax Measures to Reduce the Demand for Tobacco) to come up with an M\&E framework using a logical framework for easier understanding.

Tob. Induc. Dis. 2018;16(Suppl 1):A654

DOI:10.18332/tid/84426

\section{2:45-14:00}

EP-201-2 Mathiwos Wondu-YeEthiopia Cancer Society (MWECS) experiences in involvement of Ethiopian Tobacco Control Policy Advocacy

ZM Abate ${ }^{1}$, TW Desta ${ }^{2}$

'Sefakto Makgatho Health Sciences University (SMU), BETA Project, Building Effective Tobacco Control Advocates in Africa (BETA) Project, Addis Ababa, Ethiopia, ${ }^{2}$ Sefakto Makgatho Health Sciences University (SMU), BETA Project, Add is Ababa, Ethiopia. E-mail: zelalemabate02@gmail.com

Background and challenges to implementation: Ethiopia is the second populous country in Africa. In 2015, $4.2 \%$ of adults smoke. Despite, the proportion is low; it is a small percentage of large population with an increasing trend. The potential growth of the tobacco market attracted a significant investment from Japan Tobacco International, which acquired $40 \%$ share. This is a threat that needs to be countered, as an increase in tobacco use will have huge public health and developmental implications. The current Tobacco control directive is not FCTC compliant as it allows for designated smoking and lacks enforcement. Ethiopia levied much weaker ad valorem excise tax, despite known effectiveness of taxation on reducing the demand for tobacco. Thus policy level advocacy on Tobacco control is being underway with MWECS mounting pressure to control the negative consequences of Tobacco. This presentation highlights CSOs advocacy for stringent TC polices.

Intervention or response: MWECS is closely collaborating with a coalition of CSOs that galvanized an advocacy effort to leverage support for stronger FCTC compliant bill. As part of building the coalition, CSOs established a collaboration and networking, with key government officials, media, celebrities, other NGOs.

Results and lessons learnt: As a result of this broader engagements, a new law is being drafted and includes $100 \%$ Smoke Free Environment, Total Advertising Promotion and Sponsorship ban, Graphic Health Warning more than $70 \%$. It is also advocating for implementation of the World Bank recommendation to establish mixed tax system, complementing the ad valorem with a Uniform Specific tax.

Conclusions and key recommendations: Legislation and implementation of FCTC compliant law is critical in Ethiopia. CSOs have the ability to advocate and support the development of stronger legislation. However, the threat of tobacco industry interference is growing with the entry of JTI. Thus, strengthening the capacity to monitor and counter tobacco industry interference is essential for the success of TC law.

\section{Tob. Induc. Dis. 2018;16(Suppl 1):A655}

DOI:10.18332/tid/84179

$12: 45-14: 00$

EP-202-2 Dutch municipalities on their way towards a smokefree generation

B de Wolf', L Houtenbos

${ }^{1}$ Dutch Cancer Society, Prevention, Amsterdam, Netherlands. E-mail: bwolf@kwf.nl

Background and challenges to implementation: In 2015 the Dutch Cancer Society, Heart and Lung foundation started the movement 'Towards a Smokefree Generation'. We aim for a society in which children can be raised without any exposure to tobacco smoke and the temptation to start smoking. We developed a roadmap that holds a low threshold for stakeholders to start participating (including governments, social partners and civil society). The Smokefree Generation is positively framed, consistent with all political colors, takes away polarization and opens conversation. The movement grew rapidly to more than 70 partners in july 2017. Municipalities play an important role in the Smokefree Generation. The Dutch Cancer Society took on the task of encouraging and facilitating municipalities to contribute.

Intervention or response: We started with research in which we examined the extent to which municipalities used smoking policies, what opportunities they saw for the Smokefree Generation and their need for support. Based on this research, we developed and spread products that met the needs: information on the risks and impact of smoking, inspirational input for agendasetting and practical opportunities to tackle. We supported municipalities actively from agendasetting to policy making and implementation. Results and lessons learnt: A movement arose on a local level in the Netherlands. Municipalities inspired each other and the four largest municipalities became precursors. More and more municipalities incorporated the Smokefree Generation into their policies and activities. In some cases also with a clear coalition formation with stakeholders, including childcare, education, sports and health care. Agreements were made to contribute to the Smokefree Generation and this way children's environments were made smokefree more easily.

Conclusions and key recommendations: By inspiring and mobilizing municipalities customized and through the positive framework of the Smokefree Generation, more and more municipalities in the Netherlands make an important contribution to this fast-growing movement.

Tob. Induc. Dis. 2018;16(Suppl 1):A656

DOI: $10.18332 /$ tid/84227

\section{$12: 45-14: 00$}

EP-203-2 Investment and getting back the reward: training different government agencies to implement the tobacco control law in Bangladesh SM Mahbubus Sobhan ${ }^{1}$, R Quader ${ }^{1}$, M Ruhul Quddus ${ }^{1}$, SM Alam $^{2}$, I Chowdhury 3

${ }^{1}$ Ministry of Health and Family Welfare, Government of Bangladesh, National Tobacco Control Cell, Dhaka, Bangladesh, ${ }^{2}$ The Union South-East Asia, Tobacco Control, Dhaka, Bangladesh, ${ }^{3}$ Ministry of Civil Aviation and Tourism, Government of Bangladesh, Dhaka, Bangladesh. E-mail: smms_lincoln@ yahoo.com

Background and challenges to implementation: Tobacco leads to unrelenting detrimental effects on health. To protect the public Health, Bangladesh government has directed all officers who have the magistracy power to conduct mobile court on different 
laws frequently at all levels. For last few years, mobile court drive was proved as a very powerful tool to implement the tobacco control law. So, it was a dire obligation to sensitize the agencies on tobacco control law who are involved in implementation of law. Intervention or response: National Tobacco Control Cell has conducted training for the Executive Magistrates, Senior Health Education Officers, Sanitary Inspectors, Industry Inspectors, Police Officers, Ansar Officer, Civil Defense Officers and other authorized officers since 2012 in different batches throughout the year and trained 443 personnel of above mentioned law enforcing agencies. Then their initiatives were monitored through collecting report from concerned offices. It was a great challenge to ensure their participation from across the country, but made possible by the kind endeavor of the stakeholder Ministries.

Results and lessons learnt: Following the training, those officers conducted remarkable amount of mobile courts in different public places and public transports and made exemplary penalties. For instance, during January to September 2015, 816 mobile courts were conducted and penalized 2,053 persons and 80 stores and companies and collected an amount of BD Taka 548,005 as fine for violating tobacco control law. It has received tremendous support from peoples from all walks of life and huge media coverage. Such action made people law abiding.

Conclusions and key recommendations: Such investment did not go in vain and found very fruitful. It gave an important message to the decision makers to continue more novel approaches for curbing the tobacco use.

Tob. Induc. Dis. 2018;16(Suppl 1):A657

DOI:10.18332/tid/84625

\section{2:45-14:00}

EP-204-2 Public consultation on new health warning labels in Brazil

M Pinho ${ }^{1}$

${ }^{1}$ Cancer Foundation, Rio de Janeiro, RJ, Brazil. E-mail: maripinho77@gmail.com

Background and challenges to implementation: Guidelines for implementation of FCTC Article 11 recommend Parties adopt health warnings and messages that be consider scientific evidence and experiences in other countries. Brazilian $\mathrm{MoH}$ has adopted health warnings that cover $100 \%$ of the face in 2001 and that cover $30 \%$ of the front face in 2015. In 2017, ANVISA opened a public consultation (PC) on new health warnings.
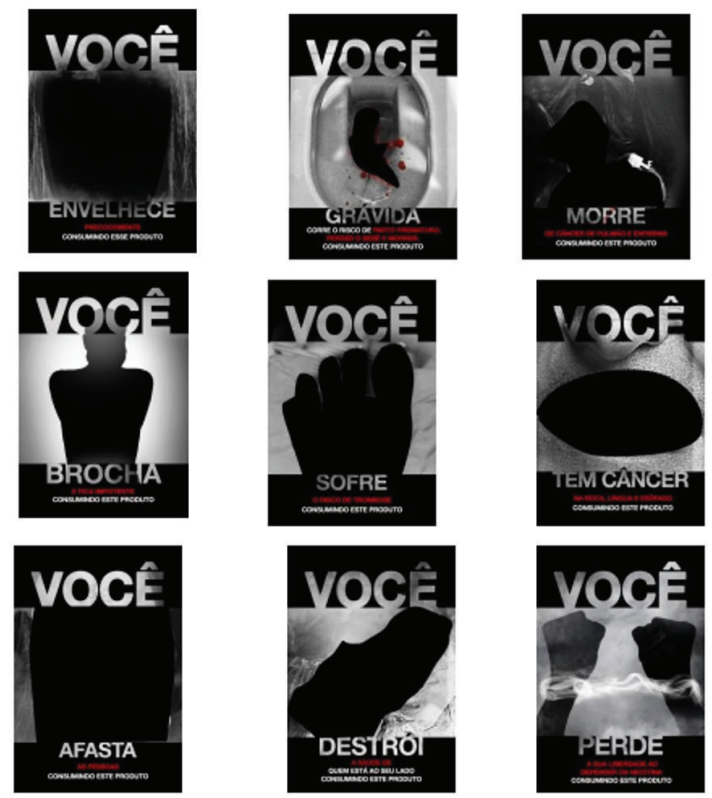

[Health Warning w/shadow under Public Consultation] Intervention or response: Civil society identified that the images proposed by ANVISA were not in accordance with the Guideline's recommendations and prepared a documentation considering all the effective design elements determined by the Guideline and submitted in the PC System.

Results and lessons learnt: There were 100 contributions, two of which were international, 39 from Rio de Janeiro and 16 from Rio Grande do Sul. There were 75 contributions made by citizens and 25 by companies. Ten contributions were explicitly made by regulated sector. The most commented articles were 6,5 and 7 . Fifty-four percent disagree with the PC's text, $52.2 \%$ believe it does not change or reduce the level of information for goods and services available to the citizen and $58 \%$ believe the proposal's impact on routines and activities will be negative.

Conclusions and key recommendations: The adoption of the new series of health warnings and messages is necessary because the current ones are already in circulation for almost 10 years and their impact on the population is reducing. PC are an important tool for social participation for implementation of the National Tobacco Control Policy. However, the contributions of the industry and its representatives are more numerous due to the economic power and influence it has in some regions and because of this, some results reflect their economic and political interests of not regulating it.

Tob. Induc. Dis. 2018;16(Suppl 1):A658

DOI: $10.18332 /$ tid/83832

\subsection{Tobacco industry interference: New tactics and counter measures}

\author{
$14: 00-15: 30$ \\ RF-1269-2 Protecting local Government Public \\ Health Policy from vested interests \\ $\mathrm{H}$ Wareing ${ }^{1}, \mathrm{H}$ Cheeseman $^{2}$, D Arnott ${ }^{3}$ \\ ${ }^{1}$ Improving Performance in Practice (iPiP), Warwick, United \\ Kingdom, ${ }^{2}$ Action on Smoking \& Health, London, United \\ Kingdom, ${ }^{3} \mathrm{ASH}$, London, United Kingdom. E-mail: Inaylor@ \\ ipip.co.uk
}

Background and challenges to implementation: Article 5.3 of the Framework Convention on Tobacco Control states that "in setting and implementing public health policies with respect to tobacco control, parties shall act to protect these policies from commercial 
and other vested interests of the tobacco industry in accordance with national law". The guidelines are applicable to government officials, representatives and employees of any national, state, provincial, municipal, local or other public institution.

The new Tobacco Control Plan for England states the government will continue to uphold its obligation under the FCTC. Local authorities are also responsible for meeting the treaty obligations. Intervention or response: The Tobacco Control Collaborating Centre (TCCC) and Action on Smoking and Health (ASH) worked with local areas to map key departments that fall under the remit of Article 5.3, then review their activities and policies for compliance with Article 5.3 and its guidelines. They used this audit to develop a strategy that will protect their public health policy from the vested interests of the tobacco industry.

Results and lessons learnt: ASH and TCCC have built a toolkit to support local government to protect public health policy. The workshop will:

- Make the case for local government to implement/amend policies in line with Article 5.3

- Discuss the approach needed to embed policies in line with Article 5.3 in local government setting and explore ways of addressing the challenges that emerge

- Present materials designed to support this process.

- Include a short case study from a local authority who piloted the materials.

Conclusions and key recommendations: Embedding policies which protect tobacco policy from the commercial and vested interests of the tobacco industry at every level of government is an important part of ensuring strong delivery of tobacco control across the whole of government. Local administration face particular pressures and challenges that need to be addressed through this process.

Tob. Induc. Dis. 2018;16(Suppl 1):A659

DOI: $10.18332 /$ tid/84233

\section{$12: 30-14: 00$}

PS-963-2 Countering tobacco industry interference/ tactics in implementation of 85\% pictorial health warnings (PHWs) on all tobacco packs through policy and political advocacy

S Gupta ${ }^{1}$

'Voluntary Health Association of India, Delhi, India. E-mail: seemagupta@vhai.org

Background and challenges to implementation: Pictorial health warnings (PHWs) effectively communicates health hazards of tobacco use - consumers see warnings thousands of times. As per law (COTPA 2003, Section 7), it is mandatory to implement PHWs across all tobacco products in India. Article 11 of the FCTC mandates that signatories should implement pack warnings within three years of ratifying the treaty.

Tobacco industry used different tactics like filing of multiple litigation's in various courts, using political clout and parliamentary committee, using livelihood card forming front groups and campaigning through paid ads in all national newspapers coercing the Government by announcing a closure of production etc.

Intervention or response: Strategies used to counter tobacco industry

- Right to Information Act to get info on representations sent to MoHFW by the industry and counter arguments.

- Mapping and creating a caucas of senstised MPs raising questions in the Parliament \& coming publicly in support

- Sensitize all the 15 members of Committee on Subordinate
Legislation (COSL) Committee on the need for large health warnings including the Chairman CoSL.

- Well coordinated civil society campaign where, letters were sent to HM and PM by 67 Public Health NGOs 500 letters sent to MoHFW, PMO, Over 650 doctors, Five widows of former tobacco users, $10 \mathrm{MPs}$ and members of Medical Associations wrote to HM and PM.

- Sharing of local, global studies/reports/evidences with the policy makers.

Timeline: Year 2014 to 2016

Results and lessons learnt: Despite all industry tactics, after a two-year battle, India implemented $85 \%$ PHWs on tobacco products package from 1st April, 2016 and moved to 3rd position out of 205 countries that have pictorial health warnings.

Conclusions and key recommendations: Government must set up a mechanism to closely monitor the compliance of pictorial health warning rules and take appropriate action against the noncompliant companies.

Tob. Induc. Dis. 2018;16(Suppl 1):A660

DOI:10.18332/tid/84365

12:30-14:00

PS-964-2 The method used to control the tobacco consumption in the post war period in Sri Lanka D Periyannan ${ }^{1}$

${ }^{1}$ Alcohol and Drug Information Center, North and East Provincial Programme, Colombo, Sri Lanka. E-mail: dinesh198604@yahoo. com

Background and challenges to implementation: In fact, we have the bitter experience of the ethnic conflict in north and east of Sri lanka for three decades. During this period the public was not educated at all about the adverse effect of the tobacco consumption. The normalcy returned in the life of the general public when the war was ended in 2009. The trade organization and multi-national companies attracted the people of north and east towards tobacco in this period. Even though the sponsorships ban in Sri Lanka, Ceylon tobacco company has build two police stations, given loan grants to famers for tobacco cultivation and encourage shop owners to sell cigarettes and some other similar strategies in 2010 and 2011. Objective was to empowering the government bodies to free people from tobacco consumption and disclosed the company strategies.

Intervention or response: We were trained public health officers, social and economic development officers and youth teams to expand tobacco prevention at grass root level. We have carried out social marketing campaigns and awareness programs to educate people through media to abolish the myths and about tobacco among the public. Our intervention carried out among the community as well as authorized officials to formulate law and regulation at the same time.

Results and lessons learnt: As results, 600 shop owners were stopped cigarette selling and it's promotions. according to the research of Alcohol and Drug Information Centre, 2011 to 2014 smoking has been reduced by $20 \%$ in Jaffna District Northern province. And available skillful resource persons located in north and east provinces and they integrated best prevention activities. authorized officers are more focused on integrating tobacco prevention activities when they are realizing the real face of Industry.

Conclusions and key recommendations: It is useful strategy to prepare existing structures to prevent tobacco industry interference at whatever level in their community.

Tob. Induc. Dis. 2018;16(Suppl 1):A661 


\section{DOI:10.18332/tid/84377}

\section{$12: 30-14: 00$}

PS-965-2 New companies act in India and Corporate Social Responsibility (CSR) by tobacco industryanother challenge for tobacco control in India

R Kumar ${ }^{1}$, RJ Singh ${ }^{2}$

${ }^{1}$ Directorate of Health Service, Shimla, India, ${ }^{2}$ The Union SEA Office, Tobacco Control \& NCD, New Delhi, India. E-mail: ravindermph@gmail.com

Background and challenges to implementation: Ministry of Corporate Affairs, Government of India (Gol) in 2013 presented a legislation called the New Companies Act 2013, which mandated every company to spend minimum of two percent of their annual profits on development agenda. Provisions of this Act were contrary to existing Indian tobacco control legislation and Article 5.3 of WHO FCTC. Tobacco companies continue to advertise by using surrogate products and brand names through CSR and pose their fair image among public and policy makers. India's three leading cigarette companies spent in excess of 677 crore in 2015-16. None of the company's CSR has a mandate to treat and rehabilitate their customers, who face serious health consequences because of tobacco use.

Intervention or response: Tobacco control advocates made representation to GoI to exclude tobacco industry from CSR ambit to stop their priviledges to carry out indirect advertisements. A Chennai based tobacco control activist group "TNPFTC" organised online signature campaign and filed a PIL in Hon'ble high court. Court issued an order to form an inter-government communications to look into the matter.

Results and lessons learnt: On May 16th, 2016, the Ministry issued a circular clarifying that companies, while undertaking CSR activities, shall not contravene any other prevailing laws of land including COTPA 2003. Incidences of direct advertisement and sponsorship are now decreased. But this is only a half battle won. Tobacco companies continue to support government-sponsored schemes and civil societies in the name of development.

Conclusions and key recommendations: Continued advertisements by tobacco companies under the garb of New Company Act is reversing the Government's efforts to denormalize the tobacco use. This is a time to put a blanket ban on the CSR activities by tobacco companies. A central Health Promotion Funds pool should be created with mandatory collection of funds from tobacco companies, so that the same could be utilized for treatment and rehabilitation of tobacco victims.

Tob. Induc. Dis. 2018;16(Suppl 1):A662

DOI:10.18332/tid/84384

\section{2:30-14:00}

PS-966-2 Tobacco industry interference with the 2017 Thai Tobacco Product Control Act

N Charoenca ${ }^{1}$, P Limstit ${ }^{2}$, V Pipattanachat ${ }^{3}$, N Kungskulniti ${ }^{1}$, S Hamann ${ }^{4}$

${ }^{1}$ Mahidol University, Faculty of Public Health, Bangkok, Thailand, ${ }^{2}$ Law Health and Ethics Center, Faculty of Law, Thammasat University, Bangkok, Thailand, ${ }^{3}$ The Thailand Health Promotion Institute (THPI), Bangkok, Thailand, ${ }^{4}$ Tobacco Control Research and Knowledge Management Center, Mahidol University, Bangkok, Thailand. E-mail: nao.naowarut@yahoo.com

Background: Thailand is among the earliest Asian countries to feel the force of the industry's influence because of trade disputes in the late $1980 \mathrm{~s}$. This provided Thailand important lessons of how to work against industry interests. These lessons have previously been researched through qualitative analysis of Thailand's policy and action.

Methods: Information from tobacco industry documents and specific policies and strategies used against the industry are illustrated and are applied to an examination of recent industry efforts: economic arguments from business interests and front groups, attempts to discredit established research findings, and using litigation to delay or block government tobacco control legislation/regulation.

Results: Counter actions were implemented early, developed continually, and shifted from being reactive to proactive as industry strategies became clear. Essential features of counter efforts included carefully monitoring tobacco industry activities, informing stakeholders of industry activities through coordinated communications, and countering industry proposals, marketing efforts, and public relations activities, including corporate social responsibility actions.

Examples of interference to delay implementation of the new 2017 tobacco control law are provided. These include how the industry established front groups such as the Thai Tobacco Trade Association (TTTA) and the Thai Tobacco Growers, Curers and Dealers Association (TTA), both exaggerating economic consequences from the new law. Interference was seen through trade organizations, media, and attempts to meet high ranking authorities prior to the passage of the new law in March 2017. Interestingly, similar tactics had been used by the tobacco industry to interfere with the adoption of the previous Thai tobacco control law in 1992.

Conclusions: Exposing how the industry seeks to undermine the very authority of state policy provides lessons about the exploitive and irresponsible nature of the industry that can serve to warn and empower politicians and all policymakers to reject CSR and other fraudulent actions of the industry.

Tob. Induc. Dis. 2018;16(Suppl 1):A663

DOI:10.18332/tid/84389

\section{$12: 30-14: 00$}

PS-967-2 Tobacco ind ustry interference challenging public health in India; time for the implementation of FCTC Article 5.3

\section{R Kumar}

'Directorate of Health Service, Shimla, India. E-mail: ravindermph@gmail.com

Background: There is an irreconcilable conflict between the tobacco industry and public health. Tobacco industry use their enormous wealth and power to influence policies to market their deadly products. Industry usually takes the shield of government's constitutions, international bilateral investment treaties, corporate property rights and adult autonomy to oppose any move by tobacco control advocates. It is important for tobacco control advocates to know the manipulative tactics of tobacco companies to get better equipped for countering the tobacco industry interference. Present study was conducted to examine the common tactics being adopted by tobacco industry in India to interfere in public health.

Methods: Investigators did a snowball retrieval research by accessing websites of major tobacco companies in India, UCSF truth tobacco industry documents online library and MOH,Govt. of India website and referred to available literature in the PubMed between 1st July 2016 to 31st December 2016.

Results: Tobacco industry manipulated political and legislative 
process; delayed notification of national legislation (COTPA 2003) and its subsequent rules. Industry took legal measures for challenging TAPS ban in the name corporate property rights, delayed new pictorial health warnings by an attempt to discredit the science. Industry intimidated government with litigation on Gutkha ban. Fronts groups of farmers, traders, media and film industry created; raised the issues of livelihood and individuals rights of expression. New Company's act 2013 is now grossly misused by tobacco companies in the name of social responsibility.

Conclusions: Tobacco industry will keep doing all possible manipulations and interference in public health to carry on their business. Tobacco control advocates and law implementers to tackle each case of industry interference using provisions in the existing legislation. It is a right time for national and state government to develop a model code of conduct and clear policy document that aligns with principle of FCTC article 5.3

Tob. Induc. Dis. 2018;16(Suppl 1):A664

DOI:10.18332/tid/84393

12:30-14:00

PS-968-2 Print media study on communicate misleading information by tobacco industry

A Dilrukshi ${ }^{1}$

${ }^{1}$ Alcohol and Drug Information Center (ADIC) Sri Lanka, Library \& Information, Colombo, Sri Lanka.E-mail: achaladil@yahoo.com Background and challenges to implementation: Objectives of this study are to; identify the new tactics of Ceylon Tobacco Company(CTC) making fabrications through media in order to decelerate the implementation of tobacco tax policies. It is proven that one of best sustainable tobacco control method is to hike cigarettes tax which reduces cigarette consumption. 2016, Sri Lankan government increased cigarettes tax thrice as never done before. CTC tried to demolish this effort by fabricating as they done before.

Intervention or response: 4 daily and 6 weekly different national newspapers which are highest circulation were selected. From 2016 January to 2017 June 2376 newspapers were analyzed in this study. The impact of perception on reader by articles, advertisement, cartoon, poem and stories of the implementation of tobacco tax hike were categorized. Under the category; reduction of smokers, discourage of smoking, increment of bidi smoking due to high price of cigarette, highlighting and pointing out that government loos the tax due to illegal cigarette, fabrication of stories that, if cigarette company shut down the number of families are more who lost their jobs and CTS's image building were identified.

The reports were sent to media institutes, politicians, policy makers and relevant authorities and made a dialogue among them. Results and lessons learnt: Identified fabricated and spread myths by CTC are; cigarette price hike turn smokers to bidi which is bad for health, illegal cigarettes will be common, if the company shut down government will be lost huge amount of tax. It also helped to identify the behavior is positive or negative of key persons in the country, media personnel, politician and policy makers. Further it revealed the media and persons who supported to CTC. Discourage the increment of cigarette tax were reported. Conclusions and key recommendations: This study helped to confirm that CTC make fabrications and spread them to demolish the law and regulations on tobacco tax hike.

Tob. Induc. Dis. 2018;16(Suppl 1):A665

DOI:10.18332/tid/84453

\section{$12: 30-14: 00$}

PS-969-2 Tobacco industry targeting Sri Lankan non-tobacco-growing farmers in corporate social investments

I Buddhika1,2, KMN Perera ${ }^{1,3,4}$, SC Lakmal1,2, CS Perera ${ }^{1}$, H Wijesooriya ${ }^{1}$, M Rajasuriya $^{1}$

${ }^{1}$ Centre for Combating Tobacco, Faculty of Medicine, University of Colombo, Colombo, Sri Lanka, ${ }^{2}$ Alcohol and Drug Information Center (ADIC), Colombo, Sri Lanka, ${ }^{3}$ Faculty of Medicine, University of Kelaniya, Public Health, Ragama, Sri Lanka, ${ }^{4}$ Tobacco Control Research Group, University of Bath, Bath, United Kingdom. E-mail: isharabuddhika@gmail.com

Background: Sustainable Agriculture Development Project (SADP) is the main Corporate Social Investment (CSI) activity of the Ceylon Tobacco Company (CTC), the subsidiary of British American Tobacco holding cigarette manufacturing monopoly in Sri Lanka. In its initiation in 2006, its target group was tobacco farmers. By 2017, its beneficiaries included farmers other than tobacco growers. This study aimed to explore the process of tobacco industry increasing its coverage towards non-tobacco growers and its apparent benefits to the industry.

Methods: A qualitative study design was used. Data were collected using document analysis (reports and presentations of CTC and CSI partners and media reports) and key informant $(\mathrm{N}=18)$ interviews (CTC CSI officers, field workers of government and non-government organisations, community leaders and beneficiaries of SADP programme). Documents from 2006 onwards were identified electronically and manually from databases, electronic archives and libraries. Search terms used were identified using an expert panel. Data were analysed using thematic analysis.

Results: By 2017 four variations of SADP existed, all targeting non-tobacco growing farmers. Activities mainly included home gardening and infrastructure development in rural communities. A variation (SADP Lite) incorporated ex-libertarian combatants as part of their rehabilitation process by the government. Activities engaged ministers, government officials, field workers and community leaders. Key informants' perceptions included; CTC functions beyond the stigmatized image of a 'tobacco company' by expansion to different activities and themes; nontobacco-growing farmers indirectly motivated to strengthen links with CTC by starting tobacco cultivation, activities are portrayed as government initiatives by incorporating government ministers, officials and field workers, and, most activities are portrayed as solutions for negative effects of tobacco cultivation (homegardening, alternative crops and agro wells) and tobacco use (nutrition and rural economy).

Conclusions: CTC uses SADP to promote their image, improve their stake in the economy and motivate farmers to grow tobacco Tob. Induc. Dis. 2018;16(Suppl 1):A666 DOI:10.18332/tid/84486

\section{2:30-14:00}

PS-970-2 Monitoring and exposing tobacco industry tactics around Conference of Parties (COP 7)

B Mathew ${ }^{1}$, B Mukhopadhyay ${ }^{1}$

'Voluntary Health Association of India, Delhi, India. E-mail: binoymathew84@gmail.com

Background and challenges to implementation: Section 5.3 of the Cigarettes and Other Tobacco Products Act (COTPA) does not allow any kind of promotion of tobacco products or its 
use. Article 5.3 of WHO-FCTC requires parties to protect the development and implementation of public health policies related to tobacco control from the commercial and other vested interests of the tobacco industry. India hosted the Seventh Session of the Conference of Parties (COP) of the WHO-Framework Convention on Tobacco Control (FCTC) in Delhi NCR (Greater Noida) from 7th to 12th November, 2016. In light of this, one of the front group of tobacco industry put huge billboards/hoardings and advertisements behind public transport showing agitation against COP 7 and tobacco control organisations working in India. This front group purported to represent the interests of the tobacco farmers in India was denied observer status by the FCTC Bureau due to its failure to submit a proper declaration of its interest.

Intervention or response: We took this issue immediately and documented the photographs of these bill boards/hoardings and the advertisements. A representation along with these photographs was shared with the district administration of the place, where the COP 7 event was taking place. We brought to the notice that displaying the promotional materials by the tobacco industry is a clear violation of WHO-FCTC treaty and requested the administration to take immediate action to ensure that these hoardings and billboards are removed.

Results and lessons learnt: The media covered the violations done by the tobacco industry extensively, exposed the tactics of the tobacco industry and further action was being taken up.

Conclusions and key recommendations: If we are to influence tobacco usage in the country and dissuade initiation, continuous monitoring and sensitization is required to counter the tobacco industry tactics and emerge victorious.

Tob. Induc. Dis. 2018;16(Suppl 1):A667

DOI:10.18332/tid/84496

\section{2:30-14:00}

PS-971-2 Advocacy for a mobile court for ban on TAPS by Japan Tobacco International (JTI) and Dhaka Tobacco Industries (DTI) by TC law

\section{MRI Milon ${ }^{1}$}

${ }^{1}$ Madok Drabya 0 Nesha Birodhi Council (MANOBIK), Tobacco \&t Narcotics Control, Dhaka, Bangladesh.E-mail: rimilon08@ gmail.com

Background and challenges to implementation: JTI and DTI jointly undertake promotional activities to market Winston cigarettes newly. Students are involved in marketing this brand. Everywhere these students make huge publicity among public by distributing Winston cigarettes including gift items such as match lights, bags, special boxes of cigarettes, umbrellas, pen drives, caps, T-shirts, mugs, etc. with Winston's logo. Due to gift distribution, new smokers are being created among the youth. In accordance with TC Act, distribution of gifts is a punishable offense. But tobacco companies do these activities in a way that they cannot be easily caught for tricky activities.

Intervention or response: These criminal activities were informed to officials and Tobacco Control Task Force. As company's promotional programs were running, magistrates operated several mobile courts accompanied by police and alert shopkeepers with financial fines and destroy fronts of flee shops with Winston brand logo. Besides, the easy to carrying shops and big umbrellas containing the brand logo were seized and dumped to remove it. Again, companies have lent money to vendors to pay for the fine. Finally, the depots are identified for stopping these initiatives of companies and duly notified to the mobile court operating magistrates. Finally all promotional activities are seized. Currently, all such activities are closed.

Results and lessons learnt: In collecting information about illegal activities of marketing of Winston Cigarette produced by JTI, the authority is properly informed. As a result, it has been possible to play a role by imposing legal penalties and punishment. It is possible to control the barriers that Company produces directly and indirectly through promotional activities.

Conclusions and key recommendations: Tobacco Companies directly and indirectly trick to broaden the use of tobacco can be stopped to protect public health with the coordination of GONGO.

Tob. Induc. Dis. 2018;16(Suppl 1):A668

DOI:10.18332/tid/84504

\section{2:30-14:00}

PS-972-2 Tobacco industry attempts to undermine tobacco control by recruiting Czech and Polish anti-communist dissidents

M Zatonski ${ }^{1,2}$, S Glantz $^{3}$

${ }^{1}$ London School of Hygiene \&t Tropical Medicine, Public Health and Policy, London, United Kingdom, ${ }^{2}$ Health Promotion Foundation, Nadarzyn, Poland, ${ }^{3}$ University of California San Francisco, Center for Tobacco Control Research and Education, San Francisco, CA, United States of America. E-mail: zatonski. mateusz@gmail.com

Background: After the collapse of communism in Eastern Europe in 1989 state-owned tobacco industry was taken over by transnational tobacco companies (TTCs). TTCs engaged in aggressive lobbying against tobacco control efforts, and targeted key politicians. In Poland and Czechoslovakia (from 1993 Czech Republic) former anti-communist dissidents often held high government office, but also enjoyed considerable prestige abroad. The objective of this study is to identify and explain the TTCs' strategy to undermine tobacco control measures through the recruitment of former anti-communist dissidents, and how they influenced tobacco control policy development in the Czech Republic and Poland in the 1990s.

Methods: An analysis of relevant documents available in the Truth Tobacco Documents Library was conducted. This was supplemented by an analysis of press coverage, industry and public health journals, as well as key informant interviews with representatives from the tobacco industry, government officials, and Polish tobacco control advocates.

Results: TTCs identified and targeted key anti-communist dissidents perceived as champions of liberty. These included Vaclav Havel in the Czech Republic and Lech Walesa in Poland, both of whom were involved in promotional efforts of TTCs internationally in the early 1990s. In 1995, as presidents of their countries, they vetoed progressive tobacco control bills. In Poland, the veto was overturned thanks to the pressure of health advocacy groups, but in Czech Republic it was upheld.

Conclusions: The strategy of TTCs to target key individuals enjoyed varying degrees of success. In the Czech Republic, it was successful in delaying the introduction of tobacco control legislation. In Poland, where health advocacy groups were actively engaged in lobbying efforts, it failed to achieve this goal. Poland's tobacco control successes in the 1990s, and the effective engagement of its anti-tobacco advocates with both policymakers and the public, can provide a reference point for countries currently undergoing market liberalisation.

Tob. Induc. Dis. 2018;16(Suppl 1):A669

DOI:10.18332/tid/84561 
12:30-14:00

PS-974-2 Tobacco industry's use of litigation to stop tobacco control measures in Brazil

C Vianna', M Molinari', R Vargas ${ }^{2}$

'The Union, Tobacco Control, Mexico City, Mexico, ${ }^{2}$ Brazilian National Cancer Institute, CONICQ, Rio de Janeiro, RJ, Brazil. E-mail: cvianna@theunion.org

Background and challenges to implementation: Brazil is the highest-performing countries in its efforts to develop policies and programs to implement FCTC.

The tobacco industry (TI) continues to hamper Brazil's efforts to fully implement FCTC, interfering in the adoption of such measures, or preventing implementation when they are adopted, challenging in national courts.

ANVISA is the regulatory agency responsible for regulating tobacco in Brazil. Two different legislations enacted by the agency (ban of additives and health warnings) are being challenged in Courts. This challenge is delaying the fully protection of Brazilian population from the harms caused by tobacco.

Intervention or response: A coalition of tobacco control partners was created, representing government health agencies, academia and health NGOs, in order to fight in this battle. Coalition members had regular meetings with the Supreme Court to urge the release of the injunction that stopped the effects of additive ban. Members of the civil society filed two amicus brief in support of ANVISA. Mobilization of legislators and public health institutions to contact the Supreme Court through letters of support was done. A dissemination of tobacco control guidelines for the Judiciary Power informing about FCTC was another strategy adopted. Technical assistance for the formal response was sent to federal prosecutors and scientific evidence to support the legislation was provided.

Results and lessons learnt: A major success of this group of initiatives was the scheduling of the hearing on the additives ban for August 17, 2018, since the lawsuit started in 2013 and the TI access to the Court had kept it off the hearing agenda. This work will provide a discussion and an analysis of the TI arguments and the counter arguments used to defend the public health laws.

Conclusions and key recommendations: It is important to create a coalition to defend against the TI use of legal strategies to prevent the implementation of the tobacco control.

Tob. Induc. Dis. 2018;16(Suppl 1):A670

DOI:10.18332/tid/84586

\section{2:30-14:00}

PS-975-2 Declaring schools tobacco free: protecting young generation to save Nation (multipronged coordinated interventions to declare 3517 schools tobacco free in Jharkhand state in India)

M Ranjan', A Pandey ${ }^{2}$, DK Mishra ${ }^{3}$

${ }^{1}$ Government of Jharkhand, Khunti, India, ${ }^{2}$ International Union Against Tuberculosis and Lung Disease, Tobacco Control, New Delhi, India, ${ }^{3}$ Socio Economic and Educational Development Society (SEEDS), New Delhi, India. E-mail: manishranjanias@ gmail.com

Background and challenges to implementation: Tobacco Industry (TI) aggressively targets young children and adolescents as new recruits. The early age of initiation for TI assures tobacco usage for longer period. As per GATS 2010, of all ever daily tobacco users (age 20-34), almost 60\% initiate tobacco use before age of 17.8. It calls for urgent intervension. As per GATS 2010, Jharkhand state in India has one of the highest total prevalence rate as $50.01 \%$ against national average of $34.6 \%$. This is disturbing. Government of India enacted its tobacco control law i.e. Cigarette and Other Tobacco Product Act, 2003 (COTPA). It prohibits smoking in all public places including schools (section 4), forbids sale to and by minors (section 6-A) and bans sale of tobacco products within 100 yards of any educational institution (section 6-b).

Intervention or response: 3.5 million students of age 14 to 18 studying in 3517 schools in Jharkhand state were protected through multipronged coordinated interventions adopted by Director of School Education, SEEDS and The UNION, technical support partners to state Government. Massive awareness program was launched. All stake holders including DEOs, teachers, media, school management committee, parents, peer groups were made sensitive and responsible ensuring that

- No sale of tobacco products around 100 Yards of schools.

- No use of tobacco in schools

- Display of two warning signages mandated under section 4 and section 6(b).

Continuous monitoring of implementation process was the key. Strict actions were also taken.

Results and lessons learnt: Visionary Zeal and regular personal monitoring resulted in high compliance of section 4 and section 6(b) ensuring 3517 Schools being declared tobacco-free in two years.

Conclusions and key recommendations: Strategic, collaborative and multipronged coodinated intervention at school level results in reducing students' exposure and their accessibility to tobacco products significantly. This minimizes chances of picking up this dangerous habit. It protects young generation. And, Protecting Young Generation means saving Nation.

Tob. Induc. Dis. 2018;16(Suppl 1):A671 DOI: $10.18332 / \mathrm{tid} / 84592$

12:30-14:00

PS-977-2 Assessing the tobacco harm reduction (THR) debate: a systematic review

Y Hendlin', M Vora', J Elias'1, C Benowitz-Fredericks', P Ling ${ }^{1}$ 'University of California, San Francisco, San Francisco, CA, United States of America. E-mail: manali.vora@ucsf.edu

Background: Tobacco Harm Reduction (THR) has become synonymous with substituting alternative tobacco products for cigarettes. However, there exists much dissension among tobacco control professionals regarding accepting harm reduction methods prolonging nicotine addiction and profiting the tobacco, e-cigarette and pharmaceutical industries. We evaluate the influence of these industries on the academic THR literature and debate.

Methods: We undertook a comprehensive review of all peerreview papers published on the topic of tobacco harm reduction between 1992 and July 2016. Our initial search yielded 5,172 relevant hits, and after screening, we double-coded 1,067 fulltext articles. Codes include the article's stand on THR (weakly or strongly pro-, anti-, or neutral/mixed), major themes, product type, country of author origin, article type (letter/commentary, RTC, longitudinal study, etc.), journal quality, and funding source. These results were analyzed in STATA.

Results: Of the 498 articles we have coded so far, 379 were included. The results show that six percent of all articles are editorials, $36 \%$ letters or commentaries, and $21 \%$ are nonempirical articles while only $31 \%$ are original research and $6 \%$ reviews. Thirty-three percent of pro-THR articles disclosed some sort of industry funding. Of these, $30 \%$ were funded by the tobacco industry, $22 \%$ by the E-cigarette industry and $48 \%$ were 
funded by pharmaceutical industries.

Conclusions: The THR debate has been influenced by scientists funded by tobacco, electronic-cigarette and surprisingly pharmaceutical industries in the favor of product substitution. Moreover, the majority of this debate is occurring over 'opinion pieces' rather than on the basis of empirical research. Thus, more robust and unbiased scientific evidence is needed to evaluate these alternative products before endorsing them for the public.

Tob. Induc. Dis. 2018;16(Suppl 1):A672

DOI:10.18332/tid/84665

\section{$12: 30-14: 00$}

PS-978-2 Confronting the challenges associated with teaching tobacco harms to students whose parents are tobacco manufactory workers

\section{JM Aloria ${ }^{1}$}

${ }^{1}$ Marikina Science High School, (Previous Engagement Is Fortune High School), Marikina, Philippines. E-mail: jossaaloria@gmail. com

Background and challenges to implementation: Fortune High School is the youngest public high school in the Marikina City, which primarily serves population within Brgy. Fortune, a barangay named after the most popular value brand cigarettes of Philip Morris Fortune Tobacco Corporation (PMFTC). The school comprises students whose parents are commonly employed by PMFTC in their manufactory just one kilometre away from the school. Students and teachers are exposed to chemicals and smoke by-products of the nearby factory, and printed advertisements are all over, especially for the new products PMFTC is testing in the market. FHS' context makes discussion smoking harms extremely needed, yet required to be balanced with clarifications on livelihood and economic impacts of tobacco control.

Intervention or response: Our school started taking on a comprehensive approach to highlight tobacco control. It recognises that the discussion can be integrated in the following teaching subjects: "Music, Arts, Physical Education, and Health, Science, and Social Studies. As a teacher in Social Studies, my role is best exemplified in discussing tobacco control as social and economic issue, and created an avenue for the students to discuss their parents' struggles in the workplace. We coordinated with Bawas Bisyo (Vice Reduction) coalition, the largest coalition of youth organizations against vices for peer-to-peer interactions and programs to impart this as a youth issue.

Results and lessons learnt: The influence of tobacco interference is huge in a community whose connections with the industry is largely economic. The coordinated efforts to teach various aspects of tobacco (health, social, and mental harms, as well as the political and legal issues surrounding it) helped in providing a holistic on tobacco.

Conclusions and key recommendations: It is recommended for the Department of Education (DepEd) to develop teaching guides for the use of all teachers as they integrated in their own subjects the complex issues surrounding tobacco.

Tob. Induc. Dis. 2018;16(Suppl 1):A673

DOI:10.18332/tid/84674

$12: 45-14: 00$

EP-191-2 Survey of child labor in the tobacco trade in Algeria

B Ouzriat ${ }^{1}$
'Directorate of Health and Population of the Wilaya of Boumerdes, Boumerdes, Algeria.E-mail: ouzriat@yahoo.fr Background: Child labor in illegal and unprotected activities, such as the tobacco trade is increasing in Algeria. In order to analyze this phenomenon, a socio-economic and bio-demographic survey on child labor in the tobacco trade was carried out in 2012. Methods: It's a socio-bio-demographic survey by interview which affected all children aged 6 - 18 years, practicing the tobacco trade in the province of Boumerdes in Algeria during the period from 01 January to 30 April 2012 .

Results:

- 523 children were enumerated and interviewed, all of them are male, with an average age of 11 years and $55 \%$ aged between 9 and 13 years

- $2 / 3$ of the respondents are no longer in school

- $75 \%$ of the children reside in the locality of the place of work. Less than $25 \%$ reside in rural areas

- $50 \%$ of the children interviewed have their father unemployed

- Only 1 in 7 mothers has a job

- $3 \%$ comes from a family victim of terrorism

- The majority of the children interviewed have begun this type of trade during the last 15 years, with an average period of 7 years of activity

- More than $33 \%$ of respondents are smokers

- The informal network is the main source of supply (65\%) And in almost $33 \%$ of cases the source of supply is not acknowledged

- $50 \%$ of these children work on behalf of their families, and more than $33 \%$ work on behalf of third parties

- The average monthly earnings per child is $60,000.00 \mathrm{D} \mathrm{A}=$ 600 US Dollars / month

Conclusions: The gravity of this phenomenon is well demonstrated. Non-compliance with child protection agreements, Risk to children's health, and ruin of the public treasury through tax evasion (informal trade, Trafficking and counterfeiting in the tobacco industry).

Tob. Induc. Dis. 2018;16(Suppl 1):A674

DOI:10.18332/tid/84219

\section{$12: 45-14: 00$}

EP-192-2 Cartography monitoring of tobacco industries in Côte d'Ivoire

T Bi Boli Francis ${ }^{1,2}$

${ }^{1}$ Felix Houphouet Boigny University, Biosciences, Abidjan, Côte D'Ivoire, 2Felix Houphouet Boigny University, Criminology, Abidjan, Côte D'Ivoire. E-mail: bolitbf@gmail.com

Background: With the end of crisis sociopolitical in 2011, Côte d'Ivoire calls for capital from diverse investors to finance its reflation. In this context, the tobacco firms could use strategies to present themselves as socioeconomic development's actors. This situation could lead a risk of thoses firms' interfering in the political and financial public decisions.

This study is part of a strategic approach based on the assumption that the better you know your opponent, the better you fight. Thus, improving tobacco control would require a better understanding of the actors providing this product for consumption: the tobacco industries. This article aims to identify the entities of the tobacco industry operating in Côte d'Ivoire throughout the chain in terms of production, processing, distribution (import, export), etc., their allies and frontal groups; to understand their interference strategies and tactics, and their marketing strategies used in our country. 
Methods: At the methodological level, a triangulation of data collection tools (documentary research, field visits and interviews) was carried out. These data were treated both quantitatively and qualitatively.

Results: The results identify the entire tobacco production chain in Côte d'Ivoire. They also highlight the inter-union organizations of employees of tobacco companies as actors that these firms use to increase their margins of maneuver and the weight of tobacco firms in the Ivorian economy. Thus, recommendations are made to improve the process of implementation of the project to support the adoption of a tobacco law in Côte d'Ivoire and limit the interference of the tobacco industry.

Conclusions: The Ivorian State made numerous efforts in the application of the WHO FCTC's requirements. However, the tobacco firms are still using strategies to interfere in the political decisions, in particular the anti-smoking law. Therfore, it is convenient to collect data on these tobacco firms to constitute arguments of plea and and counter measures.

Tob. Induc. Dis. 2018;16(Suppl 1):A675

DOI:10.18332/tid/84270

12:45-14:00

EP-193-2 The right of governments to enact public health laws - the Brazilian tobacco additives ban case

D Alves ${ }^{1}$

${ }^{1}$ Ministry of Heath of Brazil, Brasilia, DF, Brazil. E-mail: dhtalves@gmail.com

Background and challenges to implementation: Tobacco industry (TI) interference is one of the key challenges to the creation and implementation of tobacco-reduction regulation. It continues to undermine control efforts globally. One example is the Brazilian proposal to ban additives in 2012. Recent files show how a Dutch tobacco industry were planning to bring the case to World Trade Organization (WTO) and block the Mercosur European Union Free Trade Agreement negations.

Intervention or response: Bring awareness of how articulated the TI is, ahead of many Governments. Use the Brazilian case to show how a Cigar Company based in Netherland was planning to convince/lobby other European Governments to prosecute Brazil internationally.

Explore the TI library, held by the University of California in San Francisco. Display the WTO fulfilled forms they pretend to use, the exchange of documents between tobacco industry CEOs and how they were planning undermine FTA negations.

Results and lessons learnt: The Netherlands are the biggest exporter of tobacco to Brazil, in particular of cigars. Together, Belgium and Netherlands imports $22 \%$ of all Brazilian tobacco. There is evidence of the close relation between tobacco industry and former Dutch Interior Minister. The TI did not followed with the process because meanwhile, the Brazilian resolution was pledged in Supreme Court, where it remains. The focus swift to Australia and they used all previous legal basis, prepared to suit Brazil, in the Australian case.

Conclusions and key recommendations: In response to these claims from the TI, Governments may rely on their sovereign right to regulate in the interests of public health. Specifically in relation to tobacco control measures, which aim is to protect human health.

Tob. Induc. Dis. 2018;16(Suppl 1):A676

DOI:10.18332/tid/84598

$15: 45-16: 45$
LB-1361-2 Passing FCTC compliant tobacco control legislation amid conspicuous industry interference: the case of Senegal

A Sylla ${ }^{1,2}$

'Ecole National d'Économie Appliqué, Dakar, Senegal, 2Institution, Programme National de Lutte Contre le Tabac, Dakar, Senegal. E-mail: aliounemariama@gmail.com

Background: Background and context: Senegal achieved significant legislative advances with the adoption of a Tobacco Control (TC) Act in 1981. However, since 1985, increasingly powerful industry lobbying resulted in important aspects of the Act being made less effective or reversed. In 2004 Senegal ratified the WHO Framework Convention on Tobacco Control (FCTC), but once again tobacco industry interference delayed preparation of a draft bill implementing the provisions of the FCTC until 2011. Methods: Aim: To use civil society advocacy to catalyze a public health movement and reduce prevalence of tobacco use through adoption and implementation of new FCTC-compliant legislation in Senegal.

Results: Strategy / tactics: LISTAB (civil society) conducted a program of advocacy activities in parallel to the three year legislative development process, from initial drafting by Ministry of Health, through parliamentary amendment and approval, and finally promulgation by the President.

Programme / Policy process: Our activities included:

- Setting up a watchdog body called Tobacco Industry Monitoring Team

- Sensitizing and training decision makers, politicians and journalists on the FCTC, particularly Article 5.3

- Lobbying religious leaders and Members of Parliament, and identifying among them TC champions

- Running a national media campaign to gain exposure for TC issues and counter tobacco industry interference

Conclusions: Outcomes / What was learned: In March 2014, the TC bill was approved by the Senegalese parliament and promulgated by the President. In supporting this achievement, the work conducted by LISTAB highlights the importance of: Capacity building of tobacco control actors in both FCTC content and advocacy techniques hence the creation of the national tobacco control program in Senegal.

Tob. Induc. Dis. 2018;16(Suppl 1):A677

DOI: $10.18332 /$ tid/84724

\section{$15: 45-16: 45$}

LB-1370-2 Brazil responses to the strategies used by the tobacco industry to resist Tobacco Control National Policy

AP Leal Teixeira ${ }^{1}, T^{1}$ Cavalcante ${ }^{1}$

${ }^{1}$ National Cancer Institute/Ministry of Health, Executive Secretariat for implementation WHO FCTC, Rio de Janeiro, RJ, Brazil. E-mail: ana.teixeira@inca.gov.br

Background and challenges to implementation: According to the publication Tobacco industry interference with tobacco control (WHO, 2008), "The diversity of these strategies demonstrates that the mission to thwart tobacco control is global", and it is not different in Brazil. Since 1996, when Brazilian government started tobacco control policies, the tobacco industry have been enforcing and spreading their influence through the government and society.

Intervention or response: Brazilian government ratified the WHO Framework Convention on Tobacco Control in 2005. Regarding article 5.3 "Parties shall act to protect these policies 


\begin{tabular}{|c|c|c|}
\hline Tactic & $\begin{array}{l}\text { Intelligence gathering: } \\
\text { Tobacco Industry } \\
\text { representatives are constantly } \\
\text { trying to attend tobacco } \\
\text { control meetings. }\end{array}$ & $\begin{array}{l}\text { Public relations: Although } \\
\text { promotion and advertising has } \\
\text { been banned, TI uses social } \\
\text { media to defend its product } \\
\text { against increased taxes, for } \\
\text { instance. Unfortunately, } \\
\text { approved as an exception in the } \\
\text { federal law, they use TV series } \\
\text { and novels to explore the image } \\
\text { of smoking characters. }\end{array}$ \\
\hline $\begin{array}{l}\text { Branil } \\
\text { response }\end{array}$ & $\begin{array}{l}\text { Government representatives } \\
\text { for tobacco control make } \\
\text { careful assessment of } \\
\text { participants at all meetings } \\
\text { and events. }\end{array}$ & $\begin{array}{l}\text { Government tobacco } \\
\text { control officials monitor any } \\
\text { violations of federal law that } \\
\text { have banned advertising } \\
\text { and publicity in the media. } \\
\text { This exception in federal law } \\
\text { indicates interference within } \\
\text { government. }\end{array}$ \\
\hline Tactic & $\begin{array}{l}\text { Consultancy: It's easy to find } \\
\text { this experts writing articles } \\
\text { and as speakers in tobacco } \\
\text { growers forums. }\end{array}$ & $\begin{array}{l}\text { Funding research, including } \\
\text { universities: TI has some } \\
\text { partner universities to } \\
\text { produce studies in defense } \\
\text { of tobacco cultivation and } \\
\text { weaken the results obtained } \\
\text { with the reduction of tobacco } \\
\text { consumption, mainly in the } \\
\text { regions of tobacco growers. }\end{array}$ \\
\hline $\begin{array}{l}\text { Branil } \\
\text { response }\end{array}$ & $\begin{array}{l}\text { Government and civil } \\
\text { society representatives for } \\
\text { tobacco control produce } \\
\text { and disseminate scientific } \\
\text { papers that strengthen } \\
\text { the implementation of the } \\
\text { National Tobacco Control } \\
\text { Policy. }\end{array}$ & $\begin{array}{l}\text { Government and civil society } \\
\text { representatives for tobacco } \\
\text { control produce and disseminate } \\
\text { scientific papers that strengthen } \\
\text { the implementation of the } \\
\text { national tobacco control policy. } \\
\text { In tobacco producer regions, } \\
\text { it has been important to warn } \\
\text { about green tobacco sickness } \\
\text { and reduce global consumption. }\end{array}$ \\
\hline Tactic & $\begin{array}{l}\text { Intimidation: They explore the } \\
\text { fact that Brazil is the second } \\
\text { largest tobacco producer in } \\
\text { the world. Tax revenue from } \\
\text { tobacco product sales reached } \\
13 \text { billion reais in } 2015 \text {. } \\
\text { More than } 150.000 \text { families } \\
\text { are depending on tobacco } \\
\text { growing. }\end{array}$ & $\begin{array}{l}\text { Philanthropy: TI sponsors } \\
\text { some museums entrance and } \\
\text { art events, not showing their } \\
\text { cigarette brands. }\end{array}$ \\
\hline $\begin{array}{l}\text { Branil } \\
\text { response }\end{array}$ & $\begin{array}{l}\text { Government representatives } \\
\text { for tobacco control conducted } \\
\text { a research that showed that } \\
\text { this } 13 \text { billion covers only } \\
33 \% \text { of the direct costs caused } \\
\text { by smoking to the health } \\
\text { system, which represents only } \\
23 \% \text { of the total expenditure } \\
\text { attributable to smoking } \\
\text { ( } 56 \text { billion/year). Brazilian } \\
\text { government developed } \\
\text { the National Program for } \\
\text { Diversification in Areas of } \\
\text { Tobacco Cultivation to support } \\
\text { families that opt to invest in } \\
\text { another plantation. }\end{array}$ & $\begin{array}{l}\text { They cannot sponsor sports, } \\
\text { concerts or cultural events by } \\
\text { federal law. This is an issue } \\
\text { that demands new regulation. }\end{array}$ \\
\hline
\end{tabular}

Political funding: Some congressmen and political groups have received official donations from Brazil's tobacco industry in recent election campaigns.

Official donation of corporate financial resources for election campaigns was banned for the upcoming elections. It's is hard to control unofficial funding.

Smokers' rights groups: There are groups of this nature and usually express themselves by the same interlocutors.

Government and civil society representatives for tobacco control advocate for public health rights through campaigns and programs in schools.

Corporate social responsibility: Souza Cruz, controlled by BAT group and market leader in Brazil, says that "sustainability is a company commitment. The company believes in the production of better and more solid economic results in the long term from the adoption of behaviors and socially responsible practices". This program seeks diversification with beans and corn, between tobacco crops season.

The National Program for Diversification in Areas of Tobacco Cultivation is based on the principles of sustainable development, food security, diversification of production and social participation. The program operates in the qualification of the production and development process in the areas of tobacco farming, as well as from the perspective of ecological production, by reducing the use of pesticides.
Lobbying: Tobacco lobbyists act at the congress defending the tobacco industry and barring tobacco control actions.

Government representatives for tobacco control advocate at the congress in defense of the public health, and count on the partnership of civil society in the same direction.

Creating alliances and front groups: These groups can be found in congress, in government areas and in forums against tobacco illicit trade.

Government and civil society representatives for tobacco control work in the same fields to defend the public health rights.

Youth smoking prevention and retailer education programmes: Souza Cruz publishes on his website: "Although there are several ways to quit smoking, such as nicotine replacement therapies, are known, Souza Cruz believes that the most important factors for effective behavior change are the personal will and selfconfidence of each individual".

The National Cancer Institute (INCA), as the Ministry of Health body, is responsible for coordination of the National Program for Tobacco Control, considering the complexity that involves addition and smoking cessation. 


\begin{tabular}{|l|l} 
Tactic & $\begin{array}{l}\text { Smuggling: TI uses the illicit } \\
\text { trade to justify tax decreasing. }\end{array}$ \\
& \\
Bravil & $\begin{array}{l}\text { Government and civil society } \\
\text { representatives for tobacco } \\
\text { control are working to ratify } \\
\text { the Protocol to Eliminate Illicit } \\
\text { Trade in Tobacco Products and } \\
\text { protect our borders from illicit } \\
\text { trade. }\end{array}$
\end{tabular}

International treaties and other international instruments: Based on the WTO and MERCOSUL tax rules, it was not possible to tax tobacco leaves exported by Brazil.

Regarding this issue, there is no action to be implemented in short term.

\author{
Joint manufacturing and \\ licensing agreements and \\ voluntary policy agreements \\ with governments strategy: \\ $\mathrm{TI}$ in Brazil is not a state \\ monopoly.
}

No action.
Pre-emption: Brazil is a large country with 26 states and a Federal District that deal with local lobbying and government pressure.

The incentive to raise VAT rates on cigarettes and increase tax revenue has been an opportunity in the States due to the economic crisis.
Brazilian government have been monitoring and working through the National Commission to Implement the WHO/FCTC (CONICQ) and protect the National Tobacco Control Policy. The civil society also plays an important role in partnering with CONICQ to protect the policy.

Results and lessons learnt: Analyzing the 16 tobacco industry tactics for resisting effective tobacco control (table 1) described on WHO, 2008 publication, we identified the Brazilian responses to each one. We cannot say that we have been nulling the TI actions, or winning the battle, but we can say that we oppose them and make the process more transparent.

Conclusions and key recommendations: Tobacco industry is always evolving, sophisticated and looking to keep up with the government's actions. But government and civil society representatives for tobacco control are also steadily struggling to contain the industry's actions. This is a battle of unequal forces and there is still much to be done together.

Tob. Induc. Dis. 2018;16(Suppl 1):A678

DOI:10.18332/tid/84701

\subsection{Implementing sustainable funding mechanisms for tobacco control}

\section{2:30-14:00}

PS-979-2 Tracking investments by financial institutions in tobacco companies (2007-2016) - what tobacco control advocates need to know and do about it? P Lal ${ }^{1}$

'International Union Against Tuberculosis and Lung Disease, New Delhi, India. E-mail: plal@theunion.org

Background and challenges to implementation: Roughly $€ 4.1$ trillion in assets and investments are managed by socially responsible investment (SRI) funds worldwide which motivate institutional investors to take a more stringent view on ethical investing. Ethical investing precludes not investing in tobacco industry or its stocks. Tobacco is a cross-cutting area where such investments are forbidden. Tracking investments made by governments and private financial investors in the tobacco sector can influence in mitigating the proliferation of the industry in the future.

Intervention or response: This research examines investments made by the largest private banks in the world during 2007-2016 including those which conform to Global SRI standards using customised, paid-for banking databases (banker.com).

Results and lessons learnt: The analysis finds that in 2007, 42 of the top 50 global bank invest and support tobacco industry domestically and in offshore projects. However in 2016, this has reduced to 38 banks, with one bank which despite a tobacco exclusion policy had defaulted.

Conclusions and key recommendations: Definition of socially responsible investments within the perspective of screening tobacco investments is perceived variably by financial institutions. Also in the absence of a watchdog institution and few disincentives for truant behaviour, investors continue to invest tobacco companies. Tobacco control advocates need to monitor investments made by large lending banks and financial institutions in tobacco industry and ensure that banks which have committed to SRI codes conform to them.

Tob. Induc. Dis. 2018;16(Suppl 1):A679

DOI: $10.18332 /$ tid/84361

12:30-14:00

PS-980-2 Innovative health financing for tobacco control: the implementation and effectiveness of India's "Movie Rules"

N Murukutla' ${ }^{1}$ V Munish Gill ${ }^{2}$, N Singh Negi ${ }^{3}$, P Puri ${ }^{3}$, P Sinha ${ }^{4}$, V Mallik', S Mullin', F Tesfaye Tullu ${ }^{4}$

'Vital Strategies, Policy, Advocacy and Communication, New York, NY, United States of America, ${ }^{2}$ World Health Organization, India, New Delhi, India, ${ }^{3}$ Vital Strategies, Policy, Advocacy and Communication, New Delhi, India, ${ }^{4}$ World Health Organisation India, New Delhi, India.E-mail:nmurukutla@vitalstrategies.org Background: Tobacco consumption is a critical concern in populous low-income countries, like India. The social acceptability of tobacco perpetuates its use: the positive portrayal of tobacco in the media has been associated with increased consumption of tobacco. Hence, to counter the pro-tobacco imagery in the visual media, the Government of India modified its tobacco control law in 2011/2012, called as "Film Rule", requiring film theaters and television channels to screen pre-approved anti-tobacco public service announcements and a disclaimer at the beginning and during the middle of programs when tobacco consumption is depicted; and, health warning scrolls on screen while tobacco is in use onscreen.

Methods: Four studies were undertaken to monitor the genesis, implementation and effectiveness of the Rule. First, a news media analysis in the lead up the implementation of the Film Rule identified industry arguments against the Rule. Two observational studies, including exit interviews of cinemagoers, monitored the implementation and effectiveness of the Rule. Finally, legal research identified legal loopholes and opportunities. 
Results: Findings from this comprehensive set of studies identified standard arguments against the Rule - such as impingement of creative freedom, arguments that health warnings are ineffective - and provide guidance for addressing these attacks preemptively. The observational studies identified over $80 \%$ compliance with the Film Rule in film theaters but less than $10 \%$ compliance by television channels. Exit interviews among film-goers also found positive impact among those exposed to anti-tobacco messaging. Legal research identified strengths in the current law, enabling implementation, and opportunities for its advancement within existing media laws and procedures.

Conclusions: The Film Rule is a novel health financing mechanism for sustained mass media campaigns at low (or no) cost to governments. Our studies show positive impact of the Rule and offer valuable lessons for strengthening the law within India and for other countries considering similar approaches.

Tob. Induc. Dis. 2018;16(Suppl 1):A680

DOI:10.18332/tid/84379

12:30-14:00

PS-981-2 Imposing 1\% health development surcharge (HDS) to protect public health due to public demand through strong policy advocacy MRI Milon'

'Bangladesh Anti Tobacco Alliance (BATA), Tobacco Control, Dhaka,Bangladesh.E-mail: rimilon08@gmail.com

Background and challenges to implementation: Notwithstanding multiple achievements in tobacco control (FCTC ratification, law enactment-2005, formation of National, District and Upazila Task Force, increasing tobacco tax regularly, law amendment-2013 and implementation of GHW), Bangladesh has been facing a challenge of sustainable funding on tobacco control. Actually tobacco control activities are managed with the help of WHO and with the help of BI which is really inadequate. Intervention or response: Representatives of Bangladesh AntiTobacco Alliance visited Health Promotion Foundation, Thailand. With gained experience they conducted advocacy with policy makers, electronic and print media, executives of the government and political leaders for imposition of $2 \%$ surcharges on all tobacco products. Moreover, various activities have been adopted for creating public opinion. At one point, in the faces of challenges from tobacco companies, the activists have been able to convince the government that surcharges should be imposed from the harmful product for long-term tobacco control. Taxes, VAT and surcharges will increase if the price of cigarettes increases but revenue will not decrease. In order to protect public health and create sustainable funding for Tobacco Control to achieve SDG target, this policy provides policycompliance with the provision of HDS.

Results and lessons learnt: Given priority to public health, Government has imposed 1\% HDS on all tobacco products in 2013-14 fiscal year. Presently the fund has risen up to 6 billion taka which will increase gradually every year. The implementation of a policy on HDS management is in the final stage. At present, financial crisis of NTCC has been removed. As a result, sustainable fund has been formed in Tobacco Control through which SDG-3 will be implemented.

Conclusions and key recommendations: $1 \%$ surcharge will accelerate implementation of SDG targets as a sustainable fund for public health development and management of tobacco control activities.

Tob. Induc. Dis. 2018;16(Suppl 1):A681

DOI: $10.18332 /$ tid $/ 84500$

\section{$12: 30-14: 00$}

PS-982-2 Tobacco free companies - a corporate social responsibility approach

VB Yetapu' ${ }^{1}$,JR Ummadi ${ }^{1}$

'VChangeU, Health and Education, Hyderabad, India. E-mail: vb@vchangeu.com

Background: Research shows that when employers implement a Tobacco free policy, tobacco users are more likely to quit. Looking after the well being of the employees is an essential and indispensable part of any organization. As Corporate Social Responsibility (CSR) has become mandatory in India, we have conducted Healthy and Happy Living workshops under CSR activity for several public and private sector companies in 3 states and 13 districts of India.

Methods: The objective was to encourage employees and their family members to focus on key health behaviors such as increasing physical activity, improving eating habits, reducing stress, and ceasing tobacco and alcohol use among addicts. Conducted workshops under CSR activity for Gujarat State Electricity Corporation Ltd, covering seven power plants located in 8 districts, delivered 72 Sessions for the employees, family members, students and villagers. Conducted similar workshops for Exel Rubber Ltd in Telangana and Andhra Pradesh covering 5 districts, delivered 28 Sessions on Tobacco and Alcohol Awareness for the employees of five manufacturing units. Conducted workshops for DQ Entertainment Ltd and delivered 20 sessions for the associates and CSR team. We have spread the awareness about harms of Smoking, Chewing Tobacco on health and environment.

Results: Awareness through creative videos and innovative posters along with effective presentation in regional language has helped in achieving a better outcome. In house medical staff has supported the addicts in quitting process. Family members who attended the workshop have decided to keep their homes Tobacco Free for protecting the other family members from SHS. The Heads of the plant have released notices to all employees and the shop owners on prohibition of smoking in public places and to ban the sale of tobacco in their plants. 


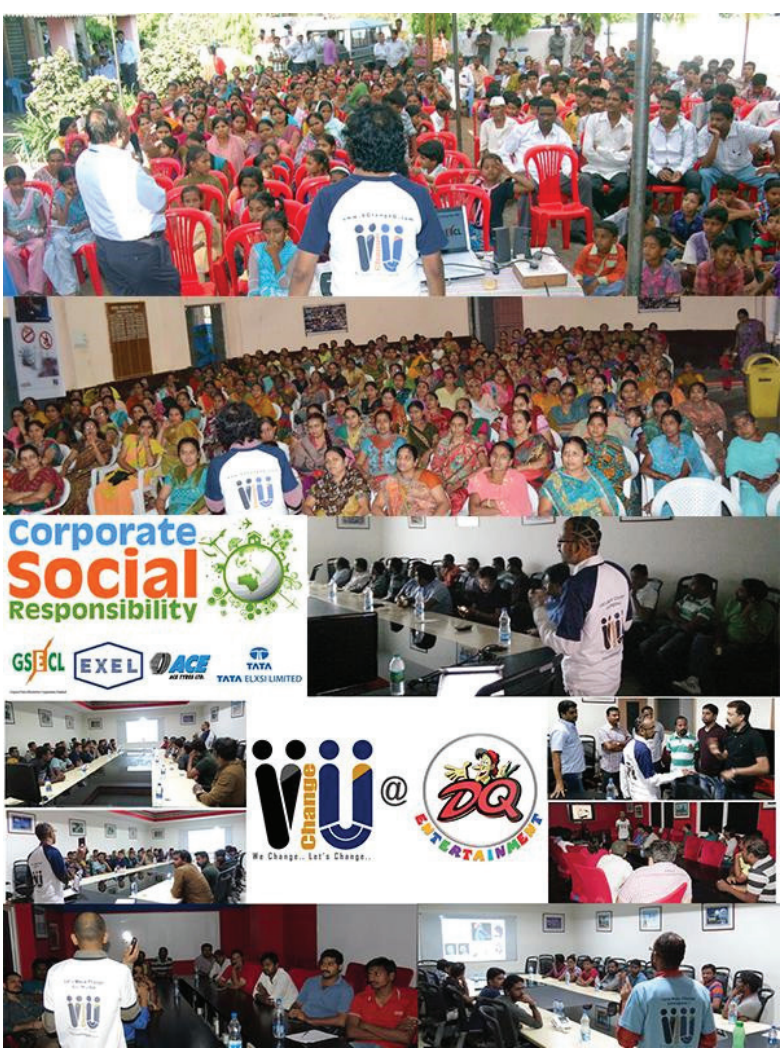

[VChangeU_CSR]

Conclusions: The success of these workshops attributed to conduct similar workshops in other companies through CSR approach.

Tob. Induc. Dis. 2018;16(Suppl 1):A682

DOI:10.18332/tid/84608

\subsection{Pricing, Taxation and countering illicit trade: Innovations and reform strategies}

\section{2:30-14:00}

PS-983-3 Political battle in increasing tobacco tax to support Universal Health Coverage in Indonesia NN Luntungan ${ }^{1,2}$, Y Meilissa ${ }^{1,3}$, A Dewi ${ }^{1}$, H Thabrany ${ }^{1,4,5}$ ${ }^{1}$ Center for Health Economics and Policy Studies University of Indonesia, Depok, Indonesia, ${ }^{2}$ National Commission on Tobacco Control Indonesia, Jakarta, Indonesia, ${ }^{3}$ Center for Indonesia's Strategic Development Initiatives, Jakarta, Indonesia, ${ }^{4}$ School of Public Health, University of Indonesia, Depok, Indonesia, ${ }^{5}$ The National Social Security Council, Jakarta, Indonesia. E-mail: nurul.luntungan@gmail.com

Background and challenges to implementation: Indonesia has been the champion of smokers and the heaven for cigarette industries. Indonesia has not sign/access FCTC. In 2014, Indonesia implemented the National Health Insurance program, to achieve Universal Health Coverage (UHC) by 2019. The UHC program is built by social health insurance principle. In the last four year, the program faced billions of dollars deficit. The government and the members/people face the increasing needs to finance its UHC. Experience from from other countries, like Phillipines, shows that tobacco tax can support UHC financing. Studies in Indonesia also show that people perception leads to desired result for public health, and revenue gain for the economy. However, policy making for tobacco control and tax is highly political. Indonesia is a country with one of the highest Tobacco Industry Interference Index. In contrast, Indonesia is having pressures from the Congress/Parliament to pass a Tobacco Bill, that protect the tobacco farmers and the cigarette industries.

Intervention or response: The authors have been studying to understand the political battles in increasing tobacco tax to expand fiscal space to finance the UHC. In-depth interviews and multi-sectoral focus group discussions among policy makers were conducted.

Results and lessons learnt: Several Ministries and including, Ministry of Health, Ministry of Finance and the National Health Insurance Agency see increasing tobacco tax will benefit public health and the economy. However, the political challenges from the Congress and some Ministries, particularly Ministry of Industry and Ministry of Labour have been blocked the people supports to raise tobacco tax.

Conclusions and key recommendations: Despite President statement about the important of tobacco control in reducing unnecessary spending among the poor, there is no movement in increasing high tobacco tax in support UHC. The proposed presentation will also include the high smoking prevalence, the rampant of cigarette advertising, and the political battles on tobacco control in Indonesia.

Tob. Induc. Dis. 2018;16(Suppl 1):A683

DOI:10.18332/tid/84069

\section{$12: 30-14: 00$}

PS-984-3 Indigenous and non-indigenous experiences and views of tobacco tax increases: findings from the ITC New Zealand Survey

A Waa ${ }^{1}$, R Edwards ${ }^{1}$, J Stanley ${ }^{2}$, S Kaai ${ }^{3}$, A C.K.Quah ${ }^{3}$, G Fong ${ }^{3,4}$ ${ }^{1}$ University of Otago, Public Health, Wellington, New Zealand, ${ }^{2}$ University of Otago, Dean's Department, Wellington, New Zealand, ${ }^{3}$ University of Waterloo, Psychology, Waterloo, ON, Canada, ${ }^{4}$ Ontario Institute for Cancer Research, Toronto, ON, Canada.E-mail: richard.edwards@otago.ac.nz

Background: Tobacco taxes are effective for reducing smoking prevalence and in New Zealand (NZ) there have been annual 10\% tax increases since 2010. There is ongoing debate in NZ about the potential unequal impact and regressive nature of these taxes for Māori (indigenous peoples of NZ). This study explored Māori and non-Māori experiences of tobacco tax increases and their support for tobacco taxation policies.

Methods: Data were drawn from the first wave (Aug 2016-April 2017) of the International Tobacco Control (ITC) NZ Survey. The sample comprised 1,023 smokers and 138 ex-smokers, and within the overall sample 358 who identified as Māori and 803 who identified as non-Māori.

Results: Around a third of participants reported recent tax increases had led them to think about quitting. Slightly more nonMāori (30\%) reported the increases had caused them to reduce tobacco consumption compared to Māori (24\%).

Despite most participants reporting tobacco tax was too high, almost a third across all ethnic groups supported ongoing tax increases. This support increased to two thirds if the tax was tied to tobacco control interventions. Almost a third of all participants supported large annual tax increases of $20 \%$ to help achieve NZ's goal of being smokefree by 2025. Support was particularly strong (90\%) for using revenue from tobacco tax to provide programmes supporting smoking cessation and discouraging smoking uptake. Conclusions: While tobacco taxes can reduce smoking, they may have slightly less effect among Māori. This may mean Māori bear a disproportionate amount of burden from tobacco tax. Despite this, there is good support among Māori and non-Māori for 
ongoing tax increases and use of tobacco tax revenue to support tobacco control interventions. There is good support among smokers for tobacco tax to be used as a strategy to help achieve NZ's tobacco endgame goal.

Tob. Induc. Dis. 2018;16(Suppl 1):A684

DOI:10.18332/tid/84075

\section{$12: 30-14: 00$}

PS-985-3 Proxy purchasing - a project to determine the awareness of the public in England of a new offence and improve practice

J Pullen', H Wareing ${ }^{2}$, P Hooper ${ }^{2}$

${ }^{1}$ Revealing People, St Austell, United Kingdom, ${ }^{2}$ Improving Performance in Practice (iPiP), Warwick, United Kingdom. E-mail: Inaylor@ipip.co.uk

Background and challenges to implementation: The introduction of an offence for proxy purchasing of tobacco in England in 2015 was well received as a means of tackling the supply of tobacco to young people.

The offence of a proxy purchase is committed by the adult that buys the tobacco product on behalf of the young person under 18 years, not the retailer that sells the product. It is not a straightforward matter for Regulatory Services to investigate or to take enforcement action on.

Little data was available to indicate the scale of the problem or how aware adults were of the offence.

Intervention or response: The project gathered insights around knowledge of the law regarding proxy purchasing; how behaviours had changed; how awareness and compliance could be improved and how communities can be encouraged to support compliance. Insight gathering was carried out systematically in two geographic areas among selected groups of the population. This method was positively received and generated additional information on other related issues.

Results and lessons learnt: The results revealed that the term a proxy purchase was not recognised by the great majority of participants; the majority of participants had experienced young people asking an adult to purchase cigarettes; proxy purchasing was seen very negatively but legislation was generally not the reason why people would not proxy purchase. It was widely recognised that proxy purchasing was only one way young people obtain tobacco. There was discussion with those interviewed about the relative risks of the ways young people obtain tobacco. Participants identified a variety of ways of informing local communities. Including targeting specific groups to ensure that individuals received relevant information in appropriate formats. Conclusions and key recommendations: When planning future actions to ensure compliance with the proxy purchasing legislation, consideration must be given to the likelihood of any unintended consequences.

Tob. Induc. Dis. 2018;16(Suppl 1):A685

DOI:10.18332/tid/84097

\section{$12: 30-14: 00$}

PS-986-3 The prevalence of illicit cigarette consumption and related factors in Vietnam: results from GATS 2015 S Dao ${ }^{1,2}, \mathrm{M} \mathrm{Hoang}^{3}, \mathrm{G} \mathrm{Kim}{ }^{4}, \mathrm{H}$ Phan $^{5}, \mathrm{H}$ Doan $^{5}, \mathrm{~L} \mathrm{Nguyen}{ }^{6}, \mathrm{~N}$

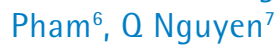

${ }^{1}$ Thuongmai University, Faculty of Economics and Law, Ha Noi, Viet Nam, ${ }^{2}$ Vietnam University of Commerce, Hanoi, Viet Nam, ${ }^{3}$ Hanoi University of Public Health, Hanoi, Viet Nam, ${ }^{4} \mathrm{Hanoi}$ Medical University, Hanoi, Viet Nam, ${ }^{5}$ Vietnam Ministry of
Health, Hanoi, Viet Nam, ${ }^{6}$ World Health Organisation, Country Representative Office, Hanoi, Viet Nam, ${ }^{7}$ General Statistics Office, Hanoi, Viet Nam. E-mail: daotheson@tmu.edu.vn

Background: Illicit trade is one of the main obstacle to tobacco tax and other tobacco control policy reform in Vietnam. The tobacco industry gives different estimates on the size of illicit trade and claims that increasing tax will only drive smokers to illicit products and increase government revenue loss. This study examines the illicit cigarette consumption in Vietnam and its related factors.

Methods: Data used in this research were from the Global Adult Tobacco Survey conducted in Vietnam in 2015. This is a national representative survey with sample size of nearly 9000 individuals. Descriptive statistics analysis was carried out to estimate the size and examine the patterns of illicit cigarette consumption.

Results: The estimated market share of illicit cigarettes in Vietnam is $26 \%$. More illicit cigarette consumption was found in urban area and in provinces in the south.

The average price of illicit cigarettes was $50 \%$ higher than the average price of legal cigarettes. This suggests that price is not the driving factor in choosing to use illicit cigarettes. This result different from other countries in the region where usually illicit cigarettes are cheaper. There is no significant difference in age, asset-index quintile between illicit and legal cigarette smokers.

$64 \%$ of illicit cigarette smokers reported buying from kiosks, 34\% reported buying from tea stalls and street vendors. This suggest illicit cigarettes can be accessed rather easily in domestic market. Conclusions: As illicit cigarettes are more expansive and price is not the driving factor, tax on cigarettes can be increase higher to reduce total consumption without having too much impact on the illicit trade market.

Smuggling control activities should be reinforced in domestic markets to reduce the easiness of smokers in seeking and buying illicit cigarettes.

Tob. Induc. Dis. 2018;16(Suppl 1):A686 DOI:10.18332/tid/84206

$12: 30-14: 00$

PS-987-3 Expected cigarette price premium on willingness to quit among men smokers in Vietnam BT Tran ${ }^{1,2}$, KM Nguyen ${ }^{3}$, DT Tran ${ }^{3}$, XT Nguyen Thi' ${ }^{1}$ MK Lim ${ }^{1,4}$, $\mathrm{S} \mathrm{ChO}^{5}$, J-K Oh $\mathrm{h}^{1,4}$

${ }^{1}$ National Cancer Center Graduate School of Cancer Science and Policy, Department of Cancer Control and Population Health, Goyang, Korea, Republic of, ${ }^{2}$ Hue University of Medicine and Pharmacy, Institute for Community Health Research, Hue City, Viet Nam, ${ }^{3}$ Da Nang University of Medical Technology and Pharmacy, Faculty of Public Health, Da Nang, Viet Nam, ${ }^{4}$ National Cancer Center, Cancer Risk Appraisal \&t Prevention Branch, Goyang, Korea, Republic of, ${ }^{5}$ Seoul National University, Graduate School of Public Health, Seoul, Korea, Republic of. E-mail: binhthang1001@ncc.re.kr

Background: Vietnamese government is targeting at achieving tobacco control goal with $10 \%$ reduction by year 2020 . In the last two decades, cigarette price in Vietnam have not been increased. According to article 6 of FCTC, raising the cigarette price is known as one of the most effective strategies to combat cigarette smoking. The aim of the study was to estimate the required price that would make smokers attempt to quit and identify the predictors of required price to quit.

Methods: A cross-sectional survey was conducted with a total of 820 current smokers who live in third largest city of Vietnam. A structured questionnaire was adopted from International Tobacco 
Control (ITC) project surve. Bayesian quantile regression and Bayes factor were applied to predict factors associate with cigarette required price.

Results: Out of $56 \%$ (461 people) of smokers were suggested price to quit with age range from 18 to 65 years. The median required prices to quit per pack, US \$2.7 IQR ( $\sim 2$ to 4.4$)$, increased 2.8 times (2.04 to 4.54) higher than usual price ( US \$ 1). If the required price reaches to at median price (higher 2.8 times), $56 \%$ of smokers suggested price tend to be quitting seriously. predictors of cigarette price were significantly associated with the lower required price including perceived awareness of warning label, smoke-free workplace and receiving advices from physicians. In contrast, high educations, having good health, heavy smoker and failure of quitting were significantly associated with the higher price across all quantiles.

Conclusions: These results suggest the potentially useful approach to setting price increase in Vietnam. Require prices have been considered various factors regarding non-tax policy and quit intention, and appropriate approach to those group with failure quitting history, high education, heavy smoker

Tob. Induc. Dis. 2018;16(Suppl 1):A687

DOI:10.18332/tid/84316

\section{$12: 30-14: 00$}

PS-988-3 Effectiveness of tobacco tax in tobacco control: evidences from India

SK Rout ${ }^{1}$, S Hooda ${ }^{2}$, VG Munish ${ }^{3}$, P Sinha ${ }^{3}$, F Tullu ${ }^{3}$, M Arora ${ }^{4}$ ${ }^{1} \mathrm{PHFI}$ IIPH Bhubaneswar, Bhubaneswar, India, ${ }^{2}$ ISID, New Delhi, India, ${ }^{3}$ WHO Country Office India, New Delhi, India, ${ }^{4} \mathrm{PHFI}$, New Delhi, India. E-mail: sarit.kumar@phfi.org

Background: Tobacco tax is proved to be the most important cost effective instrument of tobacco control. There are ample evidences suggesting the role of tax in reducing tobacco consumption. However, there are limited evidences regarding the effectiveness of tobacco tax in tobacco control in India. This study has been undertaken to address the current knowledge gap.

The objectives are to:

- examine tax structure, tax rates and price trends across tobacco products

- estimate affordability of tobacco products due to rise in income

- examine excise and total tax burden across tobacco products Methods: This study used secondary data from Government publications: Annual Survey of Industry (ASI), Ministry of Finance, Labour bureau, during 2005-06 to 2015-16. It compared wholesale and retail price indices of tobacco products with the general price indices to estimate the relative price increase of tobacco products. Affordability was measured comparing rise in per capita income and inflation rate of tobacco products. For tax burden, the share of excise tax in the retail price was calculated. Results: The relative Whole Sale price Index (WPI) of tobacco products showed increasing trend between 2005-05 and 201516. Across products, the relative WPI was lowest for bidi. Affordability of cigarette increased during 2005-06 to 2012-13 and declined during 2013-14 to 2015016. For bidi and chewing tobacco, affordability increased over the years. The excise tax burden was $40 \%$ for cigarette and was only $4 \%$ for bidi in 2015 16. Total tax burden on bidi and cigarette was $20 \%$ and was $59 \%$ respectively.

Conclusions: This study suggests to increase prices of all tobacco products to raise the excise burden comparable to the international standard to make it more effective from a tobacco control perspective. More importantly, the tax rate on bidi has to be substantially increased for which affordability is declining.

Tob. Induc. Dis. 2018;16(Suppl 1):A688

DOI:10.18332/tid/84321

\section{2:30-14:00}

PS-989-3 Media advocacy for building support for taxation of all tobacco products at the highest rate under the new Goods \& Service Tax (GST) regime B Mathew

'Voluntary Health Association of India, Delhi, India. E-mail: binoymathew84@gmail.com

Background and challenges to implementation: Article 6 of the FCTC enlists price and tax measures as an essential strategy for reducing demand for tobacco products. Raising taxes on tobacco is the single most effective way to encourage tobacco users to quit and prevent youth from starting tobacco use.

Goods and Services Tax (GST) is a new system of taxation in India aims to streamline the taxation structure in the country and replace the complete range of indirect taxes with a singular GST to simplify the taxation procedure. Tobacco products should be subjected to the highest level of tax under GST $(28 \%$ + cess) with no segmentation.

Intervention or response: VHAI engaged the media through press releases, Op-eds and keeping the media updated with the developments happening at policy level. One to one interactions were held with the journalists covering health and financial news. Social media was used as a tool for outreach through twitter and Facebook.

Results and lessons learnt: Over 563 earned media stories on GST generated by VHAI during the period from March 2016 - June 2017. Timely Op-Eds in key national dailies on tobacco taxation, media outreach nationwide for higher taxes and cess on tobacco covered in all the national dailies and 13 vernaculars. As a result, tobacco is taxed at the highest demerit rate of $28 \%+$ Cess. Conclusions and key recommendations: Keeping the tobacco products at the highest GST rate - 28 percent plus higher cess will have a major impact on the prices of the tobacco products which will encourage users to quit and youth from taking up this habit.

Tob. Induc. Dis. 2018;16(Suppl 1):A689

DOI:10.18332/tid/84416

12:30-14:00

PS-990-3 Public support on increasing cigarette prices and excise to finance UHC in Indonesia

H Thabrany ${ }^{1}$, RI Wardani ${ }^{1}$, Z Laborahima ${ }^{1}$

${ }^{1}$ Universitas Indonesia, Center for Health Economic and Policy Studies, Depok, Indonesia. E-mail: khafidlu@gmail.com

Background: Indonesia has the highest prevalence of adult men smokers with $67 \%$. Cigarette related disease will burden JKN (UHC) which currently has burden claim ratio of $115 \%$. The difficulties in collecting contribution from non-salaried workers are blamed to contribute the deficit. Many countries have earmarked cigarette excise to supplement financing health program. This study explored the possibility public support on increasing cigarette prices and excise to meet financial shortage of the JKN.

Methods: The study used telephone polling conducted from December 2015 to January 2016. 1.000 respondents was selected using systematic random sampling by the interval of 20.000 of mobile phones numbers. Analysis was focused on how various groups support increasing cigarette prices and excise 
and the allocation to finance JKN. The final analysis was logistic regression to assess any difference in supporting the price and excise increase.

Results: The polling showed, in total, $80.3 \%$ respondents support increasing cigarette price and exice to supplement health financing of JKN. The proportion of non smokers who supported the earmarking was higher (83.4\% ) compared to smokers $(75.9 \%)$, but the difference is not significance in the final model. There are plenty of room to mobilize money through increasing prices and excise of cigarettes since more than $72.3 \%$ of smokers said that they would stop smoking if the price of cigarette is above IDR 50,000 per pack; far above current prices. If the prices of cigarettes are double and the excise level reaching maximum allowable levels, there is potential to increase revenue up to IDR 70 Trillion that is almost equivalent to estimated all claims of JKN in 2016.

Conclusions: This study found that large majority (80\%) of nonsmokers and $76 \%$ smokers supported increasing cigarette prices and excise to supplement financing for the JKN. The potential money to supplement $\mathrm{JKN}$ is double of the current revenue of JKN.

Tob. Induc. Dis. 2018;16(Suppl 1):A690 DOI:10.18332/tid/84450

\section{$12: 30-14: 00$}

PS-991-3 Neoliberal policy impact: supply-side growth and emergence of duality in Turkish tobacco product market

\section{E Evrengil}

'Turkish National Coalition on Tobacco or Health, Istanbul, Turkey.E-mail: efzagil@yahoo.com

Background: In Turkey, adoption and implementation of MPOWER strategies were accompanied by a neoliberal tobacco policy framework aiming at supply-side growth, initiated in 1980's and culminating in Law No 4733 in 2002, which solidified liberalization, privatization, and market efficiency rules for tobacco manufacturing and trade, and guaranteed oligopoly conditions for transnational tobacco companies (TTCs). This study employs empirical market dynamics data to argue that demand reduction strategies cannot be pursued effectively in tandem with neoliberal policies.

Methods: Legal market dynamics are gauged with official data (2003-2016) on licenced tobacco products. The magnitude of illicit product market is assessed by employing prevalence data and estimations in secondary sources.

Results: During 2003-2016,

(a) Manufacturing and exports of licenced products have risen sharply (Cigarette manufacturing by 3 billion sticks/year; 2 new cigarette brands licenced per month). The declining trend in legal cigarette sales since 1999 was disrupted during last 5 years and was pushed upward.

(b) In addition to illicit cigarettes (market share $7.5 \%$ ), the staggering growth in illicit RYO tobacco, estimated at 15,000 tonnes for 2016 by Tobacco Experts Association, represents 20.5 billion cigarette equivalents, which explains legal sales of 19 billion macarons (empty cigarette tubes) in 2016. Estimated share of illicit products in total consumption has thus reached unprecedented level of $27 \%$. Furthermore, using prevalence data, WPT market is estimated as $99 \%$ illicit.

Conclusions: Both legal and illicit tobacco product markets are growing in Turkey, indicating neoliberal framework has had far larger de facto impact than demand reduction efforts, and Law No 4733 is failing, given emergent duality between legal and illicit markets epitomised by TTCs and domestic outfits, respectively. This picture is in clear defiance of FCTC objectives, principles, and obligations, and calls for reversal of neoliberal legacy and incorporation of supply-side measures to control tobacco manufacturing and trade and increase demand reduction effectiveness.

Tob. Induc. Dis. 2018;16(Suppl 1):A691

DOI: $10.18332 /$ tid $/ 84530$

\section{$12: 30-14: 00$}

\section{PS-992-3 Economic contributions of the bidi} manufacturing industry in India

S Bhaumik', P Datta ${ }^{1}$, M Arora ${ }^{1}$, A Barman ${ }^{1}$, GP Nazar ${ }^{1}$, VG Munish², PK Sinha², F Tullu²

${ }^{1}$ Public Health Foundation of India, Gurgaon, India, ${ }^{2}$ World Health Organisation - India, New Delhi, India. E-mail: soumyadeep. bhaumik@phfi.org

Background: Bidi is the most popular tobacco smoking product in India and commands an overwhelming market share of $85 \%$. Perceptions of potential economic loss is a major impediment against any bidi control measure. Previous research has focussed on unregistered bidi sector and at a single time point. We estimated the economic contribution made by the entire bidi industry at three time points.

Methods: We conducted secondary analyses from data of nationally representative cross-sectional surveys conducted in 2000-01; 2005-06; 2010-11: 1) Annual Survey of Industries 2) National Sample Survey (NSS) and 3) NSS Employment and Unemployment Survey. The total gross value added (GVA) has been adjusted for inflation by Wholesale Price Index using 20042005 base price.

Results: We found that there was a decline in overall bidi industry GVA in 2005-2006 from 2000-2001 by ₹ 13.7 billion but it has grown back to 2000-2001 levels of ₹ 48.2 billion in 2010-2011(0.65\% of the GVA contributed by the manufacturing sector). Ratio of GVA contributed by unregistered to registered bidi firms has decreased by half in between 2005-2006 and 2010-11, although the proportion of employment provided by the unregistered firm remained constant at $89-81 \%$ during this period. There has been a gradually shift to smaller-size units in between 2000-2001 and 2010-2011.

Conclusions: The bidi industry does not make any significant economic contribution to the Indian economy. Employees in unregistered sector receive no welfare benefits. The shift towards smaller-size units might be reflective of contractualisation to enjoy excise tax exemption which was prevalent during the pre-Goods and Services Tax (GST) era. Better regulation of the sector by mandatory registration of bidi firms together with high GST taxes without exemption will lead to improved revenue, improvement of work conditions and act as a tobacco control measure too as consumption is price elastic in nature.

Tob. Induc. Dis. 2018;16(Suppl 1):A692

DOI: $10.18332 /$ tid $/ 84602$

$12: 30-14: 00$

PS-993-3 Social media sentiment analysis of trending topic cigarette price issue 


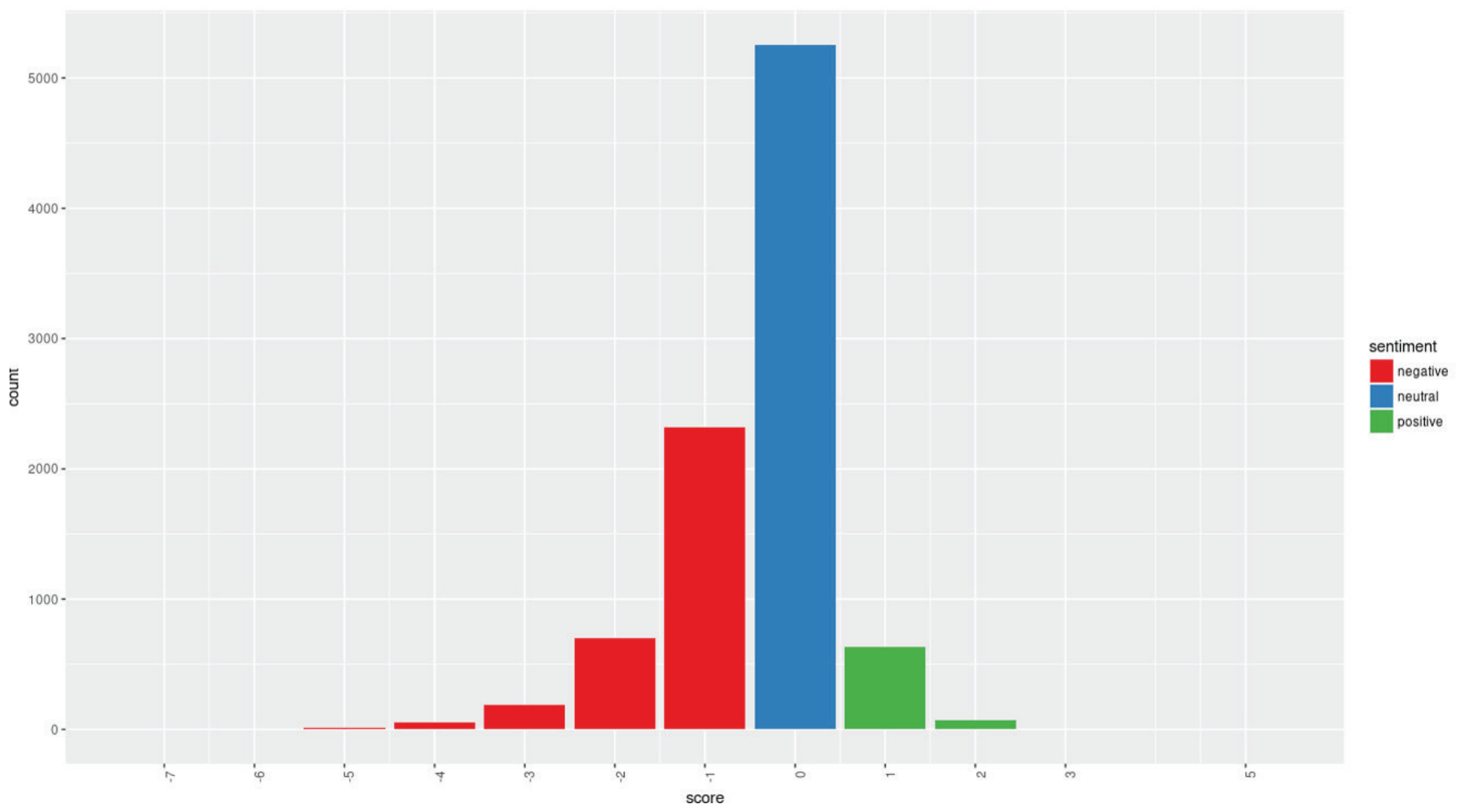

\section{A Andalan ${ }^{1}$, Z Zahrina' ${ }^{1}$, F Firhat ${ }^{2}$}

${ }^{1}$ Center for Health Economics and Policy Studies, Depok, Indonesia, ${ }^{2}$ Universitas Indonesia, Depok, Indonesia. E-mail: aldiandalan@gmail.com

Background: Cigarette price in Indonesia was increasingly booming in 2016. It all started from a controversial survey that conducted by CHEPS UI about public opinion and support toward cigarette price increase. The survey shows that 72 percent smokers agree to increase cigarette price until IDR (Indonesian Rupiah) 50.000.

Nevertheless, this survey creates diverse responds throughout various media, TV channels, newspapers, and online media such as twitter. Hence, this research curious and want the answer from how twitter social media users responds to this issue from time to time and which words that frequently appear to the social media.

Methods: This research use sentiment analysis and word cloud to analyze tweet texts using Python 2.7 and R It use convenience sampling (availability sampling), with 12.349 tweets are obtained. The time frame of data is from 24th June 2016 until 28th January 2017.

Results: In sentiment analysis we define sentiments into three categories of response to the cigarette price increase. Three categories are negative (opposing stance), positive (supporting stance), and neutral (can be canceled each other or none of them). Positive stance includes lexicon of keywords, such as health, household concern, national concern, politics, convenience, support, beliefs, and public figure.Negative stance includes keywords such as beliefs, unrest, uncertainty or rumor, farmer, brands, propaganda, ineffective, alternative, opposition, persuasive, tobacco build law, and culture. Besides the most obvious keywords relating to the issues (tobacco,cigarette, price), the economic issues relating to the immediate fate of tobacco farmer is one of the primary concern of netizens.

Conclusions: The result shows that negative sentiments still overwhelm the positive ones. Tweets tend to very booming on
August and September 2016 and the issues slowing again after that. Words that frequently occur despite cigarette price and tobacco is farmer.

Tob. Induc. Dis. 2018;16(Suppl 1):A693

DOI: $10.18332 /$ tid/84660

[Histogram of sentiment analysis]

\section{$15: 45-16: 45$}

LB-1359-3 The economics of tobacco control in Nigeria: modelling the fiscal and health effects of a tobacco excise tax change in Nigeria

C Onyekwena ${ }^{1,2}$, P Chukwuemelie Akanonu ${ }^{1}$, J Ishaku

${ }^{1}$ Centre for the Study of the Economics of Africa, Abuja, Nigeria, ${ }^{2}$ The African Capacity Building Foundation, Harare, Zimbabwe. E-mail: conyekwena@cseeafrica.org

Background: The study examines potential for tobacco tax to contribute to mobilizing domestic resources, and improving public health in Nigeria.

Methods: Primary and secondary data were obtained from domestic sources. Cigarette prices were collected through surveys of randomly selected retailers in six states across six geopolitical zones in Nigeria. For analyses, two tax simulations models were used: a home-grown model and the Tobacco Excise Tax Simulation Model (TETSim). Each model was run under two scenarios: Scenario 1-a 275 percentage increase in tobacco taxes, in line with WHO excise tax benchmark of 75 percent of retail price; Scenario 2 - a less stringent 150 percentage increase in line with a 50 percent benchmark.

Results: A more stringent increase in excise tax (Scenario 1) in both models would allow for the maximization of public health and government revenue within a one year period. Under scenario 1: total cigarette consumption will fall by 19.6 percentage points (short-run) and 71.4 percentage points (long-run); 4 million people (short-run) and 11 million people (long-run) will likely quit or fail to initiate smoking; 1 million (short-run) and 4 million (long-run) of those who quit could be saved from smoking-related deaths. Under scenario 2: cigarette consumption will fall by only 
12.7 percentage points, 2 million people will likely quit or fail to initiate smoking, 664,000 lives could be saved from smokingrelated deaths. In terms of fiscal impact, an increase in excise tax would create a 114.5 percent increase in government revenue under scenario 1 -from approximately N1.48 billion to N3.2 billion. Under scenario 2 increase by 75.7 percent - N2.6 billion. Conclusions: An effective tobacco control tax policy in Nigeria will entail a 275 percent increase in excise tax, a change in tobacco tax structure-from ad valorem to specific; and stronger tax administration and revenue-collecting agencies to yield the optimal results.

Tob. Induc. Dis. 2018;16(Suppl 1):A694

DOI:10.18332/tid/84729

\section{$15: 45-16: 45$}

LB-1365-3 Burden of disease attributable to tobacco use and impact of different taxation schemes in Latin America

A Bardach 1 , A Palacios ${ }^{1}$, MB Rodríguez ${ }^{1}$, A Alcaraz ${ }^{1}$, A Ciapponi ${ }^{1}$, S Virgilio' ${ }^{1}$, F Augustovski ${ }^{1}$, A Pichón-Riviere ${ }^{1}$

${ }^{1}$ Institute for Clinical Effectiveness and Health Policy, Buenos Aires, Argentina.E-mail: abardach@iecs.org.ar

Background: Tobacco use is the leading cause of preventable deaths worldwide. The objective of this study was to estimate the burden of disease attributable to tobacco use and to evaluate the potential impact of increasing cigarette's price through taxes in Argentina, Bolivia, Brazil, Chile, Colombia, Costa Rica, Ecuador, Honduras, Mexico, Paraguay, Peru and Uruguay-countries which encompass $84 \%$ of Latin America's population.

Methods: A microsimulation model was used to estimate the chances people have to get sick or die from 17 smoking-related conditions. An extensive review was conducted to identify key epidemiological and cost data relevant to each country.

Results: Every year, smoking is responsible for 293,364 deaths, 862,484 COPD cases (Chronic Obstructive Pulmonary Disease), 832,791 cardiovascular events, 131,037 cancer diagnoses and 28,098 USD millions in direct medical costs to the health systems in these countries. The proportion of health expenditure that these burden represent range from $3.2 \%$ in Honduras to $16,6 \%$ in Uruguay; and the proportion of the total GDP (Gross Domestic Product) ranged from $0.3 \%$ to $1.5 \%$ respectively. The percentage of attributable health expenditures recovered through tobacco taxes also varies widely: Mexico, Honduras, Argentina and Chile recover over 45\%; in Ecuador tax revenues reach 40\%; Brazil, Costa Rica, Paraguay and Uruguay recover less than $30 \%$, and Bolivia, Colombia and Peru, less than $10 \%$.

Different scenarios of cigarette price increases through taxes were modeled for each country. An example -the case for Brazil- is shown in the table below.

\begin{tabular}{lccc} 
Oigarette price increase & & & \\
Ilnrougl taves & $25^{\circ}$ & $50^{\circ}$ & $100 \%$ \\
\hline Avoided deaths & 68,241 & 136,482 & 272,964 \\
Avoided events & 336,099 & 672,199 & $1,344,398$ \\
Direct costs avoided (USD millions) & 4,866 & 9,732 & 19,464 \\
Indirect costs avoided (USD millions) & 2,995 & 8,980 & 8,411
\end{tabular}

[Potential benefts on different scenarios, Brazil]

Conclusions: The burden of disease and related direct health expenditure in Latin America is very high and tax revenues from the sale of tobacco products are far below the levels needed to cover it. Increasing cigarette's price through taxes is an effective intervention to reduce tobacco-attributable burden of disease and health expenditures in Latin America.

Tob. Induc. Dis. 2018;16(Suppl 1):A695

DOI: $10.18332 /$ tid/84750

\section{$15: 45-16: 45$}

LB-1373-3 Affordability of cigarettes products in the WHO Report on the Global Tobacco Epidemic, 2017

R Cherukupalli ${ }^{1}$, A-M Perucic ${ }^{2}$

1Johns Hopkins University, New York, NY, United States of America, ${ }^{2}$ World Health Organisation HQ, Geneva, Switzerland. E-mail: rcheruku@jhu.edu

Background: The idea of whether cigarettes have become cheaper or more expensive over time can be answered in two ways, one with reference to real prices alone (i.e whether prices change in relation to the prices of other goods and services in a country) and the other with reference to smokers' ability to purchase cigarettes (i.e. whether cigarettes became more or less expensive relative to income).

Methods: The affordability of cigarettes for each of the years 2008, 2010, 2012, 2014 and 2016 was measured by the per capita GDP required to purchase 2000 cigarettes of the most sold brand reported in that year, as also the cheapest and premium brands. To assess whether affordability changed on average since 2008 , the average annual percentage change in affordability was calculated as the least squares growth rate for all countries with three or more years of data, including data for 2016. In addition, analysis of the direction and magnitude of year-by-year changes in affordability was conducted.

Results: Cigarette price increases outpaced per capita GDP growth in 80 countries and did not significantly differ from per capita GDP growth in 73 countries, but fell short in 23 countries. The WHO Euro region, with the greatest prices and tax shares, has also seen the most success in reducing affordability of cigarettes. Countries at the top category of achievement in taxation in 2016 were also the most successful in ensuring that cigarettes, on average, became less affordable in the 8-year period since 2008. Few countries are able to make cigarettes less affordable in every successive year.

Conclusions: Countries with the highest tobacco excise taxes have been the most successful at reducing the affordability of cigarettes. Measures of affordability are easy to compute and track policy change, but are most informative when averaged over multiple years given high annual variation.

Tob. Induc. Dis. 2018;16(Suppl 1):A696

DOI:10.18332/tid/84762

\subsection{Advances in protection from tobacco pollution, second- and third-hand smoke}

\section{2:30-14:00}

PS-1000-3 Achieving the very first successful smoke free city in Myanmar: a case study of Shan State

SWZ Hlaing', MM Cho ${ }^{2}$

${ }^{1}$ Ministry of Health and Sports, State Health Department, Taungyi, Myanmar, ${ }^{2}$ SEATCA, Health Promotion Fund, Bangkok, Thailand.E-mail:swinzh@gmail.com

Background and challenges to implementation: Myanmar became the party of FCTC in 2004. The National Programme on Tobacco Control was officially launched in January 2000 before becoming the party. Despite of the commitment that Myanmar 
government has and the national law prohibits smoking in many areas including public, the implementation is a huge challenge when there is no funding specifically for tobacco control.

Intervention or response: Starting form 2014, Tobacco Control Cell, Ministry of Health Myanmar in collaboration with Southeast Asia Tobacco Control Alliance conducted national smoke free workshop. With the guidance of National Programme on Tobacco Control, it was decided that smoke free cities are needed to develop in Myanmar. Inle region and Pindaya cave from Shan State were selected to implement, which are tourist attractive areas, during the workshop.

Results and lessons learnt: Different advocacy meetings with several stakeholders were conducted. After getting the political commitment, different strategies were discussed in detailed with major stakeholders who will be involving with the campaign. The campaign started with the education and awareness raising period using the smoke volunteers who are trained with the standard manual. Several coordination meetings were conducted for better

\begin{tabular}{|c|c|c|c|c|}
\hline & & $\begin{array}{l}\text { Ban } \\
\text { smoking in } \\
\text { the outside } \\
\text { seating of } \\
\text { restaurants }\end{array}$ & $\begin{array}{l}\text { Ban } \\
\text { smoking } \\
\text { in the } \\
\text { outside } \\
\text { seating of } \\
\text { bars }\end{array}$ & $\begin{array}{l}\text { Ban } \\
\text { smoking } \\
\text { in busy } \\
\text { strects }\end{array}$ \\
\hline \multirow{4}{*}{$\begin{array}{l}\text { THS } \\
\text { increases } \\
\text { risk of } \\
\text { respiratory } \\
\text { diseases in } \\
\text { children }\end{array}$} & $\begin{array}{l}\text { Disagree or } \\
\text { Don't know } \\
(\%)\end{array}$ & 63.5 & 42.7 & 79.2 \\
\hline & Agree (\%) & 78.0 & 66.3 & 86.6 \\
\hline & $\begin{array}{l}\text { Crude OR } \\
(95 \% \mathrm{Cl})\end{array}$ & $\begin{array}{c}2.04(1.30- \\
3.19)^{* *}\end{array}$ & $\begin{array}{c}2.63(1.71- \\
4.05)^{* * *}\end{array}$ & $\begin{array}{c}1.70(1.00- \\
2.90)\end{array}$ \\
\hline & $\begin{array}{l}\text { Adjusted OR } \\
(95 \% \mathrm{Cl})\end{array}$ & $\begin{array}{c}2.13(1.35- \\
3.34)^{* *}\end{array}$ & $\begin{array}{c}2.91(1.87- \\
4.53)^{* * *}\end{array}$ & $\begin{array}{c}1.86(1.08- \\
3.20)^{*}\end{array}$ \\
\hline
\end{tabular}

$\mathrm{Lai}^{3}, \mathrm{TH} \mathrm{Lam}^{2}$

'University of Hong Kong, School of Nursing, Hong Kong, Hong Kong, ${ }^{2}$ University of Hong Kong, School of Public Health Hong Kong, Hong Kong, ${ }^{3}$ Hong Kong Council on Smoking and Health, Hong Kong, Hong Kong. E-mail: yongdang@hku.hk

Background: The harms of thirdhand smoking (THS) are being discovered, but limited studies investigated people's knowledge on these harms, especially in Asian population. Knowledge on harms of THS may increase support on tobacco control, as shown in the case of recognizing harms of secondhand smoking. We examined the association between knowledge on harms of THS and support on smokefree policies.

Methods: In the Tobacco Control Policy-related Survey 2015 using random digit dialing, 902 of 1834 never smoking Hong Kong citizens aged 15+ were randomly selected to report their knowledge on harms of THS and support on smokefree policies. Respondents were asked if they agreed "THS increases risk of respiratory diseases in children". Respondents also indicated their support on an overall extension of smokefree zones and banning smoking in 8 different public areas. Adjusted odds ratios (AORs) from separate logistic regressions showed the associations between knowledge on harms of THS and support on smokefree policies in never smokers, adjusting for socio- collaboration among different stakeholders. The campaign was successful launched on 10 September 2015.

Conclusions and key recommendations: Therewere many challenges and obstacles along the way including but not limited to funding, political support, ownership problem and interest from other related sectors. The main successful factor is gaining the political commitment from the state government. Dr. U Myo Tun, the State Social Welfare Minister, is the champion of the smoke free Shan movement. Keeping the lessons learnt from the past few years, Shan is planning to move forward with more places and cities in the near future.

\section{Tob. Induc. Dis. 2018;16(Suppl 1):A697}

\section{DOI:10.18332/tid/84324}

\section{2:30-14:00}

PS-1001-3 Knowledge on harms of thirdhand smoking is associated with greater support on smokefree policies

Y Wu${ }^{1}$, MP Wang ${ }^{1}$, DYT Cheung ${ }^{1}$, DSY $\mathrm{Ho}^{2}, \mathrm{AC} \mathrm{Kwong}^{3}$, VW

\begin{tabular}{|c|c|c|c|c|}
\hline $\begin{array}{l}\text { smokers } \\
\text { using }\end{array}$ & & Ban & Ban & Ban \\
\hline baceo & Ban & in public & in areas & in all \\
\hline Ilking & & stops or & meters & areas in \\
\hline the & pedestrian & wailing & of office & housing \\
\hline
\end{tabular}

$\begin{array}{lllll}63.5 & 83.3 & 89.6 & 73.7 & 76.0\end{array}$

$\begin{array}{ccccc}77.1 & 87.1 & 96.5 & 85.1 & 89.6 \\ 1.94(1.24- & 1.35(0.76- & 3.23(1.52- & 2.04(1.24- & 2.71(1.61- \\ 3.03)^{* *} & 2.40) & 6.88)^{* *} & 3.35)^{* *} & 4.56)^{* * *} \\ 2.19(1.38- & 1.43(0.80- & 3.42(1.59- & 2.11(1.27- & 2.75(1.63- \\ 3.47)^{* *} & 2.58) & 7.37)^{* *} & 3.50)^{* *} & 4.65)^{* * *}\end{array}$

demographic characteristics

Results: Overall 81.0\% respondents agreed "THS increases risk of respiratory diseases in children". Knowing harms of THS was associated with greater support on an overall extension of smokefree zones (AOR 2.13, 95\% CI 1.32-3.45) and banning smoking in 7 out of 8 different public areas (AOR 1.86-3.42). The strongest association was found for public transport stops or waiting areas $(3.42,1.59-7.37)$, followed by outside seating of bars $(2.91,1.87-4.53)$ and all public areas in housing estates $(2.75,1.63-4.65)$.

Conclusions: Knowledge on harms of THS was associated with greater support on smokefree policies. Future prospective studies should be conducted to investigate whether increasing never smokers' knowledge on harms of THS could raise their support on smokefree policies.

\section{Tob. Induc. Dis. 2018;16(Suppl 1):A698 DOI:10.18332/tid/84369}

[THS harms knowledge \& support on smokefree policy]

12:30-14:00

PS-1002-3 Increasing smoke free public places \& creating tobacco free educational institutions S Gupta ${ }^{1}$ 
${ }^{1}$ Voluntary Health Association of India, Delhi, India. E-mail: seemagupta@vhai.org

Background and challenges to implementation: Second Hand Smoking, early age of initiation of tobacco use and the lack of awareness regarding the Cigarette \& Other Tobacco Products Act (COTPA), 2003 amongst the community are among the key challenges in tobacco control and health promotion.

Intervention or response: In order to educate and empower the community and to initiate a systematic streamline and administrative mechanism \& to gauge public opinion regarding awareness of the law and implementation of smoke free rules, a compliance survey on Section $4 \& 6$ of COTPA was conducted. The interventions included engaging with key stakeholders - District Administration, Community, Media, Village Council Institutions, Education Department, Health Department, Home Department and volunteers; sensitization and capacity building \& awareness building campaigns. This was aimed to ensure ownership and sustainability of the cause. Technical support and guidance was extended to the govt. administration to institutionalize the enforcement mechanism. A post-intervention survey was also conducted by an independent agency to evaluate progress and monitor changes, if any.

Results and lessons learnt: Post-interventions the compliance in Budgam district (J\&K state), went up from 14.8\% to $91 \%$, in Srinagar (J\&K state), from $43.3 \%$ to $80 \%$, in Golaghat (Assam), from $7.6 \%$ to $42 \%$, in Jagatsinghpur (Orissa), from $23.3 \%$ to $96 \%$, in Jhunjhunu (Rajasthan), from $5.6 \%$ to $78 \%$ and in Shahjahanpur (Uttar Pradesh), from $1.6 \%$ to $88 \%$. Other results were Tobacco Free Educational Institutions' declaration in five districts by the Department of Education and a strong enforcement mechanism set up in 8 districts in 5 settings. The Department of Education in Golaghat Assam \& Budgam, J\&K declared all education institutions as Tobacco Free Institutions. Multi-sectoral approach, multi-stakeholder engagement and community ownership was key in ensuring sustainability which was important to ensure significant progress in the project.

Conclusions and key recommendations: There was a variance in the success and progress across the 10 districts due to different socio-political and administrative factors.

Tob. Induc. Dis. 2018;16(Suppl 1):A699

DOI:10.18332/tid/84376

12:30-14:00

PS-1003-3 Environmental damage from tobacco pollution of air and water on Thailand beaches

S Hamann ${ }^{1}$, N Kungskulniti ${ }^{2}$, N Charoenca ${ }^{2}$

${ }^{1}$ Tobacco Control Research and Knowledge Management Center, Mahidol University, Bangkok, Thailand, ${ }^{2}$ Mahidol University, Faculty of Public Health, Bangkok, Thailand.E-mail: slhamann@ gmail.com

Background: Cigarette butts are known to be dangerous since they are mostly non-biodegradable plastic and contain dangerous chemicals embedded in filter material. Pollution from secondhand smoke on congested beaches and from cigarette waste on beaches in low-and middle-income countries (LMIC) has received relatively little attention, and its assessment is important for environmental protection and human health.

Methods: We conducted an observational investigation to assess availability of cigarettes, population of smokers, warnings not to smoke, and number of cigarette butts on and adjacent to two popular public beaches in Thailand. We also took 88 measures of PM 2.5 levels in beach lounge areas on these beaches using equipment and protocols previously used for the assessment of secondhand smoke in outdoor locations.

Results: Total, smoker and child populations varied with up to 100 total population, 4 smokers and 15 children in a 400 square meter area. This translates to 50 or more smokers along a 250 meter beach front area. In less than eight hours of cigarette butt collection, 2,810 cigarette butts were collected at one beach and 3,666 butts collected at the second beach with $56 \%$ collected in the beach lounge area. Peak PM 2.5 air pollution from smokers lounging on these beaches reached as high as 716 and 1,335 $\mu \mathrm{g} /$ $\mathrm{m} 3$ taken within 2 meters from active smokers. Cigarette retail sales were widely available close to the beaches, and only one beach had any "No smoking" signs posted.

Conclusions: Pollution of beaches is evident by the number of cigarette butts and the level of secondhand smoke exposure in Thai beach areas. In Thailand and similar LMIC where beaches are heavily used, we recommend a consumer education approach which highlights pollution dangers to children and the natural environment, followed by a ban of smoking on beaches.

Tob. Induc. Dis. 2018;16(Suppl 1):A700

\section{DOI:10.18332/tid/84383}

12:30-14:00

PS-1004-3 Against the tide: a tobacco-free university in Lebanon

R Nakkash¹, M Asser ${ }^{2}$, M Chaaya ${ }^{3}$, M El Hallak ${ }^{4}$, M Khairallah $^{5}$, M Romani ${ }^{6}$

${ }^{1}$ American University of Beirut, Health Promotion and Community Health, Beirut, Lebanon, ${ }^{2}$ American University of Beirut, Communication Office, Beirut, Lebanon, ${ }^{3}$ American University of Beirut, Epidemiology and Biostatistics, Beirut, Lebanon, ${ }^{4}$ American University of Beirut, Neighborhood Initiative, Beirut, Lebanon, ${ }^{5}$ American University of Beirut, Human Resources, Beirut, Lebanon, ${ }^{6}$ American University of Beirut, Family Medicine, Beirut, Lebanon. E-mail: rn06@aub. edu.lb

Background and challenges to implementation: Although Lebanon ratified WHO's Framework Convention on Tobacco Control in 2005, smoking is rampant in the country. With high smoking rates among adults and youth, it is estimated that about 4,010 people die annually from tobacco caused diseases. Laws stipulating smoking bans in public places, including educational establishments, are not observed.

Intervention or response: In March 2017, the president of the American University of Beirut (AUB) in the Lebanese capital initiated its transformation from a campus with designated smoking areas into a tobacco-free university by January 2018 . This presentation describes how AUB went about this initiative and outlines lessons learned. The president formed a universitywide task force made up of staff, faculty, and students, including smokers, to guide the development, implementation, enforcement, and evaluation of the tobacco-free policy. The task force's work concentrated on impact and process evaluation, mapping, policy and enforcement program development, evidence-based communication campaign, and initiating a free smoking cessation program. It developed a consultation plan to enable all members of the community to voice their opinions over the policy. The consultation process consisted of a series of town hall meetings, discussions with smokers and non-smokers in pre-existing smoking designated areas, involvement of student clubs and student representative committees, and the University StudentFaculty Committee. 
Results and lessons learnt: Feedback revealed support, but also heavy concern over how the transformation would play out. Counterarguments were addressed within the communication campaign and informed the planning process.

Conclusions and key recommendations: In a country with a clear pro-smoking environment, establishing a tobacco-free university is bound to be faced with challenges. Although this has been a top-down mandated initiative, a participatory approach to implementation ensured that concerns were mitigated and allowed the community to gain ownership of the policy. The lessons learned from this process will inform other universities, in Lebanon or in other countries operating in a similar environment.

Tob. Induc. Dis. 2018;16(Suppl 1):A701

DOI: $10.18332 /$ tid/84390

\section{2:30-14:00}

PS-1005-3 Implementing comprehensive tobacco control polices in medical education institutions and associated teaching hospitals - a case study from a northern state in India

R Chand ${ }^{1}$

${ }^{1}$ Indira Gandhi Medical College, Hospital Administration, Shimla, India.E-mail: dr.rameshigmc@gmail.com

Background and challenges to implementation: GATS India 2009:10 estimated that, $2.7 \%$ of adults are exposed to second hand smoke in health care facilities in Himachal Pradesh state in north India. Department of medical education, Government of Himachal Pradesh provides professional medical education as well as clinical services through a network of 5 medical, 4 dental and 35 nursing schools and associated teaching hospitals. Indian tobacco control legislation (COTPA) bans smoking in public places and sale of tobacco products in and around an educational institution; however the implementation of the law has been suboptimal for a long time. The present case study enumerates the steps taken by the Department of Medical education to implement tobacco control policies in these institutions.

Intervention or response: With an administrative order from the department, high level institutional core committee for tobacco control were constituted in each institutions. Institute wise comprehensive action plan was developed. Nodal officers for enforcement in each institute were notified. A circular was shared to all stakeholders including the staff associations to comply with the law. Specified signages and anti-tobacco messages were displayed at all prominent places. Strict law enforcement was ensured, till date more than 800 violators has been punished and fine amount of near 1.5 lakh INR has been collected.

Results and lessons learnt: There is an overwhelmingly positive response from all stakeholders. The smoking has been literally banned. Tobacco products are not available inside and within 100 yards of the premises. Institute authorities are doing regular monitoring of enforcement activities. There is an increase in no. of tobacco users seeking tobacco cessation services.

Conclusions and key recommendations: Medical education institutions and associated teaching hospitals in Himachal Pradesh state in India demonstrated successful implementation of tobacco control policies. Following these strategies, other institutions in India or in similar settings in other countries can also be made tobacco free educational institutions to promote public health.

Tob. Induc. Dis. 2018;16(Suppl 1):A702

DOI:10.18332/tid/84411

$12: 30-14: 00$
PS-1006-3 Malaysian health promotion board (MySihat) smoke-free city initiative

M Krishnan ${ }^{1}$

${ }^{1}$ Malaysian Health Promotion Board, Ministry of Health Malaysia, Cyberjaya, Malaysia.E-mail:manimaran@moh.gov.my

Background and challenges to implementation: Malaysia became a signatory to the WHO-FCTC on 23 September 2003 and ratified on 16 September 2005. National comprehensive smoke-free laws are ideal to protect the citizens from exposure to second hand smoke but are still a work in progress by the Ministry of Health. Nonetheless, regulatory measures have been introduced to protect people from exposure to tobacco smoke through sub-national jurisdictions that ban smoking in public places the Control of Tobacco Product Regulations 2004 under the Food Act 1983. Under this provision, no-smoking ban has been expanded to 23 public spaces. Hence, states, city and local authorities can adopt laws or other available legal instruments to prohibit tobacco smoke in public places under their respective by-laws.

Intervention or response: Under this purview, MySihat together with partner organisations are championing the smoke-free city initiatives in Malaysia. The objective is to protect the public from second-hand smoke by implementing effective smoke-free policies, in line with provision of Article 8, WHO-FCTC.

Results and lessons learnt: The effort involves persuading the stakeholders, facilitating the plan of actions, building the capacity of organizations including NGOs as well as empowering our people to voice their rights for cleaner air. Legal instruments will not be effective without assertive self-enforcement and education by the public. The smoke-free cities initiative started with Melaka in 2010, followed by Johor, Penang, Kelantan, Terengganu, Kuala Lumpur, Putrajaya, Sabah, Sarawak and Selangor.

Conclusions and key recommendations: The initiative should encompass the advocacy, capacity building, networking, law and enforcement, quit smoking service as well as monitoring and evaluation measures. All of these would not be meaningful without the ownership of the programm by the state and local goverments, backed by the support from public, private and nongovernmental sectors. MySihat envisage a Smoke-Free Malaysia by 2045 as outlined by the National Strategic Plan for Tobacco Control and a healthier future for our younger generation.

Tob. Induc. Dis. 2018;16(Suppl 1):A703

DOI: $10.18332 /$ tid/84413

12:30-14:00

PS-1007-3 Effective way to prevent tobacco consumption of husbands through pregnant mothers C Herath $^{1}$

${ }^{1}$ Alcohol and Drug Information Center (ADIC) Sri Lanka, Social Mobilization, Colombo, Sri Lanka.E-mail: wasanthi.chandi@ gmail.com

Background and challenges to implementation: During the pregnancy period the husband is a lot concerned about health of his wife and child. Therefore he is very flexible to change towards the goodness during this period. Therefore we got this opportunity to influence the husbands through the pregnant mothers, to encourage their husband's to quit their tobacco consumption and to make a tobacco free environment.

Alcohol and Drug Information Center (ADIC) implemented direct interventions with pregnant mothers in $03 \mathrm{MOH}$ areas to help their husbands to quit smoking and to make a tobacco free environment.

The biggest challenge was changing the husband's smoking behavior within nine months. It was also difficult to have 
meetings with mothers to keep a sustainability after the baby was born.

Intervention or response: Pregnant mothers were trained by ADIC once a month in their clinics when they came to check their health condition. They support us for prevention and spread the messages among the target community. They used materials such as hand bills, stickers distributed by ADIC. ADIC staff conducted short sessions during their clinics and parent's meetings, empowering women's groups in the community.

Follow up's were done by conducting field visits and by making telephone calls. To overcome the biggest challenge we created small community groups near pregnant mother's houses and we held meetings with them twice a week until the baby was born. Then we did follow up with them by telephone calls to continue the progress till two years.

Results and lessons learnt: According to the pre and post evaluation tobacco free houses were improved from $23.18 \%$ to $93.75 \%$. Number of husbands who reduced smoking were improved from $18.84 \%$ to $57.69 \%$. Number of husbands who quit smoking were improved from $18.84 \%-30.76 \%$

Conclusions and key recommendations: Addressing a husband thorough pregnant mother's is a very effective way of reducing the use of tobacco in a community.

Tob. Induc. Dis. 2018;16(Suppl 1):A704

DOI:10.18332/tid/84420

\section{$12: 30-14: 00$}

PS-1008-3 Social inequalities in exposure to secondhand smoke in households with children under 12 in Spain

MJ López ${ }^{1,2}$, T Arechavala ${ }^{1}$, X Continente ${ }^{1,2}$, A Schiaffino $^{3}$, M Pérez-Ríos ${ }^{4}$, E Fernández ${ }^{5}, \mathrm{P}$ Lletjòs $^{6}$

${ }^{1}$ Public Health Agency of Barcelona, Barcelona, Spain, ${ }^{2}$ CIBERESP, Madrid, Spain, ${ }^{3}$ Departament de Salut de la Generalitat de Catalunya, Barcelona, Spain, ${ }^{4}$ Galician Directorate for Public Health, Galician Health Authority, Xunta de Galicia, Barcelona, Spain, ${ }^{5}$ Catalan Institute of Oncology (ICO), Bellvitge Biomedical Research Institute (IDIBELL), Hospitalet de Llobregat (Barcelona), Spain, ${ }^{6}$ Universitat Pompeu Fabra, Barcelona, Spain. E-mail: efernandez@iconcologia.net

Background: Children are particularly vulnerable to the effects of secondhand smoke (SHS), mainly due to presenting a respiratory and immune systems in development and a faster respiratory rate. The objective of this study is to describe the potential social inequalities in SHS exposure in households with children under 12 in Spain.

Methods: Cross-sectional study in a representative sample of population under 12 years of age in Spain. The sample size was 2,411 individuals, being proportional by regions, size of municipality of residence, sex and age groups. A telephone survey was administered to parents or legal tutors in 2016. It included sociodemographic variables and variables related to SHS exposure at home. A descriptive bivariate analysis of the main SHS exposure variables according to educational level and social class (based on occupation) was carried out.

Results: $25.8 \%$ of children are exposed to SHS at home (smoking reported either inside or on balconies or terraces). An exposure gradient is observed according to the level of studies of the household's main earner, being the prevalence of exposure higher as the educational level decreases $(37.9 \%$ in primary studies or lower, $27.8 \%$ in secondary studies and $20 \%$ in university studies, $\mathrm{p}<0.05)$. This reverse gradient is maintained by social class
(31.1\% in class V-VII (most deprived), 23.4\% in class III-IV and $21.7 \%$ in class I-II (most affluent), $\mathrm{p}<0.05$ ). There are no significant differences according to the sex of the child.

Conclusions: In Spain, one out of every four children is exposed to SHS at home. This exposure presents a clear socioeconomic gradient, being children living in more deprived families at higher risk of SHS exposure. Interventions aiming to reduce SHS exposure in children, with a perspective of equity, are urgently needed.

Tob. Induc. Dis. 2018;16(Suppl 1):A705 DOI:10.18332/tid/84432

\section{$12: 30-14: 00$}

PS-1009-3 Outdoor hospitality venues: a real challenge for tobacco control policies

X Sureda ${ }^{1}$, R Santuy 1 , U Bilal ${ }^{1,2}$, R Valiente $^{1,3}$, A Navas-Acien $^{4}$, E Fernández ${ }^{5}$, FJ Escobar ${ }^{1,3}$, M Franco ${ }^{1,6}$

${ }^{1}$ University of Alcalá, Social and Cardiovascular Epidemiology Research Group, School of Medicine, Alcalá de Henares, Spain, ${ }^{2}$ Drexel Dornsife School of Public Health, Philadelphia, PA, United States of America, ${ }^{3}$ University of Alcalá, Department of Geology, Geography and Environmental Sciences, Alcalá de Henares, Spain, ${ }^{4}$ Columbia University Mailman School of Public Health, Department of Environmental Health Sciences, New York, NY, United States of America, ${ }^{5}$ nnstitut Català d'Oncologia, Tobacco Control Unit, Cancer Control and Prevention Programme, Hospitalet Ilobregat, Spain, ${ }^{6}$ Johns Hopkins Bloomberg School of Public Health, Department of Epidemiology, Baltimore, MD, United States of America. E-mail: francisca.sureda@uah.es

Background: After the implementation of smoke-free policies in indoor hospitality venues, smokers may have displaced to their outdoor areas. Authors aimed to describe smoking visibility and second-hand smoke (SHS) exposure in outdoor hospitality venues.

Methods: An observational study was conducted in Madrid city. We collected information, through direct observation, on signs of tobacco consumption on entrances and terraces of hospitality venues. We also measured airborne nicotine in terraces with a monitor by active sampling during 30 minutes. We calculated the medians and the interquartile ranges (IQR) of nicotine concentrations. We computed an analysis stratified by the possible explanatory variables and compared the nicotine concentration using the Kruskal-Wallis test for independent samples.

Results: We characterized 256 entrances of hospitality venues, 174 measured between May and September 2016 (hot season) and 82 between October and December 2016 (mild season). 204 entrances showed signs of tobacco consumption: 97 had ashtrays; 166 had cigarettes butts; in 66, tobacco smell was perceived; and, in 67 entrances, smokers were observed. There were no differences in signs of tobacco consumption observed between seasons $(p=0.155)$. We measured nicotine concentration in 92 terraces with an overall median of $0.42 \mu \mathrm{g} / \mathrm{m} 3$ (IQR: $0.14-1.59 \mu \mathrm{g} /$ $\mathrm{m} 3)$. Nicotine concentration in terraces increased with number of cigarettes smoked from $0.03 \mu \mathrm{g} / \mathrm{m} 3$ (IQR: 0.03-0.91 $\mu \mathrm{g} / \mathrm{m} 3$ ) when no cigarette was litten to $3.83 \mu \mathrm{g} / \mathrm{m} 3(0.97-4.70 \mu \mathrm{g} / \mathrm{m} 3)$ when more than eight cigarettes were lighted $(p=0.001)$. We observed differences in nicotine concentration according to the number of covers from $0.37 \mu \mathrm{g} / \mathrm{m} 3$ (IQR: $0.15-1.59 \mu \mathrm{g} / \mathrm{m} 3$ ) in terraces with no cover to $2.40 \mu \mathrm{g} / \mathrm{m} 3$ (IQR: $0.64-13.36 \mu \mathrm{g} / \mathrm{m} 3$ ) on closed terraces $(p=0.006)$.

Conclusions: Outdoor hospitality venues are areas where nonsmoking population continues to be highly exposed to SHS. These spaces should be considered in future tobacco control interventions. 
Tob. Induc. Dis. 2018;16(Suppl 1):A706 DOI:10.18332/tid/84445

\section{$12: 30-14: 00$}

PS-1010-3 Protection from exposure to second hand SLT use in public places - spitting a public health concern or a public nuisance?

A Yadav ${ }^{1}$, K Chandan $^{1}$, DN Sinha ${ }^{1}$, R Mehrotra $^{1}$, A Chandra $^{1}$

${ }^{1}$ National Institute of Cancer Prevention and Research (NICPR), WHO FCTC Global Knowledge Hub on Smokeless Tobacco, Noida, India.E-mail: advocateamit@msn.com

Background: Until 1990, spitting was an offence in United Kingdom carrying a §5 fine. Spitting was also banned in other developed countries especially in Europe early in the 20th Century as a preventive measure against tuberculosis and later to maintain cleanliness. Several health experts consider spitting a public health concern and smokeless tobacco in all its forms induces spitting, thereby causing risk for communicable diseases like tuberculosis.

Methods: A comprehensive review of existing literature on prohibition of spitting in public places was undertaken. Legislative and policy publications were reviewed for the reasons of such prohibition and punishments thereon.

Results: Spitting has been considered a public inconvenience and a nuisance. Spitting of chewing and other smokeless tobacco stains and defaces public vehicles, buildings and other public property and is a public nuisance. In a scientific study done in India the most frequent cited reason for quitting tobacco use was spitting and ash dropping which was embarrassing in-front of others. Several states in India have banned spitting by law. Nepal has banned use of any kind of tobacco products in public places. Bhutan prohibits smearing lime and spitting doma in public area. Hangzhou- a province in China has banned spitting in public places. Countries like Singapore, Myanmar and Australia also have imposed ban on spiting in public and impose hefty fine for violation of the provisions.

Conclusions: Several countries and cities have promulgated norms against spitting in public places, including spitting after SLT use. There is a need to undertake further research to study impact of spitting ban on SLT use.

Tob. Induc. Dis. 2018;16(Suppl 1):A707

DOI:10.18332/tid/84503

12:30-14:00

PS-1011-3 Making hospitality sector of Kashmir Division smoke free - India

S Jain', S Gupta ${ }^{2}$

'Voluntary Health Association of India, New Delhi, India, ${ }^{2}$ Voluntary Health Association of India, Delhi, India. E-mail: sukriti.jain86@gmail.com

Background and challenges to implementation: Tourism is amongst the largest industries of Kashmir Division - 10 Million tourists annually. With over 120 million smokers in Jammu \& Kashmir, exposure to Second Hand Smoke (SHS) is highest in India (67.8\%). Smoking is culturally accepted and the stale smoke odour is trapped in closed carpeted homes and hotels. To protect locals and tourist from SHS, enforcement of Cigarette and Other Tobacco Products Act, 2003 Section 4, prohibiting smoking in all public places was strengthened.

Intervention or response: All stakeholders in the Hospitality Sectors were mapped and sensitized - Policy makers, Government officials, Private Associations like

- Kashmir House Boat Owner's Association (KHBOA) - 900+
Houseboats

- Kashmir Hotel and Restaurants Association (KHARA) $1200+$ hotels and restaurants,

- Kashmir Shikara and Taxi owner's Association - 1900+ Shikara (Boat Taxis) and Taxis

High level political leaders like Chief Minister and Minister of Tourism and Culture extended commitment. Orders were issued by General Manager, J\&K Tourism Development and Secretary of KHOBA and KHARA. Executive members from unions/associations were sensitized. They launched COTPA implementation drives by issuing letter to all members, sensitizing them on need of compliance and printed over 5000 signages for members. This was followed by Self Declaration by Associations of COTPA compliance by all members. Regular media coverage was coordinated applauding their efforts. Funds have been allocated by association/unions to sustain these initiatives.

Results and lessons learnt: Smoke Free Hospitality Sector of Kashmir Division (10 Districts) by Self Declaration.Behavior change and ownership via associations with over 4000 members was pivotal.

Conclusions and key recommendations: For hospitality sector to prioritize public health over perceived tourist preference required time and persistence in intervention. Widespread distribution and large scale of the industry makes compliance monitoring difficult but the sense of pride amongst Associations of being Smoke Free has proven most effective to ensure compliance and sustainability.

Tob. Induc. Dis. 2018;16(Suppl 1):A708

DOI:10.18332/tid/84519

\section{$12: 30-14: 00$}

PS-1012-3 Multilevel intervention in reducing inhome secondhand smoke exposure among pregnant woman in North Lombok district, Indonesia

Y Prabandari ${ }^{1}$, RS Padmawati ${ }^{1}$, B Suryo Bintoro ${ }^{1}$

${ }^{1}$ Universitas Gadjah Mada, Health Behavior, Environmental and Social Medicine, Yogyakarta, Indonesia. E-mail: yayisuryo@ ugm.ac.id

Background and challenges to implementation: SHS exposure among pregnant women is highly correlated with low birth weight (LBW). SHS exposure in homes can be reduced by a package of "smoke-free home" interventions, particularly in the high prevalence of LBW area as North Lombok, Indonesia. Evidence from previous research showed that smoke free home could change smoking behavior among men in Yogyakarta, Indonesia. However, different condition, site and culture may bring different approach to implement smoke free home for protecting the health of pregnant mother.

Intervention or response: Smoke free home interventions consisted of three activities; 1) media campaign (above and below the line), 2) individual contract (each husband signed contract for not smoking at home while his wife pregnant) and 3) community commitment to make all pregnant mother house become smoke free home. We collaborated with district health office in distributing media campaign, including working with midwifes association to reach pregnant woman. We advocated the head of districts and sub district in West Nusa Tenggara for developing community commitment to make smoke free home village. Primary health care officer were actively involved in facilitating smoke free home village commitment.

Results and lessons learnt: Majority of pregnant women husband signed the individual contract for not smoking at home and media were distributed widely. The activity was worked 
well by involving health care providers. It was challenging to have people gathering since they have not had existing group meeting. Therefore, we carried out formal meeting with head of village and community leaders.

Conclusions and key recommendations: Message through media campaign increasing awareness of husbands to protect their wife from passive smoking by signing individual contract. Community commitment of smoke free home supported individual contract. In a country that have limited tobacco control policy, community and individual based intervention are needed.

Tob. Induc. Dis. 2018;16(Suppl 1):A709

DOI:10.18332/tid/84590

\section{2:30-14:00}

PS-1013-3 The association between the awareness of the effects of smoking/secondhand smoke and the desire to quit

I Petroulia' ${ }^{1}$ C Vardavas ${ }^{1}$, F Filippidis ${ }^{1}$, T Peleki ${ }^{1}$, P Behrakis ${ }^{1}$, ACK Quah'², GT Fong ${ }^{2}$, Y Tountas

${ }^{1}$ National and Kapodistrian University of Athens, Athens, Greece, ${ }^{2}$ University of Waterloo, Waterloo, ON, Canada. E-mail: ipetroul@med.uoa.gr

Background: The harmful effects of smoking are well documented. Moreover, public knowledge and beliefs about the health risks of smoking and secondhand smoke exposure have increased substantially over the past several decades.

Methods: The aim of this study is to examine the association between the awareness of the health risks of smoking and secondhand smoke exposure with the desire to quit smoking. The Wave 1 (2016) ITC 6 European Country (6E) Survey within the framework of EUREST-PLUS Project covered both urban and rural areas of the country's 13 geographical regions. The survey sample consisted of 1,000 individuals smokers aged 18 and older in Greece, one of the six ITC 6E countries.

Results: The majority of Greek smokers agreed that smoking causes lung cancer (95.4\%), heart diseases (94.4\%), throat cancer (87.9\%), bronchitis (87.7\%) and mouth cancer (84.8\%), however, $51.7 \%$ smokers disagreed that smoking could cause blindness, tuberculosis (37.8\%), impotence (33.8\%), stroke (24\%) and heart attacks to non-smokers (27.3\%). Chi-square and spearman correlation coefficient were fitted to investigate the association between the awareness of the effects of smoking and the desire to quit. The percentages of the awareness regarding blindness and stroke were less among smokers who reported moderate difficulty to completely quit $(\mathrm{p}<0.05)$. Moreover, smokers reported slight difficulty to successfully quit, reported less awareness that smoking could cause bronchitis and tuberculosis $(p<0.01)$. The levels of the awareness approximately to all health risks were higher among smokers reported that they want to quit "a lot" $(\mathrm{p}<0.01)$.

Conclusions: Our findings highlighted the lack of knowledge regarding the harmful effects of smoking, mostly among those less motivated to quit. Hence, more effort will be needed by health policy-makers and public campaigns in order to inform smokers about the smoking risks and promote tobacco treatment delivery.

Tob. Induc. Dis. 2018;16(Suppl 1):A710

DOI:10.18332/tid/84622

\section{2:30-14:00}

PS-1014-3 Implementing smoke-free laws in lowand middle-income countries: a systematic literature review and proposed research Agenda

MJ Byron ${ }^{1}$, J Cohen ${ }^{2}$, S Frattaroli ${ }^{3}$, J Gittelsohn ${ }^{4}$, D Jernigan ${ }^{5}$
'University of North Carolina, Health Behavior, Chapel Hill, NC, United States of America, ${ }^{2}$ Johns Hopkins Bloomberg School of Public Health - Institute for Global Tobacco Control, Health, Behavior and Society, Baltimore, MD, United States of America, ${ }^{3}$ Johns Hopkins Bloomberg School of Public Health, Health Policy and Management, Baltimore, MD, United States of America, ${ }^{4}$ Johns Hopkins Bloomberg School of Public Health, Center for Human Nutrition, Department of International Health, Baltimore, MD, United States of America, 5Johns Hopkins Bloomberg School of Public Health, Health, Behavior and Society, Baltimore, MD, United States of America. E-mail: jbyron@unc.edu

Background: Globally, secondhand tobacco smoke causes over 365,000 deaths each year. To fulfill their obligations under the WHO Framework Convention on Tobacco Control, low- and middle-income countries (LMICs) are increasingly enacting smoke-free laws covering public places. These policies have proven effective in high-income countries, but some LMICs have struggled to implement smoke-free laws and attain compliance. We present a systematic review and a research agenda for improving implementation of smoke-free laws in LMICs.

Methods: We conducted a systematic review of the literature on implementation of smoke-free laws in LMICs through January 2017. The search of 10 databases yielded 3,894 unique articles, which reduced to 1,409 after title screening, 236 after abstract screening, and 66 after full text review. We also reviewed reports from WHO and international tobacco control NGOs and citations in sources. The total number of sources that met inclusion criteria was 131 . We analyzed these materials for common themes.

Results: We found that many of the health and economic aspects of smoke-free laws in high-income countries carry over to LMICs: the tobacco industry aggressively opposes smoke-free laws, there are commonalities in lessons learned in implementing laws across various LMICs, and a number of obstacles to successful implementation are faced in LMICs, especially in terms of resources and political will.

Conclusions: Based on these findings and our own experience in the field, we suggest four central topics on which research is urgently needed:

(1) determining the most efficient methods of working with limited resources;

(2) learning how to increase political will among political leaders and enforcement officers;

(3) finding methods for increasing public compliance in settings where laws have already been passed but not achieved success; and,

(4) understanding the social and behavioral processes underlying smoke-free laws. Investigation into these topics can inform more effective implementation of smoke-free laws in LMICs.

Tob. Induc. Dis. 2018;16(Suppl 1):A711

DOI:10.18332/tid/84668

\section{2:30-14:00 \\ PS-1015-3 Smoking topography in Korean smokers S Kim ${ }^{1}$, S Yu ${ }^{1}$, S Lee ${ }^{1}$ \\ 'Soonchunhyang University, Asan, Korea, Republic of. E-mail: sungroul.kim@gmail.com}

Background and challenges to implementation: Actual absorption dose of nicotine or tar from a cigarette could be different according to smokers' smoking topography. Different topographic characteristics, i. e., puff volume or puff frequency are applied to a standard operating procedure for intense simulation test of 
smoking in Canada, US, and ISO regimen. However, in South Korea, lack of smoking topography information limits estimation of intake dose of toxic or harmful chemicals from smoking and its health effect. In this study, we measured Korean smokers' topography and evaluated its characteristics.

Intervention or response: Under a convenient sampling design, we recruited 300 adult smokers (male: 250, female: 50). Using CReSS pocket device (BORGWALDT, Richmond, VA 23237, USA), we obtained distributions of puff volume, puff duration, puff interval, etc. from their cigarettes smoked. For those who completed his/ her topograph test, we collected urine samples for measurement of cotinine, OH-cotinine and NNAL using LCMSMS.

Results and lessons learnt: Smokers using cigarettes with higher amount of nicotine (HAN) ( $>0.1 \mathrm{mg})$ tend to have lower puff counts $(15.0(13.0 \sim 18.0)$ than those smokers $(17.0(15.0 \sim 21.0)$ smoked cigarettes with lower amount of nicotine (LAN) $(\leqslant 0.1 \mathrm{mg})$. Controlling for the number of cigarettes smoked, Korean smokers smoked with shorter inter puff interval than Whites. Total puff volume per day were similar between male and female smokers indicating the amount of toxic components inhaled from smoking might be similar between male and female smokers.

Conclusions and key recommendations: This study provides quantitative evidence that Korean smokers smoking cigarettes with shorter interval.

Tob. Induc. Dis. 2018;16(Suppl 1):A712

DOI:10.18332/tid/84683

\section{2:30-14:00}

PS-994-3 Second-hand smoke exposure in private and public places in Italy: data from the TackSHS pan-European survey

A Lugo ${ }^{1}$, X Liu' ${ }^{1}$, S Gallus

IIRCCS - Istituto di Ricerche Farmacologiche Mario Negri, Milan, Italy. E-mail: alessandra.lugo@marionegri.it

Background: Exposure to second-hand smoke (SHS) has serious adverse health effects, causing more than 600,000 deaths among non-smokers each year worldwide. Following the recommendations of the World Health Organization, most European countries adopted -more or less comprehensive-smokefree legislations. Our aim was to quantify SHS exposure among non-smokers in selected European countries.

Methods: Within the TackSHS project, we are conducting a face-to-face survey, including information on exposure to SHS in selected private and public indoor places, and outdoor places. The fieldwork has already been conducted in Italy in December 2016 on a representative sample of 1059 Italians aged $\geq 15$ years, and will soon be conducted in other 11 European countries (i.e., Bulgaria, England, France, Germany, Greece, Ireland, Latvia, Poland, Portugal, Romania, and Spain).

Results: In Italy, $81 \%$ of survey participants were non-smokers $(n=859)$. Of these, $27 \%$ were daily exposed to SHS in indoor places for a median time of 2 hours/day. The proportion of nonsmokers reporting to have seen people smoking in indoor public settings was $23 \%$ in restaurants, $20 \%$ in indoor train stations or subway stops and $17 \%$ in disco clubs, and was $20 \%$ in private vehicles without minors, and $14 \%$ in presence of minors. A high proportion of non-smokers reported to have seen people smoking in various outdoor settings, including beaches (79\%), outdoor areas of hospitals (71\%) or schools (58\%), and parks (68\%).

Conclusions: More than one out of four Italian non-smokers is daily exposed to SHS. We recognize the dramatic fall in SHS exposure thanks to the comprehensive Italian smoke-free legislation adopted more than 10 years ago. This notwithstanding, it is still not rare to observe people smoking in violation of the current legislation in Italy. Data from other 11 European countries with different smoke-free policies will allow cross-country comparisons.

Tob. Induc. Dis. 2018;16(Suppl 1):A713

DOI:10.18332/tid/84243

$12: 30-14: 00$

PS-995-3 Passive exposure to electronic cigarette aerosol in Italy: data from the TackSHS panEuropean survey

$X$ Liu $^{1}$, A Lugo 1 , S Gallus ${ }^{1}$

'IRCCS - Istituto di Ricerche Farmacologiche 'Mario Negri', Milan, Italy. E-mail: xiaoqiu.liu@marionegri.it

Background: Passive exposure to electronic cigarette aerosol, which contains various toxic chemicals, has potential adverse health effects in non users. The aim of this study was to quantify such passive exposure in different European countries.

Methods: Within the TackSHS project, we are conducting a faceto-face cross-sectional study on adults in 12 strategically selected European countries (i.e., Bulgaria, England, France, Germany, Greece, Ireland, Italy, Latvia, Poland, Portugal, Romania, and Spain). The survey includes a specific section on electronic cigarette use and passive exposure to its aerosol. The fieldwork has already been conducted in Italy on a sample of 1059 individuals, representative of the population aged $\geq 15$ years ( 52.4 million).

Results: Overall, $1.1 \%$ of our Italian population were current electronic cigarette users. Among non-users, 13.7\% have been daily exposed to electronic cigarettes in various indoor settings, with a median exposure time around 1 hour and half/day. The proportion of non-users who observed (the last time over the previous 6 months) people using electronic cigarettes was $25 \%$ in bars, $20 \%$ in restaurants, $18 \%$ in disco clubs, $21 \%$ in indoor train/ metro stations, $20 \%$ in airports, $13 \%$ in private vehicles $(7 \%$ in presence of a minor). It was frequent to observe people vaping in several outdoor settings, including restaurant or bar patios (43\%), outdoor areas of hospitals (41\%) and schools (33\%), children's playgrounds (28\%), and also while driving motorbikes/scooters (21\%), in violation of the traffic laws.

Conclusions: Current use of electronic cigarette is still relatively low in Italy, but passive exposure to its aerosol is far to be negligible. Our data suggest that, in indoor settings, more than 7 million Italians are daily exposed to the aerosol exhaled by less than 600 thousand electronic cigarette users. Data from other 11 European countries on the issue will allow cross-country comparisons.

Tob. Induc. Dis. 2018;16(Suppl 1):A714

DOI: $10.18332 /$ tid/84268

$12: 30-14: 00$

PS-996-3 Knowledge and risk perceptions of israelis towards combustable cigarettes: the need for immediate remedial action

L Rosen ${ }^{1}$, D Rier ${ }^{2}$, M Talitman ${ }^{3}$, R Schwartz ${ }^{4}$ L Z Zwanziger $^{5}$

${ }^{1} T e l$ Aviv University, Dept. of Health Promotion, School of Public Health, Ramat Aviv, Israel, ${ }^{2}$ Bar Ilan University, Ramat Gan, Israel, ${ }^{3}$ Tel Aviv University, Dept. of Statistics, Ramat Aviv, Israel, ${ }^{4}$ University of Toronto, Toronto, ON, Canada, ${ }^{5} \mathrm{Tel}$ Aviv University, Medical School, Ramat Aviv, Israel. E-mail: rosenl@post.tau.ac.il

Background: With the introduction of potentially harm-reduced tobacco products and harm reduction claims by the tobacco industry in various countries, the public health community is 
confronted with dilemmas in how to present information about the new products to the broader public. Yet, many populations may still be poorly informed about risks due to use of the conventional lethal combusted cigarettes. This paper presents knowledge and perceptions about active smoking and tobacco smoke exposure (TSE) among Israeli adults.

Methods: A nationally representative phone survey of the Israeli adult population was conducted with 505 participants (response rate $=60 \%$ ). Knowledge and risk perception questions about active smoking and tobacco smoke exposure (TSE) were included. Multi-variable models were used to identify variables associated with knowledge and risk perceptions.

Results: Among respondents, only $15.8 \%$ accurately answered all four knowledge questions. Just $28.6 \%$ knew that smoking causes more damage than traffic accidents, many (30.3\%) were unaware that TSE causes both lung cancer and heart disease, $27.7 \%$ did not know that smoking shortens life and injures quality of life and $31 \%$ did not know that all or most heavy smokers will suffer health problems from smoking. Current smokers had the lowest risk perceptions and never smokers the highest. Socio-economic status was associated with more accurate knowledge, and smoking status was associated with risk perception.

Conclusions: Knowledge about risks of smoking combustible cigarettes are low in the Israeli population. Effective mechanisms for educating the broad public, including smokers and nonsmokers, about the full extent of known harms from smoking, tobacco, and nicotine products are essential to controlling tobacco use and damage in populations.

Tob. Induc. Dis. 2018;16(Suppl 1):A715

DOI:10.18332/tid/84276

\section{$12: 30-14: 00$}

PS-997-3 Advancing towards tobacco-free state: institutionalizing tobacco control programme in Indian state of Bihar

R Kumar ${ }^{1}$, D Mishra ${ }^{2}$, A Pandey ${ }^{3}$

${ }^{1}$ District Magistrate, Gopalganj, India, ${ }^{2}$ Socio Economic and Educational Development Society (SEEDS), BIHAR, New Delhi, India, ${ }^{3}$ International Union Against Tuberculosis and Lung Disease, South-East Asia Office, New Delhi, India. E-mail: rahulias6@gmail.com

Background and challenges to implementation: According to GATS 2009-10 prevalence of tobacco use in Bihar among adults is $53.5 \%$ ( $n=30.8$ million) much higher than the national average i.e. $34.6 \%$.

In 2008-09, Indian government rolled-out National Tobacco Control Programme (NTCP) including 02 districts of Bihar. With inclusion of another 06 and 11 districts in 2014 and 2015 respectively, currently 19 out of 38 districts of Bihar are covered under NTCP. Main key activities under NTCP are India's tobacco control law's enforcement, training, awareness generation, school health programs and cessation.

Intervention or response: To establish and initiate tobacco control programme in all 38 districts, Government of Bihar in collaboration with SEEDS-Bihar (an NGO) and The Union implemented a Bloomberg Initiative project on tobacco control.

Through planned interventions, NTCP has been rolled-out in entire states as an institutional framework. Broadly three pronged strategies were adopted i.e. establishing NTCP implementation mechanism, intensive capacity building of government officials, robust reporting and monitoring from districts. Systematic efforts were made at both state and district level to engage for other stakeholder especially media. Additionally nodal officers were identified in all concerned department at state level and three district nodal officers in Health, Police and General Administration were appointed to develop synergy through better coordinated efforts.

Results and lessons learnt: Awareness and commitment of program managers, law-enforcers and media have noticeably increased for tobacco control. Following were the distinctive outcomes:

- Enforcement mechanism was established in all 38 districts (both NTCP and non-NTCP)

- Periodic programme review by top bureaucrats of concerned departments quarterly at state level and monthly at district level

- Enforcement status review in monthly crime review meeting by police department

Conclusions and key recommendations: Through intensive advocacy, capacity building and robust monitoring mechanism an efficient model for implementation of National Tobacco Control Programme can be institutionalised within existing system. Multistakeholder engagement especially media is very important for better monitoring and sustainability.

Tob. Induc. Dis. 2018;16(Suppl 1):A716

DOI: $10.18332 /$ tid/84283

\section{2:30-14:00}

PS-998-3 Second hand smoke during the pregnancy I Rakoczi ${ }^{1}$, A Fogarasi-Grenczer ${ }^{2}$, P Takacs ${ }^{3}$, K Foley ${ }^{4}$, P Balazs ${ }^{5}$ ${ }^{1}$ University of Debrecen, Faculty of Health, Nyiregyhaza, Hungary, ${ }^{2}$ Semmelweis University, Department of Family Care Methodology, Budapest, Hungary, ${ }^{3}$ University of Debrecen, Nyiregyhaza, Hungary, "Wake Forest School of Medicine, Comprehensive Cancer Center, Winston-Salem, NC, United States of America, ${ }^{5}$ Semmelweis University, Institute of Public Health, Budapest, Hungary. E-mail: rakoczi32@gmail.com

Background: Prenatal secondhand tobacco smoke (SHS) is a significant probleminHungary's fourunderdeveloped northeastern counties with a considerable ethnic Roma population. There are $33.2 \%$ of non-smoker pregnant women exposed to SHS.

Methods: Data were collected among mothers delivered with live-born babies 2009-2011 ( $\mathrm{N}=16,859)$. The neonates' biometric parameters (birth weight, body length, head and chest circumferences) were obtained from hospital records. The response rate was $74.5 \%$. We conducted binary logistic regression analysis of maternal variables for SHS exposure, frequency analysis for central tendencies and dispersion and t-probes for comparing the means of neonatal measurements using significance level $\mathrm{p}<$ 0.05 (IBM-SPSS v. 23 software).

Results: In the non-smoking sample ( $\mathrm{n}=8,497)$, pregnant women exposed to SHS were typically less educated (OR $=3.32,95 \% \mathrm{CI}$ = 2.64-4.18), of Roma ethnicity $(\mathrm{OR}=1.71,95 \% \mathrm{CI}=1.36-2.15)$, living without amenities $(\mathrm{OR}=1.70,95 \% \mathrm{CI}=1.37-2.11)$ and of extramarital status $(\mathrm{OR}=1.52,95 \% \mathrm{CI}=1.27-1.82)$. The negative difference of birth weight following fetal SHS exposure was 154.9 grams $(95 \% \mathrm{CI}=-188.5-121.3)$, the difference of body length $0.8 \mathrm{~cm}(95 \% \mathrm{CI}=-1.06-0.6)$, and of head and chest circumference $-0.5 \mathrm{~cm}(95 \% \mathrm{CI}=-0.6-0.3$ and $-0.7-03$, respectively). Gestational age was shortened by mean 0.4 week.

Conclusions: At-home SHS exposure has a serious impact on biometric and obstetrical parameters of newborn babies. The reduction of SHS exposure, especially targeting the most vulnerable pregnant populations should be one of the primary 
aims of smoking cessation public health programs.

Tob. Induc. Dis. 2018;16(Suppl 1):A717

DOI: $10.18332 / \mathrm{tid} / 84295$

\section{$12: 30-14: 00$}

PS-999-3 Comparing particulate emissions between electronic nicotine delivery devices: context for smoke-free indoor air quality

ZR Dunbar' ${ }^{1}$ L Vogl', ET Jensen ${ }^{2}$, ML Goniewicz ${ }^{1}$, MJ Travers ${ }^{1}$ ${ }^{1}$ Roswell Park Cancer Institute, Health Behavior, Buffalo, NY, United States of America, ${ }^{2}$ University at Buffalo, Buffalo, NY, United States of America. E-mail: zachary.dunbar@roswellpark. org

Background: Smoke-free indoor air policy has been widely adopted in some regions of the United States in order to protect bystanders from the deleterious effects of indoor tobacco smoke exposure; however, similar legislation has widely not been applied to ENDS devices. This study investigated differences in PM2.5 matter emitted into the indoor environment from a selection of ENDS products under controlled conditions.

Methods: Sixteen smokers were recruited to vape in seven individual sessions (one visit per week). During each visit, participants vaped using one of seven different ENDS products. All vaping occurred within a dedicated exposure chamber. Volunteers drew twenty puffs on their assigned devices over a ten-minute exposure period. ENDS products tested included: disposable, e-cigar, vaporizer, rechargeable, e-pipe, and e-Go devices. TSI SidePaks were used to record both ambient and ENDS-associated unadjusted PM2.5 before, during, and after each exposure period. Statistical analysis was performed using IBM SPSS Statistics Version 23.

Results: The rechargeable device emitted the highest amount of PM2.5 $(\mu \mathrm{g} / \mathrm{m} 3)$ on average $(\mu=21.6, \sigma=7.82)$. The disposable device emitted the lowest amount of PM2.5 on average $(\mu=4.14$, $\sigma=1.09$ ). The difference in PM2.5 matter in the smoking chamber during each session compared to ambient levels before each session was significant $(\mathrm{p}<0.05)$ by paired t-test for all devices except e-pipe, which was borderline significant $(p=0.053)$. The mean difference in PM2.5 emissions between the rechargeable device was significantly from e-cigar $(p=0.048)$, e-Go $(p=0.048)$, and disposable $(\mathrm{p}=0.021)$ products.

Conclusions: This study found that ENDS devices emit levels of particulate matter into the indoor environment that are significantly higher than ambient PM2.5 levels. Further, significant emission differences were also detected between ENDS products. These findings suggest that incorporating ENDS products into existing smoke-free indoor air policy would protect non-users from sidestream exposure to ENDS aerosol.

Tob. Induc. Dis. 2018;16(Suppl 1):A718

DOI:10.18332/tid/84296

\section{2:45-14:00}

EP-253-4 Commercial presentation of waterpipe tobacco poses fundamental impasse for tobacco control: Web-based search results for Istanbul, 2017

E Evrengil ${ }^{1}$

${ }^{1}$ Turkish National Coalition on Tobacco or Health, Istanbul, Turkey. E-mail: efzagil@yahoo.com

Background: Tobacco control literature alerts us to increasing prevalence of venues commercially presenting waterpipe tobacco (WPT). In Turkey, they are rapidly growing in numbers and offer a multitude of hospitality and entertainment functions for youth. Methods: Database of restaurant search service www.zomato. com/tr was used to identify 1,272 venues commercially presenting WPT in Istanbul as of 12 June 2017. 350 establishments, ranked as most popular, were further scrutinized with additional information from venue websites and www.trfoursquare.com, for photographic evidence (2015-present) of (a) indoor smoking, (b) health warnings on waterpipe devices, (c) small children's presence as clientele, and (d) illicit products mentioned in WPT menus.

Results: Out of 1,272 venues, $56.4 \%$ were restaurants, $30.6 \%$ cafés, $6.2 \%$ bars, $5.9 \%$ fast-food places, and $0.9 \%$ patisseries. Geographic distribution indicated increased diffusion to suburban settings (55.1\%). Out of 350 venues, $90 \%$ featured indoor smoking, only $1.1 \%$ compulsory health warnings on devices, $31.1 \%$ attendance of toddlers and small children, $7.4 \%$ kids menus, $5.1 \%$ children's events, $1.7 \%$ playrooms, and $4.9 \%$ playgrounds. WTP menus available on Internet (75.1\%) mentioned illicit brand names (63.5\%) and flavours/mixtures (90.1\%).

Conclusions: Three unresolved, pressing problems emanate from commercial presentation of WPT:

(a) Practical, easy, and profitable, the commonplace use of illicit WPT, additional ingredients, on-site manufacturing and mixtures make product presentation virtually impossible to regulate, inspect, and standardize effectively.

(b) Given its duration, WPT smoking is practiced as an indoor activity particularly in cold weather, resulting in permissive management policies and non-compliant spatial configurations.

(c) In a mixed use environment, children and other non-smokers inadvertently get exposed to tobacco smoke and the WPT culture. Innovative regulation is needed to address these problems based on serious questioning legal and social legitimacy of commercial presentation of WPT.

Tob. Induc. Dis. 2018;16(Suppl 1):A719

DOI: $10.18332 /$ tid/84510

\subsection{Mind the gaps: loopholes in the Ban on Sponsorships, Advertising and Promotions}

$12: 30-14: 00$

PS-1016-3 How compliant is Kolkata Metro to prohibition of tobacco advertisement, promotion and sponsorship legislation? A case study from India

N Mukherjee ${ }^{1}$, B Pal $^{2}$, S Ghosh $^{2}, \mathrm{P} \mathrm{Lal}^{3}$

${ }^{1}$ MANT, Tobacco Control, Kolkata, India, ${ }^{2}$ MANT, Research and MCtE, Kolkata, India, ${ }^{3}$ International Union against Tuberculosis and Lung Disease, Tobacco Control, South-East Asia Office, New Delhi, India. E-mail: mant.kolkata@gmail.com

Background: Indian youths aged 13-15 years, are exposed to cigarette advertisements on billboards, increased from $71.6 \%$ in 2006 to $74.4 \%$ in 2009 as revealed by GYTS 2006 and 2009. In West Bengal $28.6 \%$ of adults are exposed to cigarette advertising and while 31.1 and 36.2 percent are exposed to Bidi and smokeless tobacco advertisements respectively as showed by GATS 2010 . However, COTPA, 2003 prohibits advertisement, promotion and sponsorship of all tobacco products under Section 5 .

Methods: 4 Boroughs (cluster of 10-115 Municipal wards) out of total 15 have been selected randomly keeping in mind their location - i.e. South, North, Central and East for collecting samples. The sample size has been enumerated at the level of $95 \%$ confidence interval with $50 \%$ compliance rate while the design effect of the study was 1.0. The Epi-info 7 software has been used for the purpose. The data was collected with help of the 
checklist prepared by The Union. The focus was on the POS advertisements only. For documentary evidences to be produced before the Kolkata Municipal Corporation photographs of POSs were taken.

Results: $64.1 \%$ POSs in Kolkata display various kinds of advertisements. 39\% POSs display board either with colour or brand name, but only $13.7 \%$ Boards are having health warnings on tobacco out of which only $19 \%$ is written in local language. $28.3 \%$ out of them promote tobacco product through attractive messages. $19.1 \%$ POSs have a full body tobacco advertisement. In 92.6\% POSs, tobacco products are visible to the minors

Conclusions: The findings are justifying the immediate need of effective enforcement of Section 5 of COTPA 2003. he documentary evidences led to the writing of letter by the Kolkata Municipal Corporation to 4 major tobacco companies to remove the POS boards.

Tob. Induc. Dis. 2018;16(Suppl 1):A720

DOI:10.18332/tid/84187

\section{$12: 30-14: 00$}

PS-1017-3 Assessing tobacco marketing regulation implementation level in Georgia: evidence from Non Communicable Disease Risk Factors STEPS Survey Georgia 2016

L Sturua ${ }^{1}$, N Maglakelidze'1, A Gamkrelidze1, M Maglakelidze² ${ }^{1}$ National Center for Disease Control and Public Health, Tbilisi, Georgia, ${ }^{2}$ Georgian Respiratory Association, Tbilisi, Georgia. E-mail: lela.sturua@ncdc.ge

Background: Tobacco use continues to be the leading cause of preventable deaths worldwide; the biggest burden of it falls on low- and middle-income countries and this trend is expected to widen further in case of inaction. The overall objective of the study is to describe and analyze the findings of the Noncommunicable Disease Risk Factors STEPS Survey Georgia 2016 related to tobacco marketing regulation.

Methods: The current study in Georgia was a population-based STEPS survey of randomly selected adults aged 18-69 within each sex and 10-year age-group. A multi-stage cluster sample design was used to produce representative data for that age range in Georgia. A total of 5554 adults participated in the current survey. We assessed level o tobacco marketing regulation implementation in Georgia.

Results: Study results reported high prevalence of tobacco use in Georgian population (31.1\% (95 \% CI: 29.0-33.1)). Study findings showed that about half of the current smokers are exposed to antitobacco information on TV or radio 52.3\% (95\% CI: 48.9 - 55.7), whereas $35.5 \%$ (95\% CI: 32.2 - 38.8) reported being exposed to cigarette marketing at points of sales of tobacco products and $4.7 \%$ (95\% CI: 3.2-6.1) to any cigarette promotions. Health warnings on cigarette packs was reported to be noticed by $88.7 \%$ (95\% CI: 85.8-91.6)) of current smokers; only 33\% (95\% CI: 25.4 - 40.7) of them reported having thought about quitting because of these warnings.

Conclusions: The prevalence of smoking in Georgia is high compared to other countries of the European Region and it is very likely that smoking related NCDs burden will increase. Stricter tobacco control policies coupled with anti-tobacco media campaigns can address this problem. Improved health warnings on tobacco packages and total ban of tobacco advertisement, promotion and sponsorship should be implemented in order to overcome prevailed tobacco industry marketing strategies.

Tob. Induc. Dis. 2018;16(Suppl 1):A721

\section{DOI:10.18332/tid/84224}

12:30-14:00

PS-1019-3 Role of the judge in defending tobacco control measures in Colombia: total ban on advertising, promotion and sponsorship of tobacco products case

LV Calderón Pinzón ${ }^{1}$

${ }^{1}$ Ministry of Health and Social Protection, Noncommunicable Diseases Office, Bogotá, Colombia. E-mail: Ivcalderonp@gmail. com

Background and challenges to implementation: Total ban of all forms of advertising, promotion and sponsorship of tobacco products is considered one of the most cost effective measures for tobacco control in the world. Colombia incorporated this measure through articles 14 to 17 of Law $1335-2009$, which entered into force on July 21, 2011. These articles were sued for limiting the right to freedom of enterprise and commercial discourse of tobacco companies.

Intervention or response: Corpus iuris international for the control of tobacco (FCTC and guidelines), requires all efforts for effective compliance, for this, is necessary to carry out the exercise of harmonisation of domestic legal system with obligations derived from international treaties. At this point, it is vital to point out that compliance with rules adopted by the country, based on international treaties, must be given in good faith, understanding that the ultimate goal is the real guarantee of the pledged commitments of all State agencies. In this case judicial power confirm the constitutionality of the measures related with total ban.

Results and lessons learnt: Colombian jurisprudence has shown that Article 16 of that rule, referring to the prohibition of "any form of promotion of tobacco products and their derivatives"; constitutes a broad clause that must be interpreted in accordance with the international instruments subscribed by the country and which include the contents of articles 1 and 13 of FCTC, as well as its implementation guidelines.

Conclusions and key recommendations: Colombia, has established a total ban on all forms of advertising, promotion and sponsorship of tobacco, as a result of the harmonization process between internal rules, mainly related to measures to protect the right to health of the population. In view of the adverse consequences of tobacco consumption and exposure to tobacco smoke and the international obligations set out in the first legally binding public health treaty within the United Nations System,: WHO's FCTC.

Tob. Induc. Dis. 2018;16(Suppl 1):A722

DOI: $10.18332 /$ tid/84315

\section{2:30-14:00}

PS-1020-3 Assessing point of sale violations in Delhi for strategic advocacy on TAPS ban

B Mathew

'Voluntary Health Association of India, Delhi, India. E-mail: binoymathew84@gmail.com

Background and challenges to implementation: The rules banning Point of Sale (PoS) Advertising under COTPA 2003 were stayed by Bombay High Court in 2005-6 and the Government of India did not make any particular efforts to get the stay vacated. In July 2013, in the matter of HFM Trust v/s Union of India and Ors., the Supreme Court vacated the stay on PoS and directed the Government of India to rigorously implement the Rules. 
The Health Ministry issued necessary directives to the State Governments to ensure steps are taken to curb PoS violations.

Intervention or response: One year after the stay was vacated, VHAI decided to conduct a survey to assess status of PoS compliance in Delhi; methods and strategies of $\mathrm{PoS}$ advertising and whether minors have easy access to products. The sample size was 50 shops chosen from each city zone, with a total of 250 shops/outlets and the methodology involved observations and questionnaire.

Results and lessons learnt: $96.4 \%$ shops displayed PoS tobacco advertisements in varied forms. Implementation was found to be weak and ineffective. All major brands of smoking and smokeless tobacco were being advertised at points of sale. $60.8 \%$ shops did not have the top front board as specified. $50.4 \%$ boards had health warning but alongside other promotional messages, brand designs (only 36 shops had a warning board as per specifications) $94.4 \%$ shops had tobacco product display, visible and accessible to minors

Conclusions and key recommendations: Urgent steps are required for effective implementation of PoS Rules across India as the industry despite a court order is using points of sale to advertise tobacco products through incentives and there is a need to capacitate and strengthen enforcement mechanism and to streamline a system of regular raids with penalties.

Tob. Induc. Dis. 2018;16(Suppl 1):A723

DOI:10.18332/tid/84391

\section{2:30-14:00}

PS-1021-3 Increase the media contribution to reduce tobacco promotion in Tamil media

AC Abdul Raheem ${ }^{1}$, N Selladurai ${ }^{1}$

${ }^{1}$ Alcohol and Drug Information Center, Tamil Media and Plantation Programme, Colombo,Sri Lanka.E-mail: raheemadic@gmail.com Background and challenges to implementation: Alcohol and Drug Information Centre (ADIC) found that Tamil media (TV, radio and websites) highly influence on attitudes and behavior on tobacco consumption. ADIC implemented a programme with media to address this issue. The main challenge was Tobacco Company also maintains PRA with the media persons to promote their product.

Intervention or response: In 2015 directly approached and make aware 455 print media and electronic media persons by 65 training programmes. Lead them to influence the community and pressure through opinion leaders and other celebrities. 300 personal discussions used carried twice a month updating them current situation as the follow-ups. Media module made by ADIC distributed among them. Develop action groups in selected communities to pressure on media when they promote alcohol and tobacco. Formed 15 opinion leaders to criticize promotions and hold discussions and update media behaviors on the spot.

Results and lessons learnt: By $90 \%$ increases the media person knowledge on tobacco promotion. 80 electronic media directly contributed for tobacco prevention activities. 25 media persons counter attacked tobacco promotions in films and it made a changed in the film "Maari". 120 prevention articles were published in news papers. 120 prevention programmes conducted by media persons. Tobacco Promotions in print media were reduced by $12 \%$ (2015) to 5\% (2016). Meanwhile Prevention activities were increased in print media.

Conclusions and key recommendations: If develop the resource persons within the media, it is the best tools to change vast community. Same time different approaches we have to use to change media persons. Because media persons have good connection with tobacco industry as well. As Tobacco Company also try to approach media persons.

Tob. Induc. Dis. 2018;16(Suppl 1):A724

DOI:10.18332/tid/84412

\section{2:30-14:00}

PS-1022-3 Tobacco advertisements, promotion and sponsorships (TAPS): tobacco companies continue the business of lies and deception in India

R Chand ${ }^{1}$

'Indira Gandhi Medical College, Hospital Administration, Shimla, India.E-mail: dr.rameshigmc@gmail.com

Background: Tobacco companies have been using all possible tricks to market their deadly products and earn profits at the expense of lost lives. Companies are continually applying manipulative tactics and circumventing the Indian tobacco control law (COTPA). It is important for tobacco control advocates and law implementers to have an understanding how tobacco companies market their deadly products. This information is important in designing tobacco control efforts and get better prepared to counter the industries tactics. The present study was conducted with the objectives to keep a track on tobacco industry's tactics and overview the range of the ongoing TAPS violations in India.

Methods: Investigator did newspaper scans, conducted a market survey and searched leading company's website and facebook pages in 2015 and 2017. The marketing materials at point of sale (PoS), outdoor media were also analyzed.

Results: Tobacco companies are utilizing PoS as a strategic location for carrying out TAPS. Companies are paying retailers to display the advertisement billboards; tobacco products are placed strategically to give an ambience of power walls. Most cigarette brands are offering price discounts and encouraging single cigarette sale. Contests, giveaways and coupons for gift catalogs are other gimmicks. Special edition packages of cigarette brands are common in India. Surrogate advertisements as trademark diversification and brandstreching are most rampant. Industry has been successful in product placement in movies and promoting tobacco through film stars. As part of the image building exercise, most tobacco companies are doing CSR activities. The industry effectively and discreetly is using social networking sites like "facebook" to promote its products.

Conclusions: Tobacco companies in India are carrying out aggressive advertising and promotion and sponsorship campaigns to glamorize its deadly product through indirect advertising and deceitful tactics. There is an urgent need to enforce legislation to contain all common types of TAPS violations in India.

Tob. Induc. Dis. 2018;16(Suppl 1):A725

DOI:10.18332/tid/84415

$12: 30-14: 00$

PS-1024-3 Marketing of Smokeless Tobacco (SLT) products through internet among the WHO FCTC ratified countries

P Ravi', K Chandan', S Grover', A Chandra', D Sinha', R Mehrotra' ${ }^{1}$ National Institute of Cancer Prevention and Research, Noida, Noida, India. E-mail: priusarav@gmail.com

Background: Marketing of SLT products through internet is banned in 42 countries. Flavouring of SLT is banned in only in a handful of countries. There is scant literature on the internet sale of SLT products and their associated flavours. This study evaluates global online market of SLT products, the number of 
websites selling these products, the type of products and the flavours mentioned in the website.

Methods: The current study was conducted in June-July 2017; online search was done for the sites selling SLT products through Google browser. First 20 pages of the Google results were visited for each country using the key words "chewing tobacco", "snuff", "snus", "smokeless tobacco" and "oral tobacco" in combination with general terms "tobacco sale", "online sale" etc. Data was obtained for the number and type of product sold and flavours mentioned for each product.

Results: Our search showed that there is a wide spread internet sale of SLT products notably in India, UK, USA and Sweden. There is lack of policy in the high SLT burden countries like India, Bangladesh, Myanmar, Pakistan and China. Policy on ban of flavoured SLT products is there in only Australia, Brazil, Colombia, Republic of Korea and Sri Lanka. Online sale of flavoured SLT product sale was extensive in India, Thailand, USA and UK. Most commonly used flavours were sweet, spice, flower, fruit, alcohol flavours and various non-specific flavours.

Conclusions: There is a wide spread sale of SLT products online. This enables the individuals to buy the products around the globe. The need of the hour is to formulate a policy to reduce the sale of online SLT products.

Tob. Induc. Dis. 2018; 16(Suppl 1):A726

DOI: $10.18332 /$ tid/84481

\section{2:30-14:00}

PS-1025-3 Removing tobacco advertisements from all shops in Kashmir division, India

\section{S Jain 1 , S Gupta ${ }^{2}$}

${ }^{1}$ Voluntary Health Association of India, New Delhi, India, ${ }^{2}$ Voluntary Health Association of India, Delhi, India. E-mail: sukriti.jain86@gmail.com

Background and challenges to implementation: Cigarette \& Other Tobacco Products Act (COTPA), 2003 Section 5 -Prohibits advertisement, sponsorship and promotion of tobacco products. The Supreme Court of India further directed strict implementation banning any type of tobacco advertisement at Point of Sale (PoS). Kashmir was unique as all shops - Stationary, grocery, fruits \& vegetable etc. sold tobacco in order to get illuminated attractive boards from the TI bearing shop and owner name in one corner. TI knowingly continued the violation of Sec. 5 and displayed their advertisements on these boards resulting in entire highways and roads of best locations lined with their ads.

Intervention or response: Voluntary Health Association of India (VHAI) launched a campaign to make the Kashmir Division 'PoS Free'. Systematic mapping of stakeholders was done. The Police was identified as key department, and the highest level of officials were sensitized and commitment for action was established. As a result, Director General of Police issued strict orders for compliance of the Act following which capacity building was conducted of officials. Coordinated and collaborated raids with health, FDA \& NGOs were conducted across the division and boards were removed. In order to ensure sustainable compliance mechanism, the details of the tobacco wholesellers/retailers was shared with the Deputy Inspector General Srinagar who then directed TI to remove all illegal boards else face legal action. This placed the onus on the tobacco sellers instead of the monitoring agency. Media was sensitized and covered the issue extensively, gaining public opinion.

Results and lessons learnt: Kashmir Region was declared 'PoS Free' with the removal of over 400 advertisement boards.
This would decrease brand recall and digress youngsters from initiating consumption.

Conclusions and key recommendations: An active enforcement mechanism along with placing the onus on the tobacco industry to ensure compliance proved to be most effective and can be applied in other settings.

Tob. Induc. Dis. 2018;16(Suppl 1):A727

DOI:10.18332/tid/84515

\section{2:30-14:00}

PS-1026-3 Enabling the way from policy to practice realizing the gaps and loopholes in ban on promotion A Rathinam ${ }^{1}$, A Savariyar ${ }^{1}$

'Pasumai Thaayagam Foundation, Chennai, India. E-mail: mailtopt@gmail.com

Background: FCTC Article 13 requires implementing comprehensive ban on tobacco promotion and Section 5 of the Indian Tobacco Law COTPA prescribes for complete ban on all forms of promotions and having policy regulations for tobacco product sale points. FCTC Article 11 and COTPA Section 7 (1) mandate that Tobacco products be sold with appropriate pictorial warning. However the outlet sale points display tobacco products in violation, and sell imported cigarettes without stipulated statutory pack warning. The authors made a study on the factual status of compliance in April 2017.

Methods: The authors assessed if there were any violations related to provision of tobacco sale points in Chennai through a field study. Sixty sale points in two zones was selected for assessing the compliance/violation. The study analyzed the compliance of COTPA Sections 5 and 7 (1) looking at the patterns of violations.

Results: Findings revealed that all outlets sold imported cigarettes without appropriate pictorial warning as mandated by Indian law. $82 \%$ of the shops did not have the display board containing words, "Tobacco products not sold to children below 18 years", and only $8 \%$ of shops had display of "smoking prohibited area". $86 \%$ shops displayed the availability of cigarettes conspicuously. All studied shops provided lighters or match boxes and allowed smoking near their shops. Smoking activity could be seen in $90 \%$ of the shops observed. None of the shops had displayed board of tobacco products sold here. The authors used the outcome of the study for advocacy with the various enforcing authorities by way of enabling them to set in motion of policies.

Conclusions: Irrespective of various restrictions tobacco companies continue looking for loopholes and try its sophisticated and clandestine campaigns targeting to lure new users which circumvent the Indian tobacco promotional ban, which needs curbing through effective advocacy efforts for stringent implementation of law.

Tob. Induc. Dis. 2018;16(Suppl 1):A728

DOI:10.18332/tid/84539

\section{$12: 45-14: 00$ \\ EP-206-3 Norway introduces standardised packaging on smokeless tobacco \\ AK Aambø ${ }^{1}$, R Lindbak', M Edbo², K Solbakken² \\ ${ }^{1}$ Norwegian Directorate of Health, Public Health, Oslo, Norway, ${ }^{2}$ Norwegian Directorate of Health, Oslo, Norway. E-mail: monica. edbo@helsedir.no}

Background and challenges to implementation: The use of smokeless tobacco has increased and is high in Norway, especially among young people. Among 16-24-year-olds, 21 per cent of 
men and 17 per cent of women are daily users. In the past $10-15$ years an increasing amount of new snus products with varied and appealing designs has appeared on the market.

Intervention or response: New regulation requiring standardised tobacco packaging, including snus, came into effect in July 2017. Norway is the first country to introduce plain snus boxes without the manufacturer's logo and colours. The overall purpose is to reduce the number of young people who start using tobacco. By limiting the packaging's advertising effect, tobacco products are made less attractive. The purpose is also to increase the effect of health warnings and minimise the risk that the package design gives a misleading impression of the health risk.

Results and lessons learnt: The Norwegian Directorate of Health launched a mass media campaign in May 2017 to inform the population about the introduction of standardised packaging of tobacco, and to increase the support and the knowledge about its purpose. The main message was that standardised packages are introduced to protect children and adolescents. The campaign showed how good product design works as advertising for tobacco. Conclusions and key recommendations: A web-based pre-test and post-test were conducted with samples of 1000 persons aged 16-60 years. There was a significant increase in knowledge of the introduction of plain packs, from 50 to 77 percent during the campaign period. Knowledge of the main purpose (preventing recruitment of young people) increased significantly from 17 to 35 percent. The support for the regulation had a significant increase from 53 to 59 percent for cigarette packs, and from 51 to 58 percent for snus boxes. Mass media campaign can be a useful tool to increase support for new regulations.

Tob. Induc. Dis. 2018;16(Suppl 1):A729

DOI:10.18332/tid/83826

\section{2:45-14:00}

EP-208-3 Effectiveness of the ban on tobacco industry sponsorship in Brazil: findings from the ITC Brazil Wave 1 to 3 Surveys (2009 to 2016 - 17) M Pinho' ${ }^{1}$ A Szklo ${ }^{2}$, C Perez ${ }^{1}, \mathrm{G}$ Fong ${ }^{3,4}, \mathrm{~L} \mathrm{Craig}^{3}, \mathrm{G} \mathrm{Li}{ }^{3}, \mathrm{M} \mathrm{Yan}^{3}$ ${ }^{1}$ Cancer Foundation, Rio de Janeiro, RJ, Brazil, ${ }^{2}$ Brazilian National Cancer Institute, Rio de Janeiro, RJ, Brazil, ${ }^{3}$ University of Waterloo, Waterloo, ON, Canada, ${ }^{4}$ Ontario Institute for Cancer Research, Toronto, 0N, Canada. E-mail: maripinho77@ gmail.com

Background: In 2000, Brazil implemented a comprehensive ban on tobacco advertising (except point-of-sale), promotion, and sponsorship, including sponsorship by tobacco brands of national sporting and arts events, then in 2005 adding international sport events. However, a loophole in the law permits company names for sponsorship. This study examines whether this loophole has been exploited by the industry via Brazilians' awareness of tobacco company sponsorship of sporting and arts events between 2009 and 2016-17.

Methods: Data were from Waves 1 to 3 (2009, 2012-13, 2016-17) of the International Tobacco Control (ITC) Brazil Survey - a cohort survey of approximately 1200 adult smokers and 600 non-smokers in Rio de Janeiro, São Paulo, and Porto Alegre. At all three waves, respondents were asked whether they had seen or heard about (1) sport or sporting events and/or (2) music, theatre, art or fashion events sponsored by or connected with cigarette companies. At Waves 2 and 3, tobacco company name recognition was tested by asking respondents whether the following are tobacco companies: Souza Cruz, Nestle, and Pirelle. Data were analyzed using GEE logistic regression models.

Results: Awareness of a sport or sporting event sponsored by a cigarette company decreased between 2009 and 2016-17 among both smokers (from $8.7 \%$ to $4.7 \%$; $=0.004$ ) and non-smokers (from $11.8 \%$ to $5.7 \% ; \mathrm{p}=0.008$ ). Awareness of an arts event sponsored by a tobacco company decreased among smokers (from $6.3 \%$ to $1.4 \%$; $\mathrm{p}<$ .001 ), but not significantly among non-smokers (from $6.1 \%$ to $3.8 \%$; $\mathrm{p}=0.235$ ). Over $93 \%$ of smokers and non-smokers correctly stated that Souza Cruz is a tobacco company and over $98 \%$ correctly stated that Nestle and Pirelle are not.

Conclusions: Although Brazil has not specifically banned tobacco company sponsorship, public awareness of tobacco company sponsorship has decreased over 7 years, possibly suggesting that the industry has not (yet) taken advantage of the loophole.

Tob. Induc. Dis. 2018;16(Suppl 1):A730

DOI:10.18332/tid/83931

$12: 45-14: 00$

EP-209-3 Exposure to electronic cigarette advertising and intention to use electronic cigarettes in Hong Kong adolescents

LT Leung ${ }^{1}$, SY Ho ${ }^{1}$, J Chen ${ }^{1}$, MP Wang ${ }^{2}$, TH Lam ${ }^{1}$

'University of Hong Kong, School of Public Health, Hong Kong, Hong Kong, ${ }^{2}$ University of Hong Kong, School of Nursing, Hong Kong, Hong Kong. E-mail: Iloktung@hku.hk

Background: Adolescent electronic cigarette (EC) use in relation to exposure to advertising has rarely been studied outside Western settings. We investigated the exposure to EC advertising in Hong Kong adolescents and its association with the intention to use ECs in never users.

Methods: In 2014/15, 40202 Secondary 1-6 (US grade 7-12) students (mean age 14.9, standard deviation 1.8; 51.5\% boys) from 92 randomly selected schools completed an anonymous questionnaire. Exposure to messages directly or indirectly promoting ECs through each of 12 sources in the past 30 days, and the intention to use ECs in the next 12 months or when a good friend offered an EC were reported. Weighted prevalence of exposure to EC advertising was calculated. Logistic regression yielded adjusted odds ratios (AORs) of intention to use ECs for the exposure to EC advertising (versus no exposure to any source) in never EC users.

Results: In all students, 38.0\% (95\% confidence interval [CI] $37.5 \%-38.5 \%$ ) were exposed to EC advertising, most commonly through television (15.1\%). In never EC users ( $n=36274)$, any exposure to EC advertising was associated with an AOR (95\% CI) of 1.50 (1.23-1.84) for the intention to use ECs, with increasing AORs for more sources of exposure ( $p$ for trend $<0.001$ ) and an AOR of 2.20 (1.55-3.12) for 4 sources or more. Exposure through social networking sites (prevalence: 9.9\%; AOR 2.58, 95\% CI $2.03-3.27)$, video sites $(4.4 \% ; 2.56,1.84-3.55)$ and other websites $(2.5 \% ; 2.26,1.45-3.53)$ had the strongest associations with the intention to use ECs.

Conclusions: Four in 10 Hong Kong adolescents were exposed to EC advertising through different sources. The exposure was dose-dependently associated with an intention to use ECs in never users. Banning EC advertising may prevent EC use in adolescents.

Tob. Induc. Dis. 2018;16(Suppl 1):A731 DOI:10.18332/tid/83932

\section{$12: 45-14: 00$ \\ EP-210-3 The IOOS campaign in Israel \\ L Rosen ${ }^{1}$, S Kislev²}

${ }^{1}$ Tel Aviv University, Ramat Aviv, Israel, ${ }^{2}$ CEO, The National Initiative to Eradicate Smoking, Tel Aviv, Israel. E-mail: rosenl@ post.tau.ac.il 
Background and challenges to implementation: Israel was once a pioneer in tobacco control. The first advertising restrictions were legislated in 1983; these included bans on advertisement on radio and television. Israel signed the FCTC in 2003 and ratified it in 2005. Words, symbols, or colors indicating harm reduction were banned in 2004. A comprehensive ban on advertising to children was passed in 2006. In 2011, the Israeli government passed a National Plan for the Reduction of Tobacco Use and Harm which included a total advertising ban. However, the plan required further legislation, and this was blocked in the Knesset. Evidence of tobacco industry lobbying against the advertising bill was recently published by Reuters on the basis of the Philip Morris Files. Following meetings between Philip Morris (PM) executives and high-level governmental officials in 2016, IQOS entered Israel as a non-tobacco product in December, 2016. An aggressive advertising campaign followed entitled "Smoke-Free Israel", first on internet/social media (Facebook) and then as full page and large advertisements in the print press.

Intervention or response: Two petitions to the Supreme Court - one from the Israeli tobacco company, Dubek, and one from the NGO Advanced Democracy - led the Supreme Court to intervene. IQOS was redefined as a tobacco product, and covered by existing advertising legislation beginning 4/4/2017. Advertising continued unabated.

Results and lessons learnt: Legal loopholes, particularly regarding social media, and partial marketing restrictions may allow for the uncontrolled advertisement of new tobacco, smoking, and nicotine products to adults and youth.

Conclusions and key recommendations: Theworld of advertising, sponsorship, and promotion has changed dramatically in recent years. Countries must carefully review and expand legislation to include new tobacco, smoking, and nicotine products, in order to protect the health of the public.

Tob. Induc. Dis. 2018;16(Suppl 1):A732

DOI:10.18332/tid/84448

\section{$12: 45-14: 00$}

EP-212-3 Retail reform. How conditions of license can be applied to strengthen tobacco control in a range of local circumstances

C Callard ${ }^{1}$

${ }^{1}$ Physicians for a Smoke-Free Canada, Ottawa, ON, Canada. E-mail: ccallard@smoke-free.ca

Background and challenges to implementation: In most developed countries, most smokers visit a retail store a few times a week to buy cigarettes, providing a hundred or more moments of contact over a year. Tobacco companies invest heavily in their relationship with retailers to control these moments of contact and to maximize influence on price, word of mouth and other promotions. Yet controls on retail are almost absent from globally-recommended tobacco control strategies, like the Framework Convention on Tobacco Control, the Bloombergfunded MPOWER measures, or the European Union Directive. None of these offer a model for tobacco retail management. This is in contrast with international guidance on comparable health concerns like alcohol, where the World Health Organization recommends a range of supply-controls, like minimum pricing, government retail monopolies and retail licensing.

Intervention or response: To address this global policy gap, researchers and advocates are independently contributing to a menu of new options for localized retail reform. These proposals draw on the experience with tobacco and other products in diverse countries.
Results and lessons learnt: Requirements can be imposed by law or conditions of license to better align tobacco retail practice with public health goals. Financial and non-financial requirements already exist in tobacco retailing, including mandatory training of retailers in health consequences of tobacco use, requirements to provide health authorities with data on local sales, requirements to distribute materials to support cessation, restrictions on locations, types of venues or types of license-holders. Other licensing requirements can be adapted from existing distribution controls currently applied to alcohol, pharmaceutical products, cannabis and other products with risks associated with public health risk.

Conclusions and key recommendations: Retail reform has been identified as a next frontier for tobacco control. A wide range of options are available for adaptation to local or national circumstances.

Tob. Induc. Dis. 2018;16(Suppl 1):A733 DOI:10.18332/tid/84583

\section{$12: 45-14: 00$}

EP-213-3 Scottish young people's perceptions of standardised packs - a qualitative study

A Macgregor ${ }^{1}$, A Amos $^{2}$, C Best $^{3}$, D Eadie $^{3}$, J Pearce $^{4}$, M Stead $^{3}$, $\mathrm{S} \mathrm{Haw}^{3}$, DISPLAY Study Group

${ }^{1}$ ScotCen Social Research, Edinburgh, United Kingdom, ${ }^{2}$ University of Edinburgh, Usher Institute of Population Health Sciences and Informatics, Edinburgh, United Kingdom, ${ }^{3}$ University of Stirling, Stirling, United Kingdom, ${ }^{4}$ University of Edinburgh, Edinburgh, United Kingdom. E-mail:amanda.amos@ed.ac.uk Background: Standardised cigarette packs were introduced into the UK in May 2016. Retailers could sell old stock until May 2017 after which only the sale of cigarettes and tobacco in standardised packs was allowed. As in Australia, pack shape, colour, opening mechanism and font are regulated, together with the size and position of health warnings and number of cigarettes in a pack. This paper explores Scottish young people's awareness of and views about standardised packs in Spring 2017.

Methods: The DISPLAY study is a five year study established to evaluate the national tobacco point-of sale (POS) promotions ban in four communities in Scotland. This paper is based on the qualitative component, annual focus groups carried out with Secondary 2 (13 year olds) and Secondary 4 (15 year olds) students in four secondary schools. 16 groups (82 students) convened in February - March 2017 explored students' perceptions of standardised packaging.

Results: There was a high level of awareness of standardised packs prior to their full implementation. Smokers had bought them, and they and other participants had seen them in possession of friends and family members, and in litter. Participants' views of the new packaging were generally negative, described as unappealing and depressing, particularly the pictorial health warnings. Packs were compared unfavourably with previous non-standardised versions. However, there was no consensus on their likely impact. Some participants argued that their impact would be widespread, while others thought that any impact would be confined to young non/occasional smokers and that established smokers would be unaffected.

Conclusions: In early 2017 young people in Scotland had high awareness and knowledge of standardised tobacco packs before their full implementation. Despite differing views about their likely impact on youth smoking, participants irrespective of smoking status overwhelmingly regarded them as unattractive and less appealing than non-standardised packs.

Tob. Induc. Dis. 2018;16(Suppl 1):A734

DOI:10.18332/tid/84253 
$12: 45-14: 00$

EP-214-3 Marketing to the selfie generation: a case study of " $A$ " cigarette brand marketing communication in Indonesia

PAS Astuti $1,2,3$, M Assunta ${ }^{1,4}$, B Freeman ${ }^{1,3}$

'School of Public Health, The University of Sydney, Sydney, NSW, Australia, ${ }^{2}$ School of Public Health, Faculty of Medicine, Udayana University, Bali, Indonesia, ${ }^{3}$ Prevention Research Collaboration (PRC), Charles Perkin Center, The University of Sydney, Sydney, NSW, Australia, ${ }^{4}$ Southeast Asia Tobacco Control Alliance (SEATCA), Bangkok, Thailand. E-mail: past4344@uni. sydney.edu.au

Background: Incomplete bans of tobacco advertising, promotion and sponsorship [TAPS] in Indonesia enable the tobacco industry to exploit both direct and indirect cigarette promotion. Alongside its annual SoundrenAline concert to promote Sampoerna A cigarette brand, PT HM Sampoerna used social media and its corporate website to develop its brand image. This study aims to describe an integrated marketing communication (IMC) strategy of Sampoerna A brand and how Instagram and the corporate website were leveraged to broaden the marketing campaign reach and to increase engagement with young people.

Methods: A case study of the A cigarette brand IMC was conducted by gathering data from three main sources. HM Sampoerna reports and company websites were reviewed. Four pairs of observers conducted an observation survey, systematically auditing and documenting tobacco promotion and advertising at the 2016 SoundrenAline concert. Social media data was obtained from a search of hashtags on Instagram. The ten most frequently used hashtags related to the concert were reviewed and documented.

Results: An IMC strategy of the A brand was conducted through consistent brand image communication across different medium including the live music event and a virtual event on the corporate websites and social media accounts. Instagram boosted event promotion with more than 25,000 posts for the two main hashtags that were endorsed during the concerts. HM Sampoerna evaded the advertising law by changing the concert name, using pictorial health warnings on the promotional material and including an age $18+$ restriction to both the concert and to the websites.

Conclusions: This case study provides evidence that PT HM Sampoerna has exploited loopholes in the incomplete Indonesian tobacco control regulation and has embraced social media and the internet to leverage its cigarette marketing. Indonesian tobacco advertising bans need to be reformed to include internet and social media advertising in order to effectively protect young people.

Tob. Induc. Dis. 2018;16(Suppl 1):A735

DOI:10.18332/tid/83992

\section{2:45-14:00}

EP-215-3 Misleading descriptors on cigarette packs in Indonesia, the Philippines and Vietnam: before and after implementation of misleading packaging regulations

J Brown ${ }^{1,2}$, J Cohen ${ }^{1,2}$, K Smith $^{1,2}$

'Johns Hopkins Bloomberg School of Public Health, Health, Behavior \& Society, Baltimore, MD, United States of America, ${ }^{2}$ Institute for Global Tobacco Control at the Johns Hopkins Bloomberg School of Public Health, Baltimore, MD, United States of America. E-mail: jbrow212@jhu.edu

Background: Tobacco companies use descriptors on cigarette packs to convey less harm. The WHO FCTC Article 11 calls for the elimination of misleading packaging. This study compares the use of misleading descriptors on packs before (2013) and after (2015/2016) regulations on misleading descriptors were implemented in three lower middle income Asian countries.

Methods: A census of cigarette packs on the market were purchased in 2013 and 2015/2016 in Indonesia, the Philippines, and Vietnam using a systematic protocol. Packs displaying the country's health warning labels in rotation at time of purchase were double coded for select misleading descriptors in 2013 and for banned descriptors in 2015/2016.

Results: In 2013, 33\%, 28\%, and $11 \%$ of packs, in Indonesia $(\mathrm{n}=215)$, the Philippines $(\mathrm{n}=98)$, and Vietnam $(\mathrm{n}=83)$, respectively, displayed "light(s)", "mild", or "low". In 2015/2016, 76 packs $(36 \%)$ from Indonesia $(n=209)$ displayed one or more of the following banned descriptors: "light", "mild", "low tar", "slim", "special", "full flavor", and "premium", 36 with those in the brand name. No packs from the Philippines $(\mathrm{n}=83)$ displayed explicitly banned descriptors. Three packs from Vietnam $(\mathrm{n}=88)$ displayed "mild" but no other banned descriptors. In the Philippines and Vietnam, alternative descriptors such as "smooth" and colors (e.g. "wind blue") have replaced "light(s)".

Conclusions: While compliance with new regulations in the Philippines and Vietnam is high, as they do in other countries, tobacco companies are using alternative descriptors to convey cigarette strength. By retaining a similar overall design, many brands previously labeled "light(s)" may still be recognizable to consumers. In Indonesia, tobacco companies are taking advantage of a loophole in regulations that allows for misleading descriptors to remain in previously used brands or trademarks. These findings reinforce that tobacco companies continue to use the same tactics to circumvent regulations on misleading descriptors and the need for plain packaging that includes restrictions on brand names.

\section{Tob. Induc. Dis. 2018;16(Suppl 1):A736} DOI:10.18332/tid/84250

\section{2:45-14:00}

EP-216-3 A global scan of policies regulating e-cigarette advertising, promotion and sponsorship A Awopegba', R Kennedy', J Cohen'

'Institute for Global Tobacco Control, Johns Hopkins Bloomberg School of Public Health, Baltimore, MD, United States of America.E-mail: aawopeg1@jhu.edu

Background: Tobacco advertising, promotion and sponsorship is an important policy domain for tobacco control. There is emerging evidence that e-cigarettes are being marketed to consumers using similar techniques and strategies deployed by the tobacco industry. This study explores the extent to which advertising, promotion and sponsorship restrictions or bans are being applied to e-cigarette products across the globe.

Methods: National policies regulating e-cigarettes were identified primarily through direct contact with representatives of Ministries of Health or tobacco control experts in approximately 130 countries. Further, media monitoring was used to identify emerging or new e-cigarette advertising, promotion and sponsorship policies/legislation, which resulted in direct contact with incountry experts. Copies of written policies were reviewed and policies/approaches were categorized. Finally, policy summaries and classifications were verified by in-country experts including Ministry of Health staff.

Results: The study identified 79 countries that enacted policies regulating e-cigarettes. Of these, 58 countries had a policy 
regulating any or all aspects of advertising and/or promotion and/ or sponsorship. In many cases restrictions include conforming to specific guidelines on the type of media where advertising may be permitted and locations where it is prohibited. Six countries apply the advertising restrictions only to nicotine-containing e-cigarettes that are regulated as medicines. Twenty countries have a policy restricting or banning sponsorship related to e-cigarettes (nicotine and non-nicotine), including eight countries that specifically prohibit cross-border sponsorship of e-cigarettes.

Conclusions: The results of this scan determine that advertising and promotion restrictions or bans are included in the majority of countries that are regulating e-cigarette products. Fewer countries regulate the practice of sponsorship. Monitoring the status of e-cigarette legislation will support further research to measure the impact or effect of policies.

Tob. Induc. Dis. 2018;16(Suppl 1):A737

DOI:10.18332/tid/84544

\section{$12: 45-14: 00$}

EP-217-3 Identifying characteristics of cigarette displays at points of sale in Brazil

F Mendes $^{1}$, C Perez ${ }^{2}$, R Feijó ${ }^{3}$, T Cavalcante $^{1}$

${ }^{1}$ National Cancer Institute, Executive Secretariat of National Comittee for FCTC Implementation, Rio de Janeiro, RJ, Brazil, ${ }^{2}$ Cancer Foundation, Rio de Janeiro, RJ, Brazil, ${ }^{3}$ FCTC Convention Secretariat, Geneva, Switzerland. E-mail: fmendes@inca.gov.br Background: Since 2011 has banned the advertising in the points of sale (POS). However, the legislation allows the exhibition of tobacco products packages in these places, accompanied by health warnings.

Due to this gap in the legislation, the exhibition of packs at the POS (bars, restaurants, bakeries, newsstands) has become the main focus of the advertising strategies of the tobacco industry.

Methods: Identify the characteristics of the display of cigarette packages at POS. The methodology used was the market research called Secret Shopper - the researcher went to 54 locations in 3 capitals and 3 inner cities during 2015 as a potential consumer. The following characteristics of the displays were evaluated: positioning, quantity, shape, size, lighting and height.

Results: Most of the displays observed are placed next to the payment cash, which makes them very visible, because every client must pass in this area. They are usually close to products for children, such as sweets and candies. The displays are also often positioned in a way to be visible outside the POS.

The displays are mostly exposed in walls at a strategic height that facilitates their view from all angles. However, the industry has expanded the number of displays and uses small totems, little displays above tables and wall clocks. Displays on the floor, often as antique furniture, were often observed to display some tobacco products (cigars and pipes) and are easily accessible for children. All displays observed had their own lighting. Even in dark places, the displays attract the attention.

Conclusions: Due to a poor detailed regulation, the industry has improved its marketing strategies in POS. There is an urgent need for a detailed regulation on the exhibition of tobacco products in Brazil. Illuminated, with different shapes, strategically positioned displays can call for more attention and increase sales.

Tob. Induc. Dis. 2018;16(Suppl 1):A738

DOI:10.18332/tid/84149

$12: 45-14: 00$

EP-218-3 Marketing of flavored cigarettes at the point-of-sale (POS) near schools in 5 Latin American cities

A Grant ${ }^{1}$, C Weiger ${ }^{1}$, J Cohen ${ }^{1}$

'Johns Hopkins Bloomberg School of Public Health Institute for Global Tobacco Control, Health, Behavior and Society, Baltimore, MD, United States of America. E-mail: ashleyigtc@jhu.edu

Background: From 2010-2015, sales of cigarettes with menthol or flavor capsules increased in some Latin American countries, amid efforts to regulate the use of additives in tobacco products, and to restrict tobacco advertising, promotion, and sponsorship (TAPS) tactics. This study describes the availability and marketing of flavored cigarettes at the POS in Argentina, Bolivia, Brazil, Chile and Peru.

Methods: Trained observers recorded information about cigarette product displays, advertising, and special promotions in January of 2017 , from 1,188 retailers located within 100-250 meters of 310 primary and secondary schools in a major city in each of the five countries. Retailers were identified by following a map to walk all streets located within the designated radius surrounding each school.

Results: Cigarette retailers were identified within $91.9 \%(n=285)$ of the radii observed, and among $69.4 \%$ of $(n=825)$ retail outlets identified by data collectors. Flavored cigarettes were for sale in $85.2 \%(n=703)$ of all cigarette retailers, $71.4 \%(n=589)$ sold cigarettes with flavor capsules, and $41.5 \%(n=342)$ sold loose cigarettes. Mint/menthol was the most frequently observed flavor descriptor $(74.1 \%, \mathrm{n}=521)$, followed by non-conventional descriptors (69.6\%, n= 489) such as "Fusion Blast", "Double Click", and "Ruby Ice". Flavored packs were displayed in $92.3 \%$ $(\mathrm{n}=649)$ of flavored cigarette retailers, typically in the cashier zone $(72.2 \%, \mathrm{n}=512)$ and near sweets/snacks $(59.2 \%, \mathrm{n}=416)$. Advertising for flavored cigarettes was observed in $34.3 \%$ $(n=241)$ of flavored cigarette retailers, and special promotions for flavored cigarettes were offered in $7.3 \%(n=51)$ of flavored cigarette retailers.

Conclusions: Mint/menthol cigarettes and packs carrying innovative flavor descriptors, which appeal to youth and facilitate smoking uptake, are frequently sold and prominently displayed near schools in these Latin American cities. These findings can inform efforts to prohibit POS marketing and ban the use of additives in cigarettes. Future research should characterize the content and appeal of flavored cigarette marketing.

Tob. Induc. Dis. 2018;16(Suppl 1):A739

DOI: $10.18332 /$ tid $/ 84670$

\subsection{Innovations in media educational campaigns and health warning labels on cigarettes and other tobacco products}

$12: 30-14: 00$

PS-1027-3 Real smokers - helping us take on a killer through the media

A Lloyd $^{1}$, A Rutter $^{1}$

${ }^{1}$ Fresh-Smoke Free North East, Durham, United Kingdom. E-mail: ailsa.rutter@freshne.com

Background and challenges to implementation: Fresh is an award winning tobacco control programme. One of our roles is the de-normalisation of smoking and advocating policy interventions. Public awareness is fundamental, but advertising budgets are always under pressure and cannot be maintained all year round. "Earned media" is highly cost-effective, reaching thousands and setting agendas among decision makers by presenting tobacco as an serious issue. Earned media can also complement and add 
depth, believability and impact to paid-for campaigns and the role of real people is an integral part.

Intervention or response: Fresh runs an "always-on", yearround programme to gain media coverage around the benefits of quitting smoking and to argue for effective regulation. This involves supplying press releases for press, online, radio and TV and increasingly news websites. Through working with partners we have added the voices of doctors and real people sharing both positive experiences of quitting and negative experiences of smoking related diseases, and supporting calls for action. This has including working with a lung cancer patient in her final months. Results and lessons learnt:

- Relationships need sensitivity and tact to develop, but people will willingly share their stories as a warning to others

- Ex-smokers and doctors as spokespeople enables us to target news media more effectively

- The harm of disease can feel remote to smokers: real stories in the media can make these more believable.

- Ex-smokers are proud of quitting, and report that positive publicity can provide them with an added incentive to stay quit, and advise others

- Ex-smokers can also become champions for legislative change. Conclusions and key recommendations: Real people's stories play an important role in communicating the consequences of smoking and there are many opportunities to share these beyond advertising campaigns. While the media is changing away from traditional print, new media brings new channels and new opportunities.

Tob. Induc. Dis. 2018;16(Suppl 1):A740

DOI:10.18332/tid/84151

\section{$12: 30-14: 00$}

PS-1028-3 Effect of visual attention according to the position of graphic health warning labels J-E Hwang ${ }^{1}$, J-E Lee ${ }^{2}$, Y-S Yang ${ }^{2}$, S-Y Lee ${ }^{2}$, Y-M Oh², S-I Cho ${ }^{3}$ 'Seoul National University, Graduate School of Public Health, Seoul, Korea, Republic of, ${ }^{2}$ Korea Health Promotion Institute, Seoul, Korea, Republic of, ${ }^{3}$ Seoul National University, Department of Public Health Science, Graduate School of Public Health, and Institute of Health and Environment, Seoul, Korea, Republic of. E-mail: Ioshjeve@gmail.com

Background: Graphic health warning labels (GHWLs) not only induce smoking cessation among smokers, but also are effective in preventing smoking initiation of non-smokers especially young people. For this reason, the WHO FCTC and its guidelines recommend each Party to introduce a warning within three years of its entry into force, preferably with the use of pictorials. And the guidelines recommend placing GHWLs at top of packs to increase visibility and legibility. Therefore, this study investigated whether the position of GHWLs affects visual attention.

Methods: Thirty daily smokers and twenty-four non-smokers participated in this study. Participants (30 male, 24 female) mean age was 32.31 years $(\mathrm{SD}=6.43)$. The eye movements of participants were recorded by an eye-tracking equipment and measured viewing time, or fixation time, in milliseconds. Participants saw 6 cigarette packs from different combinations of 3 positions (top, middle, bottom) and 2 concepts (skin aging, toxic constituents). Size of the GHWL was $30 \%$ of the pack while tobacco branding covered $50 \%$ of the pack.

Results: Overall, Participants spent more time looking at the health warning area than in the tobacco branding area. When GHWL was displayed at the top of the pack, participants spent longer time to look at the health warning area compared to the bottom position.

Conclusions: GHWLs displayed at the top of the pack appear to increase visual attention of health warning while tobacco brand area getting less attention. Findings suggest that positioning GHWLs at the top of the pack is more visible and legible than at the bottom.

Tob. Induc. Dis. 2018;16(Suppl 1):A741

DOI: $10.18332 /$ tid/84180

\section{2:30-14:00}

PS-1029-3 Multimedia campaign aimed at increasing support among the Dutch population for measures to protect children from the temptations of tobacco M Carlier ${ }^{1}$, L Houtenbos ${ }^{1}$

'Dutch Cancer Society, Prevention, Amsterdam, Netherlands. E-mail: mcarlier@kwf.nl

Background and challenges to implementation: As part of a comprehensive strategy (2015-2018), aimed at protecting children from the temptations of tobacco, cross-media communication is an important instrument for the Dutch Cancer Society to increase knowledge, understanding and support among the Dutch population. With a high level of social support, the government will be more likely to take the necessary measures to protect children from tobacco. A successful part of the cross-media communication is the campaign 'That's actually quite ridiculous, right?', which focuses on the target group 30-50 year olds.

Intervention or response: The campaign is divided into phases and works towards 3 matching goals:

1. Awareness, urgency and indignation have been created about the temptations of tobacco;

2. Support among the Dutch population for the necessary measures has increased;

3. Society and government are inspired, mobilized and activated to protect children

from the temptations of tobacco.

Phase 1 has taken place with the key message that it's "quite ridiculous" how we still regard cigarettes to be very normal in our society. 5 campaign flights are deployed over $1 \frac{1}{2}$ years (20152016), via TV commercials, radio spots, outdoor and online.

Results and lessons learnt: The results of phase 1 measured among the target group, show:

- Average report rate: 7.7 ;

- Prompted awareness: $82 \%$;

- $86 \%$ of the target group believes that government should take measures to prevent children from starting smoking (baseline $68 \%)$

- $71 \%$ of the target group is willing to do something to ensure that children do not start smoking (baseline 65\%).

Conclusions and key recommendations: The campaign has demonstrably contributed to increasing the level of support in Dutch society for protecting children from the temptations of tobacco. This encourages Dutch government to implement the necessary measures. The campaign's success factors include the positive frame, not alienating the "smoker" and appealing to the target group by creating awareness, after which people turn to thinking.

Tob. Induc. Dis. 2018;16(Suppl 1):A742

DOI:10.18332/tid/84240

$12: 30-14: 00$

PS-1030-3 Effectiveness of pictorial health warnings on cigarette packs among adults in a rural Sri Lankan community 


\section{S Zawahir ${ }^{1,2}$, M Madhushika', H-H Yong ${ }^{3}$}

${ }^{1}$ Faculty of Medicine, University of Ruhuna, Galle, Sri Lanka, ${ }^{2}$ The Faculty of Pharmacy, Sydney, NSW, Australia, ${ }^{3}$ Cancer Council VictoriaPatients Aid Association, Melbourne, VIC, Australia.E-mail: shukry2010@gmail.com

Background: Pictorial health warnings (PHWs) on $80 \%$ of the cigarette packs were introduced in 2015 as the most recent method of communicating health risk of smoking in Sri Lanka.

This study aimed to evaluate the effectiveness of the newly introduced PHWs pictorial health warnings on cigarette packs among adults in a rural community.

Methods: Cross sectional data from 360 adults ( $56 \%$ male) smokers and non-smokers (68\%) randomly selected from rural households to complete a pre-validated self-administered questionnaire were analysed. The questionnaire collected sociodemographic data, smoking history/behavior, and exposure to antismoking media messages and newly introduced pictorial warnings, knowledge about risks of smoking and attitudes on smoking.

Results: Majority of the respondents (91\%; $87 \%$ smokers and $99 \%$ nonsmokers) reported being exposed to the pictorial health warnings (PHW) on cigarette packs.

Those who reported noticing PHWs on cigarette packs, noticing anti-smoking media messages, older age and higher education were significantly associated with higher knowledge about health risks of smoking $(\mathrm{p}<0.05)$. As expected, reported noticing PHWs and knowledge about the health risks of smoking were significantly and positively associated with having negative attitudes towards smoking in both adjusted and un-adjusted regression analyses. PHWs encouraged $54 \%(n=131)$ of the non-smokers to discuss about the dangers of smoking with their family members $(\mathrm{p}=0.002)$ and $25 \%(\mathrm{~N}=29)$ of the smokers to have intentions to quit smoking ( $\mathrm{p}>0.05)$.

Conclusions: PHW on cigarette pack is a most efficient health communication tool in rural Sri Lankan community. The findings suggest that the newly introduced PHWs that cover $80 \%$ of the cigarette packs can help to increase knowledge about the harms of smoking among both smokers and nonsmokers, stimulate more conversation with significant others about the dangers of smoking among nonsmokers and encourage quitting among smokers, thus providing some support for its effectiveness as a health communication tool in rural Sri Lankan community.

Tob. Induc. Dis. 2018;16(Suppl 1):A743

DOI:10.18332/tid/84308

12:30-14:00

PS-1031-3 International best practice adopted in China: evidence-based mass media campaigns to support city-level $100 \%$ smoke free laws

Y Chen ${ }^{1}$, T Carroll ${ }^{2}$, N Singh Negi ${ }^{3}$, S Mullin ${ }^{4}$

${ }^{1}$ Vtial Strategies, China Office, Beijing, China, ${ }^{2}$ Vital Strategies, Policy and Communications, Sydnedy, NSW, Australia, ${ }^{3}$ Vital Strategies, Policy, Advocacy \& Communication, New Dehli, India, ${ }^{4}$ Vital Strategies, Policy, Advocacy \& Communication, New York, NY, United States of America. E-mail: wchen@ vitalstrategies.org

Background and challenges to implementation: On June 1st, 2015, a remarkable $100 \%$ Smoke Free Law came into effect in Beijing. On January 1st, 2017, Shenzhen became 100\% smoke free city and on March 1st, 2017 the Shanghai smoke free law came into effect. As a result, over 60 million population in China are now protected from second-hand smoke (SHS) exposure in public places, work places and public transportation.
Intervention or response: Vital Strategies has collaborated with city governments, including Beijing, Shenzhen and Shanghai, to conduct evidence-based tobacco control mass media campaigns, incorporating message testing research to guide development of communication materials, integrated media planning utilizing a mix of media communication channels and undertaking evaluation surveys to measure campaign impact.

To support implementation of the Beijing 100\% smoke free law a campaign was designed to increase understanding of the harms of SHS exposure, particularly to children, and to support enforcement of the law. The campaign featured a new television ad, Smoke-free Restaurant, highlighting specific dangers of SHS exposure for children, and emphasizing the benefits of the SF law in providing protection from these harms.

Results and lessons learnt: A population door-to-door evaluation survey was conducted in urban and peri-urban areas of Beijing from July 1st to August 12th 2015 in a total of 900 households. The survey found the majority of smokers who saw the ad said it made them concerned about the effects of their smoking on their family's health (89\%) and more likely to avoid exposing others to their cigarette smoke (94\%). Almost all respondents said seeing the ad made them more likely to reduce their children's exposure to SHS (smokers and non-smokers both $97 \%$ ). Similarly, $87 \%$ of non-smokers said the ad made them more likely to protect themselves from SHS exposure.

Conclusions and key recommendations: The results reflect the important role that mass media campaigns can play in supporting implementation of tobacco control policies.

Tob. Induc. Dis. 2018;16(Suppl 1):A744

DOI: $10.18332 /$ tid/84335

\section{2:30-14:00}

PS-1032-3 Exposing tobacco industry tactics in implementation of $85 \%$ GHW's through media advocacy

B Mathew ${ }^{1}$

'Voluntary Health Association of India, Delhi, India. E-mail: binoymathew84@gmail.com

Background and challenges to implementation: Graphic health warnings (GHWs) are an effective measure to warn tobacco users of the harm of tobacco use. GHWs on packaging of tobacco products is legally mandated as per India's national tobacco control legislation Cigarettes \& Other Tobacco Products Act 2003. GHWs were notified on 15th October, 2014 \& effective from 1st April, 2015 - pictorial warning to cover $85 \%$ area on both sides of tobacco packs. However the notification was kept in abeyance in March 2015, due to tobacco industry pressure.

Intervention or response: Using earned media to expose tobacco industry tactics towards delaying the implementation of 85 percent graphic health warnings. The strategy was to ensure that news items or stories come out to attract the attention of the government and the public. To do so, VHAI decided to increase consumer awareness about the issue of pictorial warnings in the news through a sustained strategy of media engagement. We increased our interactions with the media, both on a one-to-one basis and through press meets during the year 2014 - 2016. Brief yet accurate press releases were also issued to the print media for stories.

Results and lessons learnt: This strategy of media advocacy resulted in nearly over 1200 earned media stories on 85 graphic health warnings. More than 100 hours' time on news channels. These stories created pressure and become a national debate. After a twoyear battle, India implemented 85 percent graphic health warnings 
on tobacco products package from 1st April, 2016.

Conclusions and key recommendations: The media was sensitized and in the process, a personal rapport began was developed with journalists. VHAI's state level network, tobacco control partners and linkages with vernacular media helped to make the GHW issue a Pan India campaign.

Tob. Induc. Dis. 2018;16(Suppl 1):A745

DOI:10.18332/tid/84418

\section{$12: 30-14: 00$}

PS-1033-3 Study on print media behavior at the time of implementing pictorial health warnings in Sri Lanka

\section{A Dilrukshi ${ }^{1}$}

${ }^{1}$ Alcohol and Drug Information Center (ADIC) Sri Lanka, Library \& Information, Colombo, Sri Lanka.E-mail: achaladil@yahoo.com Background: In 2012, Sri Lankan government announced the enforcement of Pictorial Health Warnings (PHWs) covering $80 \%$ of the cigarette packet. Subsequently the court reduced it to $60 \%$, after an interim order sought by the industry. In 2015 , new legislation was enforced to cover $80 \%$ of cigarette packet by PHWs. The tobacco industry showed its manipulation against PHWs through media. It was important to study the behavior of median order to investigate and ensure the enforcement of PHWs. Methods: Newspaper articles analysis was the method which used in this study. Four daily newspapers and 6 weekly newspapers were selected for the study based on the popularity. Analysis conducted during January 2013 to June 2014 by using 2376 Newspapers. articles, cartoons, poems and stories related PHWs were considered and categorized according to the influence on readers in the analysis to measure the degree of acceptance for PHWs. In addition the study focused on Ceylon Tobacco Company (CTC) responses on PHWs. At the end a report was shared with media, politicians, policy makers and other responsible personnel to create a discussion.

Results: The study helped to identify whether the behaviors of politicians, policy makers, journalists and other related officials who are accountable for enacting PHWs are negative or positive. There were articles which had negative perception on PHWs and those were indirectly related with CTC. This analysis proved that the supportive and encouraging articles related to PHWs were higher than the opposed ones.

Conclusions: This study proves that the majority of the community accepts the FCTC recommendation of implementing PHWs to create a healthy community for the development of the country. It also shows that the CTC uses shrewd methods on authoritative people and institutes who have the decision making powers related to law enforcement, for its advancement.

Tob. Induc. Dis. 2018;16(Suppl 1):A746

DOI:10.18332/tid/84425

\section{2:30-14:00}

PS-1034-3 Fight for 85\% pictorial health warnings on tobacco product packages - a success story from India

\section{R Singh ${ }^{1}$, AK Pandey ${ }^{2}$}

${ }^{1}$ Government of India, Ministry of Health and Family Welfare, New Delhi, India, ${ }^{2}$ International Union Against Tuberculosis and Lung Disease, Tobacco Control, New Delhi, India. E-mail: apandey@theunion.org

Background and challenges to implementation: The pictorial health warning (PW) is the most cost effective measure to create awareness about adverse health consequences of tobacco usage. Section 5 of Indian Tobacco Control Act (COTPA-2003) requires printing of specified pictorial health warning on all tobacco product packages. Though PW were first notified in 2006, its implementation was deferred time and again due to the challenge posed by Tobacco Industry (TI). First ever PW (40\% on one side of pack only) was printed from May 2009 on direction of the Supreme Court. But its insufficient size and placement it had failed to have the deterrent effect on tobacco users. Thereafter, on recommendation of the Expert Committee, the Government revised PWs and notified the new set of field tested PWs covering 85\% display area on October 2014. But as expected, its implementation was delayed by multiple court cases by TI and representations and appeals from their front groups. An Indian parliamentary committee and a High Court also issued adverse orders.

Intervention or response: Indian Government took firm stand against these challenges and defended the new PWs before Supreme Courts as well as several High Courts. It was also unflinchingly responded to the committees' queries in support of the PWs with scientific evidences and legal provisions. Indian civil societies coordinated efforts and intense media advocacy provided further strength to the Governments' stand.

Results and lessons learnt: The interventions and response from the Government and Civil Society resulted in the High Courts and the Supreme Court of India passing orders supporting the enforcement of the new warnings from 1st April 2016.

Conclusions and key recommendations: The case discussed confirms that with strategically planned approach, robust scientific evidence, legal provisions, judicial precedents, will collaborated efforts of all tobacco control stakeholders, it is possible to counter any TI interference to deflect the Government's initiatives.

Tob. Induc. Dis. 2018;16(Suppl 1):A747

DOI:10.18332/tid/84442

\section{2:30-14:00}

PS-1035-3 Observational study to check the compliance of implementation of $85 \%$ graphic health warnings on tobacco products in India from April 1, 2016

S Gupta ${ }^{1}$, B Mathew ${ }^{1}$

'Voluntary Health Association of India, Delhi, India. E-mail: seemagupta@vhai.org

Background and challenges to implementation: Graphic health warnings effectively communicates health hazards of tobacco use - consumers see warnings thousands of times. Evidence shows that large graphic health warnings on tobacco packs are efficient and cost-effective. As per law (COTPA 2003, Section 7), it is mandatory to implement pack warnings across all tobacco products in India. Since April 1, 2016, India requires 85 percent graphic health warnings to be printed on both sides of all tobacco product packets.

Intervention or response: Two months after implementation of rules, a study was undertaken by $\mathrm{VHAI}$ and seven partners to monitor the compliance of 85 percent graphic warning labels (GWLs) in 8 states on three types of tobacco product packaging. This included - Cigarettes, Bidis and Smokeless.

The tobacco packets were collected in 2 commercial areas (Low and High Income Zones) from kiosks, tobacco specialists, street vendors, independent small groceries, supermarkets, and other venues selling tobacco.

The sample included 786 cigarette packs (24 brands, 4 tobacco companies), 665 packs of smokeless tobacco (7 types of products, 
48 brands, 36 tobacco companies, 20 brands of non-identified tobacco companies), 539 bidi packs (23 bidi companies and 50 brands of non-identified bidi companies.

Results and lessons learnt: $67 \%$ cigarette brands, $16 \%$ bidi brands and $46 \%$ smokeless brands were fully complying with the new 85 percent graphic health warnings.

Conclusions and key recommendations: The Government must strictly enforce the HWL's rules as health warnings on tobacco packages are a direct, cost-effective means of communication to inform the health risks of tobacco consumption to the consumers/ new users/illiterate population.

Tob. Induc. Dis. 2018;16(Suppl 1):A748

DOI:10.18332/tid/84447

\section{2:30-14:00}

PS-1038-3 Building support for the implementation of $85 \%$ GHW's through social media

B Mathew ${ }^{1}$, B Mukhopadhyay ${ }^{1}$

'Voluntary Health Association of India, Delhi, India. E-mail: binoymathew84@gmail.com

Background and challenges to implementation: Graphic health warnings (GHWs) are an effective measure to warn tobacco users of the harm of tobacco use. GHWs on packaging of tobacco products is legally mandated as per India's national tobacco control legislation Cigarettes \& Other Tobacco Products Act 2003. GHWs were notified on 15th October, 2014 \& effective from 1st April, 2015 - pictorial warning to cover $85 \%$ area on both sides of tobacco packs. However the notification was kept in abeyance in March 2015, due to tobacco industry pressure.

Intervention or response: Using social media to garner support for the implementation of 85 percent graphic health warnings. Used online \& on-ground audience voices to reach out to the decisions makers, created awareness around $85 \%$ graphic health warnings on tobacco products. Communicated the missions of the campaign i.e., increase size of pictorial warnings (Save Lives, Increase the size), used the voice of a cancer victim to lead the campaign (petition), online petition + supported by on ground activation to reach the youth, connected with influencers/groups across different fields to gain more support for petition, used creative graphics to attract audience attention \& created connect and emphasized on need to have larger warnings, straight from the public's voice.

Results and lessons learnt: Intensive social media campaign on picture health warnings resulted in 38,735 people signing the online petition on Change.org and 30,000 on-ground signatures from youth and public. The social reach was around 5,759,331 people. Conclusions and key recommendations: Social media campaigns is also becoming one of the tool to gain support from larger audience and are among the most effective ways to warn about the dangers of tobacco use and to create support for tobacco control policies.

Tob. Induc. Dis. 2018;16(Suppl 1):A749

DOI:10.18332/tid/84502

\section{$12: 30-14: 00$}

PS-1039-3 Examining the insertion of pictorial health warning on cigarette packets in Tehran city B Valizadeh', M Yunesian²

${ }^{1}$ Ministry of Health and Medical Education, Environmental and Occupational Health, Tehran, Iran, Islamic Rep. Of, ${ }^{2}$ Tehran University of Medical Sciences, Tehran, Iran, Islamic Rep. Of. E-mail: behzadradvali@gmail.com
Background: Following to insertion of pictorial health warning on cigarette packages in 2009, in order to know the effects of this policy, a qualitative - quantitative study has been performed in Tehran City. Methods: Focus group discussions (FGD) with six groups of smokers and nonsmokers aggregated by sex, educational level and smoking status totaling 46 people as well as random dialing method in Tehran citizens totaling 850 individuals was performed. Results: FGDs showed that using a still image and not changing it over time caused in lowering effects of health messages. Most people think that the quality of the image has an impact on non-smokers as a barrier to start smoking. Telephone interview showed that these images could prevent from starting smoking (57.2\%) and $36.8 \%$ were stated that these images are ineffective in preventing the onset of smoking. $67.6 \%$ stated that these images led them to discuss about the hazards of smoking, $71.3 \%$ encouraged their families and friends to quit smoking and $63.9 \%$ announced that these images caused in preventing their associates from being smoker. In total, about 84 percent of people was supported the insertion of pictorial health warnings on tobacco packages $(83.9 \%)$ and $10 \%$ (85 individuals) were against this policy. More than half of smokers following to see these images, decreased their cigarette consumption, and less than half of smokers reported no change in their smoking rates ( $55.1 \%$ against $43.3 \%$ ). Considering the effect of these pictures on the cessation, about 38 percent claimed that made them think about quit smoking, $63.8 \%$ of smokers said it caused their associates to ask them for quitting and $12 \%$ claimed that it made them quit.

Conclusions: This policy applies to a positive change in the attitude and behavior of the smokers and people around them and necessary steps to increase the efficiency of these images are required.

Tob. Induc. Dis. 2018;16(Suppl 1):A750

DOI:10.18332/tid/84529

12:30-14:00

PS-1040-3 Pre-test of pictorial health warnings on tobacco products to test their effectiveness study based in India

P Krishnatray', S Agnihotri', VG Munish ${ }^{2}$, A Singh², S Velu³, F Tullu²

${ }^{1}$ Centre for Communication and Change India, New Delhi, India, ${ }^{2}$ World Health Organisation - India, New Delhi, India, 3Johns Hopkins University Center for Communication Programs, Baltimore, MD, United States of America.E-mail: munishvg@ who.int

Background: In 2014, the Government of India's Ministry of Health \& Family Welfare constituted an expert group for development of pictorial health warnings for tobacco products. Accordingly, six prototypes were developed with warnings displayed on the front and back occupying 85 percent of the space. The packs visually showed the adverse impact of tobacco consumption.

The prototypes were pretested. The objective of the pretest was to assess the effectiveness of the health warnings and messages on different target groups in terms of noticeability, comprehension, informativeness, credibility, legibility, clarity of color \& design, and intention to change behavior. The main task of the study was to exhibit six types of pack warnings developed separately for cigarette, bidi, and smokeless tobacco users and non-users and rank them based on their reactions to each of the attributes listed above.

Methods: The pre-testing was undertaken in one district each of the three states of India. In all, the study area covered 28 locations. The total number of respondents was 300 comprising of tobacco users and non-users. The sample included adult women and 
men. The study used qualitative methodology for pre-testing the pack warnings on cigarette, bidi and smokeless tobacco packs. It conducted: 28 focus group discussions and 20 in depth interviews. Results: The study concluded that the image on cigarette, bidi or smokeless tobacco packs is the main source of information. Pack warnings which showed damage on the face or a disease were more impactful and understood. Cigarette/ Bidi are often bought loose, especially by those who are in the age group 18-25 years. Hence, the likelihood of noticing the pack warning is much lower by this age group.

Conclusions: The study recommended that the image and the written warning on the pack must match and must be in English and an Indian language.

Tob. Induc. Dis. 2018;16(Suppl 1):A751

DOI: $10.18332 /$ tid/84531

\section{$12: 30-14: 00$}

PS-1041-3 Celebrities' voice for pictorial health warnings on cigarette packets at 'television opera' award ceremonies

R Manohari ${ }^{1}$

${ }^{1}$ Alcohol and Drug Information Centre (ADIC), Colombo, Sri Lanka. E-mail: rasika@adicsrilanka.org

Background and challenges to implementation: Sri Lanka(SL) has signed and ratified FCTC treaty in 2003 and bounded to enforce the pictorial health warnings on cigarette packets (PHW$\mathrm{CP})$. The Health Minister(HM) notified the enforcement of PHW-CP by a gazette on 2012-Aug-08. But Ceylon Tobacco Company(CTC) instituted a legal action challenging the gazette notification. ADIC started island wide campaigns empowering citizens to support for the enforcement of PHW-CP.

'Sumathi Tele Award Ceremony' is an annual glamour event conduct by the Sumathi Group of Companies to appreciate the talents of stars on television screen. Around 200 celebrities gather for this shining night.

Intervention or response: ADIC reached this Ceremony officially from 2010-2015; conducting various campaigns on alcohol and tobacco prevention(ATP) among celebrities. In 2012 and 2013; campaigns theme was 'celebrities' support for the enforcement of PHW-CP'. Glamour signature boards of a petition were displayed at the entrance; quick discussions had with each celebrity. Video documentary of celebrities' statements for supporting to enforce PHW-CP were disseminated among them; published on social media. ATP messages, information on tobacco industry influences included organizers/diaries were distributed.

Results and lessons learnt: Celebrities joined to work for ATP. In 2012 and 2013 respectively 180 and 141 celebrities including artists, political leaders, journalists and mass media persons signed for the petition and approximately 50 leading celebrities gave supporting statements empowering the health minister. Signed petition boards and video documentary on statements of celebrities were handed over to HM. Finally Court of Appeal refused the appeal made by CTC to quash the gazette issued by $\mathrm{HM}$ and implemented PHW covered $80 \%$ of the surface of cigarette packaging.

Conclusions and key recommendations: Television Award Ceremony is a stage to make ATP in glamour, effective on aware and get support of celebrities at once. Their voices and signatures are powerful and can be enforced on tobacco control policies.

Tob. Induc. Dis. 2018;16(Suppl 1):A752

DOI:10.18332/tid/84542

\section{2:30-14:00}

PS-1042-3 Impact of pictorial warning and advertisement among tobacco users in India S Veeraiah ${ }^{1}$, V Elangovan ${ }^{1}$, R Sudhakar ${ }^{1}$, S Chidambaram ${ }^{1}$, D Subramani ${ }^{1}$

${ }^{1}$ Cancer Institute (WIA), Psychooncology and RCTC, Chennai, India.E-mail: suren.psy@gmail.com

Background: Considering the increasing burden of tobacco related morbidity and mortality, various interventions are being formulated and implemented frequently. Advertisement through media and pictorial warning on tobacco products are one of the innovative interventions successfully put into practice across various nations. Although the government of India is allocating a significant amount to the media awareness, the efficacy of the anti-tobacco advertisements among the tobacco users and its contribution to the area of tobacco control has not been stated clearly. As a part of a large statewide population based survey, the impact of the advertisements and pictorial warning among the tobacco users were assessed and reported in this study.

Methods: A large statewide household survey (Tamil Nadu Tobacco Survey-2016) conducted among 99,825 individuals in India during the year 2015-2016 to monitor the tobacco usage and track the key outcome indicators for tobacco control. Of the participants enrolled, 5208 (current tobacco users) were asked four questions on the impact of the pictorial warning and anti tobacco advertisement on their tobacco usage, as one of the indicator of tobacco cessation and control.

Results: Almost $67.3 \%$ of the tobacco users reported to have noticed the pictorial warning on the tobacco packs, of which $45.6 \%$ of them reported that they were motivated by the warnings, to quit the usage. There is a significant impact of the pictorial warnings on the packages in creating a demand for tobacco cessation $(p=0.00)$. Over $88.5 \%$ of the tobacco users noticed warning advertisements and messages in various sources.

\begin{tabular}{lcc} 
Sources of media & Voticed warnings & $\begin{array}{c}\text { Thought of } \\
\text { quithing }\end{array}$ \\
\hline TV Films & $4151(79.7 \%)$ & $756(14.5 \%)$ \\
& $1056(20.3 \%)$ & $184(3.5 \%)$ \\
Newspaper & $647(12.4 \%)$ & $87(1.7 \%)$ \\
Billboards & $652(12.5 \%)$ & $90(1.7 \%)$ \\
Radio & $425(8.2 \%)$ & $84(1.6 \%)$
\end{tabular}

[Source of Adverticements]

Visual media reaches a larger population and holds a significant role in motivating the users to quit.

Conclusions: The anti tobacco advertisements and messages through visual media is an effective method which reaches larger population and motivates the tobacco users to reduce and quit the habit.

Tob. Induc. Dis. 2018;16(Suppl 1):A753

DOI: $10.18332 /$ tid $/ 84558$

\section{2:30-14:00}

PS-1043-3 Driving behavioural change and building public opinion through effective partnership and sustained media advocacy for tobacco demand reduction across Delhi-NCR

C Ramakrishnan ${ }^{1}$, P Sehrawat ${ }^{1}$, R Singh $^{1}$

${ }^{1}$ Avian Media, Advocacy, CSR and Development Communication, New Delhi, India. E-mail: chandra@avian-media.com 
Background and challenges to implementation: As per Global Adult Tobacco Survey (GATS), prevalence of tobacco use among people between 15 and 24 years has reduced from $18.4 \%$ in 2009 10 to $12.4 \%$ in 2017. Despite implementation of COTPA Act 2003 in India, every fifth adult uses smokeless tobacco; every 10 th adult smokes tobacco. Youth and adolescents are especially vulnerable to tobacco use which causes deaths and disabilities. Quality stories covering health aspects, industry tactics and lack of enforcement around tobacco use are a challenge in Delhi-NCR. Intervention or response: Pro-active media advocacy with English media and vernaculars was conducted in partnership with Fortis Hospitals. Editors and correspondents were sensitized on tobacco use harms through press conferences, press releases, media interactions with oral cancer patients and exhibits of case studies. Topics on tobacco use pitched were around oral, lung cancers and diseases caused by tobacco use, busting myths around tobacco, women and children affected by tobacco use, weak law enforcement around Sec 6, new treatments to treat cancer, collaboration with traffic police to ban sale of tobacco near educational institutions.

Results and lessons learnt: Total number of stories published in 2016-2017 were 230 including online, print and electronic media. Journalists were effectively sensitized and quality coverage appeared in The Hindu, Statesman, Tribune, Sunday Guardian, Pioneer, Asian Age, DNA, Financial Express, Health and Nutrition, Hindustan, Navbharat Times, Dainik Jagran, Amar Ujala, Rashtriya Sahara and news wires such as PTI, ANI and IANS.

Conclusions and key recommendations: Effective partnerships combining communication skills, health expertise and right advocacy can lead to authentic, factual, quality media stories and buildi public opinion against tobacco use. Right messages with facts given to media can transform them into advocates for behavioral change leading to reduction in tobacco use. Quality stories can be powerful tools to build awareness and and discourage new users and youths.

Tob. Induc. Dis. 2018;16(Suppl 1):A754

DOI:10.18332/tid/84582

\section{2:45-14:00}

EP-220-3 Indigenous language as a tool for MPOWER component 4

B Mercy', O Ogunnika²

'Educare Trust, Ibadan, Nigeria, ${ }^{2} \mathrm{TaBHealth}$ Initiative, Ibadan, Nigeria. E-mail: goodmercy22@gmail.com

Background: Over the last decade, tobacco use in Nigeria has been on the increase due the growth of illicit trade of tobacco products and a lack of political will to enact and enforce comprehensive tobacco control laws. The numerical increase in tobacco users can be attributed to a low understanding of its health risks regardless of the health warnings on cigarette packs.

Methods: Though there is paucity of data on mass media campaigns to warn about the dangers of tobacco, observational research revealed the existence of billboards along some highways in rural Nigeria. This paper attempts to highlight the use of mass media such as billboards and buses as an education tool for component 4 of MPOWER which seeks to warn about the dangers of tobacco.

Results: The billboards depicting the consequences of tobacco use are positioned in regions of low literacy levels hence its message is not understood. $65 \%$ of respondents aged 14-42 in these regions do not understand the message of the billboards while $35 \%$ knew the 'No smoking' symbol but not the words. Almost all the respondents could read the words of a political aspirant as it was written in their local language. Only about 13\% of the respondents believed that smoking was a good habit. $75 \%$ percent of the respondents aged 14-30 admitted to not continuing tobacco use into their old age but had no idea how to quit.

Conclusions: It will be beneficial that mass media tobacco control warnings are written and broadcast in the native languages of the target audience to boost its impact. Billboards, Automobiles and walls should be utilized as tools for education and behavior change communication in rural regions of Nigeria and Africa. This will accelerate the objective of the implementation of the Nigerian Tobacco Control Law.

Tob. Induc. Dis. 2018;16(Suppl 1):A755

DOI: $10.18332 /$ tid/84658

\section{$12: 45-14: 00$}

EP-221-3 Pictorial health warnings on waterpipe tobacco packs: rating of efficacy in comparison to an alternative waterpipe-specific set

A Mostafa ${ }^{1}$, HT Mohammed ${ }^{1,2}$, A Aboul Fotouh'

${ }^{1}$ Faculty of Medicine, Ain Shams University, Department of Community, Environmental, and Occupational Medicine, Cairo, Egypt, ${ }^{2}$ School of Pharmacy, University of Waterloo, Waterloo, ON, Canada.E-mail: aya.kamaleldin@med.asu.edu.eg

Background: We measured the efficacy of the existing pictorial health warning (PHW) set displayed in the Egyptian market on Waterpipe tobacco packs (WTP) in comparison to an alternative one. We aimed to examine their possible effect in encouraging cessation among waterpipe smokers and preventing initiation among non-smokers.

Methods: A convenience sample of 2014 adult participants of both genders were surveyed within rural and urban regions of Egypt. Socio-demographic data and tobacco use were recorded. Participants were shown at random one of the existing PHWs on WTPs, then one of the alternative set. The alternative set was designed to display pictures and text that were more waterpipe specific, and occupied around $80 \%$ of the WTP with plain packaging features. Participants were asked to rate both PHWs' efficacy on a scale from 1 to 10 regarding their salience, depth of processing, affective reactions, credibility, relevance, perceived behavioral control and quit intentions. Univariate and multivariate statistical analyses were performed.

Results: Participants were on average 35 years old, with $10 \%$ females, $74 \%$ were current waterpipe smokers. Paired mean efficacy ratings of the alternative PHW set were significantly higher than those of the existing one regarding all measures, yet both sets collectively scored modestly $(5.8 \pm 1.7$ versus $5.1 \pm 2.1, \mathrm{p}<0.001)$. The highest mean score differences were noted for grabbing attention, inducing affective reactions, making participants look closely at the PHW, and in encouraging quit attempts. Participants rated both PHW sets of possible relatively high efficacy in discouraging waterpipe tobacco smoking $(6.3 \pm 3.4$ versus $5.7 \pm 3.7, \mathrm{p}<0.001)$. Furthermore, significantly higher mean efficacy scores were reported by non-smokers $(6.2 \pm 1.6$ versus $5.6 \pm 1.8, \mathrm{p}<0.001)$, males $(5.8 \pm 1.7$ versus $5.1 \pm 2.3, \mathrm{p}<$ $0.001)$, younger adults $(6.0 \pm 1.7$ versus $5.7 \pm 1.8, \mathrm{p}<0.001)$, and rural residents $(6.4 \pm 1.3$ versus $4.9 \pm 2.0, \mathrm{p}<0.001)$.

Conclusions: There is an urgent need to research evidencebased effective PHW content and design that address different population subgroups.

Tob. Induc. Dis. 2018;16(Suppl 1):A756

DOI:10.18332/tid/84653

$12: 45-14: 00$ 
EP-222-3 Larger picture health warnings on tobacco packages - overview of international momentum and innovative approaches

R Cunningham

'Canadian Cancer Society, Ottawa, ON, Canada. E-mail: rcunning@cancer.ca

Background and challenges to implementation: There is tremendous international momentum regarding package health warnings, with more than 110 countries/territories requiring picture warnings, and with countries significantly increasing warning size. Roughly 100 countries/territories require a size of at least $50 \%$ of the package front/back, more than 45 require a size of at least $65 \%$, and more than 12 requiring a size of $75 \%$ to $90 \%$. Package health warnings are highly cost-effective. Package warnings provide educational messages that reach all tobacco consumers many times per day. Governments determine the messages, and tobacco companies pay the cost. This low cost is especially important in low and middle income countries.

Health warning effectiveness increases with size, as FCTC Guidelines recognize. Moreover, health warnings with graphic images of the health effects are far more effective than text-only warnings. A picture does indeed say a thousand words.

Intervention or response: This presentation will report on dramatic worldwide progress at increasing package warning size, and requiring pictures. In part, this presentation will outline the results of the fifth edition of the Canadian Cancer Society report, Cigarette Package Health Warnings: International Status Report, as well as ongoing international developments.

This presentation will also report on innovations in health warnings, including regarding new message content on health effects, messages encouraging quitting to save money, and enhanced messages on lateral sides of packages. Also, more countries are requiring a minimum surface area for warnings (in square centimetres), in addition to a minimum percentage of the front/back, to increase size and impact.

Results and lessons learnt: Global progress is being made despite continuing tobacco industry opposition including, in some countries, legal challenges.

Large picture health warnings are a highly effective measure, whether or not implemented with plain packaging.

Conclusions and key recommendations: Countries worldwide should accelerate efforts to improve warnings by increasing size, by requiring pictures, and by implementing innovative approaches.

Tob. Induc. Dis. 2018;16(Suppl 1):A757

DOI:10.18332/tid/84662

12:45-14:00

EP-223-3 Does 85\% pictorial health warning on the tobacco products have the impact among tobacco users in India?

M Bincy', E Vidhubala ${ }^{2}$, R Priyadharshini ${ }^{2}$

'Indian Institute of Sports Medicine, Psychology, Chennai, India, 'Indian Institute of Sports Medicine, Chennai, India. E-mail: annbinc@gmail.com

Background: After persistent civil society and judicial interventions, 85\% Pictorial Health Warning (PHW) was implemented in India since April, 2016. The previous 40\% PHW had negligible impact in preventing new users and motivating existing users to quit tobacco. The current PHW printed on smoking products is picture of a throat cancer and on smokeless tobacco products is cancer of oral cavity and this should cover $85 \%$ of the outer panel of the tobacco products. Text message as "Smoking causes cancer, Smoking kills" also printed on the packs.
The present study aimed at understanding the impact of $85 \%$ PHW on motivating tobacco users to quit.

Methods: Tobacco users $(\mathrm{n}=99)$ were chosen by convenient sampling from Chennai, Tamil Nadu. One cigarette packet and one chewing tobacco packet printed with $85 \%$ PHW was shown to them and interviewed using a semi structured questionnaire. The questions included were, their current tobacco use, comprehension of text and PHWs printed on tobacco products and its ability to motivate the users to quit.

Results: Majority of the tobacco users $(84.8 \%)$ noticed the health warnings on tobacco products (PHW-13.1\%, Text warning- $14.1 \%$, Both-57.5\%). Overall 67.6\% tobacco users(Smoking-46.4\%, smokeless-21.2\%) were able to identify the picture correctly and $55.5 \%$ related the picture to health problems. A few users (8\%) couldn't read the text warning since it was printed in English. The understanding is better if they could read the text warning also. The $52.5 \%$ of the users reported that the PHW made them to think about quitting and $72.7 \%$ reported that PHW will motivate the users to quit. The users (38.3\%) reported total ban as the best measure for tobacco control.

Conclusions: The $85 \%$ PHW has positive impact in motivating the tobacco users to quit. However, printing the text warning in regional language will be effective for the people who cannot read English.

Tob. Induc. Dis. 2018;16(Suppl 1):A758 DOI: $10.18332 /$ tid/84498

$12: 45-14: 00$

EP-224-3 Evidence of the continuing weak impact of China's health warnings: longitudinal findings over nine years (2006 to 2013 - 15) from the ITC China project

G Sansone', GT Fong ${ }^{1,2}$, M Yan'1, L Craig', S Xu1', ACK Quah'1, G Feng $^{3}$, Y Jiang ${ }^{3}$

'University of Waterloo, Psychology, Waterloo, ON, Canada, ${ }^{2}$ Ontario Institute for Cancer Research, Toronto, ON, Canada, ${ }^{3}$ Chinese Center for Disease Control and Prevention, Tobacco Control Office, Beijing, China. E-mail: gfong@uwaterloo.ca Background: China's health warnings do not meet the FCTC Article 11 guidelines. They are small

( $<35 \%$ of the pack) and do not include pictorial images, which research has shown to have greater impact. Four rounds of warnings have been implemented between 1992-2016. This study examines warning impact over 9 years (2006-15), including the third round (April 2012), which increased font size while maintaining overall warning size and replaced the English text with Chinese on the back.

Methods: Data are from Waves 1-5 (2006-15) of the International Tobacco Control (ITC) China Survey, a cohort survey of 800 adult smokers in each of five cities, adding five rural areas at Wave 5 (2013-15) (total $\mathrm{N}=8000$ ). GEE logistic regression models tested changes in key indicators of warning impact over time.

Results: Over 9 years, there has been little change in impact of the Chinese warnings. Indeed, the percentage of smokers who noticed warnings 'often' actually DECREASED from 51\% (2006) to $38 \%(2013-15)(p<.001)$, and fewer than one-third of smokers reported cognitions or behaviours related to quitting because of the warnings. The April 2012 revision did not improve any of the warning impact indicators. Warning noticing/salience did not differ between cities and rural areas, but rural smokers were more likely to think 'a lot' about harms of smoking ( $17 \%$ vs $11 \%$, $\mathrm{p}<.001)$ and about quitting ( $14 \%$ vs $6 \%, \mathrm{p}<.001)$ because of the 
warnings.

Conclusions: China's text-only warnings continue to be extremely weak. The minor changes in 2012 did not increase warning impact. Global evidence is clear that large pictorial warnings depicting specific harms of cigarettes would greatly enhance warning impact in China, including increasing knowledge and motivation to quit among the $300 \mathrm{M}$ Chinese smokers. These benefits would be especially important for the large rural population, where there are fewer other sources of health information.

Tob. Induc. Dis. 2018;16(Suppl 1):A759

DOI:10.18332/tid/83927

\section{$12: 45-14: 00$}

EP-226-3 From anti-smoking activist to archivist and back again: how museum exhibitions on the history of tobacco control are helping to educate a new generation

A Blum ${ }^{1}$

${ }^{1}$ University of Alabama, Center for the Study of Tobacco and Society, Tuscaloosa, AL, United States of America. E-mail: ablum@ua.edu

Background and challenges to implementation: The University of Alabama Center for the Study of Tobacco and Society was founded in 1998 as a repository for the comprehensive collection amassed over 30 years by the author and fellow tobacco control activists of photographic, audio, video, newsprint, and internet materials documenting tobacco marketing, the tobacco industry, and the anti-smoking movement over the past century. Continuously maintained since 1974 , the collection has served researchers, legislators, students, journalists, public health professionals, and the public alike via a website www.csts.ua.edu and a physical trove of over 250,000 original items divided into more than 100 subject categories (eg, the targeting of minorities; tobacco sales in pharmacies; the history of smoking cessation). It is the largest tobacco-related collection at any university.

Intervention or response: Unique among tobacco control information resources, the Center has produced 12 exhibitions for museums, libraries, universities, and conferences on compelling, ironic, and controversial aspects of the tobacco pandemic and anti-smoking activism.

Results and lessons learnt: To commemorate the 50th anniversary of the landmark 1964 US Surgeon General's landmark report on smoking and health, the Center created "The Surgeon General vs. The Marlboro Man: Who Really Won?" which comprised more than 130 original artifacts and was displayed at the Lyndon Baines Johnson Presidential Library. Other exhibitions include "When More Doctors Smoked Camels: A Century of Health Claims in Cigarette Advertising"; "Canaries in the Mine: The Flight Attendants' Battle for Smokefree Airlines"; "The American Medical Association's History of Collaboration with the Tobacco Industry: The Unfiltered Truth"; "Cartoonists Take Up Smoking" at the National Museum of Health and Medicine in Washington, DC; and "The World's Anti-Smoking Stamps."

Conclusions and key recommendations: This illustrated presentation provides excerpts from these exhibitions, some of which are available for use by health organizations to inspire the next generation of anti-tobacco activists. Online versions are also being created.

Tob. Induc. Dis. 2018;16(Suppl 1):A760

DOI:10.18332/tid/83980

\section{$12: 45-14: 00$}

EP-227-3 Effectiveness of 85\% graphic health warning on cigarette packs on smoking behaviours of Thai teenagers

N Sirichotiratana ${ }^{1}$, D Sujirarat ${ }^{2}$, M Sompopcharoen ${ }^{3}$, C Viwatwongkasem ${ }^{4}$, PSatitvipawee ${ }^{4}$, N Auemaneekul ${ }^{5}$, PSilpasuwan ${ }^{5}$ ${ }^{1}$ Mahidol University, Public Health Administration, Bangkok, Thailand, ${ }^{2}$ Mahidol University, Epidemiology, Bangkok, Thailand, ${ }^{3}$ Mahidol University, Health Promotion \& Behavioural Sciences, Bangkok, Thailand, " Mahidol University, Biostatistics, Bangkok, Thailand, ${ }^{5}$ Mahidol University, Public Health Nursing, Bangkok, Thailand.E-mail: nithats@gmail.com

Background: Thailand's policy on increasing graphic health warning areas on cigarette packs, from $50 \%$ to $55 \%$ to $85 \%$ has the objective of increasing knowledge and perception of harmful health effects of cigarette smoking. The objective of this field experiment study is to find out the effectiveness of the $85 \%$ graphic health warning label.

Methods: Factorial design was implemented after the policy regulation was implemented in 2014. Recruitment of 724 students in 4 high schools and 5 colleges in Bangkok metropolitan area were chosen. Perceptions on intention to quit smoking were tested after introducing the students to 8 mock-up cigarette packs. The study was conducted in June of 2015 and 80 students per school were chosen. Results: Results indicated that $85 \%$ graphic health warning cigarette

EP-228-3 Tobacco packaging and labeling in the WHO African region: progress after the adoption of WHO FCTC's Article 11 guidelines

\begin{tabular}{|c|c|c|c|c|c|c|c|c|c|}
\hline $\begin{array}{l}\text { INWIs } \\
\text { type \& } \\
\text { sine }\end{array}$ & $\begin{array}{l}\text { IIWLs } \\
\text { on top of } \\
\text { the PDAS } \\
(n-6)\end{array}$ & $\begin{array}{l}\text { Vlultiple } \\
\text { IIWLs } \\
(n-18)\end{array}$ & $\begin{array}{l}\text { Rotating } \\
\text { IIWLs } \\
(\mathbf{n}-17)\end{array}$ & $\begin{array}{l}\text { IIWLs in } \\
\text { principal } \\
\text { language s } \\
\text { (n 22) }\end{array}$ & $\begin{array}{c}\text { Quit } \\
\text { information } \\
(n-1)\end{array}$ & $\begin{array}{l}\text { Qualitative } \\
\text { constituents } \\
\text { \& emissions } \\
\text { information } \\
(\text { (n-9) }\end{array}$ & $\begin{array}{l}\text { Ban on } \\
\text { misleading } \\
\text { descriptors } \\
\text { (n- 23) }\end{array}$ & $\begin{array}{l}\text { Ban on } \\
\text { emission } \\
\text { yiclds } \\
(\mathbf{n}-8)\end{array}$ & $\begin{array}{l}\text { Ban on } \\
\text { expiration } \\
\text { date (n-1) }\end{array}$ \\
\hline $\begin{array}{l}\text { Text-only } \\
\text { less than } \\
30 \%(n=10)\end{array}$ & 2 & 4 & 2 & 4 & 1 & 1 & 3 & 0 & 0 \\
\hline $\begin{array}{l}\text { Text-only } \\
\text { at least } \\
30 \%(n=11)\end{array}$ & 2 & 3 & 4 & 6 & 1 & 1 & 7 & 3 & 1 \\
\hline $\begin{array}{l}\text { Pictorial } \\
\text { less than } \\
50 \%(n=2)\end{array}$ & 0 & 1 & 1 & 1 & 0 & 1 & 1 & 1 & 0 \\
\hline $\begin{array}{l}\text { Pictorial at } \\
\text { least } 50 \% \\
(n=13)\end{array}$ & 2 & 10 & 10 & 11 & 2 & 6 & 12 & 4 & 0 \\
\hline
\end{tabular}


pack significantly increased fear arousal, and intention not to smoke, among non-smoking students. Among smoking students, particular type of picture and increasing size of graphic health warning interact significantly to increasing fear arousal and intention to quit.

Conclusions: The impact of increasing size of graphic health warning on cigarette pack has significant influences on intention to quit among smoking- as well as non-smoking youths. This implied that plain packaging would be an ultimate strategy to increase awareness and perception of harmful health effects of cigarette smoking.

Tob. Induc. Dis. 2018;16(Suppl 1):A761

DOI: $10.18332 /$ tid/83827

\section{$12: 45-14: 00$}

EP-228-3 Tobacco packaging and labeling in the WHO African region: progress after the adoption of WHO FCTC's Article 11 guidelines

E Sebrie ${ }^{1}$, K Walter ${ }^{1}$, K Donley $^{2}$, B Camara ${ }^{3}$, M Carmona $^{1}, \mathrm{M}$ Muggli², R Nathan², P Lambert ${ }^{2}$

${ }^{1}$ Campaign for Tobacco-Free Kids, International Research, Washington, DC, United States of America, ${ }^{2}$ Campaign for Tobacco-Free Kids, International Legal Consortium, Washington, DC, United States of America, ${ }^{3}$ Campaign for Tobacco-Free Kids, International Advocacy, Washington, DC, United States of America. E-mail: esebrie@tobaccofreekids.org

Background: In 2008, the World Health Organization (WHO) Framework Convention on Tobacco Control (FCTC) Article 11 Guidelines were adopted. The objective of this study was to: (1) describe the current status of packaging and labeling in all 47 countries of the WHO African Region, and (2) assess the improvement of health warning labels (HWLs) since adoption of the Guidelines.

Methods: We analyzed over 225 pieces of legislation from the African Region in Tobacco Control Laws, a database of the International Legal Consortium at the Campaign for TobaccoFree Kids. In addition, we reviewed the 2017 WHO MPOWER Report. We assessed HWLs type (text-only vs. pictorial), size as a percentage of the principal display areas (PDAs), and other packaging and labeling requirements.

Results:

As of July 2017, 11 countries have no laws requiring HWLs; 10 require text-only HWLs of less than $30 \%$; 11 require text-only HWLs of at least $30 \%$; 2 require pictorial HWLs of less than $50 \%$; and 13 require pictorial HWLs of at least $50 \%$. The number of countries fulfilling other requirements is shown in the table below.

\section{[Number of Countries with Additional Requirements]}

Since adoption of the Article 11 Guidelines, the total number of countries requiring HWLs of at least $30 \%$ has increased more than 5 times (from 5 to 26); HWLs of at least $50 \%$ has spiked (from 1 to 15 ); HWLs of at least $75 \%$ has gone from none to 1. The number of countries with pictorial HWLs has jumped from none to 15 .

Conclusions: Few countries in the African Region have fulfilled key HWLs provisions. Almost half ban misleading descriptors, but less than one fifth ban emission yields or require qualitative constituents and emission information. Adoption of the Guidelines contributed to an increase in effective HWLs. However, only 13 of 47 countries have achieved their recommendations of pictorial HWLs on at least $50 \%$ of the PDAs.

Tob. Induc. Dis. 2018;16(Suppl 1):A762

DOI: $10.18332 /$ tid/83851

$12: 45-14: 00$
EP-229-3 Exposing the inconvenient truth of tobacco in China: a nationwide exhibition of graphic pack warnings

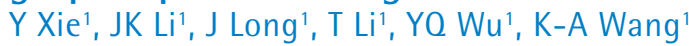

'ThinkTank Research Center for Health Development, BeiJing, China.E-mail: xieyuthinktank@sina.com

Background and challenges to implementation: Implementation of FCTC in China has been slow, especially pack warning. After three upgrades, China's current pack warnings are still limited to only generic health impact text with no graphs. The major opposition comes from China's tobacco industry, which is itself also a government agency in charge of policies regarding labelling. This is the inconvenient truth of China's tobacco control work. The Chinese public need to see the distances and differences between China's cigarette packing and other's countries', China's FCTC commitment on graphic warnings, and more importantly, this inconvenient truth. Considering China's size, this work can only be done with massive public support via an effective volunteer network.

Intervention or response: ThinkTank Research Centre for Health Development launched an exhibition of pack warnings from different countries. The exhibition went onto a nationwide tour to educate the public about China's weak implementation of this FCTC commitment, as well as tobacco industry's interference. This nationwide network combined both government agencies, the China Centre for Disease Control, and volunteers, who could download a tool kit for local satellite exhibitions. The offline physical and online virtual exhibitions reached more public.

Results and lessons learnt: The tour exhibition has reached China's 31 mainland provinces, receiving over 500 million direct visitors. More than 300 core volunteers have downloaded the tool kits, produced their own exhibitions, and run regular local tobacco control activities. This public momentum resulted in 12 label-related proposals by legislators and congress members at China's annual congress conferences.

Conclusions and key recommendations: Tobacco control work goes beyond policy makers, especially when one of the policy makers is in fact the tobacco industry. It is important to mobilize volunteers that include real smokers, real SHS victims, smoke-free champions. Their call for stronger pack warnings, as well as other MPOWER policies, will send strong signal to policy makers.

Tob. Induc. Dis. 2018;16(Suppl 1):A763

DOI:10.18332/tid/84340

$12: 45-14: 00$

EP-230-3 Low knowledge among Zambian smokers and the need for large pictorial health warnings: findings from the ITC Zambia Wave 2 survey

SC Kaai ${ }^{1}$, F Goma ${ }^{2}$, R Zulu ${ }^{2}$, M Phiri ${ }^{2}$, K Chirwa ${ }^{2}$, L Craig ${ }^{1}$, ACK Quah ${ }^{1}$, GT Fong ${ }^{1,3}$

${ }^{1}$ University of Waterloo, Waterloo, ON, Canada, ${ }^{2}$ University of Zambia, Lusaka, Zambia, ${ }^{3}$ Ontario Institute for Cancer Research, Toronto, ON, Canada. E-mail: skaai@uwaterloo.ca

Background: Many studies have shown that pictorial health warnings (PHWs) are more effective in increasing knowledge about the many harms of cigarettes. Zambia currently has a single text-only English warning covering less than 3\% of pack. This study is the first to assess health knowledge and the effectiveness of warnings in Zambia.

Methods: Data were from 1,171 smokers in the International Tobacco Control (ITC) Zambia Wave 2 Survey (2014), a 
longitudinal survey of a nationally representative sample of Zambian adults. Key variables analyzed were knowledge of specific harms of smoking and validated indicators of warning effectiveness.

Results: Knowledge among Zambian smokers was very low compared to other ITC countries: only $45 \%$ knew that smoking causes stroke (2nd lowest among 20 ITC countries), heart disease (74\%-3rd lowest among 14 ITC countries), and lung cancer (79\%-lowest among 12 ITC countries). The Zambian text-only warning was very ineffective: $58 \%$ of Zambian smokers reported "never" or "hardly ever" noticing the warning; only $24 \%$ reported closely reading the warning. 75\% reported that warning "never" stopped them from smoking; 70\% reported that warning did not make them more likely to think about health risks; $66 \%$ reported that warning "never" made them think about quitting. When shown the warning, $55 \%$ of smokers were not able to easily read it. And yet $71 \%$ thought the packs should have more health information and $86 \%$ wanted the government to do more about harms of tobacco use.

Conclusions: Knowledge of tobacco-related harms is very poor among Zambian smokers and the single-text only warning provides no help, particularly among the many low literacy smokers in Zambia. There is a clear need for Zambia to implement pictorial health warnings, as they are obligated to do as a Party to the FCTC and as other African countries-Mauritius and Kenya-have already done successfully.

Tob. Induc. Dis. 2018;16(Suppl 1):A764

DOI:10.18332/tid/83944

\section{$12: 45-14: 00$}

EP-231-3 Using social media to mobilize grassroots advocates and raise awareness about tobacco harms in Indonesia

E Aditjondro', A Rachfiansyah², Y Rabindanata², D Svenson ${ }^{3}$, S Hamill ${ }^{3}$, S Mullin ${ }^{3}$

${ }^{1}$ Vital Strategies, Jakarta Timur, Indonesia, ${ }^{2}$ Vital Strategies, Jakarta, Indonesia, ${ }^{3}$ Vital Strategies, New York, NY, United States of America.E-mail: eaditjondro@vitalstrategies.org

Background and challenges to implementation: Indonesia has one of the highest smoking rates in the world (57.1\% men / $41 \%$ boys / $3.6 \%$ women) and is described as "Disneyland" for big tobacco firms. There are limited smokefree policies, minimal tobacco taxes, and cigarette companies advertise to and target youth.

Intervention or response: Vital Strategies launched a digital awareness campaign - \#SuaraTanpaRokok (Voices Without Cigarettes) reaching out to youth and empowering real victims of tobacco to tell their stories through social media. The campaign also shifted offline, raising awareness and attendance at tobacco control events held in partnership with cities in Indonesia. The campaign gives a voice to those affected by tobacco and has built up an online following of grassroots anti-tobacco advocates.

Results and lessons learnt:

- Facebook page with 65,000+ followers, with content reaching over 2 million Indonesians/month

- Victim story telling campaign through Facebook and Instagram, featuring local tobacco victims and their families

- Discovered tobacco victim Robby who died aged 27. Told his story through a national TV campaign featuring his parents

- Offline events which attracted widespread traditional and social media coverage including: Anti-tobacco artwork murals on homes in the town of Kali Code in Yogyakarta that had previously been painted in tobacco industry colors. Event drove significant traditional media coverage and was \#1 national trending topic on Twitter; Anti-tobacco murals on "Angkot" public transport vans in Bogor for World No Tobacco Day in collaboration with local artists and the city Mayor.

- Campaign microsite http://suaratanparokok.co.id constantly updated with new statistics, victims stories, latest campaign content and photos

- Cross promotion online of our national mass media campaigns in Indonesia

Conclusions and key recommendations: \#SuaraTanpaRokok shows how social media can be used to build a group of online advocates, find tobacco victims and help tell their stories, and raise awareness of and drive attendance to offline events. It also amplified anti-tobacco mass media campaign messaging to youth audiences through social media.

Tob. Induc. Dis. 2018;16(Suppl 1):A765

DOI:10.18332/tid/83967

\section{2:45-14:00}

EP-233-3 ASEAN: world's first geopolitical region to implement pictorial health warnings

YL Tan ${ }^{1}$

'Southeast Asia Tobacco Control Alliance (SEATCA), Bangkok, Thailand. E-mail: yenlian@seatca.org

Background and challenges to implementation: Pictorial health warnings (PHWs) on tobacco products are the most cost-effective means to convey harms of tobacco use to smokers and the public. By 2017, all ten Association of Southeast Asian Nations (ASEAN) countries had legislated PHW requirements in accordance to World Health Organization Framework Convention on Tobacco Control Article 11 Guidelines. Strong opposition came from tobacco companies to undermine PHWs implementation at the country level. These challenges were identified and countered successfully.

Singapore and Thailand were the first ASEAN countries to implement PHWs in 2004 and 2005, followed by Brunei (2008), Malaysia (2009), Vietnam (2013), and Indonesia (2014). In 2016, Philippines (March), Cambodia (July) and Myanmar (September) implemented PHWs. Lao PDR was the latest country to implement (April 2017).

In most countries, the tobacco industry interfered by lobbying policymakers to adopt smaller, ineffective PHWs and delaying the implementation. They also mobilized allies and third parties and misled governments with false claims that PHWs violate international treaties on trademarks and intellectual property rights.

Intervention or response: Industry claims and tactics were exposed and countered with evidence-based arguments and international best practices. Southeast Asia Tobacco Control Alliance (SEATCA) provided technical support, display kits of sample health warnings from other ASEAN countries, and free use of copyrighted PHWs via a regional image bank.

Results and lessons learnt: ASEAN is the only region where all member states have successfully implemented PHWs on tobacco packs. The power of teamwork in fighting the industry through a regional platform and sharing experience and resources led to this success.

Conclusions and key recommendations: The tobacco industry uses a wide range of tactics to oppose implementation of effective tobacco control measures, particularly introduction of prominent 
PHWs. Industry interference remains a huge threat to public health policy in the region.

Tob. Induc. Dis. 2018;16(Suppl 1):A766 DOI:10.18332/tid/83867

\section{2:45-14:00}

EP-256-4 Evaluation of health warnings for waterpipe tobacco smoking among university students in the Eastern Mediterranean region

R Nakkash' ${ }^{1}, \mathrm{~N}$ Abu-Rmeileh ${ }^{2}, \mathrm{Y} \mathrm{Al-Farsi}{ }^{3}, \mathrm{R}_{\text {Hamadeh }}{ }^{4}, \mathrm{~K}$ Kheirallah $^{5}$, A Mostafa ${ }^{6}$, A Yusufali ${ }^{7}$, R Salloum $^{8}$

${ }^{1}$ American University of Beirut, Beirut, Lebanon, ${ }^{2}$ Birzeit University, Birzeit, State of Palestine, ${ }^{3}$ Sultan Qaboos University, Muscat, Oman, ${ }^{4}$ Arabian Gulf University, Manama, Bahrain, 5Jordan University of Science and Technology, Irbid, Jordan, ${ }^{6}$ Ain Shams University, Cairo, Egypt, 'Dubai Medical College, Dubai, United Arab Emirates, ${ }^{8}$ University of Florida, Gainesville, FL, United States of America. E-mail: rsalloum @ufl.edu

Background: Waterpipe tobacco smoking is a global public health problem that originated in the Eastern Mediterranean region (EMR). It is highly prevalent among university students, and has been increasing in popularity despite mounting evidence showing it is harmful to health. The aim of this study was to evaluate responses to various health warning messages and their location on waterpipe devices, among young residents of EMR countries.

Methods: Adult university students who were waterpipe smokers completed an Internet-based survey across 5 EMR countries (Egypt [N=728], Jordan [N=790], Oman [N=117], Palestine $[\mathrm{N}=772]$, and UAE $[\mathrm{N}=180])$. The survey evaluated their responses to text-only and pictorial waterpipe-specific health warnings and their location on waterpipe devices.

Results: Among both text-only warnings and text + pictorial warnings, messages that communicated harm to children were rated highest in raising concerns about the health consequences of smoking and in motivating smokers to think about quitting. In terms of warning location, the mouthpiece was rated as most noticeable to smokers.

Conclusions: This study is the first to compare responses to waterpipe-specific health warnings across EMR countries. Requiring health warnings on waterpipe devices may be an effective policy to educate smokers about the consequences of waterpipe use.

\section{Tob. Induc. Dis. 2018;16(Suppl 1):A767 \\ DOI:10.18332/tid/84636}

12:45-14:00

EP-258-4 An evidence based public campaign against waterpipe / hookah smoking in Turkey MPT Durgut ${ }^{1}$

'Turkish Green Grescent Society, Istanbul, Turkey. E-mail: erdebirtuba@hotmail.com

Background and challenges to implementation: Hookah use has been spreading rapidly in epidemic proportions in Turkey Intervention or response: Both qualitative and quantitative pre-campaign research has been conducted in order to define the perception, attitudes and behaviors of the target population regarding hookah. In this stage we conducted focus group studies and a baseline survey encompassing interviews with 1288 people. As a result of the pre-campaign analyses, we observed that the level of awareness on the dangers of hookah was very low compared to that of cigarettes, that hookah was not perceived as a tobacco product, that it was preferred due its scents and flavors and that many false beliefs were propagated such as its smoke being less dangerous due to passing from water. Measurable objectives of our campaign, campaign strategies and concepts were defined according to the data collected through the precampaign analyses. We applied our campaign in four phases. In each phase of our campaign we delivered different messages and the public was gradually informed of hookah facts through the use of TV and radio ads, outdoor materials, newspaper inserts, brochures and banners, PR studies as well as the use of internet and social media.

Results and lessons learnt: Following the campaign we surveyed 1266 people.The results of the post-campaign evaluation show that the campaign was successful in establishing perceived risk of hookah, changing perception and attitudes and developing the intention of quitting smoking hookah among the target population. Conclusions and key recommendations: Restrictions of flavored hookah tobacco would positively impact our struggle against prevalent hookah use, since the analyses of target population showed that $88 \%$ of users preferred flavored hookah. Legal restrictions on the fast spreading hookah cafes would also reduce the prevalence since hookah is perceived as a means of socialization in hookah bars. Legal and environmental regulations and restrictions are necessary in order to prevent the hookah epidemic.

Tob. Induc. Dis. 2018;16(Suppl 1):A768 DOI: $10.18332 /$ tid/84293

\section{2:45-14:00}

EP-263-4 Effectiveness of pictorial health warnings on the waterpipe device and tobacco packs: a qualitative study

A Mostafa', HT Mohammed ${ }^{1,2}$, W Hussein ${ }^{1}$, M Elhabiby $^{3}$, W Safwat 4,5, S Labib 6 , A Aboul Fotouh ${ }^{1}$

${ }^{1}$ Faculty of Medicine, Ain Shams University, Department of Community, Environmental, and Occupational Medicine, Cairo, Egypt, ${ }^{2}$ School of Pharmacy, University of Waterloo, Waterloo, ON, Canada, ${ }^{3}$ Faculty of Medicine, Ain Shams University, Department of Psychiatry Medicine, Cairo, Egypt, ${ }^{4}$ Egyptian Tobacco Control Coalition, Cairo, Egypt, ${ }^{5}$ International Committee Chair of Association for Treating Tobacco Use and Dependence, Cairo, Egypt, ${ }^{6}$ Egyptian Ministry of Health, Tobacco Control Unit, Cairo, Egypt.E-mail: aya.kamaleldin@med.asu. edu.eg

Background: Despite the global increase in waterpipe smoking, current evidence is limited in assessing health warning labeling practices on waterpipe tobacco (WT) products. This qualitative study aimed to

a) understand how participants perceive pictorial health warnings (PHW) on WT packs

b) explore their attitude towards a newly designed set and

c) explore their opinion of placing PHWs on the waterpipe device and accessories.

Methods: Ten focus group and ten in-depth interview sessions were conducted in Egypt with 90 waterpipe smokers and nonsmokers, of both genders, and different age-groups (18-24 and $25+$ ) within rural and urban regions. Verbatim transcripts were analyzed using thematic analysis.

Results: Participants thought that the current PHW set triggered affective reactions; still some were unclear, or unrealistic. The majority considered it attractive because of the bright colorful designs of fruits and flavors. In contrast, participants thought that the newly designed set had more positive elements that might 
help in preventing smoking initiation or inducing quit attempts, such as the absence of attracting flavors, the contrasting dark background, the believable contents, and the larger label size. Participants' views of effective warnings were leaning towards those posing proximal health risks. Most of the participants believed that inserting PHWs on waterpipe device may enhance their effectiveness. Participants equally favored placing them on the glass body, or on the mouthpiece and hose.

Conclusions: Findings implicate a policy need to extend WT labeling regulations to the waterpipe device and employ evidencebased practices to customize PHWs' content, design, and placement on WT products.

Tob. Induc. Dis. 2018;16(Suppl 1):A769

DOI: $10.18332 /$ tid/84640

\section{5:45-16:45}

LB-1360-3 South African adult smokers perception of pictorial warnings on quitting behaviour

F Senkubuge $^{1}$, OA Ayo-Yusuf ${ }^{2}$

${ }^{1}$ University of Pretoria, School of Health Systems and Public Health, Pretoria, South Africa, ${ }^{2}$ Sefako Makgatho Health Sciences University (SMU), DVC, Pretoria, South Africa. E-mail: flavia. senkubuge@up.ac.za

Background: There is no current nationally representative study to inform whether pictorial warnings will have an influence on smoking among South Africans. This study therefore sought to determine the perception of South African adult smokers of pictorial warnings on quitting behaviour.

Methods: The study involved a nationally representative sample of adults $\geq 16$ years $(\mathrm{N}=3,063)$ who participated in the $2016 / 2017$ South African Social Attitudes Survey (SASAS). Using a self-administered questionnaire, data obtained include sociodemographics, tobacco use and notice of health warnings. Also obtained were participants' perception of counter displays on encouraging youth smoking, and if participants felt current text warning can make smokers think about quitting. The respondents were then showed model pictorial warning packs and asked the extent they felt this could make smokers think about quitting.

Results: Current smoking among South African adults in 2016 was $19 \%(95 \% \mathrm{CI}=16.9-21.3 ; \mathrm{n}=597)$ and only $35.3 \%(\mathrm{n}=1120)$ frequently noticed current text health warnings, while $58.2 \%$ $(n=1794)$ agreed that counter displays will encourage youth smoking. Of the smokers, only $50.5 \%(n=338)$ plan to quit and $60 \%(n=361)$ had attempted to quit. Although, $85.7 \%$ agreed that text warnings were easy to understand, only $15.1 \%$ felt this would make a smoker think of quitting. However, $41.9 \%$ felt that the pictorial warnings would make smokers think of quitting. Those who felt pictorial would prompt quitting were also more likely to believe sales counter displays may encourage youth smoking $(\mathrm{OR}=3.13 ; 95 \% \mathrm{CI}=1.82-5.37)$, have made a quit attempt in the last 12 months $(\mathrm{OR}=1.88 ; 95 \% \mathrm{CI}=1.07-3.30)$ and less likely to be current e-cigarette user $(\mathrm{OR}=0.21 ; 95 \% \mathrm{CI}=0.06-0.83)$. Compared to those with Grade 12 education, those with $<$ Grade $12(\mathrm{OR}=1.72 ; 95 \% \mathrm{CI}=1.00-2.96)$ and $>$ Grade $12(\mathrm{OR}=3.78$; $95 \% \mathrm{CI}=1.50-9.53)$ were more likely to feel pictorial warnings would prompt quitting.

Conclusions: These findings suggest that adding pictorial warnings to the current cigarette packs is more likely to prompt quitting.

Tob. Induc. Dis. 2018;16(Suppl 1):A770

DOI: $10.18332 /$ tid/84760

\subsection{Opportunities for regulating tobacco}

\section{products' content and emissions to reduce harm and addiction potential}

\author{
$12: 30-14: 00$
}

PS-1044-3 Classification, labelling and packaging of the most popular e-cigarette refill liquids across 9 European countries

C Girvalaki¹, A Vardavas'1, M Tzatzarakis', P Stivaktakis¹, A Tsatsakis', P Behrakis ${ }^{2,3}$, C Vardavas ${ }^{1,3}$

${ }^{1}$ Medical School, University of Crete, Toxicology Laboratory, Heraklion, Greece, ${ }^{2}$ Biomedical Research Foundation of the Academy of Athens (BRFAAA), Athens, Greece, ${ }^{3}$ Institute of Public Health, American College of Greece, Athens, Greece. E-mail: charis@tobcontrol.eu

Background: Classification, Labelling and Packaging (CLP) of the e-cigarette refill liquids adopts the UN Globally Harmonised System of Classification and Labelling of Chemicals (GHS) into the EU. Regarding article 17 where a substance and mixture classified as hazardous must bear a label and article 35, where substances and mixtures must acquire packaging requirements, our study aimed to evaluate the level of compliance of these products within the European market.

Methods: Through cross referencing of two data sources, we identified examples of the most popular websites and their marketed brands, as also the most popular point of sale e-cigarettes, in nine European Member States (Greece, France, Poland, Germany, Holland, the United Kingdom, Spain, Romania and Hungary). An optical evaluation from two independent researchers followed for all the e-liquids $(n=122)$. Evaluation was conducted on the vial, on the external package and leaflet if included.

Results: Results showed that almost all products evaluated were in near compliance with the two articles based on CLP regulations. The $89.3 \%$ (109) of the samples contained hazard pictograms while $80.3 \%$ (98) contained hazard statements. Additionally, the use of child-resistant fastening (CRF) systems and tactile warnings of danger (TWD) were frequently found on the vials and external packages ( $90.2 \%$ vs. $80.3 \%$, respectively).

Conclusions: This is the first study to evaluate the labelling/ packaging practices and the technical design/safety features of e-cigarettes in 9 EU MS before the implementation of Tobacco Products Directive.

Funding: This work was supported by a grant from the European Commission (Horizon2020 HCO-6-2015; EUREST-PLUS: 681109).

Tob. Induc. Dis. 2018;16(Suppl 1):A771

DOI:10.18332/tid/84616

12:30-14:00

PS-1047-3 Evaluating compliance of labelling on tobacco packets in countries across the Middle East H Mir ${ }^{1,2}$, S Yusuf ${ }^{1,2}$

${ }^{1}$ McMaster University, Medicine, Hamilton, ON, Canada, ${ }^{2}$ Population Health Research Institute, Population Health, Hamilton, ON, Canada. E-mail: hassan.mir1@gmail.com

Background: Despite knowledge of harms, smoking rates remain high and continue to rise in Middle Eastern Countries. Initiatives such as the FCTC were developed to address the tobacco epidemic through health policy. Despite its positive impact, implementation remains a challenge. In this study, we assess compliance of labelling on tobacco packets from twelve Middle Eastern countries with national legislation and FCTC recommendations.

Methods: Investigators from twelve Middle Eastern Countries collected at least 10 unique packets of the most commonly 
consumed and cheapest brands of cigarettes between January 2015 and November 2016. The countries included Bahrain, Israel, Kuwait, Oman, Qatar, Saudi Arabia, United Arab Emirates (High-Income Countries - HIC), Jordan, Lebanon, Turkey (UpperMiddle Income Countries - UMIC), Egypt, and Palestine (LowMiddle-Income Countries - LMIC). A total of 140 packets were inspected using a structured data collection tool; all labels were assessed for content, size, and location.

Results: Health Warnings were present on the Principal Display Area (PDA - front and back panel) on 98\% of packets. All countries except for Palestine met or exceeded the WHO minimum recommendations that $30 \%$ of the packets PDA should be covered by a health warning label. However, only Bahrain, Israel, Kuwait, Qatar, and Turkey met their own national legislation about the minimum area of the packets PDA that must be covered by a health warning label.

Promotional labels were present on all packages. Deceptive terms such as 'light' and 'blue' were found on $55 \%$ of all packs.

Conclusions: Most countries were compliant with WHO recommendations on health warning labelling. However, there is poor compliance and implementation of national legislation in these countries. Promotional and deceptive labelling were present on packets from all countries despite being banned accordingly to WHO recommendations and national legislation. Monitoring labelling on tobacco packets with country-specific feedback may help improve compliance and implementation.

Tob. Induc. Dis. 2018;16(Suppl 1):A772

DOI:10.18332/tid/84645

\section{2:30-14:00}

PS-1049-3 Brazil's additive ban: understanding the importance of packaging

C Washington ${ }^{1}, \mathrm{~K}$ Welding ${ }^{1}$, K Smith'1, J Cohen

'Johns Hopkins Bloomberg School of Public Health - Institute for Global Tobacco Control, Health Behavior and Society, Baltimore, MD, United States of America. E-mail: cwashin8@jhu.edu

Background: Flavored cigarettes tend to be perceived as more palatable, less harmful, and more attractive to youth. In 2012, the Brazilian government issued a regulation banning the use of additives in all tobacco products, including sugar and flavorings. Industry interference has impeded implementation. Although not legally required, tobacco companies in Brazil use the pack to advertise cigarette ingredients. We examine the impact the additive ban would have on packaging and the cigarette market in Brazil.

Methods: In 2016, we purchased and coded 147 unique cigarette pack presentations from three cities in Brazil: São Paulo, Manaus, and Salvador using a systematic protocol. Two independent coders recorded the ingredient list and imagery or words advertising a flavor on each pack.

Results: Eighty percent of the packs' ingredient lists had at least one additive that is specified in the additive ban: sugar (77\%), flavoring agents (24\%), plant extract (24\%), clove (11\%), and menthol (10\%). Tobacco companies also used the pack to promote specific flavors including cinnamon, clove, mint, menthol, and cherry; $20 \%$ of the packs had at least one of these flavors. In some cases, flavors advertised on the pack were not included on the ingredient list: 23 packs were coded for menthol or mint but only 8 (35\%) had menthol as an ingredient. British American Tobacco (BAT) and Philip Morris (PM) accounted for $78 \%$ of the sample. An additive was listed in the ingredients on $83 \%$ of the 77 BAT packs and $100 \%$ of the 37 PM packs.

Conclusions: Based on this sample, implementation of the additive ban would entail most brand variants available in Brazil needing to remove additive and flavor advertisement on the pack. Historically, the industry's response to restrictive packaging requirements is more innovation in packaging design. Plain packaging should be considered with the additive ban to help reduce the appeal of cigarettes.

Tob. Induc. Dis. 2018;16(Suppl 1):A773

DOI:10.18332/tid/84667

\section{2:45-14:00}

EP-205-3 E-cigarettes: scientific and political controversies

\section{Y Saloojee}

${ }^{1}$ National Council Against Smoking, Johannesburg, South Africa. E-mail:ysalooje@iafrica.com

Background and challenges to implementation: The e-cigarette is touted as a disruptive technology that could make combustible cigarettes obsolete. The product, its science and politics though are controversial.

Intervention or response: Published journal articles, reports and conference presentations on e-cigarettes were located using PubMed, plus conference, industry and other websites. These were critically reviewed.

Results and lessons learnt: A number of contentious issues regarding e-cigarettes were identified:

Its use is recommended by some public health bodies (UK Royal College of Physicians) but not by others (World Health Organization). The product may not be fit-for-purpose, with low consumer acceptance. In 2016, $72 \%$ of those who had tried an e-cigarette in the UK no longer used it, A majority of users combined smoking with vaping and are thus unlikely to obtain significant health benefits. E-cigarettes do not deliver nicotine as rapidly and effectively as combustible cigarettes. Vaping was reportedly less "satisfying" than smoking., The development of 'heat not burn' and other novel products by the industry is testimony to its deficiencies. E-cigarettes have divided the antismoking community, with some regarding the tobacco companies as essential partners while others shun it. Finally, the profit-driven multinationals regard affluent countries as the prime market for novel products, while simultaneously seeking to maintain and expand the conventional cigarette market in poor continues.

Conclusions and key recommendations: There are many unresolved scientific, policy and political concerns about the role of e-cigarettes in tobacco control. A continued focus on supply and demand reduction instead of harm reduction is the best option for low-income countries.

Tob. Induc. Dis. 2018;16(Suppl 1):A774

DOI:10.18332/tid/84680

\section{$12: 45-14: 00$}

EP-207-3 Evaluation of chemical constituents of E-cigarette liquid refills marketed through online in India using NMR analysis

CV Divyambika1', E Vidhubala², G Vani

'Sri Ramachandra University, Department of Oral Medicine and Radiology, Chennai, India, ${ }^{2}$ Indian Institute of Sports Medicine, Chennai, India, ${ }^{3}$ University of Madras, Department of Bio-Chemistry, Chennai, India. E-mail: cvdivyambika@ srirmachandra.edu.in

Background: Electronic cigarette (e-cigarette) is an emerging class of electronic nicotine-delivery system claimed by the industries as a quitting aid. Laboratory testing of some refill liquids for e-cigarettes(e- 
liquids) contain impurities and toxic substances, or are not filled true to label. The additional impurities in the liquids or vapour (e.g., polyaromatic hydrocarbons (PAHs), Tobacco specific nitrosamines (TSNAs), aldehydes and acrolein), unexpected toxins and/or unreliable nicotine in different glycols mixed with concentrated flavours, warrants a thorough evaluation of the chemical constituents of these E-liquids. Previous research in the evaluation of these constituents involved gas or liquid chromatography (GC, LC) combined with mass spectrometric (MS) detection for the analysis in combination with $1 \mathrm{H}$ - Nuclear Magnetic Resonance (NMR) analysis. This study aimed to evaluate the chemical composition of e-liquid refills using 1H- NMR, 13C-NMR along with DEFT, GCMS and IR spectroscopic analysis.

Methods: A total of 6 samples marketed and sold highly in India through online, claimed to have $0,3,6,12$ and 18 milligrams of nicotine and one sample claiming no nicotine/no tar were identified and purchased. The presence of Tobacco Specific Nitrosamines (TSNAs), tobacco alkaloids, Polycyclic Aromatic Hydrocarbons (PAHs), Volatile Organic Compound (VOCs) were analysed using 1H- NMR, 13C-NMR along with DEPT, Gas Chromatography Mass Spectroscopy (GCMS) and IR spectroscopy.

Results: Significant levels of VOCs were noted which included glycerin, propylene and ethylene glycol. Acrolein and PAH were not detected in the samples. However, presence of additional organic compounds including nitrosamines, and nicotine were detected by 13C- NMR, DEPT and IR spectroscopy.

Conclusions: NMR spectroscopy is a useful and rapid method to simultaneously detect several ingredients in e-liquids, and GCMS , IR and DEFT also provided additional information on the constituents of e-liquid products. Systematic evaluation of chemical constituents of the e-cigarette may hence throw light on its possible short term and long term health effects.

Tob. Induc. Dis. 2018;16(Suppl 1):A775

DOI:10.18332/tid/84528

\section{2:45-14:00}

EP-211-3 Sensory analysis of characterizing flavors in tobacco products using a trained expert panel E Krüsemann ${ }^{1,2}, M$ Lasschuijt ${ }^{2}, S$ Boesveldt ${ }^{2}, K$ de Graaf ${ }^{2}, R$ Talhout $^{1}$

${ }^{1}$ National Institute for Public Health and the Environment (RIVM), Centre for Health Protection, Bilthoven, Netherlands, ${ }^{2}$ Wageningen University, Division of Human Nutrition, Wageningen, Netherlands. E-mail: erna.krusemann@rivm.nl Background: The European Tobacco Product Directive 2014/40/ EU prohibited cigarettes and roll-your-own tobacco having a characterizing flavor, which is defined as "a clearly noticeable smell or taste other than one of tobacco [...]". To practically distinguish between products with and without a characterizing flavor, some questions need to be answered. For example, which products should be used as reference? Which sensory method should be used? Should we smell or smoke tobacco? Should we use consumer or expert panels? Which statistical cut-off value should be used to define 'clearly noticeable'? Considering these issues, we developed a sensory method to identify characterizing flavors for regulatory purposes.

Methods: An expert panel $(n=18)$ was trained to evaluate the smell of twenty tobacco products using self-defined odor attributes. Three types of training sessions were distinguished: attribute training, consensus training, and performance monitoring. Products were tested using Quantitative Descriptive Analysis during six test sessions at Wageningen University (the
Netherlands). Principal component analysis (95\% and 99\% confidence interval) and hierarchical clustering (four and six clusters) were used to find differences and similarities between tobacco products based on odor attributes.

Results: The final attribute list contained thirteen odor descriptors. Panel consensus was reached after 14 training sessions. Attribute discrimination ability was significant for each attribute $(\mathrm{p}<0.001)$. Products marketed as unflavored that formed one cluster were considered reference space. Four clusters distinguished cherry-, vanilla-, and menthol-flavored products from reference products. Six clusters additionally divided these reference products into roll-your-own, commercially available, and additive-free products. Conclusions: Using a trained expert panel is a successful example of assessing characterizing flavors in tobacco products. Regulatory decisions should be made on significance level and products representing the reference space, as these directly influence strictness of the method and thus the number of products that imparts a characterizing flavor compared to the reference space.

Tob. Induc. Dis. 2018;16(Suppl 1):A776

DOI:10.18332/tid/84112

$12: 45-14: 00$

EP-219-3 Nicotine content in flavored electronic cigarette liquids using three unique samples

P Fagan ${ }^{1,2}$, M Guy $^{2,3}$, P Pokhrel $^{4}$, T Herzog ${ }^{4}$, E Soule ${ }^{2,5}$, S Maloney ${ }^{2,5}$, S Palafox ${ }^{4}$, T Eissenberg ${ }^{2,5}$

'Univerity of Arkansas for Medical Sciences, Health Behavior and Health Education, Little Rock, AR, United States of America, ${ }^{2}$ Virginia Commonwealth University, Center for the Study of Tobacco Products, Richmond, VA, United States of America, ${ }^{3}$ Virginia Commonwealth University, African American Studies, Richmond, VA, United States of America, ${ }^{4}$ University of Hawaii, Honolulu, HI, United States of America, ${ }^{5}$ Virginia Commonwealth University, Department of Psychology, Richmond, VA, United States of America. E-mail: pfagan@uams.edu

Background: Electronic cigarette (e-cigarette) use is increasing, but consumers may be unaware of the nicotine concentrations in e-cigarettes or discrepancies in product advertised versus measured nicotine in e-cigarette liquids. Levels of nicotine, the addictive and toxic chemical in e-cigarette liquids, can influence consumers' exposure, dependence on the product, and/or success in switching from cigarettes to e-cigarettes. This study quantified the levels of nicotine in three unique samples: national sample of 147 flavored e-cigarette liquids purchased in the USA; 66 samples purchased in the state of Hawaii, USA; and 40 samples purchased in Virginia and Hawaii, USA.

Methods: Flavored e-cigarette liquid samples were purchased from manufacturers/distributors in 2015 for the Hawaii and Virginia samples and in 2016 for the national sample. For each flavor, staff purchased three or four concentrations of nicotine depending on manufacturer/distributor availability: $0 \mathrm{mg} / \mathrm{ml}$; 3,6 or $8 \mathrm{mg} / \mathrm{ml}$; 11 or $12 \mathrm{mg} / \mathrm{ml}$; and 18,24 or $25 \mathrm{mg} / \mathrm{ml}$. We measured nicotine concentration in each sample using liquid chromatography mass spectrometry and calculated the percent or relative difference for each.

Results: For the national sample of e-cigarette flavored liquids labeled as $0 \mathrm{mg} / \mathrm{ml}, 100 \%$ contained detectable levels of nicotine; $88 \%$ of Hawaii samples; and $0 \%$ of the combined Hawaii and Virginia samples in the third study. For all three unique samples, we found high levels of discordance between advertised $(3 \mathrm{mg} / \mathrm{ml}$ or greater) versus measured nicotine. In some samples, levels of nicotine were higher, and in others, lower than that advertised on 
the labels. Discordance was not associated with flavor type.

Conclusions: Discrepancies between the manufacturer's labeling and measured nicotine could potentially influence nicotine delivery and toxicity exposure. Studies are needed to determine what standards are needed to ensure accurate labeling and quality controls that protect consumers, including those who are not aware that they are being exposed to nicotine.

Tob. Induc. Dis. 2018;16(Suppl 1):A777

DOI:10.18332/tid/84629

\subsection{Prevalence of tobacco and nicotine related products' use: Traditional and novel products}

\section{$12: 30-14: 00$}

PS-1050-4 How effective is gutka (a smokeless tobacco) ban in West Bengal, India? A case study N Mukherjee', B Pal'2, S Ghosh², S Goel ${ }^{3}, \mathrm{P} \mathrm{Lal}^{4}$

'MANT, Tobacco Control, Kolkata, India, ${ }^{2}$ MANT, Research and M\&tE, Kolkata, India, ${ }^{3}$ Post Graduate Institute of Medical Education and Research, Health Management, School of Public Health, Chandigarh, India, ${ }^{4}$ International Union against Tuberculosis and Lung Disease, Tobacco Control, South-East Asia Office, New Delhi, India. E-mail: mant.kolkata@gmail.com Background: Under the India's food legislation, adding tobacco or nicotine to anything that is consumed is prohibited, following this in May 2014, the Ministry of Health announced a ban on the sale of gutka, a popular packaged mixture comprising betelnut, condiment, lime and tobacco mixture. We conducted a compliance of the ban on gutka in the state of West Bengal, which has a tobacco use prevalence of $15 \%$ adults using smokeless tobacco (or more than 9 million people).

Methods: Three districts were selected purposively for the study. In every district, district headquarter i.e. municipality selected purposively while two Blocks were selected randomly. Based on population dispersion model, 450 consumers were divided in three clusters in each district and for every 5 consumers 1 vendor i.e. 90 vendors were interviewed. The interview schedule was pre-tested in Kolkata and after feasibility assessment of the same it was finalized. The study was conducted during September December 2016.

Results: Effect of ban on gutka has been limited and disappointing. Only $17.8 \%$ of vendors knew that selling of gutka is punishable offence while $68.5 \%$ vendors replied that gutka is easily available to them. $75.3 \%$ vendor have sold gutka to the minors and only $1.1 \%$ vendors were raided. However, almost $90 \%$ vendors felt that gutka ban is helpful in quitting the deadly habit. $16.9 \%$ vendors answered that they faced a loss in their business due to gutka ban and $15.7 \%$ vendors shifted to other businesses after the ban was enforced. It is also true that sale of gutka declined after ban as $53.5 \%$ consumers reported that they have shifted to other tobacco products.

Conclusions: Gutkha ban has been ineffective in the absence of stricter enforcement at producer, wholesaler and vendor level. To make the ban effective immediate attention is required on enforcement across the supply chain.

Tob. Induc. Dis. 2018;16(Suppl 1):A778

DOI:10.18332/tid/84190

\section{2:30-14:00}

PS-1051-4 Spatial pattern of risky health behavior in India: evidence from recent National Family
Health Survey 2015 - 16

A Kumar ${ }^{1}$

'Population Council, Delhi, India. E-mail: abhi85_iips@ redffmail.com

Background: Association between risky health behavior (consumption of tobacco/alcohol) and lung diseases is well stablished. India is one the countries with increasing burden of lung diseases. Using the most recent evidence available in the country, this paper examines the spatial pattern of risky health behavior among men and women.

Methods: We used most recent round of the National Family Health Survey data conducted in India during 2015-16. The survey is in tune with internationally recognized Demographic Health Survey (DHS) and provide information from all 36 states and union territories of India. We analyzed two key outcomes consumption of tobacco and alcohol separately among men and women. First, we examined spatial clustering in the risky behavior in the country. Further, we examined the association between the risky behavior and background characteristics such as education, household condition of men and women. We used bi-variate and descriptive statistics in the analysis.

Results: Prevalence of tobacco consumption was 7 percent among women and 45 percent among men in the country. However, there is stark variation across the states - among women the prevalence varied from $2 \%$ in Haryana to $59 \%$ in Mizoram. Moreover, there is spatial clustering is tobacco consumption in tribal states (northeastern states) of the country. Among men the prevalence varied from $27 \%$ in Andhra Pradesh to $80 \%$ in Mizoram. Consumption of alcohol was $2 \%$ among women and $28 \%$ among men in the country, with huge spatial variation. These risky healthy behaviors are significantly and positively correlated with poor education and household living conditions.

Conclusions: The evidence generated through this paper is important from policy makers to identify the pockets of risky healthy behavior in India. This identification would be helpful to combat with the lung diseases in the country and to achieve the Sustainable Development Goal of achieving health live for all.

Tob. Induc. Dis. 2018;16(Suppl 1):A779

DOI:10.18332/tid/84199

\section{2:30-14:00}

PS-1052-4 Trends in smoking prevalence in South Africa for 1998 - 2014 - overcoming the challenge of multiple data sources

C Janse van Rensburg', P Groenewald ${ }^{2}$, O Awotiwon ${ }^{2}$, B Nojilana², J Joubert ${ }^{2}$, V Pillay van-Wyk2 ${ }^{2}$ D Bradshaw ${ }^{2}$, S Manda', the South African Smoking Risk Factor Work

'South Africa Medical Research Council, Biostatistics Unit, Cape Town, South Africa, ${ }^{2}$ South Africa Medical Research Council, Burden of Disease Research Unit, Cape Town, South Africa. E-mail: charl.jansevanrensburg@mrc.ac.za

Background: Monitoring prevalence of smoking is essential for evaluating the impact of national tobacco control policies. This study aims to estimate national pooled smoking prevalence trends over a 16 year period for adult South Africans.

Methods: Smoking prevalence by age, sex and race from 14 South African population and national household surveys conducted between 1998 - 2014 were used to generate trends over time by

1) performing a series of transformations of the aggregated prevalence data,

2) investigating interactions with race/time, and

3) using a posthumous regression. Bias within surveys were 
adjusted for by using a risk-of-bias weight.

Results: The overall observed smoking prevalence decreased from $23.7(22.4 ; 24.9)$ in 1998 to $20.3(19.3 ; 21.4)$ in 2014. Downward trends were observed in both males and females, although it was steeper in males. There were significant differences between race groups, as well as within race groups across age, most notably Asian males, which also seemed to drive the steep decline among males. Whilst the downward trend was evident in older ages $(25+$ years), the youngest group (15 - 24 years) had a non-linear trend differing from the linear trend in the older group.

Conclusions: Pooling estimates from different national surveys over time, gave an effective picture of trends in smoking in South Africa. Although not directly observable, the trends reported indicate that the South African Tobacco Control Act of 1993 had influenced and maintained decreasing smoking trends in the last couple of decades, albeit to a lesser extent among females.

Tob. Induc. Dis. 2018;16(Suppl 1):A780

DOI:10.18332/tid/84201

\section{2:30-14:00}

PS-1054-4 Declining trend of tobacco use in a rural community of Bangladesh, 2006-2013

T Zissan', K Parvin², MM Zaman², J Ahmed², SR Choudhury² ${ }^{1}$ Ekhlaspur Center of Health $(\mathrm{ECOH})$, Non Communicable Diseases, Chandpur, Bangladesh, ${ }^{2}$ Ekhlaspur Center of Health (ECOH), Chandpur, Bangladesh.E-mail: tzissan@gmail.com

Background: Tobacco use in Bangladesh especially in rural area has been high, but report on its trend is lacking. The aim of this study was to examine tobacco use trend in a rural area of Bangladesh concomitant to a simple but persistent intervention.

Methods: The study was done in Ekhlaspur village located in Chandpur district that has about 1000 households. Four crosssectional surveys between 2006 and 2013 were done among adults (aged 25 to 64 years) to assess the trend of tobacco use in this village. One adult per household was selected targeting 400 to 1000 randomly selected households. Concomitantly an intervention package has been ongoing that included yard meetings, facility based counselling tagged with clinical services, observance of no tobacco days, special lectures in schools, and engagement of community organizations and opinion leaders. Prevalence of tobacco use and their 95\% confidence intervals (CI) were obtained. Linier trend analysis was done using Excel to examine the trend of tobacco use.

Results: Participants' mean age varied between 41 and 44 years in all four surveys. About half of them completed a five years of primary education. Six in 10 people at the beginning were found to use tobacco in any form in the village. A consistent decline in tobacco use has been observed from $62 \%$ (95\% CI $57 \%$ to $68 \%$ ) to in 2006 to $32 \%$ (24\% to $41 \%$ ) in 2013 . The prevalence of smoking (from $35 \%$ to $14 \%$ ) and smokeless tobacco (from $40 \%$ to $20 \%$ ) showed a similar declining trend. Sex-specific results also showed similar trends (Figure 1).

Conclusions: Community interventions in a sustainable manner can control tobacco use in rural communities of Bangladesh. Similar interventions can be replicated all over the country using lower tier of health system, public or private.

Tob. Induc. Dis. 2018;16(Suppl 1):A781

DOI:10.18332/tid/84208

12:30-14:00

PS-1055-4 Current tobacco use and its associated factors among adults in Georgia: findings from Non-Communicable Disease Risk Factors STEPS Survey Georgia 2016

N Maglaklelidze'1, L Sturua', A Gamkrelidze'1, M Maglakelidze² ${ }^{1}$ National Center for Disease Control and Public Health, Noncommunicable Diseases, Tbilisi, Georgia, ${ }^{2}$ Georgian Respiratory Association, Tbilisi, Georgia. E-mail: n.maglak@ gmail.com

Background: Tobacco surveys of past decades show that tobacco use prevalence is high in Georgia; According to nationwide Noncommunicable Diseases (NCDC) Risk Factors STEPS Survey $201030 \%$ of adult population are current tobacco users. Another Nationwide Tobacco Survey 2014 reported $28 \%$ of tobacco use prevalence among Georgian adults. However, there has been relatively little progress in systematic study of the factors associated with this high tobacco use. The current study aimed to assess the prevalence of tobacco use and its associated sociodemographic, behavioral and environmental factors in Georgia.

Methods: The current study in Georgia was a populationbased STEPS survey of adults aged 18-69. A multi-stage cluster sample design was used to produce representative data for that age range in Georgia. The. A total of 5554 adults participated in the survey. The overall response rate was $75.7 \%$. We assessed sociodemographics, behavioral and other health-related factors.

Results: The prevalence of current overall tobacco use was $31.1 \%$ (95 \% CI: 29.0-33.1) which comprised of smoked tobacco use, smoked cigarettes and use of smokeless tobacco, 31.0\% (95\% 28.9-33.0) smoked tobacco, 29.9 (95\% CI: 27.9-32.0) smoked cigarettes and 0.3 (95\% CI: 0.0-0.6) use of smokeless tobacco. Smoking prevalence was significantly higher in men $57.1 \%$ (95\% CI: 53.7-60.4) compared to women 7.1\% (95\% CI: 5.9-8.4), especially in younger age groups and with other substance abuse history (predominantly alcohol).

Conclusions: Despite of some efforts in the field of tobacco control, tobacco use (particularly smoking) was high in Georgia. Males, younger age groups, and population with addictions to other substances (especially alcohol) should be the primary target of behavioral interventions; The stricter implementation of tobacco control measures, including comprehensive ban of tobacco marketing and smoking in public places, improved health warnings on tobacco packages and anti-tobacco communication campaigns can have a significant positive impact on reducing growing tobacco epidemics in Georgia.

Tob. Induc. Dis. 2018;16(Suppl 1):A782

DOI: $10.18332 /$ tid $/ 84218$

12:30-14:00

PS-1058-4 Social determinants of tobacco smoking in Mexico stratified by sex and age. Mexico, Global Adult Tobacco Survey (GATS) 2009 and 2015

L Zavala Arciniega', WC Paz Ballesteros', SC Mejia Gil', LM Reynales Shigematsu'

${ }^{1}$ National Institute of Public Health in Mexico, Department of Tobacco Research, Cuernavaca, Mexico. E-mail: luis.zavala@ insp.mx

Background: Mexico joined the Framework Convention on Tobacco Control in 2005 and introduced the general law of tobacco control in 2008. Monitoring tobacco consumption, addressing sex and age in relation to social context, is important to plan tobacco control strategies. This study was aimed at assessing social determinants of smoking in Mexico using data from GATS 
2009 and 2015.

Methods: Data from GATS Mexico 2009 and 2015 was used to perform a secondary analysis. Weighted descriptive statistics and stratified multivariate logistic regression models were executed.

Results: 13,617 people in 2009 and 14,664 in 2015 were interviewed. Prevalence of current smoking was higher among men than in women $(24.8 \%$ vs $7.8 \%$ in 2009 and $25.2 \%$ vs $8.2 \%$ in 2015). In 2015, women living in urban areas were more likely to be current smokers (OR: 3.1 ; CI: $1.5,6.8$ in young and OR: 4.9; CI: 3.3,7.2 in adult women). In young women, higher odds of being current smoker were found in those exposed to secondhand smoke at home (OR: 3.5; CI: 1.9,6.4). Adult women with high socioeconomic status were more likely to be current smokers (OR: 2.5; CI: 1.2,5.0). Among young men, likelihood of being a current smoker increased with age (OR: 5.5; CI: 3.1,9.9 for 21-24 group, reference group 15-17) and in those exposed to second hand smoke at home (OR: 1.6; CI: 1.0,2.6). In adult men, likelihood of being a current smoker decreased with age (OR: $0.3, \mathrm{CI}: 0.2,0.5$ age $\geq 65$ reference group 25-34) and in those with high education level (OR: 0.5; CI: 0.3,0.8) while living in urban areas increases the likelihood (OR: 1.6; CI: 1.3,1.9). The results were similar for 2009.

Conclusions: Being young and living in urban areas remain important determinants of tobacco use in Mexico. Other factors vary according to sex and age, and these should be considered for control policies.

Tob. Induc. Dis. 2018;16(Suppl 1):A783

DOI:10.18332/tid/84328

\section{$12: 30-14: 00$}

PS-1059-4 Trends of tobacco smoking among males in Sri Lanka in the new millenium

PR Vithanage ${ }^{1}$

${ }^{1}$ Alcohol and Drug Information Center (ADIC) Sri Lanka, Research \&t Evaluation Programme, Colombo, Sri Lanka. E-mail: peshanirisna@gmail.com

Background: Since 2000, Alcohol and Drug Information Centre (ADIC) conducted a bi-annual survey to determine prevalence and trends of tobacco smoking in Sri Lanka. It is the only trend monitoring survey of tobacco prevalence which is widely recognized in Sri Lanka.

Objective: To determine patterns and trends in tobacco smoking among males above fifteen years in Sri Lanka.

Methods: The survey initially recruited participants from five districts selected randomly. Since 2012 the survey covered ten districts. Two hundred and fifty males above 15 years from each district were recruited using a nonrandom, accidental sampling method validated using a multi stage cluster sampling method. Data on consumption, initiation, frequency, attitudes are collected by trained interviewers. Structured interviews are employed to collect data using Statistical Package for Social Sciences.

Results: The current smoking prevalence reduced by $6.9 \%$ (July 2000; $\mathrm{N}=1136 \mathrm{P}=39 \%$; July 2016; $\mathrm{N}=2321 \mathrm{P}=31.9 \%$ ). The decreasing rate increased during the period July 2006 to July 2008 . Since July 2007 , the major initiating age group was year 16 20 (Min; 50\%, Max; 60\%). Initiating before the age of 20 shows a decreasing (from $82 \%$ to $67 \%$ ) trend. Most smokers (72\%) state they are daily smokers and the trend has remained static over the years. The most frequent number of cigarettes per day is 4 sticks. The prevalence of occasional smokers shows little fluctuation and the overall trends remain static. Tobacco users expressed that they used tobacco as a habit. Most common reason for never using tobacco was dislike/ unpleasant feeling towards tobacco use.

Conclusions: Smoking tobacco is decreasing among males in Sri Lanka. Initiation age group seems to be shifting from 20 and below years to above 20 years.

\section{Tob. Induc. Dis. 2018;16(Suppl 1):A784 \\ DOI:10.18332/tid/84329}

\section{2:30-14:00 \\ PS-1060-4 Smoking and smokeless tobacco use among urban women and men In Nepal \\ B Bajracharya ${ }^{1}$ \\ 'Support to the Health Sector Program, Lalitpur, Nepal. E-mail: bksbaj@gmail.com}

Background: Tobacco consumption causes diseases and can lead to death. Every year 16,000 deaths are attributable to tobacco smoking and smokeless tobacco in Nepal. The study aims to determine the prevalence and socio-demographic determinants of tobacco smoking and smokeless tobacco among urban women and men in Nepal.

Methods: The study is further analysis of the Nepal Demographic and Health Survey 2011 using urban subsets of women's and men's individual datasets. The study is based on 1,819 women and 717 men (weighed number) aged 15-49 years residing in urban areas. The smoking tobacco included cigarettes, pipe and bidi and smokeless tobacco included chewing tobacco, snuff and other forms of tobacco.

Results: Prevalence of smoking and smokeless tobacco was $25.2 \%$ and $30 \%$ among urban men and $4.8 \%$ and $2.5 \%$ among urban women respectively. Smoking and smokeless tobacco is highest among older age 45-49 (men 34.8\%, 44.4\%; women 16.3\%, 7\%), illiterate (men $52.4 \%, 62.8 \%$; women $15.8 \%, 7.2 \%$ ), poorest (men $57.1 \%, 56.2 \%$; women $15.6 \%$, 11.1\%), Dalit (men $38.6 \%$, $42 \%$; women $8 \%, 5.8 \%$ ), Terai region's men $(26.5 \%, 36.3 \%)$, Mountain region's women $(9.1 \%, 4.3 \%)$ and residing in Mid Western region (men 30\%, 42\%; women $6.7 \%, 4.5 \%$ ) respectively. Smoking and Smokeless tobacco is increasing with increasing age and demonstrated a negative association with education and wealth quintile.

Conclusions: In conclusion, urban men consume more smoking and smokeless tobacco than urban women. Urban men consume more smokeless tobacco whereas urban women consume more smoking tobacco. There is high rate of smoking and smokeless tobacco among older, illiterate, poorest, Dalit, and urban women and men from Terai and Mid-western region and are significantly associated. There is urgent need of anti-tobacco campaigns to older, illiterate, poor and people residing in Terai and Mid Western region of the country.

Tob. Induc. Dis. 2018;16(Suppl 1):A785

DOI:10.18332/tid/84331

12:30-14:00

PS-1061-4 Flavored smokeless tobacco use in India and Bangladesh

S Das ${ }^{1}$

${ }^{1}$ Central Institute of Psychaitry, Ranchi, India. E-mail: cipsagarika@gmail.com

Background: Flavor improves palatability of smokeless tobacco products (SLT) and attracts youth. There is limited evidence on flavored SLT use in India and Bangladesh, the two high SLT consuming countries in the world. This study maps the use of flavored SLT in India and Bangladesh.

Methods: Tobacco products assessed in the first Global Adult Tobacco Survey (GATS) of India and Bangladesh were analysed to identify flavored SLT product use and map it. Tobacco product specific assessment as well other tobacco products assessed for 
daily and less than daily users were considered. Operationally, any additional ingredient (except slaked lime) that alters or modifies the taste of plain tobacco, was considered as flavored one. SPSS used to assess the weighted prevalence estimates

Results: The flavored SLTs used in India and Bangladesh were Betel quid with tobacco or Zarda, gutkha, pan masala, Zarda, gul or gudakau, snuff, bajjar, kiwam, and dentifrice with tobacco. Prevalence of flavored SLT use in India and Bangladesh were $17 \%$ and $27 \%$ respectively. Overall use of SLTs in India and Bangladesh were $24 \%$ and $27 \%$ respectively. Zarda(18\%), and Betel quid tobacco (10\%) were commonly used flavored SLTs in Bangladesh. In India, Gutkha (8\%) and betel quid(7\%) were commonly used flavored SLTs.

Conclusions: Most SLTs are flavored one and this may aid in continuation of tobacco epidemic in India and Bangladesh. However there is an opportunity to limit SLT use by implementing legislation targeting the attractiveness of tobacco products.

Tob. Induc. Dis. 2018;16(Suppl 1):A786

DOI:10.18332/tid/84338

\section{$12: 30-14: 00$}

PS-1062-4 The ugly truth about tobacco: a permissive factor for periodontal disease among bus drivers D George ${ }^{1}$, R Shetty $^{1}$

${ }^{1} \mathrm{~A} J$ Institute of Dental Sciences, Dept. of Public Health Dentistry, Mangalore, India. E-mail: georgedona2010@gmail.com

Background: Recently, road transport has emerged as dominant segment in India's transportation sector. The most widely utilized road transport system in Karnataka is the Karnataka State Road Transport Corporation (KSRTC) which includes bus drivers who spend most of their time on wheels. This environment wrecks their schedules, disrupts their family life and supplies constant hassle and stress predisposing them to periodontal diseases. They are more likely to use tobacco products. Thus the present study was undertaken to determine the periodontal status of tobacco users and non users among bus drivers of KSRTC, Mangalore division. Methods: A cross sectional study was conducted among 328 bus drivers after obtaining clearance from institutional ethical committee. Pilot study was conducted to estimate the sample size and feasibility of the study. Oral examination was done after obtaining informed consent. Data related to prevalence of tobacco use, type of tobacco used and periodontal status was assessed using Community Periodontal Index (CPI).

Results: Among 328 bus drivers, 167 were tobacco users; 73 used smoked form and 94 used smokeless form of tobacco. Bleeding and calculus was found among $52.7 \%$ and $24 \%$ of tobacco users respectively. Among 161 non users, bleeding was found in $41 \%$ and calculus was found in $22 \%$ of the study population. The number of subjects with loss of attachment of 4-5 mm was more among tobacco users than non users.

Conclusions: Prevalence of smokeless form of tobacco among bus drivers was quite high and periodontal status was poor among tobacco users compared to non users. More studies needs to be conducted to find out the underlying cause of predisposition of tobacco usage among bus-drivers. Other parameters like dental caries, mucosal lesions and prosthetic status of the employees needs to be assessed so as to address their treatment needs and to provide comprehensive oral care to this population.

Tob. Induc. Dis. 2018;16(Suppl 1):A787

DOI:10.18332/tid/84346

$12: 30-14: 00$
PS-1064-4 Effects of cigarette tax increase of smoking rate reduction according to income status and residential area

SA Kim¹, NR Kim ${ }^{1}$, CK Choi ${ }^{1}$, M-H Shin ${ }^{1}$

${ }^{1}$ Chonnam National University Medical School, Preventive Medicine, Hwasun-gun, Korea, Republic of. E-mail:sunakim88@ hanmail.net

Background: Korea raised the cigarette tax in 2014, raising the price per cigarette from 2500 won $(1100$ won $=\$ 1)$ to 4500 won. The purpose of this study was to evaluate whether the effects of cigarette price increase on smoking reduction were affected by socioeconomic status and residential areas in Korean men.

Methods: The study used Korean Community Health Surveys from 2008 to 2015. Since 2008, the Korean Community Health Survey has been conducted on 220,000 adults aged 19 or older every year. Since women in Korea have low smoking rates, this study only included men. We assessed the trends of the 8-year smoking rate change by socioeconomic status and residence area. The smoking rate was standardized by using the Korean standard population in 2005. We conducted a statistical analysis considering the complex sample survey design.

Results: The age-standardized smoking rate declined from $48.6 \%$ in 2008 to $39.7 \%$ in 2015 . From 2008 to 2014 , it decreased by $1.0 \%$ each year, but decreased by $3.1 \%$ between 2014 and 2015 when the cigarette tax was raised. Smoking rate decreased by $2.5 \%$ in the group with monthly household income less than 2 million won, but decreased by $3.1 \%$ in the group with monthly household income more than 2 million won. Smoking rates decreased $2.9 \%$ in rural areas and $3.2 \%$ in urban areas. In conclusion, this study showed that the effect of reducing the smoking rate due to cigarette tax increase was higher in the high income group than in the low income group, and larger in the urban area than in the rural area. Conclusions: In conclusion, this study showed that smoking rate decline due to cigarette tax increase was greater in the high income group than in the low income group, and in the urban area than in the rural area.

Tob. Induc. Dis. 2018;16(Suppl 1):A788 DOI: $10.18332 /$ tid/84397

\section{$12: 30-14: 00$}

PS-1065-4 Tobacco consumption among class III CtIV employees of health care setup in Gujarat: a cross sectional study

H Nayak' ${ }^{1}$ R jadeja ${ }^{2}$, A Kaneria ${ }^{2}$, B kotecha ${ }^{2}$, K Khunt ${ }^{2}$, M ladwa ${ }^{2}$, B Karangia ${ }^{2}$, S Mahla²

'AMC MET Medical College, Community Medicine, Ahmedabad, India, ${ }^{2}$ AMC MET Medical College, Ahmedabad, India. E-mail: drhknayak@yahoo.co.in

Background: Both smoking and smokeless form of tobacco is widely prevalent \& serious public health problem in India. Hospital workers are usually aware of dangers of tobacco products. Present study was conducted to describe prevalence \& pattern of tobacco consumption, cessation practices, exposure to media \& health morbidity among health care employees in Gujarat.

Methods: A cross sectional study was conducted among Class III and IV employees of two big government hospitals of Ahmedabad \& Rajkot city. Total 200 workers were included by stratified random sampling. Personal interview was conducted \& Information about socio-demographic profile, tobacco use, health morbidities etc. was collected using W.H.O.'s tobacco questions for surveys (TQS). Data was compiled in excel \& analyzed by appropriate statistical methods. 
Results: Overall prevalence of tobacco use was $82.5 \%$. Prevalence of smoking \& smokeless tobacco was $31.5 \%$ (31\% male \& $0.5 \%$ female) \& $59.5 \%$ ( $47 \%$ male \& $12.5 \%$ female) respectively. Mean age of smokers \& nonsmokers was $43.0 \pm 11.8 \& 36.5 \pm 13.0$ years respectively $(\mathrm{Z}=3.51, \mathrm{P}<0.001)$. Majority $(73.0 \%)$ were smoking daily \& $62.2 \%$ were using smokeless tobacco daily. Majority(60.3\%) were not smoking inside home but $63.5 \%$ were consuming tobacco at work place. $54 \%$ of smokers \& $44.5 \%$ of smokeless tobacco users had tried to quit during past year, $23.8 \%$ were consulted doctor for the same $\& 81 \%$ of them were advised to quit smoking. Workers have noticed dangers of tobacco quitting information in TV (81.5\%), Newspaper (72\%) and Cigarette package $(43.5 \%)$ while $19 \%$ have noticed signs of cigarette promotion. Smoking is significantly associated with history of pneumonia (85.7\%) \& Asthma (77.8\%).

Conclusions: Prevalence of tobacco consumption among health care employees is very high compared to general population. Intervention program should be planned for health care employees to reduce burden of tobacco related morbidity \& to set up example for community visiting health care facilities.

Tob. Induc. Dis. 2018;16(Suppl 1):A789

\section{DOI:10.18332/tid/84407}

\section{$12: 30-14: 00$}

PS-1066-4 Electronic Nicotine Delivery System (E-cigarettes) marketing, sale and availability - an emerging challenge for tobacco control in India R Kumar ${ }^{1}, \mathrm{P}$ Lal $^{2}$

'Directorate of Health Service, Shimla, India, ${ }^{2}$ The Union SEA Office, Tobacco Control \& NCD, New Delhi, India. E-mail: ravindermph@gmail.com

Background: Electronic Nicotine Delivery System (E-cigarettes) are being advertised as novel products in all media and platforms across the globe. Despite the fact that these products are still not evaluated for safety and effectiveness by any regulatory body in most countries including India; their advertisements claiming the e-cigarettes to be health friendly is on rampant especially in the internet media. To explore the availability of e-cigarette brands for Indian existing and potential consumers and to understand their distribution network and marketing tactics, the investigator did the internet search.

Methods: Investigator performed the keyword search on Google in May 2014 and November 2016. Brand websites were examined for specifics about each product (flavor and nicotine strength), ingredients, and their claims about the safety of the products and usefulness in smoking cessation. Distributor's network and kiosk selling these products were also searched for.

Results: Total 112 brands of different flavor (12 types) and different nicotine strengths (9 types) of the e-cigarettes were found. In majority brands (95\%), most common ingredients were chemical nicotine, propylene glycol, water and flavours. $10 \%$ websites claimed that their product are useful as smoking cessation devices. Most brands claimed their product to be healthier and safer (90\%), suitable to use in public places (92\%) and an economical option (70\%) than conventional cigarettes. Near half of the websites gave their distribution details in the websites. 12 websites offer free shipping services, 27 websites offers the web chat options for marketing the products.

Conclusions: ENDS (e-cigarette) poses another challenge for tobacco control in India. The claims (especially healthier option and useful for cessation) of the websites marketing these products are questionable and needs further research. Ongoing advertisements on internet are the gross violations of Indian tobacco control legislation, and warrants stronger enforcement from the authorities.

\section{Tob. Induc. Dis. 2018;16(Suppl 1):A790 DOI:10.18332/tid/84436}

\section{2:30-14:00 \\ PS-1067-4 Tobacco products use among secondary school students in Uganda \\ J Kasibante1, C Tumwine1, Secondary School Students 'Gulu University, Gulu, Uganda. E-mail: johnkasi93@gmail.com} Background: To determine the experience, exposure, access and current use tobacco products use among secondary schools in Uganda.

Study question: what is the level of use of tobacco products among secondry school students in Uganda?

Problem statement: Uganda is struggling with tobacco control. The young people are the major target for tobacco companies. Early use of tobacco leads to continued use and thence tobacco related diseases whose cost of treatment outweighs government revenue.

Methods: Cross-sectional study, involving 1470 students between 14-21 years, selected using randomised cluster sampling from 21 schools in three regions of Uganda. Data collected using structured questionnaire google form administered to the responders by trained interviewers, results accessed as EXCEL tables which were transferred to SPSS version 23 for analysis. Univariate analysis was done.

Results: Total of 795 students have been interviewed, 498 (64.1\%) boys, mean age 16.7 years. 134(17\%) experimented with smoking, majority 50 (37.3\%) having tried it before the age of 12 years. $40(5.5 \%)$ were currently using a tobacco product. Yet majority 730 (95.9) were aware of tobacco use harm, still 214 students had been passive smokers in concluded week yet $702(95.4 \%)$ were aware of the harm of passive smoking. 552 (77.4\%) were in favour of banning the sell and use of tobacco products in public places, yet of the 65 students who tempted to purchase a tobacco product, 43 of them were successful because irrespective of their low age, this did not limit them from buying it. 716(93.7\%) had been taught about the dangers of tobacco smoking.

Conclusions: Tobacco exposure is high among students yet they know the harm. Proper policies and measures should be put in place to limit this. This study was able to show the burden of tobacco use among students and how measures like education are not proving effective in its control.

Tob. Induc. Dis. 2018;16(Suppl 1):A791

DOI:10.18332/tid/84459

12:30-14:00

PS-1068-4 Patterns of tobacco use and quit attempts in rural India

PD Rent ${ }^{1}$, J Purushotham ${ }^{1}$

${ }^{1}$ Nitte University, Public Health, Mangalore, India. E-mail: priyankadsouza@nitte.edu.in

Background: India is the second largest consumer of tobacco and accounts for about $1 / 6$ th of the global deaths due to tobacco. The objectives of this study were to determine the patterns of tobacco use and the association of tobacco use and quit attempts with socio-demographic variables in a rural community.

Methods: The study was carried out among 580 men and women aged 15 years and above who visited rural health care centres in two districts of Karnataka, India during July-August 2015 and 
reported ever using tobacco. Primary data was collected using a semi-structured interview schedule, administered by a trained medical professional after taking informed consent. Univariate and bivariate analysis, and multiple logistic regressions were carried out using SPSS.

Results: Majority of our respondents (73.4\%) were Current daily users of Tobacco. The most common form of tobacco used in both genders was smokeless tobacco but a significantly higher proportion of women (97\%) used smokeless tobacco compared to men $(45 \%)$. The most popular tobacco product among both genders was betel quid with tobacco. Age of initiation of tobacco use was highest in the 20-34 age groups for both genders. About $29 \%$ of the respondents reported attempting to quit tobacco while $62.4 \%$ said they were not interested in quitting tobacco.

Conclusions: Most Public Service announcements and media campaigns in India are centred around the concept of 'Smoking Kills' and only target men but as seen from our study the burden of smokeless tobacco in the community is higher than smoking tobacco, more so among women. The low proportion of people wanting to quit tobacco indicates that people may still be unaware about the ill-effects of tobacco. For tobacco prevention and control highlighting the ill-effects of smoking as well as smokeless tobacco among both genders and providing cessation support are advised.

Tob. Induc. Dis. 2018;16(Suppl 1):A792

DOI:10.18332/tid/84469

\section{2:30-14:00}

PS-1069-4 Tobacco use among young Qatari population: profile, prevalence and predictors

\section{Dogra ${ }^{1,2}$}

${ }^{1}$ Formely at Ministry of Public Health, Health Information and Intelligence Section, Doha, Qatar, ${ }^{2}$ Save the Children, Knowledge Management, Zirakpur, India.E-mail:vani1825@gmail.com Background: Increasing tobacco consumption among young and productive population is a serious public health concern worldwide. Our study reports the profile, prevalence, and predictors of tobaccouse among young population (Age, 15-35 years) of Qatar.

Methods: We used Qatar's Global Adult Tobacco Survey (GYTS) 2013 data set. Tobacco users were defined as people reporting current use of smoking and smokeless tobacco daily or less than daily. Logistic regression models were used to establish the association between tobacco use and other predictor variables such as age, gender, place of residence, education, employment status and nationality. Ministry of Public Health, Doha approved the study protocol.

Results: We found a sample of 4291 people aged 15-35 years. We found a sample of 493 tobacco users. Prevalence of tobacco use among young population (15-35 years) of Qatar is $11.5 \%$ (10.8\% smokers; $0.7 \%$ smokeless users). Among tobacco users, $463(96.7 \%)$ were smokers and few 30 (6.2\%) were smokeless tobacco user. Very few were dual users 14 (2.9\%). Majority of the tobacco users (68\%) had tried to quit the tobacco consumption in past six months. Tobacco use was significantly $(\mathrm{p}<0.000)$ different among males (10\%) compared to females (1.1\%) The likelihood of tobacco use was significantly associated with age (adjusted OR 2.9 95\%CI 1.8-4.2, education (adjusted OR 6.1 95\%CI 3.4-9.7), gender (adjusted OR 13.6 95\%CI 10.1-18.3), and employment (adjusted OR 8.1 95\%CI 5.3-12.5).

Conclusions: Preventing tobacco use among young population is one of the important elements of anti-tobacco campaigns, since it is easier to prevent younger people using tobacco than to break the habit of dependency. Government of Qatar should develop and implement tobacco quitting strategies for the young and productive population.

Tob. Induc. Dis. 2018;16(Suppl 1):A793

DOI: $10.18332 /$ tid/84470

$12: 30-14: 00$

PS-1070-4 The comparative analysis of Global Youth Tobacco Survey 2003, 2008, 2014 in the Kyrgyz Republic

C Bekbasarova ${ }^{1}$, A Shergalieva $^{2}$, M Kasimova $^{2}$, M Beisheeva $^{3}$, S Bekbasarov ${ }^{3}$, N Novoselova ${ }^{2}$, N Aidarova ${ }^{2}$

${ }^{1}$ Ministry of Health of the Kyrgyz Republic, Bishkek, Kyrgyz Republic, ${ }^{2}$ Republican Centre for Health Promotion, Bishkek, Kyrgyz Republic, ${ }^{3}$ Public Health Protection Foundation, Bishkek, Kyrgyz Republic. E-mail: chbekbasarova@gmail.com

Background: The GYTS, a component of the Global Tobacco Surveillance System, is a global standard for systematically monitoring youth tobacco use (smoking and smokeless) and tracking key tobacco control indicators. GYTS is a nationally representative school-based survey among students aged 13 to 15 years. GYTS-Kyrgyzstan was conducted in 2003, 2008 and 2014 by support WHO and CDC USA.

Methods: GYTS uses a global standardized methodology that includes a two-stage sample design with schools selected. In all GYTS of Kyrgyzstan was used the standard core questionnaire with a set of optional questions that permited adaptation to meet the needs of the country on tobacco use and key tobacco control indicators. In 2003, 4270 students participated in GYTS of Kyrgyzstan, the response rate was $98.8 \%$; in 2008 - response rate $-93.2 \%$ (4038 students) and in 2014 the response rate was $85.9 \%$, total - 4273 students participated.

Results: The comparative analysis of results of GYTS in 2003, 2008 and 2014 shows, that cigarette smoking level among youthboys aged 13-15 years decreased form 2 times from 2003 to 2014, among girls it decreased from $4 \%$ in 2003 to $1 \%$ in 2014 . However, nasvay (smokeless tobacco) using among boys increased from $3,2 \%$ in 2003 to $7,6 \%$ in 2014 and among girls from $1 \%$ in 2003 to $3 \%$ in 2014 or more than 3 times. If $65 \%$ of students were exposed to tobacco smoke at home in 2003, in 2014 this level decreased to $13,5 \% .73 .6 \%$ of students definitely thought other people's tobacco smoking is harmful to them. $79.9 \%$ of students favor banning smoking inside enclosed public places.

Conclusions: The results of GYTS 2003, 2008 and 2014 in Kyrgyzstan demonstrated that tobacco control policy in Kyrgyzstan should be changed and nasvay control should be strong same as cigarettes control.It will assist in effective implementation of FCTC in Kyrgyzstan.

Tob. Induc. Dis. 2018;16(Suppl 1):A794

DOI: $10.18332 /$ tid/84478

12:30-14:00

PS-1071-4 Tobacco use and interest in quitting among patients hospitalized in Mumbai, India

G Kruse ${ }^{1,2}$, Thawal $^{3}, \mathrm{H} \mathrm{Gupte}^{3}$, L Chaudhuri $^{3}$, S Pradhan ${ }^{4}, \mathrm{~N}$ Rigotti ${ }^{1,2}$

${ }^{1}$ Massachusetts General Hospital, Medicine, Boston, MA, United States of America, ${ }^{2}$ Harvard Medical School, Boston, MA, United States of America, ${ }^{3}$ Narotam Sekhsaria Foundation, Mumbai, India, ${ }^{4}$ Prince Aly Khan Hospital, Mumbai, India. E-mail: gkruse@ mgh.harvard.edu

Background: India has an estimated one million tobaccoattributable deaths annually. Tobacco cessation treatment is effective among hospitalized patients, but little is known about tobacco use among hospitalized patients in India. This study aims 
to measure tobacco use, interest in quitting, and cessation after discharge at a Mumbai hospital.

Methods: Cross-sectional survey of patients 15 and older at a Mumbai hospital. Of 7,889 patients admitted from 11/201510/2016, 5,038 were eligible, 2,894 were approached and 2,764 participated. Characteristics of smokers and smokeless tobacco (SLT) users were compared and self-reported cessation was assessed 6 months post-discharge.

Results: Overall, 6.5\% ( $\mathrm{N}=179)$ of subjects were current smokers and $10.2 \%(\mathrm{~N}=284)$ were current SLT users $(\mathrm{N}=37$ were dual users). Most smokers (81.7\%) and SLT users (94.3\%) reported daily use. The mean number of tobacco products used was 2 (standard deviation=1). Compared to smokers, SLT users were more often older (52 versus 48 years, $\mathrm{p}=0.002)$, female $(37 \%$ versus $1 \%, \mathrm{p}<0.001)$, illiterate $(14 \%$ versus $6 \%, \mathrm{p}=0.01)$, and less often employed ( $52 \%$ vs $75 \%, \mathrm{p}<0.001)$. SLT users less often reported a past year quit attempt (39\% versus $54 \%, \mathrm{p}=0.004)$ or a plan to stay quit after discharge ( $42 \%$ versus $54 \%, \mathrm{p}=0.04)$. There were no differences in importance of quitting, confidence, receipt of advice to quit, or treatment use. Just 3\% reported evidencebased treatment use. Compared to smokers, SLT users less often agreed that tobacco has harmed them ( $57 \%$ versus $70 \%, \mathrm{p}=0.01)$. Overall, $28 \%$ of tobacco users (25\% of smokers and 29\% of SLT users) reported having quit at 6 months post-discharge.

Conclusions: Roughly half of hospitalized tobacco users planned to stay quit after discharge and $28 \%$ reportedly succeeded. Interventions to connect hospitalized tobacco users with treatment are clearly needed. SLT users may be especially challenging to engage because SLT is viewed as less harmful.

Tob. Induc. Dis. 2018;16(Suppl 1):A795

DOI:10.18332/tid/84490

\section{2:30-14:00}

PS-1072-4 Prevalence and correlates of tobacco smoking, smokeless tobacco and passive smoking in Georgian adults: results of non-communicable disease risk factors STEPS survey Georgia 2016 M Maglakelidze ${ }^{1}$, N Maglakelidze², L Sturua², A Gamkrelidze ${ }^{2}$ ${ }^{1}$ Georgian Respiratory Association, Tbilisi, Georgia, ${ }^{2}$ National Center for Disease Control and Public Health, Tbilisi, Georgia. E-mail:maka_mag@hotmail.com

Background: Despite the growing problem of tobacco epidemic in Easter Europe and Former Soviet Union, there is a lack of national based scientific data that could serve as an evidence for appropriate policy level interventions.

Methods: A population-based national cross-sectional survey was conducted using theWHOSTEPwise approach to non-communicable disease risk factor surveillance in aged 18-69 age group. A multi-stage cluster sample design and weighting were used to produce a national representative data for that age range. Total number of participants who took part in the survey was 5554 .

Results: The prevalence of tobacco use, both smoking and smokeless together was $31.1 \%, 57.1 \%$ in men and $7.1 \%$ in women respectively. $29.9 \%$ of current tobacco users reported smoking cigarettes, among them $55.3 \%$ men and $6.6 \%$ women respectively. Biggest majority of current cigarette smokers were also defined as daily smokers, $51 \%$ men and $6.2 \%$ women respectively. Mean age of starting smoking was reported 17.8 in men and 22.4 in women. Smokeless tobacco was used only by $0.3 \%$ of adult Georgian population, among them $0.6 \%$ men and $0.1 \%$ women respectively. $43 \%$ of all participants were exposed to passive smoking at home, about $16 \%$ at the workplace.
Conclusions: Tobacco use and second hand exposure to tobacco smoke was a significant public health concern in Georgia. Cigarette smoking was identified as almost exclusive form of tobacco use in Georgia. This study provides the data which can be used by policy makers to shape evidence-based policies and targeted interventions.

Tob. Induc. Dis. 2018;16(Suppl 1):A796

DOI: $10.18332 /$ tid/84492

\section{2:30-14:00}

PS-1073-4 Use of a technophilia index to address e-cigarette trial and use in Mexican youth

I Barrientos-Gutierrez', P Lozano², P Morello ${ }^{3}$, R Mejia ${ }^{3}$, E ArilloSantillan' ${ }^{1}$ J Thrasher ${ }^{2}$

${ }^{1}$ National Institute of Public Health, Tobacco Control, Mexico City, Mexico, ${ }^{2}$ University of South Carolina, Columbia, SC, United States of America, ${ }^{3}$ Centro de Estudios de Estado y Sociedad (CEDES), Buenos Aires, Argentina. E-mail: inti.barrientos@ insp.mx

Background: E-cigarettes have had a strong growth in Mexico, especially among adolescents. These products are advertised as technology advanced products and their increased use may reflect a tendency to adopt new technologies. The concept of "technophilia" draws attention to the "pleasure, affection, and emotional qualities" that accompanies new technology. The aim of this study is to explore and validate a technophilia index among a cohort of Mexican youth, and to evaluate the association with e-cigarette trial and use.

Methods: We analyzed cross-sectional data from 8747 students from the three largest cities in Mexico. To develop the technophilia index we evaluated eight questions related to internet use. To validate this scale, we used an explanatory factor analysis on the overall item set. We used linear and multinomial logistic regressions to evaluate the association between the technophilia index and e-cigarette trial/use, and having used e-cigarette as the first tobacco product.

Results: Study results showed a dose-response association between technophilia index and e-cigarette trial $(\mathrm{OR}=1.48,95 \%$ CI 1.26-1.75, Quartile 4 vs. Quartile 1). Moreover, Mexican adolescents with high levels of technophilia were more likely to have used e-cigarettes as their first tobacco product $(\mathrm{OR}=1.78$, 95\% CI 1.31-2.42, Quartile 4 vs. Quartile 1).

Conclusions: Results from this study suggest that e-cigarette trial is characterized by greater technophilia among youth. This study highlights the need for research in this area, so that effective strategies may be developed to address these risk factors.

Tob. Induc. Dis. 2018;16(Suppl 1):A797

DOI:10.18332/tid/84591

12:30-14:00

PS-1074-4 Nicotine dependence and readiness to quit tobacco among past and present alcohol abusers in Chennai, India - a comparative study AK Sundaragopal', PE Chaly ${ }^{1}$, S Sivasamy ${ }^{1}$

${ }^{1}$ Meenakshi Ammal Dental College and Hospital, Chennai, India. E-mail: ak.s29@live.com

Background: Tobacco and alcohol are among the top causes of preventable deaths in the world, in most cases both these substances are used together. This study aims to compare the differences in nicotine dependence and the readiness to quit among past and present alcohol abusers.

Methods: Snowball sampling methodology was used to recruit past alcohol abusers from an Alcohol Anonymous group in 
Chennai, of the 25 people who were contacted over phone only 10 were ready to participate in the study and they were all grouped as past alcohol abusers, as they absented themselves from consuming alcohol for more 60 days, And to recruit present alcohol abusers two Alcoholic bars was selected and the patrons were questioned before they could consume alcohol, of the 24 people contacted 20 were ready to participate, for the subjects who participated in both the groups the levels of nicotine dependence were recorded using Fagestrom test for nicotine dependence and their readiness to quit tobacco was recorded using readiness to quit tobacco ladder which is available online, both these were recorded by the interviewer.

Results: Group 1 are past alcohol abusers, of which 3 had low dependence, 3 had moderate dependence and 4 had high dependence and only one of them was ready to quit tobacco within 30 days, in group 2 which consists present alcohol abusers, 8 had low dependence, 10 had moderate dependence and 2 had high dependence, of which 2 people have actually absented themselves from using tobacco for 10 days but worry about slipping back, 4 of them have strong determination not to quit tobacco abuse at all. Conclusions: From the pilot study it was found that 4 past alcohol abusers were highy nicotine dependent,and many of the present alcohol abusers were in pre-contemplation stage compared to past alcohol abusers.

Tob. Induc. Dis. 2018;16(Suppl 1):A798

DOI:10.18332/tid/84594

\section{2:30-14:00}

PS-1075-4 The prevalence of oral conditions and tobacco use among dental patients at princess Marina Mental Clinic, Botswana

B Mbongwe ${ }^{1}$, P Mphahudi ${ }^{1}$, R Tapera ${ }^{1}$, P Erick $^{1}$

'University of Botswana, Department of Environmental Health, Gaborone, Botswana.E-mail: bontle.mbongwe@gmail.com

Background: Several studies have associated smoking with oral conditions such as tooth staining and bad breath, periodontal diseases, impaired healing of wounds, precancer and oral cancer. These effects are often visible and potentially reversible after cessation of smoking. Dentists are frequently in contact with the general population and therefore provide an opportunity for early intervention in smoking cessation counseling compared to other health professionals. This study profiled patients with oral conditions, to assess their knowledge on oral conditions and tobacco use.

Methods: A cross-sectional survey was carried out at Princess Marina Dental Clinic in Gaborone. The study population included all dental patients visiting the clinic. Only dental patients from the age of 18 years old and above participated in the study. Patients were interviewed using a structured questionnaire and investigated clinically for the presence of oral conditions by a qualified dentist.

Results: The prevalence of Tobacco use was $21 \%$ among the patients. The prevalence was significantly higher among patients aged 25 and $36(\mathrm{p}<0.05)$. Dental carries was the most common condition affecting $45 \%$ of patients followed by periodontal disease affecting $21 \%$ of patients. The age group $25-36$ years was the most affected by oral conditions $(\mathrm{p}<0.05)$. Current smokers had a higher prevalence of tooth staining (26\%) compared with never smokers (11\%). Periodontal disease was prevalent in $32 \%$ of tobacco users and $20 \%$ of past tobacco users. Overall, $25 \%(n=68)$ of patients were not aware of the relationship between smoking and oral health.
Conclusions: Tobacco use prevalence is high among dental patients in this study. The lack of awareness on tobacco use and effects on oral health is also concerning. On account of their frequent contact with the general population, dentists could provide a window of opportunity for early detection of oral conditions associated with smoking, provide patient education, counseling and smoking cessation advice.

Tob. Induc. Dis. 2018;16(Suppl 1):A799

DOI:10.18332/tid/84605

$12: 30-14: 00$

PS-1076-4 E-cigarettes use behaviour, perceptions and barriers among Indian adults: pilot qualitative research study

V Mohanty ${ }^{1}$

'Maulana Azad Institute of Dental Sciences, Public Health Dentistry, New Delhi, India. E-mail: vikrantmohanty@gmail.com

Background: There has been an exponential increase in the prevalence of e-cigarette use worldwide, particularly among youth. There is no current evidence of ENDS use in India, but the sale online and in retail outlets has grown due to lack of regulations and experimentation with an option to quit or reduce conventional smoking. There is currently no qualitative study carried out among Indian adult E-cigarettes users to understand their behaviour, perceptions and barriers to use. Such information is needed to develop regulatory, preventive as well as cessation strategies.

Methods: Forty Two e-cigarette users in Delhi, India voluntarily participated in the semi-structured individual in-depth interview which assessed behaviour, perceptions and barriers to use of e-cigarettes. Information was also obtained on sociodemographic aspects, access to cessation services, reasons for initiating and continuing use along with barriers of e-cigarettes use in India. Verbatim transcripts were analyzed after being captured by voice recording and themes were created.

Results: Participants reported several aspects of e-cigarette experimentation and curiosity as compared to approved cessation treatment options. They expressed that flavour and taste, safer alternative, moderation in nicotine content and satisfaction were factors affecting their continued use. Participants were divided on the issue of cost and availability. Most of them were not aware of any regulations currently on the e-cigarettes in India. E-cigarettes complemented standard cigarettes in the sense that the users satisfied their desire to smoke in areas where it was not permitted. Conclusions: Understanding e-cigarette users perspectives provides us an insight into their current practices along with understanding the barriers to e-cigarette use. Regulatory and policy initiatives will need to counter the marketing strategies of e-cigarette companies with a prospect of improving coordinated cessation services, increasing awareness and improving health outcomes.

Tob. Induc. Dis. 2018;16(Suppl 1):A800

DOI: $10.18332 /$ tid $/ 84620$

\section{2:30-14:00}

PS-1077-4 Tobacco consumption patterns across gender and socioeconomic groups in Cameroon Z Njoumemi'

'The University of Yaoundé I, Faculty of Medicine \& HEREG, Public Health, Yaoundé, Cameroon. E-mail: njoumemiz@gmail. com

Background: Cameroon has substantial prevalence of both 
smoking and smokeless tobacco consumption, as well as of relative weak dual consumption. Cameroon's tobacco consumption appears to have geographical both urban and rural influences. The gender also seems to play an important role in influencing patterns of tobacco consumption nationwide. This study analyzes the determinants of smoking and smokeless tobacco consumption in Cameroon.

Methods: This study uses the 2011 Cameroon Demographic and Health Survey to analyze both smoking tobacco and smokeless tobacco consumption, stratified by gender and residence (urban/rural). Multivariate logistic regression models were used to evaluate the relationship between gender-based tobacco consumption and residence (urban/rural), age, marital status, education, socioeconomic quintile, and ethnic group.

Results: The analysis indicates that both men and women consume two distinctly different tobacco products. Tobacco is almost exclusively consumed by large proportions of men in both urban (12,23\% for smoking tobacco and 1,52\% for smokeless tobacco) and rural (19,15\% for smoking tobacco and 3,94\% for smokeless tobacco). The prevalence of tobacco consumption is less than $1 \%$ for women in both urban and rural areas for both smoking and smokeless tobacco. Conversely, while tobacco consumption does not appear to be related to socioeconomic status for women, it is likely that both the age and economic quintile influence the tobacco consumption of men in both rural and urban areas of the country. These tobacco consumption patterns are a reflection of the different production and market distribution for smokeless tobacco and smoking tobacco in Cameroon.

Conclusions: Distinct geographical by urban and rural, gender and socioeconomic distribution of tobacco consumption patterns are explored in order to begin the understanding of the tobacco epidemic environment in Cameroon.

Tob. Induc. Dis. 2018;16(Suppl 1):A801

DOI:10.18332/tid/84634

\section{$12: 30-14: 00$}

PS-1078-4 Demographic and socioeconomic determinants of smoking in the Biyem-Assi health district, Yaoundé, Cameroon

Z Njoumemi ${ }^{1,2}$

${ }^{1}$ Health Economics Research and Evaluation for Development Results Group (HEREG), Research on Tobacco Control, Yaoundé, Cameroon, ${ }^{2}$ University of Yaoundé I, Faculty of Medicine, Public Health, Yaoundé, Cameroon.E-mail:njoumemiz@gmail.com Background: There is a rise in the trend of tobacco smoking in Cameroon. The objective of the study was to investigate the demographic and socioeconomic determinants of smoking behaviour in the population of Biyem-Assi health district thereby estimating the prevalence of smoking in the district.

Methods: A descriptive cross sectional study was conducted on a sample size of 510 people. Data were collected using structured questionnaires on the demographic and socioeconomic characteristics of smoking in the Biyem-Assi health district. A multiple logistic regression analysis was conducted at $95 \%$ $(p<0.05)$ confidence interval to identify the characteristics of smoking.

Results: The age range for the sampled population was 18-75. The study showed smoking prevalence of $38.75 \%$. From the multiple logistic regressions, men were more likely to smoke than women (AOR: 6.02; 95\% CI: 2.24-16.17). The respondents less than or equal to 35 years were $55 \%$ less likely to smoke than those above 35 (AOR: 0.45; 95\% CI: 0.22-0.93), while those working with the informal sector were more likely to engage in smoking than those of the formal sector (AOR: 2.59; 95\% CI: 1.25-5.38). The respondents who had friends or relatives who smoke were more likely to smoke (AOR: 24.56; 95\% CI: 8.92-67.63) and those who believed public campaigns could prevent smoking were $75 \%$ less likely to smoke (AOR: 0.26; 95\% CI: 0.12-.053). The respondents who were targeted by the regulation of smoking-free public places were $83 \%$ less likely to smoke $(0.17 ; 95 \% \mathrm{CI}$ : 0.07-.039).

Conclusions: The prevalence of smoking in the Biyem-Assi health district is high. Gender, age, occupation and having friends and relatives who smoke are highly associated with smoking behaviour.

Tob. Induc. Dis. 2018;16(Suppl 1):A802

DOI: $10.18332 /$ tid/84643

12:30-14:00

PS-1079-4 Pattern of smoking and nicotine dependence in Lagos State Nigeria

0 Uti $^{1}, 0$ Sofola ${ }^{1}$, W Adeyemo ${ }^{2}, 0$ Ogundana ${ }^{3}$

${ }^{1}$ College of Medicine, University of Lagos, Dept of Preventive Dentistry, Lagos, Nigeria, ${ }^{2}$ College of Medicine, University of Lagos, Dept of Oral and Maxillofacial Surgery, Lagos, Nigeria, ${ }^{3}$ College of Medicine, University of Lagos, Dept of Oral Pathology, Lagos, Nigeria. E-mail: omolaraza@yahoo.com

Background: Tobacco use has been a recognized risk factor for a many diseases including Oral Cancer. Social disparities have been found to exist in smoking patterns in most populations but there has not been a concerted effort to quantify or describe the pattern of tobacco use and Nicotine dependence from tobacco use at the population level in Nigeria.

Methods: This was part of a larger population based cross-sectional study in Lagos State Nigeria in which the subjects were selected from the three Senatorial districts of Lagos state in a multistage sampling technique. Data was collected with a structured selfadministered or interviewer administered questionnaire, an oral examination and validation of tobacco use with Bedfont Pico smokerlyzer. Fagerstrom test for Nicotine dependence was used to determine level of dependence.

Results: A total of 2429 subjects were interviewed and examined. They were aged between 10 and 86 years with a mean age of 34 (sd 14.29). There were $58.4 \%$ males and $42.6 \%$ females. Prevalence of ever smoked was $28.55 \%$ while prevalence of current smoking was $9.2 \%$ of total and $32.25 \%$ of ever smoked. Of the current smokers, $57 \%$ were males and $43 \%$ were females. Nearly half of smokers (49\%) commenced the habit by age 18years. Daily smoking was reported by $73.6 \%$ while $62.2 \%$ smoked 10 sticks or less per day. Public places were the most common sites (28.1\%) for smoking. Most (78\%) smokers purchased cigarettes as individual sticks. About half (49.7\%) of the smokers showed low levels of Nicotine dependence while $75 \%$ desired to quit smoking.

Conclusions: The prevalence is higher than national average even though most smokers are light smokers and have low dependence. Health education and strict implementation of anti-smoking policies of the Federal Government of Nigeria is recommended.

Tob. Induc. Dis. 2018;16(Suppl 1):A803 DOI:10.18332/tid/84644

12:30-14:00

PS-1080-4 Global youth tobacco survey in Ecuador 2016-2017

D Ceron ${ }^{1}$, P Analuisa ${ }^{2}$, MD Valdivieso ${ }^{2}$, MJ Mendieta ${ }^{2}$

${ }^{1} \mathrm{MoH}$ Ecuador, Health Promotion, Quito, Ecuador, ${ }^{2} \mathrm{MoH}$ Ecuador, Quito,Ecuador.E-mail: dianacerondiaz@outlook.com 
Background: Ecuador has developed several national surveys in order to monitor tobacco consumption and tobacco control measures. Nonetheless, this country needed to update this information as well as to obtain data which can be comparable at regional and international levels. Therefore, the Ministry of Health, with support from the Pan American Health Organization, has undertaken the Global Youth Tobacco Survey (GYTS) in 2016, studying second hand smoke exposure, TAPS, knowledge regarding effects of tobacco consumption.

Methods: Ecuador implemented same design and methodology established by GYTS, which consists on a uniform basic questionnaire, applied to 13 - 15 years old students. Specifically, this country decided to include questions related to tobacco use, access and availability, cessation, among others. Questionnaires were applied anonymously among students from private or public schools in Quito, Guayaquil and at a National Level, who were chosen randomly. GYTS was previously conducted in 2001 and 2007 in Quito, Guayaquil and Zamora.

Results: Main results included: ever cigarette consumption reduced in Quito from $58.5 \%$ in 2001 to $56 \%$ in 2007 and to $42.2 \%$ in 2016 . In Guayaquil, this percentage did not change from 2001 to 2007; however, it decreased to $17.5 \%$ in 2016 . Additionally, current cigarette consumption in Guayaquil was at $9.1 \%$ in $2001,12.2 \%$ in 2007 and $6 \%$ in 2016. In Quito, this percentage was at $20.5 \%$ in $2001,20.5 \%$ in 2007 and $12.1 \%$ in 2016. The new data collected at national level presented current cigarette consumption in Ecuador among this population group is at $13 \%$ and current e-cigarette consumption is at $10.7 \%$.

Conclusions: Concluding, tobacco consumption among 13 to 15 years old Ecuadorian population has significantly decreased throughout the last 15 years. Finally, it is important to mention that this country ratified WHO FCTC in 2006 and approved a national comprehensive tobacco control law in 2011.

\section{Tob. Induc. Dis. 2018;16(Suppl 1):A804} DOI:10.18332/tid/84650

\section{2:30-14:00}

PS-1081-4 Comparison of concomitant use of water pipe and cigarette to exclusive cigarette smoking Z Hessami ${ }^{1}, \mathrm{M}$ Aryanpur ${ }^{2}, \mathrm{H}_{\text {Sharifi }}{ }^{2}, \mathrm{MR}$ Masjedi ${ }^{2}, \mathrm{H}$ Jamaati ${ }^{2}$ ${ }^{1}$ Shahid Beheshti University of Medical Sciences, Tobacco Prevention and Control Research Center, National Insitute Tuberculosis and Lung Diseases, Tehran, Iran, Islamic Rep. Of, ${ }^{2}$ Shahid Beheshti University of Medical Sciences, Tehran, Iran, Islamic Rep.0f. E-mail: zahra_hessami@yahoo.com

Background: In spite of increasing prevalence of concomitant use of water pipe and cigarette there is no enough information in this field. So the concomitant use of water pipe and cigarette and its comparison to only cigarette smoking were evaluated in this study.

Methods: This cross-sectional study was carried out through stratified multistage sampling in several districts of Tehran. The trained interviewers collected the data about water pipe and cigarette smoking by going to the houses randomly.

Results: The study had 1830 participants among which 243 participants only consumed water pipe, 120 cases only smoked cigarette and 76 cases (4.2\%) were dual users. There was no significant difference in both groups by sex. The mean age of dual users and exclusive cigarette smokers were $33.1 \pm 11.3$ and $46 \pm 16.5$ years respectively $(p \leqslant 0.001)$.

Comparing to cigarette smokers (35.2\%), most of the dual users $(64.7 \%)$ believed that water pipe is not addictive $(p=0.001) .13 .2 \%$ of dual users and $5.3 \%$ of cigarette users believed that water pipe smoking is safer than cigarette smoking $(\mathrm{p}<000)$. While $54.1 \%$ of dual users and $78.5 \%$ of cigarette smokers believed that waterpipe consumption could cause serious diseases.

Conclusions: Younger age and misconception about safety of water-pipe in comparison to cigarette were significantly more in dual users than exclusive cigarette users. It is necessary to promote the knowledge about harms of water-pipe smoking in society especially among youth.

\section{Tob. Induc. Dis. 2018;16(Suppl 1):A805 \\ DOI:10.18332/tid/84678}

\section{2:30-14:00}

PS-1082-4 Mediating and moderating influences of demographic factors in adult tobacco use L Chhakchhuak', L Chhakchhuak ${ }^{2}$

${ }^{1}$ Tripura University, Psychology, Agartala, India, ${ }^{2}$ Government of Mizoram, Directorate of Health Services, Agartala, India. E-mail:afaki210@gmail.com

Background: The popularity of tobacco and the lack of taboo regarding its use is deeply rooted in the cultural practices of the Mizo people. Global Adult Tobacco Survey 2009-2010 shows that Mizoram have the highest percentage of tobacco user in India at $67.2 \%$, here $61.6 \%$ comprises of female tobacco users. Demographic factors have been found to play a major role in tobacco use. In the current study mediators and moderators of demographic factors in tobacco use behavior among male and female Mizo adults were examined.

Methods: 300 participants, 150 male and 150 female adult tobacco users were randomly selected from Aizawl City, the capital of Mizoram with their age ranging between 35-45 years. Demographic factors were framed, recorded and analysed.

Results: Those with low education levels (48.9\%) were more likely to use tobacco, believed less in the negative health effects of tobacco, believed more in the positive psychological benefits of tobacco, had lower value on a healthy lifestyle, and had more friends who used tobacco. Although all of these factors significantly mediated education effects on tobacco use behavior, number of friends who smoke or use other forms of tobacco was the most powerful of these mediators. Compared with men, tobacco use among women was more strongly related to perceived psychological benefits of tobacco use, number of friends who use tobacco, and health beliefs about tobacco.

Conclusions: The study has given us baseline results of the mediators and moderators of demographic factors in tobacco use. For effective interventions targeting vulnerable groups like women, children and people from low education background, interventions should include training, cessation treatments and awareness assimilated through the church, Young Mizo Association, primary schools and health care centers. Focusing on these key areas will be very important in customizing and enhancing tobacco interventions among the present population.

Tob. Induc. Dis. 2018;16(Suppl 1):A806

DOI:10.18332/tid/84682

\section{$12: 30-14: 00$}

PS-1083-4 How is the use of e-cigarettes sustained in the customers with serious health symptoms?

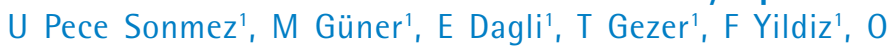
Elbek $^{1}, \mathrm{P} A \mathrm{y}^{2}, \mathrm{M}$ Ceyhan ${ }^{1}$

${ }^{1}$ Health Institute Association, Istanbul, Turkey, ${ }^{2}$ Marmara University, Istanbul, Turkey. E-mail: elifzdagli@gmail.com 
Background: In Turkey there are no legal sales of electronic cigarettes however illegal sales continue through internet and social media forum. The customers of these sites frequently post serious health complaints related to the use of product but do not quit. This study was carried out to understand how the e-cigarette social forum adminstrators respond to the health issues and what they recommend.

Methods: A survey was carried out at a social media forum with more than 60 thousand members. The titles and messages on health complaints and responses were analyzed.

The messages with unauthoritized health recommendations to prevent the customers quitting were evaluated.

Results: Social forum had a section on the complaints of the products and related health problems with 166 headings and 9588 messages. The symptoms were reported 3 weeks to 3 months after the use of e-cigarettes. The most frequent symptoms were related to respiratory system, oral cavity and gums. 2830 of the messages were responded by an anonymous admin(s). Of these responses $48 \%$ recommended the change of ingredients, \% 24 claimed that health complaints were not related with e-cigarette use, $\%$ 13 attributed the symptoms to quitting classical cigarettes, $6 \%$ reassured that the problem will soon disappear and recommended the continuation of e-cigarettes, $7 \%$ proposed herbal medicine, 5 $\%$ actual pharmaceutical drugs, only $5 \%$ to consult to a medical expert.

Conclusions: Members of this internet forum who used a product which is not legally available, they tended not to apply to regular health clinics in case of harmful effects. The forum administrators tried their best to sustain the addiction in spite of very serious complaints.

Control over the websites and social forums must be part of the tobacco control enforcement strategies.

Tob. Induc. Dis. 2018;16(Suppl 1):A807

DOI:10.18332/tid/84684

\section{2:30-14:00}

PS-1084-4 Comparison of long-term exposure of C57BL/6 female mice's lungs to three different types of cigarette smoke available in Brazil

P Corrêa' ${ }^{1}, 0$ Zeidan², F Bezerra ${ }^{3}$, V Capelozzi ${ }^{4}$

${ }^{1}$ Universidade Federal de Ouro Preto - UFOP, DECPA, Ouro Preto, MG, Brazil, ${ }^{2}$ Reutlingen University, Reutlingen, Germany, ${ }^{3}$ UFOP, DECBI, Ouro Preto, MG, Brazil, ${ }^{4}$ USP, Pathology Department, Sao Paulo, SP, Brazil. E-mail: paulocontroletabaco@gmail.com Background: Chronic obstructive pulmonary disease (COPD) is caused primarily by the inhalation of cigarette smoke, a very complex mixture of some $9000+$ constituents. The continuous inhalation of cigarette smoke is known to trigger impairment in pulmonary elasticity and airway-parenchymal remodeling.

Some studies have suggested that women are more susceptible to the effects of tobacco smoke than men, leading to more severe disease for the equivalent quantity of cigarettes consumed.

As there are different types of cigarette commonly smoked in Brazil and there is a great need of studies in female subjects, we designed a long-term exposure of C57BL/6 female mice's lungs to three different types of cigarettes available in Brazil (two filtered and one unfiltered).

Methods: Exposure experiments were conducted for 60 sequential days, each group ( $n=10$ mice) exposed to 12 cigarette a day divided into three sessions.

The three different types of cigarette were: commercial cigarette "Marlboro"(MG), Fumo de Corda cigarette without filter brand
"Souza Paiol"(SPG), and Fumo de Corda cigarette with filter brand "3 Lobos" (LG), while the forth group was the control group (CG). For histological analysis, were selected all animals in each group.

Results: Lung tissue samples from animals exposed to SPG and MG showed significant increase septal interstitium thickness when compared to LG and CG.

Collagen fibers were significantly increased in septal interstitium of SPG animals compared to CG. Interestingly, a significant decrease in collagen fibers of septal interstitium was found in MG group coincident with increased hyperdistension of the alveoli when compared to compared to CG group. In bronchiole from animals exposed to MG tobacco, a significant increase of mucinous PAS+ positive cells were found when compared to $\mathrm{CG}$, SPG and LG.

Conclusions: This lung pathology specimens study have showed differences in the effects produced by long-term exposure to different kind of cigarettes in female mice.

Tob. Induc. Dis. 2018;16(Suppl 1):A808

DOI:10.18332/tid/84686

\section{2:45-14:00}

EP-249-4 Prevalence and correlates of waterpipe use among adolescents in 60 countries

Y Yang ${ }^{1}$, M Jawad ${ }^{1}$, F Filippidis ${ }^{1}$

${ }^{1}$ Imperial College London, Public Health Policy Evaluation Unit, School of Public Health, London, United Kingdom. E-mail: f.filippidis@imperial.ac.uk

Background: The worldwide popularity of water pipe usage has sharply increased in recent years, especially among young adults and youths.

Methods: In this study, we conducted a secondary analysis of the school-based Global Youth Tobacco Survey (GYTS). GYTS included questions about waterpipe tobacco use in a total of 60 countries between 2007 and 2015. We analysed data of 182,154 students aged 13 to 15 years from these 60 countries (sample sizes ranging from 1,221 to 8,789 ), to estimate the prevalence of ever and current (within the last 30 days) waterpipe smoking. We also used logistic regression to assess the association of sex and amount of weekly allowance money (proxy for family finances) with waterpipe use.

Results: The prevalence of current waterpipe use among youths varied significantly between countries, from $2.7 \%$ in Gabon to $35.2 \%$ in West Bank and 37.0\% in Lebanon. The percentage of youths who have ever tried waterpipe was highest in Lebanon (55.1\%). In 39 out of 54 countries that had data on current waterpipe use, males were significantly more likely to smoke waterpipe than females, with adjusted Odds Ratios (AOR) ranging from 1.29 to 9.51 . In contrast, female students were significantly more likely to use waterpipe in Djibouti and Maldives. Students receiving the largest amount of weekly allowance were more likely to be current users of waterpipe in the majority, but not all, of the countries assessed, compared to those in the category of the lowest amount. However, AOR varied widely, ranging from 0.58 to 12.55 .

Conclusions: Waterpipe use among youths is high in many regions, in particular within Eastern Mediterranean and Eastern European countries. Sex and socioeconomic equalities were identified in most countries. Systematic surveillance of waterpipe use would allow closer monitoring of the trends in use over time.

Tob. Induc. Dis. 2018;16(Suppl 1):A809

DOI:10.18332/tid/84497 


\section{$12: 45-14: 00$}

EP-252-4 Evaluation of concurrent use of tobacco products (water pipe and cigarettes) and associated factors in adults in Tehran

Z Hessami', GR Heydari ${ }^{2}$, H Sharifi $^{3}$, MR Masjedi $^{3}$, S Sobhani ${ }^{4}$ ${ }^{1}$ Shahid Beheshti University of Medical Sciences, Tobacco Prevention and Control Research Center, National Insitute Tuberculosis and Lung Diseases, Tehran, Iran, Islamic Rep. Of, ${ }^{2}$ Shahid Beheshti University of Medical Sciences, Tobacco Prevention and Control Research Center, National Research Institute Tuberculosis and Lung Diseases, Tehran, Iran, Islamic Rep. Of, ${ }^{3}$ Shahid Beheshti University of Medical Sciences, Tehran, Iran, Islamic Rep. Of, ${ }^{4}$ Iranian Anti Tobacco Association, Tehran, Iran, Islamic Rep. Of. E-mail: zahra_hessami@yahoo.com

Background: Dual use of tobacco products such as cigarettes and water pipe may be associated with increased risk of nicotine dependence and smoking-related complications. Accurate statistics are not available in this regard in Iran. Thus, this study sought to assess the prevalence of dual cigarette/water pipe smoking and its related factors in Iran.

Methods: This cross-sectional study was conducted on Tehran residents over 15 years of age selected via cluster, multi-stage randomized sampling from different geographical districts of Tehran in November and December 2014. The data were collected using the water pipe section of the Global Adult Tobacco Survey (GATS) questionnaire.

Results: A total of 1,830 individuals participated in this study; of which, 243 (13.3\%) were exclusive water pipe smokers, 76 (4.2\%) were dual smokers of cigarettes and water pipe and $120(6.6 \%)$ were exclusive cigarette smokers. Of dual smokers, $86.8 \%$ were males and $13.2 \%$ were females $(\mathrm{P}<0.001)$. The mean age was $28.01 \pm 8.7$ yearsin exclusive water pipe smokers and $33.1 \pm 1.1$ yearsin dual smokers $(\mathrm{P}<0.001)$. Male sex (adj. OR: 3.8$)$ and older age (adj. OR: 1.06) increased the odds of dual smoking.

Conclusions: The prevalence of dual smoking and exclusive water pipe smoking was $4.2 \%$ and $13.3 \%$, respectively. Exclusive cigarette smoking had a prevalence of $6.1 \%$. The frequency of dual smoking of cigarettes and water pipe was considerably high. Dual smokers had a higher mean age than exclusive water pipe smokers and they were mostly males. The mean age at initiation of water pipe smoking in dual smokers was lower than the mean age at the onset of cigarette smoking. In other words, dual smokers started water pipe smoking sooner than cigarette smoking. Future studies with different methodologies are required to further scrutinize the relationship of water pipe and cigarette smoking.

Tob. Induc. Dis. 2018;16(Suppl 1):A810

DOI:10.18332/tid/84675

12:45-14:00

EP-254-4 Water-pipe tobacco (shisha) use among undergraduate health professional students College of Health Sciences, Nairobi University Kenya, 2014

A Karagu', D Kiptui², G Gatheca ${ }^{3}$

${ }^{1}$ National Cancer Institute of Kenya, Nairobi, Kenya, ${ }^{2}$ Ministry of Health, Division of Non-Communicable Diseases, Nairobi, Kenya, ${ }^{3}$ Ministry of Health, Division of Non Communicable Diseases, Nairobi, Kenya. E-mail: karagumaina@gmail.com

Background: Tobacco use contributes to more than 6 million annual deaths globally. A growing body of knowledge shows a rise in the use of water pipe tobacco (shisha), spreading from the traditional Eastern Mediterranean and Northern African regions to other parts of the world. We assessed the prevalence of and factors associated with shisha smoking among undergraduate health profession students in the College of Health Sciences, University of Nairobi, Kenya.

Methods: We conducted a cross-sectional study among final year undergraduate students enrolled in the medicine, nursing, pharmacy and dentistry programmes in August 2014. Students completed a questionnaire adapted from the Global Tobacco Surveillance System. Information on ever and current use of shisha, social demographic variables, alcohol and cigarette use was obtained. Data was analyzed using Epi Info 3.5.1.

Results: A total of 246 students were interviewed with a mean age of 23 years. Majority were female 145(59\%) and in the Medicine program $128(52 \%)$. Fifty three $(21.5 \%)$ of the respondents were current shisha users, 84(34.1\%) had ever used shisha in their lifetime and 11(4.5\%) were current cigarette smokers. Majority $36(69 \%)$ smoked weekly and at entertainment spots $47(89 \%)$. Concurrent alcohol and cigarette use among current shisha users was $47(90 \%)$ and $8(15.4 \%)$ respectively. Factors associated with current shisha use were catholic faith $(\mathrm{OR}=2.03 ; 95 \%$ CI 1.04,3.96), residence in a rented house (OR $=2.65 ; 95 \%$ CI $1.25,5.61)$, alcohol use ( $\mathrm{OR}=13.46 ; 95 \% \mathrm{CI} 5.47-33.06)$ and family member who smokes shisha (OR= $6.4395 \%$ CI 3.32-12.43).

Conclusions: The use of shisha as an alternative form of tobacco is high among university students undertaking health professional courses. There is need for initiatives geared towards behavior change among these students to boost tobacco control efforts among the youth in Kenya.

Tob. Induc. Dis. 2018;16(Suppl 1):A811

DOI: $10.18332 /$ tid/84005

\section{2:45-14:00}

EP-255-4 Reasons for use, potential use, or discontinued use of hookah among us young adult college students S Kothari', C Berg'

'Emory University, Atlanta, GA, United States of America. E-mail: cjberg@emory.edu

Background: Existing research regarding hookah use among young adults highlights the influences of perceptions of harm, social factors, and the draw of product design and marketing. However, limited research has quantitatively examined who is most likely to initiate or sustain hookah use, the characteristics of hookah use among users, or the range of potential reasons for hookah use or discontinued use. This study addresses these gaps by assessing:

1) differences in sociodemographics and other substance use among current, never, and former hookah users;

2) use characteristics among current users (e.g., types of devices/ flavors used, quit intentions); and

3) reasons for use, potential use, and discontinued use among young adult current, never, and former hookah users.

Methods: We analyzed data from a cohort study of students aged 18-25 recruited from seven United States colleges/universities.

Results: Of 2,865 participants, $56.3 \%$ were never users, $12.4 \%$ current (past 4-month), and 31.3\% former. Correlates of being a current (vs. never) hookah user included being "other race" (vs. White), attending an HBCU (vs. a technical college), and use of any other tobacco product, marijuana, or alcohol. Correlates of being a former (vs. never) hookah user included being older, being "other race" (vs. White), attending an HBCU (vs. a technical college), and use of any other tobacco product (except cigars), marijuana, or alcohol. Among current users, $73.7 \%$ reported 
no intention to quit; only $26.2 \%$ reported attempting to quit in the past 12 months. Two factors regarding current hookah use included:

1) instrumentality (e.g., flavors); and

2) social reasons. One factor emerged regarding potential use among never users, reflecting influences related to social factors and flavors. Three factors regarding discontinued use included:

1) inconvenience (e.g., messy/expensive);

2) anti-tobacco attitude (e.g., unhealthy); and

3) social reasons.

Conclusions: These results may inform policy and interventions to address hookah use in young adults.

Tob. Induc. Dis. 2018;16(Suppl 1):A812

DOI:10.18332/tid/84118

\section{$12: 45-14: 00$}

EP-257-4 Waterpipe (Shisha) smoking: pattern and predictors of use among University of Ibadan undergraduate students

A Faloye ${ }^{1}, 0$ Akinyemi ${ }^{1}$, F Adeniji ${ }^{1}$, H Shuaibu $^{2}$

'University of Ibadan, Department of Health Policy and Management, Ibadan, Nigeria, '2University of Ibadan, Department of Community Medicine, Ibadan, Nigeria. E-mail: faloyeayobamigbe@gmail.com

Background: Tobacco consumption has been described as a global epidemic common in developing countries. Some of these countries barely have tobacco control policies and treatment for nicotine dependency, and where they do, these policies are mostly centered on cigarette smoking and are not fully implemented. Hence, this study was therefore carried out to determine the prevalence, pattern of use and predictors of an emerging method of tobacco consumption known as Waterpipe Smoking (WPS) among undergraduate students in University of Ibadan.

Methods: A cross sectional descriptive study was conducted among 390 undergraduate students residing in the halls at the University of Ibadan. Data were obtained using a semi-structured questionnaire and analysed with SPSS 21. Bi-variate analysis was used to test for associations while logistic regression was carried out to determine predictors of WPS.

Results: The study revealed that mean age of initiation of WPS was $18.5 \pm 2.7$ years, $7.8 \%$ were ever users while $3.9 \%$ were current users and the pattern of use was intermittent. Ever and current cigarette smoking, smoking of other products aside cigarette, having divorced parents, having all close friends as smokers and having a smoker in the family, having shisha smoker among siblings and close friends, acceptability of shisha smoking in the family and considering of shisha as smoking were all significant correlates of current use of WP after bivariate analysis was conducted. Ever cigarette smoking [O.R:12.9; $\mathrm{p}=0.013 ; 95 \% \mathrm{CI} ; 1.7-84.4]$, smoking of other products aside cigarette [O.R:12.7; $\mathrm{p}=0.012 ; 95 \% \mathrm{CI}$; 1.7-94.0], having all close friends as smokers [O.R:65.5; $\mathrm{p}=0.016 ; 95 \% \mathrm{CI}$; 3.3-129.4], having divorced parents [O.R:27.0; $\mathrm{p}=0.002 ; 95 \% \mathrm{CI} ; 3.5-216.0$ ] and shisha smoking among siblings [O.R:94.0; $\mathrm{p}=0.001 ; 95 \% \mathrm{CI}$; 2.0-124.1] were predictors of current WP smoking after logistic regression was conducted.

Conclusions: WPS is practiced among University of Ibadan, undergraduate students. Hence, specific laws should be formulated to regulate its use.

Tob. Induc. Dis. 2018;16(Suppl 1):A813

DOI:10.18332/tid/84655

\section{$15: 45-16: 45$}

LB-1367-4 Knowledge and perception of waterpipe harm among university students in five Eastern Mediterranean countries: a cross sectional study N Abu-Rmeileh 1 , O Khufash ${ }^{2}$, K Kheirallah ${ }^{3}$, A Mostafa $^{4}$, Y Alfarsi ${ }^{5}$, A Yusufali ${ }^{6}$, R Nakkash $^{7}$, R Salloum ${ }^{8}$

'Birzeit University, Ramallah, Jordan, ${ }^{2}$ Hacettepe University, Ankara, Turkey, ${ }^{3}$ Jordan University of Science and Technology, Irbid, Jordan, ${ }^{4}$ Ain Shams University, Cairo, Egypt, ${ }^{5}$ Sultan Qaboos University, Oman, Oman, ${ }^{6}$ Dubai Medical College, Dubai, United Arab Emirates, ${ }^{7}$ American University of Beirut, Beirut, Lebanon, ${ }^{8}$ University of Florida, Gainesville, FL, United States of America. E-mail:nrmeileh@birzeit.edu

Background: Waterpipe tobacco smoking (WTS) prevalence in the Eastern Mediterranean Region (EMR) is among the highest in the world and it is increasing with time. This paper aims to investigate waterpipe harm believes among university students who smoke waterpipe in five EMR countries.

Methods: This study was conducted in 2016 across five EMR countries: Egypt, Jordan, Palestine, Oman, and the United Arab Emirates (UAE). Participants were recruited from among young adult students (18-29 years) at selected universities in each country. Data were collected using an Internet-based survey. Participants were included if they were waterpipe tobacco smokers and at least 18 years of age (including users of cigarettes, WTS, and dual users).

Results: In most countries, participants (70\%-80\%) had initiated WTS with a friend or several friends with the exception of Palestinians where $31 \%$ started smoking with family members and Egyptians where 22\% started smoking alone. More than 70\% of the participants from Oman, UAE and Egypt started smoking in a café or restaurants while around $50 \%$ of the participants from Jordan and Palestine started smoking at home or someone else's home. Sixty percent had smoked waterpipe and $44.5 \%$ had smoked cigarettes in the past 30 days. Most participants from the five countries were aware that WTS can cause harm during pregnancy. Around $30 \%$ of participants had misperceptions about waterpipe as a cause of cancer or death. Participants from Egypt seemed to have the least knowledge about WTS harm. Those who smoked cigarettes in the past 30 days had generally good knowledge about WTS harms compared to waterpipe smokers.

Conclusions: Misperception of waterpipe harm is common among university students in the five EMR countries. Culturally adapted awareness champagnes targeting young adults including university students are needed in all countries

Tob. Induc. Dis. 2018;16(Suppl 1):A814

DOI: $10.18332 / \mathrm{tid} / 84718$

\subsection{Burden and Disparities in tobacco related diseases: Non-communicable diseases (NCDs) and communicable diseases (TB and IIV/AIDS)}

\section{$11: 00-12: 30$}

FO-390-4 Smoking and HIV in sub-Saharan Africa: a 25 country analysis of the demographic and health surveys

J Murphy ${ }^{1,2}$, B Liu $^{3}$, M Parascandola ${ }^{4}$

${ }^{1}$ National Cancer Institute, Epidemiology and Genomics Research Program, Rockville, MD, United States of America, ${ }^{2}$ University of North Carolina at Chapel Hill, Epidemiology, Chapel Hill, NC, United States of America, ${ }^{3}$ National Cancer Institute, 
Surveillance Research Program, Rockville, MD, United States of America, ${ }^{4}$ National Cancer Institute, Behavioral Research Program, Rockville, MD, United States of America. E-mail: paramark@mail.nih.gov

Background: In high-income countries, being HIV positive is associated with higher rates of smoking. This is important to public health because evidence suggests that HIV/AIDS patients who smoke have poorer treatment and survival outcomes. Moreover, both smoking and HIV are risk factors for comorbidities such as tuberculosis, which is highly prevalent in some low-and middleincome countries. The HIV-smoking relationship is understudied in sub-Saharan Africa, where tobacco use patterns and HIV prevalence differ greatly from other world regions. This study is an effort to fill this gap in the literature.

Methods: This study examined cross-sectional data from the Demographic Health Surveys (DHS) and AIDS Indicator Surveys. Data from 25 sub-Saharan African countries were pooled ( $n=286,850)$, and the association between cigarette smoking and HIV status was analysed through hierarchical logistic regression models. As a secondary aim, this study also examined the relationship between smokeless tobacco use (chew and/or snuff) and HIV status.

Results: Overall, men who had HIV/AIDS had a significantly ( $\mathrm{p}<$ $0.0001)$ higher smoking prevalence $(25.90 \%)$ than men who did not (16.09\%), as did women who had HIV/AIDS compared with women who did not $(1.15 \%$ vs. $0.73 \%$; $<<0.001)$. Multivariate logistic regression analysis revealed that people living with HIV/AIDS were $12 \%$ more likely to smoke than people living without $(\mathrm{OR}=1.12$, 95\% $\mathrm{CI}=1.04,1.21 ; \mathrm{p}<0.001$ ) when adjusting for socioeconomic, demographic, and sexual risk factors. Similarly, multivariate logistic regression revealed that $\mathrm{HIV}$-positive individuals were $34 \%$ more likely to use smokeless tobacco than HIV-negative individuals (adjusted OR $=1.34,95 \% \mathrm{CI}=1.17,1.53$ ).

Conclusions: This study complements evidence from other world regions showing that HIV infection is associated with a higher likelihood of cigarette smoking as well as smokeless tobacco use in subSaharan Africa, even when controlling for demographic, socioeconomic, and sexual risk factors.

\section{Tob. Induc. Dis. 2018;16(Suppl 1):A815 \\ DOI:10.18332/tid/83905}

\section{$11: 00-12: 30$}

F0-391-4 Tuberculosis remains the leading cause of death attributed to smoking in South Africa: results from the South African death certificate study

P Groenewald ${ }^{1}$, R Laubscher ${ }^{2}$, V Pillay van Wyck ${ }^{3}$, W Msemburi ${ }^{4}$, D Bradshaw ${ }^{3}$, F Sitas $^{3,5,6}$

'South Africa Medical Research Council, Burden of Disease Research Unit, Cape Town, South Africa, ${ }^{2}$ South African Medical Research Council, Biostatistics Unit, Cape Town, South Africa, ${ }^{3}$ South African Medical Research Council, Burden of Disease Research Unit, Cape Town, South Africa, ${ }^{4}$ University of Cape Town, Statistical Department, Cape Town, South Africa, ${ }^{5}$ University of Sydney, School of Public Health, Sydney, NSW, Australia, ${ }^{6}$ University of New South Wales, School of Public Health, Sydney, NSW, Australia.E-mail: pamela.groenewald@ mrc.ac.za

Background: The question 'Was the deceased a smoker five years ago?' was introduced in mid-1997 on a revised South African death notification form to provide a direct estimate of tobacco attributed mortality, using a case-control method for ongoing monitoring of the tobacco epidemic. This paper updates previous reports (1999 to 2007).

Methods: This analysis included 772,975 deaths from 2000 to 2013, for persons 35 to 74 years with information about age, year of death, sex, province of death, educational grade, marital status, population group and smoking status (current smoker vs. not). Cases comprised deaths due to diseases expected to be associated with smoking; controls comprised deaths from selected conditions expected to be unrelated to smoking. Deaths due to HIV without TB, external causes, cirrhosis, mental disorders, maternal and perinatal conditions and diabetes were excluded.

Smoking-associated relative risks were assumed to be the case/ control versus smoker/ex \& non-smoker odds ratios, adjusted for 5-year age group, education, province, year of death, marital status. Deaths were extrapolated to the NBD national annual estimates for 2012.

Results: Smoking-associated relative risks were highest for lung and upper aero-digestive cancers and COPD amongst the white and coloured (mixed race) populations reflecting their relatively higher smoking prevalence. When extrapolated to NBD estimates for 2012, smoking-attributable deaths accounted for approximately 14329 out of 284949 total deaths in persons aged 35 - 74 years (5\%). Tuberculosis remains the leading cause of smoking-attributable deaths (approx 24\%) followed by COPD (approx 19\%) and lung cancer (approx 17\%).

Conclusions: Different population groups in South Africa are at different stages of the tobacco epidemic and ongoing monitoring by population group is required. The cause profile of smokingattributable mortality in South Africa differs from that in developed countries and suggests that tobacco cessation should be incorporated into the South African TB programme.

Tob. Induc. Dis. 2018;16(Suppl 1):A816

DOI: $10.18332 /$ tid/84203

\section{$11: 00-12: 30$}

F0-392-4 Estimation of the impact of tobacco smoking factor on the morphological, molecular and genetic parameters of the lung cancer tumor G Smagulova', A Zhylkaidarova'

${ }^{1}$ Kazakh Institute of Oncology and Radiology, Almaty, Kazakhstan. E-mail:alma_zh@inbox.ru

Background: Tobacco smoking (TS) remains the most established cause of lung carcinogenesis and other disease processes. In Kazakhstan lung cancer (LC) occupies by far the first position in the cancer mortality structure. Identification of the morphological and immunohistochemical features of tumors among smokers may enable individualized treatment and reduce mortality.

Methods: Morphological, histological, molecular genetics, immunohistochemical methods were used. The slides of 286 patients (204 smokers and 82 nonsmokers) with LC were studied. For molecular genetic studies tumor blocks of 47 patients ( 27 smokers and 20 nonsmokers) were used.

Results: There are differences in the histotype structure of LC among smokers and nonsmokers. Squamous cell carcinoma prevails among men as adenocarcinoma among women. It was found that smokers had squamous cell carcinoma histotype predominant, which share above 1.4 times $(\mathrm{p}<0.01)$, and small cell carcinoma 2.0 times ( $\mathrm{p}$ $<0.05)$. Adenocarcinoma is 3.5 times $(\mathrm{p}<0.001)$ more likely to develop in the group of non-smokers. Activating of epidermal growth factor receptor mutations were detected in $21.3 \%$ of the patients with non-small cell LC. Mutations occur most often among women (70\%) than among men (30\%) and most among non-smokers than among 
smokers (90\% and 10\% respectively). Immunohistochemical studies of LC tumor have shown that smokers have a higher proliferative activity of tumor cells, indicating the adverse effects of smoking for the LC prognosis.

Conclusions: The data obtained allow to individualize the postoperative radiation therapy and adjuvant chemotherapy to improve the efficiency of LC treatment. Considering that the presence of the mutations associated with the possibility of targeted therapy, LC treatment prognosis in smokers less favorable than that of nonsmokers. Advising the patient to quit by clinicians is an important preventive technology for prevention of LC morbidity and premature mortality.

Tob. Induc. Dis. 2018;16(Suppl 1):A817

DOI:10.18332/tid/84603

\section{$11: 00-12: 30$}

F0-393-4 Tobacco exposure in adolescence and the risk of nasopharyngeal carcinoma: preliminary results from a multi-center case-control study in Hong Kong, China

JH Lin ${ }^{1,2}$, ZM Mai ${ }^{1,2}$, TH Lam ${ }^{1,2}$

'The University of Hong Kong, School of Public Health, Hong Kong, China, ${ }^{2}$ The University of Hong Kong, Center for Nasopharyngeal Carcinoma Research, Hong Kong, China. E-mail: kawongl@hku.hk

Background: Incidence of many cancers increases with age, but the incidence of nasopharyngeal carcinoma (NPC) in highrisk areas (e.g. Hong Kong) peaks at age 40-55 years. The early onset suggests an important role of early childhood exposures in NPC development. In our ongoing NPC case-control study, we examined the relationship between tobacco exposure especially in adolescence and NPC.

Methods: We analysed 777 histologically confirmed NPC cases (mean age $=52.8, \mathrm{SD}=12.0$ ), and 1,690 frequency matched non$\mathrm{NPC}$ controls (mean age $=50.4, \mathrm{SD}=13.4$ ) recruited during July 2014 to July 2017 from 5 of the largest hospitals $(n=1,460)$ and 3 Red Cross Blood Transfusion Centres $(n=230)$ in Hong Kong. Information on smoking and other factors was collected via a computer-assisted self-administered questionnaire. Reinterviewing of the 140 subjects showed good agreement of smoking history (Kappa or ICC: 0.65-0.99). Unconditional logistic regression was used to estimate odds ratios (ORs) of NPC for tobacco exposure, adjusting for 5-year age groups, sex, housing type at age 10, and alcohol drinking status.

Results: Compared with never smokers, the OR (95\%CIs) was 1.62 (1.32-1.98) for ever-smokers. The ORs were 2.27 (1.38-3.75) for starting smoking at 6-12 years and 1.65 (1.30-2.10) for 13-18 years ( $\mathrm{p}$ for trend=0.001). For those who started at $6-18$ years, the OR was 1.23 (0.79-1.93) for smoking 1-5 cigarettes/day, 1.71 (1.21-2.41) for 6-10 cigarettes/day and 1.78 (1.26-2.53) for $11+$ cigarettes/day ( $\mathrm{p}$ for trend $<0.001$ ).

Conclusions: Our preliminary results have shown that tobacco exposure in adolescence were associated with higher risk of NPC in a high-risk area. Further analysis will be conducted after case (target $=800$ ) and control (target $=1900)$ recruitment is completed. Tob. Induc. Dis. 2018;16(Suppl 1):A818 DOI:10.18332/tid/84207

$11: 00-12: 30$

F0-394-4 Differences in African American and White mortality caused by cigarette smoking in the U.S
P Mowery', T Pechacek²

${ }^{1}$ Biostatistics, Inc., Sarasota, FL, United States of America, ${ }^{2}$ Georgia State University, School of Public Health, Atlanta, GA, United States of America. E-mail:moweryp@bellsouth.net Background: The objective of this study is to compare African American and White excess mortality caused by smoking cigarettes. Methods: Estimates of relative risks and smoking attributable fractions were made using U.S. nationally representative data. We constructed a longitudinal cohort by linking respondents to the U.S. National Health Interview Surveys with death records. Smoking history was obtained on respondents to 11 annual waves from 19992009. Data from 200,000 respondents were linked to the National Death Index with follow-up through December 31, 2011.

Relative risks comparing current and former smokers to lifetime nonsmokers were estimated. Estimates were adjusted for possible confounders including income, health insurance, access to health care, physical activity, obesity, hypertension, alcohol use, and birth country (U.S. or other). Attributable fractions showing the proportion of deaths caused by smoking were estimated.

Results: Among African American men, $82 \%$ of lung cancer, $78 \%$ of COPD, $26 \%$ of ischemic heart disease, $28 \%$ of other heart disease, and $18 \%$ of cerebrovascular disease deaths were caused by smoking. Patterns among African American women were similar but the attributable fractions were lower. The African American male attributable fraction for total cancer deaths was $22 \%$ higher than for white men. The attributable fraction for ischemic heart disease was 30\% higher for African American men. The attributable fraction for all smoking-related causes was 6\% higher for African American than White men. Attributable fractions for African American women were higher than those observed for White women for chronic obstructive pulmonary disease, ischemic heart disease, other heart disease, cerebrovascular disease, and all cancers.

Conclusions: Large mortality disparities exist for African Americans compared with Whites. The disparities cannot be explained by smoking rates alone. Other factors that may contribute include greater smoke intake per cigarette, obesity, hypertension, diabetes, exposure to environmental toxins, and a higher rate of menthol cigarette use.

Tob. Induc. Dis. 2018;16(Suppl 1):A819

DOI: $10.18332 /$ tid/84635

$11: 00-12: 30$

F0-395-4 Productivity loss by tobacco in Mexico in 2014

CM Guerrero-López ${ }^{1}$

${ }^{1}$ National Institute of Public Health, Cuernavaca, Mexico. E-mail:caosomatik@gmail.com

Background: Tobacco use causes acute and chronic diseases such as acute myocardial infarction (ICD-10: I21), cerebrovascular diseases (ICD-10: I60-I69), lung cancer (ICD-10: C34) and chronic obstructive pulmonary disease (ICD-10: J40-J44) that lead to direct and indirect costs. The latter include lost productivity due to premature death and absenteeism, among others. The objective of this study was to estimate lost productivity attributable to tobacco by premature death and absenteeism caused by hospitalizations. Methods: I used mortality microdata published by the National Institute of Statistics and Geography and hospital discharge microdata by the Directorate General of Health Information for 2014. In order to estimate productivity, I used the National Occupation and Employment Survey for 2014. Productivity was estimated using the human capital approach, calculating the 
expected wage by sex, age, region and quarter. Productivity loss by premature death was calculated as the sum of discounted future flows of wages until expected age of death in persons that died by the diseases of interest defined above. Productivity loss by absenteeism was calculated as the product of days at hospital and mean wage, according to sex, age, region and quarter. Population Attributable Fractions from previous literature for Mexico were used to estimate the costs attributable to tobacco.

Results: I estimated a total of 2859.49 million USD productivity loss by premature death and 7.56 million USD by absenteeism caused by hospitalization in 2014 .

Conclusions: In addition to medical expenditures, tobacco use causes enormous productivity loss. These results do not include productivity loss by absenteeism caused by doctor visits or presenteeism, which can be much larger.

Tob. Induc. Dis. 2018;16(Suppl 1):A820

DOI:10.18332/tid/84536

\section{$11: 00-12: 30$}

FO-396-4 Healthcare costs attributable to smoking in California, U.S. for different racial/ethnic communities

W Max', B Stark'1, H-Y Sung ${ }^{1}$

'University of California San Francisco, Institute for Health \& Aging, San Francisco, CA, United States of America. E-mail: wendy.max@ucsf.edu

Background: Smoking is a leading cause of preventable death internationally, but it impacts some population groups more than others. While the highest smoking rates in California are for Korean, Vietnamese, and African American males, current estimates of the resulting smoking-attributable healthcare costs are not available.
Methods: We developed econometric models of smokingattributable healthcare costs using US national data and then applied the models to California-specific data from the California Health Interview Survey. Healthcare costs were estimated for hospitalizations, ambulatory care, prescriptions, and home health care, and for Whites, Blacks, Asians (Chinese, Korean, Vietnamese, Filipino, and Other Asians), Hispanics, and Other/ MultiRacial. Costs were estimated using an excess cost approach that compares smokers with nonsmokers who have all the same characteristics as smokers except they don't smoke. The difference in costs between these groups is attributed to smoking.

Results: Annual healthcare expenditures attributable to smoking in California totaled $\$ 10.7$ billion for 2014 , including $\$ 5.7$ million for men and $\$ 5.0$ million for women. Costs were greatest for Whites (\$6.5 million) followed by Hispanics ( $\$ 2.1$ million) and Blacks (\$1.0 million). Among Asians, the highest healthcare costs were for Filipinos $(\$ 191,000)$ and Chinese $(\$ 157,000)$. Healthcare costs were $\$ 276$ per resident but were highest for Blacks ( $\$ 443$ per resident) and Whites (\$438 per resident). Hospital care accounted for $53 \%$ of this total, followed by ambulatory care services (25\%), prescription drugs (14\%), and home health care (7\%).

Conclusions: Healthcare costs attributable to smoking differ substantially by racial/ethnic group, reflecting differences in population size, smoking prevalence, and smoking patterns (e.g. daily vs. nondaily smoking and menthol use). Tobacco control efforts to reduce the economic burden of smoking need to take into account these differences in developing effective programs targeted to specific groups.

Tob. Induc. Dis. 2018;16(Suppl 1):A821

DOI:10.18332/tid/84304

\begin{tabular}{|c|c|c|c|c|}
\hline $\begin{array}{l}\text { Ilousehold annual amount of } \\
\text { smoky coal used, } 10 \text { years ago }\end{array}$ & $\begin{array}{l}\text { Total consumption of cigarettes from born to } \\
10 \text { years ago (reference group: never smoker) }\end{array}$ & $\beta$ & SE & OR $\left(95^{\circ} \circ \mathrm{CI}\right)$ \\
\hline Never & $\begin{array}{l}<130000 \text { cigarettes } \\
\geq 130000 \text { cigarettes }\end{array}$ & $\begin{array}{l}0.0885 \\
0.7863\end{array}$ & $\begin{array}{l}1.2933 \\
1.3245\end{array}$ & $\begin{array}{l}1.09(0.09,13.78) \\
2.20(0.16,29.44)\end{array}$ \\
\hline$<3$ tons & $\begin{array}{l}<130000 \text { cigarettes } \\
\geq 130000 \text { cigarettes }\end{array}$ & $\begin{array}{l}-0.6172 \\
-2.5037\end{array}$ & $\begin{array}{l}1.9881 \\
2.1385\end{array}$ & $\begin{array}{l}0.59(0.03,11.37) \\
0.18(0.01,4.82)\end{array}$ \\
\hline$\geq 3$ tons & $\begin{array}{l}<130000 \text { cigarettes } \\
\geq 130000 \text { cigarettes }\end{array}$ & $\begin{array}{l}-0.1636 \\
-0.2078\end{array}$ & $\begin{array}{l}1.4050 \\
1.6086\end{array}$ & $\begin{array}{l}0.93(0.32,2.72) \\
1.78(0.30,10.67)\end{array}$ \\
\hline
\end{tabular}

\section{2:30-14:00}

PS-1085-4 The association between tobacco use and lung cancer risk: difference caused by smoky coal use

\section{Liu', X Wan'1, G Chen'1, X Ma², B Ning², G Yang}

${ }^{1}$ Institute of Basic Medical Sciences, Chinese Academy of Medical Sciences \& School of Basic Medicine, Peking Union Medical College, Dept. Epidemiology and Health Statistics, Beijing, China, ${ }^{2}$ Xuanwei Center for Disease Control and Prevention, Xuanwei, China.E-mail: yangghuan@vip.sina.com

Background: Xuanwei is one of the towns with highest lung cancer mortality in China. Residents there frequently use smoky coal for domestic cooking and heating. The smoking prevalence there is also high.

Methods: We conducted a population-based case-control study. 388 lung cancer cases and 388 gender-age-area-matched controls were surveyed. The exposure levels to potential lung cancer risk factors of multi-aspects 10 years ago were collected. After checking Spearman correlations between variables and referring to previous studies, we built conditional logistic regression model adjusting education, family history of malignancy, diet, alcohol consumption, occupational history, housing type and exposure to second hand smoke. We then introduced household annual consumption of smoky coal and total consumption of cigarettes from born to 10 years ago to the model in the form of ordered categorical variable (coal level 1-3: never use, less than 3 tons per year, 3 tons or more per year; tobacco level 1-3: never use, less than 130 thousands cigarettes, 130 thousands or more cigarettes). Results: There were $27 \%$ and $31.2 \%$ ever-smokers in case and control group, respectively. Without interactive item, the effect of tobacco use on lung cancer was (all comparing with level 1 , with $95 \%$ confidence intervals) level 2 : $\mathrm{OR}=0.90(0.22,3.67)$, level $3: \mathrm{OR}=1.59(0.45,5.70)$. After adding interactive item of coal and tobacco in the model, the effect of tobacco use among people never used smoky coal was level2: $\mathrm{OR}=1.09(0.09,13.78)$, level3: $\mathrm{OR}=2.20(0.16,29.44)$; among people using relatively 
small amount of coal was level2: $\mathrm{OR}=0.59(0.03,11.37)$, level3: $\mathrm{OR}=0.18(0.01,4.82)$; among people using large amount of coal was level2: $\mathrm{OR}=0.93(0.32,2.72)$, level3: $\mathrm{OR}=1.78(0.30,10.67)$. Conclusions: Tobacco use may lead to risk of lung cancer among people never using smoky coal, and the effect increases according to total amount of cigarettes consumption. Smoky coal use may negatively moderate the relationship between tobacco and lung cancer.

\section{Tob. Induc. Dis. 2018;16(Suppl 1):A822 DOI:10.18332/tid/84138}

[Effect of tobacco use on lung cancer: diff]

12:30-14:00

PS-1086-4 Tobacco and cancers - burden and sites of cancers associated with the use of tobacco in India RC Chauhan ${ }^{1}$, NS Chauhan ${ }^{2}$

${ }^{1}$ All India Institute of Medical Sciences (AIIMS), Community Medicine and Family Medicine, Bhubaneswar, India, ${ }^{2}$ Pondicherry Institute of Medical Sciences, Puducherry, India. E-mail: rcchauhan21@gmail.com

Background: Consumption of tobacco is the leading cause of cancers in India and is responsible for about 40 to $50 \%$ of cancers in men and about $20 \%$ of cancers in women. This study was done to assess the proportion and anatomical sites of tobacco related cancers (TRCs) in India.

Methods: The data related to TRCs from the 25 population based cancer registries (PBCRs) was reviewed and analysed. The incidence and relative proportion of specific sites of cancer associated with the use of tobacco was calculated.

Results: Among all the PBCRs, East Khasi Hills District of Meghalaya (69.3\% among males, 43.0\% among females) had the highest relative proportion of cancers associated with use of tobacco. The proportion was lowest in Sikkim state (31.4\%) and Thiruvananthapuram (10.3\%) for males and females respectively. Among various sites for TRCs, mouth $(22.6 \%$ - $39.6 \%)$, lungs $(24.7 \%-48.9 \%)$ and oesophagus $(19.9 \%$ $45.1 \%)$ were the leading sites in ten, eight and seven registries respectively for males.

Among females, mouth cancer, oesophageal cancer and lung cancer were the leading sites among TRCs. Mouth cancer and lung cancer contributed to $48.2 \%$ of TRCs in Chennai whereas mouth cancer and oesophageal cancer contributed to $66.6 \%$ of TRCs in Nagaland. Lung cancer contributed to $67.9 \%$ and $72.8 \%$ of TRCs in Manipur and Mizoram states respectively. The proportion of TRCs shows an increasing trend in recent years as compared to last decade.

Conclusions: The incidence and relative proportion of specific sites of TRCs varies according to the type of tobacco and the manner of its consumption, and need tailored approach to tackle this public health issue.

Tob. Induc. Dis. 2018;16(Suppl 1):A823

DOI:10.18332/tid/84171

\section{2:30-14:00}

PS-1087-4 Association between smoking, tuberculosis and diabetes-TB: an analysis on Indian TB patients A Bhardwaj ${ }^{1}$, M Singh $^{2}$, D Kumar ${ }^{3}$

${ }^{1}$ Dr S Radhakrishnan Govtt Medical College, Hamirpur, India, ${ }^{2}$ ESIC Medical College, Faridabad, Faridabad, India, ${ }^{3}$ DRPGMC Kangra, Kangra, India. E-mail: ashoknonu@gmail.com

Background: India is currently carrying the double burden of Communicable diseases like Tuberculosis (TB) and Non communicable diseases (NCDs) like Diabetes Mellitus. Tobacco is one of the established risk factors for NCDs and India has around 28.6\% of current tobacco users (GATS-2016-17). Evidence suggests association between tobacco smoking and TB in some populations. We aim to evaluate the association between tobacco smoking and TB and TB-DM both.

Methods: The cross sectional study on 400 patients with TB from district Kangra, Himachal Pradesh was carried out in 2014-15. In this analysis the demographic details of TB patients above the age of 18 years were included along with history of tobacco smoking in form of bidi and cigarette. The criteria used for diagnosing type 2-DM was American Diabetes Association criteria or with a diagnosed history of DM and or on oral hypoglycemic drugs/ insulin. The MDR-TB patients and patients suffering from type 1 DM, HIV and leprosy were excluded from the study.

Results: At the time of diagnosis of TB 13.5\% (54/400) were diagnosed with DM. More than one third (34.4\%) of the TB patients without DM were current smokers and $16.5 \%$ were ex-smokers at the time of diagnosis. Among the patients suffering from both DM-TB equal proportion were current and ex-smokers (31.4\% each). The distribution was statistically significant (p:0.03). The median duartion of bidi/cigarette smoked in years was significantly higher among DM-TB group (20) as compared to TB alone (0.5) (p: 0.03). On multivariate logistic regression analysis only history of current smoker has a significant positive association with TB without DM (OR (95\%CI): 2.49 (1.09-7.87).

Conclusions: Current smoking was independently associated with TB only. For development of diabetes other factors also play a major role.

\begin{tabular}{|c|c|c|c|c|}
\hline Smoking & $\begin{array}{l}\text { DV with TB } \\
\text { (known } \\
\text { newly } \\
\text { diagnosed) } \\
51 \text { (13.5) }\end{array}$ & $\begin{array}{l}\text { Non DV } \\
\text { with TB } \\
316(86.5)\end{array}$ & $\begin{array}{l}\text { Total } \\
(100)-100 \\
(100)\end{array}$ & P value \\
\hline Non Smoker & $20(10.5)$ & 170 (89.5) & $190(100)$ & 0.03 \\
\hline Current & $17(12.5)$ & 119 (87.5) & $136(100)$ & \\
\hline Ex-Smoker & $17(33.0)$ & $57(77.0)$ & 74 (100) & \\
\hline $\begin{array}{l}\text { Median } \\
\text { duration } \\
\text { of smoking } \\
\text { (IOR) in years }\end{array}$ & $20(30)$ & $0.5(25)$ & $3(65)$ & 0.03 \\
\hline
\end{tabular}

[Prevalence of Smoking with TB DM]

Tob. Induc. Dis. 2018;16(Suppl 1):A824

DOI: $10.18332 /$ tid/84191

12:30-14:00

PS-1088-4 Global Bridges Healthcare Alliance for Tobacco Dependence Treatment: implementing evidence-based smoking cessation treatment in mental health and addiction care units in Brazil and Portugal

J Castaldelli-Maia ${ }^{1}$

${ }^{1}$ Clima Clinic, Sao Paulo, SP, Brazil. E-mail:.jmcmaia2@gmail.com Background and challenges to implementation: The prevalence of smoking among mental health and addiction (MHA) patients is 2-4 times higher than it is in the general population, yet this patient population has received little help to combat smoking. Considering this, MHA care units are strategic locations for integrating tobacco dependence treatment (TDT) into existing 
treatment activities.

Intervention or response: Our team trained the staff of MHA care units that have not been providing specialized TDT for smokers. Our curriculum included the following topics focused on the implementation of treatment for MHA smokers: management; epidemiology; medications; psychotherapy; and smoking/mental health assessment instruments.

Results and lessons learnt: Our team trained the staff of MHA care units in Brazil and Portugal - which included more than 200 health professionals. There were many barriers encountered as we provided this training. A summary of problems we faced were: resistance to incorporating TDT in addiction/mental health care units; resistance to the implementation of cognitive-behavioral therapy (CBT) (psychodynamic therapy and harm reduction were preferred); treatment for smoking is already implemented in primary care network; resistance to the use of medication in addiction treatment (a preference for psychotherapy and psychosocial approach).

Conclusions and key recommendations: We learned a number of important lessons as we worked to improve the delivery of TDT to MHA patients: provide clinicians an opportunity to explore how they feel/think about providing TDT to their clients before sat the very outset of the training; rather than focusing on a specific type of behavioral therapy for TDT (such as CBT), which some may find objectionable, use more generic descriptions of behavioral therapy such as "supportive counseling"; include in training professionals who open to other forms of behavioral therapy in addition to psychoanalysis; and discuss the important impact that MHA units can have in improving the quality of life for their patients who smoke.

Tob. Induc. Dis. 2018;16(Suppl 1):A825

DOI: $10.18332 /$ tid/84232

12:30-14:00

PS-1089-4 The life-shortening effect of smokers when accompanied by other common risks

S-P Tsai ${ }^{1,2}$, C-P Wen ${ }^{3},{ }^{4}$, P-J Lu ${ }^{3}$, M-K Tsai ${ }^{3}$, C Wen ${ }^{5}$, J-H Lee ${ }^{3}$, C-L Lin ${ }^{6}$

${ }^{1}$ Texas ActM School of Public Health, College Station, TX, United States of America, ${ }^{2} \mathrm{MJ}$ Health Management Institution, Taipei, Taiwan, ${ }^{3}$ National Health Research Institutes, Miaoli, Taiwan, ${ }^{4}$ China Medical University Hospital, Taichung, Taiwan, ${ }^{5}$ Long Beach VAMC Hospital, University of Irvine Medical Center, Irvine, CA, United States of America, 6 John Tung Foundation, Taipei, Taiwan. E-mail: shan.tsai99@gmail.com

Background: Smokers usually feel fine and accept the commonly cited risks. However, many smokers are unaware that they carry additional risks that would further shorten their lives.

Methods: The cohort, 434,496, was recruited from participants in a health surveillance program between 1994 and 2008. Blood and urine were collected and analyzed. Mortality was followed up by matching with National Death file. Hazard ratios (HR) and loss of life expectancy were calculated, based on the reference group of those without the risk factor(s).

Results: Male smokers as a group had their life expectancy shortened by 5.66 years. When smokers were inactive, life shortened by 8.92 years. When accompanied by proteinuria or diabetes, years of life shortened became 14.8 and 15.4 respectively. When smokers have both proteinuria and diabetes, life years lost became 23.85. Life years lost from 2or 3 risks were found to be additive.

\begin{tabular}{|c|c|c|c|c|c|}
\hline Ileart Rate & $\mathbf{V}\left({ }^{\circ} \circ\right)$ & Deaths & IIIR $\left(95^{\circ} \% \mathrm{CI}\right)$ & $\begin{array}{l}\text { Life } \\
\text { expectancy } \\
\text { at age } 30\end{array}$ & $\begin{array}{l}\text { Life expectancy } \\
\text { difference from } \\
60-69\end{array}$ \\
\hline $60-69$ & $29,735(34.6 \%)$ & 889 & 1 (Reference) & 54.27 & (Reference) \\
\hline$\geq 90$ & $4,110(4.8 \%)$ & 419 & $2.53(2.25-2.84)^{*}$ & 42.57 & 11.70 \\
\hline 80-99 (Compared with smokers with heart rate $60-69$ ) & $15,785(18.4 \%)$ & 969 & $1.60(1.46-1.76)^{*}$ & 48.03 & 6.24 \\
\hline 80-99 (Compared with nonsmokers with heart rate 60-69) & $15,785(8.4 \%)$ & 969 & $2.69(2.42-2.99)^{*}$ & 48.03 & 13.42 \\
\hline
\end{tabular}

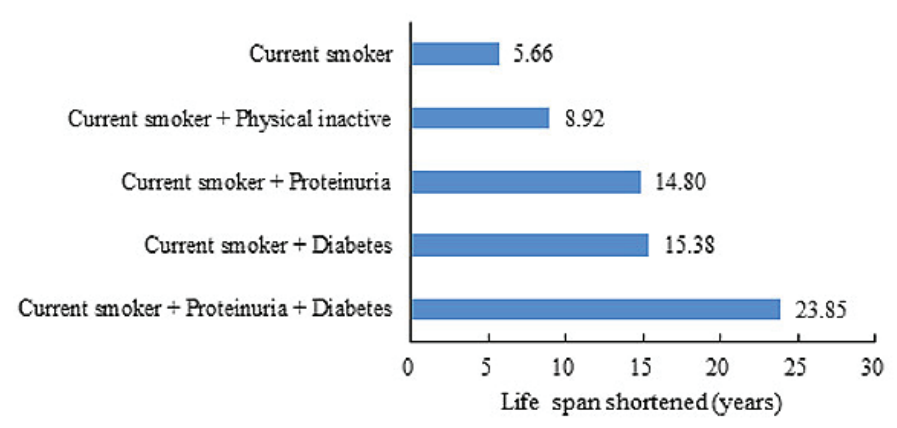

[Years of life lost from multiple risk in male]

Conclusions: The life-shortening effect of additional risk was surprisingly large, and could be used to motivate them to quit to extend their lives.

Tob. Induc. Dis. 2018;16(Suppl 1):A826

\section{DOI:10.18332/tid/84343}

$12: 30-14: 00$

PS-1090-4 Smokers with a high normal heart rate (80-99/min) found their life span shortened by 13 years

C-P Wen ${ }^{1,2}$, P-J Lu', S-P Tsai ${ }^{3},{ }^{4}, J$ Chiu', M-K Tsai', J-H Lee', $\mathrm{T}-\mathrm{C} \mathrm{Su}{ }^{5}, \mathrm{C}$ Wen $^{6}$

${ }^{1}$ National Health Research Institutes, Miaoli, China, ${ }^{2}$ China Medical University Hospital, Taichung, China, ${ }^{3}$ Texas ActM School of Public Health, College Station, TX, United States of America, ${ }^{4} \mathrm{MJ}$ Health Management Institution, Taipei, China, ${ }^{5}$ National Taiwan University, Institute of Occupational Medicine and Industrial Hygiene, Taipei, China, ${ }^{6}$ University of Irvine Medical Center, Long Beach VAMC Hospital, Irvine, CA, United States of America. E-mail: cwengood@nhri.org.tw 
Background: Not all smokers are alike in their mortality risk. Heart rate is not routinely considered as a CVD or mortality risk when the rate is less than $100 / \mathrm{min}$, and both smokers and doctors are unaware of any risk from normal heart rate.

Methods: The cohort, 434,496, was recruited from participants in a health surveillance program between 1994 and 2008. Mortality was followed up by matching with National Death file. Resting heart rate was measured by EKG. Hazard ratios (HR) and life expectancy were calculated, based on the reference group of those with heart rates 60-69/min.

Results: Smoking prevalence among male was $40.6 \%$ with 85,996 smokers in the cohort. Mortality risks increased incrementally with increasing heart rate from 70-79, 80-89, to $\geq 90$. HR with high normal heart rate, $80-99 / \mathrm{min}$, was 1.60 compared to smoker with $60-69 / \mathrm{min}$ and 2.69 compared to nonsmoker with $60-69 / \mathrm{min}$. Conclusions: Smokers with high normal heart rate, constituted nearly one fifth of smoking population, shortened life by 13 years compared to nonsmokers. Two out of three smokers in this group were expected to die from smoking related causes. Finding rapid heart rate among smokers could be used to further motivate smokers to quit, by sharing its high mortality risk.

\section{Tob. Induc. Dis. 2018;16(Suppl 1):A827}

\section{DOI:10.18332/tid/84373}

[Mortality and life expectancy for smoker]

\section{2:30-14:00}

PS-1091-4 Mind the gap: cigarette smoking rate is higher among adolescents and young adult males with HIV than those without HIV in sub-Saharan Africa

\section{O Uthman ${ }^{1}$, C Nduka ${ }^{1}$, T Moradi $^{2}$, A-M Ekestrom ${ }^{2}$}

${ }^{1}$ University of Warwick, Coventry, United Kingdom, ${ }^{2}$ Karolinska Institutet, Stockholm, Sweden.E-mail: olalekan.uthman@ki.se Background: While high cigarette smoking rates in people living with HIV are an established phenomenon in high-income countries, little or no information exist in low- and middle-income countries. We used nationally representative data from 2006 and 2017 across twenty countries in sub-Saharan Africa, in order to provide a reliable source of information about the differential in rates of cigarette smoking among men living with and without HIV.

Methods: We calculated odds ratios (OR) using logistic regression to examine if cigarette smoking was associated with HIV status. The following potential confounders of the association between HIV status and cigarette smoking were considered: age, education attainment, wealth index, employment status and place of residence. We used meta-analysis calculate pooled OR across countries.

Results: The median prevalence of cigarette smoking among HIV negative and HIV positive individuals was $18 \%$ and $24 \%$ respectively among these men. In unadjusted analysis, the prevalence of cigarette smoking was more than twice as high in adolescents and young adult males living with HIV than among those not infected $(\mathrm{OR}=2.07,95 \%$ confidence interval [CI] 1.70 to 2.51). After adjustment for age, education attainment, wealth index, employment status and place of residence the association was greatly attenuated $(\mathrm{OR}=1.24,95 \% \mathrm{CI} 1.03$ to 1.50$)$. However, among older HIV-positive adult male respondents ( $\geq 40$ years), the proportion of cigarette smoking was lower than among the uninfected, in both unadjusted ( $\mathrm{OR}=0.85,95 \% \mathrm{CI} 0.74$ to 0.97 ) and adjusted analyses (OR $=0.87,95 \% \mathrm{CI} 0.76$ to 1.00$)$.

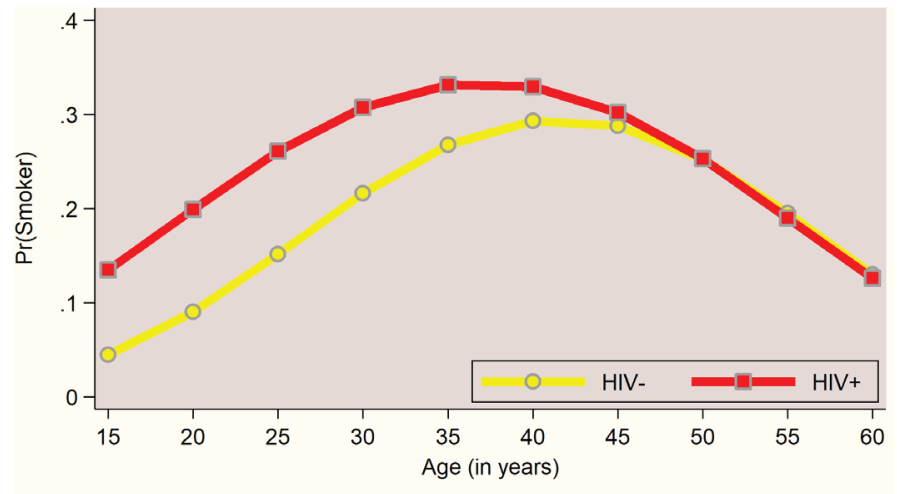

Conclusions: We found that the prevalence of cigarette smoking was more than twice as high in adolescents and young adult males with HIV than among those not infected with HIV in sub-Saharan Africa countries. Widespread and effective smoking cessation strategies in people living with HIV are needed to prevent the emerging burden of CVD and other non-communicable diseases.

Tob. Induc. Dis. 2018;16(Suppl 1):A828

DOI: $10.18332 /$ tid $/ 84463$

12:30-14:00

PS-1092-4 National framework on TB-Tobacco collaborative activities: most cost-effective way to manage TB-Tobacco comorbidity and extend tobacco cessation servcies

AK Pandey ${ }^{1}$, RJ Singh ${ }^{1}$, L Swasticharan ${ }^{2}$

${ }^{1}$ International Union Against Tuberculosis and Lung Disease, Tobacco Control, New Delhi, India, ${ }^{2}$ Ministry of Health and Family Welfare, Government of India, Directorate General of Health Services, New Delhi, India. E-mail: apandey@theunion.org Background and challenges to implementation: India, globally, has two disreputable distinctions of having the highest TB burden in the world with $2.8 \mathrm{M}$ incidence / year (Global Tuberculosis Report 2016) and also housing 2nd largest tobacco consumer with 267 million tobacco users.

Several studies have documented that tobacco consumption may contribute to TB transmission by transforming a dormant infection into active and more infectious disease, can complicate the success of TB treatment, and can increase TB mortality rates. India is implementing two national health programmes to control TB and Tobacco i.e. Revise National Tuberculosis Control Programme (RNTCP) and National Tobacco Control Programme (NTCP). It is important to integrate these two to reduce comorbidity by collaborative activities.

Intervention or response: In 2010, RNTCP and NTCP with in collaboration with The Union piloted an intervention of introducing brief advice on tobacco cessation during treatment of TB patients in Vadodara district of Gujarat. The results of were encouraging. Subsequently a working group was formed with representatives of RNTCP, NTCP WHO-India and The Union to develop collaborative framework for TB and other co-morbidity and implementing the same to cover the entire country.

Results and lessons learnt: In the leadership of the Ministry of Health \& Family Welfare, the national framework on TB-Tobacco collaborative activities was developed and released in a national level event. This is to be followed by series of training of work force involved in both the programmes.

Conclusions and key recommendations: $\mathrm{RNTCP}$ will be benefited by this collaboration to manage the comorbidity due to tobacco use among TB patients. This also leverages the robust 
programme structure of RNTCP to extend brief advice on tobacco quitting without any further investment.

Tob. Induc. Dis. 2018;16(Suppl 1):A829

DOI:10.18332/tid/84476

12:30-14:00

PS-1093-4 Tobacco use and presumptive tuberculosis in Uganda: findings from the Uganda National TB Prevalence Survey 2014 - 2016

D Kadobera', RK Majwala ${ }^{1}$, BJ Kirenga ${ }^{2}$, FR Mugabe ${ }^{1}$, S Ndyanabangi

${ }^{1}$ Ministry of Health Uganda, Kampala, Uganda, ${ }^{2}$ Makerere University, College of Health Science, School of Medicine, Kampala, Uganda. E-mail: dkadobera@gmail.com

Background: Tuberculosis (TB) patients who smoke tobacco are at an increased risk for adverse TB treatment outcomes. The association between smoking and TB is well known but has not yet received sufficient attention in terms of TB care standards in Uganda. This study describes prevalence of smoking among presumptive TB patients and risk factors associated with presumptive TB among tobacco smokers in Uganda.

Methods: This was a national cross-sectional population-based study among respondents aged 15 years and above. We defined a presumptive TB patient as any person with cough ( $\geq 14$ days) and abnormal chest $x$-ray.We collected information on current and past tobacco use, socio-economic and demographic status using structured interviewer-administered questionnaires. Data analysis entailed descriptive statistics, followed by multivariate logistic regression with backward elimination, adjusted for clustering by facility.

Results: Detailed interviews were conducted for 45294 individuals; prevalence of presumptive TB was $11.8 \%(95 \%$ $\mathrm{CI}=11.5-12.1), 12.7 \%(95 \% \mathrm{CI}=12.4-13.0)$ had a history of tobacco smoking (both previous and current tobacco smokers). Prevalence of current tobacco smoking was $7.3 \%(95 \% \mathrm{CI}=7.1$ 7.6) and prevalence of past tobacco smoking was $7.1 \%(\mathrm{CI}=6.9$ 7.4). History of tobacco smoking among presumptive TB patients was $26.7 \%$ (95\% $\mathrm{CI}=25.5-27.9)$, presumptive TB among current tobacco smokers was $13.7 \%(95 \% \mathrm{CI}=12.8-14.7)$ while for past tobacco smokers was $15.0 \%(95 \% \mathrm{CI}=14.0-16.0)$. Not having any education AOR (Adjusted odds ratio) 2.90 (95\% CI=2.34-3.6), primary education OR $1.47(95 \% \mathrm{CI}=1.22-1.76)$ and being male AOR $8.6(95 \% \mathrm{CI}=7.3-10.2)$ were the key risk factors for history of smoking among TB patients.

Conclusions: Tobacco smoking was associated with presumptive TB in Ugandans. Smoking cessation interventions should be included in the current TB care standards at various levels of the TB health care system In Uganda.

Tob. Induc. Dis. 2018;16(Suppl 1):A830

DOI:10.18332/tid/84538

\subsection{New tools for monitoring policy impact and tobacco use surveillance}

\section{2:30-14:00}

PS-1094-4 Compliance with indoor and outdoor smoke-free ban in Catalan hospitals: what do patients think?

L Antón ${ }^{1,2}, \mathrm{M} \mathrm{Fu}^{1,2}, \mathrm{Y}$ Castellano ${ }^{1,2}, \mathrm{~A} \mathrm{Riccobene}^{1,2}, \mathrm{E} \mathrm{Fernández}^{1,2}$, C Martínez ${ }^{1,2}$, Study Group on Smoking in Hospitalized Patients and Interventions to Quit Smoking (E-THIF)

${ }^{1}$ Catalan Institute of Oncology (ICO), L'Hospitalet de Llobregat, Spain, ${ }^{2}$ Bellvitge Biomedical Research Institute (IDIBELL),
L'Hospitalet de Llobregat, Spain.E-mail: Ianton@iconcologia.net Background: In Catalan hospitals, compliance with smoke-free regulation has been evaluated by direct (air markers of tobacco consumption) and indirect methods (surveys to healthcare workers); however, patients have never been asked about their insight on this matter. The objective is to describe the perception of hospitalized patients about compliance with indoor and outdoor smoke-free ban in hospitals.

Methods: Cross-sectional study in 13 hospitals of the Catalan Network of Smoke-Free Hospitals (Catalonia, Spain) in 2015. The sample size considered the number of beds in each hospital (41-205 patients; 1047 patients in total). We included adult patients hospitalized $>24$ hours. A face-to-face questionnaire was conducted to collect information about patients (age, sex, reason for admission, smoking status) and smoke-free regulation (having received information about the smoke-free regulation, level of knowledge and perception of compliance). A descriptive analysis was performed and a logistic regression model was calculated to assess the association between the perception of compliance by patients and individual and hospital characteristics (type of hospital, care unit, workers' prevalence of tobacco use, etc.).

Results: $57.6 \%$ of the participants had seen information banners about the smoke-free ban; $4.8 \%$ of the participants reported having been verbally informed; $6.4 \%$ reported receiving written information. $72.8 \%$ of the participants perceived that people respected smoke-free ban in the perimeter of the hospital, but $3.0 \%$ had seen smoking indoors and $53.7 \%$ outdoors. $65.0 \%$ were aware of the current regulation indoors and outdoors. The belief in the compliance with smoke-free ban was higher among older participants. Former $(\mathrm{OR}=2.08,95 \% \mathrm{CI} 1.29-3.36)$ and never smokers $(\mathrm{OR}=2.49,95 \% \mathrm{CI} 1.53-4.03)$ were the ones who most believed in ban's compliance.

Conclusions: In Catalan hospitals, the compliance with the smoke-free ban is high, according to hospitalized patients. Despite of this, there is room for improvement, especially in outdoor areas. The communication of smoke-free bans addressed to patients should be improved.

Tob. Induc. Dis. 2018;16(Suppl 1):A831

DOI:10.18332/tid/84116

\section{$12: 30-14: 00$}

PS-1095-4 Interest in electronic cigarettes in 5 mayor Spanish speaking countries in Latin America P Morello ${ }^{1}$, I Barrientos ${ }^{2}$, P Lozano ${ }^{3}$, R Mejia ${ }^{1}$, J Trasher $^{3}$, R Salloum ${ }^{4}$

${ }^{1}$ CEDES, Caba, Argentina, ${ }^{2}$ Instituto Nacional de Salud Pública, Cuernavaca, Mexico, ${ }^{3}$ University of South Carolina, Arnold School of Public Health, Columbia, SC, United States of America, ${ }^{4}$ University of Florida, Department of Health Outcomes and Policy, Miami, FL, United States of America. E-mail:paolamorello@hotmail.com

Background: E-cigarette use is spreading in Latin America both in adults and adolescents. However, little is known about how interest in them varies over time and across different countries. Countries have adopted different policies for e-cigarettes. When banned, consumers tend to use the Internet to search for and buy them. Google Trends is a publically available index of Internet search activity that has been used for generating public health insights. This study uses Google Trends analyses to assess variations in e-cigarette searches across 5 large Spanish-speaking Latin-American countries over the past five years.

Methods: We used Google Trends to assess the topic "electronic 
cigarette" in Argentina, Chile, Colombia, México and Perú from June 2012 through June 2017. We selected these countries based on population size, tobacco use prevalence and legislation regarding e-cigarettes. We also searched for terms of specific brands among the top 25 e-cigarette related searches in the five countries.

Results: Searches regarding e-cigarettes have increased over the last five years in all 5 countries. There are clear differences between countries. Argentina and Mexico, the two countries where e-cigarettes are banned have the highest frequency of searches, together with Chile that has the highest prevalence of tobacco use in the Region. It is noticeable a peak in searches in Argentina during May 2016, which is explained by national press coverage of the weak enforcement of the e-cigarette ban during this time period. Popular brands appeared in the top 25 related search terms and included general e-commerce sites, e-cigarette specific marketplace sites, and product manufacturer specific sites.

Conclusions: Identifying search trends around e-cigarettes can enhance the evidence base for the changes in the public perceptions of e-cigarettes in Latin America. Comparisons across countries with different tobacco control strategies could be important for understanding how to regulate this emerging product.

Tob. Induc. Dis. 2018;16(Suppl 1):A832

DOI:10.18332/tid/84147

\section{2:30-14:00}

PS-1096-4 A proportional mortality study on smoking and lung cancer using different causes of deaths for dead controls

Z-M Mai ${ }^{1}, \mathrm{~S}-\mathrm{Y} \mathrm{Ho}^{1}, \mathrm{~T}-\mathrm{H}$ Lam $^{1}$

${ }^{1}$ The University of Hong Kong, School of Public Health, Hong Kong, China. E-mail: maizm@hku.hk

Background: Proportional mortality studies using reliable death registration and smoking data can be used to assess the effects of smoking and quitting. Deaths from smoking-induced causes are treated as cases, but the selection of other causes to define controls differs. We conducted a proportional mortality study on smoking and lung cancer using four definitions of controls.

Methods: We included $81 \%$ of all deaths aged $30+$ years in 1998 in Hong Kong. Cases were lung cancer deaths ( $\mathrm{N}=2909)$. We defined controls as deaths from non-smoking related causes according to:

i) Liu (Mainland) $1998(\mathrm{n} 1=4898)$,

ii) Alam (India) $2013(\mathrm{n} 2=2944)$,

iii) Sitas (South Africa) $2013(\mathrm{n} 3=6076)$ and

iv) US Surgeon General's Report 2014 (USSG) ( $\mathrm{n} 4=4365)$.

Logistic regression yielded AORs of lung cancer, adjusting for sex and age at death.

Results: In middle age (35-64 years), AOR (95\% CI) for lung cancer was 2.79 (1.58-4.92) in ex-smokers (5+ years) and 2.65 (1.87-3.78) in current smokers (ptrend: 0.26) using controls based on Liu. The corresponding AORs were 1.87 (1.00-3.56) and 2.37 (1.58-3.58) ( $\left.\mathrm{p}^{\text {trend: }} 0.009\right)$ for Alam, 2.40 (1.42-4.04) and 4.31 (3.00-6.24) ( $\left.\mathrm{p}^{\text {trend }}:<0.0001\right)$ for Sitas, and $1.99(1.14-3.46)$ and 3.39 (2.31-5.01) ( $\left.\mathrm{p}^{\text {trend }}:<0.0001\right)$ for USSG. In old age (65$84)$, the corresponding figures for Liu, Alam, Sitas and USSG were 3.31 (2.54-4.34), 5.51 (4.26-7.17), 3.27 (2.44-4.41) and 5.40 (4.04-7.24) in ex-smokers, and 3.39 (2.63-4.37), 5.38 (4.23-6.87), 3.19 (2.44-4.18) and 5.35 (4.12-6.97) in current smokers (all $\mathrm{p}^{\text {trend}}$. $<0.0001)$.

Conclusions: The effect size of smoking on lung cancer using different controls was similar in old age but varied in middle age.
Benefits of quitting were observed in all except Liu's controls. For proportional mortality studies, the choice of the most appropriate causes of deaths to define controls needs to be cautious, as the effects or lack of effects of smoking on different diseases vary in different populations.

Tob. Induc. Dis. 2018;16(Suppl 1):A833

DOI: $10.18332 /$ tid/84213

\section{$12: 30-14: 00$}

PS-1097-4 Online informatics system of key outcome indicators to support tobacco control planning, surveillance and reporting

S O'Connor ${ }^{1}$, R Luk ${ }^{1}$, R Schwartz ${ }^{1}$, Ontario Tobacco Research Unit ${ }^{1}$ University of Toronto, Dalla Lana School of Public Health, Toronto, ON, Canada.E-mail: shawn.oconnor@camh.ca

Background and challenges to implementation: Formative research suggested that many stakeholders do not have ready access to reliable and up-to-date information on population survey results due to a variety or reasons including knowledge, permission to access, technical skills to analyse surveys and cost. Intervention or response: Adynamic database-driven informatics website-the Tobacco Informatics Monitoring System, or TIMSwas built with a user friendly and easy-to-navigate front end to facilitate stakeholders' use of tobacco survey data in their program planning, decision making, surveillance and reporting.

Results and lessons learnt: The live site, tims.otru.org, contains over 140 key indicators organized under broad themes including Tobacco Use, Prevention, Cessation, Protection, Public Opinion and Electronic Cigarettes. From these indicators, users can navigate to tens of thousands of unique data points including obtaining results by population (age, sex, education, occupation, income), geography (national, provincial, sub-provincial), and multiple surveys spanning multiple years. Data can be displayed in tables or in line/bar charts, with dynamic capability to sort/ group, print, or download into presentation ready results.

With the advent of evidence-based decision making, the TIMS site has played a key role in providing up to date information on key outcome indicators.

Conclusions and key recommendations: By providing access to regional and national survey results, the TIMS site has the potential to enable new insights into emerging issues and trends. The site facilitates links between health outcome results and the decision making process such as a consideration of health equity issues focusing on priority sub-populations and high-risk geographical areas.

Tob. Induc. Dis. 2018;16(Suppl 1):A834

DOI:10.18332/tid/84235

\section{2:30-14:00}

PS-1098-4 Monitoring population nicotine consumption, is wastewater analysis the new frontier?

J Bowden ${ }^{1,2}$, J Dono ${ }^{1,2}$, K Martin $^{1}$, C Miller ${ }^{1,2}$

'South Australian Health and Medical Research Institute, Adelaide, SA, Australia, ${ }^{2}$ University of Adelaide, Adelaide, SA, Australia.E-mail: bowden.jacquie@gmail.com

Background: Wastewater analysis is a method of routinely sampling the wastewater within a city to detect drug metabolites. Smoking by-products such as nicotine enter the sewerage network via urine excretion of people consuming cigarettes. Concentrations of nicotine and cotinine are calculated at sewerage treatment plants and converted to population consumption estimates. A similar approach has been routinely used to estimate illicit drug 
use for some time. This presentation will outline the pros and cons of wastewater analysis for population nicotine consumption estimates.

Methods: Nicotine (cotinine) sampling occurred every two months from four wastewater treatment plants across the city of Adelaide, South Australia. Simultaneously, a South Australian telephone monitoring survey ( $n=600$ per month) was used to calculate bi-monthly tobacco cigarette consumption among metropolitan residents. Data collection occurred from December 2011 and will include data until December 2017. The trends of both data sets will be compared.

Results: The wastewater and population survey data trends corresponded reasonably well until early 2015. Since then the trends have diverged, with the wastewater trend showing a small but steady decline in nicotine consumption whereas the population survey data trend shows large fluctuations in cigarette consumption. Potential reasons for the divergence include: wastewater analysis not distinguishing the source of nicotine (e.g. tobacco cigarettes, NRT, or e-cigarettes) and large confidence intervals for monthly population survey monitoring.

Conclusions: Nicotine and its metabolite cotinine are nonspecific for tobacco smoking. Therefore, wastewater analysis for tobacco smoking may supplement population monitoring of nicotine consumption but is not sufficient for detecting changes in quitting activity and smoking prevalence. Further research is needed to determine whether other tobacco-specific metabolites can be monitored to address these limitations. One advantage of wastewater analysis is that it provides an objective measure that can be compared across jurisdictions.

\section{Tob. Induc. Dis. 2018;16(Suppl 1):A835 DOI: $10.18332 /$ tid/84363}

\section{2:30-14:00}

PS-1099-4 Evaluating individual and context factors in tobacco cessation interventions

Y Castellano ${ }^{1,2}$, A Andrés $^{3}$, A Feliu' ${ }^{1,2}, \mathrm{M} \mathrm{Fu} u^{1,2}, \mathrm{M}$ Ballbé ${ }^{1,2}, \mathrm{~L}$ Antón $^{1,2}$, E Fernández ${ }^{1,2,4}, \mathrm{C}$ Martínez ${ }^{1,2,5}$

${ }^{1}$ Catalan Institute of Oncology (ICO), Tobacco Control Unit, WHO Collaborating Centre for Tobacco Control, Hospitalet de Llobregat, Spain, ${ }^{2}$ Bellvitge Biomedical Research Institute (IDIBELL), Hospitalet de Llobregat, Spain, ${ }^{3}$ National Institute of Physical Education of Catalonia (INEFC), Barcelona, Spain, ${ }^{4}$ University of Barcelona, Department of Clinical Sciences, School of Medicine, Hospitalet de Llobregat, Spain, ${ }^{5}$ International University of Catalonia, Medicine and Health Sciences School, Sant Cugat del Vallès, Spain. E-mail: ycastellano@iconcologia. net

Background: Previous research has identified that smoking cessation interventions are poorly implemented into the routine practice. Both, provider and organizational factors could intervene as facilitators or barriers. This study aimed to develop and test a questionnaire that includes factors "Knowledge, Attitudes, Behaviours, and Organizational (KABO)" that affect providers and organizations in implementing smoking cessation practices in hospitals.

Methods: An initial pool of 44 items was developed to assess individual knowledge, attitudes and beliefs of health professionals towards smoking cessation practices according to the 5As' intervention model, and organizational barriers and opportunities for its implementation into daily practice. Specific items were drawn mostly from the literature. All items were measured in a
11 -point scale $0=$ "not at all/never" to $10=$ "completely/always". The data were collected form health workers $(n=702)$ in Catalonia (Spain) using a web-based survey. The validity of the instrument was measured in the following ways:

(a) analyzing the items;

(b) assessing the internal structure;

(c) estimating the internal consistency; and

(d) analyzing the relationship between this tool and the 5As' intervention model.

Results: The estimated Cronbach's a coefficient of 37 items for reliability was .909 , indicating good internal consistency. Using exploratory factor analysis, seven factors were extracted including: individual skills, attitudes and beliefs, positive organizational support, beliefs about patient wish/readiness to quit, organizational resources, individual commitment, and organizational endorsement. These seven factors explained $61.58 \%$ of the variance, and a new refined 36 -item version of the questionnaire was obtained.

Conclusions: Psychometric testing supports that the KABO questionnaire is reliable and valid for assessing main barriers and facilitators of smoking cessation practices. Up to know, individual factors explained better the implementation of smoking cessation interventions in hospitals. The seven dimensions identified can be used for investigating how changes on individual and organizational dimensions have an impact on smoking cessation implementation services.

\section{Tob. Induc. Dis. 2018;16(Suppl 1):A836 \\ DOI:10.18332/tid/84457}

\section{$12: 30-14: 00$}

PS-1100-4 Influence of recent tobacco control policies and campaigns on Quitline call volume in Korea

J-J Park' ${ }^{1}$ LN Minh1, J-K Oh ${ }^{1,2}$, EH Yun ${ }^{1,2}$, D Lee $^{1,2}$, MK Lim ${ }^{1,2}$

${ }^{1}$ Graduate School of Cancer Science and Policy, National Cancer Center, Goyang, Korea, Republic of, ${ }^{2}$ National Cancer Control Institute, National Cancer Center, Goyang, Korea, Republic of. E-mail: jinjupark@ncc.re.kr

Background: Many tobacco control policies and media campaigns have recently been adopted and implemented in Korea. The effectiveness of these policies and campaigns can be measured by monitoring the inbound call volume of the Quitline, which has been in operation since 2006 .

Methods: We reviewed Korean government documents and regulations originating between 2007 and 2016 to summarize tobacco control policies and mass media campaigns. The corresponding incoming call volumes of the Quitline were monitored over the same periods and compared with the baseline call volumes in 2007 and 2008.

Results: Peak call volume occurred from October 2010 to March 2011, when the Quitline was directly promoted on television; during these periods, the volumes were 2.5 and 5.2 times higher than the average monthly call volumes in 2007 and 2008, respectively. A relatively gradual upward trend in call volume was found from 2013 to 2016, when the Quitline number was included on cigarette packs, a fear-oriented anti-tobacco campaign appeared in the mass media, and a tax increase on tobacco was implemented; average monthly call volume was approximately 4-5 times higher than those in in 2007 and 2008.

Conclusions: It appears that the continuous efforts to add up of tobacco control policies in the context of a nationwide antitobacco mass media campaign that includes the Quitline number 
is the most effective approach to maintaining the upward trend in smoking cessation intentions. Based on the Korean experience, Quitline data may be useful for measuring the impact of tobacco control policies and campaigns in Asian Pacific countries.

Tob. Induc. Dis. 2018;16(Suppl 1):A837

DOI:10.18332/tid/84458

\section{2:30-14:00}

PS-1101-4 Tobacco retail and publicity at points of sale (PoS) around schools in three major cities in Mexico (2014-2016)

WC Paz Ballesteros ${ }^{1}$, R Pérez Hernández ${ }^{1}$, JF Thrasher LaFontaine ${ }^{1,2}$, N Fleischer ${ }^{3}$, EJ Arillo Santillán ${ }^{1}$, R Santos Luna ${ }^{1}$, LM ReynalesShigematsu

${ }^{1}$ National Institute of Public Health of Mexico, Tobacco Research, Cuernavaca, Mexico, ${ }^{2}$ University of South Carolina, Department of Health Promotion, Education \& Behavior, Arnold School of Public Health, Columbia, SC, United States of America, ${ }^{3}$ University of Michigan School of Public Health, Department of Epidemiology, Ann Arbor, MI, United States of America. E-mail:wayracpaz@gmail.com

Background: Mexico has regulation addressing advertising, promotion and sponsorship of tobacco products. Social environment and availability must be considered to actualize tobacco control policies targeting young people. We aimed to examine changes on tobacco retail and publicity around secondary schools in Mexico.

Methods: 57 secondary schools where randomly selected by strata across three major cities in Mexico (Mexico City -CDMX-, Guadalajara and Monterrey) on 2014; urban marginalization and registered PoS according to the NAIC System were used. Tobacco retail, external and internal publicity were evaluated in each $\mathrm{PoS}$ within a ratio of 300 meters around schools and standardized questionnaires were fulfilled by trained observers; this exercise was repeated on 2016. Paired sample T test and tests on the equality of proportions were executed. This study was funded by the National Institute of Health, University of South Carolina through CODICE collaboration.

Results: Total number of PoS reduced from 796 (Density: 2816.5/km2) in 2014 to 597 (Density: 2112.3/km2) in 2016 ( $\mathrm{p}<$ 0.05 ), no reduction was found in Monterrey. Street vendors were found almost exclusively in CDMX. Sales of single cigarettes reduced only in CDMX (71.4\% to $57.1 \%$; $<<0.05)$ and we were able to detect contraband sales in less than $5 \%$ of stores. Sale of flavored capsules was found in more than half of stores (2016). External publicity: was found in less than $2 \%$ of stores in both measurements. Internal publicity: announcements at the high of children eyes reduced in Monterrey (90.7\% to 30.8\%; $\mathrm{p}<0.05$ ), promotions were found in less than $4 \%$ of stores and counterpublicity augmented in Guadalajara from $0.4 \%$ to $14.0 \%$ ( $\mathrm{p}<$ 0.05 ); in contrast, display of cigarettes augmented in CDMX from $22.3 \%$ to $34.2 \%$; $(\mathrm{p}<0.05)$.

Conclusions: Tobacco retail remain present around secondary schools and control policies should target display of tobacco cigarettes and reinforce control of contraband and single cigarettes sales.

Tob. Induc. Dis. 2018;16(Suppl 1):A838

DOI:10.18332/tid/84632

$12: 45-14: 00$

EP-251-4 Global reporting of waterpipe tobacco policy in online news articles in 2015: a crosssectional analysis

\section{El Kadi', M Jawad², R Nakkash}

${ }^{1}$ American University of Beirut, Faculty of Health Sciences, Beirut, Lebanon, ${ }^{2}$ Imperial College London, Public Health Policy Evaluation Unit, London, United Kingdom. E-mail: mohammed. jawad06@imperial.ac.uk

Background: Policy discussions are often articulated through the news media. The aim of this study is to understand how waterpipe tobacco policy is narrated and developed over time in online news media.

Methods: We conducted a cross-sectional analysis of online news articles published in the year 2015. We used the online news content retriever service 'Google Alerts' to receive weekly emails on any news article that contained 'waterpipe' or its synonyms in the title or body of English news articles. We coded text into one of the following themes: taxation, smoke-free law, regulation of content and emissions, health warning labels, bans on advertising, promotion and sponsorship, cessation programs, restriction of sale to minors, zoning or licensing, prohibition, and other waterpipespecific policy.

Results: We included 567 news articles from 33 countries. We elicited 14 themes, which we counted 1309 times (mean 2.3 themes per article). The five most common themes were licensing or zoning (24.7\%), Smoke-free law (20.9\%), prohibition (13.4\%), waterpipe-specific policy (9.4\%), and sales to minors (7.0\%). We report on the dominant policy narratives in six countries: Canada, India, Pakistan, the UAE, UK, and the US.

Conclusions: Geographical variation in policy narratives can be a useful tool for future policy dialogue around waterpipe tobacco control and prevention.

Tob. Induc. Dis. 2018;16(Suppl 1):A839

DOI:10.18332/tid/84421

\subsection{Tobacco dependence treatment and smoking cessation: Innovations in Practice and Opportunities for scaling}

14:00-15:30

F0-397-4 Sustaining a system wide intervention to promote increased smoking cessation rates among pregnant women

R Bell' ${ }^{1}$, M Willmore ${ }^{2}$, E Milne ${ }^{3}$, S Jones $^{4}$, S Hamilton $^{4}$, V AraujoSoares $^{1}$, A Rutter $^{5}$, M White ${ }^{6}$, Babyclear evaluation group

${ }^{1}$ Newcastle University, Institute of Health and Society, Newcastle upon Tyne, United Kingdom, ${ }^{2}$ NHS England, Newcastle upon Tyne, United Kingdom, ${ }^{3}$ Newcastle City Council, Newcastle upon Tyne, United Kingdom, ${ }^{4}$ Teesside University, Middlesbrough, United Kingdom, ${ }^{5}$ Fresh North East, Durham, United Kingdom, ${ }^{6}$ Cambridge University, Cambridge, United Kingdom. E-mail: ruth.bell@ncl.ac.uk

Background and challenges to implementation: In 2011/12, more pregnant women smoked at delivery in North East England (population 2.6 million) than the national average (21\% vs $13 \%$ ). Work with midwives across the region identified barriers to implementing national guidance for universal carbon monoxide (CO) screening and referral for smoking cessation (SC) support. These included lack of equipment, and midwives' concerns that quit advice was not worthwhile and might undermine relationships with women.

Intervention or response: A system wide approach, babyClear®, was commissioned across 12 local government areas involving eight NHS hospital maternity services and associated smoking cessation services. The intervention provided equipment and 
training for universal CO monitoring, opt-out referral to SC services and enhanced support to quit. Independent evaluation assessed effectiveness and implementation. Service data for more than 37,000 deliveries in 2013-14, including 10,500 smokers, was analysed for impact on quit rates. Interviews with more than 90 healthcare staff were conducted. Routine smoking at delivery data for local commissioning areas was monitored 2012-2017.

Results and lessons learnt: Quit rates nearly doubled (aOR $1.8,95 \%$ CI 1.5-2.2) and referrals for SC support increased by 2.5 fold (95\% CI 2.2-2.8) after initial implementation (Bell et al, 2017). There was variation in effectiveness between maternity services. Process evaluation indicated that effective SC advice was deliverable within routine maternity care and acceptable to women and maternity staff. Diversity of SC service delivery models, variable resourcing and organisational stability influenced implementation. Regional smoking at delivery rates declined steadily from $21 \%$ to $16 \% 2012-2017$, but some localities experienced recent increases, suggesting variable sustainability.

Conclusions and key recommendations: The intervention improved quit rates through system wide training and action to improve identification and referral of pregnant smokers. Local contexts have a critical role to play in both implementing and sustaining such initiatives into practice. Identifying core intervention ingredients and potential for flexibility and tailoring is recommended.

\section{Tob. Induc. Dis. 2018;16(Suppl 1):A840 DOI: $10.18332 /$ tid/84013}

\section{4:00-15:30}

F0-398-4 Impact of a tobacco cessation intervention on adherence to tobacco use treatment guidelines among village health workers in Vietnam

N Nguyen ${ }^{1}$, T Nguyen ${ }^{1}$, N Siman², N VanDevanter ${ }^{3}$, D Shelley ${ }^{2}$ ${ }^{1}$ Institute of Social Medical Studies, Ha Noi, Viet Nam, ${ }^{2} \mathrm{New}$ York University School of Medicine, Department of Population Health, New York, NY, United States of America, ${ }^{3}$ New York University College of Nursing, New York, NY, United States of America. E-mail:nvd2@nyu.edu

Background: Community health workers (in Vietnam referred as village health workers - VHWs) have potential to play a key role in expanding access to evidence-based tobacco use treatment. VHWs can help smokers quit by asking patients about tobacco use, advising them to quit, and offering cessation assistance (3As). We are conducting a $\mathrm{NIH}$ funded cluster randomized controlled trial comparing two strategies for implementing tobacco use treatment guidelines (TUTG) in commune health centers (CHCs) in Vietnam: brief provider counseling + self-help materials (BC) vs. $\mathrm{BC}+3$ sessions of in person counseling delivered by a VHW. This paper presents data on the effect of the intervention on VHWs' practice patterns and attitudes towards TUTG.

Methods: We examined changes in adherence to TUTG before and 12 months after the intervention with $89 \mathrm{VHWs}$ working in 13 $\mathrm{CHCs}$ in Vietnam. Adherence to each of the 3As was measured using a 5-point likert scale: from 1-none (did not ask any patients in past month if they smoke), to 5-all or most of patients. Response scales of questions measuring attitudes and self-efficacy range from 1-strongly disagree to 4 -strongly agree. Factor analysis was used to form two scales measuring attitudes and self-efficacy.

Results: The mean adherence level to each of the TUTG increased significantly: Asking about tobacco use increased from $1.79 \pm 0.57$ to $2.61 \pm 0.96$; advising to quit from $1.93 \pm 0.60$ to $2.90 \pm 1.11$; assisting from $1.53 \pm 0.62$ to $2.65 \pm 1.19$. Perceived barriers to treating tobacco use decreased significantly. Self-efficacy and attitudes towards TUTG improved significantly. Increased adherence to TUTG was associated with positive attitudes towards TUTG.

Conclusions: The implementation strategies were effective in improving VHWs' adherence to TUTG. Extending VHWs' role to include smoking cessation could offer a sustainable supporting resource for tobacco smokers who wish to quit.

\section{Tob. Induc. Dis. 2018;16(Suppl 1):A841}

\section{DOI:10.18332/tid/84132}

\section{4:00-15:30}

F0-399-4 Healthcare providers' concerns regarding smoking cessation pharmacotherapies in pregnancy: calls to a teratology information service

ML Lee ${ }^{1}$, A Havard ${ }^{1}$, DT Tran 1 , D Kennedy ${ }^{2,3}$, A Welsh ${ }^{3}$

${ }^{1}$ Centre for Big Data Research in Health, UNSW, Sydney, NSW, Australia, ${ }^{2}$ MotherSafe, Royal Hospital for Women, Sydney, NSW, Australia, ${ }^{3}$ School of Women's and Children's Health, UNSW, Sydney, NSW, Australia.E-mail:m.lee@student.unsw.edu.au Background: While pregnant smokers are receptive to using smoking cessation pharmacotherapies (SCPs), few actually use prescription SCPs during pregnancy. Due to the uncertainty regarding SCP safety during pregnancy, it is hypothesized that caution among healthcare providers (HCPs) contributes to low SCP utilisation. This study examined the extent of HCPs' concerns regarding $\mathrm{SCPs}$, relative to other medications, using data from MotherSafe, the telephone-based Australian teratology information service.

Methods: 71,473 pregnancy-related calls (2001-2016), for which a medication was the primary reason for the call, were categorised as regarding nicotine replacement therapy [NRT], bupropion varenicline or other medicines, which were categorised according to Australia's classification of risk during pregnancy: category A (low risk), B1, B2, B3, C , D or $\mathrm{X}$ (teratogenic). Calls were grouped into those made by HCPs (GPs, midwives, obstetricians or pharmacists) and consumers. Separate logistic regression models examined the odds for $\mathrm{HCPs}$ to call about each SCP relative to all other categories of medications.

Results: HCPs were more likely to call about bupropion $(n=12)$ than other medications in the same (B2) and higher (B3 and C) risk categories (B2: odds ratio [OR] 3.07; 95\% CI 1.38-6.83, B3:2.48; 1.11-5.53, C: $2.36 ; 1.06-5.26)$. HCPs were more likely to call about varenicline $(n=27)$ than other medications in the same category B3 $(2.74 ; 1.57-4.78)$, and higher risk categories (C: $2.67 ; 1.53-4.67, \mathrm{D}: 1.89 ; 1.08-3.32, \mathrm{X}: 1.93 ; 1.02-3.66)$. There was no difference in HCP's likelihood to call regarding NRT relative to its corresponding category (D) $(1.20 ; 0.94$ 1.52).

Conclusions: HCPs had a greater level of concern regarding bupropion and varenicline than other medicines in the same and higher risk categories. As these reservations may be limiting the use of SCP during pregnancy, research is needed to better understand and help correct this imbalance. This includes better quality evidence regarding the safety of SCP during pregnancy.

Tob. Induc. Dis. 2018;16(Suppl 1):A842

DOI: $10.18332 /$ tid/84029

14:00-15:30

F0-400-4 Effectiveness of face-to-face counseling with follow-up in primary care settings in India S Mahapatra ${ }^{1}$, R Panda $^{2}$ 
'Indian Institute of Public Health, Bhubaneswar- Public Health Foundation of India, Research, Bhubaneswar, India, ${ }^{2}$ Public Health Foundation of India, Gurgaon, India. E-mail: sandeep@ iiphb.org

Background and challenges to implementation: Few studies in India have investigated whether face to face counseling sessions with follow-up can increase quit rates of tobacco, and whether this strategy is feasible and effective for promoting tobacco cessation is still unclear in Indian settings. We conducted a study to test the real world effectiveness of behavioral counselling on tobacco cessation among a cluster of tobacco users. This paper highlights key findings from the same study.

Intervention or response: We compared the quit rates of one group which received brief face-to-face counseling (FC) alone (one session of 1-3 minutes) to another group which received the same face-to-face counseling plus 3 follow-up sessions (FCF) of face-to-face counseling (3-5 min each) at 1 week, 1 and 3 month follow-up, using a non-randomized controlled study. From March 2016 to October 2016, tobacco users who sought treatment in primary care settings of two high tobacco burden states in India were invited to participate in the study. Eligible tobacco users $(\mathrm{N}=535)$ were divided into two groups: $\mathrm{FC}(\mathrm{n}=132)$ and FCF $(\mathrm{n}=394)$. Main outcomes was self-reported abstinence at 3 month follow-up.

Results and lessons learnt: By intention to treat, at 3 month follow-up, the quit rates of FC and FCF were $21 \%$ and $31 \%$, and respectively. Chi-square test showed that there was significant association between type of intervention and quit rate. The odds of quitting was $1.7(95 \% \mathrm{CI} 1.1,2.7 ; \mathrm{P}<0.05)$ times higher in the FCF group compared to the FC group.

Conclusions and key recommendations: To the best of our knowledge, we have provided the first evidence in India that additional follow-up sessions can increase the effectiveness of a face-to-face counseling session and can be adopted in routine clinical practices. This data should be used to leverage further work and expansion to other districts and states of the country.

Tob. Induc. Dis. 2018;16(Suppl 1):A843 DOI:10.18332/tid/84368

\section{4:00-15:30}

F0-401-4 Effects of integrative telephone counseling in the smoking cessation system at the primary care, Thailand

K Thanomsat ${ }^{1}$, J Yunibhand', S Klumchim ${ }^{1}$

'Thailand National Quitline, Phayathai, Thailand. E-mail: kamollabhu@gmail.com

Background and challenges to implementation: Even though, there are various ways to assist smokers to quit smoking in Thailand, the number of Thai smokers who can stop smoking continuously still less than the expectation of the National Strategic Plan for Tobacco Control. This study aims to investigate the integrative telephone counseling in the smoking cessation system at the primary care among Thai smokers.

Intervention or response: The study design was a quasiexperimental design which divided the subjects into experimental $(n=85)$ and control $(n=67)$ groups. The experimental group received combination interventions, including brief intervention by Village health volunteers (VHV) and stage-matched individual behavioral counseling by the Thailand National Quitline, whereas, the control group received brief intervention and follow up by the VHV. Quit attempt, start quitting, and point prevalence abstinence at 30 days were performed. In addition, relative risk was used to analyze the ratio of the probability of the outcomes between experimental and control group.

Results and lessons learnt: The findings illustrated that quit attempt was $76.47 \%$ in the experimental group, $52.24 \%$ in the control group [RR $2.97(95 \% \mathrm{CI}=1.49,5.95)]$, start quitting was $60.00 \%$ in the experimental group, $25.37 \%$ in the control group [RR $4.41(95 \% \mathrm{CI}=2.19,8.89)]$, and point prevalence abstinence at 30 days was $44.71 \%$ in the experimental group, $17.91 \%$ in the control group [RR $3.71(95 \% \mathrm{CI}=1.74,7.90)]$. It is considered that the interventions can effectively facilitate Thai smokers in the community to quit smoking.

Conclusions and key recommendations: The integrative telephone counselling increased the quit rate among Thai smokers. Consequently, the interventions through brief intervention along with stage-matched intervention are beneficial for Thailand.

Tob. Induc. Dis. 2018;16(Suppl 1):A844

DOI:10.18332/tid/84236

\section{4:00-15:30}

F0-402-4 Feasibility of an integrated complex intervention targeting tobacco and alcohol cessation among patients with tuberculosis in three South African provinces

A Janse van Rensburg ${ }^{1}, \mathrm{M}$ Engelbrecht ${ }^{1}, 0$ 0mole ${ }^{2}, \mathrm{~J} \mathrm{Tumbo}^{3}$, N Morojele $^{4}$, G Louwagie ${ }^{5}$, K Siddiqi ${ }^{6}, 0$ Ayo-Yusuf ${ }^{7}$

${ }^{1}$ University of the Free State, Bloemfontein, South Africa, 'University of Witwatersrand, Johannesburg, South Africa, ${ }^{3}$ Sefako Makgatho Health Sciences University, Pretoria, South Africa, ${ }^{4}$ South Africa Medical Research Council, Cape Town, South Africa, ${ }^{5}$ University of Pretoria, Pretoria, South Africa, ${ }^{6}$ University of York, York, United Kingdom, ${ }^{7}$ Sefako Makgatho Health Sciences Universitity, Pretoria, South Africa. E-mail: jvrensburgandre@gmail.com

Background: South Africa's protracted battle with the dual epidemic of HIV and TB is well-documented. TB patients who smoke tobacco are often faced by low socio-economic status and alcohol dependence, with poor TB treatment outcomes. This paper reports on findings that inform the feasibility of scaling an intervention based on motivational interviewing and mhealth, delivered by lay health workers (LHWs), towards achieving tobacco and alcohol cessation and improving TB and HIV treatment adherence.

Methods: A theory-driven, qualitative feasibility study was conducted in the Free State, North-West and Gauteng provinces of South Africa. Thirteen semi-structured interviews were conducted with district, provincial and national TB managers, and nine focus group discussions were conducted with LHWs and TB patients at the study sites. Transcribed data was thematically analysed in NVivo 11 and guided by Normalisation Process Theory.

Results: The intervention package and its importance were generally understood among participants, though some had difficulty differentiating it from past and current government initiatives (Coherence). The intervention package does not require extensive re-alignment in terms of work roles, and participants were well-aligned to the introduction of LHWs in existing work spaces (Cognitive Participation). Little difficulties were foreseen in different stakeholders working together in adopting new practices, and the intervention was perceived to align well with government policies. It was noted that the LHWs would require extensive training and support (Collective Action). Measuring the effects post-intervention was perceived to possibly be hampered by fragmented health information systems (Reflexive Monitoring). 
Conclusions: The intervention package was suggested to have a fairly high level of feasibility, being well-supported by stakeholders and aligning well with key health system elements. The findings also illustrate the precarious nature of the challenge under focus, and suggest the necessity of a fair amount of sustained support, dynamism and flexibility in different sites for intervention success.

Tob. Induc. Dis. 2018;16(Suppl 1):A845

DOI:10.18332/tid/84491

\section{2:30-14:00}

PS-1102-4 Dental specialist trainees' competence in instituting tobacco cessation services in Nigeria A Dedeke ${ }^{1}, 0$ Popoola ${ }^{2}$, A Adebiyi ${ }^{2}$

'University of Lagos, Preventive Dentistry, Lagos, Nigeria, ${ }^{2}$ University of Ibadan, Community Medicine, Ibadan, Nigeria. E-mail: doctordedeke@yahoo.ca

Background: Oral health problems arising from tobacco use have been reported and some of these conditions can be prevented through Tobacco Cessation (TC). Dentists provide tobacco cessation services to patients. In Nigeria, little is documented on the knowledge of TC and its attending challenges experienced by dentists in carrying out TC services. This study was conducted to assess this among dentists in Southwest Nigeria.

Methods: A cross sectional study was carried out in eight tertiary healthcare facilities within the six states of the Southwest geopolitical zone. A total population survey involving two hundred and five dentists undergoing postgraduate training and/ or providing services in the tertiary hospitals was conducted. A pre-tested, semi structured self-administered questionnaire was used to collect information on socio-demographics, knowledge of TC methods, the 5As of cessation and challenges in TC. Knowledge questions regarding TC were graded using a 12 point Likert scale. A score of $\geq 9$ was assessed 'good', 5-8 'fair' and $\leqslant 4$ 'poor'. Data were analysed using descriptive statistics and logistic regression at $\mathrm{p}=0.05$.

Results: The population comprised of 117 (57.1\%) males, 145 (69.3\%) trainees and 129 (62.9\%) junior officers. Age and years of working experience were $33.8 \pm 5.2$ years and $6.1 \pm 4.4$ years respectively. Seventy-two $(35.5 \%)$ respondents reported good knowledge of TC methods. Fifty-six (27.9\%) respondents reported knowledge of clinical practice guidelines regarding TC. Lack of perceived efficacy and training as well as a lack of system support were the most important challenges mentioned by $60.2 \%$ and $54.1 \%$ of respondents respectively. Most (89.7\%) never had prior formal training on TC however 165 (81.3\%) were willing to be trained. Non-trainees were less likely to undertake formal TC training (OR 0.91, 95\% CI 0.43-1.96).

Conclusions: Knowledge of tobacco cessation was poor among dentists and challenges abound. Formal training to improve knowledge of Tobacco Cessation and provision of systems support are needed in Nigeria.

Tob. Induc. Dis. 2018;16(Suppl 1):A846

DOI:10.18332/tid/84205

\section{2:30-14:00}

PS-1103-4 Tobacco Control Training Programme for health professionals of the Catalan Network of Smoke-Free Hospitals: 11-year experience M Margalef ${ }^{1,2}$, M Ballbè ${ }^{1,2}$, L Antón ${ }^{1,2}, 0$ Guillén ${ }^{3}$, C Martínez ${ }^{1,2}$, $M \mathrm{Fu}^{1,2}, \mathrm{JM}$ Suelves ${ }^{4}, \mathrm{E}$ Fernández ${ }^{1,2,5}$

'Catalan Institute of Oncology (ICO), Tobacco Control Unit. WHO Collaborating Centre for Tobacco Control, L'Hospitalet de Llobregat, Spain, ${ }^{2}$ Bellvitge Biomedical Research Institute (IDIBELL), L'Hospitalet de Llobregat, Spain, ${ }^{3}$ Catalan Institute of Oncology (ICO), Teaching and Training Unit, L'Hospitalet de Llobregat, Spain, ${ }^{4}$ Health Department of the Catalan Government, Public Health Agency of Catalonia, Barcelona, Spain, ${ }^{5}$ University of Barcelona, Department of Clinical Sciences, School of Medicine, Campus Bellvitge, L'Hospitalet de Llobregat, Spain.E-mail:m.margalef@iconcologia.net

Background and challenges to implementation: The Catalan Network of Smoke-Free Hospitals (XCHSF), with 82 hospitals of Catalonia, is coordinated by the Catalan Institute of Oncology and supported by the Health Department of the Catalan Government. This public health programme is intended to design, scale down, and evaluate tobacco-control policies within its members. In this context, we aim to describe the Tobacco Control Training Programme initiated in 2006 addressed to health professionals to provide knowledge and skills to effectively uphold tobacco cessation services.

Intervention or response: The XCHSF's Training Programme is based on the identified needs detected in a baseline study conducted in 2005. It offers specific contents provided annually by a qualified group of experts in the field. Three modalities have been launched since 2006: a) face-to-face training courses on specific topics about smoking cessation intervention, b) a Train-the-Trainer Programme, and c) an on-line course about brief intervention on smoking cessation. Courses information is spread among hospital coordinators and posted online. All courses are accredited by the Official Administration and a certificate of completion is provided after participants' knowledge assessment. Participants are also required to evaluate the course and the trainer/s.

Results and lessons learnt: Since its beginnings in 2006, the XCHSF's Training Programme has increased its offer from 1 to 7 different topics of courses. Results on participation up to July 2017 indicate that 1,427 professionals have attended face-to-face courses since 2006; more than 2,700 professionals have been trained through the Train-the-Trainer Programme since 2009, and 1,474 professionals have finished the online course through several editions since 2014. The overall satisfaction reaches $\geq 8.5 / 10$.

Conclusions and key recommendations: Offering a wide and continuous range of training activities is essential for having skilled health professionals in smoking cessation in hospitals. The XCHSF's Training Programme is an educational resource that reinforces public health promotion in the territory and fosters the implementation of tobacco control strategies in hospitals.

Tob. Induc. Dis. 2018;16(Suppl 1):A847

DOI:10.18332/tid/84215

12:30-14:00

PS-1104-4 Physical activity was associated with unassisted quitting: cross-sectional and prospective findings from the Hong Kong Population Health Survey

Z-M Mai', S-Y Ho ${ }^{1}$, M-P Wang ${ }^{2}$, L-M Ho ${ }^{1}$, T-H Lam ${ }^{1}$

'The University of Hong Kong, School of Public Health, Hong Kong, China, ${ }^{2}$ The University of Hong Kong, School of Nursing, Hong Kong, China. E-mail: maizm@hku.hk

Background: In Hong Kong, only $18.9 \%$ of current smokers have tried smoking cessation services and most smokers (83.2\%) quit without any assistance, which is less well studied. Physical activity may reduce tobacco cravings hence increase quitting. Little was known about behavioral influence on unassisted quitting in a general population. This study investigated the associations of 
physical activity with intention to quit and quitting in Chinese adult in Hong Kong, which is among the lowest smoking prevalence regions.

Methods: In the Hong Kong Population Health Survey, 7084 landbased non-institutionalized subjects were interviewed face-to-face at baseline in 2003/04 and follow-up in 2006. Ethical approval was granted by a local institutional review board. Information on quit intention ('planned to quit in the next one month' versus 'none'), quitting ('ex-smokers' vs 'current smokers' in cross-sectional analysis, and 'new quitters' vs 'continuing smokers' in prospective analysis), and physical activity ('exercised any physical activity in the past 1 month' vs 'none') was collected. Logistic regression yielded ORs of quit intention and quitting for physical activity, adjusting for sex, 5-year age group, education, place of birth, income and co-habitant smoker(s). Among 1678 ever smokers at baseline, 323 were followed and were included in the prospective analysis.

Results: Physical activity was non-significantly associated with an OR of 0.94 (95\% CI: 0.68-1.30) for quit intention in current smokers at baseline. Physical activity was cross-sectionally and significantly associated with higher odds of ex-smoking (OR: 1.70, 95\% CI: 1.36-2.14, $\mathrm{p}<0.001)$ at baseline. Physical activity predicted higher but non-significant odds of quitting $(1.67,0.80$ $3.49, \mathrm{p}=0.17$ ) at follow-up.

Conclusions: This was first study found that physical activity was associated with quitting in Chinese smokers. Prospective studies with better measurement of physical activity and larger sample size of smokers are needed to confirm the results.

Tob. Induc. Dis. 2018;16(Suppl 1):A848

DOI:10.18332/tid/84222

\section{2:30-14:00}

PS-1105-4 Stoptober: an effective way for smokers to quit for $\mathbf{2 8}$ days, with five times more chance to stop permanently

I Asruf'1, L Houtenbos'

'Dutch Cancer Society, Prevention, Amsterdam, Netherlands. E-mail: iasruf@kwf.nl

Background and challenges to implementation: In 2016, based on the concept of Stoptober in the United Kingdom the third Stoptober campaign started in the Netherlands. This campaign is organised by the Dutch Ministry of Health \& Welfare, the Dutch Cancer Society and other non-governmental organisations. Stoptober encourages smokers to quit for 28 days in October. Smokers who quit smoking for 28 days are 5 times more likely to stop permanently. The burden to quit also becomes lower because smokers are encouraged to stop for a manageable period of 28 days. This period is however long enough to change attitude towards quitting positively, and behaviour permanently. In 2016, the goal was set on 50.000 participants.

Intervention or response: The campaign consisted of several communication tools. Besides traditional communication tools like radio and TV, Stoptober focused on social media and online tools, like the Stoptober application, the website and the interaction with rokeninfo.nl (managed by the Netherlands Institute of Mental Health and Addiction). To improve publicity, the campervan tour was further improved and healthcare professionals were asked to promote Stoptober to their patients. Lastly, Stoptober was focused on improving the duration of participation and the counselling of the participants. The budget of Stoptober was $€ 600.000$.

Results and lessons learnt: 53.303 people participated in 2016 .
$92 \%$ participated because they wanted to stop permanently, instead of only 28 days. $54 \%$ of the participants came in contact with Stoptober through radio and TV, $20 \%$ through social media and $12 \%$ through friends and family. \pm 140.000 people visited the website, while the application was downloaded \pm 48.000 times. $70 \%( \pm 37.000)$ of the total participants achieved to quit smoking for 28 days. Of those, $73 \%$ still stopped after 3 months.

Conclusions and key recommendations: Despite of a relatively low budget, Stoptober reached its targeted population. Stoptober is effective in changing people's attitude towards quitting positively and supporting smokers to quit for 28 days.

Tob. Induc. Dis. 2018;16(Suppl 1):A849

DOI: $10.18332 /$ tid/84246

\section{2:30-14:00}

PS-1106-4 Inter professional initiative using service training to enable a fisher folk community to quit tobacco habits

RM George ${ }^{1}$

${ }^{1}$ A.J. Institute of Dental Sciences, Public Health Dentistry, Mangalore, India. E-mail: drmariamgeorge@gmail.com

Background and challenges to implementation: Fisher folk are said to use tobacco products to avoid sea sickness and to stay awake during night, while working at sea. The people in this community have only minimal education, low income and are unaware of harmful effects of tobacco. The present dental curriculum does not emphasise on training undergraduates on anti-tobacco counselling. Dentists are in a strategic position to diagnose oral cancerous and precancerous lesions and facilitate tobacco cessation. As tobacco addiction is based on physical, psychological and behavioural factors, it could be managed through multifactorial approach by inter professional collaboration. The aim of study was to assess the effectiveness of inter professional initiative using service training to enable a fisher folk community to quit tobacco habits.

Intervention or response: A training module was formulated by an inter professional team consisting of psychiatrist, public health dentist, tobacco interventionist and primary health care workers. Thirty dental interns participated in training programme on lifestyle of fisher folk, tobacco epidemic, harmful effects of tobacco use and tobacco cessation. The interns were also trained for role play in local language, conveying the same message, to empower them to conduct awareness programmes in the community. A house to house survey was conducted by primary health workers in 208 houses of the community to assess knowledge and attitude of fisher folk towards tobacco use before and after tobacco Intervention Programme

Results and lessons learnt: There was highly significant increase in knowledge and skills of dental interns after Tobacco Intervention Training programme and $52 \%$ increase in the number of smoke free homes in the community.

Conclusions and key recommendations: The inter professional initiative empowered interns to incorporate tobacco cessation in routine dental practice and effectively enabled fisher folk community in adopting smoke free home policy. This initiative can be incorporated into the dental curriculum for raising future generation of dentists trained in tobacco cessation.

Tob. Induc. Dis. 2018;16(Suppl 1):A850

DOI:10.18332/tid/84249

12:30-14:00

PS-1107-4 Tobacco consumption prevalence among nursing students and their knowledge in tobacco addiction: ECTEC study 
A Baena ${ }^{1,2}, \mathrm{C}^{2}$ Martínez ${ }^{1,2}, \mathrm{M} \mathrm{Fu}^{1,2}$, Y Castellano ${ }^{1,2}, \mathrm{M}^{2}$ Margalef ${ }^{1,2}$, E Gavilan ${ }^{3}$, E Fernandez ${ }^{1,2}$, ECTEC Research Group

${ }^{1}$ Catalan Institute of Oncology, Tobacco Control Unit, Hospitalet de Llobregat, Spain, ${ }^{2}$ Bellvitge Biomedical Research Institute (IDIBELL), Cancer Control and Prevention Group, Hospitalet de Llobregat, Spain, ${ }^{3}$ General Hospital of Vall d' Hebron, Surgical Area, Barcelona, Spain. E-mail: abaena@iconcologia.net

Background: Nurses can positively influence in tobacco prevention and treatment. It is during undergraduate-years when students should acquire knowledge, and skills in smoking cessation; however, nursing students' knowledge has not been yet assessed in Catalonia. Therefore, the objective of our study was to analyse the smoking prevalence of nursing students and the determinants, consumption pattern, training received and knowledge in tobacco control.

Methods: Multicentre prevalence study by self-completion questionnaire, directed to all students enrolled in the 15 nursing schools in Catalonia during 2015-16. The $57.2 \%$ of the students participated in our study $(\mathrm{N}=4,381)$. The questionnaire included questions about tobacco use, training and knowledge on smoking cessation. Logistic regression models (OR and $95 \% \mathrm{CI}$ ) were used to assess the association within tobacco consumption and the independent variables (age and studying year).

Results: The participation by nursing schools was heterogeneous, varying from $35.4 \%$ to $81.9 \%$. The $83.2 \%$ of the sample were women. The $31.2 \%$ of the students smoked. Differences in prevalences were only found by schools $(22.4-41.5 \%$; $<<0.01)$. Most students received training on tobacco consumption: smoking risks (80.6\%) and secondhand smoke (86.3\%), but not on treatment (32.4\%). The $98.1 \%$ knew that smoking was an addiction and the $98.4 \%$ that secondhand smoke was unhealthy. Being smoker was related to an older age $(\mathrm{OR}=1.25 ; \mathrm{CI}: 1.05-1.49$ from 20 to 21 years old; $\mathrm{OR}=1.82$;CI:1.54-2.16 in $\geq 22$ years old), higher level of self-reported knowledge (OR=1.31,CI:1.11-1.55) and with a lower evaluated knowledge ( $\mathrm{OR}=0.81, \mathrm{CI}: 0.67-0.97)$.

Conclusions: The smoking prevalence among nursing students in Catalonia is high and increases with age, without differences by sex. Although students affirmed having received training on tobacco consumption during its degree, their level of knowledge is low for treatment. Smokers have overestimated their training since they had lower evaluated knowledge. It would be recommendable to impart training that qualifies nursing students to help smokers to quit.

Tob. Induc. Dis. 2018;16(Suppl 1):A851

DOI:10.18332/tid/84254

\section{2:30-14:00}

PS-1108-4 Effects of SMS to quit on quit rate and satisfaction among smokers calling Thailand National Quitline (preliminary analysis)

J Yunibhand', S Rojnawee ${ }^{2}$

'Thailand National Quitline, Bangkok, Thailand, ${ }^{2}$ Chulalongkorn University, Faculty of Nursing, Bangkok, Thailand. E-mail: yuni_jintana@hotmail.com

Background: Thailand National Quitline (TNQ) provides reactive and proactive counseling without nicotine replacement therapy as well as information to callers to help persons quit smoking. TNQ began providing service on 2009. Since 2011, the TNQ call system and data-base system have been continuously developed for service improvement. TNQ's created an interventions delivered via text messaging (short message service, SMS) may increase access to the services that promote smoking cessation across diverse populations.

Methods: This quasi-experimental study aims to study the effect of SMS to Quit on quit attempt, quit rate over 7 days and 1 months, and satisfaction among smokers who called the Thailand National Quitline (TNQ) between September 2016 to February 2017. A sample of 650 smokers was voluntary recruited. Data were collected from TNQ's database and telephone interviewing throughout September 2016 to June 2017. Research instruments included 1) demographic data and smoking screening, 2) the Quit Smoking Questionnaire, and 3) the Satisfaction Questionnaire. From 650 participants, there were 386,143 , and 121 smokers who received both SMS to quit and telephone counseling, SMS to quit, and counseling, respectively. The majority of participants (85.85\%) were males. Almost fifty percent of the participants were aged between 25 and 44 years. The average cigarette used per day was 14 cigarettes $(\mathrm{SD}=6.72)$ and average duration smoked was 9.23 years $(\mathrm{SD}=1.67)$. Data were analyzed with descriptive statistics and logistic regression.

Results: The participants who received SMS had stop smoking for at least 24 hours (quit attempt) and continuous abstinence rates over 1 month statistically significant higher than those who received telephone counseling and both SMS and counseling $(\mathrm{p}<.05)$. A difference in significance did not show on analysis of caller's satisfaction with those in three groups.

Conclusions: SMS to quit was effective for helping smokers were able to change their behaviors.

Tob. Induc. Dis. 2018;16(Suppl 1):A852

DOI:10.18332/tid/84261

\section{$12: 30-14: 00$}

PS-1109-4 A follow up of activity and clinical experience among Swedish tobacco cessation counsellors 1-12 years after training

H Gilljam ${ }^{1}$

${ }^{1}$ Karolinska Institutet, Stockholm, Sweden. E-mail: hans. gilljam@ki.se

Background: Sweden has one of the lowest smoking rates among developed countries but according to The Tobacco Control Scale 2016 Sweden shares a disappointing 9th place among European countries in implementing tobacco control. Earlier studies of smoking cessation activities have been few and inconsistent. By an NGO initiative in 2005, a comprehensive national training standard for tobacco cessation was established. Since then 930 individuals have completed the training and have been certified.

Methods: In 2016 an anonymous e-mail survey was sent to the 21 certified training centres and forwarded to cessation specialists certified from 2005. Twenty per cent of the addresses were no longer in use leaving 740 possible responders.

Results: A total of 542 valid responses were received, or $73 \%$. Responders were $97 \%$ female and $3 \%$ male. Most $(80 \%)$ were active in cessation. A majority, or $58.7 \%$ worked $0.5-2 \mathrm{hrs}$./week with smokers, $23,7 \%$ spent $2.5->20 \mathrm{hrs}$./w and $17.7 \%$ were inactive. No one reported demand for snus cessation. Almost all $(90 \%)$ counselled patients individually. Three out of four responders were nurses or midwifes, working in primary care. Fifteen per cent worked in hospitals. The support for smoking cessation in their organisation was judged as "good/very good" by $43 \%$, "neither good nor bad" by $44 \%$ and "bad" by $5 \%$. It was calculated that on average, a cessation expert treats 22 patients/ year.

Conclusions: This is the first national report on the clinical experiences of the large number of smoking cessation counsellors 
1-12 years after their certification. The results show a high response rate and 4 out of 5 responders are clinically active in smoking cessation. The results are encouraging and much more convincing than earlier studies. Still, better standardisation and continuous monitoring could further improve the service.

Tob. Induc. Dis. 2018;16(Suppl 1):A853

DOI:10.18332/tid/84264

\section{$12: 30-14: 00$}

PS-1110-4 Could be applied genetic markers to smoking cessation treatment?

PV Gaya' ${ }^{1}$, S Scholz'1, JR dos Santos², PRX Tomaz ${ }^{2}$, TM0 Abe ${ }^{1}, \mathrm{M}$ Nassif Junior ${ }^{1}$, AC Pereira ${ }^{2}$, PC dos Santos Junior ${ }^{3}$

${ }^{1}$ Instituto do Coração - HCFMUSP, Prevenção, São Paulo, SP, Brazil, ${ }^{2}$ Instituto do Coração - HCFMUSP, Laboratório de Genética, São Paulo, SP, Brazil, ${ }^{3}$ Universidade Federal de São Paulo, Departamento de Farmacologia, São Paulo, SP, Brazil. E-mail: jaquelineincor@yahoo.com.br

Background: The interruption of smoking is the best way to prevent diseases. The perspective of using genetic markers, which indicate more effectiveness to the tobacco treatment, can change this scenario bringing more objectivity in the treatment of this disease. Pharmacogenomics of smoking treatment was evaluated in over 1000 patients in our Smoking Cessation Center between 2009 to 2014 period. We found the association of the CHRNA4 rs 1044396 polymorphism with varenicline therapy. Patients with CT or TT genotypes had a higher chance of success than $\mathrm{CC}(\mathrm{OR}=1.67 ; \mathrm{CI}=1.10-2.53 ; \mathrm{p}=0.02)$. We also found the association of the CYP2B6 rs2279343 polymorphism with bupropion therapy. Patients with AA genotype had a higher chance of success compared to AG or GG genotypes (OR = 1.92; $\mathrm{IC}=1.08-3.42 ; \mathrm{p}=0.03)$. It was a retrospective study, so we started in 2015 a prospective study to identify the frequency of these polymorphisms in smokers who looks for smoking cessation programs.

Methods: We collected data and blood sample from 127 smokers (october 2015 to december 2016) to investigate Bupropion CYP2B6 rs2279343 and Varenicline CHRNA4 rs 1044396 polimorphisms.

Results: The average of age was $50+9$ (20 -74 years), $80 \%$ caucasian, $14 \%$ afro descend, $6 \%$ yellow. The polymorphism favoring Bupropion (AA) was found in 58.2\% (74) and favoring to varenicline (CT and TT) in $70 \%$ of the sample (89). Important to say that 42 participant had both polymorphisms favoring to varenicline and to Bupropion (33\%).Only 12 smokers presented both unfavorable polymorphisms to any of these drugs.

Conclusions: The prospect of choosing the drug based on this finding may have a relevant economic impact, eventhough trials shows that varenicline is more efective than bupropion, Bupropion is cheaper than varenicline. We already started a randomized trial to test the cost-effectiveness of this hypothesis.

Tob. Induc. Dis. 2018;16(Suppl 1):A854

DOI:10.18332/tid/84277

\section{2:30-14:00}

PS-1111-4 Association of smoking opinions and risk perception with smoking status in Serbia adults B Kilibarda ${ }^{1}$, M Milovanovic ${ }^{2}$, N Martinson ${ }^{2,3,4}$, K Foley $^{5}$ ${ }^{1}$ Institute of Public Health of Serbia, Office for Smoking Prevention, Belgrade, Serbia, ${ }^{2}$ University of the Witwatersrand, Perinatal HIV Research Unit, Soweto, South Africa, ${ }^{3}$ Johns Hopkins University School of Medicine, Center for Tuberculosis
Research, Department of Medicine, Baltimore, MD, United States of America, ${ }^{4}$ SoMCHAT MRC Collaborating Centre for HIV/AIDS and TB, Johannesburg, South Africa, ${ }^{5}$ Wake Forest School of Medicine, Department of Social Sciences and Health Policy, Winston-Salem, NC, United States of America. E-mail: kilibarda_b@yahoo.com

Background: Overall, Serbian adults are aware of the association of smoking with malignant diseases. However, it is not known whether adult smokers perceive this a risk for future concern. The study objective is to understand how smoking related opinions and risk perception in Serbian adults is associated with smoking status in order to support planning of targeted interventions.

Methods: Secondary analysis of data drawn from a 2014 cross sectional, National Survey on Lifestyles of Citizens in Serbia. Multivariate logistic regression was used to determine the association of smoking opinions and risk perception with smoking status.

Results: A total sample of 5385 adults aged 18-64 years were analysed with a smoking prevalence of $39.8 \%$. Tobacco use ranked 15th of 17 listed problems in Serbia by its perceived importance. Opinions on tobacco use as a problem significantly differs by smoking status $(\mathrm{p}<0.001, \mathrm{x} 2=90.04)$. Among those $(75.5 \%)$ who report tobacco use as rather important or an important problem, $44.5 \%$, are non-smokers, $36.5 \%$ are current smokers and $19.1 \%$ ex-smokers. There is also a significant difference $(\mathrm{p}<$ $0.05, \mathrm{x} 2=329.22$ ) in risk perception according to smoking status, $66.5 \%$ of adults perceived smoking one or more packs of cigarettes per day to be great risk, of which $48.9 \%$, are non-smokers $31.7 \%$ are smokers and $19.4 \%$ are ex-smokers. Those who think tobacco is an important problem compared with people who think it is not a problem (OR 1.6, p< 0.001) and those who perceive smoking as not risky $(\mathrm{OR}, 7.0, \mathrm{p}<0.001)$ compared with those who think it is a great risk, have higher odds of being smokers.

Conclusions: Despite high prevalence, smoking is not recognized as important problem in society. Among current smokers, risk perception is low. Findings call for interventions aimed at changing risk perception and opinions on importance of tobacco control.

Tob. Induc. Dis. 2018;16(Suppl 1):A855

DOI: $10.18332 /$ tid/84280

\section{$12: 30-14: 00$}

PS-1112-4 Impact of smoke-free home on smoking intensity in low- and middle-income countries

D Owusu1, M Quinn², K-S Wang ${ }^{2}$, J Aibangbee², RM John³, HM Mamudu ${ }^{4}$

${ }^{1}$ Georgia State University, School of Public Health, Atlanta, GA, United States of America, ${ }^{2}$ East Tennessee State University, Biostatistics and Epidemiology, Johnson City, TN, United States of America, ${ }^{3}$ Indian Institute of Technology, Jodhpur, Rajasthan, India, ${ }^{4}$ East Tennessee State University, Health Services Management and Policy, Johnson City, TN, United States of America.E-mail: dowusu2@gsu.edu

Background: Smoke-free policy is associated with smoking reduction; however, it is unknown if the reduction in smoking intensity occurs across the stages of smoking cessation, and there is paucity of literature on the relationship between home smoking rule and smoking intensity in low- and middle-income countries (LMICs). The aim of this study was to conduct a cross-country evaluation of the relationship between home smoking rule and smoking intensity among smokers in different stages of smoking cessation.

Methods: Data from 39,204 current smokers from the Global 
Adult Survey (GATS), 2009-2012 from 17 LMICs were analyzed. Weighted multiple linear regression analyses were conducted using the log of smoking intensity as the outcome variable with home smoking rule as the main independent variable. Adjusted regression coefficients $(\beta)$ with associated $95 \%$ confidence intervals $(\mathrm{CI})$ were estimated.

Results: Overall, the average smoking intensity was approximately 15,14 , and 13 for home smoking allowed, smoking restriction and smoke-free rules, respectively. There was a $12.7 \%(95 \% \mathrm{CI}=7.6 \%$ $17.8 \%)$ and a $22.5 \%(95 \% \mathrm{CI}=17.1 \%-28.0 \%)$ reduction in smoking intensity among adults in precontemplation from homes with smoking restriction and smoke-free rules, respectively. Among adults in contemplation, smoking restriction and smoke-free rules were associated with a $21.5 \%(95 \% \mathrm{CI}=6.0 \%-36.9 \%)$ and an $18.6 \%(95 \% \mathrm{CI}=9.0 \%-28.2 \%)$ reduction in smoking intensity, respectively. For adults in preparation, smoke-free rule was associated with a $19.4 \%(95 \% \mathrm{CI}=3.9 \%-34.9 \%)$ reduction in smoking intensity.

Conclusions: Smoke-free homes are associated with a significant reduction in smoking intensity across the first three stages of the transtheoretical model. This suggests that, banning public smoking may have added benefit by reducing smoking intensity, since evidence suggests that people who work in smoke-free environments are more likely to live in smoke-free homes.

Tob. Induc. Dis. 2018;16(Suppl 1):A856

\section{DOI:10.18332/tid/84286}

\section{2:30-14:00}

PS-1113-4 Incentive-based interventions for smoking cessation: early findings from the SMILE Trial in Thailand

\section{J White ${ }^{1}$, N Srivirojana $^{2}$, A Jampaklay ${ }^{2}$, W Dow $^{3}$}

'University of California San Francisco, Philip R. Lee Institute for Health Policy Studies, San Francisco, CA, United States of America, ${ }^{2}$ Mahidol University, Institute for Population and Social Research, Salaya, Thailand, ${ }^{3}$ University of CaliforniaBerkley, School of Public Health, Berkeley, CA, United States of America. E-mail: justin.white@ucsf.edu

Background: Smoking cessation services are not widely available in many low-resource settings. In this study, we tested several incentive-based interventions in a scalable workplace setting in a middle-income country.

Methods: We undertook a cluster randomized controlled trial of factories located in the metropolitan area of Bangkok, Thailand. We randomly assigned 101 worksites from 84 companies ( $n=4,241$ smokers) to treatment arms that varied on two crossrandomized factors: presence of a deposit contract and type of bonus (none, individual, or team). The deposit contract required a minimum deposit of about $\$ 3$ in a tamper-proof personal box, with voluntary contributions thereafter. A participant forfeited all deposits if he or she failed to abstain from smoking at 3 months. The individual bonus involved a payment for abstaining of either $\$ 20$ or $\$ 40$, randomly varied by worksite. The team bonus involved a $\$ 40$ payment if the person and a randomly assigned teammate each abstained. An additional arm included a deposit contract plus a teammate, but without any further incentives. All arms received smoking cessation counseling. All incentives depended on smoking status at 3 months, with biochemically verified abstinence collected at 3,6 , and 12 months.

Results: Intervention take-up was high across all arms, notably $>50 \%$ in deposit arms. Biochemically verified 7-day smoking abstinence at 6 months was highest in the $\$ 40$ individual bonus arm, a $72 \%$ (9 percentage point) increase relative to the control group. There were no strong interaction effects between deposits and the bonus, nor large team effects. Relapse was low following the end of the incentives.

Conclusions: This study advances the literature on the use of deposit contracts and social and monetary incentives to promote smoking cessation in low-resource settings. It provides some of the first rigorous evidence on the value of supplementing a basic deposit contract with individual and social incentives.

\section{Tob. Induc. Dis. 2018;16(Suppl 1):A857}

DOI: $10.18332 /$ tid $/ 84300$

\section{2:30-14:00}

PS-1114-4 A factor analysis of the Fagerstrom Test for Nicotine Dependence for bidi smokers and smokeless tobacco users (FTND): findings from India

D Persai ${ }^{1}, \mathrm{R}$ Panda ${ }^{2}$

${ }^{1}$ Public Health Foundation of India, Gurgaon, India, ${ }^{2}$ Public Health Foundation of India, Delhi, India. E-mail: dpersai@ gmail.com

Background: Fagerstrom Test for Nicotine Dependence (FTND) is widely used screening instrument for physical nicotine dependence. Psychometric properties of FTND have been validated among cigarette smokers, but the reliability and validity of its variant for smokeless tobacco users and bidi smokers is not well documented. The present study aims to evaluate reliability, construct validity, and structure model of FTND among bidi smokers and smokeless tobacco users.

Methods: A cross sectional survey was conducted among 1350 patients visiting primary care facilities in two states of India in 2016. FTND contains six items that evaluate the quantity of tobacco consumption and dependence. Cronbach's coefficient alpha was estimated to evaluate reliability of the FTND scale. We applied exploratory factor analysis (EFA) with principal component extraction and varimax with Kaiser Normalization rotation to ascertain the factor structure of the FTND scale. Eigen values greater than 1 were used as a criterion for factor extraction.

Results: Out of 1350 tobacco users 406 (30\%) were smokers, 818(60\%) were smokeless tobacco users with mean FTND scores of $4.29 \pm 1.67$ and $4.42 \pm 1.72$ respectively. Cronbach's alpha coefficients were low for FTND among bidi smokers (FTND-0.47) and smokeless tobacco users (FTND-0.32). FTND score did not predict the intention to quit tobacco use. The results of exploratory factor analysis suggest eigenvalues greater than 1 for item 1(How soon after you wake up do you smoke your first cigarette) and Item 4 (How many cigarettes per day do you smoke?). The other items in the scale add no relevant information.

Conclusions: Items of the FTND are best modeled as two correlated factors on tobacco use. A short version of FTND i.e. Heaviness of smoking index may represent an alternative to FTND. Future research should focus on refining questionnaires that more precisely measure nicotine dependence in bidi and smokeless tobacco users in primary care.

Tob. Induc. Dis. 2018;16(Suppl 1):A858

DOI: $10.18332 /$ tid $/ 84303$

12:30-14:00

PS-1115-4 Systems approach in tobacco dependence treatment through hospitals - a one year study from Jaipur, India 
R Gupta', S Narake ${ }^{2}$

${ }^{1}$ Rajasthan Cancer Foundation, President, Jaipur, India, ${ }^{2} \mathrm{Healis}$ Sekhsaria Institute for Public Health, Biostatistics, Navi Mumbai, India.E-mail: rakesh.gupta.acs@gmail.com

Background: Systems approach in tobacco dependence treatment is missing in India despite low quit rates. The attitude of the users and healthcare professionals is a major barrier. The objectives of this study were to determine whether:

(1) tobacco dependence treatment can be delivered in a hospital setting; and,

(2) its results will provide higher quit rate vs. no treatment.

Methods: This one year study (2013) undertaken at a tertiarycare multi-specialty hospital at Jaipur, India identified currently tobacco using patients. Those willing were assisted to quit through brief intervention (BI). Those desirous or referred for intensive treatment (II) were treated in Tobacco Cessation Clinic (TCC). All were followed-up telephonically after 1 week, 1 month, 6 months and in April 2014. The responders were categorized as Successful quit, Failed to quit and Relapsed.

Results: This study enrolled 1264 patients out of 19657 (6.43\%). In $43.4 \%$ patients $(549 / 1264)$ who consented for the treatment, while overall quit rate was $26.1 \%$ (CI: 23.8- 28.6), in the BI group it was $54.2 \%$ (CI: 49.7-59.0) and in the II group it was $84.9 \%$ (CI: 78.4-92.0) vs. zero quit rate in untreated. The use of pharmacotherapy improved overall quit rate by $14.6 \%(71.1[63.0,78.1]$ in those treated with pharmacotherapy vs. $56.5[51.7,61.2]$ who were counseled only); the quit rate was significantly higher in smokers and dual users vs. users of smokeless tobacco (SLT users). The quit rates did not differ for the type of tobacco user except in smokeless tobacco (SLT) users in first six months of follow-up.

Conclusions: This study achieved both its objectives of being able to deliver tobacco dependence treatment in a hospital setting; and, achieving higher quit rates. Hence, a replication of this model is recommended to establish the envisioned utility in the country as well as other low- and middle- income countries.

Tob. Induc. Dis. 2018;16(Suppl 1):A859

DOI: $10.18332 /$ tid $/ 84320$

\section{2:30-14:00}

PS-1116-4 Characteristics of clients with nicotine dependence and short-term abstinence: findings from the USM Tobacco Quitline Service in Malaysia MF Razali', S Samsudin', M Omar', B Tangiisuran², A Misnan', NA Abd. Rani', NF Abd. Ghani', N Badaruddin'

'Universiti Sains Malaysia, Clearinghouse for Tobacco Control, National Poison Centre, Minden, Malaysia, ${ }^{2}$ Universiti Sains Malaysia, School of Pharmaceutical Science, Minden, Malaysia. E-mail: fadhli_razali@usm.my

Background: Globally, quitlines have been shown to be an effective means for smoking cessation. The success of quitting among smokers is contributed by many factors such as nicotine dependence, motivation, income and educational levels, previous quit attempts, environmental characteristics and quit methods. This study will determine the characteristics of clients with nicotine dependence and short- term abstinence (one month duration) among those registered with the USM Tobacco Quitline Service.

Methods: Secondary analysis of USM Tobacco Quitline clients with the age range between 18-80 years old who completed initial assessment (one week pre Quit Date (QD)) and have been followed-up within one month post QD. The Fagerstrom Test for Nicotine Dependence (FTND), which is a validated six-item scale, was used to assess the nicotine dependence among those that registered for Quitline service one week prior to QD. Continuous abstinence (relapse or continue quitting) data was obtained for one month post QD.

Results: Of the 418 registered clients, $68.2 \%(n=285)$ confirmed their registration. Sixty four clients completed their initial assessment and were followed-up within one month post QD. Majority (93.8\%) of them were males with the mean age of 44 . All clients with high FTND score and $95.5 \%$ clients with moderate FTND score successfully achieved one month smoke- free (shortterm abstinence) higher than clients with mild FTND score.

Conclusions: The findings showed that smokers with high and moderate FTND scores were able to achieve better short-term abstinence compared to those with mild FTND score. Further research needs to be conducted to understand the reason for this behaviour. This result does not take into account other abstinence factors. A long-term abstinence data would provide an insight on the ways to improve the service particularly in the diverse client background.

Tob. Induc. Dis. 2018;16(Suppl 1):A860 DOI: $10.18332 /$ tid/84326

\section{2:30-14:00}

PS-1117-4 GB smoking cessation training for health care providers in LA: midterm confidence in competencies and estimation of population impact

G Zabert $^{1,2,3}$, B Champagne ${ }^{1,4}$, E Cruz $^{1,5}$, E Estevez ${ }^{1,6}$, D Buljubasich $^{1,7}$, R Sansores ${ }^{1,8}$, P Cortez $^{1,9}$, F Zabert $^{10}$

'Global Bridges, Rochester, MN, United States of America, ${ }^{2}$ Asociacion Latinoamericana del Torax, Montevideo, Uruguay, ${ }^{3}$ Universidad Nacional del Comahue, Facultad de Ciencias Medicas, Neuquen, Argentina, ${ }^{4}$ Interamerican Heart Foundation, Dallas, TX, United States of America, ${ }^{5}$ nstituto Nacional de Salud Pública, Cuernavaca, Mexico, ${ }^{6}$ Fondo Nacional de Recursos, Montevideo, Uruguay, ${ }^{7}$ Asociacion Argentina de Medicina Respiratoria, Rosario, Argentina, ${ }^{8}$ Asociacion Latinoamericana del Torax, Mexico, Mexico, ${ }^{9}$ nter-American Hearth Foundation México (FIC Bolivia), La Paz, Bolivia, 'OCET, Neuquen, Argentina. E-mail: gzabert@gmail.com

Background: Between 2011 and 2016, Global Bridges (GB) in Latin America successfully trained health care providers (HCP) in declarative and procedural knowledge and confidence in competencies (Moore`s level 3A, B and 4). However, a study that explored patients' reported HCP performance failed to show effect.

Methods: Cross-sectional study among all trainees between 2011 and 2016. The survey was electronically conducted (Survey Monkey) with 26 questions that explored demography, confidence in skills and practices in smoking cessation. A sample size of 400 individuals was required. Inclusion criteria were: attended at least one day session and a valid email. A mathematical model of impact estimation was developed (subrogate of level 5 of Moore). Abstinence rates for brief advice and intensive intervention of were appraised from Cochrane Review.

Results: Of 1915 trainees with valid e-mail, 402 surveys were obtained in 2 recruitment rounds. The response rate was different for the two periods 2011-2014 (17\%) and 2014-2016 (56\%). Countries (Argentina 28\%, Uruguay 20\% and Mexico $17 \%$ ) and occupation (physicians $61 \%$ and psychologists 18\%) were unevenly represented. Among the responses, female gender prevailed (64.7\%). $79.4 \%$ stated GB training was extremely/ very useful and more than $80 \%$ expressed to be very confident/ confident to provide SC support. Respondents declared to assist 
2.1 smokers per day (CI 1.6-2.6), and to offer brief advice and intensive advice, with or without drugs, in $80 \%$ and $69 \%$ of cases respectively. The model estimated that 1,564 trainees would have achieved 12,012 quitters with brief advice and 38,788 quitters due to intensive intervention in the following year after training, in the worst case scenario (lower $\mathrm{CI}$ ).

Conclusions: GB's SC program in Latin America exhibited to maintain trainees' confidence in competences in the medium term and to return at least 50,800 quitters in the following year of the educational intervention.

Tob. Induc. Dis. 2018;16(Suppl 1):A861

DOI:10.18332/tid/84327

\section{2:30-14:00}

PS-1118-4 Strengthening quit smoking services in Malaysia through Malaysia Quit (mQuit) Program N Hassan ${ }^{1}, \mathrm{~N}$ Baharom ${ }^{1}$, ND Dawam ${ }^{1}$, N Ismail ${ }^{1}$, CE Festus ${ }^{1}, 0$ Mihat $^{2}$, CK Chong ${ }^{3}$

${ }^{1}$ Malaysia Ministry of Health, Tobacco Control Unit \& FCTC Secretariate, Putrajaya, Malaysia, ${ }^{2}$ Malaysia Ministry of Health, Non-Communicable Disease Section, Putrajaya, Malaysia, ${ }^{3}$ Malaysia Ministry of Health, Disease Control Division, Putrajaya, Malaysia.E-mail: noraryana@moh.gov.my

Background and challenges to implementation: Base on the Malaysian National Health Morbidity Survey in 2011, 48.6\% of smokers have made quit attempt in the past 12 months but only $32.4 \%$ visited health care provider (HCP). One of the main challenges of getting professional advice in quitting is due to limited cessation services within public clinics and hospital. To overcome this and in line with Article 14 WHO Framework Convention on Tobacco Control (FCTC), Malaysia has developed a holistic and structured program under Malaysia Quit or mQuit services.

Intervention or response: The mQuit services was inaugurated on 27th November 2015 through public-private partnership with the objective to make smoking cessation services accessible throughout the public and private sectors. The services were further enhanced with a quitline counselling system and a website to promote and facilitate registration of smokers to cessation program through www.jomquit.moh.gov.my. Both mQuit providers in public and private sectors have to fulfil standard criteria set by the Ministry of Health before accreditation given and the list is made available in the jomquit website. To date, 160 private $\mathrm{HCP}$ and 764 government health clinics and hospitals have become mQuit providers.

Results and lessons learnt: The number of registered patients have increased from 7757 in 2015 to 10791 in 2016 . Since services start in private sector, the total number registered with private mQuit providers has increased from 10 patients in January 2017 to total of 394 patients until June 2017. The total number of registration through jomquit website was 420 patients. The implementation of the mQuit encountered a few challenges at first. Challenges and recommendations are discussed with all partners and remedial measures were applied to improve the services.

Conclusions and key recommendations: The mQuit services has increased accessibility to smoking cessation services in Malaysia.

Tob. Induc. Dis. 2018;16(Suppl 1):A862

DOI:10.18332/tid/84344

12:30-14:00

PS-1119-4 Cessation attempts in dual users (smoking plus smokeless): findings from two states in India R Panda ${ }^{1}$, D Persai ${ }^{1}$
${ }^{1}$ The Public Health Foundation of India, Research and Policy, Gurgaon, India. E-mail: raj.panda@phfi.org

Background: Concurrent use of smoking and smokeless tobacco products is common, but little is known regarding the association of smokeless tobacco use with smoking cessation. The present study aims to compare intention to quit and quit attempts among current smokers who also use smokeless tobacco (dual users) and exclusive smokers

Methods: A cross-sectional study was conducted among 1360 respondents (542 smokers and dual users; 818 smokeless tobacco users) visiting 22 primary care facilities in two high tobacco burden states of India in 2016. The health facilities and patients were chosen using systematic random sampling. We compared intention to quit between dual users and exclusive smokers, using multivariate regression model

Results: Out of 542 tobacco users, about $70 \%$ (378) were smokers and 30\% (164) were dual tobacco users. Exclusive Smokers (62\%) were more likely than dual users (38\%) to have an intention to quit tobacco even after adjusting for demographic differences and tobacco dependence levels (OR:1.75; 95\% CI 1.19-2.57). About $27 \%$ of smokers made an attempt to quit as compared to $23 \%$ of dual users. The quitting attempt last for less than 6 months for about $20 \%$ of smokers (19\%) and smokeless tobacco users (17\%). Less than $10 \%$ of smokers and smokeless tobacco users used counseling technique for tobacco cessation.

Conclusions: Smokers are more likely to intend to quit smoking and have recently made quit attempts as compared to dual users. Findings document a high level of interest in quitting among smokers than dual users suggesting the need to develop targeted interventions for dual users and smokeless tobacco users to enhance cessation attempts. Further population prospective studies are needed to understand the issues faced by dual users as they make an attempt in quitting tobacco.

Tob. Induc. Dis. 2018;16(Suppl 1):A863

DOI:10.18332/tid/84349

\section{2:30-14:00}

PS-1120-4 Increasing reach for smoking cessation service in primary care setting

J Yunibhand $^{1}$, K Thanomsat ${ }^{1}$

Thailand National Quitline, Phayathai, Thailand. E-mail: yuni_jintana@hotmail.com

Background and challenges to implementation: Thailand National Quitline (TNQ) has been established in order to provide smoking cessation service for Thai smokers. Almost a decade, the TNQ has been promoted based on television advertising which were very costly. Consequently, this study aims to develop the consolidation between the TNQ service and primary care service in order to Increasing reach for smoking cessation service in primary care setting in accordance with the policy of The WHO Framework Convention on Tobacco Control (WHO FCTC). Furthermore, this will be a new approach for effective and sustainable quitline promotion strategy.

Intervention or response: The study design was a quasiexperimental design which divided the subjects into experimental $(\mathrm{n}=123)$ and control $(\mathrm{n}=108)$ groups from primary health care community centers. For the experimental group, the subjects received brief intervention aimed at increasing intention to quit by village health volunteers who were trained and equipped with smoking cessations toolkits for providing smoking cessation service in primary care settings, whereas the control group the 
subjects received usual care. Relative risk was used to analyze the ratio of the probability of the outcomes between experimental and control group.

Results and lessons learnt: The findings revealed that participants in the experimental group, $62.60 \%$ in the control group, $5.56 \%$ [RR $28.46(95 \% \mathrm{CI}=11.56,70.04)]$ could access to smoking cessation service by the TNQ. It is considered that the new intervention can successfully Increasing reach for Thai smokers in the community, thus, Increasing smoking service accessibility.

Conclusions and key recommendations: Promoting the TNQ service in the primary care setting significantly increased the proportion among Thai smokers to access the smoking cessation service. Consequently, the expanded reach to smoking cessation service in primary care setting are advantageous for smokers in community.

Tob. Induc. Dis. 2018;16(Suppl 1):A864

DOI:10.18332/tid/84352

\section{2:30-14:00}

PS-1121-4 Introducing tobacco free workplace policy in workplaces in India - challenges and learning

H Gupte'1, G Mandal ${ }^{2}$, V Thawal' ${ }^{1}$, L Chaudhuri $^{1}$

${ }^{1}$ Narotam Sekhsaria Foundation, Mumbai, India, ${ }^{2}$ Salaam Bombay Foundation, Mumbai, India. E-mail: himanshu@nsfoundation. co.in

Background and challenges to implementation: While smoking is prohibited in India in public places and indoor workplaces, there is no regulation about smokeless tobacco use in workplaces as per the Cigarette and Other Tobacco Products Act. Very few companies in India have a documented tobacco free workplace policy addressing smokeless tobacco use.

Narotam Sekhsaria Foundation was selected for implementing Clinton Global Initiative's Tobacco free workplace policy challenge project focusing on India and including smokeless tobacco use. Five workplaces in Maharashtra with total 5000 employees were approached to be a part of this project.

Intervention or response: Activities involved a situational analysis, provision of cessation service and formulation and implementation of a policy document according to the guidelines "Smokefree-in-a-box" toolkit developed by the Global Smokefree Partnership.

Results and lessons learnt: The policy was launched and implemented in only one workplace during one year and 332 employees were provided cessation counselling.

Lessons learnt:

- Organizations are willing to get involved on a pilot basis in representative units and not willing to for the entire company

- They are interested in the tobacco free policy from the point of view of employee wellness

- Organizations are not ready to commit for policy before the cessation services

- Management employees are reluctant to enroll for the cessation services

- Coordination with a number of departments at the company level is required

- Proper and effective communication to all employees regarding the tobacco free policy by the decision makers is required

- Integration of tobacco free workplace activities with their internal processes (e.g. Occupational Health and Safety, Annual Medical Checkup etc.) ensures acceptability.
Conclusions and key recommendations: Implementing a tobacco-free workplace policy in India is required but challenging. It creates an environment conducive to behavioural change and cessation among employees. Integrating it with internal systems and effective communication from the decision makers makes it feasible to implement.

Tob. Induc. Dis. 2018;16(Suppl 1):A865

DOI: $10.18332 /$ tid/84353

\section{2:30-14:00}

PS-1122-4 Why are male Chinese smokers unwilling to quit? - A cross-sectional study on smoking rationalization and intention to quit

P Zheng ${ }^{1}, X$ Huang ${ }^{1,2}$, W Fu ${ }^{1}, \mathrm{H} \mathrm{Li}^{3}, \mathrm{Y}$ Yang $^{4}, \mathrm{H} \mathrm{Fu}{ }^{1}, \mathrm{D} \mathrm{Ding}{ }^{5}, \mathrm{~S}$ Chapman $^{5}$

${ }^{1}$ Fudan University, Shanghai, China, ${ }^{2}$ Pudong New Area Center for Disease Control and Prevention, Shanghai, China, ${ }^{3}$ Guangxi Medical University, Nanning, China, ${ }^{4}$ Mudanjiang Medical University, Mudanjiang, China, ${ }^{5}$ University of Sydney, Sydney, NSW, Australia. E-mail: 13211020016@fudan.edu.cn

Background: While having the largest population of smokers in the world, China is among countries with the lowest percentage of male smokers who have an intention to quit. Smoking rationalization is negatively associated with quit intention in developed countries. Our previous study has showed smoking rationalization is common among Chinese male smokers and some beliefs of smoking rationalization seem to be peculiar to China. Whether the association is generalizable to Chinese smokers remains unknown. This study aims to examine the association between smoking rationalization and quit intention among a sample of male Chinese smokers.

Methods: A cross-sectional survey was conducted among male smokers in China in 2013. Smoking rationalization was assessed through a newly developed Chinese rationalizations scale (including 26 items under six subscales, smoking functional beliefs, risk generalization beliefs, social acceptability beliefs, safe smoking beliefs, self-exempting beliefs and quitting is harmful beliefs). Items in the scale were rated on a 5 -likert scale ( $1=$ totally disagree to $5=$ totally agree). Multivariable logistic regression was performed to examine the relationship between rationalization and intention to quit, adjusted for sociodemographic variables and smoking-related variables.

Results: A total of 3710 participants were included in the analyses. Smoking rationalization was prevalent (mean score 3.32 ). With 1 point increase in total rationalization scale, the odds for smokers intention to quit in the next 6 months decreased $48 \%(\mathrm{OR}=0.52$, 95\%CI: $0.44-0.61 ; \mathrm{p}<0.001)$. Separate logistic regressions for six subscale of rationalization showed consistent associations with intention to quit (all p-values < 0.001). Particularly the "social acceptability beliefs" was the strongest predictor $(\mathrm{OR}=0.62$, 95\%CI: 0.55-0.71; p<0.001).

Conclusions: Smoking rationalization beliefs could be important barriers for male Chinese smokers to develop quit intention. Some beliefs have stronger association with quit intention than others. Tackling rationalization beliefs endorsed by smokers is a potential strategy for smoking cessation intervention.

Tob. Induc. Dis. 2018;16(Suppl 1):A866

DOI:10.18332/tid/84364

12:30-14:00

PS-1123-4 An examination of tobacco initiation and its implications for tobacco control strategies S Mahapatra', R Panda ${ }^{2}$ 
${ }^{1}$ Indian Institute of Public Health, Bhubaneswar- Public Health Foundation of India, Bhubaneswar, India, ${ }^{2}$ Public Health Foundation of India, Gurgaon, India. E-mail: sandeep@iiphb.org Background: Age of tobacco initiation is an important determinant leading to future tobacco dependence. The present study attempted to summarize the age of initiation of tobacco among users and its importance in formulating tobacco control measures.

Methods: A cross-sectional study was conducted among 1360 respondents visiting 22 primary care facilities in two high tobacco burden states of India in 2016. The health facilities and patients were chosen using systematic random sampling. We summarized the age of initiation of the tobacco users and its implications to health. We considered the variable "number of visits to physicians in the last 12 months" as a proxy for the health condition of the respondents.

Results: The average age of initiation of the respondents was 19 years. More than $65 \%$ respondents reported to have started using some form of tobacco (smoking and/or smokeless) between the ages of 16 to 25 years. Majority of these respondents reported to have visited a physician at least 3 to 5 times in past 12 months. Further the findings showed a decreasing trend in tobacco initiation after 25 years.

Conclusions: The study suggests that initiation of tobacco products uptake starts around the age of 16 years and has adverse health implications. Hence, the efforts to prevent initiation of tobacco should not only focus on adults, but also on older youth and young adults. So far the tobacco programs and interventions in India have focused on the adults who are already users. We also need to intervene before tobacco use is taken up as a habit, stressing on not only preventive but also primordial care. Consistent and inescapable interventions should be devised to cater this group to reduce the uptake of tobacco.

Tob. Induc. Dis. 2018;16(Suppl 1):A867

DOI:10.18332/tid/84370

\section{2:30-14:00}

PS-1124-4 Development of an evaluation tool for smoking cessation clinics and services

T-H Lam ${ }^{1}$, Y He${ }^{2}, \mathrm{CO} \mathrm{Jiang}^{3}$, YTD Cheung ${ }^{4}, \mathrm{SY} \mathrm{Ho}{ }^{1}, \mathrm{HCW} \mathrm{Li}^{4}, \mathrm{KK}$ Cheng, Nan Jiang, Shenghua Su ${ }^{5,6,7}, Y$ Tang, Yan Yang, Yuan Jiang, Weihua Zhu, Peiru Lin $8,9,10$

${ }^{1}$ The University of Hong Kong, School of Public Health, Hong Kong, China, ${ }^{2}$ Chinese PLA General Hospital, Institute of Geriatrics, Beijing, China, ${ }^{3}$ Guangzhou No. ${ }^{12}$ Hospital, Guangzhou, China, ${ }^{4}$ The University of Hong Kong, School of Nursing, Hong Kong, China, ${ }^{5}$ University of Birmingham, Institute of Applied Health Research, Birmingham, United Kingdom, ${ }^{6}$ New York University School of Medicine, Department of Population Health, New York, NY, United States of America, ${ }^{7}$ Cadre Health Center of Guangdong Province, Guangzhou, China, ${ }^{8}$ The First Affiliated Hospital of Guangzhou Medical University, Guangzhou, China, ${ }^{9}$ China Tobacco Control Office, Intervention Research Center, Beijing, China, '0Guangzhou Medical University, Guangzhou First Municipal Peoples Hospital, Guangzhou, China. E-mail: hrmrlth@hku.hk

Background and challenges to implementation: Many smoking cessation clinics are passive and dependent on motivated smokers to proactively seek help. Also, smoking cessation clinics are not well publicized. Most healthcare professionals are unaware of the existing smoking cessation services, and rarely refer smokers to these clinics. Many smokers who want to quit do not proactively visit these clinics or call the quitline. Most if not all these clinics and services are not receiving the expected number of smokers and some are dormant or closed down quietly.

Intervention or response: This project, commissioned by the Hong Kong Tobacco Control Office, was to develop and publish an evaluation tool (E-tool, version 1.1) for smoking cessation clinics and services. The project team gathered documentations and data from smoking cessation clinics in Hong Kong, Guangzhou and Beijing. Stakeholders discussed and agreed on what would be the most appropriate items for the E-tool. The E-tool draft was pilot-tested by 27 smoking cessation clinics and hospitals in Hong Kong, China Mainland and Southeast Asian countries (from the WHO Fellowship Programme on Tobacco Control, organized by Hong Kong Tobacco Control Office).

Results and lessons learnt: The finalized E-tool (version 1.1) consists of 33 most basic and essential items in 4 assessment components: Organization assessment (17 points), Environmental assessment (14 points), Process assessment (28 points) and Outcome assessment (11 points). The E-tool and user manual have English, traditional Chinese and simplified Chinese versions. All materials can be freely accessed in https://mrct.sph.hku.hk/ web/.

Conclusions and key recommendations: The E-tool for smoking cessation clinics and services can be used to evaluate the quality and outcomes of existing clinics and services, and provide an outcome-oriented guidance for setting up new clinics and services. We invite all kinds of smoking cessation clinics and services in any territories to use this E-tool, and to provide feedback for the development of more advanced E-tools in the future.

\section{Tob. Induc. Dis. 2018;16(Suppl 1):A868}

DOI:10.18332/tid/84398

$12: 30-14: 00$

PS-1125-4 Explore to operating mode of smoking cessation clinic services in China

Y Nan ${ }^{1}$

${ }^{1}$ Tobacco Control Office, China CDC, Beijing, China. E-mail: haversian@163.com

Background and challenges to implementation: Facing the large amount of smokers and the prevalence trend in China, there is a huge potential demand for smoking cessation services. Using smoking cessation clinic services is recommended by the WHO.

Intervention or response: From 2014, three smoking cessation clinics were asked to set up in each province by the National Health and Family Planning Commission.

Requests of the work include:

1. All the clinics get their personnel trained by Tobacco Control Office, China CDC.

2. Follow the Intervention process developed by the Office.

3. Establish the referral system in the hospital and provide publicity.

4. Conduct effect evaluation and process evaluation.

Results and lessons learnt:

1. Intervention process lasted from July 2014 to June 2016. In the first year, all the clinics were asked to follow the original intervention procedure, which includes one registration and three follow up. The intervention procedure was simplified to one registration and one follow up during the second year.

2. 6286 smokers received the original intervention services, while 7662 smokers received brief intervention.

3. For the original intervention, one-month reporting quitting rate is $23.6 \%$, and continued quitting rate $22.8 \%$. For the 
brief intervention, $30.7 \%$ of the smokers were reported quit smoking during one-month.

4. $52.1 \%$ of the patients were referred by the referral system, $11.4 \%$ by their family members or friends, and $27.9 \%$ by publicity.

Conclusions and key recommendations:

1. The average proportion of both one-month quitting rate reported by the smokers and continued quitting rate were over $20 \%$. Smoking cessation clinic services in China can effectively help smokers quit smoking.

2. The referral system effectively helped smokers access to the clinic and better increased the outpatient volume. Publicity must be conducted in the future work.

3. Brief intervention procedure was more acceptable, compact and feasible. It should be adopted as a standard procedure in the future.

Tob. Induc. Dis. 2018;16(Suppl 1):A869

DOI:10.18332/tid/84417

\section{$12: 30-14: 00$}

PS-1126-4 Effectiveness of a brief, self-determination intervention for smoking cessation (immediate or progressive) among people attending emergency departments: a randomised controlled trial

HCW Li', KY Ho ${ }^{1}$, MP Wang ${ }^{1}$, SSC Chan'1, TH Lam²

${ }^{1}$ The University of Hong Kong, Nursing, Hong Kong, Hong Kong, ${ }^{2}$ The University of Hong Kong, Public Health, Hong Kong, Hong Kong.E-mail:william3@hku.hk

Background: Smokers who are in physical discomfort attended to emergency departments present an excellent 'teachable moment' for smoking cessation interventions. Nevertheless, most cessation programmes generally take 20 to 30 minutes or more to implement and are thus not feasible in busy clinical settings. This study aimed to address the gap in existing literature by evaluating the effectiveness of using a brief, self-determination intervention on smoking cessation among people attending emergency departments.

Methods: A multi-centre randomized controlled trial was conducted in emergency departments of four acute hospitals in Hong Kong. A total of 1571 patient were recruited, with 787 being randomized into the experimental group and 784 into the control group. Subjects in the experimental group were allowed to select their own schedules of quitting (immediate or progressive). They received a leaflet plus a brief intervention on smoking cessation. Subjects in the control group received a leaflet on smoking cessation. Four consecutive (1, 3, 6 and 12 months) followups were conducted over the telephone. The primary outcome measure was biochemically validated abstinence at 6 months. Secondary outcomes included

(i) biochemically validated abstinence at 12 months and

(ii) self-reported 7-day point prevalence of abstinence at 6 and 12 months. Intention-to-treat analysis was adopted.

Results: Subjects in the experimental group had a statistically significantly higher self-reported quit rate than those in the control group at 12 months $(11.2 \%$ vs $6.8 \%, \mathrm{p}=0.03)$. The biochemically validated abstinence was also statistically significantly higher in the experimental group than in the control group at $6(6.6 \%$ vs $2.6 \%, \mathrm{p}<.001)$ and 12 months $(6.0 \%$ vs $3.0 \%, \mathrm{p}=0.04)$.

Conclusions: The brief, self-determination intervention was shown to be effective in promoting smoking cessation for people attending emergency departments. Such intervention should therefore be a more cost-effective and sustainable approach to helping smokers quit smoking, and consequently may save more lives.

Tob. Induc. Dis. 2018;16(Suppl 1):A870

DOI: $10.18332 /$ tid/84430

$12: 30-14: 00$

PS-1127-4 Missed teachable moments for promoting smoking cessation in primary care: a qualitative study in Armenia

A Abrahamyan ${ }^{1}$, A Harutyunyan ${ }^{1}$, V Petrosyan ${ }^{1}$

${ }^{1}$ American University of Armenia, Gerald and Patricia Turpanjian School of Public Health, Zvart Avedisian Onanian Center for Health Services Research and Development, Yerevan, Armenia. E-mail: aabrahamyan@aua.am

Background: Teachable moments (TM) have been advocated for endorsing health behavior change in a variety of settings. While primary healthcare settings have been the most potential venue for providing smoking cessation, physicians miss many TM to discuss smoking with their patients at every medical visit. The study aimed to reveal what influences primary healthcare physicians' (PHPs) decision to utilize TMs to facilitate smoking cessation counselling with patients.

Methods: The study team implemented a qualitative research through focus group discussions with PHPs using a semi-structured guide. Purposive sampling was used to recruit participants $(n=23)$ from two Armenian cities (the capital city Yerevan and the second largest city Gyumri). We transcribed the collected data and analyzed by the directed content analysis technique.

Results: The study results illustrated several misconceptions that hamper PHPs to utilize TM for providing smoking cessation counselling to their patients. Majority of PHPs reported that they preferred discussing smoking only with those patients who expressed explicit concern about smoking, as they were afraid of harming physician-patient relationship. PHPs' believed that asking patients about their smoking status could be intrusive and lead to conflict situations. Some of PHPs were considering smoking as a culturally sensitive issue and preferred checking smoking status of men rather than women. Physicians also tend to miss the opportunity to discuss smoking with special patient subgroups (elderly patients, patients with other co-morbidities) because of the misbelief that smoking "already harmed" them and their health problems take precedence over smoking cessation counseling.

Conclusions: Physicians missed opportunities to employ TMs for smoking cessation counseling with patients. Physicians appear to prioritize smoking cessation counseling based on patients' sociodemographic characteristic (age, gender), as well as diagnosis at the time of the visit. Specific interventions should be implemented to instruct physicians' to capitalize on TM and discuss smoking cessation during routine consultations with all patients.

Tob. Induc. Dis. 2018;16(Suppl 1):A871

DOI:10.18332/tid/84433

12:30-14:00

PS-1128-4 The overlooked role of physical activity in mitigating the mortality risk in smokers and nonsmokers with COPD

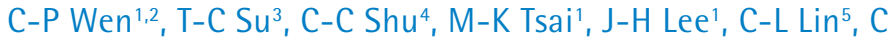
$\mathrm{Wen}^{6}, \mathrm{C}-\mathrm{W} \mathrm{Ka0^{7 }}$

'National Health Research Institutes, Institute of Population Science, Miaoli, China, ${ }^{2}$ China Medical University Hospital, Taichung, China, ${ }^{3}$ National Taiwan University, Department of Internal Medicine, Taipei, China, ${ }^{4}$ National Taiwan University, 
Department of Traumatology, Taipei, China, ${ }^{5}$ John Tung Foundation, Taipei, China, ${ }^{6}$ Long Beach VAMC Hospital, University of Irvine Medical Center, Irvine, CA, United States of America, ${ }^{7}$ Taipei Medical University, Master Program in Global Health and Development, Taipei, China. E-mail: cwengood@ nhri.org.tw

Background: Chronic obstructive pulmonary diseases (COPD), found in smokers and nonsmokers, are known to have increased mortality, but most people with COPD are unaware of their condition. They have a reduced exercise capacity and in general are less physically active, even though long-term benefits of physical activity have been demonstrated.

Methods: A total of 386,304 people participated in a health surveillance program between 1994 and 2008 in Taiwan. Lung function, blood tests, and medical history data, including smoking and exercise habits, were collected. Corresponding mortality and cancer data were retrieved from the National Death File and the National Cancer Registry, respectively. COPD was diagnosed by spirometry, as recommended by GOLD standard

Results: In a mean of 8.8-year follow-up, the prevalence of smokers and non-smokers with COPD was $5.3 \%$ and $3.7 \%$ respectively. Using Cox proportional hazard regression with factor adjustment, hazard ratio (HR) were 1.56 (1.46-1.66) in smoker, 1.48 (1.361.62) in those with COPD and 2.14 (1.97-2.34) in smokers with COPD. In those with COPD, fully active LTPA (>30 min/ day), regardless of history of smoking, can reduce the all-cause mortality compared with inactive LTPA ( $<15 \mathrm{~min} /$ day). For allcause mortality in those without COPD or cardiovascular diseaserelated mortality in those with COPD, low active LTPA (15-30 min/day) can decrease the HR compared with the corresponding group. The active LTPA may extend the estimated life expectancy about 3 years in participants with COPD.

Conclusions: COPD is a systemic disease, causing non-lung related excess mortality such as CVD and cancer, and shortening life by 6 years. These excesses could be substantially mitigated by regular physical activity for 30 or more minutes/day of moderate intensity.

\section{Tob. Induc. Dis. 2018;16(Suppl 1):A872 DOI:10.18332/tid/84438}

\section{2:30-14:00}

PS-1129-4 Social environmental factors as predictors of late relapse: prospective follow up results among Quitline users in Republic of Korea

MK Lim¹,2, BY Jung ${ }^{2}$, J-J Park¹, J-K Oh ${ }^{1,2}$, EH Yun ${ }^{1,2}$, E-Y Park ${ }^{2}$ ${ }^{1}$ Graduate School of Cancer Science and Policy, National Cancer Center, Goyang, Korea, Republic of, ${ }^{2}$ National Cancer Control Institute, National Cancer Center, Goyang, Korea, Republic of. E-mail:mickey@ncc.re.kr

Background: Although Quitlines have provided services for cessation with easy accessibility, convenient counseling, and high effectiveness, little attention has been paid to research on predictors of delayed relapse.

Methods: Among the data of 45,368 quit attempts from 1st January 2007 to 31 December 2015, only 16,729 data which is the first quit attempt of each user aged more than 19 years old and with the complete data on baseline and followed up until 31 December 2012 were included in the final analysis.

At the baseline, information on socio-demographic characters, smoking related behaviors, and motivational characteristics were collected. Undergoing the Quitline protocol, self-reported cessation status and reasons of relapse have been asked during the 1 year of follow up. Cumulative abstinence and the effect size of related factor on relapse were estimated by Kaplan-Meier method and Multinominal logistic regression.

Results: In the end of prospective follow up, $80.0 \%$ of participants were in relapse, and $15.5 \%$ of them were relapsed 4 weeks after starting their quit. The most common reason for late relapse was the stress caused by social context of daily living. Subjects induced to smoke when they drink coffee or alcohol (OR 1.359, 95\% CI 1.218-1.516) or when they saw others' smoking scenes (OR 1.422, 95\% CI 1.193-1.695) were more relapsed with the reason of temptation of environment, and subject who were induced to smoke when they were under stress (OR .479, 95\% CI 1.289-1.696) were more relapsed with the reason of stress caused by social context of daily living than withdrawal symptom. Both reasons of relapse were also more frequent in higher education and more frequent drinking group, while lesser in high nicotine dependence and low self-efficacy groups.

Conclusions: Development of relapse prevention program regarding the meaningful portion of late relapse and its' social environmental predictors is in need for effective Quitline service.

Tob. Induc. Dis. 2018;16(Suppl 1):A873

DOI:10.18332/tid/84439

\section{2:30-14:00}

PS-1130-4 Using synergised social media approaches to support tobacco control policy and cessation related behaviours in Bangladesh

T Turk' ${ }^{1}$ N Singh Negi ${ }^{2}$, D Svenson ${ }^{1}$, SMM Hussain ${ }^{3}$, MS Islam4, S Mullin ${ }^{1}$

'Vital Strategies, Policy Advocacy and Communication, New York, NY, United States of America, ${ }^{2}$ Vital Strategies, Policy Advocacy and Communication, New Delhi, India, ${ }^{3}$ Zanala Bangladesh, Marketing, Dhaka, Bangladesh, ${ }^{4}$ Vital Strategies, Policy Advocacy and Communication, Dhaka, Bangladesh. E-mail: tturk@vitalstrategies.org

Background: Social media, synergised with mass media, may provide a powerful, low-cost channel of communication for public health interventions, including promotion of new policies such as graphic health warnings on tobacco packs. However, the evidence on the efficacy of social media to support tobacco control within low -and middle- income countries, is sparse. An innovative website was developed to examine the potential of social media to increase bridging and bonding of social capital and self-efficacy to quit, given the importance of providing cessation support services, which are seen as the 'weakest link' in WHOs MPOWER framework.

Methods: A population level, mass media campaign; "People Behind the Packs" was synergized with social media to raise awareness of new graphic health warnings and support tobacco cessation in Bangladesh. One website page contained four public service announcements featuring patients suffering from diseases depicted on new pack warnings. Another, "I can quit" page, provided quit tips from a doctor. Site metrics were reviewed and an online Survey Monkey questionnaire administered to explore attitudes and cessation related behaviors of tobacco users who accessed the webpages.

Results: Site metrics identified 100,410 (65\% of website visitors) viewed the quit page immediately following the intervention, with around 24,000 (15\%) accessing patient testimonial messages, and $20,474(13 \%)$ signing the petition. Viewing by gender was balanced ( $46 \%$ females and 54\% males) with 4.8 million "Likes" on campaign content on Facebook over the 6-week campaign 
period, with the top five posts reaching 1.68 million people. 1225 respondents completed the self-administered survey. Responses identified good discussion with others about quitting or cutting down (23\%), intentions to cut-down (36\%) or quit smoking (45\%). Conclusions: Social media can provide low-cost interventions to engage audiences, promote discussion, and support policy and cessation related behaviors. This impact may be increased when linked to other mass media campaign components.

Tob. Induc. Dis. 2018;16(Suppl 1):A874 DOI:10.18332/tid/84449

\section{2:30-14:00}

PS-1131-4 Results of one year monitoring of the National telephone quitline on smoking cessation in the Kyrgyz Republic

C Bekbasarova', M Kasimova², G Kurmnabek Kyzy ${ }^{2}$, N Aidarova ${ }^{2}$, N Seitkazieva ${ }^{3}$, I Akunova ${ }^{2}$, T Djamangulova ${ }^{3}$, E Boronchieva ${ }^{2}$ ${ }^{1}$ Ministry of Health of the Kyrgyz Republic, Bishkek, Kyrgyz Republic, ${ }^{2}$ Republican Centre for Health Promotion, NSCO, Bishkek, Kyrgyz Republic, ${ }^{3}$ Public Health Protection Foundation, Bishkek, Kyrgyz Republic.E-mail: chbekbasarova@gmail.com Background and challenges to implementation: In 20102011 Public Health Protection Foundation in collaboration with Republican Health Promotion Centre (RCHP) of the Ministry Health $(\mathrm{MoH})$ has conducted pilot project on providing smoking cessation consultations by phone (quitline). The Pilot project recommended the Kyrgyz $\mathrm{MoH}$ to establish a national smoking cessation quitline service under the RCHP.

Intervention or response: The National quitline service was established in 2016. Link to the quitline website and telephone were displayed on 12 Pictorial Health Warnings (PHW) on cigarette packages, which were implemented in Kyrgyzstan since January 2016. National quitline service works from 9 a.m. till 9.p.m daily, except Sunday and national holidays. Total of 4143 smokers (93,7\% men and 6,3\% women) were consulted during 15 months (in 2016 - 2017). 50\% of smokers had 3rd degree of tobacco addiction (DTA), 32\% - 2nd DTA and 18\% - 1st DTA. $80 \%$ of smokers were $15-34$ years old.

Results and lessons learnt: During January-June of 2017, quitline service consultants made proactive calls to the telephone subscribers, who received advices and information in same period of 2016 for conducting one-year monitoring of work. More than $60 \%$ of respondents were not reached by phone and $40 \%$ (580 people) were interviewed. $27,8 \%$ of people $(28,5 \%$ males and $18,6 \%$ females) managed to quit tobacco use and $72,2 \%$ of them (71,5\% males and $81,4 \%$ females) could not quit. Among the smokers who quit smoking during the last 12 months - $46 \%$ had 1st, 34\%-2nd and 20\% - 3rd DTA.

$89 \%$ were $15-44$ years old. $94 \%$ of respondents answered that quitline helped them to quit tobacco smoking. $37 \%$ of smokers who couldn't quit tobacco were not ready do it, $32 \%$ indicated withdrawal symptoms as the underlying reason for their failure.

Conclusions and key recommendations: Results of one year monitoring indicate, that quitline is efficient population level smoking cessation intervention and should be continued and improved with the $\mathrm{MoH}$ support.

Tob. Induc. Dis. 2018;16(Suppl 1):A875

\section{DOI:10.18332/tid/84467}

12:30-14:00

PS-1132-4 Who should have the strongest quit smoking willingness and much easier to persuade? Y Gao' ${ }^{1}$ R Ding ${ }^{2}, \mathrm{Lu}^{3}$
${ }^{1}$ Chinese Association of Tobacco Control, Beijing, China, ${ }^{2}$ People Hospital of Peking University, Beijing, China, ${ }^{3}$ Chinese Society of Cardiovascular Prevention \&t Rehabilitation, Beijing, China. E-mail: yalilu@china-heart.org

Background and challenges to implementation: Base on the Report on cardiovascular diseases (CVD) in China in 2016. In China, the prevalence of CVD is continuously increasing. Currently, an estimated 290 million individuals suffer from CVD. Rates of cardiovascular morbidity and mortality are higher among smokers and passive smokers than among non-smokers.

Intervention or response: Put the "smoking history questionnaire" and "assessment of patient willingness to quit smoking survey" into process of 4154 cardiovascular disease patients diagnosis, treatment and rehabilitation. Increasing the frequency of intervention from none or 1 time to 3 times (intake, Discharge and follow-up) within one year in 27 difference hospitals from random province in China. Keep tracking the quit smoking willingness of the patients during the period from acute phase to recovery phase, and to maintenance phase.

Results and lessons learnt: Continuing to increase the awareness that cigarette smoking causes HEART DISEASE and other serious diseases in this group of people. $90.15 \%$ patients were becoming increasingly clear to the harm of cigarette smoking to their whole body, not only the lung. Base on the quit line success rate, more than $2 \%$, in 2015. Strengthen patients' quit smoking willingness is easier than non-disease smokers. The number of patients who has the willing for quit smoking increase from $21(0.5 \%$, acute stage $)$ to $282(6.7 \%$, follow up) within one years. The patients who have strong will to quit smoking is 75 individuals (1.8\%).

Conclusions and key recommendations: Quitting smoking was found to substantially reduce the risks of death. Our retrospective study shows tremendous persuade successful rate in patient's population who have been educated by our cardiovascular doctors and their medical teams. We should start the quit smoking process from the "weakness" (willpower and physical) patients who are fearful of the disease may destroy his life or even take away his life and more dangerous groups. It will give us much more clinical outcomes than the normal person.

Tob. Induc. Dis. 2018;16(Suppl 1):A876

DOI:10.18332/tid/84475

\section{2:30-14:00}

PS-1134-4 Experience establishing National Quitline Service in Ukraine

O Stoyka

${ }^{1}$ City Health Center, Health Care Department of Kyiv City, Kyiv, Ukraine. E-mail: otto.stoyka@gmail.com

Background and challenges to implementation: $41 \%$ of people who were smokers during the previous year attempted to quit. Out of these, only $7 \%$ were successful as estimated within the year of the quit attempt. The volume and quality of smoking cessation assistance at the primary health care level is not enough: only $42 \%$ of smokers who visited hospitals, doctors asked about there smoking; $77 \%$ of all smokers consider creating a professional service to help stop smoking necessary. $17 \%$ of smokers would like to use a Quitline. 13\% of smokers would like to use the SMS of e-mails.

Intervention or response: Therefore, in order to meet these needs, for the first time, a national smoking cessation assistance service in Ukraine was organized. It is based on the best scientifically proven practices and consultations of international experts. The telephone line is organized in conjunction with a site where you 
can register and undergo smoking testing, degree of dependence and motivation to throw. The site and telephone line are linked by a CRM system for rapid response to customers. You can provide on-line chat and e-mail.

Results and lessons learnt: During the first month of work 437 reactive consultations were given, the average duration was 10-15 minutes. Lost 190 calls. Consultants provide services in accordance with WHO guidelines for telephone counseling. Among the consulted 290 men and 140 women.

Conclusions and key recommendations: The service is well received by ordinary smokers. The existence of the Service encourages medical staff to provide advice on the existence of this service and provide short consultations on smoking cessation. Encourages smokers to call the telephone line on the advice of a professional counselor and on the site for self-help and selfmotivation. This is a good first example of a successful modern intervention in the prevention of non-communicable diseases in the public health system of Ukraine.

Tob. Induc. Dis. 2018;16(Suppl 1):A877

DOI:10.18332/tid/84493

\section{2:30-14:00}

PS-1135-4 Child hospitalization due to respiratory illness: an opportunity for parental/family smoking intervention

\section{Chhakchhuak', L Chhakchhuak ${ }^{2}$}

'District Hospital, Kolasib, Pediatrics Department, Kolasib, India, ${ }^{2}$ Tripura University, Department of Psychology, Agartala, India. E-mail: arini1910@gmail.com

Background: It is estimated that $20-50 \%$ of adult smokers reside with children, and the majority of these smokers(70\%) continue to smoke inside their homes despite adverse health effects of second hand smoking for their children. Majority of the smokers are the male figures in the family. Such kind of smoking is associated with increased rate of childhood respiratory illnesses. Children are usually brought to hospitals by their mothers, which gives us less chance to counsel the rest of the family members who smoke. Child's hospitalization gives us chance to meet up all family members for cessation counselling. The objective is to evaluate the feasibility of implementing a smoking intervention for a smoker parent/family member at the time of child's hospitalization for respiratory illnesses.

Methods: A prospective cohort study of parents/families who have a child admitted to District Hospital, Kolasib, Mizoram for respiratory illnesses between January-March, 2017. They were invited to complete questionarres and acceptability of cessation interventions - written materials, Nicotine Replacement Therapy, Behavioural Therapy. 1 month follow-up outcome were - quit attempts, cessation, NRT use, visits to Tobacco cessation clinic, household smoking prohibition.

Results: A total of 55 children have parents/family members who smoke, out of which 30(54.5\%) were enrolled. Only 20\% had ever participated in smoking cessation programme, and only $16.7 \%$ had ever use medications to quit smoking. On 1 month follow up, $57.1 \%$ made a quit attempt lasting for at least 24 hours, $17.8 \%$ reported not smoking for last 7 days, $25 \%$ reported use of NRT, $35.7 \%$ had visited Tobacco cessation clinic and the proportion of families who use smoking prohibition increased.

Conclusions: At the time of their child's hospitalization due to respiratory illnesses, parents/families were willing to enroll in smoking intervention, which makes it feasible to take this opportunity as a Pediatrician, a teachable moment to address smoking at home.

Tob. Induc. Dis. 2018;16(Suppl 1):A878

DOI: $10.18332 /$ tid/84499

$12: 30-14: 00$

PS-1136-4 An innovative smoking cessation program using Facebook reaching 7'000 Swiss smokers

J-P Humair' ${ }^{1}$, A Dubuis², F Joliat ${ }^{3}$, S Brun ${ }^{4}$, C Wahl'1 R Savioz ${ }^{3}$ ${ }^{1}$ Carrefour addictionS, CIPRET-Geneva, Geneva, Switzerland, ${ }^{2}$ CIPRET Valais, Sion, Switzerland, ${ }^{3}$ Ibrid Communication, Sion, Switzerland, ${ }^{4}$ Swiss Federal Office of Public Health, Tobacco Control Fund, Bern, Switzerland. E-mail: jean-paul.humair@ cipret.ch

Background: Tobacco smoking causes multiple diseases, premature mortality and is a major public health threat. In Switzerland, smoking prevalence is high (25\%) but stable since 2011. Half of smokers want to quit but they underuse effective smoking cessation services. Programs using social networks are a new smoking cessation approach potentially able to reach many smokers. We designed, implemented and tested an innovative large-scale smoking cessation program using Facebook for 7 months in 2016 in 4 states of Switzerland.

Methods: 7008 smokers were enrolled within 2 months through Facebook advertising. The program created 4 communities of smokers of each state interacting on a Facebook page. 2400 Facebook publications, which triggered 67000 comments, stimulated interaction. After a 3-week preparation providing information and advice, all participants quit smoking together on March 21, 2017. Then, they received mutual support from peers and professional help daily during 6 months. Professional support included 3 levels: community managers watching and regulating the activity; counsellors providing personalised smoking cessation counselling; physicians providing personalised counselling for complex and medical issues. Personalised counselling at 2nd and 3rd levels included help for cessation plans, relapse prevention strategies and use of nicotine replacement. Evaluation surveys were conducted at 1 week, 3 and 6 months among 1270 participants, of whom $37 \%$ responded.

Results: $81 \%$ of participants attempted to stop smoking. At 6 months, $13.5 \%$ reported total smoking abstinence while $19 \%$ were trying to quit despite occasional consumption. Smoking cessation was not associated with socio-demographic factors. The community dynamics, a strong intention to quit and relapse prevention strategies predicted success while high nicotine dependence, exposure to smokers and negative affect were negative predictors.

Conclusions: A large-scale smoking cessation program using Facebook, providing mutual and professional support seems effective to reach large numbers of smokers, stimulate smoking cessation attempts and promote smoking abstinence at 6 months.

Tob. Induc. Dis. 2018;16(Suppl 1):A879

DOI:10.18332/tid/84505

\section{2:30-14:00}

PS-1137-4 Predictive factors of 6 month continuous abstinence at the beginning of treatment

E Esteves ${ }^{1}$, G Saona ${ }^{1}$, M Baldizzoni ${ }^{1}$, I Wald ${ }^{1}$, N Rey $^{1}$

${ }^{1}$ National Fund of Resources, Montevideo, Uruguay. E-mail: eesteves@fnr.gub.uy

Background: National Fund of Resources (FNR), public nonstate agency of Uruguay, finances highly-specialized medical procedures and high-cost drugs. Given the impact of tobacco use as a serious Public Health problem and as a risk management 
strategy, in 2004 FNR implemented a Tobacco Dependence Treatment Program. It provides professional training, financing pharmacological treatment with nicotine substitutes and bupropion, provides a computerized clinical record system and performs periodic analysis of process and outcome indicators. The present work analyzes the samples studied in two consecutive evaluations.

Methods: The objective is to detect variables, at the onset of the program, that predict the probability of success, measured in continuous abstinence for 6 months. A logistic regression was performed with: sex, age at the beginning of treatment, age at onset of tobacco use, daily consumption, maximum consumption, medication use, educational level and number of controls in the first 30 days of treatment.

Results: 824 patients were included in the analysis, the variables retained by the model were the number of controls as a factor that favors abstinence $(\mathrm{OR}=1.33,1.17-1.51)$, and mean daily consumption at admission ( $\mathrm{OR}=0.97,0.95-0.99)$ and the maximum consumption ( $\mathrm{OR}=0.99,0.97-1.0)$ as the factors that do not favor. Significant effect was obtained for abstinence rate, from 4 contact with the team (OR $=1.94,1.10-3.41)$.

Conclusions: The magnitude of consumption at admission is a well-known predictor, which is related to dependence degree, however the fact that in the model was maintained independently of maximum consumption, suggests that consumption at admission reflects if there are control measures implemented before onset program, indirect indicator of motivation. On the other hand, the results suggest that it is justified to concentrate time and resources necessary to increase adherence in the first month, to achieve at least 4 patients' contacts with the program.

Tob. Induc. Dis. 2018;16(Suppl 1):A880 DOI:10.18332/tid/84514

\section{2:30-14:00}

PS-1138-4 Exposure to secondhand smoke as a risk factor for severe tobacco smoking among young healthy men in South Africa

M Milovanovic ${ }^{1}$, L Lebina $^{1}, \mathrm{~K}$ Otwombe ${ }^{1}, \mathrm{~K}$ Hlongwane $^{1}, \mathrm{P}$ Abraham ${ }^{1}$, J Golub², N Martinson 1,2,3

${ }^{1}$ University of the Witwatersrand, Perinatal HIV Research Unit, Soweto, South Africa, ${ }^{2} J o h n s$ Hopkins University School of Medicine, Center for Tuberculosis Research, Department of Medicine, Baltimore, MD, United States of America, ${ }^{3}$ SoMCHAT MRC Collaborating Centre for HIV/AIDS and TB, Johannesburg, South Africa. E-mail: milovanovicm@phru.co.za

Background: Despite public health intervention, exposure to secondhand smoke, specifically in the home, remains high. Secondhand smoke has been linked to several health outcomes however there is limited information on how secondhand smoke impacts on severity of smoking.

Methods: We report interim results of a cross-sectional study among young men (10-34 years) attending five Voluntary Medical Male Circumcision (VMMC) clinics in South Africa. Data were collected on participant and parental smoking status; exposure to secondhand smoking and alcohol consumption. A qualitative urine test for cotinine and a breathalyser test for Carbon monoxide measured in parts per million (COppm) were administered. COppm scores were classified into two categories: moderate/ mild and severe. Secondhand smoking was defined as exposure to indoor and family members smoking. Logistic regression was used to determine risk factors associated with severe smoking.

Results: Of the 2465 participants, median age 20 (IQR: 13-
27) years, $32.5 \%(785 / 2418)$ tested positive for urine cotinine of which $98.6 \%(774 / 785)$ had a COppm result. COppm result showed 53.2\% (412/774) were severe smokers, 46.8\% (362/774) were moderate/mild smokers. A total of $46.3 \%$ (189/408) of severe smokers reported often having someone smoking in their home of whom $27.8 \%$ (72/259) reported that their mother and $59.9 \%(100 / 167)$ report that their father smokes. Overall, $80.3 \%$ (330/411) of severe smokers self-reported drinking alcohol. Multivariate analysis adjusting for age and alcohol use showed that severe smoking was associated with often exposure to indoor smoking at home (OR: $1.61295 \% \mathrm{CI}$ : 1.195-2.173) and to family members that smoke (OR: 3.495 95\%CI: 1.413-8.646).

Conclusions: Family members who smoke and alcohol use put young men at risk of severe smoking. Rules and social norms must encourage smokers to smoke outside of their houses. Policy interventions should focus on highlighting the risks and reducing the prevalence of secondhand smoking.

Tob. Induc. Dis. 2018;16(Suppl 1):A881

DOI:10.18332/tid/84525

\section{$12: 30-14: 00$}

PS-1139-4 Bridging the gap: challenging attitudes towards smoking in pregnancy among healthcare professionals

M Rodnay ${ }^{1}$, S Karni ${ }^{1}$, T Kessel ${ }^{1}$, L Valinsky¹, F Hershkovitz¹, A Birenbaum ${ }^{1}$, I Vered ${ }^{1}$, D Mossinson ${ }^{1}$

1Meuhedet Health Services, Tel Aviv, Israel. E-mail: mrodnay@ gmail.com

Background: Clean Air for Babies is a Global Bridges project to reduce smoking in pregnancy in a healthcare organization, 'Meuhedet', with $1.2 \mathrm{M}$ members. One aim is empowering health professionals to provide brief smoking cessation interventions. In this study we assessed the effectiveness of an education program for nurses and ultrasound technicians.

Methods: The program consists of 8 hours, and includes smoking and ETS effects during pregnancy, practical tools and behavioral interventions. Evaluation includes before and after questionnaires based on Global Bridges tools, and focus groups. We are at the initial stage of analysis. We will conduct another round of questionnaires 3 months post intervention.

Results: We conducted 6 courses for 120 maternal health nurses and 60 technicians. Qualitative analysis demonstrates ambivalence regarding ability to intervene ("This will damage the therapeutic space") and identification with smokers ("I have seen how difficult it is to stop, my husband became unbearable."). A second theme was hesitance in approaching Muslim or JewishOrthodox spouses due to gender-based hierarchies in the cultural context ("In our culture it is hard for the women to tell a man what to do..."). A common theme among technicians was insecurity regarding their role in smoking cessation.

We completed analysis of 29 nurses' questionnaires pre and immediately post intervention. Using paired-t-test analysis we found a significant improvement in the level of knowledge (mean knowledge items 3.27 to 4.38 on a scale of $5, \mathrm{p}<0.001$ ). No difference was found in self efficacy or reported behavior.

Conclusions: Initial results indicate that it is possible to identify specific barriers among caregivers that create resistance to smoking cessation interventions. Addressing them creates an opportunity for real organizational change. The improvement in knowledge is indicative of the appropriateness of the training sessions. We expect that improvement in self efficacy and reported behavior at the 3 month evaluation. 
Tob. Induc. Dis. 2018;16(Suppl 1):A882

DOI:10.18332/tid/84548

\section{2:30-14:00}

PS-1140-4 Smoking cessation counselling - taking family in a stride

M Chopra ${ }^{1}$, D Bhattacharrya ${ }^{1}, \mathrm{M}$ Chopra ${ }^{1}$, Smokers

'Army Hospital (Research \& Referral), Pulmonary Medicine,

Delhi, India.E-mail: drmanuchopra@gmail.com

Background and challenges to implementation: Smoking as a menace is realized by smoker at one or another point in time but the family suffers this menace day in and out. Counselling a smoker regarding quitting is beneficial, but still failures are seen. Families are usually left bystanders in this practice. In developing countries like India, where-in, family bonding and social support is relatively high compared to Western countries, involving families in smoking cessation counselling is likely to yield better results.

Intervention or response: 194 male smokers reporting to a tertiary care hospital over 1 year for various illnesses were enrolled in study. The individuals were matched regarding age, educational qualifications and social status. 96 patients were counselled in presence of their family members especially wife with or without children. 98 patients were counselled traditionally regarding smoking cessation. Both groups were followed up for 1 year

Results and lessons learnt: Out of family group (n-96), 63 (65\%) admitted to be abstinent from smoking after 1 year, however only 32 (33\%) of the traditional group (n-98) successfully quit smoking. Smoking cessation counselling with family involvement results in better quit rates compared to counselling only the smoker..

Conclusions and key recommendations: Smoking affects not individuals but families. Blaming and letting only smoker decide his future yields poor quit rates. Involving families in making decisions regarding quitting leads to multi prong attack, and especially in societies with strong family bondage, respect and dependence, quitting smoking by smoker becomes a duty rather than choice. Thus, it's strongly recommended to involve family members in smoking cessation counselling.

Tob. Induc. Dis. 2018;16(Suppl 1):A883

DOI:10.18332/tid/84560

\section{2:30-14:00}

PS-1141-4 Evaluation of smoking cessation training in Armenia

A Harutyunyan'1, A Abrahamyan'1, V Hayrumyan'1, V Petrosyan' ${ }^{1}$ American University of Armenia, Gerald and Patricia Turpanjian School of Public Health, Zvart Avedisian Onanian Center for Health Services, Research and Development, Yerevan, Armenia. E-mail:vhayrumyan@aua.am

Background: Healthcare providers play a central role in promoting smoking cessation. Patients getting advice from physicians are 1.6 times more likely to quit, and trained physicians are twice as likely to offer assistance to patients. This study aimed to design, implement, and evaluate the first smoking cessation training for primary healthcare physicians (PHP) in Armenia.

Methods:Werecruited58PHPsforatwo-daytraining(intervention group) and 51 PHPs in the control group from the two biggest cities. We utilized a quasi-experimental design to evaluate the training effectiveness using a self-administered questionnaire at baseline and 4-months follow-up. Practice score was calculated by awarding 1 point when the recommended practice was reported as being "Always" performed in physicians" everyday work. We performed paired analysis to compare baseline and follow-up data using paired t-test and Wilcoxon test.

Results: Overall, 105 PHPs (57-intervention, 48-control) participated in both baseline and follow-up surveys. The selfreported mean practice score significantly increased in the intervention group (10.34 vs. $14.96 ; \mathrm{p}<0.001)$ but not in the control group ( 10.03 vs. $10.25 ; \mathrm{p}=0.739$ ). The improvements from baseline to follow-up in the intervention group were observed regarding most of the selected evidence-based recommendations. However, the most vivid improvements were observed pertaining to practices in assisting patients to quit. At follow-up, more intervention group PHPs were always proposing their help to patients in quitting $(45.61 \%$ vs. $85.96 \%, \mathrm{p}<0.001)$ and prescribing pharmacological aids such as Nicotine replacement therapy (5.36\% vs. $24.56 \%, \mathrm{p}<0.001)$, Cytisine (1.75\% vs. $24.56 \%, \mathrm{p}<$ $0.001)$ and Varenicline (3.51\% vs. $8.77 \%, \mathrm{p}<0.001)$.

Conclusions: Training of healthcare providers can greatly improve compliance with the evidence-based smoking cessation recommendations. Similar tobacco dependence treatment trainings should be applied for all primary healthcare physicians in Armenia as well as adapted and implemented for other healthcare professional groups.

Tob. Induc. Dis. 2018;16(Suppl 1):A884

DOI:10.18332/tid/84572

$12: 30-14: 00$

PS-1143-4 Material of support to community health agents for the tobacco approach of "family health" in Brazil

V Borges ${ }^{1}$, V Cunha ${ }^{1}$, A Reis' ${ }^{1}$ A Mesquita ${ }^{1}$, M Fernandes ${ }^{1}$, R Lamy ${ }^{1}$ ${ }^{1}$ Ministry of Health / National Cancer Institute, Tobacco Control Division, Rio de Janeiro, RJ, Brazil.E-mail:vborges@inca.gov.br Background and challenges to implementation: In Brazil, the Community Health Agent (ACS), inserted in the Basic Attention Program, due to the characteristics of its work, maintains a close relationship with families, which is especially conducive to an awareness-raising approach to adolescents, pregnant women and the elderly about the damages caused By this disease. It can even perceive the difficulty of cessation in the brief modality, advise and refer to treatment by other professionals in the intensive mode in a health unit. In view of these considerations, the National Cancer Institute through the National Program of Tobacco Control developed specific material for the community health agent.

Intervention or response: The Community Health Agent received pilot training for a short-term approach in a municipality in the south of Brazil with face-to-face classes. After that, they received a booklet with guidelines on smoking in pregnant women, adolescents, passive smoking, smoking cessation benefits, among others. The material was used 30 days during home visits. At the end of this period, they received a questionnaire for evaluation with questions related to size, weight, language, relevance and contents of the booklet.

Results and lessons learnt: In the face of the positive evaluation, the booklet became available to all states in the country as an important support tool for ACS, which complemented the training, and was inserted in the Smoking Control for Brief Approach.

Conclusions and key recommendations: The provision of material to ACS as the booklet allowed more people to have access to information about smoking, especially those residing in places that often only the community health agent can reach a country, given the size of Brazil.

Tob. Induc. Dis. 2018;16(Suppl 1):A885 


\section{DOI:10.18332/tid/84615}

\section{2:30-14:00}

PS-1144-4 Dental institutional model of a tobacco cessation clinic: six years of success story

V Mohanty'

${ }^{1}$ Maulana Azad Institute of Dental Sciences, Public Health Dentistry, New Delhi, India. E-mail:vikrantmohanty@gmail.com Background and challenges to implementation: Global Adult Tobacco Survey India (GATS) 2016-17 revealed that 28.6\% adults use tobacco in one form or the other. Nearly $55 \%$ smokers and $50 \%$ smokeless tobacco users are planning or thinking to quit. Dental professionals can play a pivotal role in diagnosing and effectively managing tobacco dependence. There are currently 314 Dental Institutions in India and the large dental manpower remain untapped resources who can be utilized if adequate training is provided.

Intervention or response: This gap was identified and Tobacco Cessation Clinic conceptualized and developed in a Public Dental Institution in New Delhi, India in 2011.The core principles on which the Clinic was established were

1. Patient care services behavioral counselling and NRT

2. Community based Awareness Campaigns and integration with Mobile Dental Clinics

3. Capacity Building and Training programs

4. Conducting Research in Tobacco Control and Cessation

\section{Results and lessons learnt:}

1. Results of Patient Care Services: A total of 1772 patients visited the Tobacco Cessation Clinic since 2011 to 2017. Most of the patients were males and $30.3 \%$ patients were in the age group of $30-49$ years, $40 \%$ were illiterate and $37.8 \%$ were unemployed. About $43.5 \%$ patients were Smokeless Tobacco users followed by $36.4 \%$ smokers and $20 \%$ consumed both forms. About $50.7 \%$ were provided structured behavioral counselling followed by $44 \%$ combination of behavioral counselling and nicotine replacement therapy. The overall quit rate was $5.98 \%$ with six month point prevalence of $30.2 \%$

2. More than 500 Screening programs have been conducted over 6 years

3. Nearly 40 Capacity building programs have been conducted and unique curriculum is being implemented at the undergraduate level

4. Two research grants as well as four travel grants have been awarded along with 3 International publications

Conclusions and key recommendations: The Dental Institutions across India provide an ideal platform which can use this methodology to collaborate and implement various aspects of tobacco control measures and improve health outcomes in the country.

Tob. Induc. Dis. 2018;16(Suppl 1):A886

DOI: $10.18332 /$ tid/84623

\section{2:30-14:00}

PS-1145-4 Tobacco cessation services by community pharmacists; a potential opportunity window

A Adeosun ${ }^{1}$, J Awogbemi ${ }^{2}$, W Erhun ${ }^{2}$

${ }^{1}$ Lighthouse Global Health Initiative, Osogbo, Nigeria, ${ }^{2}$ Obafemi Awolowo University, Faculty of Pharmacy, Ile Ife, Nigeria. E-mail: abayomi.adeosun@gmail.com

Background: Tobacco smoking has been proven to be addictive, and smokers would require some form of assistance to quit. Most smokers in Nigeria are unaware of cessation services nor have access to them. The involvement of Community Pharmacists, who are the first point of call for most health care seekers, in tobacco cessation intervention could have a significant impact on cessation success rates, and a reduction in smoking prevalence. This study sought to answer two main questions:

1. Are tobacco smokers willing to quit smoking?

2. Would tobacco smokers consult community pharmacists for smoking cessation intervention?

Methods: The study was carried out in Ile-Ife and Ibadan both in southwestern Nigeria. Both cities have federal universities, tertiary hospitals and registered community pharmacists capable of providing quality health care. A total of 146 smokers were studied. Questionnaires were administered to cigarette smokers who gave their consent and who were visibly smoking in locations where smokers are populated, such as night clubs, hotel bars, brothels, garage parks, etc. Data collected was analyzed using descriptive statistics.

Results: About $75 \%$ of respondents have had failed tobacco cessation attempts and $71 \%$ of them were not assisted in their cessation attempts. Majority (81.4\%) of respondents still have intentions to quit smoking.

About $60 \%$ of those who intend to quit smoking indicated that they needed assistance. Almost half (48\%) would consult a community pharmacist, $74 \%$ of whom are willing to pay for the service.

Conclusions: This study suggests that tobacco cessation services provided by community pharmacists has a potential for high patronage among smokers. Since community pharmacists are quite accessible to most of the population, their involvement in tobacco cessation will provide ready access to smokers across the country. We recommend a program designed to enrol and empower community pharmacists in tobacco cessation services.

Tob. Induc. Dis. 2018;16(Suppl 1):A887

DOI:10.18332/tid/84656

$12: 45-14: 00$

EP-250-4 Effectiveness of waterpipe smoking cessation intervention with youth

S Alzyoud ${ }^{1}$, V Sreenivas ${ }^{2}$, L Pbert ${ }^{3}$, Tobacco Control Research Group - Jordan

'The Hashemite University, Zarqa, Jordan, ${ }^{2}$ University of Texas Medical Branch, Galveston, TX, United States of America, ${ }^{3}$ University of Massachusetts Boston, Worcester, MA, United States of America. E-mail: sukainaalzyoud@gmail.com

Background: Waterpipe tobacco smoking [WPS] is the most prevalent form of smoking among youth. Few studies were reported that tested interventions of WPS cessation among youth, and none employed the use of behavioral counseling. The current study aims to evaluate the effectiveness of a culturally adapted, school-based 5As-guided counseling intervention for waterpipe smoking cessation.

Methods: A Randomized Clinical Trial [RCT] was conducted to test the effectiveness of a behavioral counseling compared to an information only waterpipe smoking cessation intervention among school students. Eight schools (4 behavioral counseling $\& 4$ information-only group) were randomly selected to participate in the study. School students who reported current waterpipe smoking and accepted to participate were recruited to the study. School counselors served as the study Interventionist. Waterpipe smoking abstinence was assessed at study entry (baseline) and at one month follow-up to assess intervention effect.

Results: The final sample consisted of 185 participants (48\% boys and $51 \%$ girls) for the pre-intervention phase and 157 participants for the post-intervention phase. The majority were 
10 th or 11 th graders with a small percentage of 12 th grade students. A decrease in number of times for waterpipe smoking is observed between pre- and post- intervention among youth. A significant association was found between study groups for last week and last one day waterpipe smoking $(\mathrm{X}=12.5, \mathrm{P}=$ $0.006 ; \mathrm{X}=18.5, \mathrm{P}=0.000$ respectively). Moreover, students who were in the information group had a higher level of nicotine dependence than students in the behavioral counseling group $(\mathrm{t}=2.5, \mathrm{p}=0.01)$

Conclusions: Our study demonstrated that behavioral counseling is effective in decreasing waterpipe smoking habits among youth. Our results also showed that behavioral counseling has an effect on youth nicotine dependence level.

Tob. Induc. Dis. 2018;16(Suppl 1):A888

DOI:10.18332/tid/84610

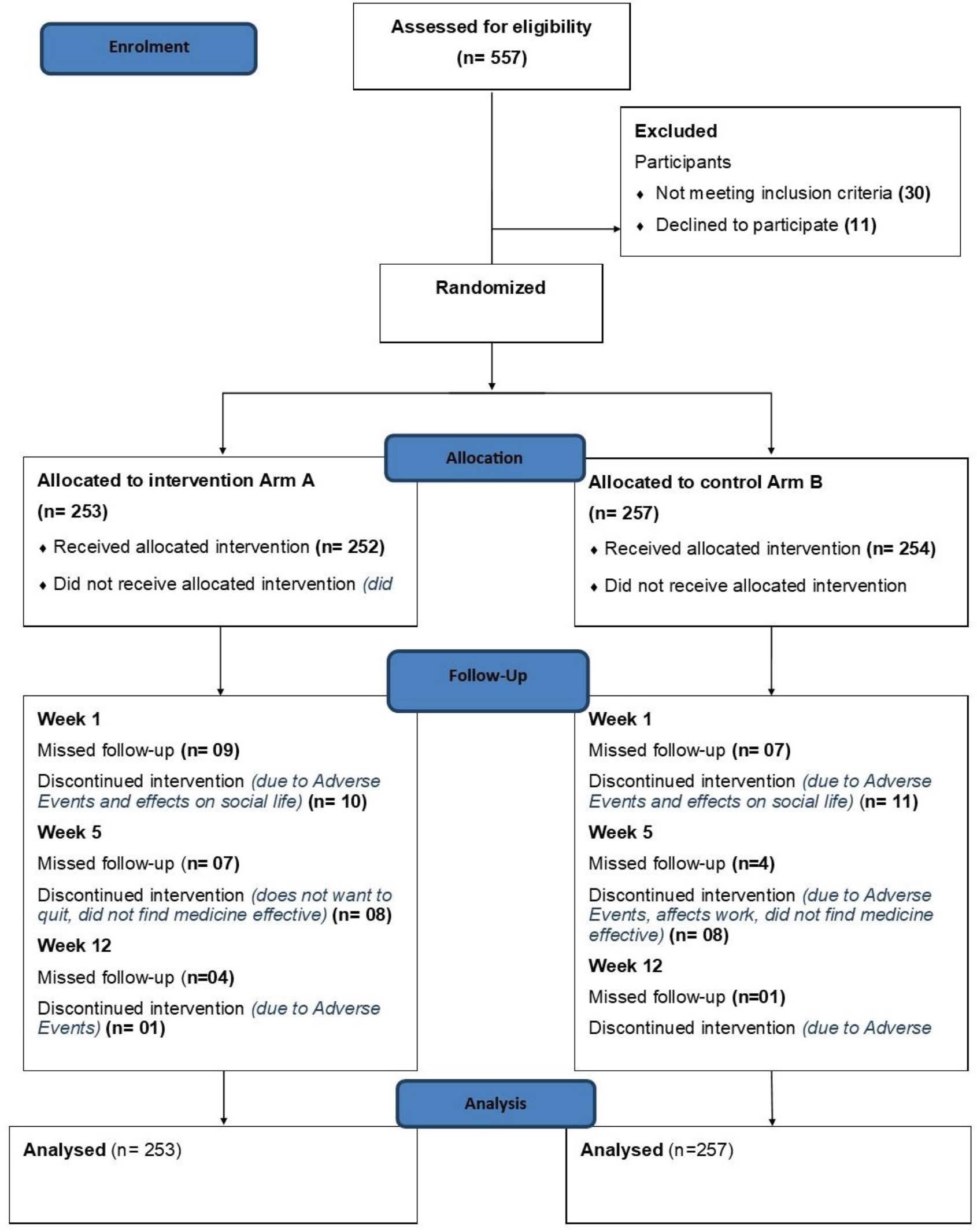

Figure 1: CONSORT DIAGRAM SHOWING FLOW OF PARTICIPANTS 


\section{2:45-14:00}

EP-259-4 Dependence and withdrawal symptoms among waterpipe tobacco smokers en rolled in a double-blind, placebo-controlled, randomised trial

M Kanaan', O Dogar1', R Zahid², S Mansoor², M Jawad², JS Ahluwalia ${ }^{4}, \mathrm{~K}$ Siddiqi ${ }^{1}$

'University of York, York, United Kingdom, ${ }^{2}$ The Initiative, Islamabad, Pakistan, ${ }^{3}$ Imperial College London, London, United Kingdom, ${ }^{4}$ Independent Consultant, Minnesota, MN, United States of America.E-mail: mona.kanaan@york.ac.uk

Background: The prevalence of waterpipe tobacco smoking has been steadily increasing worldwide. Whereas cigarette smoking dependence and withdrawal scales have been developed and used extensively, this is not the case for waterpipe smoking. Our objective was to explore correlates of the Mood and Physical Symptoms Scale (MPSS), a withdrawal scale, and smoking dependency using the Lebanon Waterpipe Dependency Scale (LWDS-11) among waterpipe smokers in South-East Asia.

Methods: MPSS and LWDS-11 were translated for use in a sample of Pakistani adults taking part in a double-blind, placebocontrolled randomised trial evaluating the efficacy of varenicline for abstinence among waterpipe smokers. Participants included adults using waterpipe on a daily basis who were willing to quit. A total of 510 participants were randomised. The scales were administered at baseline and at week 25, the last follow-up that was just completed on May 23, 2017.

Results: Among the 510 trial participants, 249 (49\%) participants used only waterpipe for tobacco smoking whereas the other 261 (51\%) used cigarettes as well. At baseline and week 25, respectively, the MPSS was completed by $501(98.2 \%)$ and 475 (93\%), whereas the LWDS-11 was completed by 505 (99\%) and 475 (93\%).

Conclusions: Complete findings will be reported at 'The 17th World conference on Tobacco or Health' in Cape Town for the first application of LWDS-11 and MPSS Scale among waterpipe tobacco smokers in Pakistan. The application of these scales in Pakistan will inform whether they can be used in practice to assess the extent of dependency and withdrawal symptoms in South-East Asia.

Tob. Induc. Dis. 2018;16(Suppl 1):A889

DOI: $10.18332 /$ tid/84638

$12: 45-14: 00$

EP-260-4 A double-blind, placebo-controlled, randomized trial of varenicline in hookah tobacco smokers in Pakistan

O Dogar', M Kanaan', R Zahid², S Mansoor², M Jawad ${ }^{3}$, JS Ahluwalia ${ }^{4}$, K Siddiqi

'University of York, York, United Kingdom, ${ }^{2}$ The Initiative, Islamabad, Pakistan, ${ }^{3}$ Imperial College London, London, United Kingdom, ${ }^{4}$ Independent Consultant, Minnesota, MN, United States of America. E-mail: omara.dogar@york.ac.uk

Background: Hookah tobacco smoking has increased worldwide yet there is little evidence for the efficacy of pharmacological therapies to achieve abstinence among hookah smokers. Our objective was to assess the efficacy of varenicline when added to behavioural support for hookah tobacco cessation.

Methods: In 2016, we conducted a double-blind, placebo controlled, randomized trial in Pakistan enrolling 510 daily hookah users who were willing to quit. All participants received behavioural support and either varenicline or placebo at randomisation. The primary outcome was 25 weeks continuous abstinence biochemically verified by a carbon monoxide level of $<10 \mathrm{ppm}$.

Results: Among the 510 trial participants, 429 (84\%) were male and 81 (16\%) female; 249 (49\%) smoked only hookah and 261 (51\%) smoked both hookah and cigarettes. 21 participants withdrew from treatment mainly due to adverse events. We recently completed the last follow-up (25 weeks) on May 23rd, 2017 with a 93\% (475/510) retention rate. The data will remain blinded for treatment allocation until the primary analysis is complete. [Trial Flow Chart]

Conclusions: We will report our primary outcome results at the

'17th World conference on Tobacco or Health' in Cape Town for the first ever trial of varenicline among hookah smokers. The findings of this pharmacotherapy trial will have major implications for knowledge translation, practice and policy regarding hookah tobacco use.

Tob. Induc. Dis. 2018;16(Suppl 1):A890

DOI: $10.18332 /$ tid/84550

\section{$15: 45-16: 45$}

LB-1371-4 5-year follow up on outcome of a smoking cessation intervention project

Y Lin', Q Shu², Q Huang ${ }^{3}$

IInternational Union Against Tuberculosis and Lung Disease (The Union), China Office, Beijing, China, ${ }^{2}$ Jinshan District CDC, TB, Shanghai, China, ${ }^{3}$ Jiangxi Provincial CDC, TB, Nanchang, China.E-mail: ylin@theunion.org

Background: Evidences have highlighted significant association between smoking and tuberculosis (TB). Smokers tend to delay accessing health services, more likely to have poor treatment outcome, and higher risk of recurrent TB than non-smokers. To address dual burden of TB and smoking, the Union published a guideline on smoking cessation intervention for TB patients, and piloted it in China. By the end of anti-TB treatment, $66.6 \%$ of current smokers quitted smoking, but we do not know the long-term outcome. We therefore conducted a follow up study to assess long-term outcome of the intervention project.

Methods: This is a longitudinal study. The cohort was established during the pilot in Xingguo and Ningdu County of Jiangxi Province, Chinain 2010-2011. Follow up visits were performed by village doctors after 5-year completion of the pilot and their anti-TB treatments.

Results: 800 TB patients were registered, including 572 male

\begin{tabular}{|c|c|c|c|c|c|}
\hline & $\begin{array}{l}\text { Von } \\
\text { smoker }\end{array}$ & $\begin{array}{c}\text { X- } \\
\text { smoker }\end{array}$ & $\begin{array}{l}\text { Recent } \\
\text { quilter }\end{array}$ & $\begin{array}{c}\text { Current } \\
\text { smoker } \\
\text { on } \\
\text { cessation } \\
\text { program }\end{array}$ & $\begin{array}{c}\text { Current } \\
\text { smoker } \\
\text { not on } \\
\text { cessation } \\
\text { program }\end{array}$ \\
\hline $\begin{array}{l}\text { Total at } \\
\text { baseline }\end{array}$ & 433 & 100 & 23 & 234 & 10 \\
\hline $\begin{array}{l}\text { No }(\%) \text { of } \\
\text { remained } \\
\text { non- } \\
\text { smoking } \\
\text { after 5-year }\end{array}$ & $\begin{array}{l}355 \\
(82.0)\end{array}$ & $\begin{array}{l}63 \\
(63.0)\end{array}$ & $\begin{array}{l}10 \\
(43.5)\end{array}$ & $116(49.6)$ & $3(30.0)$ \\
\hline $\begin{array}{l}\text { No }(\%) \text { of } \\
\text { continuing } \\
\text { or back to } \\
\text { smoking } \\
\text { after } 5 \text {-year }\end{array}$ & $17(3.9)$ & $\begin{array}{l}13 \\
(13.0)\end{array}$ & $4(17.4)$ & 63 (26.9) & $6(60.0)$ \\
\hline $\mathrm{X} 2$ & 104.239 & & & & \\
\hline$P$ value & $<0.001$ & & & & \\
\hline
\end{tabular}

\subsection{Tobacco and Women: The gendered}




\section{nature of tobacco}

\section{2:30-14:00}

PS-1147-5 Smoking addiction among young women working at night at International call centres in India A Gupta

${ }^{1}$ Tata Institute of Social Sciences, Mumbai, India. E-mail: amritagupta7@gmail.com

Background: Indian women are actively involved in occupations which were regarded as a taboo such as night work. Working at night for international call centres is a significant step in moving ahead of patriarchal control over women's mobility in India. The job brings about lifestyle changes among employees such as late night partying, smoking, and boozing. The women employees are mainly fresh graduates. The study brings about the prevalence and smoking behaviour among these young women employees.

Methods: The study is based on primary data collected from 203 women employee of international call centres employees in Mumbai. The study uses appropriate bi-variate and multivariate techniques to bring about the smoking behaviour among the women.

Results: Thirty percent women smoked cigarettes and the mean duration of smoking was 2.98 years. The duration of smoking varied from less than 2 years (35\%), 2-5 years (50\%) and 5 years or more (15\%). The reasons for smoking initiation were to beat work stress (38\%), style statement (30\%), peer pressure (27\%) and to remain awake at night (10\%). Though $87 \%$ women were aware of the health impacts of smoking they continued smoking as it acted as stress buster (50 percent), addicted to smoking (25 percent), to remain awake at night (17 percent) and it gave a sense of freedom (8\%). The mean number of cigarettes smoked per day was 2.9. The number of cigarettes smoked per day varied from 1-2 (60\%), 3-5 (30\%) and greater than $5(10 \%)$.

Conclusions: Smoking among the young women is a matter of grave concern as night work, and job-related stress takes a toll on their health and smoking further affects their health. Interestingly most of the women being aware of the ill effects continued smoking. Organisations should take efforts to provide proper counselling to enlighten the women on the ill effects of smoking.

Tob. Induc. Dis. 2018;16(Suppl 1):A892

DOI:10.18332/tid/84388

12:30-14:00

PS-1148-5 Factors associated with short and long term successful quit among adults female smokers: prospective follow up of Quitline users in Republic of Korea

MK Lim ${ }^{1,2}$, BY Jung ${ }^{2}$ SH Shin², JW Lee', J-K Oh ${ }^{1,2}$, EH Yun ${ }^{1,2}$, E-Y Park ${ }^{2}$

${ }^{1}$ Graduate School of Cancer Science and Policy, National Cancer Center, Goyang, Korea, Republic of, ${ }^{2}$ National Cancer Control Institute, National Cancer Center, Goyang, Korea, Republic of. E-mail:mickey@ncc.re.kr

Background: Female has been targeted as new consumers of tobacco products by tobacco companies, and female smoking prevalence has been increased in the world including recent increasing in some Asian countries. However, there are lack of studies to investigate factors associated with the successful quit among female smokers regarding their gender specific characteristics and response to sociopsychological environments for quitting.

Methods: Between 2006 and 2015, 3,360 female smokers registered users of Quitline in Korea, aged more than 19 years old, and have complete information in need were followed for 1year and included in the final analysis to investigate which factors associated with the short and long term successful quit (30 days and 1 year abstinence of smoking). Baseline information on age, education level, marital status, body mass index (BMI), frequency of alcohol drinking per month, nicotine dependency, self-efficacy for smoking cessation, presence of quit supporter, and mental illness were collected upon Quitline registration. Multiple logistic regression analysis was applied to identify the factors associated with successful quit and its' maintenance.

Results: $24.4 \%$ and $8.3 \%$ of 3,360 female users showed successful quit rate for 30 days and 1 year, respectively. The significant association with short term cessation was found in lower nicotine dependency, higher self-efficacy, and presence of a quit supporter, and higher education level and higher self-efficacy for quit was identified as key factors increase long term cessation. Having mental illness significantly decreased the short and long term cessation. Long term cessation showed the decreasing trend by the increase of frequency of alcohol drinking and level of nicotine dependency.

Conclusions: Increase self-efficacy for quit, having quit supporters, and intervening negative health behaviour such as frequent alcohol drinking should be included in the tailored cessation program for female smokers. Development of intensive cessation program for smokers having mental illness might be considered.

Tob. Induc. Dis. 2018;16(Suppl 1):A893

DOI:10.18332/tid/84454

12:30-14:00

PS-1151-5 Are poor women in the reproductive age group more prone to consume tobacco: a study of the factors that lead to tobacco consumption in India D Vyas ${ }^{1}$

'Institute of Health Management Research University, Public Health Management, Jaipur, India. E-mail: divya.vyas1@gmail. com

Background: Female smokers tend to have higher rates of pregnancy problems and reach menopause about two years earlier than non-smokers. Heavy smokers using the contraceptive pill have much higher heart disease rates than non-smokers on the pill. Smokers have higher cervical cancer rates and low bone density and fractures among postmenopausal women have been linked with smoking. The WHO FCTC treaty stresses to reduce the supply and demand of tobacco and support women's right to health as a human right. The purpose of the study is to identify tobacco consumption among women in the reproductive age group and the influencers of uptake of tobacco.

Methods: The study undertakes the data from The Global Adult Tobacco Survey (GATS), India, 2009-2010. GATS India was a nationally representative household survey $(n=79,690)$ on tobacco use and other tobacco control indicators using a standardized questionnaire. Data was adjusted for non-response and weighted to produce nationally representative estimates.

Results: The results from the analysis depicts that women who ever consumed tobacco were 17 times more likely (95\% confidence interval [CI]: 15.7-18.8) to consume tobacco than their counterparts. Women who were emotionally or lonely were 44.5 times more likely (95\% CI: 43.5-46.5) to consume tobacco than women who were emotionally stable. Furthermore, women who had other women who consumed tobacco in their social circle were 7.8 times more likely (95\% CI: 5.4-9.2) t consume tobacco then their other counterparts. Women who were more exposed advertising and media were more likely $21.3 \%$ (95\% CI: 17.9-24.5) to consume tobacco then other women who were not exposed to media and advertising. 
Conclusions: Consumption of tobacco varies from personal factors, psychological factors, psychosocial factors, socio-cultural factors and environmental factors. Increasing tobacco control mechanisms and identifying the factors that lead to encouraging women to consume tobacco behavioral studies needs to be undertaken.

Tob. Induc. Dis. 2018;16(Suppl 1):A894

DOI:10.18332/tid/84578

\section{$12: 30-14: 00$}

PS-1152-5 Prevalence of violence against women and negative predictors in female smokers attended in a smoking cessation unit in Buenos Aires city: a cross-sectional study

S Braun', K Agolino', A Ángel', D Sánches Gelos'

'University of Buenos Aires, Internal Medicine, Buenos Aires, Argentina. E-mail: karina.agolino@gmail.com

Background: Smoking and violence against women (VAW) are both major public health issues that result in severe consequences. Researches describe smoking as a maladaptive coping mechanism for VAW and determined the existence of a medium composite effect size for this relationship. In spite that smoking cessation treatments are cost effective, they can be less effective in victims of VAW because their higher prevalence of negative predictors as anxiety or depression among others.

Our main objective was to assess the prevalence of victimization in female smokers receiving tobacco dependence treatment at our smoking cessation unit. Also, we assessed the prevalence of nonpsychotic mental disorders and depression and compared these outcomes between the groups.

Methods: Between 2012 and 2016, a systematic sample of 532 female smokers was chosen from women who seek care at our smoking cessation unit. We implemented the instruments developed by International Planning Parenthood Federation to explore VAW. Also, we explored non-psychotic mental disorders and depression with the Self Reporting Questionnaire-20 (SRQ$20)$ and the Beck scale, respectively. Additionally, demographic data and characteristics of the smoking behavior were evaluated. Results: Almost all women agreed to participate (99\% response rate). The median age was 53 (IQR 16). Sixty one percent (328) were not married. The median year of education was 13 (IQR 4). The lifetime prevalence of VAW was $66.73 \%$ (355) and 15\% reported current violence. Women who reported VAW were more frequently single, had an unstable employment situation and smoked more cigarettes/day.

Logistic regression showed that VAW was associated with highest SRQ-20 scores's (OR= 1.15, 95\%IC 1.07 - 1.22) adjusted for age, employment and marital status, Beck's scores and cigarette/day. Conclusions: The VAW prevalence was high in this sample and positively associated with non psychotic mental disorders. More studies are needed to determine if VAW is a negative predictor for smoking cessation treatments.

Tob. Induc. Dis. 2018;16(Suppl 1):A895

DOI: $10.18332 /$ tid/84652

\section{2:30-14:00}

PS-1153-5 Attitudes of women from Kazakhstan regarding tobacco control policy as cancer prevention A Zhylkiadarova ${ }^{1}$, T Khaidarova $^{2}$

'Kazakh Institute of Oncology and Radiology, Almaty, Kazakhstan, ${ }^{2} \mathrm{Al}-$ Farabi Kazakh National University, Almaty, Kazakhstan. E-mail: alma_zh@inbox.ru

Background: Tobacco related cancers are a substantial public health problem, taking a leading position in morbidity and mortality. For more than 10 years, Kazakhstan has implemented a tobacco control policy. The aim of this study was to examine factors that influenced women's attitudes in relation to the smokefree policy as cancer prevention measures.

Methods: The results of the Global Adult Tobacco Survey (GATS) and interviews of 637 women who received cancer prevention consultations in oncological dispensaries (2015-2016) were used. GATS was conducted in Kazakhstan in 2014 that surveyed 4425 men and women aged 15 years or older. Logistic regression models were used to obtain odds ratios with $95 \%$ confidence intervals.

Results: Most women, regardless of the history of smoking, objected to smoking in public transport, closed shopping centers, hospitals and other premises. Despite the smoking ban in public places, $11.5 \%$ of non-smoking women were exposed to secondhand smoke (SHS), mainly (70.9\%) in nightclubs and bars, 17,2-22,3\% - in public transport and universities, 8,8\% - in hospitals. Only a third of women who are exposed to SHS have objected to the ban violation. A low (47\% and lower) awareness of women about the causal relationship of smoking and cancer of other localizations, except lung cancer was noted. During visiting to doctor, only $41.1 \%$ of respondents were asked about smoking history and $27.9 \%$ received advise to quit smoking.

Conclusions: In general, there is a very high support for the national smokefree law that covers public places and transport (84.8\%). Most current smokers among women (67.4\%) are ready to quit when they will receive relevant information and help from medical professionals. For this it is necessary develop and implement the women and health professionals addressed cessation programs. It is important to motivate health professionals to stop smoking and to increase population awareness on cancer prevention.

Tob. Induc. Dis. 2018;16(Suppl 1):A896

DOI: $10.18332 /$ tid/84659

\section{$12: 45-14: 00$}

EP-261-4 Assessment of exclusive and dual cigarette and hookah smoking among a sample of pregnant women in Egypt

O El Shahawy ${ }^{1,2,3}$, K Labib $^{1}$, E Mead ${ }^{4}$, A Hamdy', S Sherman ${ }^{2,3}$, C Oncken ${ }^{4}$

${ }^{1}$ Ain Shams University, Cairo, Egypt, ${ }^{2}$ New York University School of Medicine, Population Health, New York, NY, United States of America, ${ }^{3}$ New York University Abu Dhabi, Abu Dhabi, United Arab Emirates, ${ }^{4}$ UConn School of Medicine, Department of Medicine, Farmington, CT, United States of America. E-mail: omar.elshahawy@nyumc.org

Background: Limited data exists regarding tobacco use during pregnancy, particularly in Middle Eastern countries. We examined tobacco use patterns and beliefs regarding secondhand smoke exposure (SHS) before and during pregnancy among Egyptian women.

Methods: Pregnant women were recruited during their last trimester from maternity hospitals in Cairo, Egypt from June, 2015 to May, 2016. Two hundred women were interviewed about their tobacco use before and during pregnancy, as well as their attitudes and beliefs regarding tobacco use and SHS.

Results: Participants had a mean age of $27(+4.6)$ years. Before pregnancy, $30 \%$ of the women reported using tobacco. Among tobacco users, $60 \%$ were dual users of cigarettes and hookah, $35 \%$ were exclusive cigarette smokers, and $5 \%$ were exclusive hookah smokers. Prevalence of daily cigarette smoking decreased during pregnancy $(-62.9 \%, \mathrm{p}<.0001)$, but daily hookah use did not change 
$(-7.4 \%, \mathrm{p}=0.215)$. Most dual users $(75 \%)$ transitioned to exclusive hookah smoking during pregnancy. Only $13 \%$ of smokers quit all products, whereas $28 \%$ continued their same smoking behaviors. Social acceptability of hookah and cigarette smoking was high among tobacco users and did not differ by product. The majority of women agreed that tobacco use is harmful during pregnancy (92\%) and SHS is harmful to a newborn (94\%), and this did not differ by smoking status. However, only $68 \%$ agreed that a pregnant woman's SHS was harmful to herself and her unborn child.

Conclusions: Among this sample of pregnant women, dual use of hookah and cigarettes was common before pregnancy. The transition from dual to exclusive hookah smoking during pregnancy suggests that hookah may be perceived as less harmful than cigarettes and could serve as a substitute for conventional cigarettes. Future work is needed to understand and address hookah smoking during pregnancy and misperceptions about the harmful effects of SHS among women of child-bearing age in Cairo, Egypt.

Tob. Induc. Dis. 2018;16(Suppl 1):A897

DOI:10.18332/tid/84601

\section{$15: 45-16: 45$}

LB-1368-5 Gender disparity in hard-core-smoking: evidence from 14 GATS countries

P Jena ${ }^{1}$

${ }^{1}$ KIIT School of Public Health, Bhubaneswar, India. E-mail: drpratapjena@gmail.com

Background: This study examined the hard-core-smoking prevalence and its gender disparity in 14 Global Adult Tobacco Survey (GATS) countries.

Methods: First wave GATS data for India, Russia, Turkey, Ukraine, Vietnam, Brazil, Bangladesh, Thailand, Uruguay, China, Egypt, Mexico, Philippines, Poland, were analyzed using descriptive statistics to estimate hardcore smoking and its gender disparity. In this study hard-core-smoking was defined as current daily smoker having inability in quitting (no quit attempt in the past 12 months of survey or last quit attempt of $<24$ hours) and with no intention to quit in next 12 months or not interested in quitting; and with high nicotine dependence (time to first smoke within 30 minutes of waking up) and having knowledge of smoking hazards.

Results: There were 88.44 millions (6.8\%) of hard-core-smokers in 14 countries consisting of $89.2 \%$ males. Hard-core-smoking (Range:-Male:1.6-19.4\%, Female:0.1-5.1\%) prevalence varied across countries. Similarly, the estimates of hard-core-smoking as a proportion of daily smoking was also varied (Range:-Male 8.6$35.2 \%$, Female $5.9-33.6 \%$ ) widely. The gender difference in hardcore smoking among adults was varied from $1.1 \%$ in Mexico to $15.8 \%$ in Russia. Gender difference in Hardcore-smoking as a proportion of current daily smoker varies from (-) $15.8 \%$ to $13.5 \%$. In Bangladesh, Egypt and Mexico, hardcore smoking as a subset of daily smoker was higher among females than their male counterparts.

Conclusions: Males outnumber females in hard-core-smoking but hardcore-smoking as a proportion of daily smoking is higher among females than males in three out of 14 countries. There is no specific trend in gender difference across countries. Further stratified analysis is recommended.

Tob. Induc. Dis. 2018;16(Suppl 1):A898

DOI:10.18332/tid/84753

\subsection{Tobacco in vulnerable populations (e.g. indigenous populations)}

\section{$14: 00-15: 30$}

F0-403-5 Smoking disparities by level of educational attainment in the United States, 1966 to 2015 $\mathrm{PCaO}^{1}$, J Jeon ${ }^{1}$, J Tam ${ }^{2}$, TR Holford ${ }^{3}$, NL Fleischer ${ }^{1}, \mathrm{R}_{\text {Meza }}{ }^{1}$ ${ }^{1}$ University of Michigan School of Public Health, Epidemiology, Ann Arbor, MI, United States of America, ${ }^{2}$ University of Michigan School of Public Health, Health Management \& Policy, Ann Arbor, MI, United States of America, ${ }^{3}$ Yale School of Public Health, Biostatistics, New Haven, CT, United States of America. E-mail: caop@umich.edu

Background: Previous studies in the U.S. show higher smoking rates among those with lower levels of education attainment. Less is known about how these smoking patterns vary by birth cohort or how they may be driven by different demographic profiles across education groups. Furthermore, limited attention has been given to differences in smoking behaviors between those with less than a high school degree and those with 8th grade education or less.

Methods: Data from the National Health Interview Survey 19662015 were utilized to obtain smoking-related information for U.S. adults aged 25 years or older. We developed age-period-cohort models with constrained natural splines to estimate smoking prevalence in groups categorized with five education levels: $\leqslant 8$ th grade, 9-11th grade, high school graduate or GED, some college, and at least a college degree. Annual probabilities of smoking initiation, cessation and intensity by age, birth cohort (1890-1990), gender, and education level were also estimated by the models. Additional regression analyses were conducted to identify sociodemographic factors that may explain smoking disparities across education subgroups.

Results: The probability of smoking initiation was highest among individuals with 9-11th grade education and lowest among those with a college degree or more. The initiation probability among those with $\leqslant 8$ th grade education decreased by birth cohort, resulting in this group having the second lowest smoking prevalence after those with a college degree or more in more recent birth cohorts. The smoking cessation probability was highest among those with a college degree or more. Additional analyses suggest that the low smoking rates among those with $\leqslant 8$ th grade education may be driven by the increasing proportion of non-US born Hispanics in this group.

Conclusions: This study identifies population characteristics that may be driving smoking disparities between levels of educational attainment, providing detailed insights into change in smoking patterns by education for different U.S. birth cohorts.

Tob. Induc. Dis. 2018;16(Suppl 1):A899

DOI:10.18332/tid/84014

\section{$14: 00-15: 30$}

F0-404-5 Urban Indigenous commercial tobacco use in Canada: our health counts Toronto

S Wolfe' ${ }^{1}$, R Maddox ${ }^{2,3}$, K O'Brien $^{2}$, C Xavier $^{2}$, C Wells², G Blais $^{2}$, G Kitching ${ }^{2}$, J Smylie 2,4 , Our Health Counts Toronto

${ }^{1}$ Seventh Generation Midwives Toronto, Toronto, ON, Canada, ${ }^{2}$ St Michael's Hospital, Well Living House, Toronto, ON, Canada, ${ }^{3}$ University of Canberra, Faculty of Health, Canberra, ACT, Australia, ${ }^{4}$ University of Toronto, Toronto, ON, Canada. E-mail: raglan.maddox@canberra.edu.au

Background: In Canada, there is a poor understanding of commercial (non-traditional) tobacco use and effective tobacco reduction strategies among the Indigenous population. This is predominately due to data collection systems that misclassify and/or exclude Indigenous peoples and weak Indigenous health service and program evaluation systems. The most recent statistics are outdated (2006) 
and underestimate the prevalence of Indigenous tobacco use. This research generated a unique primary dataset in a major urban area in one of Canada's largest Indigenous communities.

Methods: Our Health Counts Toronto uses Indigenous community driven processes to generate a comprehensive health information platform to understand and address critical gaps in urban Indigenous health and tobacco use. Trained local interviewers implemented a survey using Response Driven Sampling among Indigenous adults in Toronto for the generation of populationlevel prevalence estimates, including information on tobacco use. Statistical analysis was used to characterize and describe the results, determining prevalence of tobacco use by age, sex, education and household income.

Results: This research recruited a sample of 935 Indigenous adults. Preliminary analysis indicated the prevalence of current daily smoking among Indigenous people in Toronto was $62.3 \%$ (95\%CI:55.0,69.6). Of current smokers, 42.7\% (95\%CI:33.1,52.3) smoked 11 sticks or more per day, and 53.1\% (95\%CI:44.1,62.1) of all Indigenous smokers had tried to quit in the last 12 months. Conclusions: Canada is a signatory to the Framework Convention on Tobacco Control which recognizes the disproportionate harm caused by tobacco use and the need to engage with Indigenous peoples in planning, delivery, and evaluation of tobacco reduction programs and policies. However, the Indigenous population in Toronto continues to experience smoking rates nearly four times greater than the general population. This research highlights the need for recent and accurate population health data to inform tobacco reduction programs and policies; reducing a completely preventable cause of morbidity and mortality.

Tob. Induc. Dis. 2018;16(Suppl 1):A900

DOI:10.18332/tid/84585

\section{4:00-15:30}

F0-405-5 A systematic approach to smoking cessation activities in shelters in Denmark

\section{Pisinger}

${ }^{1}$ Rigshospitalet - Glostrup University Hospital, Research Center for Prevention and Health, Glostrup, Denmark. E-mail: charlotta. pisinger@regionh.dk

Background and challenges to implementation: World-wide shelter users have an alarmingly high smoking prevalence; more than $70 \%$ of homeless people smoke. The burden of tobacco related diseases as cancer, heart disease and chronic obstructive disease is very high in this population. The aim of this study was to develop systematic smoking cessation activities in the shelters of Copenhagen, Denmark and thereby improve the health of shelter users.

Intervention or response: All available $(n=13)$ shelters in Copenhagen were included. There was a development/test period and a period with implementation of the revised intervention. Shelter-staff completed a questionnaire at up-start meetings. Municipal smoking cessation counsellors were trained to work with shelter users. Three types of smoking cessation activities were developed -these should be tailored, flexible and on-site.

Results and lessons learnt: The mean smoking prevalence was $79 \%$ among users of shelters. The level of smoking control in shelters varied and only two shelters had smoking cessation activities at baseline. All shelters implemented at least two out of three offered smoking cessation activities (1. participation in a house-meeting 2. quit smoking café with individual counselling, and 3. tailored groupbased smoking cessation with free nicotine replacement therapy). All but one shelter implemented the group-based intervention: approx. $13 \%$ of smokers signed up and $26 \%$ of those who signed up were abstinent after approx. 12 weeks, as confirmed by staff.

Conclusions and key recommendations: Smoking cessation activities in shelters are wanted by staff and users, they are feasible and even very vulnerable persons are able to quit. Based on experiences from all shelters in the Capital of Denmark, we propose a systematic approach for future smoking cessation initiatives, where the smoking activities should be driven by the municipality, be pro-active, on-site, tailored and flexible, and the smoking cessation counsellors should be trained to work with the target group.

Tob. Induc. Dis. 2018;16(Suppl 1):A901

DOI:10.18332/tid/84221

\section{4:00-15:30}

F0-406-5 Characteristics of individuals that complete treatment v. discontinue among criminal justice smokers S Schiavon', K Cropsey

'University of Alabama at Birmingham, Birmingham, AL, United States of America. E-mail: sschiavon@uabmc.edu

Background: Tobacco kills over 7 million people annually worldwide and is the leading cause of preventable deaths in the United States. Individuals in criminal corrections are at increased risk for tobacco-related diseases and represent a particularly vulnerable group of tobacco smokers. Within the criminal justice system, there are elevated smoking rates (70-80\%) and reduced availability of smoking cessation interventions. Further, these individuals experience significant individual- and social networklevel barriers to completing interventions. The purpose of this study was to examine differences among individuals who completed treatment as compared to those who discontinued treatment prematurely among individuals within criminal corrections.

Methods: The present study utilized data from a one-year clinical trial which randomized participants to four-weeks of Bupropion medication and/or behavioral counseling treatment. Participants $(\mathrm{N}=500)$ were recruited from the criminal justice population ( $\mathrm{M}$ age $=$ $37.4,67 \%$ male, $68 \%$ non-white). A battery of questionnaires assessed smoking characteristics and mental health history. A one-way analysis of variance (ANOVA) and chi-square analyses were conducted to determine differences among individuals who completed up to 1 month, 3 months, 6 months or 12 months of treatment.

Results: Individuals were more likely complete all 12 months of treatment if they were African American, demonstrated good medication compliance, never married, not receiving disability services, and never been treated for substance abuse. Strong smoking urges were associated with lower completion at 1 and 3 months, however, high nicotine dependence was associated with lower completion rates for all time points. A strong social support network was associated with higher rates of completing 12 months of treatment.

Conclusions: This study contributes to our understanding of a vulnerable group of smokers. While smoking urges are related to lower completion initially, nicotine dependence, social support and medication compliance lead to long-term success in treatment completion. Treatments would benefit from strengthen these factors to improve long-term outcomes.

Tob. Induc. Dis. 2018;16(Suppl 1):A902

DOI:10.18332/tid/84142

\section{$14: 00-15: 30$}

FO-408-5 Impact of social inclusion on tobacco use and wellbeing of street children: learning from field experiences in India

\section{Dogra ${ }^{1}$}

'Save the Children, Knowledge Management, Chandigarh, India. 


\section{E-mail:vani1825@gmail.com}

Background and challenges to implementation: India has largest pool of street children in the world. Lack of proper parental care and adult supervision make them highly vulnerable for tobacco and drug abuse. Save the Children India in 2013 launched a five year long program aiming empowering such children through inclusive education to help them escaping vicious cycle of substance use, diseases, and poverty. This study highlights the important findings and experiences while implementation of this program.

Intervention or response: Nine educational activity centers for street and working children (aged 5-14 years) were opened each in Delhi and Kolkata. Children were provided life skill training and taught minimum level of learning curriculum to ensure effective integration into formal schooling. Diff-in-diff method was used to measure the program performance.

Results and lessons learnt: A total of 765 children were included in our analysis (476 in Delhi; 289 in Kolkatta). Tobacco consumption (57\%), gambling (29\%) and drugs (4\%) were the self-reported habits at baseline enrolment. Bidi (47\%), Gutkha (33\%) and pan masala (20\%) were the mainly consumed tobacco products. Use of smokeless tobacco was significantly different among Delhi $(47 \%)$ and Kolkata $(26 \%)$ children $(\mathrm{p}<0.000)$. At the end of second year, majority of enrolled children (80\%) experienced a positive change in their day to day life. Tobacco consumption (10\%), gambling (3\%) and drugs consumption (< $1 \%)$ reduced significantly compared to the baseline $(\mathrm{p}<0.000)$. We learnt that meaningful social engagement of street children plays an important role in improving the overall health behavior and reduces the harmful practices such as tobacco use.

Conclusions and key recommendations: Mainstreaming of street children can help reduce the tobacco and drug use among street children. Government of India should allocate resources for providing inclusive education to street children and view street children as an asset in nation's building and growth.

Tob. Induc. Dis. 2018;16(Suppl 1):A903

DOI:10.18332/tid/84198

14:00-15:30

F0-409-5 Tobacco control policy and smoking cessation and intensity in a longitudinal sample of US older adults (1992 - 2014)

\section{Kalousova $^{1}$}

'University of Michigan, Department of Sociology, Ann Arbor, MI, United States of America. E-mail: luciekal@umich.edu

Background: US Older adults have lower smoking rates than adults in other age groups; only about eight percent of people 65 and older smoke. These lower smoking rates have limited research into the effects of tobacco control policy on smoking cessation in later life, but the lack of attention is misguided. Quitting even relatively late in life confers health and life expectancy benefits. This study addresses two questions: were changes in average cigarette prices and smoke-free laws associated with an increased likelihood of cessation and/or decreased smoking intensity among older adults? Did the effects of tobacco control policies on the likelihood of cessation and/or decreased smoking intensity vary by education and/or race?

Methods: Geocoded longitudinal data from the 1992 to 2014 waves of the Health and Retirement Study including smokers 51 and older were used to assess the relationship between changes in $100 \%$ smoke-free laws and average cigarette pack price and the likelihood of smoking cessation or change in the number of cigarettes smoked daily. All analyses were stratified by gender.
Results: The enactment of a non-hospitality workplace law was associated with an increased likelihood of cessation among men, but not among women. Transition to other smoke-free laws was not associated with increased cessation or lowered intensity. High school graduates decreased their smoking intensity in response to cigarette price change more than those with less than high school. The most consistent predictor of increased probability of cessation and decreased smoking intensity among these older adults was a negative change in health.

Conclusions: These results suggest that older adults' smoking is less sensitive to changes in smoke-free policies or increased cigarette prices than that of younger adults.

Tob. Induc. Dis. 2018;16(Suppl 1):A904

DOI: $10.18332 /$ tid/83943

$12: 30-14: 00$

PS-1154-5 Smoking among migrant factory workers in Thailand

N Kungskulniti ${ }^{1}, \mathrm{~N}$ Charoenca ${ }^{1}$, NKT Chaw

${ }^{1}$ Mahidol University, Faculty of Public Health, Bangkok, Thailand. E-mail:nipapun123@yahoo.com

Background: In Thailand, Samut Sakhon province is the location of thousands of factories that employ Myanmar migrants to process seafood and in the production of other goods. Our aim was to characterize migrants' smoking status and the factors that are important to smoking-related behaviors of this worker population. Methods: A questionnaire was used to evaluate sociodemographic, personal and psychosocial factors related to smoking status. Variables assessed included smoking status, knowledge and behavior. Sample size was calculated using experience from a previous study of migrant workers in this province. A convenience sample of 300 migrant workers at one factory was collected.

Results: Most migrant workers were males, with $90 \%$ current smokers. Most workers were married, but many did not have their families with them since they came as low-wage day workers. Mean age of smoking initiation was 18.59 years. Male gender, limited education, poor knowledge of smoking harms, smoking by friends, and having 6 or more of their 10 closest friends smoking were significant predictors of smoking ( $\mathrm{p}$-value $<0.05$ ). While these variables were significant, the psychosocial variables of workplace and community circumstances (separation from family and isolation from the larger Thai community) resulted in a very high smoking rate among the male workers interviewed (94\%). There was limited encouragement to quit smoking in the migrant work setting or community.

Conclusions: Our results and analysis shows high conformity to peer and workplace conditions which enabled and reinforced smoking behavior. Policies and actions to inform migrants of the dangers of tobacco use and the establishment of smoke-free environments in the workplace and community when working in Thailand should be developed within the migrant community. Since interventions have been developed for Thai factory workers, similar projects in the migrant community should be developed and implemented to promote better health for migrant workers.

Tob. Induc. Dis. 2018;16(Suppl 1):A905

DOI:10.18332/tid/84273

12:30-14:00

PS-1155-5 Tobacco use and other problem behaviours among street youths in South Western Nigeria

0 Uchendu $^{1,2}, 0$ Olabumuyi ${ }^{2}$

${ }^{1}$ University of Ibadan, Department of Community Medicine, Ibadan, Nigeria, ${ }^{2}$ University College Hospital, Community 
Medicine, Ibadan, Nigeria. E-mail: laideadigun@gmail.com Background: Young people are at a vulnerable stage of life that has been associated with initiation and addiction to tobacco and other substances. Street youths compared to their peers are prone to behavioural problems. However the association of smoking with occurrence of health-risk behaviour among street youths has been poorly documented. This study was conducted to determine the association between tobacco use and the occurrence of healthrisk behaviours street youths in South-Western Nigeria.

Methods: A community-based cross-sectional survey was conducted among 946 consenting street youths aged 15-25 years using multi-staged sampling technique. Independent association between tobacco use and other problem behaviours was assessed using logistic regression at $5 \%$ level of significance.

Results: The mean age was 20.4 years $(\mathrm{SD}=3.2)$, about two third were males and half of the study population were single with a partner. A higher proportion of street youths who had spent $\geq$ 1 year on the street were using tobacco compared to those that had spent $<1$ year but it was not significant. Respondents with tobacco use had 9.3 higher odds of using marijuana $(95 \% \mathrm{CI}=4.3$ 20.2), 3.7 higher odds of using injectable drugs $(95 \% \mathrm{CI}=1.2$ 12.0), 2.4 higher odds of having had sex under alcohol influence $(95 \% \mathrm{CI}=1.3-4.5), 1.8$ higher odds of having unprotected sex (95\% CI $=1.0-3.1), 2.6$ higher odds of having $>10$ sexual partners (95\% CI=1.2-5.4) and 2.2 higher odds of having had sex with commercial sex worker $(95 \% \mathrm{CI}=1.2-3.9)$ but 3.3 times less likely to use kolanut $(95 \% \mathrm{CI}=0.15-0.56)$.

Conclusions: Tobacco use increases the risk of street youths engaging in other health-risk behaviours.

Tob. Induc. Dis. 2018;16(Suppl 1):A906

DOI:10.18332/tid/84318

\section{2:30-14:00}

PS-1156-5 Tobacco addiction and it's consequences on oral health of indigenous and rural people - a cross sectional study from central India

N Meshram'1, A Bhandarkar', R Meshram', R Bhandarkar'

'Society for Oral Cancer and Health, Bramhapuri, India. E-mail: societyfororalcancerandhealth@gmail.com

Background: Chewing of tobacco related stuff has gained the social acceptance due to which there is dramatic increase in the incidence of oral cancer and oral precancer. As we proceed more towards the rural areas incidence is increases. It is almost skyrocketing in the tribal area as the tobacco consumption is their social custom. Tobacco is used as pacifier in the tribal population. So keeping in mind this increase prevalence of the oral cancer and oral precancer SOCH ( Society for Oral Cancer and Health ) has conducted a population based survey in the Chandrapur and Gadchiroli districts showing increasing incidence from the urban - rural - tribal area.

Methods: A cross sectional study was done in 4 villages and one tahsil place comprising of rural and tribal population. Target groups were school going children, population of productive age group and population habituated to tobacco. Door to door survey including intra oral examination was done to detect the disease at early stage. Results: Total 1027 individuals were screened for tobacco related oral precancer and cancer. 447 peoples were found addicted to 'Kharra' a form of smokeless tobacco. 331 patients were suffering from oral precancer like oral sub-mucous fibrosis, leukoplakia and erythroplakia. 1 patient of oral malignancy was detected in one of the rural village in the sample.

Conclusions: The prevalence of tobacco habits increases from urban to rural and tribal area which warrants strong tobacco control measures with population based screening for oral cancer and precancers.

Tob. Induc. Dis. 2018;16(Suppl 1):A907

DOI:10.18332/tid/84341

$12: 30-14: 00$

PS-1157-5 A national collaboration on reducing tobacco use in the behavioral health population in the United States

C Douglas ${ }^{1,2}$, B Sinha $^{1}$

${ }^{1}$ American Cancer Society, Atlanta, GA, United States of America, ${ }^{2}$ University of Michigan School of Public Health, Health Management and Policy, Ann Arbor, MI, United States of America. E-mail: cliff.douglas@cancer.org

Background and challenges to implementation: One of the greatest disparities is high smoking prevalence in the behavioral health $(\mathrm{BH})$ population - individuals with mental illness and/or substance use disorders. Despite progress in lowering the rate of tobacco use overall, smoking prevalence in the $\mathrm{BH}$ population in the US is $34 \%$, more than double the national average. The BH population accounts for 240,000 of the 540,000 annual deaths from smoking. BH sufferers die 10 to 25 years earlier than the general population, mainly from smoking-related diseases. Traditionally, health professionals, governmental agencies and advocacy groups have not identified $\mathrm{BH}$ sufferers as a priority population for promoting smoking cessation.

Intervention or response: The American Cancer Society (ACS) and the Smoking Cessation Leadership Center (SCLC) at the University of California, San Francisco, launched a multisectoral partnership in 2016 at a summit attended by 16 national health professional groups, federal government agencies, civil society groups and individual experts in tobacco control and BH. The participants established a new initiative to promote prevention and cessation in the $\mathrm{BH}$ population through networking, education and clinical guidance. ACS and SCLC lead this collaboration and are developing the necessary infrastructure to sustain it. Every participant commits to taking specific actions to enhance collective communications and educate and guide their constituencies of professions and consumers. Results and lessons learnt: The alliance of organizations reviewed smoking prevalence and health impact on the $\mathrm{BH}$ population, and best practices for reducing tobacco use. It then adopted the ambitious goal to reduce smoking prevalence in the behavioral health population in the US from 34\% to $30 \%$ by the year 2020 .

Conclusions and key recommendations: 22 million US smokers suffer from BH conditions. Reducing their prevalence to $30 \%$, or 19.5 million smokers, would result in 2.5 million fewer smokers, saving over a million lives. This unique collaboration endeavors to significantly reduce smoking prevalence and has the potential to be replicated globally.

Tob. Induc. Dis. 2018;16(Suppl 1):A908

DOI:10.18332/tid/84347

\section{2:30-14:00}

PS-1158-5 Tackling tobacco: addressing high smoking prevalence among mental health consumers through systems change

S Walsberger1, L Twyman 1,2, C Watts ${ }^{1}$, A Sobhan'1, R Ireland', B Bonevski²

${ }^{1}$ Cancer Council NSW, Cancer Prevention and Advocacy Division, Woolloomooloo, NSW, Australia, 'University of Newcastle, School of Medicine and Public Health, Newcastle, NSW, Australia.

Background and challenges to implementation: Smoking 
prevalence is five-times higher among Australian mental health consumers than the general population. Smoking cessation support is not routinely provided in community mental health services.

Intervention or response: Cancer Council NSW's Tackling Tobacco program partners with not-for-profit community mental health services to assess opportunities for change and develop an action plan for systems change: data collection and monitoring; leadership; policy; supportive systems; staff training and provision of quit support. A simple survey-type tool was developed to identify opportunities for change and completed pre- and post-project to track progress. Pre- and post-surveys have been completed in 11 of services since 2014. Projects aim to achieve at least four systems changes across the six elements. Results and lessons learnt: $100 \%$ of the 11 services where preand post-surveys were conducted have achieved at least four changes, such as developed a written smoking policy, trained staff, routinely recorded smoking status, and assisted consumers and staff to reduce or quit smoking. The six elements have clarified expectations for systems changes more specific practice guidelines have the potential to improve the quality of smoking care delivered and smoking outcomes for consumers further.

Conclusions and key recommendations: Tackling Tobacco has successfully achieved systems changes in all mental health services. Future plans include developing evidence based guidelines to support systems change to address smoking in community mental health services. A consensus process and clustered randomised controlled trial is planned to validate the guidelines and the revised survey, set benchmarks for systems change to address each guideline, and to examine the effectiveness of the systems change intervention at reducing smoking rates amongst consumers attending community managed mental health services.

Tob. Induc. Dis. 2018;16(Suppl 1):A909

DOI: $10.18332 /$ tid/84375

\section{2:30-14:00}

PS-1159-5 Predicting factors of smoking status in adult schizophrenia smokers

O Khongtor ${ }^{1}$, J Yunibhand ${ }^{2}$, S Preechawong ${ }^{2}$

${ }^{1}$ Royal Thai Navy College of Nursing, Bangkok, Thailand, ${ }^{2}$ Chulalongkorn University, Bangkok, Thailand. E-mail: k.orrawan@hotmail.com

Background: Smoking status defined as self-report of smoking indicated that smokers use the cigarettes per day. Comparison with the general population, schizophrenia smoke more cigarettes per day. Schizophrenia smokers who made quit attempt and reduced the number of cigarette per day were more likely to succeed in quitting smoking. The benefit of identification of factors significantly associated with smoking status maylead to mental health care providers conducting the smoking cessation intervention for this population. This study aiming to examine the direct and indirect relationship of the predicting factors of smoking status in schizophrenia smokers.

Methods: Multistage random sampling was used in the Department of Mental Health of Thailand. Six tertiary psychiatric hospitals were randomly selected from all regions, and 400 schizophrenic smokers were selected by the purposive sampling technique. All participants completed 12 questionnaires, with reliability ranging from 0.72 to 1.00 , and Validity ranging from 0.86 to 1.00 . The acquired data was analyzed using descriptive statistics and Path analysis.

Results: The findings revealed that the hypothesized model fit the empirical data and could explain $48 \%\left(\mathrm{R}^{2}=.48\right)$ of the variance of smoking status. Moreover, nicotine dependence and depression had a significant negative direct effect on smoking status $(B=-.21$, $B=-.52$ ). Readiness to quit had a significant positively direct effect $(B=.39)$ on smoking status and intensity of smoking cessation intervention had a significant positively indirect effect $(B=.14)$ on smoking status through readiness to quit.

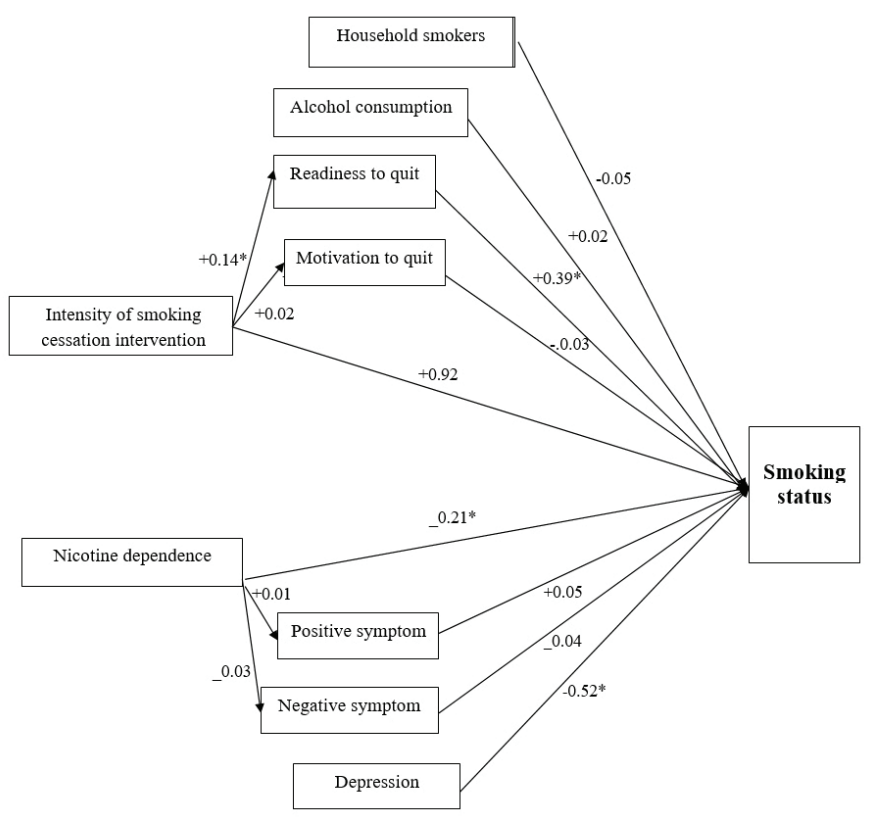

$\mathrm{X}^{2}=35.48, \mathrm{df}=36, \mathrm{p}$ value $=0.49, \mathrm{RMSEA}=0.00$

[Final model and goodness of fit statistics of smok]

Conclusions: The results demonstrated that the factors influencing smoking status were readiness to quit, nicotine dependence, intensity of smoking cessation intervention, and depression. Identifying these variables can be used to develop appropriate smoking cessation interventions to help smokers with schizophrenia success quitting smoking.

Tob. Induc. Dis. 2018;16(Suppl 1):A910

DOI: $10.18332 /$ tid $/ 84387$

12:30-14:00

PS-1160-5 Evaluation of a targeted intervention to most at risk populations of tobacco users through a tea-shops community signage program

T Turk ${ }^{1}, \mathrm{~N}$ Singh Negi ${ }^{2}$ MS Islam ${ }^{3}, \mathrm{M} \mathrm{Hossain}^{4}, \mathrm{~N} \mathrm{Murukutla}^{2}$, S Mullin ${ }^{1}$

${ }^{1}$ Vital Strategies, Policy Advocacy and Communication, New York, NY, United States of America, ${ }^{2}$ Vital Strategies, Policy Advocacy and Communication, New Delhi, India, ${ }^{3}$ Vital Strategies, Policy Advocacy and Communication, Dhaka, Bangladesh, "WBB Trust, Research and Evaluation,Dhaka,Bangladesh.E-mail: tturk@ vitalstrategies.org

Background: The, "Leaving No One Behind" report to address the Sustainable Development Goals, recommends approaches to reduce long-term vulnerability and generate sustainable, meaningful change. Evidence from Bangladesh points to a higher burden of tobacco related morbidity and mortality in "most at risk populations" with vulnerability largely due to social and economic disparities. Challenges in addressing tobacco with most at risk populations are lower access to information, low risk perceptions toward tobacco related diseases, and poor self-efficacy perceptions 
toward quitting. Targeted interventions to vulnerable groups may provide cost effective approaches to address these inequalities.

Methods: A tea-shop signage intervention was utilised in slum areas of Dhaka city. A street-intercept survey evaluation comprised a sample of 600 male and female tobacco users, aged 18 - 55-years randomly selected from 24 sites.. Measures included recall of the tea-shops signage and resultant knowledge, attitude, intention and cessation related indicators. Chi-square analyses and independent measures t-tests explored differences in predictor variables of vulnerability, income, education, gender and location.

Results: Findings identified high recall of the tea-shops lung cancer message (83\%) and strong agreement by campaign aware groups that the signs: Make tobacco users see the real harms of their tobacco use (96\%); Discuss the message with others (62\%); Know about serious illnesses caused by tobacco (75\%); Try to persuade others to stop using tobacco (64\%), and; Makes me more confident to try to quit (76\%). Comparisons of cessation related behaviours by campaign awareness; Tried to stop smoking during the past 2 months (59\% vs $40 \%$ ); Tried to stop smokeless tobacco ( $49 \%$ vs $35 \%$ ); identified significant improvements in quit attempts $(<95 \%)$ and a number of positive correlations according to vulnerability indicators.

Conclusions: Targeted interventions in community settings to most at risk populations of tobacco users, can provide costeffective approaches to support cessation related behaviors.

Tob. Induc. Dis. 2018;16(Suppl 1):A911 DOI:10.18332/tid/84404

\section{2:30-14:00}

PS-1161-5 Integrating cessation strategies into psychiatric treatment for the vulnerable

L Chhakchhuak', L Chhakchhuak ${ }^{2}$, L Hriatpuii ${ }^{3}$, Mizoram

${ }^{1}$ Tripura University, Psychology, Tripura, India, ${ }^{2}$ Government of Mizoram, Directorate of Health Services, Aizawl, India, ${ }^{3}$ Mizoram University, Law, Aizawl, India. E-mail: afaki210@gmail.com

Background: Researches have shown that tobacco use is significantly higher in people with mental illnesses than in the general population. Increased morbidity and mortality in this population is due in large part to treatable medical conditions associated with modifiable risk factors like tobacco. It is likely that tobacco cessation interventions that take into account a history of mental illness and offer a treatment programme that addresses the needs of these people may reverse the disparities seen in treatment outcomes. The aim was to find out the presence and importance of Integrating Cessation Strategies into Psychiatric Treatment.

Methods: 140 participants having mild to serious psychological problems were selected from Aizawl using purposive random sampling with age ranging between 17-30 years. Close ended questions were framed and interview was done.

Results: Out of 140 participants $100 \%$ used some forms of tobacco. $42 \%$ had used tobacco prior to their illness. 58\% used tobacco following their illness, $84 \%$ were high dependent tobacco users. $98 \%$ admit using tobacco because of their psychological problems. $96 \%$ admit feeling better using it. $54 \%$ of the participants have never been advised by their doctor to quit, more than $92 \%$ of these participants were willing to receive treatment for tobacco dependency. At least $42 \%$ have received indirect advice regarding tobacco cessation. No participants received direct advise. 93\% of the participants admit if advised, they will consider cutting down and even quitting.

Conclusions: The results have suggested the need of Integrating Cessation Strategies into Psychiatric Treatment - Training mental health care providers in tobacco cessation strategies and integrating it into mental health treatment plans will come a long way in protecting this vulnerable population. In terms of lives saved, quality of life, and cost efficacy, treating tobacco use is considered to be one of the most important activities a clinician can do.

Tob. Induc. Dis. 2018;16(Suppl 1):A912

DOI:10.18332/tid/84405

\section{2:30-14:00}

PS-1163-5 Characterising smoking among cancer patients in Poland - a cross-sectional pilot survey A Herbec ${ }^{1}$, A Sztuder ${ }^{2}$, I Skrzypczynska², E Garwacka-Czachor ${ }^{2}$, A Maciejczyk², P Krysiak², K Janik-Koncewicz ${ }^{1}$, W Zatonski ${ }^{1}$ ${ }^{1}$ Health Promotion Foundation, Nadarzyn, Poland, ${ }^{2}$ Dolnośląskie Centrum Onkologii (DCO; Lower Silesian Oncology Center), Wroclaw, Poland.E-mail: aaherbec@gmail.com

Background: Smoking among cancer patients is related to poor treatment outcomes and lower quality of life. Intensive treatment of cancer patients in secondary care offers opportunities to deliver more intensive cessation support. As a first step in creating appropriate stop smoking interventions it is important to characterise smoking patterns among cancer patients.

Methods: We conduct an ongoing cross-sectional survey among adult cancer patients treated at the Dolnoślaskie Centrum Onkologii (DCO; Lower Silesian Oncology Center) in the city of Wroclaw, Poland. Data are being collected on smoking status, dependence, quitting history and motivation, and cessation support received.

Results: Among 62 cancer patients surveyed through a pilot questionnaire to date, 47 were ever smokers, of whom $50 \%$ were current daily smokers (52\% women), smoking on average 17 cigarettes per day (range 6-40). A majority of current smokers (70\%) wanted to quit, $56.5 \%$ tried to quit smoking at least once a year in the past 10 years, $100 \%$ tried to quit at least once and $30 \%$ tried to quit more than 3 times or more in the past 12 months. Only $30 \%$ has ever received any advice on quitting from a healthcare professional, $35 \%$ ever used any cessation medications, and only $17 \%$ believed that smoking cessation support available to them was of sufficient quality. Among current daily smokers, $52 \%$ lived with other smokers, $52 \%$ did not know when to find support with sustaining abstinence, and $40 \%$ were not sure how to manage crises moments in abstinence on their own. Data collection continues and updated results will be presented at the conference. Conclusions: Secondary care cancer patients in Poland who are smoking tend to be moderately dependent and are motivated to quit. However, most are not aware of the available cessation support, and only about a third has ever received support with quitting or used cessation pharmacotherapy.

Tob. Induc. Dis. 2018;16(Suppl 1):A913

DOI:10.18332/tid/84508

12:30-14:00

PS-1165-5 Deciding protection or provocation: helping young Siddi women translating knowledge into action

A Arya'

IIndependent Educationalist and Consultant, Research, Bilaspur, India.E-mail: ankitaapj@gmail.com

Background: Tobacco consumption in nonsmoking forms is culturally accepted even among scheduled tribal women. Hence, smokeless forms of tobacco use by women are widely prevalent.

Methods: A cross-sectional study was conducted in six tribal villages of Gujarat, India. Data was collected by interview from 240 Siddi women by home visits. A quasi-experimental study design 
with two measurements at baseline and 6 months post-intervention to investigate an intervention in 15-25-year-olds. The intervention programme consists of three 1 hour interactive modules and control group. The study questionnaire measured tobacco smoking and consumers, tobacco related cognitions, and gender, social and environmental aspects was designed and pre-tested in advance. The study compared prevalence of smokers and consumers in the two study arms at 6 months after the intervention.

Results: Prevalence of tobacco use (all forms), smokeless tobacco use and smoking were $54.45 \%, 53.41 \%$, and $23.14 \%$, respectively. Prevalence of tobacco use was more in late adolescent period. Education showed significant protective effect on tobacco use. Bidi was commonly used for smoking, while pan masala and gutka were preferred smokeless tobacco. Almost all smokers were also using smokeless tobacco. Around 69\% women have heard of tobacco prevention message, but only twenty could interpret it correctly. $79 \%$ of the study participants successful stopped using tobacco along with significant reduction in frequency or quantity of tobacco consumption observed in all the study participants. The key barriers to quitting were identified as social norms followed by workload and stress.

Conclusions: Anti-tobacco activities need to scale up for tribal people, with more emphasis on behavior change through group or personal approach. School programs may have some limitation in tribal area due to high school dropout, and low enrolment. Prevention activities need more focus on smokeless tobacco use and bidi smoking.

Tob. Induc. Dis. 2018;16(Suppl 1):A914

DOI:10.18332/tid/84552

\section{$12: 30-14: 00$}

PS-1166-5 Strategies in engaging special populations for Tobacco Cessation in South Florida, USA - success models

\section{A Aftab}

'University of Miami, Miller School of Medicine, Family Medicine, Miami, FL, United States of America. E-mail: aaftab@med. miami.edu

Background and challenges to implementation: The University of Miami (UM) has been working on a State Funded Tobacco cessation program since 10 years conducting free in person cessation group counseling in South Florida from Miami to Key West in a very unique and diverse population ethnically as well as socioeconomic profiles from poorest of the poor and richer to the richest. Homeless and LGBT are the other special populations with a high prevalence of tobacco use.

Intervention or response: On one hand we deal with cultural diversity like Spanish, Haitian and African American population and on the other hand dealing with the highest tobacco user populations like homeless shelters, prison work release rehabilitation centers, LGBT community centers and HIV clinics. Challenges faced were:

- Trust

- Safe spaces

- Staff and patient turnover

- Inability to contact participants before or after program Healthcare provider misconceptions and apathy - "tobacco users don't want to quit".

Results and lessons learnt: UM tobacco cessation program have been able to take many challenges and have achieved a high quit rate between $46-49 \%$ with a highly addictive population. Some of the lessons learned are:
Administration support is necessary

Finding a champion

On-going communication with facility staff

- Staff education dispels myths

- Offering recurring groups

- Consistent presence increases trust and encourages culture change

- Convenience/flexibility,

Offer groups during high traffic times

- Allocate extra time on-site

- Train facility staff to do groups

- Include as part of position description and annual evaluations

- Utilize community partnerships/collaborations

- Can provide services not offered by TFF/AHEC, e.g. transportation

- Highlight program benefits to facilities

Conclusions and key recommendations: Tobacco is the most addictive substance in the Universe and quitting is very difficult but following cessation program features are essential:

- Persistence

- Roll with resistance

- Adapt to meet needs/restrictions

- Understand your population and the unique challenges/ barriers they experience

Tob. Induc. Dis. 2018;16(Suppl 1):A915

DOI:10.18332/tid/84556

\section{2:30-14:00}

PS-1167-5 Tobacco usage among tribal population of Nilgiris, Tamil Nadu - a cross sectional study V Murugaboopathy ${ }^{1}$

'Indira Gandhi Institute of Dental Sciences, Public Health Dentistry, Pondicherry, India.E-mail:drvikneshan@gmail.com Background: Tobacco use is attributed for deaths of 3.5 to 4 million people globally, which is expected to increase to about 10 million during 2020 and around more than two-third will be occurring in developing countries as they are showing an increasing trends of tobacco use. Anti-tobacco messages or campaigns do not reach the Tribal regions. Tobacco use is ingrained in many of the cultural practices of tribal people. This study aimed to assess the prevalence of tobacco usage among tribal populations of Nilgiris region, Tamil Nadu.

Methods: A cross sectional study was conducted among 4 villages of Conoor and Kothagiri districts of nilgiris region. A total of 363 people were screened and their tobacco usage status was assessed using Interview method. A trained interviewer along with help of community leader collected information about the usage of tobacco among the populations. Oral health status and presence of oral mucosal lesions were also recorded.

Results: Smokeless tobacco usage was high among the subjects. Majority of the males between 21-40 years were having both smoking and smokeless tobacco. Bidis was the most common form of smoking tobacco and gutka was the commonest smokeless tobacco used. More than 32\% females in the age group of 30-45 years were tobacco users.

Conclusions: Tobacco usage was high among the tribal populations. Interestingly, the number of tobacco users among females was more prevalent. Customized tobacco cessation education programs has to be carried out for effective control of tobacco usage among the tribal population of Nilgiris region.

Tob. Induc. Dis. 2018;16(Suppl 1):A916

DOI:10.18332/tid/84588

$12: 30-14: 00$ 
PS-1168-5 How to approach the question of smoking in health units with a tuberculosis program in Brazil?

VCunha', VL Borges', RH Meirelles'², A Gomes ${ }^{3}$, M Evangelista ${ }^{4}$, R Lamy $^{1}$ 'Instituto Nacional de Cancer / Ministry of Health, Divisão de Controle do Tabagismo, Rio de Janeiro, RJ, Brazil, ${ }^{2}$ Associação Brasileira de Medicina, Rio de Janeiro, RJ, Brazil, ${ }^{3}$ Pan American Health Organization, Washington, WA, United States of America, ${ }^{4}$ Ministerio de Salud, Programa Nacional de Controle da Tuberculose, Brasilia, DF, Brazil. E-mail: valeriac@inca.gov.br Background: In Brazil, tuberculosis is a serious public health problem, every year approximately 70,000 new cases are reported and 4,500 deaths occur due to the disease. The major global guidelines on tuberculosis do not highlight the need for tobacco control to better control tuberculosis. In Brazil, tuberculosis is treated free of charge and provided by the Unified Health System. In order to guide the approach to smoking in health units with a tuberculosis program, a manual has been developed to empower services, health professionals and managers.

Methods: Elaboration of the Manual "How to address the issue of tobacco control in health units with a tuberculosis program in Brazil?" Was based on the Clinical Protocol and Guidelines on Nicotine Dependence advocated by the Brazilian Ministry of Health. He participated in the elaboration of the National Tobacco Program of the Ministry of Health, National Tuberculosis Program of the Ministry of Health, Representative of PAHO Brazil and representative of the Brazilian Medical Association.

Results: Manual has been finalized and will be distributed to all health units that attend the National Tuberculosis Program. It is hoped to contribute to organize the articulation of tuberculosis services in the health units with the tobacco control program, to establish better care practices for the patient with tuberculosis who wants to abandon tobacco use.

Conclusions: The manual "How to approach the issue of tobacco control in health units with a tuberculosis program ?" Will instrumentalize health professionals to approach smoking cessation among the population with tuberculosis and optimize that this approach occurs preferentially in the attention Mainly in areas of greater poverty and misery. Thus, units should be able to apply at least the minimum approach or that have all the guidance to refer patients with tuberculosis who demonstrate motivation to quit smoking to units licensed for smoking cessation in the municipality.

Tob. Induc. Dis. 2018;16(Suppl 1):A917 DOI:10.18332/tid/84618

\section{2:30-14:00}

PS-1169-5 Of Mice and Menthol: Rethinking Wellintentioned but Insufficient Strategies to Counteract Tobacco Use and Promotion in Minority Populations A Blum ${ }^{1}$

'University of Alabama, Center for the Study of Tobacco and Society, Tuscaloosa, AL, United States of America. E-mail: ablum@ua.edu

Background and challenges to implementation: Mentholated cigarettes have become an advocacy issue because they have been disproportionately promoted to African-American and Hispanic minority groups for decades. These populations have become the largest consumers of menthol brands. Although San Francisco banned the sale of menthol cigarettes in 2017, the net impact of opposing menthol has been more symbolic than successful. The need to fight for a menthol ban could have been avoided had it been included in legislation passed in 2009 by the US Congress to let the Food and Drug Administration regulate tobacco products, but cigarette maker Philip Morris threatened to withdraw its support. Intervention or response: Castigating tobacco companies for targeting menthol brands to minorities has overshadowed the public health goal of reducing tobacco use and promotion as a whole. Campaigns that condemned specific cigarette brands marketed to minorities (eg, Uptown, Menthol X, and Kool Mixx) have focused so intensely on the aspect of racial targeting that they insufficiently emphasized the health and economic harmfulness of smoking any brand of cigarettes. Thus little has been accomplished to reduce smoking's longterm impact.by the focus on menthol.

Results and lessons learnt: Scant new federal or state legislation on smoking has been proposed or enacted to help reduce smoking in minority communities. Existing programs have largely failed to elucidate the relative impact of smoking on minority communities compared to other emerging health threats such as obesity, drug use, and AIDS. Few minority business leaders have been enlisted in the effort to reduce smoking in minority communities. Groups such as the NAACP, the Urban League, and La Raza continue to accept tobacco industry funds and have not supported aggressive anti-smoking campaigns. Leading black publications such as EBONY continue to accept cigarette advertising and have never published articles on smoking.

Conclusions and key recommendations: Rethinking strategies to counteract tobacco use and promotion in minority populations is urgently needed.

\section{Tob. Induc. Dis. 2018;16(Suppl 1):A918 DOI:10.18332/tid/84672}

\section{$12: 45-14: 00$}

EP-234-5 Afghan frontier: understanding tobacco practices among migrant population in India

R Mehra', V Mohanty', A Y Balappanavar', K Mehra'2, S Kapoor'1, V Gupta ${ }^{1}$

'Maulana Azad Institute of Dental Sciences, Public Health Dentistry, Delhi, India, ${ }^{2}$ Max Super Speciality Hospital, Saket, Pulmonology, Delhi, India. E-mail:rm17111991@gmail.com

Background: According to the World Refugee Survey by US Committee for Refugees and Immigrants, migrants from Afghanistan constitute the 4th largest group of migrants to India. No previous studies have been conducted to assess the tobacco consumption status and pattern among this migrant population. Hence the aim of our study was to understand the tobacco use patterns and barriers among the migrant population from Afghanistan.

Methods: A cross-sectional study was conducted among a convenience sample of 127 Afghan tobacco users residing in Delhi, India in the month of June, 2017. A close-ended selfadministered validated questionnaire translated in Persian language was circulated among Afghan tobacco users visiting local cafés and restaurants. The questionnaire assessed their socio-demographic factors, tobacco use patterns and barriers to tobacco cessation. Data was entered into digital spreadsheets and descriptive inferences were drawn. (SPSS v.21)

Results: A total of 127 male Afghan tobacco users with mean age of $33.49 \pm 11.97$ years voluntarily completed the questionnaire. Better work opportunities were the most common reason for migration. Most of them (69\%) smoked tobacco and 15.5\% only used Naswar. Half (52\%) of the respondents continue to use tobacco products manufactured in Afghanistan with $62 \%$ procuring the product through social means (friends/family). Higher cost of tobacco products and stringent law enforcements in India were common barriers to tobacco use. Personal barriers 
to quitting included loss of social circle and fear of withdrawal symptoms among the respondents. Strikingly, 93\% were unaware about the availability of local cessation services.

Conclusions: Majority of the Afghan respondents continue to use products manufactured in Afghanistan, probably as an attempt to hold fast to their culture and social practices. Since most of the respondents are unaware about the availability of cessation services, attempts should be made to generate awareness and motivate them to quit.

Tob. Induc. Dis. 2018;16(Suppl 1):A919 DOI:10.18332/tid/84549

\section{$12: 45-14: 00$}

EP-237-5 Tobacco retail outlet density, local smoking norms and maternal smoking during pregnancy: a case crossover study

J Pearce ${ }^{1}$, T Clemens ${ }^{1}$, N Shortt ${ }^{1}$, C Dibben $^{1}$

${ }^{1}$ University of Edinburgh, School of GeoSciences, Edinburgh, United Kingdom. E-mail: jamie.pearce@ed.ac.uk

Background: International evidence suggests local tobacco retailing is associated with individual smoking behaviours. However, most evidence is cross-sectional. This longitudinal study examines the effects of exposure to high densities of tobacco retailers on maternal smoking during pregnancy.

Methods: Scottish maternity records (2000-2015) were extracted. Information included maternal smoking and postcode of residence at delivery which enabled linkage to a GIS-based measure of residential tobacco retail density. Because the full population of births was available ( $\mathrm{n}=\mathrm{c} .750,000)$, it was possible to capture multiple births to the same mother and hence to examine the extent to which changes in smoking behaviour between pregnancies was associated with changes in tobacco retail density exposure through residential moves. A neighbourhood measure of "social norms" (maternal smoking rates) was also linked. A case crossover approach was adopted which treats each mother as their own control and removes all confounding by time-invariant characteristics of the mother.

Results: Fixed-effects analysis suggests the risks of smoking during different pregnancies to the same mother are around $40 \%$ higher (OR 1.37, CI 1.15, 1.63) for those living in the highest density tobacco retailing areas compared to zero density areas. Including the local social norms variable slightly strengthened the observed effects with an excess risk of $39 \%$ in the highest density quintile (OR 1.39, CI 1.17,1.66).

Conclusions: This longitudinal study show the significant role of the tobacco retailer environment in influencing smoking during pregnancy. Findings emphasise the need to target the supply of tobacco products as a means of reducing smoking rates.

Tob. Induc. Dis. 2018;16(Suppl 1):A920

\section{DOI:10.18332/tid/84262}

\section{$12: 45-14: 00$}

EP-242-5 Cigarette demand and supply in Africa $\mathrm{N}$ Vellios ${ }^{1}, \mathrm{H}$ Ross $^{1}$, A-M Perucic ${ }^{2}$

'University of Cape Town, Economics, Cape Town, South Africa, ${ }^{2}$ World Health Organization, Geneva, Switzerland. E-mail: nicolevellios@gmail.com

Background: Since the tobacco epidemic is moving from developed to developing countries, it is important to understand trends in cigarette demand and supply. We focus on the African market since it offers the tobacco industry one of the best expansion potentials.

Methods: A large variety of data from commercial (Canadean,
Euromonitor International, tobacco industry reports), governmental (United Nations Comtrade, national statistics), and academic sources (Cigarette Citadels map and journal articles) were used to study cigarette demand in Africa from 1990 to 2012. We focus on cigarette production and international cigarette trade to detect structural changes in cigarette supply over the past few decades. We contrast data from these different sources.

Results: Cigarette demand and supply data in Africa is limited and is sometimes inconsistent across different sources. Given this caveat, we found that the overall demand for cigarettes (measured by sales) in the 22 countries covered by Canadean, which covers $80 \%$ of Africa's population, increased by $44 \%$ (from 165.6 billion cigarettes to 238.5 billion cigarettes) from 1990 to 2012 . This higher demand has been met by an increase in cigarette production in these 22 countries by $106 \%$ during the same period. As result, Africa has transitioned from being a net importer to a net exporter of cigarettes. At the same time cigarette production has become more concentrated as the tobacco industry has strategically identified certain countries as production hubs.

Conclusions: Monitoring the production, consumption and trade of cigarettes by improving the quality of surveillance is important to understand the demand and supply of cigarettes not only in Africa, but also globally.

Tob. Induc. Dis. 2018;16(Suppl 1):A921

DOI: $10.18332 /$ tid/84008

\section{$12: 45-14: 00$}

EP-243-5 Prevalence and correlates of ENDS use among adults being treated for chronic lung disease M Moran', S Biswal², J Cohen², R Henderson², J Holbrook², V Sidhaye $^{3}, \mathrm{R}_{\text {Wise }}^{3}$

Johns Hopkins Bloomberg School of Public Health, Health, Behavior \& Society, Baltimore, MD, United States of America, 2Johns Hopkins Bloomberg School of Public Health, Baltimore, MD, United States of America, ${ }^{3}$ Johns Hopkins University School of Medicine, Baltimore, MD, United States of America. E-mail: mmoran22@jhu.edu

Background: Chronic lung disease such as asthma or COPD may be exacerbated by electronic nicotine device (ENDS) use. Despite this, little is known about the extent to which adults with chronic lung disease use ENDS and what factors are associated with use. Methods: We analyzed data from the second wave of the Population Assessment of Tobacco and Health (PATH) study. The PATH study recruited 28,362 U.S. adults over the age of 18 using a multi-stage randomized sampling protocol. Our analysis was restricted to adults who reported being treated for COPD or asthma in the past 12 months $(\mathrm{N}=1,748)$. Among these individuals, we examined prevalence of ever and current ENDS use, intensity of use, and motivations for use.

Results: Approximately one-quarter (24.72\%) of individuals being treated for COPD or asthma reported ever using ENDS; $4.09 \%$ were established users and $3.99 \%$ were experimental users. Established e-cigarette users were more likely to be white and younger. The average number of puffs from an e-cigarette on the last day used was 18.05. Key motivations for ENDS use among this population include: perceptions of less harm to the user $(79.72 \%)$ and to other people (78.84\%), because they can be used in places where people can't smoke (74.2\%), perceptions that they help people quit smoking $(73.94 \%)$, perceived acceptability to nonsmokers than cigarettes (70.37\%), and appealing flavors (64.0\%). Conclusions: A significant number of individuals being treated for lung disease have or are currently using ENDS, and many of 
these individuals use these products for health-related concerns. Healthcare providers treating these patients should ask about ENDS use and offer counseling and treatment to help these individuals become tobacco free.

Tob. Induc. Dis. 2018;16(Suppl 1):A922

DOI:10.18332/tid/84019

\section{$12: 45-14: 00$}

EP-244-5 Self-initiated pre-quit smoking reduction among community correction smokers

K Cropsey ${ }^{1}$, S Schiavon ${ }^{1}$

${ }^{1}$ University of Alabama at Birmingham, Psychiatry, Birmingham, AL, United States of America. E-mail: kcropsey@uabmc.edu Background: Smoking remains the leading cause of preventable death in the United States. While smoking prevalence in the general population has declined in the U.S., smoking remains highly prevalent in prison populations with approximately $70 \%$ reporting current smoking. Individuals within the criminal justice system represent a vulnerable group of smokers with limited access to traditional healthcare or smoking cessation treatments. This study examined differences in smoking characteristics based on smokers' self-initiated pre-quit reductions in cigarettes per day (CPD).

Methods: This study utilized data from a randomized clinical trial comparing four sessions of smoking cessation counseling to brief physician advice only to quit. All participants received 12 weeks of bupropion. Participants $(\mathrm{N}=500)$ were recruited from community corrections supervision $(\mathrm{M}$ age $=37.4,67 \%$ male, $68 \%$ non-white). Questionnaires assessed smoking history and participants were seen at baseline and one week later to begin treatment. The present study compared two groups of smokers based on whether they self-selected to reduce smoking by at least $25 \%$ between baseline and first treatment session $(n=121)$ or whether they increased smoking or did not reduce $(n=365)$.

Results: Chi-square and one-way analysis of variance revealed that individuals who reduced smoking pre-quit were more likely to be Black and lower educated with at least one prior quit attempt. Additionally, individuals who reduced smoking were older when first become a daily smoker, reported higher expectations for self-help materials and group therapy, as well as reported lower withdrawal and withdrawal expectations. No reduction in smoking related to lower likelihood of achieving at least one quit attempt during the one-year study period as compared to either increased or reduced smoking.

Conclusions: Action pre-cessation resulted in successes postquit. Overall, individuals who self-initiate pre-cessation smoking reduction exhibit higher expectations for some smoking cessation treatments and lower expectations for withdrawal improving likelihood of smoking cessation.

Tob. Induc. Dis. 2018;16(Suppl 1):A923

DOI:10.18332/tid/84136

\section{2:45-14:00}

EP-245-5 National estimates and correlates of cigarette smoking among Hispanic/Latino construction workers in the US

T Asfar ${ }^{1}, K_{\text {KL Arheart }}{ }^{1}$, AJ Caban-Martinez ${ }^{1}$, D Sierra ${ }^{1}$, EC RuanoHerreria $^{1}$, LA McClure ${ }^{1}$, KD Ward ${ }^{2}$, DJ Lee ${ }^{1}$

${ }^{1}$ University of Miami, Miller School of Medicine, Miami, FL, United States of America, ${ }^{2}$ University of Memphis, Memphis, TN, United States of America. E-mail: tasfar@miami.edu

Background: Smoking prevalence among construction workers (CWs) is nearly twice the national average of smoking in the US. In 2014, there were more than 2.6 million Hispanic/Latino CWs in the US, representing nearly a third of the US construction workforce. In this study, we used a national sample of US adults to examine the prevalence and predictors of cigarette smoking among Hispanic/Latino CWs.

Methods: Data were pooled from the 2001-2010 (5 cycles) National Health and Nutrition Examination Survey. All respondents $>20$ years who self-identified as Hispanics/Latino and were working in the construction industry were selected $(n=430)$. We calculated prevalence rates and $95 \%$ confidence intervals of the demographic characteristics of workers stratified by smoking status (current, former, never smoker). Logistic regression analysis were performed with adjustments made for the complex survey design.

Results: Overall, 99.0\% of Hispanic/Latino CWs were males, $17.9 \%$ were non-US born, $42.4 \%$ spoke only Spanish, $61.3 \%$ did not complete high school, $32.0 \%$ were without health insurance, $30.5 \%$ fell below the poverty level, and $31.0 \%$ reported smoking. The mean age at initiation of smoking was $15.1(\mathrm{SD}=13.7-16.4)$, and the mean of number of cigarettes smoked per day was 12.1 (4.5-19.7). Compared to workers who do not smoke, smokers were significantly more likely to be between the ages of $20-45$ (78.8\% vs. $90.2 \%$ ), have higher exposure to mineral dusts at work ( 38.4 vs. $66.0 \%)$ and secondhand smoke at home $(4.9 \%$ vs. $23.3 \%)$ and work $(29.5 \%$ vs. $43.3 \%)$, and higher level of depression $(2.9 \%$ vs. $8.8 \%)(\mathrm{P}<0.05$ for all).

Conclusions: Smoking prevalence is high among Hispanic/Latino CWs. This group was identified as a high-risk group for smokingrelated health problems and injuries, and should therefore be a prime focus for smoking cessation efforts. Future smoking cessation interventions need to be adapted to their culture and work/life circumstances.

Tob. Induc. Dis. 2018;16(Suppl 1):A924

DOI: $10.18332 /$ tid/84546

$12: 45-14: 00$

EP-246-5 Secondhand smoke and its impact on nicotine dependence and smoking cessation among individuals with mental illnesses

C Okoli 1,2

'University of Kentucky, Nursing, Lexington, KY, United States of America, ${ }^{2}$ Eastern State Hospital, Nursing, Lexington, KY, United States of America. E-mail: ctokol1@uky.edu

Background: Individuals with mental illnesses (MI) are a vulnerable population with high tobacco use and related morbidity and mortality. However, few studies examine factors associated with successful cessation among them. Secondhand smoke exposure (SHSe), for example, is an important predictor of greater nicotine dependence (ND) and poor cessation (SC) outcomes. However, little is known about the impact of SHSe on ND or SC in persons with MI. Hence, the objectives of our study were to:

1) Quantify SHSe in this population and

2) examine its association with ND and SC attempts.

Methods: A cross-sectional survey (15-20 minutes) was conducted of 118 tobacco-users in an inpatient psychiatric facility. Information on demographics, tobacco use and SHSe history, motivation to quit smoking, ND (on a scale of 0-10), and SC attempts (in the past year) were obtained. Participants' reported environmental (past 7 days), peer and family, and perceived SHSe (scale of 0-10) were described. Multivariate linear regression analyses were used to examine the association between SHSe and ND while logistic regression analysis assessed the associations between SHSe and SC attempts (yes vs. no). 
Results: The primary sources of environmental SHSe were from the car $(63.6 \%)$ and home (51.7\%); primary sources of peer and family SHSe were close friends (67.8\%) and parents (65.3\%); and the perceived SHSe was a mean score of $6.2(\mathrm{SD}=3.5)$. Although perceived SHSe was significantly associated with ND $(\beta=.39, \mathrm{p}<$ $.0001)$ in multivariate analyses, no SHSe variables was associated with $\mathrm{SC}$ attempts. However, motivation to quit smoking variables were associated with SC attempts.

Conclusions: Hospitalized patients with MI report high SHSe. Given that SHSe is associated with higher ND in this study, routine screening for SHSe may be incorporated as part of health assessment in this population. In addition, targeted interventions and policies should be examined to reduce SHS in this population.

Tob. Induc. Dis. 2018;16(Suppl 1):A925

DOI: $10.18332 /$ tid/83817

\section{$15: 45-16: 45$}

LB-1364-5 Longitudinal association of forced migration with cigarette smoking

T Korhonen', M Piirtola' ${ }^{1}$, J Kaprio

'University of Helsinki, Helsinki, Finland. E-mail: jaakko.kaprio@ helsinki.fi

Background: Stressful life events associated with forced migration increase vulnerability for adverse health consequences. Intermediate factors, such as smoking, can shed light on the mechanisms underlying this process. We investigated if and how forced migration from Ceded Karelia due to the World War II is associated with subsequent cigarette smoking within the nationwide Finnish Adult Twin Cohort.

Methods: Total of 12,933 were born before 1945 and included in this study. The exposure (forced migration due to war, categorized as 'no', 'once', '2+') used following measures: the municipality of birth and whether the respondent had moved municipality ever and if so, for what reason (10 options, including war). Of the respondents born in Ceded Karelia 83\% replied that they had had to move due to war, with the corresponding percentages varying from $5 \%$ to $20 \%$ in other provinces. Detailed smoking questions were used to create two smoking status variables: 1) 'ever smoker' versus 'never smoker' and 2) 'current smoker' versus 'non-current smoker'. Logistic regression with correction for sampling of twins as twin pairs was used for analyses.

Results: Those who had experienced forced migration due to World War II showed higher likelihood of being ever smoker (age, sex, education, and birth area adjusted Odds Ratio $=1.70,95 \%$ Confidence Interval $=1.43-2.02 ; \mathrm{p}<0.001)$ than those without forced migration experience. Among 1155 individuals born in Ceded Karelia increased likelihood for current smoking was found (adjusted Odds Ratio=1.73; 95\% Confidence Interval=1.08-2.78; $\mathrm{p}=0.023$ ) in those with migration experience.

Conclusions: Forced migration experience as a stressful life event seems to increase vulnerability for use of addictive substances, such as cigarette smoking. A further analysis shall explore whether cigarette smoking is a mediator between forced migration and mortality by the end of 2016 .

Tob. Induc. Dis. 2018;16(Suppl 1):A926

DOI:10.18332/tid/84736

\section{5:45-16:45}

LB-1372-5 Use of tobacco and related health problems among urban homeless women

M Chourase $^{1}$

${ }^{1}$ International Institute for Population Sciences (IIPS), Mumbai,
India.E-mail: mithlesh.1303@gmail.com

Background: Smoking tobacco doubles the risk of suffering from tuberculosis. A large proportion of world's population with tuberculosis and tobacco users are from India. No study is available on use of tobacco and prevalence of TB among the homeless women in urban India. This study attempts to understand the pattern of tobacco use and its linkages to suffering from TB among the homeless women living in New Delhi, India.

Methods: Primary data was collected among the homeless women above 15 years in New Delhi city to understand the risky behaviour and prevalence of TB. The data was collected during July-December 2015. A total of 300 homeless women were interviewed on various issues like causes of homelessness, living condition, substance use and related health issues. Frequency tables, chi-square test and logistic regression are used to full fill the objectives.

Results: Of 300 homeless women, 59\% (176) women are engaged in use of smokeless and smoking tobacco, and almost all are the daily users. The study found that $7.4 \%$ among tobacco users are suffering from tuberculosis compared to $1.6 \%$ among those tobacco non-users. The chi-square test shows differences are statistical significant. Similarly, the tobacco users are suffering from tobacco related health issues such as rupture of cheeks, oral infection and staining of teeth. About 13\%, 10\% and $26 \%$ of tobacco users are suffering from rupture of cheeks, oral infection and staining of teeth compared to $3 \%, 3 \%$ and $6 \%$ among tobacco non users respectively. With reference to tobacco non-users, adjusting for other socio-economic, living in shelter or non-shelter and years of homelessness, the odds of suffering from tuberculosis is 5.66 [95\% CI:2.9-10.0, $\mathrm{p}=<0.01]$ among the tobacco users.

Conclusions: The present study indicates urgent need of raising awareness of tobacco related health issues.

Tob. Induc. Dis. 2018;16(Suppl 1):A927

DOI:10.18332/tid/84708

\subsection{Tobacco, inequality and poverty}

\section{2:30-14:00}

PS-1170-5 Family and school socioeconomic status as predictors of tobacco and e-cigarette use in adolescents: a study from a perspective of material, human, and social capital

A Pérez ${ }^{1,2}$, P Morello $^{1}$, S Braun ${ }^{1}$, J Thrasher ${ }^{3}$, R Mejía $^{1,4}$

${ }^{1}$ Centro de Estudios de Estado y Sociedad (CEDES), Buenos Aires, Argentina, ${ }^{2}$ Facultad de Ciencias Exactas y Naturales, Universidad de Buenos Aires, Buenos Aires, Argentina, ${ }^{3}$ University of South Carolina, Department of Health Promotion, Education, and Behavior, Columbia, SC, United States of America, ${ }^{4}$ Universidad de Buenos Aires, Hospital de Clínicas, Buenos Aires, Argentina. E-mail: adrianaperez000@gmail.com

Background: The contradictory findings about the role of the socioeconomic status (SES) in accounting for substance use in adolescence can be explained by the lack of consensus on how best to conceptualize and measure SES. Based on Coleman's social theory, this study examines the association of family and school SES with tobacco and e-cigarette use.

Methods: Data came from a cross-sectional, school-based survey of middle-school early adolescents attending 33 schools in three Argentinian cities $(n=3,110)$. Family SES was evaluated using: 1) family affluence scale (FAS) for material capital, 2) parent education for human capital, and 3) nuclear family and parentadolescent communication for social capital. A school SES index was computed aggregating FAS and parent education from 
students. Multilevel logistic regression models regressed current tobacco use (in the past 30 days) and ever e-cigarette use on family and school-level SES, controlling for sex and age.

Results: $17.7 \%$ students were current tobacco smokers and $7.7 \%$ have ever used e-cigarette. At family level, material capital was unassociated with tobacco smoking, but positively associated with e-cigarette use; human capital was unassociated with both tobacco smoking and e-cigarette use; and social capital was inversely associated with both tobacco smoking and e-cigarette use. After adjusting for family SES, students from schools with higher SES had lower likelihood of tobacco smoking ( $\mathrm{OR}=0.25,95 \% \mathrm{CI} 0.09$ $0.74)$ and higher likelihood of e-cigarette use $(\mathrm{OR}=4.36,95 \% \mathrm{CI}$ 1.25-15.23) than those from schools with lower SES.

Conclusions: Family capital measures are differentially related to student substance use, pointing out that SES indicators are not interchangeable. School SES has an independent effect on substance use above and beyond that of family SES. These findings emphasize the need for SES conceptualization and consistent selection of indicators in order to study the effect of SES on health research and its role in identifying students at-risk of substance use.

Tob. Induc. Dis. 2018;16(Suppl 1):A928

DOI:10.18332/tid/84237

\section{$12: 30-14: 00$}

PS-1171-5 "Leaving no one behind": the vulnerability of children from low SES households suffering from tobacco-related harm in light of human rights and social justice

MEC Gispen ${ }^{1}$

'University of Groningen, International Law, Groningen, Netherlands.E-mail:m.e.c.gispen@rug.nl

Background: Children from low SES households are particularly vulnerable to tobacco-related harm having adverse effects on their health, development, and wellbeing. Their disadvantaged position to positive health outcomes is caused by an intersectionality of medical, child developmental, and socioeconomic factors. Human rights law is a key vehicle to achieving sustainable development, health equality and ultimately social justice and as such an important mechanism to support the rights of children from low SES households in tobacco control interventions. However, the principle of vulnerability is also contested in human rights scholarship. Against this background, the objective of this paper is to generate new knowledge on the potential role of vulnerability as legal principle in protecting children from low SES households against tobacco-related harm. The central question of this study is to what extent vulnerability can inform the specific state obligations flowing from the human rights of children to adequately address the situation of children from low SES households in tobacco control interventions.

Methods: The paper design is a legal analysis. The paper reflects a normative analysis of the scope and content of vulnerability as principle in human rights law and locates the principle in the interpretation of specific rights. Methods include literature research, document analysis (primary and secondary legal sources) and treaty interpretation.

Results: The results are that vulnerability may be of benefit in the progressive interpretation of human rights law. It can inform the application of specific obligations flowing from at least the health and interest rights of children.

Conclusions: The paper concludes that governments have obligations to promote the underlying determinants of health, actively support the wellbeing of children from low SES households in particular, and counter their systematic health inequalities, including in tobacco control mechanisms.

Tob. Induc. Dis. 2018;16(Suppl 1):A929

DOI: $10.18332 /$ tid $/ 84288$

\section{2:30-14:00}

PS-1172-5 Socioeconomic differences in secondhand smoke exposure among adolescent in South Korea: focusing on exposure at home

S Kwon', S Jeon

'Korea Health Promotion Institute, National Tobacco Control Center, Seoul, Korea, Republic of. E-mail: soontack@khealth. or.kr

Background: Secondhand smoke(SHS) is known to be one of the major causes of illness and death. Children and adolescents in smoking households are more likely to be exposed to SHS. They have a high incidence of upper respiratory infection and other respiratory symptoms. Also, disparity in SHS exposure from socioeconomic status could be main reason for health inequalities. Even if SHS has been shown to be a major contributor to health inequalities, there were few previous studies considering SHS exposure and socioeconomic status in South Korea. The aim of this study is to investigate the association between socioeconomic status and adolescent SHS exposure at home.

Methods: The data was based on the 12th Korea Youth Risk Behavior Web-based Survey(2016) from the Ministry of Education and of Health and Welfere of Korea containing case of 59,095 non-smoking adolescent aged 12 to 18 years. The dataset was analyzed using descriptive statistics and logistic regression.

Results: The adolescent who reported SHS exposure at home accounted for $28.5 \%$ among the study population. It was more likely to be exposed to SHS stepwise from high to low household income level $(\mathrm{x} 2=356.80, \mathrm{p}<0.001)$. The lower level of parental education, adolescents were more likely to be exposed to $\operatorname{SHS}(\mathrm{x} 2=568.59, \mathrm{p}<0.001)$. The low $(\mathrm{OR}=1.20, \mathrm{CI}=1.09-1.32)$ and very low $(\mathrm{OR}=1.23, \mathrm{CI}=1.04-1.44)$ income level groups were significantly associated with adolescent SHS exposure. The group of whose mother's education was higher than college was less likely to be exposed to SHS than the group of lower than middle school $(\mathrm{OR}=0.84, \mathrm{CI}=0.78-0.99)$.

Conclusions: Inequalities in SHS exposure exist among adolescent in South Korea. Especially in low income group, adolescent were more likely to be exposed to SHS at home. This study implies that public health strategies are urgently needed to protect vulnerable social group from SHS exposure.

Tob. Induc. Dis. 2018;16(Suppl 1):A930

DOI:10.18332/tid/84395

\section{2:30-14:00}

PS-1173-5 Community empowerment to stop smoking at selected villages in Hambantota, Sri Lanka $N$ Gamage

'Alcohol \& Drug Information Centre, Social Mobilization, Colombo, Sri Lanka. E-mail: nisansalagamage78@gmail.com Background and challenges to implementation: Tobacco smoking is the cause of many preventable diseases in the world. According to the WHO tobacco kills up to half of its users. Sri Lanka as a middle income country, tobacco smoking is one of the biggest economic threats especially in rural settings. The intervention was carried out with the intention to reduced smoking among villages. ADIC implemented the intervention in collaboration with Women 
Development Federation, Hambantota (WDF) which is the largest women organization in Sri Lanka by mobilizing women voice against violence related drugs and women rights.

Intervention or response: ADIC believe women can play major role in the field of drug prevention. ADIC trained 50 women trainees as community mobilizes to implement activities in village level. Initially target villages were 50 . Community mobilizes were selected from respective villages and trained them as trainers. Mobilizes capacity was developed to conduct small group discussion with smokers, calculating tobacco usage and real harm. Further we implemented door to door visits, informal discussions with vendors. Progresses meeting at village level were carried out by mobilizes. During the project period poster, leaflet and stickers were used to create environment where villagers to stop smoking. Progress discussion and necessary technical input was giving time to time. Building up a network with government and community organization has taken to continue the process.

Results and lessons learnt: There were 52 users have stopped their smoking and 102 smokers reduce their smoking during year 2016. 52 ex-daily users could able to save LKR 2,340,000 money within 6 months period. As the impact of this intervention, increased families happiness, build their houses, start new business, brought vehicles and home applications. Education of children of was increased.

Conclusions and key recommendations: Community and households have stopped their smoking or they have reduced their smoking ability. As a result of that they have increased their happiness and their economy enhance.

Tob. Induc. Dis. 2018;16(Suppl 1):A931

DOI:10.18332/tid/84410

\section{2:45-14:00}

EP-235-5 Smoking uptake, transitions and inequalities in Scottish young adults - a mixed methods study A Macgregor ${ }^{1}$, S Hinchliffe ${ }^{1}$, H Delaney $^{1}$, A Amos $^{2}$

'ScotCen Social Research, Edinburgh, United Kingdom, ${ }^{2}$ University of Edinburgh, Usher Institute of Population Health Sciences and Informatics, Edinburgh, United Kingdom. E-mail: amanda.amos@ed.ac.uk

Background: In recent years there has been a major decline in the smoking prevalence of under-16 year olds in Scotland, but relatively little decline smoking among 16-24 year olds. In addition, smoking uptake continues until the mid-20s, with smoking rates in 20-24 year olds higher than in 16-19 year olds. However, we know little about who is most at risk of becoming a smoker in this age group, what influences this, and how this relates to inequalities and smoking. This mixed-methods study aimed to address these questions.

Methods: Detailed secondary analysis of the annual Scottish Health Surveys (SHeS) 2012-15 examined smoking behaviour in 16-24 year olds by key sociodemographic factors including gender, socio-economic status (area deprivation and educational/ employment status) and other relevant health-related data including drinking behaviour and mental health status. After the quantitative analysis, qualitative purposive sampling of $\mathrm{SHeS}$ respondents was used to recruit a diverse sample (by smoking status, age, gender, economic activity) for in-depth qualitative interviews. Twenty-five interviews were conducted in 2016/17.

Results: Smoking prevalence increased between 16-19 and 2024 in both genders and all SES groups, and was significantly associated with SES, ranging in 16-24 year olds from $42 \%$ among those not in education, employment or training, to $24 \%$ among the employed and 13\% among full-time students. Qualitative interviews highlighted the importance of educational and occupational transitions (to and from school/college and the workplace), social contexts and alcohol use in shaping smoking behaviour including uptake, consumption levels and quit attempts.

Conclusions: Understanding the smoking beliefs, behaviour, social and occupational contexts and transitions of 16-24 year olds is vital for developing effective policies to reduce smoking and inequalities in smoking in this key age group. This research demonstrates the nature of inequalities in smoking in Scottish young adults, which has implications for smoking prevention and cessation strategies.

Tob. Induc. Dis. 2018;16(Suppl 1):A932

DOI: $10.18332 /$ tid/83770

\section{$12: 45-14: 00$}

EP-236-5 Social differential effects of a schoolbased smoking intervention

A Andersen ${ }^{1}$, LS Bast ${ }^{1}$, P Due $^{1}$

${ }^{1}$ University of Southern Denmark, National Institute of Public Health, Copenhagen, Denmark. E-mail: anan@niph.dk

Background: Smoking remains the leading risk for the total burden of disease in Western Europe, and smoking is more prevalent among adolescents from lower socioeconomic positions (SEP). Only eight percent of 15-year old Danish adolescents from higher social classes smoke daily or sometimes, whereas up to $37 \%$ of adolescents whose parents live from welfare benefits are smokers. The X:IT study has been the first large-scale smoking intervention to be effective in reducing uptake of smoking in adolescents in Denmark, but in order to develop effective interventions which work across social groups, it is important to study the social patterning of the effect.

Methods: We used data from a Danish cluster randomized trial including 4041 year- 7 students from 51 intervention and 43 control schools. Outcome measure 'current smoking' was dichotomized into smoking daily, weekly, monthly or more seldom vs do not smoke. We performed multilevel, logistic regression analyses of available cases and intention-to-treat (ITT) analyses, replacing missing outcome values by multiple imputation. The analyses were stratified by family socioeconomic position (SEP).

Results: Analyses on imputed cases showed that overall, the OR for smoking among students at intervention schools compared with control schools was 0.67 (0.50-0.89). Among students from high SEP the OR was 0.60 (0.37-0.98), from medium SEP the OR was 0.65 (0.45-0.96), and from low SEP the OR was 0.78 (0.49-1.26).

Conclusions: The X:IT smoking intervention showed significant effects among students from high and medium SEP, but not among students from the lowest SEP pointing at a social differential effect. Mechanisms behind inequalities in smoking interventions are understudied, and it seems important to examine which intervention strategies have the potential to improve healthrelated behavior among children and adolescents from lower socioeconomic groups.

Tob. Induc. Dis. 2018;16(Suppl 1):A933

DOI:10.18332/tid/83841

$12: 45-14: 00$

EP-238-5 Influence of cigarette prices and social environment smoking on allocation of consumption allocation of consumption of food groups: analysis of Susenas 2014 Indonesia

T Djutaharta ${ }^{1}$

'University of Indonesia, Economics and Business, Depok, 
Indonesia.E-mail: triasihdj@yahoo.com

Background: The study of cigarette consumption is still partial by looking at how environmental influences are smoked against individual cigarette consumption / household, or crowding out effect due to price increase. The price of cigarettes is a fairly effective instrument in reducing tobacco consumption. The study question of how the effect of cigarette price changes affects the allocation of expenditure of food groups and household cigarettes by considering the social environment of cigarette society. The purpose to find out how the influence of cigarette prices and the social environment smokes its effect on the allocation of expenditure of 11 food groups, including cigarettes to households. Methods: The method using Almost Ideal Demand System model, data data used Susenas 2014. Price is estimated from unit value by eliminating the effect of difference of fan quantity premier quality. Heckman procedures to address households that do not consume food / cigarette groups. The smoking social environment is projected from the prevalence of smoking at the district level from Riskesdas 2013 data. Variable controls are social and demographic characteristics of households, household expenditures, and the existence of district-level prohibition regulations.

Results: The estimation results show that by incorporating the social environmental variables smoking, the price of cigarettes has less effect as price changes than the estimate that does not consider the social environment of smoking. The price of cigarettes will also decrease the allocation of household expenditure for certain commodity groups and decrease the allocation of household expenditure for some commodity groups.

Conclusions: The conclusion of the study, the effect of cigarette prices on cigarette consumption and other commodity consumption becomes different when considering the social environment of community smoking. The policy of cigarette control not only with economic variables but also considering social factors of society.

Tob. Induc. Dis. 2018;16(Suppl 1):A934

DOI:10.18332/tid/84487

\section{$12: 45-14: 00$}

EP-239-5 Educational inequalities in smoking uptake and cessation: a birth cohort analysis of the German GEDA study

B Kuntz $^{1}$, J Hoebel ${ }^{1}$, J Zeiher ${ }^{1}$, T Lampert ${ }^{1}$

${ }^{1}$ Robert Koch Institute, Department of Epidemiology and Health Monitoring, Berlin, Germany. E-mail: b.kuntz@rki.de

Background: In most developed countries today the prevalence of smoking is higher among men and women with lower education levels. Furthermore, educational differences in smoking have increased over the last decades. As smoking prevalence is influenced by both smoking initiation and quitting, we analyzed the extent of educational inequalities in smoking uptake and cessation for different birth cohorts in Germany.

Methods: The analyses were based on pooled data of three waves $(2009,2010,2012)$ of the nationwide telephone health survey "German Health Update" (GEDA) ( $n=62,606$ aged 18 years and older). Seven 10-year birth cohorts (1920s-1980s) were included ( $\mathrm{n}=59,757)$. Smoking behaviour was assessed by several questions on current and previous smoking status. Educational status was assessed by the highest school-leaving certificate and classified as low, middle, and high.

Results: For men and women born since the 1950s, the share of ever smokers was higher among those with lower education levels. The educational gradient in smoking initiation increased in younger cohorts among both sexes. In the 1980-89 birth cohort the share of ever smokers was about 30 percentage points higher in lower than in higher educated men $(78.8 \%$ vs. $47.6 \%)$ and women (74.9\% vs. $44.9 \%)$. Quitting rates, however, were higher among the higher educated compared with the less educated, especially in women born in the 1960 s (54.8\% vs. $30.6 \%)$ and 1970s (48.9\% vs. $23.3 \%$ ).

Conclusions: Particularly in the younger birth cohorts, lower educated men and women were more likely to start smoking and less likely to quit than higher educated men and women. When implementing tobacco prevention and cessation initiatives, efforts to reach also the lower educated population groups are necessary. If educational inequalities in smoking are not reduced, educational inequalities in tobacco-related morbidity and mortality are likely to increase in the future.

\section{Tob. Induc. Dis. 2018;16(Suppl 1):A935 \\ DOI:10.18332/tid/83803}

\section{$12: 45-14: 00$}

EP-240-5 Smoking during pregnancy among primary health care clients in Argentina. Identification of resources and stressors

E Alderete ${ }^{1,2}$, I Bejarano ${ }^{3}$

${ }^{1}$ National Council for Scientific and Technologic Research (CONICET), Centro de Investigaciones Sociales y Regionales (CISOR), S.S. de Jujuy, Argentina, ${ }^{2}$ Instituto de Ciencia y Tecnología Regional (ICTER), S.S. de Jujuy, Argentina, ${ }^{3}$ Universidad Nacional de Jujuy, Facultad de Humanidades y Cs. Sociales, S.S. de Jujuy, Argentina. E-mail: alderetew@gmail.com

Background: Cigarette smoking increases the risk of perinatal disorders like premature rupture of membranes, placenta previa, abruptio placenta, stillbirth, preterm delivery, and low-birth weight. Rates of smoking during pregnancy (SDP) vary, from $9.9 \%$ in Japan to $17 \%$ in Australia and $30-35 \%$ in Spain. To determine variations in the risk of SDP and tailor interventions, understanding predictors is crucial.

Methods: This study was conducted in 2014, among participants of free maternal-child health services in four cities of Northwest Argentina. Mothers of children from birth to 5 years of age responded to a questionnaire $(\mathrm{N}=1107)$ in 16 randomly selected clinics. We conducted bi- and multivariate analysis of SDP by psychosocial risk factors.

Results: A 10.3\% of respondents reported SDP, with lower rates among Indigenous vs. non-Indigenous women (4.7\% vs. $11 \%)$. A greater percentage of mothers who did not report SDP had full term babies, and reported that the child's health was excellent or very good, but no significant differences were reported regarding child's health problems at birth. In bivariate analysis, educational level, occupational status, concern about household income, externalizing behavior, number of smokers in the household and the number of cigarettes smoked in the previous month, were significant risks. In multivariate analysis, Indigenous women were less likely to report SDP (OR 0.2); complete elementary or incomplete high school education were protective factors (OR 0.3 ) but higher educational status was not significant, compared with having less than elementary education. Students (OR 2.8), the unemployed or those with informal work (OR 9.6) were at risk compared with the formally employed.

Conclusions: Understanding social norms, resources and stressors among women of different ethnic groups or educational and occupational status, could increase the efficacy of prevention strategies. We propose that close to zero exposure to cigarette 
smoke in utero is achievable and should be a Tobacco Free Generations primordial objective.

Tob. Induc. Dis. 2018;16(Suppl 1):A936 DOI:10.18332/tid/83909

\section{$12: 45-14: 00$}

EP-241-5 Smoking inequality and health expenditures: a case study of Namibia

Z Chisha', J Ataguba ${ }^{2}$

${ }^{1}$ Economics of Tobacco Control Project, Southern Africa Labour Development Research Unit, Cape Town, South Africa, ${ }^{2}$ University of Cape Town, Health Economics Unit, Cape Town, South Africa. E-mail:zundac@gmail.com

Background: Many parts of the world, specifically Low and Middle Income Countries (LMICs), have experienced increased smoking prevalence. This portends higher future smoking-related disease prevalence. Because the largest burden of smoking-related diseases is likely to fall among the sub-populations least able to pay for healthcare services, this poses a serious threat to the fight against poverty in LMICs. This study contributes to developing an understanding of the socioeconomic disparities in smoking prevalence and intensity in Namibia, a middle income country in Africa, and the potential association with per capita health-related expenditures.

Methods: Data come from the 2013 Namibia Demographic and Health Survey. The study focuses on out-patient disease (OPD) costs, inpatient disease (IPD) costs and total out-of-pocket (OOP) payments. Concentration curves and indices are estimated for all three variables as well as for smoking intensity and smoking prevalence. Further, three Tobit regression models are run to examine the associations of the different healthcare costs with smoking intensity.

Results: The concentration index of smoking prevalence is estimated at -0.05 compared to -0.18 for smoking intensity. In contrast, the concentration index of OPD healthcare costs is estimated at 0.34 compared to 0.65 for IPD healthcare costs reflecting disproportionately higher healthcare costs among the rich. The concentration index of the overall total annual OOP payments is 0.55 . Tobit regression analysis, however, does not find any statistically significant relationship between the smoking intensity and the amount spent on healthcare.

Conclusions: The study finds that both smoking prevalence and intensity are only slightly higher among the poor compared to the rich. This will likely exacerbate the overall disease burden among the poor. Healthcare expenditures, in contrast, are more reflective of the country's income inequality. It is conceivable that as the country's economy grows and income inequality reduces, cigarettes may become affordable which may increase consumption across the wealth quintiles.

Tob. Induc. Dis. 2018;16(Suppl 1):A937

DOI:10.18332/tid/83922

\section{2:45-14:00}

EP-247-5 Employment and wages in the bidi industry in India: a gender-focused analysis

M Arora', P Datta1', S Bhaumik1, GP Nazar'1, A Barman', VG Munish $^{2}$, PK Sinha ${ }^{2}$, F Tullu²

${ }^{1}$ Public Health Foundation of India, Gurgaon, India, ${ }^{2}$ World Health Organisation - India, New Delhi, India. E-mail: monika. arora@phfi.org

Background: Majority of bidi manufacturing in India is in the unorganised sector which makes its regulation and taxation difficult. Bidi industry is highly labour-intensive and there is high home-based employment of women and children as bidi workers. Bidi workers are known to get inadequate social security and welfare benefits, and are paid low wages. This study highlights gender inequalities in employment and wages earned in the bidi industry, not previously studied.

Methods: Three data sources were used: 1) Annual Survey of Industries - for organized sector 2) National Sample Survey (NSS) Unorganized Manufacturing Enterprises Survey (2000-01; 2005-06; 2010-11) and 3) NSS Employment and Unemployment Survey (2011-12). Share of wages, nature and type of employment in organised and unorganised sectors were studied.

Results: In the organised as well as unorganised sector, the number of female workers was higher than that of male workers. Female workers made up $82.8 \%$ of the total unorganised sector bidi manufacturing employment in 2010-11. In 2000-01, average per capita wage of a bidi worker in organised sector was only $25 \%$ that of average per capita wage observed in the entire manufacturing sector. This figure declined to $20 \%$ in $2005-06$ and $17 \%$ in $2010-11$. In 2000-01, female workers earned INR 7,737.7 less per year than males, on average; in 2005-06, the wage disparity between male and female workers was lower at INR 6,327.8. In 2010-11 however, the wage disparity increased with male workers earning INR 6,610.7 more on average. Contractual workers were paid least wages and managerial workers were paid higher vs. those directly employed.

Conclusions: Although more females are employed in organised as well as unorganised bidi manufacturing sectors vs. males, the average annual wages of female workers are much lower than those of male workers, increasing inequalities. The system of crontractualisation further exacerbates inequity.

\section{Tob. Induc. Dis. 2018;16(Suppl 1):A938}

DOI:10.18332/tid/84587

\section{$15: 45-16: 45$}

LB-1363-5 Do tobacco using households have greater risk of catastrophic healthcare expenditure? Implication for tobacco control policy

F Adeniji ${ }^{1}$

'University of Aberdeenlbadan, Health Policy \& Management, Ibadan, Nigeria. E-mail: folashayoadeniji@gmail.com

Background: Tobacco consumption precipitates ill-health and the financial cost of increase in demand for medical care can be substantial and catastrophic, especially for households in the lowest income stratum. This paper extends what is known about the poverty impact of tobacco use by estimating the increased risk of incurring higher catastrophic health expenditure as a result of tobacco consumption. Methods: The data for the study was drawn from the Harmonized Nigerian Living Standard Survey (HNLSS) conducted in 2009/2010 by the National Bureau of Statistics. In predicting the health expenditure attributable to tobacco consumption, two log-linear models of tobacco consumption were estimated. The experience of catastrophic health expenditure (CHE) was estimated using the standard 40 percent threshold of household total non-food expenditure.

Results: Smokers had higher health expenditure (46.5\% and 44\%) compared to non-smokers for the two models estimated. Excess average health expenditure attributable to tobacco consumption was highest among smokers that are moderately poor and lowest among non-poor smokers. Also, extremely poor smokers incurred higher medical expenditure attributable to tobacco consumption compared to non-poor smokers. Excess medical expenditure attributable to tobacco consumption increased the burden of $\mathrm{CHE}$ by $3.83 \%$ among households living in rural location. Likewise, 
non-poor households experienced CHE, $23.87 \%$ for households in rural settings and $13.62 \%$ for non-poor urban residents.

Conclusions: Essentially, due to the sub-optimal nature of health care financing infrastructure in Nigeria, smoking will aggravate the financial hardship of households as a result of increase in out-of-pocket payment for health care services in the short and long run. Therefore, with health care policy makers battling to increase the coverage of social health care financing, policies that curtails tobacco consumption are highly supported by the evidence provided in this study.

Tob. Induc. Dis. 2018;16(Suppl 1):A939

DOI: $10.18332 /$ tid/84693

\subsection{Tobacco farming: Opportunities and experiences in facilitating alternative livelihoods and preventing tobaceo adverse environmental impact}

\section{$12: 30-14: 00$}

PS-1174-5 Moving to tobacco cultivation free livelihoods in Sri Lanka: lessons learned from India and Kenya

PSOS Cooray

${ }^{1}$ Alcohol and Drug Information Center (ADIC) Sri Lanka, Colombo, Sri Lanka.E-mail: obinsimoni@gmail.com

Background: The tobacco cultivation extend is 4480 Hectares (in year 2000) which account to $0.19 \%$ total agricultural land which it is not a government supported agriculture sector in Sri Lanka. Majority of the farmers are small scale farmers and do farming in tenure basis. The existence and sustainability of sector depend on the support provided by Ceylon Tobacco Company (CTC) which holds the monopoly of market. To make country free from tobacco cultivation, introduction of alternative livelihoods is mandatory. Therefore applicability of lessons from implementing Framework Convention on Tobacco Control recommendations in Kenya and India are discussed in the paper.

Objective: To make recommendations in adopting alternative livelihoods for tobacco farmers in Sri Lanka.

Methods: Data collected from secondary data sources were reviewed. The model was developed analyzing the existing statistics, case studies, paper articles, expert opinions and other reliable information sources. Conceptual framework was developed to address the issue.

Results: A Model was developed to make recommendations to initiate alternative livelihoods for current tobacco farmers in the country. Model is based on changing the psychological way of thinking of farmers for the sustainable adoption to change in their livelihood. This involves the improve understanding of the negative impacts to their families (wife, children) while showing viable commercial options where there are higher economic, health and emotional gains. The myths ingrained in farmers mind via tobacco industry should be defeated.

\section{Conclusions:}

- Tobacco industry uses farmers as a front group in protecting the industry while misleading the media.

Initiation of alternative livelihoods for tobacco farmers is a long process which takes time to adopt people with the change of their mindset.

Tob. Induc. Dis. 2018;16(Suppl 1):A940

DOI:10.18332/tid/84464

$12: 30-14: 00$
PS-1175-5 Defeating attempt made to introduce tobacco cultivation for farmers in Sri Lanka through sustainable agriculture development programme: a case study of Kandy district

PSOS Cooray ${ }^{1}$

${ }^{1}$ Alcohol and Drug Information Center (ADIC) Sri Lanka, Colombo, Sri Lanka. E-mail: obinsimoni@gmail.com

Background: Sustainable Agriculture Development Programme is the main corporate social investment project of Ceylon Tobacco Company (CTC). Kandy district is well known for its rich biodiversity in Kandian home gardens. A hidden strategy of the project identified as it introduce tobacco cultivation in home garden level after promoting few other cash crops \& animal husbandry for selected people with subsidies and technical support. This study was carried out to reveal the need for new counter strategies to address the negative impacts of the project and mitigate those to discourage tobacco cultivation.

Objective: To recommend counter measures against tobacco cultivation promotion through SADP project.

Methods: Case study was carried out in Kandy district where SADP has implemented. The data was collected through secondary data sources such as CTC annual report, Newspaper articles, websites, and books. The expert opinions were obtained and group discussions held with farmers to make recommendations in combating the negative impacts of SADP project.

Results: The recommended counter measures consists of informing government officials regarding the negative impacts of SADP, make people aware about the CTC strategies on how they persuade people for tobacco farming, make people aware on consequences of tobacco farming related economic, social and health issues in long run.

Conclusions:

- CTC attempts to promote tobacco cultivation in home garden level for some farmers in selected communities.

The social, economic and environmental cost is high if the negative impacts of SADP are not mitigated.

Tob. Induc. Dis. 2018;16(Suppl 1):A941

DOI: $10.18332 /$ tid/84472

\section{2:30-14:00}

PS-1176-5 The plight of tobacco farming under hegemony of transnational tobacco companies in Turkey: repercussions and remedies

E Evrengil ${ }^{1}$

TTurkish National Coalition on Tobacco or Health, Istanbul, Turkey.E-mail: efzagil@yahoo.com

Background: The shift from State oversight to contractual farming mandated by law in 2002 as a structural adjustment conditionality has had destructive impact on Turkish tobacco agriculture: Transnational tobacco companies (TTCs) gained control by being able to impose unilateral conditions; tobacco farmers were forced to face three options: prices below subsistence, migration, or turning to illicit market. A heated debate about illicit domestic tobacco market recently surfaced in news media.

Methods:

(a) Literature review,

(b) trend analysis of Turkish leaf tobacco output and trade as compared among 12 major tobacco growing countries, using official data (2003-2016), FAOstat (1961-2014), UNdata (1989-2015),

(c) review of relevant news coverage (2016 - June 2017).

Results: Over the last 25 years, Turkey was worst hit among the 12 
countries: Leaf tobacco output dropped sharpest by $78 \%$, and trade balance was drastically reversed. Between 2003-2016, cigarettes manufactured rose by $32.2 \%$, leaf tobacco imports by $46.6 \%$, whereas number of tobacco farmers declined by $82.4 \%$, domestic output by $39.4 \%$, and exports by $53.6 \%$. Domestic tobacco used in cigarettes decreased from $42.1 \%$ to $13.3 \%$. Tobacco agriculture vanished in certain regions or reduced to supplementary source of income undertaken by elderly and children. Domestic tobacco lobby seeks legalization, lower tax rates, and quotas for compulsory use of domestic tobacco in manufacturing. TTCs fervently oppose these positions and demand severe countermeasures.

Conclusions: TTCs ability to source leaf tobacco at lowest possible prices through their affiliates from around the world under liberalized trade and contractual farming regimes greatly harms agricultural activities realized by income and employment generating small family holdings, and thus national economies. In Turkey, TTC hegemony devastated farmers' livelihoods, caused market imbalance and duality, and disrupted regulations and taxation. Agriculture policy must be aligned with consumption reduction strategies through strategic planning for economically viable alternatives and effective oversight of tobacco cultivation.

Tob. Induc. Dis. 2018;16(Suppl 1):A942

DOI:10.18332/tid/84522

\section{$12: 30-14: 00$}

PS-1177-5 Analysis of the work process and suicide in the largest smoking regions in Brazil

$\checkmark$ Borges $^{1}$

${ }^{1}$ Ministry of Health / National Cancer Institute /, Division of Tobacco Control, Rio de Janeiro, RJ,Brazil.E-mail:vborges@ inca.gov.br

Background and challenges to implementation: Even though Brazil's balance of trade accounts for the real economic benefits of tobacco production. There is annual spending of $\mathrm{R} \$ 56.9$ billion in medical expenses and loss of productivity, tax collection of $\mathrm{R} \$$ 13 billion, covering only $23 \%$ of the expenses with the epidemic. Equally worrisome is the health of tobacco producers, who in the country almost 200,000 family farmers, inserted in a type of work that demands knowledge as part of health care, and Article 18 of the Framework Convention. This scenario motivated a qualitative research, aiming to evaluate the work process and to identify elements that can cause damages to the physical and mental health in farmers, even with suicide outcome.

Intervention or response: This study was organized in two stages: a) analysis of the work process in the producing regions in the south and northeast of the country, based on information from studies published since 1990; b) analysis of the occurrence of registered suicide cases from 2004 to 2013 in official databases in 52 municipalities.

Results and lessons learnt: The productive cycle can take 14 months absorbing until $12 \mathrm{~h} /$ day. Different fumes require working modalities in sheds or open air. Being plant susceptible to pests, there is intense use of pesticides and toxicities, concentrating on the leaves with nicotine that will be manipulated and stored by the farmer. The price paid at the end may not cover the investments, including industry loans, resulting in the accumulation of debts with the company and your property risk. Databases reported suicides in all tobacco producing municipalities with the largest number in the regions of highest production in the south (Rio Grande do Sul state) and northeast (Arapiraca).

Conclusions and key recommendations: The many stages in the work process and aggressors to physical and mental health point to reconsideration about the real benefits to the country's economy.

\section{Tob. Induc. Dis. 2018;16(Suppl 1):A943 DOI: $10.18332 /$ tid/84566}

\section{2:30-14:00}

PS-1178-5 Health education programs for tobacco workers: reflecting on principles for empowering farmers

\section{Lino ${ }^{1}$, AP Oliveira ${ }^{2}, \mathrm{R}$ Dantas $^{3}$, C Silva $^{4}$, M Moreno $^{2}$}

'Souza Marques School of Medicine, Rio de Janeiro, RJ, Brazil, ${ }^{2}$ Oswaldo Cruz Foundation - Fiocruz, Center for Studies on Tobacco or Health, National School of Public Health Sergio Arouca, Rio de Janeiro, RJ, Brazil, ${ }^{3}$ Oswaldo Cruz Foundation - Fiocruz, Workers' Health Service, Rio de Janeiro, RJ, Brazil, ${ }^{4}$ Secretariat for Social Assistance and Human Rights (SASDH), Niterói, RJ, Brazil. E-mail: carinarglino@gmail.com

Background: World Health Organization Framework Convention on Tobacco Control (WHO FCTC) in its article 18 recommends Parties to protect environment and human health against impacts of tobacco farming. Last Conferences of the Parties of WHO FCTC have made recommendations to the development of educational programs to the dissemination of harmful health and environmental impacts of tobacco farming among tobacco growers. The intention of this study was to propose guidelines for the development of health education programs addressed to tobacco growers, based on the historical, social and cultural context of tobacco farming in southern Brazil.

Methods: In this exploratory study, secondary data were analyzed from a research project conducted by Oswaldo Cruz Foundation in a small tobacco-growing municipality, in 2013. The original study described women tobacco farmers' opinions, beliefs and concerns on relationship between tobacco farming, environment, and health. Data were analyzed qualitatively, looking for elements to support health education programs.

Results: Main elements identified were: knowledge and naturalization of health risks; knowledge of negative impacts of tobacco farming on environment, but without believing that they have the potential to compromise their health; disbelief in performance of public services (government).

Health education programs in tobacco growing areas should be based on the reality of each territory (historical, social and cultural context). Dialogic processes should be the basis of programs, leading to the empowerment of farmers as protagonists of their personal issues. Social organization should be organized in the tobacco growing areas to strengthen local support networks and social control over public policies. Strengthening social organization can aid to weaken tobacco industry interference with the lives of farmers. Health education programs should be developed from an intersectoral and integrative perspective.

Conclusions: Health education programs should be guided by the concerns and needs experienced by tobacco growers to strengthen their autonomy and promote reflection on healthier livelihoods.

Tob. Induc. Dis. 2018;16(Suppl 1):A944

\section{DOI:10.18332/tid/84576}

\section{2:30-14:00}

PS-1179-5 Alternative crop for tobacco farming in Lebanon - an effective tool to change and educate on tobacco environmental and economical impact Z Redwan', Green Hand - MEA Commission ${ }^{1}$ Green Hand Organization, Aley, Lebanon. E-mail: zaher. 


\section{redwan@gmail.com}

Background and challenges to implementation: Several aspects are reasons to work for finding alternative crops for tobacco in Lebanon. As this agriculture is important for the economy of the country, but there are many impacts on the environment, economy, human rights and health of farmer growers.

Tobacco growing cause soil erosion and degradation as well as loss of biodiversity. The excessive use of chemicals causes water pollution and increases in atmospheric carbon dioxide. Tobacco crops deplete soil nutrients by taking more nitrogen, phosphorous and potassium than other major crops.

Farmer growers are all with their family in working hard in this agriculture to receive living wages. Child labourers and females are also at risk of nicotine toxicity caused by handling tobacco leaves without protection during harvest and production with absence of health insurance.

Due to the monopoly, "Regie Libanaise des Tabacs et Tombacs" is the only company ready to get these tobacco products. So, it controls prices in very low levels. There are many crops that could be an alternative agriculture for tobacco, one of most successful crops is Zaatar: Origanum syriacum.

Intervention or response: An intervention was made with 15 tobacco farmers in the South of Lebanon, where tobacco farming is one of the most dominant agricultural practice with a cultural and traditional dimension.

The program was initiated by establishing an economic survey, that reflected a high economical shock upon the extensive work of the whole family without consideration to gender and child abuse, as well as the illustration of economical/environmental/health value with the alternatives.

Results and lessons learnt: A total success of 10 farmers complete shift to alternative cropping and 5 other partial moving to medicinal plants.

Conclusions and key recommendations: Essential need of support to move on with program and creating a great lobby among the producers to move on to ecological, more beneficial cropping.

Tob. Induc. Dis. 2018;16(Suppl 1):A945 DOI: $10.18332 /$ tid/84630

\section{2:30-14:00}

PS-1180-5 Human trafficking and forced labour in Malawi's tobacco growing sector

M Otañez ${ }^{1}$, R Sandramu², A McGill ${ }^{3}$

'University of Colorado, Anthropology, Denver, CO, United States of America, ${ }^{2}$ Tobacco and Allied Workers Union of Malawi, Nkhotakota, Malawi, ${ }^{3}$ International Labor Rights Forum, Washington, DC, United States of America. E-mail: marty.otanez@ucdenver.edu

Background: Youth as young as five years old pick tobacco and are denied an education and basic food requirements in Malawi's tobacco growing sector. Children and adults are trafficked to tobacco farms both within Malawi and in neighboring countries of Zambia, Tanzania and Mozambique. Tobacco workers and their families remain impoverished through tobacco company practices such as collusion over leaf prices and unfair contract arrangements with tobacco companies. The study of trafficked persons requires a critical approach to sensationalized "modern day slavery" narratives that circulate in tobacco control and human rights discourses.

Methods: Astructured questionnairewas administered to 40 farmers and farm workers for health, social and economic information. We conducted content analysis of national and regional newspapers and interpretive narrative analysis of in-depth ethnographic interviews with five tobacco farmers and farm workers.

Results: A representative case of trafficking involves a man, his wife and two children who receive from an illegal labor recruiter a promise of paid work. Labourers are transported over 800 kilometers. Ultimately, parents send their children to the fields instead of schools and the family remains indebted to the farm owners due to insufficient or non-payment of earnings. Farm owners sell their tobacco to global leaf buyers who, in turn, sell to companies such as Philip Morris. Most study participants indicated they have no finances to exit tobacco farming or return to home. Some individuals acknowledge their workplace dissatisfaction while others are determined to stay on with plans to be independent tobacco farmers.

Conclusions: Trafficked persons and forced labourers respond in diverse ways to their conditions. An informed approach to labor trafficking seeks to improve health outcomes for workers and challenge tobacco industry behavior that perpetuates exploitation. Global tobacco control needs counter-narratives of tobacco farm workers as resilient and courageous, instead of as victims and targets of policymaking.

\section{Tob. Induc. Dis. 2018;16(Suppl 1):A946 \\ DOI:10.18332/tid/84669}

\section{2:30-14:00}

PS-1181-5 Tobacco farming in Indonesia: facts and myths

T Triningsih'1, SM Rahma', DW Kurniawan'

'Indonesian Institute for Social Development, Program, Jakarta, Indonesia. E-mail: sekretariat.iisd@gmail.com

Background and challenges to implementation: Indonesia is the third amongst the countries with the highest smoker's population and first rank for male smoker globally. Tobacco Farmer livelihood and welfare are issues that used by tobacco industry to encounter tobacco control in Indonesia. The average income of tobacco farmers are less than 1 million in a month. FCTC ratification in Indonesia always been questioned by government for its relation to tobacco farmer's welfare. Government and parliament member still believe that tobacco control will give impact to tobacco farmer's livelihood. Therefore, in 2016 Parliament member proposed to draft bill on tobacco that opposing the existed tobacco control policy.

Our policy advocacy program that supported by The Union focus on advocacy government and parliament members for FCTC accession through providing data and information about the correlation of tobacco farmers welfare with tobacco control. This policy target about 261 million of Indonesian people. While the case studies for tobacco farmer welfare was taken in Central Java (Yogya and Temanggung) and East Java (Jember, Bondowoso).

Intervention or response:

- Case studies at Central Java and East Java through interview, survey and video documenter.

- Developed policy document to show the fact that tobacco farmer's welfare.

- Direct advocacy meeting with related stakeholders.

- Media advocacy in providing the witnesses of tobacco farmer.

Results and lessons learnt:

- Policy document has been delivered to president secretariat office and parliament member.

- Media and public awareness was important to reduce misleading information on tobacco farming.

- Tobacco industry interference and lobbying is very strong 
especially in the parliament.

Conclusions and key recommendations:

- The Fact that tobacco Farmers, who had diversification into other crops such as coffee, rice, are more prosperous.

- The fact that tobacco control does not give impact to the farmer's welfare.

- FCTC ratification is opportunity for tobacco farmer to improve their welfare. The government should support alternative for the farmer.

Tob. Induc. Dis. 2018;16(Suppl 1):A947

DOI: $10.18332 /$ tid/84677

\section{$15: 45-16: 45$}

LB-1362-5 Alternative crops to tobacco: a gateway for tobacco farmers Ruvuma region, southern Tanzania LK Kagaruki ${ }^{1}$

'Tanzania Tobacco Control Forum, Dar es Salaam, Tanzania, United Rep.. E-mail: lutgardk@yahoo.com

Background: Tanzania is second in Africa in tobacco production after Malawi. Despite the increased production, Tanzania remains a poor country with tobacco farmers getting poorer and the country losing more than 61,000 hectares of forests annually due to tobacco growing and curing.

Methods: Since 2006, Tanzania Tobacco Control Forum (TTCF) has been working closely with tobacco farmers of Namtumbo District in Ruvuma Region, Southern Tanzania to adopt alternative crops. A survey was carried out in Namtumbo in 2011, using a Focus Group Discussion (FGD) and a structured questionnaire to determine the extent of adoption of alternative crops.

Results: Results indicated that, more than $70 \%$ of the farmers had adopted alternative crops. Current data from Namtumbo District Council indicate that, between 2006 and 2014, there has been increased tonnage in both food and cash crops and a decrease in tobacco production. For example, maize and rice production increased by $452 \%$ and $377 \%$ respectively, while pigeon peas and sesame increased by $5,839 \%$ and $1,302 \%$ respectively. Tobacco production increased by $583 \%$ from 2006 to 2009 , but dropped by $491 \%$ between 2010 and 2014 .

Conclusions: Increased farmers' sensitisation coupled with sustainable and viable markets, could enable total replacement of tobacco with alternative crops in Ruvuma Region.

Tob. Induc. Dis. 2018;16(Suppl 1):A948

DOI: $10.18332 / \mathrm{tid} / 84721$

$15: 45-16: 45$

LB-1366-5 Successful experience with tobacco crop substitution in Yuxi, Yunnan, China

S Tang ${ }^{1}$, VC Liं ${ }^{2}$ Y Cui ${ }^{3}$, J Sun ${ }^{3}$, K Khow ${ }^{3}$

${ }^{1}$ Kunming Medical University, Kunming, China, ${ }^{2}$ University of California Los Angeles, Los Angeles, CA, United States of America, ${ }^{3}$ World Health Organisation - China, Beijing, China. E-mail:khowk@who.int

Background: China is the largest tobacco producer globally, with Yunnan province being the largest tobacco-producing region. In China there are 20 million tobacco farmers. Showing that income from crop substitution can exceed that from tobacco growth is essential to persuading farm families to stop planting tobacco. In Yuxi Municipality, collaborators from Yuxi Bureau of Agriculture, University of California at Los Angeles School of Public Health, and Kunming Medical University initiated a tobacco crop substitution project. At 3 sites, 458 farm families volunteered to participate in a new, for-profit cooperative model. This project successfully identified an approach engaging farmers in cooperatives to substitute food crops for tobacco.
Methods: The current follow-up project utilized official data from the Yuxi Municipal Bureau of Agriculture. Three pilot counties Hongta District, Chengjiang County, Eshan County - were selected for information collection on acreage of tobacco, vegetables, fruits and other crops, and net income of farmers.

Results: Compared to 2012, Yuxi tobacco planting area has dropped significantly from 763,494 Chinese mu (one Chinese mu equals 0.16 acres) to 586,404 Chinese mu in 2015 , whereas the area devoted to vegetables and fruits has increased $24 \%$. The net income change directly linked to the tobacco crop substitution was an increase of more than 3000 yuan (\$484) per capita in Yuxi city and the three pilot project areas, observed from the 2012-2015 data. By mid-2014 and for the first time, farmers' per capita income passed 10,000 yuan (\$1613).

Conclusions: The tobacco substitutition pilot project in Yuxi reduced the density of tobacco planting and increased planting area of vegetables and fruits. The results not only increased the economic status of farmers, but also benefited the health of the public by reducing the availability of tobacco in China. The diffusion of tobacco crop substitution in Yunnan provides a good example for China.

Tob. Induc. Dis. 2018;16(Suppl 1):A949

DOI:10.18332/tid/84725

\subsection{Youth tobacco experience}

12:30-14:00

PS-1182-5 Flavor-related descriptors on economypriced flavored cigarette packs in five Latin American countries

J Brown ${ }^{1,2}$, A Grant $^{2}$, C Weiger ${ }^{1,2}$, J Cohen ${ }^{1,2}$

'Johns Hopkins Bloomberg School of Public Health, Health, Behavior \& Society, Baltimore, MD, United States of America, ${ }^{2}$ Institute for Global Tobacco Control at the Johns Hopkins Bloomberg School of Public Health, Baltimore, MD, United States of America. E-mail: jbrow212@jhu.edu

Background: Flavored cigarettes are popular among youth and are associated with smoking initiation. Flavor capsule cigarettes contain capsules inside the filters that smokers can press to release a liquid that flavors smoke. Argentina, Chile, and Peru are three of four countries with the highest flavor capsule cigarette market share in the world. We describe the descriptors used to indicate flavor or flavor release on economy-priced flavored cigarette packs in five Latin American countries.

Methods: 310 sampling areas within a 100-250 meter radius of primary and secondary schools in one major city in Argentina, Brazil, Bolivia, Chile and Peru were identified in January 2017. At each retailer in the sampling areas, observational data on cigarette advertising were collected. Additionally, the cheapest non-flavored, menthol-flavored, and other flavored cigarette packs were purchased at one retailer in each sampling area. Packs were coded for attributes such as brand, flavor, and descriptors.

Results: 320 flavored cigarette packs were purchased across five countries. 92.8\% $(\mathrm{n}=297)$ of packs displayed non-conventional flavor descriptors (i.e., describe a flavor or function associated with release of flavor but do not denote a characterizing flavor like menthol or cherry). Of those packs with non-conventional flavor descriptors, $99.3 \%(n=295)$ displayed one or more descriptors indicating functionality to release flavor such as "capsule", "mix", "crush" and "expand". 82.5\% ( $\mathrm{n}=245)$ displayed terms that connote a technological function related to flavor release such as "activate", "click", and "switch". In regards to flavor, $36 \%$ $(n=107)$ of packs indicated a cooling effect with descriptors like 
"cool", "fresh", and "ice". Examples of other non-characterizing flavors include "ruby burst" and "purple crush".

Conclusions: Among economy-priced flavored cigarettes sold in five Latin American countries, non-conventional descriptors are often used to describe capsule presence and flavors. These findings will inform advocacy efforts that call for restrictions on flavored tobacco and inform future research on capsule cigarettes. Tob. Induc. Dis. 2018;16(Suppl 1):A950

\section{DOI: $10.18332 /$ tid/84244}

\section{2:30-14:00}

PS-1184-5 Cigarette smoking and reasons for leaving school among school dropouts in South Africa

R Desai ${ }^{1,2}$, LAG Mercken ${ }^{3}$, RAC Ruiter ${ }^{2}$, J Schepers ${ }^{4}$, PS Reddy ${ }^{1,5}$ ${ }^{1}$ Human Science Research Council, Population Health, Health Systems and Innovation, Cape Town, South Africa, ${ }^{2}$ Maastricht University, Department of Work \& Social Psychology, Maastricht, Netherlands, ${ }^{3}$ Maastricht University, Department of Health Promotion and Care and Public Health Research Institute, Maastricht, Netherlands, ${ }^{4}$ Maastricht University, Department of Methodology and Statistics, Maastricht, Netherlands, ${ }^{5}$ University of the Western Cape, Faculty of Community and Health Science, Belville, South Africa. E-mail: rachana.desai.rd@gmail.com

Background: School dropouts are those between 13-20 years old, have not completed their schooling and are not currently enrolled in school. School dropouts are at heightened risk of tobacco use and developing tobacco related disease and disability compared to in-school learners. The aim of this paper was to examine the relationship between reasons for leaving school and past month cigarette smoking, taking into account possible gender differences. Methods: Multiple logistic regression with gender as an effect moderator was used to analyse survey data from 4185 school dropouts. Province and area (urban, rural and peri - urban) were later incorporated into the analysis as possible effect moderators. Results: Past month cigarette smoking was reported by $50.4 \%$ of the respondents with boys (61.6\%) smoking considerably more compared to girls (33.9\%). We did not find any significant association between the reasons for leaving school and tobacco smoking when gender was tested as an effect moderator. However, dropping out of school for 'not being able to pay school fees', was found to be associated with less cigarette smoking, but only among girls residing in urban areas.

Conclusions: Given the high prevalence of tobacco smoking among school dropouts, more research is needed to further explore the relationship between tobacco smoking and reasons for leaving school that were not considered in this study.

Tob. Induc. Dis. 2018;16(Suppl 1):A951

DOI:10.18332/tid/84255

\section{2:30-14:00}

PS-1186-5 Attempt to quit smoking by electronic cigarette use among smoking adolescents in Korea E Yuk ${ }^{1}$, SI Cho ${ }^{1,2}$

${ }^{1}$ Institute of Health and Environment, Seoul National University, Seoul, Korea, Republic of, ${ }^{2}$ Graduate School of Public Health, Seoul National University, Department of Public Health Science, Seoul, Korea, Republic of. E-mail: eunu.yuk@gmail.com

Background: Adolescent smoking has remained a major challenge. With strengthening of tobacco control policy, smoking rates have consistently declined over the past decade. In contrast, the use of electronic cigarette increased because companies promoted the e-cigarette as smoking cessation aid. There is a controversy whether e-cigarettes have an effect on quitting smoking. This study examines whether e-cigarette is effective as a smoking cessation aid.

Methods: This study used Korea Youth Risk Behavior Webbased Surveys(KYRBS) in $2015 \sim 2016$. For analysis, types of smokers were categorized into 2 groups; cigarette only user and dual user. Experience of attempting to quit smoking was used as the response variable. To examine the association between attempt to quit smoking and e-cigarette use among cigarette smoker, frequency analysis, Chi-square test and logistic regression were used.

Results: The lower school performance, the higher the weekly allowance, the more alcohol they drink and stress perceived, the more smoking friends, the higher was the frequency of attempt to quit smoking among dual users. As the smoking amount increases, the proportion of attempt to smoking cessation increased among dual users. The association of smoking cessation among dual user versus cigarette only user by frequency was 2.30 (95\% $\mathrm{CI}=1.76-3.00)$ at 20-29 days. When smoking 6-9 amount a day, the association of attempt to smoking cessation was the highest $(\mathrm{OR}=2.0495 \% \mathrm{CI}=1.90-2.64)$.

Conclusions: As cigarette use increased, attempt to smoking cessation was also higher. Cigarette smoking days were higher in dual users even though they tried to quit smoking. It means e-cigarette actually works in synergy with the use of smoking conventional cigarettes and has little effect on smoking cessation. To prevent the spread of misguided cessation culture in social experience such as part-time work and smoking friends, proper education on smoking cessation is necessary.

Tob. Induc. Dis. 2018;16(Suppl 1):A952

DOI:10.18332/tid/84311

$12: 30-14: 00$

PS-1187-5 Second hand smoke: awareness and acceptability among school going adolescents In India

N Rustagi', M Verma', A Kumar², L Norbu ${ }^{3}$

${ }^{1}$ AlIMS, Community Medicine \&t Family Medicine, Jodhpur, India, ${ }^{2}$ Ministry of Health \&t Family Welfare, Delhi, India, ${ }^{3}$ Natinal Center for Disease Control and Public Health, Delhi, India. E-mail: drneetigoswami@gmail.com

Background: Exposure to second hand smoke among school going adolescents is a public health problem and is associated with higher prevalence of tobacco use. It is important to understand the possible sources and the attitude among adolescents which increases the risk of such exposure.

Objectives: To assess exposure and attitude of school going adolescents towards second hand smoke (SHS).

Methods: Cross-sectional survey of school going adolescents from class VII to XII was conducted for a school in India which is supported by para- military forces to ensure quality education to children of para-military personnel's. Simple random sampling was used to select single section from classes VII to XII and data was collected for all students in selected section using Global Youth Tobacco Scale (GYTS).

Results: Exposure to second hand smoke in past one week inside home was found to be $45 \%$ and in public places $76.6 \%$. Majority participants (50.3\%) reported smoking by one or more close friends. Though smoking from other people was reported to be harmful ( $89 \%$ ) and discussion about harmful effects of smoking is carried out by school authorities (73.2\%) and families (75.9\%) of adolescents yet attitude towards SHS among adolescents is favorable and is 
considered helpful in enhancing socialization among boys (73.10\%) and making personality attractive among girls.

Conclusions: Second hand smoking either due to exposure in home or outside home is substantially high in school adolescent in India. Strategies designed to reduce smoking among school adolescents need to specially target positive image associated with smokers.

Tob. Induc. Dis. 2018;16(Suppl 1):A953

DOI: $10.18332 /$ tid/84314

\section{2:30-14:00}

PS-1188-5 Trend in smokeless tobacco use among youth in SEA region (2003-2016)

J Kishore ${ }^{1}, \mathrm{P}$ Jena $^{2}$

'Vardhman Mahavir Medical College \&t Safdarjung Hospital, Community Medicine, New Delhi, India, ${ }^{2}$ KIIT Public Health School,Bhubaneswar, India.E-mail:drjugalkishore@gmail.com Background: Tobacco ban is imposed in many countries however its impact on use of tobacco is less studied. This study assesses and compares the smokeless tobacco (SLT) burden among youth in South-East-Asia (SEA) region from 2003 till 2016.

Methods: Data from Global Youth tobacco Surveys (GYTS) from 2003 to 2016 in SEA region was analyzed. GYTS data from Bangladesh, Bhutan, India, Indonesia, Maldives, Myanmar, Nepal, Sri Lanka, Thailand, and Timor Leste was examined. Country and gender wise trend analysis was done.

Results: Overall prevalence of SLT use among youths in SEA was varied from $2.1 \%$ to $21.6 \%$. Bhutan youth both males $(25 \%)$ and females (18.9\%) topped the list. Indonesia male youth (3\%) and Sri Lankan female youth (0.5\%) had chewed SLTs the least. Male youth chewed more SLTs than their female counterparts, except in Timor Leste. There is decreasing trend of SLT use in SEA region except Nepal and Bhutan.

Conclusions: Decreasing trend of SLT use in SEA region except Nepal and Bhutan represents successful tobacco control efforts in most part of the SEA region. Despite complete ban on tobacco products, high burden of SLT use in Bhutan is a concern. This study emphasize on intense and sustained tobacco control efforts in Bhutan and Nepal.

Tob. Induc. Dis. 2018;16(Suppl 1):A954

DOI: $10.18332 /$ tid $/ 84317$

12:30-14:00

PS-1190-5 Trends in adolescent and young adult smoking in India (2009-2017): patterns and implications

P Lal', D Sharma², A Pandey ${ }^{1}$

'International Union Against Tuberculosis and Lung Disease, New Delhi, India, ${ }^{2}$ Government Medical College Chandigarh, Chandigarh, India.E-mail: plal@theunion.org

Background and challenges to implementation: Smoking initiation is a key driver to understand future trend of the tobacco epidemic. Prevalence data from the Global Adult Tobacco Survey (GATS 2009 and 2017) suggest that substantial uptake of smoking occurs after 15 years of age, including among young adult males aged 18-24 years.

Intervention or response: We calculated the proportion of new initiation by age and compare this for 2009 and 2017. We estimate how many would become lifelong smokers by age at baseline using current quit rate by age.

Results and lessons learnt: Initiation between 2009 and 2017 was $14.2 \%$ for $15-17$ year olds, $7.0 \%$ for $18-19$ years, $3.1 \%$ for 20 -
24 years and $1.4 \%$ for $25-34$ years, with low levels of initiation (< $1.0 \%$ ) among older age groups.

Conclusions and key recommendations: Although GATS 2017 reveals an increase in the age of initiation and minor decrease in intensity of smoking and improvement in quit rates, in absolute terms the numbers have increased significantly. We find that there were strong age-related gradients in smoking initiation within these three age groups. Substantial initiation occurs among younger youth and young adults, and tapers after the age of 24 . Efforts to prevent initiation of smoking should focus not only on adolescents but also on older youth and young adults. This calls for raising the age of purchase and use to 21 years, and also ensure cessation services targeted at early users.

Tob. Induc. Dis. 2018;16(Suppl 1):A955

DOI: $10.18332 /$ tid/84332

12:30-14:00

PS-1191-5 Prevalence and correlates of smoking at home in adolescent smokers - a cross-sectional study

SY Ho ${ }^{1}$, J Chen', LT Leung'1, MP Wang'1, TH Lam

'The University of Hong Kong, Hong Kong, China. E-mail: chenjianjiu@gmail.com

Background: Being able to smoke at home may facilitate adolescent smoking and nicotine dependence. However, this has rarely been studied. We studied the prevalence of smoking at home, its risk factors and potential effect on nicotine dependence in Hong Kong adolescent smokers.

Methods: In a cross-sectional school-based survey in 2014/15, 10932 secondary school students (mean age 14.8 years; $46.8 \%$ boys) indicated whether they smoked at home during 8 periods on weekdays and weekends, analysed as yes (any period) vs no. Also measured were urges to smoke (UTS, range 0-5, 5 =greatest urge) based on the frequencies and strength of smoking urges; cigarettes smoked per day (CPD); past 30-day (current) smoking; and sociodemographic and other smokingrelated characteristics. We investigated smoking at home's (1) risk factors and (2) associations with UTS and CPD (outcomes) in current smokers.

Results: Of 461 current smokers, 50.3\% (95\% CI 45.7-54.9) smoked at home, with "weekend mornings" being the most common (20.6\%). Smoking at home was associated with having 2 or more co-residing smokers (vs none) and home thirdhand smoke exposure for 4-7 days/week (vs 0) with adjusted odds ratios of $1.38(95 \%$ CI $1.09,1.76)$ and 1.66 (1.23, 2.23). Smoking at home was also associated with UTS and CPD with adjusted $\beta$ coefficients of $1.78(1.17,2.39)$ and 2.63 (1.27, 3.99).

Conclusions: About half the Hong Kong adolescent smokers smoked at home. Smoking at home was associated with living with smokers and a home environment with residual tobacco smoke, which probably make adolescent smoking difficult to detect. Smoking at home was also associated with higher nicotine dependence. Whether smoking cessation of family members and home smoking bans can reduce adolescent smoking at home and nicotine dependence should be further studied.

Tob. Induc. Dis. 2018;16(Suppl 1):A956

DOI:10.18332/tid/84334

$12: 30-14: 00$

PS-1192-5 How behavioral norm and social influence affect smoking in young adulthood: 
the experience of Korean young adults

$\mathrm{H} \mathrm{Kang}^{1}$, J Cho ${ }^{1}$, S-I Cho ${ }^{1,2}$, S Yoo ${ }^{1,2}$

'Department of Public Health Science, Graduate School of Public Health, Seoul National University, Seoul, Korea, Republic of, ${ }^{2}$ Institute of Health and Environment, Seoul National University, Seoul, Korea, Republic of. E-mail: syoo@snu.ac.kr

Background: This qualitative study explored the smoking behavior and its socio-environmental contexts of Korean young adults aged 19-30 years, the age group with an upward trend of smoking.

Methods: 8 focus groups with 63 participants in Seoul discussed the meaning, behavior, and experience of smoking and its environment. The groups were formed by the current status of smoking(daily, social, and former), gender, and occupation. Thematic analysis was performed on all focus groups.

Results: The exposure to paternal and peer smoking in childhood lowered resistance and increased access to smoking. It particularly created a belief that smoking is a behavioral option to release stress. Smoking also meant an opportunity to have a break at work, school, and military service among that allows time to be alone or to socialize with other smokers. Drinking alcohol facilitated smoking to get drunk faster or better, and to be part of drinking occasions and members, which increased the amount of smoking. The young adults were sensitive to social atmosphere and thus conscious about their smoking in public places under the current policies. Whilst they supported the policy that separates smoking areas not to harm non-smokers, they wanted their choice to smoke to be respected as well. Those who perceived quitting smoking to be easy tended to think that they might smoke again but then could quit again easily. High accessibility to cigarettes in the community was a challenge for quitting smoking.

Conclusions: Parental smoking, solicitation to smoke among friends and colleagues, and a high availability and accessibility to cigarettes in the community are focal topics for tobacco free generation. Clear and rational explanation of tobacco policy and environmental approaches would facilitate controlling tobacco use of young population. This work was supported by the Research Program funded by the Korea Centers for Disease Control and Prevention(2016P3500300).

Tob. Induc. Dis. 2018;16(Suppl 1):A957

DOI:10.18332/tid/84348

12:30-14:00

PS-1193-5 The association of electronic vapor product use and conventional cigarette smoking with Korean adolescents' oral health

K-S Lee ${ }^{1}, \mathrm{H} \mathrm{Gu}^{2}$

'The Catholic University of Korea, Preventive Medicine, Seoul, Korea, Republic of, ${ }^{2}$ Graduate School of Public Health, The Catholic University of Korea, Seoul, Korea, Republic of. E-mail: leekangs@catholic.ac.kr

Background: Smoking is more harmful to the physical health of adolescents than it is for adults. The demand for studies on electronic cigarettes as a substitute for tobacco has increased, but there is a lack of research on its harmful effects on the oral health. Therefore, we investigated the characteristics of conventional cigarette smoking, electronic cigarette use, and the dual use among adolescents and examined the relationship between oral health factors according to smoking patterns.

Methods: The subjects of this study were 65,528 adolescents who completed a questionnaire of the 12th online survey of the youth health behaviors(2016). The types of smoking were analyzed by conventional cigarette smoking, electronic cigarette use, and nonsmoking. A chi-square test and logistic regression analysis were performed.

Results: There were significant differences in general characteristics such as gender, grade, school type, scholastic performance, location, perceived socioeconomic status, and drinking status according to type of smoking. There were significant difference in the number of brushing days, oral hygiene auxiliary products and treatment, and subjective oral health awareness. There was a significant difference also in oral disease symptoms.

The relationship between type of smoking and oral health was as follows: "Chipped or broken tooth", "Tooth pain when eating", or "Aching or throbbing in tooth", it was significantly higher in dual users and conventional cigarette only smoker, and it was higher in dual users in conventional cigarette only smoker.

Conclusions: It was found that there was a difference in oral health effects according to the type of smoking. Tailored education programs and policies should be developed to promote oral health and prevent smoking in adolescents.

Tob. Induc. Dis. 2018;16(Suppl 1):A958

DOI: $10.18332 /$ tid $/ 84382$

\section{2:30-14:00}

PS-1194-5 Anti-smoking legislation in educational institutions in India: opinion of stakeholders and challenges in implementation

N Sharma ${ }^{1}$, C Kohli ${ }^{2}$, S Dhaked ${ }^{2}$, C Vankhuma ${ }^{2}$, T Anand ${ }^{2}$, MM Singh ${ }^{1}$

'Maulana Azad Medical College, New Delhi, India, ${ }^{2}$ Maulana Azad Medical College, Community Medicine, New Delhi, India. E-mail:kohlicdoc17@gmail.com

Background and challenges to implementation: Tobacco use usually begins in adolescence, and the risk of tobacco use is highest among those who start early and continue its use for a long period. Global Youth Tobacco Survey-2009, revealed that nearly $15 \%$ of youth in India use tobacco. In the wake of international efforts and growing consciousness about harmful effects of tobacco use, the Government of India enacted "The Cigarettes and Other Tobacco Products (Prohibition of Advertisement and Regulation of Trade and Commerce, Production, Supply and Distribution) Act, 2003" (COTPA), in May 2003 with a view to protect public health by prohibiting smoking in public places, banning advertisements of the tobacco products, banning sale of tobacco products to minors and near educational institutions, prescribing strong health warnings including pictorial depiction on tobacco products, and regulation of tar and nicotine contents of tobacco products.

Intervention or response: Delhi government started the "Smoke Free Delhi" initiative in 2009. The rising trend of tobacco smoking among students in the country makes it imperative to provide a smoke-free environment in the educational institutions. The current study was undertaken with the objective to assess the status of implementation of anti-smoking legislation and perception among stakeholders in educational institutions in Delhi.

Results and lessons learnt: Out of the total 596 participants, 125 (21\%) reported to be current smokers. $64.8 \%$ of the participants were aware about the COTPA, but the awareness about the organizations implementing the act was very low, being only 7 (5.6\%) among smokers, and 23 (4.9\%) among nonsmokers. Regarding the awareness of places designated as "Smoke Free 
Zones," majority said educational institutions, followed by airport, shopping malls, and buses/trains. A majority of the smokers (85.6\%) supported ban on smoking at public places.

Conclusions and key recommendations: The level of awareness about antismoking legislation was high and majority support ban on smoking. However, the mechanism of implementation of the law was unclear to most.

Tob. Induc. Dis. 2018;16(Suppl 1):A959

DOI:10.18332/tid/84385

\section{2:30-14:00}

PS-1195-5 Introduce new methods to realize real harms of tobacco among youngsters

AC Abdul Raheem ${ }^{1}$, N Selladurai ${ }^{1}$

${ }^{1}$ Alcohol and Drug Information Center (ADIC), Tamil Media and Plantation Programme, Colombo, Sri Lanka.E-mail: raheem@ adicsrilanka.org

Background and challenges to implementation: Tamil films highly promote tobacco to among youth (ADIC Tamil film research 2015 http://www.nidarshanam.lk/wp-content/uploads/2016/03/ tamil-film-research-change.pdf) .Especially Tamil speaking people highly engage with south Indian Tamil films. Even in the Tamil media they highly conduct prevention programmes but it is also doing show the real harms on tobacco among youth. Youth don't like to participate in the prevention programmes eventhough lot of prevention programmes available in the community. Therefore we thought to introduce a new way to realize the real harms on tobacco use among youngsters.

Intervention or response: Developed the massive campaign as competition target on the Valentine 's Day. Theme is "Why young girls don't like to start relationship with smoker"?, 1st of February 2016 lounged the campaign. Conducted 15 direct campaigns in tuition classes in 02 districts and published 28 free advertisements in 02 national papers. Broadcasted 40 advertisements and telecasted 25 advertisements. Displayed 50 banners in the textiles and other youth related shops. Spread the message through social media. On Valentine "s Day conducted the special morning show as competition in 02 electronic media and the direct competition where youth gather especially on valentine's day.

Results and lessons learnt: Able to get more than 10000 creative answers from youth.

According to the analyzing able to make aware on tobacco's real harm for $65 \%$ of youth in selected location. (Used Lot Quality Assurance sampling method)

Youngsters participation was highly enthusiastic to the competition.

Through the media coverage nearly more than 100000 youngsters were made aware.

Conclusions and key recommendations: If developed creative and youth attractive activity, message of the theme will be delivered automatically among youngsters.

The collaboration also will increase whether developed the suitable activity for the suitable target group.

Tob. Induc. Dis. 2018;16(Suppl 1):A960

DOI:10.18332/tid/84386

$12: 30-14: 00$

PS-1196-5 Tobacco prevention in Greek school community

M Demi ${ }^{1}$, V Evangelopoulou ${ }^{2}$ VK Vyzikidou ${ }^{2}$, D Vogiatzi ${ }^{1}$, A Lyroni', A Tzortzi ${ }^{1,2}$, C Vardavas $^{1}$, P Behrakis ${ }^{1,2,3}$
${ }^{1}$ Institute of Public Health, The American College of Greece, Athens, Greece, ${ }^{2}$ George D. Behrakis Resarch Lab, Hellenic Cancer Society, Athens, Greece, ${ }^{3}$ Biomedical Research Foundation, Academy of Athens, Athens, Greece. E-mail: mdemi@acg.edu Background and challenges to implementation: Tobacco prevention and cessation are two key approaches of tackling the tobacco epidemic. Nearly all first use of tobacco occurs before secondary school graduation; hence if adolescents can be kept away from smoking, most of them will never start it. The school community, though, consists of a range of age groups with different needs and approach requirements.

Intervention or response: Substantial public health efforts in Greece have begun since 2009 to prevent tobacco use focusing especially on youth. An educational campaign, called "SmokeFreeGreece" which emphasizes the importance of following a healthy lifestyle linked to non- smoking one has been developed and implemented in Greece. This campaign includes intervention seminars about tobacco use for school students between 10-17 years old, "train the trainer" workshops for teachers, annual school student conferences called "Education for A Smoke Free World", the production of educational material addressed to both students and teachers and collaboration with Symplefsi, an NGO, that improves the living conditions of the inhabitants of the remote Greek islands. More specifically, three individual interventions were created to address tobacco use in ages $10-12,13-15$ and $16-17$ years old. This methodological framework indicates the importance of creating a "tailor fitted programme".

Results and lessons learnt: The educational seminars offered in specially designed centers have served over 26,000 students and 1,750 teachers. The material has been distributed to date more than 285,000 Greek school students, throughout Greece including visits to 13 remote islands of the Aegean.

Conclusions and key recommendations: The educational campaign has reached a substantial percentage of the Greek school population. As a motivational buffer to continue our efforts, during the years of the campaign, smoking prevalence among 16- 24 year olds has decreased by $33.3 \%$ (Hellenic Statistical Authority, 2016) and we do believe that our activities are related with this reduction.

Tob. Induc. Dis. 2018;16(Suppl 1):A961

DOI:10.18332/tid/84392

12:30-14:00

PS-1197-5 Impact on patterns of tobacco and its initiation among children of conflict area in Jammu and Kashmir (India): a school based study MA Dar ${ }^{1}$, DS Rehman², A Pandey ${ }^{3}$

${ }^{1}$ Government of Jammu and Kashmir, Health Services, Budgam, India, ${ }^{2}$ Government of Jammu and Kashmir, Health Services, Srinagar, India, ${ }^{3}$ International Union Against Tuberculosis and Lung Disease, Tobacco Control, New Delhi, India. E-mail: uberaltaf@yahoo.co.in

Background: School going children are becoming target of Tobacco Industry and is a serious issue in disturbed areas of India particularly Kashmir. The early age of initiation underscores the urgent need to intervene and protect this vulnerable group from falling prey to this addiction. The present study was thus undertaken to assess the prevalence of tobacco habits (Patterns) among school children, determine the age \& major reasons of initiation of these habits, and compare the age of initiation between students who were more than 15 and $\leqslant 18 \mathrm{yr}$ of age.

Methods: Through cluster and random sampling method, 
private and government schools of Kashmir valley (10 Districts) of Jammu and Kashmir were identified for the study. In identified schools a structured questionnaire was administered among students of class 7 to 12 (aged 11-19 yr). The data entry was done in further descriptive and exploratory data analysis was done manually.

Results: It is found that $18.4 \%$ students; 375 (14.1\%) were 'ever smokers (including current smokers), 111(4.1\%) were 'ever tobacco chewers (including current chewers)', (11.94\%) were 'exclusive smokers' and (3.5\%) were 'exclusive tobacco chewers'. 512 (19.3\%) children belong to families affected by unrest and suffered economic loss from last 3 years. The mean age of initiation of these habits was around 10.4 year. Nearly $75 \%$ of boys and $6 \%$ of girls $\varsigma 15 \mathrm{yr}$ initiated the habit of tobacco before the age of $12 \mathrm{yr}$. Conclusions: The study concludes that Tobacco use in Kashmir (State J\&K) among children is higher than the national average, the conflict in the region may be the one of main reason behind it. It also highlight the alarming fact that age initiation among these areas is much lower than the state average. Customized policy intervention is required to control the tobacco use among children in conflict area.

Tob. Induc. Dis. 2018;16(Suppl 1):A962

DOI:10.18332/tid/84396

\section{2:30-14:00}

PS-1198-5 Tobacco - a threat to development in BiH A Sahačić ${ }^{1}$, U Bakh ${ }^{1}$

${ }^{1}$ Association PROI, Sarajevo, Bosnia and Herzegovina. E-mail: asahacic.proi@gmail.com

Background and challenges to implementation: Bosnia and Herzegovina - $\mathrm{BiH}$ is placed on 8th position on World's Smokers List and according to the World Bank, almost half of the adultan smoke and $77,3 \%$ of youth are exposed to secondhand smoke. $\mathrm{BiH}$ is composed of two political entities, RS and $\mathrm{FBiH}$ and in both there are Tobacco control laws, but they are old, with no ban on smoking and with lots of legal gaps. Till the end of 2015 there are no registered law amendments or any new law proposal. There are no CSOs working in this field and there is a lack of general public awareness about the harmful effects of tobacco.

Intervention or response: "Smeta Mi "campaign was initiated in 2016 by the Association PROI with the aim to ensure protection of human rights. "Smeta Mi“" is based on innovative strategies such as social media and creative ways such as letters from children to their parents demanding their right to clean air, photo campaign of support for the new Law, innovative work methodology "Unplugged" used for the first timein $\mathrm{BiH}$.

Results and lessons learnt: "Smeta Mi" is the most famous public campaign in $\mathrm{BiH}$, reached 3.531 .159 persons particularly members of vulnerable groups, has more than 479 media coverage, 16,663 followers on facebook, 16 campaign spokespersons and brought together 60 organizations, trained them as tobacco control activists and founded Smoke Free Partnership.

PROI signed a Memorandum of Cooperation with the Ministry of Health, set up a working group that completed draft of the new tobacco law harmonized with FCTC and the EU Directive. At the moment the law is in the parliamentary procedure.

Conclusions and key recommendations: "Smeta $\mathrm{Mi}$ " is a successful campaign that can give a positive example of a lowincome country in which one organization raised the awareness of citizens about tobacco and will stand behind the changes of legal framework.

Tob. Induc. Dis. 2018;16(Suppl 1):A963

\section{DOI:10.18332/tid/84409}

12:30-14:00

PS-1200-5 Socio-economic status as predictors of initiation and persistence of youth smoking in Mizoram

\section{Chhakchhuak ${ }^{1}$, L Hriatpuii ${ }^{2}$, L Chhakchhuak ${ }^{3}$}

${ }^{1}$ Government of Mizoram, Pediatrics, Directorate of Health Services, Aizawl, India, ${ }^{2}$ Mlizoram University, Law, Aizawl, India, ${ }^{3}$ Tripura University, Psychology, Agartala, India. E-mail: arini1910@gmail.com

Background: Mizoram have the highest tobacco prevalence in India with half of the population using some forms of tobacco. GATS 2009-2010 report 67.2\% of people in Mizoram use tobacco. In this century, nations have experienced an epidemic of diseases that are caused, either primarily or partly, by tobacco use. The success of tobacco control lies in prevention and cessation of tobacco use and identifying predictors have continued to prove most essential in countering initiation or maintenance. Socioeconomic status have been found to play a major role in tobacco use. The aim of the study is to identify and compare predictors of adolescent smoking initiation and persistence among High, Middle and Low socio-economic status adolescents.

Methods: 300 adolescents were randomly sampled from Aizawl. Family, youth, peer and sociodemographic risk and protective factors were analyzed using SPSS package.

Results: Low SES adolescents reported the highest rates of smoking initiation and persistence; Middle SES adolescents scored the lowest. Multivariate analyses revealed mostly common and few SES-specific predictors of smoking initiation and persistence. For initiation, maternal current smoking, child age, child problem behavior, and perceived peer pressure to smoke have greatest prediction in low SES groups followed by middle and high SES group; ineffective parenting were predictive among all SES groups. For persistence, child age, child problem behavior and perceived scholastic competence were predictive across all SES groups as well.

Conclusions: More common than unique factors predict smoking initiation and persistence among adolescents of different socio-economic status. Social factors are more important for smoking initiation, whereas individual factors are more important for persistence, although child problem behaviors are common determinants both of initiation and persistence. With few exceptions, universal anti-smoking interventions should be targeted to youths of different socioeconomic backgrounds. Intervention programs should target the social environment of the youth in order to have the most profound effect.

Tob. Induc. Dis. 2018;16(Suppl 1):A964 DOI:10.18332/tid/84461

\section{2:30-14:00}

PS-1201-5 Three year school based intervention for Tobacco prevention in the region of Sousse: quasi experimental design 2009-2014

F Ouni', S Bhiri', J Maatoug', R Ghammam ${ }^{1}$, J Sahli', H Ghannem ${ }^{1}$ 'University Hospital Farhat Hached, Epidemiology Department, Sousse, Tunisia.E-mail: ferdaousradaoui@gmail.com

Background: Tobacco use is one of the biggest public health threats the world has ever faced. Tunisia is one of the countries suffering from the heavy burden of tobacco use. It is in this context that the Chronic Disease Prevention Research Center 
in Sousse established a school based intervention for tobacco control and prevention. Our objective was to evaluate the feasibility and effectiveness of our intervention in tobacco control and prevention by the evaluation of smoking behavior among schoolchildren at pre and post assessment in both intervention and control groups.

Methods: We conducted a three years school based quasiexperimental study with an intervention and a control groups. We enrolled a representative sample of schoolchildren from all colleges of intervention and control areas. We have carried out a pre-assessment before the intervention in 2009-2010 and a post assessment of the same parameters at the end of the intervention in both groups in 2013-2014. We used a pre tested self administered questionnaire to assess tobacco use.

Results: Overall, 4003 schoolchildren were included: 1929 in the intervention group and 2074 in the control group. After the intervention, 4275 schoolchildren participated in the evaluation with respectively 2170 and 2105 in each group. The age of the schoolchildren varied between 11 and 16 years. There was a decrease in tobacco use in the intervention group from $5.7 \%$ to $4.8 \%$ contrary to an increase in the control group from $7.5 \%$ to $9.2 \%$. Among boys, the prevalence of tobacco use decreased in both groups. However, among girls, in the intervention group a decrease was noticed in the contrary to the control group where a significant increase was observed from $1.1 \%$ to $5.2 \%$.

Conclusions: Tobacco prevention needs multi-sectored approach and an extended intervention to be effective. That's why a political will is important for such interventions.

Tob. Induc. Dis. 2018;16(Suppl 1):A965

DOI:10.18332/tid/84482

\section{2:30-14:00}

\section{PS-1202-5 Dare or dream: helping young people} of Delhi slums to stop using tobacco

\section{Sahu ${ }^{1}$}

IIndependent Consultant, Research, Bilaspur, India. E-mail: skdevesh@gmail.com

Background: Tobacco consumption is a risk factor for various diseases and leads to the highest number of avoidable deaths worldwide. India is home to the most adolescents in the world. Out of these, $60-80 \%$ live in low resource settings. The onset of tobacco use occurs in adolescence and Youth of lower socio-economic status use tobacco at rates twice as high as youth of higher socioeconomic status. There is a need for low-cost, community-based interventions for socio-economically disadvantaged young people living in urban slums in India.

Methods: A quasi-experimental study design with two measurements at baseline and 6 months post-intervention to investigate an intervention in 10-15-year-olds. The intervention programme consists of five 60 -min community based interactive modules and control groups. The study questionnaire measuring cigarette smoking and consumers, smoking-related cognitions, and gender, social and cultural aspects was designed and pretested in advance. The primary end point was the prevalence of smokers and consumers in the two study arms at 6 months after the intervention. The percentage of smokers and consumers in the two groups was studied.

Results: All study participants used tobacco related products, out of which $41.2 \%$ indulged in smoking alone. Availability, cost, family and peer influence plays a central role in initiating tobacco use. The primary reasons for tobacco initiation and maintenance were identified as social influence, stress, boredom and misconception that tobacco is good for health. $63 \%$ of the study participants successful stopped using tobacco along with significant reduction in frequency or quantity of tobacco observed in all the study participants. The barriers to quitting were identified as stress, violence and abuse.

Conclusions: Young people living in urban slum are vulnerable to variety of risks including use of tobacco. Helping them learning and practicing life skills can better prepare them staying away from risks related to use of tobacco.

Tob. Induc. Dis. 2018;16(Suppl 1):A966

DOI:10.18332/tid/84521

\section{2:30-14:00}

PS-1203-5 Youth response to pictorial warning on tobacco products in Chandigarh India

A Rao $^{1}, \mathrm{~S} \mathrm{Goel}^{1}$

${ }^{1}$ PGIMER, School of Public Health, Chandigarh, India. E-mail: achika_rao@yahoo.com

Background: Pictorial warnings are among the most prominent and cost-effective health communications available.There are substantial literature that pictorial warning are effective among older tobacco user, who have experienced symptoms of illness but does not have preventive effect on youth who reportedly see the messages as lacking relevance and realism .Use of tobacco product is prevalent in all ages but youth have been particularly vulnerable and tobacco use among them means exposure will be longer with subsequent high adverse consequences. Prevalence of tobacco use in Chandigarh among youth (15-24 yrs) is $20 \%$. Therefore knowledge of pictorial warnings among youth becomes essential to monitor and assess the effectiveness of tobacco controls efforts. Adolescents and young adults are particularly vulnerable to influences of use tobacco use.

Methods: A cross sectional survey was conducted among 1000 students A set of six pictorial warnings were shown to students and they were asked to score between 1(low) to 5 (high) for seven indicators (accuracy, noticeable, scary,informative, reduce tobacco consumption, helps to quit and stop initiation). The mean of set of six pictures were measured, for each of 7 indicators

Results: Among all six pictures, Picture 4 (Face cancer)and Picture 6 (mouth cancer)were considered most accurate by study population. Picture 2 (cancer of mouth in young boy) was perceived as most noticeable, while Picture 6(mouth cancer) was most scary. Overall Picture 4 (Mouth Cancer) was perceived most informative, helps to reduce tobacco consumption, helps to quit and stop initiation of tobacco use. A majority of participants reported that pictorial warning on tobacco product is scary

Conclusions: This study concludes that pictorial warning have significant effect on tobacco prevalence and quit attempts in Chandigarh,India .Pictorial warning arouse fear feelings among participants. More the scarier the pictorial warming more will be impact on youth in quit intentions

Tob. Induc. Dis. 2018;16(Suppl 1):A967

DOI: $10.18332 /$ tid $/ 84570$

\section{2:30-14:00}

PS-1205-5 Second hand smoking among schoolchildren in the region of Sousse

F Ouni ${ }^{1}$, J Maatoug ${ }^{1}$, S Bhiri ${ }^{1}$, G Rim ${ }^{1}$, Z Nawel ${ }^{1}$, H Ghannem ${ }^{1}$ 'University Hospital Farhat Hached, Epidemiology Department, Sousse,Tunisia.E-mail: ferdaousradaoui@gmail.com

Background: Secondhand smoke (SHS) contains an estimated 4000 toxic chemicals and is a serious health hazard to non-smokers. 
Exposure to tobacco smoke causes extremely serious damages, placing exposed persons at increased risk of a number of adverse health outcomes, such as asthma, lung cancer and cardiovascular diseases. In this context, this work was carried out in order to evaluate exposure to smoking among schoolchildren in the region of Sousse.

Methods: We conducted a cross sectional design study among a representative sample of students from all colleges in the delegation of Sousse Jawhra and Sousse Erriadh. We used an Arabic, pre tested and self administered questionnaire to collect data about Socio -demographic characteristics and student's smoking behavior.

Results: Our population was composed of 2170 students. Girls accounted for 1100 (51.3\%) and 1070 (48.7\%) boys. The mean age of our population was $13.23 \pm(1,19)$. The prevalence of smoking in our population was $4.8 \%$.

In the month preceding our study $75 \%$ of our population was exposed to a second-hand smoking. $36.7 \%$ was exposed at home and almost one third (27.3\%) at school. But second hand smoking was affecting smoking and non smoking students. We noticed that $74.5 \%$ of non smoker were exposed to secondhand smoking despite the that the majority $(92.0 \%)$ knew that second hand smoking is dangerous. We highlighted also that non-smokers were significantly more in favor of implementing tobacco control laws $(94.6 \%$ vs $94 \%$ of smokers ; $\mathrm{p}=0.02)$.

Conclusions: Exposure to an involuntary smoke is an urgent issue, because there is no safe level of SHS exposure, even minimal exposure is harmful. And as we noticed the Second hand smoking is very high among students in the Sousse region. Therefore there is an urgent need for a program to prevent and control this scourge of active and passive smoking among schoolchildren.

Tob. Induc. Dis. 2018;16(Suppl 1):A968

DOI:10.18332/tid/84626

12:30-14:00

PS-1206-5 Waterpipe (Shisha) smoking: the experience and harm perception as narrated by University of Ibadan Undergraduate Students

A Faloye $^{1}, 0$ Akinyemi ${ }^{1}$, F Adeniji $^{1}$, H Shuaibu ${ }^{2}$

${ }^{1}$ University of Ibadan, Department of Health Policy and Management, Ibadan, Nigeria, ${ }^{2}$ University of Ibadan, Department of Community Medicine, Ibadan, Nigeria.E-mail:nubcy345@ yahoo.com

Background: According to World Health Organisation (WHO) tobacco kills up to half of its users and this is about 6 million people yearly. More so, the global rise in tobacco smoking using a waterpipe (Hookah, Narghile, Shisha) among young people, has made understanding its trend, harm perception and pattern of use among young people imperative.

Methods: A qualitative study using non-probability sampling technique was conducted among University of Ibadan undergraduate students residing in the halls of residence. This study being a part of a larger study made use of the first detected shisha smoker as the index respondent. Through a snowballing technique other thirteen (13) WP smokers were identified and voluntarily participated in In-depth Interviews and Focused Group Discussions according to their gender. Data was analysed using NVIVO version 8. In total, two (2) In-depth Interviews (IDI) were conducted among a male and a female while a Focused Group Discussion (FGD) was conducted among six (6) males and six (6) females.

Results: The study revealed that WPS practice was practiced among both male and female undergraduate students, and was perceived as less harmful than cigarette smoking. Also established through the study was that friend's house parties and clubs served as the place of initiation for most respondents, and there was a wide availability of WP at most clubs and lounges around the metropolis. WPS was described as more pleasant than cigarette smoking, especially among female WP smokers. Furthermore, majority of study respondents smoked WP with no knowledge of its preparation process.

Conclusions: The practice of WPS among University of Ibadan undergraduate students was established. Hence, more research should be conducted among young people in Nigeria to ascertain the epidemiological trend of this new tobacco consumption behaviour among them. The National Tobacco Acts should be amended to harbour specific WPS laws and be better enforced.

Tob. Induc. Dis. 2018;16(Suppl 1):A969

DOI:10.18332/tid/84671 